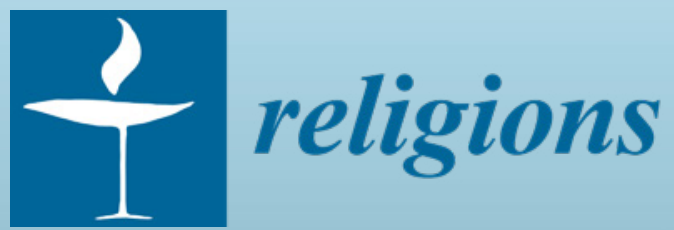

\title{
Domestic
}

Devotions in

Medieval and

Early Mor

Edited by

Salvador Ryan

Printed Edition of the Special Issue Published in Religions

www.mdpi.com/journal/religions

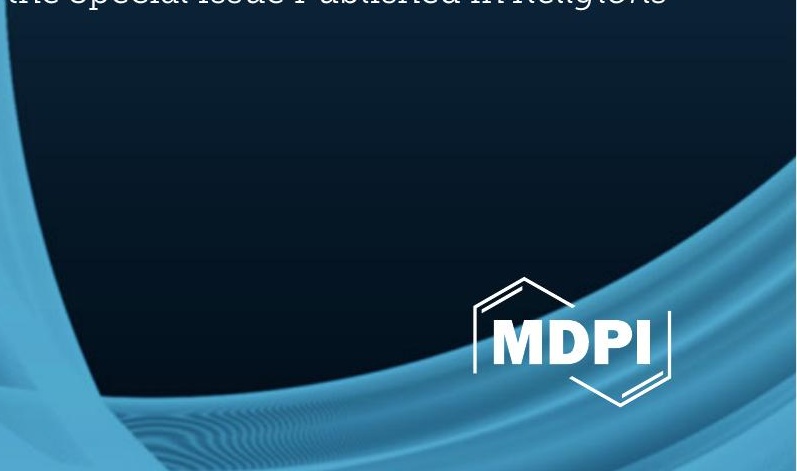


Domestic Devotions in Medieval and Early Modern Europe 



\section{Domestic Devotions in Medieval and Early Modern Europe}

Special Issue Editor

Salvador Ryan 
Special Issue Editor

Salvador Ryan

Professor of Ecclesiastical

History, Pontifical University,

St Patrick's College

Ireland

Editorial Office

MDPI

St. Alban-Anlage 66

4052 Basel, Switzerland

This is a reprint of articles from the Special Issue published online in the open access journal Religions (ISSN 2077-1444) from 2019 to 2020 (available at: https:/ / www.mdpi.com/journal/religions/special_ issues/devotion).

For citation purposes, cite each article independently as indicated on the article page online and as indicated below:

LastName, A.A.; LastName, B.B.; LastName, C.C. Article Title. Journal Name Year, Article Number, Page Range.

ISBN 978-3-03928-913-4 (Pbk)

ISBN 978-3-03928-914-1 (PDF)

(c) 2020 by the authors. Articles in this book are Open Access and distributed under the Creative Commons Attribution (CC BY) license, which allows users to download, copy and build upon published articles, as long as the author and publisher are properly credited, which ensures maximum dissemination and a wider impact of our publications.

The book as a whole is distributed by MDPI under the terms and conditions of the Creative Commons license CC BY-NC-ND. 


\section{Contents}

About the Special Issue Editor $\ldots \ldots \ldots \ldots \ldots \ldots \ldots \ldots$ vii

\section{Salvador Ryan}

Introduction to the Special Issue of Religions-“Domestic Devotions in Medieval and Early Modern Europe"

Reprinted from: Religions 2020, 11, 154, doi:10.3390/rel11040154 _ . . . . . . . . . . . . . 1

\section{Albert Kohn}

Furnishing Piety: Beds in High Medieval Jewish Domestic Devotion

Reprinted from: Religions $\mathbf{2 0 1 9}$, 10, 471, doi:10.3390/rel10080471 . . . . . . . . . . . . . . 7

\section{Ragnhild M. Bø}

Sculptures and Accessories: Domestic Piety in the Norwegian Parish around 1300

Reprinted from: Religions 2019, 10, 640, doi:10.3390/rel10110640 . . . . . . . . . . . . . . .

\section{Juliana Dresvina}

What Julian Saw: The Embodied Showings and the Items for Private Devotion

Reprinted from: Religions 2019, 10, 245, doi:10.3390/rel10040245 . . . . . . . . . . . . . 38

\section{Matko Matija Marušić}

Hereditary Ecclesiae and Domestic Ecclesiolae in Medieval Ragusa (Dubrovnik)

Reprinted from: Religions 2020, 11, 7, doi:10.3390/rel11010007 . . . . . . . . . . . . . 58

\section{Marta Crispí}

The Use of Devotional Objects in Catalan Homes during the Late Middle Ages

Reprinted from: Religions 2020, 11, 12, doi:10.3390/rel11010012 . . . . . . . . . . . . . . . 72

\section{Karen Ralph}

Performance, Object, and Private Devotion: The Illumination of Thomas Butler's Books of Hours

Reprinted from: Religions 2020, 11, 20, doi:10.3390/rel11010020 . . . . . . . . . . . . . . . 105

\section{Anna Dlabačová}

Printed Pages, Perfect Souls? Ideals and Instructions for the Devout Home in the First Books

Printed in Dutch

Reprinted from: Religions 2020, 11, 45, doi:10.3390/rel11010045 . . . . . . . . . . . . . . . . 131

Catherine Lawless

'Make Your House like a Temple': Gender, Space and Domestic Devotion in Medieval Florence

Reprinted from: Religions 2020, 11, 120, doi:10.3390/rel11030120 . . . . . . . . . . . . . 152

\section{Sarah Blick}

Bringing Pilgrimage Home: The Production, Iconography, and Domestic Use of Late-Medieval Devotional Objects by Ordinary People

Reprinted from: Religions 2019, 10, 392, doi:10.3390/rel10060392 . . . . . . . . . . . . . . . 173

\section{Margarita Voulgaropoulou}

From Domestic Devotion to the Church Altar: Venerating Icons in the Late Medieval and Early Modern Adriatic

Reprinted from: Religions 2019, 10, 390, doi:10.3390/rel10060390 . . . . . . . . . . . . . . . 199 


\section{Kaja Merete Haug Hagen}

Crux Christi Sit Mecum: Devotion to the Apotropaic Cross

Reprinted from: Religions 2019, 10,603, doi:10.3390/rel10110603 . . . . . . . . . . . . . . . . . . 240

\section{Zuzanna Sarnecka}

Experiencing La Verna at Home: Italian Sixteenth-Century Maiolica Sanctuaries and Chapels Reprinted from: Religions 2020, 11,6, doi:10.3390/rel11010006 . . . . . . . . . . . . . . . . . 272

\section{Grażyna Jurkowlaniec}

Masterpieces, Altarpieces, and Devotional Prints: Close and Distant Encounters with Michelangelo's Vatican Pietà

Reprinted from: Religions 2019, 10,309, doi:10.3390/rel10050309 . . . . . . . . . . . . . . . . . 293

\section{Milena Ulčar}

"Equal Rites": Fragmenting and Healing Bodies in the Early Modern Bay of Kotor Reprinted from: Religions 2019, 10,606, doi:10.3390/rel10110606 . . . . . . . . . . . . . . . . . 313

\section{Torsten Wollina}

Devotional Annotations: Preserving the Family's Memory in Arabic Manuscripts

Reprinted from: Religions 2019, 10,376, doi:10.3390/rel10060376

\section{Jonathan Parkes Allen}

Sanctifying Domestic Space and Domesticating Sacred Space: Reading Ziyāra and Taṣliya in Light of the Domestic in the Early Modern Ottoman World

Reprinted from: Religions 2020, 11,59, doi:10.3390/rel11020059 . . . . . . . . . . . . . . . . . . 346

\section{Jantina Ellens}

Devotions in the Ancient Way of Offices: Medieval Domestic Devotion in the Seventeenth Century Reprinted from: Religions 2019, 10,546, doi:10.3390/rel10100546 . . . . . . . . . . . . . . . 382

\section{Terese Zachrisson}

The Saint in the Woods: Semi-Domestic Shrines in Rural Sweden, c. 1500-1800

Reprinted from: Religions 2019, 10,386, doi:10.3390/rel10060386 . . . . . . . . . . . . . . . . 395

\section{Aleksandra Sulikowska-Bełczowska}

The Cult of Old Believers' Domestic Icons and the Beginning of Old Belief in Russia in the 17th-18th Centuries Reprinted from: Religions 2019, 10,574, doi:10.3390/rel10100574 . . . . . . . . . . . . . . . . . . 410

\section{Valeria Viola}

Devotion, Paintings, and the House: The Collections of Ercole and Giuseppe Branciforti, Princes of Scordia (Palermo, 1687-1720)

Reprinted from: Religions 2020, 11, 39, doi:10.3390/rel11010039 . . . . . . . . . . . . . . . . . . . 427 


\section{About the Special Issue Editor}

Salvador Ryan (Professor of Ecclesiastical History) teaches the History of Christianity at the Pontifical University, St Patrick's College, Maynooth, where he writes and researches in the area of late medieval and early modern popular religion and ritual. He is Review Editor of Irish Theological Quarterly and a member of the Editorial Boards of British Catholic History, The Journal of Baroque Studies and Archivium Hibernicum. He has edited/co-edited thirteen books and has authored numerous journal articles and book chapters on the subject of popular belief. Among his recent publications are The Materiality of Devotion in Late Medieval Northern Europe: Images, Objects and Practices (Four Courts Press, 2016); Death and the Irish: A Miscellany (Wordwell Press, 2016); Remembering the Reformation: Martin Luther and Catholic Theology (Fortress Press, 2017); The Cultural Reception of the Bible: Explorations in Theology, Literature and the Arts (Four Courts Press, 2018); Marriage and the Irish: A Miscellany (Wordwell Press, 2019). He is currently co-editing (with Henning Laugerud and James E. Kelly) Northern European Reformations: Transnational Perspectives for Palgrave. 

Editorial

\title{
Introduction to the Special Issue of Religions-“Domestic Devotions in Medieval and Early Modern Europe"
}

\author{
Salvador Ryan \\ Faculty of Theology, Pontifical University, St Patrick's College, Maynooth, County Kildare, W23 F2H6, Ireland; \\ Salvador.Ryan@spcm.ie
}

Received: 22 March 2020; Accepted: 24 March 2020; Published: 27 March 2020

This Special Issue of Religions focuses on lived religion and devotional practices as found in the domestic settings of late medieval and early modern Europe. More particularly, it investigates to what degree the experience of personal or familial religious practice in the domestic realm and the more public expression of faith in liturgical or communal settings intersected.

When I first chose this theme, my aim was to build on the significant research that has been undertaken in recent years on domestic devotion in the early modern period, most notably the volumes produced by the European Research Council funded interdisciplinary project Domestic Devotions: The Place of Piety in the Italian Renaissance Home (see, for example Brundin et al. 2018; Corry et al. 2017; Corry et al. 2018; and Faini and Meneghin 2018), but also in other studies such as Martin and Ryrie (2012). More broadly, in 2012-2013, the Ecclesiastical History Society chose for its 50th volume of Studies in Church History the theme Religion and the Household, which contains, among others, at least twelve contributions on the early modern period (Doran et al. 2014).

The specific topic of medieval domestic devotion has been slower to generate significant treatments such as those mentioned above, although there have been fine edited collections such as Spicer and Hamilton (2005), which contain a number of medieval essays, and helpful article contributions by Kolpacoff Deane (2013) and others. This collection aims to respond, in part, to the final section of that article, which sets out some directions for future research. Therefore, in its call for contributions, it especially welcomed contributors who wished to consider the relationship between domestic religious practice across medieval Christianity, Judaism and Islam, or to focus in particular on any one of the three faiths. Elisheva Baumgarten's Practicing Piety in Medieval Ashkenaz (Baumgarten 2014) and Megan H. Reid's Law and Piety in Medieval Islam (Reid 2013) serve as excellent exemplars of this kind of study. While welcoming contributions on early modern domestic devotion, it was my hope that a number of the essays gathered in this collection would shed much-needed light on this topic within the broad field of medieval studies. One of my other hopes for this collection was that it would contribute towards a broadening of the geographical range of enquiry: thus, while welcoming contributors writing on Western Europe, articles which examined aspects of domestic devotion in Central and Eastern Europe were especially encouraged. ${ }^{1}$

The response to the call for contributions was very heartening, with a final number of twenty articles published. I am very happy with the geographical spread of contributions, and particularly pleased to see a fine representation of articles from Slavic language regions, whose complementary scholarship in this area is much needed in English language publications. I am also delighted to

1 On this point, I am looking forward to the publication of important forthcoming studies such as that of Suzanna Ivanič whose monograph, The Materiality of Belief: the Spiritual World of Early Modern Prague, which will be published by Oxford University Press. Ivanič has already contributed important essays such as "Amulets and the Material Interface of Beliefs in Seventeenth-Century Prague Burgher Homes" in Domestic Devotions in the Early Modern World (Faini and Meneghin 2018). 
publish two essays on aspects of domestic devotion within Islam during the Ottoman period, and also an essay on domestic practice within medieval Judaism.

\section{The Essays}

Albert Kohn uses Hebrew texts from thirteenth-century Southern Germany, primarily Sefer Hasidim, to further analyse the role of beds in shaping medieval domestic devotion and to show how Jewish notions about the social, moral, and sexual significance of the bed also reflect those identified in late medieval Christian culture. There were certain practical considerations to be observed when reciting the shema, for instance, if sharing a bed with another. Also, the mixing of the sacred (a holy text, for example) with the profane bed could only occur if the bed had been newly laundered, to ensure that its sheets had not become polluted by bodily fluids.

Acknowledging that there is very little documentary and/or physical evidence of sensorial engagements with religious artefacts, such as kissing, licking, or stroking, in medieval Norway, Ragnhild M. Bø turns her attention to two church inventories for the parish churches in Hålandsdalen (1306) and Ylmheim (1321/1323). These offer a glimpse of what may have been a semi-domestic devotional practice related to sculpture, namely the embellishing of wooden sculptures in parish churches with silver bracelets and silver brooches. Her aim is to use a few preserved objects and archaeological finds as well as medieval sermons, homiletic texts and events recorded in Old Norse sagas, to tease out the significance of the silver items mentioned in the two inventories by exploring the interfaces between devotional acts, decorative needs, and possibly gendered experiences, as well as object itineraries between the domestic and the religious space.

Juliana Dresvina's article seeks to trace the visual sources of the English mystic Julian of Norwich's (1343 and 1416) Revelations or Showings, and suggests that many of them come from familiar everyday devotional objects such as Psalters, Books of Hours, or rosary beads. It approaches Julian's text from the perspective of neuromedievalism, combining more familiar textual analysis with some recent findings in clinical psychology and neuroscience.

Matko Matija Marušić explores domestic devotional practices in Ragusa (modern-day Dubrovnik) from the late thirteenth through the fourteenth and fifteenth centuries. Considering that important advancements in the understanding of domestic devotions in major Mediterranean cities have recently been made-particularly in Venice-the scrutiny of Ragusan sources enables further reflections on the same phenomena in minor Adriatic centres. Given the paucity of preserved objects, and the fact that no late medieval domestic space of that time has survived in Dubrovnik, he turns to archival sources to answer questions pertaining to the arrangements and uses of spaces of domestic worship. Three aspects are analysed in his essay: privately owned chapels-adjoined to the dwellings of urban nobility - prayer areas and holy images inside the houses, and relics in the possession of individuals.

Marta Crispí examines domestic devotion in Catalonia in the thirteenth, fourteenth and fifteenth centuries, based on the information provided by numerous post-mortem inventories and texts written by coetaneous spiritual authors such as Ramon Llull, Francesc Eiximenis and Saint Vincent Ferrer. Among the objects recorded in the inventories, pieces of furniture and devotional objects laypeople and clergymen used in their pious practices as "material" aid for personal prayer stand out. They were in keeping with the strong visual culture that pervaded the later Middle Ages. There were retables, oratories and images of religious themes. However, the inventories also listed lesser known but equally recurring objects such as paternosters and Agni Dei. Painted cloths depicting religious scenes that decorated the homes of numerous wealthy Catalan-Aragonese families at that time were also present. Spiritual books such as books of hours and psalters, biblical texts, Legenda Aurea, etc., were mentioned as well. They were part of the incipient libraries of the laity in the Late Middle Ages.

Karen Ralph considers the major cycles of illumination in two Books of Hours belonging to Thomas Butler, seventh Earl of Ormond (c. 1424-1515). The article concludes that the iconography of the two manuscripts reflects the personal and familial piety of the patron and was designed to act as a tool in the practice of devotion. A prominent figure in English and French public life with an Irish heritage, the products of his patronage were influenced by the various facets of his world; marked, as 
they are, by English prayers, French designs, Irish saints, borrowed folia, and some individual artistic flair. Thomas Butler's Books of Hours were performative objects, family records, "archives of prayer" and material aids to devotion.

Anna Dlabačová studies the role of the earliest books printed in the Dutch vernacular in the religious practice of lay individuals and the devout home. Many of the texts disseminated in these early printed books have received little attention and scholars have tended to view them within the sphere of the Devotia Moderna, even though often there is no direct link to this religious reform movement. This article attempts to show that the first books printed in Dutch offer an interesting lens through which to study domestic devotion in the Low Countries in the last decades of the fifteenth century. It argues that these books bridged the gap between catechetical instruction and the private home, literally bringing home many of the ideals and instructions that the clergy would have offered in church and thus increasingly 'textualizing' the lives of the late medieval laity.

Taking late medieval Florence as her focus of study, Catherine Lawless discusses domestic devotions by framing them in terms of devotions carried out in the home, defined by its opposition to ecclesiastical, consecrated space. It examines how women, considered the laity par excellence through their inability to ever attain sacerdotal authority, were advised spiritually by mendicant friars on how to lead a Christian life according to their status as wives, widows or virgins. It looks at the devotional literature that was widespread in mercantile homes and the devotional images designed to move the soul. This discussion attempts to show the tensions between ecclesiastical and domestic spaces-between the clergy and the laity, and between the corporeal and spiritual worlds of late medieval devotion. It argues that, despite clerical unease with the female and domestic space, the importance accorded to female piety by the mendicant orders at the close of the Middle Ages was such that women were entrusted with key educational roles in the family, even leading to the astonishing affirmation of them as 'preachers' within the borders of their households.

Sarah Blick uses material culture to examine late medieval domestic devotional practices. These survivals ranged from very fine examples to their low-quality, mass-produced counterparts, demonstrating that similar devotional interests and practices spanned all social strata. They included pilgrim badges; amulets inscribed with sacred names or prayers; three-dimensional figures; tiny folding pewter triptychs or diptychs; lockets, chains and cylinders; and even bells, whistles and rattles which could acquire a quasi-devotional function.

Margarita Voulgaropoulou's contribution, on icons in the late medieval and early modern Adriatic, examines how icon veneration became deeply rooted in the Catholic societies of the broad Adriatic region, and particularly in domestic households. However, some important crossovers can be identified, especially in instances where these household icons were donated to churches as votive offerings and therefore entered another devotional space as a result, becoming focal points of more public devotion.

Kaja Merete Haug Hagen examines a late medieval paper amulet containing prayers to St Dorothy and the Holy Cross which was found under the floorboards in a demolished part of a medieval wooden stave church in Torpo, Norway. From the perspective of materiality and sensory-based religious practices, this article explores the connection between the textual amulet found in Torpo and its relation to the now-lost large wooden cross in Torpo church, and to crosses believed to be wonderworking or miraculous in its proximity. The amulet may have been placed in the church, but this was not its original home. Here, once again, we have a connection between what may have been a domestically possessed religious item and its final destination, which was a formal ecclesiastical space.

Zuzanna Sarnecka turns her attention to the function of small-scale maiolica sanctuaries and chapels created in Italy in the sixteenth century. The so-called eremi encouraged a multisensory engagement of the faithful with complex structures that included receptacles for holy water, openings for the burning of incense, and moveable parts. They depicted a saint contemplating a crucifix or a book in a landscape and, as such, they provided a model for everyday pious life. The reduced scale made the objects portable and stimulated a more immediate pious experience. 
Focusing on the response to the Vatican Pietà and perversely using as a point of departure a 1549 remark on Michelangelo as an "inventor of filth", Grażyna Jurkowlaniec aims to present Michelangelo as an involuntary inventor of devotional images. The article explores hitherto unconsidered aspects of the reception of the Vatican Pieta from the mid-sixteenth into the early seventeenth century. The material includes mediocre anonymous woodcuts, and elaborate engravings and etchings by renowned masters: Giulio Bonasone, Cornelis Cort, Jacques Callot and Lucas Kilian. A complex chain of relationships is traced among various works, some referring directly to the Vatican Pietà, some indirectly; none were designed or perceived as reproductions of it, but were conceived as illustrations of the Syriac translation of the New Testament, of Latin and German editions of Peter Canisius's Little catechism, of the frontispiece of the Règlement et établissement de la Compagnie des Pénitents blancs de la Ville de Nancy-but, above all, they were widespread as single-leaf popular devotional images.

Milena Ulčar, focusing on the early modern Bay of Kotor, in modern-day Montenegro, explores how the use of illegally acquired body parts (through grave exhumation, for example) for healing in the domestic sphere mirrored the function and use of official saints' relics in more controlled ecclesiastical settings. By borrowing and adopting iconographic and ritual elements from each other, the church authorities and common people participated in a shared devotional world in which the fragmented body and its creative (mis)use possessed healing powers.

Examining Arabic texts of the Ottoman period, Torsten Wollina shows how appropriating the margins and blank spaces for notes of a sacred or revered text with one's own domestic or family life, any 'academic' or 'religious' manuscript could be turned into an object of domestic devotion. Placing notes - and thus the names of dear ones - in close proximity to revered texts and the handwriting of esteemed individuals was, in itself, an aim of a devotional practice.

Shrine visitation (ziyāra) and devotion to Muhammad (such as expressed in tașliya, the uttering of invocations upon the Prophet), both expressed through a range of ritualized practices and material objects, were at the heart of everyday Islam for the vast majority of early modern Ottoman Muslims across the empire. While both bodies of practice had communal and domestic aspects, Jonathan Parkes Allen focuses on the important intersections of the domestic with both shrine-visitation and Muhammad-centered devotion as visible in the early modern Ottoman lands, with a primary emphasis on the eighteenth century. While saints' shrines were communal and "public" in nature, a range of attitudes and practices associated with them, recoverable through surviving physical evidence, travel literature, and hagiography, reveal their construction as domestic spaces of a different sort, appearing to pious visitors as the "home" of the entombed saint through such routes as wall-writing, gender-mixing, and dream encounters. Devotion to Muhammad, on the other hand, while having many communal manifestations, was also deeply rooted in the domestic space of the household, in both prescription and practice. Through an examination of commentary literature, hagiography, imagery and objects of devotion, particularly in the context of the famed manual of devotion Dalä'il al-khayrät, Allen demonstrates the transformative effect of such devotion upon domestic space and the ways in which domestic contexts were linked to the wider early modern world, Ottoman, and beyond.

Jantina Ellens demonstrates the malleability of some textual aids to domestic devotion. She focuses her attention on Devotions in the Ancient Way of Offices, a Catholic primer first published in 1668 , which highlighted the continual usefulness of medieval devotional practice, but which was subsequently adapted for use by late seventeenth and early eighteenth century English Protestants. This article demonstrates how attempts to reclaim early church faith practices through the recuperation of medieval devotional methods was a significant feature of seventeenth century confessional debates.

Terese Zachrisson, in an article on seventeenth century Sweden, notes how it is a common saying in parts of rural Sweden, when discussing someone lacking in piety, that they went to neither church nor cross. This saying reflects the practice of placing shrines in the fields, along the roads and in the woods as a communal, semi-domestic site that complemented official church space. In the remote woodland areas of Sweden, the distance between parish churches could be considerable, and many parishioners were not able to attend church on a regular, weekly basis. Here, then, parishioners could kneel and 
make their prayers when unable to attend church service. These sites could also be used as points of contact when communicating with the divine about domestic issues. For instance, votive offerings were often left at the shrines by those hoping for deliverance from disease and difficult childbirths. Zachrisson's article positions itself at the interface between domestic and ecclesial religious practice.

Aleksandra Sulikowska-Belczowska examines the role of domestic icons in the seventeenth and eighteenth centuries as a material foundation of the identity of the Old Believers movement in Russia, and how icons became a source of heated controversy between Old Believers and the Patriarch Nikon who forbade icons after the 'Western style' to be painted or to be held in people's houses. Likewise, the Old Believers' prayer houses were always full of icons, but most of them were domestic icons, i.e., icons of relatively small size and of specific iconography, with a definite predominance of images of the Mother of God, Christ, and angels or saints, especially St. Nicholas the Miracle Worker. Here, again, we see the crossover between domestic and ecclesial space.

By way of a precise case study, i.e., Palazzo Scordia in Palermo (Sicily) in the late seventeenth and early eighteenth century, Valeria Viola examines familial devotion and its relationship with parts of the house other than the chapel. It aims to problematize the issue of the devotional/non-devotional use of paintings inside the household. Of particular note, especially when considered in relation to Albert Kohn's article on the medieval Jewish bed, is Giulio Mancini's Alcune considerazioni appartenenti alla pittura come di diletto di un gentiluomo (1617-1621), which prescribes the bedroom as among the most suitable locations for "le cose di devotione" ("items for devotion"). The article illustrates that the path approaching the chapel from the rest of the house was characterized by the crossing of numerous consecutive thresholds marked by an increase in religious images hanging on the walls. Viola concludes by cautioning that, after years in which the early modern house has been assessed as a secular place alien to devotional practices, the risk is now to go towards the opposite extreme; that is, the univocal interpretation of the house as a "devotional place", thus neglecting the complexity and the instability of meanings that spaces, images, and objects sustained in the domestic environment.

Some Concluding Remarks

In conclusion, it is my hope that this collection of articles has managed to achieve what it set out to do, at least for the most part. Just over half of the contributions deal wholly, or in large part, with the later Middle Ages (fulfilling the desideratum of increasing the number of studies of medieval domestic devotion); there is a fairly wide geographical range (which includes several welcome contributions from Eastern Europe); both Judaism and Islam are represented (although I would have liked to have seen some more contributions regarding medieval Judaism and Islam). However, if the collection as a whole inspires others to build further studies on the research gathered here, it will have made an important contribution.

I am enormously grateful to all the scholars who responded to the initial call for contributions and I owe an immense debt of gratitude for the timeliness of their contributions, and also the professionalism and generosity of the numerous anonymous readers of these articles.

Warm thanks are also owed to the dedicated team in the Religions Editorial Office whose courtesy and efficiency have been a joy throughout this process.

As I write this introduction, the global community is in the midst of the COVID-19 pandemic and most people are currently working from their homes and practicing "social distancing". As a result of this crisis, places of worship across the world have had to close their doors and, to prevent further infection, faith communities can no longer gather for communal worship in person. It seems appropriate to conclude with the thought that scholars for whom the communal expression of faith is an integral part of their personal lives now have reason to consider the term "Domestic Devotion" with more than an academic interest.

Conflicts of Interest: The author declares no conflict of interest. 


\section{References}

Baumgarten, Elisheva. 2014. Practicing Piety in Medieval Ashkenaz. Philadephia: University of Pennsylvania Press. Brundin, Abigail, Deborah Howard, and Mary Laven. 2018. The Sacred Home in Renaissance Italy. Oxford: Oxford University Press.

Corry, Maya, Marco Faini, and Alessia Meneghin, eds. 2018. Domestic Devotions in Early Modern Italy. Leiden: Brill. Corry, Maya, Deborah Howard, and Mary Laven, eds. 2017. Madonnas \& Miracles: the Holy Home in Renaissance Italy. London: Philip Wilson Press.

Doran, John, Charlotte Methuen, and Alexandra Walsham, eds. 2014. Studies in Church History 50. Religion and the Household. Woodbridge: Boydell.

Faini, Marco, and Alessia Meneghin, eds. 2018. Domestic Devotions in the Early Modern World. Leiden: Brill.

Kolpacoff Deane, Jennifer. 2013. Medieval Domestic Devotion. History Compass 11: 65-76. [CrossRef]

Martin, Jessica, and Alec Ryrie, eds. 2012. Private and Domestic Devotion in Early Modern Britain. Farnham: Ashgate. Reid, Megan H. 2013. Law and Piety in Medieval Islam. Cambridge: Cambridge University Press.

Spicer, Andrew, and Sarah Hamilton, eds. 2005. Defining the Holy: Sacred Space in Medieval and Early Modern Europe. Aldershot: Ashgate. article distributed under the terms and conditions of the Creative Commons Attribution (CC BY) license (http://creativecommons.org/licenses/by/4.0/). 
Article

\title{
Furnishing Piety: Beds in High Medieval Jewish Domestic Devotion
}

\begin{abstract}
Albert Kohn
Department of Jewish History and Contemporary Jewry, Hebrew University of Jerusalem, Jerusalem 9190501, Israel; Albert.Kohn@mail.huji.ac.il

Received: 9 July 2019; Accepted: 1 August 2019; Published: 7 August 2019

Abstract: In recent years, pre-modern beds have generated extensive scholarly interest. Their social, religious, and economic importance has been rightfully highlighted in the study of domestic piety. Yet, concern has primarily focused on beds in late medieval English homes. This essay uses Hebrew texts from thirteenth-century Southern Germany, primarily Sefer Hasidim, to further this analysis of the role of the bed in shaping medieval domestic devotion. Jewish notions about the social, moral, and sexual significance of the bed reflect those identified in late medieval Christian culture. These ideas inspired numerous rituals practiced in Jewish homes. Yet, the bed and the remnants of sex assumed to be found in it also frustrated Jewish attempts to perform domestic devotion. These findings highlight the complicated nature of the home and how medieval people had to navigate both its opportunities and challenges in order to foster a rich culture of domestic devotion.
\end{abstract}

Keywords: domestic piety; medieval Ashkenaz; beds; Sefer Hasidim; bedtime prayers; family life

\section{Introduction}

For those interested in pre-modern domestic life, the bed has rightfully generated intrigue. As a central domestic furnishing and one of the most expensive items in the medieval home, the bed demands attention. Focused primarily on England during the Later Middle Ages, recent scholarship has explored the symbolic significance of the bed as a sign of one's wealth, status, and moral worth (French et al. 2016; Gilchrist 2017). Others have taken up the embodied experience of the bed by examining its role in transforming the home into a ritual space (Brundin et al. 2018; Morgan 2017). Building upon these previous studies, this essay will explore the role the bed played in Jewish domestic piety in thirteenth-century Southern Germany. Unsurprisingly, there is general continuity between Christian and Jewish ideas about the bed. The bed was a sign of one's status and character, which at the same time represented sexuality. In the Jewish community, these ideas inspired several rituals that were organized around the domestic furnishing. While most scholarly discussion of the bed and domestic piety has highlighted the comfort and privacy available to devotees in their beds, the Jewish sources reveal an interest in engaging ritually with the domestic furnishing itself. The bed, though, was not always receptive to the pious intentions of its owner. Thirteenth-century Hebrew sources imagined the bed as a hub for domestic impurity, and some consequentially sought to distance sacred objects and activities from it. Jewish discussions of the bed in Sefer Hasidim and contemporaneous literature provide a case study of how medieval people navigated the simultaneous opportunities for and challenges to piety embedded in their homes.

In everyday life, Jews and Christians would have used their beds very similarly. The most basic use of the bed was, of course, for sleeping. Like their Christian neighbors, most Jewish households would have had multiple family members sharing beds. The suffocation of children during the night that resulted from sharing beds inspired rabbis, like their neighboring Christian leaders, to advocate against the practice (Orme 2001, p. 78). The author of one Hebrew responsum wrote that "[i]f at 
all possible, it would be good if children would not sleep next to them [their mothers] once it appears to them [their mothers] that they no longer need to sleep with them [their mothers] in the bed" (Emanual 2012, vol. 2, p. 744; Baumgarten 2004, pp. 176-78). The author's inclusion of the phrase "if at all possible" (בכל היכולת) highlights that sharing a bed in many homes was a result of the economic impossibility of having multiple beds. Some texts suggest that the norm was for gendered sleeping arrangements, with newborns staying with their mothers in the women's bed (Yacovavitz and Eliezeri 1936, p. 114). In more financially prosperous homes, however, there would have been more than enough beds for everyone to sleep comfortably alone. Sleeping, though, was not the only practical use of beds. Beds were an enshrined space for intimacy between spouses. In addition to sexual intimacy, which both Jewish and Christian norms only permitted in bed, Hollie L.S. Morgan has highlighted how beds and bedrooms facilitated verbal intimacy between spouses (Morgan 2017, pp. 77-84). One exemplum from Sefer Hasidim describes, in a similar vein, a "scholar who would speak with his wife in bed about matters until his wife mentioned attractive men and he did not want to have sex until she spoke of other matters" (Sefer Hasidim Project 2015, Parma 3280H, 1908). While this exemplum is framed as a warning about appropriate thoughts to have before sex, it reveals how the bed was a place for intimate and substantive conversation between spouses.

More than these practical uses, recent work on the bed has emphasized the sheer variety of cultural values tied up with it. French, Smith, and Stanbury, for example, describe the "range of familial, emotional, and spiritual valences that accrued to this item of furniture" (French et al. 2016, p. 84). Such associations have been revealed through the close reading of literary, visual, and economic sources. They come out most clearly in various lifecycle events—-such as birth, marriage, and death-in which the bed played an important role. Yet, because the focus has been on late medieval beds in a Christian context, a study of the Hebrew sources can supplement and nuance our understanding of this domestic object. The overlap of ideas about the bed serves as a reminder that notions sometimes linked to Christianity were, in fact, more ubiquitous in medieval Europe.

The texts to be discussed come primarily from Sefer Hasidim, a thirteenth-century collection of Jewish law and lore attributed to Judah the Pious (1150-1217), who lived in Regensburg in Southern Germany. Long considered to be the classical work of the Hasidei Ashkenaz, this work includes scores of exempla that describe the realia of Jewish life in medieval Europe. While earlier scholarship saw Sefer Hasidim as representative of an isolated school of Jewish thought and practice, more recent scholarship has highlighted its importance for understanding the everyday lives and practices of Jews in medieval Germany (Baumgarten 2014, pp. 214-19; Shyovitz 2017, pp. 4-7). Though Sefer Hasidim contributes little to our understanding of the material make-up of Jewish beds, it frequently includes them in exempla describing the conduct of pious individuals. As Elisheva Baumgarten argues, we should read these sources not as expressions of a unique pietistic worldview of an independent sect but, rather, "as a window onto the repertoire of Ashkenazic Jews who wished to elevate the practice of piety in their lives", specifically, in this context, through the use of their beds (Baumgarten 2014, p. 216). The appearances of the bed in Sefer Hasidim thus reveal the diverse range of cultural significances and uses of beds in the domestic life and piety of medieval German Jews. Unsurprisingly, though, these Jewish notions about the bed reflect those already attributed by scholars to late medieval Christian culture.

\section{Jewish Beds: Ideas and Rituals}

Likely the most consistent theme in the explorations of the bed has been its power to convey to both domestic residents and visitors the prestige of its users. French, Smith, and Stanbury explain that the "kind of bed one slept in reflected one's identity and household position" (French et al. 2016, p. 63). Servants, who generally either slept on cheap mats or crowded mattresses, would have been reminded of their diminutive status each time they helped prepare their employer's well-built bed. Laying eyes upon their beds, visitors would similarly be forced to confront the prestige of their hosts. This signaling was a natural outgrowth of the fact that the bed was generally one of the most expensive objects in the 
pre-modern home and was frequently included in dowries. A householder's luxurious bed revealed access to stores of wealth and/or valuable family connections. Penelope Eames has written about how canopied beds specifically were "inseparably associated with prestige, honour, power, wealth and privilege" (Eames 1977, p. 86). This would have been highlighted by the expensive fabrics and bedding with which affluent medieval people decorated their beds.

Such a social impact of the bed is similarly reflected in Sefer Hasidim. One exemplum highlights how the bed and its decorations signaled status to those both inside and outside of the home:

There was a story of a man who was a repairer of combs who became rich and the town leader gave his daughter to this man's son. [This man, though,] hung the tools of his profession over his table and his bed and even though the town leader requested that he remove these tools of his profession, he refused ... The [newly] rich man explained "I hang the tools of my previous profession before me so to remember the kindness that the Holy Blessed One He did for me and I thank and praise God for the fact that He did good for me and gave me wealth so that my heart will not grow haughty upon me. (Sefer Hasidim Project 2015, Parma 3280H, 1030)

This exemplum depicts the bed-along with the table-as a domestic site with a special ability to impart social status to both residents and visitors. Because of its importance, attaching symbols of a humble past to the bed was simultaneously helpful to the newly rich man, who was fearful of pride, and deeply troubling to his well-established in-laws, who were trying to maintain the public perception of their status. An identical fear of lavish beds fostering arrogance was voiced a century later by Christine de Pizan (1364-1430) in her instructions to noble women (Fresco 2003, p. 191). The personal experience of this newly rich householder in bed reflects Glenn Burger's assessment that the bed was an "at once privileged and anxious location for self-identification" (Burger 2018, p. 114). Even though the pious man most likely slept on a lavish bed like other wealthy individuals, this householder's bedroom was where he struggled to clarify for himself and his guests the contrast between his humble origins and his new identity as an elite.

The bed's function as a signal of status and wealth inspired certain pious practices to be organized around the bed in Jewish communities. As part of the weekly preparations for the observance of the Sabbath, medieval Jews made-up their beds as a way of honoring the holy day. In Sefer Hasidim, distinguished bedroom items are the first ones mentioned in a long list of one pious man's special Shabbat decorations. The exemplum describes the "beautiful bed, chair, sheets and room" prepared and designated specifically for the Sabbath (Sefer Hasidim Project 2015, Parma 3280H, 628). It was these domestic objects that showcased the home as appropriately prepared and adorned for the holy day. This interest in preparing the bed stemmed from a popular Talmudic teaching:

Rabbi Yossi bar Yehuda said: Two angels accompany a person from the synagogue to his home, one good and one bad. When they arrive to his home and find a lit candle, a set table and a made bed, the good angel pronounces, 'let it be this way on the next Sabbath' and the bad angel is compelled to respond 'Amen. (Babylonian Talmud, Tractate Shabbat 119b)

Building upon this myth, it was deemed important by some rabbinic voices for Jews to prepare their beds each Friday evening as the Sabbath approached. Eleazar of Worms (1176-1238) instructed that on Friday afternoon "close to the afternoon prayer, one should make-up the bed" (Elazar of Worms 1960, p. 37). While rooted in the Talmud, the specific focus on the bed fits within its medieval European context as well. Similar to late medieval householders who would decorate their bedrooms and beds when welcoming visitors, Jews did the same for the Sabbath and its accompanying angels. One teaching from Sefer Hasidim describes a man who, every Friday, would hurry "as one who heard that a queen is coming to stay at his home" and "say to his servants, 'Prepare the home, clear it and aggrandize it. Prepare the beds because an honored one is coming" (Sefer Hasidim Project 2015, Bologna Print $1538,149)$. Just like in the above-cited exemplum, in which the wealthy community member expected 
his new in-laws to have an appropriately adorned bed, the bed's understood role as a signifier of prestige ascribed to its adornment a privileged status when preparing to welcome in the Sabbath and its accompanying angels. During somber lifecycle events, such as the death of a family member, this same idea was reversed, as those in mourning were known to-according to the comments of a student of Rabbi Yehiel of Paris (d. 1264)_ "lie down on the ground and arrange their bedding and sheets upon straw on the ground" (Yacovavitz and Eliezeri 1936, p. 113). In this way, the bed was a means by which to ritually display the state and mood of the home.

Deeply linked to its importance for social status was the moral significance attributed to the bed during the Middle Ages. Particularly, literary sources reveal how the bed "affirm[ed] in a fundamental way status, standing, and reputation" (French et al. 2016, p. 63). Mary Carruthers explains that English sources frequently describe "honest beds" that were believed to reflect well upon the dignity of those who slept in them (Carruthers 2013, pp. 113-14). While not entirely independent of the physical construction and decoration of one's bed, the moral significance of the bed was rooted in the idea of it. Above all else, the domestic furnishing represented the sanctity of marriage and the honor of the entire household. This explains why, as Morgan notes, literary depictions of adultery generally take place in beds (Morgan 2017, p. 158). It is there that the dishonor directed at the wronged spouse and family is most emphatic. To impinge on a couple's bed is to discredit their marriage and (the husband's) character.

A similar notion appears in the rabbinic tradition in the hope for a Mitah Shleimah or a "complete bed". This idea, which had its roots in the Talmud, was generally disconnected from the physical object and instead focused on the idea of the bed. In an anonymous work attributed to the students of Judah ben Samuel of Regensburg (1150-1217), one finds the term in use:

It is expounded in the Midrash: Why is the word "even also" ( Abraham and Isaac? As it is written [Then I will remember my covenant with Jacob] and even also my covenant with Isaac and even also my covenant with Abraham (Leviticus 26:42). It was written as such because the progeny of Abraham and Isaac angered the Holy Blessed One, yet Jacob had a complete bed. (Stal 2009, p. 98)

This teaching builds on the idea that the quality of one's offspring reflects the piety of their progenitors. Unlike Abraham and Isaac, who each begot one deviant child, Jacob's children were all deemed to be righteous; therefore, their father is said to have had a complete bed. In this way, the moral quality of a family-both parents and children-was wrapped up in perceptions of the bed. A contemporary elucidation of the famed Biblical commentary of Shlomo Yitzchaki (1040-1105), better known as Rashi, connects this idea to Jacob's physical bed. Discussing the scene in Genesis 28:11 in which an on-the-run Jacob collects stones upon which to sleep, Rashi suggests that God fused the plentitude stones together as Jacob slept. A thirteenth-century supercommentary explains that this was done to indicate to Jacob that, even though he would have four wives, all his children would be righteous. The commentator wrote that Jacob "took numerous stones and they became one as a hint that Jacob would have a complete bed." ${ }^{1}$ Here, we see this same idea of the bed representing honor, but it is rooted in Jacob's actual bed. The fused stones upon which Jacob slept are understood as an indicator of the righteousness of his future family. Similar to Christian scholars, Jews saw a respectable bed as a sign of honor and stability.

These moral implications of the bed inspired requests for this idealized "complete bed" at certain liturgical moments. While going to sleep each night, Jews prayed that their beds might be deemed "complete" before God (Goldshmidt 2004, vol. 1, p. 162). This request simultaneously carries the hope that not only will one be free of impurifying seminal emissions during the night but also-as

1 Asher ben Jehiel and Samuel ha-Levi ibn Yuli. 1986. Sefer Hadar Zek. New York: ha-Makhon le-hafatsat perushe ba'ale ha-Tosafot 'al ha-Torah. p. 64 
the broader context of the prayer suggests - that God will lead the devotee down the path of morals and piety. Though this aspiration grew out of the idea rather than the physical construction of the bed, it was verbalized in the actual bed and thus could not be separated from the domestic object. This phrase may have also been used to bless relationships. One Hebrew folktale describes how a father walked in on his daughter asleep in bed with a man. When he noticed a sword lying between the lovers though, he recognized that no misdeed had occurred and that the young man "acted solely out of their love". Speaking to both parties asleep in bed together, the father declared "May your bed (מטתכם) be complete before the God of Israel" (Kushelevsky et al. 2017, pp. 294-95). While not a ritual per se, this was a blessing for the couple. The father referred to the bed both to note the honor of their relationship thus far and to give well-wishes for its future. Such notions were not foreign to Christian practice. Many Christian wedding ceremonies included blessings of the new couple's marital bed in the hope of setting them off in the right direction (Rivard 2009, p. 14). These practices all show how the interlocking of ideas about honor with the bed generated ritual interaction with the physical beds residing in every home.

While these cultural associations with the bed are found in Hebrew literature, the most dominant idea linked with the bed by Jews of the High Middle Ages was sex. Such an association is unsurprising given its parallel pervasiveness in Christian culture. Morgan explains that "the concept of the bed in late medieval cultural imagination had inherent sexual meanings, even when sex was far from the bedfellows' minds" (Morgan 2017, p. 146). This is a natural result of the bed being the only place deemed appropriate for sexual relations among both Jews and Christians. Looking at the bed, one could not but reflexively see sex (Duby 1988, pp. 62-63). This link manifested itself linguistically. In Hebrew sources, the primary means for referring to sex is the euphemism Tashmish HaMittah, or 'service of the bed'. Particularly in Sefer Hasidim, a text abundantly concerned with sexuality, one is constantly confronting this linguistic overlap. A similar semantic link is evident in Middle English literature from the fourteenth century in which-like in modern English—the verb "to bedde" refers to having sex.

This association in Jewish thought contributed to several bedside pieties concerning sexual purity. As has been well documented, the Jewish ritual literature of medieval Ashkenaz encouraged anxiety about menstruating women (Koren 2011, pp. 43-62; Baumgarten 2014, pp. 21-50). These fears manifested themselves in precautions taken to maintain distance between a man and his menstruating wife. Because the most serious prohibition concerned having sex during the menstrual period, these fears reached fever pitch when spouses were together in bed. The sexualization of the bed generated an assumption that being together in bed could not but result in coitus. To prevent sin, some rabbis demanded that spouses deviate from the usual practice of sleeping in the nude and repose fully dressed during the period of impurity. Others went even farther and expected spouses to sleep in separate beds. An exemplum from Sefer Hasidim displays how these changes could be a form of domestic piety:

There was once a pious man who would not lie on his bed each night that his wife was a menstruant. Rather, he would either sit or recline and sleep. For he said, 'If I lie on my bed comfortably, I will find myself sleeping a lot and perhaps I will have a seminal emission. Rather, I will sleep without a pillow and in discomfort so that I will not have an emission. (Sefer Hasidim Project 2015, Parma 3280H, 48)

In this exemplum, the protagonist's adjusted conduct in bed was a form of piety. Because of the sexualization of the bed, he was hyper cognizant of his conduct in bed and deviated from normal domestic activity when sex was not permitted. By altering his sleeping position as well as his bedding, his sexual drive could be kept in check even when in bed. Such domestic precautions taken during the menstrual period made up an important portion of Jewish domestic piety (Baumgarten 2014, pp. 27, 33; Karras 2017, pp. 101-2).

In understanding the ideas and practices of the bed, the gendered aspects of the furnishing cannot be ignored. Much of the recent scholarship has emphasized that, in many contexts, the bed was gendered female (Duby 1988, pp. 62-63; Morgan 2017, pp. 181-88). This was a natural result of women frequently bringing the bed into the marriage as part of their dowry and maintaining it as 
part of their domestic duties. According to the Talmud, one of the wife's numerous conjugal duties is to prepare the bed (Babylonian Talmud, Tractate Ketuvot 59b). Elazar of Worms praised the piety and character of his daughter by referencing how she "made my bed and removed my shoes each evening" (Haberman 1945, p. 166). The gendered nature of making the bed suggests that those piously preparing the bed before the Sabbath were almost certainly women. A sense of gendered ownership of the bed is also reflected in the above exemplum about the man who piously distanced himself from the bed while his wife was menstruating (Sefer Hasidim Project 2015, Parma 3280H, 48). In this case, the bed was understood to belong to the woman's domain. The woman's impurity extended itself onto the bed, and the man was expected to deviate his conduct relative to this female space. A similar sense of ownership was on display in Jewish rituals after the birth of a child, in which postpartum mothers were sequestered to their beds (Baumgarten 2004, pp. 101-2). Yet, this does not mean that beds held strictly female significance. The Hebrew folktale that references the idea of a 'complete bed' emphasizes with its use of the plural Mitatkhem that the bed belonged to both spouses (Kushelevsky et al. 2017, pp. 294-95). It was their shared future marriage that the father implicitly blessed. Similarly, in the exemplum about preparing for the Sabbath, it was the man who came in and called for the beds to be prepared in honor of the Sabbath (Sefer Hasidim Project 2015, Bologna Print $1538,149)$. While women were almost certainly those who prepared the actual bed, men still had a stake in the practices organized around it. The man's investment, though, was primarily in the ideas that the bed represented. For him, a prepared bed signified a dignified marriage, family prestige, and a patriarchally organized domestic life. In both daily life and ritual, though, the women were the ones primarily engaged with the actual domestic furnishing.

\section{Object of Filth: Jewish Perceptions of the Bed as Impure}

While there is general continuity between Jewish and Christian perceptions of and practices relating to the bed, Sefer Hasidim assigns an impurity to the bed that is less pronounced in Christian sources. Because pietistic literature is known to be exceptionally concerned with purity, it is unsurprising that discussions about the impurities hiding in the bed are scattered throughout Sefer Hasidim (Shyovitz 2017, pp. 164-71). Some of the ideas are simple reformulations of notions originally stated in earlier rabbinic texts. One such teaching instructs that one should not store food under the bed, "because the spirit of impurity resides there" (Sefer Hasidim Project 2015, Parma 3280H, 376). ${ }^{2}$ Yet, this idea is carried forward when the bed is compared to "an instrument of filthiness" (Sefer Hasidim Project 2015, Bologna Print 1538, 815). This association of the bed with impurity explains a unique rabbinic dictate about interior decoration: "Whoever resides in a town in which they are renting out a building in which to live, he should not establish a prayer space in a room in which there was previously the bed of a gentile" (Sefer Hasidim Project 2015, Parma 3280H, 535). While the main concern was almost certainly the bygone gentile residency in the home, the text focuses its anxiety on the bed out of all the potential domestic goods because of its association with impurity. The bed was deemed to be a hub and amplifier of domestic impurity.

This perception stemmed partially from the bed's varied cultural associations with the chief impurities: sex and death. It was already explored above how medieval people sexualized the bed, but both Christians and Jews also linked it to death (French et al. 2016, pp. 75-83). In Sefer Hasidim and other Hebrew texts, the same word, Mittah, is used to refer to both beds and to coffins. In this way, Jews - in a certain sense - entered their coffins each night and were placed in their beds upon their death. Because medieval people understood sleep as a quasi-form of death, that the coffin and the bed shared terminology was entirely natural. The association could only then be amplified by the commonality of dying while asleep in one's bed. With such ideas hovering in the background, it is unsurprising that both Jews and Christians felt some apprehensions about the bed.

2 For the rabbinic formulation of this idea, see Babylonian Talmud, Pesachim 112a and Babylonian Talmud Bava Batra 58a. 
Even more influential than these cultural associations, though, was the most common explanation for the impurity of the bed: the bodily fluids that were known to congregate there. Semen and flatulence were all believed to endanger one's purity and were assumed to be hiding in the bed. Worried about such residue, Sefer Hasidim describes a "pious one for whom it was accustomed that whenever he would sleep or cohabit, he would wear a particular cloak. For he would say lest some semen stick to it [my general cloak] when I go to synagogue" (Sefer Hasidim Project 2015, Parma 3280H, 1063). This pious man was not concerned with the mere associations between the bed and sex but, rather, with the very tangible impurity, namely semen, which he assumed resided in the object. While especially emphatic in Sefer Hasidim, this concern with the impurity of semen in particular has a long history in Jewish culture and should not be understood as a unique pietistic concern (Shyovitz 2017, pp. 173-74). This protagonist developed his own domestic piety by which to protect himself from the semen and other impurities hiding throughout his home in places such as the outhouse and the bed. Another ruling warns about the impurity of the bed, which stemmed from the fact that "there is semen and a person flatulates in bed" (Sefer Hasidim Project 2015, Parma 3280H, 7). Because of the activities that happen there, namely sex and sleep, the bed in Sefer Hasidim was assumed to be infested with the fluids excreted during those bodily processes.

Beyond being a literary trope, the impurity of the bed impacted how domestic devotion was practiced in the medieval Jewish home. One exemplum from Sefer Hasidim displays the thirteenth-century perspective on the status of the bed and how it impacted domestic devotion:

Two people were removing books from a large chest. One placed the books on a bed on which man and women lie and the other placed them on the floor. The one who placed them on the bed said: "Why do you dishonor the books by putting them on the floor?" to which the other responded: "You disgrace the Torah by placing them on a bed where man and his wife cohabit. The ground is pure and I do not have a cloth to put on the ground." The first man said in response: "But don't people read the Shema [in bed] with his wife next to him? And words of Torah do not become impurified." The second man responded: "This is true while they have still not had sex, but if the remnant of semen is on his body or on his clothing or on the sheets upon which he lies, he will not read [the Shema]. Further, in reading there is not impurification—as it says: 'Are not these words like fire?' - but to place [books] on something that has semen without it being laundered, that is a disgrace." (Sefer Hasidim Project 2015, Parma 3280H, 640)

This constructed dialogue about the status of the bed highlights the roots of medieval Jewish perceptions of its impurity. The voice critical of allowing presumably sacred books to touch the bed began by rooting such an opinion in the sexualization of the bed but quickly transitioned to the issue of the semen left behind after sex. As our sources have suggested, the bed's impurity was both a function of the activities and fluids one could find in it. Yet, the concluding claim that piety was acceptable only in recently laundered beds highlights that lingering bodily fluids were the root concern.

This exemplum, though, also highlights how domestic life could hamper attempts to bring the sacred into the home. The understanding that the bed was the natural home to both sex and its accompanying excretions meant that this central domestic furnishing could not interact with sacred objects. Other rulings emphasize this same point. One prohibits the placement of books on beds because of the semen and flatulence assumed to be present (Sefer Hasidim Project 2015, Parma 3280H, 7). Another chastises a person for storing a bed right next to a Torah scroll (Sefer Hasidim Project 2015, Parma $3280 \mathrm{H}, 1633 \mathrm{a}$ ). While recent work on domestic piety has emphasized how books and sacred objects were brought into the homes to foster domestic devotion (Brundin et al. 2018, p. 314), these rulings reveal how they could be deemed an unnatural fit with certain aspects of the medieval home. According to these teachings, beds were unreceptive to the sacred. This notion is integral to understanding pre-modern domestic piety, because unlike outhouses, which would have been segregated from domestic life, in most homes, the bed was a central and unavoidable domestic furnishing. Notable, as well, is that this inhospitality was not the result of some extenuating 
circumstances but, rather, of the regular use of the bed for sex between spouses. Medieval Jews were not expected to be abstinent but, rather, to be aware of the hurdles that regular domestic life placed before their pious pursuits. Unless it was recently laundered, the bed was assumed to be a site of impurity that repelled the sacred objects with which medieval Jews practiced their domestic piety.

\section{Bedtime Prayers in the Medieval Bed}

Unlike books and Torah scrolls, which could simply be kept away from beds, the Jewish bedtime ritual was, by its nature, forced to actively confront the impurities emanating from the bed. As a devotional practice whose most natural home was the bed itself, this ritual provides a case study of the challenges high medieval Jews faced when bringing piety into the domestic space. Jewish bedtime rituals all reference back to Deuteronomy 6:6-7, which commands the following: "Take to heart these instructions with which I charge you this day. Impress them upon your children. Recite them when you stay at home and when you are away, when you lie down and when you get up." These verses are understood to obligate the recitation of this very section of the Bible-known as, the Shema - at both the morning and evening services, as well as before bed each night. "Rabbi Joshua ben Levi said: Even though one recited the Shema in the synagogue, it is a commandment to do so on one's bed" (Babylonian Talmud, Tractate Berakhot 4b). Throughout the Talmud, this practice is understood variously: as a genuine ritual obligation, as an attempt to foster positive thoughts before bed, and as a form of protection during the night. These prayers-along with most other Ashkenazi prayers-were codified into the European Jewish liturgy via the writings of Amram Gaon (d. 875) and were accepted as normative in the Ashkenaz community throughout the Middle Ages (Israel Ta-Shma 1992, p. 311-14).

In the Talmud, it was recognized that reciting prayers in one's bed could be complicated by the realities of domestic life. Due to the prohibition on reciting certain prayers in the presence of a naked body, the pre-modern practice of sleeping in the nude in shared beds endangered one's ability to say the bedtime Shema. The Talmudic rabbis argued about whether one was permitted to recite this bedtime prayer when sharing a bed with a spouse, children, or others (Babylonian Talmud, Tractate Berakhot, 24a). By the Middle Ages, it was agreed that one was permitted to do so as long as certain precautions were taken. A common instruction, here authored by the late thirteenth-century Rabbi Hayim Eliezer ben Yitzchak, concludes that:

Two people who are sleeping in one bed should not recite the Shema unless there is a sheet between them. And if his wife is sleeping in bed with him, he should turn his face away and recite; our teacher Eliezer of Worms required additionally the separation via a sheet with one's wife. (Isaac ben Moses of Vienna and Hayyim ben Isaac 2010, vol. 1, p. 126)

These guidelines required that bedfellows separate themselves with bedding so as not to see one another's genitalia when reciting their prayers. With one's wife, one was encouraged to look away entirely. Though these practical precautions allowed bedfellows to circumvent the prohibition of reciting prayers in the presence of an uncovered body, a contemporary text from Italy still warns that one must be vigilant lest a spouse's mere presence inspire sensual thoughts during one's prayer. After listing nearly identical precautions to those cited above, the text explains that "it is appropriate for a fearer of heaven to be cautious when reciting the Shema on his bed if his wife is there with him lest he be taken by licentious fantasies" (Moshe ben Yekutiel 1973, p. 192). Clearly, the realities of sharing a bed were recognized to be a potential obstacle to the appropriate practice of piety.

The impurities believed to reside in the bed similarly impacted the performance of bedtime rituals. Starting in the Talmud, it was assumed by nearly every ritual text that bedtime prayers would be recited in one's bed. In the oldest manuscripts of Mahzor Vitry, a foundation stone of Ashkenaz liturgy compiled at the start of the twelfth century, it is instructed that "when one enters one's bed to sleep, one must recite the Shema" (Goldshmidt 2004, vol. 1, p. 162). Because it is a prayer that is believed to protect the devotee while he or she is asleep, its choreographical placement in one's bed while falling asleep is entirely natural. Given the impurities associated with the bed, though, this generated some 
tension. Some saw it as inappropriate to say prayers in a bed stained with semen or other bodily fluids. In the above-cited exemplum from Sefer Hasidim, one character explains that "if the remnant of semen is on his body or on his clothing or on the sheets upon which he lies, he should not read [the Shema]" (Sefer Hasidim Project 2015, Parma 3280H, 640). Such a ruling is unsurprising given the frequent concern in pietistic literature about praying in cleanliness. This same concern about potential impurities found in the bed likely inspired the instruction found in Mahzor Vitry directing one to cleanse before reciting the bedtime prayer. Goldschmidt notes that nearly every manuscript opens the section for the bedtime Shema by instructing that " $[\mathrm{w}]$ hen one goes to sleep, one should cleanse and purify and then recite the Shema" (Goldshmidt 2004, vol. 1, p. 162). Especially because one was likely to have sex before going to sleep, it was deemed important to wash off any potential contaminants accrued while in bed before reciting these prayers.

Some, though, went even further and deemed saying the prayer merely in the presence of one's bed a problem. Sefer HaTadir, penned by the thirteenth-century Italian Rabbi Moses ben Yekutiel, deals extensively with the issues that arise when reciting prayers in one's bed. Similar to many other ritual texts, he outlined how one can navigate properly reciting prayers even when surrounded by one's naked spouse and children. Though, like others, he ultimately assumed that the prayer would be said in one's bed, he included an exemplum that reflects a clear ideal that it not be. He wrote the following:

I saw that one of the scholars of the generation had in his home a synagogue and every night when he would want to go to sleep he would go specifically from the room of his bed to recite the Shema in the synagogue in order that he would recite it in purity and in cleanliness. Appropriate for him was the honor of having a synagogue to protect his home from demons. Who is such a great nation that has such righteous laws and statutes? Happy are the people who have it so! (Moshe ben Yekutiel 1973, p. 192)

According to this text, the ideal was to not recite the bedtime prayer in one's bedroom, being that it was assumed to be impossible to say it there in "purity and cleanliness". That this was a function of the general association of the bed with impurity is made clear by the emphasis that this pious man ran away not from his generic bedroom but, from his "room of the bed" (מחדר המיטה). It was the bed that was at the heart of this concern for purity. He, like other Italian rabbinic figures, was likely influenced by pietistic teachings when formulating this ideal that understood the bed as impure.

Though not as clearly attributed to impurity, other texts codify a similar separation between prayers and the bed. In one manuscript of Mahzor Vitry, according to Goldschmidt's apparatus, the Talmudic guidelines that bedtime prayers be said in the bed were replaced with instructions that the Shema be said when "one enters one's home to sleep" (Goldshmidt 2004, vol. 1, p. 162). This decentralizing of the bed is unexplained, and the language does not seem to have a precedent. In Leket Yosher, a work that collects the customs of Israel Isserlein (1390-1460), one finds a strict formulation by which the Shema is said outside of one's bed: "Someone who forgot to recite the bedtime Shema, if he remembers before falling asleep he must arise from his bed and recite it" (Joseph ben Moshe 2010, vol. 1, pp. 94, 95). According to this teaching, one should not say the Shema in one's bed and must go so far as to actively extricate himself from it in order to recite the bedtime prayer. Though neither of these passages mentions the impurity of the bed, it is likely that the changes were inspired by the realities of the pre-modern bedroom, including bodily fluids and naked bedfellows. It is clear that the norm was for the Shema to be recited in bed, yet these sources-when taken in conjunction with the anxieties around the bed-may indicate an underlying desire to distance the ritual from the bed.

This idea that prayers should be kept away from the bed seems to be more prevalent in Hebrew texts from the High Middle Ages, but it was not entirely absent from Christian practice. Peter Damian (1007-1072) recorded two cautionary tales about a sick priest and a pilgrim who recited the Compline service- a form of bedtime prayer-while lying in their beds and were punished for such misconduct (Damian 1983, vol. 3, p. 130-31). Similarly, high medieval works from both England and France encourage devotees to sprinkle holy water on the bed when reciting bedtime prayers, as if to purify 
it before transforming it into a ritual space. ${ }^{3}$ Later, in the fifteenth century, Florentine Girolamo Savonarola (1452-98) explicitly warned about the polluted nature of the bedroom and advised against praying there (Trexler 1991, p. 160). Though not as explicit as in Sefer Hasidim, these Christian bedtime instructions point to a wider recognition of the potential devotional challenges posed by the bed.

\section{Conclusions}

The diverse ideas and practices with which high medieval Jews linked their beds further validates the recent work on the centrality of the bed to pre-modern culture and domestic piety. As full members of medieval European society, Jews shared their Christian neighbors' appreciation for the import of the bed and used it to inform their own domestic rituals. These Hebrew sources emphasize the previous observation of the sheer variety of meanings that the bed accrued in pre-modern culture. In Jewish homes, the bed simultaneously signified the wealth and dignity of a family while also representing sexuality and domestic impurity. This diversity of connotations also influenced how beds were used for piety. The domestic furnishing provided not just comfort but also the context for the observance of sexual norms and important liturgical ideas, as well as a canvas upon which to display one's reverence for holy days. Such multifunctionality of the bed with respect to piety extended to the entire home. The home was not merely a neutral space in which ritual was performed but, rather, an environment with which ritual actively engaged. Especially in the Jewish tradition, which mandated the observance of numerous rituals in the domestic sphere, ritual interacted in diverse ways with the various features of the home.

Yet, the Jewish concern with the potential impurities embedded within the bed highlights how domestic objects could pose a challenge to domestic devotion. Beds were not neutral objects; they were impacted by the activities performed in them and exerted influence over the environment in which they resided. The Jewish bed's ability to accumulate domestic impurity and project it outwards onto the rituals and objects with which it came into contact is an example of the agency of pre-modern objects explored by Roberta Gilchrist and others (Gilchrist 2017, p. 216-17). Although beds offered a private space in which householders could feel comfortable while practicing their domestic devotions, beds could also repulse residents who were all too aware of the contaminants that lay beneath their covers. This evidence serves as a useful counterpoint to some recent interpretations in the literature that have construed the bed as a "religious object with a similar function to a set of rosary beads" (Morgan 2017, p. 47). This observation, though, holds true for the entire home, as impurities similar to those found in the bed existed throughout the domestic sphere. David Shyovitz highlights this when he describes how pietistic literature was especially concerned with impurities that could be passed to devotees via their children (Shyovitz 2017, p. 165). Where is one more likely to interact with children than in one's home? When thinking about domestic piety, then, it is essential to remember that the home was not a blank slate upon which piety could simply be overlaid, as some understandings of domestic religion have suggested. It was a complicated maze whose contours had to be navigated so that ritual and the sacred could reside alongside the banalities and filth that characterized much of domestic life.

The potential disruptiveness of domestic life was already recognized in the eleventh century by Heloise when she warned Abelard about the impossibility of achieving their scholarly goals in a domestic context. She asks: "Scholars and nursemaids, writing desks and cradles, a book and a distaff, a pen and a spindle—what harmony can there be in that?" (Abelard and Héloïse 2007, p. 15). Recent work on domestic devotion makes clear that Heloise's concerns in no way prevented piety from taking root in the home. Nevertheless, the people, objects, and activities that made the home bustle provided opportunities as well as challenges to those who wanted to turn their homes into

3 See Bibliothèque nationale de France, Françaises, MS 1802, 85r; Bibliothèque nationale de France, Nouvelles acquisitions françaises, MS 5232, 182r. Hollie L.S. Morgan offers an alternative interpretation of this practice (Morgan 2017, pp. 96-97). 
ritual spaces. It was this multidimensional character of the home that shaped how domestic devotion was performed. As a centerpiece of the pre-modern home, the bed exemplifies this complicated relationship between domestic life and piety. Their rich cultural significance inspired rituals, whereas their practical use occasionally repelled them. Further study of domestic piety requires a keen focus on how medieval people were able to synthesize their devotions with the potentially disruptive realities of their domestic life.

Funding: This project has received funding from the European Research Council (ERC) under the European Union's Horizon 2020 research and innovation programme (grant agreement no. 681507), Beyond the Elite: Jewish Daily Life in Medieval Europe, directed by Elisheva Baumgarten.

Acknowledgments: The author would like to thank Elisheva Baumgarten for her support in this project.

Conflicts of Interest: The author declares no conflict of interest.

\section{References}

Abelard, Peter, and Héloïse. 2007. The Letters and Other Writings. Translated by William Levitan. Indianapolis: Hackett Pub.

Baumgarten, Elisheva. 2004. Mothers and Children: Jewish Family Life in Medieval Europe. Princeton: Princeton University Press.

Baumgarten, Elisheva. 2014. Practicing Piety in Medieval Ashkenaz: Men, Women, and Everyday Religious Observance. Philadelphia: University of Pennsylvania Press.

Brundin, Abigail, Deborah Howard, and Mary Laven. 2018. The Sacred Home in Renaissance Italy. Oxford: Oxford University Press.

Burger, Glenn. 2018. Conduct Becoming: Good Wives and Husbands in the Later Middle Ages. Philadelphia: University of Pennsylvania Press.

Carruthers, Mary J. 2013. The Experience of Beauty in the Middle Ages. Oxford: Oxford University Press.

Damian, Peter. 1983. Monumenta Germaniae historica Die Briefe der deutschen Kaiserzeit. Edited by Kurt Reindel and Die Briefe des Petrus Damiani. 4 vols. München: Monumenta Germaniae Historica.

Duby, Georges. 1988. The Aristocratic Households of Feudal France. In A History of Private Life: Revelations of the Medieval World. Edited by Georges Duby. Cambridge: Harvard University Press, pp. 33-85.

Eames, Penelope. 1977. Furniture in England, France and the Netherlands from the Twelfth to the Fifteenth Century. London: The Furniture History Society.

Elazar of Worms. 1960. Sefer HaRokeach HaGadol. Jerusalem.

Emanual, Simcha, ed. 2012. Teshuvot Maharam me-Roțenburgṿe-haveraṿ. 2 vols. Jerusalem: ha-Igud ha-'Olami le-mada'e ha-Yahadut.

French, Katherine L., Kathryn A. Smith, and Sarah Stanbury. 2016. An Honest Bed: The Scene of Life and Death in Late Medieval England. Fragments: Interdisciplinary Approaches to the Study of Ancient and Medieval Pasts 5.

Fresco, Karen L. 2003. Gendered Household Spaces in Christine de Pizan's Livre des trois vertus. In The Medieval Household in Christian Europe. Edited by Cordelia Beattie, Anna Maslakovic and Sarah Rees Jones. Turnhout: Brepols, pp. 187-97.

Gilchrist, Roberta. 2017. Medieval life: archaeology and the life course. Rochester: Boydell Press.

Goldshmidt, Aryeh, ed. 2004. Mahzor Vitry. 3 vols. Jerusalem: Otzar HaPoskim.

Haberman, Abraham M., ed. 1945. Sefer Gezeirot Ashkenaz ve-Zarfat. Jerusalem: Tarshish.

Isaac ben Moses of Vienna and Hayyim ben Isaac. 2010. Sefer Or Zarua. Edited by Yacov Farbstein. 3 vols. Jerusalem: Mekhon Yerushalayim.

Joseph ben Moshe. 2010. Sefer Leket Yosher. Edited by Amihai Kinarti. Jerusalem: Machon Yerushalayim, vol. 1.

Karras, Ruth Mazo. 2017. Sexuality in Medieval Europe: Doing unto Others, 3rd ed. London and New York: Routledge Taylor \& Francis Group.

Koren, Sharon Faye. 2011. Forsaken: The Menstruant in Medieval Jewish Mysticism. Waltham: University Press of New England.

Kushelevsky, Rella, Ruchie Avital, and Chaya Naor. 2017. Tales in Context: Sefer Ha-Ma'asim in Medieval Northern France. Detroit: Wayne State University Press. 
Morgan, Hollie L. S. 2017. Beds and Chambers in Late Medieval England: Readings, Representations and Realities. Woodbridge: York Medieval Press.

Moshe ben Yekutiel. 1973. Sefer HaTadir. In Shitat HaKadmonim. Edited by Moshe Yahuda Blau. New York: Editor, pp. 179-318.

Orme, Nicholas. 2001. Medieval children. New Haven: Yale University Press.

Rivard, Derek A. 2009. Blessing the World: Ritual and Lay Piety in Medieval Religion. Washington: Catholic University of America Press.

Sefer Hasidim Project. 2015. Princeton University Sefer Hasidim Database. Princeton: Princeton University. Available online: https://etc.princeton.edu/sefer_hasidim/manuscripts.php (accessed on 19 June 2019).

Shyovitz, David I. 2017. A Remembrance of His Wonders: Nature and the Supernatural in Medieval Ashkenaz. Philadelphia: University of Pennsylvania Press.

Stal, Yacov Yisrael, ed. 2009. Sode Humash ve-Shar. Jerusalem.

Ta-Shma, Israel M. 1992. Minhag Ashkenaz ha-kadmon: heker ve-‘iyun. Yerushalayim: Hotsa'at sefarim 'a. sh. Y.L. Magnes, ha-Universițah ha-'Ivrit.

Trexler, Richard C. 1991. Public life in Renaissance Florence. Ithaca: Cornell University Press.

Yacovavitz, Aharon, and Shmuel Eliezeri, eds. 1936. Otzar Rishonim: Moed Katan. Jerusalem: Machon Harry Fischel, pp. 1-116.

(C) 2019 by the author. Licensee MDPI, Basel, Switzerland. This article is an open access article distributed under the terms and conditions of the Creative Commons Attribution (CC BY) license (http://creativecommons.org/licenses/by/4.0/). 


\title{
Article \\ Sculptures and Accessories: Domestic Piety in the Norwegian Parish around 1300
}

\author{
Ragnhild M. Bø \\ Department of Archaeology, Conservation and History, University of Oslo, P.O. Box 1008 Blindern, 0315 Oslo, \\ Norway; r.m.bo@iakh.uio.no
}

Received: 29 October 2019; Accepted: 14 November 2019; Published: 19 November 2019

\begin{abstract}
Eagerly venerated and able to perform miracles, medieval relics and religious artefacts in the Latin West would occasionally also be subject to sensorial and tactile devotional practices. Evidenced by various reports, artefacts were grasped and stroked, kissed and tasted, carried and pulled. For medieval Norway, however, there is very little documentary and/or physical evidence of such sensorial engagements with religious artefacts. Nevertheless, two church inventories for the parish churches in Hålandsdalen (1306) and Ylmheim (1321/1323) offer a small glimpse of what may have been a semi-domestic devotional practice related to sculpture, namely the embellishing of wooden sculptures in parish churches with silver bracelets and silver brooches. According to wills from England and the continent, jewellery was a common material gift donated to parishes by women. Such a practice is likely to have been taking place in Norway, too, yet the lack of coherent source material complicate the matter. Nonetheless, using a few preserved objects and archaeological finds as well as medieval sermons, homiletic texts and events recorded in Old Norse sagas, this article teases out more of the significances of the silver items mentioned in the two inventories by exploring the interfaces between devotional acts, decorative needs, and possibly gendered experiences, as well as object itineraries between the domestic and the religious space.
\end{abstract}

Keywords: holy crosses; wooden crucifixes; pendants; silver arm rings; parish churches; gift-giving; popular piety; devotional practices

\section{Introduction}

In the Middle Ages, people's engagement with sculptures took many forms. Sculptures, as well as manuscript pages, relics and reliquaries, could be grasped, stroked, kissed and tasted; at times they were felt as becoming living flesh under the hands of the devout (Freedberg 1989; Bynum 2011; Rosler et al. 2013) and they could be dressed and undressed (Trexler 1991; Genovese 2011). Sculptures, and in particular crucifixes, could also act themselves (Tripps 2000; Kopania 2010). Appearing to be animated, they took on a "practical mediatory role ( . . ) in the social process" (Gell 1998, p. 6) and participated in a phenomenological animation, that is the animation which takes place in the beholder's response to and encounter with them (Lohfert Jørgensen 2017, p. 266; also see Pongratz-Leisten and Sonik 2015). Regardless of perceived animations, however, sculptures were at times recipients of precious accessories, often as the result of a pious bequest (Marks 2004; French 2008). Although source material for people's engagements with sculptures in medieval Norway is very scant, such bequests are possibly what are referenced in two unique documents from the fourteenth-century, namely the inventories from the parish churches of Hålandsdalen (1306) and Ylmheim (1321, additions from 1323).

Hålandsdalen (today Holdhus) and Ylmheim (today Ølmheim) were both under the jurisdiction of Bjørgvin bishopric and situated at the western coast of Norway, yet more than 200 kilometres apart. Unfortunately, none of the churches for which the inventories were made are preserved; the present churches date from 1726 and 1863 respectively (Hoff 1997). However, as words have outlived the 
monuments, we can appreciate matchless windows into the contemporary medieval parish church interior. Commencing with the liturgical textiles, the inventories list chasubles, alba, antependia (frontals), dossals and altar cloths-in fact, the entire choir of Hålandsdalen was filled with textiles, ræflum and tioldum. ${ }^{1}$ Church bells, liturgical bells, censers, chalices and corpora follow suit as well as candlesticks, crosses, bearskins and liturgical manuscripts such as missals, manuals, psalters and vitae. The inventories largely converge, but there are more items of each category in Ylmheim and a slight variation in the number of paintings and sculptures. However, the object from Hålandsdalen described as "one large cross with Mary and John with six silver rings, three on each arm, and one silver brooch" (DN 21,7) is matched in Ylmheim by "a cross dressed in pæll with two silver rings around the waist and six around the arms" (DN 15,8). ${ }^{2}$

The early fourteenth-century compilers recorded virtually all the churches' possessions through brief descriptions, yet also included more qualitative judgements such as the weight of the censer (ærtog fa[t]t a vii. aura ${ }^{3}$ ), the different size and age of the manuscripts (small, old) and the different fabrics (pellz, camelot, fustane ${ }^{4}$ ) and colours (red and green) of the liturgical textiles. One of the antependia is even characterized as useless. Moreover, both inventories disclose a detail—and possibly a related devotional act - namely the embellishing of crucifixes and sculptures with silver bracelets and silver brooches. Although these descriptions are brief, it is intriguing that the only fourteenth-century church inventories which have been conserved for posterity both mention crucifixes with silver arm rings (silfr spongum). Furthermore, these are the only instances of such wording in the entire corpus of preserved medieval documents from Norway, and indeed Scandinavia.

The last decade has seen an increasing interest in medieval inventories and other documents listings things. They are valuable sources for the reconstruction of collections now lost or dispersed (Keane 2016) and they can reveal a number of cultural practises involving material culture such as debt-collection (Smail 2016), the supply of artistic materials (Kirby et al. 2010), gift-giving (Algazi et al. 2003) and pious bequests (Smith et al. 2001; French 2008). The brevity of the inventories from Hålandsdalen and Ylmheim, however, complicates the matter; apart from noting the presence of the silver items, they are silent about their appearance and function. Were the arm rings an integral part of the sculpture from the time of its execution, or were they later additions? Were they placed as embellishments, votive gifts, or heirlooms? Or, were they placed on the crucifix in order to create a portable amulet which could be relocated from the religious to secular or domestic sphere when needed?

This article aims to reach a better understanding of the bracelets and the brooch by analysing preserved crucifixes and related material culture as well as examining how the possession of religious object in households and the care for objects in local churches are portrayed in saga literature, normative texts and wills. Comparing the finds from early fourteen-century Norway with devotion to the cross and crucifixes, patterns of pious bequests and the laity's engagement in parish churches in England and on the continent allows for further assumptions about the silver items. The comparison appears particularly appropriate if the silver items are considered to be acts of domestic devotion; not, however, in the form of devotions carried out in a private home (Brundin et al. 2018; Corry et al. 2018), but as a way the laity would integrate themselves into parish communities through domestic and secular objects.

1 Rxflum/refil is a rectangular, horizontally cut textile often with iconographical motifs; tioldum/tjeld are textiles hang on the wall in larger pieces.

2 "Jtem æin kross stor cum mari et Johanne med sex silfr spongum priar vm huarn arm ok [æ]in silfr sylgia" and "Jtem æinn kross klædr med ok pæll tuæimr silfr spongum vm mitt ok vi vm armanna."

3 Eight öre but one örtug. Öre $=1 / 8$ mark and örtug $=1 / 24$ mark. In 1287, one mark $=214.3$ g, cf. Nils Ludvig Rasmussen, "Mark" in Kulturhistorisk leksikon for nordisk middelalder (KLNM) Vol.11:424 and Swen Owe Jansson, "Vikt" in KLNM, Vol. 20: 58 (Karker et al. 1956-1978).

4 Pellz/pell probably refers to silk fabrics; camelot refers to a woven fabric that might have originally been made of camel or goat's hair, and fustane is a fabric made of cotton. 


\section{Crucifixes and Crosses in Medieval Norway}

When the vicar Nils Sverdrup made an inspection par curiosité of the then damaged cathedral in Hamar in 1707, he revealed that the cathedral's crucifix had a cavity in Christ's head and swamp-like material in the area around the eyes (Hamar-krøniken 1937, pp. 90-91). These features-interpreted by Sverdrup as pia fraus, a pious fraud-testify to a sculpture able to cry, possibly serving as a prop in liturgical plays. The tears may also have been sought for healing of ailments, meaning the crucifix functioned in analogous manner to the more famous miracle-working crucifix in Røldal (c.1250) (Figure 1). Purportedly sweating during Midsummer, the Røldal crucifix caused pilgrims to flock to the mountain church to touch it with linen cloths (Bondevik 1935; Blindheim 2004b).

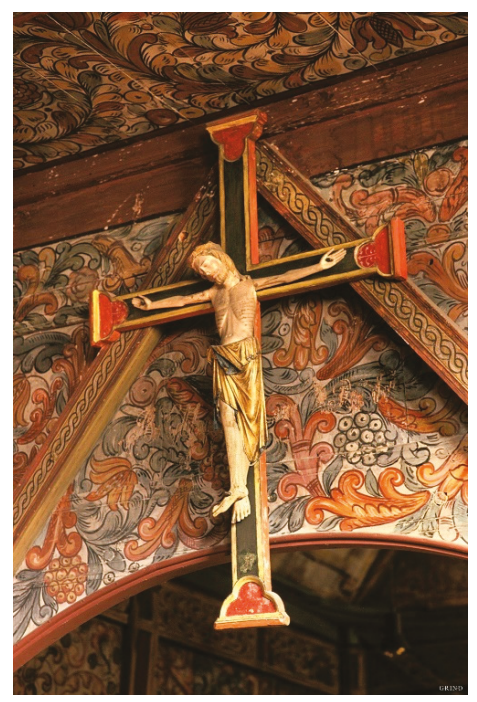

Figure 1. Crucifix from Røldal. Polychrome oak, c.1250. Røldal church. Photo: Svein Nord/Grind.

Triumphal crosses could also work miracles. Although no actual miracles are actually recorded, the large Triumphal Cross (c.1200) in the Borre parish church was a tremendously popular pilgrimage site in medieval Norway (Figure 2). The cross received testamentary gifts (DN 11,41) and was among the holy crosses mentioned as sites that Queen Margrethe (d.1412) wanted to be visited on her behalf after her death, according to a letter from 1405 (Jørgensen 1909, p. 39; Blindheim 2004b). Allegedly, the Borre cross had relics in the form of pieces from the True Cross inserted in the crossing of its wooden structure (Hohler 1994), thus it would be most efficient in bestowing the medieval creation with the features of its holy prototype (Fisher 2006, p. 49). ${ }^{5}$

5 Apart from the two crucifixes in Hamar and Røldal and the Borre cross, there is also a cavity on top of the head and incised tear channels in the figure of St Anne in the late medieval imported sculpture group of Virgin and Child with St Anne (c.1520, of unknown original display, now preserved at the Museum of Cultural Heritage, Oslo), betraying agencies similar to the ones ascribed to the crucifix from Hamar. This perceived animation, however obviously post-date the kinds of devotional activities considered for this essay. 


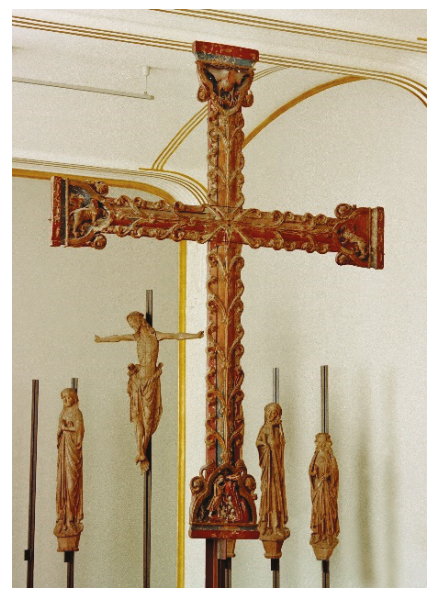

Figure 2. Triumphal Cross from Borre. Polychrome oak, c.1200. Oslo, Museum of Cultural History, Inv. No. C6130. Photo: Ann-Christine Eek (CC BY-SA 4.0).

Although the crucifixes from Hålandsdalen and Ylmheim are not preserved, more than 100 crucifixes made in Norway between c.1150 and c.1350 are still extant (Blindheim 1998; Blindheim 2004a; Brendalsmo et al. 2001). Some of these are still in situ, and others are in museum collections. Differing in style, material and iconography as well as in the amount of gilding and polychromy, they all share the feature of not comprising silver arm rings or other kinds of jewellery. Moreover, due to repainting and even replacement of arms, they hardly betray any traces of such accessories. The crucifix from Dal (c.1225-50) is remarkable in having shoes, a belt with a buckle, gloves and bracelets all painted in red bolus with black contours; the bracelet on Christ's right arm is both carved and painted (Figure 3). A mutilated crucifix from Sandeid has traces of similar decoration, one bracelet around the arm and one around the wrist. (Figure 4). The crucifix from Aulstad (c.1250) could be indicative, too. Placed within a circular frame, the Christ figure has one cut in the elbow area on each arm, which may correspond to the position of actual silver rings, although differing from the carved or painted bracelets in the Christ figures from Dal and Sandeid (Figure 5).

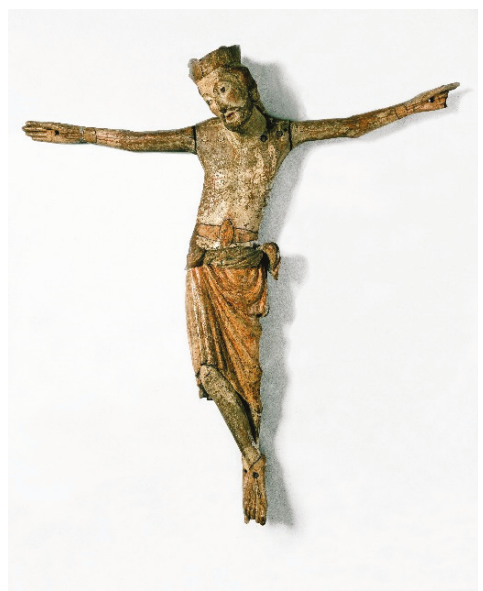

Figure 3. Crucifix from Dal. Polychrome oak. Polychrome oak, 1225-50. Oslo, Museum of Cultural History, Inv. No. C6130. Photo: Eirik Irgens Johnsen (CC BY-SA 4.0). 


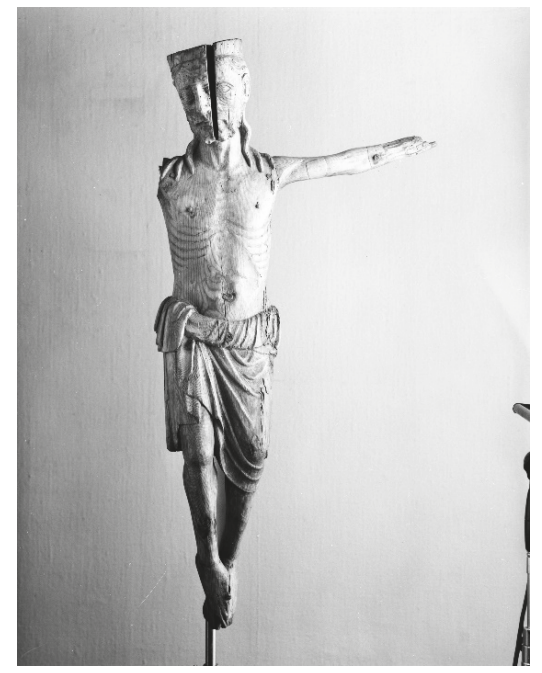

Figure 4. Crucifix from Sandeid. Polychrome birch, 1225-1250. Bergen, University Museum. Photo: Ørnulf Hjort-Sørensen (CC BY-SA 4.0).

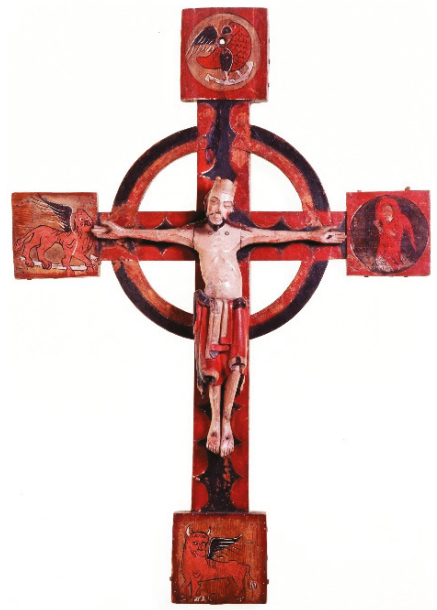

Figure 5. Crucifix from Aulstad. Polychrome oak, 1200-1220. Oslo, Museum of Cultural History, Inv. No. C33287. Photo: Erik Irgens Johnsen (CC BY-SA 4.0).

The Sandeid crucifix also wears carved shoes and there are remnants of metallic shoes on the crucifix from Gjerstad (Jernæs 2016, p. 14). Shoes also appear on a handful of crucifixes from medieval Sweden, namely Säterbo, Kiaby, Linde, Anga and Stenkumla (Blindheim 1986, p. 84). Moreover, a crucifix in Kliplev in Denmark, referred to as St Saviour in Queen Margrethe's letter, but only known from post-medieval descriptions, apparently had a crown of gold as well as golden gloves and shoes (Jørgensen 1909, p. 40). Shoes are also found on the Volto Santo in Lucca and the many replicas of it. It is difficult to tell how the pxll in Ylmheim was arranged; it may have formed part of the loincloth drapery or made up a full liturgical vestment similar to the painted tunica found in the mid- to late twelfth-century crucifixes in places as distant as Väversunda in Sweden and the region of Ripollès in Catalonia (Kroesen et al. 2019, pp. 138-40). A small crucifix from Limoges found in Oslo also wears 
tunic (Figure 6). ${ }^{6}$ Whereas shoes, loincloths or liturgical vestments feature in crucifixes in various parts of Europe, the painted/carved insignia of Dal and Sandeid-and the silver bracelets indicated as parts on the now lost crucifixes in Hålandsdalen and Ylmheim-have no equals neither in Scandinavia nor in Europe.

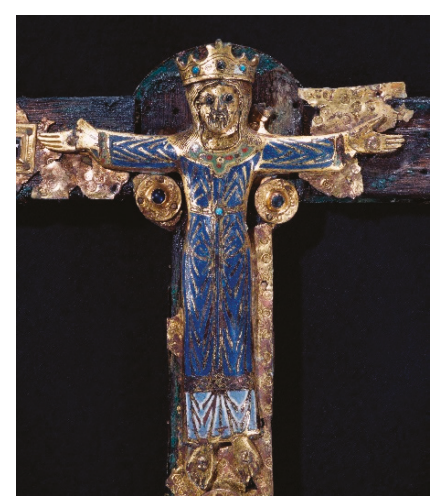

Figure 6. Limoges crucifix. 13th century. Oslo. Museum of Cultural History. Inv. No. 21300. Photo: Not identified (CC BY-SA 4.0).

Before turning to medieval devotion to the cross and/or to crucifixes, a few small scale metal crucifixes pre-dating the inventories by two centuries will be brought into the discussion insofar as they are material witnesses to early medieval practices of sculpture embellishments. The Birka-crucifix (Figure 7), a silver filigree pendant from the tenth century, is allegedly the oldest representation of Christ in Scandinavia. It was found in a grave in 1879. Due to the materials employed, it is difficult to decipher if the rings on the arms are intended as bracelets, as part of an intentional clothing, or if they facilitated the inclusion of fingers (Staecker 1999, pp. 491-93). They may even indicate that Christ was tied to the cross in the same way as does the rings on the pendant crucifix from Lilla Klintegårda indicate (Staecker 1999, p. 478). Two eleventh century pendants found in Trondheim are equally elusive, yet the one with a less static pattern could have intentionally been shaped as a crucifix with bracelets (Figure 8). The Danish Åby-crucifix, made of wood and gilded bronze around 1100, has no signs of bracelets, but it clearly wears a large collar (Figure 9), thereby indicating crucifixes could at times be invested with jewellery. Or, more specifically, it indicates accessories in the form of jewellery could be included as an integral part of a gilded crucifix. A conceptual correspondence between the collar and the silver bracelets in Hålandsdalen and Ylmheim would thus suggest that the silver bracelets were of an inalienable nature, included as part of the polychromy and the gilding.

6 Post-dating the inventories, two entries from the Icelandic diploma allows us to identify two crucifixes in the church of St Mary at Alftanes, «one cross dressed and one undressed» (1354) and one in Vallanes which was fitted with a suitable chasuble and amictus (1448). 


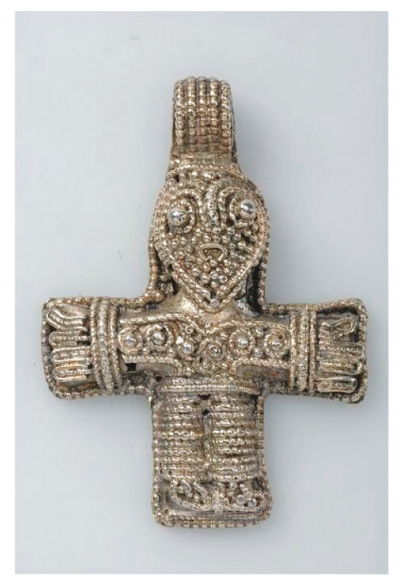

Figure 7. Crucifix from Birka. Silver pendant, 9th century. Stockholm, SHM, Inv. No. 34 000. Photo: Gabriel Hildebrand (CC BY 2.5. SE).

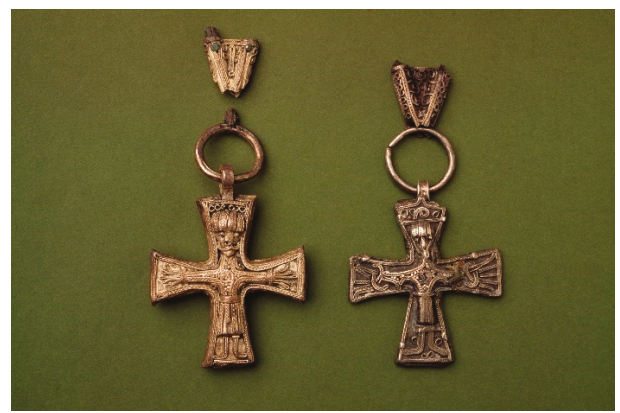

Figure 8. Crucifixes from Trondheim. Silver pendants, 11th century. Trondheim, NTNU University Museum, Inv. No. T16978A and T16978B. Photo: Per E. Fredriksen.

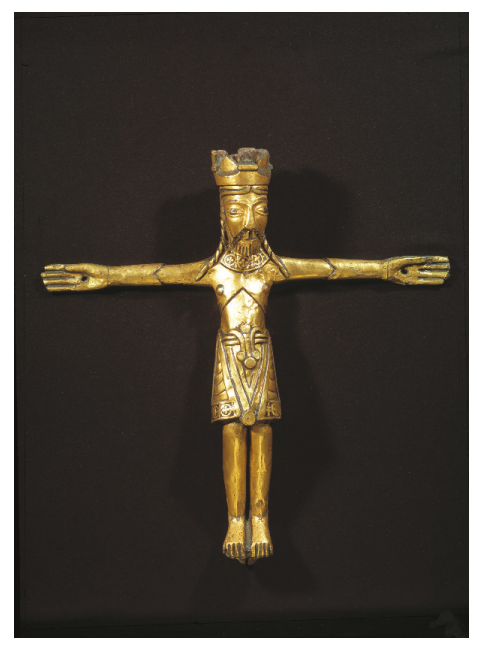

Figure 9. Crucifix from Åby. Gilded silver on wood, c.1100. Copenhagen, National Museum of Denmark, Inv. No. D629. Photo: Lennart Larsen (CC BY SA). 
We cannot exclude the possibility that the silver bracelets were included as modest attempts of investing the two crucifixes with the same delight of sensory experience as was the collar on the Åby crucifix. Such delight is known also in the form of multi-coloured gems on early medieval processional crosses, such as the ones known as the Cross of Justin II, the Desiderius Cross and the Lothair Cross, or even the now lost cross from Saint-Denis, eulogized by Abbed Suger in his De Adminastrione (Carruthers 2013, pp. 39-41). It is more likely, however, that the bracelets were a post-productional, alienable addition. Indeed, even the painted and carved bracelets in Dal appears as being secondary to the Christ-figure itself. The silver brooch (silfr sylgia) mentioned as part of the Calvary group (the crucifix with the Virgin and St John) in Hålandsdalen appears to be alienable, too. Medieval costume tradition in general and the placement of brooches made of rock crystal or polished glass, for example the ones incorporated in the Virgin in the golden altar from Lisbjerg (c.1100) or the Grong Madonna (13th century, Figure 10), suggest the silver brooch on the Calvary group in Hålandsdalen was positioned on the chest of the Virgin.

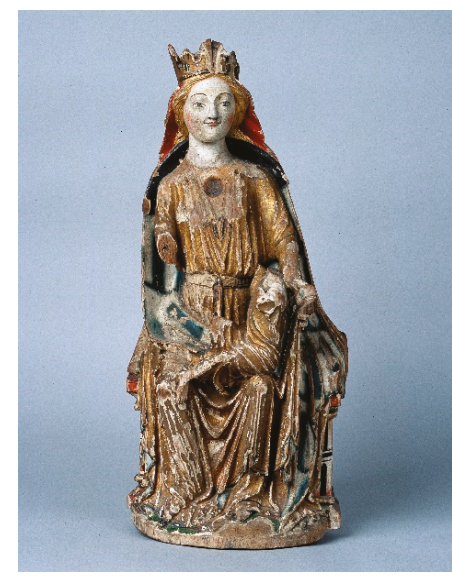

Figure 10. Madonna from Grong. Polychrome oak, 13th century. Trondheim, NTNU University Museum, Inv. No. T1947. Photo: Per E. Fredriksen (CC BY-SA 4.0).

\section{Medieval Devotion to the Cross}

The liturgy of the medieval parish church was codified in the Latin West in the early 13th century, emphasizing the doctrine of the transubstantiation established in 1215 . The ritual space where all celebrations took place followed suit in order for ecclesiastics to better communicate the significance of the Eucharist (Rubin 1991; Catto 2000). The liturgical centrality of the crucifix in the church would also prevail also in the following centuries, although in terms of image devotion, a number of complementing devotional practices revolving around images appeared from the late fourteenth century onwards. These practices were often related to private prayer and indulgences and favoured images such as the Man of Sorrows, the Wounds of Christ and the Virgin of the Rosary (Belting 1981; Ringbom 1984; Duffy 1992; Van Os et al. 1994; Kaspersen and Hastrup 2004; Marks 2004; Skinnebach and Laugerud 2007; Laugerud et al. 2016; Blick 2019).

In the Old-English Poem The Dream of the Rood, however, the Cross is the protagonist, the victory-beam into which the narrator trusts his hope of protection, assured that devotion to the Cross or crucifix would ensure entry to heaven. The poem has been situated in connection to liturgical drama, such as the Easter Adoratio Crucis from the Winchester Regularis Concórdia (c.973) (Holloway 1984; Ó Carragáin 2005). Applied for the celebration of Good Friday, the liturgy was also adopted in Norway as attested by two preserved twelfth-century manuscript fragments in the University Library in Bergen, the Mi 12 and the MS 1549, 2 (Gjerløw 1970). It is very likely the ritual was still current in 
the early fourteenth century and celebrated in Hålandsdalen and Ylmheim alike, yet in a style more suitable for smaller parish churches (Helander 2001).

Moreover, as the cross was both the instrument of Christ's death as well as the bringer of eternal life, in sculptural form crucifixes would serve "as paths of divine power of those who venerated them" (Smith et al. 2001, p. 599). The very famous Volto Santo in the cathedral of Lucca cathedral (Figures 11 and 12) is but one of many medieval crosses on public display that offered paths of divine power and generated fervent devotion. It is included here not as an example of an animated crucifix or as a prop in religious processions, but as a reference point for continental devotion to the cross as the Volto Santo proper and its miraculous capacities were known also in fourteenth-century Scandinavia.

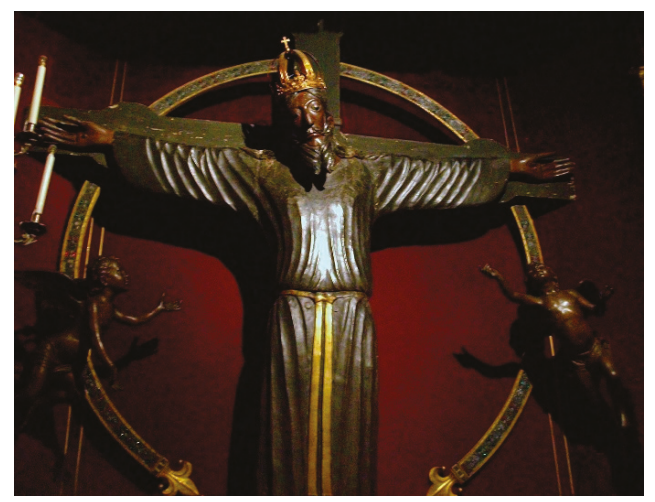

Figure 11. The Volto Santo. Polychrome wood, c.1200. Lucca cathedral. Photo: Wikipedia Commons.

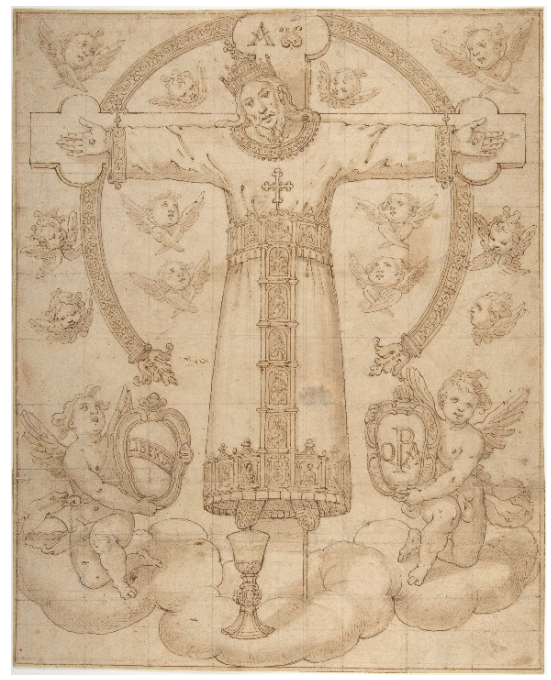

Figure 12. The Volto Santo in Lucca cathedral. Anonymous artist. Drawing, 16th century. New York, The Metropolitan Museum of Art, Inv. No. 61.211. Photo: The Metropolitan Museum of Art/Creative Commons.

Enjoying great popularity from the end of the eleventh century, possibly occasioned by the consecration of the rebuilt cathedral in 1070, the legend has it that the Volto Santo is an acheiropoietoi, an object not made by human hands. The present crucifix, however, is thought to have been carved by an artist from the circle of Benedetto Antelami around the turn of the thirteenth century. The Christ 
figure is of the triumphant type, accentuating the majestic, royal and divine aspects of Christ at the expense of his suffering and human nature. There is a cavity between the shoulders at the back, which, according to the Leobinus legend, previously held a portion of the Crown of Thorns, Christ's sudarium and an ampulla with Christ's blood. The miracle-working potential of the Volto Santo stemmed both from the crucifix being a true image and from its reliquary aspect (Kurz 1997; Il Volto Santo 2003; Matyjaszkiewicz 2018). The crucifix itself and illustrations telling the legend of its arrival in Lucca were both widely diffused from the turn of the fourteenth century to large parts of Europe (Martinelli 2016). Jean-Claude Schmitt has even proposed that the diffusion of the crucifix allowed for the sculpted simulacrum of Christ's true face image to be repeatedly recreated in a manner similar to the way the consecrated host constantly transubstantiates into the body of Christ (Schmitt 1995).

The distance between Lucca and the parish churches in Hålandsdalen and Ylmheim may seem too far for the former to have been able to influence the perception of crucifixes as clothed and/or miracle-working sculptures in the latter two. Nevertheless, the cathedral and the Volto Santo would have been known in the Nidaróss archbishopric at the time when the crucifixes were carved and the inventories were written as they are both mentioned in the Leiðarvisir, an itinerarium compiled by Nikulás Bergsson after a pilgrimage from Iceland to the Holy Land. The pilgrimage took place in the earlier parts of the 1150s. Nikúlas returned to Iceland in 1154 and became abbot at Munkapverá the following year. Listing the various places one would stop along the route, and the distances between them, the itinerarium also contains a mix of Christian themes and pagan legends. From the cathedral in Lucca, Nikúlas highlights the crucifix: “ $(.$. ) the crucifix made by Nicodemus from Christ's true face; the crucifix has spoken twice; one time it offered shoes to a poor man and another time it testified on behalf of an accused man" (Symbolae 1832, p. 20). ${ }^{7}$ Nikulas also mentions his compatriots' involvement in the city: "He [king Eiríkr] left an endowment in Lucca, so that anyone speaking Nordic languages could drink fully and freely, and he built a hospice eight miles south of Piacenza, where they could refresh themselves" (Symbolae 1832, pp. 27-28). ${ }^{8}$

As it were, Nikulas transferred knowledge of the cathedral Lucca, the Volto Santo and two miracles evoked by it and encouraged his countrymen to travel there, advocating the hospitality everyone speaking Danish would receive. It is probably this house or one of similar status that is mentioned in a letter from Pope Martin V to Archbishop Jon Raude in 1282 (DN 6, 46). Unfortunately, there is no information in the travelogue about how devotional acts would take place in its vicinity. Nonetheless, information about later indulgences such as the 40 days indulgence granted by Bishop Enrico in 1309 and contemporary devotional activities in the form of money and material goods given to support the maintenance of the cathedral and the chapel (Il Volto Santo 2003, pp. 131-72; Matyjaszkiewicz 2018) may have spread north as connections between Lucca and Norway continued in the thirteenth and fourteenth century (DN 19, 322; DN 19, 325 and others). The shoes found on some of the Scandinavian crucifixes may relate to the shoes on the Volto Santo, either as they come fore in visual replicas spreading from the early fourteenth century or through the diffusion of texts telling the miracle stories.

\section{Arm Ring Symbolism and Miracle Working Crosses in Old Norse Texts}

In her book Medieval Life: Archaeology and the Life Course, Roberta Gilchrist has appropriately evidenced how domestic settings housed a wide range of material objects with religious content, for example clasps from prayer books, spoons and brooches with inscriptions, pendants and rings. Many of these objects also have inscriptions and were to be carried close to the body (pendants), or to be

7 Dagför or Lunu til Luku; par er Biskopsstoll at Mario kirkiu [i.e., San Martino], par er roda su er Nichodemus let gera eptir Gudi sialfum; hon hefir ii. sinnum mælt, annat sinn gaf hon sko sin aumun manni, enn annat sinn bar hon vitni rægdum mann

8 Hann Eirikr Danakonungr Sveinsson lagdi fé til i Luku, at, hverr madr skylldi drecka vin okeypiss ... einu af danski tunugu ok hann let gera spital viii milum sudr fra Plazinzoborg. 
momentarily close to the body as utility goods (spoons, jugs) (Gilchrist 2012, pp. 154-68). Excavations from Perth have shown similar patterns of dispatched items with religious content in household areas (Hall 2011). On the other hand, presumably secular items may have been invested with religious meaning in ways similar to how objects such as swords were considered as animate in certain contexts in the Late Iron and Viking Ages (Lund 2017 with references). For this article, arm rings and golden rings are important cases in point.

What did it mean to have a ring around one's arm? In Viking Age Scandinavia, arm rings in precious metal were both a symbol of power and a payment method. Dated to the third quarter of the ninth-century, the Hoen hoard demonstrates some of the richness and typologies of Viking metalwork, containing one necklace, two neck-rings, three arm-rings, one finger-ring and a brooch formed of a reused strap mount (Fuglesang and Wilson 2006). From the Old Norse sagas we know of several examples of arm rings used by the one exercising power. In the Saga of the Ere-Dwellers, the events unfold at the end of the 10th and the beginning of the 11th centuries (written down only in the 13th century), Thorolf erects a house on Iceland and "off the inmost house was there another house, of that fashion whereof now is the choir of a church, and there stood a stall in the midst of the floor in the fashion of an altar, and thereon lay a ring without a join that weighed twenty ounces, and on that must men swear all oaths; and that ring must the chief have on his arm at all man-motes" (The Saga of the Ere-Dwellers, chapter 4). Arm rings are also mentioned in the scene when Gudleif and his men are allowed to leave the land onto which they sailed without cause "and or ever he and Gudleif parted, he drew a gold ring from off his arm, and gave it into Gudleif's hand, and therewithal a good sword, and then spake to Gudleif: 'If it befall thee to come back to thy fosterland, then shalt thou deliver this sword to that Kiartan, the goodman at Frodiswater; but the ring to Thurid his mother'" (Saga of the Ere-Dwellers, chapter 64).

From medieval Norway, arm rings appear, for example in Fagrskinna, the genealogical enumerating of Norwegian kings (written around 1220). “Now there came to light a lump of gold as big as a man's head. King Haraldr took it up and said: 'Kinsman, where is the gold to be exchanged for this knob?' Then the king replied, 'Unrest and heavy levies have brought it about that almost all the gold and silver has been given to the retainers, and now there is no more gold in my possession than this ring,' and he took it from his arm and gave it to Haraldr" (Fagrskinna 2004, p. 196). Arm rings were among the foremost symbols of worldly power whereas finger rings were given as gifts. Archaeological evidence sustains these assumptions in as far as rings prefigure more often in graves (Staecker 2006; Glørstad and Wenn 2017).

Miracle-working crosses are also known from the Old Norse saga literature. A relic from the Holy Cross was brought to the north by King Sigurðr which he placed in a church he erected in Konungahella (Magnússona saga 2015, pp. 156-57). Consecrated when Sigurðr had been king for twenty-four years, Krosskirkja was consecrated and embellished with a golden altar frontal (Magnússona saga 2015, p. 169). During an attack by Wendish forces led by King Réttiburr, the church was sacked and burnt; yet, the priest Andreas was allowed to keep the Holy Cross and some other valuable items. When the heathen parted, however, they were struck with fear by a portent as the priest had appeared with the Holy Cross, explaining: "And there is so much power in the Cross that such miracles have often taken place upon heathens, in some cases much plainer ones, when they have taken it in their hands" (Magnúss saga blinda 2015, p. 181).

In addition to saga literature, there are also traces of both power structure and divine power in sacred texts. The Old Norse Homily book was written around 1200 in Bergen, possibly at the cathedral chapter. Given its content, it is now better considered a homiletic handbook, its content often being more similar to sermons; sermons for both the Feast Days concerning the Cross-the Invention of the Cross (3 May) and the Exaltation of the Cross (14 September)-are included. From the Invention of the Cross, the sermon concludes with the following lines:

The Holy Cross is a shield for all danger, support for all misery, in sorrow it is a comfort and in joy an encouragement for the good; it is protection from danger, healing from ailment 
liberation from all ties, counsel from sin, victory in battle, strength against temptation, powerful for the poor and a chieftain for the powerful (stjórn auðigra) $(\ldots)$. All this is due to God's mercy, for the one who on the Cross redeemed mankind from death-Jesus Christ. Him be praised with the Father and the Holy Spirit per omnia saecula saeculorum amen.

(Gammelnorsk homiliebok, 109)

The section does mention an array of the functional uses of a cross. The reference to Christ as the 'chieftain of the powerful' (stjórn auð̇igra) is compelling as it seemingly integrates the ruling aspect of Old Norse society in a text legislated by the church. Nevertheless, Christian ideas would also occasionally occur in the literary production of Snorri Sturlusson and other thirteenth-century writers, even if sagas in general maintained the societal values emphasized in Old Norse literature (Bagge 1998, pp. 66-69; Bagge 2010, p. 153). The silver bracelets, therefore, could be considered a materialized coupling of the two cosmologies' ruling systems in visual terms, and the power symbols of chieftain may have been recognised as such when attached to the Christ figure on the cross.

Due to the dates given in the inventories, the crucifixes with silver bracelets must pre-date 1306 and 1321 respectively. It is thus likely that the crucifixes would have been similar to the Dal, Sanneid or Aulstad ones, having the Christ figure standing upright with parallel legs, one nail in each foot, arms horizontally stretched to either side and carrying a crown; that is as Christus triumphans, as the victorious king. Accordingly, Blindheim considered the insignia of Dal and Sandeid as reflecting practices related to contemporary kings, that is the investiture of insignia at the coronations of Norwegian kings in the second half of the 12th and the first half of the 13th centuries (Blindheim 1986, p. 90). The material value and mobility of the silver bracelets, however, allow for further interpretations. Even if the silver items did indeed reflect the coronation of a king, the act of placing the items on the arms of Christ may still have been a devotional act.

\section{Silver Bracelets-Devotion, Gifts and Gender}

As it were, material gifts were often offered to sculptures in churches as pious donations and according to contemporary wills, predominantly by female testators. In 1325, a Tournois woman stated in her will that she wanted "my good tissue to care for the image of Notre-Dame in Tournai." ${ }^{9}$ Richard Trexler almost laconically notes this kind of testamentary gift "n'est pas atypique" (Trexler 1991, p. 196) and a similar pattern of pious bequests in wills can be traced across medieval and early modern Europe (Howell 1996; Kamerick 2002, pp. 69-105; Marks 2004, pp. 183-85; French 2008; Jürgensen 2018, pp. 501-12). In her work on English parish churches, Katherine L. French has analysed not only wills, but also churchwarden accounts and guild accounts. Her findings, too, proved women's relation to the parish to be structured by their ability to offer their labour and donate household goods such as clothing, jewellery and knives (French 2008, pp. 37-48).

Also from medieval Norway a few practices come to light, although from a slightly later date than the inventories. In 1349, a woman called Ingebjørg Munansdatter donated a headscarf (velum), not to her parish church but to the Munkeliv monastery, in order for the convent to be able to wrap God's body (DN 11, 7). It has been suggested this textile was a Hungertuch, intended to cover the crucifix during Lent as the sight was supposed to abstain from bodily participation in the divine pleasures during this observance (Stang 2015, p. 151). It could, however, be Ingebjørg was actually donating a corporal, a cloth intended to cover the host. As testified by the inventories from Hålandsdalen and Ylmheim, the majority of the entries concern textiles. In a will dated 14 July 1400, however, Jon Marteinsson had a sculpture of St Lawrence in Foss Church and a sculpture of St Margaret in Lund church "coloured," probably meaning that he paid for the repainting or refurbishing of two sculptures worn and torn (Stang 2015, p. 152). These two documents alone cannot be given statistic value, but as

9 "Men boin tissut pour caindre de ymagène Notre-Dame di Tournay." 
it happens, they are in agreement with the late medieval European trend of complementing devotional gifts to the cross or to Christ's body with donations to images of saints (Marks 2004; French 2008, p. 43).

Caroline Bynum summarized the gendered dynamics of gift-giving as "men gave up money, property and progeny; women gave up food" (Bynum 1988, p. 193). For Ylmheim we are fortunate enough to have a preserved diploma from 1336 evidencing a transaction of lands from a lay person to the church and its priest (DN 15,16). Similar transactions are preserved for other parishes and priests; most transactions were initiated by men, but there were also a significant number of women who addressed these property issues. Documents from Oslo bishopric, however, show that women gave lands to the chapter and nunneries, whereas men gave properties to parishes, possibly because they were expected to demonstrate their power in their local communities (Emanuelsson 2005; Andersson 2010). From the sources available, people's engagement with their parish include to have sculptures repainted when they were losing colour, to donate velum to protect either the crucifix or the host, and to donate properties. There is not enough surviving evidence to ascertain whether lay people made pious bequests to their parish crucifixes in the form of silver arm rings.

One reason people would give portable wealth such as money, jewellery and items of metal was that these gifts were more flexible than land in as far as they could be bartered, melted down, refashioned or reminted. Moreover, placing family jewellery in the church meant the objects were not available for gift-exchange or trade in the secular sphere and were likewise out of circulation as inter-generational objects. As evidenced in the 1920s by anthropologist Marcel Mauss in his seminal work "Essay sur le don," gift-giving-also when in guise as pious bequest-is as much about reciprocity as about charity (Mauss 1923). Mauss' text paved the way for researchers from a range of academic disciplines in establishing the importance and political statement of gift-giving within different communities across temporary and geographical boundaries. In later anthropological frameworks, reciprocity appears as the more superficial dynamic, whereas the more powerful aspect of gift-giving lies in the politics of "keeping-while-giving" (Weiner 1992), or indeed as "an intimately political and double-faced discourse" (Palsson 2010, p. 24).

In the Saga of the Ere-Dwellers, Thorgunna opts for the keeping-while-giving strategy when dividing her property; and she does so in a manner worth exploring in more detail.

This would I have done: I would be borne to Skalaholt if I die of this sickness, because my mind tells me that that stead will be for one while the most worshipped stead in the land; and I wot also," says she, "that there will be priests to do the singing over me; so I pray thee to bring me there, and of my goods shalt thou [Thorod] have so much as that thou wilt have no loss thereby; but from my undivided goods shall Thurid have the scarlet cloak that I own; and this I do to the end that she may be content that I see to my other goods in such wise as I will; but I will that thou take for the cost thou hast for me that which thou wilt, or that pleases her, from such things alone as I leave thereto. A gold ring I have which shall go to church with me, but I will that my bed and my bed-hangings be burned up with fire, for that they will be of no good to any man; and I say this not because I grudge anyone to enjoy those good things, if I knew that they would be of good avail to any; but now I say so much thereover," says she, "because I deem it ill that folk should have so much heavy trouble from me, as well I wot will be, if ye turn away from that which I now ordain."

\section{(Eyrbyggja saga, chapter 51)}

The actual meaning of bringing 'the gold ring to church with her' may be disputed, but it seems likely that she gave it to the church when compared to the many examples of English women giving rings to the parish (French 2008, pp. 48,67). As such, it was not given to another individual for personal use in the same way as was her scarlet cloak, nor is it decomposed as should be the bed-hangings. It is possible that the silver bracelets documented on the Christ figures on the two crucifixes had, at one point, gone to church upon someone's death, too. The silver bracelets may also have come as part of distributed goods in the manner evidenced in the form of wills and letters (Bagge 2010, p. 
153) - and the brief mention in Grettir's saga "But Thorstein and Spes divided their share of the goods, and some they gave to churches for their souls' health, and some they took with them. Then they betook themselves Romeward, and many folk prayed well for them" (Grettir's saga 1900, chapter 94).

Whereas precious objects were given to the church for spiritual benefits on part of the benefactor, gifts to the images in the church also invested the image with an increased material as well as spiritual value. As posited by Ralph Dekoninck in relation to images of the Virgin, due to the rule of decorum, it is advised not only to honour images of the Virgin but also to embellish them with the most precious objects in order to materially express this honour. Moreover, such honouring practices would partly conceal the more mundane materiality of the sculpture itself (wood), in turn allowing the material surplus (silver bracelets) to enhance the power of the images (Dekoninck 2012, p. 162). Dekoninck thus follows Thomas Golsenne who contended that "[a]dornment does not follow the miracle, but makes possible the belief that a miracle has occurred through a given image" (Golsenne 2010, p. 82).

If the crucifixes in Hålandsdalen and Ylmheim evoked miracles, these were never recorded, neither in public documents nor in local folklore. This silence, however, does not mean the crucifixes were not efficacious for some people asking for assistance, and thus later wishing to reciprocate the crucifixes by having some family valuables given to them. Roberta Gilchrist noted that "People came to believe in the quasi-magical properties of a particular object because it has been consecrated by a priest or had been in close physical proximity to statues of saints" (Gilchrist 2013, p. 179). There is evidence from England that such gifts could periodically be lent back to the domestic sphere, for example as amulets during childbirth (Gilchrist 2013, p. 178), at times even abusing this privilege (French 2008, pp. 31-32), but we lack evidence of similar displacements of objects in medieval Norway. The embellishments of the crucifixes in Ylmheim and Hålandsdalen could thus be considered as domestic devotional interventions in the religious material culture; firstly in order to make the images more powerful in their religious settings, secondly to allow for this power to be deposited to domestic settings when needed. Ultimately, the crucifixes may have been embellished with bracelets and brooches as "decoration is often essential to the psychological functionality of artefacts" (Gell 1998, p. 74). A rather suggestive idea to be drawn from this is that the bracelets-and the brooch-were given to the crucifixes, not only to invest the crucifix itself with power or decorum, but also to have the bracelet receive the already inert power of the crucifix.

\section{Concluding Remarks}

The anonymous compilers who penned down the inventories of Hålandsdalen and Ylmheim both took their time to comment on the silver arm rings adorning the arms of the crucifixes in either church, and also to note that it was a brooch attached to the Virgin in Hålandsdalen. Although the crucifixes themselves have not been preserved for posterity, these entries allow us to deduce that such adornment was once a feature enjoyed by at least these two and probably more crucifixes that were made before 1320. The inventories' rather elusive nature obscures what the full significance of the silver bracelets and the silver brooch were, and our understanding is further complicated by the fact that not only are the crucifixes in question long gone, but also by the fact that none of the preserved crucifixes dating from $c .1150-c .1320$ have silver arm rings around their arms.

Although the inventories offer sparse information about the crucifixes, their appearance, their role in the celebration of Mass and their role in devotional activities may be hypothesized from cross-references with other Norwegian medieval crucifixes known to have been able to cry or to evoke miracles. Furthermore, the painted and carved bracelets on the crucifixes from Dal and Sandeid, as well as the cuts on both arms across the elbow area in the Christ figure on the crucifix from Aulstad, may be taken as material witnesses of the descriptions in the inventories. The extensive and long standing documentation of cultic practices relating to crucifixes elsewhere in the Latin West, such as the Volto Santo in Lucca cathedral, and analogies drawn from devotion to the crucifix and devotion to the cross as implied in Old Norse homiletics, also add to our understanding of the two crucifixes. 
Moreover, analogies with the symbolism of arm rings in saga literature and with the practice of gift-giving and creation of heirlooms and amulets puts the crucifixes into a relief.

The silver bracelets may have been included when the Christ figure was made, as alienable parts of its feature and equal to its polychromy. It seems more likely, however, they were placed there at a later point in time as a way of updating or embellishing the Christ figure, or as a strategy of investing the figure with a powerful symbol from the secular world. They may also have been inalienable, given as gifts or placed on the arms as a way to keep precious objects out of circulation, or to invest them with the spiritual power inherent in the holy sculpture. In as much as they decorated the church interior or the Lord's table, the crucifixes seem to have also acted as keepers of treasures, but there is no way of knowing if the silver items were deliberately relocated to the secular sphere or if they were sold, melted or stolen.

Funding: No external funding was received for the writing of this article.

Acknowledgments: The author would like to thank Salvador Ryan for his encouragement and patience, the anonymous reviewers for their questions and comments, and Ildar Garipzanov, Max Naderer, Ole-Albert Rønning Nordby and Kristina Dziedzic Wright for valuable and much appreciated feedback.

Conflicts of Interest: The author declares no conflict of interest.

\section{References}

\section{Primary Sources}

Diplomatarium Islandicum. 1857-1976. Reykjavik and Copenhagen: Hið íslenska bókmenntafélag. https: //baekur.is/search.

Diplomatarium Norvegicum. 1847-2011. Oslo: Riksarkivet. https://www.dokpro.uio.no/dipl_norv/diplom_felt.html. Fagrskinna: A Catalogue of the Kings of Norway. 2004. Translation and introduction by Alison Finlay. Leiden: Brill. Gammelnorsk homiliebok. 1972. Translation by Astrid Salvesen and introduction and comments by Erik Gunnes. Oslo: Universitetsforlaget.

Leiðarvísir, an Old Norse itinerarium: A Proposal for a New Partial Translation and Some Notes about the Place-Names. Translation and Comments by Luana Giampiccolo. https://skemman.is/bitstream/1946/14573/ 1/Lei\%C3\%B0arv\%C3\%ADsir.pdf.

Grettir's saga. 1900. Translated by William Morris and Eirikr Magnusson. https://sagadb.org/grettis_saga.en.

Hamar-krøniken med andre kilder til kunnskap om det gamle Bispesete ved Mjøsen. 1937. Published by Arne Arnesen. Oslo: Foreningen for Norsk Bokkunst.

Magnússona saga. 2015. In Snorri Sturluson. Heimskringla, Vol. III. Translated by Alison Finlay and Anthony Faulkes. London: University College London, pp. 147-69.

Magnúss saga blinda ok Haralds gilla. 2015. In Snorri Sturluson. Heimskringla, Vol. III. Translated by Alison Finlay and Anthony Faulkes. London: University College London, pp. 170-86.

Páls biskups saga. 1858. In Biskupa sögur. Vol. 1. Copenhagen: Hið íslenska bókmenntafélag, pp. 125-48.

Eyrbyggja saga. 1989. Translated by Hermann Pálsson and Paul Edwards. Harmondsworth: Penguin Classics.

Symbolae ad geographiam medii aevi, ex monumentis islandicis. 1832. Publication, introduction and comments by Erik Christian Werlauff. Copenhagen: Gyldendal.

\section{Secondary Sources}

Algazi, Gadi, Valentin Groebner, and Bernhard Jussen. 2003. Negotiating the Gift: Pre-Modern Figurations of Exchange. Göttingen: Vandenhoeck and Ruprecht.

Andersson, Catharina. 2010. Andliga gåvor i världsligt bruk-Om den religiösa gåvan som social och maktskapande handling [Spiritual gifts in secular contexts-About the religious gift as social and powerful act]. In Gaver, Ritualer, Konflikter. Et Rettsantropologisk Perspektiv på Nordisk Middelalderhistorie [Gifts, Rituals, Conflicts. An Anthropological Perspective on Nordic Medieval History]. Edited by Hans Jacob Orning, Kim Esmark and Lars Hermansson. Bergen: Fagbokforlaget, pp. 39-75. 
Bagge, Sverre. 1998. Mennesket i middelalderens Norge: Tanker, tro og Holdninger 1000-1300 [People in medieval Norway: Thoughts, belief and attitudes 1000-1300]. Oslo: Aschehoug.

Bagge, Sverre. 2010. From Viking Stronghold to Christian Kingdom. State Formation in Norway, c. 900-1350. Copenhagen: Museum Tusculanums Forlag.

Belting, Hans. 1981. Das Bild un sein Publikum. Form und Funktion früher Bildtafeln der Passion [Likeness and Presence: A History of the Image before the Era of Art]. Berlin: Mann Verlag.

Blick, Sarah. 2019. Bringing Pilgrimage Home: The Production, Iconography, and Domestic Use of Late-Medieval Devotional Objects by Ordinary People. Religions 10: 392. [CrossRef]

Blindheim, Martin. 1986. Skandinaviske krusifiks med verdighetstegn [Scandinavian crucifixes with insignia]. In Festskrift til Martin Blindheim [Tributes to Martin Blindheim]. Edited by Irmelin Martens, Bjørn Myhre, Eldrid Straume, Per Jonas Nordhagen and Erla Hohler. Oslo: Universitetets Oldsaksamling.

Blindheim, Martin. 1998. Painted Wooden Sculpture in Norway c.1100-1250. Oslo: Scandinavian University Press.

Blindheim, Martin. 2004a. Gothic Painted Wooden Sculpture in Norway 1220-1350. Oslo: Messel forlag.

Blindheim, Martin. 2004b. The Cult of Medieval Wooden Sculptures in Post-Reformation Norway. In Images of Cult and Devotion: Function and Reception of Images in Medieval and Post-Medieval Europe. Edited by Søren Kaspersen and Ulla Hastrup. Copenhagen: Museum Tusculanum Press, pp. 47-59.

Bondevik, Kjell. 1935. Valfarting og lovnader [Pilgrimage and promises]. In Museet i Haugesund: 10 års melding 1925-1935 og avhandlinger om vest-norsk kultur [Haugesund Museum: A Report after 10 Years 1925-1935 and Essays on the Culture of Western Norway]. Edited by Heming Skre, C. Magne Rønnevik and John A. Døsseland. Bergen: John Griegs boktrykkeri, pp. 65-77.

Brendalsmo, Jan, Tine Frøysaker, and Jørgen H. Jensenius. 2001. Kors og krusifiks: tre utsnitt av deres historie [Crosses and crucifixes: three aspects of their history]. Oslo: NIKU.

Brundin, Abigail, Deborah Howard, and Mary Laven. 2018. The Sacred Home in Renaissance Italy. Oxford: Oxford University Press.

Bynum, Caroline Walker. 1988. Holy Feast and Holy Fast: The Religious Significance of Food to Medieval Women. Oakland: University of California Press.

Bynum, Caroline Walker. 2011. Christian Materiality: An Essay on Religion in Late Medieval Europe. New York: Zone Books.

Carruthers, Mary. 2013. The Experience of Beauty in the Middle Ages. Oxford: Oxford University Press.

Catto, Jeremy. 2000. Currents of Religious Thought and Expression. In The New Cambridge Medieval History. Volume VI c.1300-1415. Edited by Michael Jones. Cambridge: Cambridge University Press, pp. 42-65.

Corry, Mary, Marco Faini, and Alessia Meneghin. 2018. Domestic Devotions in Early Modern Italy. Leiden: Brill.

Dekoninck, Ralph. 2012. Between denial and exaltation: The materials of the miraculous images of the Virgin in the Southern Netherlands during the seventeenth century. In Meaning in Materials, 1400-1800 (Netherlandish Yearbook for Art History 62). Edited by Ann-Sophie Lehmann, Frits Scholten and H. Perry Chapman. Leiden and Boston: Brill, pp. 148-75.

Duffy, Eamon. 1992. The Stripping of the Altars: Traditional Religion in England 1400-1580. New Haven and London: Yale University Press.

Emanuelsson, Anders. 2005. Kyrkojorden och dess Ursprung. Oslo Biskopsdöme Perioden ca. 1000-ca. 1400 [The Church Land and its Origin: Oslo Bishopric c.1000-c.1400]. Göteborg: Göteborgs universitet.

Fisher, Annika Elisabeth. 2006. Cross Altar and Crucifix in Ottonian Cologne: Past Narrative, Present Ritual, Future Resurrection. In Decorating the Lord's Table: On the Dynamics between Image and Altar in the Middle Ages. Edited by Søren Kaspersen and Erik Thunø. Copenhagen: Museum Tusculanum Press, pp. 43-62.

Freedberg, David. 1989. The Power of Images: Studies in the History and Theory of Response. Chicago and London: The University of Chicago Press.

French, Katherine L. 2008. The Good Women of the Parish: Gender and Religion after the Black Death. Philadelphia: University of Pennsylvania Press.

Fuglesang, Signe Horn, and David Wilson. 2006. The Hoen Hoard: A Viking Gold Treasure of the Ninth-Century. Rome: Bardi Editore.

Gell, Alfred. 1998. Art and Agency: An Anthropological Theory. Oxford: Clarendon.

Genovese, Valeria. 2011. Statue vestite e snodate. Un percorso [The Dressing and Undressing of Statues. An Outline]. Pisa: Edizione della Normale.

Gilchrist, Roberta. 2012. Medieval Life: Archaeology and the Life Course. London: Boydell Press. 
Gilchrist, Roberta. 2013. The Materiality of Medieval Heirlooms: From biographical to sacred objects. In Mobility, Meaning and Transformations of Things: Shifting Contexts of Material Culture through Time and Space. Edited by Hans Peter Hahn and Hadas Weis. Oxford and Oakvill: Oxbow Books, pp. 170-82.

Gjerløw, Lilli. 1970. Missaler brukt i Bjørgvin bispedømme fra misjonstiden til Nidarosordinariet [Missals used in Bjørgvin bishopric from the time of missionaries to the Nidaross ordinarium]. In Bjørgvin bispestol: Byen og bispedømmet [Bjørgvin: The City and the Bishopric]. Edited by Per Juvkam. Bergen: Universitetsforlaget, pp. 73-115.

Golsenne, Thomas. 2010. Parure et culte [Orament and cult]. In La Performance des Images [The Performance of Images]. Edited by Alain Dierkens, Gil Bartholeyns and Thomas Golsenne. Brussels: Éditions de l'université de Bruxelles, pp. 71-84.

Glørstad, Zanette T., and Camilla C. Wenn. 2017. A view from the valley: Langeid in Setesdal, South Norway-A Viking Age trade station along a mercantile highway. In Viking-Age Transformations: Trade, Craft and Resources in Western Scandinavia. Edited by Zanette T. Glørstad and Kjetil Loftsgarden. London: Routledge, pp. 191-211.

Helander, Sven. 2001. The liturgical profile of the parish church in medieval Sweden. In The Liturgy of the Medieval Church. Edited by Thomas J. Heffernan and E. Ann Matter. Kalamazoo: Michigan University Press, pp. 146-86.

Hohler, Erla Bergendahl. 1994. Borre-korset [The Holy Cross from Borre]. In Fra hammer til kors: 1000 år med kristendom. Brytningstid i Viken [From Hammer to Cross: 1000 Years of Christianity. Transitional Times in Viken]. Edited by Knut B. Bjerva and Jan Ingar Hansen. Oslo: Schibsted, pp. 173-82.

Hoff, Anne Marta. 1997. Holdhus kyrkje. In Norges kirker: Hordaland [Churches in Norway: Hordaland]. Edited by Hans Emil Lidén, Anne Marta Hoff and Ola Storsletten. Oslo: Land og kirke, Volume 2, pp. 8-24.

Holloway, Julia Bolton. 1984. The Dream of the Rood and Liturgical Drama. Comparative Drama 18: 19-37. [CrossRef]

Howell, Martha C. 1996. Fixing Movables: Gifts by Testament in Late Medieval Douai. Past and Present 150: 3-45. [CrossRef]

Jernæs, Nina Kjølsen. 2016. Tre middelalderskulpturer fra A 265 Gjerstad kirke. Undersøkelse og behandling av et krusifiks og to helgenskulpturer [Three Medieval Sculptures from A 265 Gjerstad Church. Examination and Treatment of one Crucifix and Two Saints]. Oslo: NIKU.

Jürgensen, Martin Wangsgaard. 2018. Ritual and Art across the Danish Reformation: Changing Interiors of Village Churches 1450-1600. Turnhout: Brepols.

Jørgensen, Ellen. 1909. Helgendyrkelse i Danmark: Studie over kirkekultur og kirkeligt liv fra det 11te aarhundredes midte til Reformationen [Veneration of Saints in Denmark. Essays in Culture and Religious Life from the Mid-11th Century to the Reformation]. Copenhagen: H. Hagerups Forlag.

Kamerick, Kathleen. 2002. Popular Piety and Art in the Late Middle Ages: Image Worship and Idolatry in England 1300-1500. London: Palgrave.

Kaspersen, Søren, and Ulla Hastrup. 2004. Images of Cult and Devotion: Function and Reception of Images in Medieval and Post-Medieval Europe. Copenhagen: Museum Tusculanum Press.

Keane, Marguerite. 2016. Material Culture and Queenship in 14th-century France: The Testament of Blanche of Navarre (1331-1398). Leiden: Brill.

Kirby, Jo, Susie Nash, and Joanna Cannon. 2010. Trade in Artists' Materials: Markets and Commerce in Europe to 1700. London: Archetype.

Kopania, Kamil. 2010. Animated Sculptures of the Crucified Christ in the Religious Culture of the Latin Middle Ages. Warsaw: Neriton.

Kroesen, Justin, Micha Leeflang, and Marc Sureda i Jubany. 2019. North \& South. Medieval Art from Norway and Catalonia 1100-1350. Zwolle: WBOOKS.

Karker, Allan, Helge Pohjolan-Pirhonen, Magnús Már Lárusson, Finn Hødnebø, and John Granlund, eds. 1956-1978. Kulturhistorisk leksikon for nordisk middelalder (KLNM) [Lexicon of the Cultural History of the Nordis Middle Ages]. Copenhagen: Rosenkilde and Bagger.

Kurz, Herbert. 1997. Der Volto Santo von Lucca: Ikonographie und Funktion des Kruzifixus in der gegürteten Tunika im 11. Jahrhundert [The Volto Santo from Lucca. Iconography and Function of the Crucifix with Belt and Tunic in the 11th Century]. Regensburg: Roderer Verlag.

Laugerud, Henning, Salvador Ryan, and Laura Katrine Skinnebach. 2016. The Materiality of Devotion in Late Medieval Northern Europe: Images, Objects and Practices. Dublin: Four Courts Press. 
Lohfert Jørgensen, Hans Henrik. 2017. Live Matter and Living Images: Towards a Theory of Animation in Material Media. Konsthistorisk Tidsskrift 86: 251-70. [CrossRef]

Lund, Julie. 2017. Connectedness with things. Animated objects of Viking Age Scandinavia and early medieval Europe. Archaeological Dialogues 24: 89-108. [CrossRef]

Hall, Mark A. 2011. The Cult of Saints in Medieval Perth: Everyday Ritual and the Materiality of Belief. Journal of Material Culture 16: 80-104. [CrossRef]

Marks, Richard. 2004. Image and Devotion in Late Medieval England. London: Sutton.

Martinelli, Stefano. 2016. L'immagine del Volto Santo di Lucca. Il successo europeo di un'iconografia medievale [The Image of the Volto Santo from Lucca. The European Success of a Medieval Iconography]. Pisa: Edizioni ETS.

Matyjaszkiewicz, Ika. 2018. Distance and Embrace: Spatial Conditions of Access to the Volto Santo of Lucca. In Place and Space in the Medieval World. Edited by Meg Boulton, Jane Hawkes and Heidi Stoner. London: Routledge (e-book).

Mauss, Marcel. 1923. Essai sur le don [Essay on the Gift]. In L'Année sociologique [Yearbook of Sociology]. Edited by Émile Durkheim. Paris: Alcan, pp. 30-186.

Ó Carragáin, Éamonn. 2005. Ritual and the Rood: Liturgical Images and the Old English Poems of the Dream of the Rood Tradition. London: The British Library and Toronto: University of Toronto Press.

Van Os, Henk, Eugène Honoré, Hans Niewdorp, and Bernard Ridderbos. 1994. The Art of Devotion in the Late Middle Ages in Europe, 1300-1500. Princeton: Princeton University Press.

Palsson, Vidar. 2010. Power and Political Communication. Feasting and Gift Giving in Medieval Iceland. Ph.D. dissertation, University of California, Berkeley, CA, USA.

Pongratz-Leisten, Beate, and Karen Sonik. 2015. The Materiality of Divine Agency. Boston and Berlin: De Gruyter.

Ringbom, Sixten. 1984. Icon to Narrative: The Rise of the Dramatic Close-Up in Fifteenth-Century Devotional Painting. Doornspijk: Davaco.

Rosler, Martha, Caroline Walker Bynum, Natasha Eaton, Michael Ann Holly, Amelia Jones, Michael Kelly, Robin Kelsey, Alisa LaGamma, Monika Wagner, Oliver Watson, and et al. 2013. Notes from the Field: Materiality. The Art Bulletin 95: 10-37. [CrossRef]

Rubin, Miri. 1991. Corpus Christi: The Eucharist in Late Medieval Culture. Cambridge: Cambridge University Press. Schmitt, Jean Claude. 1995. Cendrillon crucifiée. À propos du Volto santo de Lucqus [The Crucified Cinderella. Some Thoughts on the Volto Santo from Lucca]. In Miracles, Prodiges et Merveilles au Moyen Âge, Actes du XXVe Congrès de la S.H.M.E.S, Orléans, Juin 1994 [Miracles, Prodigies and Wonders in the Middle Ages. Proceedings from the 25th Congress of the S.H.M.E.S. in Orleans, June 1994]. Paris: Publications de la Sorbonne, pp. 241-69.

Skinnebach, Laura Katrine, and Henning Laugerud. 2007. Instruments of Devotion: The Practices and Objects of Religious Piety from the Late Middle Ages to the 20th Century. Aarhus: Aarhus Universitetsforlag.

Smail, Daniel Lord. 2016. Law and the Uncertainty of Value in Late Medieval Marseille and Lucca. In The Dark Side of Knowledge: Histories of Ignorance, 1400 to 1800. Edited by Cornel Zwierlein. Leiden: Brill, pp. 51-69.

Smith, Mary Francis, Robin Fleming, and Patricia Halpin. 2001. Court Piety in Anglo-Saxon England. The Catholic Historical Review 87: 569-602. [CrossRef]

Staecker, Jörn. 1999. Rex Regum et Dominus Dominorum: Die wikingerzeitlichen Kreutz- und Kruzifixanhänger als Ausdruck der Mission in Altdänemark und Schweden [Rex Regnun et Dominus Dominorum: The Cross- and Crucifix Pendants of the Vikings as Missionary Symbols in Old Denmark and Sweden]. Stockholm: Almqvist and Wiksell.

Staecker, Jörn. 2006. The Cross Goes North: Christian Symbols and Scandinavian Women. In The Cross Goes North: Processes of Conversion in Northern Europe, AD 300-1300. Edited by Martin Carver. Woodbridge: Boydell Press, pp. 463-82.

Stang, Margrethe C. 2015. Bildedonasjoner og donatorbilder. Lekfolks gaver til kirken som visuell kultur [Image Donations and Donator Images. Lay People's Gifts to the Church as Visual Culture]. Collegium Medievale 28: 145-62.

Trexler, Richard C. 1991. Habiller et déshabiller les images, esquisse d'une analyse [Dressing and Undressing Images, an Analytical Sketch]. In L'image et la Production du Sacré [The Image and the Production of the Sacred]. Edited by François Dunand, Jean-Michel Spieser and Jean Wirth. Pairs: Méridiens Klincksieck, pp. 195-231.

Tripps, Johannes. 2000. Das Handelnde Bildwerk in der Gotik [The Acting Image in the Gothic Period]. Berlin: Gebrüder Mann Verlag. 
Il Volto Santo. 2003. La Santa Croce di Lucca: il Volto Santo: Storia, Tradizioni, Immagini. Atti del Convegno, Villa Bottini 1-3 Marzo 2001 [The Holy Cross from Lucca: the Volto Santo. History, Traditions, Images. Proceedings from Villa Bottini 1-3 March 2001]. Edited by Marzia Zingoni. Empoli: Editori dell'Acero.

Weiner, Annette. 1992. Inalienable Possessions: The Paradox of Keeping-While-Giving. Berkeley: University of California Press.

(C) 2019 by the author. Licensee MDPI, Basel, Switzerland. This article is an open access article distributed under the terms and conditions of the Creative Commons Attribution (CC BY) license (http://creativecommons.org/licenses/by/4.0/). 
Article

\title{
What Julian Saw: The Embodied Showings and the Items for Private Devotion
}

\author{
Juliana Dresvina \\ History Faculty, University of Oxford, 41-47 George St, Oxford OX1 2BE, UK; juliana.dresvina@history.ox.ac.uk \\ Received: 28 February 2019; Accepted: 29 March 2019; Published: 2 April 2019

\begin{abstract}
The article traces potential visual sources of Julian of Norwich's (1343-after 1416) Revelations or Showings, suggesting that many of them come from familiar everyday devotional objects such as Psalters, Books of Hours, or rosary beads. It attempts to approach Julian's text from the perspective of neuromedievalism, combining more familiar textual analysis with some recent findings in clinical psychology and neuroscience. By doing so, the essay emphasizes the embodied nature of Julian's visions and devotions as opposed to the more apophatic approach expected from a mystic.
\end{abstract}

Keywords: revelations; mysticism; ekphrasis; neuromedievalism; neuroarthistory; psychohistory; Julian of Norwich; visions; sleep paralysis; psalters; books of hours; rosary beads

\section{Introduction}

This paper has a very simple thesis to illustrate: that a lot, if not most of theology, found in the writings of Julian of Norwich (1343-after 1416) — a celebrated mystic and the first English female author known by name-comes from familiar, close-to-home objects and images. Images are such an integral aspect of our existence that the famous neuroscientist Rodolfo Llinás, and many after him, claimed that our brain is about making images (Llinás and Paré 1991; Damasio 2010, pp. 63-88). However, such complicated private visual experiences as dream-visions or mystical revelations are insufficient to synthesise knowledge per se, particularly if understood as aimed at a broader community. The images which constitute such visions are often familiar, yet, when spelled out in words, described and reinterpreted, they produce new meanings. This paper attempts to consider Julian's narrative as an "imagetext", based on easily recognisable and readily accessible late-medieval devotional objects yet employed to convey a complex and multifaceted experience, however much misremembered and culturally filtered. Here, rather than focusing on theological and philosophical readings of Julian's text or examining it from the perspective of medieval theories of vision, I read Julian through the developing work on medieval ekphrasis (Barbetti 2011; Fraeters et al. 2013; Johnson et al. 2015) combined with the elements of neurohumanities, especially neuroarthistory Despite the recently voiced criticism of "neuromania" in the Humanities, the view best presented in (Tallis 2016), neuroscience is now entering the field of vision of medievalists, see (Chance 2012) - the whole journal issue dedicated to 'neuromedievalism', especially Jane Chance's own introduction to it, pp. 247-61; also (Blud 2016), the work on neurology of scribes by Deborah Thorpe, e.g., (Thorpe 2015), the forthcoming collection by (Dresvina and Blud forthcoming), and, to a certain extent (Karnes 2011; Morgan 2013), which includes a brief section on the role of the mirror neurons in our understanding of mystical experiences, and (Kroll and Bachrach 2005).

Such research continues the growing historiographic tradition, which considers how religion relates to the surroundings that define and contextualize human lives. Material culture plays an important role in this exploration as, "since medieval people constructed their spiritual or imagined worlds with familiar earthly building blocks, those objects matter" (Deane 2013, p. 67). Diana Webb, discussing late-medieval domestic devotion stresses the importance of this familiarity and the comfort 
deriving from familiar words, often centred around the Psalms, and/or from objects, images, and books, which became more accessible within people's homes as the Middle Ages progressed (Webb 2008, p. 31). This familiar, domestic, "homely" appeal of Julian's text was highlighted by A. C. Spearing in his introduction to one of the most popular translations of her Revelations, pointing out how Julian likens the drops of blood coming out of Christ's pierces forehead to beads, raindrops, and herring roe, and his discoloured body the clothes on the washing line and to a worn-out board (Julian of Norwich 1998, pp. xix-xx). Caroline Walker Bynum suggests that the tradition of hanging a pyx in the shape of a dove above the altar could have triggered some mystics' eucharistic visions of the Holy Spirit winging toward them; such increased use of the everyday imagery, evident in late-medieval "lived religion", is grounded in the realisation of human embodiment, its limitations and perhaps its advantages (Bynum 1991, p. 60; also Ringbom 1969; Ziegler 1992).

Recently, works by metadisciplinary scholars have started to appear discussing what our recently-acquired knowledge about our brain may truly mean for understanding our culture and spirituality, as a part of a larger discussion of the role biology plays in religion (such as Wilson 2002; Burkert 1996; Newberg et al. 2001; Malik 2001, to name a few). The most ambitious of them is perhaps Iain McGilchrist's The Master and His Emissary (McGilchrist 2010). An Oxford literary graduate and a neuroscientist working in a clinical environment, McGilchrist produced a book that has been labelled "controversial" within the left-brain academe. As Rupert Read puts it,

The "master" of the title is the brain's right hemisphere; the "emissary", the left. The brain hemisphere specialisation has been noticed a long time ago (the left usually credited as the locus for speech, dismembering analysis, mechanical fragmentation, linear logic, adherence to the familiar, and utilitarian intellect, while the right is known for its intuiting, holistic approach, handling of paradoxes, initial digestion of the new experiences, and emotional intellect). McGilchrist's basic thesis is that most neurological events and processes need to begin in the right hemisphere with its ability to see what is new, and end there too, since this is where we are able to relate, vitally, humanly, and as a part of a whole. His idea is that the left hemisphere is "essentially there to be the right hemisphere's servant or emissary, but the left hemisphere, with its obsession with analysis and its tendency to denial, has usurped the leading role and no longer relinquishes the power assigned to it for a specific purpose (Read 2011, p. 119).

Importantly, McGilchrist does not maintain that the two hemispheres are the loci for different things or activities-that would be, as he puts it, itself an overly left-brained idea. Rather he suggests, drawing from experimental and clinical evidence, that the hemispheres differ in the way they see and handle things, the kind of world they present. The world of the right hemisphere is wonderfully illustrated in a book by a neuroanatomist Jill Bolte Taylor, whose experience of suffering a rare form of stroke when the whole of her left hemisphere went "offline" does not only corroborate McGilchrist's views but also, at least in parts, is strikingly reminiscent of many mystics' recorded experiences. Her "journey into the formless abyss of a silent mind" where the essence of her being "became enfolded in a deep inner peace", shifting into "a perception that [she] was at one with the universe" would be the envy of any follower of Pseudo-Dionysius (Bolte Taylor 2009, pp. 1, 13). Julian would have recognised the laconic and reassuring answer to dying Jill's spontaneous prayer within her silent mind: "Hold on. Be quiet. Be still. Hold on", her own verbal sparseness as the language centre in her left hemisphere got affected, "thinking in pictures", or, indeed, the beginning of Jill's miraculous recovery through feeling all wrapped up in her (natural) mother's arms and love (Bolte Taylor 2009, pp. 61, 75, 87). Apart from the autobiographical narratives of scientists, there is a growing interest in the neuro-psychological research of modern-days "mystical experiences" conduced with a great deal of sympathy, even up to the point of calling the recipients "unusual but sound minds" (Farias et al. 2012). A particular popular interest is attracted to the research into consumption of psychoactive substances leading to "spontaneous walking visual and kinesthetic transformative imagery narratives", so intense and meaningful that they "often related to psychological and physical healing, problem solving, knowledge acquisition, 
creativity, spiritual development, divination, community cohesion, and encounters with disincarnate entities or beings" (much of these consequences can be applied to Julian herself) (quotations are from Echenhofer 2011, p. 153).

Part of the scholarly appeal of such an iconic and much-studied corpus as Julian's Showings or Revelations is its transcendental subject-matter resulting in a rich, complex, infuriatingly ambiguous and notoriously hard-to-understand text. Together with her writing, Julian's elusive figure itself is a hotly debated object of academic, religious, or political partisanship: Julian the proto-protestant, proto-feminist, proto-Catholic modernist, undercover heretic, undercover academic, undercover dissident, witness to the vitality and flexibility of late-medieval devotional science, and so on (Morris and Holloway 2010). Her choice to write in the vernacular on a topic for which she often had to invent the English nomenclature-with great care and a varying degree of success-particularly appeals to the logo-centric culture, academic in particular and Western European in general (McGilchrist 2010, p. 295). If we are to believe modern editors and commentators, Julian's writings almost entirely consist of complicated cultural cross-references, hidden quotations and disguised arguments, frequent puns and "word-knots" to use Ross and Gillespie's term (Gillespie and Ross 1992, p. 69; Elisabeth Dutton, perhaps more realistically, says, when suggesting that Julian may have read medieval theological compilations: "if such authorities influenced the Revelations in any way, they might then be unnamed because Julian was unaware of their identity, or because she left them unacknowledged in imitation of some compilers' practice", Dutton 2008, p. 97). Yet the paradox is that Julian, in her own words, is writing for those who are simple, for the wise know it all already (LT 9), and she calls herself "a simple creature that cowde no letter" (LT 1: The Long Text is cited by chapters as LT and the Short Text as ST. The editions used are Julian of Norwich 2001 and 2006). Pages are written to prove that this phrase is used as a standard medieval modesty topos and signifies that she did not get a formal, University training (a useful discussion of the views on Julian's literacy can be found in Dutton 2008, pp. 6-11). Saying that she did not get the formal Latinate University education would be stating the obvious, and should not be seen as mere editorial back-covering. Her text is clever and carefully designed, that is granted; but even if Julian was a prodigy and somehow received the best education available in her time (which is highly debatable), her text cannot possibly be what it is often seen like-a cerebral compendium of veiled commentaries on contemporary theological and intellectual issues.

\section{Discussion}

I would like to take Julian back to the late-medieval pious laity and enquire how her text could be approached by a simple reader, perhaps quasi-literate, whose main religious experiences were predominantly aural and visual. Even though there is no definitive evidence of such a readership, this is the "simple" audience Julian apparently anticipates. It would be unwise to ignore the existing body of scholarly work on Julian, particularly by those authors who persuasively argue that Julian ultimately tries to move away from the images into the realm of the analytical, abstract, and even apophatic, although to my taste the abstractness is occasionally overstated (Gillespie and Ross 1992; Aers David 1996, pp. 93, 97). The ultimate aim of a mystic (at least theoretically) to move away from the concrete physical images into the great spiritual silence of the divine presence does not mean that we should not be examining images a mystic employs. Without recognising these images' conventionality (or, at times, un-conventionality), we cannot appreciate the way Julian first introduces them and then takes them away from the traditional expectations, formed by the authorized Meditationes vitae Christi-type devotion, "frustrating" her audience's desires for "bodily engagement" (Aers David 1996, pp. 82-94; quotation at p. 93). Put simply, when the devotee was expected to imagine her or himself as a character of a fanfiction within the established canon of the sacred history, the creativity of his or her pious fantasising was still moulded by numerous literary and visual aids (see Karnes 2011).

Whatever was the nature of Julian's "experiences" which triggered the composition of the Revelations, these had to be expressed in the body and through culturally-informed means. In the 
current state of being it is impossible to avoid the fact that the mind is embodied and sustains the rest of the bodily organs that interact with the brain, determining our mental state. Long before the discovery of the neuron networks it was known that the embodied mind is capable of eliciting emotional responses from imaginary stimuli (Blud 2016, p. 458). Furthermore, we now know that much of what we see (or we think we see) we are filling in from memory, relying on our previous experiences (Kosslyn 1994). The visually-driven nature of these mystical experiences suggests that we are most likely to find a lot of this cultural context in contemporary visual media. However, the survival of medieval art in Norwich and East Anglia, although better than in many other English regions, is still patchy, and even with the amount of research already dedicated to it we are still not sure what exactly people like Julian actually saw around them (Nichols 2002; Lasko and Morgan 1974; also Gunn 2008).

Norwich is famous for several types of art, first of all, its cathedral bosses and misericords, although these, apart from the ones in the nave, were reserved for the monks of the cathedral priory and Julian is not likely to have seen them-or not often. Another obvious suspect is the local school of stained glass, flourishing in the fifteenth century but originating in the fourteenth (Woodforde 1950; King 2010, http:/ / www.cvma.ac.uk). However, surviving examples are mostly too late-with no complete fourteenth-century stained glass surviving in Norwich-and also they are too high up (a good review of the extant examples of the stained glass in Norwich can be found at http:/ / www. norfolkstainedglass.co.uk/Norwich/home.shtm). One wonders if people in the Middle Ages were all far-sighted.

A third artistic medium known to be popular in Norwich and East Anglia is the religious theatre, such as the precursors of the N-Town plays and its likes (Scherb 2001; Granger 2009; or the classic Gibson 1989). It is likely that Julian saw such plays, because Margery Kempe, a generation later, clearly knew quite a few of them (the ones in York at least), but, again, the information for the fourteenth century, which is Julian's time before her illness in May 1373, is far from sufficient. Even though some of Julian's visionary expectations could have come from contemporary drama, the way she does "close viewing" of only one object at a time makes the religious theatre a less likely dominant source of her visionary experience. In the late-medieval drama the necessity to have larger involvement of the community members and thus to have several actors, as well as the need to animate scenes where otherwise nothing much happens, caused various elaborations on familiar scenes, for example in some Nativities Joseph is dishing out soup as Mary lies with the Christ Child in the manger (Penketh 1997). Julian's visions, however, are never crowded with figures, they are focused on one, maximum two objects at one time.

To complicate matters, the art that Julian witnessed may not necessarily have been purely East Anglian. In the late Middle Ages the region displayed strong economic and cultural links to the Continent. Margery Kempe's son famously spent so much time on the continent that his writing was "neithyr good Englysch ne Dewch" - if he was indeed her first scribe and the initial manuscript was not copied by his wife from Gdansk. The Flemmings Johns Asgers, father (also mayor of Norwich in 1426) and son, whose tenement was occupied by one of the city's beguinages, held dual English-Flemish "citizenship" and must have been fluent in both languages (Tanner 1984, p. 65; on aliens in late-medieval England in general see Ormrod et al. 2017). It is unclear how much exactly the exiled English nuns, whose main languages of communication were French and Latin and who did not necessarily know Middle English, actually interfered with Julian's own narrative while copying it, but the texts we now have are linguistically challenging - up to the point of suspecting that Julian had direct access to Continental devotional writings, with Flemish, Dutch, or French being the main suspects (see Dutton's discussion of Julian's use of 'mean' in Dutton 2008, pp. 73-75; it has also been suggested that the support for Julian's reference to John of Beverley's sin in his youth (LT 38) can only be found in the Flemish apocryphon surviving as a chapbook Jan van Beverley (1512); notably, many Books of Hours and some Psalters begin with calendars, and this may be the source of Julian's recollection of John, whose feast day is on 7 May, immediately before her vision of bleeding Christ, see Deighton 1993; Parsons and Jongenelen 2012). This does not necessarily mean 
that Julian was of Continental birth—after all, more vernacular inscriptions in women's books were made in Anglo-Norman than in Middle English until the beginning of the fifteenth century, and Julian may well have been of upper-class origin and equally proficient in French, as perhaps suggested by her 'granmercy' in responding to God (Scott-Stokes 2006, p. 21; LT 41). However, multilingualism would certainly facilitate cultural exchange with the Continent. According to England's Migrants 1330-1550 database statistics (England's Immigrants 2019), Norfolk had the third largest immigrant population in England after London and Kent, with over $90 \%$ recorded individuals originating from the modern Low Countries, Belgium and the north of France, and residing in Norwich. Tradewise, "the link between Norwich and the Low Countries, based on commercial dealings and dynastic ties, was deeply entrenched by the late fourteenth century" (Dunn 2004, p. 227). The only communities resembling beguinages in England-a kind of informal devotional sorority, popular in the Low Countries in the late Middle Ages-were established in Norwich (Tanner 2004, pp. 140-41; Tanner 1984, pp. 64-66). At least from the fifteenth century, if not earlier, East Anglian art displayed evidence of growing influence from the Continent, particularly the Flanders (Lasko and Morgan 1974, p. 32; Dennison 2013; Mitchell 2000). We do know of later examples of Norfolk families commissioning paintings for their houses and churches such as the Ashwellthorpe Triptych (c. 1519, now in Norwich Castle Museum); the presence of Continental art in late-medieval East Anglia further testifies to the established links between the two regions, although, admittedly, the survival rate for such works of art is not better than that of the domestic examples.

The visual nature, though, of at least some of Julian's revelations suggests a different medium, which allows close-and repeated-examination: that of the book. The "close and repeated examination" engrains the observed images in the memory, which then presents them to our "mind's eye", particularly under stress (this is obviously a very brief and simplified description of a complex neural process, which has not yet been fully understood—see, for example, a discussion of the "mind's

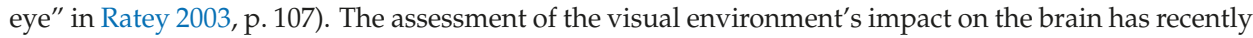
been both embraced as a prevailing explanatory tool, as in John Onians's work on neuroarthistory (Onians 2007; Onians 2016), and cautioned against as a dominant methodology by scholars like Matthew Rampley (Rampley 2016; Rampley 2017). I am inclined to keep to the middle and agree that humans tend to be predisposed, yet not bound, to replicate their visual experience, and that the way we re-live familiar imagery is often changed by physical and psychological distress. Julian's mystical experience was triggered by her acute illness, but we know from her own words that she was already spiritually inclined and therefore had spent time contemplating religious images in a reasonably stressful environment, with thoughts of Christ's sufferings and of her own inadequacy and guilt (on devotional gazing see, for instance, Lentes 2006). Moreover, as Sarah Salih reminds us, liveliness was "the normal condition of devotional art" in the later Middle Ages, and that "movement of either the viewer or the object was built into the situations in which people looked at it", hence Julian was culturally conditioned to expect these familiar images come to life (Salih 2019).

An illustrated book a fourteenth-century reasonably well-off woman could have had, or at least had had access to, would be a Breviary or a Missal type of codex, but much more likely a Psalter or a Book of Hours. The archidiaconal visitations of St Julian's church, Norwich, indicated that in 1368, five years before Julian's visions, the church had four Psalters in its possession, although we do not know whether illustrated or not (Upjohn and Groves 2018, pp. 78-79). At least one illuminated psalter was in possession of Carrow Priory, in whose jurisdiction St Julian's lay; at least two more are known to have been kept in the Priory (Salih 2019; Bell 1995, pp. 126-27). Margery Kempe may have had a Book of Hours, perhaps a simpler yet not cheap copy, but she must have seen lavish ones, status symbols as well as devotional aids, at the homes of her aristocratic and wealthy acquaintances. Margery recalls having "his boke in hir hand" during the frightful episode of the fall of the fragment of St Margaret's ceiling on 9 June 1413, described in Chapter 9 of the Book of Margery Kempe (Kempe 2004; see also Hill 2006). Given the position of Margery's father amongst Lynn's oligarchy, she may well have had an illustrated book for her private devotions, and perhaps not even one (Parker 2004, pp. 56-57). So if we 
can assume at least a mercantile or gentry background for Julian, even if she did not have one or the other herself, she must have seen copies on a regular basis too (Scott-Stokes 2006; Gunn 2008, p. 33).

Psalters were perhaps the most popular book for lay devotions until the late fourteenth century, when they were superseded in this function by Books of Hours. Ancrene Wisse contains references to the Psalter, from which the anchoresses were expected to recite versicles (Ancrene Wisse, Part 1:26). The psalms in the Books of Hours were so well known that they are often indicated only by their incipits; they were also employed as learning means, even containing ABCs (Scott-Stokes 2006, p. 8; LT, ch. 51). There was a strong tradition of fourteenth-century illustrated East Anglian and Lincolnshire Psalters, with a plethora of richly illuminated books for (presumably) aristocratic patrons, of which the most famous are perhaps the recently published facsimiles of the Luttrell Psalter and the Macclesfield Psalter, and a number of Continental ones must have been in the country by the fifteenth century (Sandler 1986; Sandler 2004). Although there was no guaranteed established visual programme generally before the end of the fourteenth century, a number of similar images appeared in many of them, such as the image of a fool for the Dixit Insipiens (Ps 52), the Trinity as identical twins (with an optional third, the dove of the Holy Spirit) for Dixit Dominus (Ps 109), or a person emerging out of the water for the Salvum me fac Deus (Ps 68/69).

By the second quarter of the fourteenth century, an illustrated English Psalter could have ten main illuminated initials: Ps 1 with the Tree of Jesse, surmounted by Mary; Ps 26, with David's Anointing; Ps 38, with David pointing to his eyes (or the Judgment of Solomon); Ps 52, with David debating the existence of God with his fool (or the devil tempting Christ); Ps 68, with David or Jonah in the water (or an image of Christ's Passion or Resurrection); Ps 80, with David playing bells (or Jacob wrestling with the angel); Ps 97, with clerics chanting (or the Annunciation to the Shepherds); Ps 109, with different versions of the Trinity. To this initially Continental division would be added Ps 51, usually with Doeg slaying the priests of Nob, and Ps 101, with the personification of Ecclesia (with or without Christ as her bridegroom) (Panayotova 2008, pp. 59-62).

There are a number of verbal allusions to the psalms in Julian, as well as to other parts of the Scripture, as indicated in recent critical editions (Julian of Norwich 2001, pp. 796-805; Julian of Norwich 2016, pp. 179-286), such as the response to her desire to have more light to see the face of Christ in LT 10 ("If God wil shew thee more, He shal be thy light; thee nedith none but Him") and the ensuing vision of the safety at the sea bottom being reminiscent of the opening line of Ps. 27 "The Lord is my light and my salvation-whom shall I fear?" She was certainly familiar with Psalms even as a devout laywoman, and must have had access to a written version in one form or another. From the late thirteenth century onwards, one could even find complete Psalters in English (metrical, prose, or paraphrased), but these were scarce and, before the multiplications of the Wycliffite translations in the early fifteenth century, often had local circulation (Sutherland 2015, esp. ch. 3). For the monolingual devotees the conventional book before the end of the fourteenth century would have been the Prymer, a close relative to the Latin Books of Hours, usually complete with 52 psalms, hymns, collects, and a litany in Middle English; 17 manuscripts of such books are still extant (Kennedy 2014; Littlehales 1895, pp. viii-x). None of these, however, contain any miniatures, even if most are decorated with flourished initials or floral borders; this, however, does not necessarily prove that their owners had to be of the Wycliffite/Lollard sympathies (Kennedy 2014, pp. 716-19).

The Books of Hours for that period had not yet acquired their more or less established canon, and their pictorial programme was still fluid in the middle of the fourteenth century. However, one may expect to see at least some of the images found in later, mass-produced versions. For example, the Hours of the Virgin were illustrated by the Infancy cycle: Matins was opened by the Annunciation, Lauds with the Visitation, Prime with the Nativity, Terce by the Annunciation to the Shepherds, Sext, the Adoration of the Magi, Nones, with Candlemas, Vespers, with the Flight to Egypt (or the Massacre of the Innocents), and the Coronation of the Virgin at Compline (Wieck 1997, p. 23). Hours made for the English market were often preceded by eight Passion scenes, from the Agony in the Garden to the Deposition in the Tomb, or they would have both cycles within one book (Duffy 2006, pp. 13-14). 
Almost any Book of Hours would have had a picture of the Annunciation, the Crucifixion, and later of such devotional, rather than narrative, images as Christ as the Man of Sorrows, the Wound of Christ (or the Five Wounds), and the Vernicle or the arma Christi (Duffy 2006, pp. 72-73).

Let us now try to establish what Julian actually saw and compare it to the surviving material object accessible as items of domestic devotion. It is surprising, given how much has been written about Julian's text as an example of medieval vernacular literature or theology, that only a few studies ever touch upon the visual side of her narrative (Windeatt 2014; Gunn 2008; Barratt 2008; Kamerick 2002, pp. 132-38; Lermack 1993; Baker 1994, pp. 40-62; Salih 2019; to a certain extent Aers David 1996; Hagen 2004, pp. 145-60; however, they provide just a couple of actual images). It is beyond doubt that the intention and meaning behind the visions in the different periods of the Middle Ages varied, and a visionary's "I saw" could mean anything from "I visualized" to "I imagined" to "I hallucinated", as Barbara Newman brilliantly demonstrates (Newman 2005). Yet the visual component of Julian's revelation remains of paramount importance. One of her most frequently used verbs is "to see" and one of the most frequent phrases is "as to my sight".

It could be fair to object that this particular phrase does not refer to the actual sight but that it should be taken figuratively, meaning to say "it seems", or "it appears", but both of these in their direct, literal meaning, too, refer to seeing. As Iain McGilchrist observes,

Probably the most important metaphor of our relationship to the world is that of sight.

'Knowing as seeing' is one of the most consistent of all metaphors, and exists in all Indo-European languages, suggesting that it developed early in the Indo-European Ursprache ... 'I see', we say, meaning 'I understand' (McGilchrist 2010, p. 161)

In order to be taken figuratively, "as to my sight" should be so familiar to a medieval English ear that the literal meaning of it is easily overlooked. However, the phrase is only attested to twice in Middle English literature outside of Julian's writings. The first instance is found in Chaucer's translation of the Roman de la Rose, 1. 740 (Chaucer 1988, p. 694-the entrance and dance of Sir Mirth and his companions: "So fair folk and so fresh had he/That whan I saw, I wondred me/Fro whennes siche folk might come,/So faire they weren, alle and some;/For they were lyk, as to my sighte,/To angels that ben fethered brighte" (1l. 737-42). Compare the original text: "S'avoit si beles genz o soi,/Et quant je les vi, je ne soi/Dont si tres bele gent pooient/Estre venu, car il sembloient/Tout pour voir anges empenez:/Si bele gent ne vit hons mez" (11. 720-25): ("And he was accompanied by such fair folk. When I saw them, I could not tell where such fair people came from, for indeed they seemed to be winged angels. I have never seen such fair folk"), Lorris and Meun 1992, p. 76). The other appears in line 241 of The Floure and the Leafe dream-poem, again, in the Rose tradition and long attributed to Chaucer (Pearsall 1990: "Next after hem came in armour bright,/All save their heads, seemely knights nine;/And every claspe and naile, as to my sight,/Of their harneis were of red gold fine" (11. 239-42). See also line 174 of the same poem: " . . and to my sight, trewly,/She lady was of the company". "To my sight" phrase is slightly more common in late ME, mostly appearing in a context of a vision/apparition, see, for instance, IMEV 3928.5, Oxford, Bodleian Library MS Ashmole 1485, Part III.ii (SC 7652), Part III, f. 46v: When busy at my booke I was vppon a certain night/this vision heare exprest appeered to my sight... (The vision of Sir George Ripley, Canon of Bridlington, f. 46v)).

Now believed to be written in the fifteenth century, perhaps by a woman and certainly in a female voice, the poem is heavily indebted, apart from Chaucer and Lydgate, to a whole plethora of French poets: Guillaume de Lorris, Guillaume Machaut, Jean Froissart, and Eustache Deschamps (Pearsall 1990; Marsh 1906, p. 7), with references to fifteenth-century songs from Normandy (11. 176-78, and, perhaps, 350). The female narrator, as with Julian, unusually does not fall asleep during her vision, but tries to make sense of it by observing intensely the arrival of the colourful companies of knights and ladies to a green meadow. It is not too unreasonable to suggest that the poem, or at least some of its parts, is also a translation, which would add extra weight to the hypothesis of Julian's Continental linguistic connections (The poem's 'sister-text', The Assembly of Ladies, still extant in the same MS and probably by the same (female) author, displays "some specific influence from the Burgundian 
styles". Another curious detail appears in line 550: The words "For wele to better" is, as Pearsall notes, "an echo, perhaps, of the idiom of the French motto, De bien en mieulx. Cf. De mieulx en mieulx, used as a motto in the fifteenth century by the Paston family of Norfolk." ("Assembly of Ladies: Introduction", and note 550 to "The Floure and the Leafe"). There are several examples of a corresponding French phrase, 'a mon avis (advis/aviz)' between 1346 and 1416, with one coming from the Letter IX of the exposition to another Rose-inspired dream-poem, La prison amoureouse (early 1370s) by Jean Froissart, as well as from his Chronicles (two cases) (Kelly 1978, p. 165; (Online Froissart 2013) https:/ / www.hrionline.ac.uk/onlinefroissart/index.jsp, citing Paris MS Fr. 2650, fols 90v and 91 r; (Dictionnaire du Moyen Français 2016) http:/ / www.atilf.fr/dmf/, citing four more examples).

What is important for our argument is that in these poems the phrase appears during moments of great surprise, of the observer's intense visual experience (as, for example, Chaucer's "I wondred me"), and the acknowledgement of the inadequacy of language to express the vision (e.g., The Floure and the Leafe, 1l. 138-40). Thus, the phrase refers to visual perception, which suggests that Julian's "as to my sight" should not be read as an exclusively figurative topos.

Three kinds of sight, or three ways of "showing" are famously described in Chapter 9 of her LT, where she is trying to explain how she actually received her revelations:

All this was shewid by thre, that is to sey, be bodily sight, and by word formyd in my understonding, and be gostly sight. But the gostly sight-I cannot ne may not shew it as hopinly ne as fully as I wolde.

These are often read as Julian building on the Augustinian textual analysis of visual perception, with the division into "corporeal", "imaginative" and "intellective" vision, acknowledging, however, that St Augustine's influence was indirect (Watson 1992, p. 85). Could this indirect influence reach Julian not via textual, but via visual channels? There is an interesting fourteenth-century visual parallel to such several types of vision in the British Library, MS Yate Thompson 11, a French devotional manuscript, which depicts, on folio $29 \mathrm{r}$, three degrees of mystical union experienced by a woman (presumably a nun) after a confession with her Dominican spiritual guide (https://www.bl.uk/ catalogues /illuminatedmanuscripts /ILLUMIN.ASP?Size=mid\&IllID=45156).

Katie Walker describes how, following Michael Camille's analysis—based, in turn, on Augustinian vision theory (Camille 1996, pp. 120-23; McGinn 2006, p. 187)—“"ii]n the first [frame], the nun kneels before her confessor and although the divine is not present, it is tangible, signalled by Christ's strangely disembodied hand blessing from the edge of the scene. Here, in this first level, the nun has only earthly sight; unable to see divinity 'in the flesh', her piety must be mediated by another" (Walker 2003, p. 3). This is similar to Julian's reliance on her curate (mentioned in the LT) before the revelation, or on the "religious person" who recites to her the life of St Cecilia in the ST. "The second frame depicts the nun kneeling before an image of the Virgin and Child. It is the statue that mediates her act of devotion; in other words, the nun recognises the statue as a signum of a higher truth." This corresponds with Julian's direct 'bodily sight'. Then "[t]he third image shows a spiritual level of perception that Michael Camille terms "imagined contemplation." Prostrate before an image of Christ as the Man of Sorrows, the nun's eyes do not look at the vision, implying that it is created in her mind. Christ himself mediates this level of perception as he, quite literally here, pours out his blood for the nun." This is the second sight in Julian, formed in her "understanding", that is her mind (Admittedly, for Julian is it oral-it is to words to which that she moves, but many mystics "often stretched or blurred Augustine's categories" (McGinn 2006)). Note also the focus on Christ's blood (something she sees often, and copiously): the physical depiction on the face of the Crucifix, the animated vision of the bleeding in her 'bodily vision', and the mental-mystical vision of his descent into Hell and ascent into Heaven in Chapter 12 of the LT. "Finally, the highest level of perception is the "mystical mode." Now, looking at the vision before her, this becomes the "pure and naked seeing of divine reality" (Walker 2003, p. 3). This last mode corresponds to Julian's 'ghostly sight', which she finds so difficult to describe through our limited human means - as opposed to the images that can easily draw on known iconography. 
There is, however, the fifth type of Julian's vision, which is remarkably different in its origin from the majority of the "showings" - the difference Julian recognises herself when she introduces the demonic appearance in Chapters 66 of LT: "This oggley shewing was made slepyng, and so was non other". This brief nightmarish episode involved all five senses, entailing strong heat, overpowering stench, and a vision of fire:

And in the slepe at the begynnyng, methowte the fend set him in my throte puttand forth a visage ful nere my face like a yong man, and it was longe and wonder lene. I saw never none such. The color was rede like the tilestone whan it is new brent, with blak spots therin like blak steknes fouler than the tile stone. His here was rode as rust evisid aforn with syde lokks hongyng on the thounys. He grynnid on me with a shrewd semelant, shewing white teeth, and so mekil methowte it the more oggley. Body ne honds had he none shaply, but with his pawes he held me in the throte and wold have stranglid me, but he myte not.

Familiar to many through Henry Fuseli's 1781 painting "The Nightmare", the phenomenon Julian describes here is sleep paralysis, believed to be a demonic visitation since late antiquity and certainly known to a medieval audience (Sharpless and Doghramji 2015, p. 59; the seminal article on the subject is (Davies 2003). Sleep paralysis is described, for example, in the legend of St Michael in the South English Legendary, see Perk 2017, p. 159). Put simply, it is an overlap between REM phase of sleep and the state of awareness, when the person is unable to move, speak, or react, and experiences terrifying hallucinations, usually accompanied by a feeling of pressure on one's chest, palpitations, difficulty breathing, and sensing a presence in the room. If there is a mirror in the room, the "intruder", being the product of the REM phase, will not be seen in it, which probably encouraged the belief, popularised by Bram Stoker, that vampires and the undead have no reflection in the mirror (Sharpless and Doghramji 2015, p. 59; Broks 2016). Medieval sleeping patterns, especially of those following the monastic timetable who regularly had their sleep interrupted, may have made people of that time particularly prone to sleep paralysis (Dudley and Goodare 2013, p. 123). As would be expected from this phenomenon, the terrifying experience was gone as soon as Julian was fully awake and able to speak and her palpitations subsided ("my harte beganne to comfort"), with her bed-side companions wetting her temples probably speeding up the process.

The implications of identifying a physiological origin for at least this particular vision is important for the justification of the use of the word "experience", applied to Julian's narrative. Finding numerous cultural parallels, combined with the human tendency to fill in gaps from culturally-affected memory, may lead us to reading Julian's text as a pure piece of pious fiction, of something that either never happened, or was entirely reconstructed years later in accordance to the familiar philosophical, theological, and iconographical expectations of her time-in which case the use of such somatically-aware methods as neuroarthistory is pointless. Of course, any attempts to come into a contact with the real live person behind the narrative are likely to fail, as anything we know about Julian's personality is derived from her text. However, being able to readily identify a phenomenon, which even today affects about $8 \%$ of the world population (with $28 \%$ in risk groups, Olunu et al. 2018), gives more credibility to Julian's text as at least a pale reflection of some genuine experiences, further confirmed by her doubts about them. The red-and-black appearance of the sharp-toothed devil may well have been suggested, at least in part, by the contemporary imagery, digested and reassembled during the vivid dreaming of the REM phase, but it is also significant that Julian is careful to include the less orthodox bits of her vision (an interesting illustration of the devil suffocating an already dead man in his bed can be seen in an early fourteenth-century French manuscript of Justinian's Digestum Vetus with glossa ordinaria, British Library Arundel 484, fol. 245r, http:/ / www.bl.uk/catalogues/illuminatedmanuscripts/ILLUMIN.ASP?Size=mid\&IllID=5122. For medieval theories about sleep paralysis see MacLehose 2013). Julian is primed, but not imprisoned by cultural expectation.

Nicholas Watson pointed out that "Julian's revelation has an imagistic sparseness and at least a surface fragmentariness to it that is largely untypical of the experiences of medieval women visionaries, 
and which must initially have been deeply confusing to its recipient" (Watson 1992, p. 85). Not only to its recipient, but to its scholars, also. However, there is imagery in Julian, although we do not notice it, as we focus on more important facets of her polysemous text. Just as, in the same way, we forget the bloodied face of Christ from Chapter 7 of the LT, which seems to be bleeding continuously-for another ten chapters. Denise Baker argues that of the seven purely corporeal visions (Christ's head wearing the Crown of Thorns, His face while being mocked; His scourged bleeding body; the discolouring of His face during the Agony, His blissful cheer, the Wound in His side and Christ in Glory) the last three "indicate the direct influence of late medieval iconography", rather than the Passion narratives designed for meditation (Baker 1994, pp. 48, 55).

David Aers remarks on the pivotal point, described in Chapter 21 of LT, of Christ's changing cheer on the cross at the anticipated moment of His death, and consequently the changing of Julian's cheer, making her full merry): "She expects to witness the death, "to have seen the body all deed" (21/379), an expectation fostered by received traditions of meditation and iconography. To her astonishment, and to her readers' astonishment, this is not what she next sees: "I saw him nott so" (21/379). He does not die and she now encounters a sudden change of his appearance and experiences overwhelming joy. This truly idiosyncratic choice disrupts the normative late medieval sequence of meditations" (Aers David 1996, p. 90). He proceeds to say that for a medieval devotee it was highly unusual see no mourners, no deposition, no burial. Instead Julian enters into a dialogue with a cheerful Christ—still on the Cross-and it is in this context, Aers rightly notes, "we meet the wound in Christ's side" (Aers David 1996, p. 91). Such a turn of the events would be "truly idiosyncratic" if we only derive the context from the textual Meditationes tradition. The cheerful Christ on the cross at the moment when He is supposed to be dead, and His active exchange with the present devotee is as an iconographic motive found in late-medieval art, especially manuscript illumination (Hamburger 1998, pp. 134-42).

The best example comes from the Psalter and the Hours of Bonne of Luxembourg (NY the Met Museum of Art, The Cloisters Collection 1969 [69.86]), made in Paris in the mid-1340s. A series of miniatures illustrating the Passion prayer in French contains several images parallel to at least some of Julian's experiences. One depicts an abbot, presumably St Bernard of Clairvaux, contemplating a realistically-rendered crucifix (fol. 295r); a second one represents real Christ, in exactly the same position, paradoxically alive in his death, gazed upon by Bonne and her husband, implying a (para-)mystical experience. Jesus is looking amicably on the aristocratic devotees while pointing to the wound in his side; His gesture as well as the praying gesture of the royal couple is simultaneously echoed by the two angels (329r). Next, we see the wound itself, looming large among the other instruments of Christ's Passion on the arma Christi image, drawing the meditator in through its entrancing gradient colouring (fol. 331r; the images are reproduced in Hamburger 1998, pp. 140-42; and on the Metropolitan Museum's website, http:/ / www.metmuseum.org/art/collection/search/471883 (click "show more")).

The first vision Julian receives is the profuse bleeding of Christ's head from under the garland of thorns, and the Passion in the first part of her vision is mostly reflected on His face, particularly through the bleeding and the changing of colour. She does not appear to see the familiar Crucifixion scene, of the full-length figure of Jesus, flanked by Mary and John, or at least this does not become one of her main visions. She indicates the awareness of the established iconography and perhaps her surprise at not being given the sight of it when she states, as she closely watches Christ's face, that at some point He looked down to the right, where, as she comments in Chapter 25, His Mother stood at the time of Passion. Christ's face as the centre of the Passion directly reflects the image of arma Christi, appearing in Books of Hours, with the head of Jesus, eerily detached from the rest of the body, surrounded by the instruments of his Passion, such as the nails, whips, and the crown of thorns (one of the most famous of these images, from the Omne Bonum Encyclopaedia (BL MS Royal 6 E. VI, fol. 15r) even appears on the cover of Elizabeth Dutton's book on Julian in recognition of its importance for the visionary process). Some of these images also contained a separate depiction of Christ's wound in one of the segments of the "coat-of-arms", and in Chapter 24 Julian relates how her 
'understanding' was led into the wound in Christ's side, where she found a wonderful space, suitable for all mankind. Images of this wound abound in late-medieval Books of Hours and solicit special devotions (e.g., Duffy 2006, p. 38. This devotion may have influenced Fra Angelico's fresco at San Marco of the disembodied torturers spitting at (as a mouth) and slapping (as a hand) the blindfolded Christ, where we sense but do not see, with Christ, his torturers).

In regards to the image of Christ's face frequently changing colour Julian helpfully gives us the clue as to where it comes from. In Chapter 10 she directly compares it to the holy Vernicle of Rome, the towel used by St Veronica to wipe off Christ's face, kept as a relic in St Peter's, which, according to pilgrim's accounts, miraculously changed colour to display Christ's Passion. Books of Hours often had an image of the Vernicle in them (on the importance of the Vernicle see Cooper and Denny-Brown 2014, especially a critical edition of "O Vernicle" by Ann E. Nichols in it; also Windeatt 2014, pp. 191-92). A particularly interesting one is found in the East-Anglian mid-fifteenth-century manuscript BL Arundel 302 on fol. 63r, where Christ's face is painted in silver and literally changes colour depending on how the light reflects on the page (http:/ / www.bl.uk/ catalogues/illuminatedmanuscripts/ILLUMIN.ASP? Size $=$ mid\&IllID=12316). It is of course too late to have been seen by Julian, but it certainly chimes in with her extended gazing at the changing face of Christ, allowing not only for a longer, but also for a more dynamic contemplation of such image. This dynamism, as well as the arma Christi imagery, also links to medieval religious drama. We know that later the craftsmen's tools became firmly associated with the instruments of Christ's Passion (the York Pinners using the nails to advertise their craft in the Crucifixion play), while actors playing God and Jesus could have their face gilded (Cooper and Denny-Brown 2014, p. 174; Muir 1997, p. 33).

Julian's discussion of the Virgin Mary focuses not so much on her visions of the Mother of God but on Julian's contemplation of these visions. However, already in Chapter 4 she describes how she was shown Mary "ghostly in bodily likeness", little older than a child, "in a stature as she was when she conceived", humbly saying to Gabriel "Lo me here, God's handmaiden". The complicated vision is an expansion the image of Annunciation, which occupied a privileged position of Books of Hours, particularly those made for women. The words from Luke 1:38, inscribed on banderols, could even be coming out of the Virgin's mouth in response to the angelic "Ave Maria". The same image is evoked in Chapter 7 when Julian sees Mary "beholding her Maker", who chose to be born of her, reminiscent of the presence of God in the Annunciation scenes.

More can be said about the presence of the Hours imagery in Julian, but let us turn now to some curious visual clues reminiscent of the Psalters. The first is a very famous one and concerns the vision of the Creation as a small round object in Chapter 5:

Also in this He shewed me a littil thing the quantitye of an hesil nutt in the palme of my hand, and it was as round as a balle. I lokid there upon with the eye of my understondying and thowte, What may this be? And it was generally answered thus: It is all that is made. I mervellid how it might lesten, for methowte it might suddenly have fallen to nowte for littil.

The word "quantity" itself (OF via Latin) is an interesting choice, apparently not found in Middle English before the very end of the fourteenth century except for one example (T. Wright, Polit. Songs Eng. (1839) 334, c. 1330: 'He wole. 3eve the gode man drinke a god quantite', cited in OED 2019, and Middle English Dictionary 2013, http:/ / quod.lib.umich.edu). It is usually translated as "the size of a hazelnut", but it can equally be the weight, bulk, mass or even, by extension, shape. Thus the "thing" corresponds with the image of Christ holding an orb—a small round object—in the palm of His hand, usually in the illustrations to Psalm 109 ("Dixit Dominus"), where the Father and the Son are depicted as identical twins sitting side by side. The orb is often decorated with tiny trees and animals, water and firmament, signifying the Creation (an amusing and enlightening dialogue between a medievalist and a planetary scientist about viewing our planet from the distance see Cohen 2016). Although these orbs appear in other media, famously in the thirteenth-century Westminster Retable, Psalters are still the most common place to find them-for example the well-known initial from the Luttrel Psalter on fol. 203r (http://www.bl.uk/onlinegallery/ttp/luttrell/accessible/images/ 
page28full.jpg). A related iconography of The Lord Enthroned (Christ on his own, or surrounded by the devotees, holding an orb) is also frequently found in Books of Hours, e.g., the Hours of the Trinity section of the De Bois Hours (c. 1325-30), made in East Anglia and owned by Hawisia de Bois (Smith 2003, pp. 103-4, 107-9). The Middle English Matins of the Virgin in the Prymer even refers to Mary as "Blessid modir bi goddis 3ifte, in whos wombe was closed he that is hi3est in all craftis and holdith the world in his fist", the final part reminiscent of the Christ with the orb image (Maskell 1846, p. 6; or Littlehales 1895, p. 2. The phrase itself also echoes Julian's metaphor of us being "enfolded" and "beclosed" in God, see LT 5). A similar image is evoked in The Fifteen Oes, where Jesus is said to be holding the earth in his hand (Windeatt 2014, p. 197).

In this particular case, we have Julian apparently referring to the Dixit Dominus imagery in Chapter 51, where she explains that although the Son is now seated at the right hand of the Father in endless peace and rest,

It is not ment that the sonne sittith on the right hand beside as one man sittith by another in this life-for ther is no such sitting, as to my sight, in the trinite. But he sitteth on his faders right honed: that is to sey, right iin the hyest nobilite of the faders joy.

Alexandra Barratt has already drawn attention to this passage's correspondence with the Dixit Dominus iconography and compared Julian's interpretation of the Trinity to the image found in a roughly contemporary manuscript. The image in question comes from the Ormesby Psalter (Bodleian Library, Douce 366, fol. 147v), made in East Anglia and kept in Norwich cathedral at some point in the late Middle Ages, where Julian could have seen it. Barratt argues that, similar to the absence of the dove in this picture, Julian finds it difficult to locate the Holy Spirit within her revelations, and that the effeminate appearance of the Deity could have contributed to her reading of God (or, rather, Christ) as our mother (Barratt 2008, p. 51; Windeatt 2014, pp. 198-99). Given that these two types of iconography seem to have been equally popular at that period, Julian may well have drawn on both of them in her text.

Another possible source of the "hazelnut" image is also related to the Psalter, although indirectly. Rosary beads and prayers (vernacular and Latin), associated with Marian Psalters, became a popular form of monastic and especially lay devotional practice in the late Middle Ages; the earliest rosaries (dating from the late thirteenth-early fourteenth century, judging by the manuscript evidence) were most likely composed in the vernacular in the Middle Dutch and Middle German-speaking areas within the wave of religious reform movement (Winston-Allen 1997, pp. 18-19). Julian was certainly familiar with this practice and expected her readers to recognise it as well, referring to "bidding of beds, which arn seid boistrosly with mouth, failing devowte entending and wise diligens the which we owen to God in our prayors", when attempting to describe the devilish audio-nightmare in Chapter 69 of the LT. Chaucer's Prioress had a rosary, and it appears in many fourteenth and fifteenth century MSS illuminations, see, for example, Hodges 2005, pp. 108-10. They also appear on English misericords as an item sometimes specific to lay female devotion, see Christchurch Priory (late 13th century), Ely Cathedral (early 14th century), St Mary's, Gayton (14th-15th century), Norwich Cathedral (15th century), St Mary's, Enville (15th century). Julian herself may have used the rosary in her daily devotions, as "life-of-Christ meditations ... have characterized the rosary since the fourteenth century" (Winston-Allen 1997, p. 16). The late 14th-century Middle English devotional poem now known as Pearl probably replicates a rosary through its structure, with every stanza connecting to the next through a repeated key word, the last line repeating the first one, with twenty "circular" sections of five stanzas each and section XV having six stanzas instead, perhaps representative of the terminal/paternoster bead (Harwood 1991).

Late-medieval rosary beads, particularly those made in Flanders and its neighbouring regions, were sometimes made of, or looked like, nuts, but more curiously, the largest bead of the rosary (the terminal bead) was known as the prayer-nut in French (noix de prière; however, the German Betnuss seems to be a post-medieval term). Sometimes these were used as a separate prayer aid on their own (see Scholten 2016 for the most recent comprehensive discussion of the prayer huts and 
related items). A number of fifteenth and early sixteenth-century examples of these survive, but there must have been many more-and probably earlier specimens too-given their highly perishable nature. A carved sphere, usually made of boxwood and about $4 \mathrm{~cm}$ in diameter, opens up to reveal two or more miniatures in high relief on devotional subjects, most commonly Crucifixion and a Marian scene (Annunciation or the Virgin with the Child). One of the smallest examples, an early sixteenth-century prayer-nut now in Hannover, Kestner-Museum measuring $2.5 \mathrm{~cm}$, when opened reveals an iconographic juxtaposition of the two arguably most important events in the human history: the Fall and the Crucifixion. Juxtaposing such scenes is not uncommon in visual arts, especially in the Books of Hours, where in the Hours of the Virgin the Infancy Cycle goes hand in hand with the Passion Cycle, and Julian's own near-conflation of the Fall and the Incarnation in LT Chapter 51, albeit theologically sound, can be at least partially inspired by medieval imagery. The Fall and the Crucifixion, Ascension, or Pentecost, and everything between them can easily qualify as "all that is made", and one wonders if Julian was in possession of, or at least closely observed, one of these objects. As Frits Scholten observes, a prayer nut is an example of a "tactile cosmology", a "pocket-sized representation of the world" (Scholten 2016, p. 67). According to Susan Stewart, one of the consequences of miniaturisations is "letting go of the narrative and concentrating on contextual information, with the result that an awareness of 'temporal closure' makes way for 'spatial closure'" - something we observe in Julian's text and in her own anchoritic enclose (Stewart 1993, p. 48, cited in Scholten 2016, p. 181).

The concept of collapsing the infinity into a tight space (seeing God in a "point", in Julian's words, LT ch. 11; "The smallest unit of measurement until the introduction of the metric system was the point, equivalent to 0.2 millimeters, which is actually the same as the point of a needle or a pin", Scholten 2016, p. 171) was familiar to medieval devotees, the most obvious example being the divinity of Christ confined within Mary's womb, as physically expressed in the statues of "vierge ouvrante" (see Gertsman 2015) — "heaven and earth in little space", as the famous Nativity lyric "There is no rose" points out, "the rose" once again linking us to the rosary. In the already-quoted passage from Chapter 5, the hazelnut's litttleness conveys an impression of "all that is made", as Julian's own text reflects her experience of infinity, composed and organized to express it as best as it can, even at the level of the text's graphic and aural presentation. Here the fragile and tiny "hesil nutt" has a near homonym, "nowte", while "littil" defines the nut both descriptively and orthographically because it is a palindrome, reflecting the closed loop of the rosary and the roundness of the "thing".

The second striking example of the imagery found in Psalters is taken from Chapter 10, where Julian says:

One tyme mine understondyng was led downe into the see ground, and there I saw hill and dalis grene, semand, as it were, mosse begrowne, with wrekke and with gravel. Than I understode thus, that if a man or a woman were under the broade watyr, if he might have sight of God, so as God is with a man continually, he should be save in body and soule and take no harme.

However unexpected this picture of sea-bottom diving may seem to us now, a user of an illustrated Psalter would immediately think of the image of David in water, beginning Psalm 68 (69), 'Unto the end, for them that shall be changed; for David. Save me, O God: for the waters are come in even unto my soul. I stick fast in the mire of the deep: and there is no sure standing. I am come into the depth of the sea: and a tempest hath overwhelmed me' (e.g., St Omer Psalter, fol. 54v, https://medieval.bodleian.ox.ac.uk/catalog/manuscript_3971; Luttrell Psalter, fol. 121v, http: / /www.bl.uk/manuscripts/FullDisplay.aspx?ref=Add_MS_42130) As the fifteenth century progressed, David could also be replaced by more generic nudes in water, male and female (Scott 1996, Tables I and III). Similar imagery is evoked in the Psalm 139:9-10, which usually had no illumination in the Middle Ages, with some notable exceptions (e.g., the eleventh-century Harley Psalter, British Library MS 603, http:/ /www.bl.uk/catalogues/illuminatedmanuscripts/record.asp?MSID=18402\&CollID= $8 \&$ NStart $=603$ ). 
Julian is surprised to be shown no Jews and no Purgatory or Hell, which contributes to her doubts about the nature and the orthodoxy of her revelation-particularly given that Doom pictures were common enough in East Anglia, primarily as west-wall church murals (Cf Psalters: Ps 38 Dixit custodiam (e.g., Macclesfield or Ormesby Psalters) depicts Christ before Pilate, with unpleasant-looking Jews.). Almost no images of Purgatory appear in English devotional manuscripts, although the locus itself was a major concern for the late-medieval believers, as demonstrated by Birgitta of Sweden's and her copycat Margery Kempe's prophecies, and it is certainly extremely unlikely to find these in Psalters and Books of Hours (see http:/ / www.medievalbooksofhours.com/learn). Betrayal scenes feature in these books only occasionally, and the earlier Crucifixes are quite laconic, with Jews or Roman soldiers usually featuring in the more elaborate compositions of contemporary altarpieces, such as the late 14th-century Despenser Altar in Norwich cathedral. Equally, the Judgment is not usually a part of a more conventional illustrative programme. When Julian states that today is "the Domesday for me" she is probably partly referencing illuminated Apocalypses of the first half of the fourteenth century and perhaps even the famous bosses of the Norwich cathedral cloister, about which she must have at least heard.

Less obvious yet plausible examples of sources of Julian's imagery found in Psalters and Hours include the just over-the-knee long, worn-out "kirtle" of the Servant. It simultaneously reminds us of the clothes of the delving Adam (most famously in the Canterbury Cathedral twelfth-century stained glass), or Christ as a gardener in the "noli me tangere" scene (more typical for the fifteenth-century continental iconography, as in British Library King's 5 of 1405, fol. 23r (http:/ / www.bl.uk/catalogues/ illuminatedmanuscripts/ILLUMIN.ASP?Size=mid\&IIlID=3579)—the English Hours and Psalters preferred depicting Christ with labarum, not spade), and of the loincloth of the Crucifixion from the late thirteenth and fourteenth century, which is more substantial than the later diaper-type, more typical from the fifteenth century onwards. It is particularly noticeable in the Deposition scene found in books, with Christ's loincloth hanging down to the ground, his floppy arms around the person below the cross, both embracing (Julian's "enfolding") and flapping like a drying cloth from Chapter 17 of the Showings. One also wonders if the famous defecation passage in Chapter 6 of the Paris manuscript of the LT could have been inspired by the manuscript marginalia similar to those found in the Gorleston Psalter and its "colleagues" (http://www.bl.uk/manuscripts/Viewer.aspx?ref=add_ms_49622_fs001r).

\section{Conclusions}

Despite her (perhaps unconscious) dependence on the images and illuminated books, Julian gently criticizes their over-use as devotional means, as she says in the same Chapter 6:

And at the same time, the custom of our prayer was brought to my mind, how that we use for un-knowing of love to make many means. Then saw I verily that it is more worship to God and more very delight that we faithfully pray to himself out of his goodness ..., than if we made all the means that heart can think of. For if we make all these means, it is too little and not full worship to God.

As she lists these means: praying to Christ's flesh, blood, Passion, death, wounds, His Mother, His Cross, the "special saints" and the "blessed company of heaven", a Book of Hours again comes to mind, with its images, its Hours of the Virgin and of the Cross and the litanies of saints, often "special", that is customised for the use of the owner. She, however, is far from dismissing them altogether, stating that "it pleases [God] that we seek him and worship him through [these] means, understanding and knowing that he is the goodness of all". This is not necessarily a sign that Julian is trying to avoid accusations of being a Lollard, who rejected devotional images; she could have been thinking of such "means" becoming luxury status symbols, used for showing off rather than true devotion, as ridiculed by her French contemporary Eustache Deschamps in the case of Books of Hours: 
A book of hours, too, must be mine, where subtle workmanship will shine, of gold and azure, rich and smart, arranged and painted with great art, covered with fine brocade of gold; and there must be, so as to hold the pages closed, two golden clasps (Panofsky 1953, p. 68)

The images of Julian's own book are not physical, gilded pictures on expensive velum, but mostly mental, which she, in turn, "forms in our understanding". This modesty continued in the manuscript of the Passion of Christ by Michael de Massa made for Julian's neighbour, Miles Stapleton (MS Bodley 758), whose daughter Emma later became an anchoress in the Carmelite priory not far from St Julian's (perhaps influenced by Julian herself, whom she must have known) and whose other daughter, Ela Brewes (grandmother to Margery Brewes of the first Valentine letter fame), inherited the book in 1443 (Hill 2006, p. 175; Meale 2009, pp. 83-84). It contains one simple monochrome illustration: the opening Crucifixion, the only colour added being the red of the blood. What is remarkable about this image is that, in keeping with Julian's words, only "Christ's friends" are present, including the medieval scribe and illuminator. In her earlier life Julian expressed a strong wish to be at the Cross with Mary Magdalen (LT 2), and here we have the hem of the saint's garment breaking the frame of the image, overhanging it towards the viewer, connecting the two dimensional with the three dimensional, the inanimate with the live, the past with the present, in a truly mystical fashion.

As an author of a contemplative text, Julian is certainly pushing beyond imagery, but the roots of her revelations are nonetheless to be found in these very means she both criticises and defends. It is difficult to accept that Julian included an apology for devotional images, found in her ST, only out of fear of being mistaken for a Lollard ("Notwithstandinge that I leeved sadlye alle the peynes of Criste as halye kyrke shewes and teches, and also the paintinges of crucifexes that er made be the grace of God aftere the techinge of haly kyrke to the liknes of Cristes passion, als farfurthe as manes witte maye reche-noughtwithstondinge alle this trewe beleve, I desirede a bodilye sight, wharein I might have more knawinge of bodelye paines of oure lorde oure savioure, and of the compassion of oure ladye, and of alle his trewe loverse that were belevande his paines that time and sithene" (Julian of Norwich 2006, p. 63). However, even if it was the case, the apology was removed from the LT altogether. Affective piety, whether its practitioners want it or not, is grounded in everyday materiality, in the inevitability of the embodied-ness, embedded-ness of the mind, of the word made flesh, and it is only natural that we may wish to study the fruit of these devotions through the workings of our own bodies, such as the ability to see, produce, and describe images. The age of multidisciplinarity increasingly means that we cannot separate the study of the human culture from the study of human physiology. Far from eliminating the mystery and trying to know "our Lords privy councell" (LT, ch. 30), or reducing Julian to a set of symptoms, reading the Revelations with psychology and mind science reminds us of how the unfathomable complexity of our organisms create the "difference in sameness" (McGilchrist 2010, p. 53), and how physical processes, affecting a brain just like ours, led to the creation of a work which has a power to amaze, comfort, inspire, frustrate, and puzzle over six hundred years later.

Funding: This research received no external funding.

Acknowledgments: Many thanks are due to Sarah Salih, Vicki Blud, Godelinde Perk, Daniel Gerrard, and Julia Bolton Holloway, who read and commented on earlier drafts of this paper.

Conflicts of Interest: The author declares no conflict of interest.

\section{References}

\section{Primary Sources}

Chaucer, Geoffrey. 1988. The Riverside Chaucer, 3rd ed. Edited by Larry D. Benson. Oxford: Oxford University Press. Julian of Norwich. 1998. Revelations of Divine Love. Translated by Elizabeth Spearing. Introduction and Notes by A. C. Spearing. London: Penguin Books. 
Julian of Norwich. 2001. Showing of Love: Extant Texts and Translation. Edited by Julian of Norwich, Sister Anna Maria Reynolds and Julia Bolton Holloway. Firenze: SISMEL, Edizioni del Galluzzo.

Julian of Norwich. 2006. The Writings of Julian of Norwich: 'A Vision Showed to a Devout Woman' and 'A Revelation of Love'. Edited by Nicholas Watson and Jacqueline Jenkins. University Park: Pennsylvania State University Press.

Julian of Norwich. 2016. Revelations of Divine Love: The Short Text and the Long Text. Edited by Barry Windeatt. Oxford: Oxford University Press.

Kempe, Margery. 2004. The Book of Margery Kempe. Edited by Barry Windeatt. Woodbridge: D.S. Brewer.

Littlehales, Henry, ed. 1895. The Prymer or the Lay Folks' Prayer Book. Early English Text Society, Original Series 105. London: Kegan Paul, Trench, Trübner \& Co.

Lorris, Guillaume de, and Jean de Meun. 1992. Le Roman de la Rose. Le Livre ide Poche: Lettres gothiques.

Online Froissart. 2013. Available online: https://www.hrionline.ac.uk/onlinefroissart/index.jsp (accessed on 1 April 2019).

Pearsall, Derek, ed. 1990. The Floure and the Leafe, The Assemblie of Ladies, and The Isle of Ladies. Kalamazoo: Medieval Institute Publications.

\section{Secondary Sources}

Aers David, Lynn Staley. 1996. The Powers of the Holy: Religion, Politics, and Gender in Late Medieval English Culture. University Park: Pennsylvania State University Press.

Baker, Denise. 1994. Julian of Norwich's Showings: From Vision to Book. Princeton: Princeton University Press.

Barbetti, Claire. 2011. Ekphrastic Medieval Visions: A New Discussion in Interarts Theory. New York and Basingstoke: Palgrave Macmillan.

Barratt, Alexandra. 2008. 'No such sitting': Julian tropes the Trinity. In A Companion to Julian of Norwich. Edited by Liz Herbert McAvoy. Cambridge: D. S. Brewer, pp. 42-52.

Bell, David N. 1995. What Nuns Read: Books and Libraries in Medieval English Nunneries. Kalamazoo: Cistercian Publications.

Blud, Victoria. 2016. Emotional Bodies: Cognitive Neuroscience and Mediaeval Studies. Literature Compass 13: 457-66. [CrossRef]

Bolte Taylor, Jill. 2009. My Stroke of Insight. London: Hodder.

Broks, Paul. 2016. Imaginal Reality. In Keynote Address at The Eye's Mind: Visual Imagination, Neuroscience and the Humanities: An International Conference at the Sainsbury Centre for Visual Arts. Norwich: University of East Anglia.

Burkert, Walter. 1996. Creation of the Sacred: Tracks of Biology in Early Religions. Cambridge, MA and London: Harvard University Press.

Bynum, Caroline Walker. 1991. Fragmentation and Redemption: Essays on Gender and he Human Body in Medieval Religion. New York: Zone Books.

Camille, Michael. 1996. Gothic Art: Visions and Revelations of the Medieval World. London: Weidenfeld \& Nicolson.

Chance, Jane. 2012. Cognitive alterities: From cultural studies to neuroscience and back again. Postmedieval: $A$ Journal of Medieval Cultural Studies 3: 247-61. [CrossRef]

Cohen, Jeffrey Jerome. 2016. For Earth Day: On Viewing a Home Planet at Great Distance. Available online: http:/ / www.inthemedievalmiddle.com/2016/04/for-earth-day-on-viewing-home-planet-at.html (accessed on 1 April 2019).

Cooper, Lisa H., and Andrew Denny-Brown, eds. 2014. The Arma Christi in Medieval and Early Modern Material Culture. Farnham: Ashgate.

Damasio, Antonio. 2010. Self Comes to Mind: Constructing the Conscious Brain. London: William Heinemann.

Davies, Owen. 2003. The Nightmare Experience, Sleep Paralysis and Witchcraft accusations. Folklore 114: 181-203. [CrossRef]

Deane, Jennifer Kolpacoff. 2013. Medieval Domestic Devotion. History Compass 11: 65-76. [CrossRef]

Deighton, Alan. 1993. Julian of Norwich's Knowledge of the Life of St John of Beverley. Notes and Queries 40: 440-43. [CrossRef]

Dennison, Lynda. 2013. Flemish Influence on English Manuscript Painting in East Anglia in the Fourteenth Century. In East Anglia and its North Sea world in the Middle Ages. Edited by David Bates and Robert Liddiard. Woodbridge: The Boydell Press.

Dictionnaire du Moyen Français (1330-1500). 2016. Available online: http:/ /www.atilf.fr/dmf/ (accessed on 1 April 2019). 
Dresvina, Juliana, and Victoria Blud, eds. Forthcoming. Cognitive Sciences and Medieval Studies: An Introduction. Cardiff: University of Wales Press.

Dudley, Margaret, and Julian Goodare. 2013. Outside in or Inside out: Sleep Paralysis and Scottish Witchcraft. In Scottish Witches and Witch-Hunters. Edited by Julian Goodacre. Basingstoke and New York: Palgrave Macmillan.

Duffy, Eamon. 2006. Marking the Hours: English People and Their Prayers, 1240-1570. New Haven and London: Yale University Press.

Dunn, Penelope. 2004. Trade. In Medieval Norwich. Edited by Carole Rawcliffe and Richard Wilson. London and New York: Hambledon and London Publisher.

Dutton, Elizabeth. 2008. Julian of Norwich: The Influence of Late-Medieval Devotional Compilations. Cambridge: D. $S$. Brewer.

Echenhofer, Frank. 2011. Ayahuasca Shamanic Visions: Integrating Neuroscience, Psychotherapy, and Spiritual Perspectives. In A Field Guide to a New Meta-field: Bridging the Humanities-Neurosciences Divide. Edited by Barbara Maria Stafford. Chicago and London: Chicago University Press.

England's Immigrants 1330-1550. 2019. Available online: https://www.englandsimmigrants.com (accessed on 1 April 2019).

Farias, Miguel, Raphael Underwood, and Gordon Claridge. 2012. Unusual but sound minds: Mental health indicators in spiritual individuals. British Journal of Psychology 17: 1-18. [CrossRef] [PubMed]

Fraeters, Veerle, María Eugenia Góngora, and Thérèse de Hemptinne, eds. 2013. Speaking to the Eye: Sight and Insight through Text and Image (1150-1650). Turnhout: Brepols Publishers.

Gertsman, Elina. 2015. Worlds Within: Opening the Medieval Shrine Madonna. University Park: The Pennsylvania State University Press.

Gibson, Gail McMurray. 1989. The Theater of Devotion: East Anglian Drama and Society in the Late Middle Ages. Chicago. London: University of Chicago Press.

Gillespie, Vincent, and Maggie Ross. 1992. The Apophatic Image: The Poetic of Effacement in Julian of Norwich. In Medieval Mystical Tradition in England: Exeter Symposium V. Edited by Marion Glasscoe. Cambridge: D. S. Brewer.

Granger, Penny. 2009. The N-Town Play: Drama and Liturgy in Medieval East Anglia. Woodbridge: Boydell \& Brewer.

Gunn, Cate. 2008. 'A recluse atte Norwyche': Images of Medieval Norwich and Julian's revelations. In $A$ Companion to Julian of Norwich. Edited by Liz Herbert McAvoy. Cambridge: D. S. Brewer, pp. 32-41.

Hagen, Susan K. 2004. The Visual Theology of Julian of Norwich. In Medieval Memory: Image and Text. Turnhout: Brepols, pp. 145-60.

Hamburger, Jeffrey. 1998. The Visual and the Visionary: Art and Female Spirituality in Late Medieval Germany. New York: Zone Books.

Harwood, Britton J. 1991. Pearl as Diptych. In Text and Matter: New Critical Perspectives of the Pearl-Poet. Edited by Robert J. Blanch, Miriam Youngerman Miller and Julian N. Wasserman. Troy: Whitson Press, pp. 61-78.

Hill, Carole. 2006. Julian and Her Sisters: Female Piety in Late Medieval Norwich. In The Fifteenth Century 6. Edited by Linda Clark. Woodbridge: Boydell.

Hodges, Laura Fulkerson. 2005. Chaucer and Clothing: Clerical and Academic Costume in the General Prologue. Cambridge: D. S. Brewer.

Johnson, Andrew James, Ethan Knapp, and Margitta Rouse, eds. 2015. The Art of Vision: Ekphrasis in Medieval Literature and Culture. Columbus: The Ohio State University Press.

Kamerick, Kathleen. 2002. Popular Piety and Art in the Late Middle Ages. New York: Palgrave.

Karnes, Michelle. 2011. Imagination, Meditation and Cognition in the Middle Ages. Chicago: University of Chicago Press. Kelly, Douglas. 1978. Medieval Imagination: Rhetoric and the Poetry of Courtly Love. Madison: University of Wisconsin Press.

Kennedy, Kathleen E. 2014. Reintroducing the English Books of Hours, or “English Primers". Speculum 89: 693-723. [CrossRef]

King, David. 2010. Corpus Vitriarum Medii Aevi. Available online: http://www.cvma.ac.uk (accessed on 1 April 2019).

Kosslyn, Stephen. 1994. Image and Brain: The Resolution of the Imagery Debate. Cambridge: MIT Press.

Kroll, Jerome, and Bernard Bachrach. 2005. The Mystic Mind: The Psychology of Medieval Mystics and Ascetics. New York and London: Routledge. 
Lasko, Peter, and Nigel Morgan. 1974. Medieval Art in East Anglia, 1300-1520. London: Thames and Hudson, Norwich: Jarrold.

Lentes, Thomas. 2006. "As far as the eye can see ... ": Rituals of Gazing in the Late Middle Ages. In The Mind's Eye: Art and Theological Argument in the Middle Ages. Edited by Jeffrey Hamburger and Anne-Marie Bouche. Princeton: Princeton University Press, pp. 360-73.

Lermack, Annette. 1993. Visual Sources in the Visions of Julian of Norwich. Master's thesis, Northern Illinois University, DeKalb, IL, USA, Unpublished work.

Llinás, Rodolfo, and Denis Paré. 1991. Of dreaming and wakefulness. Neuroscience 44: 521-35. [CrossRef]

MacLehose, William F. 2013. Fear, Fantasy and Sleep in Medieval Medicine. In Emotions and Health, 1200-1700. Edited by Elena Carrera. Leiden: Brill, pp. 67-94.

Malik, Kenan. 2001. Man, Beast and Zombie: What Science Can and Cannot Tell Us About Human Nature. London: Phoenix.

Marsh, George L. 1906. Sources and Analogues of The Flower and the Leaf. Modern Philology 4: 121-68, 281-328. [CrossRef]

Maskell, William, ed. 1846. Monumenta ritualia Ecclesiae Anglicanae. London: William Pickering, Vol. II.

McGilchrist, Iain. 2010. The Master and His Emissary: The Divided Brain and the Making of the Western World. Yale: Yale University Press.

McGinn, Bernard. 2006. Theologians as Trinitarian Iconographers. In The Mind's Eye: Art and Theological Argument in the Middle Ages. Edited by Jeffrey Hamburger and Anne-Marie Bouche. Princeton: Princeton University Press, pp. 186-207.

Meale, Carol M. 2009. Women's Voices and Roles. In A Companion to Medieval English Literature and Culture c.1350-c.1500. Edited by Peter Brown. Chichester: Wiley-Blackwell.

Middle English Dictionary. 2013. Available online: https:/ /quod.lib.umich.edu/m/med/ (accessed on 1 April 2019).

Mitchell, John. 2000. Painting in East Anglia around 1500: The Continental Connection. In England and the Continent in the Middle Ages: Studies in Memory of Andrew Martindale. Edited by John Mitchell and Matthew Moran. Stamford: Shaun Tyas.

Morgan, Ben. 2013. On Becoming God: Late Medieval Mysticism and the Modern Western Self. New York: Fordham University Press.

Morris, Teresa, and Julia Bolton Holloway. 2010. Julian of Norwich: A Comprehensive Bibliography and Handbook. Lewiston and Lampeter: Edwin Mellen Press.

Muir, Lynette R. 1997. Playing God in Medieval Europe. In The Stage as Mirror: Civic Theatre in Late Medieval Europe. Edited by Alan E. Knight. Cambridge: D. S. Brewer.

Newberg, Andrew, Eugene D'Aquili, and Vince Rause. 2001. Why God Won't Go Away. New York: Ballantine Books.

Newman, Barbara. 2005. What Did It Mean to Say "I Saw"? The Clash between Theory and Practice in Medieval Visionary Culture. Speculum 80: 1-43. [CrossRef]

Nichols, Ann E. 2002. The Early Art of Norfolk: A Subject List of Extant and Lost Art Including Items Relevant to Early Drama. Kalamazoo: Western Michigan University.

Oxford English Dictionary. 2019. Available online: http://www.oed.com/ (accessed on 1 April 2019).

Olunu, Esther, Ruth Kimo, Esther Olufunmbi Onigbinde, Mary-Amadeus Uduak Akpanobong, Inyene Ezekiel Enang, Mariam Osanakpo, Ifure Tom Monday, David Adeiza Otohinoyi, and Adegbenro Omotuyi John Fakoya. 2018. Sleep Paralysis, a Medical Condition with a Diverse Cultural Interpretation. International Journal of Applied and Basic Medical Research 8: 137-42.

Onians, John. 2007. Neuroarchaeology and the Origins of Representation in the Grotte de Chauvet: A Neural Approach to Archaeology. In Image and Imagination: A Global Prehistory of Figurative Representation. Cambridge: University of Cambridge, pp. 307-20.

Onians, John. 2016. European Art: A Neuroarthistory. New Haven and London: Yale University Press.

Ormrod, Mark, Nicola McDonald, and Craig Taylor, eds. 2017. Resident Aliens in Later Medieval England. Turnhout: Brepols Publishers.

Panayotova, Stella. 2008. The Macclesfield Psalter. London: Thames \& Hudson.

Panofsky, Erwin. 1953. Early Netherlandish Painting. Cambridge: Harvard University Press.

Parker, Kate. 2004. Lynn and the Making of a Mystic. In A Companion to Margery Kempe. Edited by John H. Arnold and Katherine J. Lewis. Cambridge: D. S. Brewer, pp. 55-74.

Parsons, Ben, and Bas Jongenelen. 2012. 'In Which Land Were You Born?': Cultural Transmission in the Historie van Jan van Beverley. Medieval English Theatre 34: 30-76. 
Penketh, Sandra. 1997. Women and Books of Hours. In Women and the Book: Assessing the Visual Evidence. Edited by Lesley Smith and Jane H. M. Taylor. London: British Library, Toronto: University of Toronto Press, pp. 266-80.

Perk, Godelinde. 2017. Was Julian's Nightmare a Māre? Julian of Norwich and the Vernacular Community of Storytellers. In Medieval Anchorites in Their Communities. Edited by Cate Gunn and Liz Herbert McAvoy. Woodbridge: Boydell, pp. 147-64.

Rampley, Matthew. 2016. Fish, volcanoes and the art of brains: Review of John Onians, European Art: A Neuroarthistory. Journal of Art Historiography 15. Available online: https:/ / arthistoriography.files.wordpress. com/2016/10/rampley-review-2.pdf (accessed on 1 April 2019).

Rampley, Matthew. 2017. Seduced by Darwin: Art, Evolution and Neuroscience. University Park: The Pennsylvania State University Press.

Ratey, John J. 2003. A User's Guide to the Brain: Perception, Attention, and the Four Theaters of the Brain. London: Abacus.

Read, Rupert. 2011. Review: The Master and His Emissary. Phenomenology and the Cognitive Sciences 11: 119-24.

Ringbom, Sixten. 1969. Devotional Images and Imaginative Devotions: Notes on the Place of Art in Late Medieval Private Piety. Gazette des Beaux-Arts 73: 159-70.

Salih, Sarah. 2019. Julian of Norwich, the Carrow Psalter and Embodied Cinema. In Visions and Voice Hearing in Medieval and Early Modern Contexts. Basingstoke: Palgrave Macmillan.

Sandler, Lucy Freeman. 1986. Gothic Manuscripts 1285-1385 (Survey of Manuscripts Illuminated in the British Isles). London: Harvey Miller.

Sandler, Lucy Freeman. 2004. Word Imagery in English Gothic Psalters: The Case of the Vienna Bohun manuscript (ÖNB, cod. 1826). In The Illuminated Psalter: Studies in the Content, Purpose and Placement of Its Images. Turnhout: Brepols.

Scherb, Victor I. 2001. Staging Faith: East Anglian Drama in the Later Middle Ages. London: Associated University Presses, Madison: Fairleigh Dickinson University Press.

Scholten, Frits, ed. 2016. Small Wonders: Late-Gothic Boxwood Micro-Carvings from the Low Countries. Amsterdam: Rijksmuseum Publications Department.

Scott, Kathleen. 1996. Later Gothic Manuscripts, 1390-1490 (Survey of Manuscripts Illuminated in the British Isles). London: Harvey Miller, vol. II.

Scott-Stokes, Charity. 2006. Women's Books of Hours in Medieval England. Cambridge: D. S. Brewer.

Sharpless, Brian, and Karl Doghramji. 2015. Sleep Paralysis: Historical, Psychological, and Medical Perspectives. Oxford and New York: Oxford University Press.

Smith, Kathryn A. 2003. Art, Identity and Devotion in Fourteenth-Century England: Three Women and their Books of Hours. London: British Library and University of Toronto Press.

Stewart, Susan. 1993. On Longing: Narratives of the Miniature, the Gigantic, the Souvenir, the Collection. Durham and London: Duke University Press.

Sutherland, Annie. 2015. English Psalms in the Middle Ages, 1300-1450. Oxford: Oxford University Press.

Tallis, Raymond. 2016. Aping Mankind: Neuromania, Darwinitis and the Misrepresentation of Humanity. London: Routledge.

Tanner, Norman. 1984. The Church in Late Medieval Norwich 1370-1532. Toronto: Pontifical Institute of Mediaeval Studies.

Tanner, Norman. 2004. Religious Practice. In Medieval Norwich. Edited by Carole Rawcliffe and Richard Wilson. London and New York: Hambledon and London.

Thorpe, Deborah. 2015. Tracing Neurological Disorders in the Handwriting of Medieval Scribes: Using the Past to Inform the Future. The Journal of the Early Book Society for the Study of Manuscripts and Printing History 18: 241-48.

Upjohn, Sheila, and Nicholas Groves. 2018. Julian's Church Norwich. Norwich: The Friends of Julian of Norwich.

Walker, Katie. 2003. The Meanings of Devotional Space: Female Owner-Portraits in Three French and Flemish Books of Hours. York Medieval Yearbook 2. Available online: http://www.york.ac.uk/teaching/history/pjpg/ devotional.pdf (accessed on 1 April 2019).

Watson, Nicholas. 1992. The Trinitarian Hermeneutic in Julian of Norwich's Revelation of Love. In The Medieval Mystical Tradition in England: Exeter Symposium V: Papers read at the Devon Centre, Dartington Hall, July 1992. Edited by Marion Glasscoe. Woodbridge: D. S. Brewer, pp. 79-100.

Webb, Diana. 2008. Domestic Space and Devotion. In Defining the Holy: Sacred Space in Medieval and Early Modern Europe. Edited by Andrew Spicer and Sarah Hamilton. Aldershot: Ashgate, pp. 27-47.

Wieck, Roger S. 1997. Painted Prayers. New York: George Braziller, Inc. 
Wilson, David Sloan. 2002. Darwin's Cathedral: Evolution, Religion, and the Nature of Society. Chicago and London: University of Chicago Press.

Windeatt, Barry. 2014. Julian of Norwich and Medieval Visual Culture. In Truthe Is the Beste: A Festschrift in Honour of A.V.C. Schmidt. Edited by Nicolas Jacobs and Gerald Morgan. Oxford: Peter Lang, pp. 185-203.

Winston-Allen, Anne. 1997. Stories of the Rose: The Making of the Rosary in the Middle Ages. University Park: Pennsylvania State University Press.

Woodforde, Christopher. 1950. The Norwich School of Glass-painting in the Fifteenth Century. London: Oxford University Press.

Ziegler, Joanna E. 1992. Sculpture of Compassion: The Pietà and the Beguines in the Southern Low Countries, c.1300-c.1600. Bruxelles: Institut Historique Belge de Rome, Turnhout: Brepols.

(C) 2019 by the author. Licensee MDPI, Basel, Switzerland. This article is an open access article distributed under the terms and conditions of the Creative Commons Attribution (CC BY) license (http:/ / creativecommons.org/licenses/by/4.0/). 
Article

\title{
Hereditary Ecclesiae and Domestic Ecclesiolae in Medieval Ragusa (Dubrovnik)
}

\author{
Matko Matija Marušić \\ Faculty of Humanities and Social Sciences, University of Rijeka, HR-51000 Rijeka, Croatia; \\ matkomatijamarusic@gmail.com
}

Received: 2 October 2019; Accepted: 14 December 2019; Published: 20 December 2019

\begin{abstract}
The present paper explores domestic devotional practices in Ragusa (modern day Dubrovnik) from the late-thirteenth through the fourteenth and fifteenth centuries. Considering that important advancements in the understanding of domestic devotions in major Mediterranean cities have recently been made-particularly in Venice-the scrutiny of Ragusan sources enables further reflections on the same phenomena in minor Adriatic centres. Considering the paucity of preserved objects, and the fact that no late medieval domestic space of that time has survived in Dubrovnik, one must turn to archival sources to answer questions pertaining to the arrangements and uses of spaces of domestic worship. Three aspects are analysed here: privately owned chapels-adjoined to the dwellings of urban nobility, prayer areas and holy images inside the houses, and relics in the possession of individuals. In light of its source-driven approach, a significant part of this paper is devoted to the issue of the terminology of devotional props in contemporary documents. On a more general level, the paper aims at showing how, although no direct evidence of domestic devotional practices survives (such as in-depth textual evidence), all indications suggest that it was a deeply family-centred matter. Accordingly, particular attention is paid to the city's most prominent families, such as Volcassio, Volzio and Sorgo. Finally, the evidence presented in this paper, gathered from both published and unpublished sources, offers valuable material for reflections on the spatial arrangements of domestic devotional spaces, not necessarily confined to the members of a single household, but, through hereditary rights, tied to specific lineages.
\end{abstract}

Keywords: medieval Ragusa; domestic devotion; devotional triptychs; ecclesiola; domestic chapel; Holy-Sepulchre chapels; jus patronatus; privately owned relics

\section{Introduction}

Valuable advancements have recently been made in the understanding of domestic devotion, both in terms of the wide variety of holy objects in homely settings, and their use in devotions (Kasl 2004; Webb 2005; Cooper 2006; Nalle 2008; Kolpacoff Deane 2013; Baldissin Molli et al. 2018). The studies have revealed a rich domestic culture of households that has previously gone unnoticed in favour of the concept of (medieval) homes as strictly secular spaces. In tackling this presumption, the interest of scholars turned to major cities, so exemplary studies were carried out for fifteenth- and sixteenth-century Florence (Lydecker 1987; Schmidt 2005; Mattox 2006). Initial endeavours aimed at investigating more expected forms of domestic devotion, such as the construction of household chapels and domestic altars, yet the complexity of scrutinising these aspects of medieval homes lies in the fact that a range of objects in everyday use could have possessed religious qualities (Musacchio 2000; Morgan 2017, pp. 215-18; Howard et al. 2017).

Sources relating to this topic regarding Venice (Morse 2007, 2013a), furthermore, have painted a rich picture of the influx of religion in the city's case, pointing to the multifaceted range of devotional props of various iconographies and settings (Morse 2007, p. 184). Interest in these topics is continuously 
growing, and the second wave of studies tends to overcome the focus on the most prominent cities by paying closer attention to lesser-studied regions. In the interest of furthering this debate, the desiderata of these studies aims at offering nuanced insights into the general and specific phenomena of certain cities and regions, which could then be compared cross-regionally, and occasionally, cross-confessionally, as well (cf. Bacci 2018).

A substantial body of documents relating to study of medieval and early modern domestic devotional art in the cities on the eastern shore of the Adriatic have been published, but these have rarely been considered in the light of practices of household piety. ${ }^{1} \mathrm{~A}$ convenient place to start is Ragusa (modern day Dubrovnik), given that the city archives store hundreds of documents (either contracts, last wills or inventories of household goods) mentioning images in the broader sense of the word. These are consistently referred to as anconae or iconae and, hence, are not necessarily "icons" and not exclusively panel paintings. These documents-the vast majority of which refer to the images in a domestic context-were collected and edited in the early 1950s (Tadić 1952, vols. I and II). How frequently these pious domestic paintings were in inventoried households is hard to tell, due to the lack of extensive quantitative research on the subject (although it has been estimated that ninety percent of Venetian households possessed at least one devotional image, cf. (Morse 2007, pp. 163-65)). Finally, Dubrovnik's medieval and early modern domestic architecture is fairly well researched-including the interiors of homes-but the sacred space within them has been left underexplored (cf. Grujić 2013).

This paper, accordingly, draws on the research carried out for other (mostly Italian) cities, aiming to reconstruct the range of domestic devotional spaces and objects in late medieval Ragusa. Considering the paucity of devotional objects preserved, this can only be done through an attentive reading of archival sources, which are abundant in number but scant in description of domestic devotional props. In this sense, one is inevitably faced with the problem of terminology in medieval notary documents, so a significant part of the paper is devoted to this important issue. Of particular interest here, however, is not the typology of devotional objects per se, but the question of their enshrinement in houses, as well as their role in the life of their beholders, and, more specifically, in the context of family values of that time. This paper, therefore, tries to scrutinise sources on the material culture of domestic devotion, as well as analyse indirect evidence on the practices of domestic piety, most notably the iconography of holy images. The paper is divided into three sections devoted to freestanding private chapels, indoor devotional places (in both well-off and ordinary households), and privately owned relics, respectively. Such an approach aims at investigating the "local religion" (French 2014) of medieval Dubrovnik through the examination of sources on the appearances and audiences of domestic chapels and household shrines-key places of everyday spiritual life.

\section{Freestanding Chapels of Private Foundations and Their Domestic Setting}

The most opulent form of domestic devotion was the ownership of a chapel with the bishop's concession to hold masses. This form of piety was restricted to the highest ranks of society, which could afford such oratories to be built next to their mansions. As can be deduced from the sources, this was a custom of earlier centuries (the first recorded examples in Dubrovnik date from the early middle ages), which was to die out by the end of the fifteenth century. Moreover, such private chapels were first and foremost a matter of the urban fabric, given that free space inside the city walls was restricted. The later chapels (starting from the late fourteen to early fifteenth century), therefore, were more often constructed inside the houses themselves, or, as we shall see, outside the city walls.

The earliest example that will be discussed is that of the Volcassio family, Pasqua (d. 1293) and Damian (d. 1295), sons of Vukas. The two brothers were arguably the most prosperous Ragusans at

1 A notable exception are the reflections on medieval devotional culture of the city of Kotor, in modern day Montenegro (Živković 2016). Apart from the documents on medieval Ragusa, which will be analysed in this paper, sources have been gathered for Zadar, and briefly discussed in (Hilje 2000). For discussion of fifteenth-century prayer books in Dubrovnik, mostly regarding their dating and decoration, see (Prijatelj Pavičić 2011). 
the close of the thirteenth century (Vekarić 2012, p. 339; Benyovsky Latin and Ledić 2014, pp. 13-15), and their testaments contain detailed accounts of household chapels. Pasqua's last will reveals that he was responsible for the construction of the Trinity chapel, adjacent to his houses. Although fully constructed, the chapel lacked an official consecration at the time of Pasqua's death, so this task was assigned to epitropi (testamentary executors). As articulated in the text of the testament, the chapel was to be supplied with liturgical utensils and a missal, both to be used daily whilst the commissioner's wife Dessa was still living. ${ }^{2}$ After her death, the funds for the church were to be obtained from the lease of his properties and administered by the city's treasurers (which were responsible for collecting and distributing the rent, according to the testament). ${ }^{3}$ The right of patronage (jus patronatus) of the Trinity chapel, as specified in Pasqua's testament, was hereditary. ${ }^{4}$

Pasqua's brother Damian made similar arrangements for a chapel erected on his private terrain, similarly adjoining his house. ${ }^{5}$ The titular of the chapel is not explicitly stated in his testament, yet it is reported that it was erected to honour God Almighty, Virgin and All Saints. This chapel was not consecrated by the time of Damian's death, and his last will does not reference any liturgical utensils for serving mass. The funds for its upkeep were procured through the rent of his house and shops in Ragusa, and (if needed) a house in Venice; the sum of money bequeathed for the chapel corresponded to 31 perpers, of which 25 were destined to Franciscan friars who were responsible for saying daily mass, while six perpers were suffice to supply the chapel with candles, and ensure its constant illumination by oil lamps. ${ }^{6}$ However, at the time of his death, Damian was not confident that the friars would carry out their appointed duty, so he suggested an alternative: if friars refused to say a daily mass, his epitropi were obliged to procure a chaplain to regularly celebrate mass for 25 perpers-that is, for an equal sum given to the friars. In case no priest could be found for day-to-day service, three or four masses could be said by the priest (for five perpers) and masses on other days could be celebrated by the friars (for 20 perpers, instead of 25 for seven masses). Regardless, the sum of the service in the chapel would not exceed 25 perpers.

Damian was particularly close to the friars; they are at the top of the list of his testamentary bequests, and he ordered his tomb monument to be erected in their church. ${ }^{7}$ Although, on his deathbed, Damian was not sure whether the friars would celebrate mass, his concerns were unnecessary, given that, almost a century later, in 1383, they were still in charge of the chapel. In the proclamation of the sale of two shops in Ragusa and half of a house, in the contrada of Sancti Maphey de Rivealtis in Venice, the levies on these real estates are listed, among which were one perper and one gross pro oleo capelle Damiani de Volcassio, and six perpers fratribus minoribus pro officiando cappellam Damian de

2 Item volo quod debeat (epitropi, op.a.) sacrari ecclesia sancte Trinitatis quam feci (cancell. laborari; edificari) iuxta domus meas pro anima mea et patris et matris mee et omnium meorum mortuorum. Et debeant fieri in dicta ecclesia paramenta et calix de argento et liber missalis et debeat celebrari missa in dicta ecclesia omni die in vita uxoris mee. (. . . ) (Čremošnik 1951, p. 230).

3 On the role of the city's treasurers, see (Lonza 2012, pp. 33-35).

4 Et volo et ordino quod ista predicta ecclesia numquam debeat esse in hereditario (Čremošnik 1951, p. 230).

5 Ordino quod ecclesia mea quam hedificari feci in territorio meo juxta domum meam propriis meis expensis ad honorem et reverentiam Dei Omnipotentis et beate Marie Virginis et Omnium Sanctorum pro salute (anime mee) ac patris et matris mee et aliorum meorum tam vivorum quam defunctorum, debeat officiari a fratribus minoribus, qui cotidie dicant unam missam in eadem ecclesia. Et missam ibi cellebraret ... pro salute anime mee et omnium predicatorum inperpetuum. (... ) Et volo et ordino, quod pitropi mei, qui per (tempora fuerint) de conductura seu pensione domus mee, que est in campo, debeant extrahere et accipere perperos XXXI annuatim. De quibus yperperis volo, quod pitropi mei debeant dare predicti fratribus minoribus pro officio dicte ecclesie mee yperperos XXV annuatim. Et sex yperperos residuos a predictis debeant dare iste ecclesie ... pro luminaribus scilicet oleo et candellis. Qui si ipsi fratres minores nollent dictam ecclesiam pro aliquo modo quo dictum est officiare, volo quod dicti pitropi mei inveniant unum honestum sacerdotem qui dicam debet cotidie officiare, cui sacerdoti de illis viginti quinque yperperis (... ) voluerit officiare ecclesiam meam predictam solummodo quatuor diebus in qualibet epdamada dicendo missam in eadem ecclesia. Volo quod ipsi fratres minores habeant de conductura domos mee predicte yperperos viginti de illis yperperis viginti quinque supradictis. Et pro residuis tribus diebus epdomade, in quibus unus sacerdos bonus et honestus debeat dictam ecclesiam officiare, dentur eidem sacerdoti quinque yperperi residui a predictis pro illis triebus diebus. Et volo, quod dicta ecclesia mea deveniat ad aliquem clericum eciam si esset de domo mea tam secundum jus patronatus quam secundam aliquam consuetudinem sicut superius ordinatum est. (Lučić 1993, p. 279).

6 Around 1300, one perper corresponded to twelve grossi, whereas several dozen perpers were an adequate sum for a wooden house; see (Živković 1985, pp. 36-37).

7 sepulturam meam eligo apud fratres minores de Ragusio (Lučić 1993, p. 279). 
Volcasso (DADU, Vend. Canc., 4, fol. $4 \mathrm{r}$ ). ${ }^{8}$ Therefore, even though the sums of the bequests do not match perfectly, the real estate sold in 1383 was the same one owned by Damian. More importantly, the chapel in question was still called by the name of its founder and, according to his wish, continued to be served by Franciscans (whether this was on a daily basis cannot be established by existing documentation). The same document, moreover, refers to the chapel as capella S. Marie de Volcasso (Vend. Canc., 4, fol. 3v), which is the first and only mention of its dedication. ${ }^{9}$ Later records, from 1422, attest that the chapel of his brother, Pasqua's Trinity chapel, was served by the friars as well, at the wish of his son, Matthew (d. before 1340). ${ }^{10}$

The evidence of the brothers' chapels reveals a great deal about them, but it leaves us with unanswered questions. Does the fact that both chapels were contiguous to the dwellings (and that they were hereditary) make them the Volcassio brothers" "private" chapels? It is beyond doubt that these sacred buildings perpetuated the memory of their founders and endowers, yet their original spatial context and use is far from certain. One is left wondering whether they were connected to the brothers' houses by internal doors, or if there was but one entrance, from the (public) street. Moreover, it is not known if these chapels were used exclusively by the members of the owners' household, or if they were public oratories, also frequented by the common congregation of the neighbourhood. In the absence of other information, we might turn to a later (seventeenth-century) situation in the former Sorgo manor (from the nineteen century, Bishop's palace). This lavish patrician house adjoined the early medieval church of Saint Cosmas and Damian, likely dating from the eleventh century (Fisković 1997). The main entrance to the church was from the public space, while the interior of the house communicated with the church through an empora (Figure 1), located just above its main altar (Belamarić 2018, pp. 28, 35). If this was, indeed, a corresponding situation to that of the much earlier Volcassio chapels, than these two early structures (referred to in testaments as "churches", yet in later sources as "chapels"), could have been both "private"/"domestic" and open to the public. The fact that they possessed a missal, and that the search for a chaplain was of prime importance to their commissioners, confirm that the edifices in question differed little from other cities' churches.

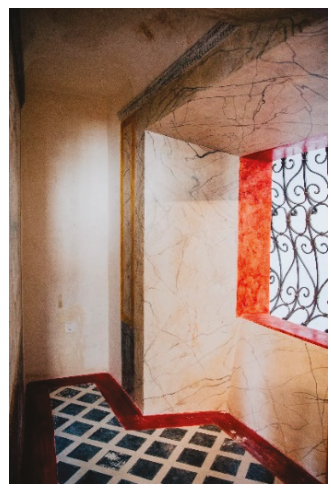

Figure 1. Empora in the Bishop's palace (former Sorgo manor); the opening with shutters is located just above the main altar of Saint Cosmas and Damian church, Dubrovnik (photo by Dean Tošović).

8 Further documents reveal that friars continued to serve Damianus' chapel, as was the case in 1461 (LAT, p. 205).

9 On the basis of later documents, it has been proposed that the chapel was dedicated to the Nativity (Beritić 1956, p. 69; taken up by Benyovsky Latin and Ledić 2014, p. 32).

10 (day unknown)-09-1422 ... dar ali frari minori de Ragusio per officiar la cappella de Sancta Trinita apresso la casa de Lauriça de Volchasso segondo el legatto del ditto Mathio (LAT, p. 201). 
Permission to hold masses in such chapels had to be obtained from the bishop, but, given that the Archives of the Archbishopric burned down in the fire that followed the disastrous earthquake of 1667, these documents are not available. Thankfully, some licences were recorded in notarial series, such as the permission granted to Jacobus Sorgo (d. 1401; cf. Vekarić 2015, pp. 165-66) at the closing of the fourteenth century, for the newly built Trinity chapel (the titular obviously fashionable among Ragusan noblemen). The cathedral chapter, represented by Deacon Dominicus Ribiça, with the consent of the Archbishop Petrus, conceded the right to hold masses and divine offices in the ecclesia seu cappella, built by Sorgo next to the communal wells (but apparently not attached to his house). According to the dedication ritual, which is described in detail, the cornerstone of the future Trinity chapel was to be blessed by the bishop in the Ragusan cathedral and laid in the ground at the future construction site of the chapel. Once the chapel was finished, the episcopal permission to hold masses was to be displayed in the church and sealed in red wax. The seal is precisely described in the licentia: its upper zone was occupied by the Virgin and Child, the central one by three unnamed saints, and the lower by a kneeling bishop and other insigniae (Div. Not., 10, fols. 133rv). ${ }^{11}$ A corresponding dedication practice can therefore be envisaged for other undocumented cases.

The most puzzling instance of privately owned oratories are the "domestic" chapels of Andreas Volzio (d. 1429). ${ }^{12}$ Tuscan humanist Philippus de Diversis, in his "Description of Ragusa" (1440), informs us that this prominent nobleman visited the Holy Land twice. Upon returning from these pilgrimages, Volzio commissioned a pair of Holy Sepulchre replicas: one in domo propria in the city, and the second one in "one of his beautiful gardens", located outside the city walls (de Diversis 2004, p. 183). ${ }^{13}$ Since both Volzio's chapels, erected ad similitudinem sanctum sepulcrum domini Jhesu, have perished, it is unknown whether their layouts reproduced the forms of the actual Holy Sepulchre. Although one could assume that both chapels were used privately, further information provided by Diversis attests to varied uses, regardless of their spatial context. The garden chapel, for instance, was frequented by numerous priests, which were hosted by Volzio "to the glory of the Saviour" (de Diversis 2004, p. 183).

In short, the case of Volzio's chapels, like the ones already discussed, palpably attests to how "domesticity" - a term that can be applied both to a (private) home and a garden-is rather slippery in discussion of these edifices (cf. Garnett and Rosser 2019, pp. 45-46). ${ }^{14}$ These hereditary cappellae and

11 Venerabilis vir Dominicus de Ribiça canonicus ecclesiae Ragusinae se confitetur quod in MCCCLXXXVI indictione VIIII die septimo Junii, dum tunc temporis esset vicarius in tempore liberis et spiritualibus reverendi in Christo patris et domini, domini fratris Petri dei et apostolice sedis gratia, archiepiscopi Ragusini, ut patet per publicum infrascriptum scripti manu Theodori Scolmafogia de Brundisio presens imperiali auctoritate notari et tunc jurament is notarius et cancellarius comunis Ragusii anno domini MCCCLXXVIIII indictione secunda die quinto mensis novembris, a me notario infrascripto iusum, lectum et plenarie intellectum dedit, cessit et concessit nobili viro ser Jacobo de Sorgo liberam licentiam et autoritatem et consensum virtute dicti vicariatus hedificandi et construendi unam ecclesiam seu capellam principaliter sub vocabulo et nomine Sancte et indivindue Trinitatis in dyocesi Ragusi et civitate iuxta puteum comunis qui dicitur illorum de Zrieva, et cum fuerit edificata in ea celebrari faciendi solemnia missarum et divina officia que in sacrosanctis aliis bulatum bulla pendenti ad cordulam facta in cera nova et ab intra cum cera rubea in cuius scultura et figura beate Virginis cum filio in superiori parte, in medio tres figure sanctorum et in inferiori parte figura unius episcopi genuflexi, cum insignis a parte. Cum niuno interferi quibus melius potuit et valuit ad augmentum divini cultus ecclesiis celebrantur, et hec licentia concessa est dicto ser Jacobo presenti et in stante petenti dicatam licentiam sibi concedi, presente reverendo in Christo patre fratre Petro ordinis minorum dei et apostolice sedis gratia episcopo soacensis epiri primum lapidem in dicta ecclesiae benedixit (et dompno Petro caput apis cancell.) in ecclesia Sancte Marie maioris et dompno Petro caput apis qui dictum lapidem portavit et in fundamento posuit ac domino Blasio dicto Vlacota Janice et aliis pluribus presentibus concessioni dicte licentie tunc facte seu concesse in quorum omnium testiomonium et cuiusdam veritatis dictus dyaconus Dominicus presens instrumentum scribi iussit et sigilli sui tunc vicariatus aprendere muniri. Presentibus nobilibus iuris ser Petro de Saracha iudice et ser Volço de Babalio testes.

12 His biography, including important duties he carried out, can be found in (Vekarić 2015, pp. 264-65).

13 (...) Andreas Martoli de Volzio nobilis (... ) qui cum bis visitasset sanctum sepulcrum domini Jhesu, illius similitudinem Ragusii in domo propria studit hedificare, et extra urbis moenia apud quoddam eius tunc pulcrum viridarium, ubi saepis sacerdotes congregans eos honore Salvatoris nostris pascebat. This instance was briefly discussed in the context of garden architecture and design in late medieval and early modern Dubrovnik, by (Šišić 2008, pp. 50-52).

14 This is further corroborated by the arrangements made by Andreas' son, Johannes, for the chapel dedicated to St Nicholas of Tolentino in 1459. The chapel, erected to honour the recently canonised saint (1446), was also located in the courtyard of his house (but inside the walls). However, the chapel was not in any way "private" or "household"; fifteenth-century sources attest that a group of tertiaries resided there, and the chapel was, in fact, adjoined to the hospital, founded in 1451 (Beritić 1956, pp. 56-57). 
ecclesiae, founded and upkept by distinguished families_even when adjoined to houses and built on the private land-could have been accessible to different and diverse audiences. After all, accessibility to such structures is linked to the commissioner's need to enhance their household prestige and secure that their souls are adequately prayed for. The conflation of public and private can also be seen in family altars, in churches where donors merged their spiritual needs with (similar) sophisticated representational strategies (cf. Bacci 2009; Murat 2014). To put it simply, hereditary chapels would not have fulfilled their purpose if they had been restricted to household members alone.

\section{Indoor Domestic Spaces and Holy Images: Iconographies and Uses}

According to the sources analysed, freestanding chapels, being limited to the social elite, were rather rare. Domestic devotional spaces, generally speaking, were confined within the walls of houses, and such examples can be found in the dwellings of urban nobility and commoners alike. For instance, in 1491, three perpers for illuminating the chapel in la casa of Dime Benessa (d. 1403), bought by Franciscus Benessa, were transferred to the altar of St Anthony in the chapterhouse of the Franciscan friary (LAT, p. 287). ${ }^{15}$ That is, the bequest for lighting a domestic church ended when the house in which it was located changed owner. In all likelihood, the chapel in the house was a room adapted to the purposes of an oratory. Apart from such rooms-chapels, devotional spaces could have been located in the courtyards. For instance, Trippe Bona, in his testament of 1463, bequeathed 40 perpers for an image of the Nativity (la istoria dela natividade del nostro Salvator) to be placed on the altar of the courtyard chapel of his caxa grande. ${ }^{16}$ The structure is referred to as capella pizola, which could imply that it was a chapel of a reduced size, most likely smaller than the ones of the Volcassio brothers, conceivably similar to the street tabernacle-like shrines that are still in place in virtually every Mediterranean city (cf. Muir 1989).

The most commonplace in-door arrangement that formed the sacred spot of houses-a devotional image protected by wooden cupboards of tabernacle-like appearance-was customarily called glesiola, a vulgarised derivative of Latin ecclesiola, literally "small church" or a "chapel". ${ }^{17}$ The contracts for such structures are rather sparsely recorded, as the structures were accessible wooden caskets, produced serially and therefore not commissioned for specific spaces and patrons. There are, nevertheless, notable exceptions. In 1369, Master Johannes Pavesarius stipulated a contract with Martinus Sorgo to deliver duas glesiolas, one smaller and the other one larger (Tadić 1952, vol. I, p. 16, doc. 48). Both glesiolae were to be painted with azurite, gilded, and ornamented with precisely 51 painted stars. ${ }^{18}$ The two decorated cupboards for holding devotional objects were commissioned for a rather modest price of six ducats. ${ }^{19}$ Given that no specific images or iconographies are mentioned in the contract, it could be presumed that Sorgo asked for a new chest for images he already possessed. There are indications that such decorated structures did not necessarily hold only one sacred object. The list of possessions of a certain Calea Greca, compiled after her death in 1382, mentions a "chest" with no less than four devotional images (una glesiola cum quatuor anconellis inter), (Div. Canc. 25, fol. 143r). ${ }^{20}$

15 1491. adi 19 giuglio. Ser Dragoie de ser Aluvise de Gozze de dar, come apar in diversse de nottaria sotto questo di, li perperi tre che se pagavano per el ditto ser Dragoie per aluminare la capela in la caxa de ser Dime de Benessa, comprada per ser Francisco St. de Benessa, sono apicade al altar de santo Antonio de Padua apresso la sepultura di quelli de Benessa in capitulo delo convento de San Francisco.

16 Voglio et ordino che in nostra capella existente in lo cortivo nostro dela caxa grande sia facta una inchona allo altare della capella pizola che io fexi construere de iperperi $X L$, et sia la istoria dela natividade del nostro Salvator.

17 It is important to note that Venetian documents of the same time almost exclusively employ the simple term "chest" (armarol, or armarol de santi), (Valenzano 2018, p. 29; Coccato 2018, p. 159). For general observations on the terminology of Florentine material, see (Schmidt 2005, pp. 50-63).

18 [facere] duas glesiolas: 1 magnam et aliam parvam, videlicet de faciendo ipsas glesiolas depingi de açuro fino, et retaies unum depingere de açuro fino et aliud cum auro de duchato. Et ponere stellas LI dictis II glesiolas copertas de auro fino.

19 For an examination of prices in fourteenth-century Ragusan painting, see (Fisković 1983).

20 In late fifteenth- and sixteenth-century records, such ensembles are more rarely referred to as "revetment" of anconae: clausura, clissura or glissura (Tadić 1952, vol. I, p. 236, doc. 487; vol. II, p. 28, doc. 804, 32, doc. 818, 97-98; Fisković 1990, pp. 149-50, doc. 978) 
In the fifteenth century, preserved contracts regularly describe simultaneous commission of images for devotional purposes and their correspondent cupboards. In 1443, prolific Ragusan painter Johannes Ugrinovich Zornea (Ivan Ugrinović) agreed to craft a wooden tabernacle (capela) containing three unnamed (saintly) figures (Tadić 1952, vol. I, p. 138, doc. 299). ${ }^{21}$ From this, it is reasonable to deduce that the commissioner, Nicolaus Bona, ordered a triptych, the prevailing design of medieval domestic imagery. ${ }^{22}$ The central field most likely represented the Virgin and Child (the other, less likely, option, is the commissioner's name-saint) while the other two figures were depicted on the inner sides of the shutters. Several years later, Ugrinovich's son, Stephanus, stipulated an analogous contract with Radissa Goicich by agreeing to craft an image of an unspecified subject (using gold, azurite, and other colours, ad laudem cuiuslibet magistri), which was incorporated in a wooden jesiola, designed by a carpenter named Petrus. ${ }^{23}$

Although these later documents reveal much about the process of commissioning and designing such devotional structures, they offer no information on their subject matter. In a similar way, household inventories, when referencing these compositions, do not comment upon their iconography, but their condition and aesthetic quality; appellations that are given are beautiful (ancona pulcra), gilded (a. deaurata), small (anconetta, a. pizola), sizeable (a. granda), etc. The term ancona greca (or alla greca / a modo greco), that has received notable attention in recent years, with regard to the devotional potential of archaizing images (Nagel and Wood 2010; Drandaki 2014; also-in the Ragusan context-Demori Staničić 2013), starts occurring in the sources only from the beginning of the sixteenth century. ${ }^{24}$

Notarial records do not specify where exactly these shrines were located, or how they were displayed-for instance, if they were hung on a wall or placed on a horizontal surface. Nevertheless, there are clues regarding the typical location of these domestic shrines in Ragusan houses, notably in references to anconae and giesiolae "da camera". ${ }^{25}$ Camera could refer to any room in the house, but the most reasonable choices are the sala grande and the bedchamber. Indeed, evidence exists for (at least) two sacred images in houses. The most illustrative example is the house of Antonius de Primo, which cherished two figurae della Madonna: one in prima camera and the other one in camera de sopra (Tadić 1952, vol. II, p. 28, doc. 801). Contrary to the situation in Florence-where devotional works were almost exclusively located in sleeping spaces (Wilkins 2002, p. 377)—evidence from Venice attests to a different placement of such images (Morse 2007, p. 161). Importantly, these "multiple locations of devotion" were not confined to spaces strictly for personal use (such as bedrooms), but to the receiving areas of homes as well (Morse 2007, pp. 161-62).

In Venetian houses and palaces, the places of devotion were often porticos (Morse 2013b). In Ragusa it was sala grande, commonly the first floor of the house. The simultaneous commissioning of two cupboards (as in the quoted case of Martinus Sorgo) is the strongest evidence of the location of such shrines in more than one room of the house. Given that the glesiolae commissioned by Sorgo differed in size, it is possible to speculate that the smaller one was intended for the bedroom, while the larger one could have occupied a more prominent and spacious place in the house of this noble family, arguably their sala grande. There are indications-albeit later-of sacred images in other parts of houses, such as the una incona dela Madonna dorada, that was hung in the kitchen (Tadić 1952, vol. II,

21 [facere] unam capelam, ligni intagliatam, de qua ipsi convenerunt ad invicem, videlicet, pulchram et laudabilem cum tribus figuris.

22 Although no example from the period in question has survived, the typology has persisted well into the modern period, see, for instance, (Gamulin 1991, pp. 68-69).

23 [facere] unam anchonam de auro fino et azuro fino et coloribus finis ad laudem cuiuslibet magistri, omnibus expensis eiusdem Stepani, et circumcirca ipsam anconam et quadrum facere jesiolam pulchram et firmam. Que anchona debet esse ad ilam mensuram, videlicet altitudinem, longitudinem et altitudinem (!) quam ordinabit Petar marangonus.

24 For instance, una figura mia alla greca, bequeathed in 1508 (Tadić 1952, vol. II, p. 33); for other contemporary denominations of domestic images, see (Musacchio 2000, p. 151; Morse 2007, p. 159). Images alla greca in Adriatic cities have received significant attention in this volume by (Voulgaropoulou 2019, pp. 14-19).

25 [inaurare] unum quadrum seu unam anchonam cum ecclesiola a camera, de bono opere et fino auro (Tadić 1952, vol. I, pp. 168-69, doc. 357), and unum quadrum de camera cum casselleta magnum et pulchrum ( ... ) et bene intagliatum quantum dare possit (Tadić 1952, vol. I, pp. 198-99, doc. 414). 
p. 33, doc. 823). ${ }^{26}$ After all, the movability of objects and chests of reduced size made it possible to relocate them according to the needs and wishes of household members, just as the sacred content of wooden cupboards could have been replaced with new devotional objects (not necessarily images, as shall be discussed below).

A surviving example offers a glimpse into what these domestic images might have looked like. The Madonna and Child (Figure 2), attributed to Blasius de Tragurio (Blaž Jurjev Trogiranin), is an emblematic painting for the purposes of personal devotion. The restoration work has proven that the outer rectangular frame is original, so the image was not supplied with hinged wings, but it could have been included in tabernacle-like structures (Demori Staničić 1986, p. 86). More importantly, the intimate iconography underlies the close connection between the Mother and Child, typical of domestic holy images. This small ancona attunes with the advice given by Giovanni Dominici in his Regola del governo familiare of 1403, in regard to the use of religious images in the upbringing of children: "It is well to have the Virgin Mary with the Child in arms, with a little bird or apple in His hand. There should be a good representation of Jesus nursing, sleeping, in His Mother's lap or standing courteously before her while they look at each other" (cited as in Musacchio 2000, pp. 147, 152). These instructions—routinely quoted in reflections on the use of objects of personal devotion—firmly place such props in the realm of "devotional pedagogy" - the moral and religious training of children (Van Os 1994, p. 91; Klapisch-Zuber 1998, p. 117; Johnson 2002).

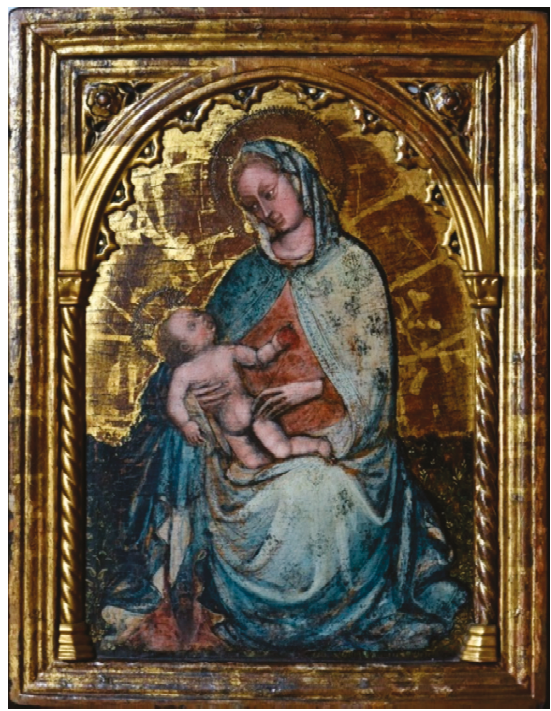

Figure 2. Blaž Jurjev Trogiranin, Madonna with Child, ca. 1421-27, tempera on wood, $64.5 \times 49.7 \mathrm{~cm}$, Bishop's Palace, Dubrovnik (photo by Božidar Gjukić).

Some of these domestic anconae were more complex, as they followed the design of church altarpieces. Besides the most radiated forms-that of a triptych or image of elementary rectangular frames-there also existed domestic panels depicting a number of saints (not necessarily in larger formats). For instance, a panel commissioned in 1440 by Michael Zamagno (from Johannes Ugrinovich), measuring $1.25 \mathrm{~m}$ in width and $1.13 \mathrm{~m}$ in height, was supposed to accommodate no less than ten saintly figures with gilded halos (Tadić 1952, vol. I, pp. 110-11, doc. 246), probably arranged in two

26 This inventory of the house of Catha, a Clarissa nun, also mentions another image, una figura de gisso tuta in una cassa depenta in sala. 
registers. ${ }^{27}$ An equally important parallel with paintings mounted on church altars was the practice of coverage, either by shutters or curtains (cf. Schmidt 2007). ${ }^{28}$ Further correlation between altarpieces in churches and those at home can be drawn from secondary visual sources, which pair domestic images with oil lamps, candles, or containers of holy water (Schmidt 2005, pp. 95-96; Morse 2007, pp. 166-69). The household image was, after all, a sacred object that was to be treated equally to those in churches. This attests to how the "laity appropriated the liturgical practice of the clergy to frame the sacred within their own home" (Cooper 2006, p. 193). In such a way, these domestic images were activated only at certain times, in moments of personal or familial prayer. However, no record from Ragusan archives thus known references an altar inside the house, including consecrated stones (pietra sagrata), on which mass could have been said (cf. Mattox 2006). The altars, accordingly, seem to have been confined to a handful of free-standing chapels with the permission to celebrate mass-such as those owned by the Volcassio, Volzio and Sorgo families.

When observing the uses of sacred installations in households, one should not fail to acknowledge the seemingly insignificant fact that they were defined as "small churches". This term is used sparingly in contemporary sources in other Adriatic cities and can, therefore, be considered a peculiarity of Ragusan documents. ${ }^{29}$ The ecclesiola/gliesiola/jesiola, apart from being a rather dry term for a range of devotional structures, was also a synonym for family in medieval Christian thought, deriving from the writings of the Church fathers (cf. Novotný 2013). In other words, the very name of the domestic shrines in question is in line with their uses, and with typically domestic iconographies. What is more, the role of the father of the family, responsible for the "organisation of religious life of his domestic community," was equated with that of a bishop (Widok 2013, pp. 170-72). All this could potentially explain not only the terminology employed, but also the emulation of forms of church altarpieces in domestic installations, which were devotional foci in dwellings and, at the same time, instruments in the religious and moral upbringing of children (Lawless 2014-2015, pp. 64-65). We should not fall into the trap of seeing domestic interiors as exclusively secular spaces (Morse 2007, p. 184); the use of devotional images was manifold, bringing together the need for the sanctification of homes and personal devotion with that of pedagogy. In this way, the small-scale domestic images, often discredited when observed against more lavish forms of church art, were the point of convergence of everyday religious experience.

\section{Privately Owned Relics}

Paintings were but one facet of the domestic culture of religion (cf. Sorelli 2001). The final part of the paper will thus briefly deal with domestic relic collecting. In general, all devotional objects were kept in chests, and occasionally incorporated into the already discussed domestic ecclesiolae. In his testament of 1442, the priest Gon Meliz from Durrës, Albania, bequeathed his casaleta e la incona e zo ch'e dentro de la caseleta, e la ghiesiola de la incuna (Tadić 1952, vol. I, p. 132, doc. 287). The formulation, "an image with other belongings in the casket," implies that several objects were stored in the chest, presumably relics or utensils for performing mass (the owner was, after all, a presbiter). Another possibility for the display of relics in households, somewhat more sophisticated, was to enshrine them in a cross (Anderson 2007, p. 117). There are a number of such references in sources, for instance, una

27 [fare] una anchona de longeza et alteza, come appar de soto, et che sia in la deta anchona santi X, como appar de soto [alta bracia $2 \frac{1}{4}$, larga brazia $2 \frac{1}{2}$ ]. Et che debia lo detto Zornella servir ben de color et de belle figure, zoe alo compartimento de soto, et le diademe far tute d'oro et li drapi de santi adornati d'oro come apartien.

28 These covering devices, however, were only occasionally recorded in sources, for instance un anchona con la cortina in the inventory of goods of Rusa, wife of Milovat Pribinich (Tadić 1952, I, p. 239, doc. 499). In his extensive archival work, Tadić was exclusively interested in "visual arts", which is why the excerpts he published from private do not acknowledge the candelabra that illuminated images. Inventories from other cities reveal that these accessories were conventional (Musacchio 2000, p. 150; Schmidt 2005, pp. 95-96).

29 For instance, liturgical utensils in Venice in the shape of minuscule church architecture were termed ecclesiola or chiesola (Dale 2010, p. 180), whilst Florentine sources use cappellanuccio / chapellinaiusso, a simple shrine (Palmer 2001, p. 78). 
croce parva argentea cum reliquiis, owned by Ratcho Priach in 1387 (Div. Canc. 25, fol. 155v). However, it is impossible to ascertain whether these were small pectoral crosses that contained particles of relics (for apotropaic and talismanic purposes) or house crosses, displayed in domestic settings similarly to other devotional artworks.

Personal inventories and last wills do not specify which relics were owned by individuals, but some evidence exists regarding private relics of the Holy Cross, owned by prominent families in the fourteenth century (namely Bona and Menze), although the subsequent whereabouts of these domestic relics are unknown (Lonza 2009, p. 290). Recent research has shed much-needed light on the city's most prominent relics (cf. Munk 2016; Trška 2017; Zelić and Viđen 2018), yet our knowledge of the importance of the private initiative in procuring them is still lacking. In fact, the information regarding relics in private hands, more often than not, derives from the time of their donation to church treasuries (otherwise, they would have remained unrecorded). ${ }^{30}$ However, we know that the most popular relics were connected to pilgrimages, either renewed spiritual journeys to Rome or the Holy Land, or some other local pilgrimage centre. In fact, pilgrims traveling to these important sacred sites often brought back a number of secondary and contact relics related to the locations of sacred events and places associated with the life of Christ (Gertsman and Mittman 2017).

An illustrative case is that of Marussa of Priboie, whose recently published testament (Lonza 2019, pp. 56-60) references the following objects: seven reliquary boxes (scatulae magnae/parvae), five Jericho roses, eight vials of water from the Jordan River, and ropes used to measure the dimensions of the sacred edifices (Lonza 2019, pp. 58, 61-63). ${ }^{31}$ Marussa's case shows how the familial (and, in this particular instance, gender) dimensions of the phenomenon were crucial-a group of women financed her pilgrimage, and she bequeathed all of her domestic devotional props to neighbours and her aunt, while the most precious among them (boxes containing relics) were given to daughters of her epitropa (Lonza 2019, pp. 42-43). Marussa, therefore, bequeathed a set of objects that were procured during a spiritual pilgrimage to the Holy Land to members of her family, and these sacred memorabilia must have been stored in proximity to other holy objects in households.

\section{Conclusions}

The archival sources discussed in this paper offer valuable insight into the appearances of sacred spaces in late medieval households of Ragusa, of the urban elite and general population alike. The evidence gathered here reveals multiple levels of familial influence on domestic devotional practices. The hereditary rights of chapels, constructed by prominent families, perpetuated the memory of their founders, and, although constructed on private terrain and privately funded, they were places of public veneration, as well. These structures were the most lavish form of domestic devotion, not only by virtue of their size, but also as a key strategy in securing the salvation of the souls of their founders and heirs. Indoor foci of prayer were equally elaborate, and a number of their features-such as the use of curtains and shutters to cover holy images, and the emulation of church altarpiece formats-testifies to the adoption of liturgical elements into domestic religious practice. This one-way influence of institutional religion on household practices is only one side of devotional practices for, as attested by later sources, a number of domestic devotional objects-due to their devotional potential and miraculous powers-passed over from domestic to public church spaces (cf. Voulgaropoulou 2019, pp. 25-32). Indeed, this interplay between the private and the public was determinant to the experience and use of the urban, household and garden chapels discussed, given that they served purposes of

30 For discussion on domestic images that have made their way to church altars, see (Voulgaropoulou 2019, pp. 25-33), for the use of donated relics in public rituals of Adriatic towns see recent discussion by (Ulčar 2015, pp. 101-3).

31 Volo quod detur una scatula Margarite filie Ruse et una alia Nicolete sorori sue scatula parva. Et Ruse detis unam craniçam seu cordellam cum qua mensuravi sepulcrum Christi et unam rusam et unam scatulam date Cuite vicini mee que stat ad parietem domus mee et unam craniçam et fili quibus consuitur quia eius sunt. Et detis unam scatolam Lubne quam vos vultis et craniçam unam et unam rosam, et unam scatulam Miladne Gradoevich que non est pulcra. (. . . ) Et date scatulam unam Goyne Velegni (?) miscitari (?). Et Ratche vicine mee dare unam uram (?) sive cimossem de scarleto que tetigit omnia loca Terre Sancte et unam rosam que est minor. 
religious and moral education, as well as their owner's representation. In this sense, one of the most promising avenues for future research is to observe the ecclesiae and ecclesiolae more attentively in the context of interfamilial networks, in order to gain a more nuanced insight into their hereditary and domestic contexts.

Funding: This research received no external funding.

Acknowledgments: I would like to thank Salvador Ryan for inviting me to participate in the special issue of Religions journal "Domestic Devotions in Medieval and Early Modern Europe". Ana Marinković helped me with Latin transcriptions; Ivan Viđen kindly provided me with photographs and offered valuable help during my research stay in Dubrovnik. I am also grateful for the comments offered by the anonymous reviewers.

Conflicts of Interest: The author declares no conflict of interest.

\section{Abbreviations}

HR-DADU State Archives in Dubrovnik (Državni arhiv u Dubrovniku)

Div. Not. HR-DADU-9 Diversa Notariae

Div. Canc. HR-DADU-15 Diversa Cancellariae

Vend. Canc. HR-DADU-16 Venditiones Cancellariae

LAT Zelić, Danko (ed.). Knjiga rizničarskih najmova = Liber affictum thesaurarie (1428-1547)

Zagreb and Dubrovnik: HAZU and Zavod za povijesne znanosti u Dubrovniku, 2012.

\section{References}

\section{Primary Sources}

Državni arhiv u Dubrovniku (Dubrovnik State Archives)

(Div. Not.) HR-DADU-9 Diversa Notariae, vol. 10.

(Div. Canc.) HR-DADU-15 Diversa Cancellariae, vol. 25.

(Vend. Canc.) HR-DADU-16 Venditiones Cancellariae, vol. 4.

(Čremošnik 1951) Čremošnik, Gregor, ed. 1951. Spisi dubrovačke kancelarije, vol. I. Zapisi notara Tomazina de Savere 1278-1282. Zagreb: JAZU.

(Lučić 1993) Lučić, Josip, ed. 1993. Spisi dubrovačke kancelarije, vol. IV. Zapisi notara Andrije Beneše 1295-1301, Praecepta rectoris II (1299-1301), Testamenta II (1295-1301). Zagreb: HAZU, Zavod za hrvatsku povijest and Filozofski fakultet.

\section{Secondary Sources}

Anderson, Caroline Corisande. 2007. The Material Culture of Domestic Religion in Early Modern Florence, c. 1480-c. 1650. Ph.D. dissertation, University of York, York, UK.

Bacci, Michele. 2009. Side Altars and Pro Anima Chapels in the Medieval Mediterranean: Evidence from Cyprus. In The Altar and its Environment, 1150-1400. Edited by Justin E.A. Kroesen and Victor M. Schmidt. Turnhout: Brepols, pp. 11-30.

Bacci, Michele. 2018. Devotional Panels as Sites of Intercultural Exchange. In Domestic Devotions in Early Modern Italy. Edited by Maya Corry, Marco Faini and Alessia Meneghin. Leiden and Boston: Brill, pp. 272-92.

Baldissin Molli, Giovanna, Cristina Guarnieri, and Zuleika Murat, eds. 2018. Pregare in casa. Oggetti e documenti della pratica religiosa tra Medioevo e Rinascimento. Rome: Viella.

Belamarić, Joško. 2018. Biskupska palača u Dubrovniku. Dubrovnik: Dubrovačka biskupija.

Benyovsky Latin, Irena, and Stipe Ledić. 2014. The Estate of the Volcassio Family in Medieval Dubrovnik. Dubrovnik Annals 18: 7-45. [CrossRef]

Beritić, Lukša. 1956. Ubikacija nestalih gradjevinskih spomenika u Dubrovniku. Prilozi povijesti umjetnosti u Dalmaciji 10: 15-83.

Coccato, Stefania. 2018. Oggetti, documenti e spazi devozionali privati nei palazzi veneziani del XIV secolo. In Pregare in Casa. Oggetti e Documenti Della Pratica Religiosa tra Medioevo e Rinascimento. Edited by Giovanna Baldissin Molli, Cristina Guarnieri and Zuleika Murat. Rome: Viella, pp. 149-61. 
Cooper, Donal. 2006. Devotion. In At Home in the Renaissance. Edited by Marta Ajmar-Wollheim and Flora Dennis. London: V\&A Publications, pp. 190-203.

Dale, Thomas. 2010. Cultural Hybridity in Medieval Venice: Re-Inventing the East at San Marco after the Fourth Crusade. In San Marco, Byzantium and the Myths of Venice. Edited by Henry Maguire and Robert S. Nelson. Washington, DC: Dumbarton Oaks Research Library and Collection, pp. 151-91.

de Diversis, Filip. 2004. Opis slavnoga grada Dubrovnika. Edited and translated by Zdenka Janeković Römer. Zagreb: Dom i svijet.

Demori Staničić, Zoraida. 1986. Cat. no. 3-Bogorodica s djetetom. In Blaž Jurjev Trogiranin: Exhibition catalogue. Zagreb: MTM, p. 86.

Demori Staničić, Zoraida. 2013. Ikona Bogorodice s Djetetom iz crkve Sv. Nikole na Prijekom u Dubrovniku. Ars Adriatica 3: 67-84. [CrossRef]

Drandaki, Anastasia. 2014. A Maniera Greca: Content, Context and Transformation of a Term. Studies in Iconography 35: $39-72$.

Fisković, Igor. 1983. Dubrovačko slikarstvo i društveni okviri njegova razvoja u XIV stoljeću. Prilozi povijesti umjetnosti u Dalmaciji 23: 75-147.

Fisković, Igor. 1990. Tipologija i morfologija oltarnih slika 15. stoljeća u Dalmaciji. Prilozi povijesti umjetnosti u Dalmaciji 29: 113-55.

Fisković, Igor. 1997. Crkvica Sv. Kuzme i Damjana u središtu Dubrovnika. Dubrovnik 8: 261-75.

French, Katherine L. 2014. Localized Faith: Parochial and Domestic Spaces. In The Oxford Handbook of Medieval Christianity [Oxford Handbooks Online]. Edited by John H. Arnold. Oxford: Oxford University Press, pp. 1-12.

Gamulin, Grgo. 1991. Pseudo-Victor, triptih u Dubrovniku. Peristil 34: 67-68.

Garnett, Jane, and Gervase Rosser. 2019. The Ex Voto between Domestic and Public Space: From Personal Testimony to Collective Memory. In Domestic Devotions in Early Modern Italy. Edited by Maya Corry, Marco Faini and Alessia Meneghin. Leiden and Boston: Brill, pp. 45-62.

Gertsman, Elina, and Asa Simon Mittman. 2017. Rocks of Jerusalem. Bringing the Holy Land Home. In Natural Materials of the Holy Land and the Visual Translation of Place, 500-1500. Edited by Renana Bartal, Neta Bodner and Bianca Kühnel. New York: Routledge, pp. 157-71.

Grujić, Nada. 2013. Kuća u Gradu: Studije o dubrovačkoj stambenoj arhitekturi 15. i 16. stoljeća. Dubrovnik: Matica hrvatska-Ogranak Dubrovnik.

Hilje, Emil. 2000. Slikarska djela u sačuvanim inventarima zadarskih građana iz 14. i 15. stoljeća. Radovi Zavoda za povijesne znanosti HAZU u Zadru 42: 65-78.

Howard, Deborah, Maya Corry, and Mary Laven, eds. 2017. Madonnas \& Miracles: The Holy Home in Renaissance Italy. London: Philip Wilson Publishers.

Johnson, Gerardine A. 2002. Beautiful Brides and Model Mothers: The Devotional and Talismanic Functions of Early Modern Marian Reliefs. In The Material Culture of Sex, Procreation and Marriage. Edited by Anne L. McClanan and Karen Rosoff Encarnación. New York: Palgrave Press, pp. 135-61.

Kasl, Ronda. 2004. Holy Households: Art and Devotion in Renaissance Venice. In Giovanni Bellini and the Art of Devotion. Edited by Ronda Kasl. Indianapolis: Indianapolis Museum of Art, pp. 59-89.

Klapisch-Zuber, Christiane. 1998. Holy Dolls: Play and Piety in Florence in the Quattrocento. In Looking at Italian Renaissance Sculpture. Edited by Sarah Blake McHam. New York: Cambridge University Press, pp. 111-27.

Kolpacoff Deane, Jennifer. 2013. Medieval Domestic Devotion. History Compass 11: 65-76. [CrossRef]

Lawless, Catherine. 2014-2015. Sensing the Image: Gender, Piety and Images in Late Medieval Tuscany. Open Arts Journal 4: 61-74. [CrossRef]

Lonza, Nella. 2009. Kazalište vlasti: Ceremonijal i državni blagdani Dubrovačke Republike u 17. i 18. stoljeću. Zagreb: HAZU-Zavod za povijesne znanosti u Dubrovniku.

Lonza, Nella. 2012. For the Salvation of the Souls, for the Welfare of the State: Ragusan Treasurers and Perpetual Trusts for Pious Purposes from the Thirteenth to the Fifteenth Century. In Knjiga rizničarskih najmova/Liber affictum thesaurarie (1428-1547). Edited by Danko Zelić. Zagreb: HAZU-Zavod za povijesne znanosti u Dubrovniku, pp. 25-41.

Lonza, Nella. 2019. Ego fui per omnia loca sancta: Maruša of Dubrovnik and her pilgrimage to the Holy Land in 1394. Dubrovnik Annals 23: 37-63. [CrossRef]

Lydecker, John Kent. 1987. The Domestic Setting of the Arts in the Renaissance Florence. Ph.D. dissertation, John Hopkins University, Baltimore, MD, USA. 
Mattox, Philip. 2006. Domestic Sacral Space in the Florentine Renaissance Palace. Renaissance Studies 20: 658-73. [CrossRef]

Morgan, Hollie L.S. 2017. Beds and Chambers in Late Medieval England. Readings, Representations and Realities. York: York Medieval Press.

Morse, Margaret A. 2007. Creating Sacred Space: The Religious Visual Culture of the Renaissance Venetian Casa. Renaissance Studies 21: 151-58. [CrossRef]

Morse, Margaret A. 2013a. From Chiesa to Casa and Back: The Exchange of Public and Private in Domestic Devotional Art. In Reflections on Renaissance Venice: A Celebration of Patricia Fortini Brown. Edited by Maria Blake de and Mary E. Frank. Milan: 5 Continents Editions, pp. 143-53.

Morse, Margaret A. 2013b. The Venetian Portego: Family Piety and Public Prestige. In The Early Modern Italian Domestic Interior, 1400-1700: Objects, Spaces, Domesticities. Edited by Erin J. Campbell, Stephanie R. Miller and Elizabeth Carroll Consavari. Farnham: Ashgate, pp. 89-106.

Muir, Edward. 1989. The Virgin on the Street Corner: The Place of the Sacred in Italian Cities. In Religion and Culture in the Renaissance and Reformation. Edited by Steven E. Ozment. Kirksville: Sixteenth-Century Journal Publishers, pp. 25-40.

Munk, Ana. 2016. Deconstructing the Myth of Byzantine Crown: The Head Reliquary of Saint Blaise in Dubrovnik. Dubrovnik Annals 20: 7-52. [CrossRef]

Murat, Zuleika. 2014. The Tomb of the beato Pacifico in the Basilica dei Frari: Personal Devotion or Public Propaganda? Hortus Artium Medievalium 20: 874-82. [CrossRef]

Musacchio, Jacqueline Marie. 2000. The Madonna and Child, a Host of Saints, and Domestic Devotion in Renaissance Florence. In Revaluing Renaissance Art. Edited by Gabriel Neher and Rupert Shepherd. London: Routledge, pp. 147-59.

Nagel, Alexander, and Christopher S. Wood. 2010. Anachronic Renaissance. New York: Zone Books.

Nalle, Sara. 2008. Private Devotion, Personal Space: Religious Images in Domestic Context. In La Imagen Religiosa en la Monarquía Hispánica. Edited by María Cruz de Carlos, Pierre Civil, Felipe Pereda and Cécile Vincent-Cassy. Madrid: Casa de Velázquez, pp. 255-72.

Novotný, Vojtěch. 2013. History of the Concept Ecclesia Domestica in Theology until the Seventeenth Century. Marriage, Families \& Spirituality 19: 157-85.

Palmer, Allison Lee. 2001. The Walter' Madonna and Child Plaquette and Private Devotional Art in Early Renaissance Italy. The Journal of the Walters Art Museum 59: 73-84.

Prijatelj Pavičić, Ivana. 2011. Prilog proučavanju dubrovačkih molitvenika XV. stoljeća. Prilozi povijesti umjetnosti u Dalmaciji 42: 205-49.

Schmidt, Victor Michael. 2005. Painted Piety. Panel Paintings for Personal Devotion in Tuscany. Florence: Centro Di.

Schmidt, Victor M. 2007. Curtains, Revelatio and Pictorial Reality in Late Medieval and Renaissance Italy. In Weaving, Veiling, and Dressing. Textiles and Their Metaphors in the Late Middle Ages. Edited by Kathryn M. Rudy and Barbara Baert. Turnhout: Brepols, pp. 191-213.

Šišić, Bruno. 2008. Dubrovnik Renaissance Gardens: Genesis and Design Characteristics. Dubrovnik and Zagreb: HAZU, Zavod za povijesne znanosti, Centar za povijesne vrtove i razvoj krajobraza u Dubrovniku, and Agronomski fakultet.

Sorelli, Fernanda. 2001. Oggetti, libri, momenti domestici di devozione. Appunti per Venezia (secoli XII-XV). Quaderni di Storia Religiosa 8: 55-78.

Tadić, Jorjo. 1952. Građa o slikarskoj školi u Dubrovniku XIII-XVI v. Belgrade: Naučna knjiga, vol. I-II.

Trška, Tanja. 2017. Miraculous Image as a Relic: The Reliquary of St Filippo Benizi in Dubrovnik's Cathedral Treasury. Radovi Instituta za povijest umjetnosti 41: 67-81. [CrossRef]

Ulčar, Milena. 2015. Relikvijar Svetog Križa i proslava Uskrsa u Perastu. Radovi Instituta za povijest umjetnosti 39: 99-108.

Valenzano, Giovanna. 2018. Gli spazi della devozione domestica. In Pregare in casa. Oggetti e documenti della pratica religiosa tra Medioevo e Rinascimento. Edited by Giovanna Baldissin Molli, Cristina Guarnieri and Zuleika Murat. Rome: Viella, pp. 15-39.

Van Os, Henk. 1994. The Art of Devotion in the Late Middle Ages in Europe, 1300-1500. Princeton: Princeton University Press.

Vekarić, Nenad. 2012. Vlastela grada Dubrovnika, vol. 3. Vlasteoski rodovi (M-Z). Zagreb and Dubrovnik: HAZU-Zavod za povijesne znanosti u Dubrovniku. 
Vekarić, Nenad. 2015. Vlastela grada Dubrovnika, vol. 6. Odabrane biografije (Pi-Z). Zagreb and Dubrovnik: HAZU-Zavod za povijesne znanosti u Dubrovniku.

Voulgaropoulou, Margarita. 2019. From Domestic Devotion to the Church Altar: Venerating Icons in the Late Medieval and Early Modern Adriatic. Religions 10: 390. [CrossRef]

Webb, Diana. 2005. Domestic Space and Devotion. In Defining the Holy: Sacred Space in Medieval and Early Modern Europe. Edited by Andrew Spicer and Sarah Hamilton. Aldershot: Ashgate, pp. 27-47.

Widok, Norbert. 2013. Christian Family as Domestic Church in the Writings of St. John Chrysostom. Studia Ceranea 3 : 167-75. [CrossRef]

Wilkins, David G. 2002. Opening the Doors to Devotion: Trecento Triptychs and Suggestions Concerning Images and Domestic Practice in Florence. In Italian Panel Painting of the Duecento and Trecento. Edited by Victor M. Schmidt. Washington, DC: National Gallery of Art, Center for Advanced Study in the Visual Arts, pp. 371-93.

Zelić, Danko, and Ivan Viđen. 2018. Inventario dela giexia di S. Maria Mazor, de tute le argentarie, aparati deli sazerdoti, insenarii, libri, tapezarie et altre robe (1531.)-najstariji imovnik liturgijske opreme, ruha i paramenata dubrovačke prvostolnice. Radovi Instituta za povijest umjetnosti 42: 19-36.

Živković, Pavo. 1985. Pariteti dubrovačkog novca u srednjem vijeku. Anali Zavoda za povijesne znanosti Istraživačkog centra JAZU u Dubrovniku 32-33: 31-39.

Živković, Valentina. 2016. The Deathbed Experience-Icons as Mental Images. Preparations for a Good Death in Late Medieval Kotor (Montenegro). Ikon 9: 221-30. [CrossRef]

(C) 2019 by the author. Licensee MDPI, Basel, Switzerland. This article is an open access article distributed under the terms and conditions of the Creative Commons Attribution (CC BY) license (http://creativecommons.org/licenses/by/4.0/). 
Article

\title{
The Use of Devotional Objects in Catalan Homes during the Late Middle Ages
}

\author{
Marta Crispí \\ Department of Humanities, Universitat Internacional de Catalunya, 08017 Barcelona, Spain; mcrispi@uic.es
}

Received: 16 November 2019; Accepted: 19 December 2019; Published: 25 December 2019

\begin{abstract}
The purpose of this article is to study domestic devotion in Catalonia in the thirteenth, fourteenth and fifteenth centuries, based on the information provided by numerous post-mortem inventories and texts written by coetaneous spiritual authors such as Ramon Llull, Francesc Eiximenis and Saint Vincent Ferrer. Among the objects recorded in the inventories, pieces of furniture and devotional objects laypeople and clergymen used in their pious practices as "material" aid for personal prayer stood out. They were in keeping with the strong visual culture that pervaded the Late Middle Ages. There were retables, oratories and images of religious themes. However, the inventories also listed lesser known but equally recurring objects such as paternosters and Agni Dei. Painted cloths depicting religious scenes that decorated the homes of numerous wealthy Catalan-Aragonese families at that time were also present. Spiritual books such as books of hours and psalters, biblical texts, Legenda Aurea, etc., were mentioned as well. They were part of the incipient libraries of the laity in the Late Middle Ages.
\end{abstract}

Keywords: domestic devotion; private devotion; material devotion; medieval art; altarpieces; retables; oratories; reliquaries; paternosters; Agnus Dei; Catalonia

\section{Introduction ${ }^{1}$}

When it dawned on Evast that his son excelled at science, was well brought up and good-mannered, his heart filled with joy. He entered their home chapel, where Aloma and he secretly prayed to God and listened to mass every day. After getting up from the table, they went there to offer thanks to God. The altarpiece was of Saint Andrew, whom Evast and Aloma deeply trusted to grant them God's grace and blessings. Inside the prayer room, Evast knelt down before the altar and made the sign of the cross as he usually did, saying the following words...". (Llull 1981-1982, p. 39)

This passage from the Llibre d'Evast $i$ Blanquerna by Ramon Llull illustrates the life of a couple, Evast and Aloma, whom the prolific Majorcan theologian introduces to his contemporaries as exemplary witnesses of Christian life. In addition to the Lullian utopia that pervades the book, the words of the Majorcan portray the religious practices in the lives of laypeople in the late medieval period: attending mass on a daily basis and praying together several times a day in their private chapel. Furthermore, Llull stresses Evast and Aloma's attitudes, which are shown as an expression of their faith: secret and sincere prayer, thanks offering and trust in God and the saints. Those attitudes are also reflected in the positions they adopt: they kneel before the altar and make the sign of the cross. All of this takes place in a domestic environment where the private chapel, in which the altar was dominated by the framed altarpiece of Saint Andrew, was a place that evidenced the importance of this personal, private, and emotional devotion praised by the Majorcan writer.

1 Text revised by Ann Swinnen. 
Llull's text also beautifully portrays those places of domestic devotion which are, together with devotional objects, the purpose of the present article. Those private places of religious practice are the settings of the daily piety of laypeople. They contain a broad assortment of objects used as physical and visual support for laypeople's prayers or as a tool or an aid in their prayers. The growing number of those devotional objects found in post-mortem inventories of the fourteenth and fifteenth centuries can be explained by the development of private piety encouraged by preachers from their pulpits. This was fully in line with the spirituality fostered by mendicant orders and later, by the influence of the Devotio Moderna.

Domestic devotion in the Late Middle Ages remains largely unexplored, although its interest and importance are unquestionable. This article aims to portray some of the characteristics of domestic devotion in Catalonia in the fourteenth and fifteenth centuries, based on the abundant information provided by post-mortem inventories published. The objective is to make an initial approach to the study of the devotional objects listed in inventories and of religious practice with the help of texts written by Catalan-Aragonese theologians and religious authors of that period. Further research including the analysis of extensive chronological series of inventories concerning protocol or parish archives will have to be carried out in the future.

\section{Post-Mortem Inventories as a Documentary Source for Art History}

The present study was carried out by scrutinising post-mortem inventories of Catalan laypeople from the thirteenth century to the year 1500 . The use of post-mortem inventories as a documentary source for socio-economic studies is a widely employed method in medieval and modern historiography. This kind of legal document intends to record the belongings of the deceased that are handed over to their heirs following the testamentary dispositions. Not all the objects of the home were itemised, only the possessions of the deceased. Actually, during the period between the deceased's death and making the inventory, it was possible that certain objects were moved from one place to another or disappeared. The inventories only listed the belongings of the deceased, often the pater familias, the head of the family, omitting those of the spouse or other members of the family. ${ }^{2}$ Inventories of women were much less common than those of men. Of a total of eighty-five inventories analysed, only seventeen were of women. The limitations and incompleteness of the inventories therefore need to be considered although they offer a highly reliable portrait of the life, habits and customs of men and women in the Late Middle Ages.

Given the legal nature of inventories, notaries were responsible for making descriptions that allowed identifying the objects for them to be donated to the heir. The objects were accurately listed providing any additional information that clarified the objects' identification such as the material, size, age or condition. Notaries could even estimate their value. A summary description of the iconography was also made in the case of images, retables and oratories. The inventories could be complemented with the transcription of the auction, that is, the sale of the objects auctioned in the public market. This last document enables us to know the final price of the sale of each object.

Other interesting information for art historians is the location of the possessions. When compiling the inventory, the notary usually walked through the different rooms of the house indicating the room in which the objects were found. In each room, he started by listing furniture, including beds, tables, benches, storage benches, boxes and safes, itemising the objects stored inside.

From the late nineteenth century onwards but more so since the 1980s, Catalan medievalists such as Soler i Palet (1916a, 1916b)³; Roca (1923, Roca 1927-1928a, Roca 1927-1928b, 1930a, 1930b);

2 For the analysis of post-mortem inventories as a primary source for the study of material culture, see the recent and complete study by Almenar about Valencia including the state of the question (Almenar Fernández 2017). See also: (Lencina Pérez 1998; Sobrado Correa 2003; Bolós and Sànchez-Boira 2015).

3 Groundbreaking work that includes the transcription of dozens of inventories. It gathered only "artistic" objects, leaving out reliquaries, books, etc. 
Batlle Gallart (1981a, 1986, 1988, 1993, Batlle Gallart 1993-1994, 1998, Batlle Gallart et al. 1989, Batlle Gallart and Cuadrada 1993); Bolós and Sànchez-Boira (2014) amongst many others, have carried out the patient task of analysing and transcribing post-mortem inventories of citizens belonging to the different estates of Catalan society in order to study social and economic aspects of life in the late medieval period.

The publication and analysis of inventories, either through full or partial transcription, provide us with first-order information about religious practices in the Late Middle Ages, this being the starting point of our work. So far, the references made to religiosity have been complementary to more global considerations on social or economic aspects. Authors such as Sabaté (1990, pp. 91-92); García Marsilla (2001, pp. 170-82); Bolós and Sànchez-Boira (2014, pp. 236-41) have introduced commentaries that are the basis of future studies on domestic devotion. In the field of history of art and private piety, post-mortem inventories have been examined in-depth by Valencian and Majorcan historians such as García Marsilla (2001); Sastre Moll (2003). In Catalonia, studies of this genre have not addressed the artistic side, except for Aurell i Cardona and A. Puigarnau, who have analysed the case of Catalan merchants (Aurell i Cardona and Puigarnau 1995; Aurell i Cardona 1995), R. Terés, who has explored the historical and mythological iconography of retables and oratories (Terés 1998) and Amenós' work (Amenós 2017).

The basis of our study corresponds to eighty-five published inventories of Catalan citizens from the thirteenth, fourteenth and fifteenth centuries. ${ }^{4}$ There are both complete inventories and partial ones that only list a specific type of object. Of the total of eighty-five inventories analysed, three correspond to the thirteenth century, twelve to the fourteenth century and the rest to the fifteenth century. Most of them are inventories of citizens that belonged to the so-called braç reial or braç popular, both in the upper estate (honourable citizens) and in the lower one (merchants, artists and craftsmen. The inventories of monarchs and members of the royal family as well as the inventories of ecclesiastical residents who resided in their own house such as canons and secular clergy (rectors and presbyters that were beneficiaries of parishes) were left out. However, their devotional objects were in line with those reflected in the laypeople's inventories. ${ }^{5}$

\section{Observations Regarding the Term Domestic Devotion}

In recent medieval and modern historiography, the use of the term "domestic devotion" has imposed itself over expressions such as "private devotion", which may present serious biases if applied to the Middle Ages considering the current meaning of private. ${ }^{6}$

Modern houses, in which separate rooms for its members provide privacy, have little in common with medieval homes, which consisted of a series of rooms where a large group of people sharing bedrooms, dining rooms, kitchens and other rooms lived together. Today's nuclear family is composed of parents and children. In the Late Middle Ages, in the case of well-off families, it used to include grandparents, married children, widowed daughters and other close relatives as well as the servants and even slaves, who shared bedrooms with the families in the limited space of houses back then. Given this situation, it is difficult to apply the term "privacy" to medieval homes in the contemporary sense of the word. The terms "personal" or "individual", reflecting the character of domestic practices,

4 These inventories have been published by: (Martorell and Valls i Taverner 1911-1912; Moliné 1912, 1922a, 1922b; Soler i Palet 1916a, 1916b; Roca 1923, Roca 1927-1928a, Roca 1927-1928b, 1930a, 1930b; Casas Homs 1970; Duran i Sanpere 1972-1975; Vinyoles 1976; Conde y Delgado de Molina 1977; Batlle i Prats 1978; Costa 1983; García Panardes 1983; Batlle Gallart 1981a, 1986, 1988, 1993, Batlle Gallart 1993-1994, 1994, 1998; Broida 1986-1987; Batlle Gallart et al. 1989; Plana i Borràs 1989; López Pizcueta 1992; Ortí i Gost 1994; Batlle Gallart and Cuadrada 1993; Cifuentes i Comamala 2000; Busqueta 2001; Fité 2001; Bolós and Sànchez-Boira 2014).

5 Regarding the inventories of the clergy, see: (Sànchez-Boira 2007, 2014). For Italy, see: (Cossar 2017).

6 For domestic devotion in the Middle Ages, see: (Webb 1990, pp. 159-73; Rigon 2001; Spicer and Hamilton 2005; Webb 2005, pp. 27-42; Goldberg and Kowaleski 2009, pp. 1-13; Kolpacoff Deane 2013, pp. 65-75; Smith 2014; Laugerud et al. 2016). Regarding the fifteenth century, see: (Corry et al. 2017, 2019). 
cannot be applied either. Although devotion could be experienced at an individual level, praying or performing rites often involved the whole family or the members of the household. Another apparent opposition is found in the terms "sacred" and "secular" in relation to homes. These are secular spaces but can become sacred in the presence of religious objects that grant the status of sacred to certain rooms or corners of the house, thus turning them into ideal spaces for devotional practice.

Domestic devotion is the expression that best fits the practice of piety at home and it avoids dichotomies that have been shown to be limited and confusing, such as those of public and private, sacred and secular or individual and collective (Kolpacoff Deane 2013, pp. 66-67, 70). When these words are used in the context of medieval homes, a certain amount of flexibility is necessary as their meanings may be overlapping or complementary (Corry et al. 2019, p. 2). Not surprisingly, recent historiography has emphasized that fluidity is a central concept in the definition of domesticity (Corry et al. 2019, p. 2).

Sixten Ringbom defined private devotion as the "devotion in private life, which is the personal relationship that the individual maintains with the Divine" (Ringbom 1997, p. 32). Today, we could enrich this definition or, at least, its practical dimension by saying: "those pious practices that medieval men and women perform in their domestic environment and which are an expression of their religious beliefs." Yet what does this domestic devotion consist of? What elements integrate it and help build and shape it? In a recent study, Erin J. Campbell, Stephanie R. Miller and Elizabeth Carroll point out that "domesticity" is made up of spaces, objects and people (Campbell et al. 2013, p. 1). When applied to domestic devotion, it provides us with a useful means for the study of domestic devotion: it is framed within a space, the home; its people are the inhabitants living in the homes and a series of devotional objects represent the material support. It goes without saying that "devotion" is not limited to the materiality of religious practices or experiences. These are merely the external expression of the personal relationship of man with the Divine, a relationship marked by religious beliefs and, in particular, of the conception of God and the holy figures that men have in each historical period. This conception evolves and achieves specific forms and connotations over time. As mentioned above, domestic devotion in the fourteenth and fifteenth centuries is marked by the spirituality that extends to the west from the mid-twelfth century and that has its final point in the Devotio Moderna. ${ }^{7}$ For this reason, a global study of domestic devotion must necessarily study the foundations on which lay devotion is built in the framework of the spirituality of the moment: the preaching from the pulpit of the churches aimed at citizens and religious texts (as well as the images accompanying them) that begin to constitute the support of pious readings.

\section{Pious Practices of Laypeople in the Domestic Environment in the Late Middle Ages}

The objective of this section is to contextualize the private expressions of lay devotion based on texts by authors at the time of the Crown of Aragon.

A prolific and influential Franciscan Catalan writer, Francesc Eiximenis (1330-1409), provides some interesting considerations on the environments that were appropriate for personal prayer in the Llibre de les dones (Book of women):

"Teach us our reverent Saviour that not only the church is a place to pray, but also the home itself. Hidden corners and obscures places are suitable as places to pray (...). And this prayer, at home, anyone can do it when they please, no one can be excused". (Eiximenis 1981, vol. 2, p. 508)

Similar words are addressed to Queen Maria de Luna (1357-1406) in Scala Dei where he recommends her the quietness of a private space in the palace as an ideal environment for prayer:

7 For a study on medieval spirituality: (Delaruelle 1975; Vauchez 2003, 2005, Vauchez 2006). 
"These wise men say that the most appropriate time for this is at night during the first wake, and even better during the second one, which is the dawn. However, sometimes someone can be in a place where contemplation is possible at another time. The place should be in the room, in the secret oratory or in the church, but in general, royal people have a chapel where they can pray secretly". (Eiximenis 1985, p. 9)

Saint Vincent Ferrer (1350-1419), a famous and popular Valencian Dominican preacher who lived during the same period as Eiximenis, expressed himself in the same terms when, in one of his sermons, he portrays a Christian life programme that helps achieve eternal life:

"And how? First, when Sunday comes, they go to Mass, they fast, and go to confession. They do not talk to anyone and do not leave Mass until the priest gives blessings. Every morning and evening they pray devoutly, etc.; Then, when Lent comes, at the beginning they go to confession, and then they go often every month, and commune ten or twelve times a year; then they often do penance, they do not wear shirts, but serge and cilices, etc.". (Ferrer 1932-1998, vol. 2, p. 81)

Both eminent preachers and theologians claimed that the private space was an optimal environment for daily personal prayer, a practice they strongly recommended in addition to participating in the sacraments the Church prescribed as a mandatory exercise from the Fourth Council of Lateran (1215). Canon 21 of the Council approved mandatory confession and annual communion for all Christians. ${ }^{8}$ Logically, the most common frame of religious life and the centre of the administration of the sacraments remained the church, the sacred and liturgical space par excellence. ${ }^{9}$ Parishes especially, but increasingly also the preaching of mendicants, put special emphasis on the personal practice of prayer that requires environments characterised by stillness and seclusion. ${ }^{10}$

Eiximenis and Ferrer refer to the domestic space for seclusion, silence and serenity to propitiate a personal relationship with God, which they consider ideal. They recommend laypeople to perform morning and evening prayers in this quiet environment.

"The devotee Petrus Damiani advised every Christian to, at least in the morning, upon rising, and in the evening, before going to bed, kneel down in a familiar and secret manner in his room, and say to our Lord God with all his heart: "I stand before You, God and Lord. I am the worst creature you have created, begging you God Most High that, through the merits of the precious death of your Glorious Son, please forgive me my sins and point out my bad life. Guide me and show me the right path". y(Eiximenis 1981, vol. 2, p. 514)

What does the content and purpose of this prayer refer to? The highest form of Christian prayer is contemplative prayer, which consists in deep intimacy of the devotee with God, a personal relationship that involves a union of knowledge and life in which neither physical or imaginative resources nor

8 (Vauchez 1993, pp. 104-105; Webb 2005, p. 28; Tanner and Watson 2006). The prescriptions of the fourth Lateran Council involve strengthening the role of parishes in the Middle Ages. Canon 21 therefore specifies that laypeople had to confess to proper priests, thus stressing the function of cura animarum of the parish rectors (Vauchez 2003, p. 184). The same author points out that it was not common for laypeople to confess in the fourteenth century, except once a year (Vauchez 1993, p. 104). Attending mass on Sunday was also supposed to be common practice. However, daily communion was only for elites and the royal family. Ramon Llull, however, explains Evast and Aloma practised daily communion. In addition to Confession and the Eucharist, the laity also had access to Baptism, Confirmation, Marriage and Anointing of the sick, sacraments that accompanied people in the Middle Ages throughout their existence from birth to death. They helped them sanctify important times of life such as marriage, illness and death. The places where they received those sacraments were usually in the church but homes were also an appropriate space in cases of illness or when death was near.

9 The religious texts explain it clearly. Francesc Eiximenis specifies: "According to the saints, the church is a suitable and recommended place to pray" (Eiximenis 1981, vol. 2, p. 505).

10 The significance of the civic and social dimension of faith during the late medieval period, expressed by means of processions, festive celebrations of important events in the liturgical calendar, and religious actions practised collectively by members of guilds and brotherhoods, may not be overlooked. 
words or images are required. However, for laypeople or for those who undertake the initial stages of the life of prayer, theologians recommend the practice of vocal prayer and mental prayer, which use texts that stimulate contemplation. It is not in vain that repeating the Paternoster, the Ave Maria and the Creed is encouraged as Saint Vincent Ferrer did from the pulpit. The powerful words of the Valencian Dominican show how vocal prayer encourages the recreation of the inner image of Jesus himself:

"And do you want to put it into practice? Tell me, you, man or woman: have you done any good deed today? You will answer: "Yes, sir, this morning, before leaving home. I prayed kneeling on the floor; joining my hands and elevating my thoughts to God. I prayed the Creed, the Paternoster, the Ave Maria, and I said the prayer to my Guardian Angel, as well as the Salve Regina. I prayed the Paternoster and the Ave Maria for the dead, thinking that I saw Jesus Christ and that He looked at me with an angry face because of my sins". (Ferrer 1932-1998, vol. 1, p. 60)

Inner strength, imagination and memory play an important role as they help bring Christians close to eternal realities, awakening their understanding and touching their hearts (Laugerud et al. 2016, pp. 1-6; Hamm 2016, pp. 10-11). Recreating the image of Jesus, as well as that of the Virgin and the saints, fosters empathy in people and it spiritually connects them to the feelings of joy and pain that filled the heart of Mary and her Son. This category of images corresponds to what St. Augustine calls "spiritual vision", which is different from the strictly corporeal one perceived by the senses and from intellectual vision. Saint Bernard considered those mental representations as a gift of God that contributed to elevating the soul and that were beneficial to people who could not achieve intellectual contemplation without images.

Apart from this common resource for vocal prayer and inner recreation of spiritual images, direct contemplation of images, whatever their support, also leads to fostering feelings of love for God and attitudes of contrition and adoration. Again, Saint Vincent Ferrer provides a good example of how images encourage contrition and repentance and thus promote sincere devotion uniting the devotee with Jesus Christ, the objective of Christian prayer.

"The second danger is from the soul, that is: when you want contrition for the sins committed and cannot have it, what should you do? I will tell you: stand in front of the crucifix, kneel down and, looking at it intently, devoutly make the sign of the cross on your heart and say: “Jesus! Jesus! Jesus!” many times; If you do so, you will feel you will receive what you ask for. And this is significant in the Passion of Jesus Christ, when he says that stones will break: your heart, hardened like a stone by sins, will break when you utter the name of Jesus; Then the monuments will open: you will see your monument will open to confession, regardless of how serious your sins are". (Ferrer 1932-1998, vol. 2, p. 63)

In the text of Saint Vincent Ferrer, crucifixes play an important role of empathy. Ringbom suggests that images, in addition to educational and cultural functions, as pointed out by Gregory the Great, may perform an empathic function (Ringbom 1997, pp. 10-11, Belting 2009, pp. 547-60). In this regard, the term "devotional image" refers to a portable piece of religious art that is painted, sometimes on both sides, and is designed to decorate the altar of a church, an oratory or a chapel, fits perfectly into the typology and iconography of numerous late medieval images that try to cause an emotional impact on the devotee in private contemplation (Belting 1981, Wirth 1989, Panofsky 1997, Ringbom 1997, p. 32, Russo 1996, Belting 2009, pp. 545-605). Devotional images are thus representations that seek to generate empathy and influence the heart and intellect of the believer who contemplates them. 
Against this background, the lawfulness of venerating images can again be considered. Saint Vincent Ferrer justifies the Christian attitude towards images following the western theological tradition saying that professed veneration falls directly on God himself, the Virgin and the saints: ${ }^{11}$

"And when you go to church, or elsewhere, outside the church, and you see an image of our Lord God, or of the Virgin Mary, or of the saints, which is made of gold, then you will love the image for the love of the one who is represented. Even if it is silver, or iron, or lead, or stone, or painted with a brush, or white, or black, you will bow. This, and even better, is what you will have to do". (Ferrer 1932-1998, vol. 1, p. 245)

Eiximenis and Saint Vincent Ferrer give special importance to body language in prayer, taking as a reference Jesus' evangelical example.

"I have searched if Jesus had other ways of praying, and I found that indeed other ways of praying exist. There are four. The first one consists in lifting one's eyes, the second one in joining one's hands, the third one in kneeling on both knees, the fourth one in lying stretched out on the ground". (Ferrer 1932-1998, vol. 1, p. 21)

Eiximenis vividly describes the propitious prayer of Jesus:

"And here, kneeling, he first glorified and testified to his Father. Secondly, he begged him to forgive us our sins. To obtain abundant mercy, grace of the kingdom of God, he rose from the ground praying, and then he kneeled and threw himself flat on the ground humiliating himself to the Father, bending over, lifting his eyes and hands to heaven. He screamed loudly, so that everything poured out of him, and with all his effort and power and thought, emptied of his essence, he fell to the ground. He showed his humanity, giving us an example of how we must worship all our will, knowledge and effort, so that our prayer, thus reinforced, reaches God Almighty". (Eiximenis 1981, vol. 2, p. 503)

Although he does not express a preference for any of the attitudes, he always mentions the spirit must be devoted (Figure 1): "Whether you are kneeling or standing, or at times kneeling and other times standing, depending on what position feels more comfortable and which one you are more devoted to" (Eiximenis 1981, vol. 2, p. 503), he recommends the Queen:

"The second point is that when the Queen is in this place at the right time, she has to withdraw and thus get away from other people, kneel and lift her hands and eyes and worship our Lord as follows". (Eiximenis 1985, p. 9)

All these excerpts from texts and preaching sermons from the fourteenth and fifteenth centuries illustrate the strength of "visual culture" in the late medieval spirituality as well as the importance of personal, private prayer of the faithful Christian to God. The solitude of an interior space, the repetition of vocal prayers and the contemplation of images encourage prayer seeking union with God through participation in feelings of joy and pain, repentance and worship. The power this visual culture reaches in the Late Middle Ages in the sermons of preachers like Saint Vincent Ferrer, whose sermons brought together thousands of people in the public spaces of large cities in the southwest of Europe, can thus be understood. ${ }^{12}$

11 Saint Joan Damascè justifies the lawfulness of images because the veneration attributed to them results in their prototypes (Ringbom 1995; 1997, p. 10). Saint Thomas Aquinas and Saint Bonaventura support this idea (Cristologia 3, qu. 25 art 3 , Saint Bonaventura Sent. lh, III, 9, I, qu 2 conl 2 (quoted in Ringbom 1997, p. 10).

12 Regarding the visual culture of Saint Vincent Ferrer: (Español 2019). 


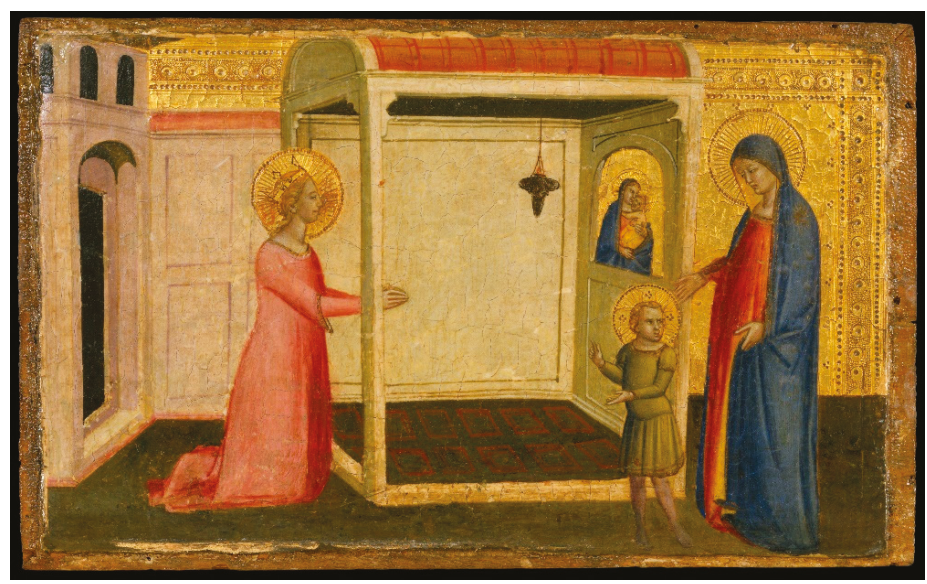

Figure 1. Saint Catherine praying before an image of Our Lady (second half of the 14th century). Metropolitan Museum of Art.

\section{Urban Houses}

Today we have detailed knowledge of medieval Catalan houses thanks to the information provided by post-mortem inventories and other documentary sources of a legal nature. Several studies have examined the structure and layout of the houses offering an accurate picture of urban and rural houses at that time ${ }^{13}$. Said studies stress that the size and layout of the houses depended on their location. In cities, the houses were usually small due to the limited urban space available within the city walls. In rural surroundings, there was more space.

The homes of the well-off classes, such as traders, merchants or bankers, were single-family houses in which a considerable number of people lived together. Couples with small children and/ or unmarried children, sometimes married children and their offspring, other close relatives and servants, often slaves, all lived under the same roof. The houses consisted of a ground floor and a first floor on top of which there was an attic, one or more rooms and a terrace. The ground floor housed a workshop, warehouses, cellars, stables and pens ${ }^{14}$ that were sometimes complemented in the back by a courtyard or a vegetable garden. The stairs to the upper floor were situated near the front door. In urban homes, which were called albergs, houses or domus (Batlle Gallart and Vinyoles 2002, pp. 109-10) in the documentation, family life was normally spent on the first floor, which contained several rooms. In the houses of wealthy families, there were up to fifteen rooms forming a network of rooms and backrooms of various sizes. The spaces dedicated to food were the kitchen, which often included a pantry, and a dining room that was also used for other family functions. The bedrooms were frequently shared. The head of the family usually had a study room, a place to work, nearby. There were also other small rooms such as rooms to knead bread and storage areas.

In Llibre d'Evast i Blanquerna Llull describes Evast and Aloma's private chapel, but except for this prayer room, did devotional spaces actually exist in medieval homes? Apart from the private chapels of kings and the members of their families and those found in castles and towers, explicit

13 It is worth noting the studies conducted by (Batlle Gallart 1983, 1985, 1993, 1998; Batlle Gallart and Vinyoles 2002, pp. 107-36; Vinyoles 1976, 2015; Bolós and Sànchez-Boira 2014, 2015). See also: (Broida 1986-1987; Ortí i Gost 1994; Bolós 2000, 2002; Fité 2001; Vercher Lletí 2005; Benito i Monclús 2008). Regarding medieval houses in the Iberian Peninsula, see: (Díez Jorge and Palazón 2015).

14 Bolós points out that some houses in Lleida had up to four floors ranging from a subterraneous floor including a cellar, to two more floors above ground, an attic and terraces (Bolós and Sànchez-Boira 2015, p. 19). Furthermore, many houses had courtyards or vegetable gardens. 
references to "private chapels" in households are scarce in the documents analysed (Broida 1986-1987, pp. 262-64). It is known that individuals with a high professional and socio-economic status, such as Arnau de Vilanova, Pere Beçet and Pere de Queralt, ${ }^{15}$ had chapels. Iglesias talks about Joan Bussot's chapel because it contained objects like retables and prayer books that were used in chapels (Iglesias 1996, pp. 110-11). However, among the well-off classes and the craftsmen, it was more common for devotional practices to be developed in the most important rooms of the house. Retables, oratories and images were found in bedrooms, study rooms or other shared spaces. The so-called prayer corner in medieval homes, the successor of the homes of early Christians, was a multi-functional space that combined private, personal, family and work uses, which was characteristic of the homes in the Late Middle Ages. ${ }^{16}$

\section{Religious Objects in Late Medieval Catalan Households}

This section is devoted to the analysis of the devotional objects founded in Catalan houses. In the eighty-five inventories analysed, two hundred and thirty-five devotional objects were listed. The religious books constituting the incipient libraries of medieval laypeople were not included because they deserve to be examined in a monographic study. ${ }^{17}$

Among those two hundred and thirty-five objects, there were fifty-two retables, fifty painted cloths, forty-seven oratories, twenty-four images, sixteen paternosters, six reliquaries, five panels, four Agni Dei, four curtains, three boards, two pieces of bright silk fabric, two cases, two chalices, two altars, two pavises and one music stand. Of all these possessions, none were from the thirteenth century. ${ }^{18}$ Thirty-four objects were from the fourteenth century and the rest were from the fifteenth century. As we are going to see, this shows that the presence of devotional objects increased considerably in the fifteenth century as a result of the enrichment of the Catalan bourgeoisie and of higher levels of wellbeing. The middle classes with higher purchasing power wanted to emulate the social and religious forms and habits of the highest estates of society (Yarza 1992, p. 29; Español 1992a, pp. 224-25; Molina 1999, pp. 43-55). This phenomenon was also observed in the large European cities of that time. The cases of Tuscany and Venice at the beginning of the Renaissance have been studied in depth. ${ }^{19}$

It is not easy to classify the number and diversity of objects found in post-mortem inventories since the function of the element listed is often unknown. They are described below, starting by those objects containing religious images that may have been the visual aid for prayer of believers such as retables, oratories, images and painted cloths. Then, other kinds of devotional objects like Agni Dei, reliquaries and paternosters will be discussed.

15 Regarding the renowned physician and theologian Arnau de Vilanova († 1311), see (Chabás 1903, pp. 189-203; Regarding Pere Beçet, see (Martorell and Valls i Taverner 1911-1912, pp. 577-656); Regarding Pere de Queralt, see (Costa 1983, pp. 115-45).

16 The existence of these spaces for devotion in homes has been acknowledged since early Christianity (Kitzinger 1954, pp. 98-99; Ringbom 1997, pp. 11-13; Webb 2005, p. 27; Herrin 2005, pp. 82-87; Kolpacoff Deane 2013, pp. 67-71; Smith 2014, p. 31; Bacci 2018, pp. 272-76). Anglo-Saxon historiography talks about the "icon corner or prayer corner" to allude to the spaces of the Byzantine houses where the laity placed icons, support for their personal and family devotion (Herrin 2005 , pp. 71-90; Bacci 2018, pp. 72-276). Those icons, which were considered prestigious because of their oriental origin, became frequent in Italian homes from the thirteenth century up to the Early Modern Period (Voulgaropoulou 2019). More recently, Smith has drawn attention to the role played by relics as devotional objects in the framework of domestic life in Western Europe in the early centuries of Christianity (Smith 2014, pp. 23-46; Blick 2019).

17 Regarding Catalan libraries, see: (Madurell i Marimon 1974; Batlle Gallart 1981b; Hernando 1994, 1995; Iglesias 1996; Alcoy 2005). For Majorcan libraries: (Hillgarth 1991).

18 The only devotional object is a prayer book that belonged to Bernat Durfort and was inventoried in 1290 (Batlle Gallart 1988 , p. 42).

19 See especially (Goldthwaite 1993). Regarding the number of objects present in Italian houses, see: (Morse 2007; Schmidt 2001, 2005; Campbell et al. 2013; Corry et al. 2017, 2019. For Italian houses, see as well: Ajmar-Wollheim and Dennis 2006; Brundin et al. 2018). 


\subsection{Devotional or Decorative Objects That Include Religious Iconography}

\subsubsection{Retables and Oratories}

These types of objects constituted the bulk of the religious belongings of laypeople in the Late Middle Ages. The figures speak for themselves: fifty-two retables and forty-seven oratories. They are, together with painted cloths, the objects that appear the most in the inventories analysed. Families often owned more than one.

The study of these pieces of religious furniture raises the difficulty of defining them. It is not easy to determine the difference between retables and oratories as both terms describe objects of a very similar kind, size and iconography.

The retable, as the etymology of the word indicates, retro-tabula, has been defined as a "structure located behind or on the altar containing figurative or history-related decoration" (Le Pogam and Vivet-Peclet 2009, p. 18), hence fulfilling a liturgical function. Historiography has pointed out the existence of a large number of small-sized retables that came in various shapes and mainly performed a devotional function in the domestic environment, without dismissing their use as complements to portable altars. ${ }^{20}$ Retables that include sculptures or paintings are currently often referred to as altarpieces. They became common from the end of the thirteenth century onwards. They were made of wood, stone, gold, silver or ivory.

It is harder to define the term oratory, which frequently appears in the abovementioned documents. The Catalan-Valencian-Balearic dictionary provides a definition that seems to match the object recorded in the inventories: "Diptych, triptych, painting of sacred images, etc., which served as a small portable retable before which prayers were said." 21 The term oratory comes from the Latin verb orare, which means to pray. Although an oratory currently refers to a private or semi-private place of worship, in the Middle Ages it was a structure similar to a retable used in houses.

Based on the information gathered in the inventories, what exactly is the difference between a retable and an oratory? The first thing that is apparent is the ambiguity of the terms used by the notaries themselves: A retable or oratory made of wood including a painting of the Virgin Mary with her son in her arms and a crucifix and other devotions on one door, on another door the Pietà and on the third one a painting of Saint Michael and saint Raphael" (Soler i Palet 1916a, p. 303). Or, when listing the belongings of Martin the Humane: "Oratory, alias retable, made of wood of Greek origin, including four foldable parts containing four stories" (Gudiol i Cunill 1902, p. 449). According to Jaume Sastre, who has studied the case of Majorcan households, oratories were foldable objects equipped with doors, which contained an image, while retables were flat open structures where the image was depicted on the front part (Sastre Moll 2003, p. 51). However, this distinction does not match the complex reality reflected in the Catalan inventories. The retables of Antoni Mura and Pere Girós, for instance, have two doors: A small golden retable made of wood with two doors, one with an image of the Pietà and the other one Our Lady and other saints (Soler i Palet 1916a, pp. 295-96) and a small three-door retable in its case (Roca 1927-1928a, p. 314). The reference of a retable shaped like a cabinet has also been preserved: A retable that has the shape of a cabinet showing the image of Our Lady, very subtle (Cifuentes i Comamala 2000). It is indeed true that, of the oratories that appear in the inventories, eight of them had doors that closed the piece and another three had the structure of a cabinet.

Another element that enables distinguishing retables from oratories is the size. Regrettably, the inventories hardly provide any information about the size of the recorded objects. Both large retables, a large retable on which Christopher is painted (García Panardes 1983, p. 175), and small ones are described. Small retables appear much more often than large ones. They are mentioned nine times. This being

20 Regarding the devotional function of small-sized retables, see: (Van Os 1994; Wilkins 2002; Schmidt 2001, 2005; Le Pogam and Vivet-Peclet 2009).

21 (Alcover and de B. Moll). 
said, what is understood by a small retable? In Pere Girgós' house, there was a small retable hanging on a wall, ${ }^{22}$ while, around the same period, a portable retable was listed: a small retable depicting the image of saint Mary and other images of saints, in its wooden case (Casas Homs 1970, p. 28).

Oratories exist in different sizes and formats. Although they are described as large, small ones also existed and there is even mention of half an oratory. In a house from the mid-fourteenth century, two oratories were found: A large oratory and a small one with images of my Lord God and of Saint Mary as well as other saints (López Pizcueta 1992, p. 53). A tanner who lived at the end of the fifteenth century had an oratory at home: a small oratory showing an image of Jesus crucified, Mary and John including a moulding and wooden frame (Soler i Palet 1916a, p. 299). This oratory contained a moulding, which was typical of oratories back then. They were occasionally placed on a base: A wooden oratory that can be closed, placed on a golden stand showing the figure of a crucifix (Soler i Palet 1916a, p. 301).

Based on the documentation consulted, it can thus be concluded that both the terms retable and oratory are used indistinctly for the same type of devotional object. They were usually small and often foldable. However, oratories, more so than retables, were articulated objects which often had doors that closed.

For a study of religious expressions present in domestic devotion, analysing the location of these objects is key. The inventories usually specify the place where the possessions of the deceased were located. They mostly appeared in the rooms where family life took place such as the bedroom of the deceased, the dining room or the study room. Occasionally, they were found in the back rooms or rooms near the entrance of the house, in the staircase or in the spaces where work was performed. A good example is the bedroom of the Barcelona merchant Guillem Ferrer where he had a small retable with two panels, on which the Crucifix of Jesus Christ and the image of saint Mary are painted, kept in a painted wooden case that hangs on the wall (Casas Homs 1970, p. 29). Another citizen from Barcelona, Ferrer Gualbes, had a similar object at the foot of his bed: Another retable containing a painted cloth, at the foot of the bed, depicting the Virgin Mary and her son in a tabernacle and two apostles, Peter on the left side and Paul on the right (García Panardes 1983, p. 183). In both cases, the retables, located in the bedroom and near the bed, portray images that Guillem Ferrer and Ferrer Gualbes would contemplate devoutly every day when saying their prayers in the morning and in the evening, in accordance with what preachers recommended at that time. The representation of the Virgin Mary is significant, and, in Guillem Ferrer's retable, the crucifix is also portrayed. Those images are the ones that appear the most in the post-mortem inventories as will be explained below.

These retables or oratories hung on the wall or on a bar that supported them as evidenced in this inventory of the Torreferrada in the municipality of Santa Eulàlia de Riuprimer: A retable including $a$ painted cloth of Saint Christopher nailed onto bars. The documents specify that they were often covered with curtains and drapes, following the medieval habit of concealing images during certain liturgical periods such as Lent or when celebrating mass in order to concentrate thoughts on the action of the priest consecrating the Eucharist (Gudiol i Cunill 1902, pp. 449-90). The inventory of sailor García Roig describes an antique retable of the Virgin Mary surrounded by a cloth (Soler i Palet 1916a, p. 294) and also a wooden oratory of medium-sized doors, showing paintings of the crucifix of Jesus Christ and the Image of the Virgin Mary and of Saint Catherine, as well as some other images, hanging on the wall (...) and a hessian towel, with blue strips at the top of the aforementioned...oratory. Already used (Roca 1923, p. 150). The act of "revealing and concealing" also played an important role in the domestic environment as it contributed to reinforcing the desire to contemplate the image, the visual support of prayer. ${ }^{23}$ These spaces of prayer, multifunctional spaces given the limited size of urban houses, were also sacralised thanks to

22 First, a small retable of two panels on which the Crucifix of Jesus Christ is painted and the image of Saint Mary, in its painted wooden case, which hangs on the wall (Roca 1927-1928b, p. 385).

23 Curtains depicting religious figures were inventoried on four occasions: Curtains of black fabric on which the lamb of Saint John is painted. There are six pieces. (Soler i Palet 1916a, p. 291). Regarding the senses in the Middle Ages, see: (Nichols et al. 2008; Palazzo 2014, 2016; Jørgensen et al. 2015). 
the light, the glass lamps burning in front of them: A retable with a painted image of the glorious Virgin Mary and a glass lamp (Soler i Palet 1916a, p. 293).

Retables, oratories and images also had an apotropaic function. This must have been the case of the numerous images, oratories and retables of Saint Christopher located in staircases or near the front door of the house: An old one of Saint Christopher at the top of the stairs (Soler i Palet 1916a, p. 294) and one of Saint Christopher carrying Jesus on his shoulders at the top of the stairs (Idem., pp. 304-5), as will later be described in the iconography.

Many of the retables and oratories recorded in the inventories are objects that were carefully stored inside all kinds of boxes, benches with drawers and trunks in the different rooms of the houses. The devotees had to take them out at certain times of the day, or at specific times of the liturgical calendar, especially during feast days or periods such as Advent, Christmas, Lent and Easter. They were kept in cases that preserved them from dust, dirt or breakage. Given the small size and the portable nature of these objects, it is necessary to consider the possibility that their owners carried them with them when they travelled for professional or devotional reasons. Perhaps this was the case of Guillem Ferrer, the merchant mentioned earlier, who owned a small retable containing the image of Saint Mary, and other images of saints, in a wooden case (Roca 1927-1928b, p. 385) or that of Antoni Mura, owner of an ivory oratory: a small ivory oratory containing images stored in a wooden case (Soler i Palet 1916a, pp. 295-96).

As for the materials, wood was the most common support for retables and oratories. Some of them were made entirely out of wood, others only had a wooden structure onto which the painted cloth or paper was stuck or inserted in, and a moulded frame: An oratory with a painted cloth showing the image of Our Lady, Saint Peter and Saint Catherine within its moulded frame (Soler i Palet 1916a, p. 299). Oratories featured a greater variety of materials and were made of marble, plaster, ivory, fabric or paper: A marble oratory shaped like a cabinet depicting Jesus crucified and in another part Our Lady with Jesus in her arms (Soler i Palet 1916a, pp. 303-4; A small plaster oratory portraying the Annunciation; An ivory oratory with doors and inside an image of Our Lady with Jesus in her arms and two images on each door (Soler $\mathrm{i}$ Palet 1916a, pp. 303-4); A paper oratory with a wooden ornament (Soler i Palet 1916a, p. 302).

With regard to the technique, they were mostly painted directly on prepared wood, on fabric or on cloth. Painted cloths frequently appear in the documents analysed: of the fifty-two retables inventoried, ten are painted on wood, eight are painted cloths. In the case of oratories, six are painted on wood and five are painted cloths of a total of forty-seven oratories. The technique used in the rest of the objects is not known. The notaries do not specify the technique although retables and oratories in the shape of a cabinet may presuppose the existence of sculptural images: A retable shaped like a cabinet showing the image of Our Lady, very subtle (Cifuentes i Comamala 2000, p. 470).

\subsubsection{Images}

In addition to retables and oratories, the inventories itemised twenty-four images: eleven of the Virgin, place the Veronica of Our Lady, six of crucifixes, four of saints, two of the Infant Jesus. It is worth noting that the term image simply refers to the representation of a religious figure and can therefore refer to both two-dimensional objects (painted on wood or cloth or in relief) and three-dimensional objects. Only the material, the reference to clothing or jewels, or the fact that the image is stored in a box or case, enables us to deduce that it is a free-standing sculpture.

The majority of the images listed corresponds to the Mother of God, eleven in total. Two were silver, two were terracotta, two were made of paper and one was made of wood. The material of the rest of the images is unknown. One of them was of the Mother of God wearing a mantle: An image of Our Lady wearing a red silk cloak and one of Jesus (Soler i Palet 1916a, p. 297). Joan Berenguer de Junyent owned a piece that was carefully stored in a valuable receptacle: A very small silver box containing a wooden image of Our Lady and two pearls (Soler i Palet 1916b, p. 592). The materials used, silver, terra cotta, tin and paper, indicate that they were small figures, ideal for private devotion. Actually, the predecessors of these images were the small silver or ivory statuettes that circulated throughout 
Europe in the thirteenth and fourteenth centuries. The two tin moulds that belonged to Narcisa, the wife of a hat maker, exemplifies how popular religious imagery was. The moulds were used in the standardised manufacturing of serialised images: Two medium-sized images or tin moulds, one of Our Lady and the other one of Jesus (Soler i Palet 1916b, p. 392).

On two occasions, the inventories specify the name of the image of the Virgin: An image of the Virgin Mary who is from El Puix (Soler i Palet 1916a, p. 291). Two gold-plated silver Virgins of Montserrat (Soler i Palet 1916b, p. 592), which shows the predilection for these two Marian sanctuaries, El Puig (Valencia) and Montserrat, the focus of numerous local pilgrimages during the Late Middle Ages. The Holy face of Our Lady that Joan Berenguer de Junyent owned was considered very old and was also of a Marian nature (see Figure 2 for an example of a Veronica of Our Lady). It is an iconography that spread widely throughout Catalonia, Valencia and Majorca and that at times assumed Immaculate Conception symbolism (Crispí 1996a, 1996b; Sureda i Jubany 2017).

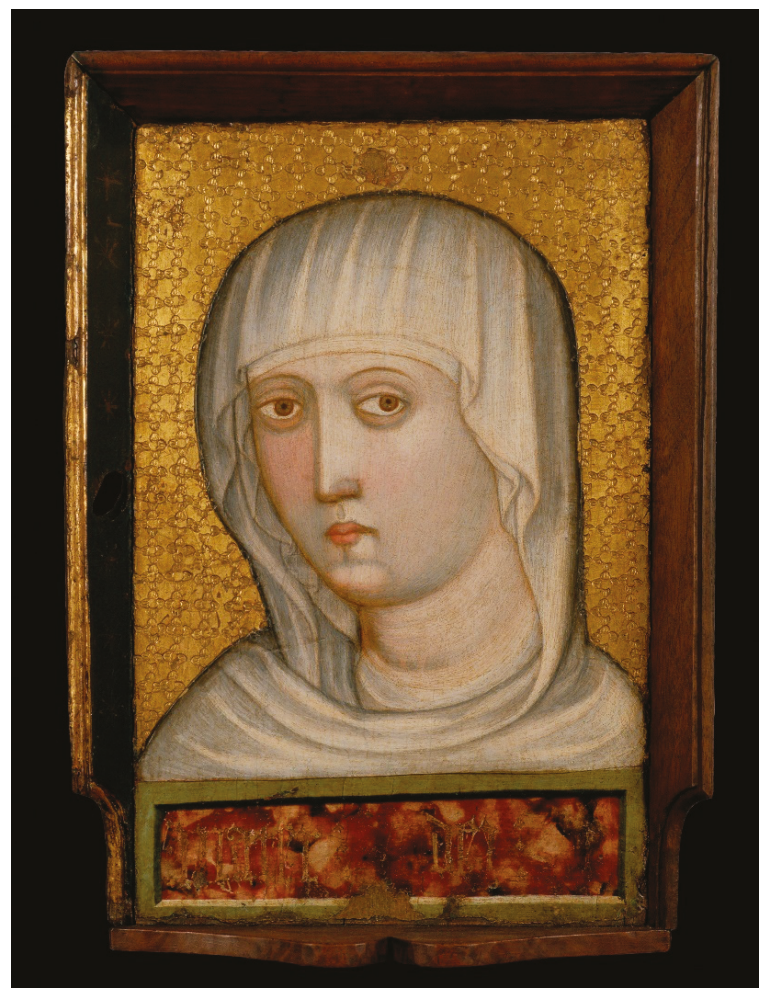

Figure 2. Veronica of Our Lady. Museu Episcopal de Vic

The inventories include six crucifixes, five of which are free-standing images. Two crucifixes are silver, one is ivory, one has a plaster figure of Christ on a wooden cross, one is not specified and the last one is painted on linen. Of all the images that appear in the inventories, there are four that are dedicated to two popular saints, a plaster figure of Saint Catherine that belonged to a tailor called Mateu Maldà and three of Saint Christopher.

\subsubsection{The Child Jesus}

Among the objects inventoried at the house of Pere Girgós in 1389, there was a small Infant Jesus painted the way Saint Christopher used to carry him, with a golden orb in his left hand, wearing a 
brocade dress in the front part and at the back, a golden braid with golden leaves (Roca 1927-1928a, p. 388). A century later, Coloma, the widow of a fuller from Barcelona, owned a wooden box or small case to put a figurine of Jesus inside in which there was a wooden Jesus wearing a tawny surcoat with a gold lace trim and a tin 'gecerant' ${ }^{24}$ (Soler i Palet 1916a, p. 302). In the same period, Joan Berenguer de Junyent, had a crib made of silver poplar wood painted green on the outside featuring the name Jesus (Soler i Palet 1916a, p. 303) and we have two references in Majorca, where notary Martí de Cedrelles had a small figure of baby Jesus (1483) in the bedroom and monsignor Deia had a small plaster figure of Infant Jesus and a shirt of Baby Jesus (1501) (Llompart 1980, p. 366). Those valuable observations prove the existence of statuettes of the Christ Child in the late fourteenth century and their number increased in the fifteenth century. It is clear from the inventories that the figures refer to free-standing wooden or plaster sculptures of the Child Jesus, often dressed. They were small-sized devotional statuettes that laypeople caressed and kissed, encouraged by the gentle and affective piety characteristic of the Devotio Moderna.

The manufacturing of these Infant Jesus figurines is related to the creation nativity scenes, inspired by the initiative of Saint Francis of Assisi to stage the birth of Jesus in Grecco on 25 December, 1223. ${ }^{25}$ The first documented nativity scene is the one Pope Nicolau III (1288-1292) commissioned for the Basilica of Saint Mary Major in Rome (Sarnecka 2019, p. 164). It is also known that Queen Sancha of Naples, the daughter of the kings of Majorca, donated a figure of a kneeling Virgin with baby Jesus to the nuns of the order of Saint Clare in Napels in 1340 (Sarnecka 2019, p. 164). It is probably no coincidence that an early sculptural ensemble from the second half of the fourteenth century of the Birth of Jesus is preserved in the monastery of the Poor Clares of Saint Mary in Pedralbes, Barcelona (Figure 3). It represents the Virgin and Saint Joseph kneeling in adoration to the Child Jesus, lying on the ground, in accordance with the description of the Meditations Vitae Christi and the Nativity of Jesus (Español 1992b, pp. 301-3; Terés 2005, pp. 65-67). Manger scenes became popular in the fifteenth century and by the first half of the sixteenth century many Italian churches had their own sculptural representations of the Nativity (Sarnecka 2019, p. 166).

In parallel to the development of nativity scenes, the first free-standing statuettes of baby Jesus appeared. It is worth noting that those figurines were used for liturgical purposes during the Christmas season. They were placed on a manger near the altar or directly on the communion table and were venerated by those attending mass (Frugoni 2012, p. 16; Siddi 2012, p. 41). The oldest Infant Jesus figures were found in the central area of Italy and seemed to follow the same prototype, possibly a sculpture by Nicola Pisano from the late thirteenth century. ${ }^{26}$ Their initial liturgical function cannot be detached from their devotional use in convents as both monks and nuns were allowed have them in their cells to encourage personal piety, following the advice of their mystical contemporaries (Sarnecka 2019, p. 169). It is not until the end of the fifteenth century that, at least in Italy, this shift to private homes is observed. Figurines of the Child Jesus as well as cribs were first documented at that time. The Child Jesus had to be put in the crib from Christmas Eve to the celebration of Candlemas, after which it had to be kept in a box or case as mentioned in the possessions of Coloma (Sarnecka 2019, p. 170). Eight wooden cribs decorated with delicate tracery dating back to the fifteenth and sixteenth centuries have been preserved. Some of them had bells that tinkled when the crib was rocked or touched (Ippel 2014, pp. 330-47; Brilliant 2017, pp. 175-76).

Joan Berenguer de Junyent's crib had the name Jesus in coloured letters written on it. It is not known if it was the whole name or the monogram JHS. This devotion to the name Jesus was encouraged by Bernardino of Siena and left a mark on the laity in the Late Middle Ages, as was corroborated by the

24 The current Catalan meaning of this word has not been found, so it could not be translated into English.

25 Regarding the origins and spreading of nativity scenes, see, amongst others: (Frugoni and Siddi 2012; Sarnecka 2019). See as well: (Van Os 1994, pp. 98-104)

26 (Siddi 2012, pp. 33-41). It features the previous biography about the origins and development of the Italian figures of the Infant Jesus of the Trecento and early Quattrocento. 
two hundred or three hundred names in terracotta of Flanders that glove maker Joan Llop had in his shop (Duran i Sanpere 1972-1975, vol. 2, pp. 298-300). This same individual, a German man who had settled in Barcelona, also traded with figurines of the Child. The inventory compiled after his death documented three hundred terracotta figurines, which shows the extent of the devotion to images of Jesus. Joan Llop's business, which also included retables, painted cloths and paper and parchment images, prospered because of the growing demand for devotional imagery of the laity and the clergy. ${ }^{27}$

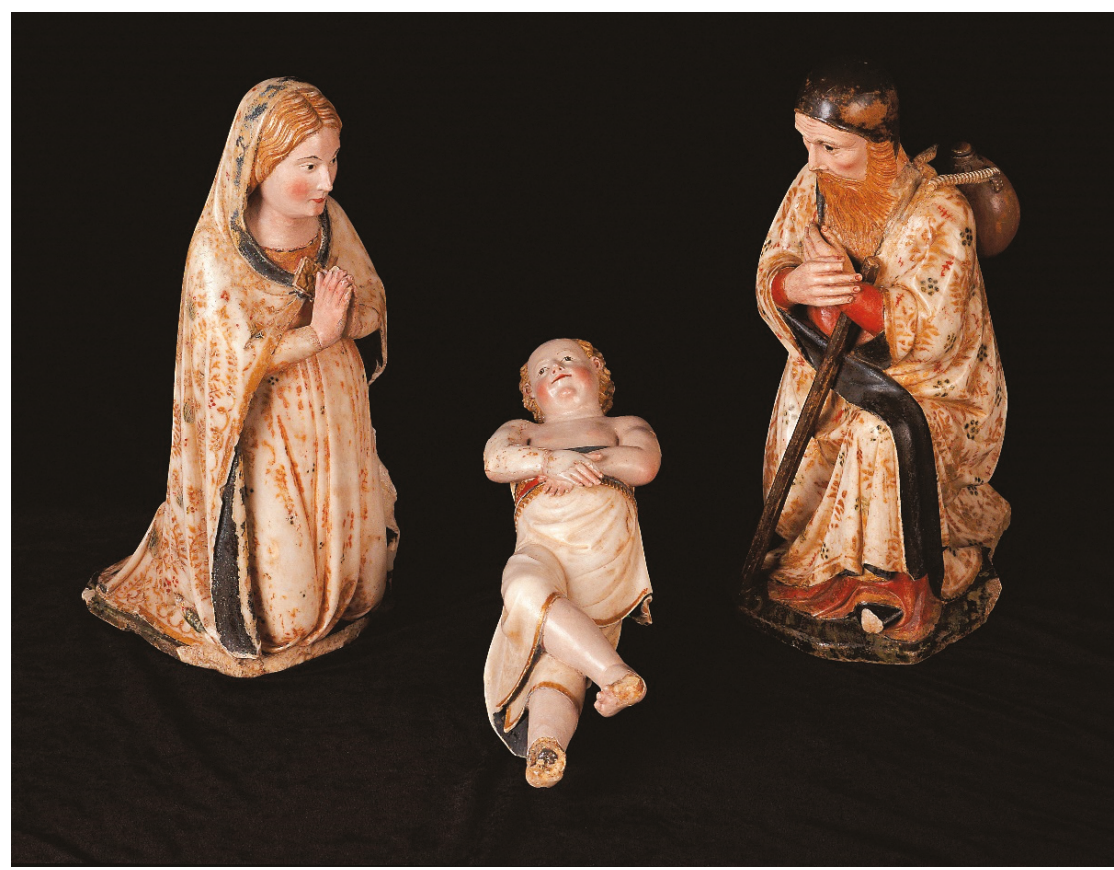

Figure 3. Nativity Scene. Reial Monestir de Santa Maria de Pedralbes. MMP 148.207. Foto Jordi Puig.

\subsubsection{Painted Cloths}

Among the objects listed in the post-mortem inventories containing religious or profane images, no less than fifty painted cloths appeared. As Gudiol pointed out, these are objects in which paint has been applied directly onto cloth, serge or hemp. Painted cloths were very popular among the middle classes because they served as inexpensive imitations of the famous tapestries from the Netherlands that began to be documented in Catalonia in the mid-fourteenth century. In Catalonia, tapestries were called draps de ras ('ras' is the simplified term for Arras, one of the major tapestry-making centres and the capital of Artois). ${ }^{28}$

Painted cloths were nailed or glued onto a wooden framework that supported them and they were hung on walls. They mainly had an ornamental function, decorating dining rooms, rooms, study rooms and other rooms in the houses of wealthy families in the fifteenth century. The size of the painted cloths,

27 His inventory also includes ten retables depicting paintings of the Virgin, three of Saint Barbara, two showing stories of the Mother of God, five large retables of the Virgin Mary with her son in her arms and eighty-six painted cloths that measured 1.25 palms the iconography of which was not specified. Two hundred unidentified sculptures, forty sheets of paper containing the name of Jesus were also mentioned (Duran i Sanpere 1972-1975, vol. 2, pp. 298-300).

28 (Gudiol i Cunill 1902, pp. 399, 495-96). Recently, painted cloths have been referred to: (Terés 1998, pp. 299-317; Molina 1999, pp. 41-43; García Marsilla 2001, pp. 187-89; Sastre Moll 2003, pp. 54-56). 
which ranged from one to two and a half canes, the equivalent of 2.3 to 3.8 metres ${ }^{29}$ leaves no doubt about their decorative use. Works of art such as a painted cloth depicting the story of Job measures around two and a half canes (Soler i Palet 1916a, p. 303) or a painted cloth portraying the Annunciation to the Blessed Virgin Mary and the Adoration of the Magi, located at the head of the room (Broida 1986-1987, pp. 253-54) must have occupied a large part of the wall of the most important rooms in late medieval homes.

The decorative nature of painted cloths explains the profane iconography of those objects representing mainly mythological, historical and literary themes. This fact contrasts with the numerous retables commissioned to coetaneous painters and sculptors. ${ }^{30}$ The fifty-two painted cloths recorded in the inventories analysed contained religious motifs showing Christological, Marian and hagiographic iconography. Not all were conceived to hang on walls. As mentioned earlier, many of them were represented on the central part of retables and oratories and had a moulded or simple frame: An ornament of blue wood surrounded by white stars containing a painted cloth portraying images of Saint Michael and Saint Anthony (Soler i Palet 1916a, pp. 295-96). Other times, the references refer to small-sized structures such as a very small painted cloth in the oratory featuring Our Lady and Jesus Christ on her lap (Soler i Palet 1916a, p. 301). This last one was a small painting of the Pietà, perhaps placed on a piece of furniture in the study or in the bedroom. It could have been the visual aid for prayer of the owner seeking to experience the pain of Mary facing the death of her Son.

\subsubsection{Iconography of Devotional Objects}

Retables, oratories, boards, images and even painted cloths inventoried in late medieval households reflect the devotional universe professed by laypeople at that time. Theoretically, they should be analogous to the wide range of Christological, Marian and hagiographic images specified in the commissions of retables for cathedrals, parishes and monasteries or conventual churches. However, the analysis of the inventories points in another direction: forty-three representations of the Virgin were identified (referred to as Madonna, Our Lady, Saint Mary, Virgin Mary, etc.) and twelve of the Mother of God (Mary with the Infant in her arms). A total of fifty-five images of the Virgin, alone, with the Infant Jesus in her arms, or surrounded by angels and saints were registered. Marian iconography is thus by far the most common, followed at a distance by the crucifixion, cited twenty-one times, and at a much greater distance, by saints and angels, as we shall see later. The first conclusion drawn is that laypeople had a clear preference for the Virgin Mary, a fact that has also been observed in Valencian and Majorcan homes (García Marsilla 2001, p. 171; Sastre Moll 2003, pp. 52-56) and that must be understood within the framework of the belief in Mary's exceptional power of intercession because of her divine motherhood. The preaching of priests, extensive Marian literature, as well as compilations of miracles, contributed to highlighting her spiritual maternity and, as a consequence, her mediating role. $^{31}$

Despite the ambiguity of the notaries' descriptions, Marian images must have been representations of the Virgin and Jesus in her arms. It was the most widespread image in the Late Middle Ages, as seen in the hundreds of Romanesque and Gothic sculptures of the Mother of God preserved in Catalonia and the numerous trecentist retables in which she is the central figure. With respect to Marian scenes, the ones that appear the most are the Annunciation, cited as the Greeting, and the Pietà. They are featured nine and eight times respectively. Both themes stress Mary's maternal role. In the Annunciation, by giving her consent, the Virgin becomes the Mother of Jesus. On Mount Calvary, Mary, afflicted by pain, holding the lifeless body of her son on her lap, shows maternal suffering. It is associated with redemptive passion. Several painted cloths illustrate longer parts of Mary's

29 The Barcelona cana, a measurement used to measure numerous inventoried painted cloths, corresponds to $1.55 \mathrm{~m}$. Another measurement used is the palm, which is the equivalent of $20.8 \mathrm{~cm}$.

30 Regarding the profane iconography of tapestries and painted cloths, see (Terés 1998).

31 For devotion to the Virgin in Catalonia, see: (Crispí 2001, pp. 39-150). 
life: two depict the well-known joys of the Virgin, ${ }^{32}$ iconography held in high esteem in Catalonia, ${ }^{33}$ and another one portrays the story of the Virgin. ${ }^{34}$ The conception, childhood and transformation of the Virgin Mary (stories related to Anna and Joachim and Mary's presentation in the Temple), narrated in apocryphal writings, also had a great impact on the laity, as evidenced by the following two splendid objects: A painted cloth portraying kings of the lineage of the Virgin Mary. On one side there are the fifteen steps Virgin Mary climbs and on the other one Joachim and Saint Anne. It is approximately three canes long and contains a cloth of black fabric and some painted branches (Soler i Palet 1916a, p. 301). Another painted cloth shows a painting of the Death of Our Lady with a yellow jug and branches on said painted cloth (Soler i Palet 1916a, p. 297).

The second most common representations after Marian images belonged to Christological iconography. Twenty-one crucifixes were listed. Six were free-standing figurines and the rest were either paintings or sculptures of crucifixes depicted in one of the panels of the retables, either on their own or flanked by the Virgin and Saint John or even the three Marys. The retables and oratories are often diptychs that combine the Crucifixion with the representation of the Mother of God: A marble oratory in the shape of a cabinet where Jesus appears crucified and on the other part Our Lady holding Jesus in her arms. (Soler i Palet 1916a, pp. 303-4).

The Christological scenes represented correspond mainly to childhood, especially Nativity scenes, which appear eight times, Epiphany, mentioned five times, and the Passion of Christ, which is listed five times. Images related to the passion and death of Jesus such as the Descent from the Cross, the Burial, the Veronica of Christ or the Passio Imaginis were also alluded to. Unfortunately, it is hard to know which image the notary referred to when describing a representation of Jesus that appears several times in the legal documentation: a built-in oratory, which is painted green on the outside and red on the inside, portraying the image of Jesus Christ and the Nativity of Jesus Christ (García Panardes 1983, pp. 172-73).

A large number of angels and saints is mentioned: Saint Christopher appears twelve times, Saint Michael and Saint Anthony five times, Saint Francis, Saint Cosmas and Damian, Mary Magdalene, Saint Catherine, Saint Barbara and Saint Eulalia three times, Saint Jerome and Saint Sebastian twice. Other saints like Peter, Helena, Anne, John the Baptist, John, Paul, Gregory, Onuphrius, Benet and Bernard were mentioned once. In some cases, the saint was represented on his/her own, but other images were narrative, depicting the events of their lives, as is the case of those dedicated to Saint Mary Magdalene and Saint Barbara, two saints that were widely venerated in the Late Middle Ages.

The most striking feature is the repeated reference to images of Saint Christopher. There are twelve images of him, more than of any other saint. Saint Christopher, a Canaanite, is considered the protector of travellers and pilgrims. The devotion to Saint Christopher became widespread in the thirteenth century in the wake of the legend that narrates how he performed service to Christ by helping a child cross a river and the child turns out to be Jesus (Rigaux 1996; García Cuadrado 2000; Buxton 2006; Manzarbeitia Valle 2009; Ondoño Rendón 2015; Thomson 2018). Curiously enough, the inventories specify twice that the images were placed at the top of the stairs, which reinforces the apotropaic function of the representation of Saint Christopher. Laypeople counted on his protection when they travelled or went on a pilgrimage. ${ }^{35}$

The painted cloths, because of their decorative nature, often depicted less conventional images. Objects dedicated to characters from the Old Testament such as Job, ${ }^{36}$ the queen of Sheba ${ }^{37}$ and

32 A very old painted cloth depicting the seven joys of the Virgin Mary (Soler i Palet 1916a, p. 302).

33 An extensive bibliography on the spreading of the joys of Mary in Catalonia, both with regard to religious texts and iconography. See two recent studies: (de Courcelles 1992; Vicens 2003, pp. 25-50).

34 Painted cloth portraying the story of the Virgin (Soler i Palet 1916a, p. 297).

35 Rigaux has stressed the representation of the saint on the exterior façades of churches in the north of Italy and the south of Germany with regard to the saint's protection to travellers and pilgrims (Rigaux 1996, pp. 246-48)

36 A painted cloth depicting the story of Job, which measures approximately two canes and a half (Soler i Palet 1916a, p. 303).

37 A retable portraying the Queen of Sheba (Soler i Palet 1916a, pp. 304-5). 
Solomon, ${ }^{38}$ were inventoried. Other illustrations that were more symbolic such as the representation of the Old and the New Testament were also listed. ${ }^{39}$ Some painted cloths, of a clear moral content, included representations of the seven capital sins, like the painted cloth owned by Francesc Rigau: A folded painted cloth that was in the dining room portrays the story of the seven mortal sins (Soler i Palet 1916a, pp. 304-5) or the large cloth dedicated to the Antichrist, which belonged to Coloma. ${ }^{40}$ The case of two retables that illustrated various invocations is also worth mentioning: First, a retable containing a painted cloth on which numerous different invocations are painted (García Panardes 1983, p. 191) and some panel paintings that represented the Ten Commandments. ${ }^{41}$ Another theme that was not very common was the destruction of Jerusalem. Its image was found in the house of a Barcelona blacksmith. It illustrated an apocryphal text of the Passion and Resurrection of Christ of which versions from the fifteenth and early sixteenth centuries exist in Catalonia. ${ }^{42}$

\subsection{Objects with a Devotional Function}

\subsubsection{Paternosters}

Among the possessions of merchant Guillem Ferrer, there was: a strip with white silk paternosters, and with blue glass paternosters (Casas Homs 1970, p. 30) and also a thick pearl necklace and seven loops of said pearls, as well as seven silver green enamel paternosters, which I, Isabel (woman) affirm to be mine (hers). A few years later, in 1408, when the belongings of sculptor Pere Sanglada were inventoried, three paternosters are described: three rosaries or paternosters: one made of jet containing five amber roses, another on made of jet containing wedges of gold-plated silver, and the third one made of coral with four silver pieces to connect (unions) and additionally, a pendant with a small cross and four small pearls, that is to say, the jewels of Saurina (Batlle Gallart 1993, p. 92). Paternosters were some of the devotional objects that appeared the most in the inventories of laypeople and clergymen in the Late Middle Ages. They were strings of beads used to pray the Lord's Prayer, as many times as the number of beads the object contained (Figure 4$).{ }^{43}$

Repeated recitation of the the Lord's Prayer and the Hail Mary spread in the thirteenth and fourteenth centuries as a pious practice. In the case of the Ave Maria, the numerous miracles attributed to the Virgin Mary by devotees who recited this prayer contributed to its success. Later, in the fifteenth century, praying the Ave Maria in sets of tens of times led to the creation of a new prayer: the rosary, which combined repeating the Ave Maria one hundred and fifty times while contemplating episodes of the life of Jesus Christ. ${ }^{44}$ At the same time, repeating the Lord's Prayer also turned into devotional practice among the laity as attested by the lay brothers of the Carthusian and Cistercian orders who prayed the Lord's Prayer in substitution of the Divine Office in the thirteenth century. ${ }^{45}$

In order to count the number of vocal prayers, a simple cord containing knots was used, an element that was also present in other religions such as Buddhism and Islam. In Christianity, there are several references to these old cords, but one of the most renowned ones is that of the noblewoman Lady Godiva of Coventry, who, in the eleventh century, bequeathed the paternoster beads she used for

38 A small painted cloth showing the story of Solomon (Soler i Palet 1916a, p. 298).

39 An old smoke grey painted cloth of the Old and New Testament (Soler i Palet 1916a, p. 298)

40 A large painted cloth showing the Antichrist (Soler i Palet 1916a, p. 301)

41 Another panel painting of the Commandments (Costa 1983, p. 126).

42 Another painted cloth regarding this theme was inventoried at the home of Arnau Guillem Samuntada, beneficiary of Santa Maria del Mar (1493): Another painted cloth of approximately two and a half canes on which the destruction of Jerusalem is painted (Soler i Palet 1916a, p. 299). Regarding the manuscripts that include the destruction of Jerusalem, see: (Hernando 1989, pp. 1-116)

43 The only two studies on paternosters in Catalonia are: (Gudiol i Cunill 1923a, 1923b; Serra i Boldú 1925). See references to said objects in: (Winston-Allen 1998, pp. 111-16; Falkenburg 2017, pp. 106-10).

44 Regarding the origin and spreading of the rosary, see the recent work by (Winston-Allen 1998).

45 (Winston-Allen 1998, pp. 14-15) (citing Thurston 1 ... ). Actually, the origin of the rosary lies in the replacement of the text of the psalm for the Ave Maria as well as the addendum of the contemplation of episodes of the life of Christ. 
praying to the religious community she had founded (Winston-Allen 1998, p. 14). The cord soon turned into a string of beads, of different materials and sizes, referred to as a paternoster, zapel, chaplet, etc. The creation of a specialised manufacture documented in London and Paris at the end of the thirteenth century ${ }^{46}$ shows how successful its use was as a devotional object. Documents have also been preserved in Catalonia. Gudiol, who carried out one of the first studies on rosaries in Catalonia, documents a paternoster at the castle of Lluçà in 1321. They are often cited in post-mortem inventories of laypeople and clergy during the fifteenth and sixteenth centuries.

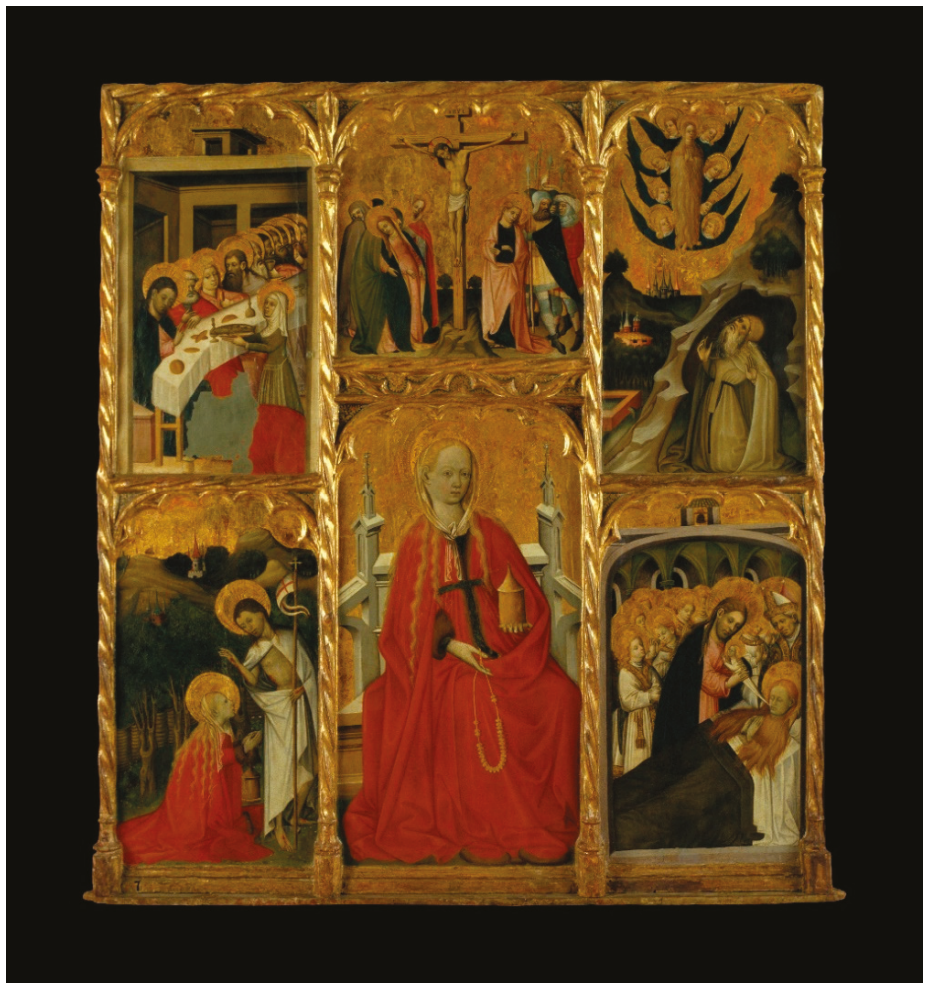

Figure 4. Altarpiece of Saint Maria Magdalena de Perella. Museu Episcopal de Vic.

Apart from the evidence found in documents, Catalan Gothic paintings and sculptures also include a large number of paternosters on coffins and tombstones where they appear in the hands of the deceased as a testimony to their piety. ${ }^{47}$ Examples are the tombstone of Nagalbos in the cathedral of Tortosa and the coffin of Saint Mary of Cervelló in the Basilica of Our Lady of Mercy in Barcelona, both dating from the fourteenth century (Serra i Boldú 1925, pp. 8 bis, 16 bis). Several fifteenth century altarpieces dedicated to Mary Magdalene depict the saint passing the paternoster. The altarpiece of Saint Magdalene of Conangle, for instance, shows the saint wearing a paternoster

46 (Winston-Allen 1998, p. 12) Étienne Boileau: Livre des métiers mentions the artisans that manufactured paternosters in accordance with the materials they worked with.

47 Serra i Boldú publishes the first ones (Serra i Boldú 1925, pp. 8 bis, 16 bis). 
containing sixteen beads and a cross. ${ }^{48}$ The panel painting of Mary Magdalene was probably part of the altarpiece depicting Saint Anne, Sant Bartholomew and Saint Mary Magdalene in Sant Martí de Pertegas (Sant Celoni, Barcelona) where the saint passes a paternoster of twenty-nine crystal or amber beads (Figure 4). ${ }^{49}$ It could also have been part of another gothic altarpiece dedicated to the same saint kept at the National Art Museum of Catalonia (MNAC). ${ }^{50}$

Serra i Boldú classifies paternosters into three different types according to their format: the first kind of paternoster is open-ended and consists of a cord ending in a tassel with beads that move freely up and down, the second one has a string of beads placed one after the other but that can move freely, and the third type corresponds to a string in which the beads are joined together with knots (Serra $i$ Boldú 1925, p. 10). Paternosters consisted of varying numbers of beads of different materials, sizes and shapes. Gudiol and Serra i Boldú mention valuable materials ranging from gold, silver, pearl, turquoise, coral, chalcedony, jasper, jet, amber, crystal, glass or ivory, to simpler ones made of bones, tin, terracotta or seeds (Gudiol i Cunill 1923a, p. 12, Serra i Boldú 1925, pp. 14-15). The beads were joined by a red, green or black silk string. Among the Catalan inventories from the fourteenth and fifteenth centuries, the most frequent ones were made of coral, glass, silver, (enamelled or not), jet, as well as plain ones made of wood or seeds. Sometimes, the size (small or large) is specified, as well as the number of beads (30, 40 or even 400 small coral beads). For instance, a string of paternosters of small coral beads, containing 400 beads. (Benito i Monclús 2008). They sometimes included complementary elements such as crosses, buttons or rings.

Although their primary function was devotional, being instruments to count prayers, paternosters were also used as clothing accessories or as jewels people wore around their necks or hung from their belts. Actually, they fulfilled an apotropaic function to ward off the devil, which is one of the factors that explains why they were popular among the laity and the clergy (Winston-Allen 1998, p. 116). According to the inventories, the paternosters are carefully kept in small boxes but it is not ruled out that they hung on walls, as seen in some fifteenth-century Flemish paintings. ${ }^{51}$

\subsubsection{Agni Dei}

According to the documentation, Agni Dei were silver or wax objects depicting the representation of the Lamb of God, attached to a blue, purple or green silk ribbon to be worn around the neck or in direct contact with the body.

Agni Dei are often mentioned in the inventories of Catalan laypeople and the clergy. The notaries specified that they were carefully wrapped and stored in boxes, cases and bags, together with reliquaries and jewels, which means they were part of the most valuable possessions of their owners. The oldest one cited, in 1308, belonged to Pere Marí. Queen Constanza's scribe owned an Agnus Dei decorated with silver (Batlle Gallart 1994, p. 252), but two other silver Agni Dei were recorded amongst the objects king James II gave to his daughter Constance in 1312 (Martínez Ferrando 1953-1954, doc. 42, pp. 58-59). In the early fourteenth century, monarchs and the people in their circle owned them. They were already considered to be valuable objects at that time. At the end of this century, these objects appeared often in the inventories as is seen in this detailed reference of 1393: Case containing a large Agnus Dei, with a blue silk ribbon and covers made of gold cloth, which belonged to Joan de Peralta, the canon and provost of the Seu Vella cathedral in Lleida (Bolós and Sànchez-Boira 2014, vol. 2, p. 556).

48 Nowadays kept at the Episcopal Museum in Vic (MEV 14). Only the main panel of the retable from the early fifteenth century See the museum record: https://www.museuepiscopalvic.com/ca/colleccions/gotic/compartiment-dun-retaule-dedicat-asanta-maria-magdalena-mev-14 (consulted on 5 August 2019).

49 Kept in the Francisco Godia collection. See: (Saura 2008, pp. 100-1).

50 MNAC 64029-CJT. From the collection of Matías Muntadas.

51 A crystal paternoster hangs on the wall of the bedroom of Giovanni Arnolfini and his wife in the painting by Jan van Eyck couple (1434). See also: (Hackenbroch 1989). 
What is the origin of Agni Dei? Initially, they were made with the wax of the Paschal candle which was lit every year at the opening of the Easter Vigil and was placed near the high altar where it stayed lit until Ascension Day. ${ }^{52}$ The Paschal candle and the light emanating from it are symbols of the Resurrection of Christ. That is the reason why the faithful took the remains of the Easter candle home ever since the sixth century. Soon after, between the eighth and twelfth centuries, candle wax was cut in the shape of lambs and later small discs were made from that same wax stamped with the image of the Lamb of God. By the twelfth century, the disc, made with a two-armed mould, was stamped on both sides and it was then that the name of Agnus Dei was attributed to it. The process of manufacturing these objects evolved from mixing the wax with holy anointing oil to the 1488 prescription, which required the wax to be pure, white and unused. The wax was mixed with holy water. Once treated, the wax received the papal blessing. From the fifteenth century onwards, the habit of Popes blessing the Agni Dei the first and seventh year of their pontificates was established. An inscription mentioning the date of the blessing and the name of the pontiff authenticated the Roman origin of the object.

The fact that Agni Dei were widespread is related to the symbolism and virtues attributed to this iconography as well as to the properties of wax. The Agnus Dei is a symbolic image of Jesus Christ himself, as John the Baptist identified him when he met him for the first time at the Jordan River. His words Ecce Agnus Dei (Here comes the Lamb of God) clearly refer to the sacrificial sense the Jewish people assigned to this four-legged animal. It was the victim the chosen people offered in sacrifice to Yahweh before leaving Egypt and crossing the Red Sea. It is remembered every year during Passover, one of the most widely celebrated Jewish holidays. To Christians, Jesus represents the new lamb that, by dying on the cross, made the final sacrifice to bring about the salvation of the human race. The Agnus Dei is a symbol of sacrifice and redemption. Additionally, the wax Agni Dei discs were made from offers of new symbolic relations that reinforce the meaning of the image. The wax, with its bright white colour and the touch characteristic of its material, shows similarities to the host, the Body of Christ, as pointed out by several theologians such as Saint Anselm. ${ }^{53}$

In the Late Middle Ages, Agni Dei were considered sacramental objects, that is, blessed objects that were intermediaries of divine grace. They were extra-liturgical objects endowed with sacredness and they also served an apotropaic function as they were believed to protect the people who wore them. This protective function is clear in the poem Pope Urban VII devoted to John VIII Palaiologos in 1362, accompanying the gift with an Agnus Dei. The pontiff's text refers to the beneficial properties of this mystically sanctified object: it helped women give birth, protected people against sudden death, the danger of fires, etc. ${ }^{54}$ The mid-sixteenth century Discorso intorno all'origine, antichità et virtù degli Agnus Dei di cera of the Dominican Bishop Vicenzo Bonardo (Cooper 2019, p. 22) also lists the circumstances in which Agni Dei provided protective powers to devotees. Agni Dei were thus considered objects of great symbolic significance as they constituted Christological images that emphasized the idea of sacrifice and redemption, thus establishing a connection with the Eucharist. The notaries referred to Agni Dei discs on chains and ribbons, which reveals that laypeople considered them valuable devotional jewels. They were worn close to the body.

The origin of Catalan Agni Dei is not specified in the inventories analysed with the exceptions of the Agnus Dei that belonged to King Martin the Humane, listed in an inventory that dates from 1410, which was a gift from the pontiff to the monarch (Gudiol i Cunill 1902, p. 469). There are only two references to Agni Dei made of wax. One is the Agnus Dei that Jaimes the Second gave to Joan Amell in 1318 (Martínez Ferrando 1953-1954, doc. 96, p. 130) and the second one belonged to Vicent Sopeira, the canon of the Seu Vella cathedral in Lleida (1482) (Bolós and Sànchez-Boira 2014, vol. 2, p. 1227).

52 Regarding Agni Dei: (Gudiol i Cunill 1902, pp. 469-70; Llompart 1966, pp. 7-25; Herradón Figueroa 1999, pp. 171-93; Cherry 2003, pp. 171-83; Musacchio 2005, pp. 139-56; Cooper 2019, pp. 220-43).

53 Quoted in (Herradón Figueroa 1999, p. 207).

54 Quoted in (Cooper 2019, p. 227). 
It seems that Agni Dei were often made of gold or silver (gold Agnus Dei and a turquoise stone) (see Figure 5 for a silver plaque with an Agnus Dei). ${ }^{55}$ They were attached to a gold chain (the one Franci de Sentmenat owned) (Broida 1986-1987, p. 256) or to a simple coloured silk ribbon. Unfortunately, we have not been able to find any medieval Agnus Dei in the collections of Catalan museums. Museu Episcopal de Vic has one Agnus Dei of the XVI century with an Ephiphany (Figure 6) and there are two wax discs that date from the seventeenth century are preserved at the Diocesan Museum of Tarragona. One is an Agnus Dei from 1630 depicting the shield of Pope Urban VIII on one side and the Coronation of the Virgin Mary on the other side and the other one is a wax medallion portraying the Lamb and Our Lady of the Rosary. ${ }^{56}$ The Frederic Marés Museum also owns several pieces.

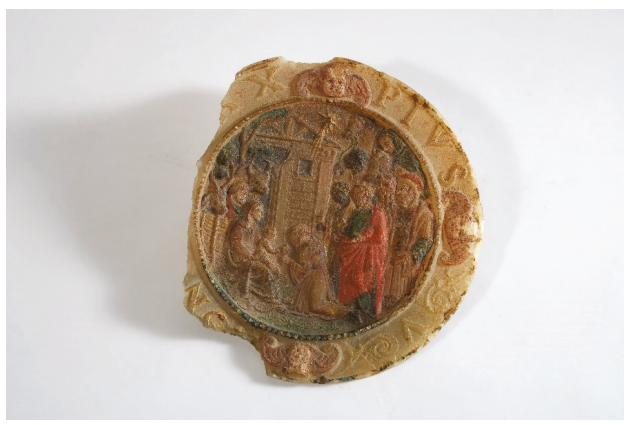

Figure 5. Agnus Dei and Epiphany. Museu Episcopal de Vic.

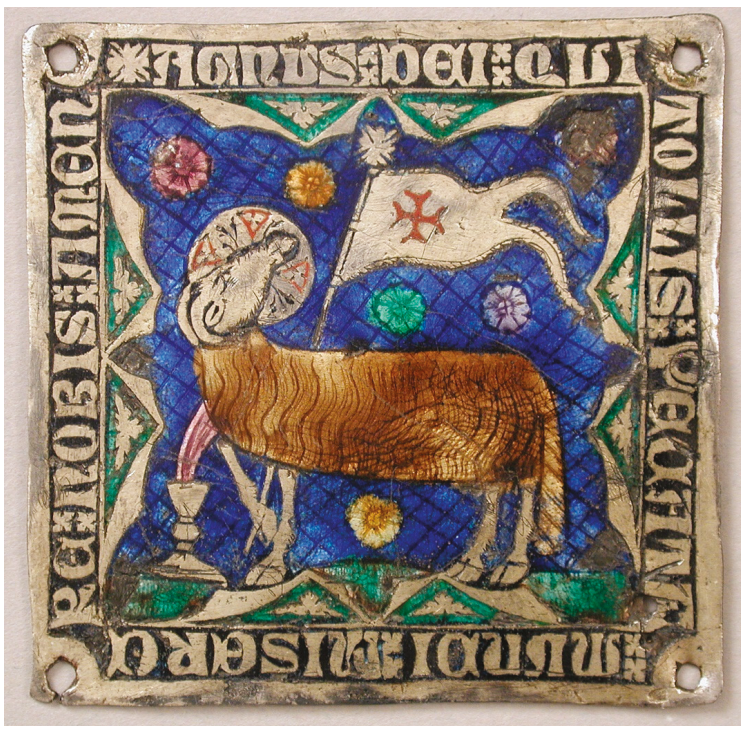

Figure 6. Plaque with Agnus Dei. Made in Catalonia. $14^{\text {th }}$ Century. Metropolitan Museum of Art.

55 Inventory of Gaspar Sanç, sacristan of the Seu Vella cathedral in Lleida (Bolós and Sànchez-Boira 2014, vol. 2, p. 1281).

56 I thank Sofia Mata, curator of the diocesan Museum in Tarragona, for the information provided on these pieces. The objects are MDT 1669 (Agnus Dei depicting the shield of Pope Urban VIII) and MDT 2366. A third Agnus Dei disc made of wax is registered in a bag containing gospels (MDT 0823). 


\subsubsection{Reliquaries}

Francesc Ferrer, a pharmacist, possessed a gold reliquary with its gold chain (Batlle Gallart 1994, p. 525) which he kept locked in a small box. Raimond Malats, a money changer from Barcelona, had a similar object: a chiseled gold reliquary containing six pearls with the image of the Pieta and a black cord (Soler i Palet 1916b, p. 390). This kind of reliquary was portable, made of gold or silver and embellished with small-sized stones, and included a chain or ribbon to hang around the neck to be worn close to the body. ${ }^{57}$ One of the earliest examples of these portable reliquaries is the Talisman of Charlemagne, although other pieces from the late medieval period have survived. At the end of the Middle Ages, those portable reliquaries were popular among kings and the nobility. In Catalonia, they were also common among members of the emerging urban bourgeoisie, as shown in the examples. ${ }^{58}$ The portable nature of the reliquaries is related to the personal experience of the sacred sought for in the Late Middle Ages. Direct contact of a sacred object with the body emphasises the personal experience of something that is holy and that, as such, also sanctifies the space or the person in contact with it. In accordance with how medieval people thought, relics transmit the virtus of the saint, in such a way that the people who touched them were imbued with it. In the case of portable reliquaries, this transmission of virtues and of the sacred became a private experience in line with the spirituality of that time.

In the above-mentioned examples, the notaries did not specify the saint the relics belonged to. It is the case of Guillem Ferrer, a merchant who owned: a yellow velvet bag containing some relics which I, Isabel (woman), affirm to be mine (hers) (Casas Homs 1970, p. 48). Although they were kept in a velvet bag, their spiritual value is evident because the widow of the deceased rushed to claim their ownership. However, it is interesting to note that the iconography of the reliquary is related to the type of relic it contains. The reliquary of Raimond Malats is a representation of the Pietà, which could be associated with certain Christological relics or with elements (soil, oil and water) from the Holy Land. This object can be related to Pere Baçet's relic holder: first, a small golden reliquary including seven pearls (... ) around it and one part has been pierced and the holes contain small pearls; the other part, which opens and closes, contains three images, one of the Crucifix and two more (Martorell and Valls i Taverner 1911-1912, p. 624). It is, once more, a small-sized piece the front part of which opens and it is decorated with pearls and a representation of the Crucifix. Although there is no mention of a chain, this kind of piece suggests that it was conceived to be worn as a pendant.

Of the documented reliquaries, the most exceptional one is the one described in the inventory of Pere de Queralt: a silver case and silver arm which contains the finger of Saint John (Costa 1983, p. 126), kept inside a box of the painted room, probably the bedroom of the couple. It is a so-called "speaking" reliquary, a kind of reliquary shaped like a body part, imitating the shape of the relic it contains. In this case, it has the shape of an arm and contains the finger of St John. Pere de Queralt thus owned a valuable relic, perhaps acquired during one of the diplomatic trips he made when he was in the service of Martin the Humane.

\section{Devotional Objects and Social Estates: A Brief Approach to Its Study}

An analysis of devotional objects in relation to the social status of their owners is outside the scope of this article. However, some preliminary issues may be pointed out. As said, the eighty-five inventories analysed correspond to the so-called braç reial or braç popular, which, like the braç militar, was divided into two large groups: the upper estate (honourable citizens) formed by urban oligarchies that held the reins of citizen power, and the lower estate, which consisted of merchants, artists

57 See examples of this kind of reliquaries in: (Treasures of Heaven 2010, cat. 72 to 76, pp. 131-33).

58 Regarding this kind of reliquary referring to the talisman of Charlemagne: (Robinson 2011, pp. 110-16). Owning reliquaries was already a phenomenon observed during the first centuries of Christianity and which reaches all the social strata in the Middle ages, see: (Klein 2010, pp. 153-75; Smith 2014, pp. 23-46; Blick 2014, pp. 110-15). 
(intellectual and liberal professions such as doctors, lawyers and notaries) and menestrals (craftsmen). The inventories analysed include a significant sample of people belonging to those four groups. There were eight honourable citizens, seventeen merchants, fourteen artists and thirty-tree menestrals. Of all the inventories studied, only twelve did not itemise any devotional objects (eight menestrals, two merchants, one apothecary and one whose social status is unknown).

In order to correctly interpret the data, it must be taken into consideration that there was a considerable increase in religious objects from the thirteenth century up to the year 1500. Having said that, figures indicate the existence of devotional objects was a generalized phenomenon that permeated all the social estates in the fifteenth century. This is shown by the fact that of the seventy-one inventories analysed from the fifteenth century, only six did not contain any devotional objects and in five cases the owners were menestrals. A direct relation is observed between the owners' wealth and the number of objects they possessed. Thus, while for artisans and artists, the average number of objects was usually one or two, despite the existence of exceptions, for merchants and honourable citizens the average number usually ranged between four and five, although there are cases in which ten to fourteen ${ }^{59}$ objects are listed. Among the honourable citizens, the cases of Pere de Queralt, who owned seven objects (1408), and of Antoni Mura, who had eleven objects (1463), stand out.

With respect to the type of objects, it is necessary to mention that retables, oratories, images, painted cloths and paternosters were inventoried in the homes of people who belonged to all the different estates. However, in the houses of merchants, a proportionally higher number of retables, oratories and images is observed in comparison to the other groups. They possessed eighteen oratories, fifteen retables and eight images, while menestrals owned sixteen oratories, nine retables and six images. However, proportions change significantly with regard to painted cloths. Twenty-six were registered in the homes of craftsmen and thirteen in the houses of merchants. Objects considered particularly valuable such as reliquaries and Agni Dei were similar in number in the homes of honourable citizens, merchants and artists but none were inventoried in the dwellings of craftsmen.

This brief quantitative overview, which should be compared with an analysis of materials and iconographies, reflects the process of the enrichment of the Catalan middle classes, in which merchants and traders played a significant part. This phenomenon was also observed in the large European cities of that time. The case of Guillem Ferrer, a Barcelona merchant who died in 1398, and that of Joan Berenguer de Junyent (late fifteenth century) serve to illustrate the variety of devotional objects as well as their devotion. Among the belongings of Ferrer, fourteen objects were registered. There were two retables, one oratory, all dedicated to the Virgin and the saints, two pavises displaying the image of Saint George, six paternosters and three valuable objects, two of them of a marked Christological nature: a bag containing relics, a silver Agnus Dei and (a cord of?) the size of the tomb of Christ. A century later, Junyent possessed a Holy Face of Our Lady, four figures of the Mother of God (two silver ones of the Virgin of Montserrat, one wooden figure and one made of terra cotta), three oratories (two of the Virgin and the third one showing the Annunciation), a gold paten and a wooden crib with the name of Jesus written on it (Soler i Palet 1916a, pp. 303-4).

\section{Conclusions}

The objective of the present study is to make an initial approach to devotional objects by means of the information gathered from post-mortem inventories. As seen above, those documents are a privileged documentary source that reflects the lives and possessions of families in the Late Middle Ages. Those extensive lists of registered objects constitute the material universe of medieval laypeople and are an eloquent expression of the beliefs, interests, habits and pastimes that filled their existence.

This final section is organized into three parts referring to the three elements that compose domestic devotion: space, objects and people.

59 This is the case of Pere Girgós (1389); Guillem Ferrer (1398); Joan Berenguer de Junyent (at the end of the fifteenth century). 
First of all, post-mortem inventories show that private chapels were uncommon and were only found in the dwellings of the wealthiest members of the braç reial. By contrast, the presence of devotional objects is observed in the rooms that constitute the most intimate spaces in the homes of laypeople: bedrooms, offices and dining rooms. Although those rooms basically performed secular functions, the presence of objects such as retables or oratories, images, reliquaries, Agni Dei or paternosters sacralised those domestic spaces, converting them into the stage of the direct, intimate and personal relationship devotees established with the Divine.

Secondly, the inventories analysed allow identifying and quantifying the devotional objects in Catalan homes at the end of the Middle Ages. They became more numerous as time went on and a considerable number of them were from the period just before the Modern Age. Of all the registered items, almost two hundred and fifty were religious objects or items containing religious images. There were retables, painted cloths, oratories, images, paternosters, reliquaries, panels, Agnus Dei, curtains, boards, pieces of bright silk fabric, cases, chalices, altars, pavises and a music stand. The most numerous objects were the domestic replicas of the large pieces of furniture that decorated the high altars and side chapels of urban and rural churches: retables, oratories and images. The difference is that in those spaces the function of altarpieces was primarily liturgical, while in the domestic environment retables, oratories and images performed a devotional function and use. Apart from this large number of items, it is worth mentioning those religious objects of personal use that helped prayer such as paternosters or those stressing God's protection or the ones conferring some kind of grace like Agni Dei. Brush cloths, which decorated the walls of dining rooms and other spaces in homes, are also worth noting. Although they were not strictly considered to be devotional objects, they contributed to making Salvation History part of laypeople's daily lives.

Thirdly, it is imperative to address the interaction between laity and devotional objects, an authentic expression of domestic piety. Inventories are a quantitative source but they do not provide any detailed information on the use of the objects or on the interaction between owners and objects and this is a limitation of the study of medieval domestic devotion. The growing number of devotional objects itemised in the fourteenth and fifteenth centuries in the homes of citizens that belonged to the braç reial shows the interest of the laity in this kind of objects. The presence of those objects in their bedrooms and the type, size and material of the retables and oratories points to their everyday use. Considering Francesc Eiximenis and Saint Vincent Ferrer recommended morning and evening prayers and preachers encouraged the practice of prayer in private and secluded spaces, there is every reason to believe that retables and images were part of laypeople's daily religious practice. It included contemplating and meditating on the life of Christ, the Virgin and the saints. The figurative representations they looked at in the retables or oratories hanging on the walls of their rooms, displayed on a piece of furniture or held in their hands were used as visual and tactile support. Interacting with those objects involved much more than simply a visual relationship. The fact of kneeling or lying on the cold ground; reaching the image to touch, caress or kiss it; covering it with a curtain and showing it later or lighting a candle entailed an intimate and personal experience that aroused their senses and touched their souls. Said actions, often accompanied by devotional readings or the repetition of vocal prayers, generated empathy and feelings of union with Jesus Christ and the Virgin in the faithful, which was the ultimate objective of Imitatio Christi. In contrast to the wide variety of hagiographic themes represented in temples, it is no surprise that the images that appear the most in the post-mortem inventories analysed are Marian and Christological. The latter depict themes related to the Passion of Christ (the Crucifixion, the Virgin's compassion, homo pietatis and the Veronica of Christ, which have also been found in Majorcan and Valencian homes. Paternosters, Agnus Dei and reliquaries are also part of this supernatural experience that penetrates and intensifies the senses such as touch and sight. The chain, ribbon or string described in the inventories indicate direct contact with the body. The fact of wearing those objects close to the heart and the possibility of touching them with their hands not only favoured a tangible experience of divine protection against the forces of evil but 
also close contact with a sacred object that transmitted sacredness and was considered a means of understanding sanctifying grace (Hamm 2016).

In summary, retables, oratories and images, as well as reliquaries and Agni Dei, had the power to act as mediators between laypeople and God. The materiality and tangibility of these objects and their understanding through touch and sight, made the experience of the Divine more intimate and personal but also more impacting and effective. ${ }^{60}$ In accordance with the value given by theologians to tangible objects, they became means to access a direct relationship with God without the need of other intermediaries as was encouraged in the Devotio Moderna. The use the laity made of those devotional objects connects with what was called the "materiality" of religion at the end of the Middle Ages: the use of images and a wide array of objects that contributed to generating a personal experience of God based on material and on the understanding of the spiritual and the supernatural.

\section{Images}

1. Saint Catherine praying before an image of Our Lady (second half of the 14th century). Robert Lehman collection. Metropolitan Museum of Art (1975.1.62)

2. Veronica of Our Lady. (C) Museu Episcopal de Vic (MEV 1885). Photo: Joan M. Díaz.

3. Nativity scene of the Pedralbes monastery in Barcelona. Reial Monestir de Santa Maria de Pedralbes (MMT 148-207). Foto: Jordi Puig

4. Altarpiece of Saint Maria Magdalena de Perella. (C) Museu Episcopal de Vic (MEV 47). Photo: Gabriel Salvans

5. Agnus Dei with Epiphany. (C) Museu Episcopal de Vic (MEV 17262). Photo: Joan M. Díaz.

6. Plaque with Agnus Dei. Made in Catalonia. 14th century. Metropolitan Museum of Art (17.190.970).

Funding: This research received no external funding.

Acknowledgments: In this section you can acknowledge any support given which is not covered by the author contribution or funding sections. This may include administrative and technical support, or donations in kind (e.g., materials used for experiments).

Conflicts of Interest: The author declares no conflict of interest.

\section{References and Notes}

\section{Primary Sources}

Eiximenis, Francesc. 1981. Lo llibre de les dones. Barcelona: Universitat de Barcelona, Departament de Filologia Catalana. Ed. Curial, 2 vols.

Eiximenis, Francesc. 1985. Scala Dei: devocionari de la reina Maria. Barcelona: Publicacions de l'Abadia de Montserrat.

Ferrer, Vicenç. 1932-1998. Sermons. Barcelona: Barcino. Els Nostres clássics. 6 vols.

Llull, Ramon. 1981-1982. Llibre d'Evast i Blanquerna. Edited by Salvador Galmés. Barcelona: Barcino.

\section{Secondary Sources}

Ajmar-Wollheim, Marta, and Flora Dennis, eds. 2006. At Home in Renaissance Italy. London: Victoria \& Albert Museum

Alcover, Antoni M., and F. de B. Moll. Diccionari Catalá-Valenciá-Balear. Available online: https://dcvb.iec.cat/ (accessed on 27 July 2019).

60 Abundant literature exists on the "materiality" and tangibility of Christianity and the relevance of understanding through the senses at the end of the Middle Ages. See among others: Bynum (2007, 2011), Rubin (2009) and Jørgensen et al. (2015) and Laugerud et al. (2016). 
Alcoy, Rosa. 2005. Libros de Casas Nobles en Cataluña y en Mediodía Francés (desde el año 1200 al Reinado de Jaume II). In Vivir en el Palacio en la Edad Media. Edited by Ramon Alberch i Figueras. Torreón de Lozoya, del 23 de marzo al 22 de mayo de 2005. Segovia: Caja Segovia, Obra Social y Cultural. pp. 191-204.

Almenar Fernández, Luis. 2017. Los inventarios post mortem de la Valencia medieval. Una fuente para el estudio del consumo doméstico y los niveles de vida. Anuario de Estudios Medievales 47: 533-66. [CrossRef]

Amenós, Lluïsa. 2017. Fuentes escritas e iconográficas aplicadas al estudio de la cultura medieval: Los inventarios de objetos de uso doméstico (siglos XIV-XV). In Exteriors i Interiors. Retalls d'história d'art Medieval. Lleida: Pagés editors, pp. 49-69.

Aurell i Cardona, Jaume. 1995. Els inventaris "post mortem" i la cultura dels mercaders medievals. Medievalia 11: 107-21.

Aurell i Cardona, Jaume, and Alfons Puigarnau. 1995. Iconografia a les llars mercantils del segle xv. Mentalitat, estética religiositat dels mercaders a Barcelona. Anuario de Estudios Medievales 25: 297-331.

Bacci, Michele. 2018. Devotional Panels as Sites of Intercultural Exchange. In Domestic Devotions in Early Modern Italy. Edited by Maya Corry, Marco Faini and Alessia Meneghin. Leiden and Boston: Brill, pp. 272-92.

Batlle Gallart, Carme. 1981a. La família i la casa d'un draper de Barcelona, Burget de Banyeres (primera meitat del segle XIII). Acta Historica et Archaeologica Mediaevalia 2: 69-92.

Batlle Gallart, Carme. 1981b. Las bibliotecas de los ciudadanos de Barcelona en el siglo XV. In Livre et lecture en Espagne et France sous l'Ancien Régime. Paris: ADPF, pp. 15-34.

Batlle Gallart, Carme. 1983. La casa burgesa en la Barcelona del segle XIII. Acta Historica et Archaeologica Mediaevalia. Annex d'História Medieval 1. La Societat Barcelonina a la Baixa Edat Mitjana 2: 69-92.

Batlle Gallart, Carme. 1985. La casa barcelonina en el segle XIII: l'exemple de la família Dufort. En la España Medieval 7: 1347-60.

Batlle Gallart, Carme. 1986. El castell de Sitges a la mort de Bernat de Fonollar (1326). Acta Historica et Archaeologica Mediaevalia, Fortaleses, Torres, Guaites i Castells de la Catalunya Medieval 3: 153-77.

Batlle Gallart, Carme. 1988. La casa i els béns de Bernat Durfort, ciutadá de Barcelona, a la fi del segle XIII. Acta Historica et Archaeologica Mediaevalia 9: 9-51.

Batlle Gallart, Carme. 1993. La casa i l'obrador de Pere Sanglada, mestre d'imatges de Barcelona (†1408). D'Art 19: 85-96.

Batlle Gallart, Carme. 1993-1994. La família i els béns de Pere Marí, escrivá de la reina Constança vers 1300. Acta Historica et Archaeologica Mediaevalia 14-15: 243-58.

Batlle Gallart, Carme. 1994. Francesc Ferrer, apotecari de Barcelona vers 1400, i el seu obrador. Miscel-Lània de Textos Medievals 7: 499-547.

Batlle Gallart, Carme. 1998. La casa de Bernat de Sarriá, canonge de la Seu de Barcelona, vers 1300. Anuario de Estudios Medievales 28: 619-34. [CrossRef]

Batlle Gallart, Carme, and Coral Cuadrada. 1993. Berenguer de Bonastre, un negociante barcelonés en el norte de África: Segunda mitad del siglo XIII. In Sardegna, Mediterraneo e Atlantico tra Medioevo ed etá Moderna. Il Mediterraneo: Bulzoni, vol. 2, pp. 129-50.

Batlle Gallart, Carme, and Teresa M. Vinyoles. 2002. Mirada a la Barcelona Medieval des de les Finestres Gòtiques. Barcelona: Rafael Dalmau.

Batlle Gallart, Carme, Àngels Busquets, and Imma Navarro. 1989. Aproximació a l'estudi d'una família barcelonina els segles XIII i XIV: Els Grony. Anuario de Estudios Medievales 19: 285-310.

Batlle i Prats, Lluís. 1978. Inventari dels béns de Guillem de Coll, apotecari especier de Girona, 1454. Estudios Históricos y Documents de los Archivos de Protocolos 6: 197-213.

Belting, Hans. 2009. Imagen y Culto: Una Historia de la Imagen Anterior a la Edad del Arte. Madrid: Akal. First published 1994.

Belting, Hans. 1981. Bild und Publikum im Mittelalter. Berlín: Mann.

Benito i Monclús, Pere. 2008. Casa rural y niveles de vida en el entorno de Barcelona a fines de la Edad Media. In Pautes de Consum i Nivells de vida al món Rural Medieval. Available online: https://www.uv.es/consum/benito.pdf (accessed on 16 November 2019).

Blick, Sarah. 2014. Common Ground Reliquaries and the Lower Classes in Late Medieval Europe. In Matter of Faith: An Interdisciplinary Study of Relics and Relic Veneration in the Medieval Period. Edited by James Robinson, Lloyd de Beer and Anna Harnden. London: British Museum, pp. 110-15. 
Blick, Sarah. 2019. Bringing Pilgrimage Home: The Production, Iconography, and Domestic Use of Late-Medieval Devotional Objects by Ordinary People. Religions 10: 392. [CrossRef]

Bolós, Jordi. 2000. La Vida Quotidiana a Catalunya a L'època Medieval. Barcelona: Edicions.

Bolós, Jordi. 2002. L'espai quotidià a la Catalunya gòtica. In La Vida Quotidiana a Través Dels Segles. Edited by María Ángeles Pérez Samper. Barcelona: Pòrtic, pp. 104-21.

Bolós, Jordi, and Imma Sànchez-Boira. 2014. Inventaris i Encants Conservats a l'Arxiu Capitular de Lleida (Segles XIV-XVI). 3 vols. Barcelona: Fundació Noguera.

Bolós, Jordi, and Imma Sànchez-Boira. 2015. La casa i els seus objectes. Una aproximació feta a partir de les fonts escrits i les fonts iconogràfiques. In La Vida Quotidiana a L'edat Mitjana: Actes del IV Seminari d'Estudis Medievals d'Hostalrich. Edited by Neus Puig i Amat and Montse Viader i Crous. Hostalric: Ajuntament d'Hostalric, pp. 13-32.

Brilliant, Virginia. 2017. Crib of the Christ Child. In A Feast for the Senses: Art and Experience in Medieval Europe. Baltimore: Walters Art Museum, pp. 175-76.

Broida, Equip. 1986-1987. Ús de l'espai en els castells i torres dels segles XIV i XV. In Castells, Guaites, Torres $i$ Fortaleses de la Catalunya Medieval. Edited by Manel Riu. Barcelona: Universitat de Barcelona, pp. 217-95.

Brundin, Abigail, Deborah Howard, and Mary Laven. 2018. The Sacred Home in Renaissance Italy. Oxford: Oxford University Press.

Busqueta, Joan Josep. 2001. L'inventari de béns dels castells i torres dels Sant Climent de lleida (1432): presentació del text. Seu Vella. Anuari d'Història i Cultura 3: 263-302.

Buxton, Sarah Victoria. 2006. Saint Christopher in Medieval Spanish Literature. Master's thesis, Durham University, Durham, UK.

Bynum, Caroline Walker. 2007. Wonderful Blood: Theology and Practice in Late Medieval Northern Germany and Beyond. Philadelphia: University of Pennsylvania Press.

Bynum, Caroline Walker. 2011. Christian Materiality: An Essay on Religion in the Late Medieval Europe. New York: Zone Books.

Campbell, Erin J., Stephanie R. Miller, and Elizabeth Carroll Consavari, eds. 2013. The Early Modern Italian Domestic Interior 1400-1700: Objects, Spaces, Domesticities. Farnham: Ashgate Publishing Ltd.

Casas Homs, Josep M. 1970. L'Heretatge d'un mercader barceloní: darreries del catorzèn segle. Cuadernos de Historia Económica de Cataluña 2: 9-112.

Chabás, Roque. 1903. Inventario de los libros, ropas y demás efectos de Arnaldo de Villanueva. Revista de Archivos Bibliotecas y Museos 9: 189-203.

Cherry, John. 2003. Containers for Agnus Dei. In Through a Glass Brightly: Studies in Byzantine and Medieval Art and Archaeology Presented to David Buckton. Edited by Chris Endwistle. Oxford: Oxbow Book, pp. 171-83.

Cifuentes i Comamala, Lluís. 2000. La promoció intel-lectual i social del: s barbers-cirurgians a la Barcelona medieval: L'obrador, la biblioteca i els béns de Joan Vicenç (fl. 1421-1464). Arxiu de Textos Catalans Antics 19: 429-79.

Conde y Delgado de Molina, R. 1977. Joan Tárrega: comerciante y hombre de negocios barcelonés del siglo XV. Miscellanea Barcinonensia 17: 55-95.

Cooper, Irene Galandra. 2019. Investigating the 'Case' of the Agnus Dei in Sixteenth-Century Italian Homes. In Domestic Devotions in Early Modern Italy. Edited by Maya Corry, Marco Faini and Alessia Meneghin. Leiden and Boston: Brill, pp. 220-43.

Corry, Maya, Deborah Howard, and Mary Laven, eds. 2017. Madonnas E Miracles: The Holy Home in Renaissance Italy. London: Philip Wilson Publishers.

Corry, Maya, Faini Marco, and Meneghin Alessia, eds. 2019. Domestic Devotions in Early Modern Italy: The Place of Piety in the Italian Renaissance Home, 1400-1600. Leiden and Boston: Brill.

Cossar, Roisin. 2017. Clerical Households in Late Medieval Italy. Cambridge and London: Harvard University Press.

Costa, Maria Mercè. 1983. L'inventari dels béns del poeta Pere de Queralt. Estudis Universitaris Catalans. Miscel-Lània Aramon i Serra 25: 115-45.

Crispí, Marta. 1996a. La difusió de les "veròniques" de la Mare de Déu a les catedrals de la Corona d'Aragó a finals de l'edat mitjana. Lambard: Estudis D'art Medieval 9: 83-103.

Crispí, Marta. 1996b. La Verònica de Madona Santa Maria i la processó de la Puríssima organitzada per Martí l'Humà. Locus Amoenus 2: 85-101. 
Crispí, Marta. 2001. Iconografia de la Mare de Déu a Catalunya (Imatgeria). Ph.D. thesis, Universitat Autònoma de Barcelona, Bellaterra, Spain.

de Courcelles, Dominique. 1992. L'écriture Dans la Pensée de la Mort en Catalogne. Les Joies, Goigs, des Saints, de la Vierge et du Christ de la fin de Moyen Age au XVIII Siècle. Paris: École de Chartres.

Delaruelle, Étienne. 1975. La Piété Populaire: Au Moyen Age. Torino: Bottega d'Erasmo.

Díez Jorge, M. E., and J. Navarro Palazón. 2015. La casa Medieval en la Península Ibérica. Madrid: Sílex.

Duran i Sanpere, Agustí. 1972-1975. Un guanter, mercader d'art. In Barcelona i la Seva Historia. Barcelona: Curial, vol. 2, pp. 298-300.

Español, Francesca. 1992a. Clients i promotors en el gòtic català. In Catalunya Medieval. Exposició, Barcelona, el 20 de maig al 10 d'agost. Barcelona: Generalitat de Catalunya, pp. 217-31.

Español, Francesca. 1992b. Naixement. Bernat Roca (?). In Catalunya Medieval. Exposició, Barcelona, el 20 de maig al 10 d'agost. Barcelona: Generalitat de Catalunya, p. 301.

Español, Francesca. 2019. La cultura visual en los sermones de San Vicente Ferrer y en los escenarios de su predicación. Anuario de Estudios Medievales 49: 103-35. [CrossRef]

Falkenburg, Reindert. 2017. Prayer Nuts Seen through the Eyes of the Heart. In Small Wonders. Late-Gothic Boxwood Micro-Carvings from the Low Countries. Edited by Frits Scholten. Amsterdam: Rijksmuseum Publications Department, pp. 106-41.

Fité, Francesc. 2001. L'alberg i l'inventari patrimonial de Rotllí Gaulter, escultor i mestre d'obres de la Seu de Lleida (1442). In Seu vella. Anuari d'Història i Cultura. Lleida: Associació d'Amics de la Seu Vella de Lleida, vol. 3, pp. 123-48.

Frugoni, Chiara. 2012. Sul Natale di Greccio e le sue rappresentazioni. In Il Misterio Goioso. Il Presepe di Greccio et le Sculture del Gesú Bambino Benedicente. Edited by Chiara Frugoni and Federica Siddi. Siena Complesso murale Santa Maria della Scala, 21. dicembre 2012, 28 febbraio 2013. Monteriggioni: Il Leccio, pp. 11-31.

Frugoni, Chiara, and Federica Siddi, eds. 2012. Il Misterio Goioso. Il Presepe di Greccio et le Sculture del Gesú Bambino Benedicente. Siena Complesso murale Santa Maria della Scala, 21. dicembre 2012, 28 febbraio 2013. Monteriggioni: Il Leccio.

García Cuadrado, María Dolores. 2000. San Cristóbal: Significado iconológico e iconográfico. Antigïedad y Cristianismo: Monografías Históricas Sobre la Antigüedad Tardía 17: 343-66.

García Marsilla, Juan Vicente. 2001. Imatges a la llar. Cultura material i cultura visual a la València dels segles XIV i XV. Recerques 43: 163-94.

García Panardes, Teresa. 1983. Los bienes de Ferrer Gualbes, ciudadano de Barcelona (hacia 1350-1423). Acta Historica et Archaeologica Mediaevalia 4: 149-204.

Goldberg, p. J. P., and Maryanne Kowaleski. 2009. Introduction. In Medieval Domesticity: Home, Housing, and Household in England. Cambridge: Cambridge University Press, pp. 1-13.

Goldthwaite, Richard A. 1993. Wealth and the Demand for Art in Italy 1300-1600. Baltimore: Johns Hopkins University Press.

Gudiol i Cunill, Josep. 1902. Nocions D'arqueología Sagrada Catalana. Edited by Imp de la Viuda de R. Anglada. Madrid: CSIC Press.

Gudiol i Cunill, Josep. 1923a. Del Rosari, Pàgina Artística. La Veu de Catalunya, 570, 7 de gener, p. 12.

Gudiol i Cunill, Josep. 1923b. Del Rosari, Pàgina Artística. La Veu de Catalunya, 575, 6 juliol, p. 5 and 576, 29 de juliol, p. 5.

Hackenbroch, Yvonne. 1989. A Paternoster Pendant in the Robert Lehman Collection. Metropolitan Museum Journal 24: 127-33. [CrossRef]

Hamm, Bernard. 2016. Types of grace mediality in the late Middle Ages. In The Materiality of Devotion in Late Medieval Northern Europe: Images, Objects and Practices. Edited by Henning Laugerud, Ryan Salvador and Skinnebach Laura. Dublin: Four Courts, European Network on the Instruments of Devotion 3, pp. 10-35.

Hernando, Josep. 1989. La destrucció de Jerusalem. La venjança que féu de la mort de Jesucrist Vespasià e Titus son fill. Miscel-lània de Textos Medievals 5: 1-116.

Hernando, Josep. 1994. Escrivans, il·luminadors, lligadors, argenters i el llibre a Barcelona, segle XIV. Documents dels protocols notarials. Miscel-lània de textos Medievals 7: 189-258.

Hernando, Josep. 1995. Llibres i Lectors a la Barcelona del Segle XIV. Barcelona: Fundació Noguera.

Herradón Figueroa, María Antonia. 1999. Cera y devoción. Los agnusdei en la colección del Museo Nacional de Antropología. Revista de Dialectología y Tradiciones Populares 54: 207-37. [CrossRef] 
Herrin, Judith. 2005. The icon Corner in Medieval Byzantium. In Household, Women and Christianities in Late Antiquity and the Middle Ages. Edited by Anneke B. Mulder-Bakker. Turnhout: Brepols, pp. 71-90.

Hillgarth, Jocelyn N. 1991. Readers and Books in Majorca, 1229-1550. Paris: Éditions du CNSR.

Iglesias, Josep Antoni. 1996. Llibres i Lectors a la Barcelona del Segle XV. Les Biblioteques de Clergues, Juristes, Metges i Altres Ciutadans a Través de la Documentació Notarial (Anys 1396-1475). Ph.D. thesis, Universitat Autònoma de Barcelona, Bellaterra, Spain.

Ippel, Iris. 2014. A Christmas Crib as a Meek Heart of the Late Mediaeval Christian. The Rijksmuseum Bulletin 62: 330-47.

Jørgensen, Hans Henric Lohfert, Henning Laugerud, and Laura Katrine Skinnebach, eds. 2015. The Saturated Sensorium: Principles of Perception and Mediation in the Middle Ages. Aarhus: Aarhus University Press.

Kitzinger, Ernst. 1954. The cult of images in the age before iconoclasm. Dumbarton Oaks Papers 8: 83-150. [CrossRef]

Klein, Holger A. 2010. Sacred Things and Holy Bodies. Collecting Relics from Late Antiquity to the Early Renaissance. In Treasures of Heaven: Saints, Relics, and Devotion in Medieval Europe. Edited by Holger A. Klein, C. Griffith Mann and James Robinson. Exhibition of the The Cleveland Museum of Art and the Walters Art Museum, Baltimore. New Haven: Yale University Press, pp. 55-67.

Kolpacoff Deane, Jennifer. 2013. Medieval Domestic Devotion. History Compass 11: 65-75. [CrossRef]

Laugerud, Henning, Salvador Ryan, and Laura Skinnebach, eds. 2016. The Materiality of Devotion in Late Medieval Northern Europe: Images, Objects and Practices. Dublin: Four Courts, European Network on the Instruments of Devotion 3.

Le Pogam, Pierre, and Christine Vivet-Peclet. 2009. Les Premiers Retables: XIIe-Début du XVe Siècle: Une Mise en scène du Sacré. Paris: Musée du Louvre Editions.

Lencina Pérez, Xavier. 1998. Espais i objectes quotidians. Els inventaris post mortem catalans en el context Europeu. Pedralbes. Revista d'història Moderna 18: 303-10.

Llompart, Gabriel. 1966. Dos notas de folklore religioso levantino: Evangelios de bautizo y peregrinos de representación. Revista de Dialectología y Tradiciones Populares 22: 7-25.

Llompart, Gabriel. 1980. Imágenes mallorquinas exentas del Niño Jesús. Boletín del Seminario de Estudios de Arte y Arqueología 46: 364-75.

López Pizcueta, Tomás. 1992. Los bienes de un farmacéutico barcelonés del siglo XIV: Francesc de Camp. Acta Mediaevalia 13: 17-73.

Madurell i Marimon, Josep Maria. 1974. Manuscrits en Català Anteriors a la Impremta (1321-1474): Contribució al seu Estudi. Barcelona: Associació Nacional de Bibliotecaris.

Manzarbeitia Valle, Santiago. 2009. San Cristóbal. Revista Digital de Iconografía Medieval 1: 43-49.

Martínez Ferrando, Jesús Ernesto. 1953-1954. La cámara Real en el reinado de Jaime II (1291-1327). Relación de entradas y salidas de objetos artísticos. Anales y Boletín de los Museos de Arte de Barcelona 11: 1-230.

Martorell, F., and F. Valls i Taverner. 1911-1912. Pere Beçet. Anuari de l'Institut d'Estudis Catalans 4: 577-656.

Molina, Joan. 1999. Arte, Devoción y Poder en la Pintura Tardogótica Catalana. Murcia: Universidad de Murcia.

Moliné, Ernest. 1912. Inventari y encant d'una especieria cerverina del segle XIV. Boletín de la Real Academia de Buenas Letras de Barcelona 6: 195-207.

Moliné, Ernest. 1922a. 1432. Inventari y encant dels béns d'un notari barceloní". Butlletí de la Reial Acadèmia de Bones Lletres de Catalunya 10: 277-85.

Moliné, Ernest. 1922b. 1432. Inventari y encant dels béns d'un notari barceloní (acabament)". Butlletí de la Reial Acadèmia de Bones Lletres de Catalunya 10: 425-27.

Morse, Margaret A. 2007. Creating Sacred Space: The Religious Visual Culture of the Renaissance Venetian Casa. Renaissance Studies 21: 151-84. [CrossRef]

Musacchio, Jacqueline Marie. 2005. Lambs, Coral, Teeth and the Intimate Intersection of Religion and Magic in Renaissance Italy. In Images, Relics and Devotional Practices in Medieval and Renaissance Italy. Edited by Sally J. Cornelison and Scott Montgomery. Tempe: Arizona Center for Medieval and Renaissance Studies, pp. 139-56.

Nichols, Stephen G., Alison Kablitz, and Andreas Calhoun. 2008. Rethinking the Medieval Senses: Heritage, Fascinations, Frames. Baltimore: The Johns Hopkins University Press. 
Ondoño Rendón, Marcela. 2015. La imagen de una oración prohibida: el culto supersticioso en torno a San Cristóbal. Studia Aurea. Revista de Literatura Española y Teoría Literaria del Renacimiento y Siglo de Oro 9: 361-90. [CrossRef]

Ortí i Gost, Pere. 1994. La casa i les possessions d'un jurista barceloní segons el seu inventari de béns: Burget de Sarrià (1338). Miscel-lània de Textos Medievals 7: 323-69.

Palazzo, Éric, ed. 2014. L'invention Chrétienne des Cinq Sens. Paris: Éditions du Cerf.

Palazzo, Éric, ed. 2016. Les Cinq Sens au Moyen Age. Paris: Éditions du Cerf.

Panofsky, Erwin. 1997. Imago Pietatis. Contribution à l’histoire des types du «Christ de Pitié»/«Homme de Douleurs» et de la «Maria Mediatrix». In Peinture et Dévotion en Europe du Nord à la fin du Moyen Âge. París: Flammarion, pp. 13-28.

Plana i Borràs, Josep. 1989. Inventari dels béns de Bernardó Benet. In Homenatge a la Memòria del Professor Dr. Emilio Sáez. Barcelona: Universitat de Barcelona-Centre d’Estudis Medievals de Catalunya-Institució Milà i Fontanals (CSIC), pp. 151-58.

Rigaux, Dominique. 1996. Une image pour la route. L'iconographie de saint Christophe dans les régions alpines (XIIe-XVe siècle). In Actes des Congrès de la Société des Historiens Médiévistes de L'enseignement Supérieur Public. Rennes: Société des Historiens Médiévistes de L'enseignement Supérieur Public, pp. 235-66.

Rigon, Antonio, ed. 2001. Religione Domestica: Medioevo, età Moderna. Quaderni di storia religiosa, 8. Caselle di Sommacampagna. Verona: Cierre.

Ringbom, Sixten. 1995. Les Images de Dévotion (XIIè-XVè Siècle). Paris: Monfort.

Ringbom, Sixten. 1997. De l'icône à la Scène Narrative. Paris: Montfort.

Robinson, James. 2011. From Altar to Amulet. Relics, Portability and Devotion. In Treasures of Heaven. Saints, Relics, and Devotion in Medieval Europe. Edited by Holger A. Klein, C. Griffith Mann and James Robinson. Exhibition of the The Cleveland Museum of Art and the Walters Art Museum, Baltimore. New Haven: Yale University Press, pp. 110-16.

Roca, Joseph M. 1923. Un cirugià barber barcelonès de la XV. centuria. Boletín de la Real Academia de Buenas Letras 11: 145-62.

Roca, Joseph M. 1927-1928a. Inventaris I. Butlletí de la Reial Acadèmia de Bones Lletres de Catalunya 13: 306-16.

Roca, Joseph M. 1927-1928b. Inventaris II. Butlletí de la Reial Acadèmia de Bones Lletres de Catalunya 13: 384-95.

Roca, Joseph M. 1930a. Inventaris III. Butlletí de la Reial Acadèmia de Bones Lletres de Catalunya 14: 244-58.

Roca, Joseph M. 1930b. Inventaris IV. Butlletí de la Reial Acadèmia de Bones Lletres de Catalunya 14: 384-89.

Rubin, Miri. 2009. Emotion and Devotion: The Meaning of Mary in Medieval Religious Cultures. Budapest: Central European University Press.

Russo, Daniel. 1996. Les fonctions dévotionnelles de l'image religieuse dans l'Italie médiévale. In L'image. Fonctions et Usages des Images dans l'Occident Médiéval. Cahiers du Léopard d'Or, Vol. 5. Paris: Le Léopard d'Or, pp. 133-53.

Sabaté, Flocel. 1990. Els objectes de la vida quotidiana a les llars barcelonines al començament del segle XIV. Anuario de Estudios Medievales 20: 53-108.

Sànchez-Boira, Imma. 2007. L'interior de les cases dels eclesiàstics de Lleida. Vida quotidiana, cultura i religiositat". In Arrels cristianes. Vol. III. Temps D'expansió. Temps de Crisi, Temps de Redefinició. L'època Moderna. Segles XVI-XVIII. Lleida: Universitat de Lleida and Pagès Editors, pp. 191-204.

Sarnecka, Zuzanna. 2019. 'And the Word Dwelt amongst Us': Experiencing the Nativity in the Italian Renaissance Home. In Domestic Devotions in Early Modern Italy. Edited by Maya Corry, Marco Faini and Alessia Meneghin. Leiden and Boston: Brill, pp. 163-84.

Sastre Moll, Jaume. 2003. L'obra pictòrica com element decoratiu, sumptuari i devocional a les llars medievals mallorquines, en el trànsit a la modernitat. Bolletí de la Societat Arqueològica Lul-liana 5: 47-88.

Saura, Mercè. 2008. Jaume Huguet. Santa Maria Magdalena. In La Col-lecció. Barcelona: Fundación Francisco Godia, pp. 100-1.

Schmidt, Victor M. 2001. Painting and Individual Devotion in Late Medieval Italy: The Case of St. Catherine of Alexandria. In Visions of Holiness: Art and Devotion in Italy. Edited by Andrew Ladis and Shelley Zuraw. Athens: Ga.

Schmidt, Victor M. 2005. Painted Piety. Panel Paintings for Personal Devotion in Tuscany 1250-1400. Florence: Centro Di. 
Serra i Boldú, Valeri. 1925. Llibre d'or del Rosari a Catalunya: Historia-Etnografia-Folklore-Arqueologia-Imatgeria-Bibliografia. Barcelona: Oliva de Vilanova.

Siddi, Federica. 2012. Le rapprezantazioni scolpite di Gesù Bambino. In Il Misterio Goioso. Il Presepe di Greccio et le Sculture del Gesú Bambino Benedicente. Edited by Chiara Frugoni and Federica Siddi. Siena Complesso murale Santa Maria della Scala, 21. dicembre 2012, 28 febbraio 2013. Monteriggioni: Il Leccio, pp. 35-41.

Smith, Julia H. 2014. Material Christianity in the early Medieval Household. In Religion and the Household. Studies in Church History 50. Woodbridge: The Boydell Press, pp. 23-46.

Sobrado Correa, Hortensio. 2003. Los inventarios post-mortem como fuente privilegiada para el estudio de la historia de la cultura material en la Edad Moderna. Hispania 63: 825-61. [CrossRef]

Soler i Palet, Josep. 1916a. L'art a la casa del segle XV. núm. 61 (gener-març). Butlletí de la Reial Acadèmia de Bones Lletres de Catalunya 8: 289-305.

Soler i Palet, Josep. 1916b. L'art a la casa del segle XV. núm. 62 (abril-juny). Butlletí de la Reial Acadèmia de Bones Lletres de Cataluny 8: 385-94.

Spicer, Andrew, and Sarah Hamilton. 2005. Defining the Holy: Sacred Space in Medieval and Early Modern Europe. Aldershot: Ashgate.

Sureda i Jubany, Marc. 2017. From holy images to liturgical devices: Models, objects and rituals around the Veronicae of Christ and Mary in the Crown of Aragon (1300-1550). Convivium 4: 194-217.

Tanner, Norman, and Sethina Watson. 2006. Least of the laity: The minimum requirements for a medieval Chistian. Journal of Medieval History 32: 395-423. [CrossRef]

Terés, Rosa. 1998. Art profà i vida quotidiana entorn 1400: Els inventaris barcelonins. Acta Mediaevalia 19: $295-317$.

Terés, Rosa. 2005. Grup del pessebre. In Pedralbes: Els Tresors del Monestir. Barcelona: Ajuntament de Barcelona, pp. 65-67.

Thomson, S. C. 2018. Grotesque, Fascinating, Transformative: The Power of a Strange Face in the Story of Saint Christopher. Essays in Medieval Studies 34: 83-98. [CrossRef]

Treasures of Heaven. 2010. Treasures of Heaven: Saints, Relics, and Devotion in Medieval Europe. Edited by Holger A. Klein, C. Griffith Mann and James Robinson. Exhibition of the The Cleveland Museum of Art and the Walters Art Museum, Baltimore. New Haven: Yale University Press.

Van Os, Henk. 1994. The Art of Devotion in the Late Middle Ages in Europe: 1300-1500. London: Merrell Holberton, Amsterdam: Rijksmuseum Amsterdam.

Vauchez, André. 2006. La Spiritualità dell'Occidente Medioevale. Milano: Vita e Pensiero. First published 1975.

Vauchez, André. 1993. The Laity in the Middle Ages. Religious Beliefs and Devotional Practices. Notre Dame: University of Notre Dame.

Vauchez, André. 2003. Esperienze Religiose nel Medioevo. Roma: Viella.

Vauchez, André. 2005. Sainthood in the Later Middle Ages. Cambridge: Cambridge University Press.

Vercher Lletí, Salvador. 2005. L'habitatge: La Casa i L'interior Domèstic. Guia Bibliográfica. Corbera: Ajuntament de Corbera, Assemblea d'Història de la Ribera.

Vicens, Teresa. 2003. Els goigs marians: un programa iconogràfic del gòtic català. Butlletí del Museu Nacional d'Art de Catalunya 7: 25-50.

Vinyoles, Teresa M. 1976. La casa i l'obrador d'un esmolet de Barcelona a finals del segle XV. Cuadernos de Historia Económica de Catalunya 15: 9-50.

Vinyoles, Teresa M. 2015. El espacio doméstico y los objetos cotidianos en la Cataluña medieval. In La Casa Medieval en la Península Ibérica. Edited by M. Elena Díez and Julio Navarro. Madrid: Sílex, pp. 613-51.

Voulgaropoulou, Margarita. 2019. From Domestic Devotion to the Church Altar: Venerating Icons in the Late Medieval and Early Modern Adriatic. Religions 10: 390. [CrossRef]

Webb, Diana. 1990. Woman and Home: The Domestic Setting of Late Medieval Spirituality. Studies in Church History 27: 159-73. [CrossRef]

Webb, Diana. 2005. Domestic Space and Devotion in the Middle Ages. In Defining the Holy Sacred Space in Medieval and Early Modern Europe. Aldershot: Ashgate, pp. 27-47.

Wilkins, David G. 2002. Opening the Doors to Devotion. Trecento Triptychs and Suggestions concerning Images and Domestic Practice in Florence. In Italian Panel Painting of the Duecento and Trecento. Edited by Victor M. Schmidt. New Haven: Yale University Press, pp. 371-94.

Winston-Allen, A. 1998. Stories of the Rose: The Making of the Rosary in the Middle Ages. University Park: Pennsylvania State University Press. 
Wirth, Jean. 1989. L'image Médiévale: Naissance et Développements, VIe-XVe siècle. Paris: Librairie des Méridiens Klincksieck.

Yarza, Joaquín. 1992. Clientes, promotores y mecenas en el arte occidental hispano. In Patronos, Promotores, Mecenas y Clientes. Actas del VII CEHA 1988. Murcia: Universidad de Murcia, pp. 15-47.

(c)

(C) 2019 by the author. Licensee MDPI, Basel, Switzerland. This article is an open access article distributed under the terms and conditions of the Creative Commons Attribution (CC BY) license (http://creativecommons.org/licenses/by/4.0/). 


\title{
Article \\ Performance, Object, and Private Devotion: The Illumination of Thomas Butler's Books of Hours
}

\author{
Karen Ralph \\ Department of Art History, New York University, 75005 Paris, France; kr68@nyu.edu \\ Received: 3 December 2019; Accepted: 20 December 2019; Published: 31 December 2019
}

\begin{abstract}
This article considers the major cycles of illumination in two Books of Hours belonging to Thomas Butler, seventh Earl of Ormond (c.1424-1515). The article concludes that the iconography of the two manuscripts reflects the personal and familial piety of the patron and was designed to act as a tool in the practice of devotion.
\end{abstract}

Keywords: private devotion; Books of Hours; illumination; Holy Trinity; pictorial cycles; Butlers; Ormond

Item I will that my sawter boke covered with whyte lether and my name writtin with myn owne hand in thende of the same wych is at my lodging in London shalbe layd and fyxed with a cheyne of iron at my tombe wych is ordeyned for me in the $[\ldots]$ church of Saint Thomas Acon there to remayne for the servyce of God in the said church the better to be hadde and done by suche persones as shalbe disposed to occupye and loke upon the same boke (will of Thomas Butler 299-300).

Shortly before his death, Sir Thomas Butler, seventh Earl of Ormond (c.1424-1515), prepared his last will and testament including the above stipulation that his Psalter bearing his name be chained to his tomb in a public demonstration of his personal piety. While the Psalter itself has not survived, the provisions of his will present an image of Butler as an active and conscientious participant in both private devotion and his own commemoration, and his surviving Books of Hours offer a personal reflection of his piety and demonstrate the importance of the material object in his practice of devotion.

Sir Thomas Butler, seventh Earl of Ormond (c.1424-1515), was the son of James Butler, fourth Earl of Ormond (the White Earl), and his wife Joan, daughter of William Beauchamp, Lord Abergavenny. Though born in Ireland, Butler spent most of his life in England and became a significant landholder there from the time of his marriage to Anne Hankeford in $1445 .{ }^{1}$ Butler had two daughters from his first marriage, Anne and Margaret, the latter of whom was mother to Thomas Boleyn, first Earl of Wiltshire and father of Anne Boleyn. Between 1495 and 1497, he married the twice widowed Lora Berkeley with whom he had a daughter, Elizabeth. Support for the Lancastrian cause and the attempt of his brother, John, the sixth Earl, to raise a Lancastrian revolt in Ireland saw Butler attainted twice and go into exile in the court of Margaret of Anjou in the duchy of Bar. Upon the death of his brother in 1477, Butler acceded to his titles and estates and was restored his English lands in November 1485. Butler attended the coronation of Richard III in 1483 where he was knighted, held positions on the privy councils of both Richard III and Henry VII, appointed lord chamberlain to Catherine of Aragon in 1509, acted as Ambassador to Brittany and Burgundy in 1491 and 1497 respectively, and is known to have been in France in the retinue of Henry VII in 1500 (Calendar of Ormond Deeds 1935, nos. 211 and 247; Beresford 1999; Cokayne 1945, pp. 131-32; Crawford 2002, p. 151; Fortescue 1869,

1 Thomas Butler and Anne Hankeford were married sometime before the 18 July 1445 when Butler sued for seisin of her lands, see Calendar of Patent Rolls, 261. 
vol. I p. 24; Sutton and Hammond 1983, p. 379). Butler died in August 1515 and was buried in the Church of St Thomas of Acre in London. At the time of his death, Butler was in possession of some seventy-two manors and diverse others lands in England, divided equally between his daughters Anne and Margaret, in addition to the Ormond lands and manors in Ireland which came under the control of a relative, Piers Butler, eighth Earl (Lodge 1754, vol. II p. 13).

I bequeth and recomend my soule unto allmyghty my maker and redemerto the moost glorius virgyn his mother our Lady Saint Mary and to the glorius martyr Saint Thomas and to all the holy colege of saintys in hevyn. And my body to be buryed in the churche of Saint Thomas Acon in London that is to wytt uppon the north syde of the hygh aulter if the high aulter in the said church where the sepulture of all mighty God is used yerely to be sett on Good Friday to thentent that by the merytes of his most precyous passion and glorius resurreccion [ ... ] I wyll ther be ordeyned and sett an epitahafe makyng memyery of me and the day and yere of my decesse. And this to be doon by the distrecyons of myn executors not for any pompe of the worled but only for a rememberance (will of Thomas Butler 298).

The instructions left by Sir Thomas Butler in his will are quite revealing in terms of his concern for action and outcome. First having already 'ordeyned' his tomb to lie in St Thomas of Acre, it seems that the seventh Earl was concerned with tangible results and that the commemorative arrangements that he had negotiated were followed through. Butler stipulates that should he die on his Essex estate or anywhere within forty miles of London that his body should be transferred to St Thomas of Acre, but he also stipulates that should he die further afield that he should be interred 'in holy sepulture wher it shall please allmyghty God to dispose of [him]' (will of Thomas Butler 298).

The Church of St Thomas of Acre was established on the site of birthplace of the martyred St Thomas Becket, archbishop of Canterbury, in the parish of St. Mary Colechurch, by an order of hospitaller knights in 1227-1228. The church became a popular burial place for Londoners wishing to associate themselves with the martyr and from the thirteenth century onwards it was accorded various grants, donations, and chantries (see Keene and Harding 1987; Steer 2013; Sutton 2008). The association of the Earls of Ormond and the Church of St Thomas of Acre dates at least to the White Earl who became an important patron of the hospital due to his supposed familial ties with St Thomas and also seemingly a political alliance and business relationship with John Neel, Master of the Hospital (Matthew 1994, pp. 258n, 263, 279n, 406; Sutton 2008, pp. 202-5). The White Earl's second wife, Joan, was interred there on her death in 1430 in the chapel of the Holy Cross. Her tomb was likely of considerable grandeur given it was included in the heraldic account of c.1504 and in John Stow's Survey of London in 1603 (Calendar of Wills 1890, p. 506; Stow 1908, vol. I p. 269). The association with Becket was later developed in 1454 when James, Earl of Wiltshire and Ormond, claimed a blood relationship with St Thomas during a petition to Parliament and also offered the estate of Hulcott in Buckinghamshire to the hospital in order to endow a chantry for the chapel in which his mother was buried (Rotuli Parliamentorum 1767-1777, pp. 257, 491-95; Watney 1906, p. 138). This belief in kinship is thought to have arisen from one of two potential misidentifications. One source of confusion could be that between Archbishop Becket and Archbishop Hubert Walter who was the brother of Theobald, the first Walter or Butler in Ireland. The second suggestion is that Theobald Butler as Lord of Hely (Ely-O'Carroll) came to be confused with Theobald of Helles, nephew of St Thomas (Brooks 1956, p. 26; Carte 1851, vol. I pp. xxxiv-xxxvi; Keene and Harding 1987, pp. 490-517). Both the Ormond interest in the hospital church, as well as some of its traditional roles, continued past the dissolution of the monasteries. The will of James, ninth Earl of Ormond (+1546), requested he be buried at 'St Thomas of Acres, somtyme so called, with others therles of Ormond' and subsequently his son, Thomas, tenth Earl (†1614), in his will dated 1576 specified that if he were to die 'within the realm of England then [his] body be buried in [his] father's tomb at St Thomas of Dacres in London.' Both men were ultimately interred along with their ancestors in the chancel of St Canice's Cathedral in Kilkenny (Calendar of Ormond Deeds, vol IV no. 352 and vol V no. 288). 
Precise instructions are also provided in Butler's will for his commemoration. Butler provided for an obit for himself, his two wives, his parents, and his grandmother, Dame Jane Beauchamp, to be performed annually for seven years on the anniversary of his death. Furthermore, he adapted his parents' annual chantry to include himself and his wives requesting that the names be 'reherce[d]' during the sermons. Butler requests that the tomb itself, be it in St Thomas of Acre or elsewhere, be situated next to the Easter sepulchre and that it bear an epitaph to record his memory and leaves funds for 'torches and other lyghts and apparell' to be brought to his tomb. The epitaph was later recorded by John Weever in his eighteenth-century account of Ancient Funeral Monuments (will of Thomas Butler 298-99; Weever 1767, p. 187). ${ }^{2}$ The appearance and commemorative nature of the tomb was obviously of some significance for Butler as it was relatively unusual for London testators to refer to the physical appearance of their tombs (Harding 1992; Steer 2013, pp. 159-69). Chaining his Psalter in which he had personally written his name to the tomb also ensured a physical and spiritual connection between individual, memory, and prayer: each time the Psalter was used the reader was encouraged to keep Butler in their prayers.

The various elements of Butler's commemoration carry an expectation of performance-physical acts-to assist in and inspire commemoration and ultimately the salvation of the souls of Butler and his wives: 'convey my body', bring the lights, 'reherce' the names, 'loke upon the [ ... ] boke', keep in their prayers and masses. This same relationship between the public life of the material object, physical performance and commemorative or devotional response is also demonstrated in the more private aspects of Butler's devotion and illumination of the two extant Books of Hours, known to have been associated with the Ormonds, mostly likely with Thomas Butler.

\section{The Butler Books of Hours}

Butler was responsible for the purchase and refurbishment of one, the Hours of the Earls of Ormond, London, British Library Harley MS 2887 [Harley], and the commissioning of a second Book of Hours, London, British Library Royal MS 2 B XV, which shall be referred to as 'the Hours of Thomas Butler' [Royal]. While Books of Hours, English manuscripts included, have been the subject of a number of publications in recent years (Duffy 2011; Reinburg 2012; Hindman and Marrow 2013; Morgan 2013; Kennedy 2014), the Butler manuscripts have received very little scholarly attention. Where the manuscripts have been the subject of discussion (Scott 1996), the focus has fallen on the inferior quality of the execution or peculiarity of the iconographical choices, rather than the creativity of the iconography, the motivation for and function of the illumination, and relationship of the manuscripts to the reader and devotional practice.

Today the Harley manuscript measures $27.5 \times 18.5 \mathrm{~cm}$ in size and is illuminated with five full-page miniatures plus one incomplete, one small miniature, seven full pages offering pictorial cycles, sixteen historiated initials, seven fully bordered pages, and ten pages bordered on three sides. Marginal notes on f.1v of the Harley manuscript record three sets of births in 1467 and 1470, those of Angneta, George, John and Annas Gower of Crooked Lane in St Michael's Parish. This suggests that the likely original owner was one Edward Gower of London. Of interest here however are only the English, or perhaps French-inspired, miniatures which were added considerably later: ff. $6 \mathrm{v}, 8 \mathrm{v}, 27 \mathrm{v}, 28 \mathrm{v}, 33 \mathrm{v}$ and $55 \mathrm{v}-58 \mathrm{v}$. A further miniature, that on $\mathrm{f} .3 \mathrm{v}$, was also a later addition but is considerably different from the others. Dutch in style, the image is associated with a group of artists known as the 'Master of the Black Eyes' and was likely imported along with the two folia directly following it (Scott 1996, vol. II pp. 299-302; Scott 1968, pp. 172, 176). One of the inserted folia, 28v, bears the blue and gold shield of the Butler family and the device is also to be found on $\mathrm{f} .15 \mathrm{v}$ of the Hours of Thomas Butler (Figure 1). The coat of arms is inserted as a preface to Matins in the Hours of the Virgin in both manuscripts. In her assessment

2 'Hic iacet Thomas filius Jacobi, comitis Ormundie, ac fratris Jacobi, comitis Wilts \& Ormundie, qui quidem Thomas obijt fecundo die $1515, \mathcal{E}$ anno regni regis Henrici octaui 37.' 
of self-representation in late medieval art Alexa Sand notes that the insertion of the patron's self-image at the invocation of Matins, 'O Lord, open my mouth', as in both the Hours of the Earls of Ormond and the Hours of Thomas Butler, ${ }^{3}$ rather than the opening of a penitential text, 'O Lord, hear my prayer' as in Psalm 101, carries an important emphasis on the 'supplicant's actions over God's perceptions' (Sand 2014, pp. 163-64). Associating the Butler arms with an active rather than passive appeal invites the reader to act upon seeing their self-image reflected on the page and to utter the words of the prayer.

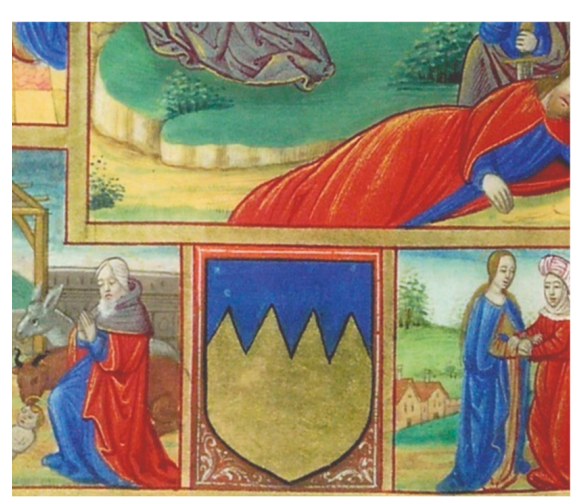

(a)

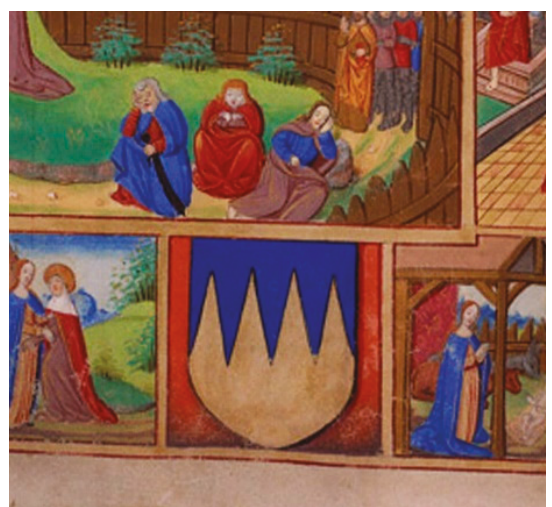

(b)

Figure 1. (a) Butler arms, The Hours of the Earls of Ormond, London, British Library Harley MS 2887, f.28v, (b) Butler arms, The Hours of Thomas Butler, London, British Library Royal MS 2 B XV, f.15v, (C) British Library Board.

The Royal manuscript measures $33 \times 24 \mathrm{~cm}$ in size and is illuminated with six full-page miniatures some with floral borders, seven half-page miniatures, four full pages offering pictorial cycles, nineteen historiated initials, eight small miniatures and marginal scenes, a space left for an image, twenty-three fully bordered pages, two pages bordered on three sides, and extensive pen flourishing throughout. This represents a relatively lavish production for an English Book of Hours of the fifteenth century. Nigel Morgan has highlighted that few high-quality illuminated manuscripts were produced after c.1430 due to the number of imported Flemish manuscripts that flooded the market (Morgan 2013, p. 70). This perhaps suggests that, in this case at least, the patron was concerned with the personalisation of his manuscript and closely involved with its production, rather than simply importing a Book of Hours. The obits of numerous members of the Butler family appear on f.2r-v: James, the fifth Earl of Ormond and first Earl of Wiltshire (†1461); Avice, his wife (†1457); Joan, first wife of James, the fourth Earl (†1452); Elizabeth, Countess of Shrewsbury and sister of the fifth, sixth, and seventh Earls of Ormond ( +1473$)$; John, the sixth Earl (†1477); Anne, first wife of the seventh Earl (†1485); Joan Abergavenny, grandmother of the fifth, sixth, and seventh Earls (†1435); and finally Lora, second wife of the seventh Earl ( + c.1501). The positioning of the obit for Countess Lora at the end of the list but incorporated into the calendar of the Royal manuscript suggests that the Harley manuscript is the earlier of the two. The Royal manuscript also bears a largely erased early sixteenth-century inscription on ff. 3-4 which entreats the reader to pray for the souls of Willelmi Froste and his wife, Juliane, who donated the book to the chapel of the Blessed Mary of Suthwycke [Southwick]. A William Frost was named as a trustee for property belonging to the seventh Earl in a patent livery of the 12th of

3 'Domine labia mea aperies' on Harley f.29r and Royal f.16r. 
December 1515 (Letters and Papers 1864, vol II p. 340; Warner and Gilson 1929, vol. I p. 49). ${ }^{4}$ The list of obits including the seventh Earl's wives, brothers, sister, mother and grandmother, but none for himself, along with the manuscript passing into the hands of one of his trustees suggests that the manuscript was commissioned for Thomas Butler rather than another member of his family. This is further supported by the original inclusion of an image, suffrage, and obit of his patron St Thomas Becket, later effaced from the manuscript (ff. $29 \mathrm{v}, 65 \mathrm{r}$, and $79 \mathrm{v}$ ), and arguably also by the miniature of a male figure kneeling in prayer with his guardian angel at his shoulder at the beginning of prayer to one's guardian angel on f.66v (Figure 2).

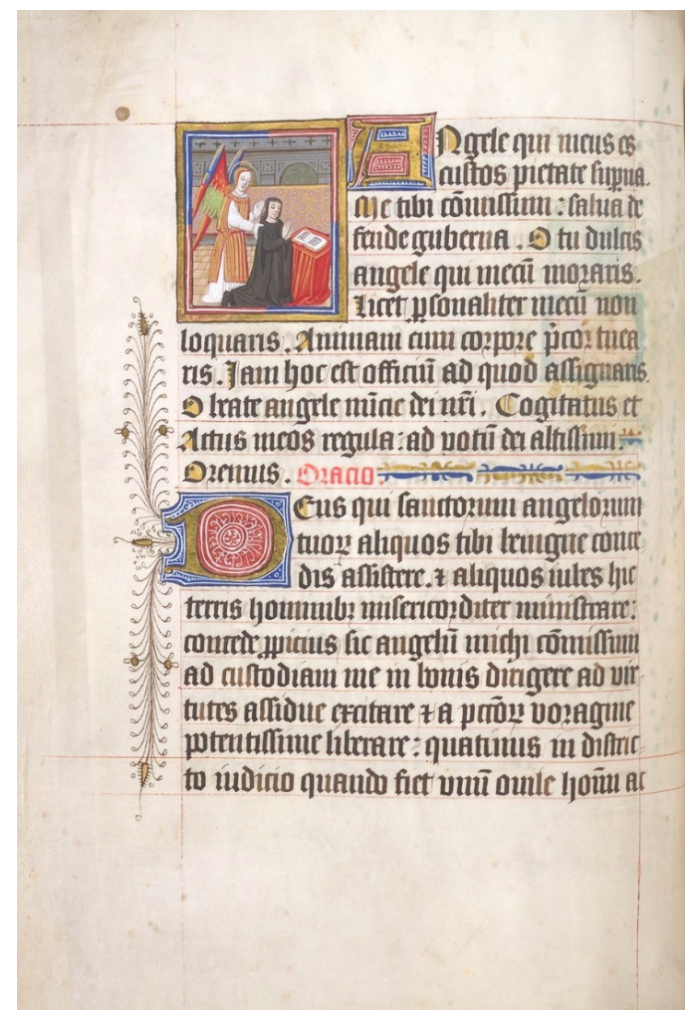

Figure 2. Miniature ahead of the prayer to one's guardian angel in the Hours of Thomas Butler, f.66v, (C) British Library Board.

In addition to the Psalter to be chained to his tomb, Butler's will refers to a further two books in his possession. The first, 'myn olde sawlter' which he keeps at Awdere, county Devon he bequeaths to the parish church of Monkeley. The second, a 'masse boke covered with russett velvett' he bequeaths to his daughter, Anne, along with a cloak embroidered in gold belonging to his wife (will of Thomas Butler 299-300). Considering the extent of the wealth and cultural capital available to him, these bequests and the surviving manuscripts believed to have been associated with him presumably represent only a portion of his library, which likely would have also included the more fashionable French and Flemish books. His would not have been the only library with multiple copies of the Book of

4 'Orate pro animabus Willelmi Froste et Juliane uxoris eius, qui hunc librum dederunt remanere imperpetuum ad orandum beate Marie in ista capella de Suthwycke', ff. 3-4, transcription from (Warner and Gilson 1929, vol. I p. 49). 
Hours as Eamon Duffy has noted that most gentry households would have had more than one copy (Duffy 2011, p. 58). Butler may also have been the 'certaine honourable auncient person [ ... ] the honnorable Erle of Ormond' who provided material for the first English biography of Henry V, written in the sixteenth century. In his enthusiasm for manuscripts, Butler was following in a family tradition as his father, the White Earl, had commissioned manuscripts in both English and Irish. In 1420 the White Earl commissioned a translation 'out of Latin and French into your mother tongue, English' of The Governaunce of Prynces, a new version of the treatise known as the Secreta Secretorum from Dublin notary James Yonge. He also commissioned an illuminated miscellany in Irish and Latin which is today known as the Book of the White Earl. Butler's cousin, Edmund MacRichard, likewise commissioned the Book of Pottlerath, an illuminated miscellany in Irish and in doing so incorporated the White Earl's manuscript into his own. John, the sixth earl, was also a keen scholar and is described by Carte as 'a perfect master of all the languages of Europe' (Allmand 1992, pp. 432-33; Carte 1851, vol. I, p. lxxxi; Kerns 2008, p. 1; Kingsford 1911, pp. 3 and xvi-xx; Matthew 1994, p. 119; Ralph 2014; Wylie and Waugh 1929, vol. III pp. 445-48). His grandmother, Joan Beauchamp, was also associated with the Tewkesbury Psalter in which her obit appears, and it seems that the manuscript was bequeathed from mother-in-law to daughter-in-law at least twice (Egbert 1935, pp. 377 and 381).

The ornamentation of Books of Hours dating to the later period generally followed a reasonably fixed scheme of standard images at the standard textual divisions or standard accompanying texts. When groups of miniatures appeared, perhaps as a double opening or as a series of scenes separated by short tracts of text, they were usually positioned as a prefatory cycle or more rarely at the major textual divisions (Morgan 2013, p. 71; Scott 1996, vol. I pp. 38 and 56). Both of Thomas Butler's Books of Hours, on the other hand, include two series of full page miniatures: the first a series of pictorial cycles which are repeated later in the manuscripts with almost identical compositions but the order reorganised, and the second a series of images dedicated to the Holy Trinity.

\section{The Pictorial Cycles}

In the Hours of the Earls of Ormond, six of the seven pictorial cycles are grouped together from ff. $55 \mathrm{v}-58 \mathrm{r}$ in front of the Fifteen Oes, a series of devotional prayers commemorating Christ's Passion, each of which begins with the exclamation ' $\mathrm{O}$ ' (Figures 3 and 4). English in origin, these prayers centred around the seven words Christ uttered on the Cross, His wounds, and the salvation of mankind through His sacrifice, and ultimately became some of the most popular prayers in late medieval England (Duffy 2005, pp. 249-56; Krug 1999). Each cycle follows the same physical layout in the form of the letter ' $\mathrm{I}$ ' with two scenes in the top register, one in the middle flanked by a floral design, and two on the bottom. The scenes are evenly sized and each is framed with a gold leaf border. Altogether, ten scenes appear in various combinations throughout the cycles: the Annunciation; Nativity; Presentation in the Temple; Christ Preaching in the Temple; Christ Carrying the Cross with the aid of the Good Samaritan; Crucifixion; Deposition; Ascension; Risen Christ Appearing to the Virgin; and Assumption; (see Figure 5 for the layout of each cycle). The group has blank outer leaves, suggesting that they are intended to function as a unit and the same ten scenes are repeated three times each over the course of three consecutive double pages with the order on f.55v reproduced exactly on f.57v and that on f.56r reproduced inverted on f.58r. This slow rotation through ten key scenes, all closely related to the Joys and Sorrows of the Virgin, encourages a ritualistic reading and conscientious reflection on the prayers they preface. The artist of the cycle is described by Kathleen L. Scott as Illustrator B and is identifiable with the so-called 'Placentius Master' whose work can be dated to $c .1485-1505$. This hand appears in numerous and diverse manuscripts, three of which were intended for Henry VII or his immediate family, suggesting that he was held in high regard by his contemporaries (Scott 1996, vol. II pp. 300-1 and 347). 


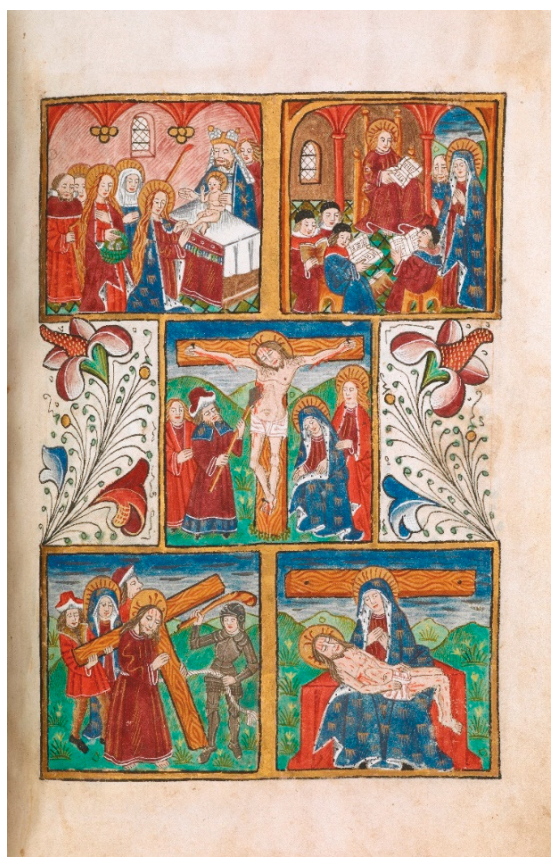

Figure 3. Pictorial cycle ahead of the Fifteen Oes in the Hours of the Earls of Ormond, f.56r, (C) British Library Board.

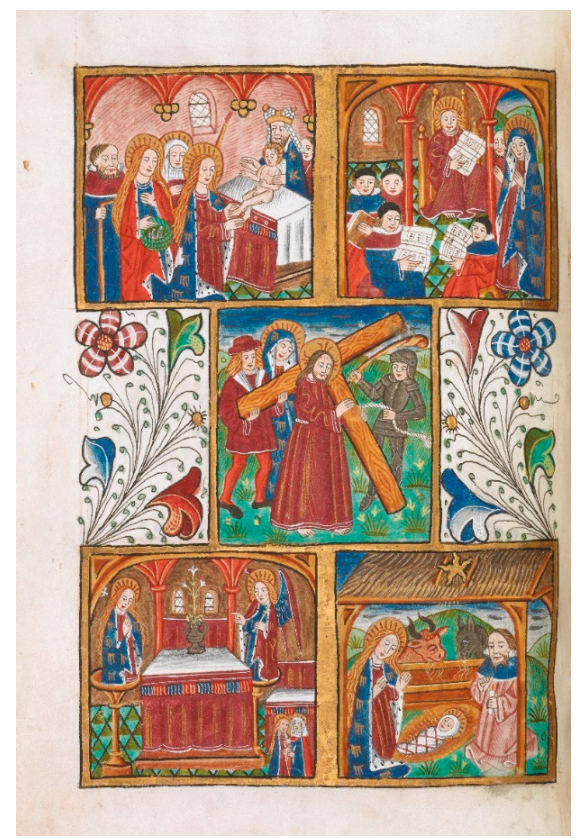

Figure 4. Pictorial cycle ahead of the Fifteen Oes in the Hours of the Earls of Ormond, f.56v, (C) British Library Board. 


\begin{tabular}{|c|c|c|c|}
\hline \multicolumn{2}{|l|}{ Assumption } & \\
\hline \multicolumn{2}{|l|}{ Ascension } & \multicolumn{2}{|l|}{ Annunciation } \\
\hline \multicolumn{2}{|c|}{ Risen Christ appearing to the Virgin } & \multicolumn{2}{|l|}{ Agony in the garden } \\
\hline Nativity & Butler arms & & Visitation \\
\hline \multicolumn{2}{|l|}{ Harley f55v } & \multicolumn{2}{|l|}{ Hader $556 \mathrm{r}$} \\
\hline $\begin{array}{l}\text { Rising from the } \\
\text { tomb }\end{array}$ & Ascension & $\begin{array}{l}\text { Presentation at } \\
\text { the temple }\end{array}$ & $\begin{array}{l}\text { Christ preaching } \\
\text { in the temple }\end{array}$ \\
\hline \multicolumn{2}{|c|}{ Assumption } & \multicolumn{2}{|c|}{ Crucifixion } \\
\hline Annunciation & Nativity & Good Samanitan & Deposition \\
\hline \multicolumn{2}{|l|}{ Haaley $556 \mathrm{r}$} & \multicolumn{2}{|l|}{ Harley $557 \mathrm{r}$} \\
\hline $\begin{array}{l}\text { Presentation at } \\
\text { the temple }\end{array}$ & $\begin{array}{l}\text { Christ preaching } \\
\text { in the temple }\end{array}$ & Ascension & Assumption \\
\hline \multicolumn{2}{|c|}{ Good Samaritan } & \multicolumn{2}{|c|}{ Rising from the tomb } \\
\hline Annunciation & Nativity & Crucifixion & Deposition \\
\hline \multicolumn{2}{|l|}{ Harley f57v } & \multicolumn{2}{|l|}{ Harley f $58 \mathrm{r}$} \\
\hline $\begin{array}{l}\text { Rising from the } \\
\text { tomb }\end{array}$ & Ascension & Good Samaritan & Deposition \\
\hline \multicolumn{2}{|c|}{ Assumption } & \multicolumn{2}{|c|}{ Crucifixion } \\
\hline Annunciation & Nativity & $\begin{array}{l}\text { Presentation at } \\
\text { the temple }\end{array}$ & $\begin{array}{l}\text { Christ preaching } \\
\text { in the temple }\end{array}$ \\
\hline
\end{tabular}

Figure 5. Layout of the pictorial cycles in the Hours of the Earls of Ormond.

The mythology surrounding the evolution of the Fifteen Oes is unclear; however, it was said that Christ revealed the Oes to a reclusive woman, sometimes said to be St Bridget of Sweden. The woman desired to know how many wounds Christ had suffered during His Passion and He responded that if she were to say fifteen Pater Nosters and fifteen Ave Marias that she would have counted and worshipped each of the wounds. In some more elaborate versions the revelation was made alongside the promise that each recitation would bring forty days of pardon and commutation of sentences to Hell and Purgatory and if they were recited every day for a year, the result would be the release from Purgatory of fifteen family members and the keeping of fifteen living family members in grace. Ultimately, Christ urged let 'every lettered man and woman read each day these orisons of my bytter passion for his sowlen medicine' (Louis 1980, pp. 264-68; Duffy 2005, p. 255; Leroquais 1927, pp. 97-99).

The legends surrounding the Oes, while offering some very enticing rewards, were also concerned with the appropriate reading of the prayers, insisting that each word be afforded due meditation and warned against the dangers of careless reading. Some introductory passages offered analogies with the permanent dulling of precious jewels as a result of careless maintenance by way of explanation. Such warnings and analogies emphasise the importance of reading the prayers rather than their recitation (Krug 1999, p. 111). The material object therefore plays a crucial role in the practice of devotion as the beholder considers the text and interacts with the folia of the manuscript. The pictorial cycles further heighten this interaction. In a world deeply immersed in religious culture and indulgences, images could trigger well known prayers such as the Pater Noster and Ave Maria with or without the presence of the text (Swanson 2007, p. 263). Acting similarly to a rosary, the cycle presents a visual anchor for each of the devotee's thirty prayers as he carefully enumerates the wounds and contemplates the suffering and sacrifice of Christ for the salvation of mankind. The devotee then arrives at, and meditates on, the Fifteen Oes themselves which relate the excruciating torment endured in vivid terms.

As in the Harley manuscript three of the four pictorial cycles in the Hours of Thomas Butler fall as a group from ff. $53 \mathrm{v}-54 \mathrm{v}$ with blanks immediately before and after (Figures $6-8$ ). The scenes are more 
numerous with twenty appearing in all and they are as follows: the Virgin and an angel flanking an altar piece depicting three saints; the Virgin and an angel flanking an altar piece of the Crucifixion; Annunciation; Visitation; Nativity; Annunciation to the Shepherds; Presentation in the Temple; Christ Preaching at the Temple; the Agony in the Garden; Betrayal; Christ Before Pilate (and the Flagellation); Christ Carrying the Cross with the aid of the Good Samaritan; Crucifixion; Deposition; Resurrection; Christ as Gardener appearing to Mary Magdalene; Risen Christ appearing to the Virgin; Ascension; Assumption; and the Coronation of the Virgin by the Trinity; (see Figure 9 for the layout of each cycle). The scenes are presented roughly chronologically beginning from the bottom of each cycle and none are repeated on facing pages thus expecting the viewer to move carefully back and forth in order to complete the cycle. Unlike the opening cycle and Harley cycles, the formatting of this grouping is less systematic with a variety of divisions and architectural frames and multiple scenes occupying the same spaces. While considerably more simplistic in execution, the mise-en-page of the cycles are reminiscent of those used in a series of pictorial cycles in fifteenth-century Parisian copies of the Defender of the Immaculate Conception of the Glorious Virgin Mary and the Golden Legend..$^{5}$ The latter is of note as two of the Golden Legend cycles also include scenes of the Trinity in Glory dressed in a collective cloak, united in their Book of Litanies, and surrounded by seraphim (in the former) and the bottom of a blue mandorla, similar to those in the Holy Trinity miniatures in the Butler manuscripts (see below). Each of the pictorial cycles in this manuscript also bears the Chourses-Coëtivy coat of arms along with the intertwined letters A and K for Antoine de Chourses and Catherine de Coëtivy (Kaplan 2016).

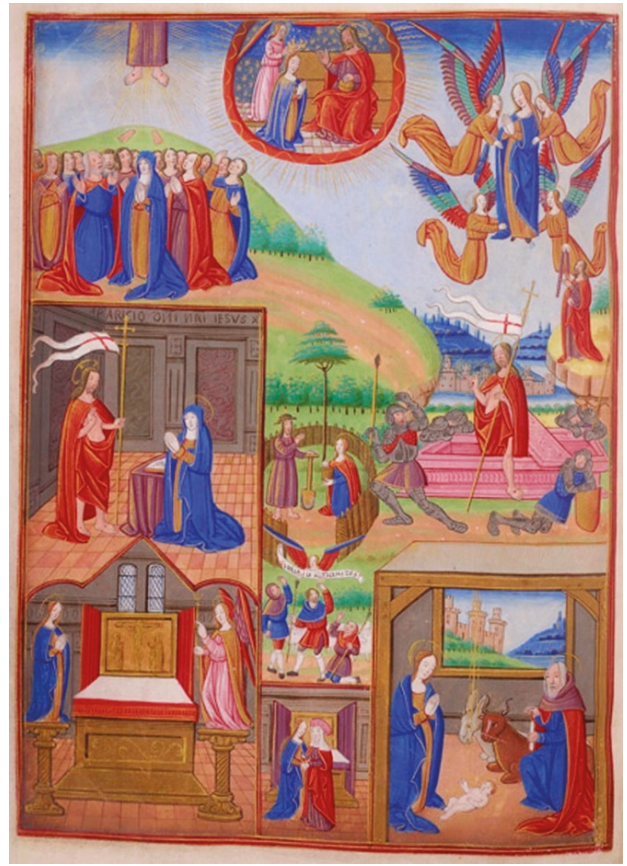

Figure 6. Pictorial cycle f.53v of the Hours of Thomas Butler, (c) British Library Board.

\footnotetext{
5 Paris, Bibliothèque Nationale de France MS Fr. 989, and Paris, Bibliothèque Nationale de France MS Fr. 244 respectively.
} 


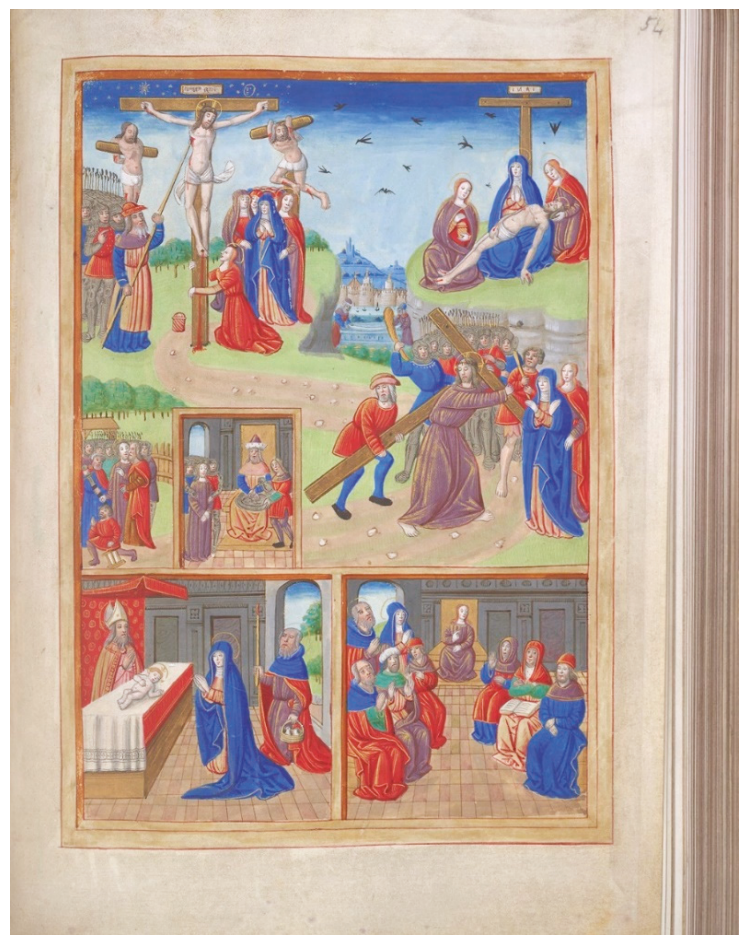

Figure 7. Pictorial cycle f.54r of the Hours of Thomas Butler, (C) British Library Board.

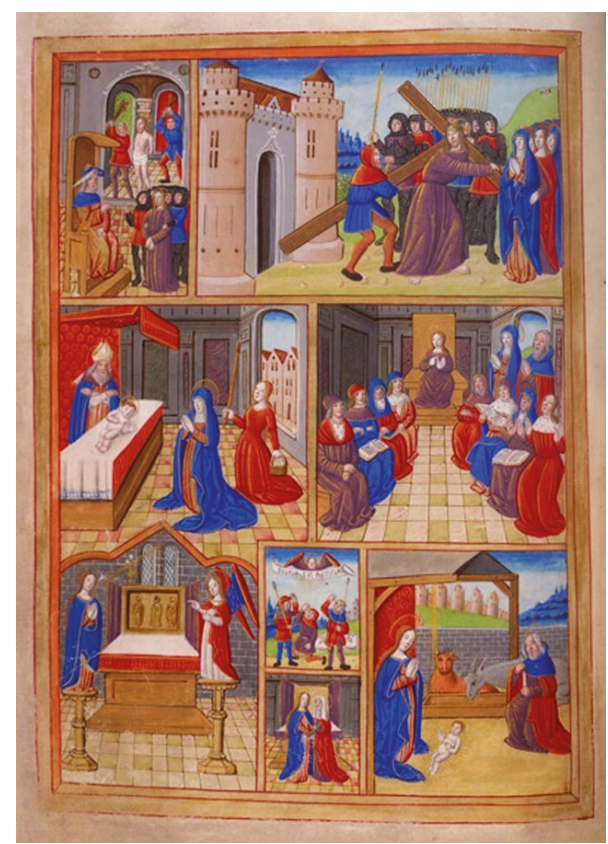

Figure 8. Pictorial cycle f.54v of the Hours of Thomas Butler, (C) British Library Board. 


\begin{tabular}{|c|c|c|c|c|}
\hline \multirow[t]{4}{*}{ Royal $\mathrm{fl} 5 \mathrm{v}$} & \multicolumn{3}{|c|}{ Coronation of the Virgin by the Trinity } & Assumption \\
\hline & \multicolumn{3}{|l|}{ Annunciation } & Ascension \\
\hline & \multicolumn{3}{|l|}{ Agony in the garden } & $\begin{array}{l}\text { Risen Christ appearing } \\
\text { to the Virgin }\end{array}$ \\
\hline & Visitation & \multicolumn{2}{|c|}{ Butler arms } & Nativity \\
\hline \multirow[t]{3}{*}{ Royal $553 \mathrm{v}$} & Ascension & \multicolumn{2}{|c|}{\begin{tabular}{|l|} 
Coronation of the \\
Virgin
\end{tabular}} & Assumption \\
\hline & $\begin{array}{l}\text { Risen Christ } \\
\text { appearing to the } \\
\text { Virgin }\end{array}$ & \multicolumn{2}{|c|}{ Christ as Gardener } & Resurrection \\
\hline & $\begin{array}{l}\text { Scene of the } \\
\text { Crucifixion flanked } \\
\text { by the Virgin and an } \\
\text { angel }\end{array}$ & \multicolumn{2}{|c|}{\begin{tabular}{|l} 
Annunciation to \\
the shepherds \\
Visitation
\end{tabular}} & Nativity \\
\hline \multirow[t]{3}{*}{ Royal f54r } & \multicolumn{3}{|l|}{ Crucifision } & Deposition \\
\hline & Betrayal & \begin{tabular}{|l|}
$\begin{array}{l}\text { Christ before } \\
\text { Pilate }\end{array}$ \\
\end{tabular} & & Good Samaritan \\
\hline & \multicolumn{2}{|c|}{ Presentation at the temple } & \multicolumn{2}{|c|}{ Christ preaching in the temple } \\
\hline \multirow[t]{4}{*}{ Royal f54v } & \multicolumn{2}{|c|}{$\begin{array}{l}\text { Christ before Pilate and } \\
\text { Flagellation }\end{array}$} & \multicolumn{2}{|c|}{ Good Samaritan } \\
\hline & \multicolumn{2}{|c|}{ Presentation at the temple } & \multicolumn{2}{|c|}{ Christ preaching in the temple } \\
\hline & \multirow{2}{*}{$\begin{array}{l}\text { Altarpiece with } \\
\text { three saints flanked } \\
\text { by the Virgin and } \\
\text { an angel }\end{array}$} & \multicolumn{2}{|c|}{$\begin{array}{l}\text { Annunciation to } \\
\text { the shepherds }\end{array}$} & \multirow[t]{2}{*}{ Nativity } \\
\hline & & Visitation & & \\
\hline
\end{tabular}

Figure 9. Layout of the pictorial cycles in the Hours of Thomas Butler

This grouping in the Royal manuscript is immediately followed by a miniature of the Coronation of the Virgin before the Trinity and the Company of Heaven (Figure 10). Collectively, the images fall between Compline and the Salve Regina and Gaude Virgo hymns on ff. 56-57. By the later Middle Ages, it had become popular to sing the Salve Regina after Compline. Supplicants were also encouraged to sing or speak the words of the hymn before an image of the Virgin Mary as depicted by the devotee with a scroll in a Book of Hours in Trinity College, Cambridge. ${ }^{6}$ The producer of the Hours of Thomas Butler anticipated the reader to be engaged in a ritualistic act involving sight, touch, thought, and speech: first, a meditation on and uttering of the prayer at Compline introduced by an image of the Entombment; 7 second, upon turning the page, a contemplation of the details of the cycle, the scenes of which are again closely related to the Joys and Sorrows of the Virgin; ${ }^{8}$ third, a contemplation on the Coronation before the Trinity; fourth, singing of the hymns with both a marginal scene of the Virgin and Child next to the words and the preceding miniature providing a visual focal point. The perceived efficacy of prayers, supplications, and gestures said or made in front of images, both in terms of rewards and as a tool to heighten devotional experience, during the late

6 Cambridge, Trinity College MS B.11.7.

7 In the Royal manuscript the hours are introduced in a relatively, though perhaps not wholly, typical manner with scenes from the Passion: the Visitation introduces Matins (f21v) though ordinarily this would offer a scene of the Annunciation; the Betrayal appears at the Lauds division (31v); Christ Before Pilate at Prime (though this is less frequent than the Betrayal at this division) (35v); Terce depicts Christ Carrying the Cross (though typically Christ Before Pilate or the Flagellation appear) (38v); Nailing to the Cross at Sext (Scott notes only two copies) (41v); Crucifixion at None (44v); Deposition at Vespers (49r); Entombment at Compline (52r); for a discussion see Scott 1996, vol. I p. 56.

8 The Seven Joys of the Blessed Virgin Mary appear on f.63v introduced by a historiated initial G with a scene of the Virgin seated before the Trinity. 
medieval period has been well documented both in England and Europe. In his account of the culture of indulgences at the time, Robert Swanson relates that praying to an image of the arma Christi every day for a year offers 6755 years, six months, and three days' remission from Purgatory. Similarly, daily recitations of multiple Aves, Paternosters, and Creeds before the Man of Sorrows could exceed 32,000 years' remission. Thomas Lentes notes the importance of 'an exact chronology of bodily poses' to be performed during the Pater Noster and recited seventy-seven times in front of an image of the Mount of Olives as 'only those who used the right gestures in front of the picture could be sure that the person in it would look at them with merciful eyes and hear their prayer' (Lentes 2006, p. 362; Swanson 2007, pp. 255, 262).

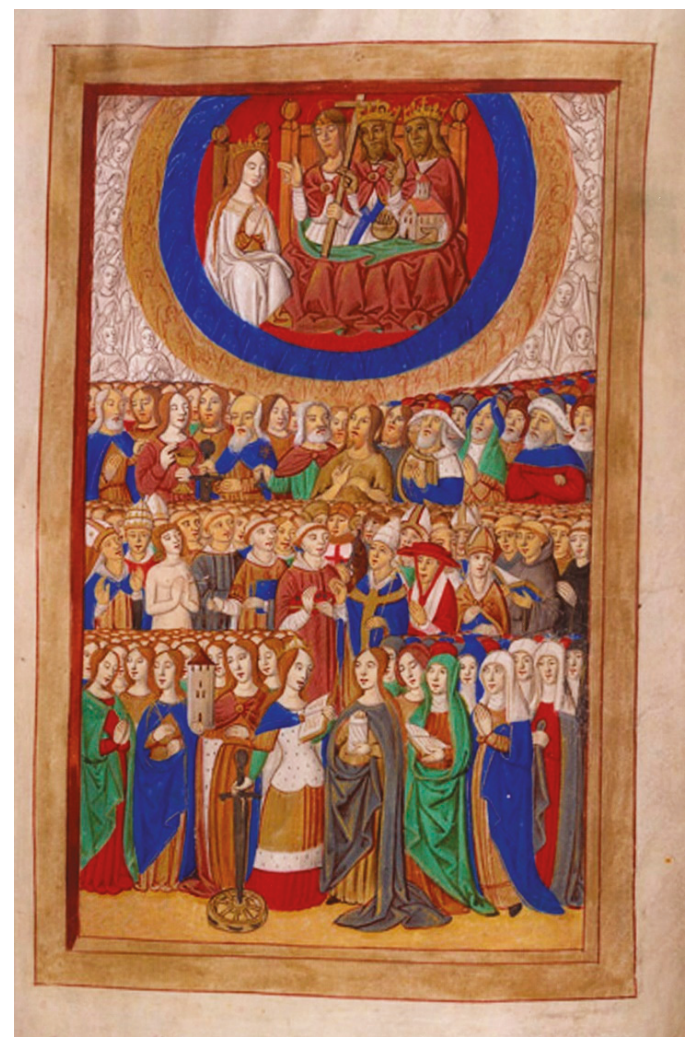

Figure 10. The Coronation of the Virgin before the Trinity, the Hours of Thomas Butler, f.55v, (C) British Library Board.

The opening pictorial cycle in the Royal manuscript occurs on $\mathrm{f} .15 \mathrm{v}$ immediately preceding the Hours of the Virgin (Figure 11). The principal scenes depict the Coronation of the Virgin by the Trinity; Annunciation; and the Agony in the Garden. These are flanked by smaller scenes (from top to bottom): the Assumption; Ascension; Risen Christ appearing to the Virgin; Nativity; and Visitation; with the Butler arms inserted between the bottom two scenes as in the Harley manuscript. The pictorial cycles therefore act as both preface and postface to the Hours of the Virgin. The opening cycle in the Harley manuscript occurs on f.28v, described by Scott as Illustrator G, and incorporates seven narrative scenes (Figure 12). In this case the two principal scenes are of the Annunciation and Agony in the Garden and these are flanked by five smaller scenes (from top to bottom): the Assumption, Ascension, Risen Christ appearing to His mother, Nativity, and Visitation, with the Butler arms inserted between the latter 
two scenes. Stylistically the Harley cycle on f.28v and Royal cycles are more refined than the group proceeding the Fifteen Oes with delicately rendered facial features, subtle modelling, and thin golden circles and delicate rays for haloes. Scott writes of the Harley manuscript that 'this glamorous insertion was clearly meant to cope with the inadequacy of the English historiated initial of Matins' (Scott 1996, vol. II 301). While this may well be true, at least in part, its insertion at this juncture also serves to unite the two series of full-page miniatures in the manuscript: drawing together the recitations demanded of the cycle (and a partial preparatory cycle on $\mathrm{f} .28 \mathrm{r}^{9}$ ) with Trinitarian imagery on $\mathrm{f} .27 \mathrm{v}$ in an active preface to Matins complete with the patron's self-image.

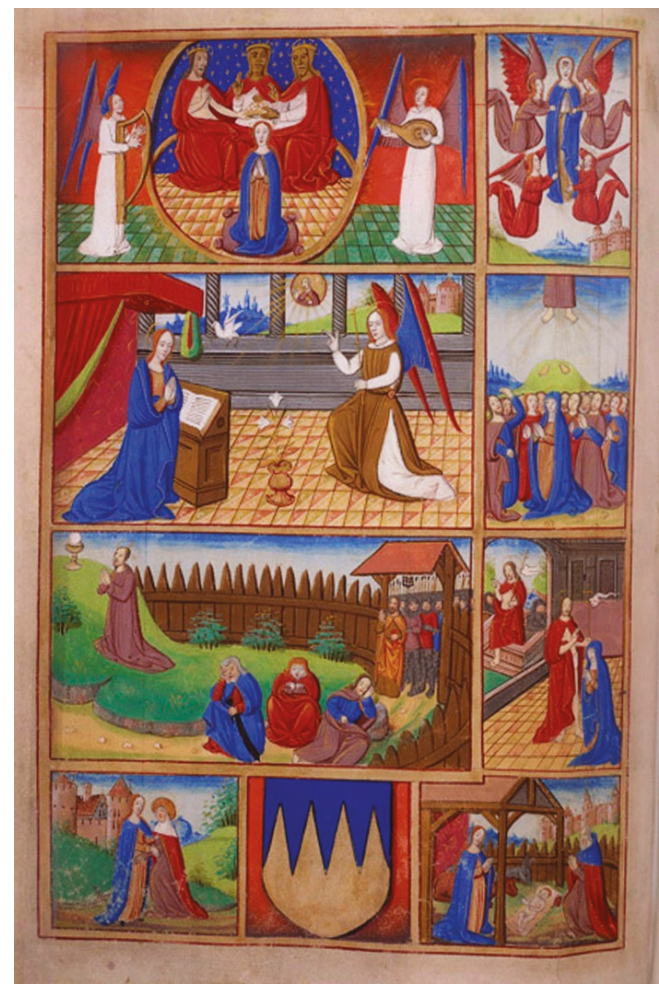

Figure 11. Opening pictorial cycle, the Hours of Thomas Butler, f.15v, (c) British Library Board.

9 Two partial preparatory scenes depict the Annunciation above the Agony in the Garden in a similar approach to the finished cycle on the verso. 


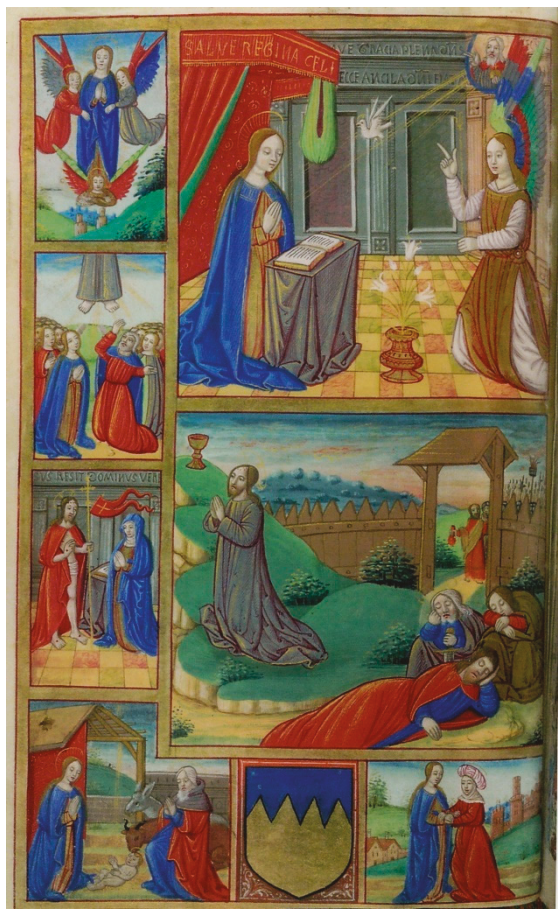

Figure 12. Opening pictorial cycle, the Hours of the Earls of Ormond, f.28v, (c) British Library Board.

\section{The Holy Trinity}

The same association between performative act and devotional image in the pictorial cycles is found in the second series of full-page miniatures which depicts the Holy Trinity. Four of the five miniatures inserted into the Hours of the Earls of Ormond depict the Triune God. In somewhat bleak terms, Scott refers to the inserted material as a footnote to a discussion of an English illumination shop in the mid-fifteenth century:

the owner apparently wished to make a luxury copy of what would have been, without pictures, merely good production. The inserted miniatures have very much the air of commercialisation about them [ ... ] The execution is poor, and the composition is identical between the repeated scenes (Scott 1968, pp. 172n-73n).

There are two similar though distinct depictions of the Holy Trinity and each is repeated once more a few folia later: pairs A (ff. 6v and 33v) and B (ff. 8v and 27v) (Figures 13 and 14). In both pairs, the figures appear as the Trinity in Glory, the three persons seated side by side in a horizontal arrangement. This type of image, though found less frequently, better preserves the equality of the three persons rather than the co-enthronement type whereby Christ and God the Father appear in line with the dove between them representing the Holy Spirit or the vertical arrangement of the Mercy Seat/Throne of Grace. ${ }^{10}$ The figures are united under a single crown and framed by the symbols of the Evangelists in the corners. Departing from the equality established in the position, the figures of God

10 For English examples of the Trinity in Glory type, see: the Wollaton Antiphonal, Nottingham, University Library MS 250, f246v dating to c.1430; a Book of Hours, New York, Morgan Library MS G 9, dating to c.1440-1450; and Camarillo, California, St John's Seminary, Edward L. Doheny Memorial Library MS $3970 \mathrm{f} 88 \mathrm{r}$ dating to c.1460-1470; for a discussion of the types of Trinitarian imagery see (MacHarg 1917). 
the Father and the Holy Spirit are depicted as a rich golden colour, unlike Christ who is much paler with smaller facial features and a narrower frame. Christ is also placed on the left of the group which is relatively atypical of the Trinity in Glory type whereby Christ is usually positioned in the centre (Price-Linnartz 2009, p. 16). The striking radiance differentiates the two pairs. Pair A shows God the Father and the Holy Spirit with strong torsos but no lower bodies alongside Christ with golden rays emanating from their collective waist filling the frame. Pair B on the other hand shows developed lower bodies for all three figures with golden rays bursting dramatically forth from the chest and back of the Trinity with a ring of cherubim and seraphim hovering between them. Scott attributes the illumination of $f .27 \mathrm{v}$ to Illustrator $\mathrm{D}$ and the other three to Illustrator $\mathrm{C}$ based on the source and finish of the parchment and the use of rose outlining. Given the iconographic pairs, this would suggest that the two artists were working very closely together and from a common exemplar or donor instruction. This use of rose ink in the Trinity scenes and decorated initials on ff. 7r-12v suggest a French influence and a possible source for the iconography (Scott 1996, vol. II p. 301).

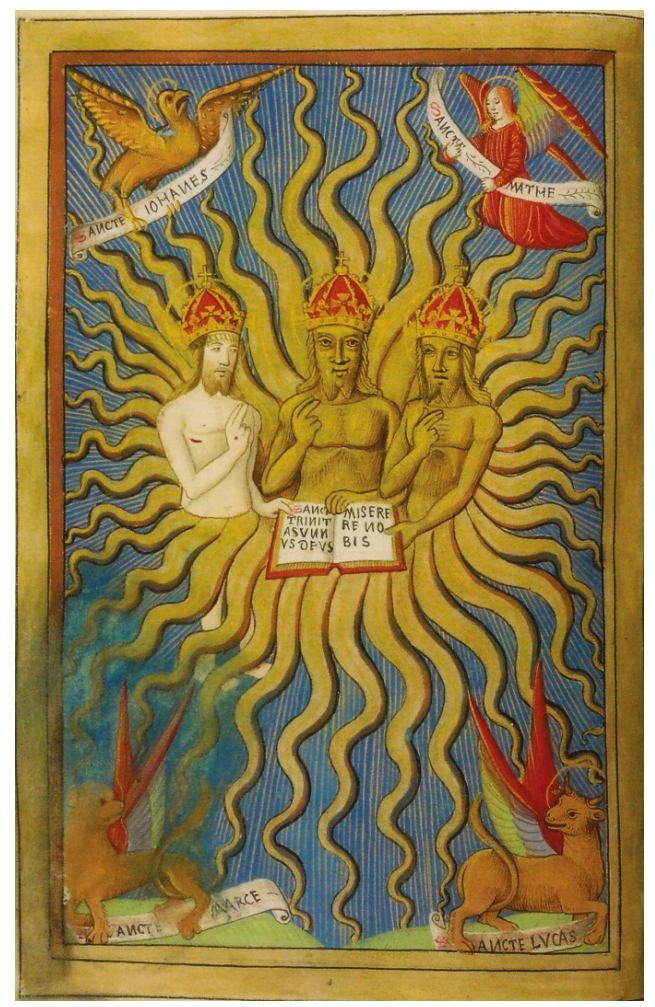

Figure 13. Holy Trinity, pair A of the Hours of the Earls of Ormond, ff. 6v and 33v, (C) British Library Board. 


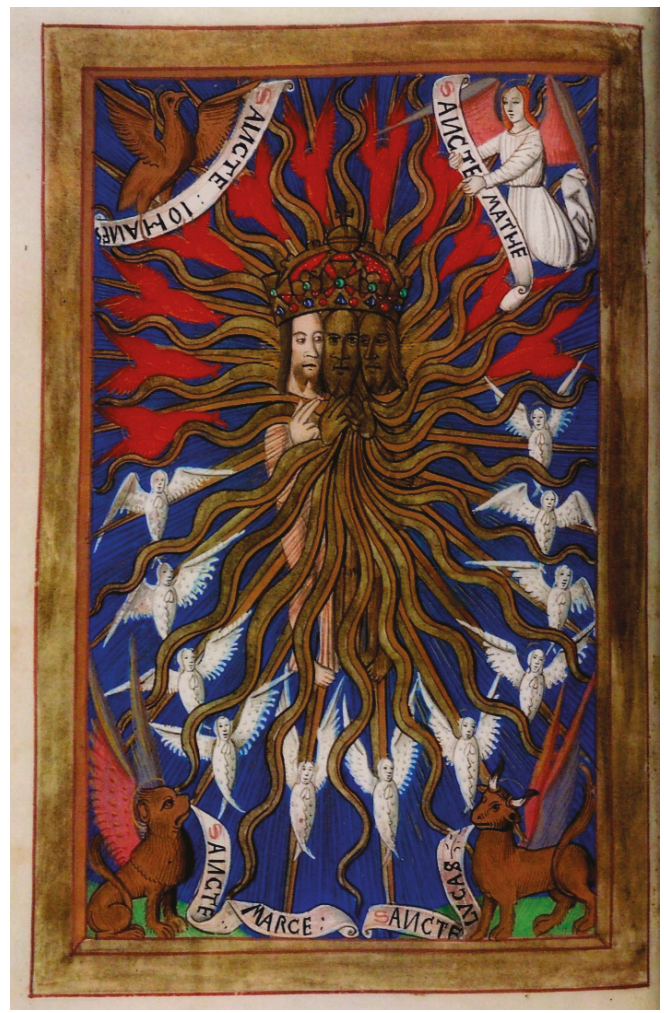

Figure 14. Holy Trinity, pair B of the Hours of the Earls of Ormond, ff. $8 \mathrm{v}$ and $27 \mathrm{v}$, (C) British Library Board.

The Hours of Thomas Butler offers two miniatures dedicated to the Holy Trinity, ff. 9v and 10v (Figures 15 and 16). ${ }^{11}$ The latter is strikingly similar in every respect with pair B from the Harley manuscript. The compositions and colouring are almost identical with very slight differences in the number of cherubim and seraphim and the colouring of St Matthew's angel. Such strong similarities in the iconography, compositions, and design choices between the two manuscripts surely suggest a coordinated approach. This prompts the question of whether the refurbishment of the Harley manuscript inspired the commission of the Royal or whether the personalised commission of the Royal manuscript was the catalyst for the insertions in the Harley. While this would be a difficult question to answer without further evidence, Scott's comment on the 'glamorous insertion' remedying the inadequacy of the opening of the Harley Matins may be useful here. Perhaps upon the commissioning of his more lavish manuscript, Butler decided to revitalise an older book with glamorous, functional imagery.

The composition of the Trinity on Royal f.9v offers a more restrained depiction of the Trinity in Glory type such as that in the Lectionnaire de l'Office de la Sainte-Chapelle de Bourges c.1410, the Chourses-Coëtivy

11 Trinitarian imagery is continued throughout the manuscript with a further miniature depicting the Virgin before the Holy Trinity and surrounded by the company of heaven on $f .55 \mathrm{v}$. The pictorial cycle on f.15v incorporates a scene of the Coronation of the Virgin by the Trinity and three initials are historiated with scenes of the Trinity: a Crucifixion type on f.12r (comparatively rare), a Throne of Grace whereby the dove is perched on the bar of the Cross on f.62r, and a Virgin before the Trinity on $\mathrm{f} .63 \mathrm{v}$ which is very similar to the miniature on $\mathrm{f} .9 \mathrm{v}$ but without the attributes. 
copy of the Golden Legend mentioned earlier, another manuscript belonging to the family, the Coëtivy Hours dating to the mid-fifteenth century or an early sixteenth-century Book of Hours possibly from Lyon. ${ }^{12}$ The three figures on Royal f.9v are depicted as full length, seated on an invisible throne. As in all the previous images God, the Father and the Holy Spirit are deeper in colour than Christ and in this case, $\mathrm{He}$ is bearing the stigmata and Crown of Thorns, and carrying the Cross. The three figures are united through their hands on a common book of litanies but also by their luxuriant scarlet cloak as in contemporary French and Flemish illumination. ${ }^{13}$ The golden rays are much more muted than in the previous images emanating from behind the throne and outwardly restrained by a blue mandorla which is in turn set within a red background. As in the case of the other five depictions, the symbols of the Evangelists occupy the corners of the frames. While perhaps a little unusual, examples of the Trinity surrounded by a mandorla and the tetramorph are to be found in the fourteenth century in the Hours of Philip the Bold by the Master of the Throne of Mercy and in the miniature with donors in the Pabenham-Clifford Hours, ${ }^{14}$ recalling alabasters and French and English Gothic ivories and their treatment of the subject. ${ }^{15}$ Both the Royal miniatures and three of the four in Harley manuscript are inserted preceding or in the middle of prayers dedicated to the Holy Trinity or the three persons of the Trinity. ${ }^{16}$ As with the pictorial cycles, the images provide a visual focus for the supplicant's devotions.

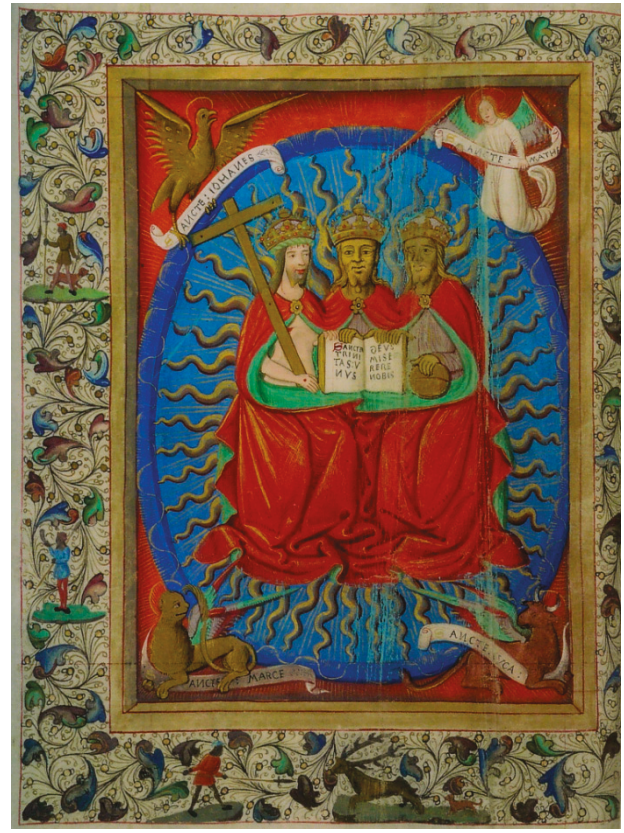

Figure 15. Holy Trinity, the Hours of Thomas Butler, f.9v, (c) British Library Board.

12 Bourges, Bibliothèque Municipale MS 35; Paris, Bibliothèque Nationale de France MS Fr. 244; Dublin, Chester Beatty Library MS W 82; and Pennsylvania, Lehigh University, Lehigh Codex 18 respectively.

13 See for example New York, Morgan Library MS 287 f134r dating to $c .1445$ and New York, Morgan Library MS M 8 f136v dating to $c .1511$.

14 Cambridge, Fitzwilliam Museum MS 3-1954 and Cambridge, Fitzwilliam Museum MS 242 respectively.

15 See for example: Toronto, Thomson Collection at the Art Gallery of Ontario 107363, an English diptych fragment; Washington, D.C., Smithsonian American Art Museum 1929.8.240.12, an English diptych; London, Courtauld Gallery O.1966.GP.2, a French diptych fragment; London, Courtauld Gallery 305, a cast of a lost French diptych, all dating to the first half of the fourteenth century. For a discussion of ivory carving see: (Gaborit-Chopin 2003; Lowden 2013; Randall 1993; Williamson 1982; Williamson and Davies 2014).

16 The miniature on Royal f.33v appears in the middle of the Te Deum hymn. 
Despite the somewhat unflattering description of the quality of execution, Scott nevertheless regards the miniatures as significant, describing them as 'unique iconographically in English illustration and worthy of note because of the Gospel symbols, the three full-length nude Trinity figures and the massive radiance as substance of the Trinity with three heads' (Scott 1996, vol. II 301). Such a strong focus on the rays raises a question about the inspiration for or origin of the imagery but also poses an interesting solution to a theological and iconographical problem. Reducing the mystery of the Trinity to visible human form is a theological hurdle and thus limitations are imposed on all figural representation. While scripture provides physical manifestations for the second and third persons in the flesh, in fire, and in the form of the dove, God the Father remains invisible (John 1:18). Given the Trinity 'is invisible in such a way that it is not seen even by the mind', Augustine of Hippo forbade all attempts to picture the Trinity, symbolic or anthropomorphic. Likewise, Pseudo-Dionysius places the concept beyond human imagination opening his De mystica theologia: 'Trinity, higher than any being, any divinity, any goodness!' (Augustine 1990, p. 136; McGinn 2006, p. 186; Price-Linnartz 2009, p. 3; Pseudo-Dionysius 1991, vol. II 141; Rorem 2015, p. 9).

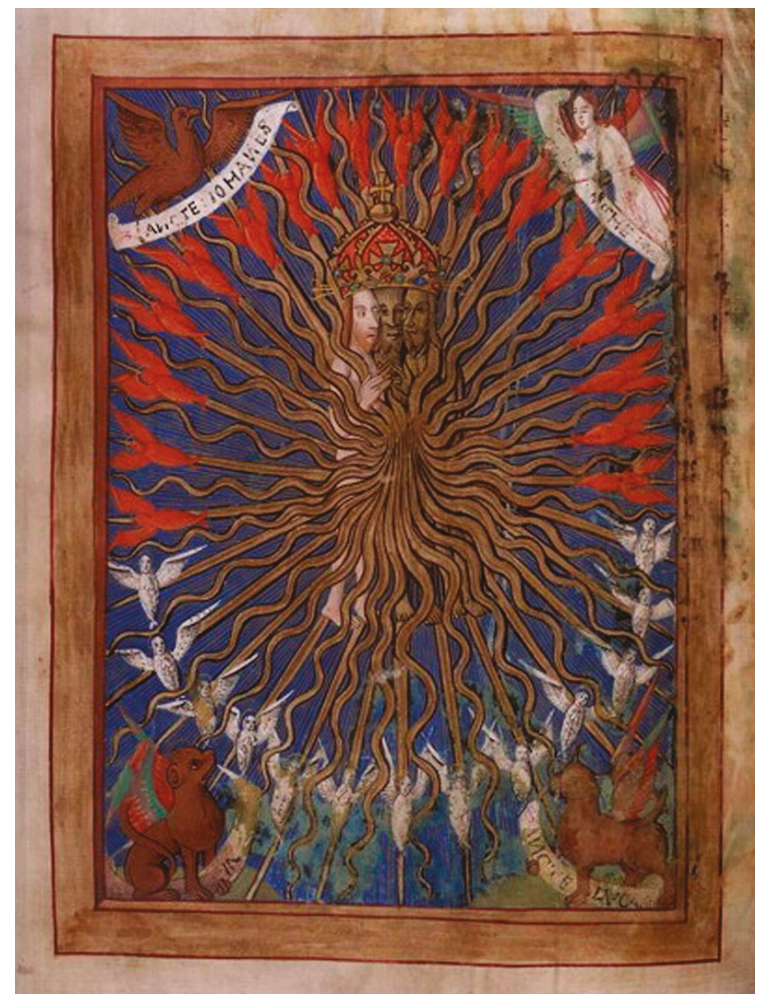

Figure 16. Holy Trinity, the Hours of Thomas Butler, f.10v, (C) British Library Board.

Obscuring the physical forms with the radiance enables the artist to surmount this theological obstacle and focus on the collective essence of the Trinity. The dazzling radiance at once presents the character of the Holy Spirit in the form of fiery tongues while simultaneously presenting the three persons as equal and co-eternal: 'together not three lights, but one light $[\ldots]$ one essence'. Both Augustine and Gregory of Nazianzus describe the intensity of the light emanating from the Holy Trinity, Augustine refers frequently to a 'so transcendent light', a 'blaze of light' and an 'ineffable light [that] beat back our gaze' and Gregory to an 'ineffable' light that 'irradiates the whole universe'. 
The radiance also however allows the artist to explore the more visionary, emotional, and personal aspects of Trinitarian writings, for example Gregory relates, '[n]o sooner do I conceive of the One than I am illumined by the Splendour of the Three; no sooner do I distinguish Them than I am carried back to the One. [ ... ] When I contemplate the Three together, I see but one torch, and cannot divide or measure out the Undivided Light' (Augustine 1886, pp. 19, 86, 109, 204; Gregory 1894, pp. 361, 375).

The drama and intensity of the Butler iconography is perhaps however more in keeping with the passionate language of the twelfth- and thirteenth-century mystics such as Hildegard of Bingen who describes how the 'bright light [representing God the Father] bathed the whole of the glowing fire [representing the Holy Spirit], and the glowing fire bathed the bright light; and the bright light and the glowing fire poured over the whole human image [Christ], so that the three were one light in one power of potential.' Gertrude of Helfa exclaims 'O ardent fire [ ... ] O consuming fire [ ... ] O burning furnace' and Mechthild of Magdeburg describes the light of the Trinity as 'God let[ting] his fiery spirit shine forth unceasingly from his Holy Trinity into this loving soul, just like a bright sunbeam shining forth from the hot sun lights up a golden shield' (Gertrude 1992, p. 105; Hildegard 1990, p. 206; Mechthild 1998, p. 179; Ray 2011). These fervent descriptions stimulate the reader's emotional, rather than rational, response to the burning mystery of the Holy Trinity. Similarly, in the Books of Hours the theatrical bursting forth of the radiance from the torsos of the three persons commands the reader's attention, elicits their personal and emotional responses, and inspires their devotional gaze.

Contemporary religious theatrical productions similarly utilised light, fire, and radiance to captivate their audiences and convey messages of divinity. Actors playing the role of God were traditionally masked or painted in gold to mark them out as divine both in England and abroad. Play expenses in York for example included gilded masks and yellow wigs for the Doomsday pageant and in Chester and Coventry the expenses list the gilding of players' faces (Muir 1997, pp. 33-35; Stevens 2013, p. 24; Twycross and Carpenter 2002, p. 220). This gilding ties in well with the golden depictions of God the Father and the Holy Spirit in the Books of Hours and with the golden face of God in stained glass of the fifteenth and sixteenth centuries, a practice described by Émile Mâle as 'a relic of the religious theatre'. Pyrotechnics and other lighting devices were also involved in the staging of plays. The Chester Pentecost, for example, directs God to send forth the Holy Spirit in the form of fire, 'in spetie ignis', and angels cast flames on the apostles while singing the antiphon Accipite Spiritum Sanctum [Receive the Holy Spirit]. Medieval accounts seem to support the idea that the apostles were showered with real fire in these scenes rather than ribbons or props. In her discussion of the staging of the York Transfiguration, Andrea Ria Stevens proposes that a metallic reflector could be used to focus and direct either sunlight or candlelight (likely augmenting an already gilded mask or effect of golden face paint) in order to create the illusion that the light of the Transfiguration was coming from within the figure of Christ himself. Such directions had been provided for the Revello [Piedmont] production of the play. The actor could also rapidly change his garments to reveal a snow-white robe to further the dramatic change (Lumiansky and Mills 1986, play 21, line 238; Mâle 1987, pp. 108-9; Muir 1997, p. 35; Stevens 2013, pp. 30-31; Twycross 2008, p. 38). This bouncing of light off a reflective plate on an actor's chest could certainly create, or at least inspire, a similarly dazzling effect to that of Butler's Trinitarian imagery. The iconography of the Books of Hours acts therefore as a catalyst to prayer and demonstrates both a rather creative and somewhat eclectic solution to the representation of a theologically and intellectually difficult subject with references to a variety of sources and styles in different media and perhaps some influence also from the performing arts.

Furthermore, though not a gruesome depiction of suffering - as in for example the Trinity of the Broken Body type which shows the dying body of Christ in an amalgamation of the Christ of Sorrows or Corpus Christi and Trinitarian imagery-the depiction of the wounds emphasises Christ's sacrifice. Such iconography is again designed to elicit an emotional response from the viewer, to move the viewer to penance and contemplation both of Christ's sacrifice for the salvation of mankind as the result of a decision by all three persons and of the fate of the viewer's own mortal soul. François Bœsflug associates the rise in funerary Trinitarian imagery across Europe from the second half of the 
fourteenth century with the intercessory power of the Holy Trinity. Devotees at their hour of greatest need seek comfort directly from the three persons and pity and understanding from a suffering Christ. This instrumental approach is also witnessed in the pleas for mercy in the Fifteen Oes, emphasising the bond between Christ's suffering and His infinite love of humanity and the promise of forgiveness (Bœsflug 2001; Duffy 2005, p. 250; Krug 1999, p. 108). ${ }^{17}$

Harley f.27v also bears the traces of pilgrim badges, pinpricks forming two circles where a badge(s) were previously pinned to the folio. Certain members of the Ormond family are known to have undertaken or at least contemplated undertaking pilgrimages. Butler's father, the White Earl for example, was granted dispensation in 1431 for a journey to Rome. Likewise, his brother John, the sixth earl, died on a pilgrimage to the Holy Land in 1477-1478 in what Thomas Carte describes as 'a fit of devotion' (Calendar of entries in the Papal Registers 1909 vol. VIII 278; Carte 1851, vol. I lxxxi). This sort of customisation of a Book of Hours through the insertion of a pilgrim badge was popular in the late Middle Ages, examples of which may be found on manuscripts originating from and belonging to families and religious communities in England, France, and Flanders such as a late-fifteenth-/early-sixteenth-century manuscript belonging to members of a family in Bordeaux or the elaborately adorned d'Oiselet Hours produced in Bruges and belonging to Claude de la Chambre of Franche-Comté. ${ }^{18}$ As Virginia Reinburg has demonstrated, pilgrim badges offered the pilgrim a tangible, tactile memory of their experience.

More than a mere memento of a trip, such souvenirs reminded returned pilgrims of their experience in a concrete way. Just as touching a relic or image — even through the mediation of tomb or shrine-conveyed the presence and power of the saint to pilgrims, so the souvenir conveyed the memory of the pilgrim's experience at the shrine. This was a culture in which memory was an art. Wearing, seeing, and handling objects provided a material memoir of the pilgrimage (Reinburg 2012, p. 81).

By incorporating his pilgrim badges into the Book of Hours, Butler had a sensory aid to memory to help him recall his experience. Just as his Psalter with his name chained to his tomb bearing his epitaph encouraged the reader to bear him in his prayers, the pilgrim badges encouraged a multi-sensory devotional experience combining his personal esoteric devotional interests with vivid imagery of an almost mystical nature and the tactile souvenir of a physical devotional experience.

Devotional imagery in English and French Books of Hours typically takes the form of the Five Wounds, Man of Sorrows, Mass of St. Gregory, arma Christi, and the Veronica, and the associated indulgences often offered magnificent rewards. These same motifs were also typical of devotional imagery in Ireland from this period (Connaughton 2012; Duffy 2011, pp. 15-16; Duffy 2005; Lewis 1993; Reinburg 2012, pp. 117-23). The tomb of Butler's distant cousin and heir, Piers, and his wife, Margaret, in St. Canice's Cathedral displays the arma Christi for example, as does a tomb of an unknown Butler knight at Gowran. Indeed, Salvador Ryan, in his assessment of the motif in late medieval and early modern Ireland, notes that in the sixteenth century the arma Christi came to replace depictions of the saints on tombs. Furthermore, Ryan notes that this devotion to the arma Christi was rather localised in Kilkenny, the heart of Butler influence (Ryan 2014, pp. 253-55). Thomas Butler does not appear to have shared in this particular interest in the arma Christi as it only appears in the older portion of the Harley manuscript introducing Psalm 21, f.105r; whereas the equivalent historiated initial in the Royal manuscript depicts a Mass of St Gregory, f.126r.

The devotional focus on the Holy Trinity seems to suggest therefore a personal choice on behalf of the patron. This choice may also reflect a familial devotional preference as in her investigation of

17 A further prayer with indulgences equalling the number of Christ's wounds granted by Pope Benedict XII, 'Precor te piissime Thesu Christi' appears on f.60r of the Royal manuscript.

18 See for example London, British Library Egerton MS 3883, f142v, a fifteenth-century manuscript with Netherlandish and English origins; Oxford, Bodleian Library MS Douce 51, ff. 45v, 57v, 58v, 59r, 74r; and d'Oiselet Hours, The Hague, Koninklijke Bibliotheek MS 77 L 60, f98r; see (Bruna 1998, pp. 127-61; Foster-Campbell 2011, vol. I pp. 234-37). 
images of the Holy Trinity from the Middle Ages and Early Modern period in Ireland, Helen M. Roe noted a particular centrality in their locations or points of origin: 'and of these it is to be remarked that no fewer than eight are or were associated with sites in Ossory, either in Kilkenny city or in the area of the modern county' (Roe 1979, p. 101). Taking into consideration only those from the fifteenth and sixteenth centuries, Roe lists five examples from Kilkenny and all of them take the form of the Throne of Grace: a stone altar piece at Callan from c. 1535 to $1541, i^{19}$ two stone cloister panels from the second half of the fifteenth century, one at each of Inistioge Priory and Jerpoint Abbey; an alabaster statue group from the first quarter of the fifteenth century at Black Abbey; and a now lost or destroyed stone panel thought to date to the late fifteenth century from St. Patrick's burial ground, known through early nineteenth-century drawings by George Millar. In addition, she lists one example from Fethard, Co. Tipperary, of a wooden statue group of the Throne of Grace from c.1490 to 1520, of which only God the Father survives (Roe 1979, pp. 110,112). As before with the arma Christi, this concentration corresponds to Butler lands, furthermore Callan was founded by Edmund MacRichard and the renovations of Jerpoint Abbey were patronised by the White Earl, Butler's cousin and father. ${ }^{20}$

\section{At the Crossroads}

Butler's private life and political career placed him at the intersection of a variety of cultural and social spheres. Prominently engaged in English public life and comfortable in the courtly circles of England and France, Butler also maintained links with his family and sphere of magnates in Ireland as touched on above. Butler's Books of Hours illustrate ties with his Anglo-Irish heritage and Gaelic-Irish culture as both manuscripts observe the feasts of St Brigit of Kildare and St Patrick in their calendars (Harley ff. 21v and 22r and Royal ff. 3v and 4r) and the Hours of the Virgin in the Royal manuscript also contains a memorial to St Patrick during Lauds f.16r and in the litany on f.80r.

Another item listed in Butler's will, a small ivory horn given to him by his father, further demonstrates his position at the juncture of Anglo-Irish and English culture:

Item wher my lorde my ffather whose soule God assoyle left and delivered unto me a lytle whyte horne of ivory garnysshed at bothe thendes with golde and a corse thereunto of whyte sylke barred with barres of gold and a tyret of golde thereuppon the wych was myn auncetours at first tyme they were called to honor and hath sythen continually remained in the same blode for wych cause my said lord and ffather commaunded me uppon his blessing that I shuld doe my devoir to cause it to contynew styll in my blode [...] I woll that myn executours delyver unto Sir Thomas Boleyn knyght sonne and heire apparaunt of my said doughter [... ] (will of Thomas Butler 301).

It is clear from the language used in the will that the horn was of significance to the Ormond household. In fact, Carte claims that the horn bequeathed was held to be the one from which Thomas Becket himself drank 'and was kept very religiously in the family till this time' and Butler proceeds to name a further heir to the horn should Thomas Boleyn die without issue (Carte 1851, vol. I p. lxxxiv). The horn, not least due to its alleged associations with Becket, was a mark of honour for the Butler family, and this was also true of other families in both England and Ireland. John Cherry has argued that horns in Britain held a symbolic importance in terms of ownership and transference of land. The Pusey Horn for example was furnished in the fifteenth century with a band with an inscription recording that: 'I kynge knowde [Cnut] gave Wyllyam Pecote [Pusey] thys horne to holde by thy land' (Cherry 1989, pp. 112-13). A reference to the so-called Kavanagh 'Charter' Horn in the Civil Survey

19 Originally from the Church of the Blessed St. Mary at Callan.

20 Edmund MacRichard Butler undertook to found the Augustinian Friary at Callan, Co. Kilkenny in 1461, although the actual establishment of the friary fell to his son James after his death in 1464, see: Calendar of entries in the Papal Registers 1909, vol. IX p. 248, vol. XII p. 25; (Carrigan 1905, vol. III p. 311); Irish Monastic and Episcopal Deeds, 1936, nos. 6 8, 15, 18. 
of Co. Carlow 1654-1956 states that the Kavanaghs were 'descended from the stock of the kings of Leinster, had a great seate and a vessel or cup to drink out of called Corne-cam-mor' suggesting a similar function as symbol of office and tenure in an Irish context. At some stage during the fifteenth century Art MacMurrough Kavanagh or his immediate descendants commissioned the construction or refurbishment of the 'Kavanagh 'Charter' Horn', an imitation buffalo horn goblet carved from an elephant's tusk. The goblet was intended for use in royal inauguration ceremonies, recalling an old Irish tradition that only those who drank from the buffalo horn of Cualu could succeed to the kingship of Leinster (Byrne 2001, pp. 152-53; Ó Floinn 1994, pp. 270, 274; Simington 1961, vol. X misc. 9; Simms 2010, p. 7). The inventory of plate belonging to the Fitzgeralds, Earls of Kildare, dated 1518, also included 'a grete rede horne, bownd \& gylt' (MacNiocaill 1992, p. 303). Thus, in addition to its contractual symbolism, the fifteenth- and sixteenth-century concern for the horn demonstrated by the Butlers, Kavanaghs and Fitzgeralds suggests a performative interest in reinvigorating the heroic past, lengthy lineage and traditions. As Katharine Simms writes of the commissioning of the Kavanagh 'Charter' Horn, it presents an '[attempt] not merely to preserve tradition, but to re-enact the past' (Simms 2010, p. 8). As with the Psalter chained to his tomb in St Thomas of Acre, this private familial object of the horn also assumed an important public role demonstrating legacy and lineage.

The Hours of Thomas Butler and the insertions into the Hours of the Earls of Ormond offer a personal reflection of their owner. A prominent figure in English and French public life with an Irish heritage, the products of his patronage were influenced by the various facets of his world. Marked by English prayers, French designs, Irish saints, borrowed folia, and some individual artistic flair and creativity these manuscripts present a combination of standard, personal, and familial devotions. A pious and devoted reader, Butler's Books of Hours also show him to be an individual concerned with tangible, measurable results, combining imagery, prayer and repetition in an active performance of piety with a particular interest in devotions to the Passion and Holy Trinity. This active participation and concern for results was also true of the more public aspects of his commemoration and the performance of lineage and tradition demonstrated in the bequest of the horn. As with the Psalter chained to his tomb and the ivory horn held dearly by generations of his family, Thomas Butler's Books of Hours were performative objects, family records, 'archives of prayer' (Reinburg 2012), and material aids to devotion.

Funding: Travel for this research was funded in part by the NYU Global Faculty Fund.

Conflicts of Interest: The author declares no conflict of interest.

\section{References}

\section{Primary Sources}

Augustine of Hippo. De Trinitate. Translated by Arthur West Haddan and Rev. William G.T. Shedd. New York: Philip Schaff, 1886.

Augustine of Hippo. Letter 120, Letters: The Works of Saint Augustine, A Translation for the Twenty First Century. Edition by John E. Rotelle. Brooklyn: New City Press, 1990.

Bourges, Bibliothèque Municipale MS 35, f1v, Lectionnaire de l'Office de la Sainte-Chapelle de Bourges.

Calendar of entries in the Papal Registers relating to Great Britain and Ireland: Papal Letters. Twemlow, J.A., ed. London: His Majesty's Stationery Office, 1909.

Calendar of Ormond Deeds, 1172-1603. Curtis, Edmund, ed. Dublin: The Stationery Office, 1935.

Calendar of Patent Rolls Preserved in the Public Record Office: Henry VI, 1429-1436. London: His Majesty's Stationery Office, 1907.

Calendar of Wills Proved and Enrolled in the Court of Husting, London. Sharpe, R.R., ed. London: Her Majesty's Stationery Office, 1890.

California, St John's Seminary, Edward L. Doheny Memorial Library MS 3970, Psalter.

Cambridge, Fitzwilliam Museum MS 242, Pabenham-Clifford Hours.

Cambridge, Fitzwilliam Museum MS 3-1954, Hours of Philip the Bold. 
Cambridge, Trinity College MS B.11.7, Book of Hours.

Dublin, Chester Beatty Library MS W 82, Coëtivy Hours.

Gertrude of Helfa. The Herald of Divine Love. Translated by Margaret Winkworth. New Jersey: Paulist Press, 1992.

Gregory of Nazianzus. Oration 40, 'Select Orations of Saint Gregory Nazianzen, Sometime Archbishop of Constantinople', Brown, Charles Gordon and James Edward Swallow (trans.), in Schaff, Philip (ed.), A Select Library of the Nicene and Post-Nicene Fathers of the Christian Church, volume VII: Cyril of Jerusalem and Gregory Nazianzen (New York, 1894: The Christian Literature Company).

Hildegard of Bingen. Scivias. Translated by Columba Hart and Jane Bishop. New Jersey: Paulist Press, 1990. Irish Monastic and Episcopal Deeds AD 1200-1600. White, Newport B., ed. Dublin: The Stationery Office, 1936.

Kew, National Archives PROB 11/18/184, Will of Thomas Butler, seventh Earl of Ormond, dated 31st of July 1515, (dated in the National Archives' catalogue as 25th of August 1515 as the date on which it was proved) transcription from Beresford see below.

Letters and Papers Foreign and Domestic in the Reign of Henry VIII. Brewer, J.S., ed. London: Longman, Roberts, and Green, 1864.

London, British Library Egerton MS 3883, Book of Hours.

London, British Library Harley MS 2887, Hours of the Earls of Ormond.

London, British Library Harley MS 3756, Kildare Rental.

London, British Library Royal MS 2 B XV, Hours of Thomas Butler.

Mechthild of Magdeburg. The Flowing Light of the Godhead. Translated by Frank Tobin, Frank. New Jersey: Paulist Press, 1998.

New York, Morgan Library MS 287, Book of Hours.

New York, Morgan Library MS G 9, Book of Hours.

New York, Morgan Library MS M 8, Breviary.

Nottingham, University Library MS 250, Wollaton Antiphonal.

Oxford, Bodleian Library Douce MS 51, Book of Hours.

Paris, Bibliothèque Nationale de France MS Fr. 244, Golden Legend.

Paris, Bibliothèque Nationale de France MS Fr. 989, Defender of the Immaculate Conception of the Glorious Virgin Mary. Pennsylvania, Lehigh University, Lehigh Codex 18, Book of Hours.

Princeton, University Library Garrett MS. 34, Tewkesbury Psalter.

Pseudo-Dionysius Areopagita. Corpus Dionysiacum. Heil, Günter, and Adolf M. Ritter, eds. Berlin: De Gruyter, 1991. Rotuli Parliamentorum ut et Petitiones, et Placita in Parliamento. London, 1767-1777.

The Hague, Koninklijke Bibliotheek MS 77 L 60, d'Oiselet Hours.

\section{Secondary Sources}

Allmand, Christopher. 1992. Henry V. London: Methuen

Beresford, David. 1999. The Butlers in England and Ireland, 1405-1515. Ph.D. dissertation, Trinity College Dublin, Dublin, Ireland.

Bœsflug, François. 2001. La Trinité à l'heure de la mort: sur les motifs trinitaires en context funéraires à la fin du Moyen Âge (m. XIVe-déb. XVIe siècle). Cahiers de Recherches Médiévales et Humanistes 8: 1-26.

Brooks, Eric St. J. 1956. Irish Possessions of St. Thomas of Acre. Proceedings of the Royal Irish Academy: Archaeology, Culture, History, Literature 58: 21-44.

Bruna, Denis. 1998. Témoins de devotions dans les livres d'heures à la fin du moyen âge. Revue Mabillon New Series 9: 127-61. [CrossRef]

Byrne, Francis John. 2001. Irish Kings and High-Kings. Dublin: Four Courts Press. First published 1973.

Carrigan, William. 1905. The History and Antiquities of the Diocese of Ossory. Dublin: Sealy, Bryers and Walker.

Carte, Thomas. 1851. The Life of the Duke of Ormond. Oxford: University Press.

Cherry, John. 1989. Symbolism and Survival: Medieval Horns of Tenure. Antiquaries Journal 29: 111-18. [CrossRef]

Cokayne, George Edward. 1945. The Complete Peerage of England, Scotland, Ireland, Great Britain and the United Kingdom: Extant, Extinct and Dormant. London: The St Catherine Press.

Connaughton, Jill. 2012. Art and Devotions to the Passion of Christ in Ireland 1450-1650. Ph.D. dissertation, Trinity College Dublin, Dublin, Ireland.

Crawford, Anne. 2002. Letters of the Queens of England. Stroud: Sutton Publishing. First published 1994. 
Duffy, Eamon. 2005. The Stripping of the Altars: Traditional Religion in England c.1400-c.1580. New Haven and London: Yale University Press. First published 1992.

Duffy, Eamon. 2011. Marking the Hours: English People and their Prayers 1240-1570. New Haven and London: Yale University Press.

Egbert, Donald Drew. 1935. The 'Tewkesbury' Psalter. Speculum 10: 376-86. [CrossRef]

Fortescue, Sir John. 1869. The Works of Sir John Fortescue, Knight. London: Chiswick Press.

Foster-Campbell, Megan H. 2011. Pilgrimage through the Pages: Pilgrims' Badges in Late Medieval Devotional Manuscripts. In Push Me, Pull You: Imaginative and Emotional Interaction in Late Medieval and Renaissance Art. Edited by Sarah Blick and Laura D. Gelfand. Leiden and Boston: Brill, pp. 227-74.

Gaborit-Chopin, Danielle. 2003. Ivoires Médiévaux: Ve-XVe Siècle. Paris: Editions de la Réunion des Musées Nationaux.

Harding, Vanessa. 1992. Burial choice and burial location in later medieval London. In Death in Towns: Urban Responses to the Dying and the Dead, 100-1600. Edited by Steve Bassett. Leicester: University Press, pp. 119-35.

Hindman, Sandra, and James H. Marrow, eds. 2013. Books of Hours Reconsidered. Turnhout: Brepols.

Kaplan, S. C. 2016. La Légende dorée, Paris, BnF, fr.244-245 (1480-1485): Un manuscript conçu pour Catherine de Coëtivy. In Les Femmes, la Culture et les Arts en Europe entre Moyen Âge et Renaissance, Women, Art and Culture in Medieval and Early Renaissance Europe. Edited by Cynthia J. Brown and Anne-Marie Legaré. Turnhout: Brepols, pp. 197-220.

Keene, Derek, and Vanessa Harding. 1987. Historical Gazetteer of London Before the Great Fire Cheapside; Parishes of All Hallows Honey Lane, St Martin Pomary, St Mary Le Bow, St Mary Colechurch and St Pancras Soper Lane. London: Centre for Metropolitan History.

Kennedy, Kathleen E. 2014. Reintroducing the English Book of Hours, or English Primers. Speculum 89: 693-723. [CrossRef]

Kerns, Lin, ed. 2008. The Secret of Secrets (Secreta Secretorum): A Modern Translation, with Introduction of the Governance of Princes. Lewiston: Edwin Mellen Press.

Kingsford, Charles Lethbridge, ed. 1911. The First English Life of Henry V Written in 1513 by an Anonymous Author Known Commonly as the Translator of Livius. Oxford: Clarendon Press.

Krug, Rebecca. 1999. The Fifteen Oes. In Cultures of Piety: Medieval English Devotional Literature in Translation. Edited by Anne Clark Bartlett and Thomas H. Bestul. Ithaca: Cornell University Press, pp. 107-17, 212-16.

Lentes, Thomas. 2006. 'As far as the eye can see ... ': Rituals of Gazing in the Late Middle Ages. In The Mind's Eye: Art and Theological Arguments in the Middle Ages. Edited by Jeffrey E. Hamburger and Anne-Marie Bouché. New Haven and London: Yale University Press, pp. 360-73.

Leroquais, Abbé V. 1927. Les Livres d'Heures: Manuscrits de la Bibliothèque Nationale. Paris: Macon, Protat Frères.

Lewis, Flora. 1993. From image to Illustration: The Place of Devotional Images in the Book of Hours. In Iconographie Médiévale: Image, Texte, Contexte. Edited by Gaston Duchet-Suchaux. Paris: Centre National de la Recherche Scientifique, pp. 29-48.

Lodge, John. 1754. The Peerage of Ireland or a Genealogical History of the Present Nobility of That Kingdom with Their Paternal Coat of Arms. London: William Johnston.

Louis, Cameron. 1980. The Commonplace Book of Robert Reynes of Acle: An Edition of Tanner MS 407. New York: Garland.

Lowden, John. 2013. Medieval and Later Ivories in the Courtauld Gallery, Complete Catalogue. London: Paul Holberton.

Lumiansky, Robert Mayer, and David Mills, eds. 1986. The Chester Mystery Cycle. Oxford: University Press. First published 1974.

MacHarg, John Brainerd. 1917. Visual Representations of the Trinity: An Historical Survey. New York: Arthur H. Crist.

MacNiocaill, Gearóid, ed. 1992. Crown Surveys of Lands 1540-41 with the Kildare Rental Begun in 1518. Dublin: The Irish Manuscripts Commission.

Mâle, Émile. 1987. Religious Art in France: The Late Middle Ages-A Study of Medieval Iconography and its Sources. Princeton: University Press.

Matthew, E. A. E. 1994. The Governing of the Lancastrian Lordship of Ireland in the Time of James Butler Fourth Earl of Ormond. Ph.D. dissertation, Durham University, Durham, UK. 
McGinn, Bernard. 2006. Theologians as Trinitarian Iconographers. In The Mind's Eye: Art and Theological Arguments in the Middle Ages. Edited by Jeffrey E. Hamburger and Anne-Marie Bouché. New Haven and London: Yale University Press, pp. 186-207.

Morgan, Nigel. 2013. English Books of Hours c.1240-c.1480. In Books of Hours Reconsidered. Edited by Sandra Hindman and James H. Marrow. Turnhout: Brepols, pp. 66-95.

Muir, Lynette. 1997. Playing God in Medieval Europe. In The Stage as Mirror: Civic Theatre in Late Medieval Europe. Edited by Alan E. Knight. Cambridge: D.S. Brewer, pp. 25-50.

Ó Floinn, Raghnall. 1994. The Kavanagh 'Charter' Horn. In Irish Antiquity: Essays Presented to Professor M.J. O’Kelly. Edited by Donnchadh Ó Corráin. Dublin: Four Courts Press, pp. 268-78.

Price-Linnartz, Jacki. 2009. Seeing the Triune God: Trinitarian Theology in Visual Art. Master of Theology dissertation, Duke University, Durham, NC, USA.

Ralph, Karen. 2014. Medieval Antiquarianism: The Butlers and Artistic Patronage in Fifteenth-Century Ireland. Eolas: The Journal for the American Society of Irish Medieval Studies 7: 2-27.

Randall, Richard H. 1993. The Golden Age of Ivory: Gothic Carvings in North American Collections. New York: Hudson Hills.

Ray, Donna E. 2011. 'There is a Threeness about You': Trinitarian Images of God, Self, and Community among Medieval Women Visionaries. Ph.D. dissertation, University of New Mexico, Albuquerque, NM, USA.

Reinburg, Virginia. 2012. French Books of Hours: Making an Archive of Prayer, c.1400-1600. Cambridge: University Press.

Roe, Helen M. 1979. Illustrations of the Holy Trinity in Ireland: 13th to 17th Centuries. The Journal of the Royal Society of Antiquaries of Ireland 109: 101-50.

Rorem, Paul. 2015. The Dionysian Mystical Theology. Minneapolis: Fortress Press.

Ryan, Salvador. 2014. The Arma Christi in medieval and early modern Ireland. In The Arma Christi in Medieval and Early Modern Material Culture. Edited by Lisa H. Cooper and Andrea Denny-Brown. Surrey: Ashgate, pp. 243-72.

Sand, Alexa. 2014. Vision, Devotion and Self-Representation in Late Medieval Art. Cambridge: University Press.

Scott, Kathleen L. 1968. A Mid-Fifteenth-Century English Illuminating Shop and its Customers. Journal of the Warburg and Courtauld Institutes 31: 170-96. [CrossRef]

Scott, Kathleen L. 1996. Later Gothic Manuscripts 1390-1490. London: Harvey Miller Publishers.

Simington, Robert C., ed. 1961. The Civil Survey 1654-1656. Dublin: Stationery Office for the Irish Manuscripts Commission.

Simms, Katharine. 2010. The Barefoot Kings: Literary Image and Reality in Later Medieval Ireland. Proceedings of the Harvard Celtic Colloquium 30: 1-21.

Steer, Christian Oliver. 2013. Burial and Commemoration in Medieval London, c.1140-1540. Ph.D. dissertation, University of London, London, UK.

Stevens, Andrea Rea. 2013. Inventions of the Skin: The Painted Body in Early English Drama. Edinburgh: University Press.

Stow, John. 1908. A Survey of London. Oxford: Clarendon. First published 1603.

Sutton, Anne. 2008. The Hospital of St Thomas of Acre of London: The Search for Patronage, Liturgical Improvement, and a School, under Master John Neel. 1420-63. In The Late Medieval English College and Its Context. Edited by Clive Burgess and Martin Heale. York: Medieval Press, pp. 199-229.

Sutton, Anne F., and Peter William Hammond, eds. 1983. The Coronation of Richard III: The Extant Documents. Gloucester: Alan Sutton.

Swanson, Robert Norman. 2007. Indulgences in Late Medieval England: Passports to Paradise? Cambridge: University Press.

Twycross, Meg. 2008. The Theatricality of Medieval English Plays. In The Cambridge Companion to Medieval English Theatre. Edited by Richard Beadle and Alan J. Fletcher. Cambridge: University Press, pp. 26-74.

Twycross, Meg, and Sarah Carpenter. 2002. Masks and Masking in Medieval and Early Tudor England. Ashgate: Aldershot.

Warner, Sir George F., and Julius P. Gilson. 1929. Catalogue of the Western Manuscripts in the Old Royal and King's Collections in the British Museum. London: Longmans, Green \& Co.

Watney, John. 1906. Some Account of the Hospital of St Thomas of Acon, in the Cheap, London, and of the Plate of the Mercer's Company, 2nd ed. London: Blades, East \& Blades. 
Weever, John. 1767. Ancient Funeral Monuments of Great Britain, Ireland, and the Islands Adjacent. London: W. Tooke. Williamson, Paul. 1982. An Introduction to Medieval Ivory Carvings. London: Her Majesty's Stationery Office.

Williamson, Paul, and Glyn Davies. 2014. Medieval Ivory Carvings 1200-1550. London: Victoria and Albert Museum.

Wylie, James Hamilton, and William Templeton Waugh. 1929. The Reign of Henry V. Cambridge: University Press.

(C) 2019 by the author. Licensee MDPI, Basel, Switzerland. This article is an open access article distributed under the terms and conditions of the Creative Commons Attribution (CC BY) license (http://creativecommons.org/licenses/by/4.0/). 
Article

\title{
Printed Pages, Perfect Souls? Ideals and Instructions for the Devout Home in the First Books Printed in Dutch
}

\author{
Anna Dlabačová \\ Leiden University Center for the Arts in Society (LUCAS), Leiden University, P.N. van Eyckhof 1, \\ 2300 RA Leiden, The Netherlands; a.dlabacova@hum.leidenuniv.nl
}

Received: 6 December 2019; Accepted: 9 January 2020; Published: 16 January 2020

\begin{abstract}
This article studies the role of the earliest books printed in the Dutch vernacular in the religious practice of lay individuals and the devout home. Many of the texts disseminated in these early printed books have received little attention and scholars have tended to view them within the sphere of the Modern Devotion, even though often there is no direct link to this religious reform movement. This article attempts to show that the first books printed in Dutch offer an interesting lens through which to study domestic devotion in the Low Countries in the last decades of the fifteenth century. It argues that these books bridged the gap between catechetical instruction and the private home, literally bringing home many of the ideals and instructions that the clergy would have offered in church and thus increasingly 'textualizing' the lives of the late medieval laity. Printers such as Gerard Leeu and his contemporaries acquainted Christians to the use of printed books for personal and practical religious instruction and knowledge and thus paved the way for developments in the sixteenth century.
\end{abstract}

Keywords: Dutch medieval literature; devotional literature; catechesis; religious instruction; lay devotion; incunabula; Gerard Leeu; manuscript and print; Dionysius the Carthusian; Der grosse Seelentrost

\section{Introduction}

With regard to devotion and religion, the impact of the early printing press is still often associated with the Protestant Reformation (Wandel 2016, p. 18; Pettegree 2015; Füssel 2003, pp. 159-93). Recently, Lee Palmer Wandel claimed that until the sixteenth century, knowledge of Christianity was mainly mediated through things, experiences and acts (Wandel 2016, pp. 9-14). For the majority of Christians the Lord's Prayer, the Apostle's Creed, the Ten Commandments, and so on, "were not ink on a page", but sounds. Moreover, "no one claimed a 'catechism' [ ... ] contained all that a Christian ought to know" (Wandel 2016, p. 11). The importance of the printed book during the Reformation cannot be denied. But while 'catechism' is a sixteenth-century word, Michael Milway has noted that sixteenth-century catechisms differ from their late medieval predecessors in style rather than contents (Milway 2000, p. 128, n. 51). Yet relatively little attention has been paid to the many printed books and booklets published in the Dutch vernacular before the start of the sixteenth century that were not pastoralia - works primarily intended to instruct the clergy (see Milway 2000, pp. 126-30). Rather, the works under discussion here instruct lay readers on how to live a pious life and offer both basic and more advanced religious knowledge, even teaching "what every Christian should know" (cf. Wandel 2016, pp. 15 and 22) ${ }^{1}$.

1 On the function and (intended) audience of Middle Dutch catechetical texts and works on moral theology, see (Warnar 1993, pp. 44-49). 
These books are still largely uncharted territory. The only two monographs that discuss a wide range of texts from the Low Countries that can be characterized-in a teleological way-as pre-Reformation catechisms date back to the first years of the twentieth century and were written from a Protestant point of view (Troelstra 1901 and Troelstra 1903). In a 1993 article, Geert Warnar focused on catechesis and "moral theology" for lay men, which resulted from the Fourth Lateran Council of 1215. Warnar discussed predominantly works transmitted in manuscript and indicated that Middle Dutch catechetical texts (from the fourteenth century onward) were intended to function independently from institutional catechetical instruction (Warnar 1993, pp. 48-49). They were mainly aimed at use within the private sphere of the household, especially in the education of youngsters. He characterized these works for a domestic setting as textualized forms of sermons and catechesis. Petty Bange's work on "the moralistic discourse" in the late medieval Low Countries, has brought together an anthology of useful information about moral instruction for lay people in Middle Dutch, both in manuscript and in print (Bange 2007). Bange, however, only included texts available in a modern edition, which significantly narrowed down her corpus, especially with regard to the many devotional texts that appeared in print ${ }^{2}$.

Herman Pleij ascribed the early printed religious literature in Dutch to a dominant role of the clergy, both on the side of production and consumption, and a demand for spiritual self-help fanned up by the religious reform movement of the Modern Devotion (Pleij 1992a, pp. 12-19; Pleij 1992b, pp. 235-44; Pleij 2010, p. 290; Warnar 1993, p. 50) ${ }^{3}$. Pleij deplores the lack of research into printed (religious) works and the role of the clergy, but nevertheless proceeds to posit that the early printing press in the Northern Netherlands was never commercial. According to Pleij commercialization only took off once printers moved south (to Antwerp) where the clergy ceased to be significant (Pleij 1992b, pp. 245 and 248; Pleij 2010, pp. 290-91). Koen Goudriaan problematizes this supposed opposition between North and South (Goudriaan 1992, pp. 203-5). Moreover, while often viewed almost exclusively as the birth-region of the Modern Devotion, religious life and literature in the Low Countries was, perhaps not surprisingly, influenced by various other religious orders and reform movements during the fifteenth century. The influence of the Devotio Moderna with regard to the introduction of the printed book has also been overestimated (Goudriaan 2013a, pp. 582-91).

Although the Modern Devotion stimulated the use of texts as the main tool for spirituality (e.g., Mertens 1993), Goudriaan has convincingly argued that the involvement of the Devotio Moderna in the production of printed books was limited (Goudriaan 2013a). In the fifteenth century, only three religious houses connected to the Modern Devotion and located in the Low Countries ran a printing press-in Brussels, Gouda, and Schoonhoven (near Gouda). Their reason for existence, however, was never the provision of vernacular books to lay people-rather, their core business appears to have been liturgical texts. Sometimes they tapped into popular devotional trends, but their production was subject to commercial laws of supply and demand (Goudriaan 2013a; Goudriaan 2010 on the press of the Gouda Collaciebroeders; Kock 2002), as with the business of secular printers. Secular, commercially operating printers collaborated with members of the secular and regular clergy. Again, the main suppliers of religious and devotional texts to these shops were not the Modern Devout, but the reformed Franciscans (Franciscan Observants)—the Carthusians came in second place (Goudriaan 2013b, 2016, pp. 280-81). The Church, however, did not adopt a single, centralized strategy towards the new medium. Vernacular devotional books that came on the market were primarily produced by commercially operating printing shops and met a demand for catechetical and devotional texts among the laity ${ }^{4}$.

2 See also her earlier work on the (rather disparate) genre of 'Mirrors': (Bange 1986).

3 Warnar states that the first printers likely thought that Middle Dutch catechetical literature would ensure sales. He also emphasizes the diversity of these kinds of texts, which shows that the various needs of lay people were addressed. Cf. (Mertens 1993, p. 13 and n. 15).

4 A similar situation occurred in Lübeck: (Andersen 2016). 
The focus of the present article is solely on incunabula (books printed before 1501) and offers an incomplete overview of religious instruction in the late fifteenth century household as illuminated by these sources ${ }^{5}$. My aim is to provide a number of examples of texts that early printers made available for the laity and the lay home and, as such, to point to their potential as sources for research into domestic devotion. The first books printed in the Dutch vernacular offer an interesting lens for the study of domestic devotion in the Low Countries in the last decades of the fifteenth century: even though the books themselves (and the surviving copies) are often the only evidence of their role in domestic devotion, the very fact they were printed implies that they met a certain demand amongst lay readership. What did the ideal pious household look like according to Dutch incunabula? In what way did they aim to instruct lay audiences? The discussion of this small sample of texts shows that the supposed caesura between the fifteenth and sixteenth centuries as ages of respectively experientially and textually—-through the printed book—oriented knowledge-transfer is not tenable. The situation in the last quarter of the fifteenth century was more complicated and the present essay hopes to invite further research into the role of commercially produced incunabula in the religious practice of lay people.

In order to meet the ideals and practical instructions given in the printed books discussed below, readers would often need (other) textual materials such as prayer collections, Books of Hours or meditative texts. While my primary focus in this essay is on the former (ideals and instructions), if we are to better understand the role of the religious printed books in lay households, the latter should not be ignored. Printed books with both kinds of texts would often have functioned as objects of domestic devotion, alongside manuscripts. Recent studies, notably by Sabrina Corbellini and Margriet Hoogvliet on the appropriation of religious texts by artisans, have pointed to lay ownership of religious works in France and Italy (Corbellini and Hoogvliet 2013). The texts one would need to live up to the ideals and/or to follow the given instructions could be directly supplemented in the very same book, or readers could find them in other (more specialized) editions. The Ten Commandments, discussed in the first section of the present essay, could be found briefly listed in the Tafel des kersteliken levens (Table of Christian Life), elaborately discussed and supplemented with exempla and prayers in the Der sielen troest (The Soul's Consolation), and yet, as we will see, to comply fully, for instance, with the third commandment (keep holy days), one might also need a Book of Hours, a treatise with an explanation of the Mass and/or a meditative life of Christ. All of these texts became readily available in print through the activity of commercial entrepreneurs.

One of the most popular and voluminous catechetical texts in late medieval printing shops of the Low Countries was originally not written in Dutch or in Latin, but in Low German. The Soul's Consolation was composed around 1350 (the oldest manuscript dates from 1403), possibly by a member of the Dominican order (Schmitt 1959; Palmer 1992, cols. 1030-40; Warnar 1993, pp. 46-47). The extant manuscript transmission of the Dutch translation of The Soul's Consolation is modest: only three manuscripts contain the full Dutch text (Deschamps 1963, 1972, pp. 193-97). The commitment to private lay devotion to which The Soul's Consolation testifies took wings with the first printed edition in the Netherlands, published as early as 1478. The Dutch text remained in print until 1800, which attests to a remarkable longevity of this work and continuity in religious instruction (Schmitt 1959, pp. 32-34, p. 117; Murjanoff 1964, pp. 206-9). Margarete Schmitt, the editor of the modern edition of the Middle Low German text, tried to relate the dissemination of The Soul's Consolation, and in particular the popularity of the Dutch translation in print, to the Modern Devotion. More recent insights into the lack of involvement of the Modern Devotion in printing-as discussed above-show that it is highly unlikely that the Modern Devout were responsible for the editions (Schmitt 1959, pp. 141-42) ${ }^{6}$. Rather,

\footnotetext{
The references to incunabula include the ISTC number, which refers to the (ISTC database).

Schmitt situates the entire text within the sphere of influence of the Modern Devotion. However, since the text predates the movement, it cannot be connected directly to the Modern Devotion. Schmitt next tries to make a case for a strong involvement of the Modern Devotion within the text's dissemination.
} 
the venture was a commercial one. I use this text and the ways it encourages readers to put the Ten Commandments into practice in their daily lives as a starting point for an exploration of domestic devotion in Dutch incunabula. I then elaborate on two central themes that can be distilled from the The Soul's Consolation: prayer and meditation, and family life within a religious household.

\section{Bringing the Ten Commandments Home}

The anonymous fourteenth-century author of The Soul's Consolation collected over 200 exempla. Hence, the text is often presented as an exempla-collection (Deschamps 1972, p. 193: the work consists "almost entirely out of stories and exempla [ ... ]"), but the frame story written per modum dyalogi is at least equally important. Although often overlooked, the dialogue between a father (confessor) and a flexible-gendered child about the Ten Commandments is the most original part of the text, and it organizes and interprets the exempla taken from a variety of sources, including the Bible, Jacobus de Voragine's Legenda aurea, Petrus Comestor's Historia Scholastica, the Gesta Romanorum and many others. It is in the dialogic parts of the text that the father explicitly answers the child's casuistic questions, explains the different ways in which the Commandments can be understood and gives direct instructions on matters of faith and domestic devotion, which is our particular concern here (cf. Palmer 1992, col. 1033).

The first commandment is introduced as "how one should love God" (hoemen god minnen sal) in the rubric. At the start of the chapter, the reader is addressed as follows: "O man, you shall not honor idols or alien gods" (O mensche, du en selste gheen afgoden of vremde goden aenbeden) ((Der sielen troest 1484, a3r-v; Schmitt 1959, p. 5, 11. 1-9) ${ }^{7}$. The father repeatedly warns against any belief in superstition and instructs the child not to keep any books on magic in his possession (Der sielen troest 1484, b1r; Schmitt 1959, p. 18). He supports his teaching with a story about a woman who believed in spirits, that takes on a particularly domestic form: every night the woman would set the table for the spirits and dish them up plenty of food in order to gain protection for her business. The woman only abandoned this ritual after Saint Germain convinced her that these are bad spirits that take advantage of people who believe only half-heartedly in the Christian God (Der sielen troest 1484, a8v; Schmitt 1959, p. 17, 11. 3-23). The faithful are instructed to let their love for God not only prevail above the love for material things-which are only too easily venerated as idols—-but also above the love for their housemates and family members: their parents, wives, husbands, children, friends and relatives (Der sielen troest 1484, b3v; Schmitt 1959, p. 24, 11. 17-27).

In the second chapter, the father tells the reader not to let his mouth get accustomed to swearing in vain as did a knight who cursed by the Lord's eyes in particular. While playing a board game his eyes fell out of their sockets and he stayed blind for the rest of his days (Der sielen troest 1484, c1v; Schmitt 1959, p. 38, 11. 1-15). Equal dangers lie in swearing an oath one cannot keep and in taunting saints, and Mary in particular (Der sielen troest 1484, c2r-c4r; Schmitt 1959, p. 38, 1. 15-p. 44, 1. 28). Jesus' name should be kept in high regard, spoken and heard with great pleasure, since it serves as a weapon against all kinds of evil: "Dear child, if you wish to keep the second Commandment so you shall gladly mention the name of our Lord Jesus Christ and gladly hear [his name] mentioned because the name of God is as virgin honey" [[L]ieve kint, wilste dat ander gebot wel houden, soe selste den naem goods ons heren Ihesus Cristus gheern noemen ende gheern horen noemen want die naem goods is als honich seem] (Der sielen troest 1484, c3v-c5v (Der sielen troest); (Schmitt 1959, p. 44, 11. 29-31)).

At the start of the third chapter, the father instructs the child not to work, nor to let your family work on holy days. Moreover, one should not dance, sing or jump, since one who dances on holy days might just as well plough or do other work (Der sielen troest 1484, d7r; Schmitt 1959, p. 69,

7 I cite the Dutch text according to the 1484 edition published in Haarlem by Jacob Bellaert (Der sielen troest 1484), followed by a reference to the relevant passage in Schmitt's edition of the Low German text (Schmitt 1959). Spelling and punctuation of the Dutch text have been modernized. 
11. 1-22). The father encourages the child not to eat or drink to excess, which is always sinful, but even more so on holy days (Der sielen troest 1484, d8r; Schmitt 1959, p. 71, 11. 33-36). Furthermore, one should go to church on feast days, and make up for what one may have neglected during the week. Above all it is important to hear Mass—not only on holy days, but—if possible—on workdays as well (Der sielen troest 1484, d8v; Schmitt 1959, p. 72, 11. 22-27). The latter lesson is supported by an exemplum about young knight Willem whose life is saved because his father taught him to attend Mass daily (Der sielen troest 1484, d8v-e1r; Schmitt 1959, p. 72, 1. 28-p. 74, 1. 2). This suggests that parents should take responsibility for religious education of this sort. As we will see, other texts do indeed elaborate on this matter.

Apart from hearing God's word eagerly, one should also dedicate oneself to a more private devotion on holy days: prayer. Nigel Palmer has already observed that the chapter on the third commandment in particular contains an instruction for prayer that is important for the history of devotion or Frömmigkeistgeschichte (Palmer 1992, cols. 1033-34). Early printers made these directions and the associated prayers and meditations widely available. As with church attendance, holy days may serve to catch up with prayer skipped during the week (Der sielen troest 1484, e3v-e4r; Schmitt 1959, p. 78, 1. 36-p. 79, 1. 2). The most important message is that the chosen prayer is less important than the mental attitude with which it is performed. You should simply choose the prayer that lends you the most fervor:

Lieve kint, wat gebet dat di alre meest innicheit gheeft dat selste alre liefste lesen ende spreken, het si dat Pater Noster ende dat Ave Maria of dat si een ander ghebet. Ende du selste dy immer vlietigen dair toe dattu innichlike ende mit pure herten bedeste, dattu ander lude niet en hinderste, dattu ymmer wat innicheit of wat geestelicheit ende wat sueticheit voelste, want beter is een Pater Noster mit innicheit gesproken dan een heel souter sonder innicheit (Der sielen troest 1484, e3v-e4r; Schmitt 1959, p. 79, 11. 3-12).

[Dear child, whichever prayer lends you the most fervor that you should read and speak preferably, whether it is the Pater Noster and the Ave Maria or [whether] it is another prayer. And you should always apply yourself to pray with inner fervor and with a pure heart, not to disrupt other people, to always feel some fervor or devotion and some sweetness, because it is better to speak one Pater Noster with fervor than an entire Psalter without fervor]

If you read the hours, you should read them slowly and enunciate each word. You should learn to commend your prayer to Our Lord, and say as many prayers as is within your power, sometimes more, sometimes less, depending on the amount of time you have, but always with fervor (Der sielen troest 1484, e4v; Schmitt 1959, p. 80, 1. 30-p. 81, 1. 4). Ideally, you should first pronounce three Pater Nosters in honor of the Holy Trinity. This instruction is followed by brief descriptions of fifteen moments from the life of Christ-from the Annunciation to the Ascension—and the recommendation to pronounce each of the moments together with a Pater Noster and an Ave Maria, or with two or three of these prayers, depending on how much time you have at hand. Should the fifteen moments be too long, then you should omit part of the list and speak five Pater Nosters and Ave Marias to Christ's five wounds, supplemented with whatever you prefer. And if you do not actually pronounce your prayers out loud, you should at least say a Pater Noster to Our Lord "interiorly" (spreken innentlike) (Der sielen troest 1484, e5r; Schmitt 1959, p. 81, 1. 5-p. 82, 1. 6).

Christ's Passion should be the central focus during prayer. Prayers should be prayed in honor of Christ's suffering, with compassion, and seven times a day (during the canonical hours, i.e., vespers, compline, matins, primes, tierce, sext, none) it should be remembered how Christ suffered for us. The Soul's Consolation provides short meditative texts for each of the hours, starting with those in the evening: the washing of feet for vespers; the arrest of Jesus for compline, and so on. While the father confessor narrates the events in third person, the prayers (originally in verse) that follow allow the devotee to repeat the events in direct speech to Christ (Der sielen troest 1484, e5v-e8v; Schmitt 1959, p. 83, 1. 14-p. 90, 1. 31). The author concludes these 'hours' with the following remark: 
Lieve kint, mit desen vij dage ghetiden selste die mertelie ons heren eren. Duncket di dese vij getide te lanc wesen, so wil ic dy een ander leren dat corter is...

[Dear child, with these seven hours you shall honor the agony of Our Lord. Should you deem these seven hours too long, so I shall like to teach you another [prayer] that is shorter ... ]

What follows is a prayer that focuses on Christ's wounds. Another prayer exercise focuses on four moments at which Christ shed bitter tears (Der sielen troest 1484, e8v-f1v; Schmitt 1959, p. 90, 1. 32-p. 95, 1. 3).

Equally elaborate directions for church attendance follow these extensive, yet flexible and pragmatic instructions with regard to prayer. Naturally, this is something that would take place outside of the domestic sphere. This does not mean to say, however, that church attendance is entirely independent from domestic devotion-after all, preparation for church attendance would take place at home. Nevertheless, a brief summary has to suffice here. When you come to church you should not speak or even whisper. When you come to hear Mass, you shall make good use of your time: you shall pray and weep, and never make yourself comfortable but alternate seated, standing, and laying positions, fall to your knees every so often, make the sign of the cross or beat your chest. The author specifies bodily positions for each part of the Mass, provides an interpretation of the hymn Gloria in excelsis Deo, a translations of Te Deum laudamus and Salve Regina, and prayers for certain moments during Mass, for example during the elevation of the consecrated host (Der sielen troest $1484, \mathrm{f} 1 \mathrm{v}-\mathrm{f} 4 \mathrm{v}$; Schmitt 1959, p. 95, 11. 4-7 and p. 95, 1. 18-p. 100, 1. 6).

After the instructions for Mass, the author-addressing the audience in the person of the father-returns to the topic of prayer and reminds the child/reader to pray as he has taught him/her, and to speak to Mary after s/he has prayed to Christ. An exemplum about a priest who prayed in remembrance of the seven joys of Mary every day introduces the short expositions and rhymed prayers on these seven joyful moments (Der sielen troest 1484, f4v-g4r; Schmitt 1959, p. 102, 1. 29-p. 120, 1. 25). This section again follows the same structure as the elaboration on the seven hours and the four moments when Christ wept: the prayer verses repeat in direct speech what has been explained and narrated, and the similes and Old Testament prefigurations that have been discussed in prose. The second joy also includes an explanation and translation of the hymn Magnificat anima (Der sielen troest 1484, f5v-f6r; Schmitt 1959, p. 104, 1. 33-p. 105, 1. 15). These seven joys 'introduce' various Marian exempla and miracles that are used to underpin the effectiveness of Marian prayer, not only of the Ave Maria (in Dutch or in Latin, however you may have the most fervor-Duytsche of in Latijn, hoe di meest innicheit hebste), but also of the sequence Ave preclara, which you shall hear gladly or read yourself (Der sielen troest 1484, g4v-g5v; Schmitt 1959, p. 120, 1. 26-p. 125, 1. 27).

The fourth commandment is explained as follows: each child who disobeys or causes grief to his parents commits a mortal sin and kills his or her own soul (Der sielen troest 1484, g6v-g8r; Schmitt 1959, p. 126, 11. 1-11). However, one should not obey his parents in things that go against God's will or follow them in their evil ways (quade dinghen). Moreover, one should always help his parents in their needs and remember that they suffered many a broken night for them (Der sielen troest 1484, g8r-h3r; Schmitt 1959, p. 129, 11. 17-18, p. 132, 11. 35-36, p. 133, 11. 19-20, p. 135, 11. 21-27). In turn, parents should manage their possessions wisely and not give everything to their children so as to not be reduced to beggary (Der sielen troest 1484, h3v; Schmitt 1959, p. 136, 1l. 26-30, p. 137, 11. 17-18). It is equally important to help your parents after their death. One of the exempla that supports this statement recounts the story of a woman who, in order to gain material wealth, promised the devil to sleep with priests and other religious persons, to take in poor people and throw them out at night, to chat in church so as to hinder others in their prayer, and never to confess again. Eventually, after her death, her son confesses her sins and does penance for seven years, which frees his mother from the pains of hell. The father advises the child to be as faithful to his own parents' souls as the son (Der sielen troest 1484, h6r-v; Schmitt 1959, p. 142, 1. 25-p. 143, 1. 23).

With good works and a devout life, you should also honor your spiritual father, Jesus Christ (Der sielen troest 1484, h7v; Schmitt 1959, 145, 1l. 11-17). Moreover, to honor the image of the cross 
is to honor Christ himself. The crucifix thus plays an important part in the domestic sphere, as we read in the story about a Christian who rented a house for a year and fixed a crucifix to the wall of his room to which he used to pray. When he moves out, he forgets to take the crucifix off the wall, and the new residents, Jews, torture the crucifix until it starts to bleed. When the blood heals their sick, however, they convert to Christianity (Der sielen troest 1484, h8r; Schmitt 1959, p. 146, 1. 13-p. 147, 1. 5). Because of the protective powers of the sign of the cross-demonstrated by a story about a Jew who does not fall prey to the devil because he crossed himself-it is advisable that every Christian, before going to bed in the evening, say his prayers and make the sign of the Cross (Der sielen troest 1484, i2r-v; Schmitt 1959, p. 150, 1. 17-p. 151, 1. 22). Besides, you should honor your heavenly father with the prayer that Christ himself taught his disciples. Here, the father translates the words of the Lord's Prayer into the (Dutch) vernacular, warns never to impede anyone praying a Pater Noster, and reminds the reader to always pray the Lord's Prayer with the greatest possible fervor (Der sielen troest 1484, i3v-i4v; Schmitt 1959, p. 154, 11. 14-24, p. 155, 11. 34-38). A woman, whom the author brings forward because of her exemplary prayer, can scarcely say a single Pater Noster during an entire Mass due to her zealous and meditative way of praying, as she explains herself:

Ic en can niet anders dan dat Pater Noster ende ic spreket in Duytsche. Dat doe ic daer om dat ic dat verstae wat ic bidde. Ende spreke icket in Latijn soe en verstae ickes niet ende soe en wert daer mi gheen innicheit of. [ . . . ] als ic dat Pater Noster beghin soe spreec ic aldus: "Vader ons". Soe neem ic dese ij. eerste woerden ende begin te dencken daer op dat die hemelsche god mijn vader wesen wil ende dat ic sijn kint werden mach of ic wil ende ic sijn erfnaem wesen sel. Ende dat mi die hemeslche vader so grote lieft ende trouwen bewesen heeft meer dan ye vader sijn kinde ende dat hi om mi een mensche gheworden is ende om minen wil soe groot armoede geleden heeft [ ... ] ende te lesten leet hi om minen wille den bitteren doot. [ ... ] Als ic dit bedencke soe ghevet mi God bi wilen soe grote innicheyt ende soe grote soeticheit in mijnre herten dat ic een heele misse toe brenghe inden enen woerde "vader mijn". Daerna bedencke ic wat daer nae volget [ ... ] soe bedenc ic alle die woerden die in dat pater noster sijn, so en kan ic onder eenre misse nauwe een pater noster spreken [ ... ] (Der sielen troest 1484, i5r-v; Schmitt 1959, p. 156, 11. 2-35).

[I only know the Pater Noster and I speak it in Dutch. I do that so that I understand what I am praying. And [if] I speak it in Latin, I do not understand it and I do not get any devotion thereof. [ . . . ] when I start the Pater Noster I speak as follows: "Our Father". And I take these first two words and start to think thereupon that the heavenly God wants to be my father and that I may become his child if I want and be his heir. And that the heavenly father showed such great love and faith, more than a father ever [showed] his child and that he has become a human being because of me and for me has suffered such great poverty [ ... ] and eventually he suffered the bitter death for me. [ ... ] When I consider this, God at times gives me such great devotion and such great sweetness in my heart that I spend an entire Mass in the only word, "Our Father". Then I recall what comes next [ ... ] in this way I recollect all the words of the Pater Noster and so during a Mass I can barely speak one Pater Noster [ ... ]]

This description of exemplary prayer is instructive and allows us to form a clearer picture of what the author means with his constant emphasis on fervor: a meditative, and visually oriented form of prayer. This exemplum also prompts the child to inquire about the relationship between the vernacular and the language of the Church and to ask whether the Pater Noster is "as good" (soe goet) in Latin as in Dutch. According to the father, a book is as good in the hands of a child as it is in the hands of a priest, but it is more profitable for the latter. Similarly, the Pater Noster in Latin is as good in a priest's mouth as in the mouth of a layman, but a priest who understands the words may receive more fervor thereof than a layman. Moreover, Jesus did not teach the prayer in Latin, but in the language of the Jewish lands-had he lived in the Low Countries, he would have taught the Pater Noster in Dutch 
(Had hi in duytschen landen gheweest, hi had duytscher tale gheleert). Nevertheless, should you prefer Latin that is fine too (Der sielen troest 1484, i5r; Schmitt 1959, p. 157, 11. 6-27).

The child further interrogates the father: is the Lord's Prayer sufficient, or should one's religious practice also include other prayers? The father draws an analogy with a domestic scene: if you come to the table and you find bread and wine only, you will not die of hunger, but it is not enough to satisfy you. The sustenance of the soul is ardent prayer, and the prayer should be as manifold and colorful as the foodstuffs that you eat. Apart from the Pater Noster you should say "the Ave Maria, the Creed, the Seven Psalms, the Book [=the Hours] of Our Dear Lady and of Our Dear Lord, of his Passion, of the Holy Cross, and of the five holy wounds" (dat Ave Maria ende Credo in deum ende die vij psalm ende den boeck van onser vrouwen ende van onsen lieven heer, van sijnre marteli, van den heylighen cruce ende vanden heilighen vijf wonden) (Der sielen troest 1484, i5r-v, quote on i5v; Schmitt 1959, p. 157, 1. 28-p. 158, 1. 17). The father provides the text of five prayers (to Christ, Mary, your guardian angel, your apostle and all apostles, and for the consolation of all Christians) and instructs the child to pray for the deceased when walking across a graveyard (Der sielen troest 1484, i5v-i7r; Schmitt 1959, p. 158, 1. 18-p. 161, 1. 34).

The fifth commandment not only applies to actual murder, but equally to 'moral' death caused by the tongue: rumors can kill over the distance of more than a hundred miles and take the soul of many a man (Der sielen troest 1484, 13r; Schmitt 1959, p. 188, 11. 27-36). If one is in charge of others, one should guide his subjects and children so that they do not corrupt their souls (Der sielen troest 1484, 14v-15r; Schmitt 1959, p. 191, 1. 33-p. 192, 1. 4). Adultery, the sixth commandment (Ex. 20:14 thou shalt not commit adultery), may not be committed in your home, yard or anywhere on your property (Der sielen troest 1484, 16r; Schmitt 1959, p. 196, 11. 1-13). Living a chaste life also involves overcoming bodily temptations and chastising your body "with hunger and with thirst, with waking and prayer, with uncomfortbale clothes and hard beds, with rods and with scourges" (mit hongher ende mit dorst, mit waken ende mit bidden, mit scerpen clederen, mit herder legheren, mit roeden ende mit gheselen) (Der sielen troest 1484, 17r; Schmitt 1959, p. 198, 11. 18-22). If you want to avoid impurity, you should keep away from the company of women (Der sielen troest 1484, 18r; Schmitt 1959, p. 206, 11. 30-35). Furthermore, the father advises the child to be a manlic man (a valiant man) and not to succumb to a bad woman because she will make you into a fool, the fate that Sardanapalus and Solomon suffered. You may, however, gladly follow the counsel of your good wife (Der sielen troest 1484, m3r-m6v; Schmitt 1959, p. 207, 1. 5-p. 215, 1. 3). The child, now taking on the role of a woman, asks what she can do if she wants to live a chaste life but her husband does not agree. She always has to follow the will of the man and keep a chaste soul-if he does something to which he is not entitled, he will receive what is coming to him, although she shall always reprimand him. If you lead a chaste life-which demands a true battle against temptations and with your own thoughts-your soul will become God's temple and a home for the Holy Ghost (Der sielen troest 1484, m7v-m8r; Schmitt 1959, p. 216, 1. 29-p. 217, 1. 9).

The chapter on the seventh commandment (Ex. 20:15, thou shalt not steal) contains many concrete queries about unjust property, which also affect the home and devout actions. For example, it is forbidden to keep a thief in your home, because that would make you complicit to theft (Der sielen troest 1484, n1v; Schmitt 1959, p. 219, 11. 2-3). The child, again taking the role of a woman, asks whether she may steal from her husband in order to give alms. Of the goods that belong to both her and her husband she may take a little, answers the father, as long as it does not harm her husband or upset him. Only in the event of an emergency-when someone is in danger of dying of starvation-may she break these rules. The same applies for children and servants. Women who actively contribute to the income of the household may give alms more freely than women who do not work (Der sielen troest 1484, n1v; Schmitt 1959, p. 220, 11. 10-29). Interestingly, a child may be stolen by a Christian from a heathen-in war and in peace time- because the Christian does not harm the child's father: he will bring the child to God whereas the heathen would have brought it eternal damnation (Der sielen troest 1484, n2r; Schmitt 1959, p. 221, 11. 22-27). Within the context of the ninth commandment, thou shalt not covet your neighbour's goods, the child asks whether a woman may possess and use goods that her husband has gained unjustly. She may only do so in dire need. If it is unclear which goods have been gained 
unjustly and which have not, then husband and wife should divest themselves of all their possession: it is preferable to go begging from door to door than to have unjust goods. Should the husband not consent, the wife may go to the bishop (Der sielen troest 1484, o3v; Schmitt 1959, p. 241, 1. 8-p. 242, 1. 9). Money or other goods won with gambling should be given away as alms. Gambling is a great sin and those who allow it in their homes sin as well (Der sielen troest 1484, o4v; Schmitt 1959, p. 243, 1. 17-p. 244, 1. 19).

The tenth commandment, thou shalt not covet your neighbour's wife (Ex. 20:17), is said to be equal to the ninth, and first focuses on man's desire for another's wife. The story about King David and Bathsheba is followed by the stories of King Abimelech, Abraham and Sarah, and Isaac and Rebecca (Der sielen troest 1484, p1r-1v; Schmitt 1959, p. 254, 1. 1-p. 257, 1. 16). These Old Testament-exempla are followed by a rather crude and cruel story aimed at women. A knight and his household witness that the wife of the stork who has his nest in their grounds consorts with another male whenever the stork is out in search of food. Usually, the female washes herself in a nearby pond after her lover's visit, but one day her access to the water is obstructed. When her husband notices what she has been up to, he calls for a number of other storks that stab her to death. The author concludes: "This shall be an example to all women" (Dit sel een exempel wesen alle vrouwen) (Der sielen troest 1484, p1v; Schmitt 1959, p. 257, 11. 17-29).

The Soul's Consolation concludes with an elaborate narration of King Alexander, who is portrayed as the epitome of a bad life (Der sielen troest 1484, p2r-p7v; Schmitt 1959, p. 258, 1. 8-p. 271, 1. 31). Here, in this life he did not observe the Ten Commandments, and now he has to obey the devil. A last direct instruction to the child summarizes the goal of the text: keep to the Ten Commandments so you will not end up as Alexander but reach eternal life. Thanks to the activity of early printers, the text's many instructions on how to implement the Ten Commandments into daily life and the household became available as printed words. Many of the issues discussed in The Soul's Consolation were taken up in other works that appeared as printed books in the last quarter of the fifteenth century. The following sections focus first on prayer and meditation and subsequently on family life within the devout home.

\section{Prayer and Meditation}

The Soul's Consolation not only contains an extensive discussion of prayer but also material one could actually use to live up to the instructions: the short meditations and prayers on the (seven hours of the) Passion, the four moments at which Christ shed bitter tears, the seven joys of Mary, a Dutch translation of the Pater Noster and several other texts and prayers to 'spice up' religious practice (see above, Section 2). Other texts that were disseminated in printed form offer many interesting parallels. Discussing the contents of these works yields a more complete picture of what incunabula in Dutch offered readers with regard to instruction and texts, how they functioned and even (potentially) interacted. Dionysius the Carthusian gives identical advice when discussing the third commandment: on holy days one should be fervent in prayer and spend the day with psalms, hymns and spiritual songs (Carthusiensis 1479, 55r-v). The words at the end of his Van den loveliken leven en de staet der echten [On the laudable life and state of marriage] also resonate with The Soul's Consolation's quest for attentive prayer in which less is more and a thorough understanding of the prayer-through the use of the vernacular-is vital:

Ende want beter ende saliger is luttel woerde des gebeets mit verstant ende andacht te spreken dan veel sonder verstant ende andacht. Daer om ist nutter ende oerberliker dat die ongeleerde menschen lesen hoer pater noster ende ander gebeden des ghelijcs in Duutscht dan in Latijn. (Carthusiensis 1479, 115v)

[And because it is better and more blissful to speak a few words of prayer with understanding and attention than many without understanding and attention. Therefore, it is more useful and profitable that uneducated people read their Pater Noster and other prayers as well in Dutch than in Latin.] 
The Tafel des kersteliken levens [Table of Christian life] instructs the reader in five points on how to lead a correct Christian life (Tafel 1478, A2r; edition of the Low German text based on Tafel c. 1475 in Bahlmann 1894, pp. 63-75, see also pp. 19-20z). After he wakes up in the morning, he is to contemplate that God has created him in His image. He should read at least one Pater Noster and one Ave Maria "with fervor of his heart" (wt ynnicheit sijns herten) "on his knees in front of his bed" (op sinen knyen voer sinen bedde), and plan to keep to the Ten Commandments. Additionally, prayer booklets such as Die gulden Litanie vander passien Cristi [The Golden Litany on the Passion of Christ] offer readers "a good blessing that one should say in the morning when one gets up" (Een goede zegheninge die men des morghens segghen sal als men op staet), directed at the Father, the Son and the Holy Spirit (Die gulden Litanie 1492, d7r-v). Afterwards, in church, he should hear Mass devoutly, contemplate Christ's Passion, and read his five wounds with five Pater Nosters and Ave Marias (Tafel 1478, A1r-A1v). Other texts, such as Dionysius' Van den loveliken leven and the Korte lering uit de schrifturen der heiliger leraars [Short teaching from the scriptures of holy teachers], elaborate on behavior in church and stress the importance of a devout, attentive and interiorized experience instead of gaping or walking around and chatting as if one were at the market (Carthusiensis 1479, 115v; Korte lering 1481-1482, h7v-i5r, has chapters on "How you should go to church" (Hoe ghi te kercken selt gaen) and "In church" (Inder kercken), which give similar advise). An interiorized experience of the Mass could be achieved using one of the many printed booklets that encourage readers to meditate on the Passion by offering so-called allegorical-rememorative explanations of the ritual (e.g., Boecxken 1479; see below Section 4; Dlabačová 2019).

At noon, before lunch, according to the Tafel, a good Christian should thank the Lord, and read, consciously and with devotion, a benediction or a Pater Noster. After the meal, he should say grace, give alms and, should it be within his powers, he should welcome a poor man once a week at his table (Tafel 1478, A1v). The fact that Dionysius enumerates the failure to say grace before and after the meal as one of the points one might want to address during confession shows that this was indeed considered standard practice (Carthusiensis 1479, 86v). Before going to bed he should reiterate his actions during the day, repent any sins and do penance according to the advice of his father confessor. When going to sleep he should first read three Ave Marias, again on his knees, pray to Our Lady to keep him safe from the devil's temptations during the night, and commend himself to the Lord, Mary, and to his Holy Angel and Apostle. This shall make him sleep peacefully and, in the morning, wake up with ever more commitment to honor God (Tafel 1478, A2r). The materials one would need to conform to the given instructions-and more-are also provided. There are translations into Dutch of the Pater Noster, the Ave Maria, and the Creed. In the next section-referred to as the register (dat register)-the author lists, amongst others, the external and internal senses, the four cardinal virtues, the Four Last Things, the seven deadly sins, the seven works of mercy, the seven gifts of the Holy Ghost, the seven sacraments, and the Ten Commandments (Tafel 1478, A2r-v).

Dionysius the Carthusian in his Van den loveliken leven gives very similar advice for (married) lay people in search of a devout life: they should pray every morning, and moreover, everything one does during the day should be commended to God. Therefore, it is advisable to pray or contemplate before going off to do bodily work, and once at work you should often direct your heart to God with short prayers and devout thoughts. Worldly work done in honor of God is virtuous and can earn eternal life: while a good, devout priest earns eternal life with reading his hours, farmers, merchants, artisans, lawyers and officers earn it through their profession as long as they work out of love for God. Before going to bed all Christians should first examine their conscience and how they fared that day (Carthusiensis 1479, 69v-71r). The anonymous Glose ofte expositie opten psalm Miserere [Gloss or Exposition on the Psalm Miserere] (the third Penitential Psalm) advises to let your consciousness be a book in which you note down all your sins during the day. At night, when you go to sleep and no one bothers you, you may open the book and read all sins you have committed with words, deeds, and thoughts (Glose 1491, b2v-b3r). King David is said to have washed off his sins with tears at night (Glose 1491, a4r). According to Dionysius, after the examination, one should say some prayers, 
especially the Lord's Prayer and Ave Maria, make the sign of the Cross, entrust oneself to God and enter the bed. Once in bed one should contemplate blissful matters, especially Christ's Passion, until one falls asleep (Carthusiensis 1479, 69v-71r). Moreover, those who can afford to do so should also go to vespers and compline in the afternoon and in their bedroom they should focus on God in their prayer or contemplation, "and study something good if they are educated" (ende wat goets studeren is dat si gheleert sijn). The remark that this applies more to the rich and noble than to poor and working people (Hier toe sijn die rijcke ende edele luden meer verbonden dan die armen ende arbeyts luden) points to differences in (the demands for) devotional practices between social classes (Carthusiensis 1479, 73v).

Both the Tafel and Dionysius prefer the bedroom as the place of prayer. Quoting from Matthew 6:1-6, Dionysius stresses that prayer should take place in the bedroom, with the door closed (cf. on the bedroom in Jewish prayer practice: Kohn 2019, Section 4): Christ advised not to pray in public because there one might seek praise from others, and because a secret, quiet place encourages devotion. This does not mean that one cannot pray elsewhere. When praying in church or somewhere else where others are present, you should not pray or sigh loudly, beat your chest more than others, or show any sign whatsoever of exceptional devotion so as not to unsettle others' prayer and resemble the hypocrites (Carthusiensis 1479, 39v). Good Christians should not only pray for themselves, but also for everyone, because one who prays for everyone benefits from the profits of everyone's prayer. Therefore, according to Dionysius, all true Christians should pray daily for the unity, peace, and reformation of the Church (Hier om sel alle waer kersten mensche alle dage bidden om eendrachticheit ende vrede ende der reformacien der heyligher kerken) (Carthusiensis 1479, 68v-69r, quote on 69r).

A one-quire leaflet that might have been useful in accomplishing the daily examination of the consciousness and subsequent atonement that both the Tafel and Dionysius mention, is the Lering van berouw en vergiffenis van zonden [Teaching on repentance and forgiveness of sins]. This text instructs the reader to go into an appropriate place, either in church or at home, to consider ten or twelve of your sins that burden your heart the most, and then to "think in your heart or speak as follows" (peysen metter herten ofte spreken soe hier na volghet) (Lering 1491-1492, a1r). What follows is a prayer script that starts with the formula "O gentle Lord Jesus Christ, you know that I have committed horrible and ugly sins with my evil will in such and such place and at such and such time with such and such persons". In the remainder of the text, the votary asks repeatedly for forgiveness while expressing his regret, considering aspects of Christ's life, and expressing his intention to better his ways (Lering 1491-1492, a1r-a3v). This text was also incorporated at the end of the edition of the Exposition on the Psalm Miserere, which, by example of King David, aims to bring readers to knowledge of and penance for their sins. It includes a special exposition of the psalm Miserere in the form of a prayer, which one should read once a day-or two or three times or at least once a week (of twe of drywerf of ten minsten eens ter weken)-in order to receive forgiveness for your sins (Glose 1491, d1r-e1r; Lering 1491-1492, e4r-e8v). Printed booklets such as these may well have found their way to late fifteenth-century bedrooms and assisted readers in their evening rituals. To find out more about repentance, one could consult a text such as Van den seven sacramenten [On the seven sacraments] where one would find an answer to questions such as "What is repentance?" and "What brings a person to repentance?" (Van den Seven Sacramenten $1484, \mathrm{a} 5 \mathrm{r}-\mathrm{a} 7 \mathrm{v})$.

After a section that focuses on the role of parents in the upbringing of their children (see below, Section 4), the text of the Tafel comes to a close (Hier eyndet die tafel des kersteliken levens). The printer, Gerard Leeu (d. 1492), or the editor of the text, however, added a poem in praise of the Holy Sacrament, the Lof van den Heiligen Sacrament. The poem was written by the famous Bruges rhetorician Anthonis de Roovere, who composed it back in 1456-57. Thus, the edition combines a text previously published in Cologne around 1475 (the Tafel) and a poetic reflection on the nature of the Sacrament. In view of the importance of De Roovere as a famous poet in his own right, this final part has attracted the attention of literary scholars, who write about the publication in terms of collaboration between the poet and Gerard Leeu (Oosterman 2018b, p. 160). Although it is true that Leeu had a connection with Bruges, it seems difficult to imagine that De Roovere would have advocated for the publication of his most 
famous work-by then a more than twenty-year-old, widely disseminated classic-as an anonymous addendum to a catechetical work transferred into Dutch from Low German. Together with the Tafel the poem would have functioned as a prayer, likely in preparation of the Mass, and could be read in church or at home. As such, Leeu's edition is an excellent example of how incunabula further bridged the distance between the private domestic sphere and the Church and could bring home devotions and devotional texts such as prayers in the most literal sense. De Roovere's praise of the Sacrament was also placed on the wall of a church (in handwritten form) and thus functioned within the public space (Mareel 2017, pp. 237-38 and pp. 243-48). Incunables such as the Tafel were intermediaries in this sense, not only between the clergy (priests) and lay people, but also between public space and people's individual homes.

Leeu published another set of poems attributed to de Roovere on the History of Salvation, the life of Christ, and the Four Last Things (Death, Judgement, Hell, Heaven) — not as a book, but as single sheets that could be cut up and either hung on the wall or held in the hand (Geirnaert and Lievens 2018; Oosterman 2018a and Oosterman 2017). These poems were likely used as decoration in the home while also functioning as objects of devotion, giving residents the opportunity to engage in prayer and meditation on Christ's life (cf. Mareel 2017 and Areford 2010, pp. 1-14). The images in the form of woodcuts that accompany each prayer would have enhanced devotion and the visual aspect of prayer, a characteristic of prayer and meditation emphasized in the description of the ideal prayer in for example The Soul's Consolation (see above, Section 2). Leeu's Rosarium Beatae Virginis Mariae editions reflect briefly on the function of woodcut images in relation to the articles or prayers that comprise the Rosary: "Each article has its own image, depicted in front of the eyes, in order that it arouses ever-new devotion in anyone looking at the image and reading the article" (Habet autem quisque articulus suam propriam figuram ante oculos depictam, ut ipsa nouam semper atque nouam figuram uidenti et articulum legenti afferat deuotionem) (Rosarium 1487, a5v-a6r; Dlabačová 2020b). The woodcuts Leeu used to illustrate De Roovere's poems would have had a similar function in the meditative practice of the votary. Gerard Leeu used the same woodcuts in a number of works that facilitated a daily meditation and prayer on Christ's life and the Four Last Things, one of which (the Devout Hours on the Life and Passion of Jesus Christ) its author explicitly directed at lay people juggling work and attention to their spiritual life and religious practice. He 'reinvented' the Book of Hours for those who "who live in the world and participate in the active life [and] spend a lot of time bustling about their livelihood, trade or business, for which reason they do not have the time or the strength to keep the seven [canonical] hours" (Dlabačová 2017, paragraph 8; Dlabačová 2020a).

The centrality of Christ's Passion and the Four Last Things to daily (domestic) devotion is well illustrated by the last chapter of Dionysius' Van den loveliken leven, where he states that all Christians should contemplate Christ's Passion once a day. Furthermore, every Christian should daily "weigh and notice how insecure, deceptive and short this live is" (te wegen ende te merken hoe onsekerlijc bedriegelic ende cort dit leven is), how after death he will be judged by God, how "intolerable and most heavy the pains of hell are" (hoe onverdrachlic ende alre swaerste die pyne der hellen sijn), and contemplate "how indescribable the beatitude and glory of the saints in the realm of the heavens is" (hoe onspredelic dat is die salicheyt ende die glorie der heylighen inden rijke der hemelen) (Carthusiensis 1479, 109r-v). Despite the claim that the heavenly joys are indescribable, a brief description of heaven follows, and short descriptions are given of the Passion and the other Last Things. Still, a book such as the already mentioned Devout Hours or a copy of Die vier uterste [The Four Last Things], which Leeu first published in 1477, would facilitate more in-depth meditations for readers who wanted to follow Dionysius' advice (Die vier Uterste 1477; Devout Hours 1482-1483).

Another work that stresses the importance of books with meditative prayers on Christ's life is the so-called Spiegel des kersten gheloefs, written around the middle of the fifteenth century. Leeu finished printing the text on 1 September 1478, only two weeks after he published the Tafel (Spiegel 1478). Like the Tafel, the Spiegel was previously published in Low German in Cologne by Johann Koelhoff the Elder (Spiegel c. 1477). The text is also known under the title Fundament van der kerstenre gheloven and based 
on sources such as the Compendium theologicae veritatis by Hugo Ripelin of Straatsburg (1210-1270) (Pansters 2007, pp. 99-101). It contains information similar to the Tafel, especially its so-called register (Spiegel 1478, a4r). In the Spiegel, many of the lists are expanded with explanations of each point, as is for example the case with the Creed in Dutch. When discussing the fourth article-the article that relates to Christ's Passion, "I believe that Jesus Christ the Son of God suffered torture from the Jews under Pilate" - it is said that "this point brings before our eyes the Passion and the suffering, and the bitter death" of Our Lord "so that we will always carry that death in our hearts and thank, and praise, and love him for that. If you shed tears while meditating on Christ's suffering, that is a good sign and those tears are salvific" (Spiegel 1478, k3r).

The printer, Gerard Leeu, may well have intended the editions of the Tafel and the Spiegel to be read together-the texts complement each other-and apparently readers did follow this suggestion: two extant copies of both editions were originally bound together (London, British Library, IA.47309 and The Hague, Museum Meermanno Westreenianum, 001 E 049). While the latter Sammelband belonged to a female religious community in Utrecht, the booklets with the Tafel themselves might well have played a role in domestic devotion just as the illustrated meditative prayers mentioned above. The short prologue on the first page tells (potential) buyers that this text encompasses all that a good Christian should know, and all good Christians should therefore have a copy in their homes for themselves, for their children, and members of their household (Tafel 1478, A1r).

A translation with extensive instructions for meditation to accompany each verse of the Lord's Prayer, not unlike the exemplary prayer of the woman who could barely read a single Pater Noster during Mass discussed in The Soul's Consolation, is included in the first chapters of the Boecxken van der officie ofte dienst der missen [Booklet on the office or the service of the Mass] (first published by Leeu on 20 July 1479). These chapters were later incorporated into the Korte lering, which later still was published as the first part of the so-called Spiegel der volcomenheit, not to be confused with Hendrik Herp's Spieghel der volcomenheit (Boecxken 1479; Spiegel 1488). As in The Soul's Consolation a thorough understanding of the meaning of the Lord's Prayer is paramount: only once one has memorized the meaning of the Pater Noster may he read the prayer "after the letter" and fully appreciate it with ardent desire (Korte lering 1481-1482, h4v). The later, extended text (Korte lering and Spiegel der volcomenheit) starts with an exercise on the life of Christ for the seven Hours. There is also a section on what one should read (Vanden daghelicsen lesen [On daily reading]). Although according to the text no general rule can be set for religious reading, since people have different needs and schedules, and it is always advisable to read the Short Hours of the Cross, each hour at the correct time, supplemented with a Pater Noster with considerations on the event of the Passion specific for that hour (Korte lering 1481-1482, g6v; similar reading instructions are given in a text written for a lay woman and transmitted in manuscript only: De Jong 2018). Those unable to read the Short Hours of the Cross can stick to seven Pater Nosters "with remembrance of the Passion" (met ghehoechnis der Passien). However, to make the most of one's religious reading, it is paramount to consult a smart, discerning father confessor for personal, customized advice (Korte lering 1481-1482, g6v-g7r).

The importance of a good father confessor is also recommended in the Twispraec der creaturen [Dialogue of creatures], a Dutch translation of the Dialogus creaturarum. This collection of fables is believed to have been written in northern Italy towards the end of the fourteenth century (Gheraert 2015). Although the prologue points to preachers first when discussing the intended audience, lay readers are not excluded:

Hier om is dit yeghenwoordighe boec nut ende profitelic allen predikers ende allen anderen goeden verstandighen menschen yeghen die verdrietlicheyt des herten der gheenre diet horen ofte lesen. Op dat also ouermits genoechlicheyt der soeter materien dye herten der simpelre menschen getoghen mochten warden tot hogher dinghen in doechden te soeken ende te verstaen (Gheraert 2015, a2r)

[Therefore, this present book is useful and profitable for preachers and all other good, capable people against the sadness of the heart of those who hear or read it. So that through the 
pleasantness of the sweet material the hearts of the simple people may be drawn to search for and understand higher things in virtues.]

In one of the stories recounted by the author, a monkey seeks a cure for her sick cub. She only finds it after a visit to the skillful Master Macer, the master of all herbs, who fixes a medicine from the roots of the plantain ${ }^{8}$. The moralization tells readers to proceed in the same way when seeking their soul's salvation: they should turn to a learned and experienced priest and father confessor who can provide customized medicine (Gheraert 2015, e4v-e5r). In his Der zondaren troost of Spiegel der conscientien [The Sinner's Consolation or Mirror of Conscience], the Franciscan Observant Johannes of Reimerswael compares the soul to the body in order to explain to the "simple, uneducated and good Christians" (simpel ongeleerde ende goede kerstene menschen) how sin corrupts the soul. Moreover, since the soul is more precious than the body one should care more about choosing a 'Master of the Soul' than a good physician (Reimerswael 1492, b3r-v and g6r).

However important religious reading, prayer and contemplation may be, it is nevertheless essential to relax every now and then. In the words of Dionysius: "Just as man cannot always do bodily work, so he cannot always be in spiritual exercise" (Ghelikerwijs als die mensche niet en mach stadelijck lichamelijck arbeyt doen, alsoe en mach hi niet altoos in gheesteliker oefeninghe wesen) (Carthusiensis $1479,98 v$ ). To provide the soul with rest, she must be entertained with honorable and moderate games. The same thoughts are expressed in the prologue of the Twispraec der creaturen where the story of John the Evangelist is recounted: just as the bow of a hunter would be weakened by bending it constantly, so man has to withdraw from contemplation every now and then in order to reach new heights with regained strength (Gheraert 2015, a2r).

\section{Family Life in a Devout Home}

All of the religious exercises discussed in the previous section and disseminated in printed form in the late fifteenth century were meant to secure the soul's salvation and to-as the father tells the child in The Soul's Consolation-prepare the soul as a temple for God (see above, Section 2). In the Der zondaren troost, written in 1492 and published by Leeu within a week after completion, Johannes of Reimerswael writes about the "house of our soul" (huys onser sielen) which should be made free of all deadly sins (Reimerswael 1492, n5r) ${ }^{9}$. He describes the soul as a home with three rooms (memory, intellect, and will) (Reimerswael 1492, o5r). To let the reader fathom how profound the purification of the soul should be, the Boec van der biechten [Book on Confession], published together with a text by the same author as On the Seven Sacraments (see n. 11), encourages readers to think how sedulously they would prepare their home if a king or bishop were to visit: they would clean so diligently that the eyes of the high ranking individual would not meet anything that could displease them (Spiegel 1480, aa1r). In order to prepare the soul for God, then, one should act as if cleaning the house: "You should do as one who sweeps his house: he searches around all corners and whatever impurity he finds therein he wipes it all out." (Du sulste doen ghelikerwijs als een die sijn huus vaghet: die soect alle die winckelen omme ende wat hij onreyns vindet daer in, dat vaghet hi al wt) (Spiegel 1480, aa1v).

Just as God's spiritual home, i.e., the soul, the actual physical home should indeed also be kept pure and free of sin. Several early printed books in Dutch show that the inner and outer home converge. Gambling and adultery have no place in a devout home, nor should you provide shelter to criminals (Der sielen troest 1484, 16r, n1v and o4v, see above, Section 2). Pilgrims and the poor, on the other hand, should be given refuge in a devout home. The father or mother should prepare a sleeping place for them in an appropriate location of the home (Carthusiensis 1479,68r). Furthermore, a devout home should be free of magic. The Soul's Consolation advised against keeping books on magic in your

8 The name likely refers to Macer Floridus, also called De viribus herbarum, an eleventh-century didactic poem and one of the standard works on herbal medicine in medieval Europe.

9 On the soul or heart as a home that should be prepared for God see (Falkenburg 2001 and Klinkenberg 2007). 
home. Similarly, Dionysius the Carthusian, in the discussion of the Ten Commandments in his Van den loveliken leven, advises against the use of texts for magical purposes. Those who seek advice from fortune-tellers who then help them "with apples in which has been written, and with notes to hang around the neck, with odd magical scripture" (mit appelen der in gescreuen is, ende met briefkens anden hals te hangen, mit vreemden carecteren), act against the first commandment (Carthusiensis 1479, 51r). On the Seven Sacraments lists five years of penance for those who mark their home with signs of magic or sorcery (Van den Seven Sacramenten 1484, d8v).

On the other hand, (printed and handwritten) books that contained texts that would assist the faithful in preparing their soul as a home for God were welcomed into the physical household and many of the editions discussed here would have functioned as domestic devotional objects. The walls and furnishings of the family home could serve various devotional functions: they could support printed prayers and images such as the ones printed by Leeu, a crucifix, but the name of Jesus would also inspire a devout home. Various media complemented each other when it came to fifteenth-century domestic devotion, and the printed book played an increasingly important part in it. Again, the contents of these works give a good impression of the kind of instructions made available in print. Dionysius ends his discussion of the Ten Commandments with remarks adopted from Deuteronomy (11:18-20): "The words of the Lord shall be in your heart and you shall tell them to your children and you shall remember them when you sit at home ... and you shall write them in a book and on the door of your home" (Die woerden der heren sellen wesen in dijnre herten ende du selstese vertellen in dinen kinderen ende seltese ghedencken sittende in dinen huse... Ende du selste scriuen in een boeck, ende inden doeren dijns huses) (Carthusiensis 1479, 64r-v). Similarly, in Vander kersten ewe [On Christian Law], published in print as Spieghel ofte reghel der kersten ghelove [Mirror or rule of Christian faith], readers are advised to paint the sweet name of Jesus in the places of their home where they come most often, because Jesus' name is powerful enough to protect all world (Bange 2000, p. 93; Dlabačová 2014, p. 90). The Soul's Consolation already mentioned this quality of the Lord's name and advised to speak and hear it often. At the end of one of the meditative lives of Christ first published by Gerard Leeu we find a song "on the most sweet name Jesus Jesus Jesus" - a vernacularization of the hymn Ihesu dulcis memoria—preceded by a woodcut of the IHS-monogram (Tboeck 1487, oo4v-oo6r; on this edition see Dlabačová 2008, pp. 330-46) ${ }^{10}$. A prayer on Jesus' name at the end of the Devout Hours, to be said everyday together with the Creed and accompanied by a woodcut of the Christ child, would ensure a devotee's daily 'dosage' (Devout Hours 1482-1483, t6v-t7v).

A devout home should also be a place of learning and the man should lead intellectually: if a woman wants to know something, she should ask her husband at home. If he does not know the answer, it should be he who consults prelates or intellectuals (Carthusiensis 1479, 14r-v). The man is the head of the 'house family' and he is in charge of the household, finances, the education of the children, their chastisement, servants, the marriage of daughters, and so on. However, the day-to-day life at home is mainly the domain of the wife (Carthusiensis 1479, 19v-20r). As Augustine already argued, the father should take on the role of the bishop of the home and be more virtuous than everyone else in the house so as to set an example (Carthusiensis 1479, 31r-v, cf. 48v).

Both parents moreover have to take care of the spiritual well-being of their children, and try to guide servants to focus on eternal life as well (Der sielen troest 1484, 14v-5r; Schmitt 1959, p. 191, 1. 33-p. 192, 1. 4; Carthusiensis 1479, 32r). Children should get to know God from an early age and learn how to love and honor him. If they can afford it, parents should not hesitate to send their children to school, at least until they have learned the Pater Noster and the Ave Maria, the articles of the faith and to read and write in Dutch - a type of 'pragmatic literacy' oriented towards a basic knowledge of Christian religion (Parkes 1991; cf. Driver 1989, p. 235). Ideally, however, they should stay in school until they

10 See Lutton 2011 on the (vernacular) cult of the Holy Name of Jesus in England, which seems to have intersections with the Dutch situation 
have mastered Latin so that they can understand what is being sung in church and can even assist at Mass. Unfortunately, many parents, especially in rural areas, do not provide their children with such an education and thus do not lead them to good morals (Carthusiensis 1479, 24r-24v).

In The Soul's Consolation, we already saw that a father saved his son's life by teaching him to attend Mass daily. The religious instruction of children within a devout household is a recurring topic in the earliest religious texts printed in Dutch. A section directed at parents and describing their responsibility in the Christian upbringing of their children is also included in the Tafel des kersteliken levens. Parents should instruct their offspring to offer their innocence and purity to God, together with the innocents massacred by Herod. Children should be taught the Pater Noster, Ave Maria, the Twelve Articles of the Creed, the Ten Commandments, and everything explained previously in the Tafel and its 'register' (Tafel 1478, A2v). Furthermore, they should be instructed on how to honor their apostle, their holy angel and other saints. They should learn how to bless themselves at night and in the morning, and always enter or leave with the sign of the Cross. Furthermore, they should be taught how to pray in the evening and morning on their knees in front of their bed, how to read their benediction and grace according to custom and fervently, to be austere with regard to food and drink, and also in chatting. They should not gossip and destroy other people's reputation-a topic elaborately discussed in The Soul's Consolation-and keep the home free from evil by not bringing new rumors to their home. If one does not teach this to children from their early youth, they will resemble old dogs that are difficult to tame (Tafel 1478, A3r-v). Parents should give a good example by living a virtuous life, punish their children when necessary, and ensure a good education at the school with the best master of an undisputed reputation (Tafel 1478, A3v-A4r).

On the Seven Sacraments also reminds its readers that parents are obligated to teach their children - or have them taught—in the holy faith, lead them to church and chastise them for their bad habits (Van den Seven Sacramenten 1484, h6v). Marriage has two aims: to create offspring and to avoid sin. Even though, as we have seen before, magic had no place in (the bookcase of) a devout household, the anonymous author of On the Seven Sacraments discusses complications in one of marriage's main aims: the possibility that the husband is made infertile through sorcery or herbs. To remedy such problems, according to this text Albert the Great advises the husband to get hold of his wife's shirt and to pee at once both through the neckband and the left sleeve (Van den Seven Sacramenten 1484, k4r-v).

A good, Christian upbringing naturally starts with the sacrament of baptism. On the Seven Sacraments, a dialogic text not previously transmitted in manuscript and probably created especially for the printing press, discusses this sacrament that was so strongly connected to procreation, one of the main aims of marriage. The author seeks to familiarize non-Latinate readers with the seven sacraments ${ }^{11}$. Many of his instructions pertain to the domestic sphere and appear to empower lay people to transfer the religious ritual not only into their own mother tongue, the Dutch vernacular, but also into their own family home. The author's remark that he asks one of his questions (i.e., how should a priest behave when he hears confession?) "for the benefit of the simple priests who are not lawyers so that they may be informed a little about this" (om der simpelre priesteren willen die welcke gheen iuristen en sijn dat sy doch hier inne wat gheinformeert moghen wesen), however, reveals that his work was at least partially aimed at a priestly readership (Van den Seven Sacramenten 1484, b7v, cf. also c4v).

According to On the seven sacraments, in case of an emergency, baptism can be performed by any person - a woman as well as a man, father or mother (soe wel wijf als man, vader of moeder) —as long as it is done in the way prescribed by the Holy Church (Van den Seven Sacramenten 1484, a1v). The author discusses the Dutch words that one should use and addresses the difference with the Latin original, which has no need for a personal pronoun: "In Latin the baptizer may say: 'baptiso te in nomine patris et cetera', which in our Dutch language would not sound well. Therefore, it is the best that one says:

11 The author came from Holland; see Van den seven sacramenten 1484, 12r, where he writes that this not the custom there. He also wrote the Spiegel des eeuwigen levens, first published in Delft in 1480 (Spiegel 1480). See (Warnar 2019, pp. 494-500). $\rightarrow$ if everything is bracketed this should be too probably (please remove this comment) 
'I baptize you et cetera'" (In Latijn soe soude den doper wel moghen segghen: "baptiso te in nomine patris et cetera", twelcke nae onsen Duytsche niet wel luden en soude. Daerom so ist sekerste dat men segghe "Ick dope di et ceterea") (Van den Seven Sacramenten 1484, a2r). In case a woman is in labor and there is fear for the child's life, the midwife should baptize the child. Only later should the child be brought to church where-if needed — the priest may rectify what failed at home (Van den Seven Sacramenten 1484, a2v). The question whether baptism is legitimate when done 'in jest or joke' (in boerten ende in iocke) seems to point to the performance of religious ritual in a playful or carnivalesque atmosphere (Van den Seven Sacramenten 1484, a3r).

About the compatibility of marriage and a devout, pious lifestyle, one could read more in Dionysius the Carthusian's De laudabili vita coniugatorum, translated into Dutch as Van den loveliken leven en de staet der echten and published by Leeu on 26 July 1479 in Gouda. Written from a male perspective, the text discusses the conduct towards the wife and family, but also offers an explanation of the Ten Commandments, advice about confession, speaking, gambling, and so on. "Learned men" (notabele gheleerden) as well as male acquaintances who live a married life have often asked Dionysius to put pen to paper and write about these matters (Carthusiensis 1479, 2v-3r).

Marriage brings many challenges to a devout, Christian life and home. Some women make their husbands' lives difficult by complaining even at night about how other women have more beautiful clothes and jewels and stand in high regard. They question their husband's behavior: "why did you look in such a friendly manner at that woman? What did you have to discuss with that virgin?" (Carthusiensis 1479, 6v: Waer om saecht ghi dat wijf so vriendeliken an? Wat haddi te spreken met dyer maghet?). In general, marriage makes it difficult to focus on fervent prayer, holy thoughts, and contemplation because it distracts the heart-the lust of the flesh makes the heart unable to care for godly things (Carthusiensis 1479, 10r).

According to Dionysius, it is better to die without children than to leave behind bad children (bose kinderen). As in the Tafel, good Christian parents should reprimand their children whenever they see anything bad in their behavior: they should keep measure in food and drink, sleeping, and in the way they dress (Carthusiensis 1479, 26r-27v). And it is not only children who should keep measure in these matters: all Christian people should beware of food and drink that is too good and beds that are too soft, since opulence darkens the heart and makes the senses blunt (Carthusiensis 1479, 71v-73v). Drinking, especially outside of lunch and dinner, should be avoided, since drunkenness breaks the soul. To bring the message home, Dionysius quotes from Ambrose: the drunk who devours wine will be devoured by it (Carthusiensis 1479, 74v-75v). As to fashion: just as religious people wear the habit of their order, so should all Christians who follow Christ's law wear clothes that show their meekness (Carthusiensis 1479, 77r-v). When discussing the last three Commandments, Dionysius expresses his annoyance at a specific kind of fashion. People tend to wear a cloak (heuke) directly over an undershirt (wamboeys) that is half open on one side which reveals the loins (Carthusiensis 1479, $62 \mathrm{r}-\mathrm{v})$. However, both women and men are allowed to make themselves beautiful within reasonable measure in order to keep their partner from going off with someone else (Carthusiensis 1479, 13v). Further, children should be kept from dishonorable dancing and from all forbidden games, especially gambling and tournaments. Girls should especially be kept off the streets and busy so that they do not get the chance to gossip or cast their eyes on men. The example of emperor Octavian who kept his daughters spinning and weaving serves to make a point about textile labors as an important part of a devout household (Carthusiensis 1479, 26r-27v; Dlabačová 2018 on printed texts that offer meditations on Christ's Passion for during spinning and weaving).

\section{Conclusions}

The distinction some scholars make between the period until 1500 as an age in which religious knowledge was "learned over a lifetime—-through acts, images, architecture, song, sound, sight, smell, and touch" - and the sixteenth century as an era in which foundational teachings became printed words (Wandel 2016, p. 7, n. 17) does not do justice to late medieval religious developments, book 
production and fifteenth-century printing in particular. The changes that occurred appear to be much more gradual, and a discussion of the contents of a small sample of incunabula shows that the two periods display more overlap with regard to written religious instruction and domestic devotion than scholars acknowledge. Many fifteenth-century believers would have seen 'basic' texts such as the Lord's Prayer or the Creed as printed words on a page. The early 'catechetical' works discussed here paved the way for developments in the sixteenth century: printers such as Gerard Leeu and his contemporaries accustomed Christians to the use of printed books for personal and practical religious instruction and knowledge. Although it might be true that "no text, singularly or in aggregate, was definitive of being 'a Christian'"' (Wandel 2016, p. 14), many texts did try to define a 'good Christian'. In this sense, the decades before the Reformation were indeed a "dawn of the Reformation" rather than an "eve of the Reformation"12.

Yet, the material discussed in this essay should prompt us to further reconsider the supposed dichotomy between the medieval and early modern period without viewing fifteenth-century printed books only as predecessors of later, sixteenth-century developments. The first books printed in Dutch offer a wealth of ideals and instructions for prayer, meditation, religious reading, and family life within the devout home. These commercially produced printed pages offer texts that generally do not pertain directly to the Modern Devotion and were often not already disseminated in manuscript. Although a few of the texts specifically addressed at a lay audience were available in manuscript copies, many other ones were never disseminated in manuscript. These printed books would have functioned as devotional objects in lay homes in dialogue with other-printed and handwritten-books containing prayers, exercises and meditations, with (printed) images and objects such as crucifixes or the IHS-monogram painted on the wall. At the same time, there was interplay with public spaces, especially the church to which many of the texts refer and to which readers would have brought their (printed) books. Prayer and meditation in a private space were distinctly different from prayer and meditation in a public space (on the 'consonance or dissonance' between private and public devotion, see, e.g., (Williamson 2013)). Moreover, these early printed books bridged the space between catechetical instruction and the private home, literally bringing home many of the instructions that the clergy would have offered in church and thus increasingly 'textualizing' the lives of the late medieval laity.

Funding: This research was partially funded by the Dutch Research Council (NWO) (Veni-project Leaving a Lasting Impression. The Impact of Incunabula on Late Medieval Spirituality, Religious Practice and Visual Culture in the Low Countries, 2018-2022, grant number 275-30-036) and the essay has been partially researched and written during fellowships at the Herzog August Bibliothek, Wolfenbüttel (2017) and at the Kluge Center, Library of Congress, Washington D.C. (2019).

Acknowledgments: I am indebted to Heather Bamford and Rijcklof Hofman for critically reading and editing the essay.

Conflicts of Interest: The author declares no conflict of interest.

\section{References}

Andersen, Elisabeth. 2016. Religious Devotion and Business. The Pre-Reformation Enterprise of the Lübeck Presses. Ons Geestelijk Erf 87: 200-23.

Areford, David S. 2010. The Viewer and the Printed Image in Late Medieval Europe. Farnham: Ashgate.

Bahlmann, P. 1894. Deutschlands Katholische Katechismen bis zum Ende des Sechzehnten Jahrhunderts. Münster: Druck und Verlag der Regensburgschen Buchhandlung.

Bange, Petty. 1986. Spiegels der Christenen. Zelfreflectie en Ideaalbeeld in Laat-Middeleeuwse Moralistisch-Didactische Traktaten. Nijmegen: K.U., Centrum Voor Middeleeuwse Studies.

12 The term 'dawn of the Reformation' was coined by Heiko Oberman and with regard to printed religious works discussed in (Milway 2000, pp. 137-40). 
Bange, Petty. 2000. Een Handvol Wijsheden. Eenvoudig Geloof in de Vijftiende Eeuw: de Spieghel Ofte Reghel der Kersten Ghelove. Nijmegen: Valkhof Pers.

Bange, Petty. 2007. Moraliteyt Saelt Wesen. Het Laatmiddeleeuwse Moralistisch Discours in de Nederlanden. Hilversum: Verloren.

Boecxken van der Officie Ofte Dienst der Missen. 1479. ISTC no. is00529000. Gouda: Gerard Leeu. Washington: Library of Congress, Rosenwald 467.

Carthusiensis Dionysius. 1479. De Laudabili vita Coniugatorum [Dutch] Van den Loveliken Leven en de Staet der Echten. ISTC no. id00249200. Gouda: Gerard Leeu, The Hague: Royal Library, 169 G 97.

Corbellini, Sabrina, and Margriet Hoogvliet. 2013. Artisans and Religious Reading in Late Medieval Italy and Northern France (ca. 1400-ca. 1520). Journal of Medieval and Early Modern Studies 43: 521-44. [CrossRef]

De Jong, Susanne. 2018. 'Read some good Dutch books.' Laypeople, Books, and Religious Reading in the Late Medieval Domestic Setting. Queeste. Tijdschrift over Middeleeuwse Letterkunde in de Nederlanden 25: 32-54.

Der sielen troest. 1484. Haarlem: Jacob Bellaert. ISTC no. is00361000. The Hague: Royal Library, 168 E 6.

Deschamps, J. 1963. De Middelnederlandse handschriften van de grote en de kleine 'Der sielen troest'. Handelingen Koninklijke Zuidnederlandse Maatschappij voor Taal en Letterkunde en Geschiedenis 17: 111-67.

Deschamps, J. 1972. Middelnederlandse Handschriften uit Europese en Amerikaanse Bibliotheken. Leiden: E.J. Brill.

Horae [Dutch] Devote Getijden van Het Leven Ons Heren. 1482-1483. ISTC no. ih00433130. [Gouda: Gerard Leeu]. San Marino: Calif., Huntington Library, inv. 100989.

Die Gulden Litanie Vander Passien Christi. 1492. ISTC no. il00228900. Antwerp: Gerard Leeu. London: British Library, IA.49852.

Die vier Uterste. 1477. ISTC no. ic00902000. Gouda: Gerard Leeu. Washington: Library of Congress, Rosenwald 462.

Dlabačová, Anna. 2008. Drukken en publieksgroepen. Productie en receptie van gedrukte Middelnederlandse meditatieve Levens van Jezus (ca. 1479-1540). Ons Geestelijk Erf 79: 321-68. [CrossRef]

Dlabačová, Anna. 2014. Literatuur en observantie. De Spieghel der Volcomenheit van Hendrik Herp en de Dynamiek van Laatmiddeleeuwse Tekstverspreiding. Hilversum: Verloren.

Dlabačová, Anna. 2017. Religious Practice and Experimental Book Production: Text and Image in an Alternative Layman's "Book of Hours" in Print and Manuscript. Journal of Historians of Netherlandish Art 9: 2. [CrossRef]

Dlabačová, Anna. 2018. Spinning with Passion. The Distaff as an Object for Contemplative Meditation in Netherlandish Religious Culture. The Medieval Low Countries 5: 177-209. [CrossRef]

Dlabačová, Anna. 2019. Seeing beyond Signs: Allegorical Explanations of the Mass in Medieval Dutch Literature. In Quid est Sacramentum? Visual Representation of Sacred Mysteries in Early Modern Europe, 1400-1470. Edited by Walter Melion, Elizabeth Carson Pastan and Lee Palmer Wandel. Leiden: Brill, pp. 199-226.

Dlabačová, Anna. 2020a. Illustrated Incunabula as Material Objects: The Case of the 'Devout Hours on the Life and Passion of Jesus Christ'. In Inwardness, Individualization, and Religious Agency in the Late Medieval Low Countries. The 'Devotio Moderna' and Beyond. Edited by Rijcklof Hofman, Charles Caspers, Peter Nissen, Mathilde van Dijk and Johan Oosterman. Turnhout: Brepols, pp. 183-224.

Dlabačová, Anna. 2020b. Marian Devotions from a Printer's Perspective. The Rosary, the Seven Sorrows, and Gerard Leeu (d. 1492). In Marian Images in Context: Doctrines, Devotions, and Cults. Edited by James Clifton, Barbara Haeger and Elliott Wise. Leiden: Brill.

Driver, Martha W. 1989. Pictures in Print: Late Fifteenth- and Early Sixteenth-century English Religious Books for Lay Readers. In De Cella in Seculum. Religious and Secular Life and Devotion in Late Medieval England. Edited by Michael G. Sargent. Cambridge: D.S. Brewer, pp. 229-44.

Falkenburg, Reindert. 2001. The Household of the Soul: Conformity in the Merode Triptych. In Early Netherlandish Painting at the Crossroads. A Critical Look at Current Methodologies. Edited by Maryan W. Ainsworth. New York: The Metropolitan Museum of Art, pp. 2-17.

Füssel, Stephan. 2003. Gutenberg and the Impact of Printing. Translated by Douglas Martin. Aldershot: Ashgate.

Geirnaert, Dirk, and Robrecht Lievens. 2018. Van zondeval tot hemel. Staties uit de heilsgeschiedenis: een reeks geillustreerde devotionele strofen van Anthonis de Roovere (Gouda, 1482). Jaarboek De Fonteine 63.

Glose Ofte Expositie Opten Psalm Miserere. 1491. ISTC no. ig00308500. Antwerp: Gerard Leeu. The Hague: Royal Library, 150 F 20 (2).

Goudriaan, Koen. 1992. Een drukker en zijn markt. Gheraert Leeu (Gouda 1477-Antwerpen 1492/3). Madoc 6: 194-205. 
Goudriaan, Koen. 2010. Apostolate and Printing. The Collaciebroeders of Gouda and Their Press. In Between Lay Piety and Academic Theology. Edited by Ulrike Hascher-Burger, August den Hollander and Wim Janse. Leiden: Brill.

Goudriaan, Koen. 2013a. The Devotio Moderna and the Printing Press (ca. 14751-540). Church History and Religious Culture 93: 582-91.

Goudriaan, Koen. 2013b. The Church and the Market: Vernacular Religious Works and the Early Printing Press in the Low Countries, 14771-540. In Cultures of Religious Reading in the Late Middle Ages. Instructing the Soul, Feeding the Spirit, and Awakening the Passion. Edited by Sabrina Corbellini. Turnhout: Brepols, pp. 93-116.

Goudriaan, Koen. 2016. The Franciscans, the Laity and the Printing Press. In Piety in Practice and Print. Essays on the Late Medieval Religious Landscape. Edited by Anna Dlabačová and Ad Tervoort. Hilversum: Verloren, pp. 272-301.

ISTC. Incunabula Short Title Catalogue. London: British Library. Available online: https://data.cerl.org/istc/_search (accessed on 4 December 2019).

Klinkenberg, Emanuel S. 2007. 'Wil diin herte bereeden gheliic eenen huze'. De binnenhuisallegorie in de geestelijke letterkunde. Queeste. Tijdschrift over middeleeuwse letterkunde in de Nederlanden 14: 126-53.

Kock, Thomas. 2002. Die Buchkultur der Devotio Moderna. Handschriftenproduktion, Literaturversorgung und Bibliotheksaufbau im Zeitalter des Medienwechsels, 2nd ed. Frankfurt am Main: Peter Lang.

Kohn, Albert. 2019. Furnishing Piety: Beds in High Medieval Jewish Domestic Devotion. Religions 10: 471. [CrossRef]

Korte Lering uit de Schrifturen der Heiliger Leraars. 1481-1482. ISTC no. il00178300. Gouda: Gerard Leeu. Antwerp: Erfgoedbibliotheek Hendrik Conscience, B 27303 [C25-42 h].

Lering van berouw en vergiffenis van zonden. 1491-1492. ISTC no. il00178600. Antwerp: Gerard Leeu. The Hague: Royal Library, 150 F 17 (2).

Lutton, Rob. 2011. 'Love this Name that is IHC.' Vernacular Prayers, Hymns and Lyrics to the Holy Name of Jesus in Pre-Reformation England. In Vernacularity in England and Wales, c. 1301-1550. Edited by Elisabeth Salter and Helen Wicker. Turnhout: Brepols, pp. 119-45.

Mareel, Samuel. 2017. Tekens aan de wand. Het ophangen, lezen en verwijderen van gebeden en gedichten in katholieke religieuze ruimtes $\left(15^{\mathrm{de}}-16^{\mathrm{de}}\right.$ eeuw). Spiegel der Letteren 59: 231-59.

Mertens, Thom. 1993. Boeken voor de eeuwigheid. Ter inleiding. In Boeken Voor de Eeuwigheid. Middelnederlands Geestelijk Proza. Amsterdam: Prometheus, pp. 8-172.

Milway, Micheal. 2000. Forgotten Best-sellers from the Dawn of the Reformation. In Continuity and Change. The Harvest of Late-Medieval and Reformation History. Essays presented to Heiko A. Oberman on His 70th Birthday. Edited by Robert J. Bast and Andrew C. Gow. Leiden-Boston-Köln: Brill, pp. 113-42.

Murjanoff, Michael. 1964. Zur Überlieferung des Seelentrostes. Beiträge zur Geschichte der deutschen Sprache und Literatur 86: 189-224.

Oosterman, Johan. 2017. Discovering New Media. Anthonis de Roovere and the Early Printing Press. In Medieval Urban Culture. Edited by Andrew Brown and Jan Dumolyn. Turnhout: Brepols, pp. 17-82.

Oosterman, Johan. 2018a. Anthonis de Roovere, Leven en passie ons Heren. In Colard Mansion. Incunabula, Prints and Manuscripts in Medieval Bruges. Edited by Evelien Hauwaerts, Evelien de Wilde and Ludo Vandamme. Gent: Snoeck, pp. 60-61.

Oosterman, Johan. 2018b. Tafel des Kersteliken levens, with: Anthonis de Roovere, Lof van den Heiligen Sacrament. In Colard Mansion. Incunabula, Prints and Manuscripts in Medieval Bruges. Edited by Evelien Hauwaerts, Evelien de Wilde and Ludo Vandamme. Gent: Snoeck, pp. 159-61.

Palmer, Nigel F. 1992. Seelentrost. In Die Deutsche Literatur des Mittelalters. Verfasserlexikon, VL2. Berlin/New York: Walter de Gruyter, vol. 8, cols. 1030-40.

Pansters, Krijn. 2007. De kardinale deugden in de Lage Landen, 1200-1500. Hilversum: Verloren.

Parkes, Malcolm. 1991. The Literacy of the Laity. In Idem, Scribes, Scripts and Readers: Studies in the Communication, Presentation and Dissemination of Medieval Texts. London: Hambledon Press, pp. 275-97.

Pettegree, Andrew. 2015. Brand Luther. 1517, Printing, and the Making of the Reformation. New York: Penguin Press. Pleij, Herman. 1992a. Drukpers, literatuur en geestelijkheid in het laat-middeleeuwse Holland. Madoc 6: 3-19.

Pleij, Herman. 1992b. De betekenis van de beginnende drukpers voor de ontwikkeling van de Nederlandse literatuur in Noord en Zuid. Spektator 21: 227-63. 
Pleij, Herman. 2010. The Printing Press as a Long-Term Revolution. In Books in Transition at the Time of Philip the Fair. Manuscripts and Printed Books in the Late Fifteenth and Early Sixteenth Century Low Countries. Edited by Hanno Wijsman. Turnhout: Brepols, pp. 287-307.

Reimerswael, Johannes. 1492. Der Zondaren Troost of Spiegel der Conscientien. ISTC no. ij00399500. [Antwerp]: Gerard Leeu. The Hague: Royal Library, 150 F 23, Paris: Bibliothèque nationale de France, D-54016.

Rosarium Beatae Virginis Mariae. 1487. ISTC no. ir00318850. Antwerp: [Gerard Leeu]. The Hague: Royal Library, 150 F 9. Schmitt, Margarete. 1959. Der Grosse Seelentrost. Ein niederdeutsches Erbauungsbuch des Vierzehnten Jahrhunderts. Köln/Graz: Böhlau Verlag.

Spiegel des Kersten Gheloefs. 1478. ISTC no. is00672000. Gouda: Gerard Leeu, The Hague: Royal Library, 169 G 94 (1). Spiegel des Eeuwigen Levens and Boec van der Biechten. 1480. ISTC is00671000. Washington: Library of Congress, Rosenwald 478a.

Spiegel der Volcomenheit. 1488. ISTC no. is00677700. Antwerp: Gerard Leeu, The Hague: Royal Library, 1084 D 4. Spiegel des kersten geloven [Low German]. c. 1477. ISTC no. is00671900. [Cologne: Johann Koelhoff the Elder]. The Hague: Royal Library, 170 F 59.

Tafel des Kersteliken Levens. 1478. ISTC no. it00010800. Gouda: Gerard Leeu. The Hague: Royal Library, 169 G 94 (2). Tafel des Kersteliken Levens [Low German] Ene guede Vermaninge en ene Tafel des kerstlyken Levens. c. 1475. ISTC no. it00010830. Cologne: Johann Koelhoff the Elder.

Tboeck vanden leven Jhesu Christi. 1487. ISTC no. il00353000. Antwerp: Gerard Leeu, The Hague: Royal Library, 171 E 39. Troelstra, A. 1901. De Toestand der Catechese in Nederland Gedurende de Vóór-Reformatorische Eeuw. Groningen: Wolters. Troelstra, A. 1903. Stof en Methode der Catechese in Nederland Vóór de Reformatie. Groningen: Wolters.

Gheraert, Leeu. 2015. Dialogus Creaturarum dat is Twispraec der Creaturen. Edited by Hans Rijns. Available online: https://www.dbnl.org/tekst/leeu002diag02_01/leeu002diag02_01_0001.php (accessed on 4 December 2019).

Van den Seven Sacramenten. 1484. ISTC no. is00015000. Gouda: Gerard Leeu, Liège: Bibliothèque Universitaire, XV.C088.

Wandel, Lee Palmer. 2016. Reading Catechisms, Teaching Religion. Leiden-Boston: Brill.

Warnar, Geert. 1993. Biecht, gebod en zonde. Middelnederlandse moraaltheologie voor de wereldlijke leek. In Boeken Voor de Eeuwigheid. Middelnederlands Geestelijk Proza. Edited by Th. Mertens. Amsterdam: Prometheus, pp. 36-51 and 372-80.

Warnar, Geert. 2019. Lady Scripture's Sacred Commitments: Dialogic Understanding in Dutch Religious Literature of the Late Fifteenth Century. In Quid est Sacramentum? Visual Representation of Sacred Mysteries in Early Modern Europe, 1400-1700. Edited by Walter Melion, Elizabeth Carson Pastan and Lee Palmer Wandel. Leiden: Brill, pp. 494-532.

Williamson, Beth. 2013. Sensory Experience in Medieval Devotion: Sound and Vision, Invisibility and Silence. Speculum 88: 1-43. [CrossRef]

(C) 2020 by the author. Licensee MDPI, Basel, Switzerland. This article is an open access article distributed under the terms and conditions of the Creative Commons Attribution (CC BY) license (http://creativecommons.org/licenses/by/4.0/). 


\title{
Article \\ 'Make Your House like a Temple': Gender, Space and Domestic Devotion in Medieval Florence
}

\author{
Catherine Lawless \\ Trinity College Dublin, The University of Dublin, Dublin 2, Ireland; lawlessc@tcd.ie \\ Received: 16 December 2019; Accepted: 2 March 2020; Published: 11 March 2020
}

\begin{abstract}
This article will discuss domestic devotions by framing them in terms of devotions carried out in the home, defined by its opposition to ecclesiastical, consecrated space. It will examine how women, considered the laity par excellence through their inability to ever attain sacerdotal authority, were advised spiritually by mendicant friars on how to lead a Christian life according to their status as wives, widows or virgins. It will look at the devotional literature that was widespread in mercantile homes and the devotional images designed to move the soul. This discussion will attempt to show the tensions between ecclesiastical and domestic spaces; between the clergy and the laity, and between the corporeal and spiritual worlds of late medieval devotion. It will argue that, despite clerical unease with the female and domestic space, the importance accorded to female piety by the mendicant orders at the close of the Middle Ages was such that women were entrusted with key educational roles in the family, even leading to the astonishing affirmation of them as 'preachers' within the borders of their households.
\end{abstract}

Keywords: mendicants; devotional texts; devotional art; female patronage; Florentine trecento

\section{Introduction}

In a well-known treatise, Regola del governo di cura familiare, written in 1403, the Dominican friar Giovanni Dominici advised Bartolomea degli Obizzi, widow of the exiled Antonio di Niccolò degli Alberti, on how to practice devotion within the home and raise her four children (Dominici 1860, p. 132; Baxendale 1991, p. 731). After having advised her to have paintings in the house to instruct the children, as 'paintings of Angels and Saints are allowed and ordered, for the lower mental levels' ${ }^{1}$, he went on to say that if she did not want to have images, or could not have, 'such paintings to make almost a temple of the home' she should have the children frequently brought to church when offices were not said, so they should become used to holy places. $^{2}$ The polarity between the ecclesiastical and liturgical space of the church and the domestic one of the home was ideally to be overcome by the transformation of the home into a church, and the (male) children were to be taught the principles of sacramental religion by being urged to dress as priests, to create little altars, to decorate them, clean them and adorn them with images (Dominici 1860, p. 146). ${ }^{3}$ There is no doubt about the superiority of the ecclesiastical space and only the boys can become its administrators: priests. The passage neatly illustrates the difference

'Però che debbi sapere sono permesse e ordinate le dipinture degli Angeli e Santi, per utilità mentale de' più bassi' .

2 'E se non vuogli, o non puoi, di tante dipinture fare quasi tempio in casa, avendo balia fa' sieno menati spesso in chiesa a tempo non vi sia tumulto, nè vi si dica ufficcio; acciò nè lor mente sia rapita dalla tumultuata gente, nè lor cianciare dia impaccio al divino ufficio.'

3 'Ma farai uno altaruzzo o due in casa, sotto titolo del Salvatore, del quale è la festa ogni domenica: abbivi tre o quattro dossaluzzi variati, ed egli, o più, ne sieno sacrestani; mostrando loro come ogni festa debbano variatamente adornare quella cappelluzza. Alcuna volta saranno occupati in fare grillande di fiori o d'erbe, e incoronare Iesu, adornare la Vergine Maria dipinta, fare candeluzze, accendere e spegnere, incensare, tenere pulito, spazzare, parare gli altari, comporre de' candelieri di fuscelli di cera, di terra; sievi la campanuzzi, corrino a sonare tutte l'ore co me sentono nelle chiese, possansi parare con le camice come accoliti, cantinvi come sanno, parinsi a dir messa, e sieno menato alcuni volta alla ch iesa e loro mostrato quel 
between the familial space, sometimes, and arguably, governed by the woman, and the ecclesiastical, governed by the male clergy.

In this contribution, I examine the domestic devotions of women as exemplifying those of a group that was lay in extremis; that is, unable ever to enter sacerdotal functions and spaces. Using the sermons and treatises of the Dominicans Giordano da Pisa (d.1310), Domenico Cavalca (d.1342), Giovanni Dominici (c.1355-1419), Antoninus Pierozzi (1389-1459) and the Franciscan Bernardino da Siena (1380-1444), I will examine the messages transmitted through these texts to women. I will then look at popular devotional texts that were demonstrably owned by women and devotional art originating, in all probability, due to scale, subject matter and format, from domestic spaces. The appearance of a female donor figure or a couple will further place these images within female and domestic concerns. The voices of women themselves remain isolated and sporadic, but I shall use that of Margherita Bandini Datini (c.1360-1423) wife of the famous merchant of Prato, Francesco di Marco Datini. Her voice is not to be discounted as an important witness of fourteenth-century life, as significant for relaying what her daily concerns were when negotiating the demands of her household and mediating between domestic life and religious requirements.

Medieval Christianity bore within it a number of tensions: those between the religious and secular, sacred and profane, male and female, virginal or celibate and married. These opposite poles were also nodes of power relations in which, in all cases, the dominant power (religious, sacred, male, virginal) was the former, the lesser was the latter (secular, profane, female, married). The highest form of life was the religious one, in which, theoretically, celibate male priests held the sole authority over the sacrament of the Eucharist, necessary, after 1215, for every Christian to consume at least once a year, in a suitable state of penitence after having been confessed (Von Moos 1996, p. 120; Rusconi 1981, pp. 68-69). Underlying my discussion is the contrast between masculine, consecrated space and its ministers, and domestic space, conceived of by these friars as feminine, or at least as a space in which women could exert significant influence. Ecclesiastical space was sharply distinct from domestic space.

Women, operating largely within a vernacular milieu, were usually responsible for the religious instruction of their children, and were seen as significant players in the late medieval piety, known as affective and penitential. In fourteenth- and fifteenth-century Florence, as elsewhere, women's spirituality and devout piety had long been recognized as powerful, but in need of regulation and guidance (Benvenuti Papi 1990, pp. 119, 205, 218, 242, 247). Of Cistercian inspiration, the penitential movement emphasized affectivity in devotional practice, and empathy in considering the Passion of Christ and the lives of his mother and the saints. Promoted effectively by the mendicant orders in the thirteenth and fourteenth centuries, the movement was particularly popular with women, already culturally linked with affectivity and the body (Bornstein 1996, p. 7; Vauchez 1993, p. 408; Bynum 1991). The literature on this feminized devotion is vast (Casagrande 1988, pp. 251-89; Vauchez 1990, pp. 206-20; Bynum 1991, pp. 181-238; Grundmann 1995; Bornstein 1996, pp. 1-27). Linked with mendicant promotion of the suffering humanity of Christ was the growth of vernaculars in the distribution and reception of religious texts (which naturally favoured women, among other groups of the 'unlettered') (Cornish 2000, pp. 166-80) and the spread of Eucharistic cults in the wake of the decrees of the Fourth Lateran Council of 1215, emphasizing transubstantiation, the requirement of the faithful to receive Communion at least once a year and to confess before communicating. As women's nature was seen as more pliable and receptive, female experiences as visionaries and mystics were, if accepted as orthodox in origin, welcomed by the clergy in the struggle against heresy and in the popularization of the Eucharist (Bynum 1991, pp. 183-87; Frugoni 1996, pp. 130-84). However, the widespread belief that women had weaker intellects and more fragile physical and mental natures assumed that they were

che fanno e' veri sacerdoti acciò imparino a contraffargli; e così variatamente quanto di può sieno occupati con amore circa il divino santuario, lasciandogli guastare le frascoline loro faranno, acciò abbin bisogno di rifarle.' 
as susceptible to diabolical influence, thus their piety had to be managed and supervised by the clergy (Bornstein 1998, pp. 82-83).

\section{The Spaces of Devotion}

The difficulties in looking at domestic devotions begin with trying to define the term 'domestic' and what it may have meant in medieval and Renaissance Florence. Recent scholarly interest in the material culture of religious devotion in the home has enriched the historiography of medieval and renaissance social life (Lydecker 1987; Webb 1990; Musacchio 1997, 1999, 2008; Ajmar-Wollheim et al. 2006; Anderson 2007; Campbell et al. 2013; Brundin et al. 2018; Corry et al. 2017, 2019). The sacred place of the altar at which Mass is celebrated is differentiated in space from the domestic space, understood here as the dwelling place and culture of the family (often consisting of patrilineal groups rather than a single nuclear one) group and its household (which could include a number of servants), and mindful of the cautions indicated by Corry, Faini and Meneghin in articulating the fluidity of the borders of the domestic (Corry et al. 2019, p. 2). As observed by Marta Ajmar-Wollheim, Flora Dennis and Ann Matchette, the idea of the early modern house as a site of privacy and seclusion has dominated historical debate. The Victorian model of private and domestic being the opposite of public and non-domestic does not suit medieval and Renaissance Florence, where houses were often the sites of business, display and diplomacy as well as being homes (Ajmar-Wollheim et al. 2006, p. 623). Morse has written of the complexity of the Renaissance casa, warning that it could not be considered a secular space, but rather, a complex environment, situated at the intersection of public and private, individual and communal, spiritual and worldly (Morse 2007, p. 184). Although written about the Venetian casa, the same could be said of the Florentine palazzo in its mix of functions, with banks and shops often forming part of the house and with business being conducted within the home.

Although borders between domestic and non-domestic are fluid, and terms such as 'public', 'private', and 'personal' must be used with care, there were clear distinctions between ecclesiastical space and other spaces, including the home or indeed liturgical spaces (chapels) within the domestic space. The ceremony of consecration circumscribed a sacred place and made it holier than the surrounding area. Careful control was exercised over when and where the sacrifice of the Mass was celebrated and the conditions necessary for that process. Mass was celebrated in a distinct space, differentiated from the domestic, even when permission was obtained for the privilege of a private chapel (Böninger 2000, pp. 335-37). Jérôme Baschet points out that there is no great boundary between secular and sacred, but rather degrees of sacredness, radiating from poles such as the consecrated altar with the presence of the Eucharist (Baschet 2008, pp. 70-71). In his I frutti della lingua, a translation of St Gregory's dialogues and the letter of St Jerome to his female disciple Eustochium, the Dominican preacher Domenico Cavalca (d.1342) made it clear that sin committed in the church was worse than if it were done anywhere else, stating that greater reverence is due to the house of God in which the sacraments are administered, especially that of the 'precious body and blood' of Christ (Cavalca 1837, p. 74). Cavalca also discusses the laws of purification which command that a woman, after childbirth, through which 'certain corporeal dirt' is contracted, should not enter the temple until after forty days, when the dirt has ceased. (Cavalca 1837, p. 77). Fra Bernardino da Siena (1380-1444) stated a similar view when he argued that, although God is everywhere, he is more present in the church than elsewhere. Bernardino railed against those who misused this sacred space, who traded or chattered and he particularly noted those women who went there to see or be seen (Bernardino da Siena 1935, pp. 85-87), words echoed by Antoninus (Pierozzi 1858, p. 141) and the Dominican Fra Girolamo Savonarola (1450-1498) (Savonarola 1845, pp. 72-73).

\section{Women's Space}

Sacerdotal authority is confined to men and ecclesiastical authority, or 'the body of specialists of religion' (Bourdieu 1971, p. 304). Within the categories of the laity, in medieval Florence, men had greater access to spaces outside the home than women, or at least the elite women who were 
the subject of behavioural manuals and who received tailored spiritual advice in the form of letters or treatises from mendicant friars. The gendered nature of the 'production of space' (Lefebvre 1991) has been explored for its implications in the medieval hierarchies allocated to burial and building by Roberta Gilchrist (Gilchrist 1999) and specifically in the Florentine context by Robert C. Davis (Davis 1998, pp. 19-38), Adrian Randolph (Randolph 1997, pp. 17-41) and Ena Giurescu Heller (Heller 2005, pp. 161-83). The role model for women was the Virgin Mary and, according to Paolo da Certaldo (fl. 1347-1370), a secular writer, women should, like her, stay indoors in a hidden and honest place (Paolo da Certaldo 1945, p. 59). The domestic space, as such, was not always 'hidden', with a workshop or shop often occupying the same building as the home (Cavallo 2006, p. 66), but women were rarely, if ever, occupants of those 'public' spaces. Women were forbidden to enter most civic buildings in Florence. They were not allowed, for instance, to enter the Palazzo del Podestà, for instance, having to instruct their notaries at its door (Laufenberg 2009, pp. 199-200).

The place for secular women was the home. As Adrian Randolph points out, 'women' and 'public' are nearly exclusive terms, yet women did worship in the public sacral space which was the church (Randolph 1997, p. 18). Women were restricted in various degrees from sacramental proximity (Randolph 1997, pp. 29, 39; Cooper 2011, pp. 92, 95, 100-1; Romagnoli 2016). They were, however, recipients of much pastoral care, particularly from the mendicant orders, not only for the purposes of instructing their children in the faith, but also due to the predominately female character of late medieval devotion and penitential piety. It is thanks to a number of works written or preached by mendicant friars that we know what they wished for in domestic devotion and when, and how, people, especially women, should pray. (Gagliardi 2013, pp.117, 125). Maria Pia Paoli has shown how spiritual treatises addressed to women, particularly those of Dominican tradition, fused with secular behavioural manuals and humanist works in promoting the role of the family as a unifying building block of civic life, with the family 'understood as a depositary of affective values' (il ruolo unificante della famiglia intesa come deposito di valori affettivi)' (Paoli, p. 1).

The confinement of women to the domestic space is evident in some of these works. In the Regola, Dominici warned Bartolomea degli Obizzi that women should obey their husbands and never stay overnight elsewhere, even on pilgrimage (Dominici 1860, pp. 87-88). If a husband forbids visiting relatives or friends, he should be obeyed; he should even be obeyed if he forbids going to church on ordinary days-the wife should pray at home instead. Only on Sundays and important feast days should women attempt to disobey, if they can, in order to attend church (Dominici 1860, pp. 90-91). He advocated caution to those who wish to live in penitence as mendicants, advising that there are many false followers of Dominic and Francis who sow discord rather than peace (Dominici 1860, pp. 98-99). Dominici expressed particular concern for a woman enclosed as a hermit, primarily because she would be unable to hear sermons and solitude can bring dangerous thoughts (Dominici 1860, p. 100).

As noted above, suspicion was voiced by Bernardino da Siena about women's motivations for going to church when he reprimanded women who only went to church to be seen, or worse, to show off their daughters to potential suitors (Bernardino da Siena 1888, III, pp. 212, 381). Caution for women was not only limited to the giving of alms, but also in going on pilgrimage. Pilgrimage was routinely cited as a space for potential danger. Dominici, as noted above, instructed Bartolomea degli Obizzi to never stay overnight outside the house, even on pilgrimage, although he concedes that husbands, too, should not remain away from their wives without their permission unless they are on a crusade (Dominici 1860, pp. 87-88). San Bernardino da Siena (1380-1444) echoed other preachers in fearing that more sin could be committed on pilgrimage than value gained (Bernardino da Siena 1884b, p. 380). Over a century earlier, the Dominican friar Giordano da Pisa (d.1310) preached in Florence that the Virgin should be a role model for women not because she fasted, or went on pilgrimages, except rarely little, or gave alms, or served in hospitals, but because she loved God above all things (Delcorno 1975, p. 72). The Vallombrosan monk Don Giovanni dalle Celle (d.1396) rebuked a religious woman, Domitilla, when he heard that she intended to go to the Holy Land, despite it having been urged by St Catherine of Siena, to whom he was otherwise devoted (Biscioni 1736, pp. 68-76). 
The advice given by friars and clergy to women to focus their devotions within the home could not overcome the tensions between a liturgical, sacramental religion in which a consecrated altar was essential, and an interiorized affective piety which could be practiced within the home. As seen above, friars preached against women going on pilgrimages and attending mass devotions, yet the evidence of Margherita Bandini Datini (c. 1360-1423) indicated that such activities were widely participated in by women. Margherita writes of how she and a party of women attended the devotions and indulgences at S. Maria Primerana, and how she went in on behalf of Francesco (Datini 2012, p. 169). Donato Velluti (1313-1370) described the piety of his wife, Bice Covoni, who went to Rome at the time of the Jubilee (1350), leaving Donato to care for their two children. He later wrote, after her death: '[She] was small and not beautiful; but wise, good, pleasant, loving, well-behaved, and of every virtue full and perfect, and who worked to love and wish good for every person: and I praised her much, as she loved and desired me with all her heart. She was very good of her soul: and it is to be believed, that Our Lord Jesus Christ will have received her in his arms, making good and the best works, alms, and praying and visiting the church, and having had, in her illness, pardon from guilt and penance from bishop Agostino Tinacci (Velluti 1914, p. 290). ${ }^{4}$ The evidence of Bandini Datini and Velluti shows the prescriptive, rather than descriptive, nature of the warnings of the clergy. In neither case do we have indications of the secular men involved (Francesco Datini, Donato Velluti) expressing disquiet about their wives' pilgrimages.

Predicatrici: 'Oh ladies, tomorrow I want to make you all preachers.' Women and devotional instruction.

Bernardino da Siena explicitly called on the women listening to his sermons to be preachers-predicatrici: 'Oh ladies, tomorrow I want to make you all preachers. ${ }^{5}$ He thus invested them with a teaching and converting role but safely circumscribed within the walls of the home, limited to the family and, indeed, the relaying of his words (Bernardino da Siena 1884a, p. 59). This echoes Dominici's advice to Bartolomea degli Obizzi, when he told her that she should preach to them, saying to them things that are not harmful, and then teach them how to preach themselves (Dominici 1860, pp. 146-147). ${ }^{6}$ This could suggest an extraordinary degree of trust on behalf of both Dominici and Bernardino, and, given clerical beliefs about the weakness and lack of intellectual capacity of women, can be explained only by the fact that it is they, the mendicants, who are telling the women what to preach, and that the women are, in effect, mediators passing the doctrine of the mendicants on to their children.. Orthodoxy could be checked by the dynamics between the domestic and the religious in the form of confession, and, it is to be noted, heretics could be discerned by their absence from confession and liturgical offices, exemplified by the trial of Giovanni da Montecatini, burned in 1450. The dangers that the home represented were shown when Giovanni was reported to have never been in seen in S. Lorenzo, his parish church, but to have declared that his own dwelling was a church where he received the Eucharist (Morçay 1914, p. 168). The home was the crucible of lay Christian formation and it was essential that its occupants were orthodox. Bernardino also, surprisingly, told women that their primary duty was to look after the household, and only after such duties were fulfilled, were they to go to the sermons. 'Is there someone who has a sick person at home? - Yes. - Do you not know how important his care his? Do not abandon him to come to the sermon. Have you children? - Yes. - Do not abandon he who has need of you, to come to the sermon. Have you husband and children, who need that they be looked after with the necessity of the family? - Yes. - Do, do it so

4 'La quale fu piccola e non bella; ma savia, buona, piacevole, amorevole, costumata, e d'ogni vertù piena e perfetta, e la quale si facea amare e volere bene a ogni persona: e io molto me n'ò lodare, chè me amava e disiderava con tutto quore. Era bonissima dell'anima sua: ed è da credere, che Nostro Signore Iesù Cristo l'abbia ricevuta nelle sue braccia, faccendo buone e ottime operazioni, limosiniera, e d'orare e visitare la chiesa, e avendo avuto, nella sua infermità, perdono di colpa e pena dal vescovo Agustino Tinacci.'

5 'O donne, domain vi voglio fare tutte predicatrici.'

6 'Insegna loro predicare poi hanno veduto alcuna volta predicare in ch iesa, e tu predicherai a quegli dicendo cose non nocive e di sollazzo, e poi facendoli predicare stando tu con la tua famiglia a sedere basso quando in alto dicono, non ridendo ma commendando, e premiando quando ha contraffatto l'ufficio spirituale.' 
you do not leave them to come to the sermon; do it that first you look after the house with those things that they need, and then, come to the sermon ... ' (Bernardino da Siena 1884b, pp. 42-43). ${ }^{7}$

After speaking about how states should be governed, Bernardino connected civic governance to how women should govern the home, addressing himself directly to his female audience: 'And as I taught how men should rule the palazzo and the city; so also I want to teach women what they should do in their homes' (Bernardino da Siena 1884b, p. 41). ${ }^{8}$ Bernardino even felt that this instruction was so important that if women had to choose between mass and the sermon, they should choose the sermon (Bernardino da Siena 1884a, p. 66). ${ }^{9}$ To Bernardino, the parents' role was clear, they were to make the children like the angels of Paradise. 'It is necessary to teach children the Ave Maria, the Pater noster, the Credo in Deum, that every Christian is obliged to know; and leaving the house they should sign themselves with the sign of the holy cross. And when one gets up, and when one goes to bed; that they should say five Pater nosters and five Ave Mariaswhen one goes to bed, and one rises the Salve Regina, and other prayers ... And all the said things the mother should teach them.' The father, instead, was to offer instruction involving other spaces: he was to teach them the hearing of mass, sermons, vespers, how to confess and then how to behave in the secular world and guard themselves against bad contracts, gambling, going to taverns, and bad company (Bernardino da Siena 1935, pp. 65-66). ${ }^{10}$

The emphasis on women's responsibilities can be seen again in the case of Margherita Bandini Datini. She was entrusted by the Dominican preacher Antonio Cancellieri with a book of the seven penitential psalms and a sheet with the prayer of St. Birgitta of Sweden, 'which she [Birgitta] made when the Body of Christ was raised' so that she could teach it to her little niece (Brambilla 2010, p. 19). Bandini Datini's letters talk of how Caterina (Tina) 'has read the Psalter; she needs a small prayer book that has the seven psalms and the Office of the Virgin with good lettering' (Datini 2012, p. 106). In the inventories researched by Christian Bec, some psalters are described as specifically for children, 'j saltero da fanciulli' (Bec 1984, p. 152). A century later, the wife of Lorenzo il Magnifico de' Medici, Clarice Orsini (1450-1488), had, to the great discomfort of the humanist Angelo Poliziano (1454-1494), undertaken the teaching of her young son, Giovanni, changing the reading prescribed by Poliziano to the Psalter: 'As for Giovanni, you will have seen for yourself. His mother has taken it upon herself to change his course of reading to the Psalter, a thing I did not approve of. While she was absent he had made wonderful progress. He was already able to select, without any help from me, all the letters and syllables in his exercise in composition' (Ross 1911, p. 216).

The domestic space was an ideal one to inculcate the piety of children with the use of fictive altars and dolls. In a famous passage cited in the introduction to this essay, Dominici urges Bartolomea degli Obizzi to instil in her children a love of the church by transposing the church into the home (Dominici 1860, pp. 130-133). The children are to dress as priests and to create little altars, decorate them, clean them, adorn them with images. Dominici was drawing on a long tradition. The miniature altars to be recreated in a domestic space are, according to Danièle Alexandre-Bidon found already in

7 'Écci chi abbia lo infermo in casa? - Sì. - Non cognosci tu quanto bene fa il governo suo? Non l'abandonare per venire alla predica. Hai figliuoli? - Sì. - Non gli abandonare di quello che hanno bisogno, per venire alla predica. Hai il marito e' figliuoli, i quali bisogna che sieno governati di quello che bisogna alla famiglia? - Sì. - Fa', fa' che non gli lassi per venire alla predica; fa' che tu prima governi la casa di quelle cosec he bisognano, e poi viene alla predica [...]'

8 'E perchè io ho insegnato come debbano règgiare gli uomini il palazzo e la città; così anco voglio insegnare alle donne quello che debbano fare alle case loro.'

9 'E se di queste due cose tu non potessi fare altro che l'una, o udire la messa o udire la predica, tu debbi piuttosto lassare la mess ache la pedica; imperò chè la ragione ci è espressa, che non è tanto pericolo dell'anima tua a non udire la messa, quanto è a non udire la predica.'

10 'Dèbbesi a' fanciulli insegnare l'Ave Maria, il Pater noster, il Credo in Deum, che ogni cristiano è tenuto di saperlo; e che a l'uscita di casa si segni del segno della santa croce. E così quando si leva del letto e quando si corica; e che dica cinque Pater nostri e cinque Ave Marie quando si va a letto, e quando si lieva la Salve Regina, e dell'altre orazioni ... E tutte le dette cose debbe loro insegnare la madre. Il padre: l'udire della messa, le prediche, il vespro, le laulde, insegnarli orare, confessare, e che si guardi da ma' contratti, e che non giuochi, e che non esca di casa la notte con bullettini o sanza. Ammunirlo che si guardi dalle taverne, dalle ghiottornie, e che digiuni e dì comandati, e che si guardi dalle cattive compagnie e dalle male usanze.' 
the twelfth-century work of Guillaume of Auvergne (Alexandre-Bidon 1998, p. 1176). Later, Giovanni Certosino recommended that women should pray in front of a small altar decorated with images (Miglio 2008, p. 235). It was perhaps at altars like these that children were instructed on how to receive holy communion. Franco Sacchetti (d.1400) wrote of how his contemporaries taught children to hear confession and to take communion by giving them unconsecrated hosts (Sacchetti 1857, p. 29).

The domestic space could be sacralized through the possession of relics. Bernardino wrote of how some at home may hold the relics of the cord of St Francis, or bits of cloth from his cape, but, again reinforcing the sacrality of the ecclesial space, he reminded his audience that the most precious relic of all was the Body of Christ (Bernardino da Siena 1884a, pp. 237-38). It is interesting to note Bernardino's concern with relics, given their scant appearance in inventories before 1560 (Anderson 2007, p. 114). Women were believed to be more prone to superstition and thus needed to be protected against believing too quickly in unlikely relics (Dominici 1889, pp. 62-63). Bernardino, too, railed against too easy belief in relics such as the milk of the Virgin and the wood of the True Cross (Bernardino da Siena 1884b, p. 375).

\section{Devotional Texts}

As seen in the advice given by friars to women, spirituality was encouraged by looking at holy images and reading or hearing devotional texts (Paoli, pp. 17-19). The relationship between women and written culture has been explored by Miglio (2008, pp. 57-62) and Kaborycha (2012, pp. 793-95) who caution against believing in the inflated figures given by Giovanni Villani for female literacy but indicate that the circulation of texts among bourgeois Florentine women shows a familiarity with and interest in written culture, at least in vernacular written culture. Inventories, such as the ones studied by Bec (1984), reveal the titles of well-known devotional texts, but Florentines also kept miscellanies, or zibaldoni, similar to English commonplace books, in which we can find examples of sermons, doctrinal treatises, instruction manuals, legends of saints, prayers and other religious writings. The ubiquity of these miscellanies can be seen in their survival in Florentine libraries and the range of materials and social categories of owners has been studied by Gill (1994), Kent (2000), Miglio (2008), Bryce (2005, 2009), Kaborycha (2012), and Corbellini (2019). Dominici's Regola, although not believed to have been widely available, was copied out by Caterina di Donato Alderighi, wife of Zanobi di Taddeo Gaddi, son of the artist, in 1410 (Boehm and Kanter 1994, p. 33).

Books of hours, rarely as lavishly illustrated as Northern European examples, were found in the inventories, sometimes described as specifically women's, such as the 'libracio di donna', listed in Piero Beni's inventory of 1431, which belonged to his wife, Mona Sandra (Bec 1984, p. 175). Margherita Bandini Datini wanted her book covered with black satin, so that it would not resemble that of a nun: 'am not happy about sending you vermilion samite for my prayer book. I want black. If you want to fob me off with the nuns' style of cover, there is no need to tell me. I could do that myself. I don't want any sort of white or pink on it because it would dazzle me. I want it black. Please get it done quickly, because it upsets me that I am not reading the hours as I used to.' (Datini 2012, p. 227).

The Lives of the Desert Fathers of St Jerome, loosely translated by Fra Domenico Cavalca, showed the exemplary lives of the desert fathers and mothers, and was a particularly useful text for the penitential movement, dominated, as it was, by women. Delcorno has compiled an index of manuscripts owned by women (Delcorno 1977, p. 679). One of these manuscript owners was Costanza, widow of Benedetto Cicciaporci, whose inscription on the text of BNCF II, III, 89 (Magl. XXI, 123) noted that she had the book written and made for the consolation of her own soul and secondarily for that of her daughters (Gill 1994, p. 97). The book was inherited by one of these daughters, Lucrezia (Bryce 2005, p. 149). The codex also contained extracts from the Fior di Virtù and Petrarch's Trionfi (Bryce 2005, p. 149).

The affective devotional text, Meditationes Vitae Christi, written for a Clarissan nun and advocating the reader/listener to imagine themselves present at various moments of the life of Christ, narrated with immediacy and charged with emotional affect (Green and Ragusa 1961; McNamer 2009; Holly 2009) is 
also found in codices owned by women, such as BNCF MS II, VIII, 25, containing the Meditationes Vitae Christi, a fragment of the life of St Elizabeth of Hungary, a passage from the gospel of St John and two laude, which at one time belonged to Antonia, wife of Daniele Canigiani (Mazzatinti 1901, p. 230). Antonia was the daughter of Tommaso di Zanobi Ginori, who married Canigiani in 1434 and died 30 December 1457 (Passerini 1876, Tav, II).

The lives of the saints were popular, such as one contained in MS Panciatichiano 39 which is inscribed at f.91r: 'Iste liber est domine chatarine, uxoris domini bonifacj de lupis, ut omnes qui habebunt hunc librum in suis manibus deprecor orent pro anima eius' (Morpurgo 1887, pp. 75-79). Its owner, according to the inscription, Caterina Franzesi, was the daughter of Antonio di Niccola dei Franzesi da Staggia and Cina di Francesco Cinughi. Widowed in 1389, she was the executor of the testament of her husband, the mercenary originally from Parma and formerly at the service of the Carrara of Padua, Bonifazio Lupi, which, among other things, established the hospital known as the Spedale di Bonifazio (Angiolini 2006). She maintained a supervisory role over the construction and decoration of the hospital. She was also a Franciscan tertiary (Passerini 1876, p. 226).

Petrucci has shown that these vernacular texts, copied and recopied by the laity, were kept with letters, account books and family documents in chests and read in the home or the shop, and that they 'belonged to the sphere of leisure and free time'; a sphere, that is, that was not ecclesiastically circumscribed (Petrucci 1984, pp. 612-13). Letters were frequently shown to others and copied. The notary Ser Lapo Mazzi, in his letter to Francesco di Marco Datini, refers to how he will show a letter from the Dominican prioress Chiara Gambacorta to Guido di M. Tommaso del Palagio (c. 1335-1399) when he has time: 'When I will have time, I will show the letter of the Sister to Guido' (Mazzei 1880, I, p. 116).

\section{Devotional Images}

Numerous small panel paintings survive from late medieval Italy, likened in their popularity by Victor Schmidt to books of hours in northern Europe (Schmidt 2005, p. 9). The function of these paintings was supposedly to help prayer and meditation and, thus, they did indeed serve a similar function to books of hours, themselves described as 'painted prayers' (Wieck 1997). Dominici did not hesitate to recommend the type of works that Bartolomea degli Obizzi should keep at home. In a frequently cited passage, he said that she could have images of the Virgin and Child, and the Massacre of the Innocents in order to teach little boys to shun violence. For little girls he wanted to see images of SS Agnes, Cecilia, Elizabeth of Hungary, Catherine of Alexandria, Ursula, and other figures that would give them a love of virginity, a hatred of sins, and a desire to flee bad company (Dominici 1860, pp. 130-31). Dominici's advice regarding the Massacre of the Innocents seems to have gained little traction, judging from the subject matter of surviving Florentine panels, but the saints he recommended do frequently appear (Lawless 2011, pp. 235-37). Dominici did not just expound on subject matter, but also on the type of painting that should be kept in the home. He did not like the use of silver, gold or precious stones, fearing that the young would become idolatrous and love the materials rather than the representations of truth depicted within the image; the older and more smoke-blackened through the maintenance of candles, the better (Dominici 1860, pp. 132-3; Freedberg 1989, pp. 11-12).

As Wilkins has pointed out, the survival of so many trecento tabernacles indicates widespread use, but little evidence suggests how or where they were used. Some, certainly, must have been used in religious houses in the private cells of the religious. He cites the important observation of Tommaso da Celano in his Vita Prima of St Francis, wherein he spoke of how Roman wives had a painted icon in their houses on which their favourite saint was painted (Wilkins 2002, pp. 371-72). This evidence is important, as it connects images of saints with women and domestic devotion, but Celano is clear that it is a custom of Roman women and we cannot be certain that the evidence can be related to Umbria and Tuscany. Anderson has demonstrated that exempla and sermons suggest that altarini were common, but that there is little other evidence to prove it (Anderson 2007, p. 37). Yet we know 
from the inventories that holy images existed in homes. Jacopo di Filippo Guidetti's inventory of 1427 included a small panel painting of the Crucifixion (Musacchio 2008, p. 198). Crisi elaborates that, in his house, he had a panel of the Virgin in the bed chamber of the ground floor, another panel 'quasi nuova' in his chamber, and another 'all'anticha' in the chamber over the garden. Further, he had a panel with 'Santa Ilaria' and a bone tablet with the Crucifixion (Cristi 2012, p. 873). In his villa at Vingone, he had three painted panels, one of St Jerome, one of the Crucifixion, and the third 'dipinta alla crecha, vecchia' (Cristi 2012, p. 874). Giovanni Boccaccio's 1374 testament left, to Sandra di Giovanni Sassetti, a little panel, on which was painted the Virgin Mary with the Child and, on the other side, a skeleton's skull (Lami 1758, I, p. 137).

The inventories show, thus, images in the home of the Virgin and Child, the Crucifixion, and various saints. Wilkins suggests the standardized iconography with the dominance of the Virgin and Child suggests an audience of women (Wilkins 2002, p. 372). Given the injunctions of the clergy on women as guardians of the faith within the home, the likelihood of tabernacles serving as the type of 'little altar' envisaged by Dominici is strong. The continuity of religious imagery being purchased or commissioned by private patrons in the Quattrocento can be seen in the ricordanze of the painter Neri di Bicci, many of whose clients were women (Gilday 2001, pp. 51-75).

Some evidence of how such images may have functioned has been gathered by Victor Schmidt in looking at depictions of St Catherine of Alexandria and the hermit. In this late medieval legend, Catherine is introduced to her future spouse by a hermit, who shows her a panel of the Virgin and Child. When the Virgin and Child appear to her in her chamber, she recognizes them as the same figures as in the panel shown to her by the hermit. The altarpiece showing the life of St Catherine by Donato and Gregorio d'Arezzo shows Catherine's mother taking the panel from the hermit, and shows it as clearly portable (Donato and Gregorio of Arezzo, St Catherine of Alexandria Vita panel, $100 \times 172 \mathrm{~cm}$, Malibu, J. Paul Getty Museum (Schmidt 2005, pp. 9-26).

Further evidence on how such a panel may have been used is shown in the life of the Florentine saint Umiliana dei Cerchi (d.1246). Umiliana is a useful example as, although she was promoted by the Franciscans immediately after death as a saint. Forbidden by her family to enter a religious order on becoming a widow, she enclosed herself within a tower of the Cerchi palazzo. Having refused remarriage, she dedicated herself to a life of prayer and charity, but some references to her children indicate that they remained with her rather than with her marital kin. We learn that Umiliana prayed in front of a painted image of the Virgin Mary to decide on her future, and then resolved that she would rather be burned in a furnace than remarry (De Luca 1977, III, pp. 370-371); that she kept a lamp burning in front of the image, as when it blew out a white dove came into the room, illuminating it by means of a vermilion rose in its mouth; another time when the lamp went out it was relit by the hand of an angel (De Luca 1977, III, p. 376). Another miracle recounted by the hagiographer was when her little daughter fell out of a window and lay on the ground as if dead. Umiliana brought the image of the Virgin and Child to the injured child and prayed, after which the Christ Child himself came out of the picture, blessed Umiliana's daughter, and she got up, completely cured (De Luca 1977, III, pp. 395-96). At her death, her companion brought her the image and we learn then that there was a precious relic inside in the form of a lock of hair of the Virgin. She lit candles and formed them in the shape of a cross and placed them with the image on Umiliana's breast, lit incense and sprinkled holy water on her head and Umiliana then, after rejecting the devil, placed the image in the silk cloth of her mantle and 'positioned it better upon her breast' (Schuchman 2009, p. 383; De Luca 1977, III, pp. 401-2). The vita shows us that the image of the Virgin was the chief focal point of the prayers and devotions of Umiliana, that it was portable, and when laid on her breast it provided comfort when she died. This can be compared with the devotion of the dying son of Giovanni Morelli in 1406, Alberto, who asked on his deathbed that he be brought the image of the Virgin and Child (Branca 1986, p. 294; Bailey 2009, p. 984). We also learn that the portability of the image lent itself to being touched and, thus, its importance was far from confined to the visual. 
Typical of many devotional panels is a small panel, now in Boston, from the workshop of Bernardo Daddi (d.1348), depicting the Crucifixion with mourners and a kneeling female donor portrait figure (Figure 1). I intend the word 'portrait' here to indicate not a naturalistic portrayal of an individual (although it may be that), but rather, as Victor Schmidt uses it, as a surrogate, performative self, engaged in eternal prayer (Schmidt 2005, p. 117). In the panel, to the left, the fainting Virgin is supported by the holy women and the Magdalen embraces the cross. To the right, the donor kneels, facing the cross and the Magdalen, her hands joined in prayer, while Longinus gestures to the cross. The crucified Christ occupies the top half of the panel, with mourning angels catching his blood. The panel is perfectly suited to meditation of the kind advocated by Dominici and others in domestic spaces. The donor figure herself, perhaps, could merge with her effigy in praying and contemplating the passion and body of Christ, or her family could pray in front of the panel and remember her soul in their prayers, thus joining the worlds of the living and the dead. The panel reminds the viewer of the importance of the sacrifice of Christ and the Eucharist, through the blood being caught in chalices by the angels. The penitent state necessary to receive the Eucharist is evoked by the Magdalen and the sorrows of the Virgin echo the words powerfully wrought in texts such as the Meditationes Vitae Christi.

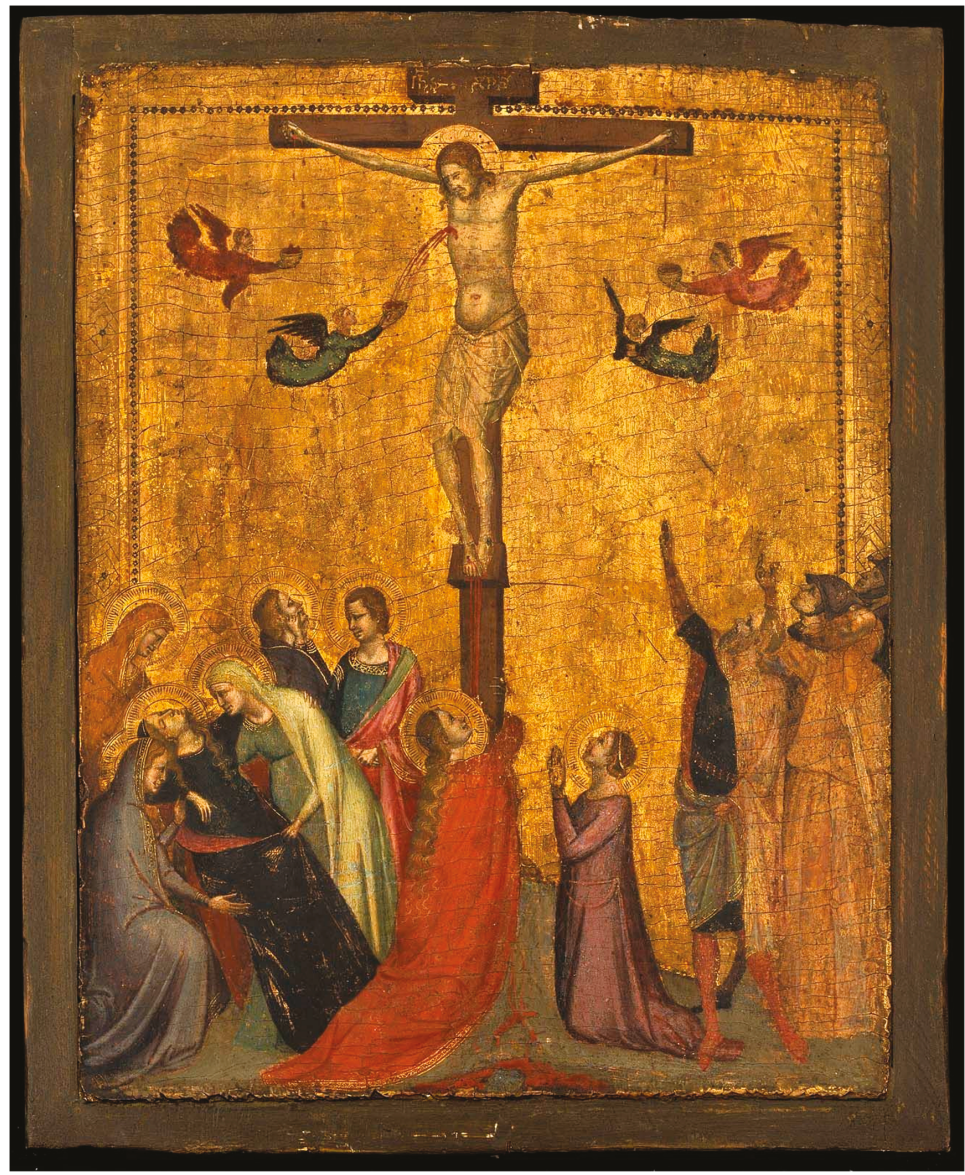

Figure 1. (Close follower of) Bernardo Daddi, Crucifixion with kneeling donor, c.1320, wooden panel, probably part of a diptych, $40 \times 28.5 \mathrm{~cm}$. Boston, Museum of Fine Arts, 23.11 . 
The workshops of artists like Bernardo Daddi and Jacopo del Casentino produced vast numbers of small panels, diptychs and triptychs for personal and domestic devotion. The popularity of saints such as Bernard, whose writings are found throughout the miscellanies owned by the devout, is echoed in the iconography of small-scale devotional works, such as the so-called Cagnola triptych, (Figure 2) the only signed work of Jacopo del Casentino (d.1349), where St Bernard is seen in the central panel with St John the Baptist flanking the Virgin Mary. On the side panel is the Stigmatization of St Francis with two virgin martyrs (probably Lucy and Margaret) and, on the other side, the Crucifixion of Christ. The entire triptych measures only $39.2 \times 42.4 \mathrm{~cm}$ (http://www.polomuseale.firenze.it/catalogo/scheda.asp, no.00282237).

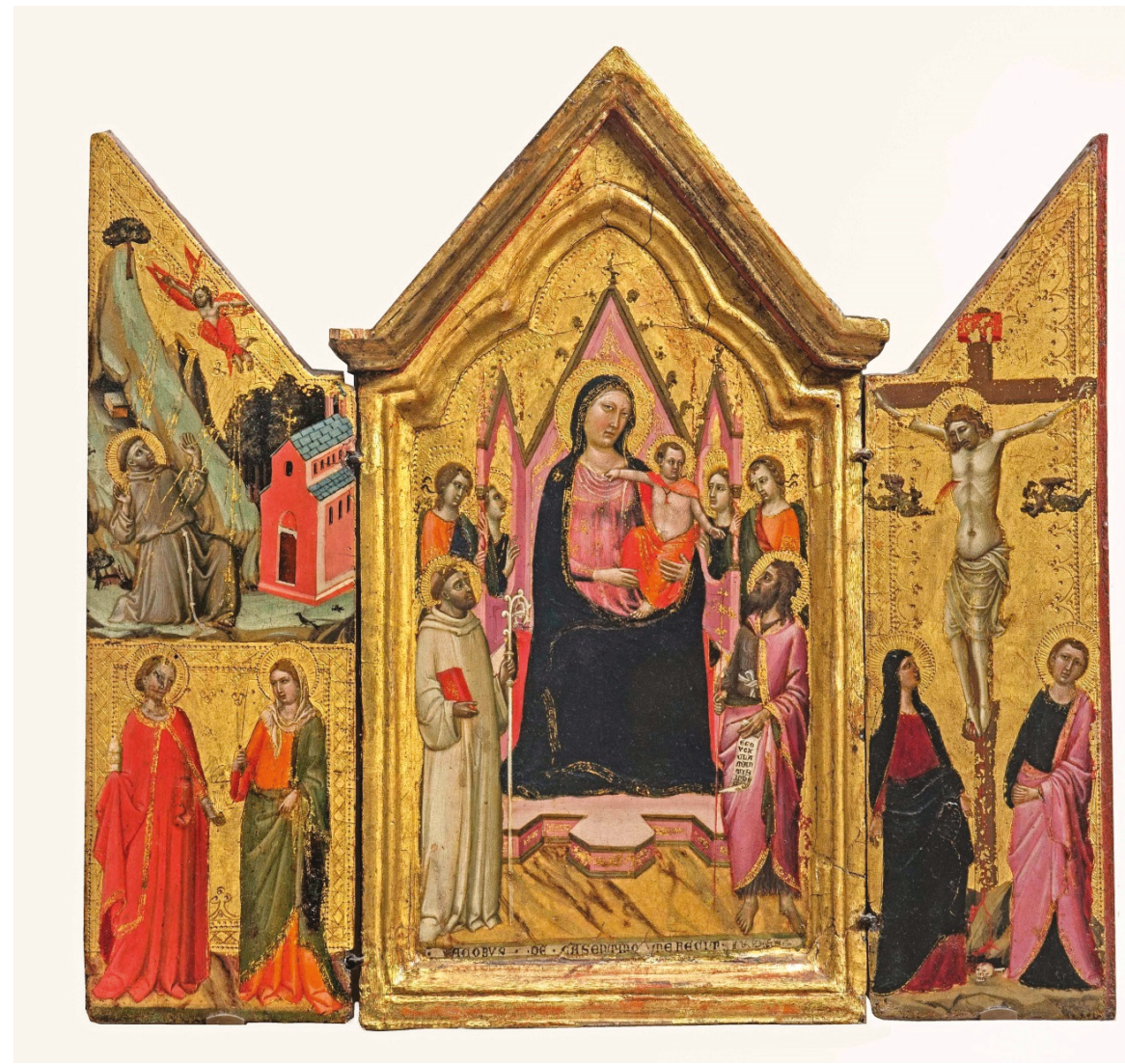

Figure 2. Jacopo del Casentino, The 'Cagnola' triptych: Virgin and Child with angels and Saints Bernard, John the Baptist; Stigmatization of St Francis; SS Lucy and Margaret; Crucifixion, panel, $39.2 \times 42.4 \mathrm{~cm}$, c.1320-1349. Florence, Uffizi, 9258.

It is, of course, impossible to know how images were 'read'. We rarely, if ever, have direct testimony of the relationship between the viewer and the viewed, but the work of Antoninus Pierozzi, in his advice to the widow Dianora Tornabuoni, although nearly a century later, could provide some useful clues, given the consistency of Dominican spirituality and instruction to pious women. The stigmatization of St Francis shows the moment in which Francis, rapt in prayer in La Verna, was imprinted with the marks of the Passion of Christ and became an alter christus, perfected through prayer, and naturally links with the Crucifixion on the other wing, a scene which devotional texts 
urge their readers and listeners to meditate upon and to move 'with the eyes of the mind' over the bleeding wounds of Christ (Pierozzi 1858, pp. 169-70; Flanigan 2014, p. 181, Lawless 2014-2015, p. 64). The angels catching the blood of Christ remind the viewer of the Eucharistic significance of the body of Christ, with the viewer, adequately prepared after confession and penitence, being encouraged to receive Communion. Tornabuoni, whether she was at home or at church, was to gaze at the crucifix and move across the body of Christ with the 'eyes of the mind', saying Ave Marias and Paternosters, dwelling on individual details such as his blood, his sputum-flecked beard, his forehead and, most particularly, his wounds (Pierozzi 1858, pp. 169-70). The presence of St Bernard, reputed author of De Planctu Beatae Mariae, or the lament of the Virgin on the Passion of Christ-a well-known text in Florentine miscellanies-could also remind the viewer of the future sacrifice of the Child he gazes at (Mâle 1986, p. 468). It could be hypothesized, with the help of Antoninus, that such a triptych could help prayer in a complex interplay of image, prayer, texts-remembered or read-and the devotee or devotees. The distinct spaces of the triptych could easily, if required, be used for different devotional exercises and compartmentalized, with the viewer moving from one image to another to culminate, perhaps, in the Virgin and Child or the Crucifixion. The Child, as in so many of these small panels, appears playful and active, reaching out to the angel or saint at his side. The saints gaze up at the Virgin, while she serves as visual interlocutor to the viewers, inviting them to join the saints in prayer.

Another small triptych, now in the Lord Methuen collection in Corsham Court (Wilts.) (Figure 3), painted by Puccio di Simone, is explicitly linked to female devotion by the presence of a kneeling female figure (Fremantle 1975, p. 92). The central panel shows the Virgin and a standing, playful child touching her chin and holding a chaffinch. Angels stand on either side of them, with their arms folded, and below is St Margaret and St John the Evangelist. In the side wings are four scenes, on the right, the Lamentation over the Dead Christ; the Mystic Marriage of St Catherine of Alexandria; the Noli Me Tangere; and the Feeding of SS Anthony and Paul in the Wilderness. It is in this latter scene that the female figure appears. Her flowing hair indicates that she is not married, and her richly lined dress, with a square-cut neck, shows that she is not a member of a religious order. She is not, thus, yet a mother or wife with a family to instruct in religious devotion but could be a young woman on the cusp of marriage, with a panel containing suitable themes for a Christian wife or for a young woman about to enter a convent. The central panel's Virgin and Christ Child is, of course, a maternal image, which is enhanced by the presence of St Margaret, patron saint of childbirth (Musacchio 1999, p. 143) and frequently represented in Florentine small-scale panels (Lawless 2011, pp. 254-55). St John the Evangelist was the most beloved of Christ, the author of the Apocalypse, and was related to Christ, being his first cousin according to the apocryphal accounts used in many Florentine miscellanies (Lawless 2010). The Lamentation scene connects directly to the Noli Me Tangere through the presence of the Magdalen. The penitence personified by the Magdalen then connects to the penitential and austere lives of SS Anthony and Paul in the desert, and the need to expiate sin by appropriate fasting and oration. St Anthony's life was popularized in Cavalca's Vite dei Santi Padri (Cavalca 1858, pp. 17-38). The association with food and fasting is particularly associated with female penitence, as shown by Walker Bynum, and is a key component in the lives of many late medieval female saints (Bynum 1987). Margherita Datini records a contemporary of hers, Maria Strozzi, a widow, whose abstinence was so severe that 'Her mother and father believed she had been sleeping only on a board and they thought she had been fasting so much that she had consumption.' (Datini 2012, p. 57). Giordano da Pisa, preaching on the feast of St Mary Magdalen, 22 July 1305, told his audience how women could be among the the greatest saints, using the example of the Magdalen, who, as she had been rewarded with angelic food exceeded other hermits such as Mary of Egypt and Paul the Hermit whose sustenance consisted of herbs (Moreni 1831, I, p. 182). 


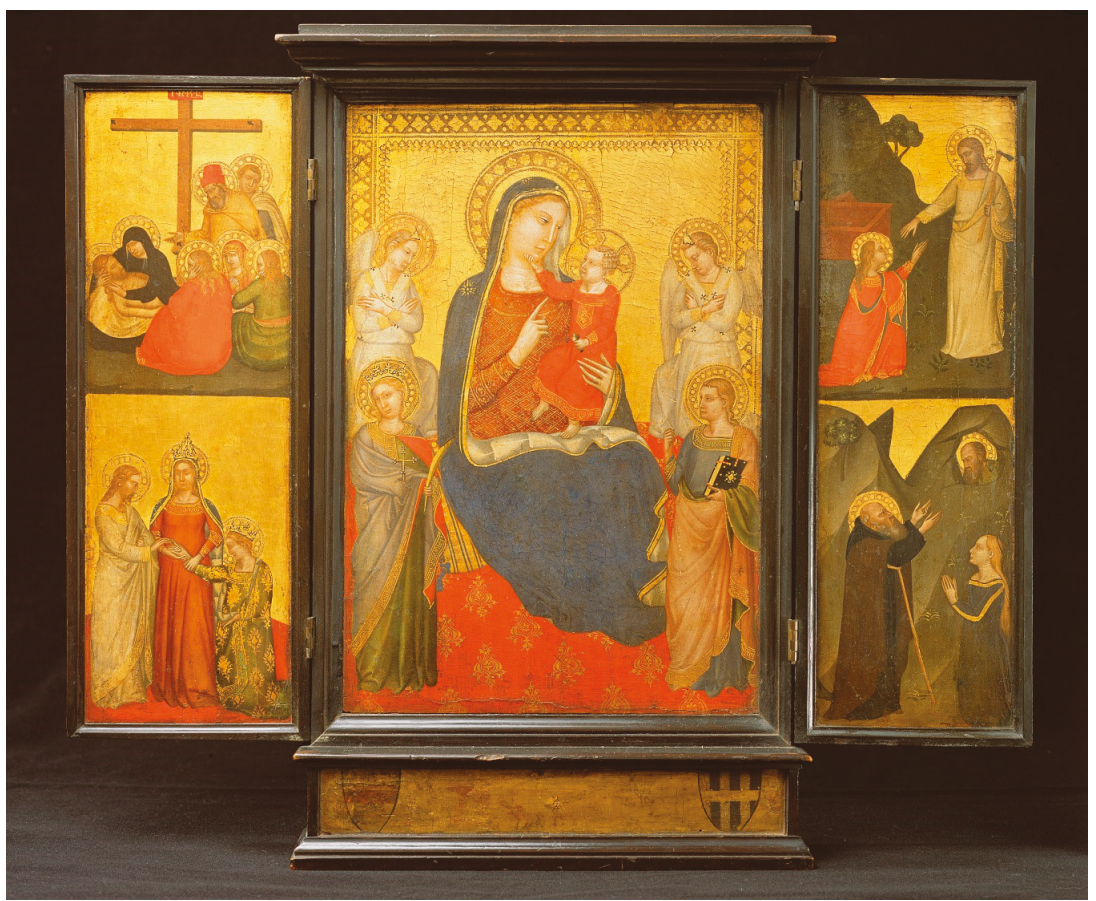

Figure 3. Puccio di Simone, Virgin and Child with angels and SS Margaret, John Evangelist; Lamentation over the Dead Christ; Mystic Marriage of St Catherine of Alexandria; Noli Me Tangere; St Anthony Abbot and St Paul the Hermit with kneeling donor, c.1350, panel, $39.5 \times 26.0 \mathrm{~cm}$ (centre), $39.5 \times 12.9 \mathrm{~cm}$ (each wing). Corsham Court (Wilts.) Lord Methuen Collection. Image courtesy of Corsham Court, Wiltshire/ Bridgeman Images.

The Mystic Marriage of St Catherine was a popular late medieval legend in which the virgin princess, Catherine, is rewarded by the Christ Child in a vision by the Virgin Mary joining him to her in marriage. Although there was a long tradition of mystic marriage within Christianity and Judaism representing a symbolic pact, perhaps between the soul and God, or the Church and Christ (Matter 1999, p. 31) the mystic marriage of St Catherine knew a particular fortune of its own, appearing first in visual imagery and only then in texts (Walsh 2007, p. 4). Schmidt points out the importance of the soul being joined to Christ as his bride in the contemplative life, and that, although married women led an active life, Giordano da Pisa had emphasised how a room or a cell could be transformed into a desert in acts of devotion (Schmidt 2001, pp. 24, 26). St Catherine is here presented by the Virgin to an adult Christ - a more destabilizing, yet popular, iconography which undermines both the role of male authority in sanctioning a union and, by placing the protagonists of the union as nubile, brings the mind more closely to marriage than the desexualised imagery of the Christ Child and St Catherine. Another possibility to be considered regarding the identity of the young woman is that she is not about to be married, but has died, and that the panel serves as a memorial and and invocation to her family to pray for her.

[This is part of the main text, not caption for the image.Main text begins here] Family devotion is suggested by the 1333 winged triptych of Bernardo Daddi now in the Museo del Bigallo in Florence (Figure 4) In the central compartment, the Virgin and Child are surrounded by fourteen male saints, and flanked by a male and female donor. On the inside left wing is the Nativity, and on the right, the Crucifixion with St Francis. In the spandrels is found the narrative of St Nicholas rescuing and restoring the little Adeodatus to his parents. St Nicholas was a particularly child-friendly saint, not only due to 
his rescuing of Adeodatus but also in his provision of dowries to three poor girls and his resurrection of three young boys who had been pickled in brine (De Voragine 1993, I, pp. 21-27). In a mercantile city whose wealth was based on foreign trade, his role as a patron against storms at sea and shipwrecks was, doubtless, also important. On the outside of the wings is found, once again, the patron saint of childbirth, Margaret, with Catherine, Martin, and Christopher. Catherine was one of the most popular saints of the middle ages, partly due to her mystic marriage, which made her the daughter-in-law of the Virgin Mary, and also for the reasons enumerated by the Dominican Jacobus de Voragine (Jacopo da Varazze) in his Golden Legend, c.1260: 'Lastly, she was admirable by reason of her privileged dignity. Some saints have received special privileges at the time of death - for instance, a visitation by Christ (Saint John the Evangelist), an outflow of oil (Saint Nicholas), an effusion of milk (Saint Paul), the preparation of a sepulcher (Saint Clement), and the hearing of petitions (Saint Margaret of Antioch, when she prayed for those who would honour her memory). Saint Catherine's legend shows that all these privileges were hers." (De Voragine 1993, II, p. 341). St Martin's generosity in giving his cloak to a beggar was a perfect example of alms-giving and charity (De Voragine 1993, II, pp. 292-301). Another perfect family saint was the giant, St Christopher, a ferryman who carried people across a river and who unknowingly carried the Christ Child, weighed down with the sins of the world (De Voragine 1993, II, pp. 10-15); He was patron saint of travelers and perhaps his most important role was that of a protector against sudden death. It was believed that whoever looked on his image was safe from sudden death for that day (Rigaux 1996, pp. 241-43). It is, perhaps, telling that the only saint to feature in the frescoes in the house of the merchant Francesco di Marco Datini was St Christopher (Schmidt 2005, p. 100; Cole 1967, p. 80).

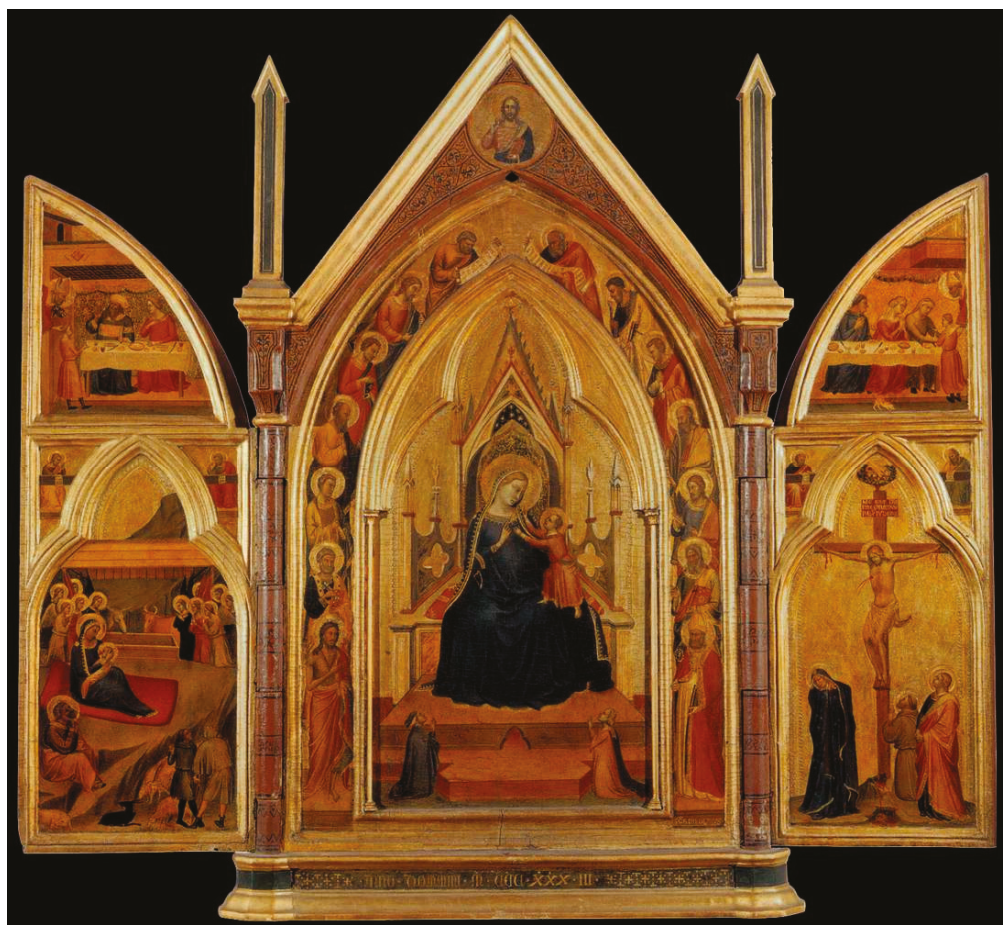

Figure 4. Bernardo Daddi, The Bigallo triptych. Virgin and Child with Saints; Nativity, St Nicholas rescuing Adeotatus from the pagan king; Crucifixion; St Nicholas restoring Adeotaus to his family, 1333, panel, $89 \times 97 \mathrm{~cm}$ (closed). Florence, Uf. 
The folding triptych in the Walters Art Gallery, Baltimore, also shows male and female donor figures (Figure 5). The central panel shows the Coronation of the Virgin with, below the Virgin, St Anthony presenting a male donor and, on the other side, below Christ, a male bearded saint (probably St Christopher) presenting a female donor figure. On the side panels are SS Nicholas and Peter, Paul and Catherine of Alexandria. Anthony Abbot was popularly invoked against not only St Anthony's fire, but a wide range of other illnesses and, in particular, the plague. On the exterior sides of the panel are SS Anthony Abbot and Christopher, with inscriptions indicating their patronal functions: 'Lucerna veri luminis, doctor humilitatis, magister et viator mirae sinplicatatis; refulsit in Egitto' ('A lamp of the true light, a teacher of humility, a master and traveller of admirable moral simplicity; he shone in Egypt') and 'Xpofori sancti spetiem quicumque tuetur, illo namque die nullo languore tenetur' (Whoever looks at the image of St Christopher will not be taken by any illness during that day') (Figure 6) (Schmidt 2005, p. 98).

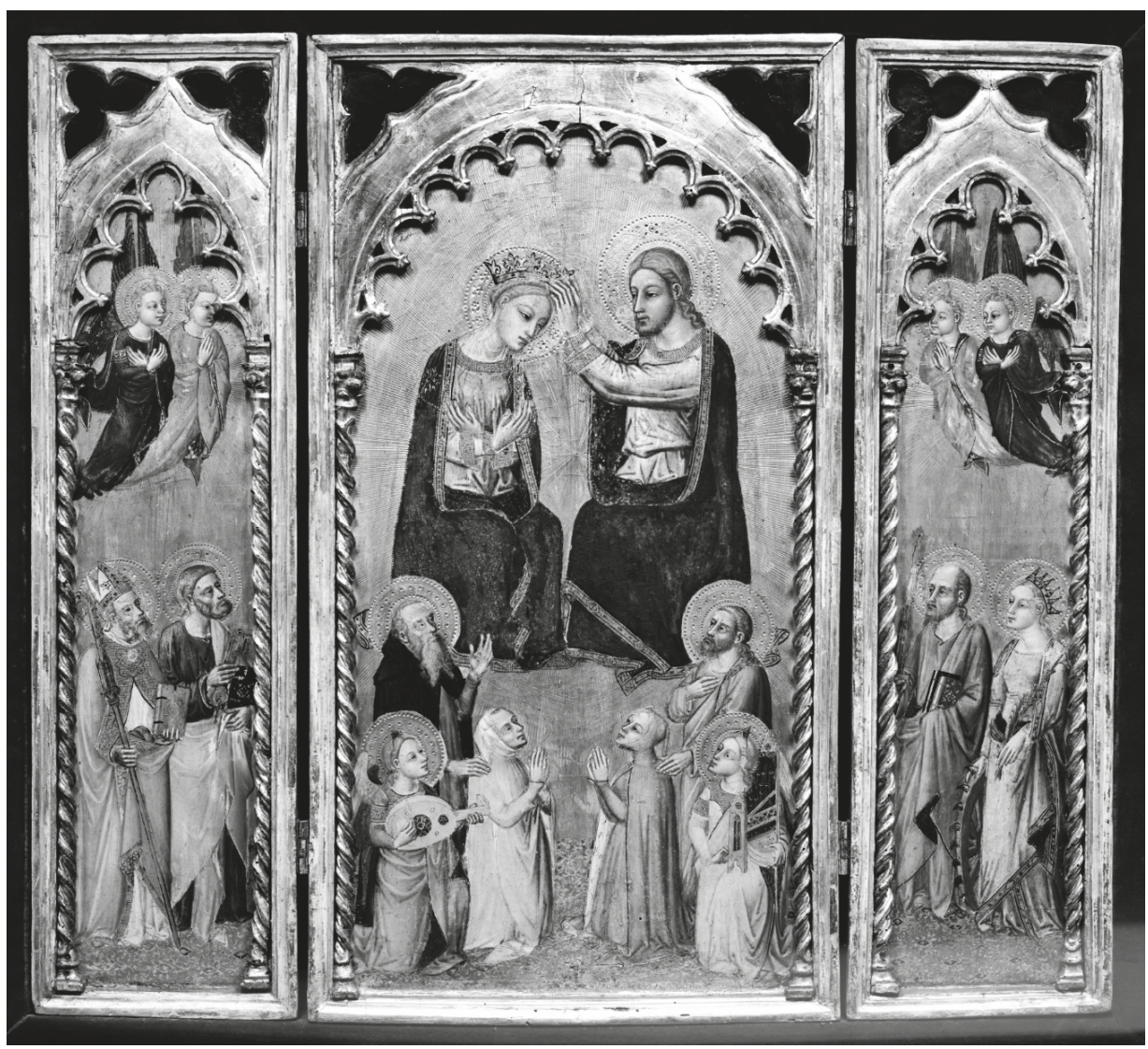

Figure 5. Niccolò di Tommaso, Coronation of the Virgin with donors and saints, c.1370, panel, $66.8 \times 36.3 \mathrm{~cm}$ (centre), $66.8 \times 18.1 \mathrm{~cm}$ (each wing). Baltimore, Walters Art Gallery, no. 718 . 


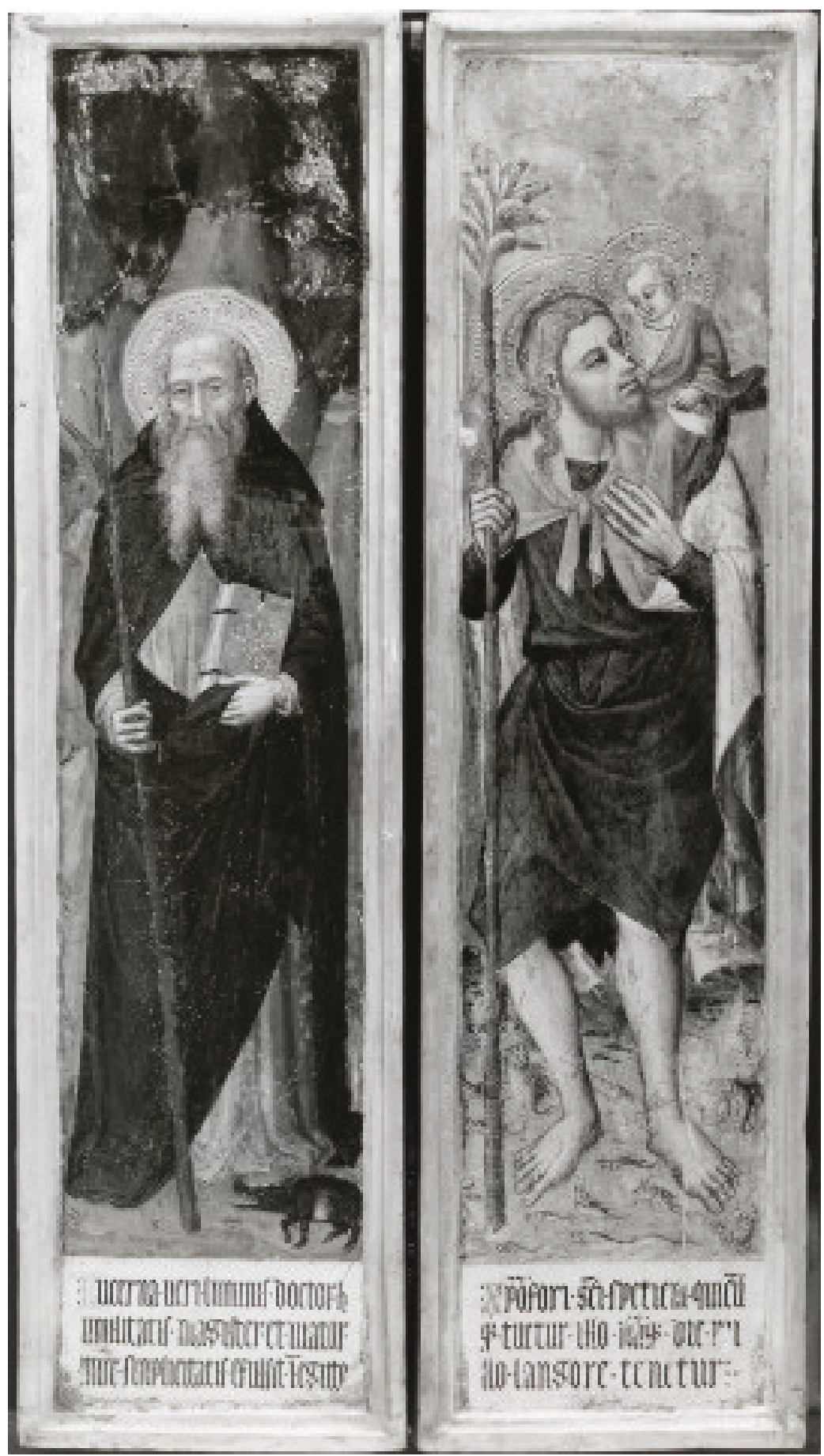

Figure 6. Niccolò di Tommaso, exterior shutters of wings of Coronation of the Virgin. 


\section{Conclusions}

Panels like these were only a small part of the domestic, devotional culture of medieval Florence, and by and large little documentation survives on their patronage or early provenance. Later in the fifteenth century, more evidence exists on the art of domestic devotional milieu for families such as the Tornabuoni (DePrano 2018) and the Medici (Gombrich 1966), as well as the rich workshop records of Neri di Bicci (1419-1492) (Neri di Bicci 1976; Thomas 1995). The mendicants advised women how to use the crucifix in their prayer. Bartolomea was encourated by Dominici to embrace the crucifix repeatedly, to bathe it with her tears and to use it as tool for meditation (Dominici 1860, p. 61). Although these panels were costly and could not have been owned by many, even the poorest could own a crucifix. The Florentine apothecary and diarist, Luca Landucci describes how, in 1473, a poor woman, 'poveretta', recommending her many children to the crucifix in her home for prayer, suddenly saw it begin to sweat. Crowds gathered, and the Carmelite friars removed the crucifix and placed it in a tabernacle in their own church, transferring it thus from domestic to sacred space (Landucci 1883, p. 13) and, in so doing, reinforcing the hierarchy of sacrality.

This essay shows that male religious figures of authority were resolute in their advice as to where and how women could practice their faith. Their treatises directed women on the management of domestic space and the instruction of children. The ecclesiastical space was managed and curated in direct and considered opposition to that of the domestic realm, where women were (ideally) restricted to certain practices and had very different responsibilities to their husbands. They were kept distinct and isolated from the holiest of spaces and, yet, were expected to fulfil the necessary guidance and instruction of their children in religious instruction. The evidence of the treatises shows us the advice directed to, in particular, pious married women such as Bartolomea Obizzi, but the devotional literature read by women, and panels containing female donor figures indicate a broader basis on which to investigate religious devotions. The caustic letters of Margherita Datini, like those of Alessandra Strozzi a generation later, show a more pragmatic and mercantile approach to religious practice and merit further exploration in the field of female emotion and mentality. Finally, both inventories and the wealth of surviving trecento and early quattrocento small tabernacles and panels show how some religious themes and devotions were given a visual form. Some of these show indications of female patronage in the form of a donor figure or a donor couple, in the latter case, almost certainly linking the panel to the domestic space. Women, both religious and lay, could be said to have had a particularized agency within the domestic sphere, an agency which was negotiated with the help of mendicant friars. This guidance was underlined in texts, images and devotional practices in a cultural milieu in which the social fabric upon which the city depended was woven through the domestic spaces of the ordo congiugatorum.

Funding: This research received no external funding.

Conflicts of Interest: The author declares no conflict of interest.

\section{References}

Ajmar-Wollheim, Marta, Flora Dennis, and Ann Matchette. 2006. Approaching the Italian Renaissance Interior: Sources, Methodologies, Debates. Renaissance Studies 20: 623-28. [CrossRef]

Alexandre-Bidon, Danièle. 1998. Une foi en deux ou trois dimensions? Images et objects du faire croire à l'usage des laics. Annales. Histoire, Sciences Sociales 3: 1155-90. [CrossRef]

Anderson, Caroline Corisande. 2007. The Material Culture of Domestic Religion in Early Modern Florence, c.1480-c.1560. Ph.D. thesis, University of York, York, UK. Unpublished.

Angiolini, Enrico. 2006. Lupi, Bonifacio. Dizionario Biografico degli Italiani. p. 66. Available online: http://www.treccani. it/enciclopedia/bonifacio-lupi(Dizionario-Biografico)/ (accessed on 29 January 2020).

Bailey, Elizabeth. 2009. Raising the Mind to God: The Sensual Journey of Giovanni Morelli (1371-1444) via Devotional Images. Speculum 84: 984-1008. [CrossRef]

Baschet, Jérôme. 2008. L'iconographie médiévale. Paris: Gallimard. 
Baxendale, Susannah Foster. 1991. Exile in Practice: The Alberti Family in and out of Florence, 1401-28. Renaissance Quarterly 44: 720-56. [CrossRef]

Bec, Christian. 1984. Les Livres des Florentins (1413-1608). Florence: Olschki.

Benvenuti Papi, A. 1990. 'In castro poenitentiae'. Figure e modelli femminili nella rappresentazione della santità (sec.XII-XIV). Santità e società femminile nell'Italia medieval. Rome: Herder.

Bernardino da Siena. 1884a. Le prediche volgari di San Bernardino da Siena I. Edited by Luciano Banchi. Siena: Tipografia di San Bernardino.

Bernardino da Siena. 1884b. Le prediche volgari di San Bernardino da Siena II. Edited by Luciano Banchi. Siena: Tipografia di San Bernardino.

Bernardino da Siena. 1888. Le prediche volgari di San Bernardino da Siena III. Edited by Luciano Banchi. Siena: Tipografia di San Bernardino.

Bernardino da Siena. 1935. Prediche Volgare Inedite, 1424-5. Edited by P. Dionisio Pacetti. Siena: Cantagalli.

Biscioni, A. M. 1736. Lettere di santi e beat fiorentini. Florence: Moucke.

Boehm, Barbara Drake, and Laurence Kanter. 1994. Painting and Illumination in Early Renaissance Florence, 1300-1450. New York: New York Metropolitan Museum.

Böninger, Lorenz. 2000. “"Altare portatile" e "cappella privata": Il caso dei Medici'. Mitteilungen des Kunsthistorisches Institut in Florenz 44: 335-37.

Bornstein, Daniel. 1996. Women and Religion in Late Medieval Italy: History and Historiography. In Women and Religion in Medieval and Renaissance Italy. Edited by Daniel Bornstein and Roberto Rusconi. Chicago: University of Chicago Press, pp. 1-27.

Bornstein, Daniel. 1998. Spiritual Kinship and Domestic Devotion. In Gender and Society in Renaissance Italy. Edited by Judith C. Brown and Robert C. Davis. London and New York: Longman, pp. 173-92.

Bourdieu, Pierre. 1971. Genèse et structure du champ religieux. Revue française de sociologie 12: 295-334. [CrossRef]

Brambilla, Simona, ed. 2010. 'Padre mio dolce.' Lettere di religiosi a Francesco Datini. Antologia. Rome: Direzione Generale degli Archivi.

Branca, Vittore, ed. 1986. Mercanti Scrittori: Ricordi nella Firenze tra Medioevo e Rinascimento. Milan: Rusconi.

Brundin, Abigail, Deborah Howard, and Mary Laven. 2018. The Sacred Home in Renaissance Italy. Oxford: Oxford University Press.

Bryce, Judith. 2005. Les Livres des Florentines: Reconsidering Women's Literacy in Quattrocento Florence. In At the Margins: Minority Groups in Premodern Italy. Edited by Stephen J. Milner. Minneapolis: University of Minnesota Press, pp. 133-62.

Bryce, Judith. 2009. Dada degli Adimari's Letters from Sant' Antonino. Identity, Maternity, and Spirituality. I Tatti Studies in the Italian Renaissance 12: 11-53. [CrossRef]

Bynum, Caroline Walker. 1987. Holy Feast and Holy Fast: The Religious Significance of Food to Medieval Women. Berkeley: University of California Press.

Bynum, Caroline Walker. 1991. Fragmentation and Redemption: Essays on Gender and the Human Body in Medieval Religion. Cambridge MA and New York: The MIT Press.

Campbell, Erin J., Stephanie R. Miller, and Elizabeth Carroll Consavari, eds. 2013. The Early Modern Italian Domestic Interior, 1400-1700: Objects, Spaces, Domesticities. Farnham: Ashgate.

Casagrande, Giovanna. 1988. Il movimento penitenziale francescana nel dibattito storiografico degli ultimi 25 anni. In Santi e Santità nel movimento penitenziale francescano dal Duecento al Cinquecento. Edited by Lino Temperini. Rome: Analecta TOR.

Cavalca, Domenico. 1837. I frutti della lingua, volgarizzamento del Dialogo di San Gregorio e dell'Epistola di S. Girolamo at Eustochio. Edited by Giovanni Bottari. Milan: Giovanni Silvestri.

Cavalca, Domenico. 1858. Vite dei Santi Padri. Edited by Bartolomeo Sorio and A. Racheli. Trieste: Lloyd Austriaco.

Cavallo, Sandra. 2006. The Artisan's Casa. In At Home in Renaissance Italy. Edited by Marta Ajmar-Wollheim and Flora Dennis. London: V \& A Publications, pp. 66-85.

Cole, Bruce. 1967. The Interior Decoration of the Palazzo Datini in Prato. Mitteilungen des Kunsthistorischen Institutes in Florenz 13: 61-82.

Cooper, Donal. 2011. Access All Areas? Spatial Divides in the Mendicant Churches of Late Medieval Tuscany. In Ritual and Space in the Middle Ages Proceedings of the 2009 Harlaxton Symposium. Edited by Frances Andrews. Donnington: Shaun Tyas, pp. 90-107. 
Corbellini, Sabrina. 2019. Creating Domestic Sacred Space: Religious Reading in Late Medieval and Early Modern Italy. In Domestic Devotions in Early Modern Italy. Edited by Maya Corry, Marco Faini and Alessia Meneghin. Leiden and Boston: Brill, pp. 295-305.

Cornish, Alison. 2000. A Lady Asks: The Gender of Vulgarization in Late Medieval Italy. PMLA 115: 166-80. [CrossRef]

Corry, Maya, Deborah Howard, and Mary Laven, eds. 2017. Madonnas \& Miracles. The Holy Home in Renaissance Italy. London and New York: Philip Wilson.

Corry, Maya, Marco Faini, and Alessia Meneghin, eds. 2019. Domestic Devotions in Early Modern Italy. Leiden and Boston: Brill.

Cristi, Lucia. 2012. Un illustre e ricco sconsciuto nella Firenze quattrocentesca. In Uomini, Paesaggi, Storie. Studi di Storia Medievale per Giovanni Cherubini II. Edited by Duccio Balestracci. Siena: Salvietti \& Barabuffi, pp. 865-75.

Datini, Margherita. 2012. Letters to Francesco Datini. Translated by Carolyn James, and Antonio Pagliaro. Toronto: Iter. Davis, Robert C. 1998. The Geography of Gender. In Gender and Society in Renaissance Italy. Edited by Judith C. Brown and Robert C. Davis. London and New York: Longman, pp. 19-38.

De Luca, Giuseppe, ed. 1977. Scrittori di religione del Trecento: Volgarizzamenti. 4 vols. Turin: Einaudi.

De Voragine, Jacobus. 1993. The Golden Legend. Edited by William Granger Ryan. 2 vols. Princeton: Princeton University Press.

Delcorno, Carlo, ed. 1975. Giordano da Pisa e l'antica predicazione volgare. Florence: Olsckhi.

Delcorno, Carlo. 1977. Predicazione volgare e volgarizzamenti. Mélanges de l'Ecole Française de Rome, Moyen Age, Temps Modernes 89: 679-89. [CrossRef]

DePrano, Maria. 2018. Art Patronage, Family and Gender in Renaissance Florence: The Tornabuoni. Cambridge: Cambridge University Press.

Dominici, Giovanni. 1860. Regola del Governo di cura Familiare. Edited by Donato Salvi. Florence: Angiolo Garinei.

Dominici, Giovanni. 1889. Il libro d' amore e di carità del fiorentino. B. Giovanni Dominici dell' ordine de' predicatori. Edited by Antonio Ceruti. Bologna: Reale Commissione pe i testi di lingua nelle provincie dell' Emilia. Collezione d' Opere.

Flanigan, Theresa. 2014. Art, Memory, and the Cultivation of Virtue: The Ethical Function of Images in Antoninus Opera a ben vivere. Gesta 53: 175-95. [CrossRef]

Freedberg, David. 1989. The Power of Images: Studies in the History and Theory of Response. Chicago: University of Chicago Press.

Fremantle, Richard. 1975. Florentine Gothic Painting from Giotto to Masaccio. London: Secker and Warburg.

Frugoni, Chiara. 1996. Female Mystics, Visions, and Iconography. In Women and Religion in Medieval and Renaissance Italy. Edited by Daniel Bornstein and Roberto Rusconi. Chicago: University of Chicago Press.

Gagliardi, Isabella. 2013. Coscienze e città: la predicazione a Firenze tra la fine del XIII e gli inizi del XV. Considerazioni introduttive. Annali di Storia di Firenze 8: 113-43.

Gilchrist, Roberta. 1999. Gender and Archaeology: Contesting the Past. London: Routledge.

Gilday, Rosi Prieto. 2001. The Women Patrons of Neri di Bicci. In Beyond Isabella: Secular Women Patrons of Art in Renaissance Italy. Edited by Sheryl G. Reiss and David G. Wilkiins. Kirksville: Truman State University Press, pp. 51-75.

Gill, Katherine. 1994. Women and the Production of Religious Literature in the Vernacular, 1300-1500. In Creative Women in Medieval and Early Modern Italy: A Religious and Artistic Renaissance. Edited by E. Ann Matter and John Coakley. Philadelphia: University of Pennsylvania Press, pp. 64-104.

Gombrich, E. H. 1966. The Early Medici as Patrons of Art. In Norm and Form. London: Phaidon, pp. 35-70.

Green, Rosalie, and Isa Ragusa, eds. 1961. Meditations on the Life of Christ. Princeton: Princeton University Press.

Grundmann, Herbert. 1995. Religious Movements in the Middle Ages. Translated by Steven Rowan. Notre Dame: University of Notre Dame Press.

Heller, Ena Giurescu. 2005. Access to Salvation: The Place (and Space) of Women Patrons in Fourteenth-Century Florence. In Women's Space: Patronage, Place, and Gender in the Medieval Church. Edited by Virginia Chieffo Raguin and Sarah Stanbury. New York: State University of New York Press, pp. 161-83.

Holly, Flora. 2009. The Devout Belief of Imagination. Turnhout: Brepols.

Kaborycha, Lisa. 2012. Brigida Baldinotti and Her Two Epistles n Quattrocento Florentine Manuscripts. Speculum 87: 793-826. [CrossRef] 
Kent, Dale. 2000. Cosimo de' Medici and the Florentine Renaissance. London and New Haven: Yale University Press. Lami, Giovanni. 1758. Sanctae Ecclesiae Florentinae Monumenta I. Florence: Ex Typographio Deiparae ab Angelo Salutatae. Landucci, Luca. 1883. Diario Fiorentino dal 1450 al 1516. Edited by Iodoco del Badia. Florence: Sansoni.

Laufenberg, Lynn Marie. 2009. The Long Arm of the Florentine Law. Two Texts (1343, 1345). In Medieval Italy: Texts in Translation. Edited by Katherine Ludwig Jansen, Joanna Drell and Frances Andrews. Philadelphia: University of Pennsylvania Press, pp. 199-200.

Lawless, Catherine. 2010. The Virgin's Grandmother. Journal of Medieval History 36: 379-93. [CrossRef]

Lawless, Catherine. 2011. Representation, Religion, Gender and Space. In Ritual and Space in the Middle Ages: Proceedings of the 2009 Harlaxton Symposium. Edited by Frances Andrews. Donnington: Shaun Tyas, pp. 232-58.

Lawless, Catherine. 2014-2015. Sensing the Image: Gender, Piety and Images in Late Medieval Europe. Touch Me, Touch Me Not. Open Arts Journal 4: 41-60. Available online: https://openartsjournal.org/issue-4/ (accessed on 9 April 2019).

Lefebvre, Henri. 1991. The Production of Space. Oxford: Blackwell.

Lydecker, John Kent. 1987. The Domestic Setting of the Arts in Renaissance Florence. Baltimore: The Johns Hopkins University Press.

Mâle, Emil. 1986. Religious Art in France: The Late Middle Ages: A Study in Iconography and its Sources. Edited by Harry Bober. Translated by Marthiel Mathews. Princeton: Princeton University Press.

Matter, E. Ann. 1999. Mystic Marriage. In Women and Faith: Catholic Religious Life in Italy from Late Antiquity to the Present. Edited by Lucetta Scaraffia and Gabriella Zarri. Cambridge: Harvard University Press, pp. 31-41.

Mazzatinti, Giuseppe. 1901. Inventari dei Manoscritti delle Biblioteche d' Italia. Forli: Bordandini, vol. XI.

Mazzei, Lapo. 1880. Lettere di un Notaro a un Mercatante del Secolo XIV, con altre Lettere e Documenti. 2 vols. Edited by Cesare Guasti. Florence: Le Monnier.

McNamer, Sarah. 2009. The Origins of the Meditationes Vitae Christi. Speculum 84: 905-55. [CrossRef]

Miglio, Luisa. 2008. Governare l'alfabeto: Donne, scrittura e libri nel Medioevo. Rome: Viella.

Morçay, Raoul. 1914. Saint Antonin. Fondateur du Couvent de Saint-Marc. Tours and Paris: Maison A. Mame et Fils; Libraire Gabalda.

Moreni, Domenico. 1831. Prediche del B. Giordano da Rivalto recitate in Firenze dal MCCCIII al MCCCVI. 2 vols. Florence: Marghera.

Morpurgo, Solomone. 1887. Catalogo dei Manoscritti Panciatichiani Della Biblioteca Nazionale Centrale di Firenze. Rome: Istituto Poligrafico dello Stato, vol. I.

Morse, Margaret A. 2007. Creating Sacred Space: The Religious Visual Culture of the Renaissance Venetian Casa. Renaissance Studies 21: 151-84. [CrossRef]

Musacchio, Jacqueline Marie. 1997. Imaginative Conceptions in Renaissance Italy. In Picturing Women in Renaissance and Baroque Italy. Edited by Geraldine A. Johnson and Sara F. Mathews Grieco. Cambridge: Cambridge University Press, pp. 42-60.

Musacchio, Jacqueline Marie. 1999. The Art and Ritual of Childbirth in Renaissance Italy. New Haven and London: Yale University Press.

Musacchio, Jacqueline Marie. 2008. Art, Marriage and Family in the Florentine Renaissance. New Haven and London: Yale University Press.

Neri di Bicci. 1976. Le Ricordanze. Edited by Bruno Santi. Pisa: Edizioni Marlin.

Paoli, Maria Pia. S. Antonino 'vere pastor ac bonus pastor': Storia e mito di un modello. In Verso Savonarola. Misticismo, profezia, empiti riformistici fra Medioevo et Età moderna. Edited by G. Garfagnini and O. Picone. Florence: Sismel, Available online: https://www.storiadifirenze.org/?biblioteca=s-antonino-vere-pastor-acbonus-pastor-storia-e-mito-di-un-modello (accessed on 9 April 2019).

Paolo da Certaldo. 1945. Il Libro di Buoni Costumi. Edited by A. Schiaffoni. Florence: Le Monnier.

Passerini, Luigi. 1876. Genealogia e Storia della Famiglia Ginori. Florence: M. Cellini.

Petrucci, A. 1984. Lire au Moyen Âge. Mélanges de l'Ecole française de Rome. Moyen-Age, Temps modernes 96: 603-13.

Pierozzi, Antonino. 1858. Opere a ben vivere di Santo Antonino. Edited by Francesco Palermo. Florence: M. Cellini. Randolph, Adrian. 1997. Regarding Women in Sacred Space. In Picturing Women in Renaissance and Baroque Italy. Edited by Geraldine A. Johnson and Sara F. Matthews Grieco. Cambridge: Cambridge University Press, pp. 17-41. 
Rigaux, Dominique. 1996. Un image pour la route. L'iconographie de saint Christophe dans les régions alpines (XIIe-XVe siècle. In in Voyages et voyageurs au Moyen Age. Actes des congrès de la Société des historiens médiévistes de l'enseignement supérieur public, 26e congrès. Aubazine 1996: 235-66.

Romagnoli, Alessandra Bartolomei. 2016. La Vita Religiosa Femminile alla Fine del Medio Evo: Temi e Problemi. In Litterae ex quibus nomen Dei componitur. Studi per l'ottantesimo compleanno di Giuseppe Avarucci. Biblioteca Seraphico-Capuccina, 104. Rome: Istituto Storico dei Cappucini, pp. 291-305.

Ross, Janet. 1911. The Lives of the Early Medici As Told in Their Letters. Boston: Richard G. Badger, The Gorham Press. Rusconi, Roberto. 1981. De la prédication à la confession: Transmission et contrôle de modèles de comportement au XIII siècle. In Modalité de la diffusion et de la réception des messages religieux du XII au XV siècle. Actes de table ronde de Rome (22-23 juin 1979). Rome: École française de Rome, pp. 67-85.

Sacchetti, Franco. 1857. I sermoni evangelici, le lettere ed altri scritti inediti o rari. Edited by O. Gigli. Florence: Le Monnier.

Savonarola, Girolamo. 1845. Prediche di Fra Girolamo Savonarola de' Predicatori. Florence: Alcide Parenti.

Schmidt, Victor M. 2001. Painting and Individual Devotion in Late Medieval Italy: The Case of Saint Catherine of Alexandria. In Visions of Holiness: Art and Devotion in Renaissance Italy. Edited by Andrew Ladis and Shelley E. Zuraw. Athens: University of Georgia, pp. 21-36.

Schmidt, Victor M. 2005. Painted Piety. Panel Paintings for Personal Devotion in Tuscany, 1250-1400. Florence: Centro Di. Schuchman, Anne M. 2009. The Life of Umiliana de' Cerchi (1246). In Medieval Italy: A Documentary History. Edited by Kate Jansen, Joanna Drell and Frances Andrews. University Park: Pennsylvania University Press, pp. 377-84.

Thomas, Anabel. 1995. The Painter's Practice in Renaissance Tuscany. Cambridge: Cambridge University Press.

Vauchez, André. 1990. Ordini Mendicanti e Societa Italiana XIII-XV secolo. Milan: Arnoldo Mondadori.

Vauchez, André. 1993. Comparsa e affermazione di una religiosità laica (XII secolo-inizio XIV secolo). In Storia dell'Italia Religiosa: 1. L'Antichità e il Medioevo. Edited by G. De Rosa, T. Gregory and A. Vauchez. Bari: Laterza, pp. 397-425.

Velluti, Donato. 1914. Cronica Domestica. Edited by Isidoro del Lungo and Guglielmo Volpi. Florence: G. C. Sansoni. Von Moos, Peter. 1996. Occulta cordis. Contrôle de soi et confession au Moyen Âge, II. Formes de la confession. Médiévales 30: 117-37. [CrossRef]

Walsh, Christine. 2007. The Cult of St Katherine of Alexandria in Early Medieval Europe. Aldershot: Ashgate.

Webb, Diana M. 1990. Woman and Home: The Domestic Setting of Late Medieval Spirituality. Studies in Church History 27: 159-73. [CrossRef]

Wieck, Roger. 1997. Painted Prayers: The Book of Hours in Medieval and Renaissance Art. New York: George Braziller. Wilkins, David G. 2002. Opening the Doors to Devotion: Trecento Triptychs and Suggestions concerning Images and Domestic Practice in Florence. In Italian Panel Painting of the Duecento and Trecento. Edited by Victor M. Schmidt. Washington and New Haven: Yale University Press, pp. 371-93.

(C) 2020 by the author. Licensee MDPI, Basel, Switzerland. This article is an open access article distributed under the terms and conditions of the Creative Commons Attribution (CC BY) license (http://creativecommons.org/licenses/by/4.0/). 
Article

\title{
Bringing Pilgrimage Home: The Production, Iconography, and Domestic Use of Late-Medieval Devotional Objects by Ordinary People
}

\author{
Sarah Blick \\ Art History Department, Kenyon College, Gambier, OH 43022, USA; blicks@kenyon.edu
}

Received: 25 April 2019; Accepted: 6 June 2019; Published: 20 June 2019

\begin{abstract}
Tracing the devotional beliefs and practices of everyday people during the late Middle Ages through documents is tricky, as most were written with other purposes in mind. To make up for this, it is necessary to examine the abundant material culture that survives from this period. By analyzing a variety of finds and comparing them with well-known objects used by the upper classes, it becomes evident that ordinary people shared the same religious views and practices. Both classes were interested in pieces that inspired active devotional and amuletic practice. They were intended to be gazed at and handled, then rested on a tabletop or nailed to a wall. Some folded, some rang, some could be blown through, while others were gazed upon. Lower quality materials and production of pieces had no impact on their ultimate use in the home.
\end{abstract}

Keywords: devotional; amuletic; pilgrimage; private devotion; reliquaries; mirrors; bells

\section{Introduction}

"It is notoriously difficult to assess how well ordinary people of the late-medieval period understood the theological significance of the mass-or indeed, the basic tenets of their faith in general" (Humpfrey 1993, p. 58; Jacobs 2013, p. 202, n. 11). In comparison to the upper classes (especially affective piety among the upper classes and nuns and monks), documents are mostly silent on how the devout among the lower classes practiced spirituality on an individual level (Belting 1994; Van Os 1994; Ringbom 1984; Brantley 2007; Hamburger 2007; Rudy 2011; Blick 2015). This essay proposes a way to begin to fill this somewhat-overlooked area by examining how mass production of interactive pieces, with similar forms and iconography as more-expensive objects, reveals that the lower classes were aware of and up-to-date on the latest developments in personal devotion and amuletic practice. Pewter, as the most popular and best surviving medium used to create such pieces, will be the focus of this article. Its malleability allowed artisans to make works that, with their intricate imagery, complex movements, and construction, ${ }^{1}$ could not be taken in at a glance; they were intended to be gazed at and handled, placed on a tabletop or nailed to a wall, rather than worn by the devotee. ${ }^{2}$ Initially

1 Not all movement was appropriate for devotional pieces. Spinning parts, found in toys and secular badges of the same period, were not used, presumably because it would have been disrespectful to watch a sacred figure spin. Some secular badges incorporate spinning, such as 15th-century pins in a tracery hexagonal shape with cusping and crockets surrounding a sun- or starburst and windmill badges with sails that rotate (Spencer 1998, p. 297; Spencer 1990, p. 103, cat. 235).

2 Some pilgrim badges with moveable parts, such as the tiny swords with scabbards produced at Canterbury as souvenirs of the martyrdom of Becket and at Mont-St-Michel as an emblem of the Archangel Michael, were not necessarily designed to create a personal devotional object (Spencer 1998, pp. 93-9, cat. 66-72c). See also the virtual exhibition http://www.inrap.fr/preventive-archaeology/Virtual_exhibitions/Virtual_exhibitions/Making_Pilgrim_Badges_ at_Mont_Saint_Michel/The_historical_context/p-1478-Pilgrims_and_the_Mount.htm. 
viewed close up and then stepped away from, this action, too, linked them to richer forms of devotional objects. $^{3}$

It should be noted that precise terminology describing these pieces, the people who interacted with them, and their perceived function is difficult because value judgments are embedded in the words typically used to discuss any of these aspects, such as "lower classes", "common people", "ordinary people", etc. Obviously, there were very poor and less poor people who were not rich, ordinary and extraordinary people who had different abilities and understanding, and so forth. Nuanced distinctions are not really possible because so few documents which reference this material name the users of these pieces; much less comment on their particulars. This essay presumes that those who purchased and used these objects were not wealthy or they likely would have chosen to buy something made of finer materials (following a hierarchy of gold, then silver, then silver gilt, etc.) and with finer workmanship. Terms like "sacred" and "secular", too, pose problems because these divisions were often non-existent and, even when weighed toward one side or the other, were liable to change, depending on how the owner of the piece viewed and used it. A pilgrim "souvenir" was sometimes regarded as merely commemorative, but there are many instances where it was viewed as a carrier of sacred power and protection. Likewise, a statue or box-no matter its medium—could be pleasantly decorative or be revered as a direct touchstone to the divinity depicted or contained therein in the form of relics. Obviously, how something was treated by its owner/viewer determined its value; a sliver of wood might be just that or it could be part of the True Cross. To that end, I will continue to use these general terms, but will endeavor to be specific when investigating these objects.

For example, the study of the production and use of pilgrim souvenirs has revealed a glimpse into the devotional interests of all classes. After pilgrims bought, displayed, and enjoyed their souvenirs, what happened to them? Their fates varied; they could be sewn into a manuscript, cast into a bell, or placed into moving water. Most were used, initially, for public display, attached to clothes, or worn around the neck. ${ }^{4}$ At times, though, their owners transformed them into private devotional objects. Some were pinned to small boards roughly fashioned into Gothic tracery (Figure 1). ${ }^{5}$ Nails driven through ampullae and badges attaching them to a wall in one's home or stable also accomplished the same thing (Figure 2). ${ }^{6}$ Going beyond pilgrim souvenirs—-for most of these pieces were not strictly connected to pilgrimage-devotees used all kinds of cheap adornment to create interactive works of art as reliquaries and sacred images to be used at home. Because not everyone had the skills to make inventive homemade devotional objects, artisans in the late Middle Ages mass-produced specially designed pieces made of low-quality metal which spurred individual prayer.

3 Denis Bruna (2006, fig. 109-15) first noted these kinds of objects and their use for personal devotion.

4 They could act as devotional objects and be a source of succor for the remainder of the pilgrims' lives (Köster 1985, pp. 94-96). Sometimes pilgrims made them more powerful by casting them into bells, morses, tankards, or baptismal fonts, or placing them in cattle troughs or fields to guard against vermin infestation.

5 Amsterdam, Historisch Museum, c. 1500 (Kammel 2000, p. 135, fig. 8). Badges are from Wilsnack and Blomberg, Amsterdam.

6 Many pilgrim souvenirs (especially ampullae, though they could be hung up by their handles) have been discovered with nail holes through them. See, for example, badges such as that of Adrian of Geraardsbergen, found in Middelburg in 1983 and now in Zeeuws Museum, inv. M84-004-04. My thanks to Hanneke van Asperen for this reference. See also http://www.findsdatabase.org.uk/hms/pas_obj.php?type=finds\&id=001482469ED018D2, http://www.findsdatabase.org.uk/ hms/pas_obj.php?type=finds\&id=00147AC7E1101B08. 


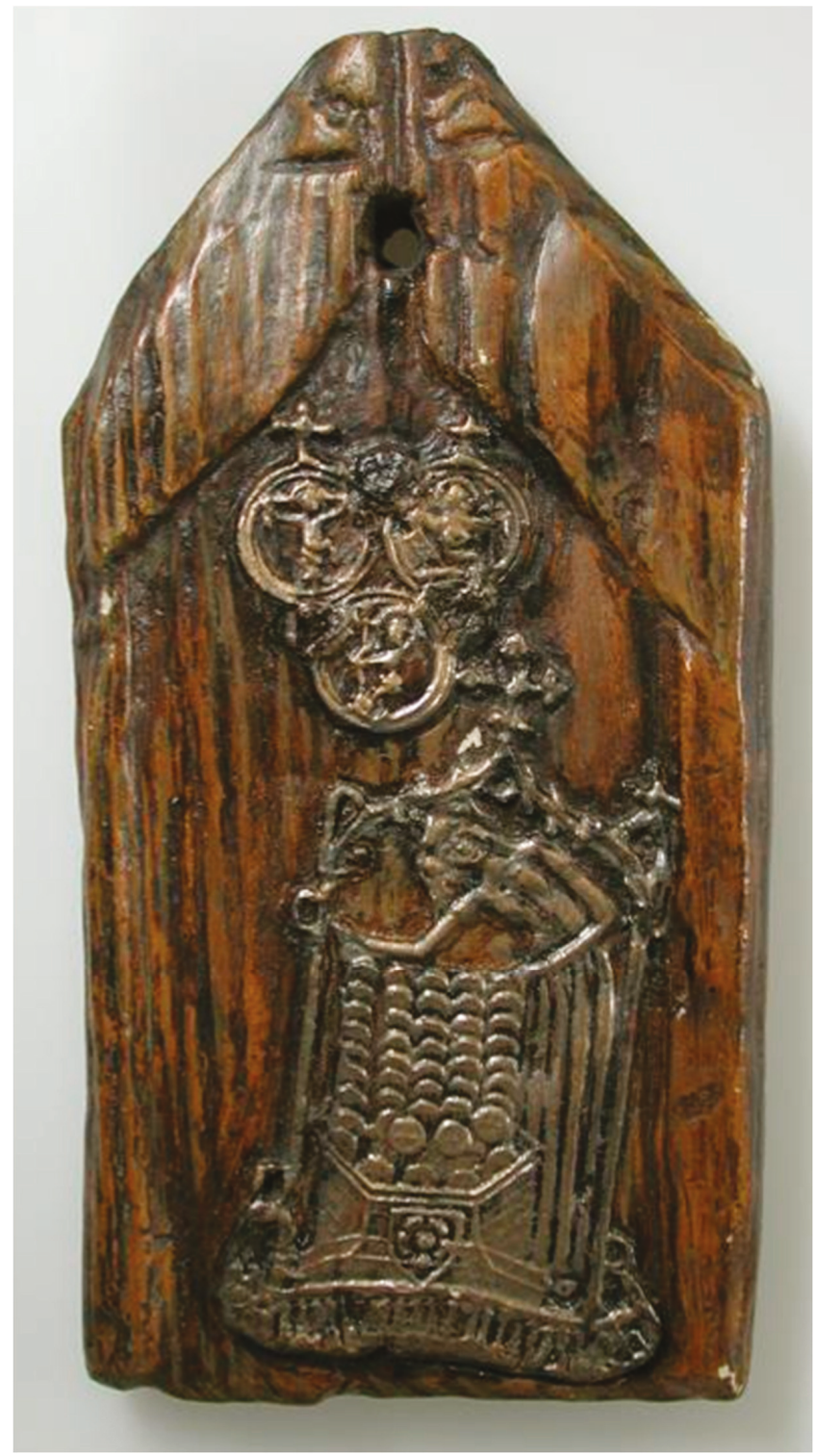

Figure 1. Carved board with pilgrim badges. Amsterdam, Historisch Museum, c. 1500. Badges are from Wilsnack and Blomberg, Amsterdam. Photo: Amsterdam, Historisch Museum. 

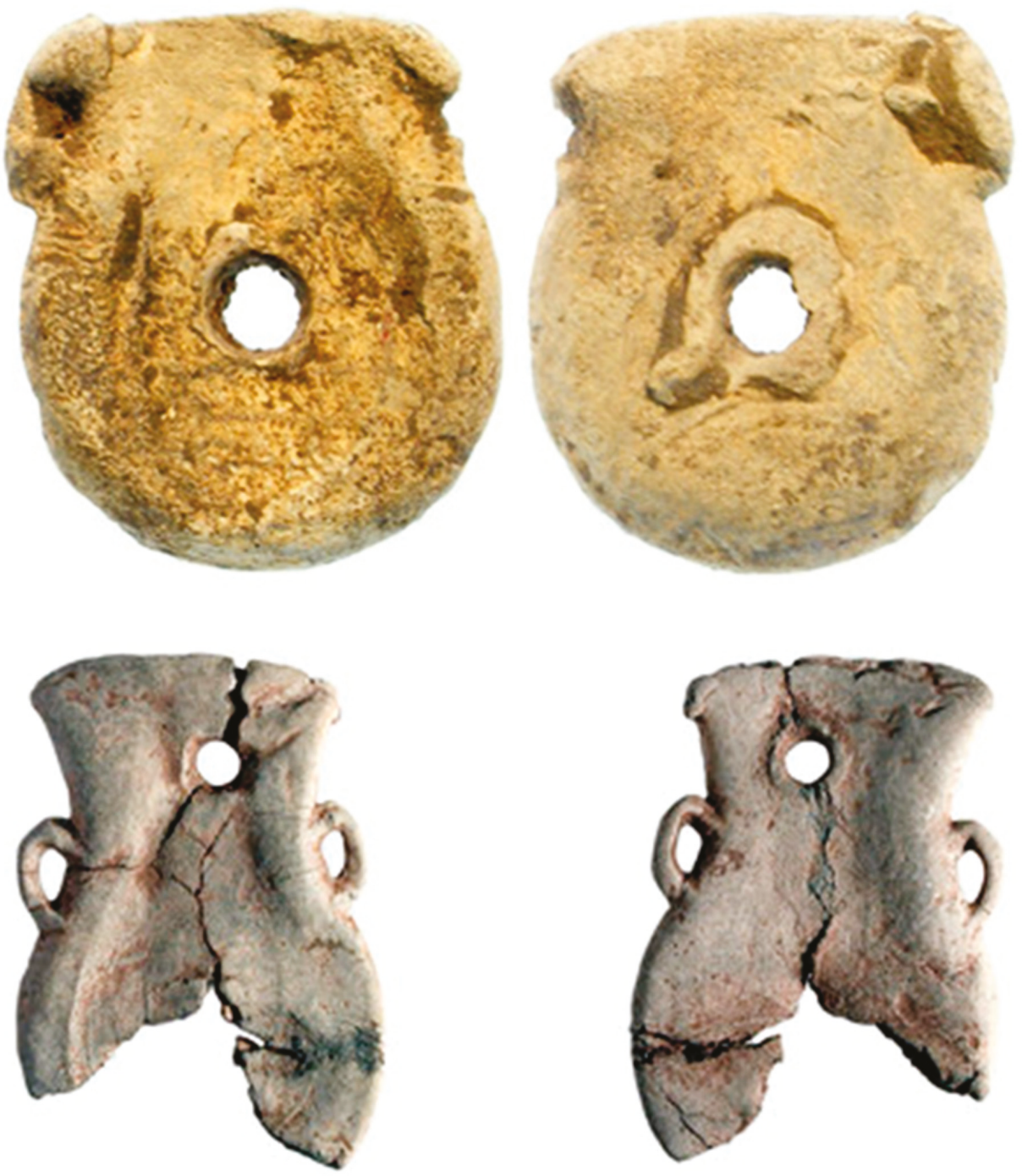

Figure 2. Pilgrim ampullae and badges with nail holes. 13th-15th century, tin/lead. Photos: Portable Antiquities Scheme.

\section{Private Devotion-Upper and Lower Classes}

The movement toward forms of private devotion for all classes in the later Middle Ages was facilitated by the production and distribution of small devotional objects that allowed the individual to ask for optimal health and salvation (Duffy 2006, pp. 57-58; Ringbom 1984, p. 19). The images were not strikingly different from those found in churches, just as the same prayers recited in church were repeated at home. Although St. Gregory (540-604) recommended the daily veneration of images to make the invisible visible and to increase emotions in the worshiper, few early-medieval examples of such pieces survive in the West. Beginning in the twelfth century, private images appear regularly in miracle stories and by the 13th century, portable images became popular throughout all 
the property-owning classes. In the 14th and 15th centuries, the lower classes begin to acquire private devotional images on a broad scale (Ringbom 1984, pp. 14, 52-58).

\section{Public versus Private Images}

Just as the wealthy used tiny richly illuminated books of hours, precious devotional jewelry, and small sculptures in their homes during prayer, so did the lower classes, using cheaper versions. For instance, an image of St. Anthony's tau cross was considered powerful whether it was made of gold or lead (Figure 3). Medieval commentators made no distinction regarding media in relation to the effectiveness of a devotional image and so, while the rich sought pieces made of the same precious metals and gems seen in public spaces, the lower classes purchased private devotional images made of pewter, pipe clay, bread dough, wax, and paper maché (Marks 2004, p. 18). ${ }^{7}$ Typically, class distinction in pilgrim souvenirs was denoted by the media used rather than the particular design. Sometimes the same badge was made from gold or silver or was hand-painted (as were the elaborate badges from Aachen and Our Lady of Undercroft, Canterbury Cathedral), while others featured colorful paper or shiny metallic backgrounds which showcased openwork badges. Some artisans copied expensive-looking items, creating tiny metal replicas of ivory mirror backs and jeweled brooches. That neither object was particularly associated with religious practice reflects the integration of sacred and secular.
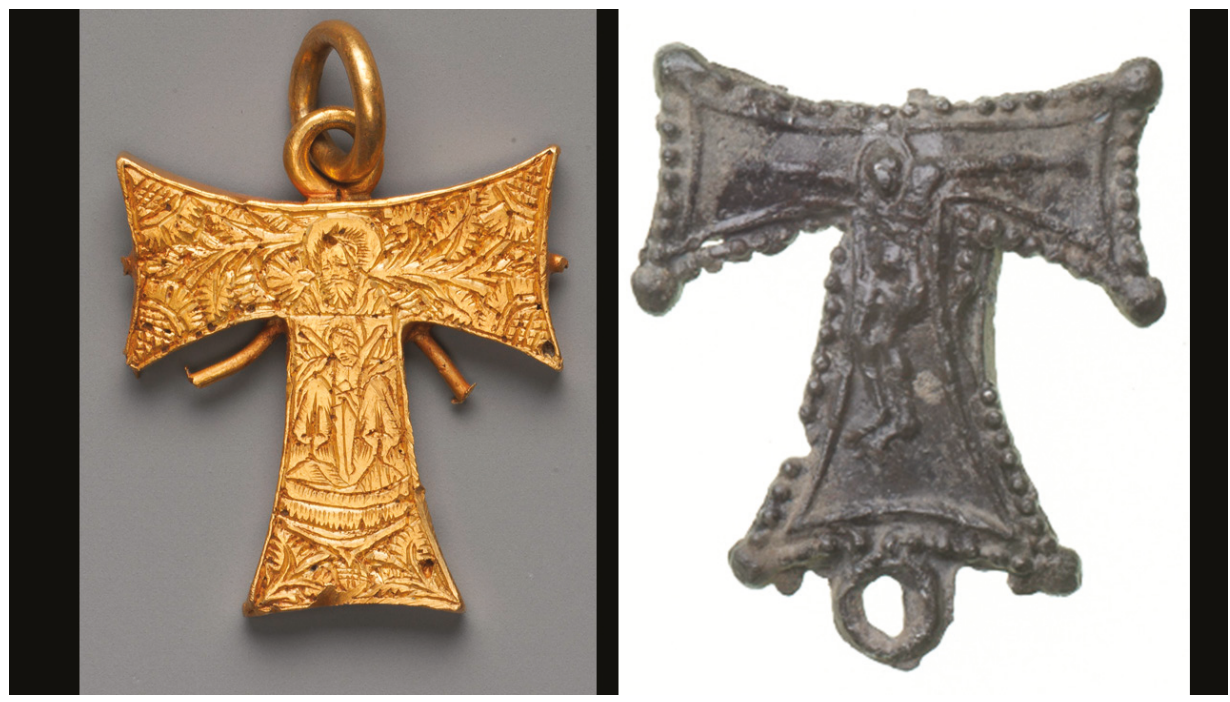

Figure 3. (Left) Pendant Tau Cross with Trinity and Virgin and Child, gold, England, c. 1485, New York: The Cloisters Collection. Photo: Cloisters Collection. (Right) Pilgrim badge tau cross with crucified Christ, pewter, England, 15th century. Museum of London. Photo: Museum of London.

Still, the small scale of devotional objects, whether rich or poor, emphasized that the action was a private one. While it could be claimed that personal ownership of images diffused the centrality of the cult image, the replication of the cult image in important ways strengthened its perceived power because worshippers wanted their own version of the public image. The reproductions held a similar capacity for the sacred power as the miraculous originals, many of which were enhanced by their role

7 On agnus dei discs, see (Jones 2000, p. 2 and Brückner 1993). Paper maché forms the central image and side roundels of a humble wooden triptych c. 1600, Berlin, Skulpturensammlung (Kammel 2000, p. 24, fig. 22). 
as contact relics, having been touched to the original piece and absorbing a kind holy radioactivity spreading outward (Finucane 1977, p. 26). Sophisticated or crude, large or small, all images were "essentially archetypes, containing levels of meaning within their histories and emblems that reified their powers of healing and salvation" (Roffey 2006, p. 27). ${ }^{8}$

Private images could be venerated anywhere. Bishop Reginald Pecock (c. 1395-c. 1461) wrote that they could be venerated "before a bare wall in a church, or in a corner of a church or of another house, or in the field" (Pecock 1860, p. 169), ${ }^{9}$ but the most popular and most recommended place for keeping devotional images (for those people who did not have the luxury of a private chapel) was the bedroom or a private secluded place (Figure 4). Here, they were nailed to the wall or placed on a domestic altar or in a niche, separated from the room with a curtain (Ringbom 1984, p. 38; Heyne 1899 , p. 271, fig. 70; Schultz 1892, p. 106.). In addition to images, miraculous wells and newly-found relics were celebrated, but not all discoveries were approved, as "stones, wood, trees, or fountains on account of anyone's dream or deception" were repeatedly banned in multiple dioceses (Golding 2001, p. 145). ${ }^{10}$ Their repetition reflects their ineffectiveness. Many church officials eventually gave up trying to ban them and instead placed them under episcopal control.

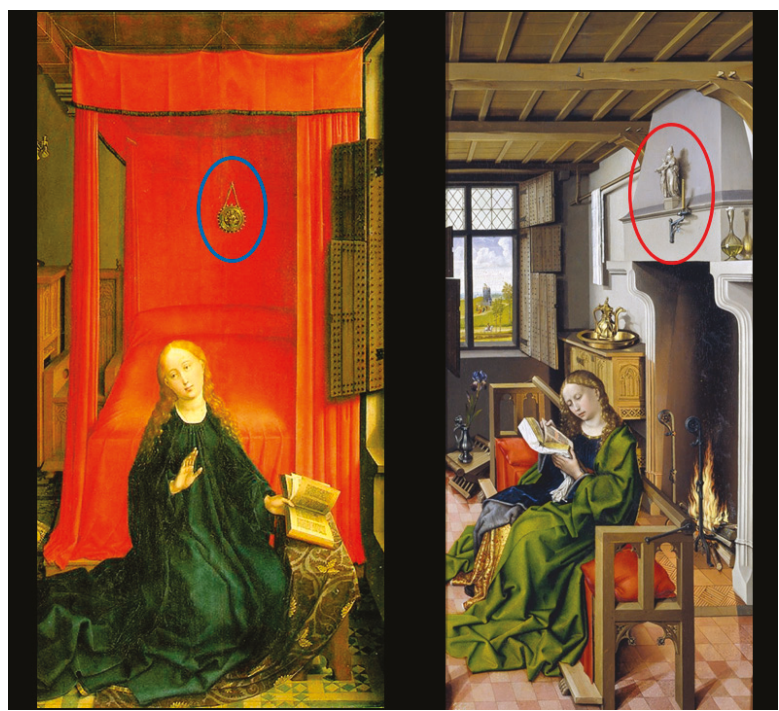

Figure 4. (left) Rogier van der Weyden, Annunciation Triptych (detail of center panel), 1440. Paris: Louvre. Note hanging devotional piece on the back bed-curtain. Photo: Wikimedia. (right) Robert Campin/Master of Flémalle, St. Barbara, wing of Werl Altarpiece, 1438. Madrid: Prado. Note image of the Trinity on the mantle. Photo: Wikimedia.

\section{Market}

To counteract the proliferation of unofficial images and relics, churches encouraged veneration of approved images. Some churches tightly controlled pilgrim souvenir production, renting out casting

8 Old Testament pictures were rarely found in private homes, but New Testament iconography was common to both church and home. With popular images, institutions could lose power, so some recalled worshipers by making their images more compelling through elaborate and sumptuous materials or through the aura of sanctity "conferred by age or authenticity" or by indulgences (Belting 1994, p. 410; Ringbom 1984, p. 53). Also portable were images in single sheets and small pamphlets, booklets, schedules, and quires (Aston 2004, p. 166; Belting 1994, p. 409).

9 English modernized.

10 Bans included Wells (1258), Exeter (1287), Lincoln (1290). 
molds and denying touch access to shrines of those badges not made in such molds (Cohen 1976). ${ }^{11}$ Money played a part in this decision because badges, commonly sold at patronal festivals, raised significant funds for various churches. When the Church of St. Andrew's, Canterbury, decided to sell "scuchons" (perhaps badges in the shape of the saltire cross) during 1519-1520, it purchased four leaves of gold paper to make the badges (at $4 \mathrm{~d}$ or pence) and paid a painter $13 \mathrm{~d}$ to create them, which they then sold individually for 3s 5d (Campbell 1998, p. 72, pl. XXVIIc; Marks 2004, pp. 83-84; Cotton 1917). Experiments today with replica molds and lead-tin alloy of the same mixture has shown that a badge a minute could be cast with the potential for thousands of badges in a single day, further increasing their profit potential. Yet, with so many badges lost or placed in fields and rivers (and found centuries later on river foreshores), it is apparent that they were not all revered equally and that every purchaser did not venerate every cheap piece of metal. This aspect of reception is the most difficult to trace. While there are many examples of deliberate placement of objects in rivers as votives from Neolithic times onward, Jennifer Lee has examined the ambiguity of such a determination for pilgrimage souvenirs. (Merrifield 1987; Lee 2014) It seems that some intangible element determined the depth of devotional reverence and how devotees handled what were, under normal circumstances, humble, almost-worthless objects.

Among these potentially worthless pieces, though, were a class of objects that demanded interaction and, by examining these, we are provided with evidence for distinctive forms of veneration among the lower classes. Their forms responded to changing needs of the faithful as they were intended to be gazed at and handled, then rested on a tabletop or nailed to a wall. The novelty of the interactive souvenirs drew customers to one's stall, as later pictorial evidence points to the sale of both religious and secular pieces at religious sites and at religious fairs, where pinwheels and noisemakers attracted attention $^{12}$ (Figure 5). For example, in order to cope with enormous crowds, the tightly-controlled monopoly on pilgrim souvenirs was opened every seven years for the jubilee at Aachen and artisans who wished to distinguish their wares from their neighbors created multi-layered badges, frames, mirror badges, and folding diptychs. These works tapped the upper end of the lower-price market, their artisans hoping to attract the pilgrim with more money and more complicated tastes by creating distinctive souvenirs.

11 Only certain artisans in Le Puy-en-Velay were allowed to sell badges; other kinds of souvenirs were not regulated.

12 Pieter Aertsen, Retour d'un pèlerinage à Saint Antoine (c. 1500), Muséedes Beaux-Arts de Belgique, Brussels, paints a stall with pilgrim souvenirs and toys, including small windmills, drums, and crossbows. 


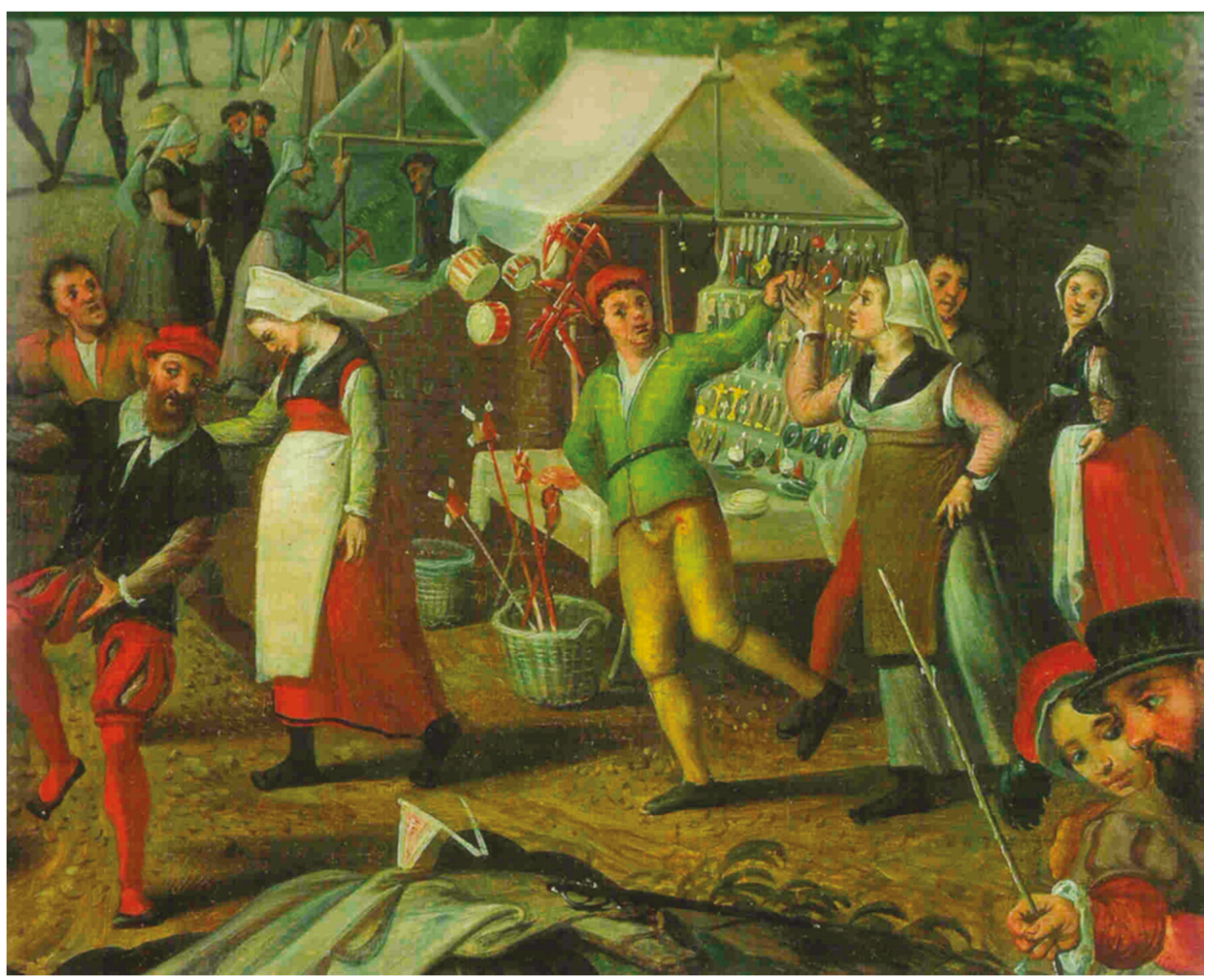

Figure 5. Pieter Aertsen, Detail of Retour d'un pèlerinage à Saint Antoine, c. 1500, Muséees Beaux-Arts de Belgique, Brussels. Photo: Author.

\section{Pilgrim Souvenirs or Private Amulets?}

Although these common devotional pieces were sold at pilgrim sites, their generalized iconography often makes it difficult to assign them to a particular church or to determine whether they were truly pilgrim souvenirs or private amulets purchased locally. Documents regarding pilgrim souvenir purchases are sparse; complicating matters, sometimes religious objects were resold elsewhere, meaning that not all pieces were acquired at a particular pilgrimage site. ${ }^{13}$ Most pilgrims at the lower end of the market visited local sacred sites rather than far-off shrines. When nearby, religious assistance could be repeatedly called upon. ${ }^{14}$ The purchaser of the piece, of course, knew whether it was obtained on pilgrimage or purchased by a peddler who brought it from a distant pilgrimage site. Either way, it is evident that they were used for both private devotional and amuletic purposes.

Belief in amuletic magical practice through the manipulation of religious images and texts also crossed class boundaries and the choice of what served as amulets was vast. Holy names, spoken or inscribed, were easily at hand. Most popular were those of the Three Kings (against epilepsy), as the Magi were believed to be magical sages (Skemer 2006, p. 62, n. 15). Names could be written anywhere: In water, butter, apples, cheese, or molded into bread and then eaten as medicine. Stones, sticks, and

13 There were attempts to regulate the trade. By the 13th century, 100 stalls were licensed to sell scallop shell pilgrim souvenirs at Santiago de Compostela, but selling them outside the town boundaries courted excommunication (Stalley 1988, p. 410; Köster 1985, p. 86).

14 http://peregrinations.kenyon.edu/vol2-1.pdf; (Bugslag 2006). 
leaves were also used, as well as pottery sherds, wooden boards, small metal sheets, printed amulets, and papal lead seals. Because the Gospels were believed to ward off evil, manuscripts, especially if their vellum had been cut from devotional books, were thought to be capable of stopping fire and flood (Skemer 2006, p. 110, 127-29). Some devotees rinsed the ink from holy books and drank the liquid as medicine (Skemer 2006, p. 137; Swartz 2001, p. 178). Chanting or listening to certain words was also thought efficacious. Rhyming words helped spur memory and prayers were intoned in multiples of three, five, seven, or some other sacred number (Skemer 2006, pp. 80,92). Written words, even if not directly read, manifested power; many amulets with inscriptions inside remained closed, because to open them might lessen their effect. This notion was mocked by reformers who particularly pointed to nonsense words used therein (p. 127). Nonetheless, wearing an inscribed amulet meant that, in a crisis, one would be protected even if one forgot to speak the holy words (p. 148).

Amulets (and sometimes pilgrim souvenirs, particularly ampullae on cords) were worn over the heart (considered the gateway to the soul and in need of protection against the devil). Sometimes called "books of the heart" (p. 135), amulets were commonly small reliquaries or phylacteria worn around the neck, or tiny metal (usually lead) plates inscribed and placed on the body for healing purposes. Alternatively, amulets and pilgrim souvenirs were pinned to walls in homes, stables, special trees, and even carefully placed in fields. William Tyndale (1492-1536) condemned itinerant friars who went door to door promising better crops or safe childbirth using the amulets they sold (Skemer 2006, p. 70, n. 127; Hopeful 2002). ${ }^{15}$ Such practices were not considered to be useless superstition, for when clerics criticized amulets and charms, they criticized their misuse rather than their effectiveness (pp. 5-6, 12-13). ${ }^{16}$ In "a world charged with signs", amulets and pilgrim souvenirs co-opted significant holy symbolism, thus amulets and souvenirs became interchangeable (Gellrich 1985, p. 62; Skemer 2006, p. 143).

\section{Types of Interactive Souvenirs}

In the late Middle Ages, many people enjoyed pranks and illusions. ${ }^{17}$ While the very wealthy purchased automatons and spinning wine fountains with bells, by the fourteenth century, advanced casting and assembling techniques made complicated pieces easier to produce, reducing their price for the lower classes. Some of this complexity appears in the secular market which sold such toys as a hollow-cast bird made with moving parts which could bob and stick out its tongue. ${ }^{18}$ Production of these intricate objects opened up possibilities for devotional interaction through movement and touch.

\section{Three-Dimensional Devotional Sculptures}

Reformation critics singled out three-dimensional pieces as having the greatest effect on the viewer (Kahsnitz 2005, p. 10; Marks 2004, pp. 18-19), a view reflected in many northern countries where bequests to parish churches of three-dimensional figures far outnumbered the two-dimensional. Whether two- or three-dimensional, devotees responded first to the figures' reputation for healing, but the notion that these figures were alive and responsive was enhanced by their apparent reality. For instance, the Salve Regina prayer asks Our Lady to "turn to us thy merciful eyes ... " (Marks 2004,

15 Churches bought batches of printed amulets, sold by itinerant peddlers, to distribute to parishioners.

16 Some believed that talismans should be kept from the uneducated as they could not understand them (Skemer 2006, p. 59, n. 111).

17 Automatons were popular adornments for late medieval public clocks, such as that on the tower of Orvieto Cathedral, cast in 1351 from the same metal composition as bells. A mechanical rooster crowed and fluttered its wings with every clock stroke (Frugoni 2003, pp. 86,89-90). There are also stories of the 16th-century king Charles V being entertained by military automata and wooden birds that seemed to fly around the room without support. (see also Truitt 2015; Roy 1980, esp. pp. 63-64; and Scot 1930, p. 198.) At fairs, jugglers used illusion to make one think that their heads were cut off and laid on a platter like John the Baptist.

18 Bird, Late 13th/early 14th century, Museum of London (Forsyth and Egan 2005, pp. 52, 56, 143, and fig. 9). Some pilgrim souvenirs ended up as toys. In 1487, a child, given a Becket pilgrim badge to play with, choked on it, and was only saved by appealing to the popular saintly figure Henry VI (Grosejean 1935, no. 133). 
p. 21; Littlehales and Bishop [1895] 1996, p. 34); perhaps the figures' realism encouraged devotees to touch, lick, and kiss such sculptures, typically starting at the feet (often shod in silver shoes), then attending to the hands and face (Marks 2004, pp. 170, 220; Aston 1988, p. 152, n. 91, p. 226). ${ }^{19}$ They were not mere blocks of wood or stone, but read as "hands", as "feet", and so on. Some have argued that the focus on the image of the divinity over relics in the later Middle Ages was meant to de-localize sanctity (Vauchez 1997, pp. 448-53), but it actually produced the opposite effect, creating multiple localities of sanctity through each church's patronal image. Having access to them at one's parish church, or even better, one's home, allowed people to touch and interact with divinity as they wished. Seeing the image repeatedly encouraged thoughts of the saint and their life and tribulations. These thoughts were meant to spur empathy and, by extension, a real connection with the holy figure. They could turn to the saint whenever they needed support, comfort, or absolution prompted by the easily accessible image.

This touch formed part of a visionary practice. In the normal course of things, objects on the altar were off-limits to the laity. To counteract this exclusivity (and hasten their time through Purgatory), the very wealthy had entire chapels created with figures, which allowed them to imagine themselves as witnesses to sacred events standing near and touching the sacred figures (Gelfand 2012). This imaginative slippage, emphasized through spiritual exercises, was facilitated by certain churches and cities which were identified as alternative Jerusalems or Bethlehems. Beyond this, the sacred event could recur, with the correct prayers, in one's home.

Three-dimensional votives adorned saints' shrines and in the homes of the wealthy; Charles V (1338-1380) owned a tableaux of small gold religious statues (Ringbom 1984, p. 36). The same demand for three-dimensionality was satisfied for a much larger population by the production of low-cost hollow cast figures (made with slush casting), either placed on pedestals or nailed atop walking staffs. For example, one could place a three-dimensional, hollow, standing figure of Thomas Becket (late 13th/early 14th century) with detailed episcopal robes on the top of one's walking stick (Lee 2014, pp. 9-10; Spencer 1998, pp. 72-73) (Figure 6). Other hollow-cast figures of Becket feature him on horseback or standing on the back of a flamboyant peacock, the latter referring to either the incorrupt nature of the saint (as peacocks were believed to never decay) or the practice of taking a vow on a noble bird (Spencer 1998, pp. 75-77). A copy of the reliquary bust of Charlemagne from Aachen ${ }^{20}$ was similarly used and a lovely seated Virgin, dating from the first half of the 14th century, was "an example of the ordinary man's devotional equivalent of the rich man's ivory" (Spencer 1990, p. 25). ${ }^{21}$ The line between toy and devotional object was not always clear. For instance, hollow busts of Christ as the Man of Sorrows and three grotesque tormentors were probably used as finger puppets (Egan 2005, pp. 64, 141-43, 146; Spencer 1998, p. 166, fig. 180b)..$^{22}$

19 One poor troubadour could give an image nothing, but "received a silver shoe slipped off and thrown to him as a gift" (Belting 1994, p. 305).

20 Spencer 1998, p. 260, no. 256; VRY89 26 (93.82) Museum of London. Mid-14th-16th century.

21 See also (Spencer 1987, p. 224, fig. 85: $8.6 \mathrm{~cm} \mathrm{~h} \times 2.8 \mathrm{~cm} \mathrm{w} \times 2.6 \mathrm{~cm} \mathrm{d),} \mathrm{private} \mathrm{collection;} \mathrm{Spencer} \mathrm{1990,} 25$.

22 These figures were possibly from the Boxley Rood pilgrimage site. (see also Egan 1997, p. 414; Egan 1998, pp. 281-82, no. 930-31.) One of these heads still retains a flat base; another has an internal second tube that enabled the head to be extended and then pushed back. 


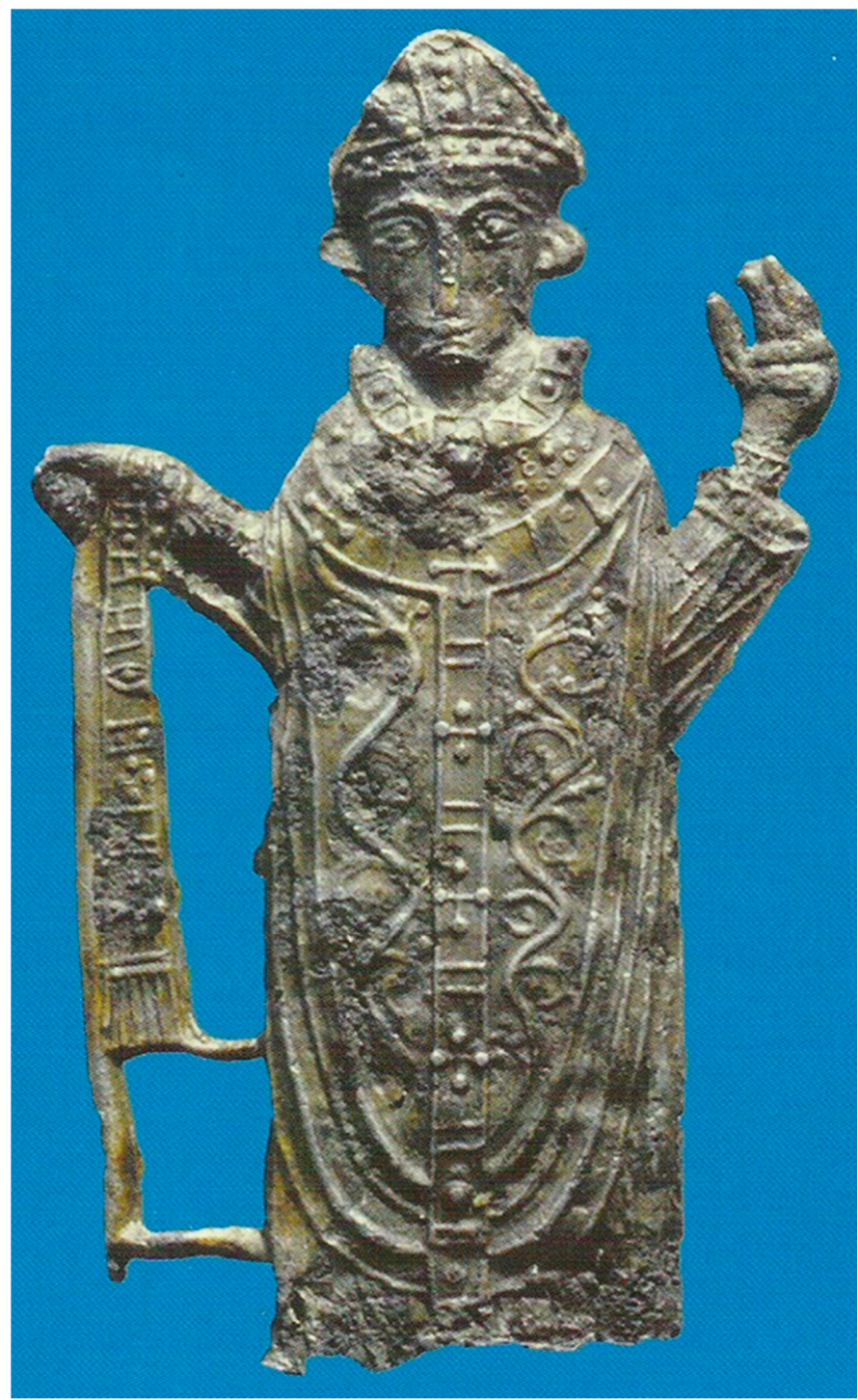

Figure 6. Hollow-cast figure of St. Thomas Becket, Canterbury, late 13th/early 14th century. Formerly Collection of Brian North Lee. Photo: Author.

Also manufactured were flat figures soldered onto round or square thin tubes that rise from a base. The imagery of holy figures posing on pedestals was something seen throughout church art, whether as sculpted jamb figures or as part of painted rood screens. ${ }^{23}$ The bases ranged in form, from eight-spoked cusped and beaded wheels to solid circles with inscriptions (AVE MARIA GRA[cia] PL[ena]) to stands with four legs cusped with trefoils (Figure 7). Most of the flat figures discovered

23 For example, the figures from the 15th-century screen at Branham Broom parish church, Norfolk: https://www.flickr.com/ photos/52219527@N00/23395013335/sizes/l. 
thus far date to the 15th century and feature a wide variety of saintly figures from a cardinal (possibly Jerome) to St. Martin, St. Barbara, and the Virgin and Child (Praha Museum of Decorative Arts 1985). ${ }^{24}$ These could be grouped together, as in the 14th-century Annunciation scene with the angel Gabriel kneeling and grasping a three-dimensional lily/fleur-de-ly on its circular stand supported by three cabriole claw feet. Measuring $8 \times 6.5 \mathrm{~cm}$, it was a significant work cast in separate pieces and soldered together. ${ }^{25}$ Another is a 15th-century piece with a Virgin and Child placed in a monstrance-shaped frame. This is, in turn, surmounted by Christ on the cross and then flanked by angels grasping chalices to capture the blood, all supported on a narrow tube soldered onto an openwork base (height $5.7 \mathrm{~cm}$ ). ${ }^{26}$

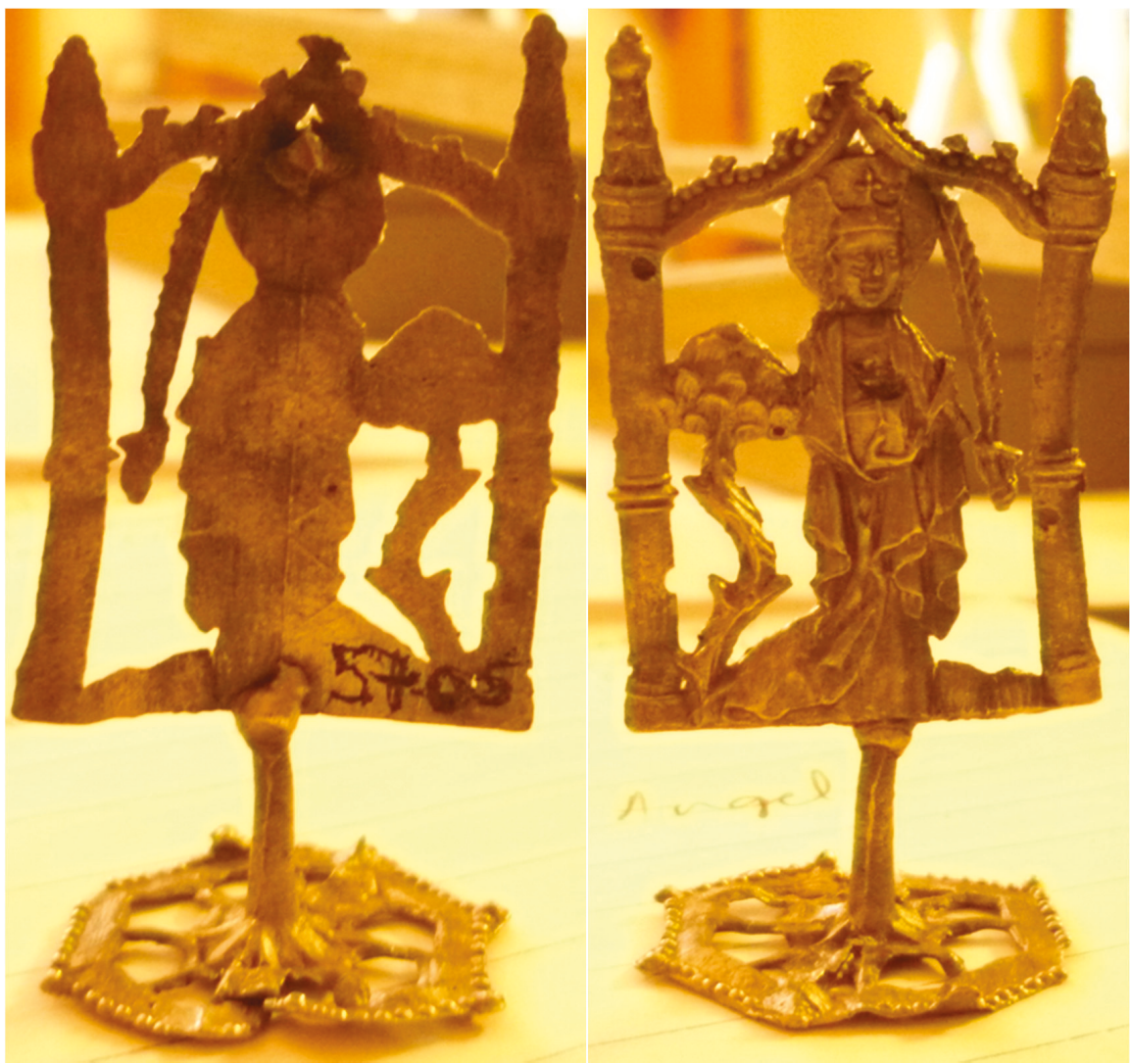

Figure 7. St. Barbara flat figure on framed stand, 15th century. Praha, Museum of Decorative Arts, Prague, Czech Republic. Photo: author.

24 Several are in Praha, Museum of Decorative Arts, including Barbara (UPM 5 705/1894), 15th c. (Praha Museum of Decorative Arts 1985), 21 no. 14, 23, no. 158; (Kühne et al. 2012, p. 94, cat. 152) and (St. Martin 1990) [UPM 5 704/1894] and p. 113, cat. 187. A cardinal (Jerome?), late 15th century, is in Paris at the Musée National du Moyen Age (17974) (Bruna 2006, p. 269). Two bases have survived without their figures in the Historisches Museum Basel (1904.2114 and 1904.2113) (Bruna 2006, p. 272, cat. 523-24). A number of unidentified figures retain pieces of the socle base.

25 Stedelijke Musea, Bruges. Another example of a free-standing souvenir is the less elaborate cross on a circular socle braced by two arcs on either side. On one side is the corpus with the INRI board above and on the other a beaded outline of the crossbeams (Bruna 2006, pp. 79-80, cat. 70).

26 Praha 1985, 25, no. 179 (UPM 5 658/1894); Kühne et al. 2012, 79, cat. 112-13. 
These statuettes could be set up on a mantle or table or placed in a carved tabernacle of their own. Some rare examples depict an entire three-dimensional scene, such as the 14th-century diorama of the Virgin and Child resting on a crib/chasse reliquary bed by an altar before which Charlemagne kneels; all is enclosed in a tiny architectural structure ${ }^{27}$ (Figure 8). That architectural frames were widely used is supported by the many surviving fragments of three-dimensional Gothic tracery. ${ }^{28}$

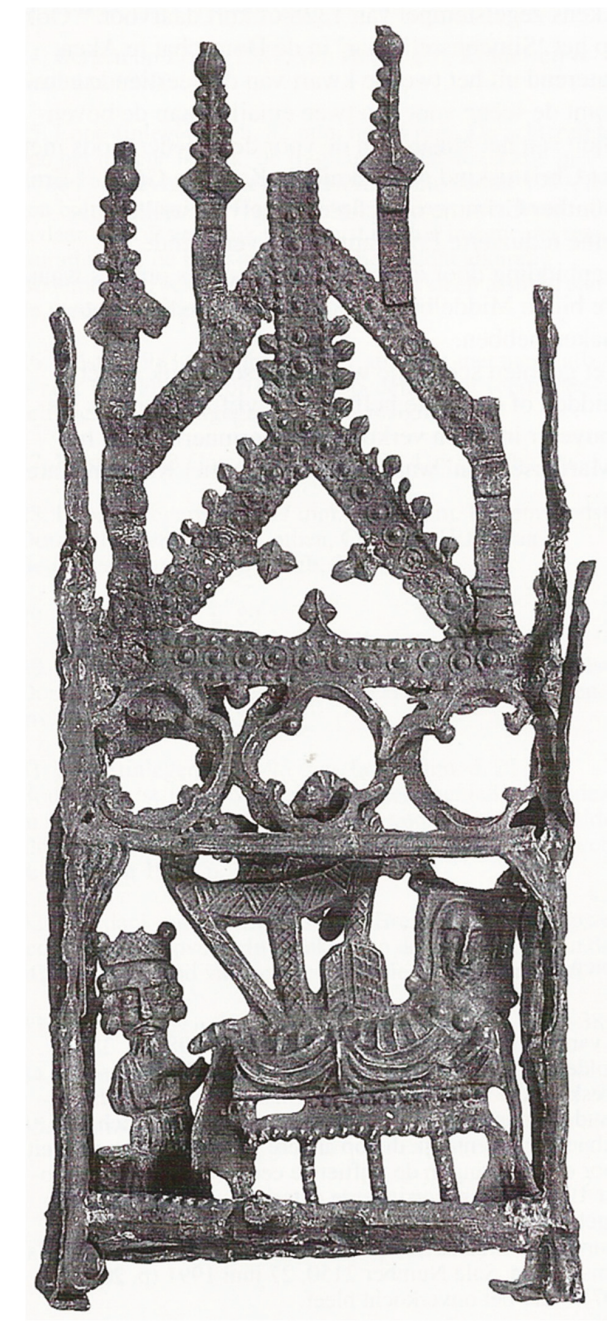

Figure 8. Pilgrim souvenir diorama with Charlemagne kneeling before the Virgin and Child, 14th century. Photo: After Koldeweij, "Karel de Grote-souvenirs", pp. 124-26, pl. 10.

$2795 \mathrm{~mm} \mathrm{~h} \times 43 \mathrm{~mm} \times 21 \mathrm{~mm}$ d. (Koldeweij 1989, pp. 124-26, pl. 10).

28 The upper portion of a traceried Gothic pinnacle measured $6.3 \mathrm{~cm}$ (Praha Museum of Decorative Arts 1985), p. 25, no. 190 [UPM 5 646/1894]). 


\section{Folding Objects}

Artisans and their customers were aware of the dramatic and emotional potential of hiding and revealing a sacred object. Objects were placed under cloths, behind screens, doors, shutters, curtains, and into special containers (Marks 2004, p. 243). By hiding the image at least some of the time, viewing became a privilege of heightened anticipation. For example, when congregations prepared parish church altars for Lent: "On the wall are curtains and hangings to be hung and raised. In the choir are to be set dosers, tapits [both ornamental hangings], and bankers (tapestry chair coverings), and a veil that was before the crucifix shall be removed and a pall put behind him because that which was hidden before the passion of Christ is now opened and showed" (Speculum 1936, p. 46 quoting Speculum 1936, p. 122).

Private devotional objects echoed the drama of concealment and exposure. As early as the ninth century, certain illuminations and decorative initials had curtains sewn over them to protect and decorate the image and to allow the reader a sense of revelation as they turned over the cloth to reveal the colorful picture beneath. ${ }^{29}$ For example, a Missal from Nuremberg, c. 1420, features a green silk curtain sewn to the top of an illumination of the crucified Christ flanked by the Virgin and St. John (Lohr 1986, p. 149, cat. 26). ${ }^{30}$ Such an image, placed facing the canon page, was kissed during the Mass (Sciacca 2007).

The same kind of suspense and privilege was found in folding retables on church altars or in wealthy private homes. In northern Europe, the retable's gold (unlike many painted Italian altarpieces) was hidden inside, to be revealed only on special feast days, while its exterior was commonly painted using neutral or grisaille coloring (Kahsnitz 2005, p. 14). As the viewer went deeper, the images often became more realistic, more colorful, and more dramatic, going from two- to three-dimensional or sculptural. Both rich and poor were enamored with the devotional possibilities of the triptych and diptych form. The wealthy had small, but sumptuously painted, ivory, alabaster, or precious metal versions, while the lower classes, as seen by many finds, had similar access to the folding and unfolding form, though done in base metal. Some of the more elaborate versions created tiny oratories, such as a late 15th century piece that features Saints Barbara and Catherine on the exterior wings and Gabriel and the Virgin Mary on their interior. The central image, once riveted to the back, is now missing (Bruna 2006, p. 274, cat. 530; Spencer 1998, pp. 154-55, figs. 165a,b; see also Bruna 275ff).

Far more prevalent were tiny folding pewter triptychs, latched in the center, often backed by colored paper and painted, and whose iconography was almost as varied as that seen on larger, fancier triptychs. ${ }^{31}$ Annunciation scenes with Gabriel on one wing and the praying Virgin on another are typical, as are heraldic shields, usually decorating both sides. Though small, these were used to illustrate whole iconographic cycles, as seen on the two panels of a triptych from the first half of the 14th century, adorned with a program of the infancy of Christ, including the Annunciation (center top), Visitation (top left), Adoration of the Magi (center with horses and groom waiting to the left), and the Massacre of the Innocents (bottom) (Spencer 1980, pp. 26-27; Tait 1955-1956, pl. XIVC). Cast as openwork (with cusped tracery), it was backed by a diaper pattern made of thin lead (Figure 9).

29 Fictive curtains, too, were used to frame important images and give the viewer a sense of ceremony when seeing Christ or Mary or other sacred personages.

30 See also Hague, National Library of the Netherlands, MS 130 E 18, fol. 86v, where a miniature of a funeral mass is still covered by an attached curtain and there are also traces of pilgrimage badges (Van Asperen 2009, pp. 324-25, no. I 43). Rudy (2015, pp. 84-85, fig. 66) suggests that the book owner treated the miniatures with curtains as altarpieces. My thanks to Hanneke van Asperen for these references.

31 Colored-paper backing can be seen on the pilgrim badges which decorate the hat of Saint Sebaldus, Saint Veits Altarpiece, by the Meister des Augustineraltars, 1487, Nuremberg, Nationalmuseum. Dabs of paint can also be seen on the badge in the upper left in Book of Hours (Use of Angers) with pilgrims' badges, in Latin, illuminated manuscript on parchment, France (Angers?), 1450-1475 http://www.textmanuscripts.com/medieval/book-of-hours-use-of-angers-96384. 


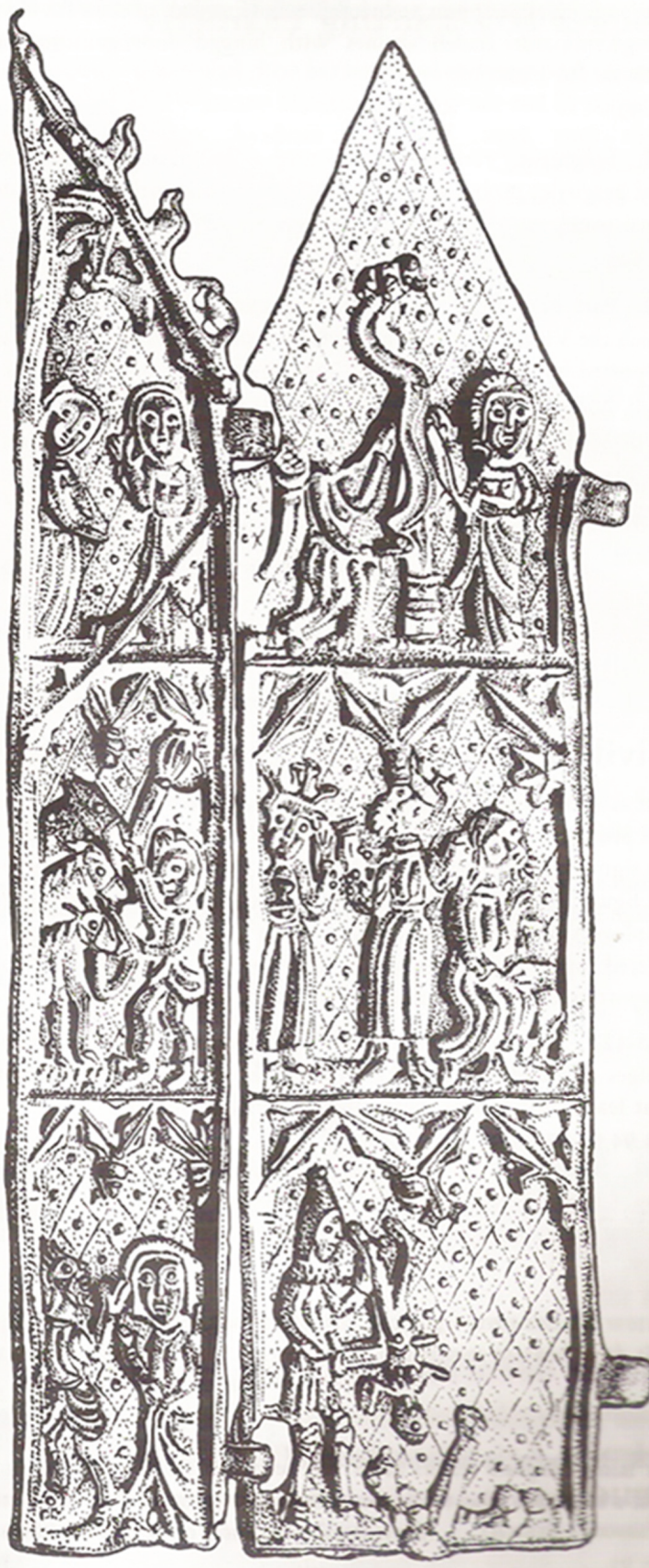

Figure 9. Pilgrim souvenir triptych, first half of the 14th century. Norfolk, Lynn Museum. Photo: After Spencer, Medieval Pilgrim Badges from Norfolk, pp. 26-27. 
The most spectacular finds of this kind are late-14th/early-15th century elaborate diptychs from Aachen. Cast of pewter with openwork elements soldered on one example pictures on its interior panels Saints Catherine and Barbara flanking the Virgin and Child and Cornelius and Anthony flanking Christ Salvator. Above the women, the Holy Tunic is repeated (this time backed by rays of light) and above the men is the nimbed vernicle portrait, with both showing the back side of the Calvary scene on top. Another example features an interior panel made of paper painted with a black-robed angel holding up the white tunic of Mary on a red background. The architectural structures of some examples display loops, allowing the devotee to wear, or more likely, hang, the folded diptych as they wished $^{32}$ (Figure 10).

Folding was integral to another form of devotional object, a sort of do-it-yourself reliquary. Only two (almost) complete examples survive, though many fragments have been found. Cast in openwork designs, they laid flat until the purchaser pulled the soft metal apart and folded the object into shape, firmly fastening it with the built-in clips. One example features two long sides made of gabled and cusped Gothic arches and a four-part quatrefoil decorated roof line. At its gable end stands a bishop saint with a pilgrim kneeling in devotion ${ }^{33}$ (Figure 11). Unfortunately, the opposite end does not survive. The hollow cage allowed one to see and rattle the object placed within (probably a pebble or other physical souvenir taken from the site). Earlier reliquary-shaped ampullae had been slush cast in the form of reliquaries which contained holy water or oil. In contrast, the folding reliquary allowed the pilgrim to place their own relic inside. ${ }^{34}$ Once filled, they could be votive gifts. Perhaps they were expressly created for children, as some late-medieval Italian preachers recommended that children should play with and decorate toy altars, and a number of fragmentary remnants appear to have once formed miniature altars. ${ }^{35}$

$32111 \mathrm{~mm} \times 57 \mathrm{~mm}$ (Van Beuningen et al. 2001, pp. 59-66, 326).

33 The other example has three vertical sides and two roof panels that could be folded into a three-dimensional reliquary châsse. Measuring $7.7 \times 3.5 \mathrm{~cm}$, done in openwork patterns with clips (Pieters et al. 1997-1998). Now in Oostende, Heemkundig Museum De Plate. See also Kühne et al. 2012, p. 150, cat. 266; and Praha 1985, p. 25, no. 186 (UPM 5 641/1894), 14th-15th century, $4.3 \mathrm{~cm}$ in height.

34 See Spencer 1998, pp. 65-71, for examples from Canterbury.

35 Fragments of tiny furniture are described in Egan 1998, p. 127. See also (Webb 1990, p. 159); and (Trexler 1980, p. 377). 

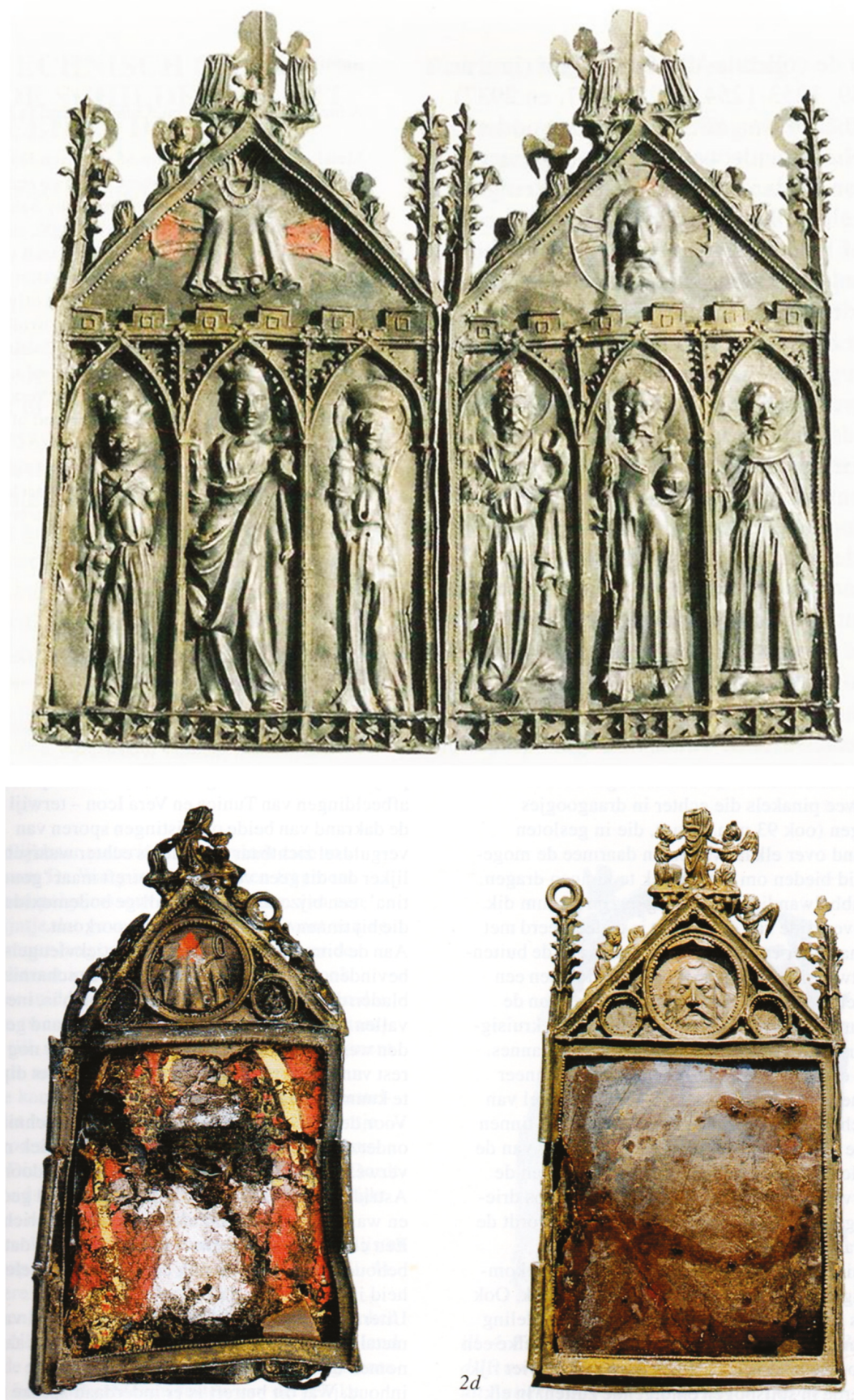

Figure 10. Pilgrim souvenir diptych from Aachen, Germany, late 14th/early 15th century, Cothen, Collection of H.J.E. van Beuningen. Photo: After Van Beuningen et al., Heilig en Profaan 2, pp. 59-66, 326. 


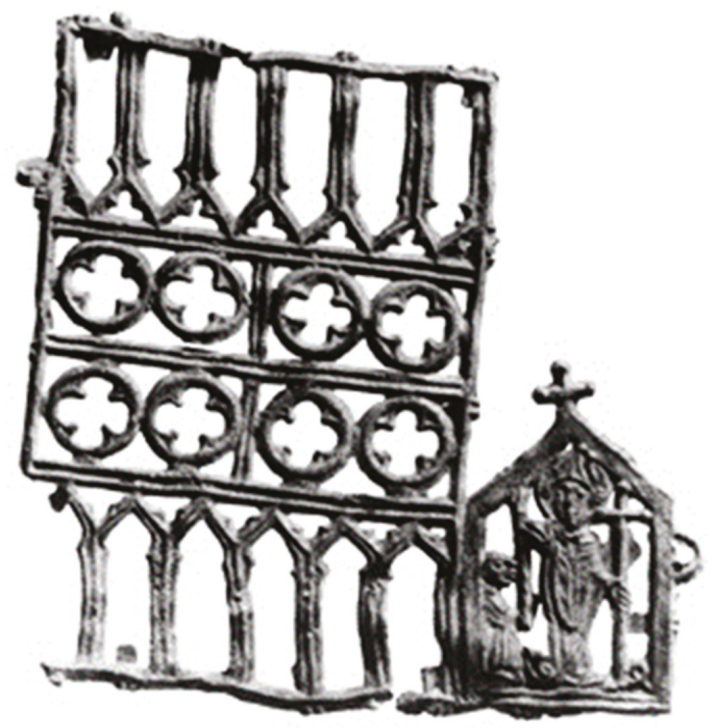

Figure 11. Pilgrim souvenir in the shape of a foldable reliquary, 14th/15th century. Praha, Museum of Decorative Arts, Prague, Czech Republic. Photo: After Kühne, Jungfrauen, Engel, Phallustiere, p. 150, cat. 266.

\section{Lockets, Chains, and Cylinders}

As the desire for ever-greater personal interaction with the divine grew, so did the demand for sacred jewelry. Of interest are those that encouraged touching or manipulation with hinged containers, lockets, chains, and loops. Most common are pins, brooches, and necklaces that featured hidden cavities filled with relics made of precious metals, enamels, and gems. Those with less means copied these ornaments in lead or copper alloy, like the Carpow Reliquary, meant to contain a tiny sliver of the True Cross. ${ }^{36}$

Lockets functioned similarly and could contain small, flat items picked up on pilgrimage. An example of this is the openwork piece from the 14th century, which shows a bishop with a crozier and a king holding a model church with the inscription +AVE MARIA GRATIA PLENA DOMINUS $\mathrm{TECV}(\mathrm{M}){ }^{37}$ Another locket—here revealing a slippage between sacred and secular—copied a mesh, pendant purse (decorated with lattice patterns of pierced quatrefoils) with fictive drawstrings and tassels. It held coins or other good luck tokens, drawing wealth to their owners (Koldeweij 2006, pp. 116-17, cat. 309-11. Koldeweij 2006, pp. 153-57, on coinage adopted for pilgrimage and amuletic reasons). These were sometimes suspended from fleur-de-lys in circular beaded and hatched frames which attached to a larger chain, though there are instances of suspensory loops dangling miniature secular objects such as scissors or a pair of pattens. Religious figures were also dangled on chains for pleasure or used for ligature charms, such as the pilgrim badges of the Virgin and Child and John the

36 Copper alloy was used more frequently in pilgrim souvenirs in the second half of the 15th century (Spencer 1998, pp. 167-70; Hall 2007, p. 77). A folded textual amulet was found in the Ingleby Arncliffe Crucifix (Skemer 2006, p. 183). Personal reliquary crosses, popular in Byzantium, were often incised with images of holy figures on their exterior. See, for example, the "Reliquary Cross with Saint George [Byzantine] (2000.526.2)" at. http://www.metmuseum.org/toah/hd/priv/ho_2000.526.2.htm. In the West, these items were made out of precious materials beginning around 1000.

37 Now in Ghent, Oudheidkundig Museum van de Bijloke (3538) (Heins 1897-1915, p. 261; Koldeweij 2006, p. 24, fig. 1.13, 16th century, $50 \mathrm{~mm}$ diameter; Van Asperen 2013, p. 230). Secular counterparts can be found too, such as a locket with Tristan and Isolde in Spencer 1998, p. 327. Van Beuningen 2001, p. 360, cat. 1516 illustrates a crude hinged locket with the Vera Icon (front) and the Agnus Dei (reverse), 1350-1400. 
Evangelist from 's-Hertogenbosch and St. George. The latter had a loop rather than a pin so that it could be wired, perhaps, to armor or connected through another charm in the shape of a Tau Cross (Spencer 1998, p. 159, cat. 176b, p. 186, cat. 206f).

\section{Rattles, Bells, and Whistles}

Less delicate and purposefully more raucous were rattles, bells, and whistles sold at pilgrim shrines and popular markets. Sweet sounds of singing and chanting were praised and considered necessary for church services, while jangling, loud noises were prized for their ability to scare off demons and threatening weather. ${ }^{38}$ Such noises grate and distract-the joyful focus of children and troublemakers-and were used in processions and at fairs and in ceremonies (Spencer 1990, pp. 62-63). The pieces that made these noises are more difficult to identify as devotional. This was probably intentional because, as mentioned earlier, some churches kept control of the pilgrim souvenir trade by renting out approved molds for badges, while allowing artisans to create non-badge objects without oversight or payment. ${ }^{39}$ Yet, the devotional was implicit too, as molds used to produce these whistles have been found with molds used to create other, more-definitively religious pieces at the same sites, such as at Mont-St-Michel. ${ }^{40}$

Rattles as noisemakers, produced in the 14th century, were commonly made of reticulated tracery in two hemispherical halves soldered together enclosing small shells (referring to pilgrimage?), pebbles, or bells (Figure 12 left). Some had handles, but most were probably meant to be worn on a cord around the neck or just carried, as their size makes them fit neatly within one's palm. The user could not only hear the contents shake, but see them rattle around. Again, objects like this are situated between the sacred and the secular; they could just be used as a rattle, but they had the potential to also call upon holy protection for the infant who was shaking it (Spencer 1990, p. 64, cat. 197-98; Spencer 1998, pp. 209-11).

Whistles both sacred and secular were even more popular; it is sometimes hard to tell for which market a whistle was made. For instance, at Rocamadour, molds were discovered for whistles in the shape of a man's head or a cockerel. The cockerel was a noisy bird who could be used for apotropaic frightening of evil (Spencer 1998, p. 207; Rocacher 1980). Other examples were cast in the shape of boatswains' whistles with a tube attached to a hollow bulb. With a loop cast as an attachment, people could run a cord or chain through it and wear it around their neck.

A whistle in the form of a man's head, keeping with the cockerel theme, has a bird perched on top of the head (Spencer 1998, pp. 207-9; Bruna 2006, pp. 296-68, cat. 564-65). Horn-shaped whistles were also popular and have been found with inscriptions that range from the holy (ave maria) to the profane (bla me or blow me) (Spencer 1998, p. 209; Spencer 1990, pp. 63-64, cat. 195-96) (Figure 12 right). A particularly extravagant type was the 15th-century whistle or sifflet, which turned when blown (Bruna 2006, pp. 345-46, cat. No. 673). Boat-shaped whistles could be purchased at coastal sites dedicated to the Virgin, like that of Our Lady of Boulogne-sur-mer, which were decorated with scales and a dragon-head prow and surmounted by a tiny figure of St. Nicolas or trilobe tracery (Spencer 1990, p. 63; Bruna 2006, pp. 89-91). Pottery horns, too, were manufactured at Aachen, Cologne, and St. Nicholas-de-Port near Nancy, France. Just as horns were loudly blown at processions and

38 Many sources exhorted the faithful to make "honeyed" sounds. Basil the Great noted that vocal music was to be like honey smeared in a cup of bitter medicine leading to tranquility and peace (Basil the Great 1857-1866, p. 212) and John Chrysostom wrote of hymns as uplifting the mind with modulated melody (Chrysostom 1857-1866, p. 156). Colossians 3:16 speaks of "singing psalms and hymns and spiritual sings with thankfulness in your hearts to God." My thanks to Rebecca Abbott for her help in suggesting sources and discussing this topic with me.

39 At Mont-St-Michel, the monks required a portion of the profits of the badges sold to be turned over to them. See the INRAP virtual exhibition referenced in note 2 .

40 http://www.inrap.fr/mont-saint-michel-une-production-d-enseignes-de-pelerins-5083. 
the exhibition of relics, a whistle such as this would have drawn attention of even the most devout passerby (Spencer 1990, p. 63; Meckseper 1985, vol. 1, pp. 408-9; Van Heeringen et al. 1988, p. 10). ${ }^{41}$
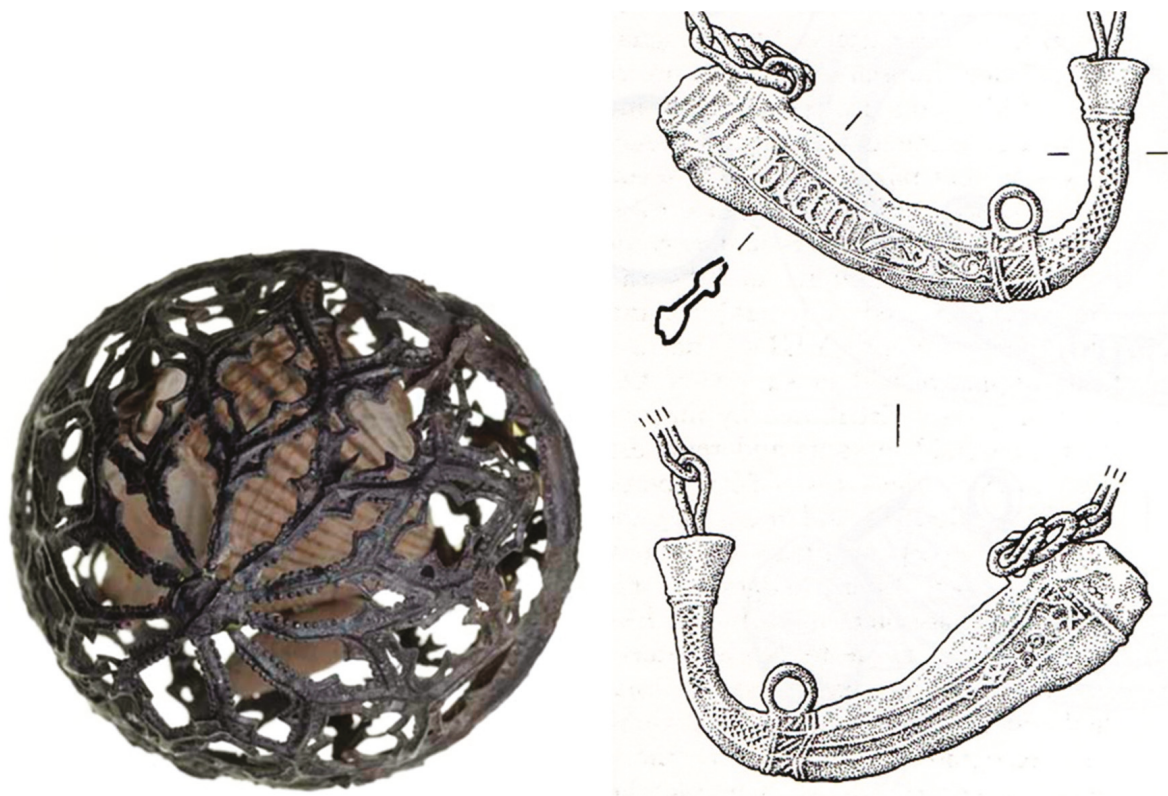

Figure 12. (left) Rattle with shells, 14th century, Museum of London, Photo: Museum of London. (right) Secular whistle, 15th century, Salisbury Museum of Art. Photo: After Spencer, Pilgrim Souvenirs ... Salisbury, pp. 63-64, cat. No. 196.

Noise from bells was also believed to scare away evil. The Golden Legend, describing the processions of the Lesser Litany, explained that devils cannot stand the sounds of the bells (called Christ's trumpets), so they flee and the storms abate. Bells were named after saints that were believed to be efficacious against bad weather, but the name could be changed to that of a demon if they proved unworthy (Ryan 1993, p. 287; Scribner 1987, pp. 14-15). Bells also accompanied the raising of the covers of some shrines. ${ }^{42}$

However, bells were more important than whistles. They were part of the church furnishings and set the rhythm of the medieval day, sounding the hours (set by sundials, hourglasses, calibrated candles, or water clocks) (Frugoni 2003, p. 86). The sacristan rang bells, calling monks and nuns to worship at certain times of the day and night and regulating meal and sleep times. Bells were integral to the daily and yearly changes in a church and both large and small hand bells were rung during services (Scribner 1987, p. 22). Sacring bells were rung when the host was elevated during the Mass. There was also the Ave Bell rung morning, noon, and night. When one heard it, one knelt and said at least one Ave Maria so that one could receive a papal indulgence, as that paid for in 1481 by Elizabeth Woodville, queen of Edward IV (Duffy 1992, p. 408; Bliss and Twemlow 1867, vol. 13, pt. 1, pp. 90-91).

Even during funerals, bells played a crucial role. They would entice those within hearing distance to pray for the souls of the deceased (French 2008, p. 55; Speculum 1936, p. 234). "The first ring was to announce that the person had died and to ask that those within hearing pray for their soul, then more notes would be rung depending on the status of the deceased." Bellmen were "to go about according to

41 For ceramic examples, see Koldeweij 2006, pp. 75-76, cat. 4.10-4.13.

42 Six silver bells accompanied the raising of the shrine cover at Durham Cathedral (Fowler 1964, p. 4). 
the use and custom of the town" on the funeral day and the seventh day, month, and year anniversary, ringing hand bells as they walked around the town. The sound of the bell was believed to scare away devils that might attack the soul (Heath 1984, p. 217; Daniell 1997, p. 53). Bell-ringers were paid more than the clergy performing the funeral service, reflecting their importance (Daniell 1997, p. 53; Burgess 1987, p. 188).

Most well-known are the souvenir bells from Canterbury (some inscribed CAPANA THOME, Thomas's bell), but bells were also sold to pilgrims at Amiens, Mont-St-Michel, and Rocamadour (Bruna 2006, pp. 263-68; Koldeweij 2006, pp. 171-75; Spencer 1998, p. 123). ${ }^{43}$ Round in section with clappers inserted and held by a split pin on the top of the bell, they were meant to be resonant. Metal analysis shows they were cast with high-grade pewter (including copper and bismuth) (Spencer 1990, p. 24). Their diminutive size would have lessened their impact on sight, but it was their tinny, clanking sound that probably brought them closer to the annoying sounds of whistles than to the sonorous, deeply meaningful large church bells. As William Thorpe, a Lollard preacher, complained in 1407, "what with the noise of their singing, and with the sound of their piping and with the jangling of their Canterbury bells, and with the barking out of dogs after them, they make more noise than if the king came there away, with all his clarions and minstrels" (Pollard 1903, p. 97). Visually, they referenced the larger bells and perhaps sought to share in the reverence created by true church bells.

\section{Mirrors}

These figures worked by stimulating the imagination through touch and through gaze. Acting on the latter were pilgrim souvenirs that featured mirrors. For example, every seven years at Aachen Cathedral, between Easter and October 1, the main relics went on display, including the nightgown that the Virgin wore on Christmas, Christ's first swaddling cloths (Joseph's stockings), Christ's loin cloth, shroud, and even the cloth that once wrapped John the Baptist's bloodied head. Initially, they were shown inside the cathedral. Later, beginning in 1322, the relics were displayed outside from the tower gallery, and scaffolding was set up to give pilgrims a good view (Spencer 1998, p. 259). By the fifteenth century, overwhelmed with pilgrims, the church opened up its souvenir monopoly every seven years to any artisan. Attracting artisans from all over (including Johannes Gutenberg), some created complex badges with tiny mirrors. Unable to directly come into contact with the relic or its shrine, the mirrors allowed the pilgrim to reflect the image of the displayed relic into their mirror, which could then reflect the image onto a piece of bread for ingestion, etc. One mirror badge illustrates the tunic of the Virgin (Aachen's main relic) and the vernicle portrait of Christ, both surmounted by tiny Calvary scenes with praying, kneeling angels. Surrounding the whole is the inscription AVE MARIA GRACIA PLENA DO[MIN]I.

With the mirror placed near the center of each badge, the act of beholding is emphasized as it was on other mirror badges from 's-Hertogenbosch and Cologne Cathedral (Figure 13). Mirrors were also believed to blind evil spirits, so they were hung from trees and were set into the evergreen Wildman costume during the Nuremberg Carnival. Some pilgrims of the same period wore coronets made of linked mirrors over their hats. ${ }^{44}$

43 Other bells were associated with St. Anthony pilgrim badges in the shape of a Tau cross (Van Beuningen and Koldeweij 1993, p. 125, cat. 35).

44 (Sumberg 1941, p. 106, fig. 18); (Koldeweij 2006, p. 44, fig. 2.51), illustrating a detail from the Legend of St. Lucy by the Master of the St. Lucy Legend. Bruges, Church of St. James, c. 1480. 


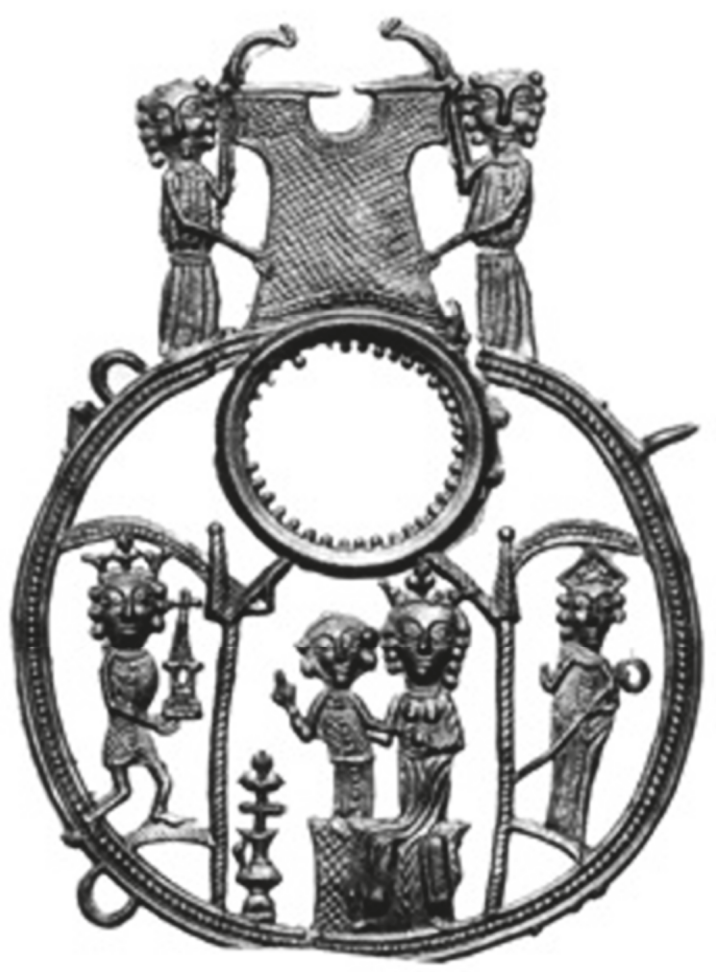

Figure 13. Pilgrim badge with mirror frame, Aachen, 1350-1400. Cothen, H.J.E. van Beuningen Collection (Inv. 3492). Photo: After Van Beuningen et al., Heilig en Profaan 2, p. 316, cat. 1347.

\section{Conclusions}

Scholars agree that personal devotion grew more intense and tactile in the later Middle Ages. ${ }^{45}$ Different kinds of devotion focused the attentions of the individual on complicated and emotional ideas (Bugslag 2016). Yet, it has been difficult to trace such devotions among the lower classes. Few documents bothered to record the actions and beliefs of such people. When mentioned, the lower classes featured mostly in terms of large crowds and the response of such crowds. Rarely recorded was a lower-class individual's devotion. Material culture can, though, give us an idea of how such people practiced their piety. The iconography and added movement of their devotional objects were, not surprisingly, inexpensive copies of the pieces prized by the upper classes. The same New Testament and saintly pictures and the same folding, looping, blowing, and peering through occurs in both. Although mass-produced, these complicated base metal objects moved and interacted with the viewer in the same manner as those made of expensive materials. Still, they had their own creativity and interest, they were not just empty echoes. Instead, whether a small triptych, hollow-cast figure, or assembled diorama, they allowed those of the lower classes to interact directly with the divine.

Funding: This research was funded, in part, by a Faculty Research Grant by Kenyon College.

45 See also James Bugslag (2016). Performative Thaumaturgy: The State of Research on Curative and Spiritual Interaction at Medieval Pilgrimage Shrines. In The Sacred and the Secular in Medieval Healing: Sites, Objects, and Texts. Edited by Barbara S. Bowers and Linda Migl Keyser. London: Routledge, pp. 219-65, pls. 8-10. 
Acknowledgments: I would like to thank Kathryn Harper's for her excellent editorial work. Other people who have discussed the ideas presented here in whole or part to whom I owe a debt of gratitude include: John Pepple, Karen Gerhart, Kathryn Harper, Laura Gelfand, James Robinson, Hanneke van Asperen, and Ann Marie Rasmussen. I am also grateful to the two anonymous reviewers who carefully read the manuscript and offered excellent, detailed suggestions.

Conflicts of Interest: The author declares no conflict of interest.

\section{References}

\section{Primary Sources}

Basil the Great. 1857-1866. Homiliae in Psalmos, I:1-2. In Patrologia Graeca. Edited by Jacques Paul Migne. Paris: Imprimerie Catholique, vol. XXIX, p. 212.

Bliss, William Henry, and Jessie Alfred Twemlow, eds. 1867. Calendar of Entries in the Papal Registers Relating to Great Britain and Ireland: Papal Letters. London: Public Record Office, vol. XIII, part 1.

Chrysostom, John. 1857-1866. Exposition of Psalm XLI. In Patrologia Graeca. Edited by Jacques Paul Migne. Paris: Imprimerie Catholique, vol. LV, p. 156.

Fowler, Joseph Thomas, ed. 1964. Rites of Durham Being a Description or Brief Declaration of all the Ancient Monuments, Rites, E Customs Belonging or Being within the Monastical Church of Durham before the Suppression, Written 1593. Durham: Bernard Quaritch.

Grosejean, Paulus, ed. 1935. Henrici VI Angliae Regis Miracula Postuma ex Codice Musei Britannici Regio 13. C. Brussels: Société des Bollandistes, Subsidia Hagiographica, no. 133.

Littlehales, Henry, and Edmund Bishop, eds. 1996. The Prymer or Lay Folks' Prayerbook. Oxford: Early English Text Society. First published 1895.

Pecock, Reginald. 1860. The Repressor of Over Much Blaming of the Clergy. Edited by Churchill Babington. London: Longman, Green, Longman and Roberts, vol. 1.

Ryan, Willian Grangerand. 1993. ed. and trans. Jacobus de Voragine. In The Golden Legend, Readings on the Saints II. Princeton: Princeton University Press.

Scot, Reginald. 1930. The Discoverie of Witchcraft (1584). Edited by Montague Summers. London: John Rodker. Speculum, Sacerdotale, ed. 1936. Edward Weatherly. Oxford: Early English Text Society.

\section{Secondary Sources}

Aston, Margaret. 1988. England's Iconoclasts, Laws against Images. Oxford: Oxford University Press, 2 vols.

Aston, Margaret. 2004. Lap Books and Lectern Books: The Revelatory Book in the Reformation. In The Church and the Book. Edited by Robert N. Swanson. Woodbridge: Boydell, pp. 163-89.

Belting, Hans. 1994. Likeness and Presence: A History of the Image before the Era of Art. Translated by Edmund Jephcott. Chicago: University of Chicago Press.

Blick, Sarah. 2015. Common Ground: Reliquaries and the Lower Classes in Late Medieval Europe. In Matter of Faith: An Interdisciplinary Study of Relics and Relic Veneration in the Medieval Period. Edited by James Robinson, Lloyd de Beer and Anna Harnden. London: British Museum, pp. 110-15.

Brantley, Jessica. 2007. Reading in the Wilderness: Private Devotion and Public Performance in Late Medieval England. Chicago: University of Chicago Press.

Brückner, Wolfgang. 1993. Christlicher Amulett-Gebrauch der frühen Neuzeit. In Frömmigkeit: Formen, Geschichte, Verhalten, Zeugnisse. Edited by Ingolf Bauer. Berlin: Deutsche Kunstverlag, pp. 89-134.

Bruna, Denis. 2006. Enseignes de Plomb et Autres Menues Chosettes du Moyen Âge. Paris: Éditions du Léopard d'or. Bugslag, James. 2006. Local Pilgrimage and Their Shrines in Pre-Modern Europe. Peregrinations 2: 7-35.

Bugslag, James. 2016. Performative Thaumaturgy: The State of Research on Curative and Spiritual Interaction at Medieval Pilgrimage Shrines. In The Sacred and the Secular in Medieval Healing: Sites, Objects, and Texts. Edited by Barbara S. Bowers and Linda Migl Keyser. London: Routledge, pp. 219-65, pls. 8-10.

Burgess, Clive. 1987. A Service for the Dead: The Form and Function of the Anniversary in Late Medieval Bristol. Transactions of the Bristol and Gloucestershire Archaeological Society 105: 183-211. 
Campbell, M. 1998. Medieval Metalworking and Bury St. Edmunds. In Bury St. Edmunds. Medieval Art, Architecture, Archaeology and Economy. Edited by A. Gransden. Oxford: British Archaeological Association, vol. 20, pp. 69-80.

Cohen, Esther. 1976. In haec signa: Pilgrim-badge trade in Southern France. Journal of Medieval History 2: 193-214. [CrossRef]

Cotton, Charles. 1917. Churchwardens' Accounts of the Parish of St. Andrews, Canterbury from AD 1485 to AD 1625. Archaeologia Cantiana 32: 181-246.

Daniell, Christopher. 1997. Death and Burial in Medieval England 1066-1550. London: Routledge.

Duffy, Eamon. 1992. The Stripping of the Altars: Traditional Religion in England c. 1400-c. 1580. New Haven: Yale University Press.

Duffy, Eamon. 2006. Marking the Hours: English People \& their Prayers 1240-1570. New Haven: Yale University Press.

Egan, Geoff. 1997. Childrens' Pastimes in Past Times: Medieval Toys Found in the British Isles. In Material Culture in Medieval Europe. Edited by Guy de Boe and Frans Verhage. Bruges: Instituut voor het Archeologisch Patrimonium, wetenschappelijke instelling van de Vlaamse Gemeenschap, Departement Leefmilieu en Infrastructuur, Administratie Ruimtelijke Ordening, Huisvesting en Monumenten en Landschappen, pp. 413-21.

Egan, Geoff. 1998. The Medieval Household, Daily Living c. 1550-c. 1450. London: The Stationery Office.

Egan, Geoff. 2005. Material Culture in London in an Age of Transition: Tudor and Stuart Period Finds c. 1450-c. 1700 from Excavations at Riverside Sites in Southwark. London: Museum of London.

Finucane, Ronald C. 1977. Miracles and Pilgrims: Popular Beliefs in Medieval England. London: J.M. Dent.

Forsyth, Hazel, and Geoff Egan. 2005. Toys, Trifles \& Trinkets: Base-Metal Miniatures from London 1200-1800. London: Museum of London.

French, Katherine L. 2008. The Good Women of the Parish: Gender and Religion after the Black Death. Philadelphia: University of Pennsylvania Press.

Frugoni, Chiara. 2003. Books, Banks, Buttons and Other Inventions from the Middle Ages. Translated by William McCuaig. New York: Columbia University Press.

Gelfand, Laura D. 2012. Sense and simulacra: Manipulation of the senses in medieval 'copies' of Jerusalem. Postmedieval: A Journal of Medieval Cultural Studies 3: 407-22. [CrossRef]

Gellrich, Jesse. 1985. The Idea of the Book in the Middle Ages: Language, Theory, Mythology, and Fiction. Ithaca: Cornell University Press.

Golding, Brian. 2001. The Church and Christian Life. In Barbara Harvey, the Twelfth and Thirteenth Centuries: Short Oxford History of the British Isles. Oxford: Oxford University Press, pp. 135-66.

Hall, Mark. 2007. Crossing the Pilgrimage Landscape: Some Thoughts on a Holy Rood Reliquary from the River Tay at Carpow, Perth and Kinross, Scotland. In Beyond Pilgrim Souvenirs and Secular Badges: Essays in Honour of Brian Spencer. Edited by Sarah Blick. Oxford: Oxbow Books, pp. 75-91.

Hamburger, Jeffrey F. 2007. Nuns as Artists: The Visual Culture of a Medieval Convent. Berkeley: University of California Press.

Heath, Peter. 1984. Urban Piety in the Later Middle Ages: The Evidence of Hull Wills. In The Church, Politics and Patronage in the Fifteenth Century. Edited by Barrie Dobson. New York: St. Martin's Press, pp. 209-34.

Heins, N. 1897-1915. Inventaire Archéologique de Gand: Catalogue Descriptif et Illustré des Monuments, Oeuvres D'art et Documents Antérieurs à 1830. 2 vols. Gand: N. Heins.

Heyne, Moriz. 1899. Fünf Bücher Deutscher Hausaltertümter, I, Das Deutsche Wohnungswesen von den Ältesten Geschichtlichen Zeiten bis zum 16. Jahrhundert. Leipzig: Verlag Von S. Hirzel.

Hopeful Dead Clutching Their Tickets to Heaven. 2002, British Archaeology, October. under "News.".

Humpfrey, Peter. 1993. The Altarpiece in Renaissance Venice. New Haven: Yale University Press.

Jacobs, Fredrika. 2013. Votive Panels and Popular Piety in Early Modern Italy. Cambridge: Cambridge University Press. Jones, Peter. 2000. Encounters with Amulets. Societas Magica Newsletter, 1-4.

Kahsnitz, Rainer. 2005. Carved Splendor: Late Gothic Altarpieces in Southern Germany, Austria, and South Tirol. Translated by Russell Stockman. Los Angeles: Getty Museum.

Kammel, Frank Matthias Kammel, ed. 2000. Spiegel der Seligkeit: Privates Bild und Frömmigkeit im Spätmittelalter. Nürnberg: Germanisches Nationalmuseum. 
Koldeweij, A. M. 1989. (Jos) Karel de Grote-souvenirs uit Aken. In Annus Quadriga Mundi. Opstellen over middeleeuwse aan Prof. Dr. Anne C. Esmeijer. Zutphen-Utrecht: De Walburg Pers- Clavis kunsthistorische monografieën, pp. 116-28.

Koldeweij, Jos. 2006. Foi \& Bonne Fortune: Parure et Dévotion en Flandre Médiévale. Bruges: Terra.

Köster, Kurt. 1985. Le Coquilles du Pèlerinage de Saint-Jacques de Compostella et les routes de Saint-Jacques en Occident. In Santiago de Compostela, 1000 ans de Pèlerinage Européen. Europalia: Centrum voor Kunst en Cultur, pp. 85-96.

Kühne, Hartmut, Carina Brumme, and Helena Koenigsmarková. 2012. Jungfrauen, Engel, Phallustiere: Die Sammlung Mittelalterlicher Französischer Pilgerzeichen des Kunstgewerbemuseums in Prag und des Nationalmuseums Prag/im Auftrag des Kunstgewerbemuseums in Prag. Berlin: Lukas Verlag.

Lee, Jennifer. 2014. Medieval pilgrims' badges in rivers: the curious history of a non-theory. Journal of Art Historiography 11: 1.

Lohr, Otto. 1986. Missal: Winter Section. In Gothic and Renaissance Art in Nuremberg 1300-1550. Munich: Prestel-Verlag, p. 149, cat. No. 26.

Marks, Richard. 2004. Image and Devotion in Late Medieval England. Gloucestershire: Sutton Publishing.

Martin, Biddle, ed. 1990. Object and Economy in Medieval Winchester. Oxford: Clarendon Press.

Meckseper, Cord, ed. 1985. Stadt im Wandel: Kunst und Kultur des Bürgertums in Norddeutschland 1150-1650. Stuttgart: Bad Cannstatt, 2 vols.

Merrifield, Ralph. 1987. The Archaeology of Ritual and Magic. London: B.T. Batsford

Pieters, M., E. Cools, J. Koldeweij, and A. Mortier. 1997-1998. Middeleeuwse en latere insignes en devotionalia uit Raversijde (gemeente Middelkerke en stad Oostende, prov. West-Vlaanderen). Archeologie in Vlaanderen 6: 261-301.

Pollard, Alfred William. 1903. Fifteenth-Century Prose and Verse. Westminster: A. Constable \& Co.

Praha Museum of Decorative Arts. 1985. Katalog Sbirky Stredovekého Umleckého Remesla Muzeum v Praze. Prague: Museum Catalogue of Praha, Museum of Decorative Arts.

Ringbom, Sixten. 1984. Icon to Narrative: The Rise of the Dramatic Close-Up in Fifteenth-Century Devotional Painting, 2nd ed. Irving: Davaco.

Rocacher, J. 1980. Les mouliers de Rocamadour. Bulletin de la Societé des Études de Lot, October-December. 284-92. Roffey, Simon. 2006. Devotional Objects and Cultural Context: The Medieval Parish Church. In Catholic Collecting: Catholic Reflection 1538-1850, Objects as a Measure of Reflection on a Catholic Past and the Construction of Recusant Identity in England and America. Edited by Virginia Raguin. Worcester: College of the Holy Cross, pp. 21-30.

Roy, Bruno. 1980. The Household Encyclopedia as Magic Kit: Medieval Popular Interest in Pranks and Illusions. Journal of Popular Culture 14: 60-69. [CrossRef]

Rudy, Kathryn M. 2011. Virtual Pilgrimages in the Convent: Imagining Jerusalem in the Late Middle Ages. Turnhout: Brepols.

Rudy, Kathryn M. 2015. Postcards on Parchment: The Social Lives of Medieval Books. New Haven: Yale University Press. Schultz, Alwin. 1892. Deutsches Leben im XIV. und XV. Jahrhundert. Vienna: Tempsky Wien.

Sciacca, Christine. 2007. Raising the Curtain on the Use of Textiles in Manuscripts. In Weaving, Veiling, and Dressing: Textiles and Their Metaphors in the Late Middles Ages. Edited by Kathryn M. Rudy and Barbara Baert. Turnhout: Brepols, pp. 161-90.

Scribner, Robert W. 1987. Popular Culture and Popular Movements in Reformation Germany. London: The Hambledon Press.

Skemer, Don C. 2006. Binding Words: Textual Amulets in the Middle Ages. Centre County: The Pennsylvania State University.

Spencer, Brian. 1980. Medieval Pilgrim Badges from Norfolk. Kings Lynn: Norfolk Museums Service.

Spencer, Brian. 1987. Pilgrim Souvenirs. In Age of Chivalry: Art in Plantagenet England, 1200-1400. Edited by Jonathan Alexander and Paul Binski. London: Royal Academy, pp. 205-24.

Spencer, Brian. 1990. Pilgrim Souvenirs and Secular Badges. Salisbury Museum Medieval Catalogue, Part 2. Salisbury: Salisbury \& South Wiltshire Museum.

Spencer, Brian. 1998. Pilgrim Souvenirs and Secular Badges: Medieval Finds from Excavations in London. London: Stationery Office. 
Stalley, Roger. 1988. Sailing to Santiago: Medieval Pilgrimage to Santiago de Compostela and its Artistic Influence in Ireland. In Settlement and Society in Medieval Ireland. Edited by John Bradley. Kilkenny: Boethius Press, pp. 397-420.

Sumberg, Samuel L. 1941. The Nuremberg Schembart Carnival. New York: Columbia University Press.

Swartz, Michael D. 2001. Magical Piety in Ancient and Medieval Judaism. In Ancient Magic and Ritual Power. Edited by Paul Allan Mirecki and Marvin W. Meyer. Leiden: Brill, pp. 167-83.

Tait, Hugh. 1955-1956. Pilgrim-Signs and Thomas, Earl of Lancaster. The British Museum Quarterly 20: 39-47. [CrossRef]

Trexler, Richard. 1980. Public Life in Renaissance Florence. Cornell: Cornell University Press.

Truitt, Elly Rachel. 2015. Medieval Robots: Mechanism, Magic, Nature, and Art. Philadelphia: University of Pennsylvania Press.

Van Asperen, Hanneke. 2009. Pelgrimstekens op Perkament. Originele en Afgebeelde Bedevaartssouvenirs in Religieuze Boeken (ca. 1450-ca. 1530). Nijmegen: Orange House.

Van Asperen, Hanneke. 2013. Annunciation and Dedication on Aachen Pilgrim Badges. Notes on the Early Badge Production in Aachen and Some New Attributions. Peregrinations: Journal of Medieval Art E Architecture 4: 215-35.

Van Beuningen, H. J. E., and A. M. Koldeweij. 1993. Heilig en Profaan. 1000 Laatmiddeleeuwse Insignes uit de Collectie H.J.E. van Beuningen. Cothen: Stichting middeleeuwse religieuze en profane insignes.

Van Beuningen, H. J. E., Jos Koldeweij, and Dory Kicken. 2001. Heilig en Profaan 2, 1200 Laat-Middeleeuwse Insignes uit Openbare en Particuliere Collecties. Cothen: Stichting Middeleeuwse Religieuze en Profane Insignes.

Van Heeringen, Robert Maarten, Adrianus Maria Koldeweij, and A. A. G. Gaalman. 1988. Heiligen uit de Modder in Zeeland Gevonden Pelgrimstekens. Utrecht: de Walburg Press.

Van Os, Henk W. 1994. The Art of Devotion in the Late Middle Ages in Europe, 1300-1500. London: Merrell Holberton. Vauchez, André. 1997. Sainthood in the Later Middle Ages. Translated by Jean Birrell. Cambridge: Cambridge University Press.

Webb, Diana M. 1990. Woman and Home: The Domestic Setting of Late Medieval Spirituality. In Women in the Church. Edited by William J. Sheils and Diana Wood. Oxford: Basil Blackwell, pp. 159-74.

(C) 2019 by the author. Licensee MDPI, Basel, Switzerland. This article is an open access article distributed under the terms and conditions of the Creative Commons Attribution (CC BY) license (http://creativecommons.org/licenses/by/4.0/). 
Article

\title{
From Domestic Devotion to the Church Altar: Venerating Icons in the Late Medieval and Early Modern Adriatic
}

\author{
Margarita Voulgaropoulou \\ Center for Eastern Mediterranean Studies, Central European University, 1051 Budapest, Hungary; \\ mvoulgaropoulou@gmail.com
}

Received: 23 May 2019; Accepted: 17 June 2019; Published: 19 June 2019

\begin{abstract}
Although traditionally associated with Eastern Christianity, the practice of venerating icons became deeply rooted in the Catholic societies of the broad Adriatic region from the Late Middle Ages onwards and was an indispensable part of everyday popular piety. The evidence lies in the massive amount of icons located today in public and private collections throughout the Italian Peninsula, Croatia, Slovenia, and Montenegro. At a time when Greeks were branded as "schismatics", and although the Byzantine maniera greca had become obsolete in Western European art, icon painting managed to survive at the margins of the Renaissance, and ultimately went through its own renaissance in the sixteenth century. Omnipresent in Catholic households, icons were very often donated to churches as votive offerings and were gradually transformed into the focal points of collective public devotion. Through the combined study of visual evidence, archival records and literary sources, this article will shed light on the socio-political, confessional, and artistic dynamics that allowed for Byzantine or Byzantinizing icons to gain unprecedented popularity throughout the Catholic milieus of the Late Medieval and Early Modern Adriatic, and become integrated into domestic and public devotional practices.
\end{abstract}

Keywords: icon painting; maniera greca; Byzantine art; Renaissance; Council of Trent; Adriatic Sea; Venice; Dalmatia; Ragusa; Candia

\section{Introduction}

On 2 March 1506 a miracle was recorded in the Italian village of Longiano, in modern-day Emilia Romagna. A Greek icon of the Madonna and Child (Figure 1a), which decorated the residence of the devout Sebastiano Barberi suddenly started to "sweat" tears ("sudore manavit"). As news of the miracle spread, Barberi's house was slowly transformed into a site of intense public devotion, swarming with visitors who came to worship the weeping Madonna. It was not long until this new devotion outgrew the domestic confines of Barberi's household, prompting him to donate the icon to the local community along with his house (Figure 1b), which was subsequently converted into a church to host the wonder-working image, henceforth known as Our Lady of Tears (Madonna delle Lacrime). ${ }^{1}$ As extraordinary as it sounds, this episode—one of many encountered in Late Medieval and Early Modern sources-illustrates the prominent place of Orthodox icons in Catholic devotional practices, while highlighting the fluid boundaries between domestic and public worship.

1 The episode is commemorated in a dedicatory inscription at the ex-church of Our Lady of Tears (Santa Maria delle Lacrime) in Longiano: D.O.M. // BEATISSIMAEQUE VIRGINI // LACRIMARUM // QUAE// ANNO CIJIJVI POSTRID. K. MAR // SUDORE MANAVIT // SEBASTIANUS BARBERIUS // DOMUM IN TEMPLUM D.D.D.S. // QUODQ. VETUSTATE COLLABENS // CONSULES LONZANI // A FUNDAMENTIS IN HANC FORMAM AEDIF. CIJIDCCLXXII. The inscription is cited and transliterated in (Turchi 1829, pp. 4-5). See also (Pasi 1986, pp. 156-58). 


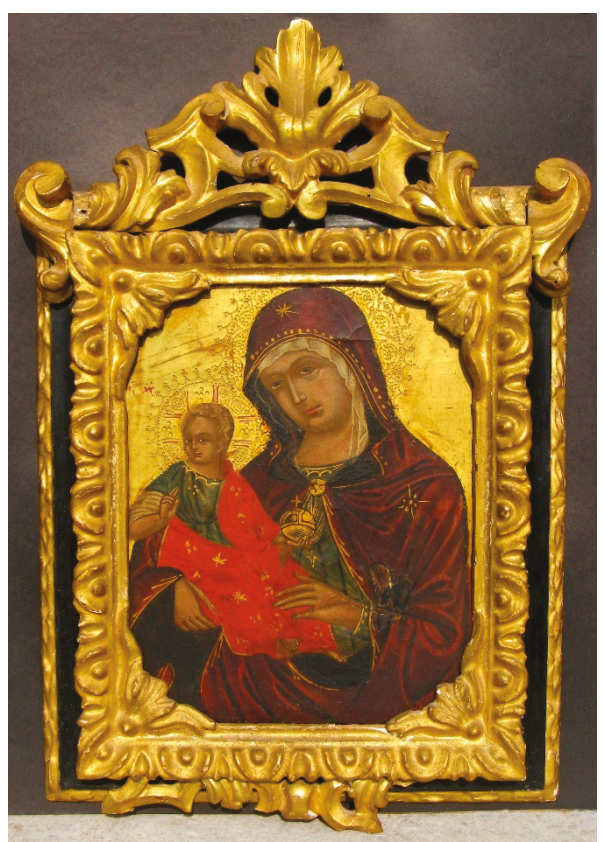

(a)

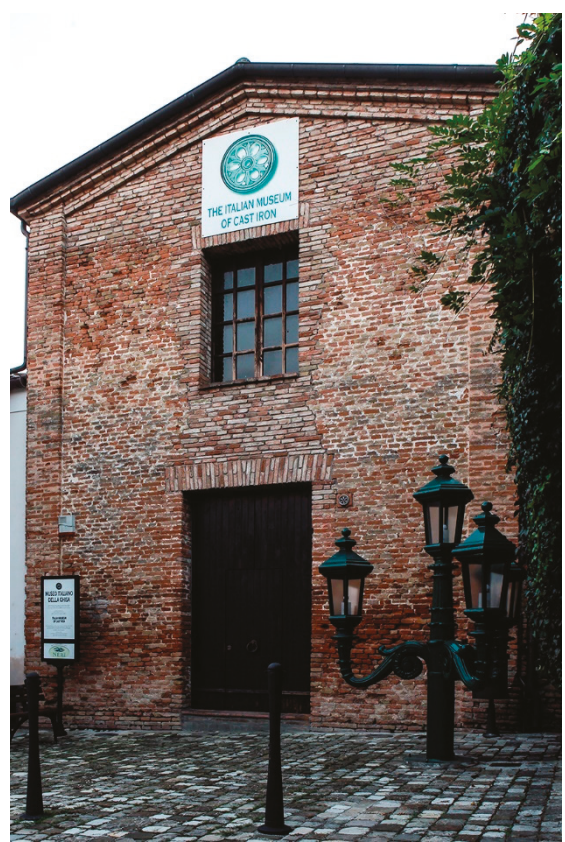

(b)

Figure 1. (a) Madre della Consolazione (Madonna delle Lacrime), ca. 1500, (c) Longiano, Museo d'Arte Sacra; (b) Ex Church of Santa Maria delle Lacrime, now Museo Italiano della Ghisa, Longiano (image in the public domain).

The past twenty years have seen an abundance of scholarship on the material culture of the Early Modern domestic interior and the devotional practices that took place in the Renaissance home. ${ }^{2}$ Drawing heavily on archival sources, previous publications have been geographically limited to the major artistic centers of Central and Northern Italy, notably to Renaissance Florence and Venice, whereas the rest of the Italian Peninsula and the Adriatic region still remain largely uncharted. In addition, extant studies have almost exclusively dealt with art objects of Western style and manufacture, while icons made in the Byzantine tradition are mentioned only briefly as a side-note or listed among items casually encountered in private collections. Departing from the limitations of previous scholarship, this article will explore the place and function of Byzantine and Byzantinizing icons in Catholic households throughout the broad Adriatic region, and at the same time, trace the afterlives of images of domestic devotion in the public space. Through the comparative evaluation of material evidence with archival and literary sources, this study seeks to outline the socio-political, confessional, and artistic dynamics that fostered a favorable setting for the reception of icon painting in the Adriatic. As this article will argue, the intense circulation of artists, artworks and artistic trends in the Eastern Mediterranean from the fifteenth century onwards, as well as the image theories promulgated during the age of confessionalization were the key factors that allowed for the survival of Byzantine artistic forms long after their presumed abandonment by Renaissance Humanism, rendering Orthodox icons the votive objects par excellence in the pre- and post-Tridentine societies of both Adriatic coasts. Due to

2 (Palumbo Fossati 1984; Lydecker 1987; Grubb 2000; Brown 2004; Palumbo Fossati 2004; Ajmar Wollheim and Dennis 2006; Currie 2006; Ajmar Wollheim et al. 2007; Anderson 2007; Cavallo and Evangelisti 2009; Henry 2011; Morse 2013; Feigenbaum and Freddolini 2014; Walsham 2014; Corry et al. 2017; Brundin et al. 2018; Corry et al. 2018). 
the scarcity of sources from the South Adriatic, this study will draw mainly from published records from Venice and its Adriatic possessions, as well as from published and new documents from the archives of Dubrovnik. However, visual evidence in support of the article's main arguments can be found throughout the Adriatic region, including less documented regions, such as Apulia, the Marche, Abruzzo and Molise, and will be presented here selectively.

\section{The Introduction, Reception and Appropriation of Icon Painting in the Catholic West}

Image worship lay at the heart of Eastern Christian devotional practices since the early centuries of Byzantium, growing increasingly in popularity after the end of iconoclasm in 843. From the mid-eleventh century onwards, painted icons gained added significance, and were promoted as "living

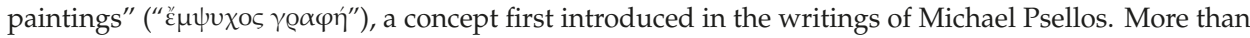
mere art objects, for the Byzantines, icons were considered to be the true likenesses and material embodiments of Christ and the saints and were often treated as equivalent to relics. In fact, certain early icons were believed to have been created not by human hands but by divine agency, often credited as the original portraits of saints painted by Saint Luke himself. These acheiropoietoi icons were often attributed thaumaturgic powers of healing and protection conferred by the sacred figures they represented. In Byzantine theology, the veneration of such images and their copies allowed the devout to communicate directly with the divine, based on the belief that the honor given to an image was transferred directly to its prototype, as expressed by Basil the Great and John of Damascus. Images representing holy figures, crafted in various sizes and a variety of media permeated all aspects of public and private life. In ecclesiastical settings, icons functioned as integral parts of liturgical rituals, decorating church interiors and being carried in public processions, whereas in official civic and military contexts, they were steadily replacing imperial images, acting as divine protectors of armies (apotropaia) and defenders of cities (palladia), roles particularly diffused during periods of intense warfare. Besides their public functions, icons were also commissioned individually for personal uses; small portable icons, such as diptychs, triptychs and polyptychs were used as visual aids for private devotion in the home, and accompanied their owners to battle or distant travels, providing them with constant protection from harm. ${ }^{3}$

Although icon worship was an integral part of Orthodox devotional practices from very early on, in Catholic Europe, the veneration of painted panels was a much later introduction. ${ }^{4}$ In the first half of the thirteenth century, during the period of Latin rule, the Archbishop of Ohrid, Demetrios Chomatenos, was writing to Constantine Kavasilas, Metropolitan of Durrës: "There are some Latins that do not seem to be completely different from our customs [ ... ] Indeed they too revere icons and they exhibit them in their churches. ${ }^{\prime 5}$ While Chomatenos raises a point of convergence between Greek and Latin approaches to images, his statement implies that the practice of venerating sacred images was not always or, at least not universally, adopted by the Catholic Church. In fact, during the Middle Ages, popular piety in the West mainly revolved around the veneration of relics, and even domestic devotion was mainly aided by textual artefacts, such as private psalters and books of hours. It was only after the Crusades and especially, after the Sack of Constantinople in 1204, that Italian and Dalmatian workshops started producing icon-like devotional images, influenced by the reputedly ancient and miraculous icons that came streaming from the East, and possibly by the

3 For the role of icons in Byzantium see (Belting 1994; Carr 2004, pp. 142-52; Vassilaki 2008, pp. 758-69; Drandaki 2013, pp. 109-14).

4 For the diffusion of icon worship in the West see (Ringbom 1984, pp. 12-22; Belting 1994; Carr 2004, pp. 142-52; Drandaki 2013, pp. 109-14; Bacci 2018, pp. 272-92).

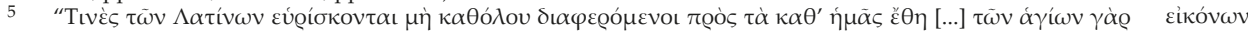

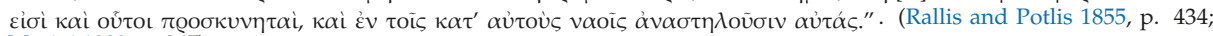
Morini 1989, p. 267). 
teachings of Byzantine painters migrating to the West. ${ }^{6}$ Images made in the so-called maniera greca appropriated the morphological and iconographic features of Byzantine icons, enriched with Crusader innovations and local pictorial elements (Figure 2). ${ }^{7}$ Over the following centuries, Eastern and Western traditions would eventually diverge into largely disparate paths, as Italian-and to a lesser extent Dalmatian-painters gradually adopted a more naturalistic way of representation, abandoning the more abstract Medieval forms.

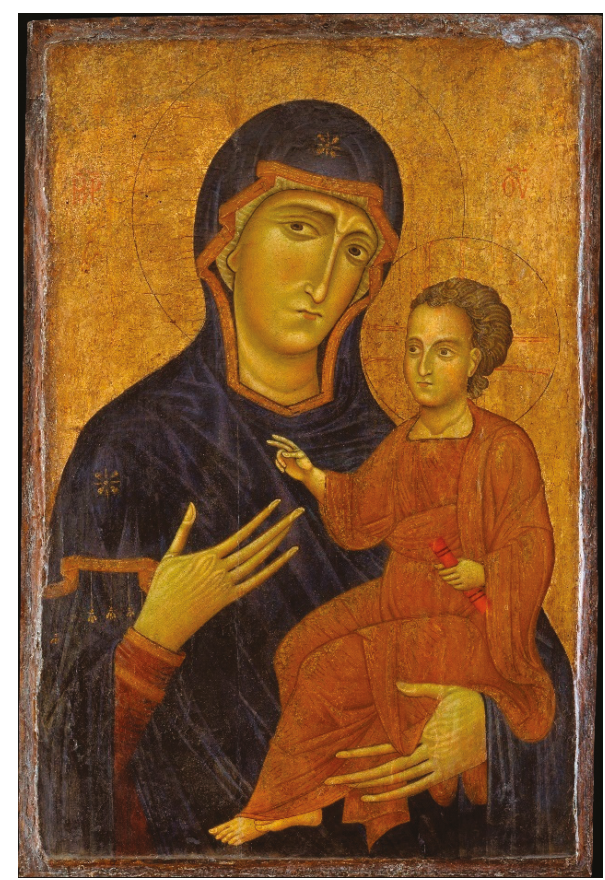

Figure 2. Berlinghiero Berlinghieri, Madonna and Child, ca. 1230s, New York, Metropolitan Museum of Art (image in the public domain).

Rejected as repetitive, distasteful, and unimaginative, the maniera greca of the Orthodox East and the Italian Duecento ended up becoming synonymous of artistic decline by the fifteenth century, representing everything a Renaissance artist should avoid. Cennino Cennini and Lorenzo Ghiberti both praised Giotto for "changing the profession of painting from Greek back into Latin" (Cennini 1960, p. 2), ${ }^{8}$ "bringing new art and abandoning the roughness of the Greeks" (Ghiberti 1998, p. 84). ${ }^{9}$ Leonardo da Vinci reproached the "followers of the Byzantine manner", claiming that they "continually imitated each other, and art declined steadily from age to age" (Leonardo 1961, p. 84;

6 In his life of Cimabue Giorgio Vasari relates the emergence of Italian panel painting to the presence of "Greek painters", who had been invited to Florence "for no other purpose than that of introducing there the art of painting, which in Tuscany long had been lost" ["Avenne che in que' giorni erano venuti di Grecia certi pittori in Fiorenza, chiamati da chi governava quella città non per altro che per introdurvi l'arte della pittura, la quale in Toscana era stata smarrita molto tempo"]. (Vasari 1550, p. 126; cited and translated in Maginnis 1994, p. 147; Concina 2002, pp. 89-96). See also Voulgaropoulou, Margarita. Forthcoming. Transcending Borders, Transforming Identities: Travelling Icons and Icon Painters in the Adriatic Region (Voulgaropoulou, Margarita. Forthcoming. Transcending Borders, Transforming Identities: Travelling Icons and Icon Painters in the Adriatic Region ).

7 For the development of Italian panel painting see (Folda 2015, pp. xxii-xxiii, with references).

8 "Il quale Giotto rimutò l'arte del dipignere di Greco in Latino e ridusse al moderno".

"Arrechò l'arte nuova, lasciò la rozeza de' Greci". 
Nagel and Wood 2010, pp. 72-73). ${ }^{10}$ Renaissance critique of the Byzantine manner culminated in the writings of Giorgio Vasari, who repeatedly dismissed it as "awkward", "coarse", "disproportionate" and even "monstrous" (Vasari 1550, pp. 59, 83, 97, 119, 244). ${ }^{11}$ Within the context of such an increasingly negative discourse, one can only wonder how images made in the Byzantine tradition managed to not only survive in a seemingly unfavorable intellectual setting, but also develop into essential parts of domestic and public devotional practices of Italian and Dalmatian Catholic populations.

A catalyzing moment for the reception of Byzantine culture and icon painting in the West, and especially in Venice, was the council for the union of the Greek and Latin Churches held in Ferrara and Florence in 1438-1439.12 In 1438, the Greek delegation, comprising of seven hundred clerics and laymen, including the Byzantine emperor John VIII Palaiologos (1392-1448) and the patriarch of Constantinople, Joseph II (1360-1439), spent a few weeks in Venice right immediately prior to setting out for Ferrara. The brief Byzantine presence in the city nonetheless left a lasting impression on the Venetians, who developed an almost exotic interest in all things Greek. At the same time, a great many Byzantine icons and luxury items were arriving in Venice, Rome and other Italian cities either by means of trade or as diplomatic gifts, filling up the treasuries of lay and ecclesiastical magnates. Cardinal Bessarion (1403-1472), for instance, donated seven mosaic icons to Saint Peter's basilica from 1454 to 1466 , although an inventory from 1489 suggests that his legacy was much larger, also including numerous painted panels (Müntz 1879, p. 298n3; Müntz and Frothingham 1883, p. 111; Antetomaso 2007, pp. 225-32; Nagel 2011, pp. 21-22; Duits 2013, p. 170). Arguably the largest collection of icons, numbering twenty-three mosaic icons ("de musayco parvissimo") and thirteen painted and sculpted ones ("ycona graeca"), was amassed by the Venetian cardinal Pietro Barbo, future Pope Paul II (Müntz 1879, pp. 201-5; Cutler 1995, p. 251; Corbo 2004, pp. 31-33; Pedone 2005, p. 102n36; Cherra 2006, pp. 184-85; Duits 2011, pp. 127-41; Nagel 2011, p. 21; Duits 2013, pp. 169-71; Menna 2015, pp. 101-11). When Barbo died in 1471, a part of his collection was acquired by Lorenzo de' Medici, who already owned numerous icons in the "Greek" style, which were registered in the Medici household inventories as early as 1463 (Furlan 1973, pp. 19-20; Spallanzani et al. 1992, pp. 27, 47-48, 63, 80; Spallanzani 1996, pp. 119, 142-44; Menna 1998, pp. 122-24; Cherra 2006, pp. 185-87; Fusco and Corti 2006, pp. 74-75; Nagel 2011, p. 21; Duits 2013, pp. 157-88). Smaller collections of icons belonged to cardinal Ludovico Trevisan (1401-1465), owner of "antique Greek panels with silver revetments" (Bagemihl 1993, p. 563; Duits 2013, p. 170), and cardinal Francesco Gonzaga (1444-1483), whose will mentions "two old Greek panels, one with the Crucifixion and one with Our Lady" (Chambers 1992; Duits 2013, p. 170). It is not a coincidence that all of these collections were in some way related to Venice: both cardinals Trevisan and Barbo were of Venetian origin, cardinal Bessarion served at the city as a papal legate, and cardinal Gonzaga is known to have acquired art objects from Venice for his Mantua palace.

A new, massive wave of icons and Byzantine artefacts swept through Venice and other Italian centers towards the turn of the sixteenth century, along with an unprecedented influx of Greek migrants and members of the Byzantine intelligentsia that followed the conquest of Constantinople in 1453, and the Ottoman expansion into former Byzantine and Venetian lands in the Eastern Mediterranean (Bianca 1999; Menna 1998, pp. 111-58; Cherra 2006, pp. 175-204; Pedone 2005, p. 100; Lamers 2015, p. 4). Carried in the baggage of church prelates and aristocratic refugees, such as cardinal Bessarion and Anna Palaiologina Notaras, these migrating heirlooms eventually entered Italian and Western European private collections; this time, however, they were not only perceived as exotic curiosities and prized relics that appealed to the antiquarian and philological interests of fifteenth-century humanists (Effenberger 2004, p. 212; Cutler 1995, p. 252; Nagel and Wood 2010, p. 105; Duits 2011, p. 135;

10 “[ ... ] come vedemo in ne' pittori dopo i Romani, i quali sempre imitarono l'uno dall'altro, e di età in età sempre mandarono detta arte in declinazione".

11 "la maniera goffa greca", "rozza maniera Bizantina", "altre mostruosità di que' Greci", "quella maniera greca goffa e sproporzionata".

12 For the Council of Ferrara-Florence see (Geanakoplos 1955, pp. 324-46; Nicol 1988, pp. 376-79; Morini 1989, pp. 267-96). 
Duits 2013, pp. 159-160n6) but also as the last remnants of the fallen Byzantine Empire and symbols of the battles of allied Christianity against Islam. ${ }^{13}$ According to Alexander Nagel, "the icon enthusiasm of the later fifteenth century led to efforts to revitalize and reauthorize religious images in the West", leading to the rediscovery of local icons and the formation of legends revolving around miracle-working images (Nagel 2011, p. 22).

The influx of Eastern icons to the West continued throughout the Early Modern Period, only it no longer concerned Byzantine artworks but rather, images created in the flourishing icon-painting centers of the Balkans and the Eastern Mediterranean, the most important being Candia, a Venetian possession from 1211 to 1669 . From the sixteenth century onwards, a vibrant trade of icons was established between Venice, Crete and other centers of the Mediterranean and Adriatic Seas, while icons kept streaming to the West, following the mass migrations of Orthodox populations during the Ottoman-Venetian wars. At the same time, numerous Greek Orthodox communities and churches were founded in Venice and other Adriatic cities, encouraging the establishment of icon-painting workshops that produced devotional images for a confessionally diverse clientele (Voulgaropoulou 2014, pp. 132-432) Therefore, it appears that the geopolitical upheavals in the Eastern Mediterranean during the Late Middle Ages and the Early Modern period resulted in an inflow of Byzantine and post-Byzantine icons in Adriatic collections, allowing for the rediscovery of icon painting and the further familiarization of Italian and Dalmatian Catholics with the Byzantine tradition. What remains to be answered, however, is the question of how exactly these icons were introduced in Catholic religious practices and accepted as devotional objects par excellence by the Roman Catholic Church, especially in a period of intense theological debates and inter-confessional conflicts.

Respect for Byzantine icons' spirituality had pervaded Western theology since the Late Middle Ages. As early as 1306, the Dominican preacher Fra Giordano da Rivalto (1260-1311) attributed "the utmost authority to images imported from Greece", for depicting the holy figures "exactly as they looked" (Giordano 1867, p. 171; Belting 1994, p. 182; Cutler 1994, p. 194; Cutler 2000). ${ }^{14}$ Almost a century later, the Blessed Fra Giovanni Dominici (ca. 1355-1419), mentor of Fra Angelico and Archbishop of Ragusa, advised the faithful to revere the "old smoky" humble images of the past ("vecchie affumate") instead of worshipping the gilded and richly ornamented religious paintings of their time, which he thought distracted them from the essence of prayer (Dominici 1860, p. 133; Nagel and Wood 2010, p. 85; Nagel 2011, pp. 20-21, 290n21; Drandaki 2014, p. 45). Although Dominici's "old smoky" icons can probably be identified with devotional images of the Italian Duecento and Trecento (see Figure 2) rather than Byzantine icons, both his and Giordano's accounts certainly denote a reverence towards Byzantinizing Medieval forms that resonated with more conservative Catholic circles of scholars and theologians.

A marked shift in the attitude of the Roman Catholic Church towards Greek icons occurred in the wake of the Council of Trent with the implementation of a set of liturgical-artistic reforms that redefined the principles of religious art. The Counter-Reformation defended the veneration of images and promulgated the moral and didactic role of art rather than its aesthetic value. ${ }^{15}$ In the post-Tridentine

13 The common Ottoman threat created a sense of solidarity between Greek and Latin Christians, especially considering that a large part of the Byzantine refugees had accepted the Union of Florence and converted to Catholicism. Although short-lived, the Union of Florence had cultivated a favorable environment for Orthodox Christianity in the Catholic West, especially in Venice, where there was more tolerance towards doctrinal differences. Quite revealing is a letter of the Camaldolese monks, Paolo Giustiniani and Vincenzo Querini to pope Leo X in 1513, claiming about the Greeks that "they are true Christians; we half-pagan" ("hi Christiani vere sunt, nos semipagani"). (Giustiniani and Quirini 1773, p. 662; Bianchini 1995, p. 75; Prodi 1973, pp. 422-23. See also Nagel 2011, p. 21; Lamers 2015, p. 5).

14 "sicchè queste dipinture, e specialmente l'antiche, che vennono di Grecia anticamente, sono di troppo grande autoritade; perocchè là entro conversaro molti santi che ritrassero le dette cose e diederne copia al mondo, delle quali si trae autorità grande, siccome si trae di libri".

15 This is explicitly decreed in the twenty-fifth and last session of the Council, which took place on 3-4 December, 1563: "[ ... ] picturis vel aliis similitudinibus expressas erudiri et confirmari populum in articulis fidei commemorandis et assidue recolendis; tum vero ex omnibus sacris imaginibus magnum fructum percipi non solum quia admonetur populus beneficiorum et munerum quae a Christo sibi collata sunt sed etiam quia Dei per sanctos miracula et salutaria exempla 
era, the artist's primary duty was to serve the triple ciceronian prerequisite "docere, delectare, movere", i.e., to educate, to delight, and to move the audience. One of the first to profess this thesis was the Archbishop of Bologna, Cardinal Gabriele Paleotti in his treatise De imaginibus sacris et profanis (1594), which epitomizes the focal points of the Tridentine decrees on art (Paleotti 1594, pp. 26, 91). Together with the educational role of art, from the mid-sixteenth century onwards the Holy See promoted an "early Christian revival", especially under the patronage of Pope Gregory XIII (1502-1585) and cardinal Cesare Baronio (1538-1607), aiming to serve Tridentine propaganda by strengthening the ties of the Roman Catholic Church with Early Christianity. ${ }^{16}$ This movement entailed the rediscovery of the works of the Church Fathers and early ecclesiastical writers, the conservation of early Christian monuments and the revival of types of Early Christian art and architecture, which instigated a pronounced interest for Christian history and archaeology, and cultivated, as a result, a favorable setting for the reception of earlier pictorial forms, as was Byzantine icon painting. ${ }^{17}$

In his monumental treatise, De Picturis et Imaginibus sacris (1570), Johannes Molanus (1533-1585) builds on the Tridentine decrees on images, stressing the necessity "to receive in all honor those pictures that are recommended by the venerable Antiquity" (Molanus 1570, p. 19; Molanus 1996, pp. 137-39). For ecclesiastical writers such as Molanus, early icons stemmed directly from the original portraits of holy figures or were considered to be acheiropoiettoi, i.e. not made by human hand. Based on the reproduction of original prototypes, Byzantine icon painting was considered to be closer to the Early Christian tradition and, therefore, represented the most authentic art form (Paleotti 1594, 74; Goffen 1975, p. 487; Chatzidakis 1977, pp. 684-85), while its austere otherworldliness allowed for an essential connection with the divine (Chastel 1988, p. 99). In this context, even though Orthodox Greeks were branded as "schismatics" and "heretics" - especially after the refutation of the Union of Florence and during the confessional conflicts of the sixteenth and seventeenth centuries (Fedalto 1967, p. 34) - their art seemed to ideally conform to the tridentine doctrines, offering the Catholic Church a useful tool in their battle against Protestant iconoclasm.

The Catholic Church's appreciation of the spirituality of Greek Orthodox iconographic tradition is commonly encountered in the art treatises of the Counter-Reformation era, often paired with the denunciation of the "paganism" and sensuality of Renaissance and Mannerist art. In his 1564 treatise, Degli errori de' pittori circa l'istorie, published just a year after the final session of the Council of Trent, Giovanni Andrea Gilio da Fabriano suggests "painting the sacred images honest, and pious, with those signs which were attributed to them by the ancients as the privilege of their sanctity; although it appears to the moderns vile, tasteless, plebeian, old-fashioned, lowly, devoid of genius and art" (Barocchi 1961, p. 111). ${ }^{18}$ Likewise, the Archbishop of Bologna and engineer of the tridentine reforms, Cardinal Gabriele Paleotti, in his Addenda de picturis, advises artists to depict the Virgin half-length with

oculis fidelium subiiciuntur ut pro iis Deo gratias agant ad sanctorum que imitationem vitam mores que suos componant excitentur que ad adorandum ac diligendum Deum et ad pietatem colendam [ ... ] Quodsi aliquando historias et narrationes Sacrae Scripturae cum id indoctae plebi expediet exprimi et figurari contigerit: doceatur populus non propterea divinitatem figurari quasi corporeis oculis conspici vel coloribus aut figuris exprimi possit". For the English translation of the excerpt see (Schroeder 2011): "by means of the stories of the mysteries of our redemption portrayed in paintings and other representations the people are instructed and confirmed in the articles of faith, which ought to be borne in mind and constantly reflected upon; also that great profit is derived from all holy images, not only because the people are thereby reminded of the benefits and gifts bestowed on them by Christ, but also because through the saints the miracles of God and salutary examples are set before the eyes of the faithful, so that they may give God thanks for those things, may fashion their own life and conduct in imitation of the saints and be moved to adore and love God and cultivate piety [ ... ] this is beneficial to the illiterate, that the stories and narratives of the Holy Scriptures are portrayed and exhibited, the people should be instructed that not for that reason is the divinity represented in picture as if it can be seen with bodily eyes or expressed in colors or figures". For the consideration of painting as Poor Man's Bible (Biblia pauperum) and live scripture see (Paleotti 1594, p. 288; Barocchi 1961, p. 408; Molanus 1570, pp. 15, 32; Molanus 1996, pp. 125-27).

16 For the so-called "early Christian revival" of the end of the sixteenth century see (Zuccari 1984, pp. 49-52, 64, 80, 94; Bianchi 2008, vol. 11, pp. 84-85; Tosini 2009).

17 "usum a primaevis christianae religionis temporibus". [following the sacred writings and the ancient tradition of the Fathers]. Sessio XXV 3-4 dec. 1563. Cf. the treatises of Molanus and Gabriele Paleotti.

18 "Dipingere le sacre imagini oneste e devote, con que' segni che gli sono stati dati dagli antichi per privileggio de la santità, il che è paruto a' moderni vile, goffo, plebeo, antico, umile, senza ingegno et arte". 
the Infant Jesus "as occurred in the past and can be seen today in Greek icons" ("et così nelle figure grece" [sic]); otherwise, the holy figures might not be recognized by the faithful (Bianchi 2008, p. 171).

Paleotti's image theory shaped the aesthetics of Catholic devotion and popular piety, and was further developed in the writings of contemporary and future scholars. Johannes Molanus, quoting the Bishop of Meaux, William Durant the Elder (ca. 1220-1296), condemned the use of nudity by his contemporary artists, pointing out that "the Greeks use images, painting them as it is said only from the navel above, and not lower, so that there is no opportunity for brute thoughts to suggest themselves" (Molanus 1570, p. 69; Freedberg 1971, pp. 229-45). ${ }^{19}$ The Archbishop of Milan, Federico Borromeo (1564-1631) shared similar opinions. In his treatise, De Pictura Sacra (1624), Borromeo echoes Paleotti's views that art should educate the public, and praises the "Christian Greeks" ("i Greci cristiani") for respectfully depicting only the upper part of the saints' bodies and veiling the lower part with a cloth, "as we can still see in many pictures" (Borromeo 1932, p. 65; Jones 1997, pp. 32, 96). Almost a century later, the bishop of Bisceglie, Pompeo Sarnelli (1649-1724) was making specific recommendations to the painter Angelo Solimena, from whom he had commissioned four religious images for personal use. In a lengthy letter, the bishop provided Solimena detailed instructions on "how the sacred images should be painted" ("come si debban dipingere le sacre imagini"), and stressed that he would prefer his icons to be "half-length, according to the old Christian custom, which has been preserved by the Greeks". Like Gilio and Molanus, Sarnelli pointed out in his letter how old Christian masters painted religious images that "inspired devotion and superhuman majesty, even though they did not conform to the rules of art", contrary to "the painters of our age, who have profaned with their style the sacred pictures to the point where, not only is it impossible to worship them, but it is also impossible to look at them with pure eyes for they have introduced nudity even into the altars" (Sarnelli 1686, pp. 273-74; Basile Bonsante 2002, pp. 110-11). ${ }^{20}$

Despite Sarnelli's expressed recognition of the spiritual merits of Greek icon painting, the iconic images he commissioned from Solimena were ultimately executed in the late Baroque style, which was fashionable during his time. In Counter-Reformation Italy, appreciation for the spirituality of Eastern Orthodox icon painting did not necessarily entail a return to Byzantine pictorial forms; instead, at the basis of Post-Tridentine image theory lay the combination of the devotional qualities of Byzantine icons with the aesthetic principles and the naturalistic vocabulary of Italian or Flemish Baroque art, a precept that even regulated the production of iconic copies. In 1597, when Federico Borromeo wanted to include the "original portraits" of Orthodox saints and Church fathers in his Ambrosiana, he entrusted his commissions to several Italian artists instead of hiring one of the numerous Greek icon-painters working in Northern Italy. However, he went to great lengths to ensure that the copies would be modeled after authentic prototypes, ideally originating from the East. ${ }^{21}$ Much more important for him was the adherence of the copies to specific iconographic prototypes, rather than the painter's origin or the faithful emulation of the original's technique. After all, for Borromeo, any direct or indirect copy

19 "His adde, quod Gulielmus Durandus Mimatensis Episcopus scribit de quibusdam Graecanicis Ecclesiis in Rationali divinorum officiorum, Graeci, ait, utuntur imaginibus, pingentes illas ut dicitur, solum ab umbilico supra \& non inferius, ut omnis stultae cogitationis occasio tollatur".

20 "Per prima io le desidero a mezzo busto. Così fù l'antico uso de' Christiani ritenuto da greci per degni rispetti [ ... ] tanto che spirano divozione e maestà sopraumana; ancorche l'opera non appaja secondo le regole dell'arte [ ... ] Pittori de nostro secolo, che hanno profanato in maniera le sacre pitture, che non solamente non si ponno adorare, ma nè men rimirare con occhio puro, havendo introdotta la nudità infin sopra gli Altari".

21 In 1597, Borromeo assigned the German artist Hans Rottenhammer and the Spanish Dominican Alphonsus Ciacconius with the task of locating the "original portraits" of Doctors of the Eastern Church in Venice and Rome, respectively, cities with strong Byzantine associations. Rottenhammer's inquiry proved fruitless, so he proposed to have the portraits commissioned from a Greek artist residing in Venice, whom he, however, dismissed as a less skillful painter ("non è troppo maestro"). On the other hand, Ciacconius informed Borromeo that he had discovered certain portraits of Orthodox saints, which had been "carried off from Constantinople". See (Jones 1997, pp. 190-92, 205n94). Although most portraits have been attributed to Giuseppe Franchi, the close resemblence of certain ones among them with popular iconographic models or types associated with Greek icon-painting workshops (for example the one of Ioannes Permeniates in Venice), suggests the possibility that the images could have been modelled after original Greek designs or executed with the assistance of a Greek-speaking icon painter. 
of a miraculous icon channeled part of the qualities of the original, regardless of style or authorship (Noreen 2005, pp. 665-66; Merriam 2009, p. 204).

By sharing the powers of authentic images, reproductions of wonder-working icons became popular in sixteenth- and seventeenth-century Europe and were used by the Catholic Church as propagandistic tools in their battle against Protestant iconoclasm. Federico Borromeo's famous cousin, Cardinal Carlo Borromeo (1538-1584), owned, in his private collection, several reproductions of miraculous icons and symbols of Roman popular piety. In 1569, at the initiative of Cardinal Francesco Borgia, who wanted a "true and living portrait" of the Virgin, Carlo Borromeo received the permission of pope Pius V (1566-1572) to issue reproductions of the miraculous icon venerated at the church of Santa Maria Maggiore under the title of Salus Populi Romani (Noreen 2005, pp. 662-72). Most copies of the miraculous icon were distributed throughout Europe to Jesuit missionaries and state leaders fighting Protestantism. One of them, however, Borromeo kept for himself and had it placed in his private chambers, as we can see in a painting from the Duomo of Milan depicting the cardinal on his deathbed. The same copy or a similar one ended up later in the property of his cousin, Federico, and is featured in another painting portraying Federico Borromeo in his study (Noreen 2005, p. 665) (Figure 3).

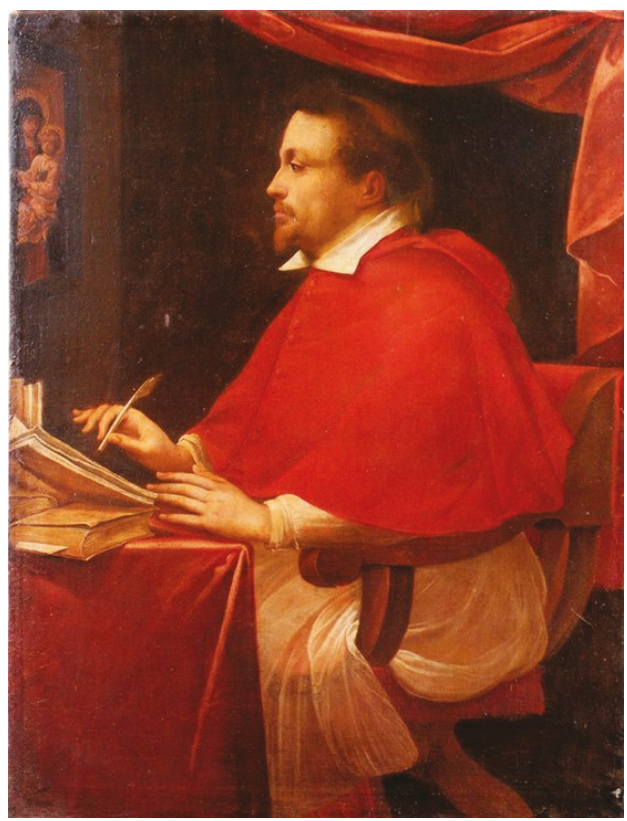

Figure 3. Giulio Cesare Procaccini, Cardinal Federico Borromeo, Milan, Museo Diocesano (image in the public domain).

In addition to the Borromeo family, a multitude of wealthy cardinals and cardinal-nephews (nipoti) owned copies of popular miraculous images or icons in the "Greek" style in their collections, usually objects of private devotion and of inferior economic or aesthetic value (Cappelletti 2014, p. 79). Cardinal Paleotti, for example, owned two small icons painted alla greca in his private residence ("un quadro con una Madonna alla greca, con sua coperta di tavola piciola [ ... ] e un altro dipinto di piccolo formato sempre alla greca") (Bianchi 2008, p. 171). A “Madonna alla greca" was listed in 1609 in cardinal Ludovico de Torres' estate in Rome (Abbate 2009, p. 283), while devotional images and copies of thaumaturgic icons were in the possession of cardinals Benedetto Giustiniani and Francesco Maria Del Monte (Jones 2004, p. 88). The domestic veneration of Greek icons was especially popular 
with the house of Barberini, as evidenced by the property inventories and wills of cardinals Francesco Barberini, his brother Taddeo, prince Maffeo, Cardinal Antonio and Don Francesco (Aronberg Lavin 1975, pp. 83, 97, 216, 231, 234, 268, 284, 313, 315-16, 343-44, 386, 392, 424).

While in Rome, higher intellectual and artistic circles often promoted the creation of iconic images in the Italian fashion, the diffusion of the cults of miraculous Byzantine icons resulted in their wider popularization and integration through means of copies in domestic settings. Contrary to pre-Tridentine times, when Byzantine icons were mainly collected as rare and precious items of prestige, in the age of confessionalization icons, alla greca, were primarily acquired for their spiritual properties, and functioned mainly as objects of devotion and piety. The spread of icons in Early Modern domestic interiors becomes even more conspicuous in the multiethnic and multiconfessional societies of Venice and the Adriatic region, where the persistence of Byzantine artistic traditions and the presence of vibrant Greek communities and artistic workshops allowed for the intense veneration of Byzantine and post-Byzantine icons in both private and public contexts.

\section{The Veneration of Greek Icons in the Italian and Dalmatian Household}

\subsection{The Archival Evidence}

As previously mentioned, during the course of the Middle Ages, Catholic domestic worship was mainly associated with manuscripts, although the presence of isolated religious images for private use is suggested in sporadic sources. In any event, in the fourteenth century, a new kind of image-centered spirituality emerged across Europe, aiming to engage the emotional involvement of the viewer, and emphasizing the importance of private piety (Ringbom 1984, pp. 12-14, 30-39). Since that time, religious images have consistently constituted the primary foci of domestic religious practices, representing, by far, the most common type of material culture encountered in Late Medieval and Early Modern Catholic households. It is estimated that ninety percent of all inventoried households in Venice possessed at least one devotional icon (Palumbo Fossati 1984, p. 131; Kasl 2004, p. 63; Morse 2007, pp. 163-66; Brundin et al. 2018, p. 139). Among these items, Byzantine and Byzantinizing icons, commonly referred to as alla greca to emphasize their Eastern origin, are encountered in sources as early as the fifteenth century, although mainly in the collections of wealthier and highly educated patrons. Nevertheless, by the early sixteenth century, their popularity increased to such a degree that they became almost ubiquitous in a vast range of households.

The prominent role of Byzantinizing icons in the Italian household is colorfully illustrated by the sixteenth-century historian, Giambattista Armenini, in his treatise titled De veri precetti della pittura (1587). During his travels through Northern Italy, Armenini reveals that he visited many mansions and palaces, all "admirably decorated, except for the paintings of holy images, which, for the most part, consisted of little panels of certain figures alla greca, very coarse, unpleasing, and entirely sootblackened" (Armenini 1587, p. 214; Armenini 1977, p. 256; Ringbom 1984, pp. 34-35). ${ }^{22}$ These icons were usually found in "the rooms where we rest and spend most of our lives", a custom which Armenini emphatically dismissed as shameful ("un gran vergogna"). But regardless of Armenini's personal sentiments, his narration reflects the proliferation of icon-worship in Catholic households, which is further confirmed by the dozens of icons located today in Italian and Croatian museums and private collections, as well as by a multitude of archival sources dating from the fifteenth, right through to the eighteenth century. Household inventories, family memoirs, wills and testaments, and other documents drawn up in the occasion of inheritances or dowaries testify to the presence of at least one or more "Greek" icons in

22 "per molti palagi e case, e fino nelle camere secrete [ . . . ] e tutte ho veduto essere con mirabil arte fornite, eccetto di pitture delle sacre imagini, le quali erano la maggior parte quadretti di certe figure fatte alla greca, goffissime, dispiacevoli e tutte affumicate". 
Catholic residences, and offer a vivid impression of the domestic uses of devotional images in the Italian Peninsula and the East coast of the Adriatic. ${ }^{23}$

In Venice, the hub of the Adriatic culture, the domestic cults of icons remained vital from the Late Middle Ages and throughout the entirety of the Early Modern period, long after the maniera greca had been abandoned from official artistic production. The vibrant commerce between Venice and its Mediterranean possessions, especially Candia, supplied the city with an abundance of devotional icons, while, from the sixteenth century onwards, the increasing local demand was supplemented by the production of Greek painters, commonly named Madonneri, who either travelled to the metropolis or settled their workshops in the city. According to Isabella Cecchini's estimates, Greek icons made up for $18 \%$ of the total art objects inventoried in Venetian collections during the years 1511-1513, faring considerably better than paintings of other foreign workshops, such as Flemish or German (Cecchini 2008a, p. 187; Corazza 2017, p. 202).

Icons of Greek provenance were almost omnipresent in the households of the Venetian nobility, with their popularity peaking towards the mid-sixteenth century. For instance, a Greek painting of the Virgin ("uno quadro de la Madonna greco") hung in one of the rooms at the residence of the nobleman and member of the Council of Ten, Domenico Cappello, and was inventoried on 22 June 1532 (Kasl 2004, p. 63; Hochmann 2005, p. 129). On 10 December 1533, a gilded icon of the Virgin ("un quadro de Nostra Donna grando dorado alla grecha") was registered among the possessions of noblewoman Elena, widow of Alvise Vitturi and, according to an inventory from 20 September 1529, a "Greek Christ" belonged to another noblewoman, Magdalena, widow of Girolamo Dedo (Hochmann 2005, pp. 121n70, 130). Likewise, a gilded icon of the Virgin ("una Nostra Donna greca dorada") was registered in the residence of Andrea Grimani on 7 June 1553 (Hochmann 2005, p. 130).

Among the wealthiest households, it was not uncommon to find more than one icon in the "Greek" style, sometimes even forming small collections. We know, for example, from a 1594 document, that Zuan Jacomo de' Agostini owned three Virgins alla greca in his impressive, for the time, collection of sixty-seven paintings (Jestaz 2001, p. 188). Three Greek icons in canvas ("doi quadri in tolla alla greca", "una madona greca in tolla con soaza dorata piccola") also belonged to the property of Doge Francesco Molin, and was inventoried on 30 November 1655 (Levi 1900, p. 15). The nobleman Alessandro da Mosto, on the other hand, kept six Greek icons of different subjects and styles ("sie quadri senza soaze greci de più sorte"), listed en masse in an inventory from November 27, 1538 (Hochmann 2005, p. 121). Similar to this was the case of the nobleman Priamo Malipiero, who had assembled a collection of various types of icons alla greca, including a triptych with "portelle" (Palumbo Fossati 2004, p. 481).

Icons alla greca also regularly appeared in the property of non-aristocratic Venetian professionals (cittadini), including lawyers, notaries, doctors, civil servants, and international merchants. Affluent, educated and socially ambitious, the cittadini had risen to a status of "second nobility", and their residences often rivalled those of the patricians (Schmitter 1997, p. 6; Jestaz 2001, pp. 186-87; Hochmann 2005, p. 120). In addition, wealthy citizens who did not share the connoisseurship of cultured aristocratic patrons often chose to decorate their dwellings with devotional icons of lower cost and quality (Cavazzini 2014, p. 89). To mention just a few, "due figure alla greca" were listed in the house of Giovanni Balarmi, "civis muriani" [sic] on 1 January 1513, and a "Madona grecha" was found in the residence of Bernardino Redaldi, duchal secretary, on 5 April 1526 (Hochmann 2005, p. 129).

It is noteworthy that, in Venice, as well as in the rest of the Adriatic, icons were not only restricted to upper-class palaces but were also commonly found in modest dwellings. In fact, one of the major innovations witnessed in sixteenth-century Venetian society was that art ownership gradually spread across all social and economic strata, and painting entered in the households of the lower classes, the popolo, as evidenced by property inventories (Henry 2011, p. 256; Corazza 2017, p. 193). According to

23 The display and uses of icons in Italian and Dalmatian households bear striking similarities with relevant practices documented in Venetian Candia. See (Constantoudaki 1975, pp. 36-74). 
Isabella Cecchini's calculations, while the percentage of art-owning patricians and citizens remained virtually stable and always at high levels during the years 1511-1615, that of craftsmen witnessed a $20 \%$ increase, whereas that of labourers was almost doubled, rising from $41 \%$ to $81 \%$ (Cecchini 2008b, Table 2). Indeed, inventory data demonstrates that $75 \%$ of popolani households contained at least one art object, mostly paintings of smaller dimensions, and of devotional rather than decorative function (Corazza 2017, p. 193). Mass-produced Byzantinizing icons perfectly fit this description. For example, a small icon of Saint Christopher ("uno S. Cristofalo alla greca piccolo depento") belonged to the grocer (aromatario) Francesco de Scoperti, as was registered in an inventory from 1528 (Molmenti 1928, p. 480). In another contemporary residence, that of Zaccaria Franchini, second-hand clothes dealer (strazzarol), there was a panel of the Madonna "alla greca indorado cum le sue arme suso" (Cecchini 2008a, p. 182). Lastly, from the inventory of the valuables of the apothecary (spezier) Giovanni Ambrosio Perlasca, compiled in 1587, we learn that a "madoneta alla greca" decorated the walls of the house's portego among other works of art (Corazza 2017, p. 194).

The presence of Greek icons in Venetian households is documented well into the seventeenth and even eighteenth centuries. To name a few examples: from an inventory drawn on 21 August 1613, we learn that a Greek icon of the Madonna ("un quadro de nostra donna soazado d'oro alla grecha") hangs on the walls of a corridor at Alvise da Ponte's mansion (Carlton 2015, p. 152n52). In addition, a Greek icon of the Virgin, which the notary assessed as "old" ("un quadro della Beata Vergine alla greca vecchio"), was found in the residence of nobleman cavalier Marin da Pesaro on 20 July 1671 (Bellavitis 1975, p. 260). Two more icons ("una Madonina greca con soaza nera d'ebano", "un quadro della Madona, pittura greca con soaze antiche dorate") were part of the collection of Andrea Morosini in 1674 (Levi 1900, p. 57). Moreover, in 1701, a Greek Madonna on canvas ("un quadro di Madonna in tolla alla Greca") decorated the house of Francesco Caffi (Cecchini 2005, p. 163). Several Greek icons of the Virgin were documented during the course of the century in the residences of Francesco Querini ("Madona Greca in tavola"), Domenico Cottoni ("effigie di una Madonna, pittura alla greca", "un quadretto con l'effiggie della Madonna alla greca con soaza negra e rotta"), and Antonio Giustinian ("Madona alla Greca in tavola") (Levi 1900, pp. 154, 184, 192).

However, despite this documented continuity of paintings alla greca in Venetian households, their records in the notarial sources grew progressively fewer as the centuries advanced. Going back to Isabella Cecchini's studies, it appears that the percentage of Greek icons in Venetian households dropped from $18 \%$ at the beginning of the sixteenth century to just $4 \%$ in the years $1560-1562$, and $1 \%$ in the period 1600-1615 (Cecchini 2008a, p. 187, Tab. 10; Corazza 2017, p. 202). One can only presume that these numbers kept decreasing in the following decades. While this data should be interpreted with caution, there are several reasons that would explain such a remarkable drop. Towards the late sixteenth century, the already outdated maniera greca, fell gradually out of fashion in favor of more naturalistic pictorial styles, and Greek icon painters started to incorporate increasingly more Western elements in their works, often creating mass-produced icons in the style of the Venetian mannerists (Figure 4). Although these works definitely found their ways into the Venetian households, they no longer bore the features that would allow notaries to detect their Eastern origin and identify them as alla greca. Another important factor that needs to be considered is the outbreak of the Cretan war in 1645 , and the fall of the centers of Chania (1645), Rethymno (1646) and ultimately, Candia (1669), to the Ottomans, which put a halt in the number of imports of Cretan icons to the Venetian markets.

Apart from the metropolis of Venice, Greek icons became widely popular throughout the whole Adriatic region, especially in the peripheral territories of the Stato da Màr, but also in regions that developed under the Venetian sphere of influence. In the Veneto and the Venetian terraferma, Greek icons were regularly encountered in household inventories. The residence of nobleman Alvise Biasio Speron in Padova offers an illuminating example, boasting of a considerable number of icons of various styles and subjects, as evidenced by an inventory compiled on 19 May 1683: an icon of the Virgin ("un'imagine della B. Vergine dorata alla greca") decorated the mezzanine room (mezato) of the house, another gilded canvas with the Madonna and Child with Saints Augustin and Jerome ("quadro greco 
in tela dorato con B. Vergine Bambino s. Agustino e s. Gerolemo") hung above the door of one of the chambers, whereas two more icons were located in another chamber, an "old" icon depicting the Madonna and Child with Saint Lucy ("quadro alla greca B. Vergine Nostro Signore e s. Lucia, vechio dorato") and a smaller one with the Coronation of the Virgin ("altro poco più picolo Incoronation della B. Vergine") (Sartori 1989, p. 166).

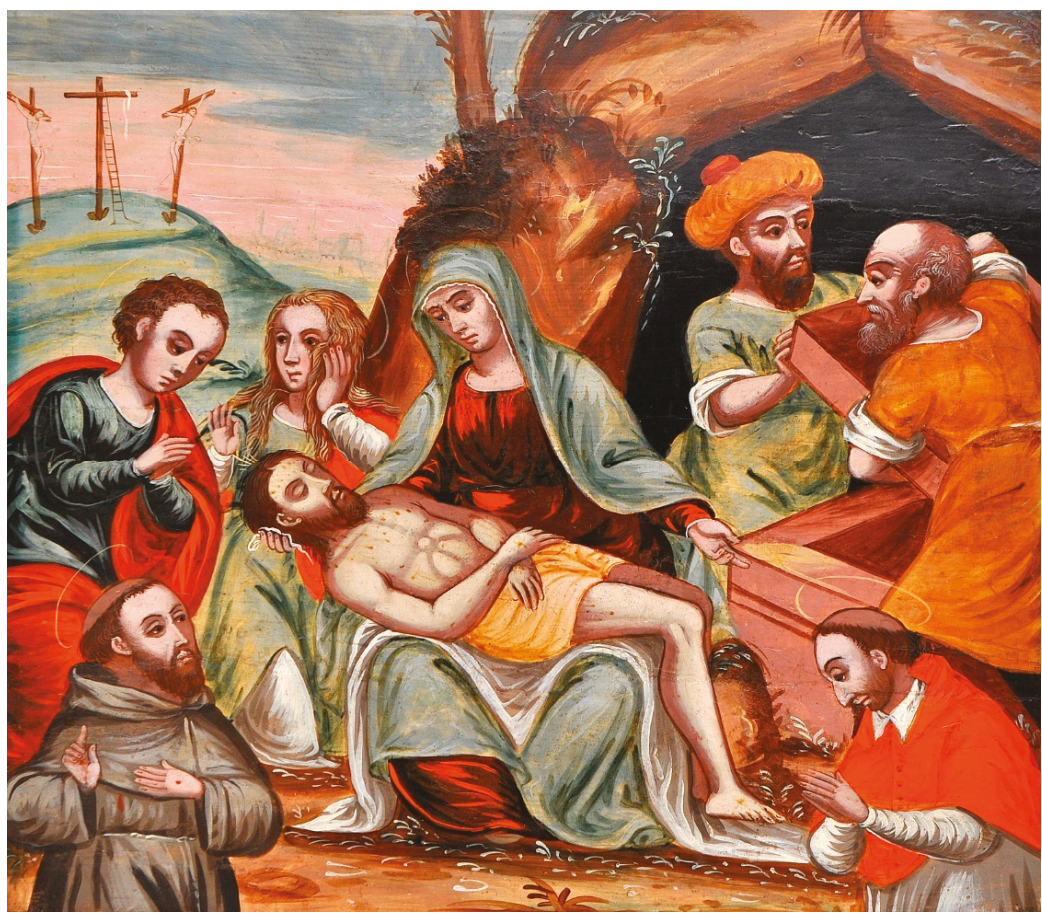

Figure 4. The Deposition (Pietà) with Saints Francis of Assisi and Carlo Borromeo, ca. 1620-1630, (C) Koper, Pokrajinski Muzej.

A similar picture is painted in the east coast of the Adriatic, although most published archival sources from the region date from before the late fourteenth century, when the practice of owning and bequething icons was still not as widely diffused as in the Early Modern period. Zoran Ladić and Valentina Živković have demonstrated the growing popularity of the veneration and bequest of artworks in Late Medieval Zadar, Trogir and Kotor; however, the cases they presented make no specific reference to icons alla greca, thus not permitting us to draw concrete conclusions (Karbić and Ladić 2001, pp. 180, 217; Ladić 2003, pp. 22-24; Živković 2016, pp. 221-30). More specific stylistic descriptions survive from later periods: for instance, in the property inventory of nobleman and doctor of law, Giovanni Casio (Ivan Kašić) from Nin, compiled after his death on 31 January 1699, there is mention of a small Greek icon ("un imagine della Beata Vergine alla grecha picola"), located in one of the rooms of his house (Katušić and Majnarić 2011, p. 240). Likewise, on 2 August 1722, a small icon of the Virgin with Christ ("un detto picolo con pitura alla grecha con l'Imagine della Beata Vergine con un Christo in mano") was listed among the artworks included in the dowry of Marietta, daughter of Antonio and Lucietta Garbellotto from Zadar (Goja 2014, pp. 136, 142). The widespread popularity of Greek icons in Dalmatia is further highlighted by Alena Fazinić, who claims that on the island of Korčula, there was no household that did not possess at least one Greek icon (Fazinić 2009, pp. 127, 133). Indeed, in her publications of the Boschi family archives, Fazinić mentions numerous icons in the Greek style and 
iconography, such as a small painting of Saint Spyridon ("un quadretto di s. Spiridion"), an icon of the Madonna and Child with a silver crown ("un quadro antico rappresentante Madona col bambino pittura greca su legno con una piccola corona con lami d'argento sopraposta alla testa della Madonna con cornice di faggio lucida in nero"), as well as a small oval panel with Saint Simeon ("un quadretto ovale in legno pittura greca rappresenta S. Simeona") (Fazinić 1980, pp. 587, 596-97; Fazinić 2009, $116 \mathrm{n} 28,119 \mathrm{n} 40,124 \mathrm{n} 47)$.

It is important to note that the veneration of icons was not only diffused in the Venetian possessions of the Adriatic, where there was plenty of interaction with Greek- and Slavic-speaking Orthodox populations; icons were equally popular in the Republic of Ragusa, where the Eastern rite was strictly prohibited until the eighteenth century. In the State Archives of Dubrovnik, there are numerous records of Greek icons in probate and dotal inventories, spanning all social and economic classes, as was the case in Venice. An icon of the Virgin in the Greek style ("una figura de Nostra Dona greca in clausura picola"), for example, decorated the house of the Ragusan Hieronymo Marini de Dimitrio, according to an inventory drawn on July 28, 1510 (Kovač 1917, p. 86; Tadić 1952, vol. 2, p. 97). Another Greek icon ("una figura alla grecha") was located in the residence of Petar Antulinović, which was inventoried after his death on 26 April 1518 (DAD, Diversa Cancellariae 108, f. 1r; Tadić 1952, vol. 2, p. 99). Likewise, an icon made "more greco" was found inside a painted cassone in the house of the leather-worker (cimator) Andrea Benchi (Benković), as registered in an inventory from 22 May 1518 (DAD, Diversa Cancellariae 108, f. 3r; Tadić 1952, vol. 2, p. 99). According to the inventory of the estate of Mirussa, wife of Natale Dobrić that was compiled on 11 May 1519, a small icon "alla greca" was found inside a big chest (cophano), while another Greek icon of the Virgin ("una inchona di nostra dona indorata alla greca") hung in one of the house's rooms (DAD, Diversa Cancellariae 108, ff. 15r, 16r; Fisković 1959, pp. 85-86n18). A mention of a small Greek icon ("una incona pizola indorata greghescha") is also found in an inventory from 23 January 1520 (Fisković 1959, pp. 85-86n18. Lastly, on 5 May 1528, a "Madonna de pinctura greca" was listed in the property of Nicoletta, daughter of Antonio Bolegnini (Tadić 1952, vol. 2, p. 143).

\section{2. "Alla Greca" in the West, "Alla Latina" in the East: The Many Styles of Greek Icons}

In Late Medieval and Early Modern archival sources, devotional images are usually described by a wide range of generic terms, including ic( $h$ )ona (and the alternative spellings anc( $h$ )ona, ycona, cona), denoting any kind of portable devotional panel or altarpiece; quadro, a term used to describe a broad range of artworks of various subject matter; tavola, which stands for a wooden panel; and, after the seventeenth century, tela or teller, signifying a painting on canvas, usually made in the Western style. Since all of these obscure terms may refer to any kind of religious painting without specification of its style and provenance, more precise terminology was required to discern Byzantinizing icons from their Western counterparts. In all the previously cited cases, the Eastern origin and Byzantine technique of the inventoried icons was explicitly noted through the use of terms highlighting their "Greekness", such as alla greca, more greco, greghescha or pittura greca. Some few documents even make more specific assessments: for example, a "quadro candioto picolo" was listed in Bernardino Redaldi's inventory in 1526, and a "quadro a la candiota" was mentioned in another Venetian inventory from 1541 (Hochmann 2005, pp. 118n21, 129). Though, undeniably, the best part of the icons that reached the Adriatic markets was imported from the workshops of Candia, it might be safer to assume that classifications such as alla candiota or alla greca rather implied the style and technique of the icons and not necessarily their actual provenance.

But what does the term alla greca precisely refer to? On an initial level, one might easily assume that an icon painted in the "Greek" style was one conforming to the rules of the Byzantine pictorial tradition, as it was preserved in the iconographic centers of the Balkans and the Eastern Mediterranean. More specifically, in order to be classified as "Greek" even by an Early Modern notary inexperienced in matters of art, an icon should observe certain stylistic and iconographic conventions, such as the use of egg tempera on a gold-ground panel, the adherence to an austere two-dimensionality, the stylized 
modelling of the faces and the linear articulation of the draperies. Based on such criteria, the author of the 1498 inventory of Paolo Morosini was able to distinguish between an old "Greek" icon and a painting “a la moderna” (Kasl 2004, p. 63).

That being said, in the culturally diverse milieu of the Venice and the Eastern Mediterranean, aesthetic definitions were not always clear or straightforward. In particular, in territories that remained long under Latin rule, as were Rhodes, Cyprus, the Ionian Islands, and especially, Venetian Crete, icon painters had grown highly familiar with Western art, and had adapted their painting style and techniques to better respond to the demands of their Catholic clientele. ${ }^{24}$ An extraordinary case study from Venetian Candia might help shed some light on this stylistic bilingualism of Cretan artists: on 4 July 1499, the dealers Giorgio Basejo from Venice and Petro Varsamà from Morea signed a series of contracts with three icon painters, named Michael Fokas (Migiel Fuca), Nikolaos Gripiotis (Nicolò Gripioti) and Georgios Miçoconstantin, commissioning them to deliver seven hundred icons of the Virgin within a period of forty-five days (Cattapan 1972, pp. 211-15; Lymberopoulou 2007, pp. 188-89). The painters were provided specific instructions on the preferred style of the commissioned icons, out of which two hundred were to be executed in the "Greek" manner ("in forma greca"), while the remaining five hundred would be painted in the Italian fashion ("tuto in forma a la latina"). Therefore, in this case, the distinction between the Eastern Orthodox and Western painting techniques is clearly noted: the term "in forma greca" corresponded to the strict Byzantinizing tradition (Figure 5a), while the definition "alla latina" indicated that the icons would be executed in a hybrid Italianate style (Figures 5b, 6 and 7), based on the combination of Byzantine elements with Late Gothic or Renaissance influences.

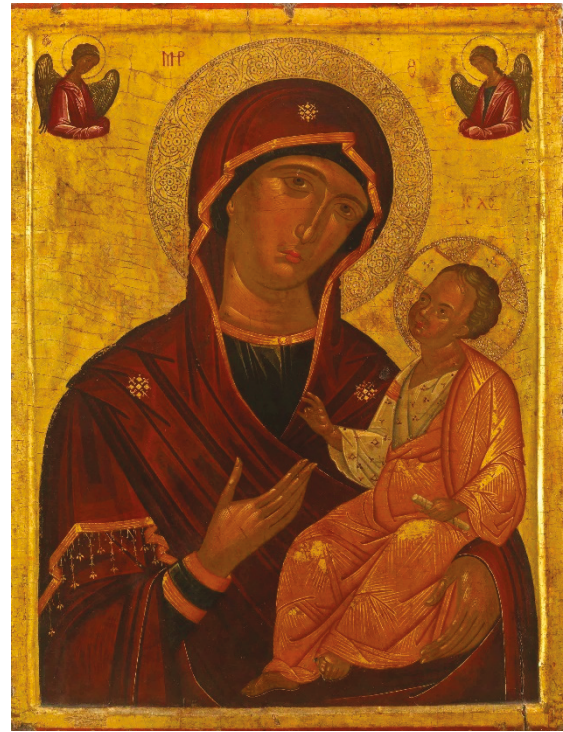

(a)

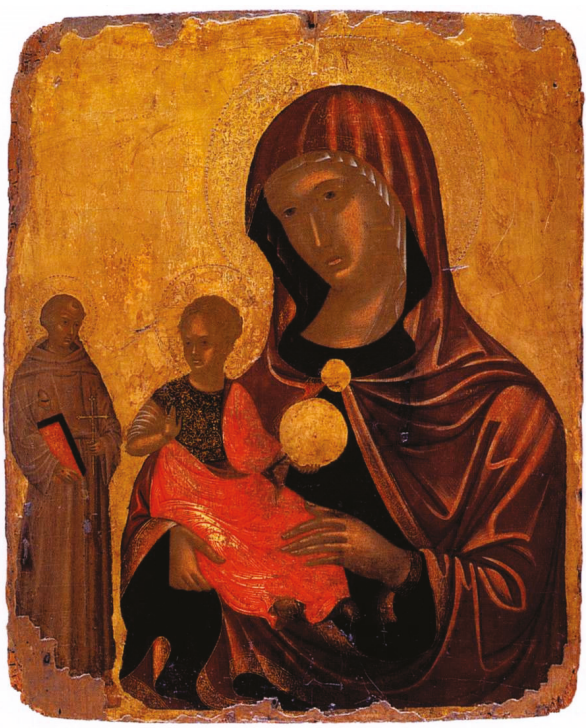

(b)

Figure 5. (a) The Virgin Hodegetria, 16th century, Venice, Istituto Ellenico di Studi Bizantini e Postbizantini (image in the public domain); (b) Nikolaos Tzafoures, Madre della Consolazione with Saint Francis of Assisi, Athens, Byzantine and Christian Museum (image in the public domain).

24 For the presence of Italian artists in Venetian Candia see (Constantoudaki 2018, pp. 30-58). 


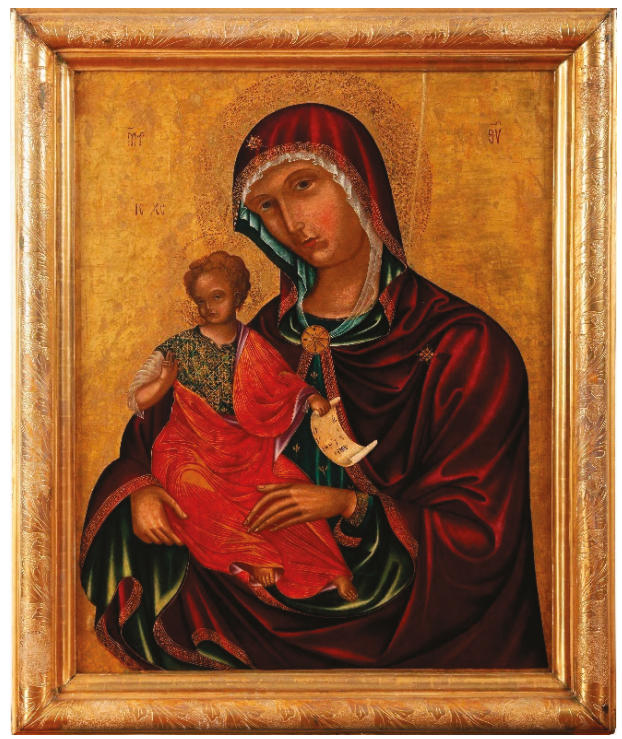

Figure 6. Circle of Nikolaos Tzafoures, Madre della Consolazione, Hvar, Biskupski Muzej (provenance: Church of the Annunciation of the Virgin) (c) Živko Bačić.

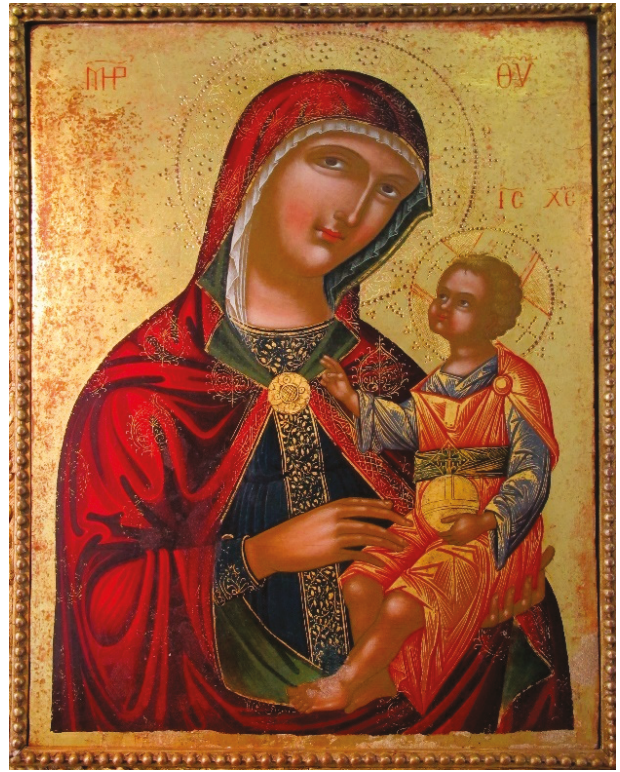

Figure 7. Ioannes Permeniates (attr.), Madonna and Child, San Marino, Museo di Stato (photograph by the author).

From the above, it appears that the identification of the different styles of icon painting depended heavily on the cultural background of the viewers and their levels of familiarity with the Byzantine tradition. What would classify as "Latin" for a Greek Orthodox viewer might be easily seen as "Greek" by an Italian or Dalmatian, who was used to viewing paintings in the naturalistic style of 
the Renaissance and Baroque. Therefore, we can presume that a good part of all these icons alla greca mentioned in the archival sources were actually painted in the Westernizing style that was then becoming popular in Latin-ruled Greek lands. After all, the bulk of the icons ordered in the aforementioned massive commission were intended to be "a la latina", and were probably destined for Western markets, a hypothesis which is also confirmed by the plethora of Italianate icons in museums and private collections throughout the Adriatic (Figures 6, 7, 9a, 17, 19, 20).

On the other hand, it is possible to assume that, due to their stylistic ambiguity, these hybrid icons of Greek manufacture were not always successfully identified in the sources, and were easily mistaken for works of the Italian trecento or quattrocento (Figure 8). While notaries were seemingly up to date on the aesthetic tendecies of their time, and able to distinguish between different painting styles, such as Italian, Greek or Flemish. However, their assessments should still be read with caution. After all, even in modern-day museum or auction catalogues, Greek icons are often mistaken for works of Italian or Dalmatian provenance, especially if they feature pronounced Western influences. Although it is difficult to know for sure, it seems highly likely that some of the ancient gilded Madonnas (all'antica, indorata) that are so often cited in the inventories might as well have been of Greek provenance.

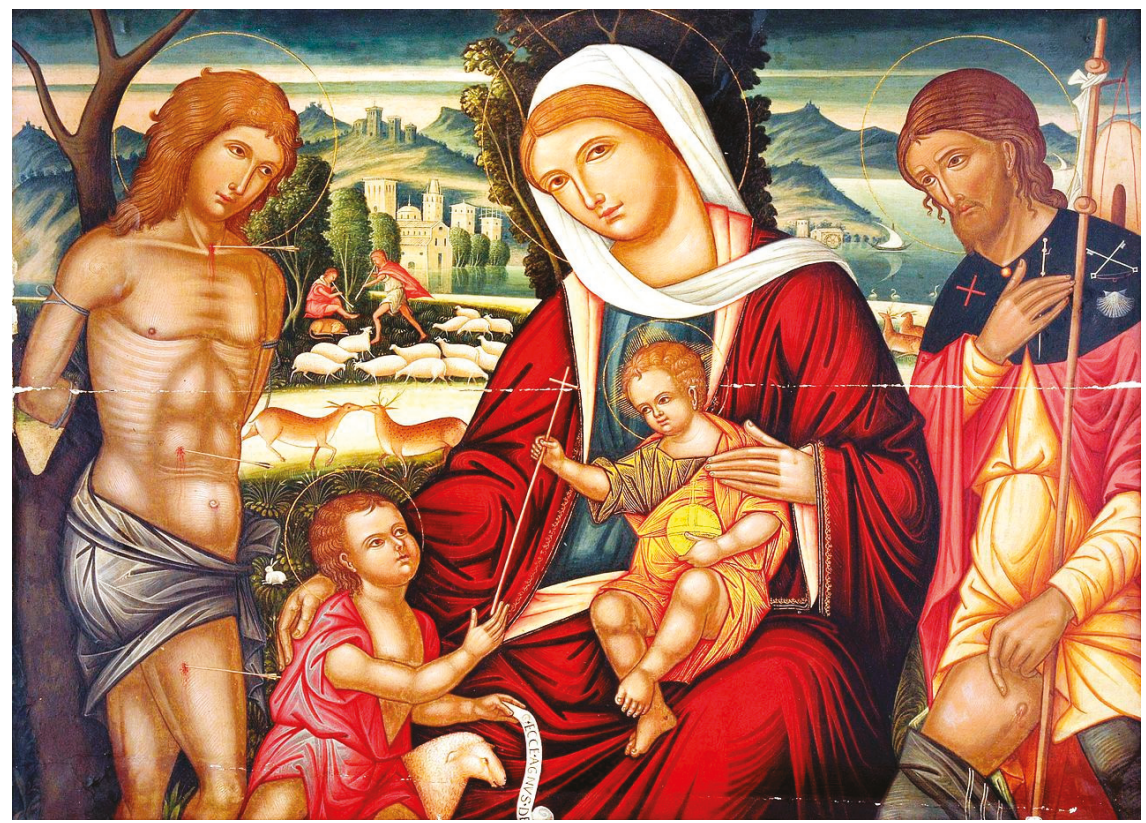

Figure 8. Permeniates (attr.), Madonna and Child with the Infant Saint John and Saints Sebastian and Roch, private collection (image in the public domain).

The identification of Greek icons becomes even harder, as icon painters were not only adopting Western stylistic elements but also replicating Western iconographic themes. The practice of reproducing popular iconographic prototypes lay at the core of icon painting, and Greek workshops systematically copied works of famous Italian masters, thus popularizing them for a mass public. Widely popular among Greek painters were the works of Giovanni Bellini and his circle: in its most concise compositional variation, Bellini's Madonna and Child with Saints from the Städel Museum in Frankfurt, a type already proliferated within the master's workshop (Heinemann 1963, pp. 239, 277, 279; Golden 2004, pp. 91-127), served as a model for an icon at the Franciscan Monastery of Hvar (Voulgaropoulou 2014, p. 758) (Figure 9a-c); even more popular was Bellini's Pietà, now at the Gemäldegalerie in Berlin (Heinemann 1963, p. 116, Fig. 85, n. 168; Belting 1985, p. 71, Fig. 32; Bock and Grosshans 1996, n. 1781), replicated 
in numerous copies in Rijeka, San Marino, Saint Petersburg, Berlin, Athens, Geneva, and in private collections (Ricci 1901, p. 131; Gamulin 1984, pp. 147-50; Piatnitsky 1993, p. 89; Pasini 2000, pp. 132-34; Kakavas 2003, p. 305; Frigerio Zeniou and Lazović 2006, Tab. 44-45; Voulgaropoulou 2007, pp. 216-19; Voulgaropoulou 2014, pp. 633, 667) (Figure 10a,b).

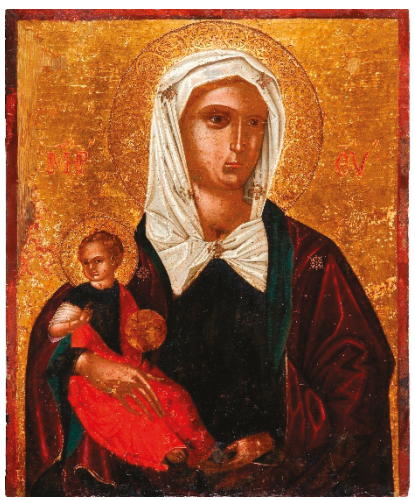

(a)

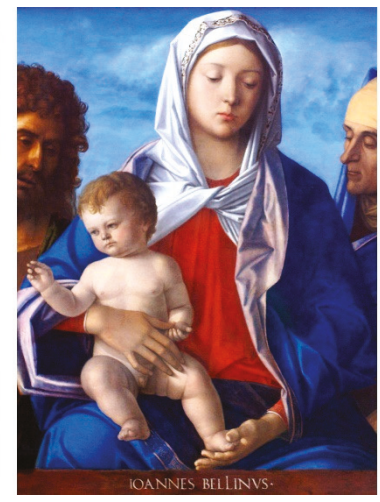

(b)

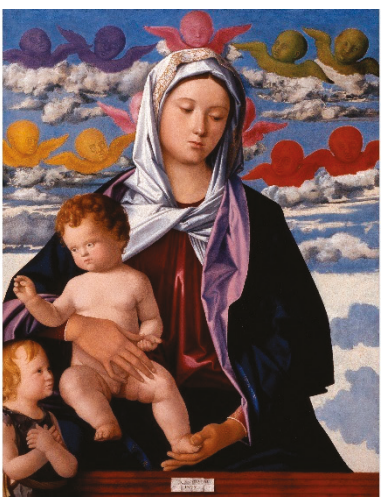

(c)

Figure 9. (a) Madonna and Child after Giovanni Bellini, Hvar, Franciscan Monastery of Our Lady of Mercy (Zbirka Umjetnina Franjevačkog Samostana Gospe od Milosti) (C Živko Bačić; (b) Giovanni Bellini, Madonna and Child with Saints John and Anne, Berlin, Gemäldegalerie (image in the public domain); (c) Giovanni Bellini, Madonna and Child with the Infant Saint John, Indianapolis, Indianapolis Museum of Art (image in the public domain).

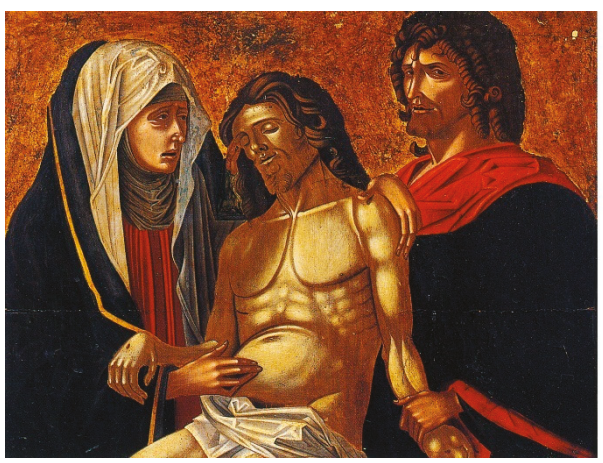

(a)

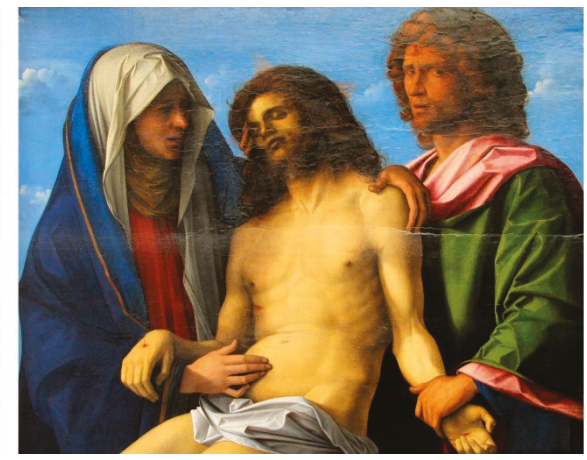

(b)

Figure 10. (a) Pietà after Giovanni Bellini, San Marino, Museo di Stato (taken from Pasini 2000, Fig. 57); (b) Giovanni Bellini, Pietà, ca. 1495, Berlin, Gemäldegalerie (image in the public domain).

In his inventory of the paintings preserved at the convent of Saint Nicholas in Zadar, the Venetian painter, Giovanni Battista Pitteri, identified several icons alla greca that were modelled after Italian prototypes, among which were an Ecce Homo and an icon of the Virgin (Brunelli 1913, p. 393). It should be noted that while the Greek icons from Pitteri's list were obviously cheaper than their Italian originals, still they were considered more valuable than other devotional images of Italian manufacture contained in the same inventory. In particular, the icons marked "alla greca" were estimated from 50 to 120 lire, whereas those "all'italiana" were only priced from 16 to 30 lire. What is more, when the painter Agustin Crivellari was asked to evaluate the paintings included in the dowry of Marieta Garbelloto, he appraised the only Greek icon on the list for 44 lire, a price much higher than all the other Italian images in the collection (Goja 2014, p. 142). Likewise, in the inventory of Pietro Gritti, compiled in 1557, 
a Greek Madonna was estimated at 6 ducats, second only to a painting of the Judgement of Solomon that cost 15, when all the other artworks in the collection had significantly lower prices (Brown 2004, p. 86; Hochmann 2005, p. 118). The same value of 6 ducats was also ascribed to another Greek Madonna in the 1553 inventory of Andrea Grimani, making it the most expensive artwork among his possessions (Hochmann 2005, p. 118).

As prices of paintings in Candia and other Mediterranean centers were substantially lower than those in Venice (Panagiotakes 2009, vol. 7, pp. 31-32; Corazza 2017, p. 163), Greek icons offered an affordable solution to collectors with a wide range of budgets, while retaining a relatively high level of quality in comparison to mass-produced images of Italian or Dalmatian workshops. When the painters Fokas, Gripiotis and Miçoconstantin were commissioned to manufacture seven hundred icons, they were asked to design them in three different sizes, which corresponded to three different prices. A month earlier, the same painter, Fokas, had employed the woodcarver Giorgio Sclavo to cut for him a thousand wooden panels, again in three different sizes and prices (Cattapan 1972, pp. 211-15). It was precisely the varying cost of Greek icons, combined with their mass manufacture, that contributed the most to their wide popularity, making them affordable even to modest households, as opposed to only to the upper and educated classes. The prices of Greek icons were the most likely to increase with time, given their actual or claimed antiquity, or their asserted miraculous and spiritual properties.

\subsection{A World of Homely Madonnas and More: The Subjects of Greek Icons}

From the examples presented in the previous sections, it becomes evident that icons intended for private use demonstrate a very limited range of subject matter. By far the most popular subjects of domestic devotional imagery were depictions of the Virgin Mary with the Christ Child. Every art-owning household contained at least one image of "Nostra Donna", and even in the humblest dwellings, where there was only one recorded painting, it would most probably be an icon of the Virgin. Although inventory entries do not offer specific iconographic descriptions, we can get a sense of the most popular types that were venerated in Italian and Dalmatian houses by looking into the icons preserved today in museums and private collections around the Adriatic. In the surviving samples, the Virgin is portrayed in a variety of iconographic types established in the Greek-Orthodox tradition, such as the Hodegetria (Figure 5a), the Galaktotrophousa (Figure 15), the Eleousa or Glykophilousa (Figure 16b), the Madonna of Passion (Figure 18), and the Virgin Threnousa. ${ }^{25}$ More popular among the Catholics, however, was the composite type of the so-called Madre della Consolazione (Virgin of Consolation), which was invented in fifteenth-century Crete in order to respond to the aesthetic preferences of the local Catholic and Latin-oriented clientele (Figures 5b, 6, 7, 17, 19). By emulating Venetian models of the fourteenth and fifteenth centuries, while retaining the morphological features of a Byzantine icon, this hybrid type appealed more to the taste of a Western audience than icons of an austere, purely Byzantine style, and was largely diffused in the Adriatic markets. We can safely assume that the five hundred icons "in forma a la latina", that were commissioned in the above-mentioned 1499 contracts, belonged to this hybrid iconographic type.

It appears, therefore, that the increasing demand for religious icons in the West had a profound impact on the art of icon painting itself, which was required, in turn, to adapt to the needs of its Latin clientele in terms of both form and content. Apart from images of the Madre della Consolazione, highly diffused in domestic interiors were hybrid iconographic subjects relating to the Passion of Christ, such as the Man of Sorrows, the Pietà or Christ carrying the Cross, themes once again borrowed from the Western iconographic tradition (Figures 10 and 11). From the mid-fifteenth century onwards, when the artistic exchanges with the West became more intensive, the iconographic repertoire of Greek icon painters was further enriched with a long list of saints worshipped in the Catholic Church. Devotional icons often featured saints that were invoked for particular purposes, bearing various attributes and

25 For icons in the collections of the broad Adriatic region see (Voulgaropoulou 2014). 
powers, and depicted either alone or accompanying the Madonna and Child, often in portable triptychs and polyptychs. For instance, very common in devotional imagery were representations of Saints Sebastian and Roch, healing saints and protectors from the plague and other contagious diseases that regularly infested the Adriatic ports (Figure 8). Icons depicting the Doctors of the Catholic Church, usually Saints Augustin and Jerome, were also popular, presumably among learned scholars and theologians. Female saints were also frequently represented in Greek devotional icons, with the most popular among them being Saint Catherine, followed by Saint Lucy, Agnes and Magdalen. Among the saints of the Orthodox Church that appeared in Catholic households, the most popular were Saints Nicholas and Spyridon, whose cults were widely diffused in the maritime societies of the Adriatic.
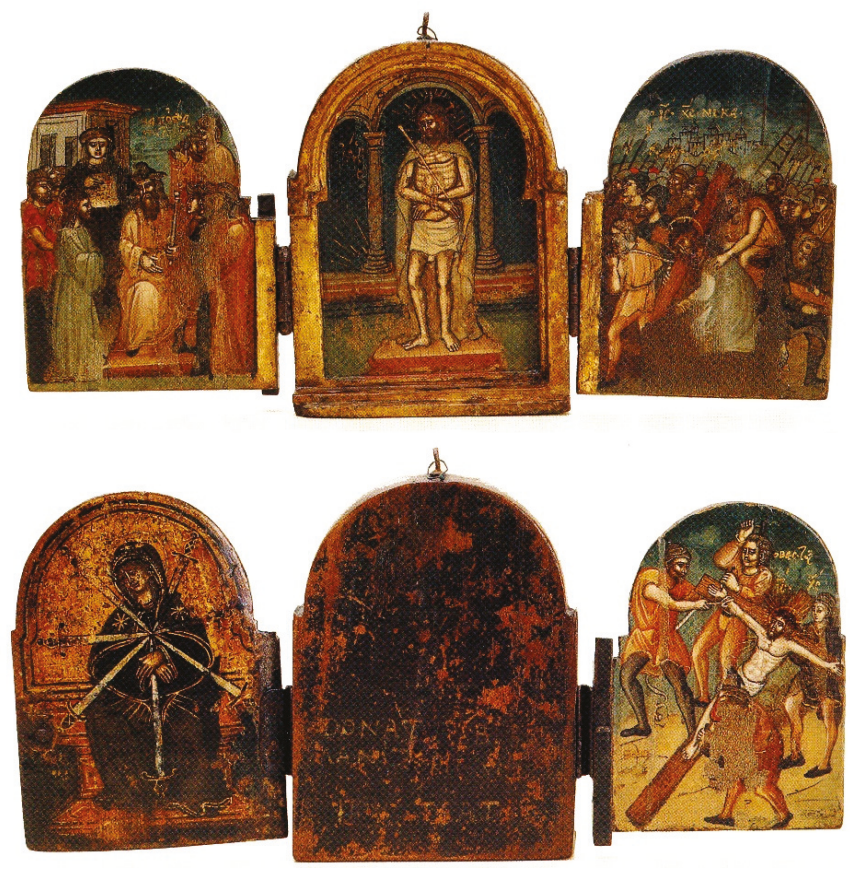

Figure 11. Donatos Bitzamanos, Triptych with Scenes of the Passion of Christ (recto and verso), painted in Otranto, San Marino, Museo di Stato (taken from Pasini 2000, Fig. 58).

\subsection{The Display and Functions of Icons in the Domestic Space}

The preference for such subject matter is directly linked to the function of devotional images in the domestic space, which was mainly to serve the needs of everyday individual piety. Most of these portable objects have been long removed from their original domestic contexts, posing critical obstacles to our understanding of their devotional roles and their place in the religious experiences of devout Catholics. Once again, information drawn from archival records, combined with insights from literary sources, in addition to painted illustrations, help us reconstruct a fairly accurate picture of the varied uses of Greek icons and their display within the Catholic household.

The devotional roles ascribed to paintings for private devotion were crystallized during the Late Middle Ages, and remained essentially unchanged through the Early Modern period, when they found their ideal representation in icons of Eastern provenance. Icons of saints were placed around the household in order to offer divine protection from harm and sickness, assistance to everyday challenges, while also serving as instruments of catechism and paradigms of virtue. More precisely, icons of the Virgin were invoked to provide fertility for the married couple, assistance to women in 
childbed, as well as comfort to the ill and dying. In his diaries (ricordi), the fifteenth-century Florentine merchant, Giovanni di Pagolo Morelli, describes how his dying son Alberto found consolation by praying before a tavola of the Madonna on his deathbed (Trexler 1980, p. 173; Gavitt 1990, p. 294; Romano 1993, p. 720n331). Young mothers were expected to model their behavior after the Virgin Mary, whereas children were encouraged to imitate the Infant Saint John the Baptist or virgin Saints. ${ }^{26}$ On the other hand, images of the Passion were designed to inspire penance, prayer, meditation, and compassion (compassio). By depicting the holy figures half-length (a mezza figura), icons accentuated the most expressive features of the human body, i.e., the face and the hands, thus achieving the emotional engagement of the believer, and enhancing their experience of worship. Further in his narration, the above-mentioned Giovanni Morelli colorfully illustrates how he used to pray before the painted image of the Crucifix, privately in his chamber, kneeling barefoot in his nightgown; at the end of this ritual, he would kiss the three figures of the Crucified Christ, the Virgin Mary and Saint John, and make the sign of the cross (Trexler 1980, pp. 159-86; Webb 2005, p. 35).

In Morelli's account, this "domestic ceremonialization", to borrow Richard Trexler's terminology, took place within the most private and secluded space of the home, the bedchamber, where the devout would retire in solitude to pray. Indeed, since the Middle Ages, the veneration of icons had usually taken place in the bedchamber (camera), making it the principal locus for informal religious practices. As early as the thirteenth century, Tommaso da Celano (1200-1265) noted the presence of small rooms (camerulae or secreta cubicula) in the residences of the Roman nobility, where the devout would pray before a devotional panel (icona or imago) (Schmidt 2005, p. 93). Likewise, in thirteenth-century Florence, the Blessed Umiliana de Cerchi (1219-1246) had converted her bedchamber into a cell, so that she could pray before a portable icon of the Virgin (Maginnis 2001, pp. 13-20; Webb 2005, pp. 32-34; Schmidt 2005, p. 95). These early accounts match later testimonies, such as the one of Giambattista Armenini, who came across Greek icons in "secret rooms" ("camere secrete") at the palaces he visited, underscoring the connection of icons with private domestic spaces, and thus illustrating the continuity of devotional practices from the Late Medieval through the Early Modern period.

Notarial acts, memoirs and inventories, such as the ones discussed in the previous sections, confirm the preponderance of Greek icons in private rooms and bedchambers of Catholic households. ${ }^{27}$ To cite yet another example, from the property inventory of the silk-spinner (filatore) Mafeo de Calvis, drawn up in Venetian-ruled Bergamo on 14 September 1540, we learn that a panel of a "Madonna alla Greca" was kept in the owner's master bedroom (camera grande) (Roncalli 1945, p. 335). The same picture is observed even in the houses of cultured and entirely conscious collectors of icons: the majority of the Byzantine icons in Lorenzo de' Medici's palace, for instance, were found in his private study (scrittoio) and in his bedchamber (camera), while one of the least costly ones was kept in the chamber of Piero de' Medici for private use (Fusco and Corti 2006, p. 110; Duits 2013, pp. 175-77).

Paintings containing illustrations of Medieval and Early Modern interiors offer an additional representation of the uses of devotional images as visual supports for prayer in the domestic space: particularly valuable in our research are Altichiero da Zevio's Dream Of King Ramirez, the scene of The Prayer of Saint Henry of Hungary from the predella of an altarpiece in the church of San Martino a Mensola, the predella of Francesco Francia's Coronation of the Virgin in the church of San Frediano in Lucca, and especially, the episodes of the Return of the Ambassadors and the Saint's Dream from by Vittore Carpaccio's Legend of Saint Ursula (Figure 12), to name some examples (Goffen 1975, p. 512; Romano 1993, p. 720; Belting 1994, p. 42; Kasl 2004, pp. 66-68; Schmidt 2005, pp. 93-94, Fig. 52;

26 In his Regola Del Governo Di Cura Familiare, Giovanni Dominici went as far as to encourage children to worship icons in private, sometimes even by engaging in ritualistic practices that involved dressing up like priests, and playing Mass around toy altars in imitation of what occurred publicly at church. (Dominici 1860, pp. 132-33). For Giovanni Dominici's pedagogical views see also (Battista 2002, pp. 126-38; Webb 2005, pp. 34-35; Morse 2006, pp. 170, 307, 313-14n135; Kolpacoff Deane 2013, p. 69; Drandaki 2014, pp. 45-46).

27 It is far from surprising that icons were also found in private chambers in the households of Greek migrants in Venice. (Vlassi 2008, pp. 96-97). 
Corry et al. 2017). These paintings reveal how the religious icon hung above the bed or was placed on a domestic altar, often covered by a curtain (cortina), lit by a candle (candelier) or lamp (cesendello), and accompanied by other devotional accessories, such as Crucifixes, rosaries, incense burners and holy water stoups (situla).

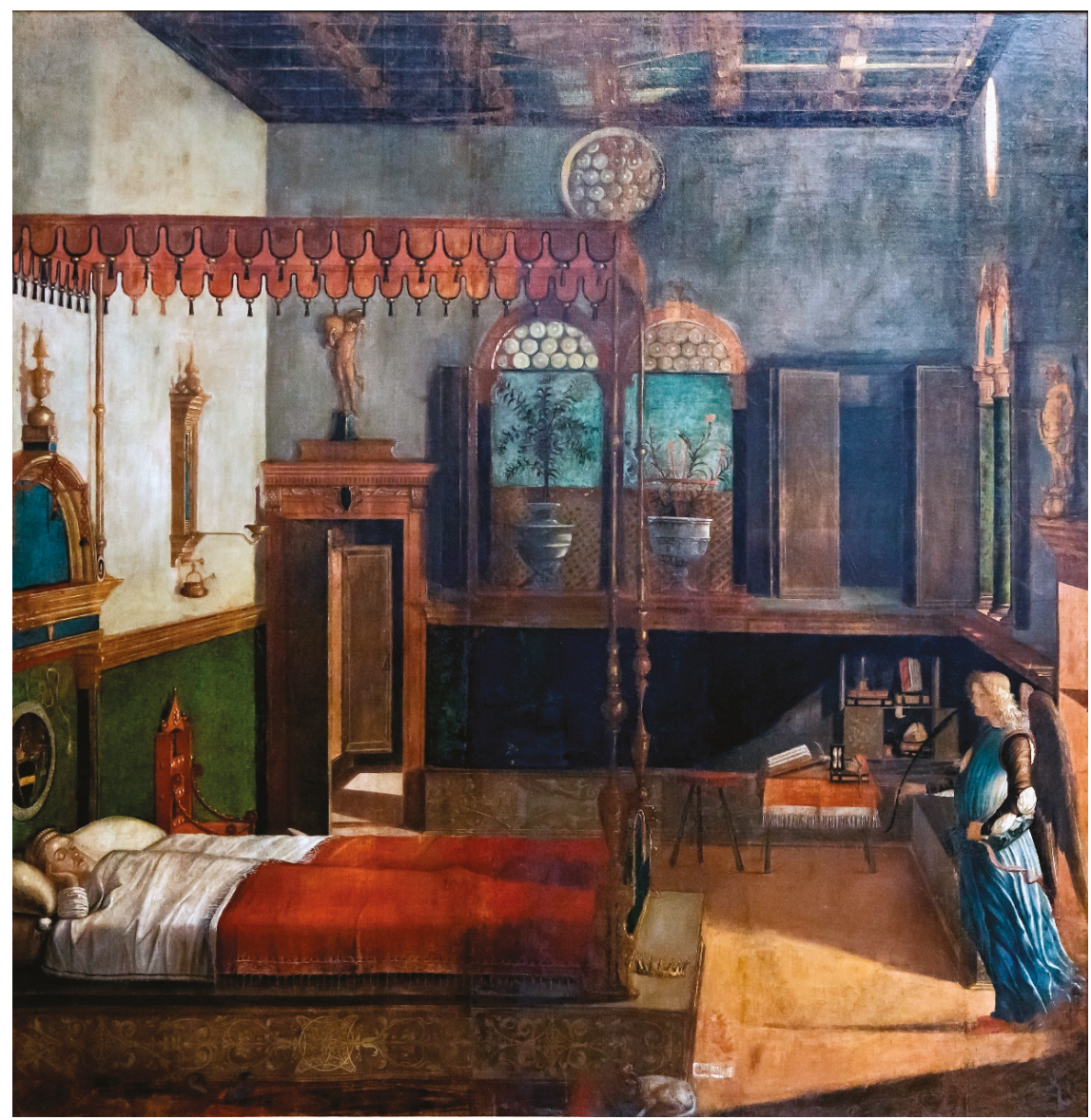

Figure 12. Vittore Carpaccio, The Dream of Saint Ursula, ca. 1495, Venice, Gallerie dell'Accademia (image in the public domain).

Archival evidence confirms the accuracy of these artistic impressions. In 1533, for instance, the Venetian Nicolò Zorzi kept a Greek icon of the Virgin in his private chamber, behind a protective curtain ("quadro uno greco de Nostra Dona d'oro con fornimento d'oro con ferro e cortina") (Hochmann 2005, p. 129). In 1538, an icon of the Virgin with its gilded candleholder ("uno quadro de Nostra Donna alla greca con suo candelier dorado") was recorded in one of the bedchambers at the residence of the Venetian noblewoman and former courtesan, Elisabetta Condulmer, accompanied by an image of the Shroud (Hochmann 2005, p. 130; Schmitter 2011, p. 729n120). Similarly, from the 1535 inventory of the artworks preserved at the palace of the Venetian nobleman Nicolò Salomon, we learn that an icon "alla greca", together with three more paintings of the Virgin and a small image of the Dead Christ were all placed next to a small metal basin for holy water and a bronze bell (Morse 2007, p. 166). At the residence of the Da Lezze family, always in Venice, an icon of the Virgin "alla greca" was located in the 
master bedroom, while in the adjacent study, there was a small altar with a wooden Crucifix and a painting of the Three Magi (Brown 2004, p. 79).

It is, therefore, evident that the domestic space was often appropriately customized in order to host the devotional icon, which stood at the center of domestic worship, emulating, in all aspects, the public setting of the church (Webb 2005, p. 32; Morse 2007, pp. 165-66). A remarkable case illustrating this merging of private and public devotion was that of the Venetian nobleman, Daniele Dolfin, who had a small chapel (giesiola) built in his palace to house a Greek icon of the Virgin ("una madonna maniera Greca"), as evidenced from a 1681 inventory (Levi 1900, p. 71). As may be expected, such domestic altars and chapels were not consecrated for public celebrations but were only used for private devotion; however, this still required special permission from the Venetian Church (Cecchini 2000, p. 42; Kasl 2004, pp. 68-70; Mattox 2006, pp. 658-73; Corry et al. 2017, p. 11).

The placement of icons in the private apartments of a household clearly reflects the emphasis placed upon their spiritual and devotional function, as well as their appreciation as rare and valuable objects. That being said, we need to keep in mind that, contrary to Florence, in Venice and its colonies, the bedchamber was not an exclusively private space, and often functioned as a semi-private reception hall or sitting room. Moreover, while in Florence or other Italian artistic centers, devotional images were almost exlcusively restricted to private domestic spaces, in Venice and the rest of the Adriatic, they were quite omnipresent throughout the household and were even displayed in the most public shared areas of the house. For example, an "old" Greek icon of the Last Supper ("una cena del nostro Signor, in tolla pittura greca antica") decorated the staff dining room (tinello) at the residence of the Venetian Angelo Bozza, as mentioned in a document from 27 May 1680 (Levi 1900, p. 65). Plenty of icons alla greca were also documented in the main reception areas of Adriatic houses, mainly in the portego, the central entry and entertainment hall of upper-class palaces, which was usually decorated with diverse objects, maps, secular portraits or large paintings of historical, allegorical or mythological themes in the Italian or Flemish fashion (Morse 2013, pp. 91, 95-96; Carlton 2015, p. 152). ${ }^{28}$

Indeed, Greek icons were among the most common types of religious paintings decorating the walls of Venetian porteghi and were often paired with other works of a religious or profane subject matter, sometimes even with erotic paintings and nudes. A large gilded icon of the Madonna and Child with a candlestick, pictured in Giovanni Mansueti's Miraculous Healing of the Daughter of Benvegnudo of San Polo (Figure 13), offers a visual representation of the display of icons in Venetian interiors, in support of what is inferred from the archival sources. A 1545 inventory reveals that a Greek Maestà hung on the walls of the portego at the residence of Hieronimo Zono in Venice, together with a Last Supper and a Flemish painting with bathing nudes (Hochmann 2005, p. 119). Another inventory, documenting the valuables of the miniaturist Gasparo Segizzi (or Segezi) after his death in 1576, reports that "an ancient Greek Madonna" was displayed in one of the reception areas of his estate together with a female nude, portraits of Roman emperors and an image of an old map; two other old Madonnas, possibly also of Greek origin, hung in the adjacent portego near the owner's portrait (Palumbo Fossati 1984, pp. 144-49; Kasl 2004, p. 64; Carlton 2015, pp. 152-53). In a period that saw the rise of collectionism, it is not surprising that certain icons of higher artistic quality were also valued as works of art, as is manifested by the inclusion of icons painted alla greca in the first private collections established in Venice during the seventeenth century (Savini Branca 1964, pp. 120, 149, 160).

28 For the Venetian quadro da portego see (Schmitter 2011, pp. 693-751). 


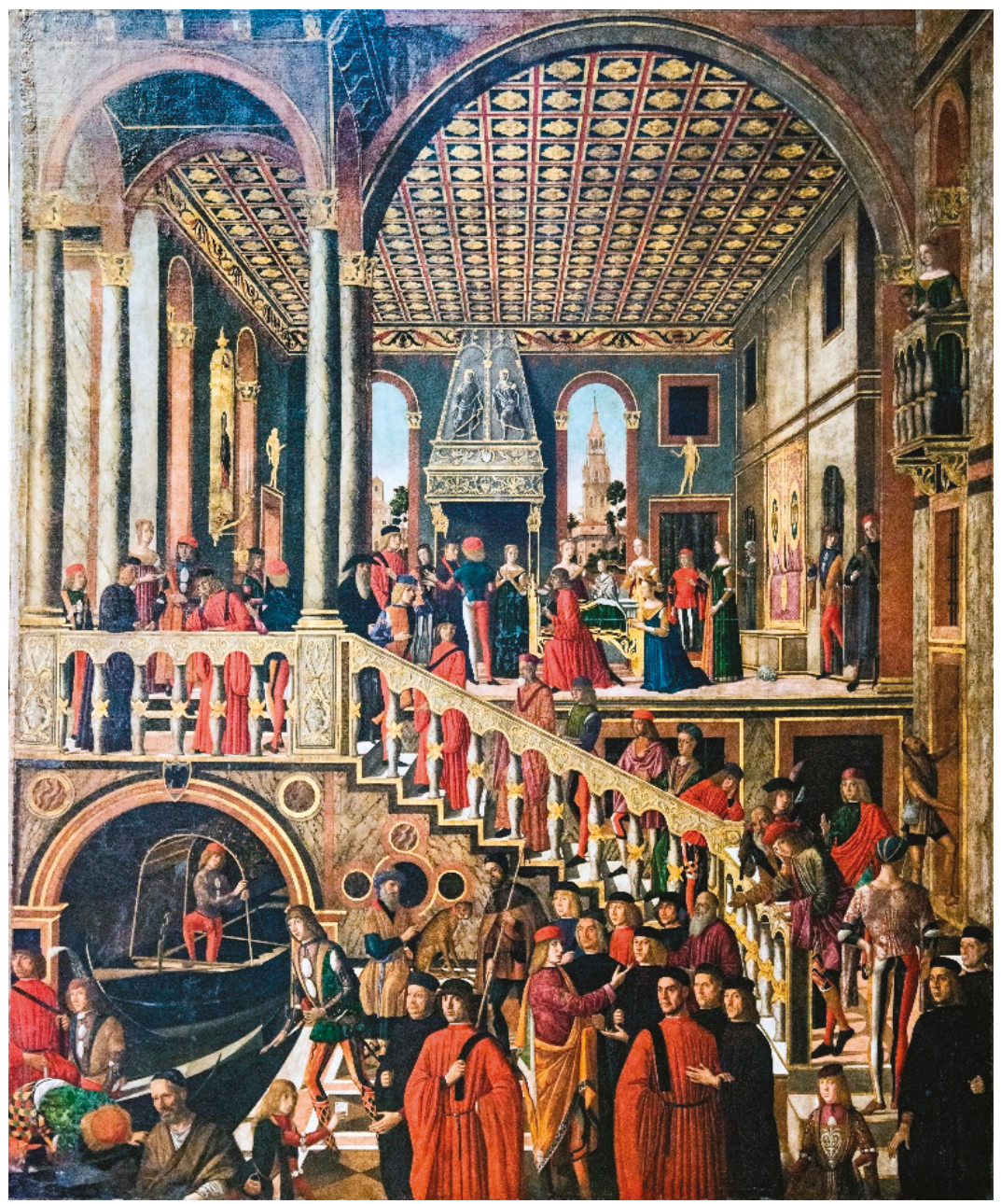

Figure 13. Giovanni Mansueti, The Miraculous Healing of the Daughter of Benvegnudo of San Polo, ca. 1505, Venice, Gallerie dell'Accademia (image in the public domain).

A quite different arrangement to those of the ones mentioned above was encountered at the palace of Girolamo Foscarini. There, Greek icons of the Virgin were paired with a paper illustration of the Siege of Candia with the coats of arms of the Foscarini family ("Madonne dipinte alla greca ed un quadro col desegno in carta dell'assedio di Candia da parte dei turchi coll'arma Foscarini"), and other items referring to the war of Crete (Levi 1900, LXV1ll). Given that the residence was inventoried in 1679 , only ten years after the fall of Candia to the Ottomans, the association of Greek icons, presumably from Candia, with other objects relating to the Cretan war was surely intended to allude to the military accomplishments of the Foscarini family, and especially, to pay tribute to the owner's namesake, Girolamo Foscarini, who had served as Provveditore Generale in Dalmatia and Capitano Generale da Màr during the Siege of Candia. It is possible to conclude, therefore, that apart from their devotional and aesthetic roles, Greek icons, especially those stemming from Venetian territories contested by the Ottomans, were also valued in the domestic space as historical tokens or miraculous guardians during periods of geopolitical unrest. Indeed, the practice of commissioning Greek icons was particularly 
popular with Venetian or Dalmatian noblemen that had participated in the Ottoman-Venetian wars, and it was intended that the images as protective talismans in the battlefield or tokens of gratitude for their survival and success, customs that were widely diffused in the Eastern Orthodox world. ${ }^{29}$ Such icons were privately venerated in domestic settings, as was the case with the Foscarini Madonnas or similar works kept in private collections; however, a large proportion of them was commonly donated to churches and monasteries, thus becoming part of public devotion.

\subsection{From the House Tabernacle to the Church Altar: Icons of Domestic Devotion in the Public Space}

The veneration of religious icons often exceeded the boundaries of domestic life and entered the sphere of public collective piety (Figures 14-20). As we have already seen, a large number of the sources documenting the presence of icons in Catholic households were actually probate inventories, dowries and last wills, recording the donation of icons to private individuals or public institutions. During the course of the Early Modern period, the practice of bequeathing devotional icons on the deathbed became increasingly common in the Catholic milieus of both Adriatic coasts (Romano 1993, pp. 713, 718-20; Belting 1994, pp. 230-33; Janeković 1996, pp. 25-34; Cecchini 2000, p. 86; Sivrić 2002, pp. 73-77, 107; Vlassi 2008, pp. 83-117; Ladić 2012, p. 306; Živković 2016, pp. 221-30). Although rarely mentioned before the mid-fourteenth century, paintings started to be included in the wills of Italian and Dalmatian testators from the late fifteenth century onwards, together with monetary legacies, bequests of land, garments, food, and liturgical objects (Ladić 2003, pp. 22-23; Ladić 2012, pp. 304-06).

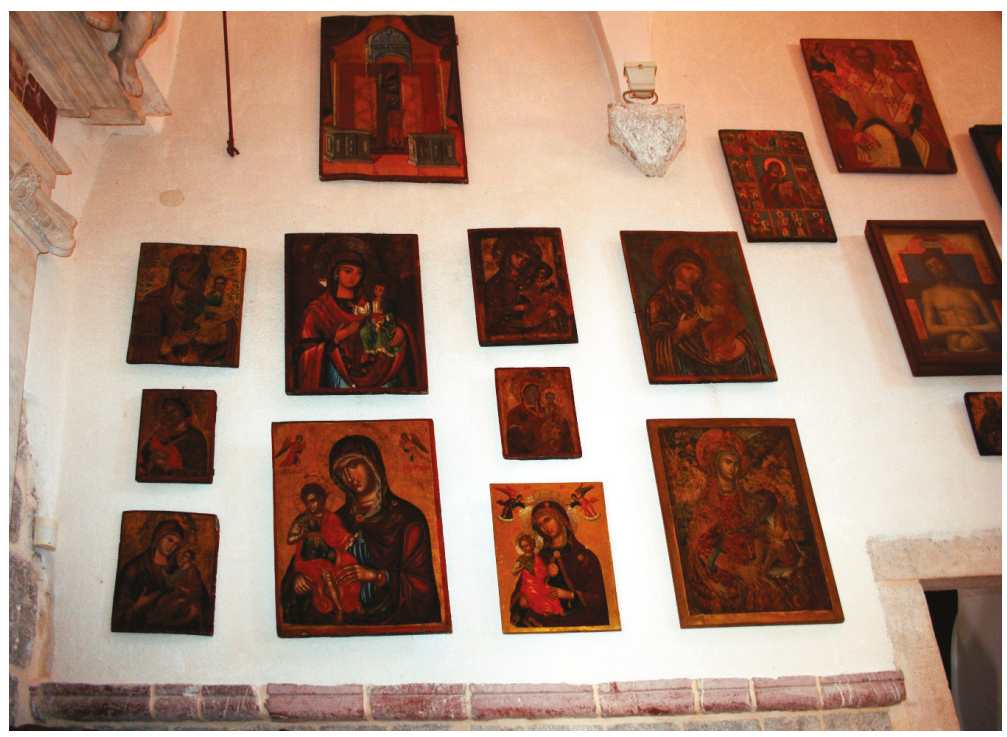

Figure 14. Icons from private collections and churches of the Boka Kotorska, Kotor, Cathedral of Saint Tryphon (Katedrala Svetog Tripuna) (photograph by the author).

Icons were usually bequeathed to close relatives, friends, as well as to faithful servants, young unmarried girls or the poor, with the request that they pray and mediate for the souls of the deceased. For instance, in 1525, Nicola de Saracha from Dubrovnik left to his cousin, Frana, the icons of the Virgin and Saint Ephrem "out of love" ("per amore"), as he explicitly mentioned in his will (Tadić 1952, vol. 2, pp. 142-43). In a similar vein, the Venetian ambassador to England, Girolamo Lando left in

29 See (Voulgaropoulou 2014, pp. 123-25, 647). 
his 1656 will, three Greek icons to his sons, Antonio and Giovanni ("una madona alla greca", "un quadro de Madonna in tolla alla greca dorado", "un quadro alla greca nominato la pietà") (Levi 1900, p. 24). Likewise, in a will that was drawn up on the Dalmatian island of Korčula in 1779, the nobleman Angelo Boschi left, to his brother Gasparo, an icon of the Virgin "di pittura greca", which was, in turn, bequeathed to him by his grandmother, Brigita Albertini (Fazinić 1980, p. 587).

Apart from bequeathing icons on a personal level, a common practice among Catholics was to donate or bequeath religious paintings to churches and other ecclesiastical institutions, often located in the places of their origin. As in the case of bequests to private individuals, icons were donated to churches with the request that the donor's name be commemorated in Mass, thereby ensuring the eternal salvation of their soul and the souls of their ancestors (pro remedio animae). Because of their spiritual properties, icons alla greca were particularly suited for pious acts compared with other art objects in the possession of Catholic families. Quite revealing is the case of Vittoria, widow of Pavao Grgurov, a Bosnian merchant living in Venice, who specifically requested in her will that all the paintings in her property be sold with the sole exception of a "Madonna Greca", which would be donated instead to the church of San Giobbe (Čoralić 2002, pp. 50, 55n65).

In the public archives of various Adriatic countries, one occasionally comes across similar records of donations and bequests of Greek icons to churches and monasteries, signed by testators from a wide range of socio-economic backgrounds. In 1507, for example, the milliner (baretarius) Juraj (Georgius) Gojković drafted his last will and testament, bequeathing a Greek icon of the Virgin ("una figura mia alla greca, la imagine de sancta Maria") to the church of Saint Luke in Dubrovnik, seat of the confraternity of the oil merchants (oleari) (Tadić 1952, vol. 2, p. 33). In a similar fashion, towards the end of the seventeenth century, the bishop of Makarska, Nikola Bijanković, donated his rich collection of icons to the town cathedral and to other nearby churches (Demori Staničić 2012, p. 171; Voulgaropoulou 2014, pp. 130, 506-8, 776; Demori Staničić 2017, pp. 319-20). In this group of icons, there was a Virgin Galaktotrophousa (Figure 15), which was given to Bijanković in 1707 by Meletios Typaldos, Greek Metropolitan of Philadelphia in Venice (. Farlati 1769, p. 202; Tomasović 2013, p. 31; Voulgaropoulou 2014, p. 508). Among the most renowned donors of Greek icons, we can also mention the Venetian painter Gentile Bellini, who, in 1506, bequeathed a mosaic icon-probably acquired during his visit to Constantinople—-to the Scuola di San Marco (Duits 2013, p. 170).

The practice of bequeathing and donating icons to churches was particularly diffused among noblemen or wealthy commoners who wished to leave their mark on the public space in order to enhance their prestige within the local communities, and at the same time, obtain spiritual benefits. In Venice alone, Alberto Rizzi counted ninety-two Greek icons throughout the city churches, many of which came directly from donations of prominent patrician families, such as the Corner, Foscolo, Pisani, Morosini, Venier, Barbaro, Pizamano and Meseri (Rizzi 1972, pp. 250-72, 253n9). Icons originating from bequests and individual donations retained much of their private devotional character in the public space, and were often placed on their owners' tombs or in churches, chapels and altars over which their donors possessed rights of patronage (jus patronato) or which they helped establish. These religious edifices usually had a purely devotional and secular rather than liturgical function, serving as an extension of the domestic space.

According to a dedicatory inscription in the Franciscan monastery of Saint Euphemia on the island of Rab, the Venetian merchant and printer Andrea Cimalarca bequeathed an icon of the Virgin Glykophilousa (Figure 16) to his sister Maria with the order to have it installed on the altar of his funerary chapel that he built in 1506 in the monastery church of Saint Bernardine (Domijan 2007, pp. 221-23; Voulgaropoulou 2014, pp. 490-91, 665). Objects of private and, at the same time, public devotion were also an icon of the Madre della Consolazione, work of the Cretan painter Emmanuel Tzanes, which decorated the chapel of the Duboković-Šprung family in the town of Jelsa on the Dalmatian island of Hvar (Demori Staničić 1990, pp. 139-40; Demori Staničić 2012, vol. 2, pp. 264-65; Voulgaropoulou 2014, pp. 522, 766; Demori Staničić 2017, pp. 359-60), as well as an eighteenth-century icon of the Madonna Hodegetria that was venerated in the chapel of the Butafogo family of merchants (crkva Svete 
Marije al Prato dei Butafogo) on the island of Cres (Fillini and Tomaz 1988; Demori Staničić 2012, vol. 2, p. 281; Voulgaropoulou 2014, pp. 492-93, 653; Demori Staničić 2017, p. 367).

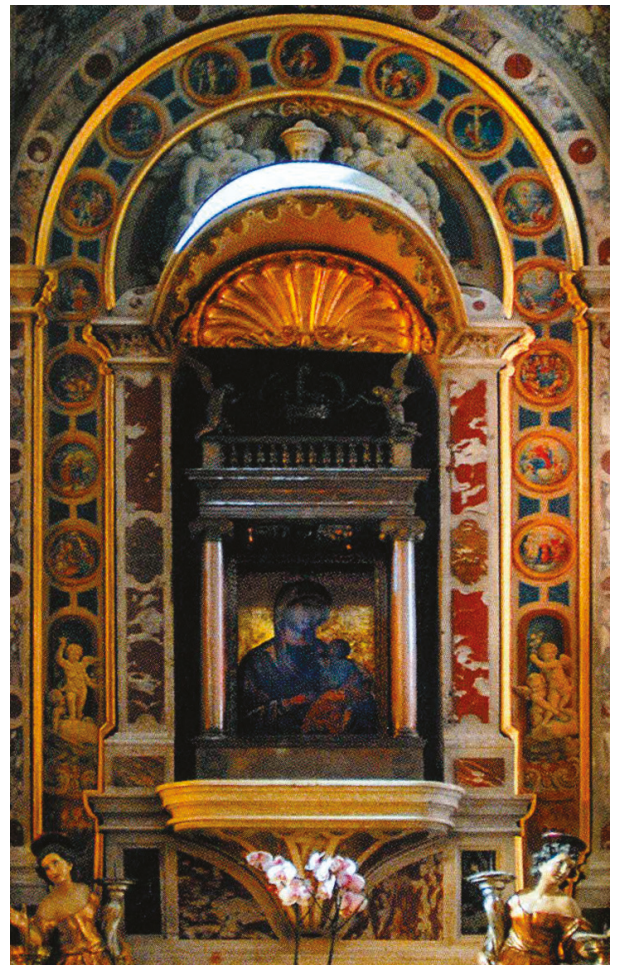

Figure 15. The Virgin Galaktotrophousa (Madonna of the Rosary), Makarska, Co-Cathedral of Saint Mark (Konkatedrala Svetog Marka) (taken from Tomasović 2013, p. 31).

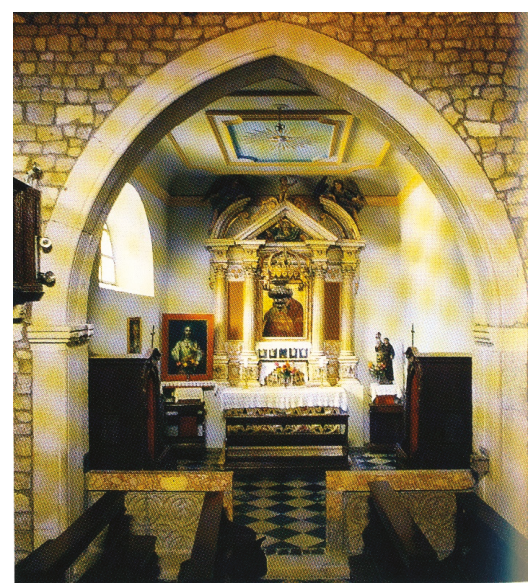

(a)

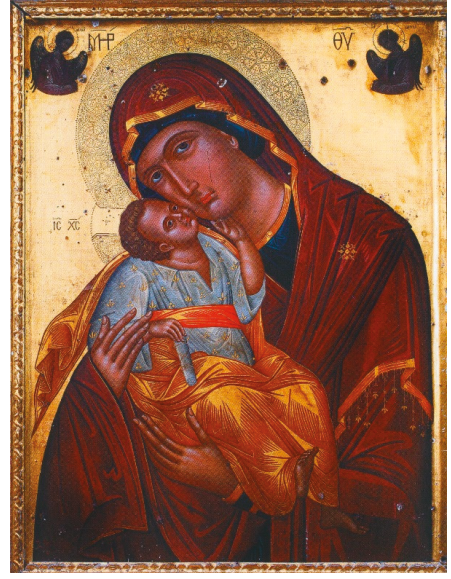

(b)

Figure 16. (a,b) The icon of the Virgin Galaktotrophousa in the funerary chapel of Andrea Cimalarca, Rab, Monastery of Saint Euphemia, Church of Saint Bernardine (Franjevacki Samostan Svete Eufemije u Kamporu, Crkva Svetog Bernardina) (taken from Domijan 2007, pp. 221-22). 
The bequest and donation of icons to funerary and family chapels or other types of private and semi-public places of worship was largely linked to their spiritual protective properties, guaranteed not only in the domestic space but also in the afterlife, and often manifested in the form of miracles. In fact, a large number of icons publicly venerated in chapels and churches made their way into the public domain after manifesting miraculous healing powers. According to Susan Merriam, "miracle-working icons proved especially useful to the Church in the aftermath of the Reformation, as they offered divine proof in support of the cult of images" (Merriam 2009, p. 204). At the beginning of this study, we mentioned the case of "Our Lady of Tears" from Longiano, an icon of domestic devotion, which became the center of a popular public cult after allegedly exhibiting miraculous properties. As striking as it sounds, the case of Longiano was hardly an isolated occurrence, as legends of wonder-working icons were extremely widespread in the entire Adriatic region, often triggering the initiation of public cults and even the establishment of religious institutions.

A similar example to that of Longiano comes from Venice, and revolves around a "devout image of old Greek art" ("una divota imagine di greca antica pittura") kept at the house of a certain Agostino around the year 1497 (Corner 1749, pp. 369-78; Corner 1761, p. 515; Concina 1998, p. 529; Walberg 2004, pp. 261-69). According to Flaminio Corner, Agostino had long undervalued the icon in question, which hung neglected in a dark corner of his house. Then, suddenly, one day, Agostino heard a "miraculous voice" reproaching him for his lack of respect towards the icon and ordering him to place it somewhere where it would receive its due veneration. Obeying the celestial command, the devout Agostino took the icon from his house, and in a solemn procession, transported it to the nearby Oratorio di San Vincenzo, which was subsequently dedicated to the Virgin in honor of the miraculous icon. News of the miracle-working icon soon reached another pious Venetian, the nobleman Alvise Malipiero, who decided to finance the construction of a new church in place of the old oratory and have it dedicated to Santa Maria Maggiore. ${ }^{30}$

Another illustrative case, ideally manifesting the blending of private and public religiosity, was that of a streetside icon of the Virgin, which belonged to the Venetian family of merchants, the Amadi, and was affixed on the external wall of their residence or of the nearby Ca' Dolce, facing a public street (Corner 1749, pp. 218-23; Corner 1761, pp. 89-92; Apollonio 1880, pp. 7-23; Boni 1887, p. 249n1; Tassini 1915, p. 234; Rizzi 1972, p. 279). Lore has it that the icon, a work of inferior artistic quality, suddenly started to perform miracles and became the object of public veneration. Acknowledging the importance of the icon for public piety, the Amadi relocated it on a small wooden altar near the ponte della Fava, but soon requested that a more suitable place be built for its veneration. Only three months later, on 10 November 1480, after verifying the miracles attributed to the image, the Venetian Patriarch, Maffeo Gerardo, granted the Amadi family and other patricians the permission to build an oratory to honor and protect the prodigious icon. The oratory was dedicated to Santa Maria della Consolazione, after the iconographic type of the icon, but was commonly referred to as Santa Maria della Fava from the nearby eponymous bridge. ${ }^{31}$ Amadi and his descendants were assigned as lifetime custodians of the church, an honor otherwise restricted to noblemen. ${ }^{32}$ It is noteworthy that, in the same year, the Amadi family was involved in yet another legend regarding a different miraculous icon in their property — this time an image in the Late Gothic style, work of a certain maestro Nicolò-whose

30 The same story with slightly differering details is reproduced by Helen Deborah Walberg. (Walberg 2004, pp. 261-69).

31 The idea to place the icon in a church was first revealed by divine inspiration to the wife of Francesco, head of the Amadi household, as evidened in the copy of an annotation in the Annali Guarnieri-Bocchi, which reads: L'anno 1480 Per rivelazione / dalla moglie di un Francesco / Amadi fu edificato l'oratorio de[lla] / Madona della fava deta anche / di consolazione il quale prima / era un cappitello depinto con una / inmagine di Maria operando / miracoli si edifico il luogo. Si crearono 6 procuratori Tre / nobili tre cittadini includendo / in questo numero gli Amadi. (Pastega 2015, p. 276). See also (Apollonio 1880, pp. 11-12).

32 Giuseppe Tassini offers an alternative account, according to which the icon hung on the walls of Ca' Dolce and not Ca' Amadi, and that in the year 1496 the parish priest of San Lio, Natale Colonna, Giacomo Gussoni, and other patricians bought out the two houses of the Dolce family to build a small church and move the icon to a more dignified place. (Tassini 1891, pp. 436-37; Tassini 1915, p. 234). 
ownership was contested at court by the neighboring Barozzi family, and whose public veneration led to the construction of the church and convent of Santa Maria dei Miracoli (Corner 1761, pp. 52-56; Apollonio 1880, p. 6; Boni 1887, pp. 236-74; Crouzet Pavan 1992, pp. 617-68; Grubb 2000, pp. 130-31; Grubb 2004, pp. 272-73; Kasl 2004, p. 60; Morse 2007, pp. 182-83).

The involvement of the Amadi family in at least two cases of miracle-working icons in the same year, and with the foundation of important religious institutions throughout the city, significantly boosted their reputation within the Venetian society and allowed them to elevate their social status, obtaining privileges usually reserved for the nobility. Indeed, owners of icons were eager to proclaim them as miraculous, and then donate them to churches, thus enhancing their social prestige, as well as the value of their bequest. In numerous acts of bequest, it is explicitly mentioned whether the image donated was famed for exhibiting miraculous properties. To name one example, an "old miraculous Greek icon of the Virgin" ("imagine miracolosa della B. Vergine, e di Pittura Greca, antica") was donated on 13 February 1671, to the church of the Redentore in Venice by the novice capuchin Fra Mariano da Venezia, born Giorgio Corner (Da Portogruaro 1930, p. 206; Rizzi 1972, pp. 254-55n14, 262). A quite different example is that of the so-called "Madonna of the Miracle" (Gospa od Čuda), an image of the Madre della Consolazione, venerated at the church of Saint Andrew at the Benedictine convent in Rab (Figure 17). The icon belonged to Lucchina de Dominis, a nun of noble birth who lived at the convent in the years 1530-1545. According to the written tradition, the nun, who had been bedridden for nine years, prayed before the icon that hung over her bed and was miraculously cured on the feast day of the Nativity of the Virgin. The miraculous recovery of the ailing nun initiated the public cult of the icon, which was immediately removed from the nun's cell, and placed on a special altar in the monastery church (Barić 2007, p. 147; Domijan 2007, p. 130; Demori Staničić 2012, vol. 2, pp. 207-8; Voulgaropoulou 2014, pp. 491-92, 666; Demori Staničić 2017, pp. 333-34).

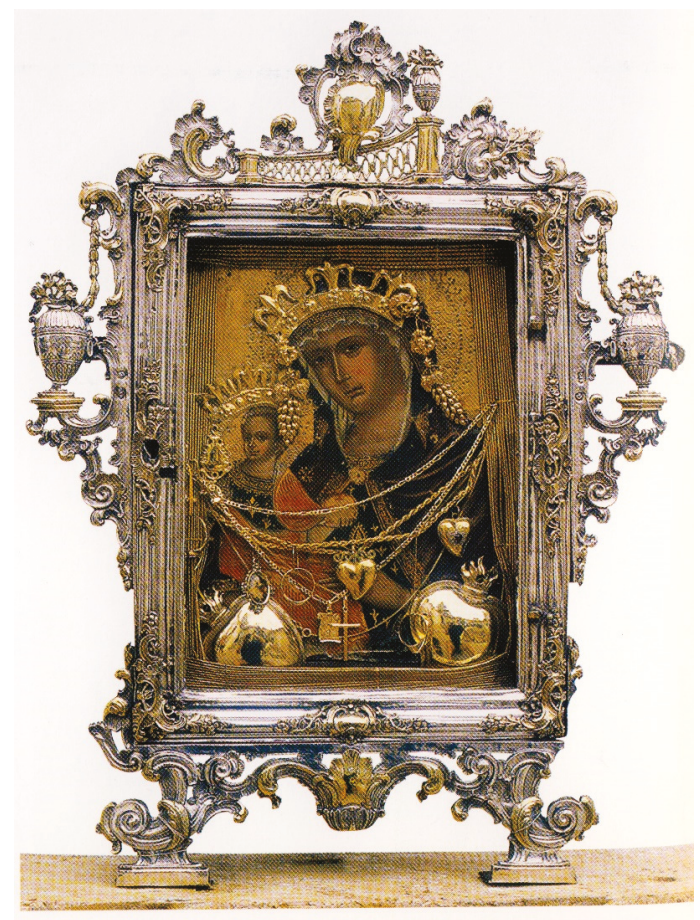

Figure 17. "Our Lady of the Miracle" (Gospa od Čuda), Rab, Benedictine Convent of Saint Andrew (Samostan Benediktinki Svetog Andrije) (taken from Domijan 2007, p. 126). 
In the period of the Counter-Reformation, legends revolving around miracle-working icons were received with more suspicion and often attracted the attention of the Inquisition. Particularly revealing is the trial of two Venetian citizens, which started because of an icon: around 1668, a Greek triptych of the Virgin was donated to the church of Santa Ternita in Venice by a poor woman named Cecilia, widow of a certain Pietro Bevilacqua, after divine revelation (Schutte 1999, pp. 153-55; Schutte 2001; Black 2004 , p. 170). At first, the rector of the church, Francesco Vincenzi, kept the icon at the sacristy, but soon, a local woman named Antonia Pesenti, daughter of a local artisan, informed him "that this image would perform miracles, and at Antonia's persuasion he [Vincenzi] hung it in the church at the altar near the sacristy". The prodigious image shortly started attracting flocks of believers, who expected to be healed from various illnesses. Worshippers of the image were distributed sanctified oil from a lamp that hung in front of the icon and carnations from a vase placed on its altar, whereas reproductions of the icon were sold in the square right outside of the church, along with "relics" from Pesenti herself, now promoted as a living saint. It was not long until the events revolving around the icon of Santa Ternita reached the Inquisitor Agapito Ugoni, who persecuted Vincenzi and Pesenti with the charges of pretense of holiness; in 1669, they were both convicted of heresy. As for the miracle-working icon, it was immediately removed from the church and placed for safekeeping in the patriarchal residence.

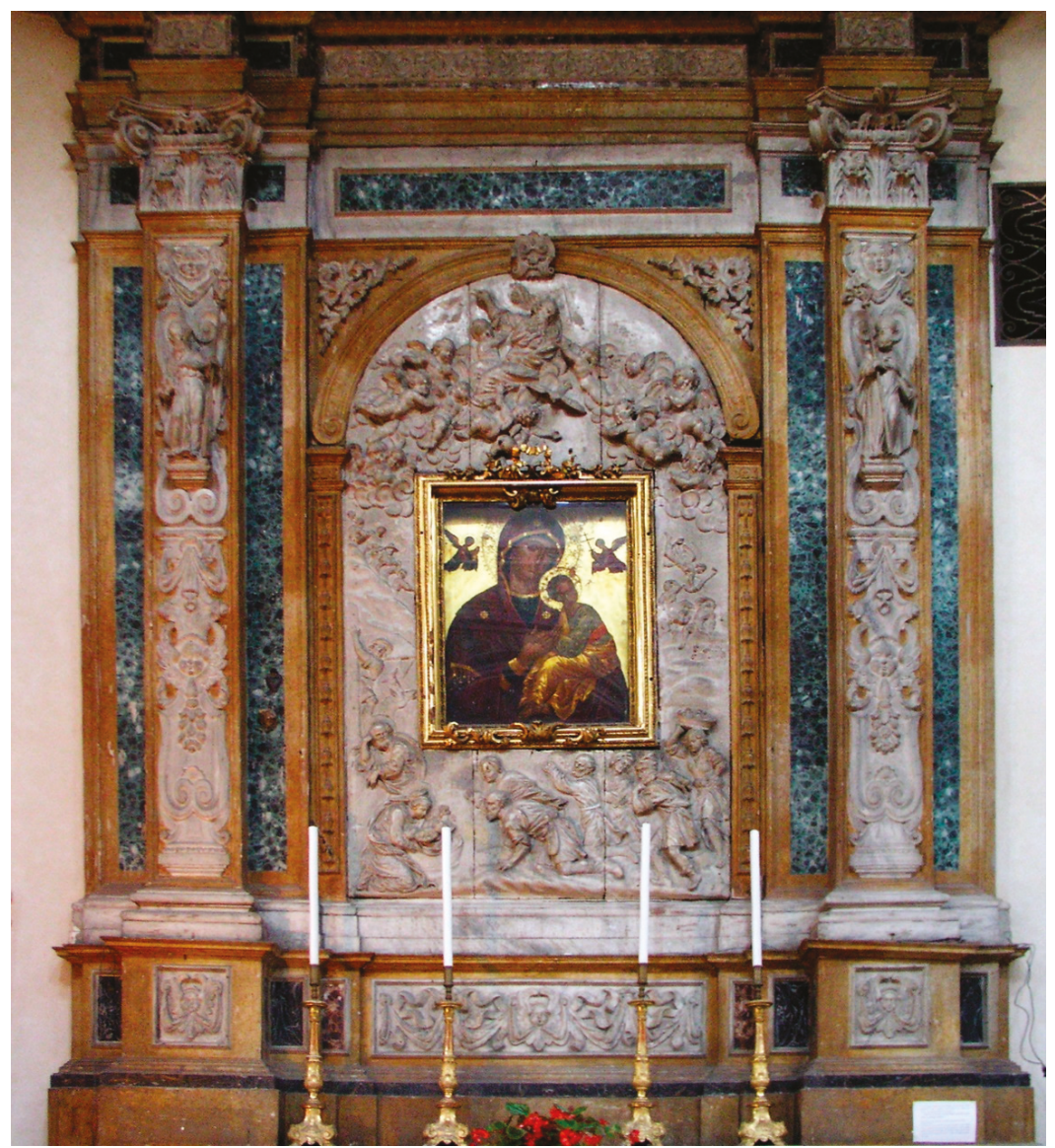

Figure 18. The Madonna of Passion, Ferrara, Church of Santa Maria in Vado (photograph by the author). 


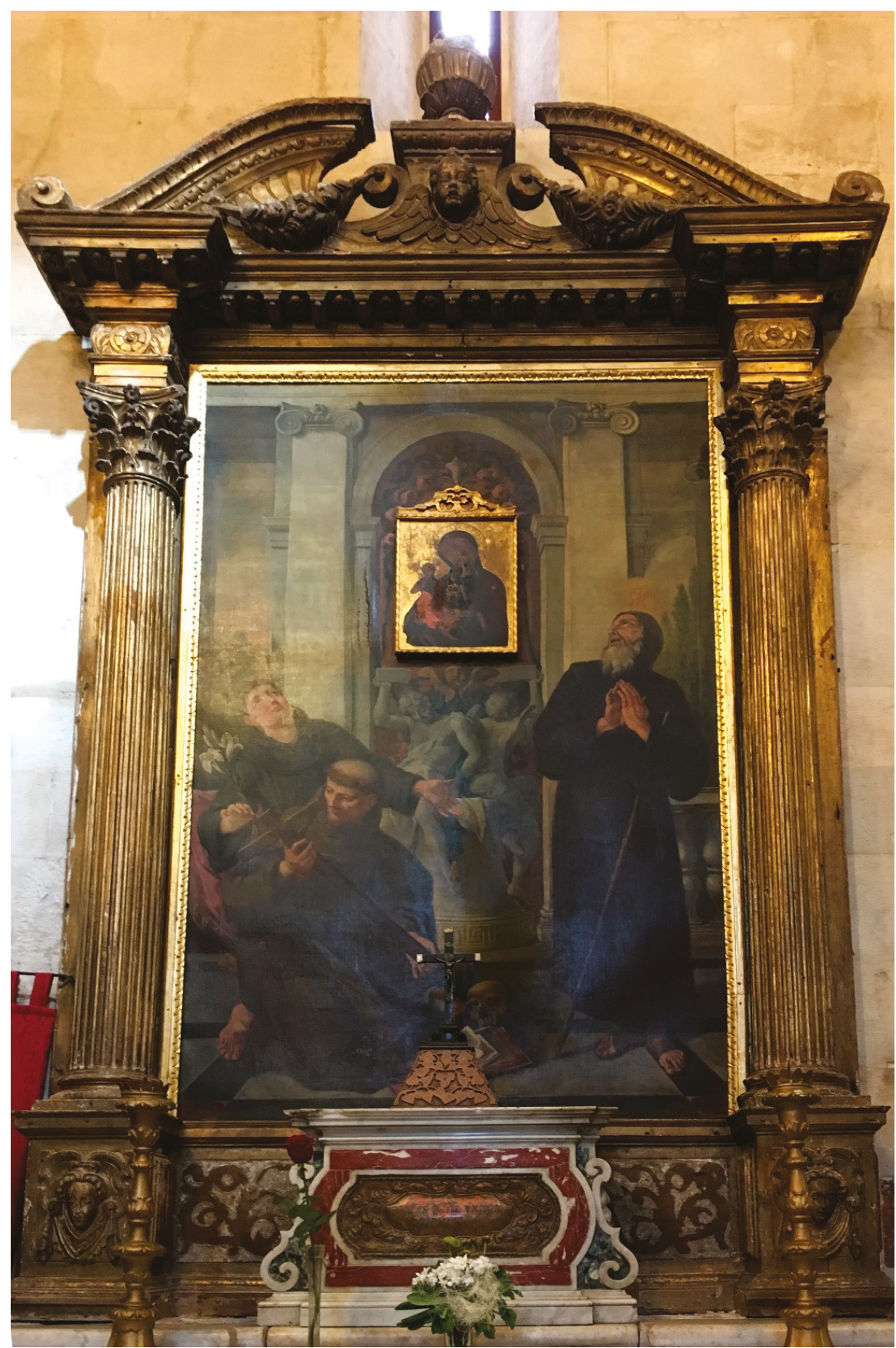

Figure 19. Icon of the Madre della Consolazione encased in an altarpiece by Giovanni Battista Agostino Pitteri, Trogir, Cathedral of Saint Lawerence (Katedrala Svetog Lovre) (photograph by the author). 


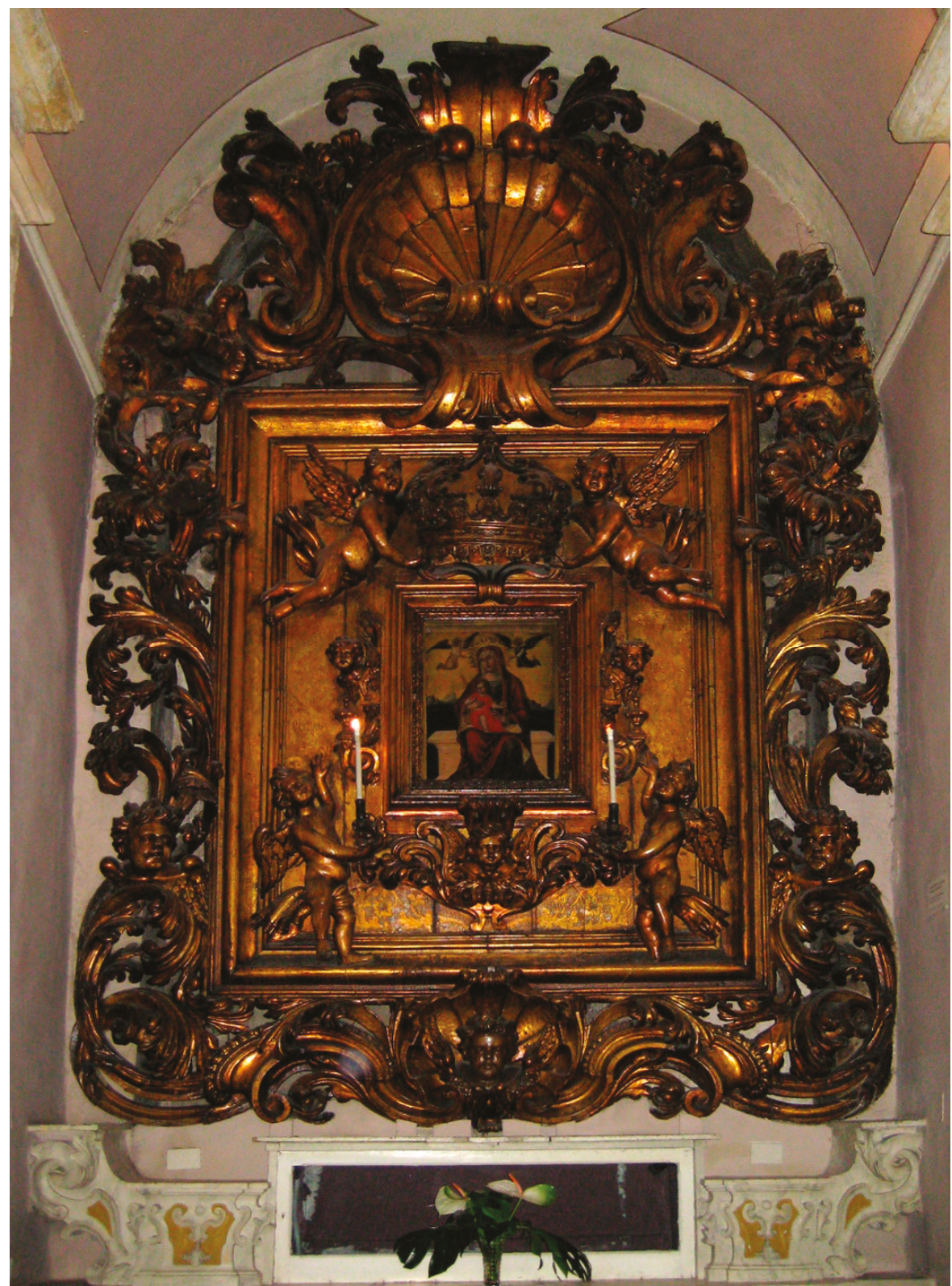

Figure 20. Madonna and Child Enthroned (Madonna di Costantinopoli), Bisceglie, Church of Saint Matthew (Chiesa di San Matteo) (photograph by the author).

The dedication of votive offerings was another channel through which icons of domestic devotion ended up in public collections. Apart from a few icons that gained popularity and became the foci of public cults, church and monastic collections throughout the Adriatic abound with Greek devotional paintings that were donated to religious institutions as petitionary and votive offerings. In the treasury of the convent of Saint Nicholas in Zadar, for example, inventoried by the Venetian painter Giovanni 
Battista Pitteri after the monastery's fusion with that of Saint Mary in 1798, there were nine icons that the artist assessed as "Greek", in all probability deriving from individual legacies:

“B. V. sopra tavola, fatta alla greca e presa dall'italiano di Sassoferrato, con cornice d'intaglio dorata lire 80; Decollazione di s. Giovan Batt. opera greca, sopra tavola, con cornice id. lire 120; S. Niccolò alla greca sopra tavola con cornice dorata lire 90; S. Stefano martire alla greca sopra tavola, con cornice antica dorata lire 50; S. Giorgio a cavallo alla greca id con cornice id lire 50; Sacra Famiglia alla greca id con cornice id. lire 80; Ecce-Homo alla greca id preso dall'italiano con cornice id. lire 80; B. V. col bambino, maniera greca sopra tavola con cornice id. lire 80; S. Spiridione maniera greca id, con cornice dorata d'intaglio lire 110. (Brunelli 1913, p. 393)

In view of the above, there is enough evidence to suggest that the majority of the Greek icons that are now located in Catholic churches and monasteries of both Adriatic coasts originated from bequests and votive offerings and were, initially, in private use. Jacob Burckhardt was the first to suggest that the Madonnas seen in Venetian churches and scuole originated from private houses, and were transferred to the public sphere due to space limitations or in the absence of heirs (Burckhardt 1898, pp. 298-99; cited in Ringbom 1984, p. 36). Apart from archival sources and inscriptions documenting private donations to ecclesiastical institutions, the domestic provenance of icons in churches is implied by their small, sometimes minute dimensions, as well as their subjects, mostly half-length images of the Madonna and Child or other themes commonly associated with private devotion (Figures 18-20). In addition to their size and iconography, the often low cost and inferior artistic quality of icons of public devotion further indicate that they were donated not because of their value and magnificence, but mainly, because of their spiritual properties that were presumably intrinsic to Byzantine and Byzantinizing icons regardless of their aesthetic qualities.

\section{Conclusions}

Serving as devotional accessories, spiritual mediators or measures of social prestige, icons alla greca were omnipresent in Italian and Dalmatian households, regardless of class or confessional orientation. At the same time, with the seal of antique authenticity and claims of possessing miraculous properties, icons of Eastern provenance often became the center of legends and popular cults that extended their veneration from the uttermost privacy of the domestic space to the heart of civic worship. The large variety of icons venerated in Catholic households and churches, ranging from paintings of high artistic value to works of mass production, reveals that their artistic quality had little impact on their consideration as holy and miraculous, and their integration into private and public devotional practices. In his discussion of the reputedly miraculous Madonna of Santa Maria della Fava, Ferdinando Apollonio not only stresses that the icon was made "without study of art and colors", but that all of the images venerated as miraculous in Venice were "artistically speaking, rough pieces of wood cut with an axe [... ], smears rather than paintings". As harsh as his assessment reads, for Apollonio, it was exactly this lack of artistic sophistication that added to the spiritual qualities of these images, and inspired the public's devotion: "the cruder they are, the greater the waves of people that surround them" (Apollonio 1880, pp. 7-8).

Perhaps more telling is the story of Franceschina, mother of the Venetian priest Andrea Ingegneri. In 1435, a merchant from Constantinople brought Franceschina a fragment of Saint Theodosia's shroud, and advised her to make an offering to the saint in order to cure an illness that had almost left her blind. Franceschina followed the man's advice, and thereby made a swift and remarkable recovery. Three years later, the devout woman received from Constantinople a painted icon of Saint Theodosia ("ex byzantio ymago sancte Theodosie depicta aportaretur"); however, her husband refused to accept it, because he believed it to be unsophisticated and overpriced. Instead, he chose to commission another, more beautiful painting, this time from a Venetian artist. The outcome of this decision was that Franceschina's health deteriorated immediately, and did not improve until after the family purchased 
the rejected Byzantine icon for double the initial price (Corner 1749, vol. 2, p. 334; Bacci 2018, p. 282; Giani 2018, p. 70).

The case of Franceschina Ingegneri demonstrates precisely the fundamental difference between religious images painted in the Western style and icons made in the Byzantine tradition, a difference that ultimately explains the survival and popularity of icon painting in the Catholic milieus of the Late Medieval and Early Modern Adriatic. Orthodox icons were never meant to rival contemporary works of Renaissance or Baroque art, but on the contrary, they were intended to cover a completely different set of market needs. As compared to the more naturalistic art of the West, Byzantinizing icons were not destined for the decoration and embellishment of lay households but were rather meant to inspire devotion and piety, responding almost exclusively to the spiritual needs of the faithful. In other words, icon painting, in spite of its humility, but also thanks to it, was able get through to the common people, sometimes even more than the "high" art of the Renaissance and the Baroque, and thus constituted an enduring and indispensable part of everyday religious practices. Thus, even though the maniera greca was banished from the official artistic production, the cult of icons managed to survive at the margins of the Renaissance, and ultimately went through its own renaissance in the Early Modern period.

Funding: This research received no external funding.

Acknowledgments: I owe many thanks to Raffaella Bassi, director of the Fondazione Neri, Museo Italiano della Ghisa, and Giorgio Buda, responsible of the Museo d'Arte Sacra of Longiano for kindly supplying me with a reproduction of the icon of the "Madonna delle Lacrime", as well as with bibliographical documentation on the icon. I also wish to thank Luka Juri, director, and Edvilijo Gardina, chief curator of the Pokrajinski Muzej Koper, who provided me with reproductions of artworks from their collections. Many thanks are due to Živko Bačić and Vivian Grisogono for authorizing me to use photographic material from the icon collections of Hvar. Last but not least, I would like to express my sincere gratitude to Salvador Ryan, editor of the special issue "Domestic Devotions in Medieval and Early Modern Europe" for his kind invitation to contribute to this volume, as well as to the external reviewers and the editorial team of the journal Religions, for their careful reading of the manuscript, and especially for their insightful, valuable and encouraging feedback.

Conflicts of Interest: The author declares no conflict of interest.

\section{References}

\section{Archival Sources}

Državni Arhiv u Dubrovniku (DAD).

\section{Published Primary Sources}

Apollonio, Ferdinando. 1880. Intorno all'Immagine e alla Chiesa di S. Maria della Consolazione al ponte della Fava. Venice: Tipografia dell'Immacolata.

Armenini, Giovanni Battista. 1587. Dé veri precetti della pittura di M. Gio. Battista Armenini da Faenza libri tre ne' quali con bell'ordine d'utili \& buoni avertimenti, per chi desidera in essa farsi con prestezza eccellente, di dimostrano $i$ modi principali del disegnare, $\mathcal{E}$ del dipignere, $\mathcal{E}$ di fare le pitture, che si conuengono alle conditioni de' luoghi, $\mathcal{E}$ delle persone. Ravenna: Francesco Tebaldini.

Armenini, Giovanni Battista. 1977. On the True Precepts of the Art of Painting. Edited and translated by Edward J. Olszewski. New York: B. Franklin. First published 1587.

Borromeo, Federico. 1932. De pictura sacra. Edited by Carlo Castiglioni. Sora: P.C. Camastro.

Cennini, Cennino. 1960. The Craftsman's Handbook (Il libro dell'arte). Edited and translated by Daniel V. Thompson. New Haven: Yale University Press. First published 1933.

Corner, Flaminio. 1749. Ecclesiae venetae antiquis monumentis nunc etiam primum editis illustratae ac in decades distributae. Venice: Typis Jo. Baptistae Pasquali.

Corner, Flaminio. 1761. Notizie storiche delle apparizioni, e delle immagini piu celebri di Maria Vergine santissima nella citta, e dominio di Venezia. Tratte da documenti, tradizioni, ed antichi libri delle Chiese nelle quali esse immagini son venerate. Venice: Antonio Zatta. 
Dominici, Giovanni. 1860. Regola del governo di cura familiare; compilata dal beato Giovanni Dominici, fiorentino. Firenze: A. Garinei.

Ghiberti, Lorenzo. 1998. Lorenzo Ghiberti: I commentarii. Edited by Lorenzo Bartoli. Firenze: Giunti.

Giordano, da Pisa. 1867. Prediche inedite del b. Giordano da Rivalto dell' ordine de' predicatori: Recitate in Firenze dal 1302 al 1305. Edited by Enrico Narducci. Bologna: G. Romagnoli.

Giustiniani, Paolo, and Pietro Quirini. 1773. B. Pauli Iustiniani Et Petri Quirini Eremitarum Camaldulensium Libellus ad Leonem X Pontificem Maximum. Edited by Anselmo Costadoni and Giovanni-Benedetto Mittarelli. In Annales Camaldulenses ordinis Sancti Benedicti, 9. Venice: Aere Monasterii Sancti Michaelis de Muriano, pp. 612-719.

Leonardo, da Vinci. 1961. The Genius of Leonardo da Vinci; Leonardo da Vinci on Art and the Artist. Edited and translated by André Chastel. New York: Orion Press.

Molanus, Joannes. 1570. De picturis et imaginibus sacris liber unus. Lovanii: H. Wellaeus.

Molanus, Johannes. 1996. Traité des saintes images. Edited and translated by François Boespflug, Olivier Christin, and Benoît Tassel. Paris: Cerf.

Paleotti, Gabriele. 1594. De imaginibus sacris et profanis illusstriss. et reverendiss. D.D. Gabrielis Palaeoti cardinalis: Libri quinque. Quibus multiplices earum abusus, iuxta sacrosancti concilii Tridentini decreta, deteguntur. Ac variae cautiones ad omnium generum picturas ex Christiana disciplina restituendas, proponuntur ... nunc primum Latine editi. Ingolstadium: Sartorius.

Sarnelli, Pompeo. 1686. Lettere ecclesiastiche di Pompeo Sarnelli. Naples: Antonio Bulifon.

Schroeder, Henry Joseph. 2011. Canons and decrees of the Council of Trent. St. Louis: Herder Book Co., Reprint Charlotte: TAN Books, trans. 1941.

Vasari, Giorgio. 1550. Le Vite de' più eccellenti architetti, pittori, et scultori italiani, da Cimabue insino a' tempi nostri. Florence: Lorenzo Torrentino.

\section{Secondary Sources}

Abbate, Vincenzo. 2009. Il contesto familiare Mattei-De Torres e una riconsiderazione della copia palermitana dell'Emmaus di Londra. In Da Caravaggio ai Caravaggeschi. Edited by Maurizio Calvesi and Alessandro Zuccari. Rome: CAM Editrice.

Ajmar Wollheim, Marta, and Flora Dennis. 2006. At Home in Renaissance Italy. London: V \& A.

Ajmar Wollheim, Marta, Flora Dennis, and Ann Matchette. 2007. Approaching the Italian Renaissance Interior: Sources, Methodologies, Debates. Malden: Blackwell.

Anderson, Caroline Corisande. 2007. The Material Culture of Domestic Religion in Early Modern Florence, c.1480-c.1650. Unpublished Ph.D. dissertation, University of York, York, UK.

Antetomaso, Ebe. 2007. La collezione di oggetti liturgici del cardinale Bessarione. In Collezioni di antichità a Roma tra '400 e'500. Edited by Anna Cavallaro. Roma: De Luca, pp. 225-32.

Aronberg Lavin, Marylin. 1975. Seventeenth-Century Barberini Documents and Inventories of Art. New York: New York University Press.

Bacci, Michele. 2018. Devotional Panels as Sites of Intercultural Exchange. In Domestic Devotions in Early Modern Italy. Edited by Maya Corry, Marco Faini and Alessia Meneghin. Leiden: Brill, pp. 272-92.

Bagemihl, Rolf. 1993. The Trevisan Collection. Burlington Magazine 135: 559-63.

Barić, Ivo. 2007. Rapska baština. Rijeka: Adamić.

Barocchi, Paola. 1961. Trattati d'arte del Cinquecento, fra Manierismo e Controriforma 2. Bari: Laterza.

Basile Bonsante, Mariella. 2002. Arte e devozione: Episodi di committenza meridionale tra Cinque e Seicento. Galatina: Congedo.

Battista, Giuseppina. 2002. L'educazione dei Figli nella Regola di Giovanni Dominici (1355/6-1419). Florence: Pagnini e Martinelli Editori.

Bellavitis, Giorgio. 1975. Palazzo Giustinian Pesaro. Vicenza: N. Pozza.

Belting, Hans. 1985. Giovanni Bellini, Pietà: Ikone und Bilderzählung in der venezianischen Malerei. Frankfurt am Main: Fischer Taschenbuch Verlag.

Belting, Hans. 1994. Likeness and Presence: A History of the Image before the Era of Art. Chicago: University of Chicago Press.

Bianca, Concetta. 1999. Da Bisanzio a Roma: Studi sul cardinale Bessarione. Rome: Roma nel Rinascimento. 
Bianchi, Ilaria. 2008. La politica delle immagini nell'età della Controriforma. Il cardinale Gabriele Paleotti teorico e committente. Bologna: Compositori.

Bianchini, Geminiano, ed. 1995. Lettera al Papa: Paolo Giustiniani e Pietro Quirini a Leone X. Modena: Poligrafico Artioli.

Black, Christopher F. 2004. Church, Religion, and Society in Early Modern Italy. Basingstoke: Palgrave Macmillan.

Bock, Henning, and Rainald Grosshans. 1996. Gemäldegalerie Berlin: Gesamtverzeichnis. Berlin: Staatliche Museen zu Berlin, Preussischer Kulturbesitz.

Boni, Giacomo. 1887. Santa Maria dei Miracoli in Venezia. Archivio Veneto 33: 236-74.

Brown, Patricia Fortini. 2004. Private Lives in Renaissance Venice: Art, Architecture, and the Family. New Haven: Yale University Press.

Brundin, Abigail, Deborah Howard, and Mary Laven. 2018. The Sacred Home in Renaissance Italy. New York: Oxford University Press.

Brunelli, Vitaliano. 1913. Storia della città di Zara dai tempi più remoti sino al 1815, compilata sulle fonti da Vitaliano Brunelli. Venezia: Istituto Veneto d'Arte Grafiche.

Burckhardt, Jacob. 1898. Beiträge zur Kunstgeschichte von Italien: Das Altarbild, das Porträt in der Malerei, die Sammler. Basel: Verlag von G.F. Lendorff.

Cappelletti, Francesca. 2014. An Eye on the Main Chance: Cardinals, Cardinal-Nephews, and Aristocratic Collectors. In Display of Art in the Roman Palace, 1550-1750. Edited by Gail Feigenbaum and Francesco Freddolini. Los Angeles: The Getty Research Institute, pp. 78-88.

Carr, Annemarie Weyl. 2004. Images: Expressions of faith and power. In Byzantium: Faith and Power (1261-1557). Edited by Helen C. Evans. New York: The Metropolitan Museum of Art, pp. 142-52.

Carlton, Genevieve. 2015. Worldly Consumers: The Demand for Maps in Renaissance Italy. Chicago: University of Chicago Press.

Cattapan, Mario. 1972. Nuovi elenchi e documenti dei pittori in Creta dal 1300 al 1500. Thesaurismata 9: 202-35.

Cavallo, Sandra, and Silvia Evangelisti, eds. 2009. Domestic Institutional Interiors in Early Modern Europe. Farnham: Ashgate.

Cavazzini, Patrizia. 2014. Lesser nobility and other people of means. In Display of Art in the Roman Palace, 1550-1750. Edited by Gail Feigenbaum and Francesco Freddolini. Los Angeles: The Getty Research Institute, pp. 89-102.

Cecchini, Isabella. 2000. Quadri e commercio a Venezia durante il Seicento: Uno studio sul mercato dell'arte. Venezia: Marsilio.

Cecchini, Isabella. 2005. Al servizio dei collezionisti. La professionalizzazione nel commercio di dipinti a Venezia in età moderna e il ruolo delle botteghe. In Il collezionismo a Venezia e nel Veneto ai tempi della Serenissima. Edited by Bernard Aikema, Rosella Lauber and Max Seidel. Venezia: Marsilio, pp. 151-72.

Cecchini, Isabella. 2008a. Collezionismo e cultura materiale. In Il collezionismo d'arte a Venezia. Dalle origini al Cinquecento. Edited by Michel Hochmann, Rosella Lauber and Stefania Mason. Venezia: Marsilio/Fondazione di Venezia, pp. 164-91.

Cecchini, Isabella. 2008b. Material Culture in Sixteenth Century Venice: A Sample from Probate Inventories, 1510-1615. Department of Economics Research Paper Series 14/08; Venice: University Ca' Foscari of Venice.

Chambers, David. 1992. A Renaissance Cardinal and His Worldly Goods: The Will and Inventory of Francesco Gonzaga (1444-1483). London: The Warburg Institute.

Chastel, André. 1988. "Medietas imaginis": Le prestige durable de l'icône en Occident. Cahiers Archéologiques 36: 99-110.

Chatzidakis, Manolis. 1977. La peinture des "Madonneri" ou "vénéto-crétoise" et sa destination. In Venezia, Centro Di Mediazione Tra Oriente e Occidente, secoli XV-XVI: Aspetti e problemi, 2. Firenze: L. S. Olschki, pp. 673-90.

Cherra, Diletta. 2006. Collezionismo e gusto per l'arte bizantina in Italia tra Trecento e Quattrocento. Bollettino della Badia Greca di Grottaferrata 3: 175-204.

Concina, Ennio. 1998. Venezia e l'icona. In Venezia e Creta. Atti des Convegno internazionale di studi, Iraklion-Chanià, 30 settembre-5 ottobre 1997. Edited by Gherardo Ortalli. Venice: Istituto Veneto di Scienze, Lettere ed Arti, pp. 523-42.

Concina, Ennio. 2002. Giorgio Vasari, Francesco Sansovino e la Maniera Greca. In Hadriatica. Attorno a Venezia e al Medioevo. Tra arti, storia e storiografia, Scritti in onore di Wladimiro Dorigo. Edited by Ennio Concina, Giordana Trovabene and Michela Agazzi. Padova: Il Poligrafo, pp. 89-96. 


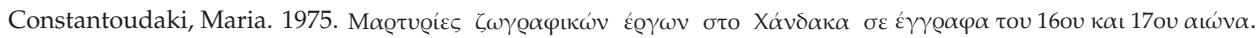
Thesaurismata 12: 35-136.

Constantoudaki, Maria. 2018. Aspects of artistic exchange on Crete: Questions concerning the presence of Venetian painters on the island in the fourteenth and fifteenth centuries. In Cross-Cultural Interaction between Byzantium and the West, 1204-1669: Whose Mediterranean Is It Anyway? Edited by Angeliki Lymberopoulou. New York: Routledge, pp. 30-58.

Corazza, Lucia. 2017. Corazza, Lucia. 2017. Il mercato di quadri nella Venezia del Cinquecento. Master's thesis, Università Ca' Foscari Venezia, Venice, Italy.

Corbo, Anna Maria. 2004. Paolo II Barbo: Dalla mercatura al papato, 1464-1471. Roma: Edilazio.

Corry, Maya, Deborah Howard, and Mary Laven, eds. 2017. Madonnas E Miracles: The Holy Home in Renaissance Italy. London and New York: Philip Wilson Publishers.

Corry, Maya, Marco Faini, and Alessia Meneghin, eds. 2018. Domestic Devotions in Early Modern Italy. Leiden: Brill.

Crouzet Pavan, Elisabeth. 1992. "Sopra le acque salse". Espaces, pouvoir et societé à Venise à la fin du Moyen Âge. Roma: Istituto Storico Italiano per il Medio Evo.

Currie, Elizabeth. 2006. Inside the Renaissance house. London: V \& A.

Cutler, Anthony. 1995. From Loot to Scholarship: Changing Modes in the Italian Response to Byzantine Artifacts, ca. 1200-1750. Dumbarton Oaks Papers 49: 237-67. [CrossRef]

Cutler, Anthony. 1994. Byzantium, Italy \& the North: Papers on Cultural Relations. London: Pindar Press, pp. 190-226.

Cutler, Anthony. 2000. La 'questione bizantina' nella pittura italiana: Una visione alternativa della maniera greca. In La Pittura in Italia: L'Altomedioevo. Edited by Carlo Bertelli. Milan: Electa, pp. 335-54.

Čoralić, Lovorka. 2002. Prilog poznavanju prisutnosti i djelovanja hrvatskih trgovaca u Mlecima (15.-18. stoljeće). Povijesni Prilozi 22: 40-73.

Demori Staničić, Zoraida. 1990. Neki problemi kretsko-venecijanskog slikarstva u Dalmaciji. Prilozi Povijesti Umjetnosti u Dalmaciji 29: 83-112.

Demori Staničić, Zoraida. 2012. Javni kultovi ikona u Dalmaciji. Ph.D. disseration, University of Zagreb, Zagreb, Croatia.

Demori Staničić, Zoraida. 2017. Javni kultovi ikona u Dalmaciji. Split-Zagreb: Književni Krug.

Domijan, Miljenko. 2007. Rab: Grad Umjetnosti. Zagreb: Barbat.

Drandaki, Anastasia. 2013. Icons in the devotional practices of Byzantium. In Heaven E Earth. 1: Art of Byzantium from Greek Collections. Edited by Anastasia Drandaki, Demetra Papanikola Bakirtzi and Anastasia Tourta. Athens: Hellenic Ministry of Culture, pp. 109-14.

Drandaki, Anastasia. 2014. A Maniera Greca. Studies in Iconography 35: 39-72.

Duits, Rembrandt. 2011. Una icona pulcra. The Byzantine icons of Cardinal Pietro Barbo. In Mantova e il Rinascimento italiano: Studi in onore di David S. Chambers. Edited by Philippa Jackson, Guido Rebecchini and David Chambers. Mantova: Sometti, pp. 127-41.

Duits, Rembrandt. 2013. Byzantine icons in the Medici collection. In Byzantine art and Renaissance Europe. Edited by Angeliki Lymberopoulou and Rembrandt Duits. Farnham: Ashgate, pp. 157-88.

Effenberger, Arne. 2004. Images of personal devotion: Miniature mosaic and steatite icons. In Byzantium: Faith and Power (1261-1557). Edited by Helen C. Evans. New York: The Metropolitan Museum of Art, pp. 209-12.

. Farlati, Daniele. 1769. Illyricum Sacrum. IV. Venice: Apud Sebastianum Coleti.

Fazinić, Alena. 1980. Pet stoljeća dominikanske prisutnosti na Korčuli (1480-1980). Croatica Christiana Periodica 4: 83-86.

Fazinić, Alena. 2009. Korčulanska spomenička i kulturna baština. Korčula: Ogranak Matice Hrvatske.

Fedalto, Giorgio. 1967. Ricerche storiche sulla posizione giuridica ed ecclesiastica dei Greci a Venezia nei secoli XV e XVI. Firenze: Olschki.

Feigenbaum, Gail, and Francesco Freddolini. 2014. Display of Art in the Roman Palace, 1550-1750. Los Angeles: The Getty Research Institute.

Fillini, Matteo, and Luigi Tomaz. 1988. Le chiese minori di Cherso. Quaderni della Comunità Chersina 7. Vicenza: Tipografia Regionale Veneta Editrice.

Fisković, Cvito. 1959. Slikar Angelo Bizamano u Dubrovniku. Prilozi Povijesti Umjetnosti u Dalmaciji 11: 72-90.

Folda, Jaroslav. 2015. Byzantine Art and Italian Panel Painting: The Virgin and Child Hodegetria and the Art of Chrysography. New York: Cambridge University Press. 
Freedberg, David. 1971. Johannes Molanus on Provocative Paintings. De Historia Sanctarum Imaginum et Picturarum. Journal of the Warburg and Courtauld Institutes 34: 229-45. [CrossRef]

Frigerio Zeniou, Stella, and Miroslav Lazović. 2006. Icônes de la collection du Musée d'Art et d'Histoire. Genève: Musées d'Art et d'Histoire.

Furlan, Italo. 1973. Le icone bizantine a mosaico. Milano: Stendhal.

Fusco, Laurie, and Gino Corti. 2006. Lorenzo de' Medici, Collector and Antiquarian. Cambridge: Cambridge University Press.

Gamulin, Grgo. 1984. Dva djela Antonija Vivarinija u Hrvatskoj. Peristil 27-28: 147-50.

Gavitt, Philip. 1990. Charity and Children in Renaissance Florence: The Ospedale degli Innocenti, 1410-1536. Ann Arbor: University of Michigan Press.

Geanakoplos, Deno J. 1955. The Council of Florence (1438-1439) and the Problem of Union between the Greek and Latin Churches. Church History 24: 324-46. [CrossRef]

Giani, Marina. 2018. Una santa orientale a Venezia. La «Passio» di Teodosia di Cesarea. Bisanzio e l'Occidente 1: 67-83.

Goffen, Rona. 1975. Icon and Vision: Giovanni Bellini's Half-Length Madonnas. The Art Bulletin 57: 487-518. [CrossRef]

Goja, Bojan. 2014. Novi prilozi o baroknom slikarstvu u Zadru. Radovi Instituta za Povijest Umjetnosti 38: 133-50.

Golden, Andrea. 2004. Creating and Recreating: The Practice of Replication in the Workshop of Giovanni Bellini. In Giovanni Bellini and the Art of Devotion. Edited by Ronda Kasl. Indianapolis: Indianapolis Museum of Art, pp. 91-127.

Grubb, James S. 2000. House and Household: Evidence from Family Memoirs. In Edilizia privata nella Verona rinascimentale. Edited by Edoardo Demo, Paola Marini, Paola Lanaro and Gian Maria Varanini. Milano: Electa, pp. 118-33.

Grubb, James S. 2004. Piero Amadi Acts Like His Betters: "Original Citizenship" in Venice. In A Renaissance of Conflicts: Visions and Revisions of Law and Society in Italy and Spain. Edited by John A. Marino and Thomas Kuehn. Toronto: Center for Medieval and Renaissance Studies, pp. 259-78.

Heinemann, Fritz. 1963. Giovanni Bellini e i Belliniani. Venezia: Neri Pozza.

Henry, Chriscinda. 2011. What makes a picture?: Evidence from sixteenth-century Venetian property inventories. Journal of the History of Collections 23: 253-65. [CrossRef]

Hochmann, Michel. 2005. Quelques réflexions sur les collections de peinture à Venise dans la première moitié du XVIe siècle. In Il collezionismo a Venezia e nel Veneto ai tempi della Serenissima. Edited by Bernard Aikema, Rosella Lauber and Max Seidel. Venezia: Marsilio, pp. 117-34.

Janeković, Zdenka. 1996. 'Pro anima mea et predecessorum meorum.' The Death and the Family in 15th century Dubrovnik. Otium 3/1-2 / Medium Aevum Quotidianum 35: 25-34.

Jestaz, Bertrand. 2001. Les collections de peinture à Venise au XVIe siècle. In Geografia del collezionismo: Italia e Francia tra il XVI e il XVIII secolo: Atti delle giornate di studio dedicate a Giuliano Briganti: (Roma 19-21 settembre 1996). Edited by Olivier Bonfait, Michel Hochmann, Luigi Spezzaferro and Bruno Toscano. Roma: École française de Rome, pp. 185-201.

Jones, Pamela M. 1997. Federico Borromeo e l'Ambrosiana: Arte e riforma cattolica nel XVII secolo a Milano. Milano: Vita e Pensiero.

Jones, Pamela M. 2004. Italian Devotional Paintings and Flemish Landscapes in the 'Quadrerie' of Cardinals Giustiniani, Borromeo, and Del Monte. Storia dell'Arte 107: 81-104.

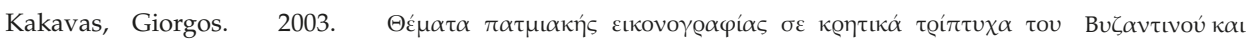

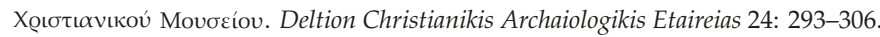

Karbić, Marija, and Zoran Ladić. 2001. Oporuke stanovnika grada Trogira u arhivu HAZU. Radovi Zavoda za Povijesne Znanosti HAZU u Zadru 43: 161-254.

Kasl, Ronda. 2004. Holy households: Art and devotion in Renaissance Venice. In Giovanni Bellini and the Art of Devotion. Edited by Ronda Kasl. Indianapolis: Indianapolis Museum of Art, pp. 58-89.

Katušić, Maja, and Ivan Majnarić. 2011. Dopo la morte dell quondam signor Giouanni Casio dotor ... Inventar ninskog plemića Ivana Kašića s kraja 17. stoljeća. Zbornik Odsjeka za povijesne znanosti Zavoda za povijesne $i$ društvene znanosti Hrvatske akademije znanosti i umjetnosti 29: 219-71.

Kolpacoff Deane, Jennifer. 2013. Medieval Domestic Devotion. History Compass 11: 65-76. [CrossRef]

Kovač, Karl. 1917. Nikolaus Ragusinus und seine Zeit. Jahrbuch Des Kunsthistorischen Institutes 11: 5-94. 
Ladić, Zoran. 2003. Legati kasnosrednjovjekovnih dalmatinskih oporučitelja kao izvor za proučavanje nekih vidova svakodnevnog života i materijalne kulture. Zbornik Odsjeka za Povijesne Znanosti Zavoda za Povijesne $i$ Društvene Znanosti Hrvatske Akademije Znanosti i Umjetnosti 21: 1-28.

Ladić, Zoran. 2012. Last Will: Passport to Heaven. Urban Last Wills from Late Medieval Dalmatia with Special Attention to the Legacies Pro Remedio Animae and Ad Pias Causas. Zagreb: Srednja Europa.

Lamers, Han. 2015. Greece Reinvented: Transformations of Byzantine Hellenism in Renaissance Italy. Leiden: Brill.

Levi, Cesare Augusto. 1900. Le collezioni veneziane d'arte e d'antichità dal secolo XIV. ai nostri giorni. Venice: F. Ongania.

Lydecker, John Kent. 1987. The Domestic Setting of the Arts in Renaissance Florence. Unpublished Ph.D. disseration, The Johns Hopkins University, Baltimore, MA, USA.

Lymberopoulou, Angeliki. 2007. Audiences and markets for Cretan icons. In Viewing Renaissance Art. Edited by Kim Woods, Carol M. Richardson and Angeliki Lymberopoulou. New Haven: Yale University Press, pp. 171-206.

Maginnis, Hayden B. J. 1994. Duccio's Rucellai Madonna and the Origins of Florentine Painting. Gazette des Beaux-Arts 123: 147-64.

Maginnis, Hayden B. J. 2001. Images, devotion, and the Beata Umiliana de' Cerchi. In Visions of Holiness: Art and Devotion in Renaissance Italy. Edited by Andrew Ladis, Shelley Zuraw and H. W. van Os. Athens: Georgia Museum of Art, University of Georgia, pp. 13-20.

Mattox, Philip. 2006. Domestic Sacral Space in the Florentine Renaissance Palace. Renaissance Studies 20: 658-73. [CrossRef]

Menna, Maria Raffaella. 1998. Bisanzio e l'ambiente umanistico a Firenze. Rivista dell'Istituto Nazionale d'Archeologia e Storia dell'Arte 3: 111-58.

Menna, Maria Raffaella. 2015. Sulla disposizione delle icone bizantine nella collezione del cardinale Pietro Barbo. In Curiosa itinera: Scritti Daniela Gallavotti Cavallero. Edited by Enrico Parlato. Roma: Ginevra Bentivoglio Editori, pp. 101-11.

Merriam, Susan. 2009. The reception of garland pictures in seventeenth-century Flanders and Italy. In Domestic Institutional Interiors in Early Modern Europe. Edited by Sandra Cavallo and Silvia Evangelisti. Farnham: Ashgate, pp. 201-20.

Molmenti, Pompeo G. 1928. La storia di Venezia nella vita privata dalle origini alla caduta della repubblica, 2. Bergamo: Istituto Italiano d'Arti Grafiche, Firsted published 1880.

Morini, Enrico. 1989. La Chiesa Greca ed i rapporti 'in sacris' con i Latini al tempo del concilio di Ferrara-Firenze. Annuarium Historiae Conciliorum 21: 267-96.

Morse, Margaret Ann. 2006. The Arts of Domestic Devotion in Renaissance Italy: The Case of Venice. Unpublished Ph.D. dissertation, University of Maryland, College Park, MD, USA.

Morse, Margaret Ann. 2007. Creating sacred space: The religious visual culture of the Renaissance Venetian casa. Renaissance Studies 21: 151-84. [CrossRef]

Morse, Margaret Ann. 2013. The Venetian Portego: Family Piety and Public Prestige. In The Early Modern Italian Domestic Interior, 1400-1700. Edited by Erin J. Campbell, Stephanie R. Miller and Elizabeth Carroll Consavari. Farnham: Ashgate Publishing.

Müntz, Eugène. 1879. Les arts à la cour des papes pendant le XVe et le XVe siècle: Recueil de documents inédits tirés des archives et des bibliothèques romaines, 2. Paris: Ernst Thorin.

Müntz, Eugène, and Arthur L. Frothingham. 1883. Il Tesoro della basilica di S. Pietro in Vaticano dal XIII al XV secolo con una scelta d'inventarii inediti. Roma: Società Romana di Storia Patria.

Nagel, Alexander, and Christopher S. Wood. 2010. Anachronic Renaissance. New York: Zone Books.

Nagel, Alexander. 2011. The Controversy of Renaissance Art. Chicago: The University of Chicago Press.

Nicol, Donald MacGillivray. 1988. Byzantium and Venice: A Study in Diplomatic and Cultural Relations. Cambridge: Cambridge University Press.

Noreen, Kirstin. 2005. The Icon of Santa Maria Maggiore, Rome: An Image and Its Afterlife. Renaissance Studies 19: 660-72. [CrossRef]

Palumbo Fossati, Isabella. 1984. L'interno della casa dell'artigiano e dell'artista nella Venezia del Cinquecento. Studi Veneziani 8: 109-53.

Palumbo Fossati, Isabella. 2004. La casa veneziana. In Da Bellini a Veronese: Temi di arte veneta. Edited by Gennaro Toscano and Francesco Valcanover. Venice: Istituto Veneto di Scienze, Lettere ed Arti, pp. 443-92. 
Panagiotakes, Nikolaos. 2009. El Greco: The Cretan years. Farnham: Ashgate.

Pasi, Silvia. 1986. Icone tardo- e postbizantine in Romagna. Felix Ravenna 131-32: 99-162.

Pasini, Pier Giorgio. 2000. Il Museo di Stato della Repubblica di San Marino. Milano: F. Motta.

Pastega, Giuseppe. 2015. Gli Annali Guarnieri-Bocchi (1745-1848): Un secolo di cronaca e storia adriese. Adria: Apogeo Editore.

Pedone, Silvia. 2005. L'icona di Cristo di Santa Maria in Campitelli: Un esempio di «musaico parvissimo». Rivista dell'Istituto Nazionale di Archeologia e Storia dell'Arte 60: 95-131.

Piatnitsky, Yuri. 1993. Из коллекций Н. П. Лихачев $a$. Каталог выст $a$ вки вГосударственном Русском музее. Saint Petersburg: ИздательствоСеда-с.

Da Portogruaro, Davide. 1930. Il tempio e il convento del Redentore. Rivista mensile della città di Venezia 4-5: 141-224.

Prodi, Paolo. 1973. The Structure and Organization of the Church in Renaissance Venice: Suggestions for Research. In Renaissance Venice. Edited by John Rigby Hale. London: Faber \& Faber, pp. 403-30.

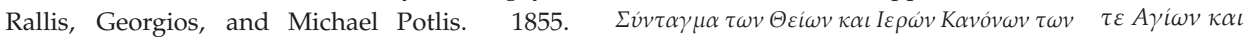

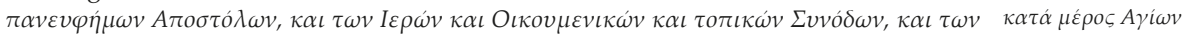
$\Pi \alpha \tau \dot{\varepsilon} \rho \omega v$,

Ricci, Corrado. 1901. La pittura a San Marino. Rassegna d'Arte 1: 129-32.

Ringbom, Sixten. 1984. Icon to Narrative. The Rise of the Dramatic Close-Up Fifteenth-Century Devotional Painting. Åbo: Åbo Akademi. First published 1965.

Rizzi, Alberto. 1972. Le icone bizantine e post-bizantine delle chiese veneziane. Thesaurismata 9: 250-91.

Romano, Dennis. 1993. Aspects of patronage in fifteenth-and sixteenth-century Venice. Renaissance Quarterly 46: 712-33. [CrossRef]

Roncalli, Angelo Giuseppe. 1945. Gli atti della Visita apostolica di S. Carlo Borromeo a Bergamo nel 1575. Vol. 2: La Diocesi. Parte 2. Fontes Ambrosiani 16. Firenze: Olschki.

Sartori, Antonio. 1989. Archivio Sartori: Documenti di storia e arte francescana. Vol. 4, Guida della basilica del Santo, varie, artisti e musici al Santo e nel Veneto. Padova: Biblioteca Antoniana, Basilica del Santo.

Savini Branca, Simona. 1964. Il collezionismo veneziano nel '600. Padova: CEDAM.

Schmidt, Victor M. 2005. Painted Piety: Panel Paintings for Personal Devotion in Tuscany, 1250-1400. Firenze: Centro Di.

Schmitter, Monika Anne. 1997. The Display of Distinction: Art Collecting and Social Status in Early Sixteenth-Century Venice. Unpublished Ph.D. dissertation, University of Michigan, Ann Arbor, MI, USA.

Schmitter, Monika Anne. 2011. The Quadro da Portego in Sixteenth-Century Venetian Art. Renaissance Quarterly 64: 693-751. [CrossRef]

Schutte, Anne Jacobson. 1999. Little women, great heroines: Simulated and genuine female holiness in early modern Italy. In Women and Faith: Catholic Religious Life in Italy from Late Antiquity to the Present. Edited by Lucetta Scaraffia, Gabriella Zarri and Keith Botsford. Cambridge: Harvard University Press, pp. 144-58.

Schutte, Anne Jacobson. 2001. Aspiring Saints: Pretense of Holiness, Inquisition, and Gender in the Republic of Venice, 1618-1750. Baltimore: Johns Hopkins University Press.

Sivrić, Marijan. 2002. Oporuke Kancelarije stonskog kneza od sredine 15. stoljeća do 1808. godine: Analitički inventar oporuka i abecedno kazalo oporučitelja. Dubrovnik: Državni Arhiv.

Spallanzani, Marco. 1996. Inventari Medicei, 1417-1465 Giovanni di Bicci, Cosimo e Lorenzo di Giovanni, Piero di Cosimo. Florence: Associazione 'Amici del Bargello'.

Spallanzani, Marco, Giovanna Gaeta Bertelà, and Simone Di Stagio dalle Pozze. 1992. Libro d'inventario dei beni di Lorenzo il Magnifico. Florence: Associazione Amici del Bargello.

Tadić, Jorjo. 1952. Građa o slikarskoj škole u Dubrovniku XIII-XVI v. Beograd: Naučna knjiga.

Tassini, Giuseppe. 1891. Il Palazzo Gussoni. Nuovo Archivio Veneto Pubblicazione Periodica della R. Deputazione di Storia Patria 1: 433-38.

Tassini, Giuseppe. 1915. Curiosità veneziane. Venice: Giusto Fuga.

Tomasović, Marinko. 2013. Mijat Sabljar (1790.-1865.) i Makarsko primorje. Makarska: Gradski Muzej Makarska.

Tosini, Patrizia, ed. 2009. Arte e committenza nel Lazio nell'età di Cesare Baronio: Atti del Convegno internazionale di studi, Frosinone (Sora, 16-18 maggio 2007). Roma: Gangemi.

Trexler, Richard C. 1980. Public Life in Renaissance Florence. New York: Academic Press. 
Turchi, Giovanni. 1829. Memorie istoriche di Longiano. Cesena: C. Bisazia.

Vassilaki, Maria. 2008. Icons. In The Oxford Handbook of Byzantine Studies. Edited by Elizabeth Jeffreys, John F. Haldon and Robin Cormack. Oxford: Oxford University Press, pp. 758-69.

Vlassi, Despina Er. 2008. Le richezze delle donne. Pratica testamentaria in seno alle famiglie greche di Venezia (XVI-XVIII sec.). In Oltre la morte. Testamenti di Greci e Veneziani redatti a Venezia o in territorio greco-veneziano nei sec. XIV-XVIII. Edited by Chryssa Maltezou and Gogo Varzelioti. Venice: Istituto Ellenico di Studi Bizantini e Postbizantini di Venezia, pp. 83-117.

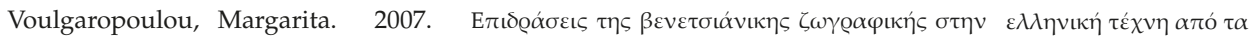

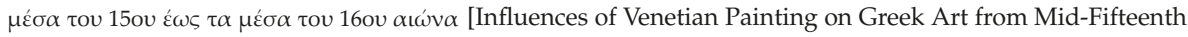
to Mid-Sixteenth Century]. Master's thesis, Aristotle University of Thessaloniki, Thessaloniki, Greece.

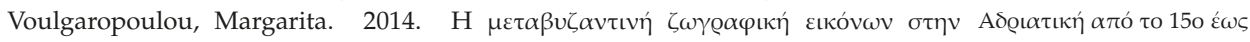

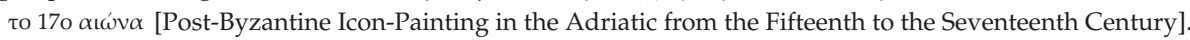
Unpublished Ph.D. dissertation, Aristotle University of Thessaloniki, Thessaloniki, Greece.

Voulgaropoulou, Margarita. Forthcoming. Transcending Borders, Transforming Identities: Travelling Icons and Icon Painters in the Adriatic Region (14th-19th centuries). In rebus, 10th Anniversary Issue, "Mobility, Movement and Medium: Crossing Borders in Art". Essex: Re·bus.

Walberg, Helen Deborah. 2004. "Una compiuta galleria di pitture veneziane": The Church of S. Maria Maggiore in Venice. Studi Veneziani 48: 259-305.

Walsham, Alexandra. 2014. Holy Families: The Spiritualization of the Early Modern Household Revisited. Studies in Church History 50: 122-60. [CrossRef]

Webb, Diana. 2005. Domestic Space and Devotion in the Middle Ages. In Defining the Holy: Sacred Space in Medieval and Early Modern Europe. Edited by Sarah Hamilton and Andrew Spicer. London: Routledge, pp. $27-47$.

Živković, Valentina. 2016. The Deathbed Experience-Icons as Mental Images Preparations for a Good Death in Late Medieval Kotor (Montenegro). Ikon 9: 221-30. [CrossRef]

Zuccari, Alessandro. 1984. Arte e committenza nella Roma di Caravaggio. Torino: ERI, Edizioni Rai.

(C) 2019 by the author. Licensee MDPI, Basel, Switzerland. This article is an open access article distributed under the terms and conditions of the Creative Commons Attribution (CC BY) license (http://creativecommons.org/licenses/by/4.0/). 
Article

\title{
Crux Christi Sit Mecum: Devotion to the Apotropaic Cross
}

\author{
Kaja Merete Haug Hagen \\ The Faculty of Theology, University of Oslo, P.O. Box 1023 Blindern, NO-0315 Oslo, Norway; \\ kajamh@icloud.com
}

Received: 27 September 2019; Accepted: 24 October 2019; Published: 30 October 2019

\begin{abstract}
A late medieval paper amulet containing prayers to St. Dorothy and the Holy Cross was found in a demolished part of a medieval wooden stave church in Torpo, Norway. This article examines the content and the function of this textual amulet by placing it in a wider Scandinavian and Western European context. From the perspective of materiality and sensory-based religious practices, this article will explore the connection between the textual amulet found in Torpo and its relation to the now-lost large wooden cross in Torpo church, and to crosses believed to be wonderworking or miraculous in its proximity. By doing so, this study will shed light on the apotropaic and healing potential that the material and immaterial cross offered the pious in late medieval Norway. The last part of this article addresses the Post-Reformation theological understanding of the amulet, and its use and function in Lutheran Norwegian society.
\end{abstract}

Keywords: material devotion; holy matter; hagiosensorium; haptic devotion; amulets; devotional literature; the holy cross; wonderworking and miraculous images; apotropaic potential; reformation

\section{Introduction}

At the end of the nineteenth century, a late medieval textual amulet was found in the wooden church at Torpo in Norway. As many other textual amulets from the medieval Latin West, the prayer written on the Torpo amulet was addressed to the Holy Cross. How are we to understand this prayer? What was its function, and why has it, at some point, been placed inside the church under the floor boards?

The protective powers of the cross could be invoked through a plethora of media in medieval culture, not only by the use of amulets but also, for example, through prayer books, benedictions, in liturgy, as well as through encounters with crosses present in churches. The aim of this article is to understand more fully the potential embodied in the cross in late medieval culture through an intertextual and intermedial examination.

In this article, I will begin by examining the formal and textual properties of the amulet, before placing the Torpo amulet in a wider Scandinavian and European context. A comparative and contextual analysis will help us identify the lacunae of the text and contribute to a fuller understanding of the amulet's content, as well as of religious beliefs and amuletic practices in its original late medieval context. The next part of the article will explore the relation between the cross addressed in the amulet and the now-missing cross in the church of Torpo, as well as miraculous crosses in Torpo's proximity. Lastly, light will be shed on continuity and change regarding amuletic practices, wonderworking crosses and religious beliefs in post-Reformation society. 


\section{A Prayer Beneath the Wooden Floor Boards}

Torpo is situated in the mountainous part of southern Norway, where the valley of Hallingdal offers a passage between the eastern and western part of the country. In 1880, the old stave church at Torpo was to be demolished and replaced by a new church. The destruction of the stave church had already commenced but was interrupted as The Society for the Preservation of Norwegian Ancient Monuments intervened and bought the remaining part of the stave church. By that time, the chancel had already been torn down, but the nave was rescued (Christie and Christie 1981, p. 118) (Figure 1).

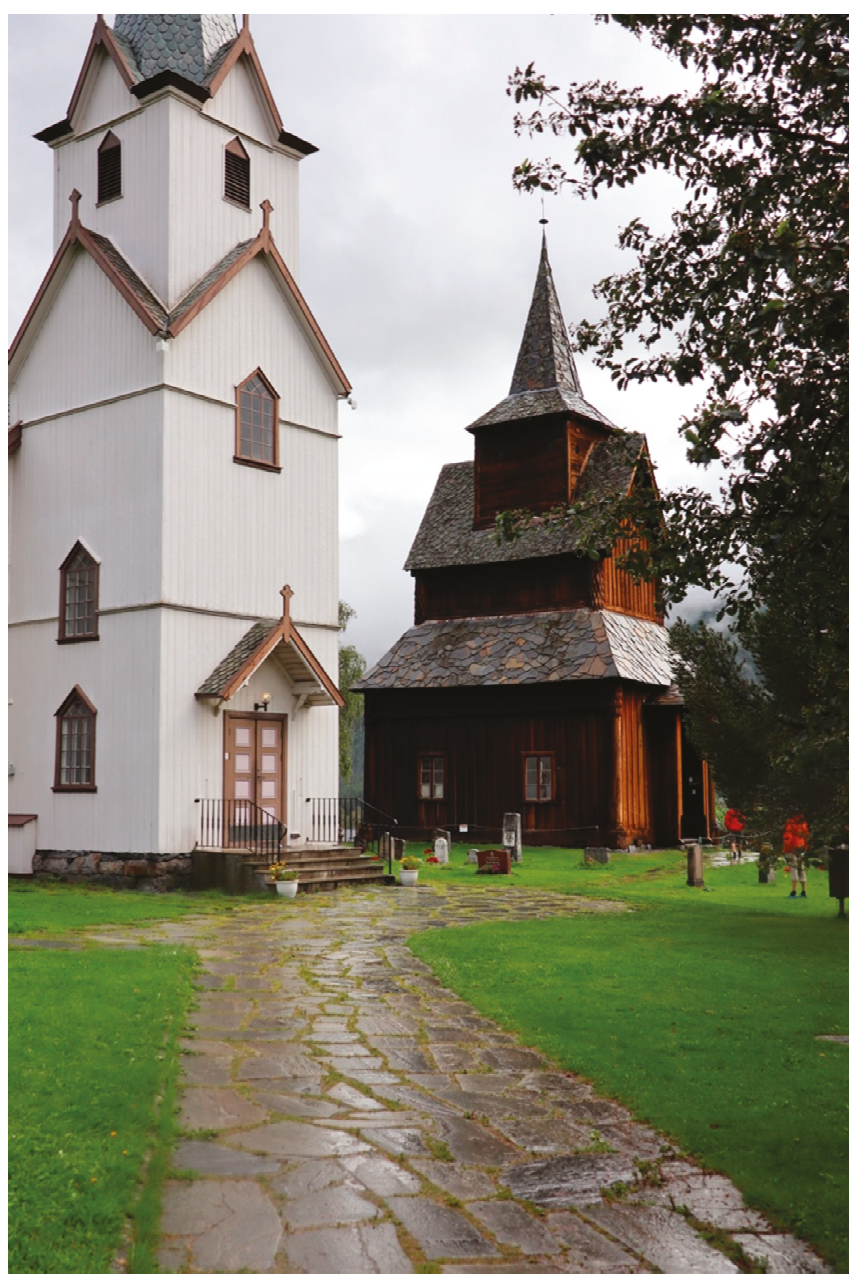

Figure 1. The wooden church of Torpo. Photo: Kaja M. H. Hagen.

During the demolition of the chancel, an old piece of paper, dated 1450-1500, was found. While the sources do not say exactly where in the chancel the paper was found, maybe it was placed beneath 
the wooden floorboards. ${ }^{1}$ On one side is drawn a sigillum Salomonis or Salomon's knot, a cross-shaped ornament with interlaced cross arms (Figure 2). The other side contains prayers in Latin (Figure 3):

[...] virtuosa Dorathea vernans rosa mundi vita patens [ ... ] pro nobis speciose interuentrix graciosa ora pro nobis beata [ ... ] ot digni efficiamur gracia Christi omnipotens sempiterne [ ... ] [beate] Dorathee virg(i)nis tue tantam gloriam contulisti [ ... ] ssum vt eius intersseteptibus et meritis (ad) misericordiam peruenir [ . . ] domini nostri amen

Crux Christi sit mecum et ecclesia nostra crux Christi est enim quam [ . . ] adoro crux Christi est vita salus crux Christi superat gladium crux Christi [ ... ] nt vincula mortis crux Christi admirabile signum crux Christi sit mihi [ . . . ] crux Christi sit mihi via virtutum cuper cruciem Christi aggrediar omne [ . . ] ] crux Christi expandit omne bonum crux Christi aufferat a me omne [ . . ] l[ . . ]um crux Christi saluet me se et hodie super me ante me post [ . . . ] ante me et post me et vbique me viderit antiquus hosttis [ ... ] [fu]giat a me jn nomine domini nostri Iesv Christi benedicat me Ihesus [ ... ] [sem-] piternus rex Judeorum [ ... ] per medium illorum ibat [ . . . ] s Ihesus Christus crucifixus et filius dei viui benedicat me erigat. [ ... ] studiat Christus defendat me et dominus auertat a me omne [... ]um jn nomine patris et filii et spiritus sanctus amen. ${ }^{2}$

(Diplomatarium Norvegicum, 12, no. 266)

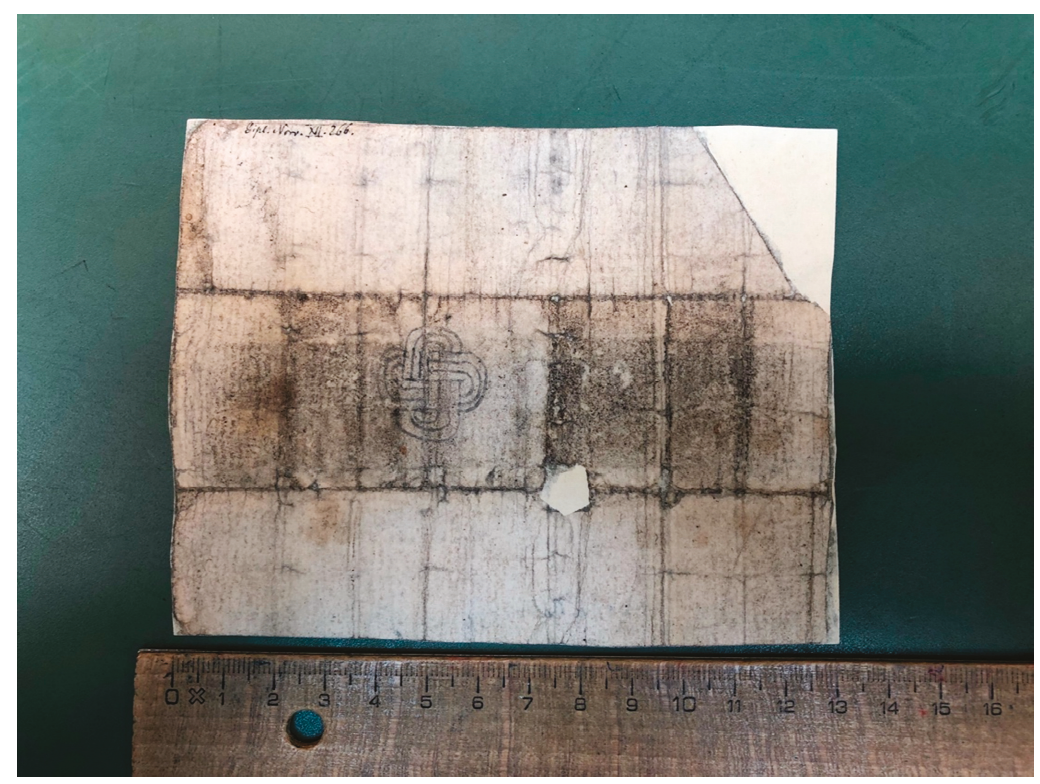

Figure 2. The amulet from Torpo, sigillum Salomonis or Salomon's knot. Ink on paper, 1450-1500. Photo: Kaja M. H. Hagen.

1 This suggestion is based on comparisons to other medieval amulets and a manuscript that, as we shall see later in the article, have been found under the floor boards in Norwegian churches.

2 I rely on the transcribed version in the Diplomatarium Norvegicum 12, no. 266.In the Diplomatarium Norvegicum 12 , no. 266, lacunae are marked by a varying number of dots, and conjectures are marked with [ ... ]. In this article, illegible text is marked with [ ... ], the conjectures suggested by the Diplomatarium are marked with $\{\ldots\}$, and conjectures suggested by Vemund Blomkvist and the author are marked with ( ... ). Conjectures are filled out based on Latin grammar and comparative material. 


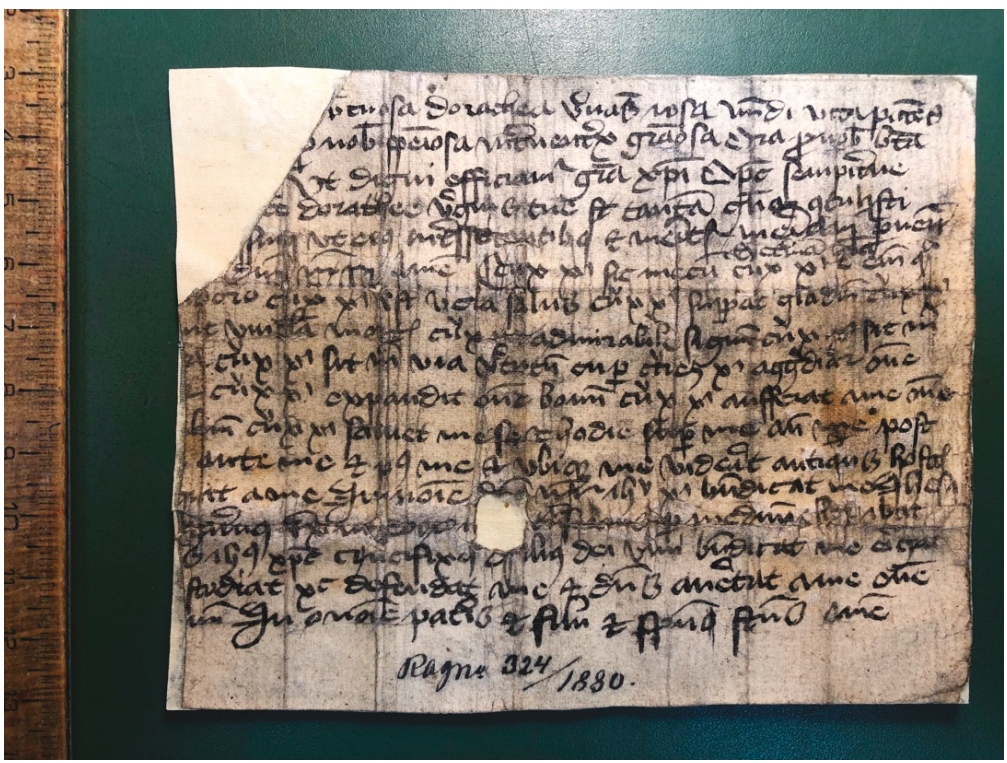

Figure 3. The amulet from Torpo, Latin prayers. Ink on paper, 1450-1500. Photo: Kaja M. H. Hagen.

Translated to English, ${ }^{3}$ the text on the Torpo prayer reads:

(Hail) virtuous (jewel), Dorothea, fresh open rose of pure life $[\ldots] !^{4}$ May you be a speaker for us, beautiful mediator full of grace. Pray for us, you blessed one [ ... ], that we may be made worthy of the grace of Christ. $\mathrm{O}$, Almighty and Eternal, you gave [ ... ] such a great honour to your virgin Dorothea; that we through her intercessions and merits may attain mercy [ ... ] of our Lord. Amen.

May the Cross of Christ be with me and our Church, for the Cross of Christ is that which I (always) honour. The cross of Christ is life salvation. ${ }^{5}$ The Cross of Christ is stronger than the sword. The Cross of Christ is stronger than the chains of death. The Cross of Christ is a wondrous sign. May the Cross of Christ be for me [... ]. May the Cross of Christ be a way to virtues. With the Cross of Christ, I will face every [ ... ]. ${ }^{6}$ The Cross of Christ bestows upon me all good things. May the Cross of Christ take away from me all (evil). The cross of Christ saves me $[\ldots]$ and today over me before me behind [ ... ] before me and behind me and everywhere [ ... ] (When) the old fiend sees (it), may he flee from me in the name of our Lord Jesus Christ. Bless me, Jesus (of Nazareth) the eternal King of the Jews (passed) through the midst of them and went away. May Jesus Christ crucified and Son of the Living God bless me and raise [ ... ] may Christ care for me and defend me and may the Lord avert from me every evil. In the name of the Father and the Son and the Holy Spirit Amen.

Several parts of the text have been worn off and other parts are illegible. Despite the lacunae, the text is recognizable as a composite of two different prayers, the first to St. Dorothy addressed as the virtuous rose of the world and intercessor between God and humans, followed by a plea for help to make the

\footnotetext{
I am most grateful to Vemund Blomkvist who helped me with the translation of the text from Latin to English. Incorrect Latin: mundi vita should most likely be mundae vitae. Incorrect Latin: vita salus should most likely be vera salus. Incorrect Latin: cruciem should most likely be crucem.
} 
supplicant worthy of divine grace. The next part is addressed to the cross-Crux Christi sit mecum, the cross of Christ be with me-affirming the cross as the way to salvation, as the breaker of the chains of death, followed by an appeal to the cross, emphasizing its protective powers. The prayer to the cross ends with the name of Jesus, addressed as eternal king of the Jews, and a fragment which, as we shall see, is a quote from the Vulgate. The text ends with an invocation to Christ with a plea for protection from all evil.

When the amulet was registered in the Diplomatarium Norvegicum in the 1880s, it was accompanied by a summary, stating that the piece of paper was a "Praise (hymn to?) St. Dorothy and the cross of Christ, seemingly used as a sorcerous spell or amulet". ${ }^{7}$ An amulet was an object carried on the body for healing and/or protection against evil and could ward off diseases, or protect the bearer against the attacks of visible and invisible enemies (Kieckhefer 1989, p. 75). This article will seek to establish that the paper prayer was indeed an amulet, but was not, however, an object of sorcery or witchcraft as suggested in the Diplomatarium. The amulet was a devotional object, connecting God and the faithful.

The amulet is today kept at the National Archives in Oslo. The paper amulet is small, measuring only $13 \times 10.5 \mathrm{~cm}$. The paper has marks from wear and tear, some pieces around the outer edges and in the middle of the paper are missing altogether. Although undocumented, the paper has been subject to conservation after its rescue in the 1880s. During conservation, worn-off pieces have been replaced with a more brightly coloured paper, before the whole sheet has been covered in rice paper.

There are several vertical and horizontal, and darker, grid-structured lines that reveal the edges of the once folded paper. The part that made up the outer part of the object when folded is significantly darker that the rest. Following the original folding pattern, we can recreate the size and the shape of the folded piece of paper which would have measured only $3 \times 2 \mathrm{~cm}$. Paper is a fragile material; the middle missing part corresponds with one of the corners of the folded piece of paper.

\section{Theological Attitudes towards Amuletic Practices}

According to Don Skemer, the medieval belief in the efficacy of the written word to heal and protect was an inheritance from Antiquity (Skemer 2006, pp. 23-30). Amuletic practices were not, however, accepted in early Christianity. The use of textual amulets was condemned by the church fathers: St. Augustine related the use of amulets containing texts and sacred symbols to signs of pagan superstition, and St. Jerome equated textual amulets to Jewish phylacteries (Skemer 2006, pp. 32-35).

From the thirteenth century onwards, there was a division between black magic-magic associated with the devil—on the one side, and natural magic on the other. While black magic was seen as a perversion of religion and a turning away from God, natural magic relied on God in order to be successful (Kieckhefer 1989, pp. 8-12). Natural magic is probably best understood as a form of religion-based pre-modern science that tried to influence the hidden powers within nature. During the Middle Ages, the term "magic" seems to have been reserved for descriptions of pagan cults and demonic activities, such as frauds, deceptions and divinations of earth, water and air (Kieckhefer 1989, pp. 8-12). Attempts to influence nature in benign ways or to seek protection from dangers and daemons with the help of God, including through the use of amulets, was-although performed in a multitude of ways and far from universally accepted-not labelled as "magic" at all.

The theological opinion towards textual amulets became more nuanced during the course of the Middle Ages, resulting in a cautious acceptance of amulets and amuletic practices. St. Thomas Aquinas (122?-1274) allowed for a limited role for textual amulets as long as they were based on Christian texts. In his Summa Theologica, Thomas writes: “Divine words are no less efficacious when written than when uttered" (Aquinas n.d.b ST II-II, Q 96, Art.4). Conversely, texts that contained demonic invocations, mysterious names, meaningless words and unknown characters as well as symbols, were forbidden

7 Translated by the author. Original text: "Lovprisning af (Hymne til?) den hellige Dorothea og Christi Kors, vistnok benyttet som Trylleformular eller Amulet." 
as these would have fallen under the category of (black) magic. The only image Thomas accepted in amuletic context was that of a cross. According to Thomas, the amulets worked not because of the words put on paper or parchment, but as a result of people's prayer and devotion. Understood in this manner, Christians could use textual amulets as emblems or tokens of their own devotion and hope for God's protection: "it seems to be lawful to wear sacred words at one's neck, as a remedy for sickness or for any kind of distress" (Aquinas n.d.b, ST II-II, Q 96, Art.4). With correct content and venerated in the accepted manner, Thomas saw the use of amuletic texts as an equivalent to relics that could be kept in the domestic sphere. A textual amulet could thus be understood to be an expression of divine agency working through the material object (Aquinas n.d.b, ST II-II, Q 96, Art.4 and Skemer 2006, pp. 58-63). Although Thomas' view was contested by other theologians, he did provide an authoritative rationale for textual amulets. By the later Middle Ages, textual amulets were a widespread and common part of the Christian community (Skemer 2006, p. 73), believed to be an apotropaic weapon (Greek apotropaios, from apotrepein to avert, from apo- + trepein to turn) - a weapon used to ward off evil-in the fight against ever-lurking daemons and disasters.

\section{Haptic Devotion and Hagiosensorium}

The nuances regarding the theologically correct understanding and function of amulets articulated through scholastic arguments were most likely beyond the comprehension of most people. How then did the late medieval bearer of an amulet understand his or her use of such an object? It is safe to assume that the use of amulets with religious content was not understood as a form of demonic magic or actions in opposition to authoritative theology, but rather as an expression of piety and devotion.

During recent decades, the material turn in the humanities has offered art historians an alternative approach to the study of medieval religious objects. Theories and perspectives of materiality emphasize matter's ability to disclose the sacred to the medieval worshipper (Bynum 2011). The holy could be revealed in multiple ways. One was by allegorical interpretations of matter and phenomena, another was by looking at the likeness between matter and God. But the divine could also be instantiated in matter, as an actual presence. That the holy works in and through matter, was theologically established in Christian doctrines:

The doctrine of creation (that matter had been created by God), of the Incarnation (that God became human in the figure of Christ), of the resurrection of the body (that the material would in some way be present in heaven at the end of time and of divine omnipotence (that God could make matter behave in ways that violated the natural laws he also established (... ) (Bynum 2011, p. 154)

First and foremost, God could be experienced as a real presence through the sacraments. During the miracle of mass, the host became the flesh of Christ. Other forms of matter could also be instantiated by the divine: relics were believed to embody the virtue and a real presence of the saint. In addition to being placed in reliquaries, relics could be placed inside images, image frames and sculptures, blurring the line between relics and images (Belting 1994, pp. 297-303). By the later Middle Ages, even images without inserted relics were understood to be capable of embodying a divine presence (Belting 1994, pp. 208-304; Bynum 2011; Aavitsland 2015; and Jørgensen 2015, p. 29).

For the late medieval worshipper, the sacred was experienced through sensory mediation of holy matter. The human sensory apparatus was activated and structured to apprehend and identify God's presence in the world. Sensory experiences of earthly matter could disclose glimpses of the divine, a paradigm of perception modern scholars have labelled hagiosensorium (Jørgensen 2015, pp. 25-31 and Aavitsland 2015). The human sensorium—sight, hearing, smell, taste or touch—was considered dynamic, overlapping and interchanging. In his commentary on Aristotle's De Anima, Thomas wrote: "[The sense of touch] appears to be the least spiritual of the senses, though it is the foundation of all the others" (Aquinas n.d.a, Book II, Chp. XI, lectio 22, p. 517). The sense of touch was thus regarded as the basic component of all other senses. A haptic or tactile connection between object and body 
seems to have been an independent and adequate way of experiencing the holy (Skinnebach 2019). Laura K. Skinnebach has examined rubrics, found in medieval Danish devotional books. A rubric is a text informing the reader of the prayer's origin, apotropaic powers and/or instructions for use. Based on a study of this material, Skinnebach concludes that a medieval prayer could be performed in different ways: heard, read, seen—or carried. Skinnebach convincingly argues that prayers did not necessarily need to be read or even looked at, but could be performed by the body. She labels this form of devotion haptic prayer (Skinnebach 2019). According to Skinnebach, "The transference of protection to the body did not happen through the eyes or by reading, but through the sense of touch" (Skinnebach 2019, p. 8). In several rubrics, the physical presence between person and object was underlined in order to make the amulet more effective. In some cases, a whole devotional book could be carried or placed upon a person, analogous to a textual amulet, or a page from a devotional book could be excised and used as an amulet (Skinnebach 2019, p. 7). Both practices were forms of devotion where the connection between the pious and the material object was understood as a form of haptic prayer.

\section{Who Made the Amulet?}

Many medieval textual amulets were produced by priests, monks and clerics to combat sickness, injuries, demonic possession or for protection of mother and child during childbirth (Skemer 2006, pp. 79-81). Written sources reveal that textual amulets were used to help heal the sick both inside monastic infirmaries and outside the monastic sphere. We know that men and women of great authority made amulets, for example St. Hildegard of Bingen and St. Francis of Assisi (Skemer 2006, pp. 81-83).

In the diary of Luca Landucci (1436-1516), an apothecary from Florence, we can read of a trader of prayers, travelling around selling amulets to lay people. In order to promote his merchandise, the prayer seller presented himself as a miracle-worker-he climbed into a burning oven without being burned, he held his hand over candles, placed burning candles in his mouth and put his hand in a boiling pan of oil, all while urging the onlookers to watch. According to the diary, the popolo did indeed regard his actions as miracles, and in his diary, Landucci has written: "And this way he sold as many of those prayers as he could make" (Trexler 1972, p. 31). When it comes to the production of amulets, it seems like there was an expectation among lay people that the manufacturer had a special bond to God or served as a mediator between God and ordinary people, either as a man or woman of God or a miracle worker such as the Florentine fire-eater.

We do not know where or by whom the Torpo-amulet was produced-it could have been written by a local priest in Torpo or the surrounding area, but it could also have been transported from afar for local use, perhaps by a returning pilgrim. In Norway, we have no traces of such activities as described in Florence above. Still, it cannot be ruled out that a similar travelling vendor came to Torpo.

The original owner of the amulet is unknown, and the amulet might have had several owners through the years. Given the fact that the prayer was placed inside the local church, it is reasonable to assume that at least one of the owners of the prayer had a connection to Torpo.

\section{Ave Gemma and the Cross-Prayer}

The text on the Torpo-amulet is lacunose, and might at first glance be challenging to recognize and contextualize. In the following, a comparison to other preserved prayers will help us to understand the Torpo text more fully. The Torpo-amulet contains two prayers. The opening words of the first prayer are missing, with the first readable words being "( ... ) virtuosa Dorathea", a prayer to St. Dorothy. The second prayer is also fragmentarily preserved, but recognizable as a prayer to the cross. Both prayers are well known from other sources. As we shall see, there are several examples of both prayers appearing in amuletic form, as well as in lay devotional literature.

There are regrettably few extant written sources that reveal much about lay religious practices in pre-Reformation Norway. In order to find equivalent prayers and contextualization, we must look 
beyond the borders of present-day Norway. The prayer to St. Dorothy is found in Danish and Icelandic medieval devotional literature, as well as in the form of a medieval hymn (Nielsen 1946-1982, V. 84). In the Danish prayer book AM $41812^{\circ}$, we find the following Latin prayer:

Ave gemma virtuosa

dorothea vernans rosa

vite mundi patens glosa

sis pro nobis speciosa

interuentrix gloriosa ${ }^{8}$ (Nielsen 1946-1982, II. 327)

An Icelandic devotional book, AM 429 12mo, known as the Kirkjubxjarbók (the book from Kirkeby), contains legends of female martyrs (AM 429 12mo, 1490-1510.). In this manuscript, the same prayer appears (fol. 59v), accompanied by an image of St. Dorothy (fol. $48 \mathrm{v}$ ) and the vita of the saint written in vernacular Norse (Wolf 1997, p. 62) (Figure 4).

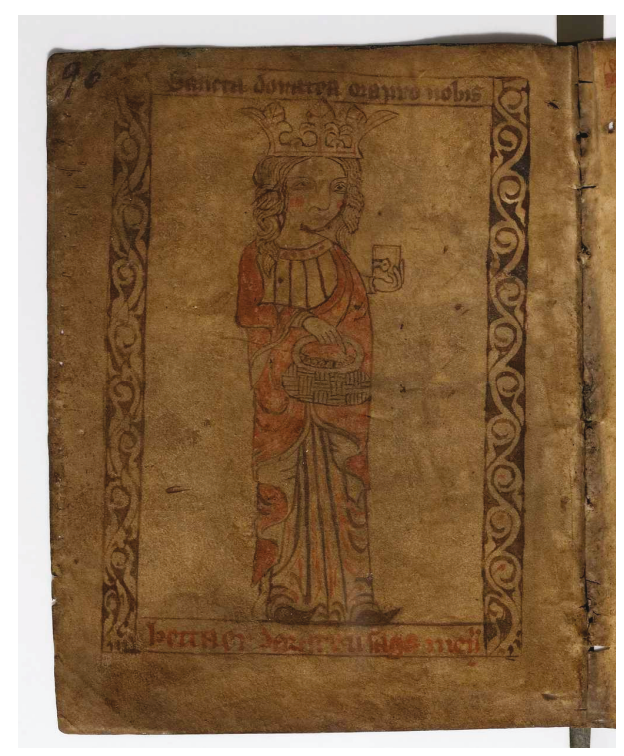

Figure 4. Image of St Dorothy, facsimile from AM 429 12mo (fol. 48v) dated between 1490-1510. Available at https://handrit.is/en/manuscript/view/en/AM12-429.

Apart from minor scribal and grammatical differences, the Danish and Icelandic versions are identical to the first part of the remaining fragments of the Torpo prayer. Based on these prayers, it is possible to suggest plausible conjectures to fill out many of the lacunae in the Ave Gemma-prayer in Torpo.

The second part of the Torpo prayer is known as the cross-prayer and was known throughout Western medieval Europe. In Denmark alone, ten versions are preserved (Skinnebach 2019, p. 5). The theme of all prayers is the same: the supplicant invokes the protection offered by the cross of Christ. Two of the extant Danish late medieval private devotional books, Anna Brade's prayer book

8 Translated to English by Vemund Blomkvist: Hail virtuous jewel, Dorothea, fresh open rose of pure life! May you be our glorious and beautiful mediator. 
(Nielsen 1946-1982, II. 33) and Johanne Nielsdatter's Book of Hours (Nielsen 1946-1982, I. 75), contain cross-prayers with striking similarities to the one found in Torpo. Although these two Danish prayers are in the vernacular, the order of phrases, rhythm and repetition show a strong resemblance to the Torpo-prayer. Anna Brade's cross-prayer reads:

The cross of our Lord Jesus Christ + be with me the cross of Jesus Christ t is the sign that I worship the cross of Jesus Christ + offers health the cross of Jesus Christ + surpasses all swords the cross of Jesus Christ + unties the chains of death the cross of Jesus Christ + is a steadfast weapon the cross of Jesus Christ + is a wonderous sign the cross of Jesus Christ + guard my life the cross of Jesus Christ + be my guide for all virtues/in the name of the holy cross I will follow everywhere the cross of Jesus Christ + means all good the cross of Jesus Christ + take away all evil + the cross of Jesus Christ + take away perpetual pain the cross of Jesus Christ theal me and be today before me/and over me/and after me so that the enemy which is the devil flees from me when he sees in me the name of our Lord Jesus Christ. ${ }^{9}$ (Nielsen 1946-1982, II. 33)

Karen Bille's book of hours contains yet another form of the cross-prayer (Nielsen 1946-1982, IV. 920). Although there are several linguistic differences between this prayer and to the two previously mentioned Danish versions and the Torpo prayer, the content is the same-all prayers call for the protection of the apotropaic cross.

If we look beyond the Danish material, the cross-prayer also appears in Latin in an extant handbook, Enchiridion Leonis Papae, supposedly written by Pope Leo III for Charlemagne. This book was printed in Mainz as late as 1633, and is a compilation of late medieval prayers that circulated in the Latin West. The prayer contains many components that also feature in the Torpo prayer:

Crux + Christi quam semper adoro, + Crux Christi sit in porta \& vera falus, + Crux Christi super et omnem gladium, +Crux Christi super \& vincula mortis, + Crux Christi sit pro me. + Crux Christi sit admirabile signum. + Crux Christi sit mihi potestas, + Crux Christi spondeat ante bonum mihi, + Crux Christi liberet me ab omni malo præsenti $\&$ future, + per hoc signum Crucis sit mihi divinæ gratix omnis interceffio, + Crux Christi auferat à me omnes adversitates hujus mundi. + Crux Christi sit mecum, \& salvet me, ante me \& post me, quia antiquus diabolous ubi te videt, à me recedat, + per Crucis hoc signum, + fugiat à me omnis malignus sprirtus + pax Helo + tetragrammaton + didai + pant aero elbry. + Jesus autem transiens per medium illorum ibat, fons, principlum, finis, veritas, omnipotens. † Amen. (Leo III. 1633, p. 119f)

The cross-prayer in the Torpo amulet ends with fragmentarily preserved text that reads: "Ihesus [ ... ] [sem-]piternus rex Judeorum [ ... ] per medium illorum ibat". This passage is a composite of two textual fragments. The first part Ihesus [ ... ] [sem-]piternus rex Judeorum recalls the inscription of the titulus of the cross of Christ, I.N.R.I. The phrase "Jesus Nazarenus rex Judeorum" was believed to be especially powerful in warding off evil. According to the commentary tradition, the titulus was associated with the words of Pilate, "what I have written, I have written". This statement has been interpreted as a testimony to the power of the written word: the text has been written, it will forever remain unchanged and it will persist (Hahn 2011, p. 196). In Torpo, the titulus-reference comes at the end of the cross-prayer, a feature consistent with Johanne Nielsdatter's Book of Hours where the cross-prayer ends with the phrase "Jesus of Nazareth, king of Jews" (Nielsen 1946-1982, I. 75). ${ }^{10}$ It

9 Translated by Laura K. Skinnebach and the author. Original text: "Wors herre ihesu christi kors + være meth mek Ihesu christi kors $+æ r$ thet tegn som iek tilbedher Ihesu christi kors + ær san helsen Ihesu christi korss + offuergaar alle swærdh Ihesu christi kors + løser døtzcens bondh Ihesu christi kors + ær eth stadelicht vapn Ihesu christi kors + ær eth vnderlicht tegn Ihesu christi kors + værie mith liiff Ihesu christi kors + være myn veylederæ til allæ dygder/i thet hellige korsses naffn tha gaar iek alle veyæ Ihesu christi korss + vthtydher alt goth Ihesu christi kors + taghe fra mek alt onth + Ihesu christi kors + taghe fra mek ewynneligh pynx Ihesu christi kors + hielæ mek oc være $i$ dagh foræ mek/ oc offuer mek/ oc effter mek so ath wvennen som ær dieffuelen flyer fra mek ther han seer mek $i$ vors herre ihesu christi naffn."

10 Translated by the author. Original text: "Ihesus nazarenus iødæ kongæ⿱", (Nielsen 1946-1982, I. 75). 
seems that this phrase enhanced the power of the cross-prayer, gave it authority and offered a verbal image of the titulus of the cross of Christ.

The latter part of the above text fragment is a biblical quote from the Vulgate, Luke 4:30, "autem transiens per medium illorum ibat" and recalls how Christ passed "through the midst of them", avoiding his enemies. This text was believed to bless the bearer with divine protection (Skemer 2006, p. 90) and is also found in other cross-prayers, for example in the Latin cross-prayer in the above-mentioned Enchiridion Leonis Papae.

\section{A Comparative Case: The Parchment Amulet}

In addition to the amulet from Torpo, there are several other extant Norwegian medieval textual amulets-some are small pieces of wood, others are made of lead. Some are in the shape of crosses, others rectangles (Diplomatarium Norvegicum 7, no. 441, Bang 1901/1902, pp. 470-74, http://www.unimus.no/arkeologi/forskning/index.php). As archaeological excavations and the use of metal detectors increase, so does the number of amuletic finds ${ }^{11}$. Some of the textual amulets have inscriptions in Norse, but the majority are in Latin.

Of special interest for this study, is an amulet made of parchment, dated around 1400-1450, thus predating the Torpo amulet (Diplomatarium Norvegicum 7, no. 441, see also Aalholm 1957, p. 63 and Bang 1901/1902, pp. 470-74). The amulet is of unknown provenance and regrettably no information regarding where and when it was found is preserved. ${ }^{12}$ The single sheet of parchment measures ca. $20 \times 14 \mathrm{~cm}$ and, just like the Torpo amulet, has traces after folding. When folded, the parchment amulet would have measured ca. $5 \times 3.5 \mathrm{~cm}$. This amulet also has traces of wear and tear-the outer part of the folded amulet is darker than the rest of the sheet, and one corner of the folded amulet is dark from friction, handling and wear.

At the vertical and horizontal edges of the parchment are tiny holes which are most likely traces of preparation for writing. Vertical holes were made in order to organize text lines on the sheet, with each hole indicating where to start a new line. The horizontal lines on the bottom of the parchment are at first glance more confusing. The parchment has, however, been identified as a palimpsest, but the original writing is not possible to reconstruct (Gjerløw 1959, p. 63). The current format of the parchment is thus most likely secondary, a fragment excised from a larger page. The bottom of the current parchment would once have made up the left margin. Just as the manufacturer was believed to be in a favorable relation to God, so was the writing material of importance-pages from sacred books were believed to be particularly effective for amuletic purposes (Skemer 2006, pp. 127-30). Perhaps this parchment was once a part of a liturgical or devotional book.

The outer part of the amulet has an illustration consisting of three concentric circles, the largest with a diameter of $5.3 \mathrm{~cm}$ (Figure 5). Between the two outermost rings is an ornament, and between the two innermost circles is text: "Sanctus sanctus sanctus dominus deus sabaoth pleni sunt ce [li] et terra Gloria tua oseanna [in] excelsis agyos ys[Chir]os tetragramaton"13. In the innermost ring, a cross has been drawn. Between the wide cross arms is text that reads "Jesus Nazarenus rex Judeorum beniora biro buro berto". The first part is recognizable as the apotropaic phrase discussed above, but the last four words do not have an apparent meaning (Aalholm 1957, p. 59). A row of small crosses is drawn in red ink at the top—or bottom if the parchment is rotated—at the long side of the document.

11 Many of the amuletic registrations do not specify whether they contain text. Many lead objects have not been examined for texts as opening of a folded lead object will damage the object see (Imer and Olesen 2018). Furthermore, some databases only register amulets with runic inscriptions such as Samnordisk Runtextbas, http://www.nordiska.uu.se/forskn/samnord.htm.

12 This amulet is kept at KUBEN, Aust-Agder museum and archive in the south of Norway.

13 I rely on the transcription by Aalholm (Aalholm 1957). Translated to English by Vemund Blomkvist: Holy, holy, holy (are you) the Lord God Sebaoth. The heavens and the earth are full of your glory. Hoseanna in the highest, holy strong tetragrammaton. The last words are in Greek. 


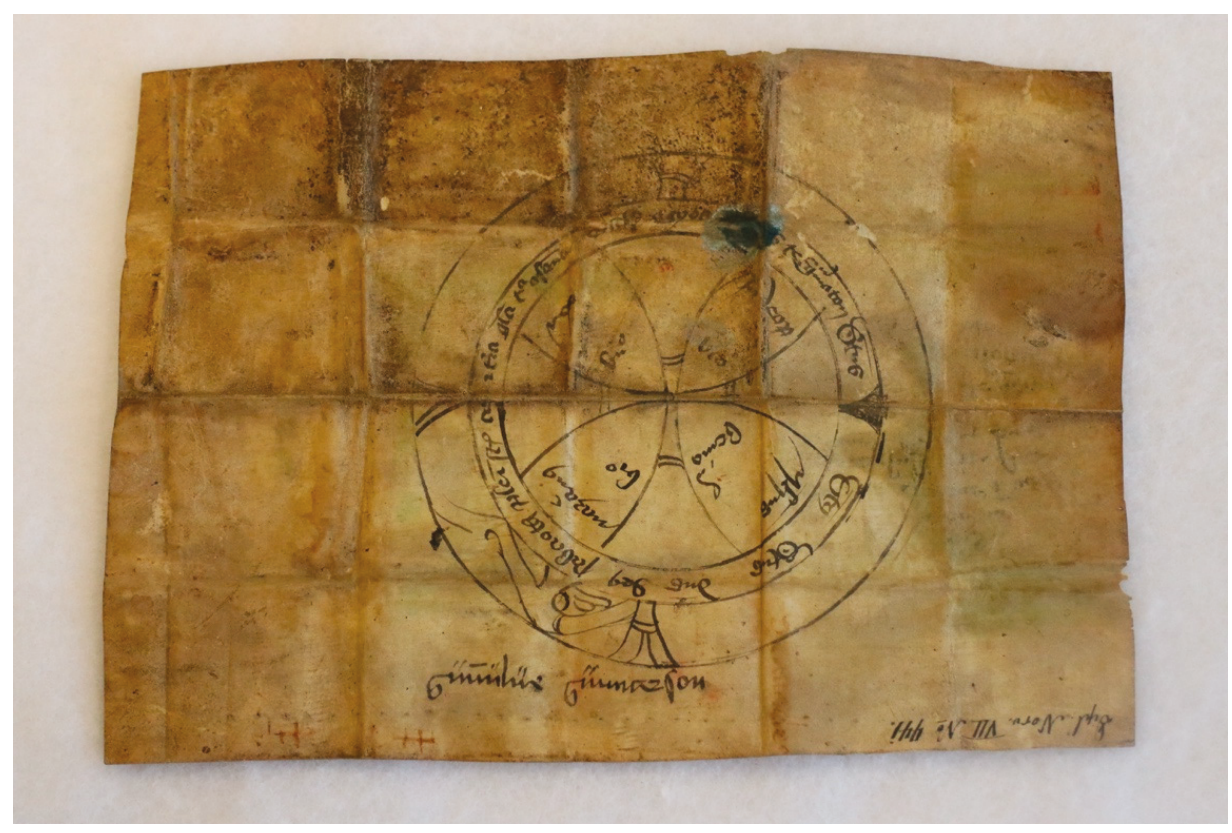

Figure 5. Amulet from Aust Agder, cross ornament. Ink on parchment, 1400-1450. Photo: Kaja M. H. Hagen.

The other side of the amulet is covered with text (Figure 6). The opening words from the Gospel of John were considered to be especially apotropaic, and the passage was frequently used in textual amulets (Skemer 2006, p. 84). The first text passage on the amulet is a paraphrase of the prologue of the Gospel of John. This passage is followed by a prayer to John the Baptist and a prayer to John the Evangelist, before a prayer to the cross appears:

crux bona crux dingna

Per omnia lingna benigna

tu me consignia

ne moriar morte malignia. ${ }^{14}$

Again, we see the cross invoked as an intercessor between God and man, capable of action, with protective qualities, able to keep a wicked death away from bearer. The text ends with an invocation to the many names of Christ in Latin and Greek.

14 Translated to English by Vemund Blomkvist: Good Cross, worthy Cross/Among all trees the most fruitful/Set your seal on me/That I not die an evil death. 


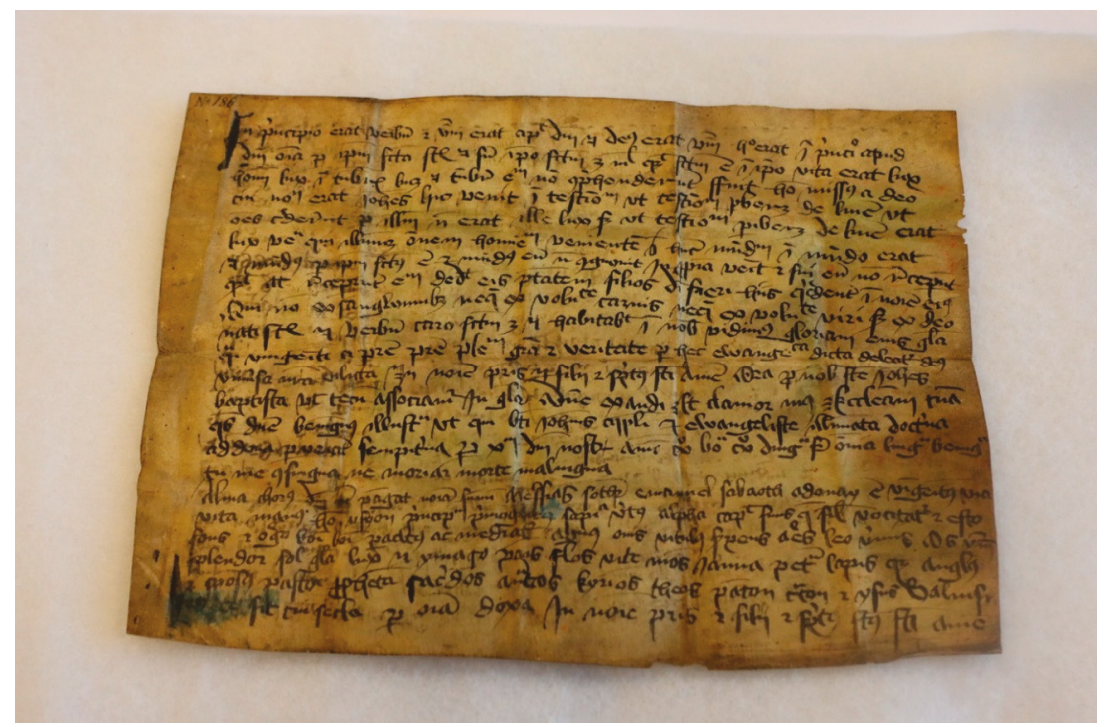

Figure 6. Amulet from Aust Agder, Latin text. Ink on parchment, 1400-1450. Photo: Kaja M. H. Hagen.

The prayer to the cross found in the parchment amulet is, however, not written in correct Latin. An explanation for the lack of proper Latin might have been the need for rhyme and rhythm which could have been regarded as more important than correct grammar. In both the Ave Gemma-prayer and the cross-prayer found at Torpo, rhyme and repetition are striking features. Rhyme, rhythm and/or repetition would have facilitated the recollection of the prayers and hence functioned as mnemotechnical strategies. We do not know if the original owner(s) of the Torpo prayer was a literate person, or if he or she knew Latin. Nonetheless, this does not imply that the bearer had no awareness of the content of the prayer. Rhyme and rhythm would have made recollection of a prayer easier, especially if the prayer had to be learned by heart in a language few had mastered. As mentioned above, many of the preserved Norwegian amulets have inscriptions in Latin. The choice of language was undoubtedly important-Latin, the ecclesiastical and sacred language, could have been believed to enhance the power of the prayer. The auditive aspects of the Latin language would have added to the words' semantic references, making the prayer a multi-sensory experience (Jessen and Sørensen 2015).

\section{Informative Rubrics: Information of Origin, Protective Powers and Instructions for Use}

As mentioned above, several of the extant Danish medieval prayers are accompanied by rubrics, explaining to the reader when, by whom and to whom the prayer was written, underlining the potential and the success of the prayer and/or providing the reader with instructions for use.

The origin of the cross-prayer is emphasized in both Anne Brade's and Karen Bille's devotional books-the texts state that this prayer was written by Pope Leo to Charlemagne. ${ }^{15}$ The supposed origin of this prayer can be traced back to the apocryphal legend of the Heavenly Letter. The legend of a holy letter accompanying certain prayers exists in different versions. In one version, the letter was written by Christ himself to King Abgar V of Edessa for protection. In other versions, the letter was sent by the Archangel Michael or St. George, and yet in others, like in the Danish devotional literature, the letter was presumedly written by Pope Leo III (Skemer 2006, pp. 99-105). The Latin cross-prayer

15 (Nielsen 1946-1982, II. 33). One of the original texts reads: "Item thenne efterscreffne bøn dictæde oc screff een helligh pawæ i rom som hedh sanctus leo/oc sæde henne til syn brodher karll magnus om var konnyngh i franckkeriigæ( ... )". 
in Enchiridion Leonis Papae was also accompanied by the Heavenly Letter. The papal origin and the protective powers of the prayers are analogous to the information found in the Danish material.

The papal-imperial origin undoubtedly served as proof of the cross-prayer's authority and the apotropaic potential of the prayer. The author of the prayer was the Holy Father himself. Needless to say, this information was of the highest importance for the understanding of the prayer as righteous, pious and effectual. That the prayer had been given to, presumably carried, and perhaps said, seen and heard by the most legendary and successful of kings in Europe, the Pater Europae Charlemagne, served as proof of its superiority, power and success. Furthermore, the prayer is presented as ancient, predating both the extant Scandinavian devotional prayers and the Torpo amulet by centuries, testifying to its established authority and power.

Not only the origin of the cross-prayer, but also its apotropaic power and instructions for use were included in both the Scandinavian devotional material and in the Enchiridion Leonis Papae. In the latter, under the heading Oraisons contre les perils du monde, it is stated that nothing bad would happen to the one who carried or read-portera ou lira—the cross-prayer (Leo III. 1633, p. 118). The Danish cross-prayers found in Anna Brade's and Karen Bille's devotional books, are accompanied by similar text ensuring the reader or bearer of its apotropaic power-the one who hears, sees or carries this prayer on his or her body, shall not drown, burn or die, and neither another person nor the devil can harm him or her (Nielsen 1946-1982, II. 33).

It was not only the cross-prayer that was accompanied by instructive rubrics or assurances of the power of the prayer. The above-mentioned Ave gemma-prayer in AM $418,12^{\circ}$ follows directly after a rubric with the following text: " $(.$.$) ) If a woman is in pain during labour, and if she has this prayer on$ her body, the child will soon be born without permanent injury". ${ }^{16}$ St. Dorothy is the patron saint of gardeners, brewers and newlyweds, in addition to being the patron saint of midwives and women in labour. Perhaps the Torpo amulet also functioned as a birth-gridle, protecting the woman if carried on her body. Interestingly, the above instruction informs us that the prayer would not necessarily need to be read at all. Perhaps it was the material presence of the amulet, its proximity to the body and hence its haptic qualities that ensured the prayer's apotropaic power (Skinnebach 2019), or perhaps the presence of the amulet reminded the bearer of the content inside.

Textual amulets could be carried on the person either in a sewn-in pocket, a pouch, a small container or in other ways fastened to the body. Based on a survey of surviving rubrics, it seems like a default placement of an amulet for overall protection was to carry it around the neck. For specific injuries, an amulet could be placed on the body parts in question. For protection during pregnancy and delivery, the instructions as to where the woman was to bind the amulet could vary, for example over the abdomen, on the right knee, below her right breast or on the back (Skemer 2006, pp. 236-239). As the Torpo prayer is not accompanied by a written instructive rubric, we are left to compare it to the surviving Danish rubrics, and to study its material traces. As mentioned, the Torpo prayer measured only $3 \times 2 \mathrm{~cm}$ when folded, and was hence suitable for portable purposes. Some of the folded edges has vanished as a result of wear and tear. Paper is a fragile material that would most likely have deteriorated without any protective cover. Although no container or pouch has survived, it seems a fair assumption that it was indeed placed in such.

Given that so few written sources regarding lay devotional activity from the Norwegian Middle Ages have survived, the textual amulets are valuable for our understanding of the spiritual environment in the Middle Ages at the most northernerly corner of Europe. The amulets' existence testifies to a shared belief in the power and potential of the written word-and in the apotropaic potential of the cross-found throughout Western Christianity. During the Middle Ages, Norway was a fully integrated part of the European Christian community. The textual amulets testify to shared devotional

16 (Nielsen 1946-1982, II. 327). My translation. Original text: “( . . ) Oc hulcen qwinnæ som meth barn pynes oc hawer hun thenne bøn paa segh - thet barn skal snarlighe fothes foruthen alt meen". 
ideals and activities found in other countries in the Latin West, the same prayers were said in Rome, in Denmark and in the remote parts of Norway.

\section{The Apotropaic Cross in Text and Image}

From the point of the discovery of the True Cross by dowager empress Helena in Jerusalem in 326, the cross was already linked to its healing capabilities. The widespread legends of testing of the True cross bear witness to the medieval understanding of its wonderworking potential. When Helena had difficulties verifying which cross was the cross of Christ, a bishop brought forward a dying woman who was miraculously healed in the presence of the holy wood. A similar story describes how a dead man was brought back to life when Christ's cross was brought forward (Jensen 2017, pp. 59-60). These legends were known all over Western Christianity, including in the north (Steinsland 2004). From the second half of the fourth century, the cross became a symbol associated with apotropaic and healing potential (Garipzanov 2018, p. 99).

The apotropaic qualities of the cross were also addressed during liturgy. In 1519, the archdiocese of Nidaros had a breviary printed, providing uniform liturgical instructions and texts for celebration of the divine office throughout the church province. The breviary illustrates how the cross was celebrated during the church year and how its apotropaic powers were underlined. For example, under In commemoratione sancte crucis the cross was revered as follows: "Hoc est namque sacrum signum, per quod vincimus malignum et fugamus vitium"17 (Sperber 2019, p. 533). That crosses could function as devotional tools or apotropaic shields was, therefore, not a trope reserved for textual amulets or devotional literature, but was also acknowledged by theological authorities, expressed in liturgical settings-as well as in images and sculpture.

The belief in the protective and healing power of the cross seems to be fundamental for the extant Norwegian amulets, and crosses were indeed present in various forms in most textual amulets, both as subject matter for the written prayer and as pictorial elements. Image and written texts seem to have had a mutually advantageous effect. Many textual amulets include drawn images of crosses, in line with Thomas Aquinas' pictorial instructions mentioned above. Crosses could take the form of simple or more ornamented graphic drawings and could be inscribed with names, such as the above-mentioned parchment amulet, while prayers could be formed in the graphic shape of crosses, and a series of different crosses could be juxtaposed (Skemer 2006, p. 200). Two of the other extant medieval Norwegian amulets are lead crosses with identical Latin inscriptions starting with "Ecce crucem domini, fugite partes adverse"18 (Bang 1901/1902, pp. 470-71). Crosses could also be drawn inside a text and could function as punctuation or, as seen above in the cross-prayer in Anne Brade's prayer book, as instruction to the reader to perform physical gestures at certain stages in the prayer, to make the sign of the cross upon the body. In this manner, the cross was present in the text, in image, and also, albeit briefly, as a bodily image.

As mentioned above, the backside of the Torpo prayer has a drawn symbol, sigillum Salomonis or Solomon's knot, a cross-shaped ornament with interlaced cross arms, measuring ca. $2.1 \times 2.3 \mathrm{~cm}$. During the Middle Ages, King Solomon was the ultimate source of wisdom, and was also believed to be capable of influencing nature (Davies 2012, p. 35). Solomon's knot had been associated with apotropaic powers since Antiquity, and was used as an ornamental motif in early Christian churches. The protective aspect of the Salomon's knot was understood as analogous to that of crosses (Garipzanov 2018, pp. 45, 103 and 110). The use of the Salomonic seal would have enabled the owner of the Torpo prayer to visualize the protective power of the amulet and hence facilitated the memory of the written content of the prayer on the inside. In this manner, seeing an image was analogous to reading, hearing or seeing the prayer inside.

17 Translated to English by Vemund Blomkvist: For this is a holy sign through which we conquer evil and dispel vice.

18 Translated to English by Vemund Blomkvist: Behold the Cross of the Lord! Flee, evil powers! 
When examining medieval devotional practices, the relationship between prayers, gestures, text and images was interdependent and interlaced. Not only amulets, but liturgical books and private devotional books also contained images of crosses and crucifixes accentuating the protective powers of the cross. In a fourteenth-century version of Speculum Humanae Salvationis is an illumination of Christ. In one hand, he holds the vexillum; in the other, he holds the cross with which he has pierced the fallen devil lying on the ground. With the holy cross, the sacrum signum, the devil is crushed and evil overthrown (Figure 7).

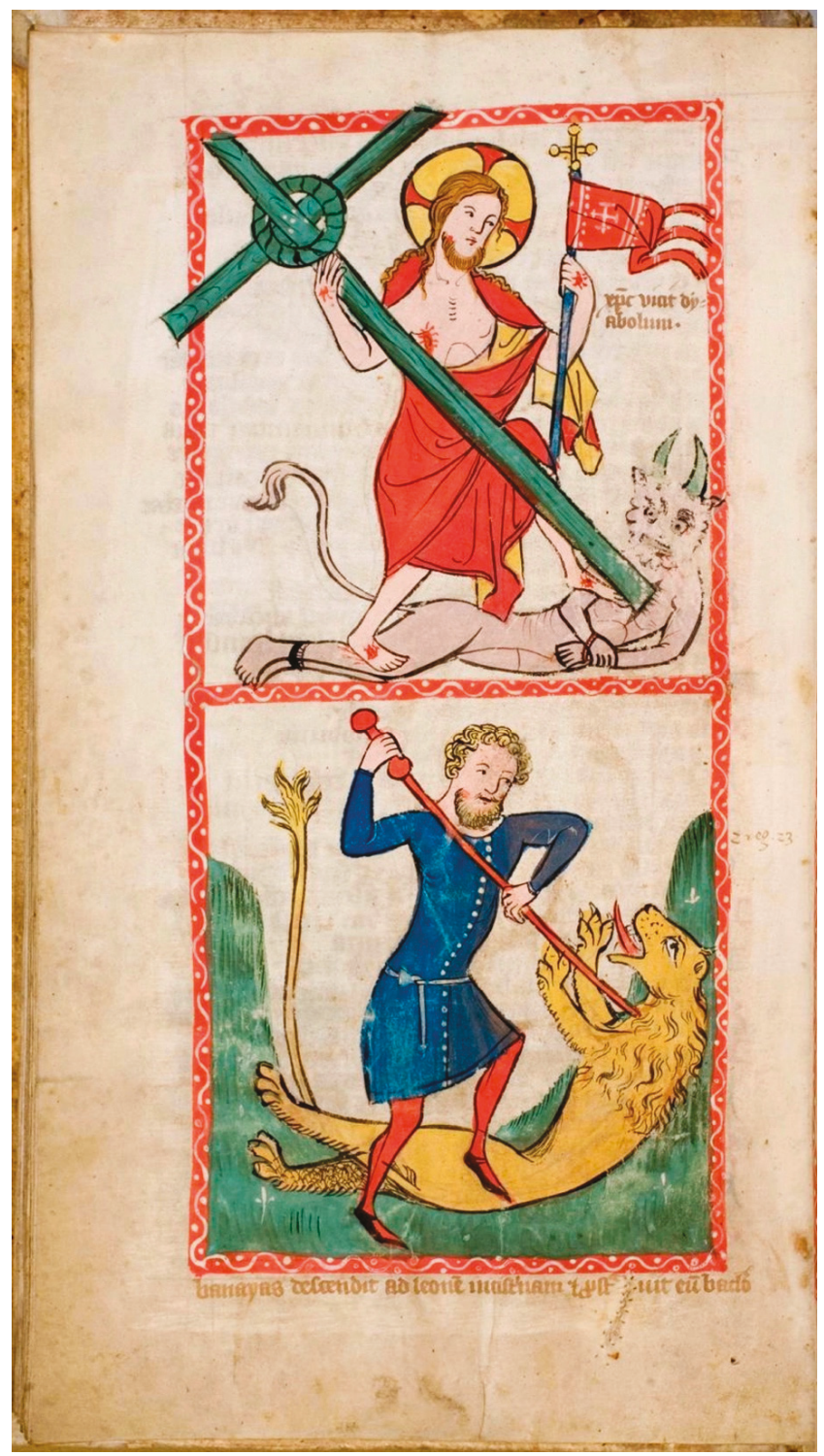

Figure 7. Christ. piercing the devil with the cross, facsimile from Speculum Humanae Salvationis, Hs 2505. Germany, ca. 1350. Available at http://tudigit.ulb.tu-darmstadt.de/show/Hs-2505/0108/image. 
According to the above-mentioned Enchiridion Leonis Papae, indulgence was given to those praying in front of an image of crucifix (Leo III. 1633, p. 154). It does not say what kind of image, but most likely a drawn image in an amulet, a painted illumination in a manuscript or a large crucifix inside a church were all understood as adequate images for gaining an indulgence. The next part of the article will further explore the Torpo text's relation to the large wooden cross in the church of Torpo and to wonderworking crosses in Torpo's proximity.

\section{The Wonderworking Crosses in Norway}

As the cross emerged as the prime Christian symbol, large crosses and crucifixes were consecrated and placed in churches and on altars. By the time the amulet in Torpo was made, the cross was omnipresent in churches across western Christianity. The cross was addressed as part of the liturgy, for example as seen above in the brevarium from Nidaros. In addition, the cross was celebrated and honoured on Good Friday and during yearly feasts of commemoration: the Invention of the Cross on 3 May and the Exaltation of the Cross on 14 September. The large crosses also served as focal points for individual devotion.

It is generally assumed that most churches in medieval Norway had a great crucifix. Within the borders of medieval Norway, around 1300 medieval churches, chapels and ecclesiastical houses are documented either in written sources or through archaeological evidence (Brendalsmo 2007; Karlsen 2013, pp. 33-34). It has been suggested that as much as 500 to 1000 churches should be added to this number, based on local tradition, church names, etc. (Bertelsen 2016, p. 54; Karlsen 2013, p. 33). These examinations indicate the medieval existence of at least 2000 churches and a corresponding number of great crosses. Today, 170 Norwegian medieval wooden polychrome crosses, crucifixes, and Calvary groups are preserved (Hohler 2017, pp. 47-55). Even if most of the Norwegian written sources from the Middle Ages are lost, there are numerous references to crosses that were connected to extra-liturgical cults or crosses believed be to wonderworking or miraculous. No less than 37 crosses owned land, received gifts and pilgrims or performed miracles (See for example Diplomatarium Norvegicum vol. 1, no. 545, vol. 2, no. 825 , vol. 11, no. 41, vol. 18 , no 164 , vol. 21 , no. 1058, vol. 23, no. 69; Regesta Norvegica vol. 8, no. 284, vol. 8, no. 389, vol. 9, no. 1439; Huitfeldt 1879 pp. 22, 45, 75, 80, 103, 108, 110, 138, 451; Munch 1834a pp. 23, 24, 60, 81, 82, Munch 1834b; p. 92; Nicolaysen 1862-1866, p. 48; Skielderup 1572/1905 pp. 53-54; Nielsen 1885, p. 517). Given the meagre body of written sources, the actual number is most likely significantly higher.

One of the most renowned crosses in Norway was found in Røldal in the south of Norway, the miraculous sweat of which healed the faithful on Midsummer's Eve (Figure 8). The wonderworking cross in Røldal received pilgrims from far and near. Both Røldal and Torpo belonged to the bishopric of Stavanger. The bearer(s) of the Torpo prayer was assumedly familiar with the stories of the renowned wonderworking crucifix in Røldal.

By the later Middle Ages, tales of wonderworking crosses and crucifixes were widespread across western Europe. It has been argued that the many legends of miraculous crosses affected the perception of crosses and crucifixes in general-any cross had the potential of becoming a miraculous or wonderworking cross that could affect the lives of the pious (Cooper 2006, p 56). Perhaps this understanding was also valid for the local crucifix in Torpo church. 


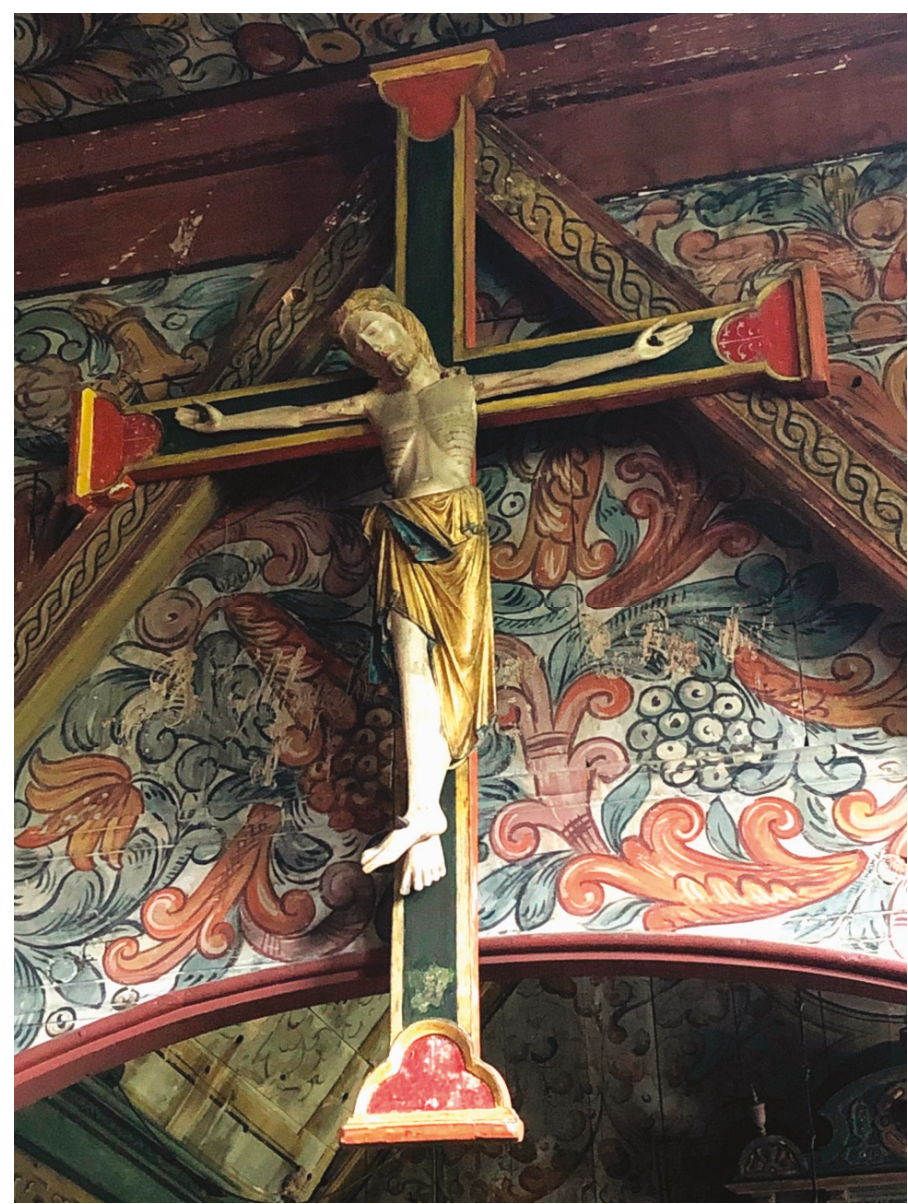

Figure 8. The Røldal Crucifix. 1240-1255. Cross H 157cm. Figure H 89 cm. Photo: Kaja M. H. Hagen.

\section{The Large Wooden Polychrome Crucifix in Torpo}

During the Middle Ages, individual devotion could take place both in the private sphere and in church, both during and outside of mass. Several extant Danish prayers instructed the reader to say specific prayers at specific times during mass (Skinnebach 2013, pp. 145-48). Instructions also show that private prayers were to be said in front of crucifixes. Such instructions likely referred to crucifixes of all forms, depicted in prayer books or amulets, hung on the wall in private homes or the large crucifix in a church. We cannot know if the owner of the Torpo prayer had some form of visual depictions of a cross or crucifix in his or her private sphere, serving as a visual aid and as a basis for recollection. We can, however, safely assume that the cross in his or her local church was visually accessible to the supplicant. Prominently displayed in a sacred surrounding in the local church, 
the large crucifix offered an elaborate setting that would have been of importance for the visual and devotional experience. ${ }^{19}$

The nave of the old wooden church in Torpo is still standing, and offers a partial glimpse of the devotional surroundings in Torpo. In the east part of the nave is a preserved vaulted canopy resting on columns, placed in front of the old entrance to the now demolished chancel (Figure 9). The canopy is the only surviving part of a medieval pulpitum..$^{20}$ The vaulted canopy has a span of $4.6 \mathrm{~m}$ and a width of $2.15 \mathrm{~m}$. The back wall measures ca. $2 \mathrm{~m}$ at its highest (Brænne 1982, p. 196). The vault is covered in lavishly painted images made during the last half of the thirteenth century (Christie and Christie 1981, pp. 130-37). In the arched ceiling, Christ in Majesty is surrounded by the symbols of the Evangelists. On each side of Christ are the apostles, six in each row. In the bottom two rows, beneath the apostles, is a series of images depicting the martyrium of St. Margaret.

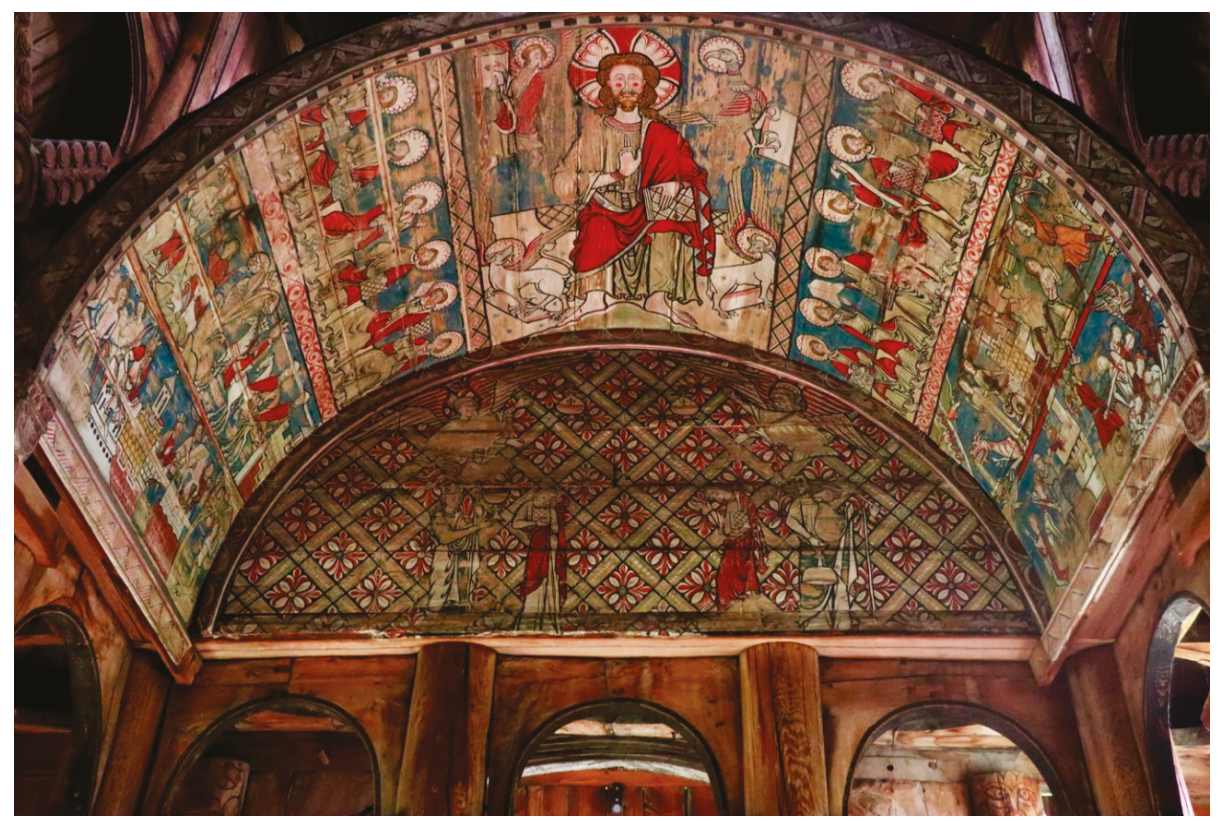

Figure 9. The Torpo canopy. Paintings from the thirteenth century. Photo: Kaja M. H. Hagen.

The back wall depicts figures from the crucifixion against a background of a square painted pattern with botanical ornamentation. To the left is the Virgin, who carries a book in her right hand, while her left hand rests against her cheek. To her right is a personification of the triumphant church, Ecclesia, holding the banner of the cross and a raised altar chalice. To the right is St. John, gesturing to the centre of the composition with one hand, carrying a book in the other. To his left is a personification of the Synagoga overthrown. Her head is bent, the pole of her banner is broken and the chalice turned upside down. Above them are facing angels holding censers in one hand while the other is pointing to the middle of the composition. The central figure, the cross or crucifix, is missing. Unlike the rest of the painted figures on the back wall, this was probably a three-dimensional crucifix placed in front of

19 According to Belting, the "crucifix was an elaboration of the preexisting cross" (Belting 1994, p. 299). In this article, I make no distinction between the cross and crucifix, as the apotropaic potential of a cross, present in both pictorial categories, assumedly would not have changed when the corpus of Christ was included.

20 Please see Linn W. Borgen's forthcoming Ph.-D. thesis. University of Oslo, Oslo, Norway. 
the painted motif where a tenon would have been tapped into the now missing vertical wall plate (Aavitsland 2016; and Stang 2008, pp. 161-66). ${ }^{21}$

Several medieval theological authorities in the Latin West have argued that crosses and crucifixes were to be treated separately from other categories of religious imagery and that such imagery required especial care and veneration (Cooper 2006, p. 48). Thomas Aquinas argued that not only images of Christ, but also of the cross, should be adored with latria, the highest mode of veneration (Aquinas n.d.b, ST, III, Q. 25 art. 3 and Laugerud 2005, pp. 66 and 72). However, both adoring an image with latria and venerating Christ himself involved a mental process that depended on recollection. For Aristotle and scholastic philosophers such as Thomas, visual images were the foundation for memory. In other words, we remember through images. When the bearer of the Torpo amulet addressed the cross in his or her prayers, either at home or in church, previously seen visual images of a cross were recollected. When venerating an image of a crucifix, previous memories and experiences of venerating both the prototype and memory of seen crucifixes were activated. In this manner, the physical images were prerequisites for mental images and a fundamental part of the devotional activity (Van Eck 2015, p. 72; Laugerud 2005). The now-lost great cross or crucifix present in the church of Torpo would have served as a focal point during prayers inside the church, but would also function as a mental imprint for the bearer of the prayer, and thus as a basis for a mental image he or she could recollect when praying to the cross outside of church.

The medieval cross was a polysemous object, embodying several layers of meaning-the cross was a symbol of the Christian faith and a referential object testifying to the crucifixion of Christ. The cross was also a mnemotechnical device serving as a mental imprint for the bearer inside and outside of church. The cross was an embellishment and an aesthetic work of art. The materials that the cross was made of could convey a meaning for the beholder: made from precious stones, materials, forms and colours invested with meaning, the allegorical potential of materials bore witness to God's presence in the world (Dahlerup 2010, p. 490 and Aavitsland 2015, p. 76). In addition, the cross could offer the pious an encounter with God and was believed to be capable of healing the supplicant or to ward off evil.

\section{The Power of the Object}

The pressing question when discussing the cross as an apotropaic or healing instrument, whether it is a textual amulet or a three-dimensional wonderworking cross, is where the power of the object was believed to come from. What made the object work? Was the object believed to be efficacious in itself, was it the immaterial divine power behind the object that made the object work-or a combination of the two?

The orthodox attitude towards images was that an image only referred to its divine prototype. An image was a sign pointing beyond itself and could be used as an aid in order to see the transcendent God. Prayers said in front of images were transferred to the holy persons depicted. This stance echoed formulations that went back to St. Basil in the fourth century, repeated at the Second Council of Nicea, by John of Damascus and Thomas Aquinas among others (Belting 1994, pp. 149-55; Laugerud 2005, pp. 64, 68; Maniura 2018, p. 64). Idolatrous warnings were reiterated by theologians throughout the Middle Ages. The authoritative theological attitude towards both textual amulets and religious imagery in respect to divine presence was the same-it was the transcendence of the object, understood as the underlying force, that made the objects work. God was the ultimate source of efficacy of both wonderworking crosses and textual amulets, and the objects were thus reduced to prosthetic aids.

21 The cross was most likely not fastened directly onto the back wall as there is neither discoloring in the pigments in the motif nor any signs of larger holes that would have been required in order to carry the weight of a large cross. There is a minor mark in the centre of the composition that might stem from a supporting mechanism for the once-standing cross. Please see Linn W. Borgen's forthcoming Ph.-D. thesis. University of Oslo, Oslo, Norway. 
The recognizable orthodox distinction between image and prototype was made by a Sienese sculptor, Lando di Piero, in 1338. He enclosed a piece of parchment in a sculpture of a crucifix he was working on that read:

Lord God made it possible for Lando di Piero of Siena to sculpt this cross from this wood in the likeness ['similitudine'], of the true Jesus Christ to recall for people the passion of Jesus Christ Son of God and of the Blessed Virgin Mary, therefore you true and holy cross of Jesus Christ Son of God, render the said Lando to God.

In addition to this plea, Lando added the following words:

The year of our Lord 1337 [1338 modern style], January, this figure was completed in the likeness ['similitudine'] of Jesus Christ crucified, true and living Son of God. And one should venerate him and not this wood. (Cooper 2006, pp. 47-48)

In line with authoritative theology, Lando urged people to look beyond the material image and venerate a prototype that was not present in the image.

One hundred and fifty years prior to Lando, in the 1230s, the bishop of Paris William of Auvergne wrote in his De legibus: "( . . ) there are many simple folks ( . . ) who make no distinction in their prayers between the images of saints and the saints themselves; nay those prayers which they should make to the saint they make to the image" (Maniura and Shepherd 2006, p. 8). William claims that an understanding of divine presence in religious imagery implied a cognitive error by "simple folks", most likely referring to (groups of) lay worshippers. William's statement indicates that lay people held opinions towards the ability of matter to disclose the divine that diverged from authoritative theology. Before discussing late medieval belief in the ontology of holy matter, let us look as William's statement concerning the various strata in society who held correspondingly different beliefs regarding the potential of objects and images.

As mentioned above, the presence of God in the world could be experienced in small glimpses on earth. Towards the later Middle Ages, the allegorical potential of images as pointers to an unseen transcendent truth was supplemented with a belief in the potential of divinity embodied in material objects, surpassing its symbolical or allegorical limitation. A multitude of images and objects all over Western Christianity were believed to be miraculous. The surviving written corpus on late medieval miracle working images is indeed vast (Freedberg 1989, pp. 299-301).

However, that the holy could reside in matter was far from universally accepted and was treated with ambivalence and sometimes condemnation by several learned theologians (Bynum 2011, chp. II). Certain cults, such as the blood-spotted wafers at Wilsnack, was criticized by several contemporary authorities (Bynum 2011, chp. II). According to Caroline W. Bynum, miraculous images made learned theologians uneasy and, as a result, they "jumped through intellectual hoops to avoid certain other implications of the idea that the holy is instantiated in matter" (Bynum 2011, p. 154). Hans Belting has stated that the philosophical and ontological difference between image and person depicted was of little interest to thinkers in the Latin west during the Middle Ages. The authority of miraculous images "lay rather in their unusual history rather than in their ability to be an incarnation of the person" (Belting 1994, pp. 304-8). Despite what motives have been assigned to late medieval theological thinkers in order to avoid the issue, the result was that the theology of holy matter was confusing and ambiguous (Bynum 2011, p. 164). According to Bynum: "Everyone condemned everyone else for misunderstanding how the divine intersected with the material, but no one denied that it did" (Bynum 2011, p. 165). It has been argued that highly intellectually and theologically defined beliefs, emphasizing the abstract, rational, systematic and dogmatic, stood in opposition to a more popular, irrational, emotional and object-based way of believing (Diedrichs 2005, pp. 34-35). But, as we shall see below, such a distinction is quite problematic.

Just as most people shared the understanding of the divine's ability to reside in matter (although by some restricted to the host and relics), the understanding of the human senses was most likely also 
shared by all strata of society. During the Middle Ages, experiences through human sensorium were believed to form the basis of all knowledge (Carruthers 2013, p. 17). As mentioned above, human sensory apparatus was structured and formed in order to apprehend the signs of God's presence in the world (Jørgensen 2015, pp. 25-31). By the later Middle Ages, a more practical way of believing, including perception and sense-based religious experiences, gained influence. People who had not necessarily had the education to access the intellectual dimension of the mysterium fidei, relied on their senses in their devotion (Diedrichs 2005, pp. 92-93). As a result of the acknowledgement of sense-based religious experiences, the role of images and objects was accentuated. Late medieval monstrances and reliquaries were designed to make hosts and relics visually accessible, testifying to the desire for visual contact between the pious and God. In a corresponding way, amulets served the need for haptic religious experiences. Images throughout western Christianity were held, kissed and in other ways venerated, in contact with human sensory apparatus. Artefacts such as images, sculptures, reliquaries and monstrances were material expressions of devotional practices that in most cases were facilitated, and thus sanctioned, within the ecclesiastical sphere. As mentioned above, amulets could also be produced by priests, monks and men and women of religious authority.

All of these examples illustrate a shared belief throughout the religious community both in the human hagiosensorium and in the potential of the holy to be instantiated in matter. Ecclesiastical authorities did indeed enable and sanction image worship and amuletic practices. Educated men and women of the church took part in the same culture as the uneducated, which makes it difficult to draw a hard line between all theologians on one side and lay people on the other. It has been argued that a distinctive line can only be drawn between a relatively small group of theologians of monastic circles whose writings have influenced our current understanding of the epoch today (Diedrichs 2005, p. 93). Instead of viewing theologians and lay people as two opposite and conflicting poles, perhaps a more nuanced and dynamic way of understanding the late medieval religious community is seeing the different arguments, understandings and practices as continuous negotiations to overcome disharmony.

When returning to the question of the ontology and perceptions as to what made an amulet effective, it cannot be ruled out that some bearers used the amulet as a prop accompanied neither by any devotional activity nor recognition of God as the working force behind the object nor a belief in a divinity present in the object. When amulets were used detached from religious understanding, the object could have been perceived as a magical device and hence in opposition to authoritative theology.

However, it must be assumed that a textual amulet containing a Christian prayer was most often used within a Christian context, with a belief in God working either in or through the object. The prototype addressed in the prayer written on an amulet was of course to be venerated. Presumably, the textual or visual content held a cognizance for the bearer, and attention was correspondingly given to the written and iconographical content of the amulet. Some preserved Norwegian amulets are made of lead, folded up in the shape of crosses and rectangles, with inscriptions inside. If opened up, the lead plates would have broken off, so any written content of such a lead amulet would not have been regularly read (Imer and Olesen 2018). In the same manner, if the carrier was illiterate, the content could not have been read by the bearer. If the content was in Latin, it is questionable if he or she fully understood the text.

However, that the words written on the amulet were not read does not necessarily mean that the carrier had no conscious awareness of its content. The prayers could have been read or explained for them when the amulet was made. Furthermore, as mentioned above, to read or hear someone else read the content of the amulet, was just one of several ways to perform a prayer. Amulets hung around the neck were visually and tactilely accessible with ease, and amulets placed in protective containers could have been taken out and seen, felt and handled as a part of a haptic devotional act. The material presence of the amulet, the feeling of the object on the body, could have served as a reminder to initiate further devotional activity. The amulet could also have served as a memory aid of the content known to the bearer. As mentioned above, rhyme and rhythm would have facilitated recollection. Perhaps the presence of the amulet would have urged the bearer to recite the verse learned 
by heart. In addition, the images drawn outside of both the Torpo and the parchment amulet, could have functioned as mnemotechnical aids of the content inside. It must then be assumed that veneration and devotion was indeed shown to God as He made the amulet effective in line with Thomas Aquinas' view discussed above.

Where does that leave the ontological status of the amulet? Was the amulet effective solely as a result of the veneration shown to the prototype? We have every reason to assume that the material presence of the amulet was believed to cause healing and an apotropaic effect. If not, then why bother with the material object at all? In order to shed light on the ontological belief in late medieval wonderworking objects, the next part of the article will draw comparable lines between amuletic practices and venerations of miraculous crosses.

\section{Amuletic Practices and Venerations of Miraculous Crosses}

In a European context, there are many stories of people addressing their prayers to a cross or crucifix and that in response, the image spoke, moved, bled or in other ways interacted with the worshipper (Bynum 2011; Freedberg 1989 and Maniura 2018, p. 64). Although the belief in divine presence was contested during the Middle Ages, it is hard to deny the belief in the agency of wonderworking images-they were, by many, understood to hold a capacity to act, influence and change the material world. Also in medieval Norway, many crosses and crucifixes were believed to be wonderworking.

Many medieval crosses were of obscure origins, often connected to acheiropoietic legends or legends where the cross had demanded to be placed in a specific church. These stories contributed to an understanding of the image as an independent agent, embodying a sacred presence. In Røldal, for example, several legends on the origins of the cross are preserved: one says that is was found in the mountains, three others that it was found at sea or in fjords. Despite their differences, all legends claim that it was the cross itself that expressed its will to be placed in Røldal church (Dalen 1960, pp. 74-77). These legends all contribute to an understanding that this image, not made by human hands, was elevated above similar depictions, and that the cross itself was able to articulate the will of God. In a comparable manner, the cross-prayer was believed to be written by pope Leo III or even Christ himself. The analogous holy origin of both wonderworking cross and the amuletic cross-prayer, served to elevate the ontology of both groups of objects.

To be in the proximity of the miraculous cross was paramount, and many holy crosses were locus for pilgrimage activity. In many cases, the cross should even be touched directly by the worshippers for increased efficacy. In Røldal for example, sweat from the crucifix would be wiped off by cloths and then pressed or fastened to the injured or sick parts of the bodies for healing. One of the most renowned miraculous crosses in Western Europe, is the Volto Santo of Lucca. The current crucifix is a thirteenth-century copy (Belting 1994, pp. 304-5 and Fishburne 2010, pp. 151-52), as the first crucifix was eroded as worshippers carved small pieces off the sculpture. The cross, and the many pieces carved from it, were thus treated like relics (Fishburne 2010). The understanding of the material presence of a wonderworking cross can therefore not be underestimated. To keep a part of the material object for personal use, carried on the body, whether a cloth with sweat or a piece of wood, was a testimony to the belief in the object per se. The similarities to amuletic practices are evident. Undoubtedly, the worshipper in Røldal believed it was God who made the sculpture sweat, it was God who made the crucifix efficacious. The prototype was not believed to be detached from the miraculous object. But at the same time, God had chosen to manifest himself through this particular object, elevating the ontology of the cross in question.

The visual difference between a small piece of paper and a large polychrome wooden cross is obvious. At first glance, the Torpo amulet is unimpressive, almost unnoticeable. Both the small size and the low-status medium of perishable paper limits its material and visual qualities. Meant for personal protection, the amulet was presumably kept inaccessible for other than the bearer. A large cross on the other hand, was invested with symbolic, referential and aesthetic value, in many cases a 
result of supreme craftmanship. A large cross was, based on the extant Norwegian material, made of wood, painted and could also be adorned with precious stones and gold and staged as the public focal point in the church.

Despite the apparent differences, a material cross and a textual amulet could conceptually express the same belief in the apotropaic powers of material objects. The apotropaic power of the cross addressed in the portable amulet was analogous to the power of the cross in church, and offered an expanded protection outside the ecclesiastical sphere, more far-reaching and personal than the immobile public cross in church, based on the proximity between bearer and object.

No one would claim that everyone in late medieval society shared a uniform and coherent opinion and set of beliefs regarding the ontology of religious objects. Rather, religious beliefs and practices were multifaceted, in some cases conflicting and constantly shifting. Many people may have believed in amulets and crosses in accordance with authoritative theology, that God worked through the object. At the same time, the potential for divine presence was understood to be there, under the surface of the material wonderworking object. The required physical connection between supplicant and material object testifies to a belief in the object itself. In this way, an amulet or a large cross in church could serve as a medium through which God could extend his power and establish a connection between Himself and the bearer. These objects were believed to make the sensuous divine accessible for humans, serving as holy locus-meeting point-between God and humans.

\section{Protection through a Plethora of Objects}

Textual amulets and large crosses were, however, not the only media that offered protection and healing. Above, we have seen that the Ave Gemma and the cross-prayer appeared both in amuletic form and in devotional books. A codex containing religious literature could be used amuletically to protect or cure and could, for example, be placed upon a sick person for healing. By the end of the Middle Ages, protection could be sought through a plethora of different objects, materials and practices, not only amulets and devotional and liturgical books, but also liturgical benedictions and relics, for example (Skemer 2006, pp. 57 and 68). There were, in other words, an abundance of complementary sources, understood as different paths all leading to the same goal.

This intermedial concept of protection can be observed in a preserved cross, now at the Museum of Cultural History in Oslo (C23299) (Figure 10). In 1923, a medieval silver double cross was found during construction work in Tønsberg, a town in the south east of Norway. The cross is only $7.1 \mathrm{~cm}$ tall with precious stones at the end of each cross arm. In the middle is a piece of wood still intact, most likely a relic from the True Cross. In the top vertical beam is a small ring through which a chain could be threaded. This object incorporates several aspects of how divine protection could be evoked-as mentioned above, the wood of the True Cross was believed to have miraculous powers already from the point of discovery. An inclusion of a relic from the True Cross would undoubtedly have enhanced the apotropaic qualities of the Tønsberg-cross. The preserved ring on top of the cross indicates that this was a pendant, meant to be carried around the neck, close to the body, analogous to that of a textual amulet. The Tønsberg-cross was thus both symbol and prototype, both a relic and amulet. 


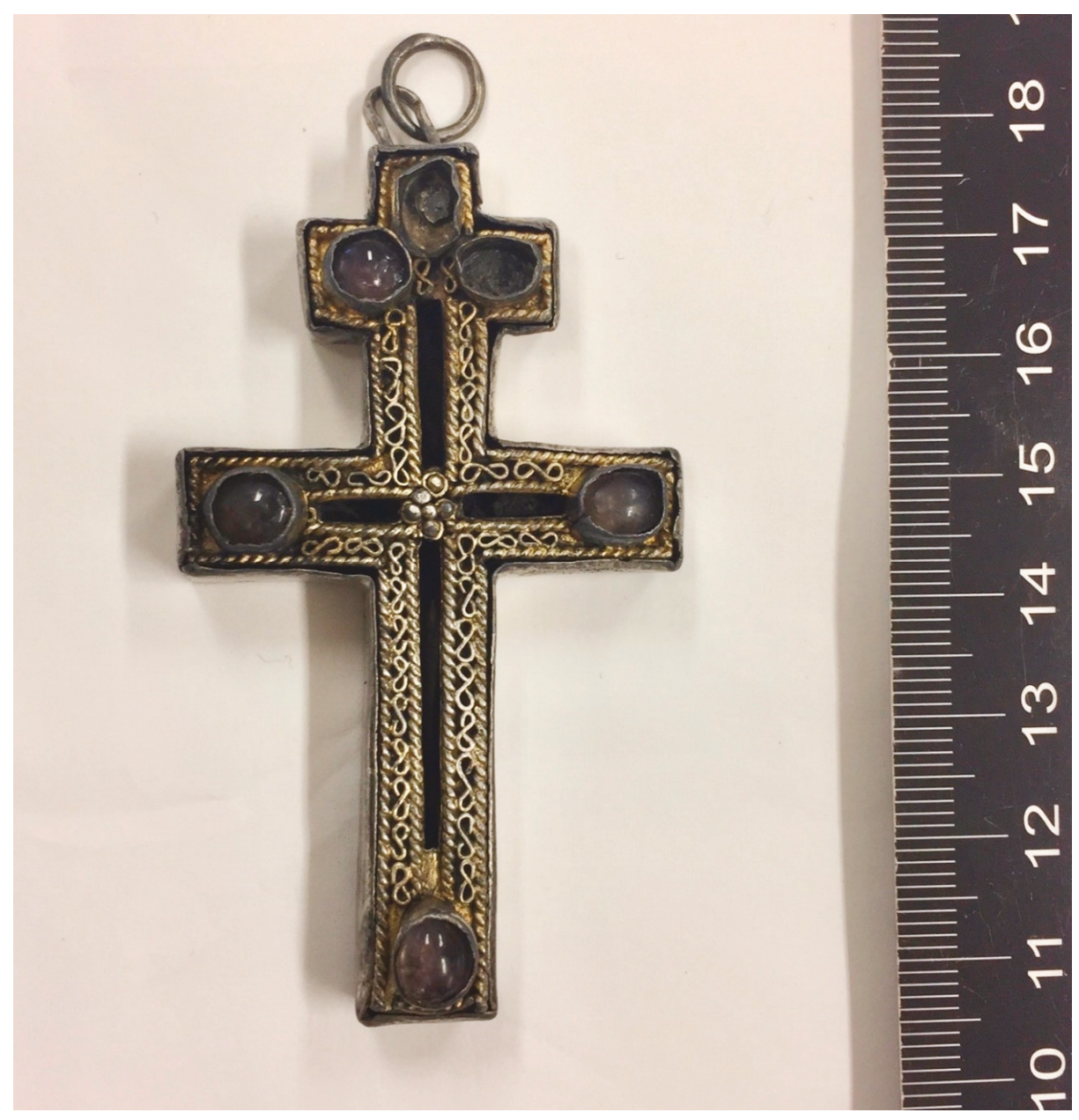

Figure 10. The Tønsberg cross. Silver and amethysts. H $7.1 \mathrm{~cm}$. In the middle of the cross is an extant piece of wood. Photo: Kaja M. H. Hagen.

\section{The Post-Reformation Understanding of Miraculous Crosses}

In 1536-1537, Norway was made subject to the Danish crown and the new Lutheran faith was implemented in the new kingdom of Denmark-Norway. The introduction of Protestantism entailed a revised attitude towards earthly matter as carrier of the divine. The medieval veneration of matter was, according to Luther, a deceitful staging carried out by the papal church in an attempt to control the faithful beholder. Yet Luther never dismissed the idea of matter as a potential carrier of the divine. Rather, his defense of the ubiquity of Christ implied an omnipresence of God, a presence without limits imposed by time or space (McGrath 1999, p. 189 and Luther 1989, pp. 64-65). By denoting Christ's own words in Matthew 26: 26, hoc est corpus meum, Luther confined God's presence to the Eucharist (Luther 1989, p. 82).

Prior to the Reformation, the anchoring of Lutheran ideas and belief was minimal among the Norwegian population and the old beliefs and practices continued for several decades, and in some cases centuries (Kolsrud 1939; Oftestad et al. 1993; Imsen 2016 and Laugerud 2018). What happened to 
the venerated images and amulets under the new paradigm? In the following, religious instruction, didactic texts, Luther's own words and studies of the Lutheran priests' practical work in early modern Denmark-Norway will shed light on the post-Reformation understanding and treatment of amulets and miraculous crosses in Norway.

As Norway became subject to the Danish crown, religious instructions and regulations penned in Denmark also applied to Norway. In line with Luther's own writings, Danish religious instructions, such as the Church Ordinance of 1537 and the visitation book written by the influential bishop and reformer Peder Palladius, stated that images that were worshipped in an incorrect manner were to be removed or burned. Palladius' visitation book offers a glimpse of what was regarded as false or wrong veneration: (i) images that were sought out, most likely referring to extra-liturgical cultic images, images that were believed to embody a divine presence and images which attracted pilgrims, (ii) images that received gifts in the form of wax dolls, understood as votive offerings, and (iii) images that the faithful hang crutches on, most likely referring to the practice of leaving crutches after healing in the presence of a miraculous image (Palladius 2003, pp. 39 and 141). All other images were to remain in church. The congregation was to be instructed in the correct understanding of images-images could be useful as didactic tools and aesthetic objects, but could not offer the pious a glimpse of the divine. An image was nothing but lifeless matter, not a medium between God and humans.

Thirty years after the Reformation, the religious and spiritual changes in Norway were limited, however (Kolsrud 1939). In 1572, in order to educate the people on the correct understanding of images, the bishop in Bergen, Jens Skielderup, published a didactic book formed as a dialogue between a peasant and a priest. Skielderup was clearly inspired by more image-hostile theologians, such as Niels Hemmingsen and John Calvin. The peasant articulates Luther's more tolerant view on images, while the priest, lecturing to the ignorant peasant, paraphrases Calvin and Hemmingsen. The peasant asks why the images cannot remain in the church, as they do neither good nor harm (Skielderup 1572/1905, pp. 34f). This argument is easily recognizable as a Lutheran adiaphora-argument. But the priest cannot accept this stance-not everyone possesses the wisdom to know that images are simply dead pieces of wood, ("døde Træstytter") deprived of sacredness. An image is indeed harmful if someone worships it, the priest explains. The peasant continues, wanting to know why images cannot remain as "biblia pauperum" in accordance with Gregory the Great's view. The priest dismisses this old and well-known justification of images, claiming that this argument was not anchored in the bible (Skielderup 1572/1905, p. 38f). "How about the crucifix? Can one have a crucifix?", the peasant presses on. And the priest reluctantly answers, "If you were to have an image on the altar, it should be a crucifix" (Skielderup 1572/1905, p. 53f). Skielderup reasons that a crucifix would be acceptable-but he dared not decide this definitely as he feared the wrong use of such an image and explicitly warns against the idolatry caused by the crosses in "Fana, Oppdal, Røldal and Austad and many other places in Norway" (Skielderup 1572/1905, pp. 53-54). ${ }^{22}$ This passage sheds light on several aspects of the early Protestant understanding of holy crosses: firstly, it testifies to the understanding that many crosses in medieval Norway were indeed venerated; secondly that the practice continued after the Reformation; thirdly, that even in the strictest post-Reformation writings, crosses and crucifixes seem to have been accepted - as long as they were not venerated.

It is questionable how much influence Skielderup's book gained. Despite Skielderup's attempt to implement a new attitude towards images and despite the theological instructions mentioned above, it seems that many of the crosses connected to unaccepted veneration remained in the churches. Most likely, their potential for didactic functions secured their prolonged ecclesiastical presence. Today,

22 Translated by the author. Original text: "Skulde mand haffue nogen Lignelse eller Billede paa Alteret/da maatte det heldst være it Crucifix/Thi efferdi alting som der handlis/bør at lyde om Christo alene/saa skulde oc Bemalingen eller Billedet være om hannem: Men her tor jeg intet beslutte/Thi den hellige Scrifft lyder intet der om/oc Christus oc Apostlerne vise oss til Ordet/mand skal oc befrycte at der kand opkomme med tiden saadan Vrangbrug der hoss/som skede udi Paffuedommit hoss kaarssit i Fane/Opdal/Rørigedal/Eustad/oc mange andre steds her i Norge." 
as mentioned above, as many as 170 crosses have survived, and among them are 10 of the crosses that the sources show as receiving pilgrims and gifts. The famous sweating crucifix from Røldal was visited by pilgrims seeking healing up to the nineteenth century, and is still in situ.

We do not know if the cross that was once a part of the Calvary group in Torpo was in any way venerated against the Lutheran instructions. Today, the cross is missing, but we do not know why and when it was removed. It has been suggested that the Calvary group once formed the backdrop for a cross altar. ${ }^{23}$ After the Reformation, the numerous altars in churches were to be removed as a church was to have one altar only (Palladius 2003, p. 39). It is a possibility that the Torpo cross was removed or relocated as the probable cross altar was deconstructed. Perhaps the cross was damaged, or it may simply have gone out of fashion and was replaced by the newer seventeenth-century crucifix currently hanging in the church. It cannot, however, be ruled out that the cross was removed as a result of unacceptable veneration. It seems strange to leave the iconographical motif, the Calvary Group-a motif that seem to have been accepted even by image-hostile Lutheran theologians-without the most central piece, the crucifix. There must have been weighty reasons for removing the crucifix and hence leaving the rest of the composition incomplete.

\section{The Post-Reformation Understanding of Textual Amulets}

The Torpo amulet was made just years before the Reformation was implemented in Norway. Not only false veneration of images, but also the use of textual amulets was strongly condemned by Luther and later Lutheran theologians. To write the opening words of John or the words 'Ihesus Nazarenus Rex Judeorum' on a piece of paper and hang it in a container around the neck-as seen in both the Torpo amulet and the parchment amulet-was used as a prime example by Luther to illustrate misuse and religious malpractice (Luther 1957, pp. 106-7). Luther associated the practice of textual amulets with witchcraft and sorcery. To believe that a piece of paper hung around the neck could protect the bearer was, according to Luther, "customary in the papacy" and a godless act (Skemer 2006, p. 67).

The same rhetoric was used by Danish theologians. In Niels Hemmingsen's 1575 book on witchcraft and demonology, Admonitio de superstitionibus magicis vitandis, Hemmingsen states that written words, signs and symbols held no power (Gilje and Rasmussen 2002, p. 249). In the same book, he also states that benedictions, either performed orally or written down, could indeed influence humans and nature, he would not deny that, but it was always the Devil who was responsible. In all forms of magic, benevolent or malevolent, the devil was the causa efficiens (Gilje and Rasmussen 2002, pp. 248-50).

During the Middle Ages, the term 'magic' was, as mentioned earlier in the article, reserved for demonic magic. After the Reformation, the term was used for both benevolent and malevolent practices, the first category encompassing all forms of religious activities not in line with Lutheran theology. Remaining medieval devotional practices-including amuletic devotion-were labelled superstitio and demonic magic according to authoritative Lutheran theology. ${ }^{24}$

Although Thomas Aquinas accepted the use of some amulets if veneration was shown to God as the working force behind the object, theological opposition to amulets could also be found in the Middle Ages (Skemer 2006, p. 58-66). Luther and Lutheran theologians' attack on the use of amulets is, however, a break with medieval theology, as amuletic practices were described as demonic sorcery

23 Please see Linn W. Borgen's forthcoming Ph.-D. thesis. University of Oslo, Oslo, Norway.

24 In an educational book from 1601, Hemmingsen and Reravius equates "blessings, measurings and writings for diseases" with ungodly acts, and the people performing such activities as corrupted by the Devil (Hemmingsen and Reravius $1601 / 1618$. Please also see Helge A. Staxrud's forthcoming Ph.-D. thesis. University of Oslo, Oslo, Norway.) The original text in the introduction to the 1618 edition of the book, Reravisu writes "Gunstige Læser/denne undervisning om den store oc gruelige Guds bespottelse/som skeer met Troldom/signelse/manelse/oc anden saadan vanbrug/er saare fornøden/vel at betracte/effterdi Dieffuelen stedse oc altid tracter at forfore Menniskene/til all vrang oc falsk Tro oc mening/til Troldom/Signelse/Maalelse/manelse/spaadom/oc anden saadan Guds allerhelligste Naffns oc Ords vanbrug/saa at vi maa vel beklage det alt formeget/mere end sanden at være/det den hellige Apostel Paulus/udi sin Epistel til de Epheser/i det andet Capitel/udi sin tid klager/saa sigendis: Dieffuelen haffuer i denne tid sin Gierning $i$ Vantroens Børn. Item/til Timothæum i den anden Epistel/Cap ii. siger hand om de Ugudelige/at de ere fangne aff Dieffuelen/effter hans Villie." 
under the new paradigm. The belief in sorcery and witchcraft in the post-Reformation society was real and people did believe in the efficacy of magic (Gilje and Rasmussen 2002, p. 242). Theological condemnation of textual amulets could be seen as an attempt to control and constrain the evil powers, but categorizations and accusations of magic, witchcraft and sorcery can also be understood as social, cultural and/or religious constructions, in line with Lutheran efforts to change the old beliefs and practices still valid among common people.

However, recent studies of the role of the early modern Lutheran priest indicate that the normative ideal expressed in the vast corpus of early Lutheran writings could be far from the practical everyday challenges facing the priests in the parishes. Despite their efforts in convincing the parishioners with persuasive arguments in favour of the new faith, old practices and beliefs, intertwined in the daily life of most people, were difficult to eradicate. Authoritative Lutheran theology was in several instances interpreted and negotiated in order to adapt to local traditions and rituals (Appel and Fink-Jensen 2009), and it cannot be ruled out that some priests looked the other way when it came to amuletic practices.

We know that many of the old religious practices continued for a long time after the Reformation, as was probably also the case with amulets. In 1584, a citizen of the high society in Stavanger sought help for his wife who was believed to have been afflicted by maleficium. In order to help her, a man known to have "magical" skills were summoned, and he carved five crosses into the woman's flesh (Gilje and Rasmussen 2002, pp. 241-43). ${ }^{25}$ This story reveals several aspects of religious beliefs in the post-Reformation society: firstly, that the apotropaic belief in the cross persisted for decades after the Reformation; secondly, that practices of haptic devotion correspondingly continued; thirdly, that the cognitive universe of haptic devotion and apotropaic crosses was shared by both high and low classes in Norwegian society also after the Reformation; and fourthly, that the understanding of how forces in the world worked remained the same across the confessional change-God was responsible for the good in the world, and the Devil for the bad, such as diseases and accidents (Gilje and Rasmussen 2002, p. 239). Amuletic practices and amulets were devotional tools that armed the bearer against the ever-present disasters, dangers and daemons both before and after the confessional change.

In 1901/1902, the Lutheran theologian Anton Chr. Bang collected the content and use of textual amulets and related devotional practices in Norway from the thirteenth century up to that point (Bang 1901/1902). The largest part of the collection are medical and practical advices. Two aspects of this work are of particular interest for this study. Firstly, many prayers and practices collected and recorded in the book from the nineteenth century do indeed resemble medieval devotional activities. Of course, it cannot be claimed that all the prayers, rubrics and formulas in Bang's collection are of medieval origin or that they are blueprints of medieval practices. Nonetheless, there are many similarities-several prayers are addressed to the Virgin, such as prayers in hope for relief from toothache (Bang 1901/1902, pp. 532-33). Furthermore, many rubrics are similar to the medieval ones. The material shows that many prayers were to be written down and carried on the body. Secondly, the book's title is Witch Formulas and Magical Recipes. ${ }^{26}$ When the amulet from Torpo was registered in the Diplomatarium Norvegicum, it was, in a corresponding manner, described as "sorcerous spell or amulet". ${ }^{27}$ To label remnants of medieval devotional activities as sorcery, seem to have an accepted principle of classification, also in the nineteenth century. In this article, I seek to argue that in its original context, the Torpo amulet was a material expression of the bearer's devotion, a medium between God and man and a belief in the healing and protective power of the cross, not an instrument of witchcraft.

25 The story of the crosses carved into the woman's flesh, reached Jørgen Eriksen, the bishop in Stavanger. He addressed this incident with King Fredrik II who issued a law against practices such as carving of crosses, benediction and readings that were to help against diseases. For practitioners of such magic, the penalty was death, without mercy (Gilje and Rasmussen 2002, p. 244). Original text: "Folk befindes, som enten at bruge eller gjøre saadanne forargelig ukristelig Middel, enten med Kors, Signelse, Lxrning og anden saadan ugudelige og ukristelig Handel, (... ) da at skulle lade dennem straffe paa Livet uden al Naade (... )."

26 Original title: Norske Hexeformularer og magiske Opskrifter.

27 Original text: "benyttet som Trylleformular eller Amulet", Diplomatarium Norvegicum, 12, no. 266. 
It is reasonable to assume that the understanding of the Torpo amulet was in continuance with medieval devotional practices for a long period after the Reformation, as we know that the old beliefs did persist, but we cannot know for how long. Disrupted from Rome, over time the Catholic religious and spiritual influence became meagre, and we cannot rule out that the once devotional tool could have been used for other ends.

In Vinje, a small village in the south of Norway, the old church was demolished and a new one erected at the end of the eighteenth century. During the demolition, medieval parchment leaves, many of them dated from around 1480, were found under the wooden boards in the chancel. These texts were later published under the name The Book from Vinje. The Eldest Book on Black Magic from the Norwegian Middle Ages (Garstein 1993). ${ }^{28}$ The first part of the book contained medical and practical advice. Some were accompanied with rubrics and incantations in order to increase the effect. The next part contained hymns to the Virgin, a fragmentarily preserved legend of the Virgin and prayers directed to saints. The greater part of this book was in other words not a book of black magic, at least not in its original context. The categorization of the book as altogether sorcery is best understood as another expression of Lutheran condemnation towards remnants of Catholic devotional practices.

The Torpo prayer was found in 1880 , but we do not know when it was placed in the church. Would it be possible for a piece of paper to lie under the floor for centuries without deteriorating? Is it possible that the Torpo prayer was placed under the floor boards shortly before the demolishment of the chancel? Could the Torpo amulet have continued to function as an amulet or perhaps as a birth-girdle for women in the community for years, perhaps centuries after the Reformation?

It is not only The Book from Vinje and the amulet from Torpo that were found in churches. Amulets have also been found under the floor in both Borgund stave church and Årdal church for example (Bang 1901/1902, p. 470). If textual amulets were condemned by the Protestant clergy and believed to be objects of black magic, the obvious question is why these objects were placed and hidden inside churches. It is hard to imagine that it was possible to place a manuscript or an amulet under the floorboards in the church's chancel without the acceptance or at least the knowledge of the act by the local priest. One explanation could be that the priest sought control over these unwanted objects. But why were the amulets simply not thrown away or burned? Could the placement inside the church be understood as a recognition of the power of the written word after all? A rubric before a prayer to St. Dorothy in the Danish prayer book AM 418, $12^{\circ}$ may shed light on the placement of the Torpo prayer. The rubric reads "In that house, where her image [St. Dorothy] is written, that house will not be damaged by fire" ${ }^{29}$ The amulet could thus have been placed inside the church for protection from fire.

\section{Concluding Remarks}

The small textual amulet in Torpo was made at the end of the fifteenth century, when Norway was still a part of the Roman church, and while the belief in apotropaic agency of both amulet and cross was still rooted among the faithful. In its original context, the Torpo amulet was an object of devotion, carried as a material expression of the cross' capacity to protect and heal the pious. After the Reformation, amulets were labelled as objects for demonic and magical use. To describe the textual amulet from Torpo as a device used in magic and witchcraft, is to disregard the function and the meanings ascribed to it in its original context.

During the Middle Ages, multiple functions and layers of meaning were incorporated in the image of the cross. The cross was a symbol of the redemption of humankind, a didactic symbol, a referential image of the crucifixion and an aesthetic embellishment. The cross was also a miraculous object offering healing and protection to the faithful. Many crosses and crucifixes in medieval Norway

28 Original title: Vinjeboka. Den eldste svartebok fra norsk middelalder.

29 (Nielsen 1946-1982, II. 327). Translated by the author. Original text: "Och huilket hws hennes belethe er screuenth wthi Thet hws maa ey worthe ild skadhe". 
were believed to be wonderworking. As we have seen, many amulets, also in a Norwegian context, had crosses inscribed, were made in shape of crosses or contained prayers addressing the cross.

Protection could be sought from a variety of practices and material objects, not only textual amulets and large wonderworking crosses. These two categories of objects seem nevertheless to have accentuated the apotropaic and healing potential offered by the cross, and both categories of objects were treated as a meeting place between God and humans with a capacity to influence the lives of the pious.

We do not know to whom the Torpo prayer belonged, neither do we know when it was placed in the chancel of the church. We do know, however, that the prayer was not thrown away or burned, but transferred back to the realm of the sacred. Perhaps this act can be interpreted as a persisting belief in the power originally ascribed to the amulet.

Funding: This research received no external funding.

Acknowledgments: I am much indebted to Laura K. Skinnebach for sharing her knowledge on devotional culture in late medieval Denmark-Norway, for insightful comments and interesting discussions during the writing process. I am also much grateful to Vemund Blomkvist who helped me translate the Latin texts to English. Many thanks are also due to Linn W. Borgen for helping me shed light on the canopy in Torpo, to Helge A. Staxrud for interesting discussions on post-Reformation magic and witchcraft, to Halfdan Baadsvik for helping me translate the Torpo amulet from Latin to Norwegian, to Karen Holmqvist for information about amulets written in runes and to Elise Kleivane for valuable input on medieval manuscript production. I am as always grateful to my supervisors Kristin B. Aavitsland and Sivert Angel for valuable comments and suggestions during the writing process.

Conflicts of Interest: The author declares no conflict of interest.

\section{References}

\section{Primary Sources}

AM 429 12mo. 1490-1510. Legends of Female Martyrs (Kirkjubæjarbók). Copenhagen: Den Arnamagnæanske Samling. Available online: https://handrit.is/en/manuscript/view/en/AM12-429 (accessed on 1 July 2019).

Aquinas, St. Thomas. 1951. Commentary on Aristotle's De Anima. Translated by Kenelm Foster O.P., and Sylvester Humphries O.P.. New Haven: Yale University Press.

Aquinas, St. Thomas. Summa Theologica, Part II-II (Secunda Secundae) and III (Tertia). Available online: http: //www.gutenberg.org/ebooks/18755andhttp://www.gutenberg.org/ebooks/19950 (accessed on 1 July 2019).

Diplomatarium Norvegicum. Available online: https://www.dokpro.uio.no/dipl_norv/diplom_felt.html (accessed on 1 July 2019).

Hemmingsen, Niels, and Rasmus Hansen Reravius. 1601/1618. En Undervisning aff den Hellige scrifft, hvad mand døme skal om den store oc gruelige Guds bespottelse, som skeer met Trolddom, Sinelse, Manelse oc anden saadan Guds hellige Naffns oc Ords vanbrug: Item 33 Propositiones imod Troldom. Der til 33 Propositiones om Spaadom. Kiøbenhaffn: Salomone Sartorio.

Huitfeldt, Henrik J. 1879. Biskop Eysteins Jordebog: (Den røde Bog). Fortegnelse over det geistlige Gods i Oslo Bispedømme omkring Aar 1400. Christiania: J. Chr. Bogtrykkeri.

Leo, Pope, III. 1633. Enchiridion Leonis Papae ... Imp. Carolo Magno ... Datum. Nuperrime Mendis Omnibus Purgatum/ Leo III] Mainz, S.n.]. Available online: https://search-proquest-com.ezproxy.uio.no/docview/ 2090367616? accountid=14699 (accessed on 1 July 2019).

Luther, Martin. 1957. Sermons on the Gospel of St. John. In Luther's Works. Edited by Jaroslav Pelikan. St. Louis: Concordia Publishing House, chp. 1-4. vol. 22.

Luther, Martin. 1989. Verker i Utvalg: 5. Edited by Inge Lønning and Tarald Rasmussen. Oslo: Gyldendal.

Munch, Peter A. 1834a. Registrum prædiorum et redituum ad ecclesias dioecesis Bergensis sæculo P.C. XIV.to, pertinentium, vulgo dictum "Bergens Kalvskind" (Bjørgynjar Kalfskinn). Christianiae: Guldberg \& Dzwonkowski.

Munch, Peter A. 1834b. Aslak Bolts Jordebog: Fortegnelse over Jordegods og andre Herligheder tilhørende Erkebiskopsstolen i Nidaros. Christiania: C. Grøndahl. 
Nicolaysen, Nicolay. 1862-1866. Norske fornlevninger. Utgivet af Foreningen til norske fortidsmindesmærkers bevaring. Kristiania: Carl Werner \& comp's bogtrykkeri.

Nielsen, Karl Martin. 1946-1982. Middelalderens Danske Bønnebøger. Copenhagen: Gyldendal, vol. 1-5.

Nielsen, Yngvar. 1885. Biskop Jens Nilssøns Visitatsbøger og Reiseoptegnelser 1574-1597. Udgivne efter offentlig foranstaltning ved Dr. Yngvar Nielsen. Kristiania: A.W. Brøgger.

Palladius, Peder. 2003. En Visitasbog. Udgivet på nud Ansk Med Inledning og Noter af Martin Schwarz Lausten. København: Forlaget Anis.

Regesta Norvegica. Available online: https://www.dokpro.uio.no/dipl_norv/regesta_felt.html (accessed on 1 July 2019).

Skielderup, Jens. 1572/1905. En Christelig Underuisning aff den Hellige Scrift. Edited by Fredrik B. Wallem. Christiania: Det Mallingske Bogtrykkeri.

Sperber, Ingrid, ed. 2019. Breviarium Nidrosiense. Oslo: National Library of Norway. Available online: https://www.bokselskap.no/wp-content/themes/bokselskap2/tekster/pdf/breviarium.pdf (accessed on 15 September 2019).

Steinsland, Gro. 2004. Draumkvedet og Tekster fra Norrøn Middelalder. Oslo: De Norske Bokklubbene.

\section{Secondary Sources}

Aalholm, Olav Anton. 1957. Er snart vår 600-årige pergament-amulett fra Bringsvær i Fjære? In Aust-Agdermuseets Årbok 1957. Arendal: P.M. Danielsens Trykkeri.

Aavitsland, Kristin Bliksrud. 2015. Incarnation. Paradoxes of Perception and Mediation in Medieval Liturgical Art. In The Saturated Sensorium. Principles of Perception and Mediation in the Middle Ages. Edited by Hans Henrik Lohfert Jørgensen, Henning Laugerud and Laura Katrine Skinnebach. Århus: Aarhus University Press.

Aavitsland, Kristin Bliksrud. 2016. The Church and the Synagogue in Ecclesiastical Art: A Case from Medieval Norway. Eologisk Tidsskrif 5: 324-39. [CrossRef]

Appel, Charlotte, and Morten Fink-Jensen. 2009. Når det Regner på Præsten. En Kulturhistorie om Sognepræster og Sognefolk, 1550-1750. Højbjerg: Hovedland.

Bang, Anton Chr. 1901/1902. Norske Hexeformularer og Magiske Opskrifter. Kristiania: I Commission Hos Jacob Dybwad.

Belting, Hans. 1994. Likeness and Presence: A History of the Image before the Era of Art. Chicago: University of Chicago Press.

Bertelsen, Dag. 2016. Kirker i Glemselens slør. Søkelys på det Norske Kirkelandskapet i Middelalderen. Jakobsli: D. Berthelsen.

Brendalsmo, Jan. 2007. Deserted Churches and Migratory Legends about Churches towards an Understanding. In Arv. Stockholm: Almqvist \& Wiksell, vol. 63.

Brænne, Jon. 1982. Torpo stavkirke-Problemer omkring konserveringen av det dekorerte middelalderlektoriet med tilhørende baldakin. In Polykrom Skulptur og Maleri på træ (Volume 2 of Kompendium). København: Nordisk Ministerråd, (Kompendium 2).

Bynum, Caroline Walker. 2011. Christian Materiality. An Essay on Religion in Late Medieval Europe. New York: Zone Books.

Carruthers, Mary. 2013. The Experience of Beauty in the Middle Ages. Oxford: Oxford University Press.

Christie, Haakon, and Sigrid Christie. 1981. Norges Kirker: 1: Buskerud. Oslo: Land og kirke.

Cooper, Donal. 2006. Projecting Presence: The Monumental Cross in the Italian Church Interior. In Presence. The Inherence of the Prototype within Images and Other Objects. Edited by Robert Maniura and Rupert Shepherd. Aldershot: Ashgate.

Dalen, Knut. 1960. Alma. 1960: Røldal Bygdebok. Røldal: Røldal kommune.

Dahlerup, Pil. 2010. Sanselig Senmiddelalder. Litterære Perspektiver på Danske Tekster 1482-1523. Århus: Aarhus Universitetsforlag.

Davies, Owen. 2012. A Very Short Introduction to Magic. Oxford: Oxford University Press.

Diedrichs, Christof L. 2005. Desire for viewing. "A Deluge of Images" in the Middle Ages. In Genre and Ritual. The Cultural Heritage of Medieval Rituals. Copenhagen: Museum Tusculanum Press. 
Fishburne, James. 2010. Renaissance Devotion and the Volto Santo of Lucca. Comitatus: A Journal of Medieval and Renaissance Studies 41: 149-66. [CrossRef]

Freedberg, David. 1989. The Power of Images: Studies in the History and Theory of Response. London: University of Chicago Press.

Garipzanov, Ildar. 2018. Graphic Signs of Authority in Late Antiquity and the Early Middle Ages, 300-900. Oxford: Oxford University Press.

Garstein, Oscar. 1993. Vinjeboka. Den Eldste Svarteboka fra Norsk Middelalder. Oslo: Solum forlag.

Gilje, Nils, and Tarald Rasmussen. 2002. Tankeliv i den lutherske stat. In Norsk Idehistorie. Oslo: Aschehoug, vol. 2.

Gjerløw, Lilli. 1959. Aust-Agder-Arkivets middelalderlige håndskriftsfragmenter. In Aust-Agder-Arv. Arendal: P.M. Danielsens Trykkeri.

Hahn, Cynthia. 2011. Inscriptions and Interactions: Text and Image on the Cloisters Cross and other Ivories. Acta ad Archaeologiam et Artium Historiam Pertinentia 24: 185-204. [CrossRef]

Hohler, Erla. 2017. Medieval Wooden Sculpture in Norway. In Collegium Medievale. Interdiciplinary Journal of Medieval Research. Oslo: Society for Medieval Studies, vol. 30.

Imer, Lisbeth M., and Rikke S. Olesen. 2018. In the Beginning was the Word: New Finds of Lead Amulets in Denmark. In Epigraphy in an Intermedial Context. Edited by Alessia Bauer, Elise Kleivane and Terje Spurkland. Dublin: Four Courts Press.

Imsen, Steinar. 2016. Da Reformasjonen Kom til Norge. Oslo: Cappelen Damm.

Jensen, Robin M. 2017. The Cross. History, Art, and Controversy. Cambridge: Harvard University Press.

Jessen, Mads D., and Tim Flohr Sørensen. 2015. Embodiment and Senses in Eleventh to Thirteenth-Century Churches in Southern Scandinavia. In The Saturated Sensorium. Principles of Perception and Mediation in the Middle Ages. Edited by Hans Henrik Lohfert Jørgensen, Henning Laugerud and Laura Katrine Skinnebach. Århus: Aarhus University Press.

Jørgensen, Hans Henrik Lohfert. 2015. Into the Saturated Sensorium. In The Saturated Sensorium. Principles of Perception and Mediation in the Middle Ages. Edited by Hans Henrik Lohfert Jørgensen, Henning Laugerud and Laura Katrine Skinnebach. Århus: Aarhus University Press.

Karlsen, Espen. 2013. Latin Manuscripts of Medieval Norway: Studies in Memory of Lilli Gjerløw. Oslo: Novus Press.

Kieckhefer, Richard. 1989. Magic in the Middle Ages. Cambridge: Cambridge University Press.

Kolsrud, Oluf. 1939. Folket og Reformasjonen i Noreg. Bergen: A.S. John Greigs Boktrykkeri.

Laugerud, Henning. 2005. Det Hagioskopiske Blikk. Bilder, Syn Og Erkjennelse I Høy-Og Senmiddelalder. Ph.D. dissertation, University of Bergen, Bergen, Norway.

Laugerud, Henning. 2018. Reformasjon uten folk. Det katolske Norge i før-og etterreformatorisk tid. Oslo: St. Olav Forlag.

Maniura, Robert, and Richard Shepherd. 2006. Presence: The Inherence of the Prototype Within Images and Other Objects. Aldershot: Ashgate.

Maniura, Robert. 2018. Agency and Miraculous Images. In The Agency of Things in Medieval and Early Modern Art: Materials, Power and Manipulation. Edited by Ika Matyjaszkiewicz, Grażyna Jurkowlaniec and Zuzanna Sarnecka. London: Routledge.

McGrath, Alister E. 1999. Reformation Thought. An Introduction. Oxford: Blackwell.

Oftestad, Bernt, Tarald Rasmussen, and Jan Schumacher. 1993. Norsk Kirkehistorie. Oslo: Universitetsforlaget.

Samnordisk Runtextbas. Available online: http://www.nordiska.uu.se/forskn/samnord.htm (accessed on 15 September 2019).

Skemer, Don C. 2006. Binding Words. Textual Amulets in the Middle Ages. University Park: Pennsylvania State University Press.

Skinnebach, Laura K. 2013. Practices of Perception. Devotion and the Senses in Late Medieval Northern Europe. Ph.D. dissertation, University of Bergen, Bergen, Norway.

Skinnebach, Laura K. 2019. Haptic Prayer. Devotional Books and Practices of Perception. In Touching. Devotional Practices and Visionary Experience in the Late Middle Ages. Edited by David Carrillo-Rangel, Delfi I. Nieto-Isabel and Pablo Acosta-Garcia. London: Palgrave Macmillan.

Stang, Margrethe C. 2008. Body and Soul: The Legend of St Margaret in Torpo Stave Church. In Ornament and Order. Essays on Viking and Northern Medieval Art for Signe Horn Fuglesang. Edited by Kristin B. Aavitsland and Margrethe C. Stang. Trondheim: Tapir Akademisk Forlag. 
Trexler, Richard C. 1972. Florentine Religious Experience: The Sacred Image. In Studies in the Renaissance. Chicago: University of Chicago Press on Behalf of the Renaissance Society of America, vol. 19. Available online: https://www.jstor.org/stable/2857086 (accessed on 3 April 2019).

Van Eck, Caroline. 2015. Art, Agency and Living Presence: From the Animated Image to the Excessive Object. Boston: de Gruyter.

Wolf, Kirsten. 1997. The Icelandic Legend of Saint Dorothy. Toronto: Pontifical Institute of Mediaeval Studies.

(c) (1) BY

(C) 2019 by the author. Licensee MDPI, Basel, Switzerland. This article is an open access article distributed under the terms and conditions of the Creative Commons Attribution (CC BY) license (http://creativecommons.org/licenses/by/4.0/). 
Article

\title{
Experiencing La Verna at Home: Italian Sixteenth-Century Maiolica Sanctuaries and Chapels
}

\author{
Zuzanna Sarnecka \\ The Institute of Art History, University of Warsaw, 00-927 Warszawa, Poland; z.sarnecka@uw.edu.pl \\ Received: 30 September 2019; Accepted: 17 December 2019; Published: 20 December 2019
}

\begin{abstract}
The present study describes the function of small-scale maiolica sanctuaries and chapels created in Italy in the sixteenth century. The so-called eremi encouraged a multisensory engagement of the faithful with complex structures that included receptacles for holy water, openings for the burning of incense, and moveable parts. They depicted a saint contemplating a crucifix or a book in a landscape and, as such, they provided a model for everyday pious life. Although they were less lifelike than the full-size recreations of holy sites, such as the Sacro Monte in Varallo, they had the significant advantage of allowing more spontaneous handling. The reduced scale made the objects portable and stimulated a more immediate pious experience. It seems likely that they formed part of an intimate and private setting. The focused attention given here to works by mostly anonymous artists reveals new categories of analysis, such as their religious efficacy. This allows discussion of these neglected artworks from a more positive perspective, in which their spiritual significance, technical accomplishment and functionality come to the fore.
\end{abstract}

Keywords: Italian Renaissance; devotion; home; La Verna; sanctuaries; maiolica; sculptures; multisensory experience

\section{Introduction}

During the fifteenth and sixteenth centuries, ideas about religious sculpture still followed two conflicting trains of thought. On the one hand, writers understood the efficacy of both sculptural and painted images at impressing the divine image onto the mind and soul of the beholder. On the other hand, especially in the context of the home, writers expressed justifiable concern about unorthodox, physical interactions with three-dimensional images (Sarnecka 2018b, p. 274; Galandra Cooper 2019). The present study focuses on the function of small-scale maiolica models of sanctuaries and chapels, a group of devotional objects that has never been studied before in relation to their specific form and medium. Created for a domestic space, to be handled and remind their beholders of the need for a pious life, these are among the most interesting devotional maiolica objects from the point of view of their function. They suggest ways in which artistic ingenuity satisfied the devotional needs of the faithful.

The devotional sculptures recorded in Renaissance inventories are rarely identified by their makers and it seems that subject matter and material were much more important aspects for fifteenthand sixteenth-century beholders than for audiences today. ${ }^{1}$ Of primary concern for original viewers was the devotional significance of the represented scene and the role of the object in everyday life, rather than its specific attributions. In the selection of themes, small-scale maiolica sculpture in part reflected the iconographies of large-scale glazed terracotta altarpieces. The iconography

1 Some exceptional examples, when the maker is specified, include Fra Saba's bust of St John the Baptist by Donatello (Thornton 1997, pp. 106-7) and two terracotta figures by 'Benedetto ischultore' (Benedetto da Maiano) acquired by Luigi Martelli in 1489 (Lydecker 1987, p. 127). 
of small-scale figures has sometimes been discussed in relation to free-standing terracotta groups painted in cold polychromy from the Po Valley region, created by such celebrated Renaissance artists as Niccolò dell'Arca (c. 1435-1494) or Guido Mazzoni (c. 1445-1518), set in purpose-made niches in various churches (Ravanelli Guidotti 1998, p. 221; Palvarini Gobio Casali 2000; Reale et al. 2008; Bonsanti and Piccinini 2009). The Nativity and the Passion scenes, which were the most popular narratives represented in these life-size terracotta groups, were also illustrated in small-scale glazed terracotta sculptures (Paolinelli 2014; Sarnecka 2018a). The relationship between large- and small-scale sculptures went beyond their common iconographies; scholars have observed the importance of mimesis for both artistic products and the common attention given to the facial expressions (Ravanelli Guidotti 1998, p. 221). The highly individual features of Guido Mazzoni's life-size sculptures moved the souls of beholders towards a more intense reliving of the sacred events (Lugli 1990, p. 32). We find the same attention given to individual characterizations of figures and settings in small maiolica figures. Unlike large-scale glazed terracotta altarpieces located on high altars or in side-chapels, which functioned primarily as a focal point of devotion during the liturgy, small-scale devotional statuettes fulfilled a variety of different roles. The decoration of everyday objects, such as inkstands, was just one of them (Piccini 2002; Marini 2007; Sarnecka 2018a).

Small, colourful glazed terracotta models of chapels welcomed more direct, physical, and intimate interactions, thus better serving the needs of family worship than large-scale altarpieces in the same medium created by artists from the specialized workshops of the Della Robbia and the Buglioni. Yet, devotional sculptural maiolica is often omitted not only from discussions of glazed terracotta sculpture, but also from studies of Italian Renaissance maiolica, which has privileged investigation of the so-called piatti istoriat - maiolica plates most frequently decorated with classical and mythological scenes. Only recently have scholars taken up sculptural maiolica (Ravanelli Guidotti 1998; Thornton and Wilson 2009; Warren 2014, 2016; Wilson 2016, 2017) and explored its role in Italian devotional practices (Paolinelli 2014; Sarnecka 2018a, 2018b). The attention given here to maiolica models of chapels and sanctuaries reveals new categories of analysis, such as their special religious efficacy, innovative artistic solutions informing devotional experiences and the agency of domestic sculpture. These maiolica objects solicited specific actions through their form, material, iconography and sometimes accompanying texts.

\section{Discussion of the Type}

During the sixteenth century the Italian city of Urbino became a centre for the production of small-scale maiolica sculptures. The Fontana workshop, active in the city from the mid-1550s (Wilson and Sani 2007, vol. 1, p. 201), gave new impetus to the development of sculptural ceramics (Watson 1986, p. 20). Towards the end of the century, the Patanazzi family, documented in Urbino from around 1575 until 1618 and related to the Fontana workshop, became involved in three-dimensional maiolica (Gardelli 1991, p. 131; Wilson 1996, p. 370; Negroni 1998). To these families, we owe the development of the small maiolica objects that show a chapel, set in a suggestive landscape, with a saintly figure kneeling in prayer or standing, whilst reading a devotional book (Table 1). These forms originated towards the mid-sixteenth century and became increasingly popular by 1600 .

These objects, described by contemporary sources as tempietti or eremi, seem to have played a significant role in shaping the domestic devotional experience (Figure 1). An inventory of the Palazzo Ducale in Urbino indicates a great number of similar objects and confirms that they were used in private residences (Sangiorgi 1976). The document, compiled in 1609, was the first such list created for the duke; the objects listed might have been in the palace for a long time before the beginning of the seventeenth century. The maiolica eremi described in the inventory include a representation of Christ praying in a garden accompanied by an angel, St Jerome in a grotto with the Crucified Christ and two angels above, St Jerome with one angel above, St Mary Magdalene praying before a crucifix below a church atop the grotto, St Mary Magdalene in her cell with four angels and the Crucifix (Figure 2), 
St Francis in prayer, and a mountain formed of three sections that included the Virgin, St Francis of Paula and St Francis of Assisi (Sangiorgi 1976, pp. 188-89).

Table 1. The surviving examples of eremi known to the author, attributed to the Patanazzi Workshop.

\begin{tabular}{|c|c|c|c|c|c|c|}
\hline & Title & Date & Size & Location & $\begin{array}{l}\text { Inventory } \\
\text { Number }\end{array}$ & Bibliography \\
\hline 1. & $\begin{array}{c}\text { Chapel with Mary } \\
\text { Magdalene Adoring the } \\
\text { Crucified Christ and Angels }\end{array}$ & $1575-1600$ & unknown & $\begin{array}{l}\text { The State Hermitage } \\
\text { Museum, St Petersburg }\end{array}$ & F 2884 & $\begin{array}{l}\text { (Ivanova 2003), } \\
\text { cat. no. } 96\end{array}$ \\
\hline 2. & St Jerome Writing & $1575-1600$ & h: $33 \mathrm{~cm}$ & $\begin{array}{l}\text { The State Hermitage } \\
\text { Museum, St Petersburg }\end{array}$ & F 2528 & $\begin{array}{l}\text { (Ivanova 2003), cat. no. } \\
\text { 97; (Kube 1976), no. } 89 .\end{array}$ \\
\hline 3. & $\begin{array}{c}\text { Chapel with St Francis and } \\
\text { the Crucified Christ }\end{array}$ & $1575-1600$ & $\begin{array}{l}\text { h: } 36 \mathrm{~cm}, \\
\text { w: } 38.5 \mathrm{~cm}\end{array}$ & $\begin{array}{l}\text { Victoria and Albert } \\
\text { Museum, London }\end{array}$ & C. $258-1926$ & $\begin{array}{l}\text { (Rackham 1940), } \\
\text { cat. no. } 854, \text { pl. } 136\end{array}$ \\
\hline 4. & $\begin{array}{l}\text { Chapel with St } \\
\text { Mary Magdalene }\end{array}$ & c. 1580 & $\begin{array}{l}\text { h: } 47 \mathrm{~cm} ; \\
\mathrm{w}: 37 \mathrm{~cm}\end{array}$ & Private Collection & unknown & $\begin{array}{l}\text { (Paolinelli 2019b), p. 30; } \\
\text { Sales catalogue Finarte, } \\
\text { Milan, November 1964, } \\
\text { lot no. } 73 .\end{array}$ \\
\hline 5. & $\begin{array}{l}\text { St Jerome Adoring } \\
\text { the Crucifix }\end{array}$ & c. 1580 & $\begin{array}{l}\text { h: } 36 \mathrm{~cm} ; \\
\text { w: } 31 \mathrm{~cm}\end{array}$ & unknown location & unknown & $\begin{array}{l}\text { Sales catalogue Finarte, } \\
\text { Milan, November 1964, } \\
\text { lot no. } 74 .\end{array}$ \\
\hline 6. & Chapel with St Francis & c. 1580 & unknown & $\begin{array}{l}\text { unknown location; } \\
\text { according to the } \\
\text { information obtained } \\
\text { from Professor Carmen } \\
\text { Ravanelli Guidotti once } \\
\text { in the Philadelphia } \\
\text { Museum of Art }\end{array}$ & unknown & unpublished \\
\hline 7. & Chapel with St Jerome & c. 1590 & $\begin{array}{l}\mathrm{h}: 36 \mathrm{~cm}, \\
\mathrm{w}: 35 \mathrm{~cm} \\
\mathrm{~d}: 22 \mathrm{~cm}\end{array}$ & unknown location & unknown & $\begin{array}{l}\text { Sales catalogue Finarte, } \\
\text { Milan, November } 1963\end{array}$ \\
\hline 8. & $\begin{array}{l}\text { St Jerome Adoring } \\
\text { the Crucifix }\end{array}$ & $\begin{array}{l}\text { end of } 16 \text { th } \\
\text { century }\end{array}$ & $\begin{array}{l}\text { h: } 54.7 \mathrm{~cm} \text {, } \\
\text { w: } 41 \mathrm{~cm} .\end{array}$ & $\begin{array}{c}\text { Musée de la } \\
\text { Renaissance, Écouen }\end{array}$ & Cluny 7605 & $\begin{array}{l}\text { (Giacomotti 1974), } \\
\text { cat. no. } 1117, \text { p. } 375\end{array}$ \\
\hline 9. & $\begin{array}{c}\text { Dominican Saint (?) } \\
\text { Praying before an Altarpiece }\end{array}$ & $\begin{array}{l}\text { late } \\
\text { XVI-early } \\
\text { XVII c. }\end{array}$ & $\begin{array}{l}\text { h: } 38 \mathrm{~cm} \\
\text { w: } 30 \mathrm{~cm}, \\
\text { d: } 20 \mathrm{~cm}\end{array}$ & $\begin{array}{c}\text { Museo di Arti } \\
\text { Applicate, Milan }\end{array}$ & 3173 & $\begin{array}{l}\text { (Wilson } 2000) \\
\text { cat. no. } 247\end{array}$ \\
\hline 10. & $\begin{array}{l}\text { Chapel with St Francis and } \\
\text { the Santa Casa di Loreto }\end{array}$ & $\begin{array}{l}\text { early } \\
\text { XVII c. }\end{array}$ & h: $38 \mathrm{~cm}$ & Palazzo Ducale, Urbino & 1990 C 416 & $\begin{array}{l}\text { (Gardelli 1987), } \\
\text { cat. no. } 26\end{array}$ \\
\hline
\end{tabular}

These objects helped to shape the devotional experiences of people who interacted with them. By viewing saintly figures such as St Jerome, St Francis or St Mary Magdalene in prayer, contemplating the Crucified Christ or reading a devotional book, the faithful who owned these objects were inspired to follow their example. At home, people used a range of different objects to enact their daily prayers. The maiolica eremi may have been employed in private devotions alongside an actual prayer book or a rosary-items depicted in the hands of the saintly figures.

Research conducted on the Renaissance practices of prayer demonstrates that people engaged with the supernatural through sensory experience, which not only involved looking at images but also touching and talking to them (Niccoli 2001, pp. 273-99; Brundin et al. 2018, pp. 179-80, 214-15). This physical interaction was not limited to children's prayers (Niccoli 2014, pp. 419-20). In fact, the types of figures included in the maiolica models of chapels suggest that they were more suitable for adults than for children. In his first regulation in the Regola del governo di cura familiare of 1419, the Dominican preacher, Cardinal Giovanni Dominici, recommended that parents instruct their children by showing them images of a Young Christ or John the Baptist or the two of them interacting (Dominici 1860, p. 130). What Dominici had to say about the subject matter and the function of paintings in domestic space also applied to statues: 'e come dico di pinture, così dico di scolture' (Dominici 1860, p. 131). Such comments imply a very specific kind of interaction with religious images at home, one that gives priority to the iconography and type of representation over the issue of media. Later, in the sixteenth century, Gabriele Paleotti's guidelines stressed that all religious works of art 
have the same origins, as all artistic creation begins with a disegno, be it a flat image or one in relief (Paleotti 2002, p. 16).

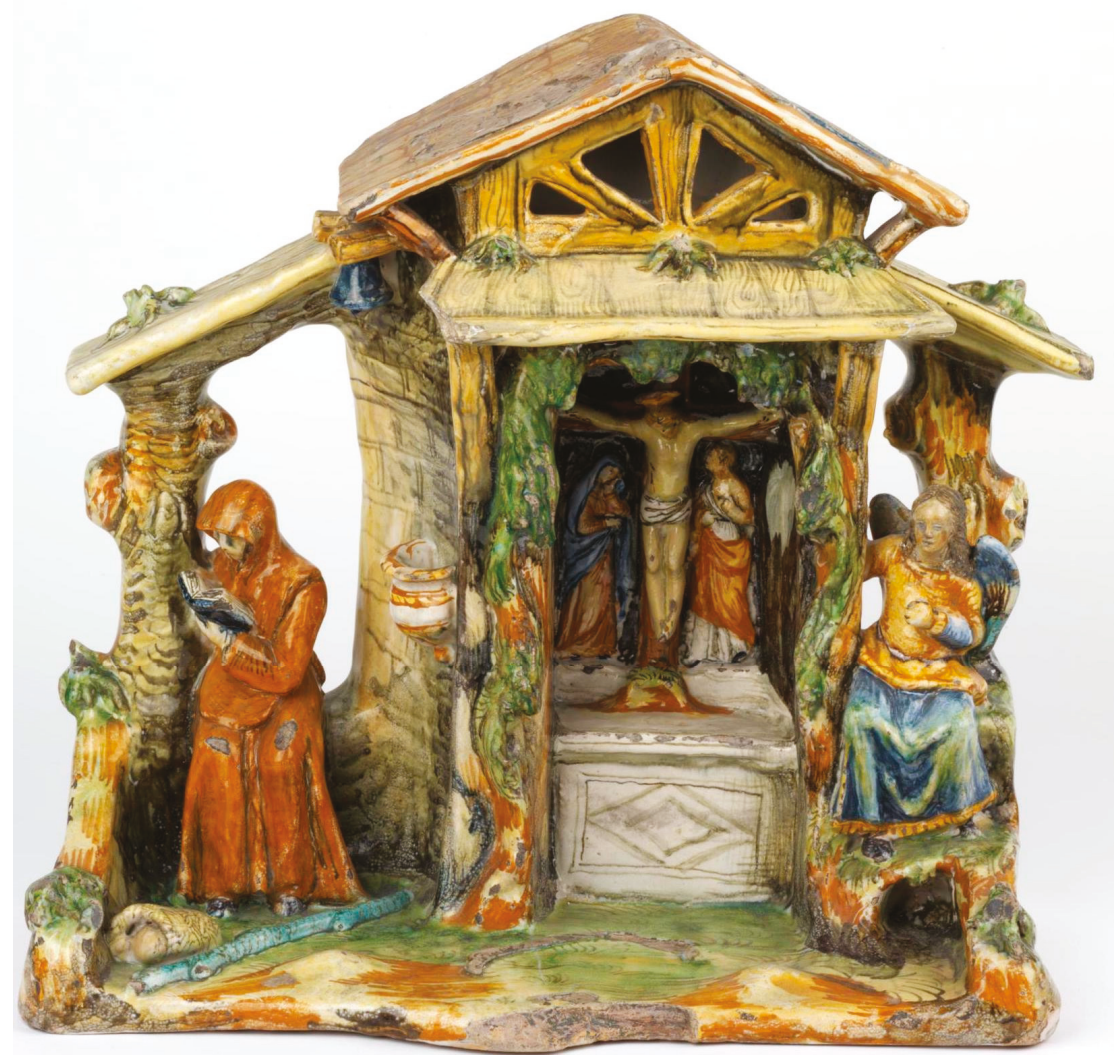

Figure 1. Urbino, Model Altar, c. 1575-1600, maiolica, h: $36 \mathrm{~cm}$, w: $38.5 \mathrm{~cm}$, Victoria and Albert Museum, London, inv. no. C. 258-1926. Photograph (C) Victoria and Albert Museum, London.

Indeed, late Medieval and Renaissance polychromed terracotta sculptures point to the close workshop ties between sculptors and painters. Scholars highlight the dilemma in describing devotional reliefs by Antonio Rossellino (1427-1479), a celebrated Florentine artist, as a 'painted sculpture' or 'painting in relief' (Gentilini 2008, p. 35; Gentilini 2012). This difficulty with the categorization of religious images was not confined to Tuscany. It was a common trend in Renaissance sculpture, linked to the increasing popularity of the medium of polychromed terracotta, which partook of both painterly and sculptural practice. In the case of glazed terracotta, its reflective surface both attracted the eye and had the potential to distract the focused gaze away from the subject matter, so that the faithful could approach the divine not only visually but also through the sense of touch, and subsequently with their minds and souls.

The interchangeability of sculpted and painted images that both Dominici and Paleotti had identified is attested in the written accounts, which shed light on the encounters with domestic devotional images. The inventories of Renaissance households, stories of domestic miracles, and inquisitional records, 
typically use ambiguous terms, such as 'cona' or 'figura' (Henry 2011). Unless a document specifies the material from which an object was made, it is impossible to determine its character. Brief terms, such as 'Item una madonna piccola' or 'Item doe madonne et uno presepio con proprio panisello' (Sarnecka 2018a, p. 170), do not allow us to make clear distinctions between a flat image, a relief and a free-standing figure. Furthermore, inventories prove that devotion at home was also practised in front of multimedia structures. Examining notarial records from Rimini, Angelo Turchini transcribed an inventory which recalled, in the camera da letto of one Francesco Bruni, aromatarius, 'una tavola depincta et dorata in la quale è depincta la figura de la gloriosa vergine Maria cum el suo dulcissimo figliolo messer Ihesu in gremio [sic, possibly mistranscribed 'grembo'] con la historia de li Magi che lo adorano, antiqua [...] un crucifisso de petra terracocta depincto sopra dicta tavola (Turchini 1980, p. 356) [A painted and gilded panel with the Virgin Mary in glory with her sweet son, Lord Jesus on her lap with the scene of the adoration of the Magi, ancient [...] a terracotta crucifix polychromed displayed above this panel]. Thus, it seems that the interaction of the painted and sculpted elements of a tabernacle, or in three-dimensional objects such as the maiolica eremi, underwrote domestic experiences of the divine.

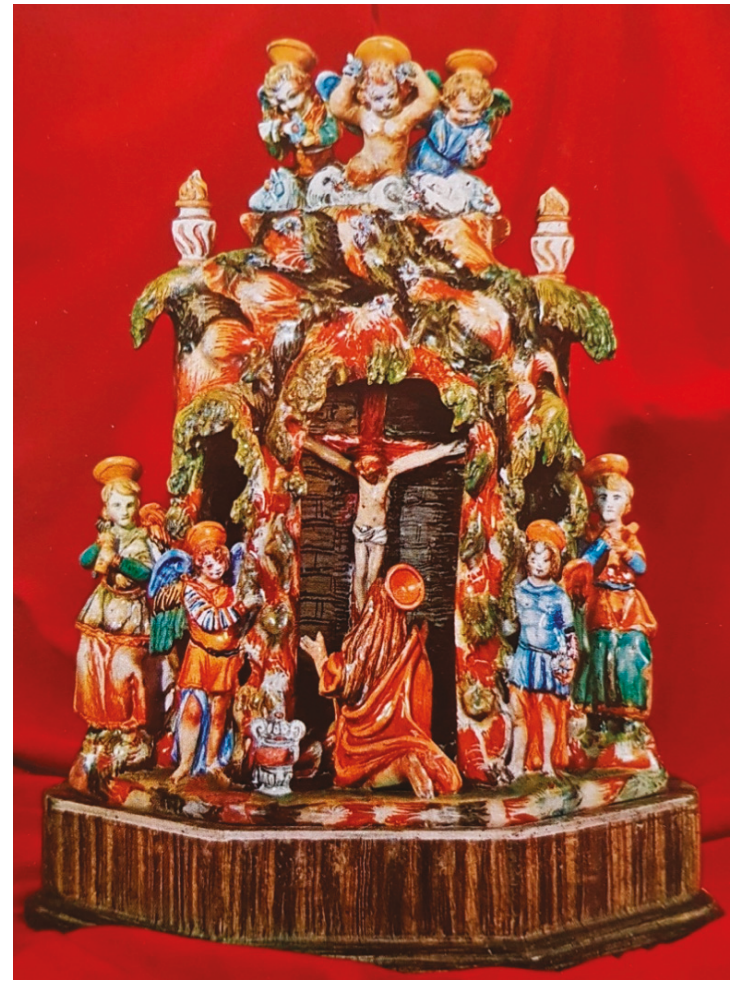

Figure 2. Urbino, Eremo with St Mary Magdalene, c. 1580, maiolica, h: $47 \mathrm{~cm}$, w: $37 \mathrm{~cm}$, unknown location, photo from a sales catalogue Finarte, Milan, November 1964.

\section{Scale, Original Collocation and Early Provenance of Maiolica Models of Chapels}

In a public space, glazed terracotta groups were typically life or nearly life-size. The figures acted as substitutes for real bodies and facilitated the identification of the viewer with the saintly figures. At home, the scale was obviously reduced for practical reasons. The domestic objects typically measured between 35 and $45 \mathrm{~cm}$ in height and width and about 20-30 cm in depth. The smaller scale ensured the 
intimate nature of the encounter within a household. That intimacy was encouraged by the vividness of the maiolica, accomplished through its surface qualities, life-like colouring, and reflectiveness, all of which animated the object.

The maiolica eremi from the Palazzo Ducale in Urbino were catalogued in a section of the inventory entitled Lista delli vasi di maiolica. The title seems to suggest that they were displayed permanently in the palazzo, together with maiolica decorative vases, perhaps in the credenze. However, it is also likely that the inventory catalogued all objects executed in the same technique regardless of their actual location at the time the inventory was drafted. As devotional objects, they could have been displayed permanently in domestic tabernacles or stored in chests to be taken out temporarily. During domestic prayers they could have been placed on a table covered by a tablecloth, with decoration inspired by church altar cloths. The maiolica image of a saintly figure praying before a crucifix, with beeswax candles lit in front of it, would have transformed ordinary furniture into an altarpiece. The centre of devotion in the Italian Renaissance home was typically the camera da letto, or a bedchamber (Brundin et al. 2018, pp. 66-69). However, the meditative quality of these objects suggests that in elite residences, with more rooms, they might have been displayed on a table in a studiolo for meditation and solitary prayer.

Existing studies of Renaissance interiors focus predominantly on Florence and Venice. Indeed, the visual evidence of the scale of buildings in those cities, such as the Palazzo Davanzati, suggests that in similar interiors a sculpture of 70 or $80 \mathrm{~cm}$ in height could have been easily displayed permanently in a tabernacle located in a bedchamber. If we compare the largest maiolica model of a chapel known to the author, namely St Jerome Adoring the Crucifix (from the Musée de la Renaissance, Écouen), which is $54.7 \mathrm{~cm}$ in height and $41 \mathrm{~cm}$ in width, with some of Antonio Rossellino's devotional relief stuccos (Horne Museum, stucco, h: $72 \mathrm{~cm}$, w: $52 \mathrm{~cm}$, Bargello, stucco h: $76 \mathrm{~cm}$, w: $51 \mathrm{~cm}$, Paris, Musée Jacquemart-André, stucco h: $79 \mathrm{~cm}$, w: $51 \mathrm{~cm}$, Opava, Slezské zemské muzeum, stucco h: $67 \mathrm{~cm}, \mathrm{w}$ : $46 \mathrm{~cm}$ ), which we know were destined for private Florentine homes, the maiolica model is significantly smaller. Moreover, Gentilini categorised Rossellino's Virgin and Child relief of over $50 \mathrm{~cm}$ in height explicitly as the 'tipo piccolo' (Paris, Musée Jacquemart-André, h: $53.3 \mathrm{~cm}$, w: $37 \mathrm{~cm}$ ) (Gentilini 2008, p. 39). Therefore, to follow the Florentine logic, maiolica chapels of approximately $35-45 \mathrm{~cm}$ in height would fit perfectly into the purpose-built tabernacles in bedchambers of even less grand structures than the urban palazzi.

The nineteenth-century provenances are often cited as an argument for the church as the original location of many maiolica devotional sculptures. Church provenances, however, should not be surprising, as many devotional objects were sold publicly when a financial need occurred, or were bequeathed to the church or to confraternities after the death of the last family member (Matchette 2006, p. 712). That domestic objects could be inherited by the Church is clear from documentary evidence. Many wills stipulated that a house, with all its moveable and immoveable contents, should be given to a certain church or a monastery after the death of the last family member, or if the family became impoverished and wished to sell the house. Such was the case in Guido Mazzoni's will (9 July 1518), which stipulated that the members of his family were prohibited from selling the house (proibisce che la casa in alcun tempo possa essere venduta) and, if they attempted the sale, then the rights to the house should be given to the monastery of Saint Peter in Modena (e se la alienavano, i Mazzoni dovevano essere privati di tale diritto e dovevano essere sostituiti nel possesso della casa dal monastero di San Pietro della città di Modena) (Venturi 1889, pp. 155-57). Similar bequests under conditions of entail were not unusual in Renaissance Italy, and the presence of small glazed terracotta sculptures inside churches may be linked to this practice, rather than commissions by the church (Cohn 2011, pp. 8-9). ${ }^{2}$ The issues of the original collocation and the early provenance of maiolica models of chapels and sanctuaries are challenging and general conclusions should be drawn with caution.

2 Samuel Cohn discussed this practice in detail and linked it to the post-Black Death mentality to protect goods from entering the general market and ensuring that they remain among family possessions or within repositories of the Church. 


\section{Function of Maiolica Models of Chapels and Sanctuaries}

The practice of owning small domestic models of chapels and sanctuaries probably developed from the communal need for devotional architectural street shrines, typically referred to as 'eremo' or 'eremitorio' (Guidotti 1976, p. 15ff; Pia Mannini 1981, p. 11). In fact, some surviving domestic examples are explicitly described as models of wayside shrines. These maiolica forms always feature at least a suggestion of a landscape. The grotto-like structure of the chapels refers to a spiritual refuge. They brought an inspiring aura of religious contemplation and piety into an elite private space, which was very unlike the rustic, derelict chapel depicted in the object (Figure 3).

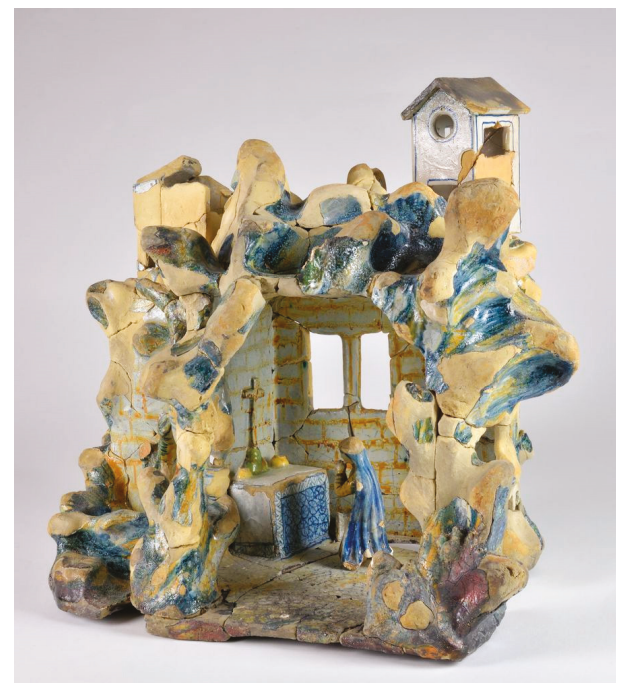

Figure 3. Urbino, Tempietto with the Praying Virgin (the figurine not originally belonging to the object), c. 1560, maiolica, h: $42.5 \mathrm{~cm}$, w: $45 \mathrm{~cm}$, d: 28 cm, Museo Internazionale delle Ceramiche, Faenza, inv. no. 10405,1. Photograph (C) Museo Internazionale delle Ceramiche in Faenza.

The maiolica hermitages may be seen as domestic extensions of the secluded romite, in which Franciscan friars prayed away from the cities. St Francis stressed the significance of nature in enacting private devotion, a reminder that became increasingly important towards the end of the sixteenth century. This may be linked to Post-Tridentine piety, which highlighted the importance of the contemplation of God in nature. Federico Borromeo (1564-1631) viewed landscape paintings as important vehicles for sending a message about Christian values and commissioned works from artists such as Jan Brueghel the Elder that showed hermits studying sacred texts in the vast landscapes, often with ruins (Jones 1988). A set of engraving—Solitudo, sive Vitae Patrum Eremicolarum—by two Netherlandish artists, Johann and Raphael Sadeler, owned by Borromeo, inspired these paintings (Jones 1988, p. 263) (Figure 4). The genre included important fifteenth-century examples, such as Paolo Uccello's The Thebaid c. 1460 (Galleria dell'Accademia, Florence), which included St Francis, St Jerome and other saint hermits portrayed in secluded spaces within a vast landscape.

The maiolica eremi share with these images a similar approach, depicting a figure who contemplates a crucifix or reads a religious book, as in the example from the Victoria and Albert Museum in London (Figure 1). In this maiolica chapel, set in nature, St Francis reads piously, and the pilgrim staff placed on the ground before him clearly divides his space from the outside world. Even the angel depicted to the right of the chapel is strikingly aware of her physical presence; she seems to hide behind the chapel's wall to avoid disturbing the saint. To assist the faithful in imagining themselves sharing the saints' space and devotional experience, the maiolica models of chapels include telling details, such as 
the bell by the standing figure of St Francis with a book (Figure 1) or a highly detailed representation of an altarpiece with the crucifix, blue and white altar cloth and two candlesticks (Figure 3). We do find bells in domestic inventories; perhaps they summoned family for prayer (Morse 2007, pp. 170-74). Thus, the maiolica prompted an imagined sound that could have been re-enacted at home with small brass bells.

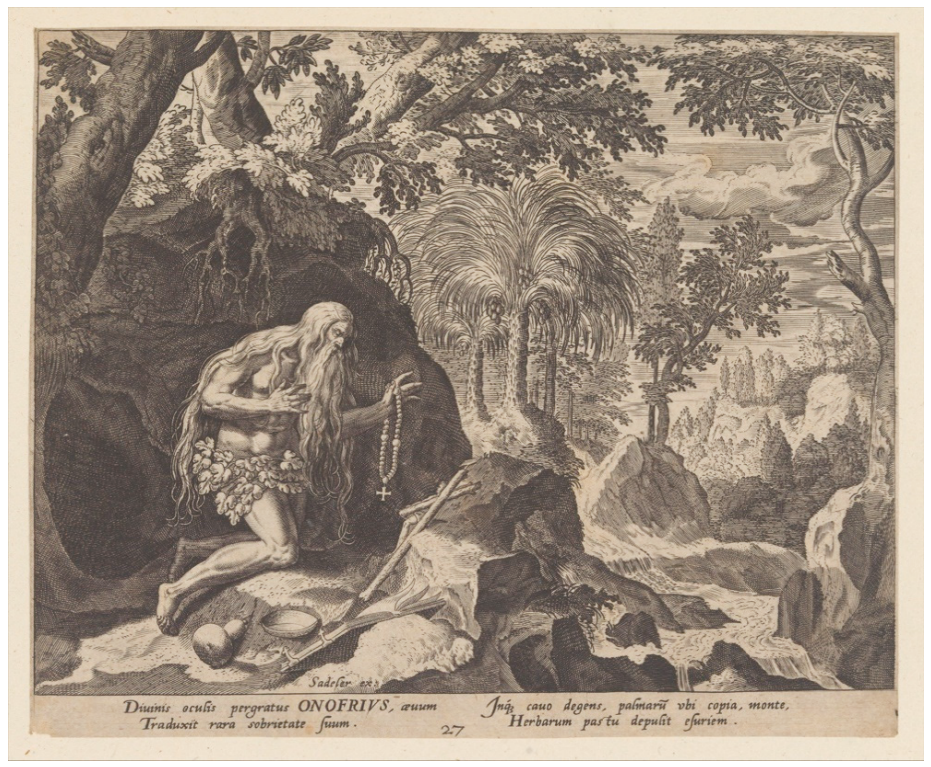

Figure 4. Johann and Raphael Sadeler, St Onofrius, from the series Solitudo, sive Vitae Patrum Eremicolarum, 1585-86, engraving, h: 17.4 cm, w: 21.3 cm. Metropolitan Museum, New York, inv. no. 49.95.1293(28). Image in the Public Domain.

Another way of merging the space of the faithful with that represented in the model of the chapel was through the use of light. The effects of candlelight upon reflective glazed surfaces would have been particularly transformative (Kupiec 2019, pp. 83-97). Furthermore, candlelight was indispensable if the viewer was to see the deeply recessed details of the statuettes. The Crucified Christ, the Virgin and St John, in the example from the Victoria and Albert Museum, are somewhat concealed within the structure; candlelight would have brought out their forms. The prominent perforations in the roof beams directly above the group would have enhanced this effect, allowing the light to be refracted, thus further animating the scene.

Most of the surviving maiolica sculptures of this type show similarly tranquil, manifestly secluded environments, where the derelict architecture is integrated with nature. The example preserved in the Museo Internazionale delle Ceramiche in Faenza (Figure 5) shows the chapel framed by other religious buildings in a unifying landscape.

The desire to touch and manually operate the figures was an important way in which the beholders interacted with the devotional sculpture in the sixteenth century, and is crucial for understanding of the popularity of the maiolica models of sanctuaries and chapels. The material properties of glazed terracotta—namely the brilliant, vivid colours, durability and portability—called out for physical contact. Moreover, the fired surface, which ensured the immutability of the colours, also reduced the risk of abrasions, even with regular and intense handling. Strength was a unique property of these religious artefacts, as at that time every other painted surface was prone to rubbing off or smudging, due to exposure to natural oils on hands or saliva on lips (Rudy 2010, 2017). Through the centuries, 
the integrity of small maiolica statuettes was threatened solely by fire and bombardment, as is clear from the present state of the example preserved in Faenza (Mazzotti 2018, p. 37, Figures 15-16 show the state of the eremo before and after the bombardment of 1944).

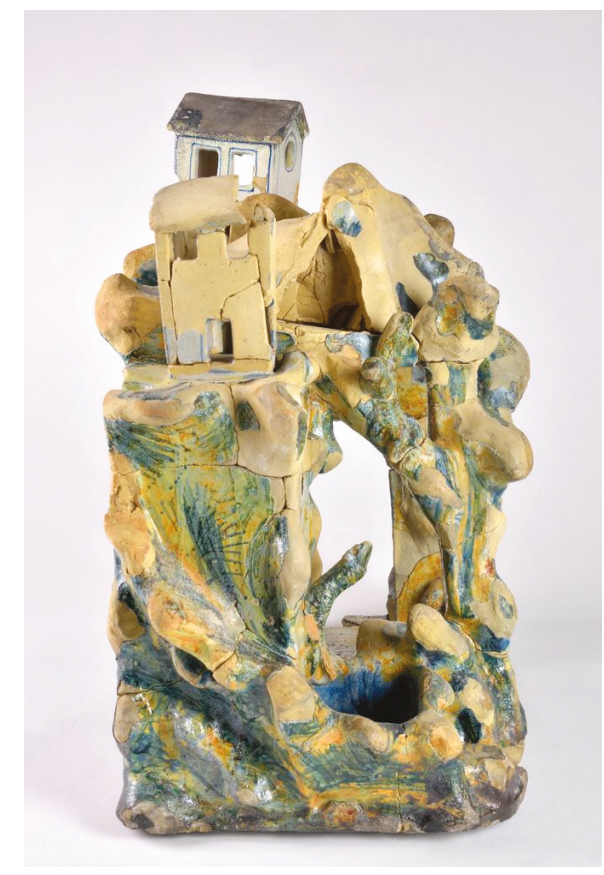

Figure 5. Side view of Figure 3.

The Faenza maiolica eremo includes an opening with painted water on the left-hand side. It appears to imitate a lavabo for handwashing before mass, but it seems that there is no system for actual water to flow through this tempietto. However, it is likely that the space served as a receptacle for holy water, as in the many maiolica acquasantiere. Water is important element in all these models, as it facilitated the experience of the imagined space as spiritually real. This feature became a tangible link between the space in which saintly figures were visualized and the space of the faithful. All surviving examples of maiolica eremi have smaller or larger containers, presumably for holy water (Figure 6).

The presence of these openings has led scholars to suggest that similar structures were table fountains, that entertained guests through sprinkling wine during dinner (Gardelli 1987, cat. no. 64 B, p. 150). Such might be true of models with secular themes, such as the Bacchus sitting on a grassy mount, flanked by two goats, pouring wine from a jug to his chalice (Figure 7). However, the prominent religious character of the eremi excludes the entertaining function. The openings, clearly designed to contain a liquid, motivated the shift in the objects' role. An eighteenth-century inventory of the guardaroba of the Palazzo Ducale in Urbino (1758) confirms that devotional structures with similar openings were used in subsequent centuries as inkstands: 'Tabernaculo uno di majolica alto, nel quale sono quattro figure di rilievo, si scompone in più pezzi con colonne, et altri ornamenti et serve per calamaio' (Oliveriana, Ms. 460, c. 89r). ${ }^{3}$ The entry highlights the original devotional role of the object, a multipart tabernacle with four figures in relief, columns and other decoration, at that

3 I am very grateful to Claudio Paolinelli for making me aware of the document. The transcription is mine. 
time used as an inkstand. The object listed in the inventory was probably more similar to a large-scale tabernacle, such as the one with St Mark and St Luke, in the collection of Palazzo Madama in Turin (inv. no. 623/C) (Maritano 2008, pp. 32-33), than to the smaller models of chapels. However, similar changes in function are reflected in the catalogue entries of eremi, for instance, the example from the collection of the Museo di Arti Applicate in Milan. According to the museum's archival documents, the maiolica statuette was initially called altarino, and subsequently calamaio, attesting to the shift in function from devotional to practical (Wilson 2000, p. 239).

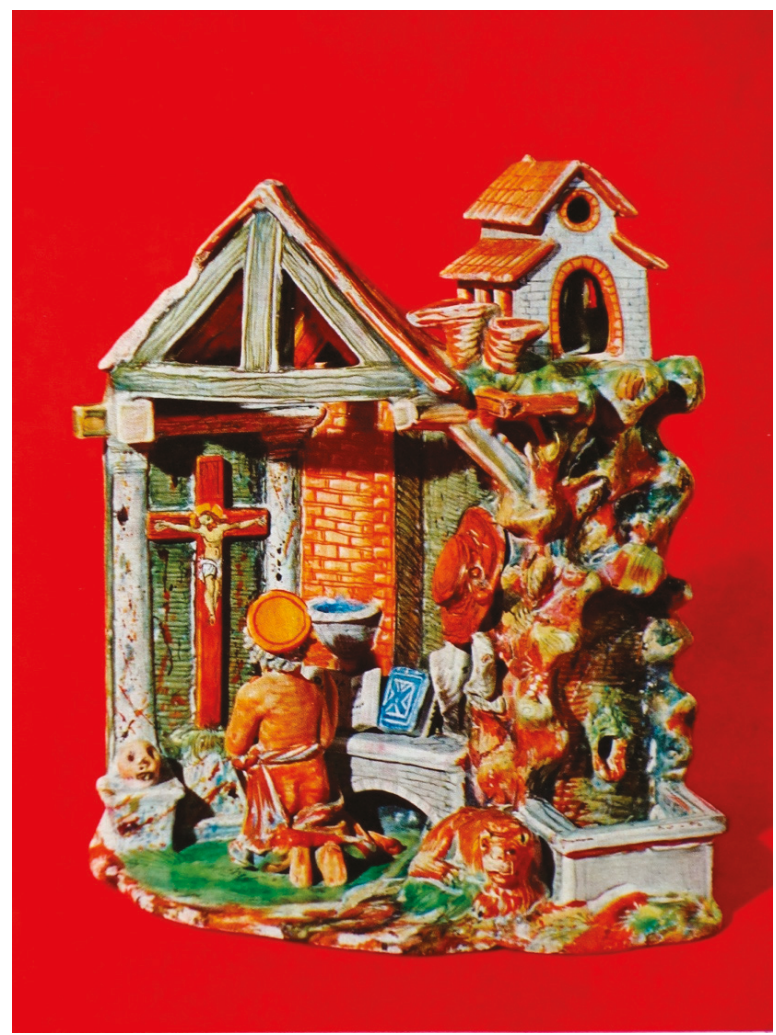

Figure 6. Urbino, Chapel with St Jerome, c. 1590, maiolica, h: $36 \mathrm{~cm}, \mathrm{w}: 35 \mathrm{~cm}, \mathrm{~d}: 22 \mathrm{~cm}$, unknown location, photo from a sales catalogue Finarte, Milan, November 1963.

The Faenza model of the chapel offers many interesting views, and different structures can be seen from the sides rather than simply from the front. Some maiolica figures were clearly meant to be viewed simply frontally, such as the statuette showing St Paul the Hermit and St Anthony the Abbot (Sarnecka 2018b, p. 271; Paolinelli 2019a), in a private collection in Cento. The group may have been inspired by a print, as the subject matter was popular in the early sixteenth century. Dürer's workshop created a woodcut version of St Anthony Visiting St Paul in the Wilderness (c. 1503-4), which includes a stream of water flowing between the two saints, dividing the space into two sections (Strauss 1980, p. 202), very similar to the one depicted in the maiolica statuette. However, the maiolica includes many attributes of the hermit saints, such as the prominent rosary of St Anthony and the book held by St Paul, which are omitted from the print. The maiolica group includes standard elements of the scene, such as the raven that brought bread every day to the hermits. The back of the maiolica group is painted white, which suggests that it was placed in a niche. The majority of maiolica figurines, 
however, are finished as if to be viewed also from the back but this might not necessarily be proof of how they were actually viewed.

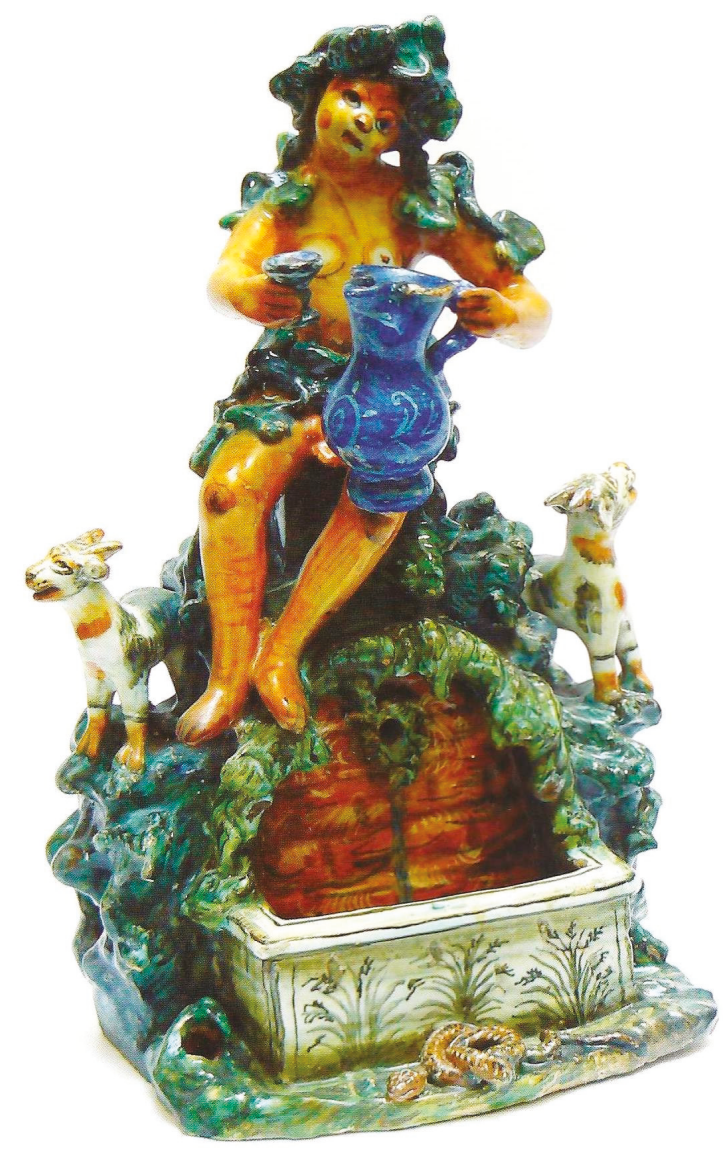

Figure 7. Urbino, Table Fountain with Drinking Bacchus, c. 1580, maiolica, h: $45 \mathrm{~cm}$. Photograph (C) Catalogue of the sale of maiolica JM Béalu \& Fils 2010.

Movement around the eremo was certainly implied in a model of the La Verna sanctuary (in the collection of the Princeton Art Museum) (Figure 8) (Prentice von Erdberg 1961). Stylistically different from the eremi discussed thus far, this model of the sanctuary seems to have originated in a monastic context. Its form stems less from the desire to represent pious activities than from the will to emphasise spiritual links between locations, and to stress affinities between geographically distant monasteries. The beholder was invited to move around the three-dimensional visualization of a monastic complex to gain a better understanding of the spatial relationships between various structures, such as the Church of Santa Maria degli Angeli, the Upper Church (Chiesa Maggiore), the Chapel of the Stigmata, and the rock from which the water miraculously sprung. With great accuracy, the model of the La Verna sanctuary shows scenes of monastic life set in the architecture of the holy place in Casentino. It is dated to 1521, exactly ninety years after La Verna was given to the Observants, and might have been commissioned to celebrate this event (Di Miglio 1568, p. xiii). This relatively early dating also proves that maiolica models of chapels and sanctuaries were not exclusively a Counter-Reformation phenomenon, but were in use long before the Council of Trent to establish spatial and spiritual links 
between various locations. The model of La Verna provides multiple views and could have invited interaction beyond the mere emulation of the represented figures praying. One could narrate the daily life of a Franciscan friar by observing each figure and his movements through the sanctuary, with its combination of churches and other religious buildings set in the surrounding landscape. The behaviour of the friars demonstrates the contemplation of God through His creation, one of the key elements of Franciscan teaching (Figure 9).

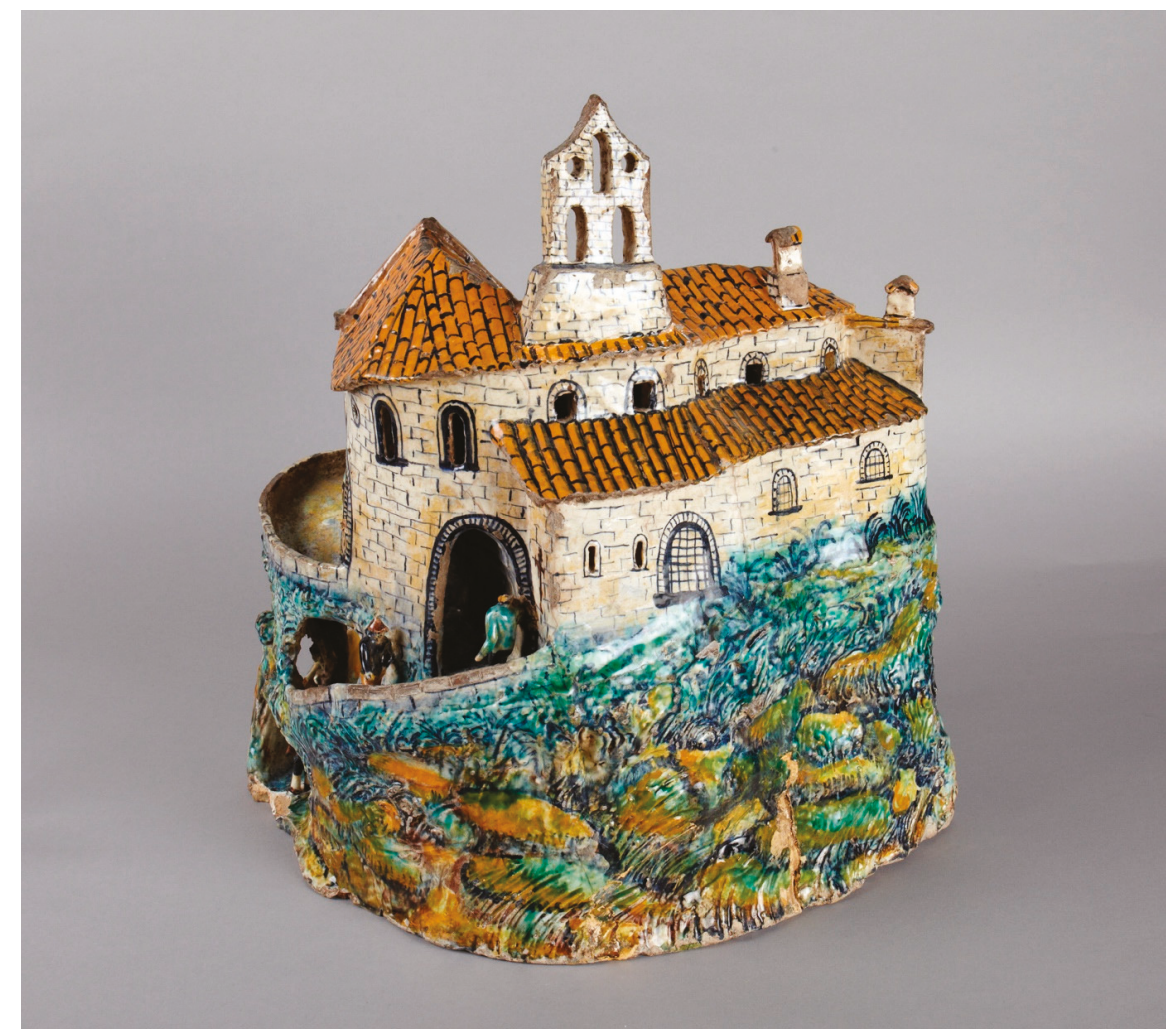

Figure 8. Urbino (?), Model of La Verna Sanctuary, 1521, maiolica, h: $35 \mathrm{~cm}, \mathrm{w}: 36 \mathrm{~cm}, \mathrm{~d}: 33.3 \mathrm{~cm}$, Princeton University Art Museum, inv. no. y1929-24. Photograph @ Princeton University Art Museum.

The holiness of a recognisable place, in this case the La Verna sanctuary, introduces sanctity into another space, by encouraging a mental pilgrimage. Scholars have discussed enclosed gardens as vehicles for mental pilgrimages-extensions of holy places (Rudy 2011, pp. 110-18). The concept of showing realistically the architecture of the place where St Francis received his stigmata was conveyed in larger glazed terracotta altarpieces, for instance in the Franciscan Observant church in Barga, Tuscany, attributed to Girolamo della Robbia (Gentilini 1983, pp. 218-20). The representation of La Verna was also included in other glazed terracotta altarpieces, such as the altarpiece in the Chapel of Vieri-Canigiani, Santa Croce, Florence; the Baglioni altarpiece, from the family chapel now in the Museo della Porziuncola in Assisi; as well as in the predella of the altarpiece of Rocca di Gradara, near Pesaro, the composition of which was repeated in the altarpiece for the Oratorio della Madonna del Buon Consiglio in Prato (Marquand 1922, pp. 37-38). These numerous altarpieces demonstrate that sculptural representations of La Verna, imbued with the aura of the miracle and with St Francis's holy presence, were popular across Central Italy. It seems possible that the tradition of creating 
small-scale, maiolica models of sanctuaries originated from the large altarpieces that visualised such places, but which did not satisfy the need for direct sensory engagement (Figure 10). The same need nourished the pious fashion for domestic maiolica models of chapels in the second half of the sixteenth century.

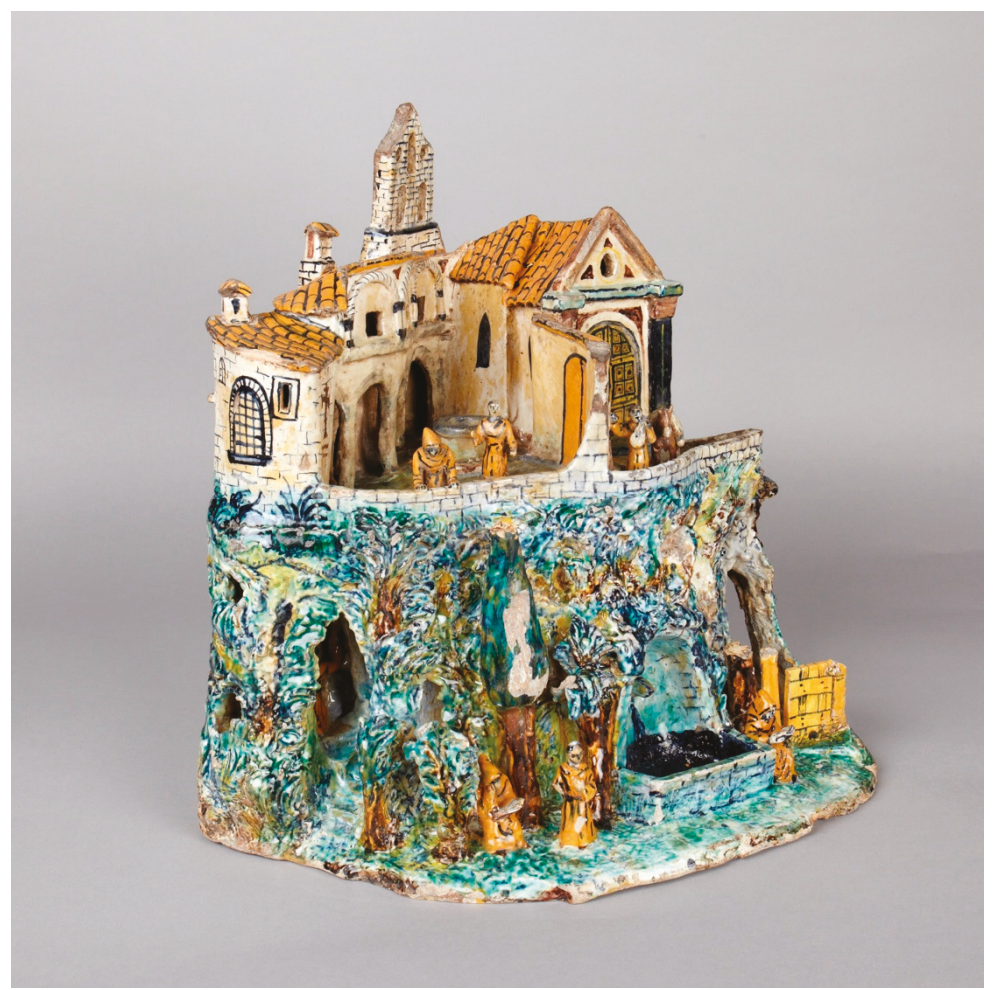

Figure 9. Frontal View of Figure 8.

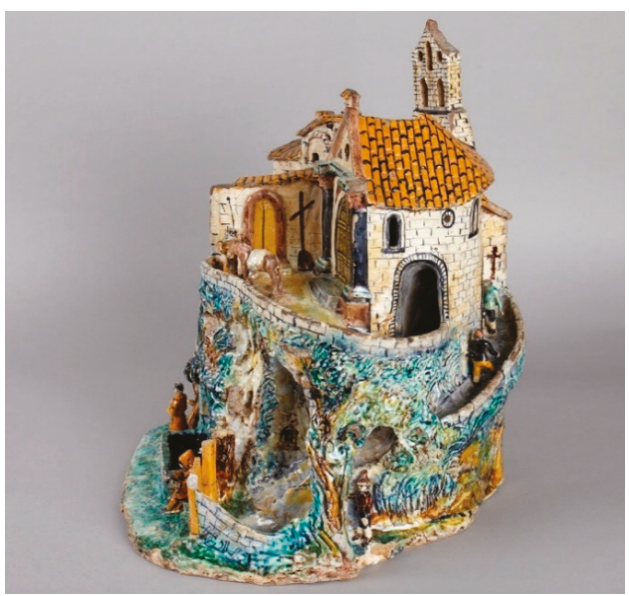

Figure 10. Side View of Figure 8. 
Another glazed terracotta eremo, in the Museum of Fine Arts in Boston, shows St Francis surrounded by nature, contemplating the crucifix and receiving the stigmata (Figure 11) (Cambareri 2007a, pp. 61-69, on p. 67). It is likely that in this example, too, the beholder was able to contemplate the importance of La Verna sanctuary, although the connection is less explicit than in the example from Princeton. The biography of St Francis recounts that he retreated to this place two years before his death. The location was defined as a heremitorio, and St Francis's solitude both before and after the stigmatization is one of the most important experiences in La Verna (Da Celano 1904, pp. 74-75). For generations, artists imagined the moment in which the saint received the stigmata and formulated the traditional elements of the scene (Goffen 1988; Brooke 2006, pp. 160-217, 281-83, 401-4; Rutherglen 2015).

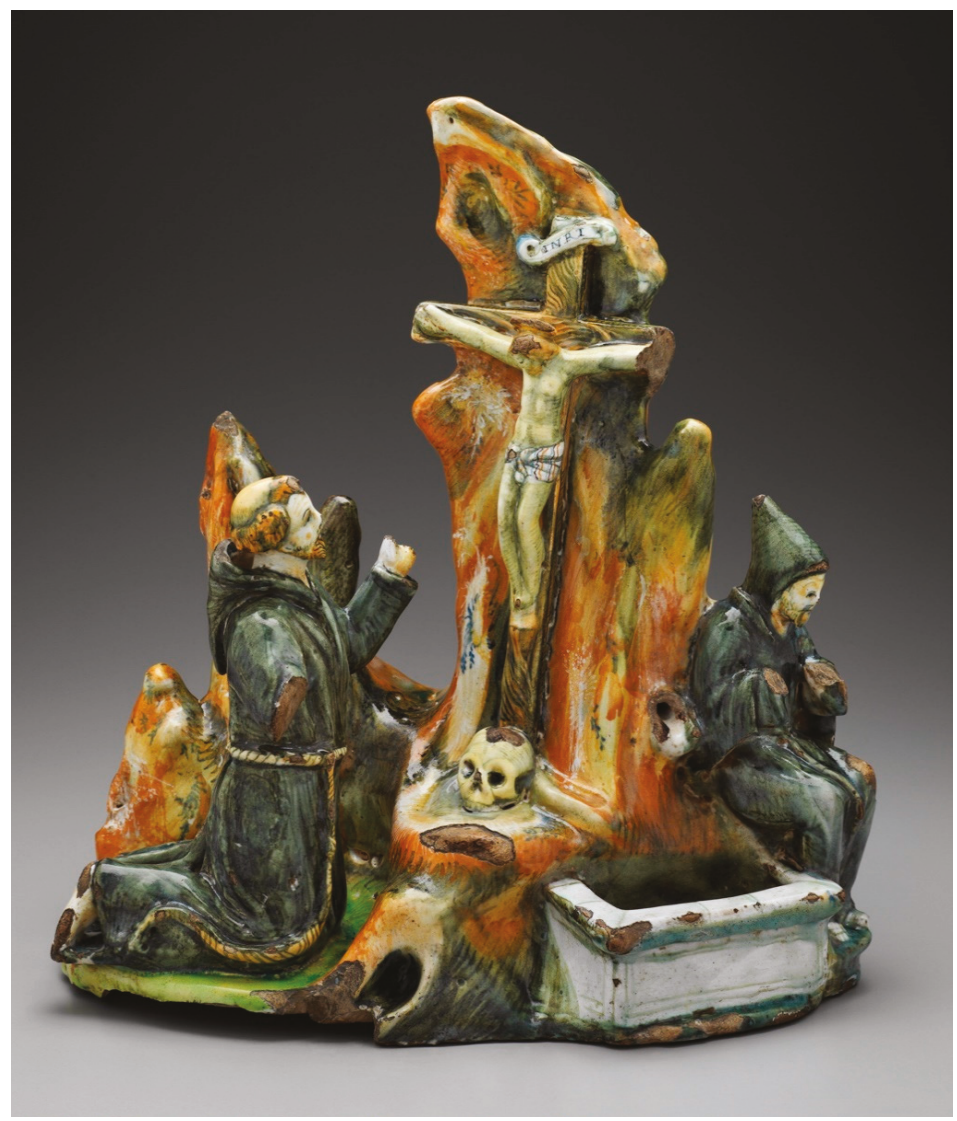

Figure 11. Urbino (?), Saint Francis receiving the Stigmata Italian, c. 1540 , maiolica, $36.8 \times 33 \times 22.9 \mathrm{~cm}$. Museum of Fine Arts, Boston, inv. no. 53.2912. Gift of Charles B. Barnes and W. D. Gooch, Executors of the Estate of George R. White Photograph (C) 2019 Museum of Fine Arts, Boston.

In the maiolica statuette, we see the Crucified Christ, but not the six-winged seraph usually depicted in the images of the stigmatization. Moreover, unlike in paintings or sculptures in relief, there are no linear rays to connect the five wounds of the Christ with the newly imprinted, miraculous openings in the flesh of St Francis. Below the maiolica Crucifix there is a skull, typically found in similar statuettes showing St Jerome but rarely included in representations of Francis's stigmatization. It clearly references Golgotha, thus characterizing La Verna as the new mountain of salvation of 
mankind. Moreover, this 'holy place' was envisaged by God as the site of the stigmatization from the beginning of time, as Bartolomeo da Pisa describes in his De conformitate vitae Beati Francisci ad vitam Domini Iesu (Fleming 1982, pp. 7-8, n. 8). Perhaps inspired by Bonaventure, Bartolomeo da Pisa emphasized the intense spiritual contemplation of the Passion and internalization of Christ's suffering that preceded the appearance of physical wounds on Francis's body. Francis's gaze, focused on the greenish tones of Christ's body set against the spiky rocks, imbues the central scene with a religious intensity that dramatically contrasts with the sleeping figure of brother Leo (Meiss 1964, p. 21). The prominent chasm in the foreground might be a reference to the belief that when Christ died on the cross La Verna was struck by an earthquake, which created its characteristically sharp-edged landscape. Bartolomeo da Pisa noted the event as: "Nam tempore passionis, ut patet in Evangelio, petrae scissae sunt; quod singulari modo in monte isto (La Verna) apparet" (Da Pisa 1906, p. 387). This element of the maiolica sculpture would thus convey the distance between the devotee and the saintly figure depicted in the centre. The meditating person was supposed to conceal her or himself mentally inside the crevasse, so as to be isolated from the saint as decorum demanded.

The composition of the Stigmatization of St Francis is likely to have been based on one of the hugely popular prints showing the scene, which had circulated widely in Italy since the end of the fifteenth century, as single sheets or as frontispieces for the Fioretti (Sarnecka 2018b, p. 267). We know that prints were widely used for the decoration of maiolica plates, and perhaps the same holds true for three-dimensional objects modelled by the same artists (Marabotti Marabottini 1982). The spiky rock behind St Francis's feet and the pose of friar Leo behind a prominent tree could have been based on the print by Marcantonio Raimondi after Dürer's woodcut. It is also possible that the artist who modelled the scene of the stigmatization had access to a northern print, as they circulated widely in Italy. The Adorno family from Genoa, residents in Bruges, had a panel by Jan van Eyck showing $S t$ Francis Receiving the Stigmata (Rohlmann 1999, p. 39). One memory of the act of contemplation of Dürer's print was described by Fra Sabba da Castiglione (c. 1480-1554), who, in a garden at the foot of the Mount Formicone, marvelled at the artist's composition "which is certainly divine, and which had recently arrived from Germany" (Thornton 1997, p. 111). That maiolica statuettes could have derived their composition from prints has already been suggested in relation to inkstands showing St George on his horse fighting the dragon, which might have been inspired by woodcut illustrations from 'battle books' or other written sources (Thornton 1997, p. 165).

The object seems to facilitate a range of devotional practices. The opening to the right of the Crucifix might have been a receptacle for holy water, as in the maiolica models of chapels. It does have a little tube through which water could have flowed, but the view of the back seems to suggest that this was only to create an illusion, and perhaps to enhance the link between the holy water kept at home and water from the La Verna sanctuary. The holy water could be used to make the sign of cross, thus marking the bodily preparations for spiritual engagement with the divine. The faithful brought blessed water from their parish churches or from various shrines as a pilgrim souvenir (Brundin et al. 2018, p. 4). The water that sprang miraculously from the rock at La Verna is one of the most vivid stories from St Francis's life, and one that clearly identifies him as the antitype of Moses and Christ. The other curious element is the structure of the back, which connects the opening to a chimney-like rock behind the Crucified Christ. It seems likely that this connection was created to allow the burning of incense and the smell would have added to the devotional experience (Sarnecka 2018b, pp. 268-69). Similarly, paper flowers could have been placed in the small holes spread across the top of the eremo (Cambareri 2007b). The glaze runs inside these holes, and therefore they must be original. It has been suggested in relation to a Florentine sculpture of St Jerome in Penitence (Ashmolean, WA 1960.64) that eighteen small holes in the rocks behind the saint 'would presumably have held representations of trees or other vegetation' and that these additions in terracotta or another material would have enriched the landscape (Warren 2014, pp. 483-84). Other reliefs showing the lives of saints, such as St John the Baptist from Budapest, also include holes and small trees (Museum of Fine Arts, Budapest inv. no. 1121) (Balogh 1975, no. 69, Abb. 93). 
The Last Supper, in the Museum of Fine Arts in Boston, is another maiolica sculpture, which does not strictly belong to the group of maiolica models of sanctuaries or chapels but shares numerous key characteristics with this category (Figure 12). Most importantly, it points to the operational potential of these objects. The maiolica models of chapels and sanctuaries invited faithful owners to animate the scene by turning the object around or to project themselves into the space occupied by the saints. In the maiolica Last Supper, movement similarly informed the devotional experience. The figurines of apostles are not simply designed to be looked at, but also to be touched and re-arranged. Close examination of the glazed terracotta group reveals that the figures could be moved to a different spot and taken off their stools (Cambareri 2007a, pp. 66-67). This physical manipulation perhaps encouraged the owners to enter into the drama of the unfolding events.

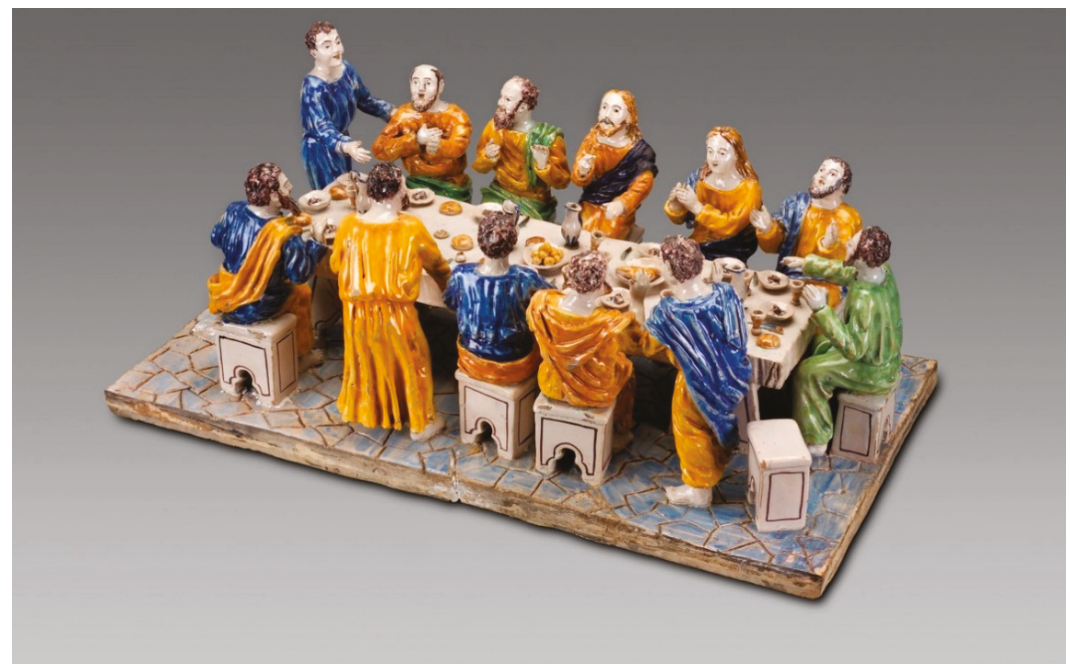

Figure 12. Faenza, The Last Supper, 16th century, maiolica, $21.6 \times 32.6 \times 58.1 \mathrm{~cm}$. Museum of Fine Arts, Boston, inv. no. 1983.61. Bequest of R. Thornton Wilson in memory of Florence Ellsworth Wilson. (C) 2019 Museum of Fine Arts, Boston.

Though scenes of the Last Supper are by no means unusual in Renaissance and Baroque paintings, three-dimensional representations of this subject are rather rare in the Early Modern period. Therefore, it is significant that we have another example of this iconography created in the medium of glazed terracotta, namely a relief attributed to Giovanni della Robbia based on a print after the celebrated composition by Leonardo da Vinci (Figure 13), in the Victoria and Albert Museum (Lambert 1987, pp. 198-99, cat. no. 215 and 218). Scholars argued that the relief was designed for the refectory of the Convent of San Francesco in Barga (Hess 1999, p. 13). If that was indeed the case, it would point to a similar relationship between the representations included in glazed altarpieces and in small, maiolica domestic models, as in the case of the La Verna sanctuary. The artist responsible for the Last Supper, in Boston, created a detailed composition, paying great attention to minutiae. The clear interest in realistic depictions of vases, cutlery and food recalls a still-life depicted in a fresco of the Wedding at Cana from the Church of San Nicolà in Tolentino. In the maiolica group, the vases are decorated with simple motifs of blue lines on white background, promoting the two basic colours of Renaissance ceramics. 


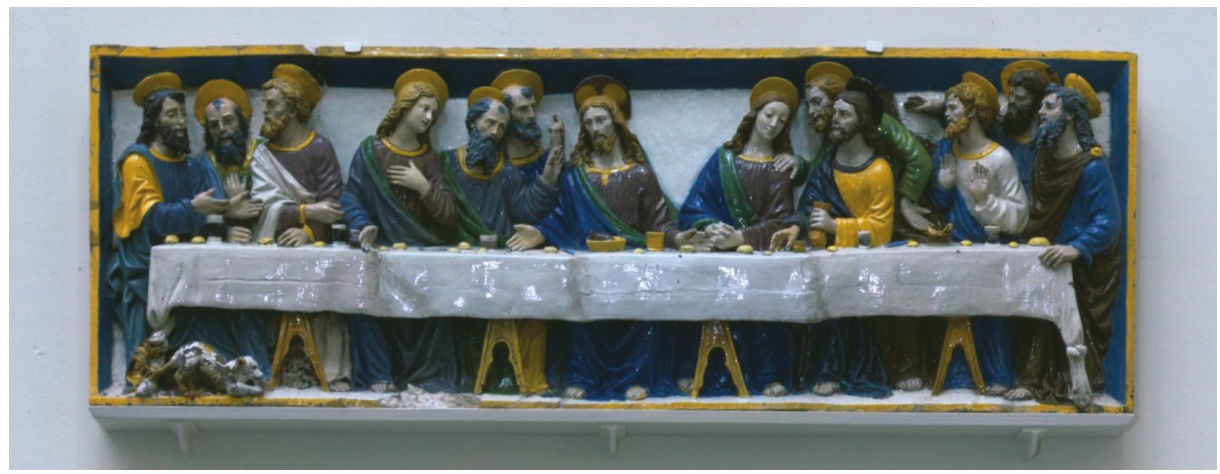

Figure 13. Giovanni della Robbia, The Last Supper, 1515-1520, tin-glazed terracotta, h: $55.88 \mathrm{~cm}, \mathrm{w}$ : $162.56 \mathrm{~cm}$. Victoria and Albert Museum, London, inv. no. 3986:1 to 3-1856. Photograph (C) Victoria and Albert Museum, London.

The artist notably used manganese purple rather than blue to delineate the facial features, and the handling resembles, to an extent, that of Giovanni della Robbia in the Last Supper and in his smaller scale reliefs, such as the Meeting of Christ Child and Young John the Baptist (c. 1510), at the Museo Nazionale del Bargello, Florence (inv. no. 15474). Moreover, the artist responsible for the maiolica paid great attention to the modelling of the hair. The firing of individual figures is very accomplished, as there is no evidence of air bubbles or crawling. The object is executed in a technique typical for the maiolica statuettes, whereby a white glaze applied to the biscuit-fired surface provides the basis for subsequent painterly decoration. Thus, white remains visible here and there underneath the blue or yellow surfaces of the robes. However, the boundaries between different colours are well defined, for instance in the necks, which remain white planes enveloped by the colourful robes. Only in some areas glazes run during the firing and colours from the robes affected elements that were supposed to remain white. The same has happened with the blue from the vases and jars on the tables, which have run into the white surface of the tablecloth.

Almost all hands and other protruding elements of the group are intact, which suggests that the object was kept in one place for a long time. This means that it was designed for a specific location, perhaps a refectory in a convent. Certainly, the iconography of this small maiolica group would be suitable for a space where eating would take place. Every apostle is highly individualized, not only by the colour of his robes, but also through varied gestures and facial expressions; some figures are clearly amazed, others are shown in adoration or in shock. Overall, the lively atmosphere, which results from the animated responses of the apostles, seems to be rather positive. The atmosphere of conviviality and joy resultant from the meeting around the table could have inspired a similarly positive attitude among the people gathered around the object, be they friars, nuns, or laypeople.

The figures do not seem to be related to the Fontana or the Patanazzi workshops active in Urbino that were responsible for the maiolica eremi discussed above. John Mallet has suggested that the Boston Last Supper should be categorised in the bianco di Faenza class, though he stressed that it did not necessarily have to have come from the city famous for its production of high quality maiolica. ${ }^{4}$ He proposed a dating 'quite far into the seventeenth century', linking the convivial representation to the little-studied compendario figure-groups. ${ }^{5}$ Subsequently, Timothy Wilson has proposed that it

4 Letter from John Mallet to Marietta Cambareri preserved in the object file at the MFA, dated 1998.

5 Ibid. 
might have been produced in Urbino around $1600 .{ }^{6}$ Wilson's proposed dating links to the inventory of possessions in the Ducal Palace in Urbino.

\section{Conclusions}

The maiolica models of chapels and sanctuaries, with their complex structures and narrative encouragement, might have inspired a multisensory experience of the divine. By representing saints kneeling before the altar or contemplating a crucifix in a landscape, they provided a model for everyday pious life. Small maiolica figures seemed to reflect a fashion for mixed-media religious objects and a desire to enact devotions with religious objects that escaped simple categorization as a sculpture or a painting. They were modelled as sculptural objects but much of their expressive quality depends on their vivid colours, skillfully and imaginatively applied to the white tin-glaze covering the surface of the biscuit-fired earthenware. Both their roles in defining space and the ways in which the viewer seems to have been expected to engage with them varied. Unlike glazed terracotta altarpieces, maiolica eremi could have originally formed a part of a more intimate and private setting. Moreover, their portability and reduced scale seem to have allowed for a more immediate and physical pious experience. Although they were less lifelike than full-size effigies, they had the significant advantage of inviting handling and touch more freely than sculpted altarpieces in church. This realisation about maiolica statuettes offers a positive and revealing perspective, in which their spiritual significance, technical accomplishment, and functionality come to the fore. Della Robbia and the minor masters had complementary specialisations; each was vital for answering specific pious and aesthetic needs. This dynamic suggests a symbiosis rather than a divergence between these two artistic products. Even if they satisfied different markets and adopted different aesthetic preferences as types of objects stimulating devotional experience, both were glazed earthenware with luminous surface, and both were treasured for satisfying devotional needs and expectations. The focus on these artefacts from the perspective of technique and devotional impact, challenges unhelpful distinctions between higher and lower art.

Funding: This research was funded by the European Research Council, grant number 319475 and by the National Science Centre, Poland, grant number 2018/29/B/HS2/00575.

Acknowledgments: I am very grateful to Deborah Howard, Claudio Paolinelli, Carmen Ravanelli Guidotti and Bella Szala for their comments and assistance with this research. Many special thanks to Marietta Cambareri, Courtney Harris, Abigail Hykin, Valentina Mazzotti, Antonietta Epifani and Cristina Maritano for their support when examining the objects from the collections of Museum of Fine Arts in Boston, Museo Internazionale delle Ceramiche in Faenza and Palazzo Madama in Turin. I am greatly indebted to the anonymous reviewer, Salvador Ryan and the editorial team.

Conflicts of Interest: The author declares no conflict of interest.

\section{References}

Balogh, Jolán. 1975. Katalog der ausländischen Bildwerke des Museums der Bildenden Künste in Budapest, IV-XVIII. Jahrhundert. Budapest: Akadémiai Kiadó.

Bonsanti, Giorgio, and Francesca Piccinini, eds. 2009. Emozioni in terracotta. Guido Mazzoni, Antonio Begarelli. Sculture del Rinascimento emiliano. Modena: F.C. Panini.

Brooke, Rosalind. 2006. The Image of St Francis. Responses to Sainthood in the Thirteenth Century. Cambridge and New York: Cambridge University Press.

Brundin, Abigail, Deborah Howard, and Mary Laven. 2018. The Sacred Home in Renaissance Italy. Oxford: Oxford University Press.

6 A written record of an oral communication with Professor Timothy Wilson during his visit at the MFA in September 2001. 
Cambareri, Marietta. 2007a. Italian Renaissance Sculpture in Maiolica and Glazed Terracotta in the Collection of the Museum of Fine Arts, Boston. In La statua e la sua pelle. Artifici tecnici nella scultura dipinta tra Rinascimento e Barocco. Edited by Raffaele Casciaro. Galatina: Congedo, pp. 61-69.

Cambareri, Marietta. 2007b. Label for the Maiolica St Francis. For the exhibition Donatello to Giambologna. Italian Renaissance Sculpture at the Museum of Fine Arts. Boston: Museum of Fine Arts.

Cohn, Samuel. 2011. Renaissance Attachment to Things: Material Culture in Last Wills and Testaments. Economic History Review 65: 1-22. [CrossRef]

Da Celano, Tommaso. 1904. Legenda Sancti Francisci. London: J.M. Dent \& Co.

Da Pisa, Bartolomeo. 1906. De conformitate vitae Beati Francisci ad vitam Domini Iesu. In Analecta Franciscana. Italy: Quaracchi, vol. IV.

Di Miglio, Augustino. 1568. Nuovo dialogo delle devozioni del sacro Monte della Verna. Florence: Ducal Press.

Dominici, Giovanni. 1860. Regola del governo di cura familiare. Edited by Donato Salvi. Florence: A. Garinei.

Fleming, John. 1982. From Bonaventure to Bellini. An Essay in Franciscan Exegesis. Princeton: Princeton University Press.

Galandra Cooper, Irene. 2019. Unlocking 'pious homes': Revealing devotional exchanges and religious materiality in early modern Naples. Renaissance Studies 33: 832-53. [CrossRef]

Gardelli, Giuliana. 1987. A gran fuoco: Mostra di maioliche rinascimentali dello Stato di Urbino da collezioni private: Sale del Castellare di Palazzo ducale. Urbino: Accademia Raffaello.

Gardelli, Giuliana. 1991. Urbino nella storia della ceramica: Nota sulla grottesca. In Italian Renaissance Pottery. Edited by Timothy Wilson. London: British Museum Press, pp. 126-35.

Gentilini, Giancarlo, ed. 2008. Dal rilievo alla pittura: La Madonna delle Candelabre di Antonio Rossellino. Florence: Polistampa.

Gentilini, Giancarlo. 1983. Le "terre robbiane" di Barga. In Barga medicea e le "enclaves" fiorentine della Versilia e della Lunigiana. Edited by Carla Sodini. Florence: Olschki, pp. 203-42.

Gentilini, Giancarlo. 2012. Scultura dipinta o pittura a rilievo? Riflessioni sulla policromia nel quattrocento fiorentino. Technè 36: 8-17.

Giacomotti, Jeanne. 1974. Catalogues des Majoliques des Musees Nationaux, Ministère de Affaires culturelles. Paris: Editions des Musées Nationaux.

Goffen, Rona. 1988. Spirituality in Conflict: Saint Francis and Giotto's Bardi Chapel. University Park and London: The Pennsylvania State University Press.

Guidotti, Paolo. 1976. La dimensione popolare delle targhe ceramiche devozionali e della poetica epigrafica nei tabernacoletti dell'area emiliano-romagnola. In Ceramiche devozionali nell'area emiliano-romagnola. Edited by Paolo Guidotti, Giovanni I. Reggi and Alfredo Taracchini. Imola: Galeati, p. 15ff.

Henry, Chriscinda. 2011. What makes a picture?: Evidence from sixteenth-century Venetian property inventories. Journal of the History of Collections 23: 253-65. [CrossRef]

Hess, Catherine. 1999. Maiolica in the Making: The Gentili/Barnabei Archive. Los Angeles: Getty Research Institute for the History of Art and the Humanities.

Ivanova, Elena. 2003. Il Secolo d'oro della maiolica. Ceramica italiana dei secoli XV-XVI dalla raccolta del Museo Statale dell'Ermitage. Milan: Electa.

Jones, Pamela. 1988. Federico Borromeo as a Patron of Landscapes and Still Lifes: Christian Optimism in Italy ca. 1600. The Art Bulletin 70: 261-72. [CrossRef]

Kube, Al'fred Nikolaevič. 1976. Catalogue no. 89. In Ital'janskaja majolika XV-XVIII vekow. Sobranie gosudarstvennogo Ermitaža. Edited by O.E. Michajlova and E.A. Lapkoskaja. Moscow: Iskusstvo.

Kupiec, Catherine. 2019. New Light on Luca della Robbia's Glazes. In The Art of Sculpture in Fifteenth-Century Italy. Edited by Amy Bloch and Daniel Zolli. Cambridge: Cambridge University Press, pp. 83-97.

Lambert, Susan. 1987. The Image Multiplied: Five Centuries of Printed Reproductions of Paintings and Drawings. London: Abaris Books.

Lugli, Adalgisa. 1990. Guido Mazzoni e la rinascità della terracotta nel quattrocento. Turin: Umberto Allemandi.

Lydecker, John Kent. 1987. The Domestic Setting of the Arts in Renaissance Florence. Ph.D. dissertation, Johns Hopkins University, Baltimore, MD, USA.

Marabotti Marabottini, Alessandro. 1982. Fonti iconografiche e stilistiche della decorazione nella maiolica rinascimentale. In Maioliche umbre decorate a lustro. Edited by Grazietta Guaitini. Florence: Nuova Guaraldi Ed., pp. 25-57. 
Marini, Marino. 2007. Catalogue entry no. 23. In Le maioliche rinascimentali nelle collezioni della Fondazione Cassa di Risparmio di Perugia. Edited by Timothy Wilson and Elisa Sani. Città di Castello: Petruzzi, vol. 2, pp. 54-65.

Maritano, Cristina. 2008. Catalogue entry. In Le ceramiche di Palazzo Madama. Guida alla collezione. Turin: Fondazione Torino Musei.

Marquand, Allan. 1922. Andrea della Robbia and his Atelier. Princeton: Princeton University Press.

Matchette, Ann. 2006. To Have and Have Not: The Disposal of Household Furnishings in Florence. Renaissance Studies 20: 701-16. [CrossRef]

Mazzotti, Valentina. 2018. 13 Maggio 1944: La distruzione e la rinascita del MIC di Faenza. Lo Stato dell'Arte 16: 33-41.

Meiss, Millard. 1964. Giovanni Bellini's St. Francis in the Frick Collection. New York: Princeton University Press.

Morse, Margaret. 2007. Creating Sacred Space: The Religious Visual Culture of the Casa in Renaissance Venice. Renaissance Studies 21: 151-84. [CrossRef]

Negroni, Franco. 1998. Una famiglia di ceramisti Urbinati: I Patanazzi. Faenza 84: 104-15.

Niccoli, Ottavia. 2001. Bambini in preghiera nell'Italia fra tardomedioevo ed età tridentina. Quaderni di storia religiosa 8: 273-99.

Niccoli, Ottavia. 2014. Pregare con la bocca, con gli occhi e col cuore nell'Italia della prima età moderna. The Italianist 34: 418-36. [CrossRef]

Paleotti, Gabriele. 2002. Discorso intorno alle immagini sacre e profane (1582). Vatican: Libreria Editrice Vaticana.

Palvarini Gobio Casali, Mariarosa. 2000. Ceramiche d'arte e devozione popolare in territorio mantovano. Mantua: Publi Paolini.

Paolinelli, Claudio. 2014. Lacrime di smalto. Plastiche maiolicate tra Marche e Romagna nell'età del Rinascimento. Ancona: Il Lavoro Editoriale.

Paolinelli, Claudio. 2019a. Gruppo plastico con San Paolo Eremita. e sant'Antonio Abate. In La Grazia dell'Arte. Collezione Grimaldi Fava. Maioliche. Edited by Carmen Ravanelli Guidotti. Cinisello Balsamo: Silvana Editoriale, pp. 68, 69.

Paolinelli, Claudio. 2019b. L'aquila e la quercia. Maioliche al Palazzo Ducale di Urbino. In Raphael Ware. I colori del Rinascimento. Edited by Timothy Wilson and Claudio Paolinelli. Turin: Umberto Allemandi, pp. 12-36.

Pia Mannini, Maria. 1981. Immagini di devozione. Ceramiche votive nell'area fiorentina dal XVI al XIX secolo. Milan: Mondadori Electa.

Piccini, Alberto. 2002. I calamai dei Mazzoni. Fimantiquari Arte Viva 30: 37-51.

Prentice von Erdberg, Joan. 1961. Outstanding Maiolica at The Art Museum, Princeton University. The Burlington Magazine 103: 299-305.

Rackham, Bernard. 1940. Victoria and Albert Museum. Catalogue of Italian Maiolica. London: Board of Education.

Ravanelli Guidotti, Carmen. 1998. Thesaurus di opere della tradizione di Faenza nelle raccolte del Museo Internazionale delle Ceramiche in Faenza. Faenza: Agenzia polo ceramico.

Reale, Giovanni, Andrea Samaritani, and Elisabetta Sgarbi. 2008. Il pianto della statua nelle sculture sacre in terracotta di Niccolò dell'Arca, Guido Mazzoni e Antonio Begarelli. Milan: Bompiani.

Rohlmann, Michael. 1999. Flanders and Italy, Flanders and Florence. Early Netherlandish Painting in Italy and its Particular Influence on Florentine Art: An Overview. In Italy and the Low Countries. Artistic Relations. The Fifteenth Century. Edited by Victor Schmidt. Florence: Centro Di, pp. 39-67.

Rudy, Kathryn M. 2010. Dirty Books: Quantifying Patterns of Use in Medieval Manuscripts Using a Densitometer. Journal of Historians of Netherlandish Art 2: 1-2. [CrossRef]

Rudy, Kathryn M. 2011. Virtual Pilgrimages in the Convent. Imagining Jerusalem in the Late Middle Ages. Turnhout: Brepols Publishers.

Rudy, Kathryn M. 2017. Eating the face of Christ: Philip the Good and his physical relationship with Veronicas. In The European Fortune of the Roman Veronica in the Middle Ages. Edited by Amanda Murphy, Herbert L. Kessler, Marco Petoletti, Eamon Duffy and Guido Milanese. Convivium: Supplementum, pp. 169-79.

Rutherglen, Susannah. 2015. "The Footprints of Our Lord": Giovanni Bellini and the Franciscan Tradition. In In a New Light. Giovanni Bellini's St. Francis in the Desert. Edited by Susannah Rutherglen and Charlotte Hale. New York: The Frick Collection, pp. 23-45.

Sangiorgi, Fert. 1976. Documenti urbinati: Inventari del Palazzo Ducale (1582-1631). Urbino: Accademia Raffaello. 
Sarnecka, Zuzanna. 2018a. "And The Word Dwelt Amongst Us". Experiencing the Nativity in the Italian Renaissance Home. In Domestic Devotions in Early Modern Italy. Edited by Maya Corry, Marco Faini and Alessia Meneghin. Leiden: Brill, pp. 163-83.

Sarnecka, Zuzanna. 2018b. Le piccole sculture maiolicate e il loro significato nelle case marchigiane del primo Cinquecento. In Pregare in Casa. Edited by Cristina Guarnieri, Giovanna Baldissin Molli and Zuleika Murat. Rome: Viella, pp. 265-78.

Strauss, Walter. 1980. The Illustrated Bartsch. New York: Abaris Books, vol. 10.

Thornton, Dora. 1997. The Scholar in His Study. Ownership and Experience in Renaissance Italy. New Haven and London: Yale University Press.

Thornton, Dora, and Timothy Wilson. 2009. Italian Renaissance Ceramics: A Catalogue of the British Museum Collection. London: British Museum Press, vol. 2, cat. no. 213.

Turchini, Angelo. 1980. Tracce di religione domestica in ambiente urbano: Il caso di Rimini fra il XV e il XVII secolo. Il Carrobbio 6: 351-64.

Venturi, Adolfo. 1889. Il testamento di Guido Mazzoni detto il Paganino o il Modanino. Archivio storico dell'arte 2: $155-57$.

Warren, Jeremy. 2014. Medieval and Renaissance Sculpture. A Catalogue of the Collection in the Ashmolean Museum. Oxford: Ashmolean Museum Publications, vol. 2, pp. 482-87.

Warren, Jeremy. 2016. The Wallace Collection. Catalogue of Italian Sculpture. London: Trustees of the Wallace Collection, pp. 60-67.

Watson, Wendy. 1986. Italian Renaissance Maiolica from the William A. Clark Collection. London: Scala Books.

Wilson, Timothy. 1996. Italian Maiolica of the Renaissance. Milan: Bocca Editori.

Wilson, Timothy. 2000. Catalogue entry no. 247. In Musei e Gallerie di Milano. Museo di Arti Applicate. Ceramiche. Edited by Raffaella Ausenda. Milan: Mondadori Electa, vol. 1.

Wilson, Timothy. 2016. Maiolica: Italian Renaissance Ceramics in the Metropolitan Museum of Art. New York: The Metropolitan Museum of Art, pp. 72-75.

Wilson, Timothy, and Elisa Sani. 2007. Le maioliche rinascimentali nelle collezioni della Fondazione Cassa di Risparmio di Perugia. 2 vols. Città di Castello: Petruzzi.

Wilson, Timothy. 2017. Italian Maiolica and Europe. Oxford: Ashmolean Museum Publications, pp. 368-79.

(C) 2019 by the author. Licensee MDPI, Basel, Switzerland. This article is an open access article distributed under the terms and conditions of the Creative Commons Attribution (CC BY) license (http://creativecommons.org/licenses/by/4.0/). 
Article

\title{
Masterpieces, Altarpieces, and Devotional Prints: Close and Distant Encounters with Michelangelo's Vatican Pietà
}

\author{
Grażyna Jurkowlaniec \\ Institute of Art History, University of Warsaw, Krakowskie Przedmieście 26/28, 00-927 Warszawa, Poland; \\ g.jurkowlaniec@uw.edu.pl
}

Received: 2 April 2019; Accepted: 1 May 2019; Published: 7 May 2019

\begin{abstract}
Focussing on the response to the Vatican Pietà and perversely using as a point of departure a 1549 remark on Michelangelo as an 'inventor of filth,' this article aims to present Michelangelo as an involuntary inventor of devotional images. The article explores hitherto unconsidered aspects of the reception of the Vatican Pietà from the mid-sixteenth into the early seventeenth century. The material includes mediocre anonymous woodcuts, and elaborate engravings and etchings by renowned masters: Giulio Bonasone, Cornelis Cort, Jacques Callot and Lucas Kilian. A complex chain of relationships is traced among various works, some referring directly to the Vatican Pietà, some indirectly, neither designed nor perceived as its reproductions, but conceived as illustrations of the Syriac translation of the New Testament, of Latin and German editions of Peter Canisius's Little catechism, of the frontispiece of the Règlement et établissement de la Compagnie des Pénitents blancs de la Ville de Nancy — but above all, widespread as single-leaf popular devotional images.
\end{abstract}

Keywords: devotional image; Pietà; Michelangelo Buonarroti; reception

\section{Introduction}

Michelangelo's contemporaries unanimously described his Vatican Pietà (Figure 1), carved between 1498 and 1500, as the work that ensured the fame of the young artist and thus paved the way for his subsequent career (Vasari [1550 and 1568] 1962); (Condivi 2009). More problematic was the devotional value of the work, as demonstrated by Pietro Aretino's criticism (Aretino 1957), and also the opinion of another author on the copy displayed in the church of Santo Spirito in Florence in 1549. The author of the Florentine Chronicle, also titled Diario del 1536 di Marucelli, regarded Michelangelo as 'the inventor of filth, saving the art but not the devotion' (de Tolnay 1948)—inventor delle porcherie, salvandogli larte ma non devotione (Cronaca fiorentina 2000). Nonetheless, this view, noted by scholars as early as the nineteenth century (Gaye 1839) and often quoted since then, must have been rather isolated. Displayed in various altars of the old and then the new Vatican Basilica through the centuries, Michelangelo's Pietà was not only described as a masterpiece, but also-despite criticism-venerated as a holy image, a status that was officially confirmed by its coronation by the Chapter of St. Peter's in 1637 (Jurkowlaniec 2015).

In the sixteenth and early seventeenth centuries, several of Michelangelo's works provoked various responses as masterpieces or as religious images. The Pietà drawing for Vittoria Colonna (Boston, Isabella Stewart Gardner Museum) was not only a typical artist's gift, a token of his skills, but also a devotional image strictly related to Michelangelo's and Vittoria's considerations of the question of divine grace, as can be inferred from the preserved correspondence (Nagel 2000); (Roman D'Elia 2006). In turn, the Last Judgment in the Sistine Chapel was perceived as a fresco that shows the power of art, but it was also vehemently criticised by Aretino, among others, as an indecent painting, which was particularly inappropriate in the pope's chapel (De Maio 1990); (Barnes 1998). The marble statue 
of Risen Christ in Santa Maria sopra Minerva in Rome was praised for its excellence, but it was also reportedly mutilated by Dominican friars who, scandalized by the figure's nudity, were said to have broken off Christ's penis (Ligorio 1549) cf. (Celio 1638). However, in the early modern period the Minerva Christ, provided with a loincloth and displayed within a tabernacle, was venerated by the faithful. Since it must have been a widespread custom to kiss Christ's feet, the General of the Dominicans, Antonino Cloche, commissioned metal sandals in 1706, to protect the marble from destruction (Panofsky 1991); (Schwedes 1998). As the devotional practices continued, one of the sandals was hardly preserved in the early nineteenth century (Stendhal 1817), cf. (Wallace 1997).

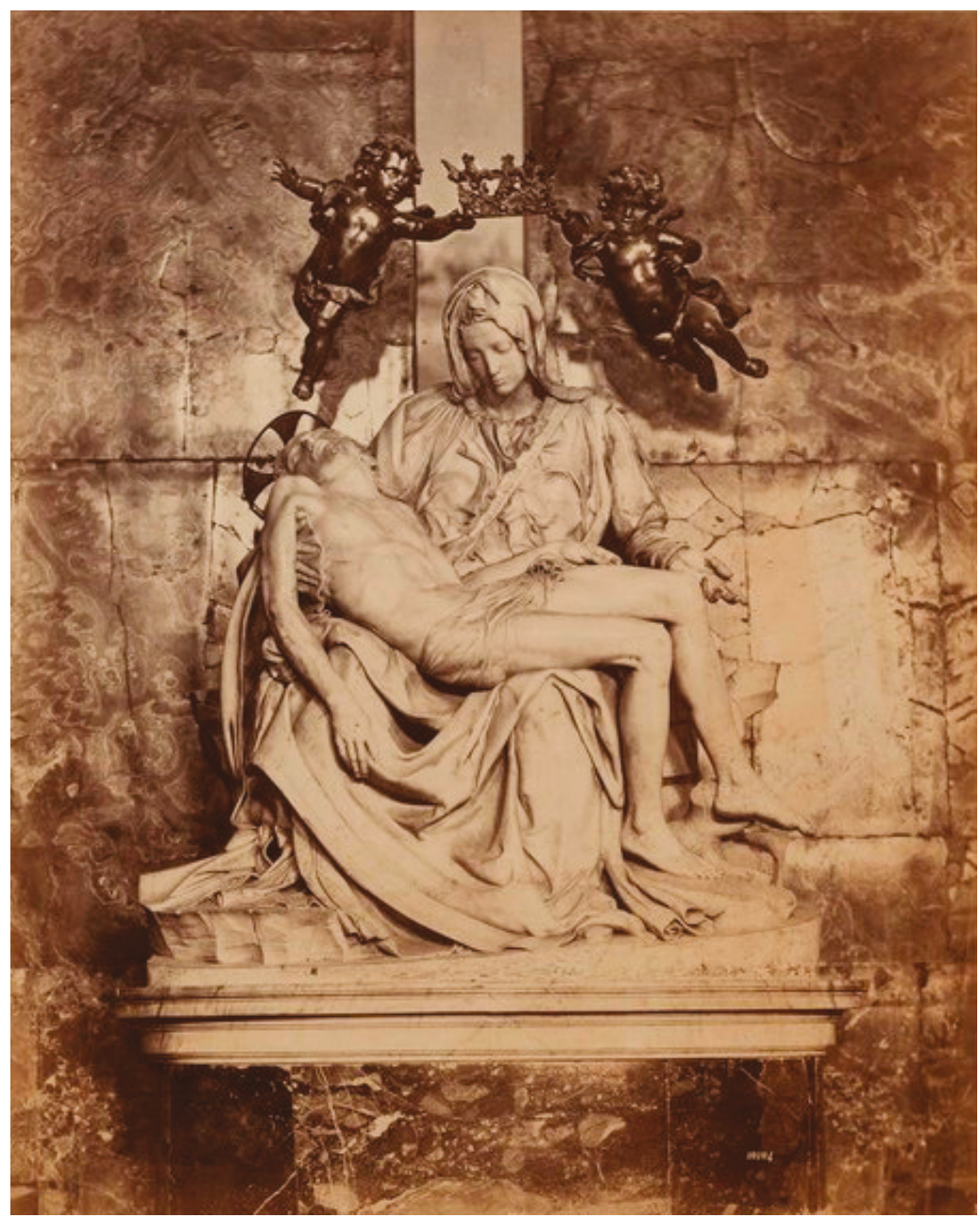

Figure 1. Michelangelo Buonarroti, Pietà, 1489-1500, marble, Rome, San Pietro in Vaticano. Archival photo, before 1927 (the date of the removal of the crown and the putti) (C) Kunsthistorisches Institut in Florenz, Max-Planck-Institut, Florence.

Michelangelo's works became famous thanks to written accounts, including eulogies, factual descriptions, and pasquinades, as well as engravings, which were used profusely at that time to reproduce famous works of art and-since the fifteenth century-to disseminate devotional images. Early modern inventories evidence that prints after Michelangelo's sculptures, paintings, and 
drawings were offered by Roman publishers, and often listed among devotional images (Ehrle 1908); (Pagani 2008a; Pagani 2008b; Pagani 2011; Pagani 2012); (Lincoln 2000); (Rubach 2016). Some of these engravings were subsequently copied and copied again and thus contributed to the dissemination of the design, and were used in various contexts.

The reception of the Vatican Pietà was ubiquitous in writings, but also in the visual culture of the sixteenth and early seventeenth centuries. Among many copies, reproductions, travesties, and pastiches executed in various techniques, some referred to the sculpture as Michelangelo's masterpiece, some depicted it as an altarpiece of St. Peter's Basilica, while some-and these are most relevant for our argument-used it as a point of departure for devotional images. In the previous research, the devotional value of the copies of the Vatican Pietà, particularly those executed in graphic techniques, has admittedly been acknowledged (Barnes 2010); (Veress 2010-2011); (Veress 2012); (Alberti 2015), but several works remain unnoticed and thus various aspects of the phenomenon have been hitherto neglected. The aim of this paper is to elaborate on the less-explored pattern of the reception of the Vatican Pietà, and thus to contribute to the discussion on how a masterpiece turns into a devotional image-sometimes for the sake of 'simple folk,' sometimes intended for a more sophisticated public -in a process in which various models are occasionally combined and the name of the original author often gets lost.

\section{Fontainebleau: A Copy for the King}

In a letter to Michelangelo, dated 8 February 1546, Francis I of France expressed his desire to possess the master's works (Carteggio di Michelangelo 1979). The King wished to purchase them via his agent in Rome, a Bolognese painter Francesco Primaticcio, whom he also asked to mould the Vatican Pietà and the Minerva Christ, wanting the copies of these sculptures to be displayed in his chapel. While the whereabouts of the copy of the Minerva Christ remain unknown, the royal accounts of the 1540s include an entry for a Pietà in the Haute Chapelle du Donjon, the Royal Chapel of the Palace of Fontainebleau (Comptes des bâtiments 1877). The 1642 description of the palace confirms that the Pietà, moulded after Michelangelo's sculpture in the Vatican, was displayed on the right side of the altar of the chapel (Dan 1642). Eventually, in 1664, the figure was moved to the Chapel of the Holy Trinity, which seems to be the last trace of this work (Cox-Rearick 1995).

Now lost, the Fontainebleau cast was made to fulfil Francis's personal desire as an art amateur, but it was also, like the copy in Santo Spirito in Florence and several others, displayed in a sacred place, even if it was not a publicly accessible church but the King's chapel in his palace. This combination of aesthetic and religious motivations, on the one hand, and the intersection of the public and the domestic spheres, on the other hand, invite us to analyse the reception of Michelangelo's sculpture as a masterpiece, as an altarpiece, and also as a model for devotional images. The Fontainebleau copy of the Vatican Pietà was perhaps the earliest one north of the Alps (for despite some similarities, I do not believe the relief, dated ca. 1520, in the epitaph of Johann von Hatstein in the cloister of Mainz Cathedral to be a descendant of the Vatican Pietà; cf. (Thode 1908)). The question arises, then, of how Francis I became familiar with particular Michelangelo works that he wanted to be copied. A possible intermediary was Primaticcio, one of several Italian artists working in Fontainebleau. Francis had already sent Primaticcio to Rome in 1540 to take casts of the most famous ancient sculptures, notably those from the Belvedere collection (Dimier 1900); (Cupperi 2010). It must remain conjecture whether the King relied on written or oral accounts, or had some visual sources at his disposal, such as drawings, rather than engravings. Prints are usually regarded as a typical medium by scholars. However, interestingly, Francis's letter to Michelangelo ranks among the earliest responses to the Vatican Pietà and the Minerva Christ and it also predates the printed reproductions of both works.

\section{Intermediaries: Italian Engravings of the Mid-Sixteenth Century}

Among the earliest Italian engravings after the Vatican Pietà are two prints dated 1547. The one attributed to Antonio Salamanca and Nicolas Beatrizet displays the group of Mary and Christ against a ruined niche and is inscribed 'Michelangelo Buonarroti of Florence divinely made from one stone 
the mother and the son for the [Basilica of] Saint Peter in the Vatican/Antonio Salamanca engraved in 1547, as much as it could be imitated' (MICHELANGALVS BONAROTVS FLOREN[inus] DIVI PETRI IN VATICANO EX VNO LAPIDE MATREM // AC FILIVM DIVINE FECIT // ANTONIVS SALAMA[n]CA QVOD POTVIT IMITATVS EXCVLPSIT 1547), which inevitably suggests to an erudite beholder that Salamanca reproduced a masterpiece that rivals ancient sculptures (Figure 2). For a less-educated and, especially, illiterate spectator, however, it may not be clear if the engraving depicts a marble sculpture or Mary holding Christ on her lap (Barnes 2010, p. 149). Thanks to this ambiguity, the print might satisfy a wide range of both learned art amateurs and faithful believers. It is, for instance, meaningful that Salamanca's engraving was copied twice, still in the late sixteenth century (Alberti 2015, nos. 311-13).

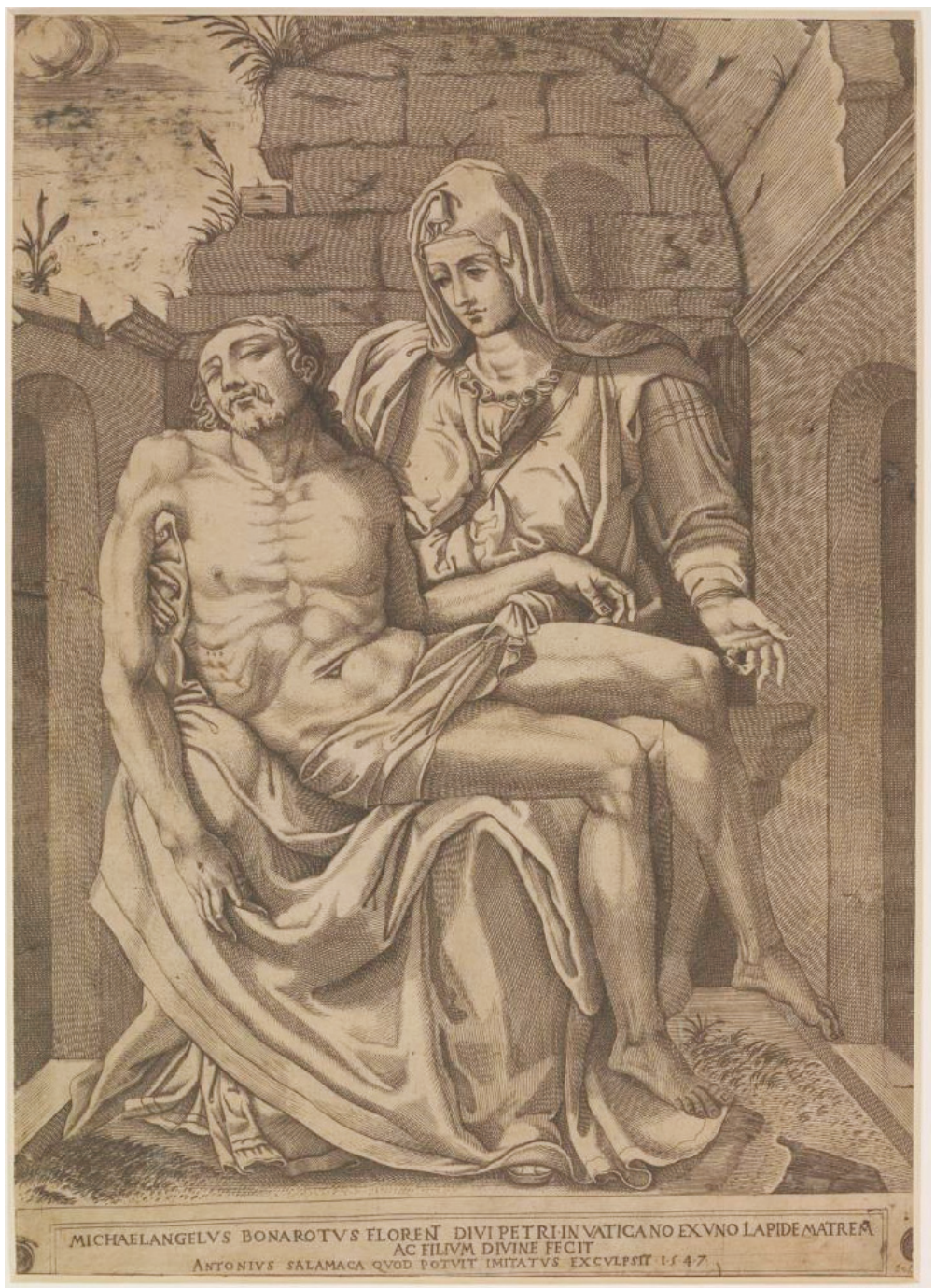

Figure 2. Antonio Salamanca and Nicolas Beatrizet, Pietà after Michelangelo Buonarroti, 1547, engraving. London, Victoria and Albert Museum, DYCE.1099 (as School of Marcantonio Raimondi) (C) Victoria and Albert Museum, London. 
More unequivocal is the contemporaneous engraving by Giulio Bonasone, inscribed MICHAELANGELVS BONAROTVS NOBILIS // FLORENTINVS INVENTOR IVLIVS BONASONIS F(ecit) (Alberti 2015, no. 314) (Figure 3). While the concise and factual inscription explicitly refers to Michelangelo as the inventor, the image obscures the material features of the original, as Mary and Christ are placed in the landscape, at the foot of the Cross among the instruments of the Passion (Barnes 2010, p. 149). Thus, Bonasone took Michelangelo's sculpture as a point of departure for his own representation of Pietà, a widespread motif of Christian iconography. Such an attitude may also be traced back in two prints that are travesties rather than reproductions of the Vatican Pietà: the engraving signed by Monogrammist PHPT, active in the late sixteenth century (Alberti 2015, no. 318), to which we shall return, and the engraving by Mario Cartaro of 1564-the year of Michelangelo's death (Alberti 2015, no. 319).

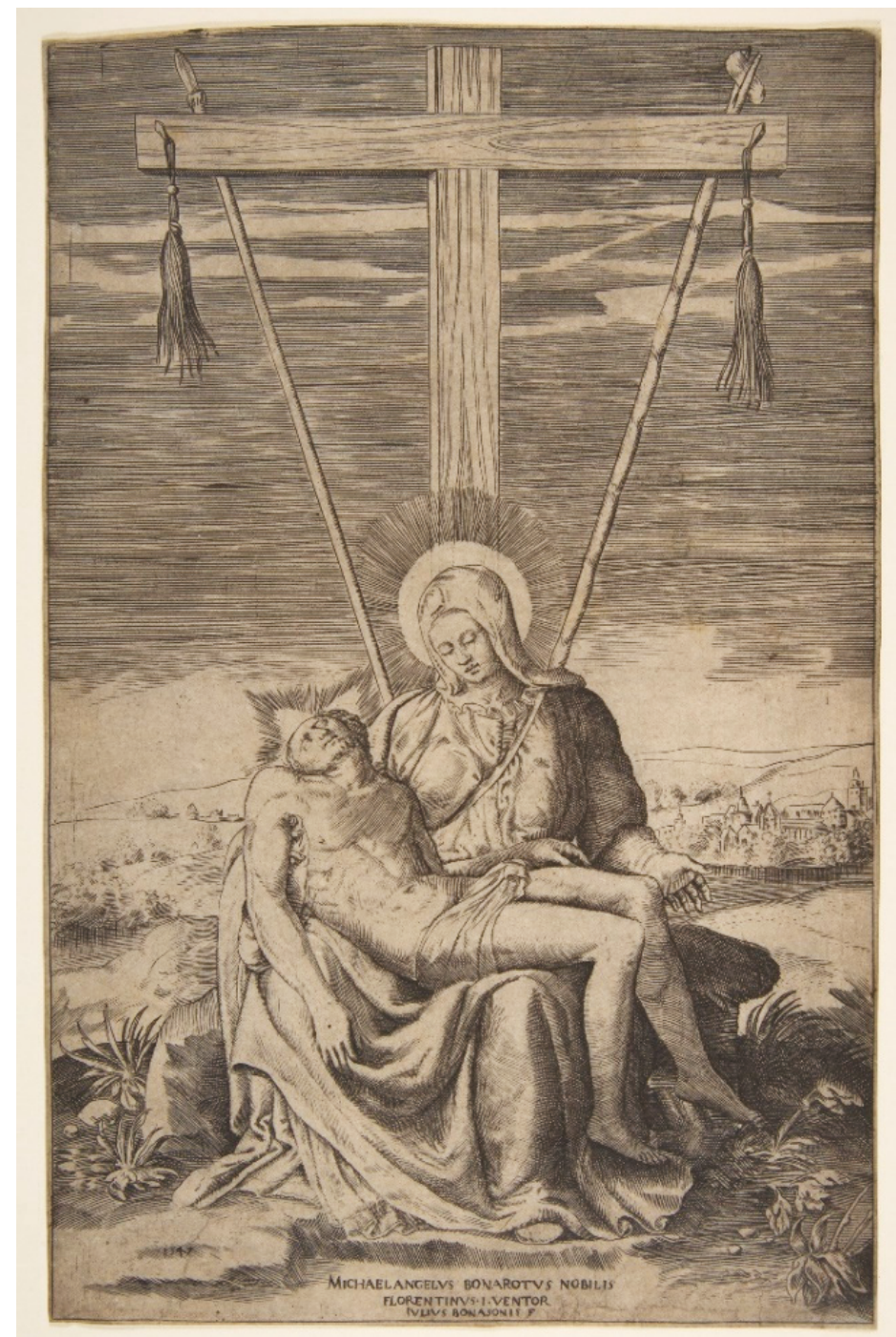

Figure 3. Giulio Bonasone, Pietà after Michelangelo Buonarroti, 1547, engraving. New York, The Metropolitan Museum of Art, acc. no. 59.595.3 (C) The Metropolitan Museum of Art, New York. 
In or soon after 1564, further engravings were produced, which testify to the development of the two different, even if occasionally merging, responses to the Vatican Pietà. Giovanni Battista de' Cavalieri admittedly showed Mary and Christ with a cross in the background, but his print clearly reproduces the sculpture, together with the rocky ground on which Michelangelo situated the figures, and the erudite caption contains homage to Michelangelo and no explicit reference to devotion (Alberti 2015, no. 316); (Jurkowlaniec 2015, p. 184). Adamo Scultori depicted the sculpture too, but he situated it in front of an opening in the rock, thus alluding to the rock-cut tomb of Christ (Alberti 2015, no. 315) (Figure 4). Moreover, in the second (out of four) state, dated 1566, Scultori's engraving was inscribed: 'Michelangelo Buonarroti accomplished these statues, which can be seen in the Vatican, so accurately, that you rather mourn the virgin Mother, scarcely breathing, pale and exhausted, and the pitiable dead body of her son, than suspect them erected of marble' (MICH(ael) ANG(elus) BONAROTVS signa haec, quae in uaticano uisuntur, ita exacte perfecit, ut potius Parentem uirginem // extremo spiritu exanguem \& confectam, et nati corpus miserabile emortuum doleas; quam de marmore positum putes). Various literary allusions to ancient poetry included in this caption are in the service of devotion (Jurkowlaniec 2015, p. 179). A beholder is invited to contemplate 'the pitiable dead body' of Christ and the 'scarcely breathing' Mary (a quote from Cicero, For Sestius 37. 79). Hence, the inscription is addressed to a pious faithful rather than to an art amateur, but even the latter, touched by the suggestive value of the representation of the Pietà, is expected to forget the material qualities of the marble statue.

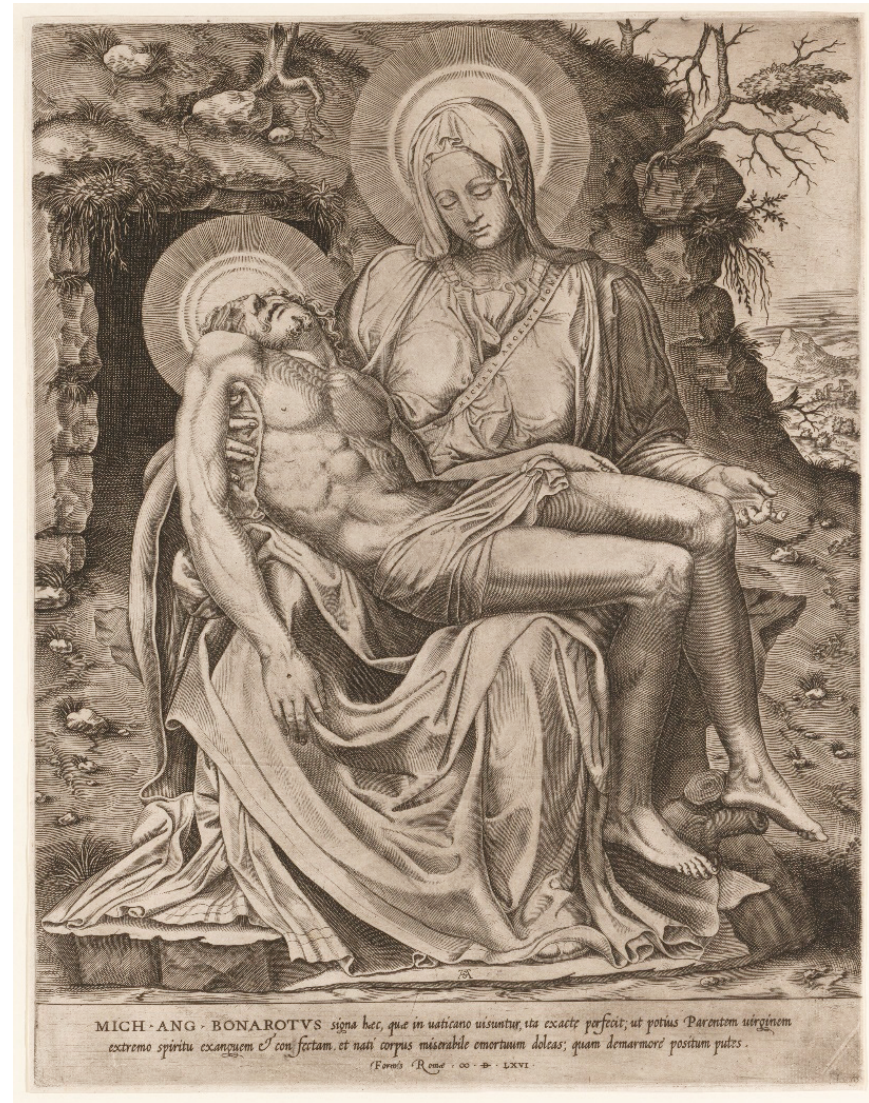

Figure 4. Adamo Scultori, Pietà after Michelangelo Buonarroti, ca. 1566, engraving. New York, The Metropolitan Museum of Art, acc. no. 58.642.3 (C) The Metropolitan Museum of Art, New York. 
Thus, the early engravings after the Vatican Pietà constantly oscillate between two genres. One may be labelled as 'reproductions of a masterpiece,' the other as 'devotional images after a famous design.' Bonasone's 1547 print is especially relevant for our further investigation, not only because it seems relatively closest to the latter group. Compared to other engravings after the Vatican Pietà, notably Salamanca's, Bonasone's print itself and, above all, its further reception in the mid- and late-sixteenth century, have not yet been studied.

\section{Vienna: Woodcuts in the Syriac New Testament and Peter Canisius's Little catechism}

In 1555, Viennese printer Michael Zimmermann produced the famous editio princeps of the Syriac New Testament, edited by Johann Albrecht Widmannstetter and financed by Ferdinand Habsburg (Ketābā d-Ewangeliyōn 1555). The publication was illustrated, but the woodcuts have remained almost hitherto overlooked in the scholarly literature, with the sole exception of The Crucifix and the Sephirotic Tree preceding and concluding St. John's Gospel on fol. $101^{\mathrm{v}}$ and [fol. 158 ${ }^{\mathrm{r}}$ ], respectively (Wilkinson 2007). Further allegorical images - two diagrams and the triumphal Cross-recur in the publication a few times each, but a special emphasis must be placed on the Pietà that concludes the section of St. Paul's epistles (Figure 5).

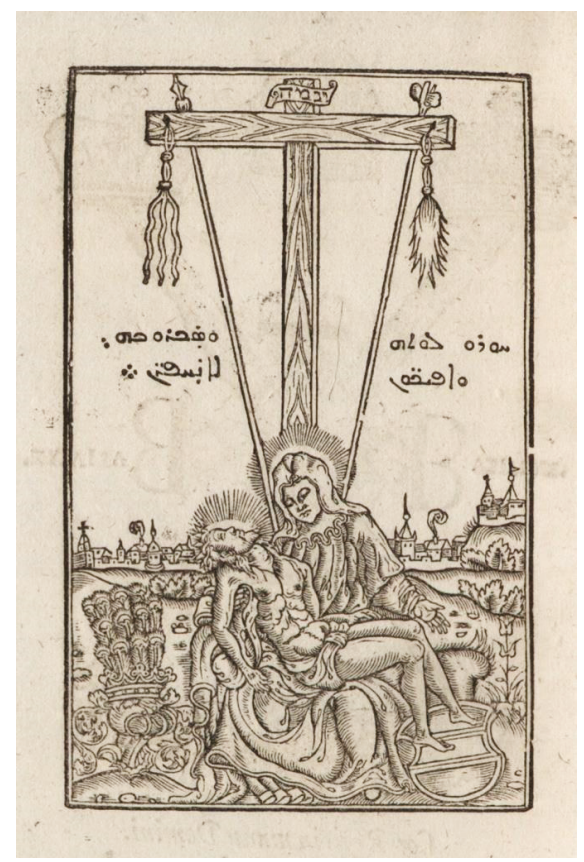

(a) Pietà after Giulio Bonasone [fol. LXXXIr]

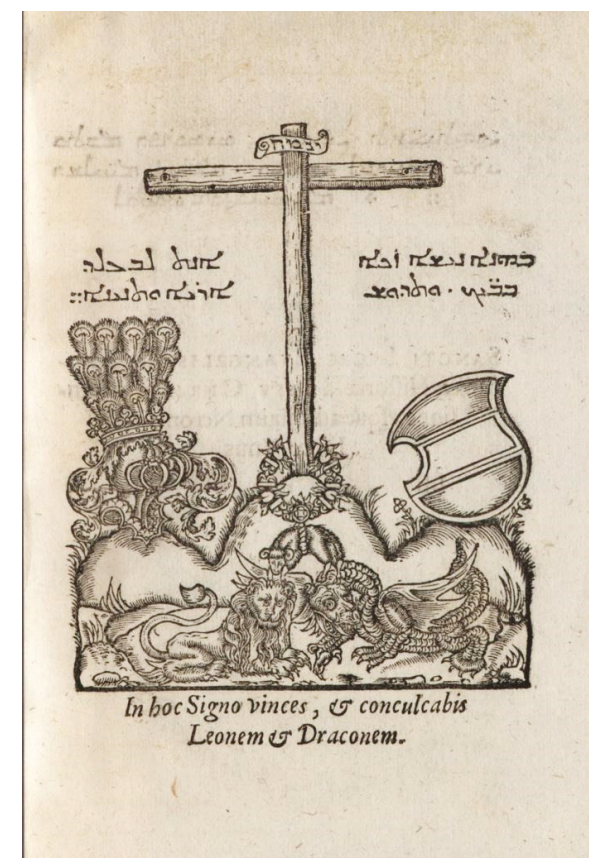

(b) Triumph of the Cross [fol. $\left.\mathrm{bb}^{\mathrm{r}}\right]$

Figure 5. Woodcuts in the Syriac New Testament (Ketābā d-Ewangeliyōn 1555). Munich Bayerische Staatsbibliothek, Rar. 155 (C) Bayerische Staatsbibliothek, Munich, urn:nbn:de:bvb:12-bsb00070810-5.

Several motifs of the Pietà-Christ's pose, Mary's gestures and dress, the shape of both nimbi, the lowered line of the horizon and the arma Christi displayed in a characteristic manner, with the scourges hanging from the beam of the Cross and, in particular, the lance and the rod with the vinegar-soaked sponge forming a V-shape-undoubtedly follow Bonasone's engraving. However, there is no reference to either Bonasone or Michelangelo. Instead, the Syriac inscription cites Psalm 34[33]:6:

$$
\text { טبونه }
$$


—that is, 'Come ye to him and be enlightened: and your faces shall not be confounded' (all English quotes from the Bible are from the Douay-Rheims Catholic Bible).

The Pietà is not a mere devotional image here, as the assumed audience of the translation of this New Testament was not only the Syriac Christian community, but above all, learned humanists. It is meaningful, for instance, that the woodcuts are inscribed in Latin and Syriac, some also in Greek, while the abbreviation of 'Jesus the Nazarene, King of the Jews' in the titulus of the Cross (cf. John 19:19) is consistently given in Hebrew (ינמה as an abbreviation of ישוע הנצרת מלך היהודים ). Finally, some motifs also allude to the dynastic or imperial identity of the patron, Ferdinand Habsburg, as a crested, mantled helmet and the Habsburg coat of arms flank not only the Pietà but also the triumphal Cross.

These two woodcuts were apparently conceived as counterparts. The Cross is associated with a triumphal wreath and the small figure of a lamb trampling upon a lion and a dragon, referring to Psalm 91(90):13. While the Syriac inscriptions vary in particular impressions throughout the publication, the Latin caption 'in this sign thou shalt conquer and thou shalt trample underfoot the lion and the dragon' (In hoc Signo vinces, \& conculabis Leonem \& Draconem) invariably combines the famous augury of Constantine the Great's victory in the Battle of the Milvian Bridge, subsequently used as a motto in diverse, usually military, contexts, with the quote from Psalm 91 (90), considered to be a prophecy of Christ's victory over death and Satan.

Consistent with common practice, the woodcut blocks used to impress the illustrations in the first edition of the Syriac New Testament were reused in the second edition published in 1562 (Ketābā d-Ewangeliyōn 1562). Before that, however, both the Pietà and the triumphal Cross also recurred in another work printed by Zimmermann: Peter Canisius's Summa doctrinae Christianae: In usum Christianae pueritiae per quaestiones recens conscripta-the so-called Parvus catechismus catholicorum, or A little catechism for Catholics.

This version of Canisius's catechism was intended primarily for students. Zimmerman published it several times, usually with illustrations (the only exception is Canisius 1558a) and invariably preceded by Ferdinand Habsburg's 24 August 1554 letter to the reader, but with no date on the title pages or the colophons. The repertoire of the woodcuts is repetitive, but not identical (Table 1) (Streicher 1933); (Palmer Wandel 2015).

Table 1. Illustrations in editions of Petrus Canisius's Summa doctrinae Christianae printed by Michael Zimmermann; dates after (VD16).

\begin{tabular}{|c|c|c|c|c|}
\hline & $\begin{array}{l}\text { Verso of the } \\
\text { Title Page }\end{array}$ & $\begin{array}{l}\text { After Ferdinand's Letter } \\
\text { to the Reader }\end{array}$ & $\begin{array}{l}\text { Before De iustitia } \\
\text { Christiana }\end{array}$ & $\begin{array}{c}\text { Recto of the Last } \\
\text { Page }\end{array}$ \\
\hline (Canisius 1555) & Crucifixion & Christ among the children (A) & Crucifixion & Triumph of the Cross \\
\hline (Canisius 1558a) & Pietà, 1556 & Christ among the children (B) & Crucifixion & \\
\hline (Canisius 1558b) & Pietà, 1556 & Christ among the children (B) & Crucifixion & \\
\hline (Canisius 1558a) & & & & \\
\hline (Canisius 1558b) & Pietà, 1555 & Christ among the children $(\mathrm{C})$ & Crucifixion & Triumph of the Cross \\
\hline
\end{tabular}

Christ among the children, impressed from three diverse matrices, appears after the preface, a clear allusion to the assumed audience of Christian youth, as declared in the title. The Crucifixion scene, in turn, consistently impressed from one woodblock of rather poor quality, recurs before the chapter on Christian justice. The same Crucifixion matrix was used to impress the illustration on the verso of the title page in what is believed to be the editio princeps. In subsequent editions, the Crucifixion was replaced by the Pietà as the opening scene, of which Zimmermann had two similar matrices at his disposal (Figure 6). One was the block known from the Syriac New Testament, modelled on Bonasone's engraving. The other, with the date 1556 in the lower left corner, is distinguished by a similar composition, but Mary's gesture, the position of Christ's arm, and both nimbi as well as various details in the background were designed independently or informed by another model. One has to remember that the Pietà was a popular iconography; many painters and printmakers situated the group of Mary and Christ in a landscape; also the V-shape of the arma Christi is far from unique. 


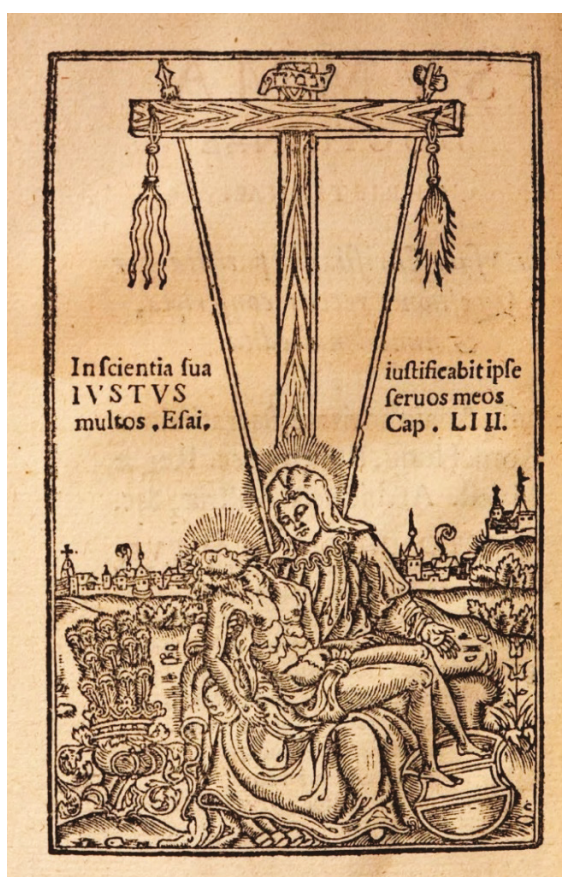

(a) (Canisius 1558b)

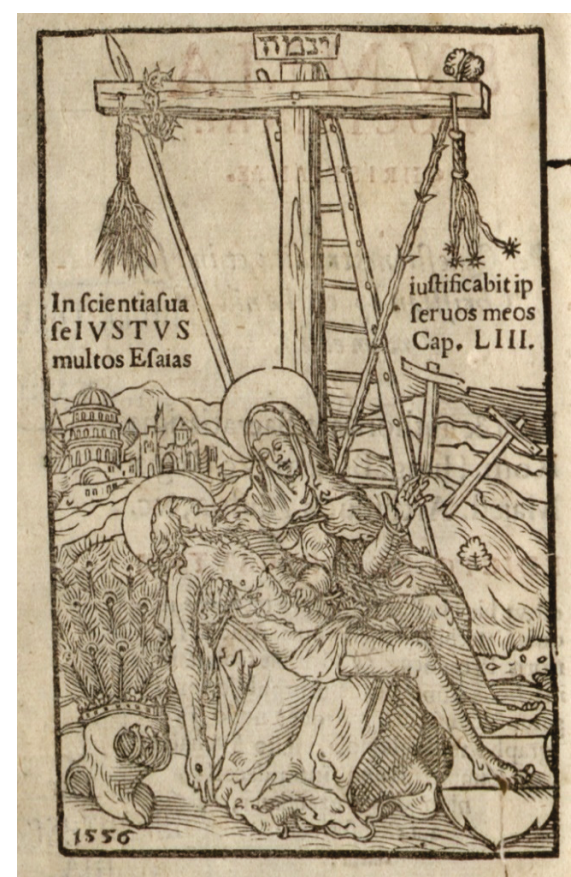

(b) (Canisius 1556a)

Figure 6. Pietàs after Giulio Bonasone, woodcuts in: Peter Canisius's Summa doctrinae Christianae (Vienna: Michael Zimmermann), fol. A1v . Regensburg, Staatliche Bibliothek, 999/Asc. 203 and Munich, Bayerische Staatsbibliothek, Catech. 89 nd (C) Bayerische Staatsbibliothek, Munich, urn:nbn:de:bvb:12-bsb11081946-4 and urn:nbn:de:bvb:12-bsb10537350-4.

Regardless of the matrix used, in the Latin editions both Pietàs were provided with almost identical inscriptions referring to Christ as the Suffering Servant from the Book of Isaiah: 'by his knowledge shall this my just servant justify many' (In scientia sua / iustificabit ipse // IVSTVS servos meos / multos. Esaias Cap. LIII-Isaiah 53:11). The 1556 Pietà was subsequently reprinted in the German edition of the catechism, this time, however, with the inscription travestying St. Paul's preaching on the crucified Christ 'who of God is made unto us wisdom, and justice' (Jesus Christus / d(er) gecreuziget // ist der anfang / und das end // unserer weis/heit un(d) gerech/tigkeit—cf. 1 Corinthians 1:26-30).

The Pietàs in the books printed by Zimmermann were provided with various Biblical verses that invite the reader to contemplate the mystery of redemption. Neither illustration appears to have been considered a reproduction of a masterpiece or even of an altarpiece image. Still, the question arises whether Zimmermann or the Formschneiders who worked for him were aware of the origins of the design. This awareness cannot be excluded in the case of the earlier woodcut, which seems to rely directly on Bonasone's engraving which, in turn, explicitly referred to the original. A conscious reference to the Vatican Pietà is much less plausible in the case of the woodcut dated to 1556. This one should be regarded as the next generation of descendants of Michelangelo's design, mediated first by Bonasone's engraving and then by the 1555 woodcut, maybe combined with another model. This sequence is plausibly the earliest, but not the only chain of works initiated by Bonasone's print and thus ultimately—even if unintentionally—anchored in Michelangelo's sculpture.

\section{Fiammingo a Roma: Cornelis Cort for the Sake of Devotion}

The undated Pietà signed by Cornelis Cort-a Dutch engraver active from 1565 until his death in 1578 in Italy, among other cities in Rome-at first fails to evoke any associations with the Vatican Pietà 
(Figure 7). Scholars consider the engraving to be 'after an unidentified artist' (Sellink and Leeflang 2000). The pose of Christ differs from that in Michelangelo's sculpture, while Mary's gestures are reminiscent of various prints after the Vatican Pietà, and specifically the Cross with the scourges seems to be an abridged version of Bonasone's design, maybe combined with another invention. In Cort's print, the Pietà is the main image, in an oval frame, set within an aedicule with a scene of the Deposition in a cartouche at the bottom. Such a composition may resemble an altarpiece, but it does not seem to represent one and the print's dimensions, only $13 \times 9.1 \mathrm{~cm}$, are typical of popular devotional images. Lorenzo Vaccari-the publisher whose address was added in the second state (Laurentium V[accarium] Formis Romae) together with the date 1580 - plausibly offered this print among the small devotional images, some of which were 'in an oval' (in ouato), an expression recurring in his 1614 inventory. However, it is impossible to point to a particular entry, because while the bigger prints in Vaccari's stock (fogli imperiali and fogli reali) are often attributed to specific engravers and, rather exceptionally, to inventors, the names only occasionally appear with respect to the medium-sized prints (mezzi fogli) and are never given in the case of smaller ones (diuersi quarti fogli). Thus, only tentatively may one consider La Madonna della Pietà or La Madonna con il Signore as possible correspondences to Cort's Pietà (Ehrle 1908, p. 66, lines 666 and 734).

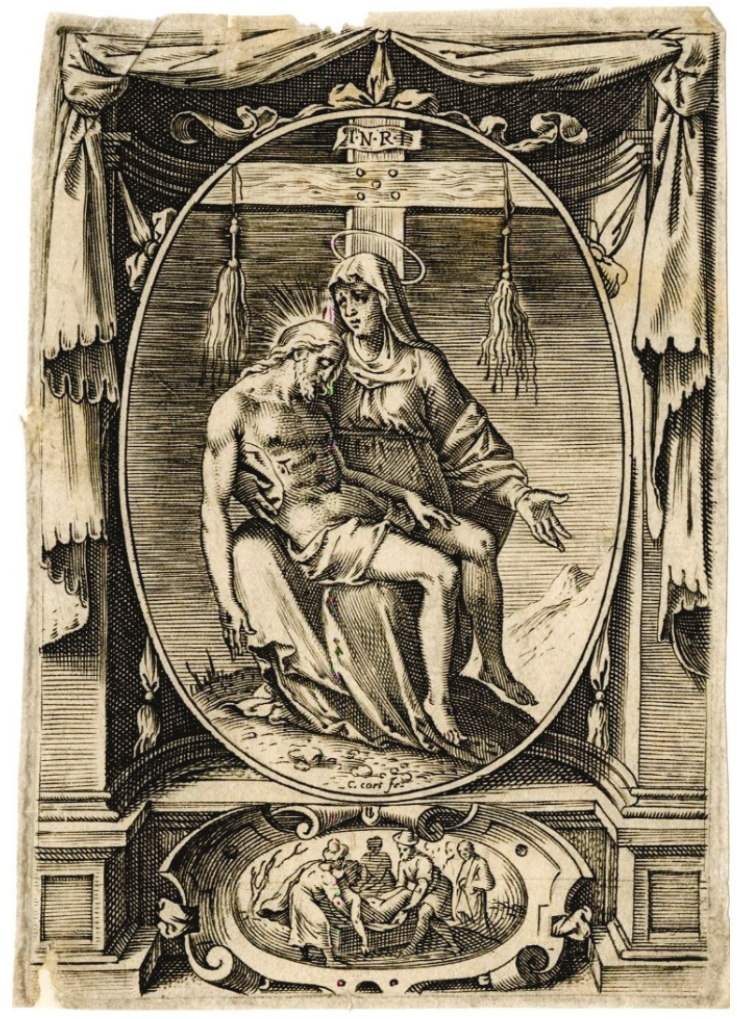

Figure 7. Cornelis Cort, Pietà, before 1578, engraving. London, British Museum, V, 8.140 @ Trustees of the British Museum.

Whatever the sources of Cort's Pietà, it became a model for later engravings. One of these is as a small print in a rectangular frame, inscribed 'Surely he hath borne our infirmities and carried our sorrows' (VERE LANGVORES N[ost]ROS IP[s]E TVLIT / ET DOLORES N[ost]ROS IP[s]E PORTAVIT-Isaiah 53:4), another fragment of the prophecy of the Suffering Servant, also quoted in the woodcuts in the Vienna editions of Canisius's catechism (Figure 8a, cf. Figure 6). Given the 
dimensions and the inscription, it can be concluded that the engraving after Cort's Pietà was conceived as a devotional image. However, some devotional objects became collectible items, as testified by the impression owned by Jan Ponętowski, a Polish nobleman who gathered an ample collection of prints between 1580 and 1587, while abbot of the Premonstratensian monastery in Hradisko, near Olomouc (Hordyński 2016). Ponętowski's collection is multifarious thematically, and it includes both artistic engravings and rather mediocre prints. The inconspicuous Pietà was impressed on one sheet together with fifteen other images, predominantly representing religious subjects (Figure 8b). Further examples of engravings after Cort's Pietà, all in oval frames, can be found in the Wolfegger Kabinett, one of the largest private collections of graphics (Kunstsammlung des Hauses Waldburg-Wolfegg, vol. 147, no 91: subscribed Attendite et videte si est dolor sicut dolor meus, Thren 1, signed F. Campion fe[cit] and Herman Weyen excud[it]; no 136: signed J[ohannes] sadeler excud[it], no 137: subscribed ATTENDITE VNIVERSI POPVLI, ET VIDETE DOLOREM MEVM, no 150 with inscriptions VENITE AD ME OMNES Matt[haeus] 11 and O quam tristis et afflicta fuit / Illa benedicta mater vnigeniti).

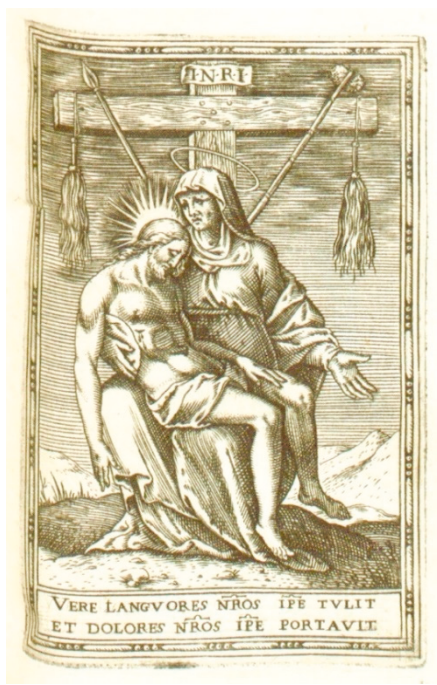

(a)

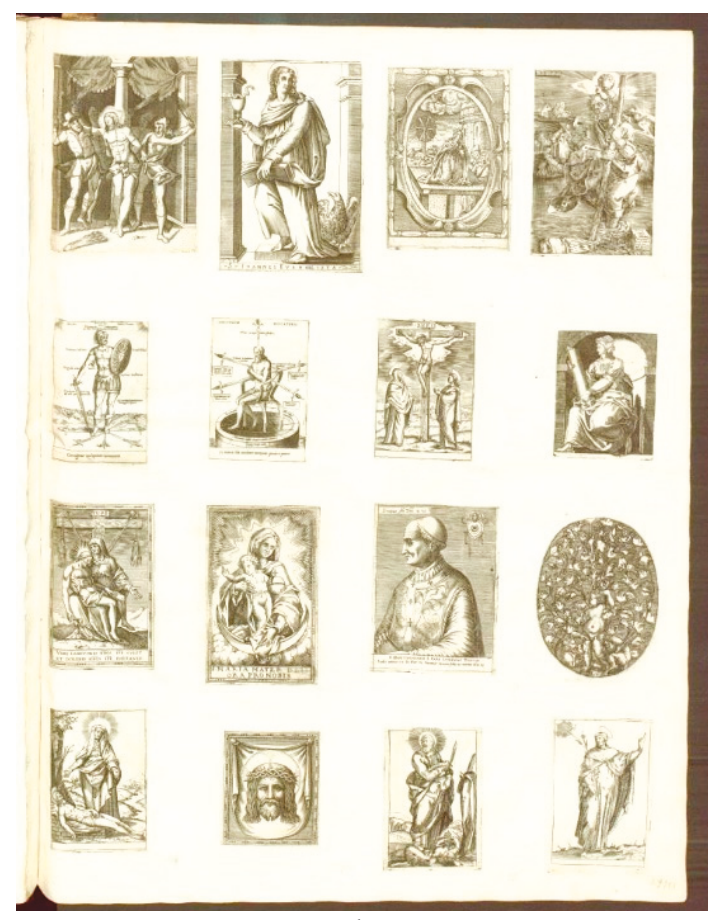

(b)

Figure 8. Pietà after Cornelis Cort, ca. 1580, engraving. Cracow, Biblioteka Jagiellońska, kolekcja Jana Ponętowskiego, box 149, no. I. 9104 @ Jagiellonian Library, Cracow.

The group of Mary and Christ drawn from Cort's engraving can also be found as the central motif of a much bigger and elaborate devotional print: the Rosarium dolorosum gloriosae Virginis signed by Giulio Roberti and Natale Bonifacio in 1579 (Iulius Robertus exc[udit] Romae Natalis Bonifacius inventor atque Fecit 1579.) An impression preserved in Anzio (Biblioteca Clementina, $470 \times 351 \mathrm{~mm}$ ) is included in a collection of devotional engravings from the stock of Antonio Lafreri's heirs (Marigliani and Biguzzi 2010, no. 85). Their inventories mention several prints representing the Rosary (Pagani 2008a; Pagani 2008b; Pagani 2011; Pagani 2012), while solely Vaccari's index includes an entry 'Sorrowful Rosary engraved by Natale Bonifacio'-Rosario doloroso con li misterij, intagliato da Natale Bonifacio (Ehrle 1908, p. 61, line 94). 


\section{Between Rome and Nancy: Jacques Callot for the White Penitents}

Yet another Pietà resembling Cort's engraving (in mirror image) is Jacques Callot's etching for the frontispiece of Règlement et établissement de la Compagnie des Pénitents blancs de la Ville de Nancy (Règlement et établissement 1635) (Figure 9), subsequently copied in the eighteenth century in the woodcut technique (Meaume 1855), (Dompnier and Vismara 2008). It is impossible to state whether Callot represented a painting or a sculpture displayed in the architectural framing and still less whether he portrayed a real altar with an existing panel, as he did on the frontispiece of the Miracles et graces de N. Dame de Bon Secours les Nancy (Miracles et graces 1630), (Choné 1992), (Châtellier 1993). The Notre-Dame de Bonsecours, a relief by Mansuy Gauvain (died after 1542), is still preserved in the Bonsecours church, but the chapel (subsequently the collegiate church) of St. Michael Archangel in Nancy, given to the White Penitents in 1634, was destroyed in 1793. In 1779, Jean-Joseph Bouvier Lionnois described the chapel of the White Penitents as having no distinguishing elements in either its exterior or interior (an opinion repeated by later authors), with the choir of the congregation decorated with a number of pious images (plusieurs tableaux de piéte); he mentioned a few interesting objects preserved in the church but no altarpiece with the Pietà (Lionnois 1779, pp. 340-42); (Lionnois 1805, pp. 215-16); (Lepage 1838).

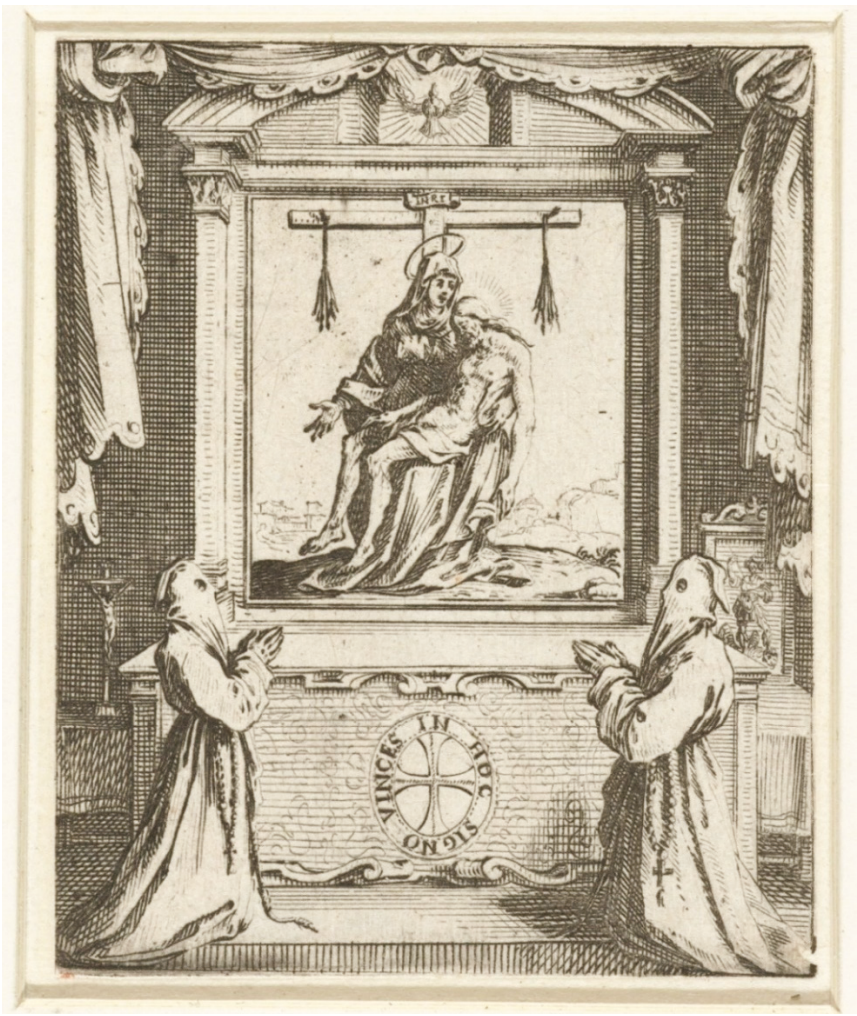

Figure 9. Jacques Callot, frontispiece of (Règlement et établissement 1635), etching. Amsterdam, Rijksmuseum, RP-P-OB-4885 (C) Rijksmuseum, Amsterdam http://hdl.handle.net/10934/RM0001. COLLECT.40271.

Whether or not a depiction of a real altarpiece image, the Pietà of the White Penitents does not seem to be intended as a reproduction of Michelangelo's sculpture. This is particularly interesting, as Callot not only knew the Vatican Pietà, but he even reproduced it in the early seventeenth century (Figure 10). Born in Nancy, Callot was active in Rome between 1608 and 1611 and his Delineationes 
picturae altarium in Ecclesiis S. Petri et S. Pauli Romae, also known as Les Tableaux de Rome or Les Eglises jubilaires, certainly included the Vatican Pietà, as it was placed in one of the privileged altars of St. Peter's Basilica (Loire 1993, pp. 98, 104-5); (Harent 2012, p. 208); (Meyer 2012). The Pietà of the White Penitents, engraved after Callot had returned to his hometown, seems rather unrelated to his Pietà from Les Tableaux de Rome, while close to the Pietà by Cornelis Cort, considering the poses and gestures of Christ and Mary, especially the scourges hanging from the horizontal beam of the Cross (Figure 7). Thus, on the title page of Règlement et établissement de la Compagnie des Pénitents blancs de la Ville de Nancy we find a third- or even fourth-generation descendant of Michelangelo's masterpiece, being at the same time an altarpiece image: mediated first by Bonasone's engraving, then Cort's engraving and-perhaps - the unpreserved altar panel in the Confraternity of White Penitents in Nancy.

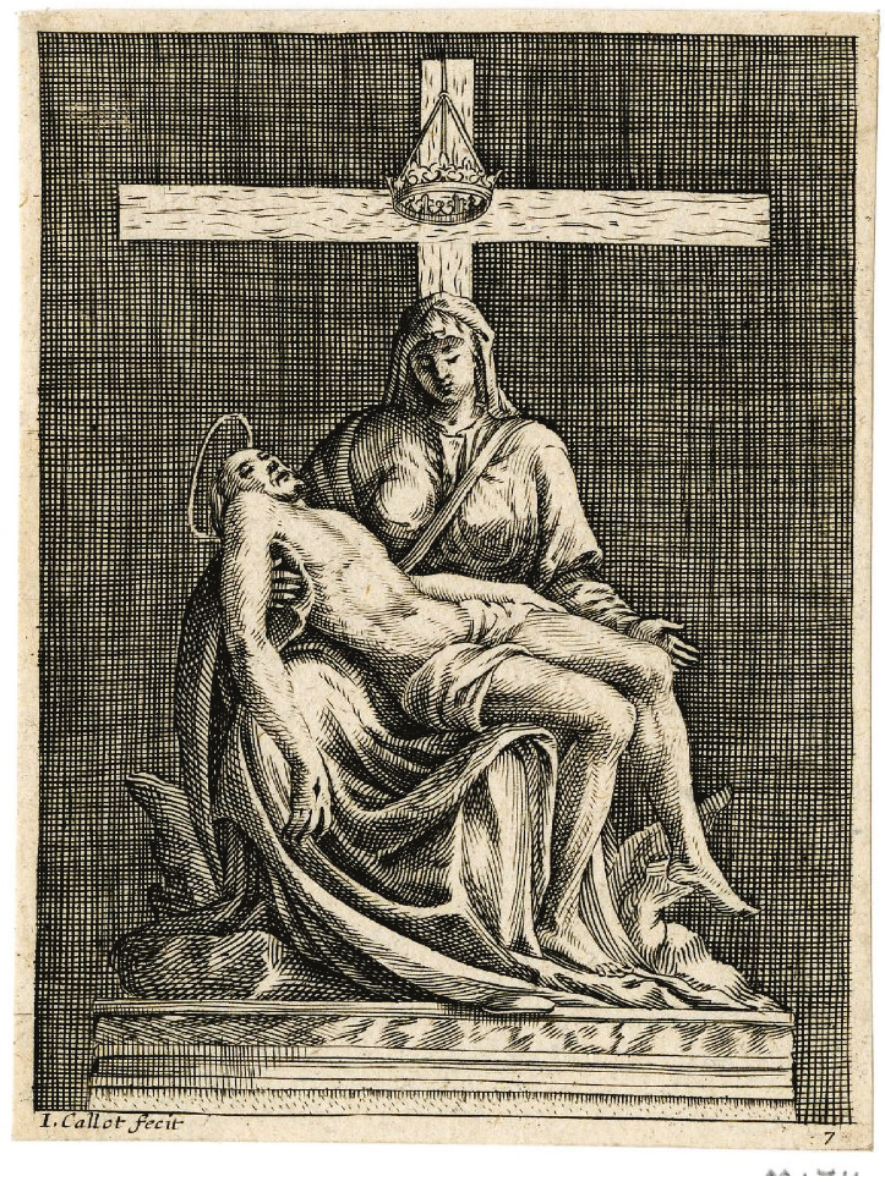

Figure 10. Jacques Callot, Pietà after Michelangelo Buonarroti, 1608-1611, engraving. London, British Museum, 1861, 0713.327 (C) Trustees of the British Museum

\section{Augsburg: Lucas Kilian for a Learned Lithuanian Cleric}

The ambiguous status of many works regarded as both masterpieces and religious images is far from unusual and a shifting back and forth between the realms of masterpieces, altarpieces, and devotional prints is well attested in the early modern period. An example is the Pietà by Hans von Aachen of ca. 1596, originally placed in a chapel of the Wilhelminische Veste, later known as 
Herzog-Max-Burg, in Munich. The painting, destroyed in 1944 and known from archival photographs (Figure 11) (Peltzer 1911-1912), was subject to various adaptations ca. 1600 (Jacoby 2000). One of these is the Pietà by Lucas Kilian, one of the most productive Augsburg printmakers of the early seventeenth century (Zijlma and Anzelewsky 1976, no. 51), (Jacoby 1996). The engraving is inscribed S(uae) C(aesareae) M(aiestatis) pictor Ioan(nes) ab Ach(en) pinxit and signed Lucas Kilian(us) Aug(ustanus) scalps(it) Venetijs (Figure 12). Thus, the work was explicitly referred to as modelled on Hans von Aachen's invention and executed in Venice, where Kilian stayed between 1601 and 1604 (Trevisan and Zavatta 2013). However, the Pietà after Hans von Aachen was not only a reproduction of a painting, but also a pious gift.

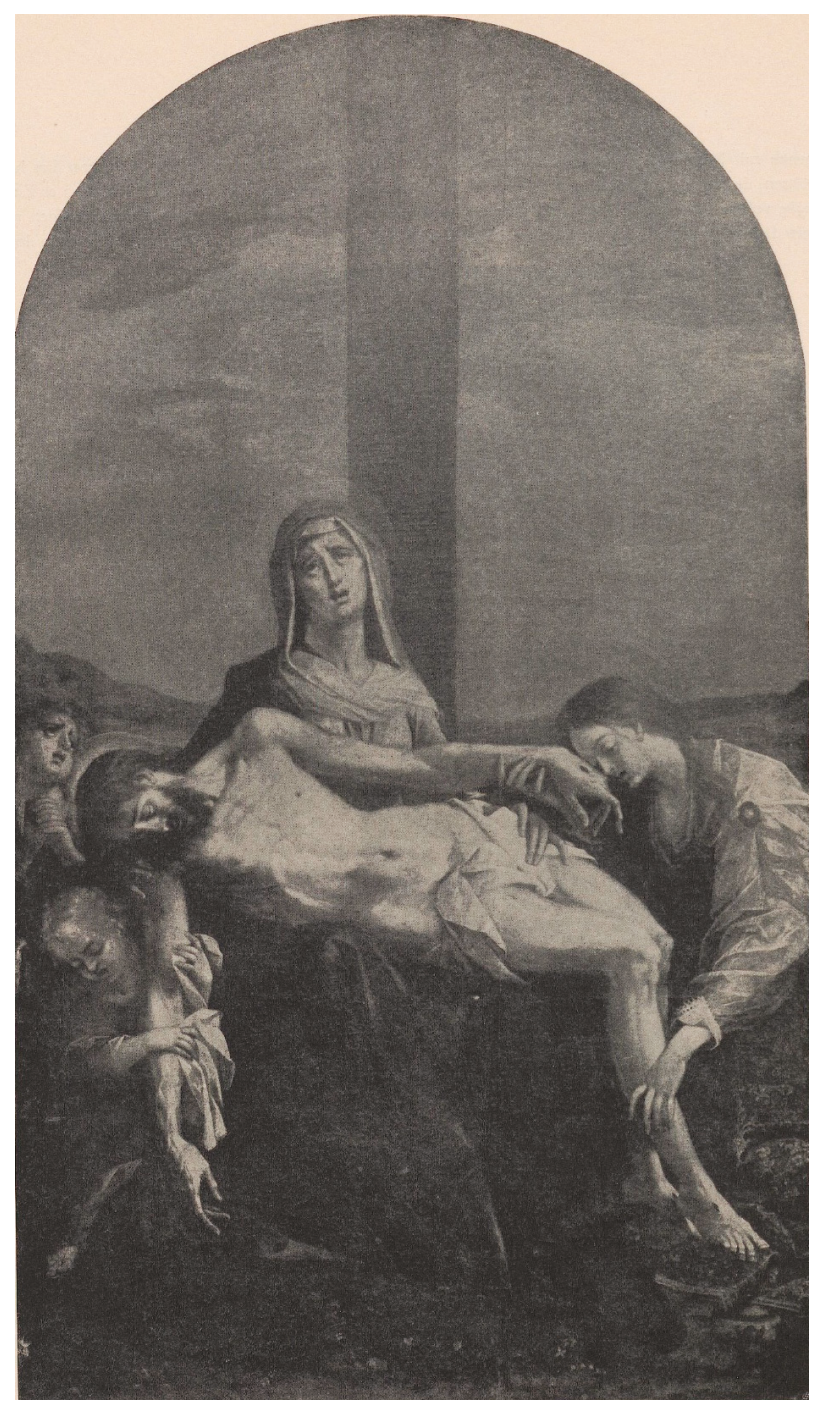

Figure 11. Hans von Aachen, Pietà, ca. 1596, oil on canvas. Destroyed in 1944. Photo after Peltzer (1911-1912) https://digi.ub.uni-heidelberg.de/diglit/jbksak1911_1912/0106 C) Universitätsbibliothek Heidelberg. 


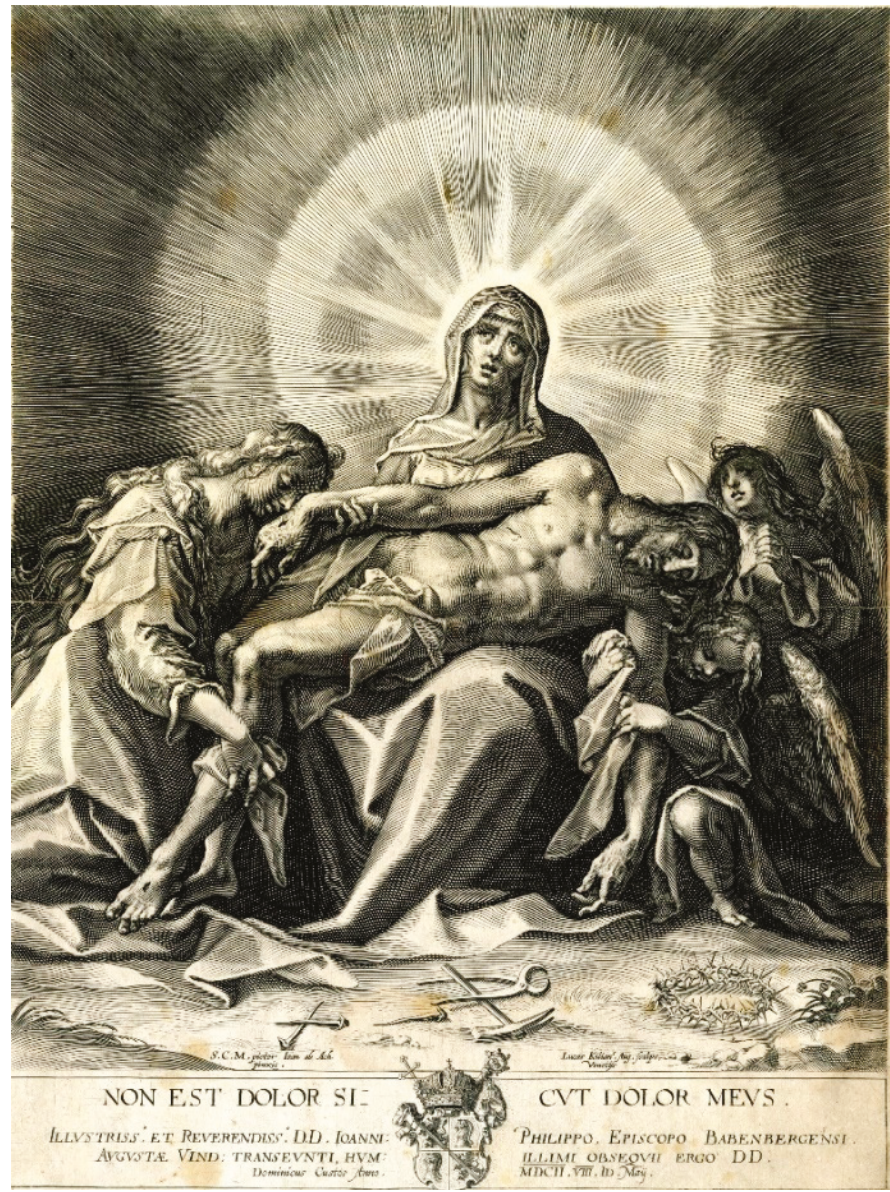

Figure 12. Lucas Kilian (engraver) and Dominicus Custos (publisher), Pietà after Hans von Aachen, 1602, engraving. London, British Museum, D, 7.8 C Trustees of the British Museum

Two further names appear in the lower margin, where the publisher, Dominicus Custos (Kilian's stepfather and teacher), dedicated the engraving to Johannes Philipp von Gebsattel, the Prince-Bishop of Bamberg, 'when he was crossing Augsburg' in May 1602. The dedication is preceded by a verse from the Lamentations of Jeremiah '[there is no] sorrow like to my sorrow' (NON EST DOLOR SI/CVT DOLOR MEVS—Lamentations 1:12). Thus, the expression ... AVGVSTAE VIND[elicorum] TRANSEVNTI ... referring to Gebsattel 'crossing Augsburg' may be understood literally, but at the same time it recalls the beginning of the verse from the Lamentations, which reads in its entirety $O$ vos omnes qui transitis per viam, attendite, et videte si est dolor sicut dolor meus! -' $\mathrm{O}$ all ye that pass by the way, attend, and see if there be any sorrow like to my sorrow!'.

Thus, Kilian used Hans von Aachen's Munich altarpiece as a model for an engraving, whereas Custos skilfully chose a Biblical quote for a dedication that responded to both the iconography and the circumstances. Gebsattel's visit to Augsburg was an opportunity that was not to be missed, as the mighty Prince-Bishop might turn into a patron. Therefore, Custos not only dedicated the Lamentation to Gebsattel but also engraved his portrait in 1602 (Amsterdam, Rijksmuseum, RP-P-1918-1075, http://hdl.handle.net/10934/RM0001.COLLECT.100185). In the early seventeenth century, offering devotional prints and portraits to prominent personalities was a common strategy for capturing 
customers among Church hierarchs and members of noble families and Augsburg attracted visitors from various countries, which brings us to the final example of indirect reception of Michelangelo's Vatican Pietà.

In early 1604, a Lithuanian magnate, Mikołaj Krzysztof Radziwiłł 'the Orphan' (Lithuanian: Mikalojus Kristupas Radvila Našlaitèlis) dispatched his sons to study in Augsburg (Chachaj 1995). They were welcomed by Eustachy Wołłowicz (Lithuanian: Eustachijus Valavičius), a protonotary apostolic, provost of Trakai, custos of Vilnius, and referendary of the Grand Duchy of Lithuania, who travelled through Europe at the time and who, encouraged by Radziwiłł, stayed in Augsburg for a few months to accompany his sons. The visit of the young Radziwiłłs proved particularly fruitful for the Augsburg engravers, who found keen customers among the Polish and Lithuanian nobles, and Wołłowicz was portrayed thrice by Kilian: first in 1604, and subsequently, as Bishop of Vilnius, in 1618 and 1621 (Zijlma and Anzelewsky 1976, nos. 492-494). The date of the earliest portrait coincides with Wołłowicz's sojourn in Augsburg, when another print dedicated to him was also created: a Pietà explicitly, even if indirectly, referring to Michelangelo (Figure 13), (Zijlma and Anzelewsky 1976, no. 52).

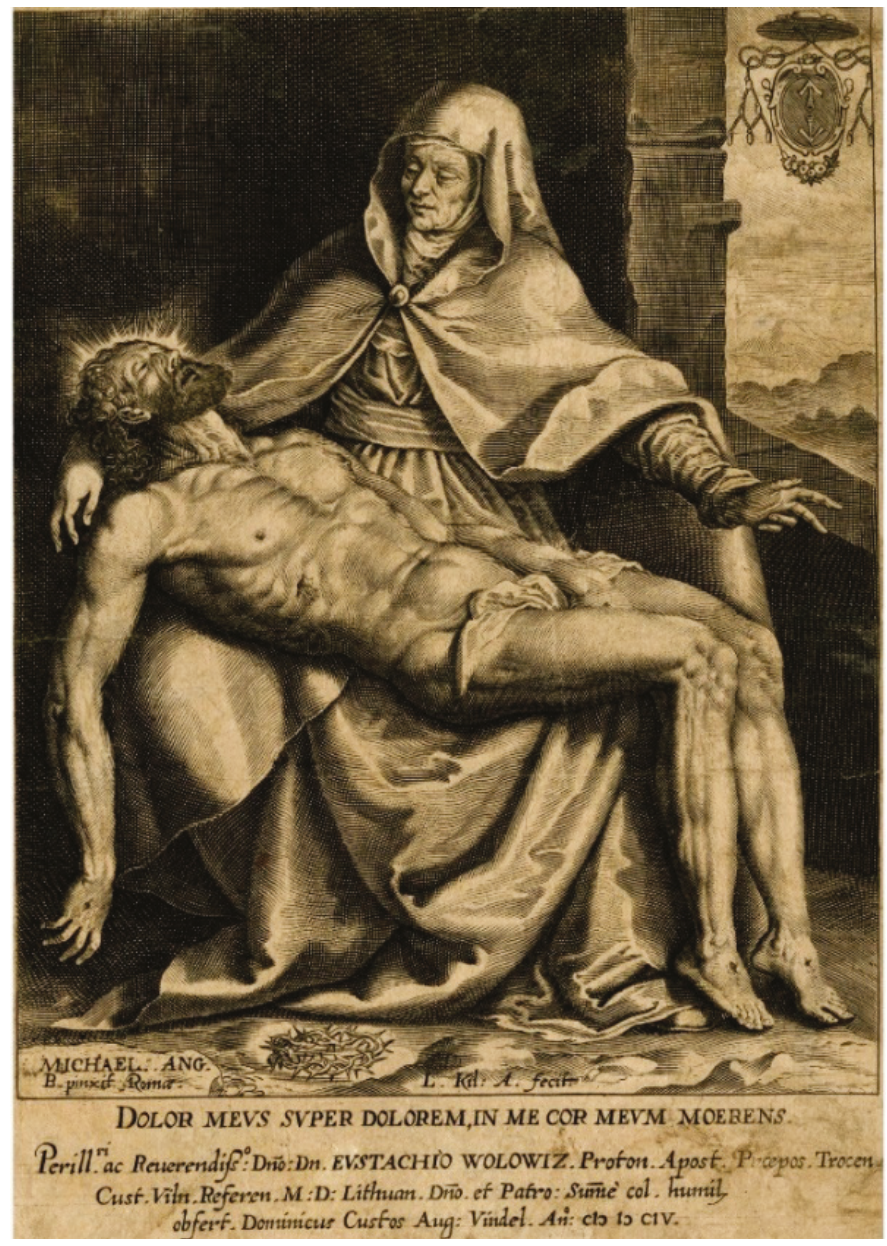

Figure 13. Lucas Kilian (engraver) and Dominicus Custos (publisher), Pietà after Monogrammist PHPT, 1604, engraving, London, British Museum, 1980, U.1445 (C) Trustees of the British Museum. 
According to the inscriptions, the engraver, Lucas Kilian (L[ucas] Kil[ian] A[ugustanus] fecit), used as a model Michelangelo's work 'painted in Rome' (MICHAEL. ANG[elus] // B[onarotus] pinxit Romae). Actually, Kilian did not reproduce the Vatican Pietà, but almost slavishly copied one of its travesties, the aforementioned late-sixteenth century print by the Monogrammist PHPT, which also includes an identical reference to Michelangelo. However, Kilian's Pietà, like his earlier Lamentation after Hans von Aachen, was additionally provided with a dedication in the lower margin, also subscribed by Dominicus Custos. The inscription begins with a caption from Jeremiah: 'My sorrow is above sorrow, my heart mourneth within me' (DOLOR MEVS SVPER DOLOREM, IN ME COR MEVM MOERENS-Jeremiah 8:18), and continues with an address to Wołłowicz dated 1604 in Augsburg.

The future Bishop of Vilnius (1616-1630) Eustachy Wołłowicz, renowned as both religious cleric and sophisticated humanist, studied in Rome from 1593 to 1596 where he was ordained subdeacon on 24 September 1594 and deacon on 13 April 1596 (Jujeczka 2018, no. 77). He had a chance to see Michelangelo's Pietà then, but it is not confirmed that he did. It is also not known how he received the Pietà dedicated to him in 1604: as a token of his past visit to Rome, as a reproduction of Michelangelo's famous work, as a devotional image - or as all of these. However, there is a record of how the Radziwiłł brothers spent time in Augsburg, studying but also devoting themselves to various religious practices, often accompanied by Wołłowicz. Learned Jesuits played a prominent role in their milieu, such as Matthäus Rader who dedicated a collection of exempla, Viridarium Sanctorum, to the Radziwiłłs on 29 September 1604 and Syntagma de statu morientium, to Wołłowicz on 30 September (Rader 1604a; Rader 1604b; VD17). Soon afterwards, in early October, Wołłowicz left Augsburg and headed again to Italy.

\section{Conclusions}

Long before its official coronation in 1637, the Vatican Pietà proved to be a direct or, admittedly much more often, an indirect model for various devotional images. Between Primaticcio's 1546 copy 'on the right side of the altar' of the Royal Chapel in Fontainebleau palace and Callot's 1635 frontispiece of the Règlement et établissement de la Compagnie des Pénitents blancs de la Ville de Nancy, perhaps representing the altarpiece of the chapel of the White Penitents, there is a wide group of graphic images. Several printmakers responded to the Vatican Pietà as early as the mid-sixteenth century, and some of their works were copied repeatedly. The engravings were not always accurate, and as they gradually moved away from the appearance of Michelangelo's sculpture, the remote original design and its author were often forgotten. In addition, the contexts and the recipients-if known-are of essence here. Even some single-sheet engravings clearly conceived as reproductions of the statue carved by Michelangelo were provided with inscriptions that appeal to believers rather than to art amateurs. Some engravings do not depict the marble sculpture, but rather represent a religious scene and name Michelangelo as the inventor. Giulio Bonasone's 1547 print is particularly prominent, as it stands at the beginning of the whole chain of interrelated works, multifarious with regard to quality, contexts, and assumed audience. There are mediocre anonymous woodcuts and elaborate engravings and etchings by renowned masters from various countries including Cornelis Cort, Lucas Kilian, and Jacques Callot. There are single-leaf prints, title page images and illustrations in Biblical books and catechisms. Some may be regarded as images for the sake of 'simple folk,' some were devised for sophisticated scholars or even specific individual addressees, but all the intended recipients were, after all, pious Christians. Therefore, Michelangelo, called 'the inventor of filth' by an otherwise unknown author of the mid-sixteenth century, at the same time turned out to be an involuntary inventor of devotional images.

Funding: This research was funded by the NATIONAL SCIENCE CENTRE, POLAND, grant number 2015/17/B/HS2/02469.

Acknowledgments: I would like to express my sincere gratitude to Prof. Luise Schorn-Schütte for her kind invitation to spend a month at the Forschungskolleg Humanwissenschaften in Bad Homburg, where I wrote the first draft of this article in August 2017.

Conflicts of Interest: The author declares no conflict of interest. 


\section{References}

\section{Bibliographies}

Verzeichnis der im deutschen Sprachbereich erschienenen Drucke des 16. Jahrhunderts. Available online: www.vd16.de (accessed on 6 May 2019).

Verzeichnis der im deutschen Sprachraum erchienenen Drucke des 17. Jahrhunderts. Available online: www. vd17.de (accessed on 6 May 2019).

\section{Primary Sources}

Aretino, Pietro. 1957. Lettere sull'arte. Edited by Ettore Camesasca. Milan: Edizioni del Milione, vol. 1, no. LXIII.

Canisius, Petrus. 1555. Summa doctrinae Christianae per quaestines tradita, et in usum Christianae pueritiae nunc primum edita. Vienna: Zimmermann (VD16 = C 722). Available online: http://gateway-bayern.de/VD16+C+722 (accessed on 6 May 2019).

Canisius, Petrus. 1556a. Summa doctrinae Christianae per quaestines tradita, et in usum Christianae pueritiae nunc primum edita. Vienna: Zimmermann (VD16 = C 723). Available online: http://gateway-bayern.de/VD16+C +723 (accessed on 6 May 2019).

Canisius, Petrus. 1556b. Frag vnd antwurt Christlicher Leer in den jaubstucken yetz neulich ... der Christichen Jugent vnnd allen aingaltigen zu nutz inn Druckh außgangen. Vienna: Zimmermann (VD16 = C 734). Available online: http://gateway-bayern.de/VD16+C+734 (accessed on 6 May 2019).

Canisius, Petrus. 1558a. Summa doctrinae Christianae per quaestines tradita, et in usum Christianae pueritiae nunc primum edita. Vienna: Zimmermann (VD16 = C 724). Available online: http://gateway-bayern.de/VD16+C+724 (accessed on 6 May 2019).

Canisius, Petrus. 1558b. Summa doctrinae Christianae per quaestines tradita, et in usum Christianae pueritiae nunc primum edita. Vienna: Zimmermann (VD16 = C 725). Available online: http://gateway-bayern.de/VD16+C+725 (accessed on 6 May 2019).

Il Carteggio di Michelangelo. 1979. Edited by Giovanni Poggi and Paola Barocchi. Florence: Sansoni, vol. 4, p. 229. Available online: http://www.memofonte.it/autori/carteggio-michelangelo-buonarroti-1475-1564-10.html (accessed on 6 May 2019).

Celio, Gaspare. 1638. Memoria delli nomi dell'Artefici delle Pitture che sono in alcune chiese, facciate, e palazzi di Roma. Naplec: Bonino, pp. 65-66.

Comptes des bâtiments. 1877. Les comptes des bâtiments du roi (1528-1571). Edited by Léon de Laborde. Paris: Comptes des bâtiments, vol. 1, pp. 193-94.

Condivi, Ascanio. 2009. Vita di Michelangelo Buonarroti. Edited by Charles David. p. 21. First published 1553. Available online: http://archiv.ub.uni-heidelberg.de/artdok/volltexte/2009/714/ (accessed on 6 May 2019).

Cronaca fiorentina, 1537-1555. 2000. Edited by Enrico Coppi. Florence: Olschki, p. 103.

Dan, Pierre. 1642. Le tresor des merveilles de la maison royale de Fontainebleau. Paris: Sebastien Cramoisy, p. 60.

Ketābā d-Ewangeliyōn qaddīšă de-Māran w-Alāhan Yēšù' Mešĭhā: Liber sacrosancti evangelii de Iesu Christo. 1555. Vienna: Zimmermann (VD16 = B 4584). Available online: http://gateway-bayern.de/VD16+B+4584 (accessed on 6 May 2019).

Ketābā d-Ewangeliyōn qaddīšă de-Māran w-Alāhan Yēšù' Mešĭhā: Liber sacrosancti evangelii de Iesu Christo. 1562. Vienna: Zimmermann (VD16 = ZV 1928). Available online: http://gateway-bayern.de/VD16+ZV+1928 (accessed on 6 May 2019).

Ligorio, Pirro. 1549. Antichità di Roma. manuscript, Oxford, Bodleian Library, ms. Canon. Ital. 138, fol. 6r.. Available online: http://ligorio.sns.it/ligorio.php?tipo=doc\&idDoc=0 (accessed on 6 May 2019).

Miracles et graces de N. Dame de Bon Secours les Nancy. 1630. Nancy: Sébastien Philippe.

Rader, Matthäus. 1604a. Viridarium Sanctorum. Augsburg: Christoph Mang (VD17 = 23:3021174D). Available online: http://gso.gbv.de/DB=1.28/CMD?ACT=SRCHA\&IKT=8002\&TRM=\%2723:302174D \%27 (accessed on 6 May 2019).

Rader, Matthäus. 1604b. Syntagma de Statu Morientium. Augsburg: Christoph Mang (VD17 = 12:100215Q). Available online: http://gso.gbv.de/DB=1.28/CMD?ACT=SRCHA\&IKT=8002\&TRM=\%2712:100215Q $\% 27$ (accessed on 6 May 2019). 
Règlement et établissement de la Compagnie des Pénitents blancs de la Ville de Nancy. Nancy: Antoine Charlot. Vasari, Giorgio. 1962. La vita di Michelangelo nelle redazioni del 1550 e del 1568. Edited by Paola Barocchi. Milan: Ricciardi, p. 19. First published 1550 and 1568.

\section{Secondary Sources}

Alberti, Alessia. 2015. La fortuna di Michelangelo nelle stampe del Cinquecento. In D'après Michelangelo. Edited by Alessia Alberti, Alessandro Rovetta and Claudio Salsi. Venice: Marsilio, pp. 204-11.

Barnes, Bernardine. 1998. Michelangelo's Last Judgment: The Renaissance Response. Berkeley: University of California Press, pp. 71-101.

Barnes, Bernardine. 2010. Michelangelo in Print. Reproductions as Response in the Sixteenth Century. Farnham: Ashgate, pp. 149-53.

Chachaj, Marian. 1995. Zagraniczna edukacja Radziwiłłów. Lublin: Wydawnictwo Uniwersytetu Marii Curie-Skłodowskiej, pp. 59-60.

Châtellier, Louis. 1993. La Vie religieuse à Nancy et dans ses environs à l'époque de Jacques Callot. In Jacques Callot (1592-1635): actes du colloque. Edited by Daniel Ternois. Paris: Klincksieck, pp. 163-77.

Choné, Paulette, ed. 1992. Jacques Callot 1592-1635 [cat. exh. Musée Historique Lorrain, Nancy, 13 May-14 September 1992]. Paris: Réunion des musées nationaux, nos. 619, 675.

Cox-Rearick, Janet. 1995. The Collection of Francis I: Royal Treasures. New York: Harry N. Abrams, cat. no. IX, 6.

Cupperi, Walter. 2010. Giving away the moulds will cause no damage to his Majesty's casts. In Plaster Casts: Making, Collecting and Displaying from Classical Antiquity to the Present. Edited by Rune Frederiksen and Marchand Eckart. Berlin: De Gruyter, p. 85.

De Maio, Romeo. 1990. Michelangelo e la Controriforma. Florence: Sansoni, pp. 17-167. First published in 1974. de Tolnay, Charles. 1948. Michelangelo. Princeton: Princeton University Press, vol. 2, p. 69.

Dimier, Louis. 1900. Le Primatice: peintre, sculpteur et architecte des Rois de France. Paris: E. Leroux, p. 333.

Dompnier, Bernard, and Paola Vismara. 2008. Confréries et dévotions dans la catholicité moderne (mi-XVe-début XIXe siècle). Rome: École française de Rome, pp. 44, 121.

Roma prima di Sisto V. La pianta di Roma Du Perac-Lafréry del 1577 riprodotta dall'esemplare esistente nel Museo Britannico. Edited by Franz Ehrle. Rome: Danesi.

Gaye, Giovanni. 1839. Carteggio indedito d'artisti dei secoli XIV. XV. XVI. Pubblicato ed illustrato con documenti pure inediti. Florence: Giuseppe Molini, vol. 2, p. 500.

Harent, Sophie. 2012. Jacques Callot et la leçon de Rome (1608-1611). In Ein privilegiertes Medium und die Bildkulturen Europas. Deutsche, französische und niederländische Kupferstecher und Graphikverleger in Rom von 1590 bis 1630. Akten des Internationalen Studientages der Bibliotheca Hertziana, Rom, 10-11 November 2008. Edited by Eckhard Leuschner. Munich: Hirmer, pp. 206-20.

Hordyński, Piotr. 2016. Kolekcja Jana Ponętowskiego. Wstęp do opisu zawartości. Biuletyn Biblioteki Jagiellońskiej 66: 69-84.

Jacoby, Joachim W. 1996. The New Hollstein German Engravings, Etchings and Woodcuts 1400-1700: Hans von Aachen. Compiled by Joachim Jacoby. Edited by Ger Luijten and Robert Zijlma. Rotterdam: Sound \& Vision Interactive, no. 39.

Jacoby, Joachim W. 2000. Hans von Aachen 1552-1615. Munich: Deutscher Kunstverlag, cat. no. 18.

Klerycy z ziem polskich, litewskich i pruskich święceni w Rzymie (XVI-pocz. XX w.)/Clerici ex terris Poloniae, Lithuaniae et Prussia Romae seu Urbe ordinati (saec. XV ad init. XX). 2018. Edited by Stanisław Jujeczka. Wrocław: Wydział Nauk Historycznych, no. 77.

Jurkowlaniec, Grażyna. 2015. A Miracle of Art and Therefore a Miraculous Image. A Neglected Aspect of the Reception of Michelangelo's Vatican Pietà. Artibus et Historiae 72: 175-98.

Lepage, Henri. 1838. Historie de Nancy. Ville-vielle et ville-neuve. Nancy: Gonet, pp. 67-69.

Lincoln, Evelyn. 2000. The Invention of the Italian Renaissance Printmaker. New Haven: Yale University Press, p. 185.

Lionnois, Jean Joseph. 1779. Essais sur la ville de Nancy. La Haye: Aux dépens de la Compagnie, pp. $324-42$.

Lionnois, Jean Joseph. 1805. Histoire des villes vieille et neuv de Nancy depuis leur fondation jusqu'en 1788. Nancy: Haener Père, vol. 1, pp. 212-19.

Loire, Stéphane. 1993. Les tableaux de Rome gravés par Jacques Callot. In Jacques Callot (1592-1635): actes du colloque. Edited by Daniel Ternois. Paris: Klincksieck, pp. 91-117. 
Marigliani, Clemente, and Giancarlo Biguzzi. 2010. La collezione sacra della bottega di Antonio Lafréry. Anzio: Topografia Marina, no. 85.

Meaume, Édouard. 1855. Recherches sur la vie et les ouvrages de Jacques Callot. Mémoires de l'Académie de Stanislas 1854: 366-67, (republished as a book: Paris: Renouard, 1860, pp. 132-33, no. 199).

Meyer, Véronique. 2012. Les graveurs professionels français à Rome entre 1590 et 1636: Audran, Callot, Mellan et les autres. In Ein privilegiertes Medium und die Bildkulturen Europas. Deutsche, französische und niederländische Kupferstecher und Graphikverleger in Rom von 1590 bis 1630. Akten des Internationalen Studientages der Bibliotheca Hertziana, Rom, 10-11 November 2008. Edited by Eckhard Leuschner. Munich: Hirmer, pp. 301-14.

Nagel, Alexander. 2000. Michelangelo and the Reform of Art. Cambridge: Cambridge University Press, pp. 169-87. Pagani, Valeria. 2008a. The Dispersal of Lafreri's Inheritance, 1581-1589 [part I]. Print Quarterly 25: 3-23.

Pagani, Valeria. 2008b. The Dispersal of Lafreri's Inheritance, 1581-1589, part II: Pietro de Nobili. Print Quarterly 25: 363-93.

Pagani, Valeria. 2011. The Dispersal of Lafreri's Inheritance, 1581-1589, part III: The De' Nobili-Arbotti-Clodio Parnership. Print Quarterly 28: 119-36.

Pagani, Valeria. 2012. Inventari di rami Lafreri-Duchet, 1598-1599. In Ein privilegiertes Medium und die Bildkulturen Europas. Deutsche, französische und niederländische Kupferstecher und Graphikverleger in Rom von 1590 bis 1630. Akten des Internationalen Studientages der Bibliotheca Hertziana, Rom, 10-11 November 2008. Edited by Eckhard Leuschner. Munich: Hirmer, pp. 63-86.

Palmer Wandel, Lee. 2015. Reading Catechisms, Teaching Religion. Leiden: Brill, p. 326.

Panofsky, Soergel Gerda. 1991. Michelangelo's Christus und sein römisches Auftraggeber. Worms: Werner, pp. 146-7. Peltzer, Rudolf Arthur. 1911-1912. Der Hofmaler Hans von Aachen. Seine Schule und seine Zeit. Jahrbuch der Kunsthistorischen Sammlungen des Allerhöchsten Kaiserhauses 30: 162.

Roman D'Elia, Una. 2006. Drawing Christ's Blood: Michelangelo, Vittoria Colonna, and the Aesthetics of Reform. Renaissance Quarterly 59: 90-129. [CrossRef]

Rubach, Birte. 2016. Ant. Lafreri formis Romae. Der Verleger Antonio Lafreri und seine Druckgraphikproduktion. Berlin: Lukas Verlag, pp. 61-63, 131-34.

Schwedes, Kerstin. 1998. Historia in statua. Zur Eloquenz plastischer Bildwerke Michelangelos im Umfeld des Christus von Santa Maria sopra Minerva zu Rom. Frankfurt/Main: Peter Lang, pp. 33-32.

Sellink, Manfred, and Huigen Leeflang, eds. 2000. The New Hollstein Dutch and Flemish Etchings, Engravings and Woodcuts, 1450-1700: Cornelis Cort. Rotterdam: Sound \& Vision Interactive, vol. 1, no. 70.

Stendhal. 1817. Histoire de la peinture en Italie. Paris: Didot, vol. 2, p. 343.

Streicher, Fridericus, ed. 1933. S. Petri Canisii ... Catechismi Latini et Germanici. Rome: Pontificia Universitas Gregoriana, vol. 1: Catechismi Latini. vol. 1, pp. 103-5.

Thode, Henry. 1908. Michelangelo. Kritische Untersuchungen über seine Werke. Berlin: Grote, vol. 1, p. 58.

Trevisan, Luca, and Giulio Zavatta. 2013. Incisori itineranti nell'area veneta nel seicento: dizionario bio-bibliografico. Verona: Università degli Studi di Verona, p. 77.

Veress, Ferenc. 2010-2011. La fortuna della Pietà di San Pietro nel Cinquecento: influsso, interpretazioni, copie e varianti. Ph.D. thesis, Sapienza Università di Roma, Roma, Italy.

Veress, Ferenc. 2012. La fortuna della Pietà vaticana nel Cinquecento: influsso, interpretazioni, copie e varianti. Arte cristiana 100: 81-90.

Wallace, William E. 1997. Michelangelo's Risen Christ. The Sixteenth Century Journal 28: 1251-80. [CrossRef]

Wilkinson, Robert J. 2007. Orientalism, Aramaic and Kabbalah in the Catholic Reformation: The first printing of the Syriac New Testament. Leiden: Brill, pp. 182-85, 174-75, note 6.

Zijlma, Robert, and Fedja Anzelewsky, eds. 1976. Hollstein's German Engravings, Etchings and Woodcuts ca. 1400-1700. vol. 17: Lucas Kilian to Philipp Kilian. Amsterdam: Van Gendt \& Co.

(C) 2019 by the author. Licensee MDPI, Basel, Switzerland. This article is an open access article distributed under the terms and conditions of the Creative Commons Attribution (CC BY) license (http://creativecommons.org/licenses/by/4.0/). 


\title{
Article \\ "Equal Rites": Fragmenting and Healing Bodies in the Early Modern Bay of Kotor
}

\author{
Milena Ulčar \\ Faculty of Philosophy, Department of Art History, University of Belgrade, Cika Ljubina 18/20, 11000 Belgrade, \\ Serbia; lena.ulcar@gmail.com
}

Received: 17 August 2019; Accepted: 25 October 2019; Published: 2 November 2019

\begin{abstract}
The aim of this paper is to examine the exchange of practices that developed when treating the bodies of ordinary laymen and those of saints. Body parts that had been obtained in unorthodox ways were used in private households in a manner strongly resembling the official methods of relic veneration. Conversely, the church authorities carried out repairs to damaged reliquaries by adopting an approach that mirrored the ways in which common people were healed in their homes (the application of holy images, use of votive gifts, etc.).
\end{abstract}

Keywords: reliquaries; body-part reliquaries; post-Tridentine religion; Bay of Kotor; Republic of Venice; healing; domestic devotion

\section{Introduction}

The main arguments in this article will be supported by a rather peculiar body of visual and archival data, including broken reliquaries (severely damaged in an earthquake that occurred in the 17 th century), incomplete verbal descriptions of long lost amulets, and testimonies given by mostly illiterate witnesses at local church court hearings. The methodological approach examines domestic practices that occurred in the Bay of Kotor in what is today Montenegro, most of which were considered blasphemous, and theoretically reframes them as logical counterparts to complementary institutional practices. On the other hand, institutionalised church practices, which were still unorthodox for their time, despite being officially approved, I will treat as an important clue to understanding the "rules" of domestic piety. The main focus will be on two related processes: the fragmenting and healing of the body in the early modern period. I will consider the act of fragmentation as not only implying use of relics and reliquaries, but also the dismemberment and manipulation of the bodies of common laymen, both living and deceased. In the same manner, I will examine healing practices as acts that applied equally to the suffering of "ordinary" bodies as to those of saints.

The topographical and chronological focus of this research is the 17th and 18th century Bay of Kotor, a small coastal area in the South-Western Balkans. Kotor, the capital of this region, and nearby villages Perast and Prčanj, were ruled by the Venetian government from the early 15th century until the fall of the Republic of Venice at end of the 18th century. Surrounding towns and villages such as Herceg Novi and Orahovac remained under the rule of the Ottoman Empire (Butorac 1998, 2000). Due to the nature of the archival and material evidence and its redistribution, I will place the focus of this analysis on the areas where the Catholic Church was dominant. In a somewhat odd turn, official archival records will serve my interpretation as the main source when analysing domestic devotional practices, whereas the parallel examination of official church practices will be approached solely through the analysis of damaged physical evidence. Similar inversions, which occur throughout the paper, add support to the main hypothesis: the dualistic nature of the domestic and the institutional, i.e., private and public, can be understood as part of the same "devotional spectrum" rather than as mutually exclusive (Ryan 2003, pp. 281-90). 


\section{Fragmentation of the Dead Body}

During the 17th and 18th century, a significant number of sermons urged believers in the Bay of Kotor not to excavate and fragment the bodies of the deceased. They described this act as a practice that "had already made two priests abandon this area". ${ }^{1}$ Despite the condemnation, the excavation and fragmentation of bodies occurred regularly, and the people who engaged in it believed they were "being healed and saved from death"2. As we learn from court records, men and women used bones and other parts taken from corpses for various rituals, which they performed at home to ward off everyday misfortune, sickness, and death. This domestic use of fragmented human bodies resembled the institutional treatment of saintly bodies. Both were treasured, used in rituals, and expected to offer protection in times of distress. However, to understand the significance of these "sinful acts", we are required to examine the nuances that the process of bodily fragmentation signified for contemporary believers in the Bay of Kotor.

The focal point of fragmentation, seen from this point of view, was the relics in the chapel of the Cathedral of Saint Tryphon in Kotor (Figure 1). Dozens of silver body parts-mainly legs, arms, and heads - have housed the bones of saints for centuries and are displayed on shelves around the casket holding Saint Tryphon's remains (Tomić 2009, p. 35; Ulčar 2017, p. 6) (Figure 2). During Saint Tryphon's Day celebrations, these reliquaries were displayed in the church and carried in processions along the streets of Kotor, where common people could observe (and touch) the fragmented bodies of their patron saint and his entourage (Stjepčević 1938). ${ }^{3}$ The silver limbs and heads, which were always displayed as a group, offered an institutionally approved image of fragmented bodies. This was crucial for ritual and devotional acts in the early modern Bay of Kotor.

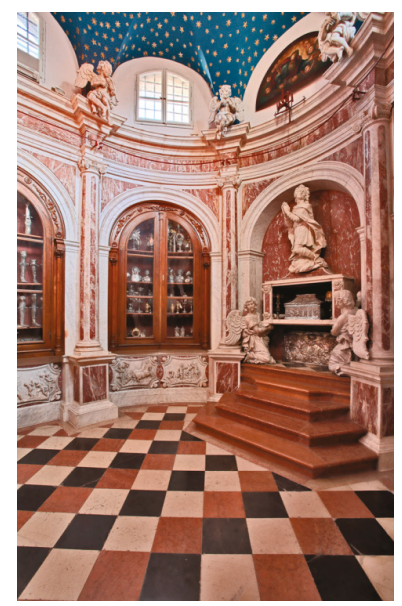

Figure 1. Saint Tryphon's relic chapel, 1704-1708, Kotor, Saint Tryphon's Cathedral (photo by Stevan Kordić).

Proporjedi. PROP VIII. p. 182. (Parish Archive in Perast NAP n.d.a).

Ibid., p. 179.

The Saint Tryphon's Day procession is described in: Timotej Cizila. BoveD'Oro, NAP R XVI. (Parish Archive in Perast NAP n.d.b). 


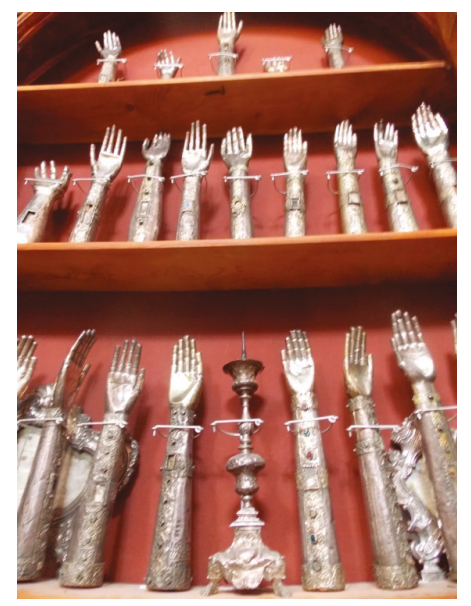

Figure 2. Reliquaries, 16th-18th century, Kotor, Saint Tryphon's relic chapel (photo by author).

By contrast, the same act of dissolution was considered sinful when other kinds of bodies came into question. As the popular sacral play of this period recalls, during his suffering on the cross, Christ's body was in danger of fragmentation. Missandro, one of the main protagonists in condemnation and crucifixion in Nenadić's play, was determined to break Christ's legs with a heavy mace and divide his body into pieces: "He should be cut/And ripped into pieces/Let them trusting (his Resurrection)/remain deluded!" Longinus opposed this suggestion strongly: "Great injustice would it be/to do that even to deceased!" (Nenadić 1975, p. 266). The fragmentation of Christ's body would result in the destruction of his identity. Had it been performed, he would have failed to fulfil his purpose. Bodies of saints, on the other hand, acquired power through an act that was similar, although usually less violent in nature (Bynum 1991).

Examples seen in the "secular" life of people in the bay correspond to the aforementioned duality of fragmentation. In official terms, the domestic use of common people's body parts was condemned. Popular 18th century catechisms by Antun Nenadić warned that excommunication awaited those who excavated corpses and desecrated deceased bodies (Nenadić 1768, p. 113). Numerous sermons of the time reflect this sentiment: "And where are those hellish souls who with wickedness, just as infidels, viciously and cursedly open the graves and tear the dead body apart?" It was detailed how people would open the tombs and "tear the dead body apart or collect the pus from the corpse and apply it on the skin of the sick." The same priest suggested perpetrators be banished "as one branch from the tree, cut from the goodness of the Holy Church." ${ }^{4}$ Therefore, the prescribed punishment for the fragmentation of deceased bodies was the excommunication of those sinners taking part in this dangerous act. The frequency with which similar statements appeared in sermons of the time indicates that the practice was widespread in the 17th and 18th centuries.

In 1630, a church tribunal in Venice accused a woman from Dalmatia of mutilating a male corpse. Two of her servants had allegedly helped her execute this sinful act. Her maid confessed that she had been forced to visit the graveyard two or three times: "a pigliar una testa del mordo (...), un osso di homo, o di spalla, o di coste (... )" (Čoralić 1998, p. 94). Once inside the household, they broke the bones with a hammer and performed rituals while uttering prayers. As will become clear later in this paper, people from different social classes resorted to manipulating the bones of the deceased to gain various benefits, with the healing of the body being the most commonly desired outcome. Concurrently, bones and

4 Propovjedi, PROP VIII, pp. 180, 181. (Parish Archive in Perast NAP n.d.a). 
particles of saints' bodies were employed in a similar manner in churches and chapels. It is apparent that there was a need to maintain and conduct the same processes in the domestic setting, as well as relying on officially approved rituals. In addition to various herbs, "dragon's leaking blood", and "claw or hoof of a large beast", an inventory discovered at Kotor's old pharmacy also listed a tooth of Saint Nicholas (Butorac 1999, p. 294). Particles of saints' bodies were obviously used for medical purposes outside the jurisdiction of the church. Once again, fragmentation was tolerated when it came to the remains of saints, but objectionable when attempted or executed on Christ's or "ordinary" deceased bodies.

More recent research reminds us that the concept of fragmentation of the holy body could be linked to another contemporaneous practice, wherein body parts considered criminal were put on public display as form of punishment (Terpstra 2015, pp. 7-52). The mutilation of "criminal" bodies was allowed across Europe, and it would be of great scholarly interest to understand the parallel perceptions that occurred in the early modern believer, e.g., the image of a powerful silver limb of a saint in the church juxtaposed against the sight of a criminal's rotting dismembered arm, leg, or head. However, as we learn from the archival materials, those kinds of punishments were quite rare in the Republic of Venice, at least when compared with other parts of Europe (Seitz 2011; Ostling 2011), and almost entirely absent from the Bay of Kotor. One exception occurred after a victory over the Ottoman army in Perast in 1654. The locals cut off the right arm of Mehmed Paša Rizvanagić, commander of the enemy troops, and sent his corpse to the Ottomans in exchange for slaves (Krušala 1996, p. 220). Through this act, they desecrated Rizvanagić's identity and humiliated his body by destroying its power and integrity. In 1538, a similar fate met don Carlo Gonzaga, Spanish commander of Herceg Novi, who was killed in retaliation for raping and murdering two young girls from Perast. ${ }^{5}$ The townsfolk cut off his right arm and confiscated his sword, which is preserved in Perast to this day. In spite of the rareness of similar occurrences, fragmenting the enemies' or criminals' bodies in the Bay of Kotor, in contrast to their saintly counterparts, always brought disempowerment and harm to their identity.

Such acts of fragmentation implied harming an individual's integrity, whether it was Christ on the cross, a defeated enemy, a common criminal, or a deceased neighbour. Tearing and cutting up the body brought humiliation, diminished its power, and endangered the prospect of life after death. And yet, when it came to relics and reliquaries, the fragmentation of bones and their placement into body-part containers was deemed a prerequisite for the opposite effect-one of gaining and distributing power and preserving identity (Hahn 1997, pp. 20-31). However, the domestic use of "common" deathly remains should not be reduced to a mere consequence of these mixed messages about fragmentation and the preservation of body parts in the early modern bay.

Fragmentation was considered a powerful yet dangerous act. Dismembering an enemy's body implied superiority and control acquired through victory over him. Missandro's desire to tear Christ's body stemmed from a similar motive. Both cases depict dismemberment and fragmentation as acts to relocate and gain power. Alternatively, body part reliquaries were always displayed as a group in chapels, church altars, and in the processional carrier along the streets. These particular types of reliquaries (as opposed to caskets, ostensory-shaped reliquaries, medallions, etc.) were meant to "work" together. To the early modern believer in the bay, they were always presented as a carefully orchestrated group of body parts, meant to create physical evidence for the concept of pars pro toto. They gained their power through a metaphorical or performative integration with the whole, whether that was a saint in heaven or other reliquaries in the chapel (Bynum and Gerson 1997, pp. 3-7; Hahn 2010, pp. 284-316). Fragmentation and integration were considered two sides of the same coin,

Прилог „Гласника народног универзитета“. 1934. Списак издожених предмета на културно историјској 5 издожби Народног универзитета Боке Которске која је свечано отворена 7. октобра 1934. г.

(The list of exhibited artifacts of The National University of Boka Kotorska, opened 7 October 1934). 
both of which were heavily co-dependent. The paradoxical (although theologically complementary) way of thinking (Bynum 2012, pp. viii-455) allowed for this institutional practice to become desirable and, more importantly, translatable to the domestic setting. This fragmentation implied power, its distribution, manipulation, or relocation.

In his Catechism, Ivan Antun Nenadić explained that a soul inhabits every limb of the human body in the same way that Christ was present in every host and chalice in the world. When death occurs, the soul separates from the "bodily temple" and transcends from the earthly domain into the realm of God (Nenadić 1768, p. 192). Nevertheless, various examples of post-mortem use of body parts in the early modern period point to a belief that human flesh and bones possessed power even after death. With veneration of relics, this belief was built upon the fact that the deceased person was holy and, as such, different from an "ordinary" human. The use of dead bodies was carefully orchestrated within the institutional context of church rituals. By contrast, manipulation of non-saintly corpses by common laymen was severely condemned. Numerous above-mentioned incidents testify that believers in the Bay of Kotor (as well as in other parts of the Venetian Republic) still excavated bodies of their neighbours from their graves and used the fragments in domestic rituals. This practice implied a strong conviction in the power of the human body to hurt or heal, even after death.

Katherine Park has argued that, during the late Middle Ages, Italians believed that "the corpse was only a corpse, the cast off of a self now definitely elsewhere." Once the being no longer occupied the body, the corpse became a mere object, in contrast to Northern European beliefs, which held that deceased bodies were "a magical and semi-animated subject."(Park 1995, pp. 115, 125) Therefore, dissection practices, burial rituals, but also theological perspectives, greatly differed between these two regions. When it came to the post-mortem use and misuse of bodily remains, it was Northern Europeans who focused on powers that bodies obtained after death, not Italians. Non-saintly bodies, such as royal ones, were treated in accordance with that belief (Đorđević 2017, pp. 1-19). In regard to the use of the "ordinary" human body after death, Michael Camille inspected the phenomenon of mumia, a substance that was derived from corpses and consumed as a drug. During the late medieval and early modern period, parts of human corpses were widely used in traditional medicine, while the sick were supposed to be healed by ingesting these substances. However, this practice was thought to work by "direct natural, rather than supernatural or divine means." (Camille 1999, p. 312) Moreover, mumia was always extracted from "pagan" corpses, obtained mostly from Egypt and Syria.

In the past, examination of dead bodies' potential was usually illustrated through the examples of royal, pagan, criminal, or holy bodies. Once again, the "otherness" of the deceased (perceived either as an affirmative or a negative feature) was needed to create the belief that their corpses could influence the living. And still, in the Venetian Bay of Kotor during the 17th and 18th century, people dissected corpses of ordinary laymen. No archival source specified the identity, social class, or gender of the excavated bodies. It appears that the quality of other-worldliness was deemed enough to achieve the desired effect.

In contrast with Park's hypothesis, the belief in the "semi-animated" corpse was still widespread in the southernmost part of the Venetian Republic, years after the Tridentine Reform. As already noted, in one of the aforementioned sermons, a priest urged his congregation not to collect pus from corpses. He mentioned how people used it "to apply it to the skin of the sick, or as protection from the deceased." This warning illuminates how a "common" corpse could both cure the sick and protect the living from mortal danger. Corpses were understood, therefore, to simultaneously heal and harm.

As the archival records do not specify the identity of corpses used in "sinful" rituals nor detail the reasons behind this unorthodox concept, its influences should be sought elsewhere. One possible explanation could be found in popular beliefs that existed in Southeast Europe at the time. The Balkan peninsula was an area characterised by a dynamic exchange between Western and Eastern cultural codes. As such, the age-old beliefs in vampires and other kinds of "undead", common in local folklore, could have influenced the use of bodily fragments within the private domain. Furthermore, from the 8th century onwards, the Bay of Kotor fashioned its identity as the "city of relics", where 
most of the civic rituals revolved around the remains of Saint Tryphon, the town's patron saint. The complex performative agency made the reliquaries and relics an integral part of everyday life for Kotor's residents. The important role holy bodies played in times of distress, in combination with the vivid imagery derived from local folklore, could have contributed to the emergence of practices of unearthing and fragmenting of dead bodies. The belief that a deceased body could heal (and not only harm) the living likely stems from the same source as the relic veneration in the Bay. Visual and ritual methods of conservation and treatment of dead bodies were shared between official and unofficial domains. By assigning power to "ordinary" bodies and placing them in private domains, people from the Bay took control over this process, although always by mirroring established institutional practices.

The differing ways in which deceased bodies were used in either an institutional or domestic setting-although the latter was condemned-should be regarded as complementary, resting on opposite sides of the same early modern devotional coin. Laymen found creative ways to put into practice the knowledge they gained in institutional contexts. Their strong belief in the dogmas of the Catholic Church triggered an impulse to refashion its form, bringing them closer to their own world (Arnold 2005). Domestication of institutional practices, despite being condemned as "magical", "superstitious", or "sinful" by church authorities, can be understood as a coherent part of the same religious mechanism. From this point of view, the fragmentation and use of body parts was widespread across various social settings. Despite existing within different contexts, it relied on a similar set of rules. Material objects used in these practices reveal a mirroring between the institutional and the domestic, official and popular, even orthodox and deviant.

\section{Healing Using the Fragmented Body}

In 1626, a woman from Kotor named Lucieta was accused before a church tribunal of performing acts of witchcraft, or strigarie. As the case unfolded, it soon became obvious that not only Lucieta, but also several other women in the town were involved in the "arte di Demonio". The names Maria from Korčula and Mariza from Ragusa were often mentioned in the records, which described how these women had been healing children, men, and women in the bay. ${ }^{6}$

The town physician Francesco Grassi was called on to inspect the evidence found in Lucieta's home. An object "in the shape of agnus dei" was found in the cupboard. Wrapped in red cloth, it held animal bones, blonde hair (strongly resembling that of Lucieta), children's nails, and various types of wood of unknown origin (Vicentius Bucchia, pp. 916,918). Additionally, it was revealed that Lucieta had given a cup of water to Maria from Korčula, which she was to serve to her master Vicenzo Bucchia. In the meantime, Lucieta was supposed to say "parole invocative di Dio" (Vicentius Bucchia, p. 920), i.e., the prayers that would complete the ritual. All three women were accused of healing children by witchcraft, a practice condemned by the Catholic Church, yet broadly accepted in the everyday life of its subjects. One witness even claimed that the women had been "doing the same for the whole town" and beyond, e.g., in the neighbouring villages of Orahovac and Ljuta (Ibid.).

The objects Lucieta used (bones, wax, body parts such as hair and nails, red cloth, and water) and the fact they were used in conjunction with rituals such as spoken prayers further elucidate the relationship between institutional and domestic devotion. Firstly, her "instruments" were stored with care, hidden in a cupboard and wrapped in red fabric. Secondly, they included specifically chosen materials, such as body parts, wax, water, and wood. Finally, they were utilised as tools in ritual performances with the aim of healing or eliciting benefits for herself and other citizens of the bay. The instruments employed in the secrecy of Lucieta's home could be seen in a strikingly similar format and more institutional setting only a few streets away, at the Cathedral of Saint Tryphon.

6 Buća (Vicentius Bucchia). 1623-1646. pp. 916-20. (Diocesan Archive in Kotor (BAK) Fond I/XVI n.d.). 
The most important reliquary in the bay, the "Glorious Head", containing the skull of Saint Tryphon, patron of the cathedral and protector of the town, has been treasured for centuries in the central part of the relic chapel (Tomić 2009, p. 125; Stjepčević 1938, p. 36) (Figure 3).

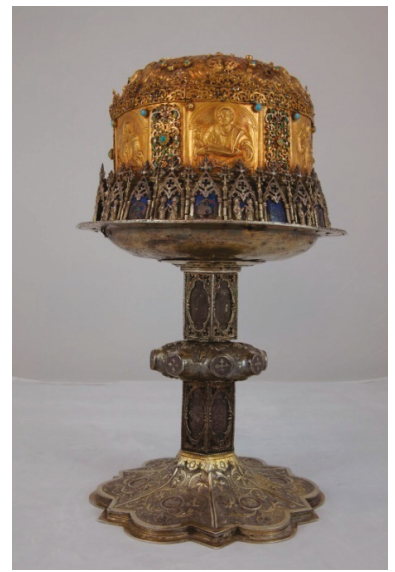

Figure 3. Reliquary of Saint Tryphon's head ("Glorious Head"), 15th-17th century, gold, sliver, enamel, rock crystal, jewels, $43 \mathrm{~cm} \times 27 \mathrm{~cm} \times 23 \mathrm{~cm}$, Kotor, Saint Tryphon's relic chapel (photo by Stevan Kordić).

The renovation of the chapel at the beginning of the 18th century formally replicated the earlier organization of the space (Milošević 2003, p. 267). The silver casket with Saint Tryphon's bones and the head reliquary were concealed in a marble box in the chapel. The entire space was enclosed with a large red iron grid, which had been recorded in the archives before the renovation. It was mentioned specifically in the 17th century, indicating that Lucieta could have witnessed its existence (Tomić 2009, p. 170$)^{7}$ (Figure 4).

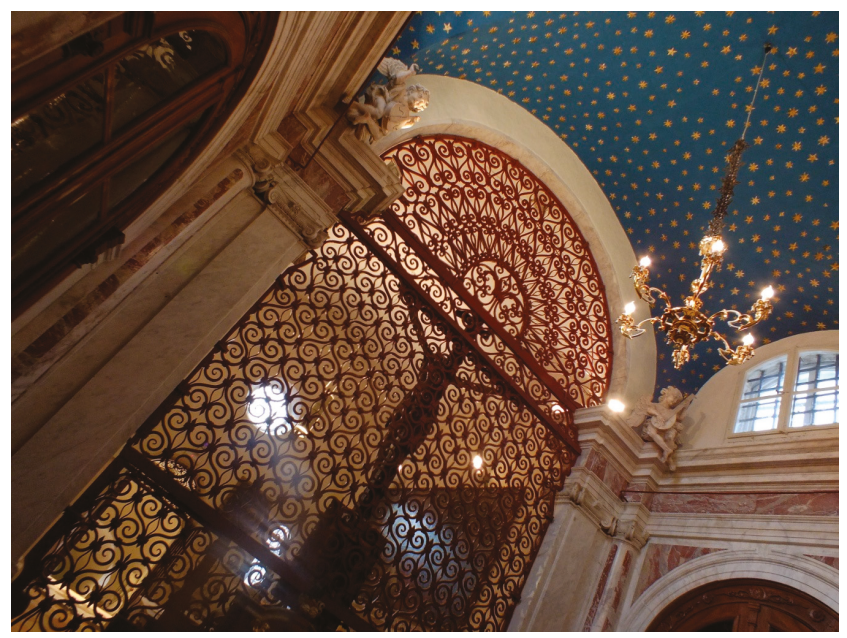

Figure 4. Grid, 17th century, Saint Tryphon's relic chapel, Kotor, photo by author.

7 The red grid was made in Venice in 1652: Tomić, Radoslav. 2009. Umjetnost od XVI do XX stoljeća. In (Tomić 2009, p. 170). 
Moreover, the cathedral inventory listed red curtains in the chapel, also evidenced by the red wrought iron hooks that lined its interior. It can be concluded that the bones of the most important saint in town were carefully stored and locked in a casket, and then fenced off with an iron grid and covered with red curtains. Furthermore, the head reliquary, ritually held in processions, was wrapped in precious red cloth and hidden from the view of the common people for the remainder of the year (Figure 5).

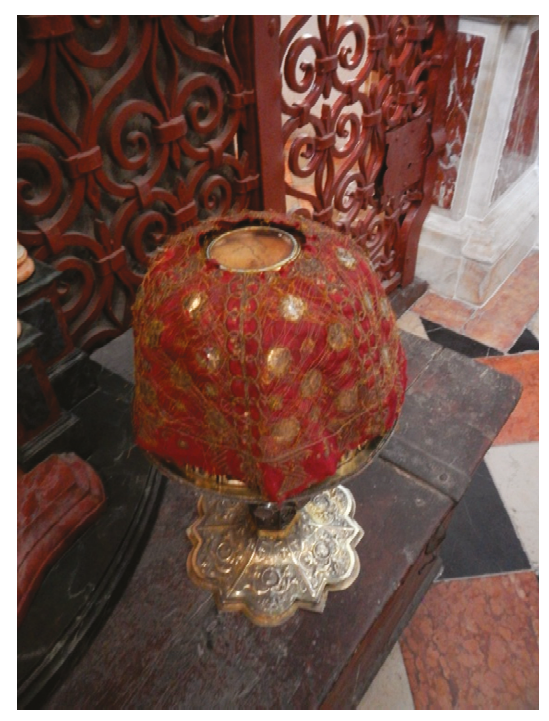

Figure 5. Cloth for the "Glorious Head", 17th century, Cathedral of Saint Tryphon, Kotor, photo by author.

On 3 February, Saint Tryphon's Day, as part of the complex ritual celebration and manipulation of the relics, this reliquary was washed with water, which was then preserved and later used by nuns, as it was believed to be capable of healing the sick. ${ }^{8}$ Ritual washing of the head using water as a precious and powerful substance, along with spoken prayers and the kissing of the reliquary, was the focal point of celebration for the common people. This was the sole instance in the liturgical year when direct physical contact with the saint was institutionally approved. In addition to being washed with water, the reliquary was dried with linen cloths attached to tiny candles. After the ceremony, these would be treasured by believers "until the hour of their death". ${ }^{9}$ Water and wax candlesticks, the objects that remained after the celebrations were over, were kept in the households of common people as precious contact relics.

The physical evidence found in Lucieta's cupboard reveals the profound influence that institutional practices had on the common people. Since the possession of saints' bones was a privilege reserved for religious institutions and wealthy citizens, she replaced them creatively with different kinds of corporeal elements obtained from animal carcasses and even living human bodies, including her own. The use of the fragmented body, along with the particular ways in which it was preserved and manipulated in healing rituals, was Lucieta's way of controlling everyday life, something she achieved by replicating institutional forms of devotion.

8 "Dal ritorno in Chiesa queste reliquie sogliono toccar un bombace e quello lavare i Reverendi Sacerdoti del Capitolo con aqua $i$ qualle poi davano alle Reverendi Suore o Monache (... ) Queli lo conservano con gran diligenza e divotione per tutto l'anno, e la distribuiscono giornalmente secondo le occasioni che gli si rappresentano ( . . . "): Timotej Cizila. Bove d'Oro, pp. 55, 56. NAP R XVI.

$9 \quad$ lbid., p. 56. 
Witnesses confirmed that the two other women, Maria and Mariza, used a similar performative framework in their "witchcraft". Maria was rumoured to be a witch because she healed "children, women, and men" by using herbs and "saying sacred words". Mariza used "certain oils" to heal children while saying "various prayers". A male witness confessed that she had healed his impotence by giving him a remedy for "poter ussar carnalmente con le Donne". ${ }^{10}$

The use of oils, water, bones, wax, or wood particles in combination with sacred words and prayers was how the three women performed healing across the Bay of Kotor. The evidence of treasuring material objects for use in "witchcraft", in the case of Lucieta, further illuminates the dynamic relationship between the official and the private. After deciding to take control of the healing process, these women simulated and appropriated knowledge that had been gained in an institutional context. It had been proven repeatedly that the fragmented bodies in the relic chapel and the church were potent. Lucieta decided not only to relocate that power by translating it to her private household, but to do so by following the rules of presentation and animation as prescribed by the church authorities; for example, by using the colour red, treasuring the bones by wrapping them in cloth, and securing them in a concealed place in her home. Their animation was triggered through the utterance of sacred words and usage in combination with other materials believed to be sacred and potent, such as wax, wood, and water.

\section{Healing the Fragmented Body}

In 1667, forty years after Lucieta's misdeeds had been brought to light, a massive earthquake caused severe damage to parts of the Cathedral of Saint Tryphon, including the relic chapel. Numerous silver body part reliquaries were broken. Archival material holds evidence detailing efforts to overcome the devastation caused to the sacred objects. Among the donors was nobleman Radeta Chiapechi, who bequeathed part of his funds to be used annually for repairs to two reliquaries in the cathedral (Tomić 2009, p. 122). However, the archival document offers no description of the exact manner in which repairs were conducted. Fortunately, physical proof of these efforts is still present in Kotor's cathedral, although it remains hidden inside the cupboards of the relic chapel, as opposed to being displayed on the shelves of the cathedral's Museum of Sacral Art.

Taking into consideration important research on animation (Bynum 2011, pp. 25-31), as well as the complex nature of reliquaries, which were seen as objects and subjects by contemporary observers (Hahn 2005, pp. 239-63), I would like to propose the concept of healing-as opposed to repairing or fixing - the physical damage caused by the earthquake to these sacred containers. Caroline Walker Bynum, in her highly influential study on Christian materiality, argues that not only did medieval devotional objects point beyond their own materiality, but also deliberately disclosed their "stuffness" to the beholders. Their problematic nature led to simultaneous proliferation and occasional iconoclasm. Both hostility and enthusiasm, thus, could be found in the attitudes of the church authorities and theologians toward different forms of holy matter. All those numerous oppositions have grown from the powerful and problematic assertion that matter was experienced as organic, fertile, changeable, and, most importantly, alive. This basic understanding of matter, shared between different social classes, although present during the previous centuries, was mostly found in later medieval phenomena. (Bynum 2011, pp. 35-40). When it comes to post-Tridentine attitudes toward the living matter, the circumstances remained problematic, although church authorities continued to warn against the "wrong" beliefs. Nenadić in his catechesis reminds the congregation that "the image does not hear our prayers, but those who are depicted there do" (Nenadić 1768, p. 111). However, during the 17th and 18th century, believers in the Bay of Kotor continued to treat devotional objects, and especially reliquaries, as if they were alive. The sacred composite of relic and reliquary was referred to as an animated (or at least semi-animated) substance. The skull of Saint Tryphon in its lavish reliquary was

10 Vicentius Bucchia, p. 917. (NAP n.d.). 
called the Glorious Head. The arm reliquary of Blessed Grazia bears on its skin the carved inscription "Il brazo di Beato Grazia" (The arm of Blessed Grazia). The semiotic inseparability of relics and reliquaries enabled relentless belief in the animated nature of the powerful composite. Reliquaries took on properties of the very things they contained, becoming "both subject to and the object of sacred contagion" (Klein 2015, p. 233). Therefore, the physical repair that broken objects endured after the earthquake in Saint Tryphon's cathedral took the form of the process of healing that injured individual went through inside the walls of domestic space.

The practice of "healing" these broken limbs sheds light on the ways in which institutional efforts simulated certain principles of private devotion, mirroring previously presented examples, which illustrated the process in the opposite direction. Several body part reliquaries from Kotor feature small, silver ex-voto plates on their surfaces, which were used as patches to cover cracked skin or missing parts. These odd-looking objects prompt the question: Why were silver votive plates used to undo the harm inflicted on the reliquaries?

In the 17th and 18th century, silver was widely used and, therefore, was visibly prominent in the churches of the bay. A great number of icons were adorned with silver casings and lit by candles inserted into large silver candlestick holders. The Cathedral of Saint Tryphon had, among other treasures, an entire altar made of silver (Butorac 1999, p. 290). The reasoning behind the use of ex-voto plates in the "healing" of the reliquaries should not be reduced to a pragmatic explanation such as a lack of other materials or the convenience of using silver for these particular shapes. Since the reliquaries were highly valued and carefully protected as the cathedral's most precious treasure (Stjepčević 1938, p. 13) $)^{11}$, any argument that implies ignorance or negligence on behalf of their guardians should also be dismissed. To answer the question posed above, it is useful to delve deeper into the function and role these plates acquired once they were removed from the walls and reused as parts of holy limbs. In the early modern era, donating an ex-voto plate after surviving an ordeal such as a shipwreck, enslavement, or sickness was common practice (Jacobs 2013; Didi-Huberman 2007, pp. 7-16). As a result, the walls of churches in the bay, especially the island church of Our Lady of the Rocks (Gospa od Škrpjela) in Perast, were covered in hundreds of silver plates depicting cured legs, arms, eyes, torsos, or breasts (Brajović 2006) (Figure 6). Others portrayed praying figures, various saints, ships caught in storms, or chained men praying before an icon.

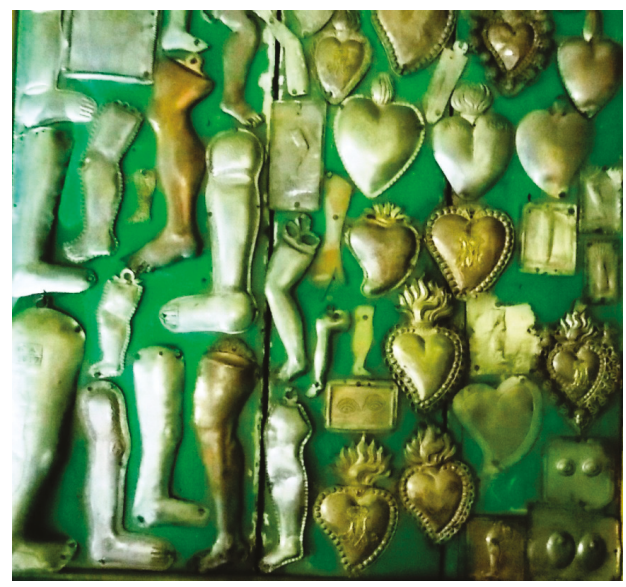

Figure 6. Votive plates, 17th, 18th century, Perast, Church of Our Lady of the Rocks (photo by author).

11 Three guardians, "thesaurarii", took care of the relics during the year and held three keys to the chapel: (Stjepčević 1938, p. 13). 
On reliquaries, the majority of ex-votos were found on round surfaces on the top or at the bottom, depending on whether the plate was added to an arm- or leg-shaped container. This particular placement has its own micro history, which can be traced back to the medieval period.

It is important to note that this miniscule area of the object was not visible to the beholder, neither in the relic chapel nor during a procession. If we compare late medieval and early modern examples, we could draw the conclusion that this particular spot served a similar purpose, even when differences in visual vocabulary and form are taken into account. It was used either for inscriptions or figural representations, which is why many of them bear the figures of saints or saints' names. Furthermore, representations of Jesus, the Arma Christi, or Christograms were commonly displayed during the early modern period (Figure 7).

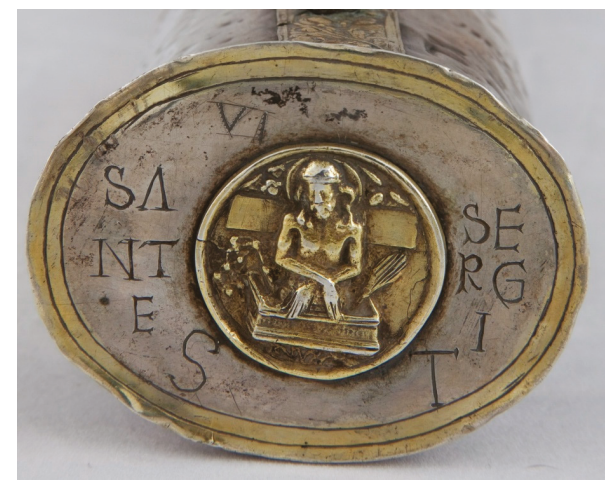

Figure 7. Bottom of an arm-shaped reliquary, 15th century, Cathedral of Saint Tryphon, Kotor, photo by Stevan Kordić.

These round spaces also contained the names or coats of arms of donors or those of the artisans and silversmiths who made the reliquaries. During the early modern period, artisans' names were also accompanied by the stamps of their workshops (Figure 8). It can be assumed that the function of this tiny silver spot was twofold. It could have been a medium used to enhance the object's sacred power by adding an image or the name of a holy person, or a prominent votive dedication through which artisans or patrons recommended themselves to God.

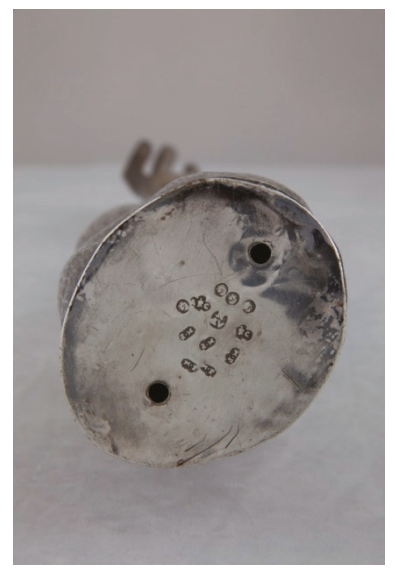

Figure 8. Bottom of an arm-shaped reliquary, 18th century, Cathedral of Saint Tryphon, Kotor, photo by Stevan Kordić. 
In some cases, the ex-voto plates that replaced the original parts of damaged reliquaries replicated the same iconographic scheme. A few of them featured representations of holy figures, such as the Virgin Mary or various saints. These depictions could be classified as motifs that served to emphasize continuity with their medieval neighbours-the image of a holy person whose task was to empower the object as a whole. More provocative, however, was the choice of votive plates that depicted the praying figures of donors. On the votive plate placed at the bottom of one arm-shaped reliquary, an anonymous man is seen praying to the figures of the Virgin Mary and a saint who is wearing military garments and holding in his left hand a bird with a ring in its beak (Figure 9).

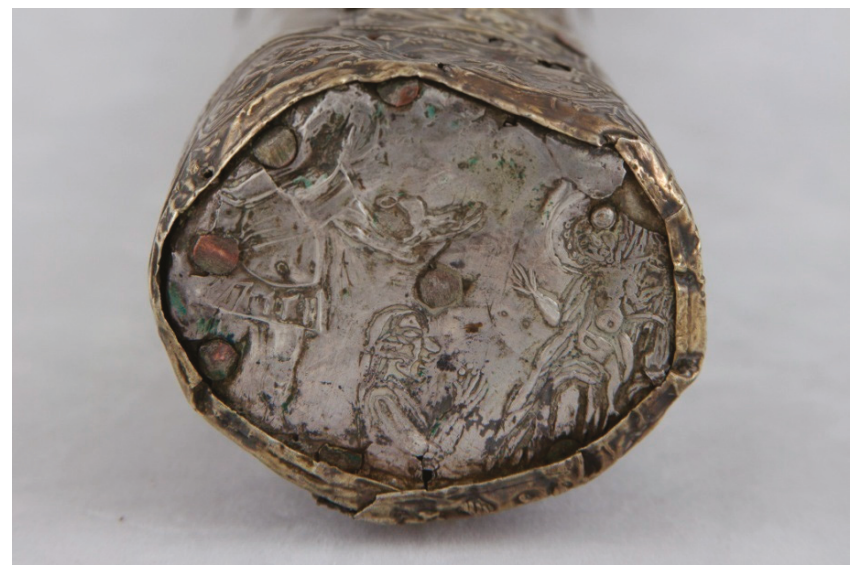

Figure 9. Bottom of an arm-shaped reliquary (15th century) with ex-voto (18th century), Cathedral of Saint Tryphon, Kotor, photo by Stevan Kordić.

This rather unusual iconography for the early modern Bay of Kotor proves less exotic once the second figure is identified as a saint whose portrait is currently housed in the Cathedral of Saint Tryphon's Museum of Sacral Art: Saint Oswald of Northumbria, a 7th century British ruler who united two kingdoms of the British Isles.

The painting likely dates to 1700 and is the work of Giovanni Venanzi, a lesser-known Venetian artist (Tomić 2005, pp. 14-16) (Figure 10). The particular iconography seen in the portrait is explained by events in the saint's vita: the raven was used as a mediator between the king and his bride, carrying the ring in both directions (Giampietro della Stua 1769). It could be that this painting was used as the model for the figure on the plate. In his right hand, Saint Oswald holds a sceptre, which can be discerned on another votive plate, placed not on top or at the bottom of the silver reliquary, but on the main surface. Although severely damaged, the saintly king in military attire can still be distinguished (Figure 11). On the same leg-shaped reliquary, another votive plate is used as a "patch", depicting a figure kneeling and praying with rosary beads (Figure 12). A similar relationship of an anonymous layman praying to the saintly king is visible on both objects, although creatively combined using separate ex-voto plates on the leg-shaped reliquary. The third example, originating from the same treasury, bears a peculiar depiction of a young nobleman with, once again, a bird and a ring (Figure 13). Although likely dating to the late Middle Ages, this metalwork is attached to the top by visible nails, suggesting it could have been added to the medieval reliquary later. However, this particular silver "patch" differs in nature from the other examples mentioned and it is probable that this image was not meant to have a votive purpose. On the other hand, its function and familiar iconography, not dissimilar to the depictions of Saint Oswald on other votive plates, could very well place it within the same group of ex-votos. 


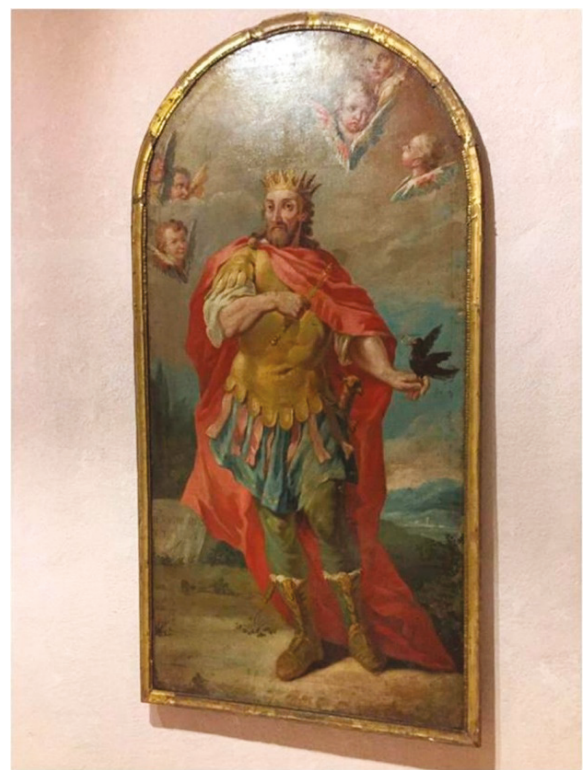

Figure 10. Saint Oswald, Giovanni Venanzi, 1700 ca., Cathedral of Saint Tryphon, Kotor, photo by author.

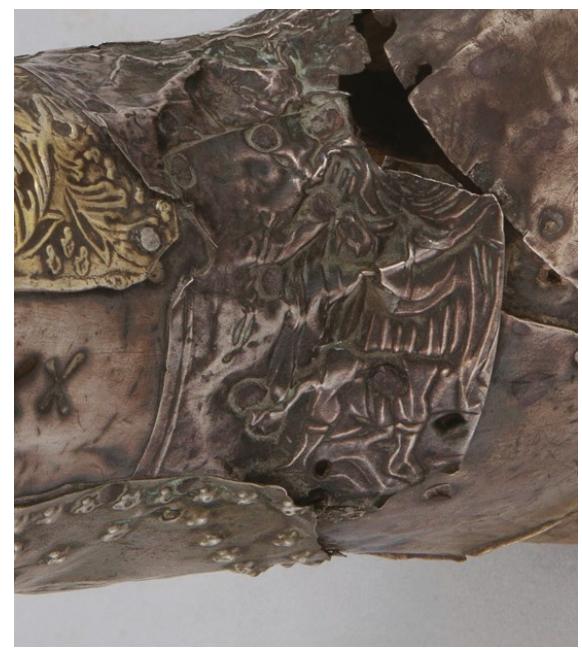

Figure 11. Leg-shaped reliquary (15th century) with ex-voto plates (18th century), Cathedral of Saint Tryphon, Kotor, photos by Stevan Kordić. 


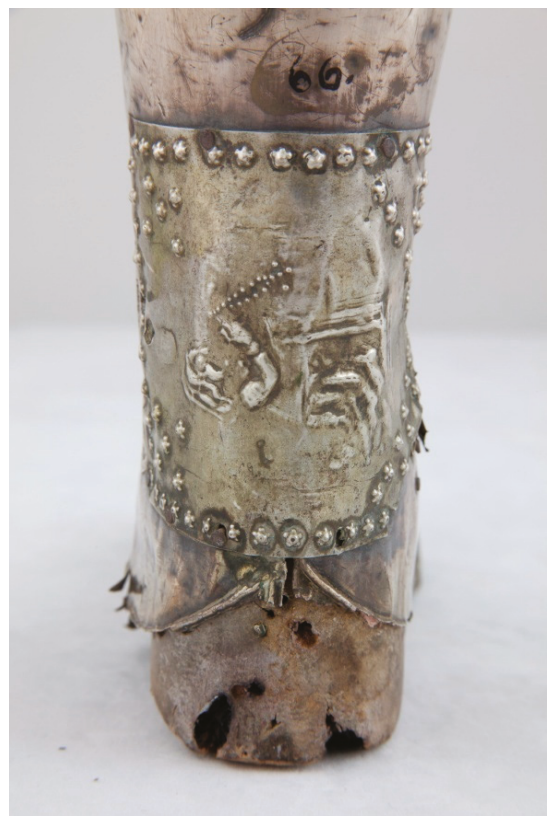

Figure 12. Leg-shaped reliquary (15th century) with ex-voto plates (18th century), Cathedral of Saint Tryphon, Kotor, photos by Stevan Kordić.

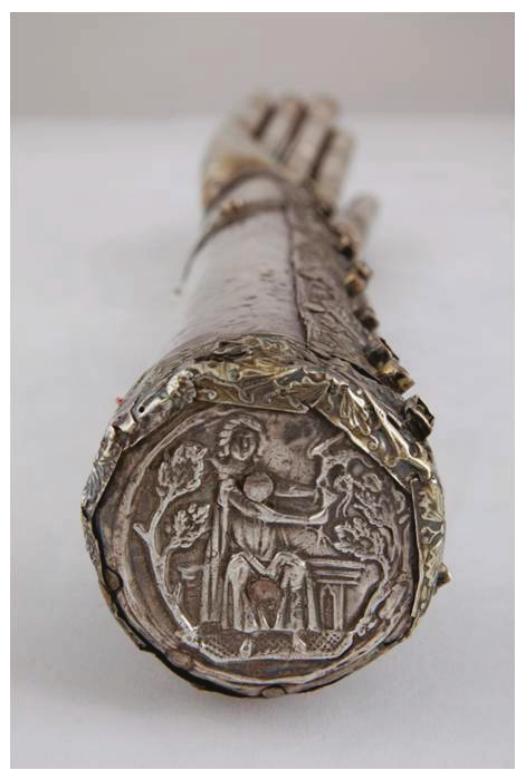

Figure 13. Bottom of an arm-shaped reliquary, 14th century, Cathedral of Saint Tryphon, Kotor.

This iconographic clarification only complicates the question posed earlier. Is it possible that Venanzi's late 17th/early 18th century painting triggered a cult of Saint Oswald in Kotor and that votive plates were offered to this particular saint? Even more curious is the reasoning behind the repeated use of Saint Oswald's image on damaged reliquaries in the cathedral. His Northumbrian 
hagiography can hardly be related to Venetian Kotor. There were no other votive plates with similar iconography recorded in churches in the bay, nor were comparable objects mentioned in archival material or secondary literature (at least to the author of this paper's knowledge). Saint Oswald's nearest and most developed cult was in Sauris, located in Friuli Venezia Giulia, which was at the time ruled by the Republic of Venice (Grassi 1782, p. 170). The church of Saint Oswald was a centre of pilgrimage during the 17th and 18th century. As we learn from the notably scarce sources, this particular saint was venerated across the Republic of Venice as a protector against disease. More precisely, he was often invoked as "saint protector of bones". Unfortunately, the physical findings of his cult remain insufficiently researched. It is known, however, from contemporary pilgrim sources, that the saint was famed for the successful and quick healing of damaged bones. ${ }^{12}$

If we recall that the reliquaries were not only instruments for treasuring the memory of saints, but themselves dynamic and present subjects throughout the past centuries, it can be argued that healing them required a process more complex than the mere fixing of cracks caused by an earthquake. The application of votive plates to the epidermal layer of broken reliquaries, together with the choice of a saint responsible for healing disease and broken bones, could lead this interpretation to the topics of animation and subject-object relations. However, this phenomenon might also offer a deeper insight into the role that "domestic" or "private" processes for healing the "ordinary" body occupied in the creative refashioning and treatment of saintly bodies in the institutional setting. As dozens of silver ex-voto plates in the bay testify, the men, women, and children of households prayed before an icon for their loved one to be healed in times of sickness. Some plates feature miniscule representations of families, united around the bed of an ailing family member, kneeling and praying under an icon of the Virgin Mary or another saint. Other plates render single body fragments-leg parts, arms, breasts, or eyes-as evidence of healed body parts. Some depict a saint or praying figure as crucial elements in the act of receiving heavenly aid. The most common timeline for the process would have been: suffering; praying to the saint; healing; donating the votive plate. Through the final element in the chain, a process that largely takes place within the domestic space of the injured person suddenly becomes part of the institutional setting (Laven 2016, pp. 191-212). As such, silver votive offerings incorporated features of both private and public devotion.

When it comes to their application to body-part reliquaries, the timeline mentioned above, which assumes a gradual process of miraculous transformation, is replaced with an instantaneous change. From the point of iconography, these composite objects-reliquary with votive plates attached-required the presence of praying figures, patron saints of the sick, and the newly cured limb. In this case, the healing was performed by integrating an object that represented aid successfully received, one that held the power of the holy presence in the everyday world. The choice of Saint Oswald, a sacred figure celebrated for curing the diseased, especially those with injured bones and limbs, conforms to the popular image of a reliquary that simultaneously involves components of the object and subject. The praying figures and the presence of carefully chosen saints were needed to affect the "subject" part of the sacred composite. Applying newly forged silver plates as "patches" rather than pre-used but more evocative ones would leave us in no doubt about the clear-cut separation of the two spheres within the institutional setting after the Reformation. However, treating the reliquaries as animate matter shows the existence of highly complex attitudes in early modern (official) devotion to the saints. Most importantly, in times of distress, the church authorities, which had to approve every contact with the reliquaries in the chapel, chose a mechanism of healing that reflected the private lives of believers. They treated the injured saintly body the same way that loved ones treated their sick family members or themselves, by using elements already familiar-from images of saints and rosary beads to performing the act of kneeling and praying, and, finally, offering a votive gift as testament to

12 "Il pellegrinaggio a Sauris (Carnia) per pregare nella chiesa dedicata a S. Osvaldo": http://www.costalissoio.it/varie/SAURIS/sauris. htm. 
the successful end of the journey. The body parts of saints, who for centuries assisted in healing and protecting believers in the bay, were themselves healed by simulating procedures usually performed in a domestic setting, even though that was sometimes done by using fragmented bodies other than those of the saints.

\section{Conclusions}

The domestic use of fragmented bodies for the purpose of healing was a consistent practice in the early modern Bay of Kotor. Instead of relics and reliquaries, common laypeople used the remains of deceased people acquired through the risky practice of grave exhumation. Others, such as Lucieta, used fragments of living bodies and animals. When questioned before a church tribunal, both the accused and witnesses emphasized their strong belief in God, which they supported by stating that they attended church regularly and took communion twice a year. The "sinful acts" that resulted in their condemnation, in terms of iconographic and performative elements, bore a strong resemblance to the institutional treatment of the fragmented bodies of saints. On the other hand, institutional efforts to repair damaged reliquaries, with the use of particular iconography and votive plates, mirrored the way in which the injured bodies of common laypeople were healed in the private domain. When a body needed to be protected from illness and damage, both parties from the different sides of the border separating the "official" and "unofficial" domains of religion reached for tools that had proved effective in yielding the desired result. By borrowing and adopting iconographic and ritual elements from each other, the church authorities and common people participated in a shared devotional world in which the fragmented body and its creative (mis)use possessed healing powers.

The examples of creative exchange in ways of healing the sacred and the ordinary human bodies, where official and private practices existed on a continuum rather than as opposites of orthodox and heretical, could be seen as a fruitful ground for further methodological treatment of similar subjects. The lack of archival data on domestic practices could be complemented, although carefully, by the existent knowledge of institutional manipulation of the body (and vice versa), allowing for the more flexible approach to the matter. Dissolution of clear bynaristic methodological structures should not leave the researcher on a shaky relativistic or pluralistic ground of postmodernism with the fear that following any particular direction would only mean creation of another fixed narrative. In this case, allowing the spectrum or continuum of religious practices, instead of familiar binary model, does not imply the lack of (always artificial) methodological symmetry. However, the ways in which domestic and institutional attitudes toward the body intersected are various and rather complex. In this paper, the chosen methodological path has followed usage of the fragmented bodies in the two domains, merging them into the vast research ground, and denying the firm line of division. In that manner, diverse historical subjects (the witch and the priest, among others) were allowed to communicate their realities as equals, in spite of differences in verbal and visual vocabularies that were both used for the purpose. Moreover, the articulations of the healed bodily image that each offered were treated as equally influential, allowing for the ideas to flow in both directions. In that way, fragmentation and healing, as the focal points in this analysis, proved to be flexible and adaptive concepts, similarly used in both official and domestic surroundings.

Funding: This research is part of a project: "Representation of Identity in Art and Verbo-Visual Culture of the Early Modern Period". This project is sponsored by the Ministry of Science and Technology, Government of the Republic of Serbia, project number: 177001, led by Nenad Makuljević.

Conflicts of Interest: The author declares no conflict of interest.

\section{References}

Arnold, John H. 2005. Belief and Unbelief in Medieval Europe. London: Hodder.

Brajović, Saša. 2006. U Bogorodičinom Vrtu: Bogorodica i Boka Kotorska-Barokna Pobožnost Zapadnog Hrišćanstva. [In Mary's Garden. The Blessed Virgin and Boka Kotorska-Baroque Piety of Western Christianity]. Beograd: Plato, Filozofski fakultet. 
Butorac, Pavao. 1998. Razvitak i Ustroj Peraške Općine. [The Development and Structure of Perast Community]. Perast: NIP Gospa od Škrpjela.

Butorac, Pavao. 1999. Kulturna Povjest Grada Perasta. [Cultural History of Perast]. Perast: NIP Gospa od Škrpjela.

Butorac, Pavao. 2000. Boka Kotorska u 17. i 18. Stoljeću: Politički Pregled. [Bay of Kotor in 17th and 18th centuries: Review of Political History]. Perast: NIP Gospa od Škrpjela.

Bynum, Caroline Walker. 1991. Fragmentation and Redemption: Essays on Gender and the Human Body in Medieval Religion. New York: Zone Books.

Bynum, Caroline Walker. 2011. Christian Materiality: An Essay on Late Medieval Religion. New York: Zone Books.

Bynum, Caroline Walker. 2012. Why Paradox: The Contradictions of My Life as a Scholar. The Catholic Historical Review 98: viii-455. [CrossRef]

Bynum, Caroline Walker, and Paula Gerson. 1997. Body-Part Reliquaries and Body Parts in the Midde Ages. Gesta 36: 3-7. [CrossRef]

Camille, Michael. 1999. The corpse in the garden: Mumia in medieval herbal illustrations. In Micrologus 7. Ilcadavere. Thecorpse. Florence: SISMEL, pp. 297-318.

Čoralić, Lovorka. 1998. Hrvati u procesima mletačke inkvizicije: Peti dio: Magija i svodništvo. Croatica Christiana Periodica 22: 71-116.

Della Stua, Giampietro. 1769. Vita di San Oswaldo, re di Nortumberland e Martire. Collastoria del Suoculto. [Life of Saint Oswald, king of Northumbland and Martyr]. Udine: Fontana.

Didi-Huberman, Georges. 2007. Ex-Voto: Image, Organ, Time. L'Esprit Créateur 47: 7-16. [CrossRef]

Diocesan Archive in Kotor (BAK) Fond I/XVI. n.d. Buća (Vicentius Bucchia). pp. 1623-46, Kotor, Montenego.

Đorđević, Jakov. 2017. Made in the Skull's Likeness: Of transi tombs, identity and memento mori. Journal of Art Historiography Number 17: 1-17.

Grassi, Niccolo. 1782. Notizie Storiche Della Provincia Della Carnia. [Historical Chronicle of the Province of Carnia]. Udine: Fontana.

Hahn, Cynthia. 1997. The Voices of the Saints: Speaking Reliquaries. Gesta 36: 20-31. [CrossRef]

Hahn, Cynthia. 2005. Methaphor and Meaning in Early Medieval Reliquaries. In Seeing the Invisible in Late Antiquity and the Early Middle Ages. Papers from Verbal and Pictorial Imaging: Representing and Accessing Experience of the Invisible, 400-1000 (Utrecht, 11-13 December 2003). Edited by De Nie Gissele, Morrison Karl Frederick and Mostert Marco. Utrecht: Brepols Publishers, pp. 239-63.

Hahn, Cynthia. 2010. What Do Reliquaries Do for Relics? Numen 57: 284-316. [CrossRef]

Jacobs, Fredrika Herman. 2013. Votive Panels and Popular Piety in Early Modern Italy. Cambridge: Cambridge University Press.

Klein, Holger A. 2015. Saints and Sacred Matter. The Cult of Relics in Byzantium and Beyond. Edited by Chang Hahn and Howard A. Klein. Washington, DC: Dumbarton Oaks Research Library and Collection, pp. 231-52.

Krušala, Ivan. 1996. Ivan Krušala, Spjevanje događaja boja Peraškogana 15. svibnja 1654. In Pjesnici Baroka $i$ Prosvećenosti. Edited by Miroslav Pantić. Cetinje: Obod.

Laven, Mary. 2016. Recording Miracles in Renaissance Italy. Past and Present 230: 191-212. [CrossRef]

Milošević, Miloš. 2003. Pomorski Trgovci, Ratnici i Mecene. Studije o Boki Kotorskoj XV-XIX Stoljeća. [Maritime Merchants, Warriors and Patrons. Studies in Bay of Kotor from 15th until 19th century]. Edited by Đokić Vlastimir. Beograd. Podgorica: Equilibrium-CID.

Nenadić, Ivan Antun. 1768. Nauk Kerstjanski. No publisher: Venezia.

Nenadić, Ivan Antun. 1975. Prikazanje Muke Jezusove. Drama u pet činova. In Иван Ivan Antun Nenadić. Slijepa Pravda. Edited by Stojović Milorad. Titograd: Pobjeda.

Ostling, Michael. 2011. Between the Devil and the Host. Imagining Witchcraft in Early Modern Poland. The Past and Present Book Series. Oxford: Oxford University Press.

Parish Archive in Perast NAP. n.d.a. Propovjedi. PROP VIII. pp. 180-82, Perast, Montenegro.

Parish Archive in Perast NAP. n.d.b. Timotej Cizila. Bove D'Oro. NAP R XVI, Perast, Montengro.

Park, Katherine. 1995. The Life of the Corpse: Division and Dissection in Late Medieval Europe. Journal of the History of Medicine and Allied Sciences 50: 111-32. [CrossRef] [PubMed]

Ryan, Salvador. 2003. 'The most contentious of terms': Towards a New Understanding of Late Medieval Popular Religion. Irish Theological Quarterly 68: 281-90. [CrossRef]

Seitz, Jonathan. 2011. Witchcraft and Inquisition in Early Modern Venice. Cambridge: Cambridge University Press. Stjepčević, Ivo. 1938. KatedralaSvetogTripuna u Kotoru. [Saint Tryphon Cathedral in Kotor]. Split: Novo Doba. 
Terpstra, Nicholas. 2015. Body Politics: The Criminal Body between Public and Private. Journal of Medieval and Early Modern Studies 45: 7-52. [CrossRef]

Tomić, Radoslav. 2005. Slika 'Sv. Osvald' GiovannijaVenantija u Kotoru. Lux-Glasnik Kotorske Biskupije 1: 14-16. Tomić, Radoslav, ed. 2009. Zagovori Svetome Tripunu: Blago Kotorske biskupije. Povodom 1200. Obljetnic eprijenosa moći svetoga Tripuna u Kotor. [Votive offerings to Saint Tryphon: Treasure of Kotor's Diocese]. Zagreb: Galerija Klovićevi Dvori.

Ulčar, Milena. 2017. Saints in Parts: Image of the Sacred Body in an Early Modern Venetian Town. Sixteen Century Journal XLVIII: 67-86.

(C) 2019 by the author. Licensee MDPI, Basel, Switzerland. This article is an open access article distributed under the terms and conditions of the Creative Commons Attribution (CC BY) license (http://creativecommons.org/licenses/by/4.0/). 
Article

\title{
Devotional Annotations: Preserving the Family's Memory in Arabic Manuscripts
}

\author{
Torsten Wollina \\ Asien-Afrika Institut, Hamburg University, Mittelweg 177, 20148 Hamburg, Germany; \\ torsten.wollina@uni-hamburg.de
}

Received: 9 May 2019; Accepted: 5 June 2019; Published: 7 June 2019

\begin{abstract}
This contribution explores a peculiar kind of annotation in Arabic multiple-text manuscripts. These manuscripts were often compiled as a personal 'one-volume library', containing copies and excerpts of a unique selection of texts. Further, they were often used for less guided writing activities. The owners left notes, lists and sometimes even sketches in the margins or on blank pages between the texts. Among these, lists of life dates of relatives are a valuable source for studies on domestic devotion. On the one hand, they give glimpses on the composition of households. How many people lived together and who were they? These lists inform us about names regardless of gender. On the other hand, the penning of these list is in itself a trace of a practice intricately tied to the familial and domestic spheres. These lists are usually the only place, in which the memory of those people is preserved.
\end{abstract}

Keywords: book history; family history; ottoman empire; Arabic manuscripts

\section{Introduction}

Among the Daiber collection in Tokyo, there is a nondescript manuscript of 92 folios measuring $16.5 \times 21.2 \mathrm{~cm}$. As so many Arabic manuscripts, MS Daiber II 146 is a majmü' $a$, a book that contains several texts that were bound into one book. Such books could either be compiled from composite materials, i.e., pages written by several different people in diverse places and at different times (=composite manuscripts). Or they were the product of one textual engagement during which a more or less deliberate selection of texts was penned and compiled to function as a "one-volume library" (=multiple-text manuscripts, short MTMs). ${ }^{1}$ Gerhard Endress describes majmü'as as "the least formal genre of books among the familiar types of collecting and organizing knowledge in medieval manuscripts" " "Not complete works or "best of" collections, nor corpus sets (the Organon of logic, described above, is not a typical instance), are united in such volumes, but treasure troves resulting from months, or even years, of activity. ${ }^{3 "}$ And these characteristics would be kept during the early modern period and beyond, as well.

Depending on their context of production, MTMs often constitute entirely unique compilations of materials, notes and even sketches which in themselves already hint at a devotional component in engagements with the written word. This is also true for MS Daiber II 146 which contains eight different textual units (described below), as well as a list of life dates of life dates of, assumedly, relatives of the former owner. What, however, distinguishes this manuscript from many other MTMs, is that we can identify the person who penned this list and thus wanted to preserve the memory of his relatives. He was the manuscript's former owner-and most probably compiler-Muhammad

On this distinction, see (Endress 2016; Schmidt 2016; Friedrich and Schwarke 2016).

(Endress 2016, p. 177).

(Endress 2016, p. 204). 
al-Qarmashlī who furthermore wrote one work in the compilation and endowed it in the Great Mosque of Diyarbakr as he tells in an endowment note on the first page (fol. 1r): "I have endowed this volume [ ... ] for my son Muhammad Sa'1̄d, then for his son Muhammad Rashīd and for both their offspring ... then for the Sunni scholars and the community; signed the endower, Muhammad al-Qarmashli, 3.12.1269 [7.9.1853]."

The concern for remembrance in writing is not particular to predominantly Muslim societies, be they pre-modern or modern. However, they developed specific modes and practices of remembrance, tackled with their own concerns about the afterlife, drew on diverse sources and reacted to particular social and material conditions. With regard to the early modern period, the increasing availability of affordable paper due to the 'European paper revolution' should be mentioned in one breath with the relatively high levels of literacy and the overall growing importance of the written word. ${ }^{4}$ At the same time, there was a great deal of continuity to the medieval period in terms of the preservation of writing. As we have seen, the endowment remained a viable mode of assuring the survival of one's book, well beyond the early modern period. Al-Qarmashlī thus participated in a long tradition concerned with remembrance, creating his own personal 'treasure trove' and using it to secure his and his family's memory.

This article connects the making of such lists to a wider framework of practices of remembrance in the early modern Middle East. It sets out by situating the use of books within the field of domestic devotion with a focus on the Ottoman period (Section 2). It then introduces several lists of life dates (Section 3). While we do not know enough of the concrete motivations for including such lists in a given manuscript, I argue that more often than not we should consider the placement of such lists as deliberate. Moreover, they were put in a specific place within a specific manuscript for a variety of reasons, as will be shown below. Finally, I will link these lists to other expressions of remembrance practices found in pre-modern Muslim cultural production, such as hadìth studies with their concern for uninterrupted oral/aural transmissions of the Prophet's deeds and sayings, the connected emergence of a rich biographical tradition, and literary genres intended to help bereaved parents to cope with the death of their children (Sections 4 and 5).

Although the lists at the center of this contribution rarely give away emotions directly, the act of writing down names and life dates in itself shows a concern over the well-being of one's descendants (be they children or adults) in this world and the next. Moreover, in contrast to the highly public nature of most large-scale biographical collections concerned with the pillars of community, those humble lists were concerned with the realm of family and thus with relations built and formed to a large degree in the domestic sphere.

\section{Ottoman Domestic Devotion}

In 2012, the journal History Compass published a special issue in which several scholars discussed the influence of Michael McKeon's The Secret History of Domesticity on public sphere theory and state building processes during the early modern period. Thereby, the contributors shed further light on the division of public and private spheres in both language and diverse social contexts. Whereas this endeavor remained restricted to a very limited imagination of European history, concurrent processes which brought domestic spaces to the foreground seem to have taken place in early modern Islamicate societies as well.

Research on the historical Middle East has usually approached the topic of domestic space in conjuncture with other topics more prominent in the field. This can be partly attributed to differences in available sources on the domestic sphere. However, in the introduction to her recent edited volume Performing Religion, Ines Weinrich has summarized that devotion more generally had long been sidelined in the philologically oriented discipline of Islamic Studies: "[ . . ] for a long time the main

4 Cf. (Hirschler 2012; Hanna 2004). 
written text in Islam was treated solely as a book, i.e., a textual composition in its entirety, and the oral character of the text and its formation was thereby largely neglected. ${ }^{5}$ Weinrich identifies a scholarly division of labor as a main cause for the neglect of practices surrounding devotional texts:

On the other hand, the study of [ ... ] the interaction between religious specialists and believers, the body or of devices aiming at the senses has often been carried out by scholars from the field of Cultural Anthropology [ ... ]. This labour division has unfortunately led to a tendency which has further deepened the division: studies of Islamic religious practice have tended to focus on the seemingly sensational, on the so-called 'different' [ ... ]. Valuable as these works are, showing the great diversity of Islamic practices and lending deep insight into individual forms of it, they have at the same time suggested-often without intention — that the use of aesthetic sensation or bodily techniques is limited to the extra-ordinary practices and groups of Islam. ${ }^{6}$

Only recently a growing literature, both contemporary and historical, has begun to explore more or less 'ordinary' devotional experiences, as well. Among those, Marion Katz's work on several pillars of religiosity is particularly noteworthy. ${ }^{7}$ This being said, a certain shift towards 'domesticity' during the Ottoman period is visible in, particularly but not restricted to, court cultures, which witnessed a transformation of domestic space itself. ${ }^{8}$ In Ottoman Egypt, the houses of higher military officers (amin/umara $\bar{a}^{\prime}$ ) gained functions that before were served by public building projects. Their residences featured a dīw $\bar{a} n$ chamber for semi-public receptions and gatherings. Likewise, the scholarly or literary sessions (majālis) enjoyed by large parts of the "bourgeoisie" were moved from communal to domestic settings. The same shift apparently affected education, including the establishment of private libraries in separate rooms or niches, where guests could read or borrow books. ${ }^{9}$

Houses thus became more private and more public at the same time. Among the most impressive manifestations of this shift are certainly the wall decorations, which encompassed the reception areas in Ottoman-era upper-class houses. Four rooms from Damascus (18th-19th centuries) and one earlier example from Aleppo (16th century) are currently on exhibition in museums in Honolulu, Los Angeles, New York, Dresden, and Berlin, all of which feature panels with inscriptions of passages from religious texts. Whereas the Damascus Rooms were presumably ordered by Muslim patrons, the Aleppo Room in the Pergamon Museum in Berlin points towards a Christian owner. ${ }^{10}$ Apparently inscriptions were selected to be inviting to people of all (monotheistic) faiths present in the region. In the more recent rooms, religion-specific phrases are avoided-neither the Prophet Muhammad nor even the basmalla (invocation of God's name) are mentioned-so that its contents would be agreeable to Christian, Samaritan, or Jewish guests. The earlier Aleppo Room proceeds in a different manner but with a similar result: while it includes passages from the psalms, it also features a quote from the Quran and an "ecumenical invocation of God". ${ }^{11}$ Furthermore, these reception halls featured a niche at the entrance where a pitcher of water and a bowl were kept for the washing before and after meals as well as for the ablution before prayers. Thus, these new domestic arrangements provided a material basis for domestic devotional practices to emerge as well as being a testimony to, and a result of, their increased importance during the Ottoman period. Last but not least, these wall decorations included spaces where books could be kept.

A higher valuation of activities in the domestic sphere is further tangible in narrative sources from Syria and Egypt between, roughly, the 14th and 18th centuries. The literary output and variety

(Weinrich 2016, p. 11).

(Weinrich 2016, p. 12).

(Katz 2013, 2014, 2019).

(Peirce 1993; Lal 2005).

(Hanna 1998, pp. 93, 97-99). See also (Pfeifer 2015).

(Ott 2008). For the New York room, see (Mathews 1997).

1 (Ott 2008, pp. 215-16). 
increased due to concurrent processes described as "textualisation" and "popularisation". 12 A number of contingencies contributed to this development. On one hand, writing seems to have gained growing importance vis-a-vis the oral tradition of Muslim education and knowledge transmission. Domestic matters appear with higher frequency in these and offer possibilities for an assessment of normative sources. In this context, literature sought out new audiences and adapted to them in format, language, style, and content. The Ottoman period further provided the necessary stability for written and material evidence to survive in larger numbers.

The proliferation of private libraries certainly benefitted the survival and transmission also of texts which could be considered miscellaneous, were unedited or were written outside the traditional circles of authors. ${ }^{13}$ The complementary relationship of private collections with endowed libraries, particularly the legal framework that ideally should secure the integrity of book endowments over time, is already visible in the short description of MS Daiber II 146 above. While the communal repositories depended upon private endowments to become and remain libraries, they also constituted a source of texts which would enter-either directly or through the book market-private collections. In turn, those collections would once again be bequeathed upon an endowed library.

Texts, books, single quires or even pages circulated between high-profile communal book repositories in mosques, Sufi convents, or madrasas, local book and paper markets, and also in the domestic spaces described above. The prevalence of these circulations is both a blessing and a curse in terms of studying domestic devotional book use. On the one hand, many manuscripts that have been used in such ways have survived due to their (repeated) endowment. On the other hand, their circulation makes it difficult to distinguish which of those traces are connected to domestic spaces and which were particular to other spaces. Still, I would reiterate that during the early modern period manuscripts would increasingly be used in domestic spaces, even if they, or parts thereof, were produced elsewhere. More importantly, books could only function as vessels of remembrance if they circulated:

Whereas it is difficult to establish which books were written in domestic settings, it is clear that they were used there. In fact, the memoria functions of books can only be understood against the background of their circulation between private and endowed libraries and a wider readership. A book was a tangible object that connected one's present to the great figures of the past or to one's forefathers-and promised to offer the same for oneself in the future. This was not to be achieved by storing it away. Its value was realized only by its future readers and copyists. ${ }^{14}$

These traces include the individual organization of texts within any given MTM. They also become visible in the annotations made by a manuscript's users and owners. By appropriating its margins and blank spaces for notes with one's own domestic or family life or other forms of annotations, any 'academic' or 'religious' manuscript could be turned into an object of domestic devotion. Using a manuscript as a seemingly arbitrary writing surface was not simply the result of scarcity of paper. Placing notes - and thus the names of dear ones-in close proximity to revered texts and the handwriting of esteemed individuals was in itself an aim of a devotional practice. Defying an easy definition, baraka, as something that was embedded in multiple practices has best been circumscribed by Fartacek and Nigst:

Baraka is seen as a force that augments the good, increases fertility and which helps people to make the right decisions; it keeps people grounded and gives strength; it makes an individual healthy and is conducive to 'sound' relations between the people. Baraka, one

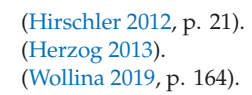


is told, is helpful in respect of remorse and forgiveness. Moreover, baraka is said to be a preventive force against the machinations of the demons and the effects of the Evil Eye. [ ...

] Baraka is thought to be an exclusively positive force and this characteristic trait bestows upon baraka a unique rank: it is of divine origin—from an emic perspective as well. ${ }^{15}$

Through their traceable circulation, manuscripts could become carriers of baraka: "The devotional value of a book was thus not only enshrined in the text but in the object itself. The transmission of baraka dependeds on direct interaction between people-and apparently between people and books. ${ }^{16 "}$ Moreover, baraka was accumulated through the same circulation which it helped fuel. The relationship between this complex notion and the modes of production, reproduction, and preservation of texts ${ }^{17}$ was thus more than complex itself. It was certainly a factor which informed al-Qarmashli's penning of a list of birth dates and bespeak of a hope that those dates and more so the names attached to them would be remembered.

\section{Simple Lists of Names and Dates?}

The following analysis explores the content and placement of three lists of life dates. This endeavor is guided by the question of whether the placement was deliberate or if it was simply predetermined by material constraints such as the scarcity of blank space. Did scribes place lists of life dates of their relatives in a particular manuscript they owned? Did they place it in a specific place within that manuscript? The manuscripts introduced here indicate that there was a wide spectrum of possibilities. Choices were influenced by social criteria such as a scribe's involvement in writerly culture and traditions of learning. They were further influenced by the difference of MTMs and composite manuscripts. Whereas the annotation of a composite manuscript always had to take the pre-existing mis en page into account, the compiler of an MTM could plan ahead and deliberately leave free space for annotation. In both cases, however, owners could include blank pages to later be filled with annotations of their own..$^{18}$ Finally, these lists were not a set writerly genre in themselves and therefore granted the respective scribe a great amount of agency.

\subsection{MS Daiber II 146}

Muhammad al-Qarmashlī penned the eight texts included in the manuscript between 1241 and 1253 (1825-26 to 1837-38), and later compiled it and endowed it in 1269 (1853). The following list gives the contents in their order:

1. Kalimat bayān madhhab al-țấ'ifa al-Yazīdiyya wa-hukmihim wa-ḥukm al-amwāl al-kā'ina bi-aydīhim (fols. 1v-7r): a text on the Yezidis and the conditions should they convert to Islam by "al-Ṣālih"; followed by a commentary by "Mụhammad al-Barqal'î".

2. Excerpts from Anwār al-Tanzīl by al-Bayḍāwī on the first three Suras of the Qur'ān (fols. 7r-v).

3. Fragment of a legal work (fols. $7 \mathrm{v}-8 \mathrm{v}$ ).

4. Risālat al-tunbāk (fols. 9r-10r) by Muhỵ̄ al-Dīn al-Jazārī: a legal treatise on smoking.

5. Al-Qașīda al-lāmiyya fī al-tawhīd (fols. 10r-31v) by 'Alī b. 'Uthmān al-Farghānī al-Ūshī: a creed; with the commentary Nafīs al-riyāọ li-i'dām al-amrāẹ by Khalīl b. 'Alā al-Dīn al-Bukhārī (ca. 750/1349)

6. Nubdha fī ta'rīf awsāf sayf 'Alī karramahu Allāh wa-ḥarasahu al-musammā bi-dhī al-faqār (fols. 32r-v) by Muhammad b. Ni'ma al-Qarmashlī (see below)

15 (Fartacek and Nigst 2016, pp. 53, 57)

16 (Wollina 2019, p. 162).

17 (Wollina 2019, pp. 161-62).

18 In the case of composite manuscripts, a rebinding of pre-existing contents with a few blank pages would create a new composite manuscript. For MTMs, blank pages could appear especially at the end of quires when a copied work did not correspond exactly with the number of leaves in that quire. 
7. Badī' al-ma'ānī fī sharh 'aqīdat al-Shaybānī (fols. 33r-69r): a commentary on al-Shaybānī's creed by the Damascene scholar Najm al-Dīn Ibn Qāẹī ‘Ajlūn (d. 866/1472)

8. Dhāt al-shifā' fī sīrat al-nabī wa-l-khulafā' (fols. 70v-93r) by Muhammad b. Muhammad al-Jazarī (d. 833/1429): poem on the biographies of the Prophet and the first four caliphs; followed a historical survey concluding with the Ottoman sultan Bayezid's conquest of Constantinople.

The composition of this manuscript can be regarded as typical for a personal compilation of the early modern period. It combines general works on Muslim faith and devotion with more topical aspects relevant to specific places-the Yezidis have been settled close to al-Qarmashli's hometown in the border regions between Iraq, Syria, and Turkey, or time periods-treatises on the permissibility of smoking proliferated since the 17th century immediately following the spread of tobacco. ${ }^{19}$ The manuscript possibly served as a form of compendium or almanac, being consulted by its owner in a variety of situations while, at the same time, revealing much of its owner's positioning within society. Most of the manuscript is written by al-Qarmashli's s hand except for parts in the beginning of the seventh work, where he restored the texts from fragments of an older copy (fols. 34a-39b). The list of life dates is placed between this title and the following on fols. $69 \mathrm{v}-70 \mathrm{r}$. I give here translated summaries (the text in brackets was added above the line):

1. Āmina, daughter of Hawwā', born 3 Ramaḍān 1276/25 March 1860

2. Fātima, daughter of Sittiyya, born on the night of Muhammad's Nightly Journey, i.e., 27 Rajab 1277/8 February 1861

3. Hawwā', daughter of Hawwā', born one hour after the evening prayer on a Wednesday night in the second half of Jumādā II 1278/December 1861

4. Ummat Allāh, daughter of Maryam, born shortly before the afternoon prayer on Saturday, 15 Ramaḍān 1278, 3 Adhār/16 March 1862

5. Raqiyya, daughter of Muhammad Sa'îd, born on Monday night, 26 Jumādā II 1280, 25 Tishrīn II/6 December 1863 [she then lived 11 nights and died]

6. Muhammad 'Azīz, son of Muhammad Ṭāhir, born in the night of the Bayram, i.e., 1 Shawwāl 1281/27 February 1865

7. Āsya, daughter of Muhammad Sa'îd, born in the night of 6 Dhū al-Hijja 1282/22 April 1866

8. Muhammad(?) on 7 [Jumādā I around the afternoon prayer 1284/6 September 1867] and he lived until 30 Tammūz, 12 Rabī' II of the following year/2 August 1868, his age was one year

9. Muhammad Badr al-Dīn, born one hour after the morning prayer on Monday, 22 Kānūn II, 9 Shawwāl 1284/3 February 1868, then died on the seventh day after his birth in his first year

10. [Fol. 70a] Hawwā, daughter of Mullā Muhammad, born around the afternoon prayer on Thursday, 3 Jumādā II, 13 Tammūz 1257/23 July 1841

11. Her sister Maymūna, born one hour after the call to the evening prayer on Thursday night, 21 Adhār, 6 Rabī' II 1262/3 April 1846

12. Muhammad Rashīd, son of Muhammad Sa'îd, born in the first half of Tuesday night, 22(?) Tishrīn II, 8 Muharram 1267/13 November 1850

13. Hafṣa, daughter of Muhammad Sa'îd [from Āmina], born in the first third of Monday night, 26 Sha'bān, 10 Kānūn 1270/24 May 1854

14. Zaynab, daughter of Muhammad Sa'îd [from Sittiyya], born in the beginning third of Monday night, 4 Jumādā I, 10 Kānūn II 1274/21 December 1857

15. Fāṭima, daughter of Ḥawwā', born just after sunrise on Friday, 11 Jumādā II, 2 Kānūn II 1277/25 December 1860

19 (Grehan 2006). 
16. 'A' 'isha, daughter of Muhammad Sa'îd from his wife Āmina, born on the last night of 1275 , i.e., 30 Dhū al-Hijja 1275/20 June 1860

17. The final entry does not give a name but only states " 10 days on $22 \mathrm{R}(\mathrm{ajab})$ ' $85 / 8$ November 1868 ” which might refer to the time of writing this list.

The positioning of this list between a creed that lays out the ritual obligations of every Muslim on the one hand and a work of Prophetic reverence on the other might be regarded as intentional as it carries symbolic meaning, placing those people close to his heart in close textual proximity to the Prophet, while asserting their adherence to the Muslim faith through the equal proximity to a work expounding the rules upheld by this faith. The dates of the entries show that this list was added to the manuscript after it had been endowed. The former owner still had access to and could even alter the manuscript. The order of the entries with the later dates having been penned on the first page (fol. $69 \mathrm{v}$ ) further indicates that this list was not continuously expanded. Rather, the entire list was connected to the manuscript's endowment. It might even have carried legal weight in that it elaborates on his endowment note on fol. 1r., several people listed were, in fact, intended beneficiaries of his book endowment, "for my son Muhammad Sa'îd, then for his son Muhammad Rashīd and for both their offspring". As will become apparent below, the list's placement within the manuscript benefitted the survival of the memory of his mentioned family members.

The list identifies who belonged to "their offspring". Thus, we can identify Muhammad Sa'îd's children as Raqiyya (no 5), Āsya (no 7), Hafșa (no 13), Zaynab (no 14), and 'Ā'isha (no 16), besides the mentioned Muhammad Rashīd (no 12). ${ }^{20}$ What is striking in this respect is the large number of entries where the mother is mentioned instead of the father. I assume that these women belonged to al-Qarmashli's family and married men outside the family. However, the other entries show that the list did not only serve to support legal claims but was meant to preserve the memory of all those members of al-Qarmashlī's family. The list also includes several entries where the person had died in infancy $($ nos $5,8,9)$ and thus raises the question what the purpose of their inclusion was.

Andreas Görke and Konrad Hischler have stated that the documentation of endowments was indeed produced with the intention of preserving one's memory. They were "as much legal records as textual spaces to celebrate [i.e., commemorate] the respective endower." ${ }^{21}$ The manuscript at hand is not in itself an endowment record (waqfiyya). But in a similar way it offers itself for the preservation of people's memory. As it was compiled by the endower and starts out with an endowment note, I would argue that we could subject the entirety of his al-Qarmashlī's annotations to a joint reading of a paratext which is scattered throughout the manuscript. Anchored by the endowment note, all his annotations constitute a joint textual space which allowed him room for maneuvering in his self-presentation comparable to that in an endowment record, if not more so. The list would thus respond to those annotations as well as to the works in the manuscript.

\subsection{Earlier Examples}

There are two other instances of such lists that I have found in the Egyptian National Library under the call numbers MS Fiqh Taymūr 58 and MS Fiqh Taymūr 511. Neither is an MTM but their blank spaces have been used in a similar way to preserve life dates of an owner's family members. At the same time, those lists are different from the one in MS Daiber II 146 in several respects. Both are placed on their manuscript's final page, after the end of the text they contain. This placement marks those lists as paratextual annotations, which are dissociated from the respective content, whereas in the former manuscript it appears on the first glance as a part of the compilation. This aspect is emphasized

20 The parentage of the entries 10 and 11 is more difficult to identify as all three generations of males share the first name Muhammad. The honorific "mullā" does not really tell us which one of them was their father. However, judging by their dates of birth it seems most likely that they too, were Muhammad Sa'îd's children.

21 (Görke and Hirschler 2011, p. 11). 
by the difference in handwriting. Al-Qarmashli's handwriting is found throughout his manuscript, whereas in these two cases the owner's/scribe's handwriting is distinct from the one which penned the text of the manuscript.

Their position is also more prominent in regard to cursory readings of the manuscript, and at least the list in MS Fiqh Taymūr 58 might have been interpreted as a proof of legal ownership. This might explain why the scribe's signature has been rendered illegible. This list consists of only two entries from the end of the 17th century on the otherwise blank verso of the text's last page. Neither entry gives a name but they refer to the birth of a "hopefully blissful" son and daughter respectively. Apart from the time and date of birth, the latter entry also gives the place of birth as "the city of God's Prophet [peace be upon him]", i.e., Medina. The two births are five years apart, the former occurring in 1104/1692 and the latter in 1109/1697.

The other list in MS Fiqh Taymūr 511 is more comprehensive in that it consists of seven entries, all of which give a name. Unfortunately, the handwriting is difficult to decipher. This 'list' consists of seemingly independent notes all around the colophon of the work included in the manuscript. Some run vertical on the page and their order is not apparent from their arrangement. I have indicated the position on the page in brackets and also where dates are difficult parts to decipher.

1. 'Abd al-Malik, born 1 Rajab 1161 (1171?)/27 June 1748 [left of the colophon, vertical script] ${ }^{22}$

2. Nafīsa, born 2 Ramaḍān 1162/16 August 1749 [left of the colophon, horizontal script, below no 1]

3. Halīma, born 27 Dhū al-Qa'da 116(4?)/17 October 1751(?) [lower left corner of the page, below no 2, horizontal script]

4. 'Abd al-Rahīm, born 16 Jumādā I 1167/11 March 1754 [right of colophon, horizontal script]

5. 'Abd al-Rahmān, born 16 (26?) Jumādā II 1167/10 (20?) April 1754 [right of colophon, horizontal script, above no 4$]^{23}$

6. Much of this entry is hidden by a paper slip carrying the stamp of the Taymüriyya Library, thus neither the name nor the person's gender can be identified. They were born in Ramadiann of one year between 1168 and 1171/1754-1758 [below the colophon, right of no 3 and below no 4 , diagonal script $]^{24}$

7. 'Abd Allāh "the last son/child", born 15 Sha'bān 1172/13 April 1759 (top right of colophon, above no 5 , vertical script)

This list discloses a different engagement with a manuscript. It was turned around for the entries to be written in different directions, which might indicate a dialogical situation of writing. It further suggests that not all of the entries were penned in one session but rather as part of a continuous practice, in particular, the final entry had to be 'squeezed in' above the others on the right side of the colophon. Neither list is as developed as al-Qarmashlī's list, which supports this notion. Nevertheless, they result from the same motivation, using the respective manuscript as a vessel to preserve the memory of one's children. When the manuscripts were endowed, this memory could be perpetuated and, with it, the memory of the familial relationships between those people-as well as between them and the scribe.

The question remains what qualifies this practice as a devotional practice and what can such a small sample tell us about domestic devotion more generally. In the remaining two sections, I will contextualize these terse lists with regards to more visible and prevalent communal practices. Although only traces of a more complex social practice, they indicate that the following saying of the Damascene scholar Muhammad Ibn Ṭūlūn (d. 1546) was widely heeded: "It is not fitting that anyone who

22 As this entry is the only one which calls the son a "blessed son", I assume it refers to the scribe's firstborn. Therefore, the earlier date would make more sense.

23 I am rather uncertain of my reading in the case of nos. 4. and 5. It might well be that both were born on the same day and that the order in which their names are written on the page recreates that of the basmalla: bi-smi llāhi al-rahmān al-rahīm.

24 For the later date, see the following entry no. 7; concerning the earlier date, I speculate that the entry's position on the page indicates that it was added after the two sons born in 1167. 
possesses even a small amount of knowledge should allow himself to be forgotten."25 Moreover, their preoccupation with kinship ties and birth dates could build on models prevalent in the wide-spread hadīth and biographical literature (Section 4). From another perspective, al-Qarmashli's inclusion of deceased children resonates with genres concerned with the consolation of bereaved parents and with their overcoming of grief (Section 5). Whereas the lists discussed here do not amount to a narrative engagement with mourning, they do bespeak of a concern over one's children in this world, and the next, and therefore can qualify as traces of devotional practices.

\section{Biographical Literature}

The longstanding concern over preserving the memory of individuals in Arabic Islamicate literature and writing more generally is epitomized in a long and broad tradition of biography, collective biography, and prosopography. ${ }^{26}$ There is consensus that the origins of those literary genres lay in the field of hadith, the discipline concerned with preserving and interpreting the deeds and sayings of the Prophet, his family, and his companions. The biographical interest lay initially in ascertaining the validity of individual traditions and, therefore, the credibility of the traditionaries themselves. Following the composition of the canonical hadith collections (the 'six books') by the 11th century, the post-canonical transmission shifted to a more distinctly devotional character with "a unique view of temporality and history based not on the passing of days, years or months, but on the successive acts of hadīth transmission, each act of transmission forming a unit of time." ${ }^{27}$ Garrett Davidson argues that within this temporality, a generation was not understood "as a group of people having been born during some period of years [ ... ] but rather as a group of people with the same number of links of transmission separating them from the Prophet." 28 As the prosopographical or collective biographical literature organized its material according to these generations, they recreated temporality itself in devotional terms:

In this understanding of a generation, the Prophet's generation is the first and each act of transmission creates a new generation, each generation consisting of all those people who had the same number of links of transmission separating them from the Prophet. In a degenerative model of temporality, the Prophet's founding generation is, of course, the best of all generations and each successive generation is of less merit than the one before it. Thus, the short chain of transmission brought "one closer to the generations of merit" and by attaching oneself to a short chain of transmission one could belong to a generation superior to the one he would be considered to belong to in a conception of generation based on years. ${ }^{29}$

Through the participation in the transmission of Prophetic traditions, one could approximate the Prophet, and by collecting short chains of transmission (isnād), the distance created by historical time itself could be bridged. Those works were themselves essentially lists of biographies with a focus on giving the name and life dates of their biographees as well as information on their place in the transmission of Prophetic traditions. However, with the development of the genre, more information as to their lives and characters was included, perhaps in service to wider and different audiences. And with the diffusion of hadith transmission, details about more and more lives would be recorded in writing, if not always in the biographical literature as such.

In both cases, those works should be understood as embedded within a wide-ranging intertextual network. On the one hand, they guided an interested person to further readings, including the works authored by a biographee or the sources on which they themselves relied. On the other hand, their

\footnotetext{
Quoted from (Sajdi 2013, p. 1)

(e.g., Robinson 2007, p. 66; Al-Qadi 2006).

(Davidson 2014, p. 12).

(Davidson 2014, p. 43).

(Davidson 2014, p. 43).
} 
reliance on external sources often comes to the surface in their own descriptions. These included oral testimonies as well as written documentation, particularly on acts of transmission (ijāza, samán'), but also other notes in the margins of manuscripts. In this, writing reproduced and continued relations between the author of such a work and the older relatives and possibly teachers of a biographee. ${ }^{30}$ Often enough, such personal connections were both the reason for a person to be included as a biographee and an aspect that was emphasized by the author, by which he increased his own standing in the transmission of knowledge. One curious case was the 'binge-reading' (as Konrad Hirschler phrases it) that the 15th century Damascene scholar Yūsuf Ibn 'Abd al-Hādī conducted in the circle of his family. The dates and attendants of the reading sessions were documented on the respective manuscript. This documentation was part of "a devotional ritual", using "textual proximity to the Prophet" in order to "come close to the Prophet himself". ${ }^{31}$ This documentation is further relevant as it simultaneously provides biographical information on Ibn 'Abd al-Hādi's s family members. In particular, it makes clear that his youngest son 'Abd al-Hādī joined these reading sessions from the day after his birth.

On the other end, people welcomed their inclusion into the biographical dictionaries, even if they were not themselves biographees. A mention of one's name meant that one would be remembered by future generations. And as the inclusion in such a work was coveted, people intentionally prepared for that possibility. This was one reason why they would keep documents that attested to birth and death dates in their possession. And it is also one reason why teaching and attendance certificates (ijäza, sam $\bar{a}^{\prime}$ ) became institutions in their own right. It might have been a motivation for some to engage in hadìth transmission - to secure their own place in the biographical literature.

The lists we encountered in the prior section attest to this impulse. At the same time, the creation of such lists pluralizes the preservation of memory. Those people might not have made it into any of the large selections of biographies, which constituted a central mechanism by which pious and scholarly communities created their shared identity, nor even into any documentation of hadith transmission. It is difficult to say without more contextual information but we could hypothesize that these lists created their own small-scale identities and communities, connected by their ancestry and by their shared experience of living in the same household. The endowment of the manuscript suggests, at the same time, that these lists might serve as 'primary sources' for an eventual inclusion in a biographical work, and the departure from that connection through the establishment of a 'textualized community' as much of personal value to the scribe as the selection of texts within his 'one-volume library'. The above-mentioned Ibn 'Abd al-Hādī also endowed his books, inscribed with the names of his family members, shortly after the end of his reading sessions. ${ }^{32}$

Whereas 'published' biographical works realized connections between people and books within each biography, al-Qarmashli created a different quality of connection through the inscription of the list in between the works he compiled into MS Daiber II 146. As with the other lists discussed above, the penning of the list could function also as a surrogate of earlier certifications of education. The inscription of the names in the manuscripts might imply the familiarity of the manuscript owner's children with the manuscript's contents. It certainly cements not only the connection between those people and the manuscript but also between them and the scribe.

\section{Consolation Literature}

The lists further resonate with two genres which have been coined 'consolation treatises' (Avner Giladi) and 'Kindertotenlieder' (Thomas Bauer). The fear of a child's untimely death is already palpable in the short list in MS Fiqh Taymūr 58 where the scribe repeatedly expresses his anxiety that the lives of his children may hopefully be blissful. Consolation treatises as a genre in its own

30 For a selection of both forms, see (Ibn Ṭūlūn n.d., pp. 20a, 25b, 82b); (Ibn Ṭūlūn n.d., pp. 30b, 44b, 52a, 90a-90b); (Ibn Țūlūn n.d., p. 59a).

31 (Hirschler 2017).

32 (Hirschler 2017). 
right emerged during the Islamic middle periods (11th-16th centuries CE) and combined in new ways, or simply in a handy format, age-old materials such as "the Koran, hadith reports, and poetry as well as sermons (mawa'iz), genealogical and biographical works, lexicography, and (other) works by authoritative religious scholars" 33 , as well as chapters in books giving ethical and pedagogical, juridical and theological, hygienic and medical information. Giladi argues that the genre developed in unique ways, focusing on "psychological problems connected with and resulting from infant and child mortality" and "on practical ways of coping with the loss of one's offspring". ${ }^{4}$ These materials were selected and organized in a way that used devotional and literary texts to speak about the unspeakable and, at the same time, would discipline expressions of grief. The central term in this respect is $s a b r$, steadfastness, with which adult Muslims should face adversities. In contrast, loud wailing and other "un-Islamic" mourning practices were declaimed by religious scholars as they implied doubt in God's judgement and in the rewards that awaited the deceased children in Paradise. ${ }^{35}$ While some recommendations can be understood from a communal perspective that valued stability in the public sphere over everything else, we can glimpse a serious concern over the individual well-being of the mourning. The "filling of the gap" left behind by a child's death was seen as the therapeutical state-of-the-art. ${ }^{36}$ Scholarly consensus held that deceased children could intercede on their parents' behalf in the Hereafter, that they would immediately be granted access to Paradise, and that Paradise specifically provided for their well-being. Whereas the term 'martyr' often carries negative connotations today, it was at the core of premodern attempts to ease the painful experience of bereaved parents, especially during the period of the Black Death. The relevance of the genre continued well into the Ottoman period, as the issuing of new works attests. ${ }^{37}$

Those ideas were disseminated widely and also inform the obituaries that the Damascene court clerk Ahmad Ibn Ṭawq (d. 1509) inserted into his diary chronicle ${ }^{38}$ During the covered period, he himself lost four daughters to recurring epidemics. Whether he practiced sabr or not, a lack of words often characterizes his entries on a child's death. Thus he restricts the obituaries for his daughters 'A $\bar{A}$ 'isha and Sāra to the time of death and burial, the cause of death, and their respective age. In the one for his daughter Fātima he speaks about the costs of her funeral. It is only in the devotional phrases in which his grief becomes palpable: "May God allow her [i.e., Fātima] immediate entry into Paradise and have her reserve a place for me"; "God created [Sāra] as a treasure for her mother and me on Judgement Day [ ... ] this world will end but the Hereafter lasts for eternity"; "Oh God, reward me for her [i.e., his fourth daughter Umm al-Fadl] misfortune and grant me compensation for her loss!"39

Practices of mourning thus emerge as a broader spectrum with one end defined by complete speechlessness and șabr, whereas on the other end we find the Kindertotenlieder studied by Bauer. Bauer identifies poetry as a means to talk about grief:

[... ] poetry-including its artistic and playful element—can be helpful in coping with the grief of the loss of one's own beloved, for poets as well as for their public. It may help the poet to prove his own abilities to create a work of art, and by way of the act of active creativity he may cope with the experience of loss. He may find relief from the experience of helplessness and passive suffering and prove to himself that he still has a share in life. Even more important may be the fact that a poem (or any other work of art) is a means to

\footnotetext{
(Giladi 1993, p. 371).

(Giladi 1993, p. 370); see also (Bauer 2003).

(Giladi 1993, p. 377).

(Wollina 2014, p. 95).

7 Giladi names another treatise from the mid-16th century. At around the same time, the Damascene scholar Ibn Tūlūn also authored "Tabrīd al-fu'ād'an mawt al-awlād" and "al-Tabyīn al-marsakh fī hịkam aṭ̂āl al-muslimīn fī al-barzakh" whose titles suggest they belonged to the same genre.(Giladi 1993, p. 371; Ibn Tülūn 1929, pp. 31-32)

38 Cf. (Wollina 2013).

39 (Wollina 2014, pp. 95-96).
} 
break the speechlessness of death, to resume communication and thereby to reassume a social role without having to interrupt the process of mourning. ${ }^{40}$

Bauer's research topic has the advantage that the expression of emotions can be traced and analyzed in a much more comprehensive manner than is allowed even by Ibn Tawq's short eulogies. In contrast to both, the scribes who inserted information on family members into manuscripts they owned, compiled, or even commissioned could not claim similar textual authority. Nonetheless, the inclusion of these lists should be viewed as a product of similar concerns and sentiments. Even though a list of dates is hardly "a work of art", it might give the scribe some feeling of control and thereby "relief from the experience of helplessness". It certainly constitutes a, however little, "means to break the speechlessness of death". Moreover, the juxtaposition of deceased children with those who lived on, might ease the feeling of loss. On the pages, they are back with their family. It does not necessarily contradict the call for șabr when the names of deceased ones are included. Rather, the terseness of those notes indicates an adherence to the notion. Even where they remain speechless, the naming of the deceased alone points at what is not being said outright. One could say, that whereas medieval scholars used biographical materials as a model for mourning, those scribes proceeded in almost the opposite way, creating biographical entries for the children they mourned. The concern with the well-being of their offspring in this world, and the next, certainly does qualify this seemingly simple practice as a devotional practice.

\section{Conclusions}

Claudine Moulin describes annotation as a prototypical act of writerly and communicative practice. ${ }^{41}$ The lists of names and life dates discussed in this contribution support this notion. Although they offer themselves, at first glance, much more for social history or family history than for the history of domestic devotion, I hoped to demonstrate, particularly in the last two sections, that they are also part of a larger metatextual web which connects them to more outspoken and better researched types of writing concerned with or being used in devotional practices concerned with preserving people's memory. As much as they were a documentation of attendance for Ibn 'Abd al-Hādī, his lists combine in their content and codicological context, devotional, and in many cases, domestic devotional practices common in the Ottoman period with other concerns, be they of a legal or social nature. They further bespeak of the same need to capture important dates in the lives of loved ones in writing as Ibn Tawq's diary. Whereas the biographical, consolation or even hadith related 'genres' offer more comprehensive narratives and descriptions of pious concerns, they are also usually placed high within a hierarchy of defining to what devotion one should conform. In contrast, these lists are more or less individual expressions of practices that navigate those normative prescriptions. In this, the lists constitute another, more elusive writerly and communicative practice.

This contribution cannot present a conclusive statement as to the nature and purposes of this practice of list making. A more systematic investigation into the temporal and spatial distribution of this kind of annotation as part of devotional practices is, however, needed to situate it within the emergence of the broader phenomenon of Ottoman period domesticity outlined above. This entails biographical studies of book collectors of the era from a domestic devotional perspective. Who were the people that engaged with those manuscripts? Where did they store their books and did they share access with others? ${ }^{42}$ Where were those books used and who was allowed to leave annotations in them? In other words, we have to ask who was able to engage in this prototypical act of writerly and communicative practice and who was not? ${ }^{43}$

40 (Bauer 2003, pp. 51-52).

41 (Moulin 2010, p. 19).

42 Cf. (Liebrenz 2016).

43 Cf. (Liebrenz 2013, particularly pp. 39-42). 
Another aspect which I have not fleshed out above is that the devotional relevance of the lists needs to take into account their specific codicological context. In turn, the placement of al-Qarmashlī's list raises the questions of reading practices as well as editorial choices. Would it have been read as a separate textual item or in conjunction with the devotional texts before and after it? Could it fulfill a function similar to hadith documentation or endowment records? Finally, was it purposefully placed at this exact position within the manuscript? Recent studies on personal libraries have suggested that the specific placement of books creates a secondary level of metatextual meaning. ${ }^{44}$ Would not the placement of texts within a compilation do the same? I would suggest that annotations can open up a door, not to discover private contents, but to trace practices which engage with either the texts or the object, or both. In order to do this, a study of texts is not enough but the layout, organization and materiality of books need to be taken into account as well. ${ }^{45}$ The real source value of such lists will only emerge if we heed Weinrich's call and approach them as traces of performative practices.

Funding: This research received no external funding.

Conflicts of Interest: The author declares no conflict of interest.

\section{References}

Al-Qadi, Wadad. 2006. Biographical Dictionaries as the Scholars' Alternative History of the Muslim Community. In Organizing Knowledge. Encyclopaedic Activities in the Pre-Eighteenth Century Islamic World. Edited by Gerhard Endress. Leiden: Brill, pp. 23-75.

Bauer, Thomas. 2003. Communication and Emotion: The Case of Ibn Nubātah's Kindertotenlieder. Mamlūk Studies Review 7: 49-95.

Daub, Frederike-Wiebke. 2016. Formen und Funktionen des Layouts in arabischen Manuskripten anhand von Abschriften religiöser Texte al-Būṣīin̄s Burda, al-Ǧazūlīs Dalā'il und die Šifā' von Qāḍ̄ 'Iyād. Wiesbaden: Harrassowitz.

Davidson, Garrett. 2014. Carrying on the Tradition: An Intellectual and Social History of Post-Canonical Hadith Transmission. Ph.D. dissertation, University of Chicago, Chicago, IL, USA.

Endress, Gerhard. 2016. One-Volume Libraries and the Traditions of Learning in Medieval Arabic Islamic Culture. In One-Volume Libraries: Composite and Multiple-Text Manuscripts. Edited by Michael Friedrich and Cosima Schwarke. Berlin, Boston: De Gruyter, pp. 171-205.

Fartacek, Gebhard, and Lorenz Nigst. 2016. Conforming to and Breaking with Social Norms: Two Contrary Modes of Baraka. In Performing Religion: Actors, Contexts, and Texts. Case Studies from Islam. Edited by Ines Weinrich. Beirut: Orient-Institut Beirut, pp. 53-74.

Friedrich, Michael, and Cosima Schwarke. 2016. Introduction-Manuscripts as Evolving Entities. In One-Volume Libraries: Composite and Multiple-Text Manuscripts. Edited by Michael Friedrich and Cosima Schwarke. Berlin and Boston: De Gruyter, pp. 1-26.

Giladi, Avner. 1993. "The Child Was Small ... Not So the Grief for Him": Sources, Structure, and Content of al-Sakhawi's Consolation Treatise for Bereaved Parents. Poetics Today 14: 367-86. [CrossRef]

Görke, Andreas, and Konrad Hirschler. 2011. Introduction: Manuscript Notes as Documentary Sources. In Manuscript Notes as Documentary Sources. Edited by Andreas Görke and Konrad Hirschler. Beirut: Orient-Institut Beirut, pp. 9-20.

Grehan, James. 2006. Smoking and "Early Modern" Sociability: The Great Tobacco Debate in the Ottoman Middle East (Seventeenth to Eighteenth Centuries). The American Historical Review 111: 1352-77. [CrossRef] [PubMed]

Hanna, Nelly. 1998. Culture in Ottoman Egypt. In The Cambridge History of Egypt. Edited by Martin William Daly. Cambridge: Cambridge University Press, vol. 2, pp. 87-112.

Hanna, Nelly. 2004. In Praise of Books. A Cultural History of Cairo's Middle Class, Sixteenth to Eighteenth Century. Cairo: The American University in Cairo Press.

44 (Stört 2010; Wieland 2015; Manguel 2018).

45 Cf. (Daub 2016). 
Herzog, Thomas. 2013. Composition and Worldview of Some Bourgeois and Petit-Bourgeois Mamluk Adab-Encylopedias. Mamlūk Studies Review 17: 100-29.

Hirschler, Konrad. 2012. The Written Word in the Medieval Arabic Lands a Social and Cultural History of Reading Practices. Edinburgh: Edinburgh University Press.

Hirschler, Konrad. 2017. Binge Reading in Fifteenth-Century Damascus. DYNTRAN Working Papers 27. Available online: http://dyntran.hypotheses.org/1992 (accessed on 25 May 2019).

Ibn Ṭūlūn, Shams al-Dīn Muhammad. 1929. al-Fulk al-mashhūn fì aḥwāl Muhammad Ibn Ṭūlūn. Edited by Șalāḥ al-Dīn al-Munajjid. Damascus: Maṭba'at at-Taraqqī.

Ibn Ṭūlūn, Shams al-Dīn Muhammad. n.d. Dhakhā'ir al-qaṣr fì tarājim nubalā' al-'așr. MS Orient A 1779. Gotha: Forschungs- und Landesbibliothek Gotha.

Ibn Ṭūlūn, Shams al-Dīn Muhammad. n.d. al-Ghuraf al-'aliyya fĩ tarājim muta'akhkhirīal-Hanafiyya. MS Şehid Ali Paşa 1924. Istanbul: Süleymaniye Library.

Ibn Ṭūlūn, Shams al-Dīn Muhammad. n.d. Ta'līqāt Ibn Ṭūlūn. MS 'āmm 3849, fols. 57-62. Damascus: Asad National Library.

Katz, Marion. 2013. Prayer in Islamic Thought and Practice. Cambridge: Cambridge University Press.

Katz, Marion. 2014. Women in the Mosque: A History of Legal Thought and Social Practice. New York: Columbia University Press.

Katz, Marion. 2019. Commemoration of the Prophet's Birthday as a Domestic Ritual in Fifteenth- and Sixteenth-Century Damascus. In Domestic Devotions in the Early Modern World. Edited by Marco Faini and Alessia Meneghin. Leiden: Brill, pp. 167-81.

Lal, Ruby. 2005. Domesticity and Power in the Early Mughal World. Cambridge: Cambridge University Press.

Liebrenz, Boris. 2013. The Library of Ahmad al-Rabbāț. Books and their Audience in 12th to 13th/18th to 19th Century Syria. In Orientwissenschaftliche Hefte 32: Marginal Perspectives on Early Modern Ottoman Culture. Missionaries, Travellers, Booksellers. Halle: Universitätsverlag Halle-Wittenberg UG, pp. 17-59.

Liebrenz, Boris. 2016. Die Rifä'ìya aus Damaskus: Eine Privatbibliothek im osmanischen Syrien und ihr kulturelles Umfeld. Leiden: Brill.

Manguel, Alberto. 2018. The Art of Unpacking a Library. The Paris Review. Available online: https://www. theparisreview.org/blog/2018/02/01/art-unpacking-library/ (accessed on 30 June 2018).

Mathews, Annie-Christine Daskalakis. 1997. A Room of 'Splendor and Generosity' From Ottoman Damascus. Metropolitan Museum Journal 32: 111-39. [CrossRef]

Moulin, Claudine. 2010. Am Rande der Blätter. Gebrauchsspuren, Glossen und Annotationen in Handschriften und Büchern aus kulturhistorischer Perspektive. In Autorenbibliotheken. Bibliotheques d'auteurs. Biblioteche d'autore. Bibliotecas d'autur. Special issue Quarto 30/31: 19-26.

Ott, Claudia. 2008. Die Inschriften des Damaskuszimmers im Dresdner Völkerkundemuseum. In Die Grenzen Der Welt. Arabica Et Iranica Ad Honorem Heinz Gaube. Edited by Lorenz Korn, Eva Orthmann and Florian Schwarz. Wiesbaden: Reichert, pp. 211-21.

Peirce, Leslie. 1993. The Imperial Harem: Women and Sovereignty in the Ottoman Empire. New York: Oxford University Press.

Pfeifer, Helen. 2015. Encounter After the Conquest: Scholarly Gatherings in 16th-Century Ottoman Damascus. International Journal of Middle East Studies 47: 219-39. [CrossRef]

Robinson, Chase F. 2007. Islamic Historiography. Islamic Historiography. Cambridge: Cambridge University Press.

Sajdi, Dana. 2013. The Barber of Damascus: Nouveau Literacy in the Eighteenth-century Ottoman Levant. Stanford: Stanford University Press.

Schmidt, Jan. 2016. From One-Volume-Libraries to Scrapbooks. Ottoman Multiple-Text and Composite Manuscripts in the Early Modern Age (1400-1800). In One-Volume Libraries: Composite and Multiple-Text Manuscripts. Edited by Friedrich Michael and Cosima Schwarke. Berlin and Boston: De Gruyter, pp. 207-31.

Stört, Diana. 2010. Johann Wilhelm Ludwig Gleim und die gesellige Sammlungspraxis im 18. Jahrhundert. Hamburg: Verlag Dr. Kovač.

Weinrich, Ines. 2016. Introduction: From Texts to Performances. In Performing Religion: Actors, Contexts, and Texts. Case Studies from Islam. Edited by Ines Weinrich. Beirut: Orient-Institut Beirut, pp. 11-21.

Wieland, Magnus. 2015. Materialität des Lesens. Zur Topographie von Annotationsspuren in Autorenbibliotheken. In Autorenbibliotheken. Erschließung, Rekonstruktion, Wissensordnung. Edited by Michael Knoche. Wiesbaden: Harrassowitz, pp. 147-75. 
Wollina, Torsten. 2013. Ibn Țawq's Ta'līq. An Ego-Document for Mamlūk Studies. In Ubi Sumus? Quo Vademus? Mamluk Studies-State of the Art. Edited by Stephan Conermann. Göttingen: Bonn University Press, pp. 337-62.

Wollina, Torsten. 2014. Zwanzig Jahre Alltag: Lebens-, Welt- und Selbstbild im Journal des Ahmad Ibn Tawq. Göttingen: Bonn University Press.

Wollina, Torsten. 2019. Between Home and Sufi Convent: Devotional Book Use in Early Modern Damascus. In Domestic Devotions in the Early Modern World. Edited by Marco Faini and Alessia Meneghin. Leiden: Brill, pp. 147-66.

(C) 2019 by the author. Licensee MDPI, Basel, Switzerland. This article is an open access article distributed under the terms and conditions of the Creative Commons Attribution (CC BY) license (http://creativecommons.org/licenses/by/4.0/). 
Article

\title{
Sanctifying Domestic Space and Domesticating Sacred Space: Reading Ziyāra and Tașliya in Light of the Domestic in the Early Modern Ottoman World
}

\author{
Jonathan Parkes Allen \\ Roshan Institute for Persian Studies, University of Maryland, College Park, MD 20742, USA; jallen22@umd.edu
}

Received: 2 December 2019; Accepted: 24 January 2020; Published: 28 January 2020

\begin{abstract}
Shrine-visitation (ziyāra) and devotion to Muhammad (such as expressed in tașliya, the uttering of invocations upon the Prophet), both expressed through a range of ritualized practices and material objects, were at the heart of everyday Islam for the vast majority of early modern Ottoman Muslims across the empire. While both bodies of practice had communal and domestic aspects, this article focuses on the important intersections of the domestic with both shrine-visitation and Muhammad-centered devotion as visible in the early modern Ottoman lands, with a primary emphasis on the eighteenth century. While saints' shrines were communal and 'public' in nature, a range of attitudes and practices associated with them, recoverable through surviving physical evidence, travel literature, and hagiography, reveal their construction as domestic spaces of a different sort, appearing to pious visitors as the 'home' of the entombed saint through such routes as wall-writing, gender-mixing, and dream encounters. Devotion to Muhammad, on the other hand, while having many communal manifestations, was also deeply rooted in the domestic space of the household, in both prescription and practice. Through an examination of commentary literature, hagiography, and imagery and objects of devotion, particularly in the context of the famed manual of devotion Dalā'il al-khayrät, I demonstrate the transformative effect of such devotion upon domestic space and the ways in which domestic contexts were linked to the wider early modern world, Ottoman, and beyond.
\end{abstract}

Keywords: domestic devotion; Ottoman religious history; shrine visitation; ziyāra; pious graffiti; Ottoman art; space; devotion to Muhammad; tașliya; Dalā'il al-khayrāt

\section{Introduction}

On a verdant hill in Istanbul's ritzy Beşiktaş district, overlooking the Bosphorus Straits, is a complex of buildings with a dome-covered saint's shrine at its center, akin in basic form and function to Islamic saints' shrines in diverse circumstances the world over (Mulder 2014; Petersen 2018). Underneath is buried one of the most popular Muslim saints of the city, Hazret-i Yahyā Efendi (1494-1570), his tomb marked by a large cenotaph, with the smaller cenotaphs of family members and important disciples clustered around him. ${ }^{1}$ On almost any given day of the year the chamber under which he lies buried is frequented by devotees who have come to venerate the saint and to ask for his intercession with God. According to early modern authorities such as the historian Peçevī (1574-1649), his popularity first emerged during the saintly shaykh's lifetime, the 'great and small' bringing votives and other gifts to his sufi lodge, around which, according to Peçevī, Yahyā Efendi planted the many

1 Yahyā Efendi, originally from Trabzon, would become famous for being the 'milk-brother' of Sultan Süleymān (the Magnificent to Western Europe but Kānūnī, the Lawgiver, to both Ottomans and to contemporary Turks), though their relationship was not entirely untroubled (Şahin 2013). 
trees whose descendants still mark the place today amidst the concrete sprawl of modern Istanbul (Peçevî 1864-1866, pp. 455-546). Another early modern visitor to the saint, the famed traveler Evliyā Çelebi (1611-c. 1683), noted that 'upon the summit of a tall hill close to the sea, within an exalted domed chamber (kubbe), is the burial place [of Yahyā Efendi], a shrine (āsitāne, lit. 'threshold') whose four walls have been covered by how many thousands of passionate lovers ('uşşākān) able to write with all manner of couplets of poetry (Evliyâ Çelebi 1996, p. 222).'

Indeed, should you visit the shrine today, once you had surveyed the busy Baroque and later decorative scheme of the interior, your eyes might fall upon the still quite legible remnants of these 'thousands' of verses. Now, fortunately preserved behind plexiglass sheets, not just 'couplets of poetry' but short prayers, names, and dates can be read, most having been written in neat, clear naskh. Some are in carefully voweled Arabic, others in Ottoman Turkish (Figure 1). While many of the inscriptions that must have once covered these walls are now gone, enough remain that while perusing them one begins to sense that despite being a communal and quite public structure, this domed room has also long been experienced as an intimate, personal, and even domestic space. Perusing this open archive of past devotees, you might begin to understand why the Arabic word ziyāra is used to describe both pious visits to such a holy place as well as for visits to the home of a friend.
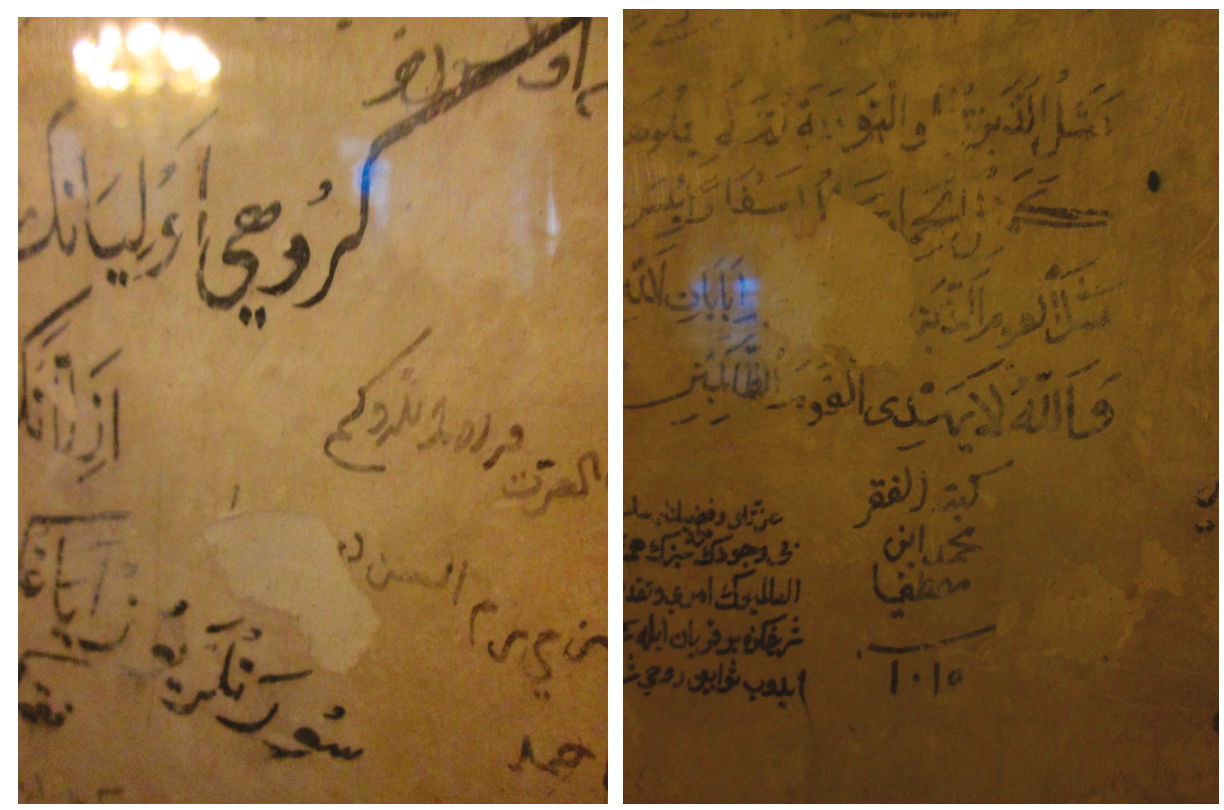

Figure 1. Examples of the wall-writing in the shrine of Yahā Efendi, here showing examples in both Arabic and Ottoman Turkish, compositions both original and reproducing other texts, particularly the Qur'an. One of the inscriptions, in the left photo, is dated AH 1015/1606-7. Photos by the author.

Were you to go back in time to eighteenth century Beşiktaş to visit the shrine of Yahyā Efendī and then accompany home one of the visiting devotees, odds are good that within her home you would find a copy of the ubiquitous devotional manual Dalä'il al-khayrāt, a compilation of prayers and blessings upon the Prophet Muhammad (șalāt 'alā al-nabì); if not the Dalä'il, then perhaps another, similar compilation of devotion to Muhammad, in Arabic or in Ottoman Turkish (or both). Historically, devotion to Muhammad has been expressed in many different ways across the Islamicate world. Amidst them all, the most fundamental was-and remains-the practice sometimes referred to in Arabic as tasliya, that is, prayer for God's blessing upon Muhammad, most succinctly expressed in the 
frequently invoked and foundational invocation, 'May the peace and blessing ${ }^{2}$ of God be upon him (that is, Muhammad).' Out of this short phrase an almost endless body of devotional material has been elaborated, the Dalä'il al-khayrāt becoming the most important and widespread textual iteration of such devotion (Rippen 2012; De La Puente 1999; Padwick 1961; see Appendix A for a sample translation from the text). In conversing with your early modern host you might learn that in addition to the daily ritual prayers and pious visits to the shrines of the saints, such litanies of blessing and prayer upon Muhammad made up a central part of her family's devotional life, crucial constitutive components of what it meant in fact to 'be Muslim.' Unlike the visits to the saints or the performance of the obligatory ritual prayer in a mosque, ${ }^{3}$ such devotion to Muhammad frequently took place inside their home, often upon waking from sleep or before going to bed, and so within the most intimate precincts of the house.

Domestic devotion to Muhammad was not limited to the textual and aural. If you were to examine the family's copy of the Dalā'il you might be struck by colorful depictions of the holy places and objects of the Hijāz such as Mecca's Ka'ba or the minbar of Muhammad in Medina, the style of the painting possibly reminding you of early modern Western European art conventions (Figure 2). You might also note within such a book or upon one of the walls of the home a curious calligraphic composition, a hilye-i ş̧eriff, a verbal icon of the Prophet (Figure 3). Families of more modest means might have a less artistically refined but still 'functional' printed copy, with little roundels showing Mecca and Medina as well. Finally, perhaps your host would modestly relate to you that Muhammad himself had once visited her home, in a dream-vision or even in 'waking life,' as a reward for the household's daily acts of devotion and love towards him. Perhaps she would ask you to smell deeply and see if you could still detect the sweet scent of musk that his footsteps had left behind. Bidding farewell to your pious host, you muse on the fact that similar households exist all across the Ottoman Empire in multiple linguistic settings, and beyond, little drops of devotion that together constitute a vast sea of early modern devotion to the Prophet. ${ }^{4}$

These two sites of Ottoman Islamic devotion-the communal space of the saint's shrine, and the domestic space of the home of an ordinary Ottoman household - with their associated practices do not at first glance seem to have had much in common beyond their centrality within the daily religious life of Ottoman Muslims. Yet on closer analysis these two sites and ritual bodies of devotion prove to have been connected through a dialectic of domestic and communal, open and closed. The communal, outwardly opened space of the saint's shrine incorporated aspects of the domestic, while the devotional regimes of the more inward facing and restricted access household brought close distant holy sites and presences within the space of the home. Practices of shrine visitation drew elements of the home into the holy place, while practices of devotion to Muhammad brought the effacious traces of the holy places into the home. Ottoman Muslims sought to perpetuate their own personal presence in the vicinity of the holy dead even after they had physically left the shrine. Similarly, they strove to reproduce the

2 Literally, 'prayer,' as in the obligatory ritual salāt; the exact meaning of this curious usage is a matter beyond our concerns here but is one which has been much discussed in Islamic exegetical literature.

3 To be sure, șalāt - here the five 'canonical prayers - could be performed virtually anywhere, save the Friday communal prayers; but the mosque was and is the preferable location, and is linked to the performance of the salät in other ways such as through the presence and activity of the muezzin announcing the times of the prayers.

4 While our imagined encounters are set in the Turkish-speaking northern tier of the empire, we could expect very similar devotional profiles had our hosts been Arabic-speaking residents of, say, Damascus or Cairo. Rural religious life would have both parallels and likely divergences, and while some aspects are accessible-veneration of saints would not be vastly different, for instance-it remains an open question what devotion to Muhammad looked like in rural areas, particularly among peasants and nomads, the vast majority of the population. Glimpses can be had here and there: For instance, during his stay in Karak Nūh in the mountains of Lebanon, al-Nābulusī (for whom see below) writes groups of people came from the village of Qar' ū 'wanting to perfom a mawlid for the Prophet, God bless him and give him peace, at night in that place (al-maqām, meaning the shrine of the Prophet Noah), so we were present with them in this hospitable reception, and through it there occured for us great joy and increase of His kindness' (al-Nābulusī 1979, p. 94). The rihla of an eighteenth century rural Kurdish scholar Taha al-Kurdī, who later settled in Damascus but who discusses rural Kurdish life at length in his autobiographical work, reveals in passing aspects of devotion to Muhammad, such as manners of mawlid performance: when Taha was born, he writes, his father was in the midst of a mawlid assembly wherein, upon being brought news of his son's birth, he proclaimed his son's name to the other attendees (al-Kurdī 2007, p. 29). 
Prophetic presence in their households through acts and material objects of devotion. Both broad bodies of practice were linked to each other through shared sensual and imaginative regimes that drew upon touch, smell, taste, sight, and hearing; and both were as much the domain of women as of men, resisting neat gendered categorizations.

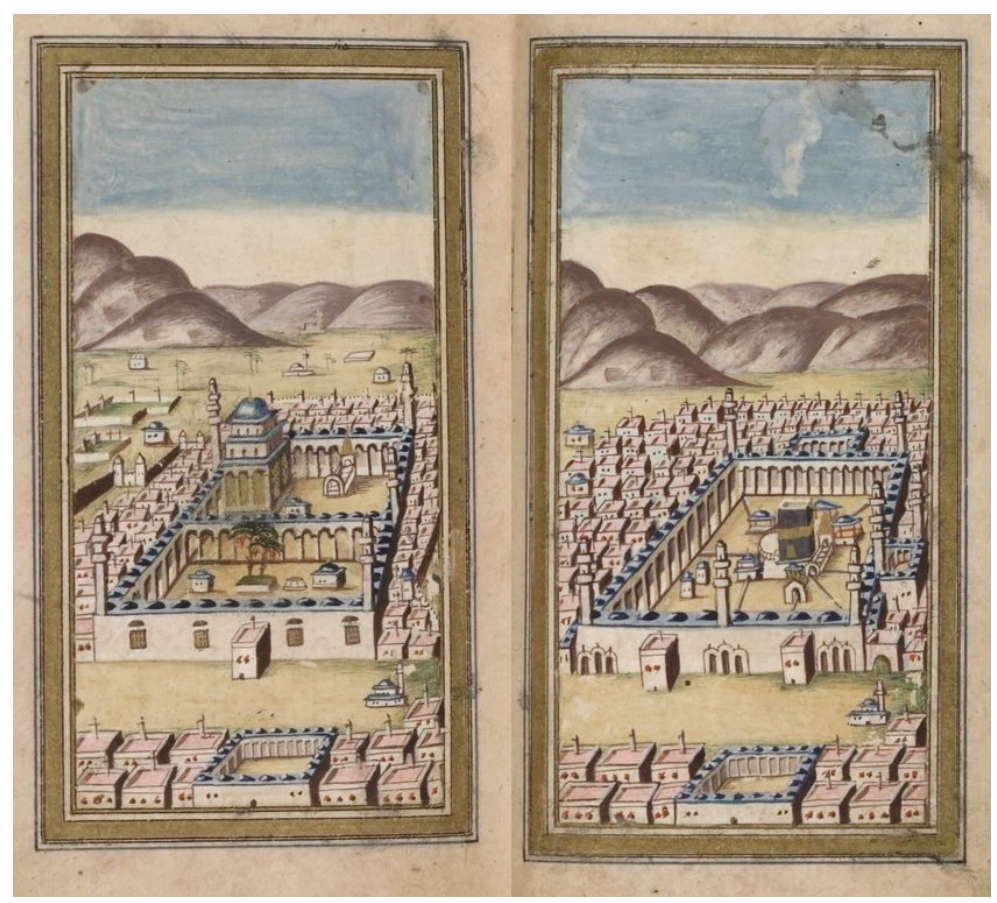

Figure 2. Cavalier perspectival views of Mecca (right) and Medina, from a 1772 copy of the Dala' $i l$ al-khayrāt, the body of the text written by one Hāfiz Muhammad ibn Hāfiz Ibrāhīm Mevlevī, imām of the Murādiye Camii in Edirne. Library of Congress, BP183.3.J39 1772 Arabic MSS, fol. 23b-24a. 


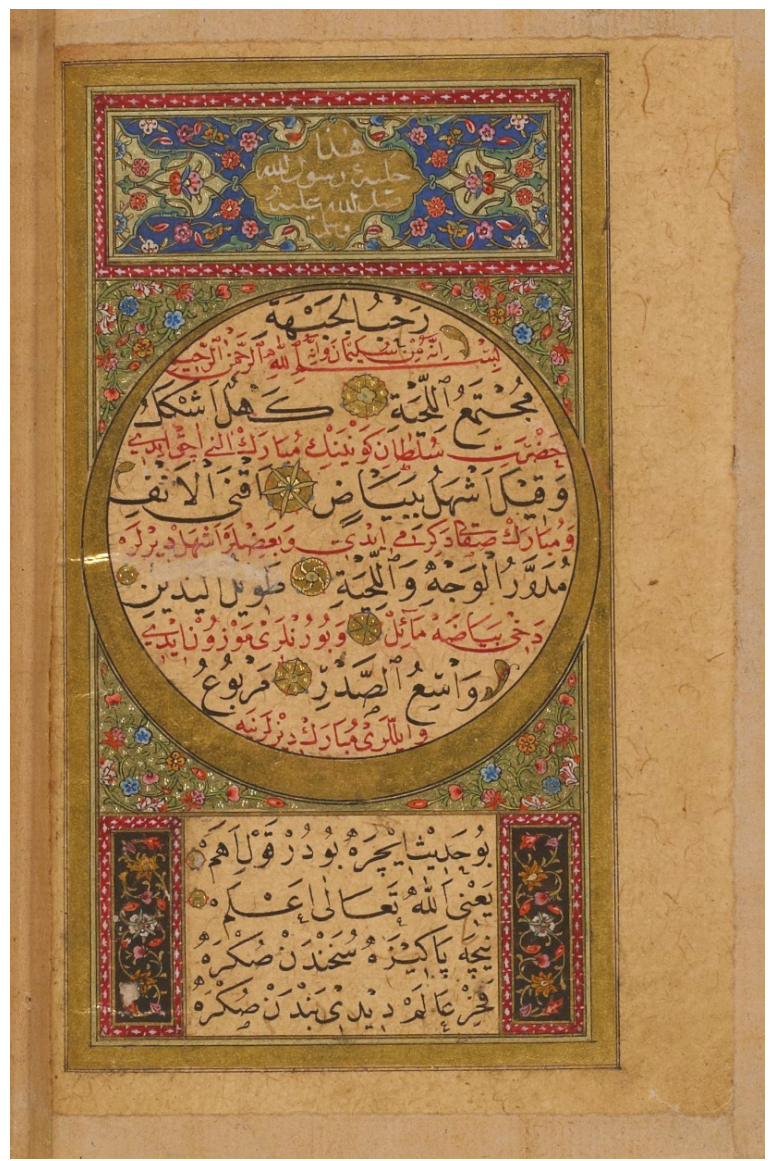

Figure 3. A hilye-i şerîf, with Ottoman Turkish interlinear translation, completed by by Ismā'îl Bagdādī in $1756 / 57$, added to a somewhat earlier copy of the Dalá' $i l$. Bibliothèque nationale de France, Département des manuscrits, Arabe 6859 i., folio $5 \mathrm{v}$.

To be sure, definite differences distinguish the devotional regimes of the shrine and the domestic household from each other, even if, as in other early modern contexts, such distinctions can only do so much work (Faini and Meneghin 2019, pp. 5-17). The shrine to which devotees made pious visitation was (and remains) oriented around a particular saint who was (usually) physically present in the form of his or her entombed body beneath a magnificent (or modest) cenotaph or another marker. When it was open, people could come and go from the shrine as they pleased, lighting candles, depositing votives, sprinkling incense. Often part of a larger complex, the shrine might have benefited from endowments and the oversight of a caretaker, or it might have been under the supervision of a community of sufis and their shaykh. In short, it was in many ways a resolutely communal and public space. By contrast, while devotion to Muhammad certainly had public, communal forms (Katz 2007; Krstic 2011, pp. 32-35; Allen 2019a), some quite prominent, such as the great mevlid rituals Ottoman sultans regularly held, in both prescriptive form and in actual daily practice tașliya and other forms of devotion to the Prophet were frequently domestic in setting. They frequently took place within the relative privacy of a house or in the private rooms contained within a madrasa, sufi lodge, or bachelor housing (Allen 2019b). The associated devotional texts and images were abundantly 
produced and relatively affordable even before the rise of typographic print in the nineteenth century, and for Turkish-speaking Ottomans the core Arabic prayers such as those of the Dalä'il were often supplemented by translations and glosses in Ottoman Turkish, as well as various independent works of devotion composed in that language. The visual repertoire of images that developed within the Dala $\bar{a}^{\prime} i$ and in other contexts did not require any sort of aural performance; rather, such images 'worked' through presence alone, with sight, imagination, and tactile participation additional modes of interaction, all especially suited for household use. In short, if we wanted to isolate the most typically domestic form of Islamic religious life in the early modern world, there is little else for which as strong a case could be made as these texts, rituals, and associated materialities of devotion to Muhammad. ${ }^{5}$

As central aspects of Ottoman Islamic identity and practice, there are many possible routes of analysis for understanding saints' shrines and household devotion to Muhammad, even from within the frame of the domestic. What I deal with here is but a selection. For the shrine, I have singled out the discrete practice of pious wall-writing, not only for its importance in shrine visitation, but also because it has been scarcely recognized in historical scholarship before. However, in thinking about the domestic aspects of the shrine, I have also sought to uncover some of the other constitutive practices and spatial imaginations of a more abstract nature, suggestive of how early modern Ottomans experienced the space of the shrine generally. So, my treatment of shrine visitation begins with an investigation into the spatial and imaginative constitution of shrines as 'homes' of saints, the context within which wall-writing and its social functions unfolded. As for domestic devotion to Muhammad in the home, while taking into account other devotional texts and objects, I have centered my analysis on the Dala' $i l$ al-khayrät in its diverse forms as a means of ritual performance in a domestic setting, ${ }^{6}$ as the subject of commentary works, and as a setting for devotional imagery and ensuing forms of participation (Abid 2017). I show how practices and imagery that originated in more communal, 'public' contexts became, in the early modern period, 'domesticated,' moving in some cases quite literally from the walls of mosques to material substrates within family homes, the varieties of participation oriented around these objects changing in the process. Where the act of wall-writing and other forms of shrine visitation cemented people's ties to a saint's shrine, aural, tactile, and visual repertoires of devotion to Muhammad entailed making the distant holy places of the Hijāz a part of everyday life within domestic space, and in so doing, 'prepared the ground' for realizing the powerful presence of Muhammad himself within individual Ottoman households.

In what follows, I have generally limited my analysis to the seventeenth and eighteenth centuries, looking further back at some points in order to uncover the genealogies of certain practices, texts, and images. I could have pressed much further back, to be sure: The history of the veneration of the saints goes back much further, while the space of the home already possessed significant resonances and meanings stretching far back into the Islamic past (Campo 1991), a heritage upon which the early modern transformations in devotional practice could build. Instead of focusing on a single location within the empire, I have drawn upon a diverse range of contexts from across the empire (though Constantinople and Damascus dominate) in both Arabic and Ottoman Turkish. While I have tried to take some note of regional particularities and points of divergence, I contend that out of all of this evidence a distinctly Ottoman pattern of devotional life and imagination emerges. While its ultimate shape was distinctly Ottoman, this pattern of devotional life was forged with resources from the wider

5 More explicitly 'sufi' practices and ritual-liturgical texts were certainly in wide circulation, often bound physically alongside copies of Muhammadan devotional material. However, at least notionally these sorts of ritual texts-awrād ('litanies') and dhikr formulas (short, mantra-like prayers of 'remembrance' of God)—were tied to a given shaykh or tarīga and took place collectively as well as at home. Ideally one received awräd and dhikr from a precepting shaykh with some degree of personal input and direction; while devotional texts were often formally transmitted, such transmission was but one of many ways of accessing the material and took place in a much less involved manner.

6 It should be noted at the outset that the Dalä'il, along with many other texts and forms of devotion to Muhammad, was not strictly domestic in its use and settings; as noted above, public devotion to Muhammad, including the incorporation of the Dala ${ }^{\prime} i l$ and other, similar tașliya texts, was common, with the various contexts of practice mutually supporting and feeding into one another. 
Afro-Eurasian early modern world, Muslim and non-Muslim, important aspects of which appear in this article in the form of texts originally produced in the Maghrib. Ottoman Muslim devotional life existed alongside and in contact with devotional cultures and currents prevailing in the other religious communities of the empire and beyond the empire, such that connections and parallels with devotional practice in Christian Europe and those of Muslim Ottomans can often be descried. My thinking has benefited from both the existence of such parallels and of the scholarship, much of it relatively recent, centered on domestic devotion in Christian Europe. While scholars such as Torsten Wollina and Marion H. Katz (both contributers to the recent Brill volume Domestic Devotions in the Early Modern World) have examined the intersection of Islamic devotion and domestic spheres, neither devotional life in its communal or domestic aspects, nor domestic religious practice in all its forms have received anything like the degree of coverage afforded in the historiography of Western Christianity (Wollina 2019; Katz 2019). While a thorough analysis is beyond the scope of this article, I will return in the conclusion to a consideration of wider early modern connectivities and contexts within which Ottoman Islamic practices of shrine visitation and household devotion inhered.

\section{The Saint's Shrine as a Domestic Space: Practices and Imagination}

Nestled within the barren stony desert that rises precipitously from the western shore of the Dead Sea towards Jerusalem is a sprawling shrine complex known as Nabī Mūsā, the rugged expanse of exposed stone surrounding a world away from the verdant environs of Yahyā Efendi above the Bosphorus (Figure 4). Within the complex of courtyards and domed rooms is a tomb that devotees venerate as the final resting place of the Prophet Moses-Mūsā in Arabic-conveyed by God from his place of death on the other side of the Jordan to this now numinously charged hilltop in the color-streaked and mostly empty desert (Aubin-Boltanski 2005; Aubin-Boltanski 2013).

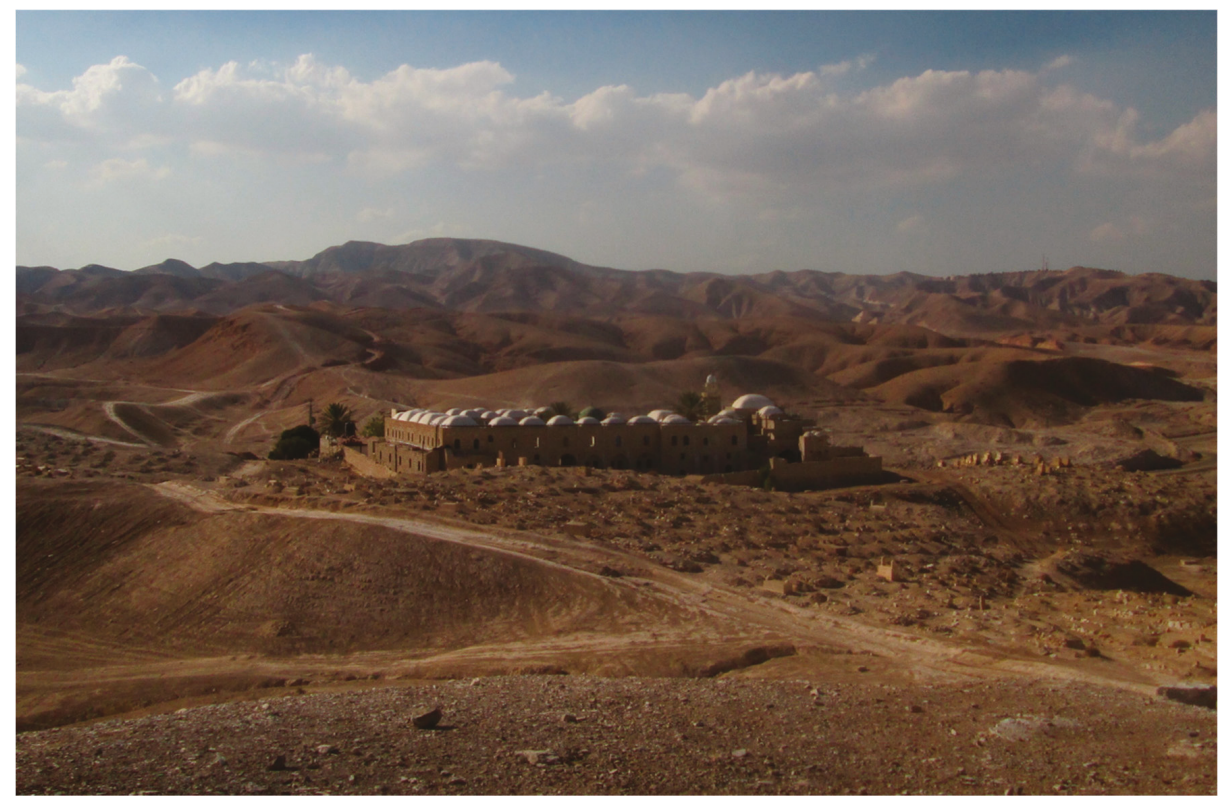

Figure 4. The shrine of Nabī Mūsā viewed from a subsidiary shrine uphill from the main complex. Photo by the author, October 2017.

Yet from the medieval period to the present, Muslim pilgrims from near and far have made the journey across the desert hills to this shrine in order to commune with the spirit of Nabī Mūsā, the 
Interlocuter of God (Kalìm Allahh) as he is called in the Qur'an. One of the many such pilgrims to this place was the great shaykh, saint, and scholar of Ottoman Damascus, 'Abd al-Ghanī al-Nābulusī (1641-1731) (Sirriyeh 2005; Allen 2019b, pp. 366-439). Along with a party of companions he made his way from Jerusalem to Nabī Mūsā in April of 1690, during the course of a two-and-a-half-week sojourn in the holy city. His account of the shrine and his pilgrimage to it in his travel narrative al-Hadra al-unsiya fí al-rihla al-Qudsìya includes a substantial discussion of its authenticity, the final resting place of Moses having long been contested among Muslim scholars. ${ }^{7}$ Alongside, and in support of, al-Nābulusī's arguments for the genuine nature of the tomb are his descriptions of his own emotional and spiritual reactions to the shrine as well as those of his companions. ${ }^{8}$ He records the lines of a praise-poem ( $\left.q a s_{i} i d a\right)$, which he composed on the spot and inscribed 'upon the qibla-facing wall so that their trace (athar) might persist there.' He then goes on to describe how 'there was with us a pious man from the folk of Egypt, named Shaykh 'Alī ibn 'Alī al-Dayșțī,' from the village of Dayst,

a village from among the villages of rural Egypt. He was illiterate, neither reading nor writing. But he said [to us]: "Write in this place from my memory [the following lines],

Good, all of it, is for those who bear hardship procured/ and the everlasting Garden is for those who in kindness are overcome.

I am a friend to people who say, 'For me proper deportment is required.'/Walk uprightly, and eyes and hearts will watch over you!"

(al-Hadra al-unsīya, pp. 224-25)

What this translation does not capture is the colloquial style of the Arabic original, which al-Nābulusī helpfully glosses for readers unfamiliar with some of the particular local usages. He then adds that in addition to having his poetic composition inscribed on the shrine's wall (perhaps right alongside al-Nābulusī's own recent addition) Shaykh 'Alī prayed to God that he might soon learn to read 'for himself.' Indeed, al-Nābulusī records, upon their return to Damascus Shaykh 'Alī busied himself with learning to read the Qur'an and after four months 'God opened for him' the ability to read, after which he memorized the entirety of the Qur'an, 'and this was due to the baraka of his supplication here, in that blessed place' (al-Nābulusī 1990, p. 225). In other words, Shaykh 'Alī's interaction with this holy place-an interaction literally written on the wall-had long-term repercussions, the baraka he activated through his 'gift' of pious poetry upon the wall following him home to Damascus. However, before departing, al-Nābulusī and his companions, like others before and after, spent a great deal of time in and around the sacred enclosure containing the great prophet's body, quite literally inhabiting it, eating and praying and sleeping within a space made all the more intimate by the empty, forbidding desert without. If their bodily sojourn there was temporary, the traces of that sojourn remained, in their memory and, through the medium of wall-writing, in the physical presence of the departed saint himself.

Before returning to shrine wall-writing itself, we must consider some of the ways in which Ottoman Muslim saints' shrines were deeply imprecated in the domestic, both in terms of everyday practices of veneration and in the ways in which these spaces were imagined and depicted. Ottoman devotees of the saints understood the saint's shrine as his or her house, containing not just the departed saint's body, but also his or her continuing presence, which simultaneously required certain practices while

7 Al-Nābulusī in fact reproduces en toto a treatise by a slightly earlier scholar, Shaykh Yūsuf ibn Mahmūd al-Maqdisī discussing the controversies and the proofs for the shrine's authenticity at some length; particularly curious is the presence, attested in the treatise and by al-Nābulusī himself, of spectral, angelic figures dancing upon the walls (which, alas, this author did not view upon his visit to the site in 2017, though admittedly much of the complex was closed off for reconstructive work) (al-Nābulusī 1990, pp. 208-23).

8 His disciple Mustafá al-Bakrī (d. 1759) would also visit the shrine and record both emotional and sensual effects, namely, the strong scent of rosewood and lavender within the space! (al-Bakrī 2009, pp. 114-16); he describes his visit elsewhere as well, including his encounter with a Maghribī named 'Abdallāh al-Sharīf, who ran about the shrine of Mūsā reciting loud dhikr, rapturously singing, and proclaiming, 'No god but God, my heart is aflame with Him!' (al-Bakrī 2015, p. 44). 
opening up the space to other possibilities. Most important in Ottoman Islamic practices of saint veneration was bodily proximity to the entombed saint accompanied by inner right intention, outward action honoring the saint (at a minimum, reciting the opening sura of the Qur'an, the Fätiha, for the soul of the saint), and the aural or inward seeking of his blessing and intercession. Just as one would act in certain ways when visiting an ordinary person's home, maintaining proper adab-here, behavior-in visiting the home of the departed saint was equally important. ${ }^{9}$ A brief clarification of the (usual) spatial arrangement of an Islamic saint's shrine is helpful here: As might be surmised from our 'visits' to the tombs of Yahyā Efendi and Nab̄̄ Mūsā, at the center of the shrine was the tomb of the saint himself (and sometimes herself, though female saints have generally been significantly less common in Islamic milieus than in most Christian ones), almost always marked by a cenotaph, the saint's body being interred in the earth below (see Figure 5). Unlike practices of saint veneration in some other religious traditions, which emphasize the production, dispersal, and display of bodily relics, the bodies of Muslim saints have generally been preserved whole, veiled, as it were, by being buried, not displayed in the way a Catholic or Orthodox saint might be. In this regard, we may detect a certain partial homology between the inner portion of a house-the haram-to which access is relatively restricted, with the saint being at once accessible and innaccesible. Regardless, in the (usual) absence of direct physical contact, the power of the saint's bodily presence in his tomb was filtered up and out, as it were. His physically grounded baraka (roughly, divinely bestowed power) was transmitted through the material of the shrine's components, including in many cases the very earth thereabouts. While a saint might visit a devotee in a dream, and a devotee might take away some soil or other artefact of proximity from the tomb, it was here in the saint's 'house' that he was most powerfully present and encountered, even if veiling intervened.

Because of the extent of his own writings and his deep investment in both venerating the saints and in defending that veneration from opponents, 'Abd al-Ghanī al-Nābulusī provides us with especially useful evidence of such homologies between domestic home and saint's shrine. During the second half of his life, he and his family lived in Damascus's Șalāhiyya quarter, practically next door to one of the most important and widely venerated saints of the Ottoman world, Muhyī al-Dīn ibn al-'Arabī (1165-1240). While today perhaps best known for his doctrine of the 'unity of existence,' for early modern Ottomans, Ibn 'Arabī was first and foremost a powerful saint of God, a status reflected in al-Nābulusī's relationship with the saint. While al-Nābulusī was a major exponent and exegete of Ibn 'Arabī's works, he also regarded the 'Greatest Master' as his intimate friend, visiting his 'home' regularly. The saint once even appeared to al-Nābulusī in a dream as being present in the latter's own house (al-Ghazzī 2012, p. 487). Al-Nābulusī would visit the saint's shrine every Tuesday before going to his regular teaching session, asking Ibn 'Arabī's permission before entering and leaving, in accordance with good $a d a b$. Once, al-Ghazzī writes, Ibn 'Arabī spoke audibly to al-Nābulusī from within the tomb, and even more extraordinarily, twice al-Nābulusī transcended the usual boundary between devotee and saint's physical body, first by placing a branch of myrtle in the miraculously outstretched hand of Ibn 'Arabī, another time by kissing the same outstretched hand (al-Ghazzī 2012, p. 519). ${ }^{10}$ In a long dream-vision that al-Nābulusī records in a compilation of visions and spiritual 'conversations' with God and departed saints, he describes asking Ibn 'Arabī with his 'spiritual tongue (al-lisān

9 Graves in general, as in many cultures across human history, were frequently coded and treated as domestic spaces by pre-modern Muslims, either as homes of the dead or as extensions of living households; the saint's shrine can be seen as both an elaboration upon a larger theme and as a distinct development in its own right, proceeding from not just the sustained presence of the departed in the tomb and his ability to interact with the living, but a much 'livelier' sense of such presence and interaction, one predicated upon the relatively minor difference between a physically living and bodily dead saint.

10 Al-Nābulusī elsewhere records moments in which physical contact of a sort persisted after death: The disciple of a recently departed saint, Dervish Murād al-Rūmī, was inspired to give al-Nābulusī a hand sculted from white fish bone with an ebony wood forearm-perhaps a manuscript pointer, or an ornament of some sort-which had been placed in the turban atop his cenotaph. 'I received it with my right hand, being made very joyful by that ... it was as if although I had not met him in the world of the living, his hand had stretched out to me in order to enact the pledge after physical death ... ' Al-Nābulusī, al-Hadra al-unsīya, 339. 
al-ruhān $\bar{\imath})^{\prime}$ - and receiving an answer from within the tomb-concerning proper bodily deportment when visiting the saint (al-Ghazzī 2012, pp. 528-29).

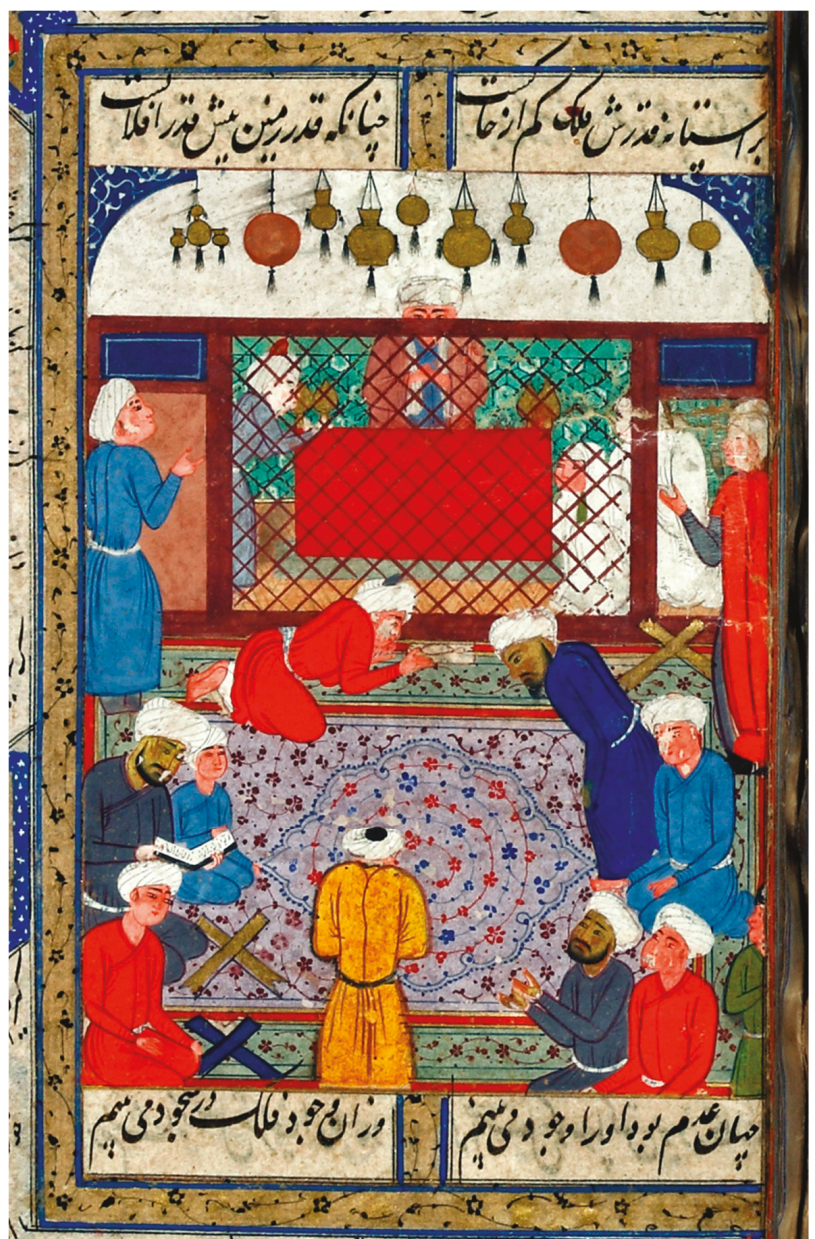

Figure 5. A typical shrine layout, a depicted in a c. 1550 copy of Kulliyat-i Mazlānāa Ahlī Shirāzī: While this image hails from the Safavid world, it nicely reflects architectural conventions and practices common to the Ottoman lands. Note the presence of women alongside men behind the grill marking the saint's tomb. David Collection, Inv. no. Isl 161.

While, like al-Nābulusī, many pious visitors sought to display proper deportment in visiting a saint's shrine-home, such concerns existed alongside another important aspect of the shrine: Its openness to practices and social arrangements quite different from that of most other public, communal spaces. Much as a visit to a friend's home might entail the enjoyment of food, drink, poetry, and music, shrines, especially more prominent ones, were often decidedly ludic and even sensual spaces. Candles, lamps, incense burners, and rich and colorful textiles filled these spaces, devotees themselves often contributing such objects on their visits. Aurally, the air would be filled with the sounds of Qur'an recitation, the prayers and supplications of visitors, poems, songs, and conversation (even as some devotees such as al-Nābulusī might seek quiet converse with the saint). Also, to the great ire of the puritanical opponents of saint veneration, men, women, and 'beardless young men' mixed quite freely 
in the saint's presence, suggestive in a different and potentially troubling way of the inner precint of the shrine as analogous to the inner spaces of the domestic house (Allen 2019b, pp. 298-321).

The movement of a saintly shaykh from the house he inhabited during life to the 'house' he inhabited upon death entailed some changes of practice and attitude, to be sure, on the part of devotees. Yet, in other ways, the movement was interpreted almost as if it were simply a matter of transferring from one room to another. In the menākı (from Ar. manāqib: The rough equivalent of a saint's vitae in the Western Christian tradition) of Şeyh Hasan Ünsī (1643-1723), a saintly shaykh of Constantinople's Hoca Paşa neighborhood, the disciple and memorializer of the saint Ibrāhīm Hās describes how even after his death the saintly shaykh remained active in his disciples' lives much as he had during his bodily life, the saint's 'room' now being the free-standing türbe (that is, tomb) that was part of the dervishes tekke (communal lodge) complex:

Even now someone who has a fearful matter or a difficult thing or a problem will go to the venerable Shaykh's noble türbe with honor and, practicing honor and graciousness, with etiquette will recite, for the dissolution of the problem will recite the [Sura] Ikhlās three times and the Fätiha once before [the saint's] pure spirit, give [the reward] to his noble spirit, and no sooner than leaving the türbe the solution to that person's problem will present itself to the heart, with God's permission, and provided the person's intention is pure.

(Hasan Ünsî Halvetî ve Menâkıbnâmesi, 351)

Sometimes the overlap of the domestic and the saint's shrine went from the homologous and imaginal to quite literal: For instance, the great early Ottoman saint and sufi of Cairo, 'Abd al-Wahhāb al-Sha'rānī (1492/3-1565) (Winter 1982; Sabra 2006), buried his preceptor-shaykh and father-figure 'Alī al-Khawwās within his familial zawiya (another type of sufi 'lodge'). After the burial of two of his sons therein, he sealed off the tomb-space to preserve a measure of exclusivety (though he himself would be buried there as well). Such burials in one's family zāwiya in this manner was not uncommon in the Ottoman world and beyond (al-Sha'rānī 2005, pp. 303-4). Two centuries later, in the village of Tillo in linguistically complex lands of what is now southeast Turkey, the scholar and lover of the saints Ismā'îl Hakkı (1703-1780) would oversee the construction of an architecturally innovative tomb-shrine directly incorporating the house of his beloved sufi master, the saint Şeyh Faḳirallāh, preserving the memory of the saint's home in both textual and diagramic form as well (İbrâhîm Hakkı 1914, pp. 507-8; Michot 2015). In both cases, the actual domestic spaces of the saints directly overlaid the space of their shrine-tombs in death, a pattern that drew ultimate inspiration and authorization from that of none other than Muhammad, who was buried in his home, a home that gradually became a full-fledged shrine and prototype for many to come.

Lived domestic space and the space of the saint's tomb could intersect in other rather literal ways as well. Ibrāhīm Hās describes the living arrangements of the türbedār, the dervish who oversaw the shrine and its use, as consisting of a room (oda) attached to the türbe itself, an arrangement that would have been found in other places as well. In two stories of türbedārs, Ibrāhīm suggests how the relationship between the türbedār's room and the inner prescint of the saint was supposed to work: In the first story, Ibrāhīm relates how the first türbedār of the shrine, a Dervish 'Alī, used in summer to sleep within the türbe itself instead of in his designated room next to it, for the very quotidian reason of a flea infestation in his room. When asked why he eventually stopped this practice, 'Alī replied that he kept seeing the saint in his dreams, brandishing a club and commanding him to stop sleeping there; over time this repeated vision had its effect, 'Ali evidently suffering through the fleas as best he could (İbrâhîm Hâs 2013, pp. 303-4). 'Alī had infringed on the special inner space of the saint, an intrusion that violated proper $a d a b$, akin to a subordinate member of a regular household deciding to sleep next to the head of the household (the presence of fleas-and the scratching and ensuing potential production of ritual impurity-excaberating the offense). That the türbedār was essentially part of the saint's household is suggested in the second story, in which a later türbedār, Derviş Yūsuf, was asked if he had ever experienced any 'signs' of Hasan Ünsī's sainthood, to which he replied, 
One day through the weakness of the flesh I commited a sin. That night I was lying in the türbe [attached] room, and I saw [in a dream] that Shaykh Ḥasan Ünsī had been manifest forth from his türbe. In his hand was a staff. With majesty and anger he advanced against me. So I went into the mosque, but I saw him following behind me! Awakening I was full of fear and took refuge in God.

(Hasan Ünsî Halvetî ve Menâkıbnâmesi, 305)

Derviş Yūsuf left the türbe for a few days and wandered the city, 'my mind remaining therein,' but upon his return, the saint was evidently pleased with his repentance and restored him to health. The foreshortening of the saint's domestic space and that of his devotees could be perilous if one were not careful.

\section{Perpetuating Presence in the House of the Saint: The Role of Pious Wall-Writing}

If a shrine custodian had the privilege—or burden, as the case may be-of literally inhabiting the domestic space of the entombed saint, other visitors had to come and go. ${ }^{11}$ Fortunately, in shrine wall-writing, devotees had potential access to a practice that allowed them to inscribe themselves into the space of the saint and to maintain a personal anchor in that space long after returning home. If in the modern world such personalized, officially unauthorized inscriptions would be classified as graffiti and potentially give grounds for prosecution, in the pre-modern Islamicate world, as in much of the rest of the pre-modern world, writing on public or semi-public substrates was not just tolerated but was an expected, indeed valorized, means of participating in public and especially sanctified spaces. Historians have, in the last few years, begun to realize the sheer extent and research potential of 'authorized graffiti' (terminology is itself problematic given the difference in attitudes between our world and prior ones), most notably in the path-breaking work of Karen B. Stern. However, the existence, much less the significance of wall-writing practices in the Islamicate world has barely been recognized thus far (Stern 2018; Ragazzoli 2018). ${ }^{12}$ Suffice to say, the practice had deep roots in the Ottoman world, with a genealogy that can be traced back to the antique and late antique milieu examined by Stern in fact, and which, by the eighteenth century, was manifest not just in Eurasia but in European colonies in the Americas as well. By our period, the composition of messages upon publicly accessible and visible surfaces was widespread and widely practiced by elite and non-elite; even lack of literacy need not preclude someone leaving his (and possibly her, though I do not-yet-have such evidence to say for sure) written mark, as this section's opening story demonstrates. Shrine wall-writing was but one aspect of the wider body of practice, as Ottoman left messages upon the walls of mosques, khans, churches, and other locations, for a range of reasons from simple indication of presence to running arguments with anonymous interlocuters.

Not all shrines received wall-writing, or at least not in equal measure. Our two most important literary witnesses, al-Nābulusī and Evliyā Çelebi, visited a rather staggering number of saints' shrines in the course of their respective monumental travels through the Ottoman lands, but only in relation to some do they note either the presence of wall-writing or their own contributions. Sometimes records of such wall-writing are incidental: al-Ghazzī mentions that his saintly ancestor al-Nābulusī had written a praise-poem, which he 'placed' (either by writing on the wall or on loose paper tacked to the wall or placed directly upon the tomb's cenotaph) in Ibn 'Arabī's shrine, a record precipitated by a dream in which a disciple of al-Nābulusī heard Ibn 'Arabī recite the very poem. Had he not recorded the dream account, al-Ghazzī might not have mentioned the poem in the shrine at all. Survival of actual

11 To be sure, there were shrines where overnight accomodations were included close by, and some wherein practices of incubation were practiced, though these were special cases and at any rate still entailed temporary lodging at most.

12 For the Islamicate context, a translation of an early medieval 'anthology' of found (or in some cases probably invented) poetic wall-writings exist, the distant ancestors of our examples and in some cases quite similar in content, though generally of a different nature and purpose, al-Iṣbahānī's Book of Strangers (al-Isbahānī 2000). 
wall-writing is even more contingent: Not only do shrines, as fundamentally 'living' structures, require frequent renovations, potentially obscuring many years of wall-writing, with modern shifts in how wall-writing (which gradually became an undifferentiated mass of 'graffiti') was perceived, most saints' shrines saw injunctures against further additions and the erasure or covering up of early modern writing, a shrine like that of Yahyā Efendi a fortunate exception. ${ }^{13}$

Because we are dependent upon such chance survivals and literary references, neither of which are at all systematic, we can only speculate on why some shrines may have been objects of wall-writing devotion and others not. Certainly, popularity, reputation, and existing custom mattered, though alternatively the presence of writing could, if initiated, help sustain a site's status as a holy place suitable for visitation. One basic factor, which seems obvious, but which proves difficult to parse further in fact, is the matter of substrates: Some surfaces are much better suited for writing than others. The ideal surface, and one which was-and is-indeed typical of many shrines in the Ottoman lands is one of smooth white plaster upon which charcoal, the preferred medium, leaves a very visible, and enduring, trace. Smooth stone (that is, stone surfaces not extensively ornamented or incised) provides the next most ameable substrate, whether in the form of a wall, architectural details, or even a tombstone. Zeynep Yürekli notes the former presence of figural pious graffiti upon the door jambs and voussoirs of the shrines of Hacı Bektāş and Seyyid Gazī, made up of 'birds, dervish bowls, ships, the sword zülfkēr, the hand of Fatima and the curious image of big fish swallowing smaller fish,' some with evident enough meanings, others quite opaque (Yürekli 2012, pp. 148-49). Such material no doubt once existed in far greater numbers than can now be observered after bouts of well-meaning modern restorations that so often eliminated etchings and writings no longer seen as appropriate. That pious graffiti might be written directly on tombstones is indicated by Evliyā Çelebi, who writes of the recently martyred Shaykh Mahmūd (here referred to be his other name in common use, Shaykh Rūmī) in Diyarbakır:

The shaykh lies buried in the Muslim cemetery outside the Rūm gate, in a grave without dome or any structure. May God bless us through this saint's miraculous powers! This humble author wrote the following lines on his tombstone:

We came as pilgrims to this station

Where reposes the great guide, Shaykh Rūmī!

(Evliyâ Çelebi in Diyarbekir, Evliyâ Çelebi 1988, p. 189)

Unlike walls of roofed shrines or mosques, the pious graffiti of an open-air de facto shrine such as this would have been relatively ephemeral in all but the driest climates, charcoal the likely medium of inscription here as elsewhere. Nonetheless, we can imagine Şeyh Murād's tombstone peppered in poems and other pieces of writing, the black of the script coursing along the surface of the stone, indicating even to casual observers the special status of the tomb's occupant even absent a typical shrine structure (which was possibly precluded at the time by this particular saint's politically controversial position). The very presence of charcoaled or etched words and texts on Shaykh Murād's helped to socially distinguish him as one of the 'very special dead' (Brown 1981).

Writing on a shrine's susbtrates-or on the grave marker of a saint not yet possessed of a shrine-certainly functioned, if implicitly, as an argument for the sanctity of the interred person. Explicating the full range of social work such writing could do, as well as the significance of its reproduction in our narrative sources, could encompass a study in itself. In terms of the intended function of shrine wall-writing, most fundamentally it was for devotees a way of remaining present in the 'home' of the saint even after one had physically left. Whether in the form of poetic or scriptural

13 As Yürekli notes about the pious graffiti at the shrines of Seyyid Gazi and Hacı Bektaş, 'unforunately almost all the graffiti on our two shrines were chiseled off during twentieth-century restorations, but those on the Seyyid Gazi shrine were fortunately recorded by Theodor Menzel in 1911' (Yürekli 2012, p. 14). 
'gifts' to the saint, requests to the saint for his intercession, or simply one's name and the date of visitation, these personal acts of writing linked the person writing (or dictating, as the case may be) with the saint through a very durable medium. We might think of such writings as a way of holding at length and at distance a conversation with the saint, a continuous expression of love and devotion, whereby the saint's baraka flowed into the devotee. Once inscribed in a substrate, the writing 'worked' without futher interventions, much as talismans written with prophylactic sacred phrases 'worked' even without being read or seen. That said, wall writings were also meant to be read by others who came to visit the saint, and in being read, reactivated in a particularly powerful way. ${ }^{14}$ Through such reactivation by other visitors, wall-writing helped to cement a peripatetic 'household' around the saint, devoted to the saint but also devoted, in a different way, to one another, praying for and reactivating the prayers of other visitors.

Shrine-writing could overlap with interventions in more conventional domestic space: al-Nābulusī visited the collective tomb of the al-Bakrī family, a saintly 'dynasty' of Cairo through whose lineage familial baraka had been transmitted over the last century, a pattern of particular purchase in Egypt (al-Bakrī 2015; Sabra 2016). Al-Nābulusī and his traveling companions were guests in the home of the current head of the family, Zayn al-'Ābidīn al-Bakrī (al-Nābulusī 1998, p. 31), and after visiting the family shrine wherein the 'lords of the Bakriyya' were entombed, al-Nābulusī composed a qașìda (a form of poetry often used for eulogistic purposes) in praise of the maqāmāt (that is, spiritual stations) of these holy dead. Zayn al-'Ābidīn had this qașīda inscribed on a wooden board and hung up inside the shrine, a contribution that lay in-between more permanent, 'architectural' inscriptions and the sort of wall-writing we have discussed so far. Here as elsewhere the categories and terms to which we in the modern world are accustomed generally lack the ideal capacity for encompassing the many possible forms these written interventions took (al-Nābulusī 1998, p. 64 ) ${ }^{15}$ It is also a challenge for us to appreciate the presence and power written words could take in a pre-typographic world in which public texts, even when monumental inscriptions are taken into account, were considerably rarer than in our text-saturated present. The social work al-Nābulusī's act of writing and Zayn al-'Ābidīn's space-modification facilitated was also complex. Al-Nābulusī contributed to the veneration of the 'lords of the Bakriyya' by inscribing their sainthood in poetry, while also reinforcing his bonds with the family and offering a sort of repayment for Zayn al-'Ābidīn's copious hospitality. Zayn al-'Ābidīn benefited, too, from the 'endorsement' of an important and himself widely venerated shaykh while further strengthening the bonds of friendship and mutual support between himself and al-Nābulusī by rendering the shaykh's qașida into a format more durable than ordinary shrine wall-writing.

In these ways and more, shrine wall-writing, then, can be understood as a means by which the domestic aspect of the saint's shrine was (further) constituted, as an intimate space of communication with the spirit of the saint and with his or her 'family' of devotees, present and future. The logic of this particular form of wall-writing followed upon the domestic logic of the shrine: As the saint's home, the devotee offered a gift to the saint and his household, a gift that also anchored his continuing presence in the vicinity of the saint. To etch an inscription on the wall or other surface of a saint's 'house' was in some ways to go beyond one' status as visitor and to instead become a sort of household inhabitant, the written traces of one's name and supplications and poetic composition abiding opposite a beloved saint, seen and read by the steady stream of other guests of the holy person entombed there.

\section{Finding the Prophet in Domestic Space: Envisioning the Effects of Devotion to Muhammad}

If a range of practices, attitudes, and imaginations worked to locate the domestic upon and within the tomb of the saint, in the homes of the living, a related if distinct set of practices, material objects,

14 Refer for instance to the neat, in some cases vocalized, writing in Figure 1.

15 It is not entirely clear what this assembly would have looked like; to my knowledge, no such inscribed wooden plaques survive, though we might look to examples of poetry written on wooden panels such as those preserved in the Metropolitan Museum of Art's famed c. 1707 Damascus Room (Met. 1970.17). 
and ritual texts brought holy presences into already existing domestic space and offered routes towards transformations of that space. While diverse saintly presences and relationships were reproduced and maintained in domestic settings, as displayed in the seventeenth century correspondences of 'Asiye Hatūn (Asiye Hatūn 1994), of greatest importance was devotion to the Prophet Muhammad. What follows will consider further two aspects of this interplay of the domestic and of the transformation of the domestic: First, a look at how tașliya, whether aurally performed using the Dalä'il al-khayrät or some other means, was envisioned as introducing the Muhammadan presence into one's home and so in a way transcending the constraints of the domestic while transforming that very space. Second, I will briefly trace the history of the images of holy places and objects that became a central part of the Dalä'il as a material object. I will demonstrate, among other things, both a 'domestication' of a previously 'public,' communally experienced visual repertoire and the place of these images in generating the Muhammadan presence within the walls of one's own home.

That the Dalā'il al-khayrāt and similar repositories of devotion to Muhammad were regarded, indeed valorized, as domestically oriented bodies of practice is evident from the commentary literature and other sources; the abundance of the many extant manuscripts and the evidence of frequent use on their pages argues that such literary observations and prescriptions in fact corresponded to actual practice. Initial forays into reconstructing the ownership and use histories of individual copies of the Dalā'il and other devotional works (with multi-volume or multi-excerpt compilations also very frequent) have established ownership of the text by both institutions and households. There is still need however to better establish the details of who owned copies of the this text and what those copies would have looked like (certainly, 'prestige' copies with full illuminative and sophisticated illustrative schemes are over-represented in most modern institutional collections) (Hanna 2003; Göloğlu 2018, pp. 231-35). My goal here is more modest: To briefly demonstrate how certain Ottoman authors envisioned Muhammadan devotion in certain modes as being uniquely domestic in nature.

We begin with the hagiography of the Dalä'il al-khayrät's compiler, Muhammad al-Jazūlī (d. 1465), venerated as a saint in both his native Maghrib and everywhere the Dala' $i l$ went, a status reinforced in hagiographic compositions as well as in the commentary (sharh) literature on the Dalä'il (Cornell 1998; Blecher 2013). Arabic-language treatments of the saint give, in varying lengths, a now classic story that explains why al-Jazūlī compiled the book. One day, while trying to retrieve water from a well in order to perform his ritual ablutions, he was stymied by the lack of a rope. A girl came along, breathed into the well, and up came the water of its own accord! Amazed, al-Jazūlī asked her what in her practice of piety had invested her with such miraculous ability, to which she replied it was her regular practice of invoking peace and blessings upon the Prophet Muhammad. Inspired, al-Jazūlī set out to compile his now classic work (al-Fāsī 1989, pp. 6-7). In his early eighteenth-century Ottoman Turkish commentary on the Dalā'il, the otherwise obscure preacher and exegete Qara Dāvudzāde Mehmed Efendi (d. 1756) elaborated upon this story further. ${ }^{16}$ The 'girl' $(k \imath z)$ expresses amazement that someone whom 'the people praise for your goodness and miracles (kerāmāt)' is unable to draw up the water. In this version, al-Jazūlī goes home determined to transform his devotional life, a determination reinforced when that night he awakes to see his wife quietly arising from bed and heading outside. Angered and jealous, he creeps off after her, and is amazed to see her go down to the seashore, encounter two wild lions, mount one, and ride him across the water to an island where she performs the ritual prayer. Upon her return, he asks her what she has done that has given her such powers, to which she replies, unsurprisingly, that it is her practice of pronouncing peace and blessings upon Muhammad (Qara Dāvudzāde 1750, pp. 2-3).

There are many potential messages encoded in these accounts (and in other stories in Qara Dāvudzāde's compendium emphasizing the superiority of a woman's piety and practice over that

16 In a late eighteenth century copy of the text, the author is described in a short text after the colophon as an 'advice-giving preacher to the community of Muhammad' and an exegete, working in Istanbul's Yeni Valide Mosque (now generally known simply as Yeni Camii, 'New Mosque,' after is completion in the late seventeenth century) (Qara Dāvudzāde 1750, p. 699). 
of a man). Central is the idea that not only can women practice devotion to Muhammad within the space of the home (and without), but that they are potentially especially powerful practicioners of this devotion, devotion whose effects might well transcend those of other more male-dominated forms of saintliness. Furthermore, if the sanctification of women's domestic lives and spaces (and those of men, who are meant in these and similar accounts to look to women for emulation) are encouraged in these accounts, the transcendence of the domestic is also implicitly suggested: While, we surmise, al-Jazūlī's wife practiced her devotion to Muhammad primarily within the house, the connection with God and his Prophet that she thereby generated and which endowed her with saintly powers enabled her to not only depart from the boundaries of the house, but even walk upon water and command wild beasts. She was quite literally transported beyond the bounds of the household through her devotion to the Prophet. Perhaps even more remarkably, there is no indication in Qara Dāvudzāde's story that she was in any way at fault in neither informing her husband of her devotional regime nor in not asking his permission to leave the house and go out walking on water and cavorting with lions. ${ }^{17}$ There is an implicit message in these stories that devotion to Muhammad is not just accessible to women in some form or another, but that it is especially suited to opening up the spiritual and otherwise horizons of women. While the women in the two above stories are shown literally transcending the limits of the domestic, implicit is the idea that even while centered on the house, within the space of the domestic, women can partake of a vigorous and powerful devotional regime, such that they might even surpass the powers of a prominent saint.

However, while it might be tempting to interpret these bodies of devotion as being uniquely gendered as 'female,' perhaps as extensions of the 'female space' of the household, other instances from the commentary literature suggest a more complex picture. Three examples from the commentary (sharh) of the most influential and most widely copied commentator on the text in Arabic, Muhammad al-Mahdī ibn Ahmad al-Fāsī (d. 1698), provide a good sense of other iterations of the domestic setting prescriptive literature suggested as normative for devotion (Dennerlein 2018). In a short story in his manāqib of al-Jazūlī, for instance, al-Fāsī relates the tale of a student in Fes who owned the Dalä'il and another, somewhat similar, compilation of blessings and prayers upon Muhammad, the Tanbih al-anām, copies of which circulated in the Maghrib and in the Ottoman world thought not, it would seem, beyond. In the story, the student would leave his copy of the Dalá'il underneath the Tanbih then leave his room in the madrasa. When he returned, the Dalä'il would have miraculously switched spots with the Tanbīh, coming out, quite literally, on top (al-Fāsī 1989, pp. 9-10)! Beyond the obivious purpose of this story in establishing the superiority of one form of Muhammadan devotion to another, the account points to domestic space-here, a student's private room—as the expected physical location devotional books would be used and stored. Such a domestic context is implied even more strongly in two stories of the effects of tasliya al-Fāsī provides in his commentary on the Dalä'il, stories that also point to the potential transformative effects of such devotions upon the spaces in which they were practiced. The first story concerns a pious craftsman —as with Qara Dāvudzāde, the emphasis is upon the broad accessibility of this devotional regime to people up and down the social ladder-who reported,

I used to perform every night by myself before going to bed a certain number of salutations and blessings upon the Prophet, God bless him and give him peace. One night I had completed this number of prayers and feel asleep. I was then dwelling in a first-floor room (ghurfa). Suddenly the Prophet, God bless him and give him peace, entered into the door of the upper room, light streaming from him. He stood up before me and said, "What is this

17 Certainly there were expectations in Ottoman Islamic society, as in other societies shaped by normative Islam, that women-women of certain classes, statuses, and ages, at least-would generally remain within the home; at the same time, here as in many other cases, that expectation was but background to women's actual public lives, either in their own movement without the house or, in the case of women who because of their elite status really did tend to remain hidden from public sight, their deployment of patronage and power through other means, including acts of devotion and piety. 
sorrow which increases ṣalāt upon me?"18 Then I was embarrased to kiss him on his mouth, so I turned my face and he kissed me on my cheek. Then I awoke with a start in that very moment, awakening my wife beside me. The house (al-bayt) was suffused with the smell of musk from his scent, God bless him and give him peace, and the scent of musk remained on my cheek some eight days, my wife finding it upon my cheek every day and night!

(Matāli' al-masarrāt, 58)

The setting here is not only determinedly domestic, it is strikingly intimate, both in the space in which it unfolds-an 'upper room,' in which the man and his wife sleep and to which others would presumably not have access, itself a part of a larger domestic complex, the bayt-and in its particular details. An almost erotic charge pervades these details, the performance of devotion to the Prophet establishing a means for him to penetrate into inner domestic space and leave his trace therein, both upon the body of the devotee and upon the space itself. The scent of him pervades the house, the physical, sensory (and indeed sensual) effect of the devotions performed within.

This sensory, even sensual effect of tasliya within the walls of the house is further reinforced in the next story al-Fāsī relates, set in late medieval al-Andalūs, and related by one Abū al-Qāsim al-Murīd:

When Shaykh Abū 'Umrān al-Barda'î came to Malaga he found therein Shaykh Abū 'Alī, that is al-Kharrāz ['the Cobbler']. The three of us met together one day in my home (dārî̀) for food I had made for them ... My father was also present, and he had come down with a cold such that his sense of smell was interrupted. Shaykh Abū 'Umrān said to Shaykh Abū 'Alī, "Yā Abū 'Alī, you have eight years [of devotional performance], so what effect has taṣliya had on you?" He replied, "Yā Sīdī, it has endowed me with such-and-such [spiritual powers]." Shaykh Abū 'Umrān replied, "This is what is manifest to children; one ought not in such manner practice remembrance of the Prophet." Then he said, "Breath upon the palm of the hand of Shaykh Abū al-Qāsim's father." He did so, and the smell of musk wafted about, but weakly. Then Shaykh Abū 'Umrān blew upon my father's palm, and by God! The smell of the musk cleaved my father's nostrils to the point that they started to bleed from the force of it! Blood flowed from his nose and the scent pervaded my home until scents of musk reached even the neighbors!

(Mațāli' al-masarrāt, 59)

Lest there be any doubt as to just whose scent the smell of musk is in this story, just before this passage al-Fāsī relates how the scent of Muhammad is directly derived from the smells of the Garden itself, a scent that he in turn bestows upon those who practice devotion to him. Through the potency of tașliya —of which the Dalä'il is presented as the finest and most accessible distillation—ordinary domestic spaces can be suffused with the scent of Paradise. Domestic space becomes marked in this rendering with the space of the eternal Garden through the presence of the Prophet breaking into that inner, intimate space of the house, opening it to himself and to the eternal.

\section{Bringing the Holy Home: Devotional Imagery in the Dalā'il and Other Contexts}

Both al-Fāsī and Qara Dāvudzāde largely limit themselves to the aural performance of devotion to Muhammad, following the text of the Dalá'il itself in this regard. However, within the original late medieval composition of al-Jazūli itself is the seed of a tacticle and visual regime of practice that would flourish in the early modern Ottoman world (and beyond), and in so doing establish another means of drawing the Muhammadan presence into domestic space through participation in the most important shared holy places of the Muslim umma, those of Mecca and Medina. These images, along

18 Suggesting that the pious man's performance of taṣliya was precipitated by some concern or anxiety in his life, which he wished to resolve through turning to the Prophet. 
with related pictoral depictions of objects associated with Muhammad, emerged in the medieval period but were either limited to elite audiences or were accessible to others through public display in communal locations such as mosques. It would be primarily through the medium of the Dalä'il and its visual repertoire that images of Mecca and Medina, along with new forms of visual representation of the Prophet's presence, would become domestically accessible. Simultaneously, the ways in which devotees interacted with such images would diversify and shift, from the tactile to more visual and imaginative modes of participation, the originally Ottoman hilye an important aspect and driver of this change. While the Turkish-speaking provinces would see the most expansive elaboration of devotional imagery, these transformations would play out in the Arabic-speaking regions as well and beyond the empire's borders both west and east. This final section will trace the trajectories of these devotional images from settings of a primarily communal and public nature to an increasing emphasis on intimate, domestically rooted use, drawing upon both material evidence and that of commentaries and devotional texts themselves.

The fully elaborated repertoire of devotional images that would become typical in copies of the Dalä'il in (primarily) the Turkish-speaking half of the Ottoman Empire had multiple points of origin, each displaying a movement from the communal to the domestic. ${ }^{19}$ Within the text of the Dalä'il itself is a short description of the most significant physical site associated with Muhammad-the so-called Rawda (literally, 'Garden') of Muhammad, the place in which he and Abū Bakr and 'Umar, the first two caliphs, were buried. The text itself envisions a clarifying diagram of the three tombs, al-Jazūli himself probably having drawn the original prototype, which was soon elaborated somewhat with additional details, a facing image of the nearby Muhammad's Minbar complementing that of the Rawda (see Figure 6 for an Ottoman Syrian example). In North Africa and some other regions, these two images, rendered in many different styles, would often constitute the only imagery. Copies made in the Arabic-speaking provinces of the empire tended to follow this pattern, though not exclusively. The presence of these two schematics-anchored in the text itself-served as an entry point for further elaborations, though the exact chronology and rationales are murky; the following is only a brief summary of the likely trajectory and of the possible interpretations we may attach to that trajectory (Rusli et al. 2016; my analysis departs somewhat from that of Witkam 2007).

19 It should be noted that different parts of the early modern Islamicate world had distinctive traditions of illustrating and using the Dala' $i l$, with some regions' practices resembling the Ottoman Turcophone, others the Maghrib. A comprehensive history of just the images used in the Dala' $i l$ remains to be written, though there are now a number of scholars working on the topic, as represented in the secondary works cited here. 


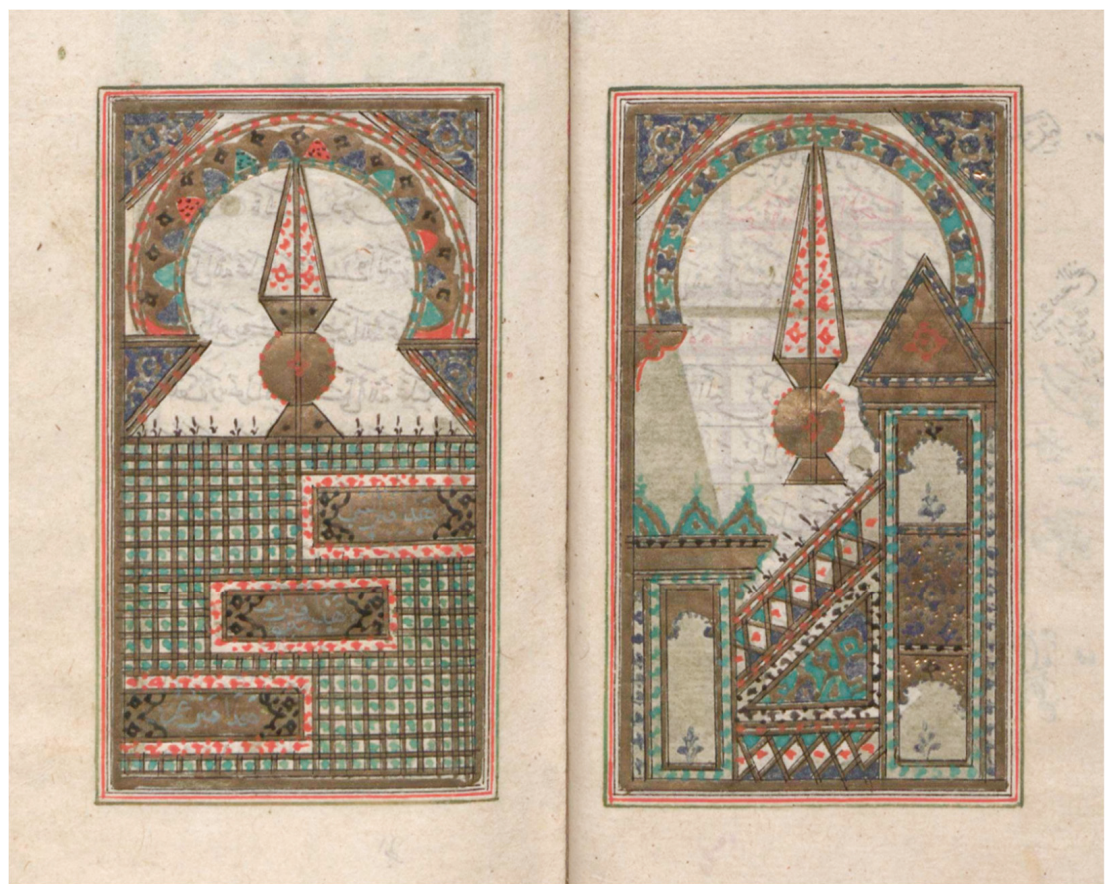

Figure 6. A fairly typical representative of the 'traditional' pairing of depictions of the Rawda with Muhammad's Minbar with decorative elaboration typical of the wider Ottoman world, from an Ottoman Syrian copy of the Dala' $i l$ completed at some point in the eighteenth century. University Library of Leipzig, Vollers 200.

Images of Mecca and Medina as a whole and of sites within or associated with the holy cities (including Muhammad's Minbar and the Rawda) long predated the composition of the Dalä'il and continued to be elaborated upon in other media and matrices as well long after the elaboration and spread of the Dalä'il and its images. Views of Mecca and Medina were developed and propagated in, among other contexts, pilgrimage certificates, often elaborate works in scroll-form that documented someone's pilgrimage to Mecca (Chekhab-Abudaya et al. 2016). While a surprising number of these otherwise rather ephemeral texts, most seemingly produced in Mecca, have survived, dating from the thirteenth century forward, their social contexts are not always obvious (Aksoy and Milstein 2000). Some show signs of having been hung up, either in full or cut down into constitutive components. We are fortunate to possess a passage from the early sixteenth century that sheds more light on the context in which these images were displayed and could have entered into wider artistic and devotional consciousness as a result. In his treatise on the proper usage of sacred spaces, the sufi scholar Shaykh 'Alwān of Hama (d. 1530) described what he saw as pernicious contemporary practices of mosque 'ornamentation (zukhruf)' that needed to be confronted (Ibn Ațiyah 2003, pp. 27-28; Allen 2019b, pp. 116-38). Among the 'reprehensible practices' of the people of Syria in mosques, Shaykh 'Alwān argues, is that

they raise up sheets of paper joined together decorated with the image of the noble and exalted Ka'ba, the noble Stone, and other than that, on which they write things such as, after 
praise to God and what follows it, 'So-and-so made the 'umra ${ }^{20}$ on behalf of so-and-so,' and 'So-and-so made the hajj on behalf of so-and-so.' They intend thereby-and God knows best-fame, eye-service, and repute, and the dissimulation of the repute of the one for whom the 'umra or hajj was made, in life and in death. They attach those to the qibla [wall or niche or both] of the mosque and elsewhere on its surfaces, causing distraction on the part of those praying the șalat through looking at the decoration.

(Asná al-maqāṣid, pp. 28-29)

While we need not accept at face value Shaykh 'Alwān's interpretation of the moral probity of people who so displayed pilgrimage scroll imagery, his supposition that they served as sources of social capital does not seem far off. These scrolls were meant to be seen, displayed in a communal setting (consider the example at Figure 7). The iconography developed primarily in these scrolls-which included both schematic views of Mecca and Medina as well as of other holy places and of iconic 'relics' such as Muhammad's sandal-print-would go in many directions from their original contexts. In the Ottoman world, the public display of images of Mecca and Medina would find more permanent form in, above all, the many Iznik tile panels presenting somewhat similar schematic depictions of either one or both holy cities of the Hijāz (see Figure 8 for an example), prominently if somewhat incongruously displayed on the qibla walls of Ottoman mosques great and small. Such images worked to link the space of the mosque to that of the Hijāz and invited the viewer to imaginatively traverse the sacred topography him or herself (Maury 2013). Relatedly, depictions of the iconic 'relics' of Muhammad, particularly his sandal or foot-print, entered tile form, across the Ottoman world and not just in the Turcophone (Milstein 2006). ${ }^{21}$ While there is at least one instance of tilework depictions in a domestic context, it is the highly rarified one of the Topkapı's Harem. More important for our purposes, images of the holy places of the Hijāz would also begin to appear within the pages of books, most significantly the Dalä'il. ${ }^{22}$ This shift would begin the movement of these devotional images from shared communal settings to also existing within domestic, household ones-which, in this case, entailed a movement from spaces in which women's participation was secondary and restricted (that of the mosque) to spaces in which women were dominant presences (that of the home).

20 That is, the 'lesser' pilgrimage, which can be carried out at any time of the year and now just during the designated pilgrimage season.

21 See for instance the beautiful mihrab panel from an eighteenth Ottoman mosque in Damascus, now preserved in the Aga Khan Museum's collections, which prominently features plain blue depictions of Muhammad's sandalprints: Aga Khan Museum, AKM585.

22 For instance, elaborations on pilgrimage scroll style images formed the visual repertoire of a popular Persian-language guide to the holy places of the Hijāz, the sixteenth century Futūh al-haramayn by Muhyi al-Dīn Larī (d. 1521?), production of which would continue in the following centuries from India to the Ottoman lands, though never on the large scale of the Dala' $i l$, in part no doubt because of the much larger (and hence more expensive) range of illustrations the text usually included (Rogers 2010, pp. 250-51). 


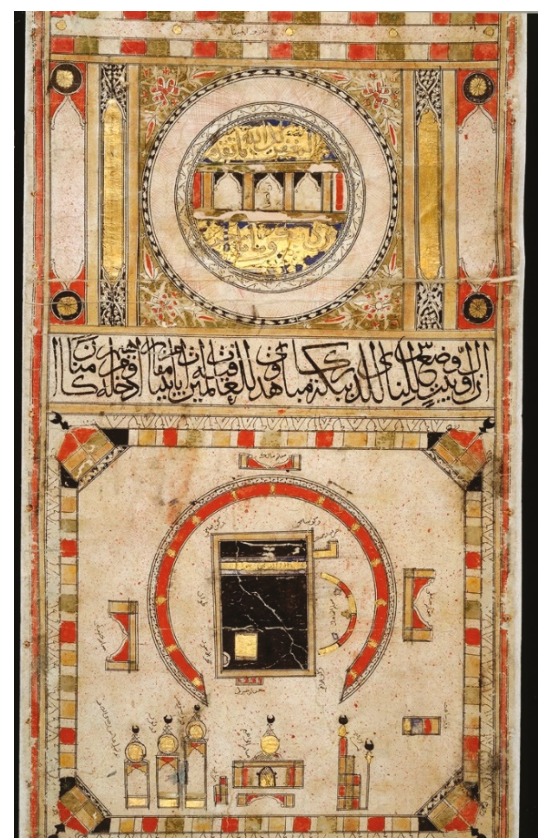

Figure 7. A section of a pilgrimage scroll showing examples of schematic depictions of holy places in the Hijāz; the entirety or portions of such scrolls would have been mounted on mosque walls for public display, a function that may well have been in the makers' minds given the scale of the depictions. This example was completed around the year 1433 and displays the increasingly sophisticated visual repertoire that developed within the genre. British Library, Add. MS 27566.

By at least the mid-seventeenth century, copies of the Dalā'il itself frequently included depictions of the two holy cities-either complementing or supplanting the original views of the Rawda and the Prophet's Minbar-with some stylistic departures from the original schematic renderings found in the pilgrimage scrolls, as is visible in one of the earliest copies of the text, produced in early seventeenth century Tunisia (probably). ${ }^{23}$ Around the start of the eighteenth century, the depictions of Mecca and Medina would increasingly (though far from exclusively) be rendered in a cavalier perspective (Figure 9) redolent of contemporary Western European art, a change primarily found in the northern, Turcophonic tier of the empire (Maury 2010), though further research will no doubt establish the exact regional makeup of this change. The ensuing visual repertoire of the Dalä'il would increasingly shape the imagery of other devotional texts. For instance, the previously cited manuscript of Qara Dāvudzāde's commentary on the Dalā'il reproduces, as a sort of establishing citation, a Dalä'il-style cavalier perspective view of Mecca in the opening illuminated frontspiece (Figure 9; see also Figure 10). Many other similar cases could be cited. ${ }^{24}$

Another major source of the Dala' $i l^{\prime}$ ' iconographic repertoire, at least in the core provinces, was the early modern transformation of long-existing physical descriptions of Muhammad (and, to a lesser

23 This copy of the Dalä'il was recently acquired by the Metropolitan Museum of Art as Met. 2017.301.

24 To give another example: a recently auctioned mid-eighteenth century copy of the Dalä'il's 'rival' devotional work, the Tanbih al-anām, not only includes the by then standard cavalier perspective views of the holy cities of the Hijāz, but also, just like many copies of the Dala' $i l$ during the period, collates other devotional imagery into its pages, such as a particularly beautiful rendering, with ample neutral space and delicate floral details, of Muhammad's sandal-print (Sotheby's London 2015). See also Figure 13 below. 
degree, other holy persons from early Islam), the shamä'il and hilya (Ottoman Turkish hilye) literature, into a discrete genre of texts and objects (Elias 2012, pp. 272-74; Gruber 2014; Gruber 2019, pp. 285-381). Most famously, these descriptions, which could be traced back to canonical hadith literature, formed the core of the aforementioned calligraphic 'icon' known as the hilye-i şerif (for examples see Figures 3 and 10). Not only were hilyes increasingly included in (primarily) Turcophonic copies of the Dalá'il, the modes of engagement associated with them, focusing on the visual, imaginative, and prophylactic, seem to have contributed to the changes in how Ottoman Muslims interacted with the imagery of the Dalā'il and related texts.

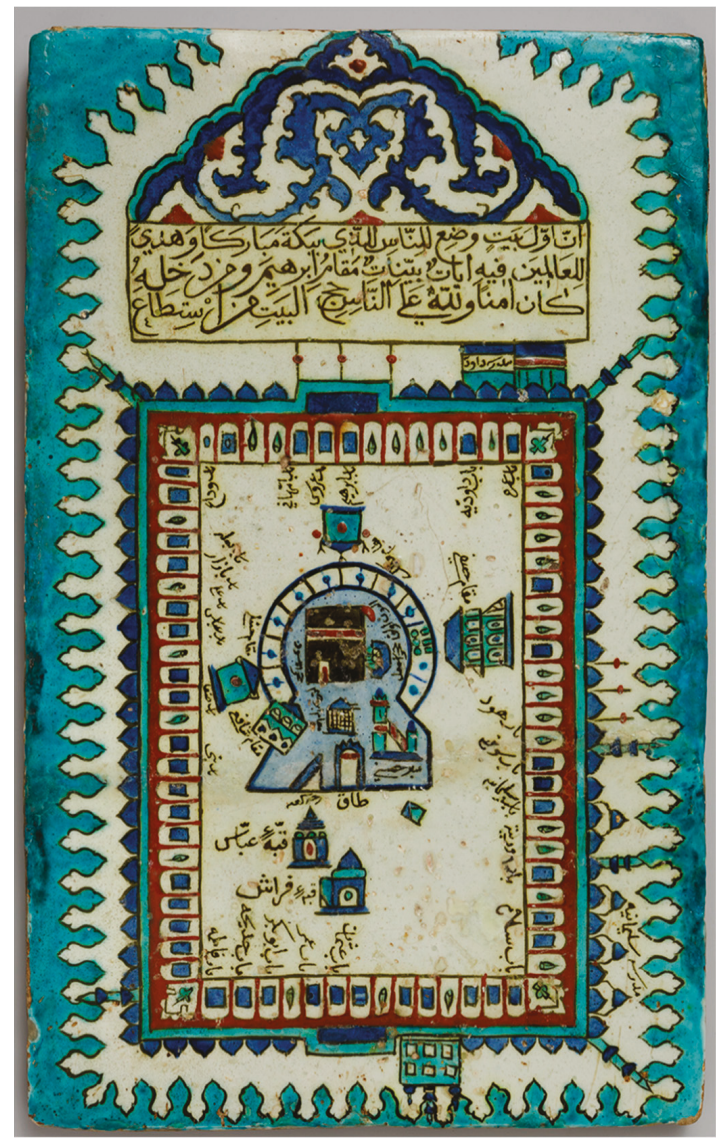

Figure 8. A circa 1640 tile depiction of Mecca with the Ka'ba at the center, formerly set in the qibla wall of a mosque. The relationship between the tile's visual vocabulary and that of the pilgrimage scroll is immediately evident. Aga Khan Museum, AKM58. 


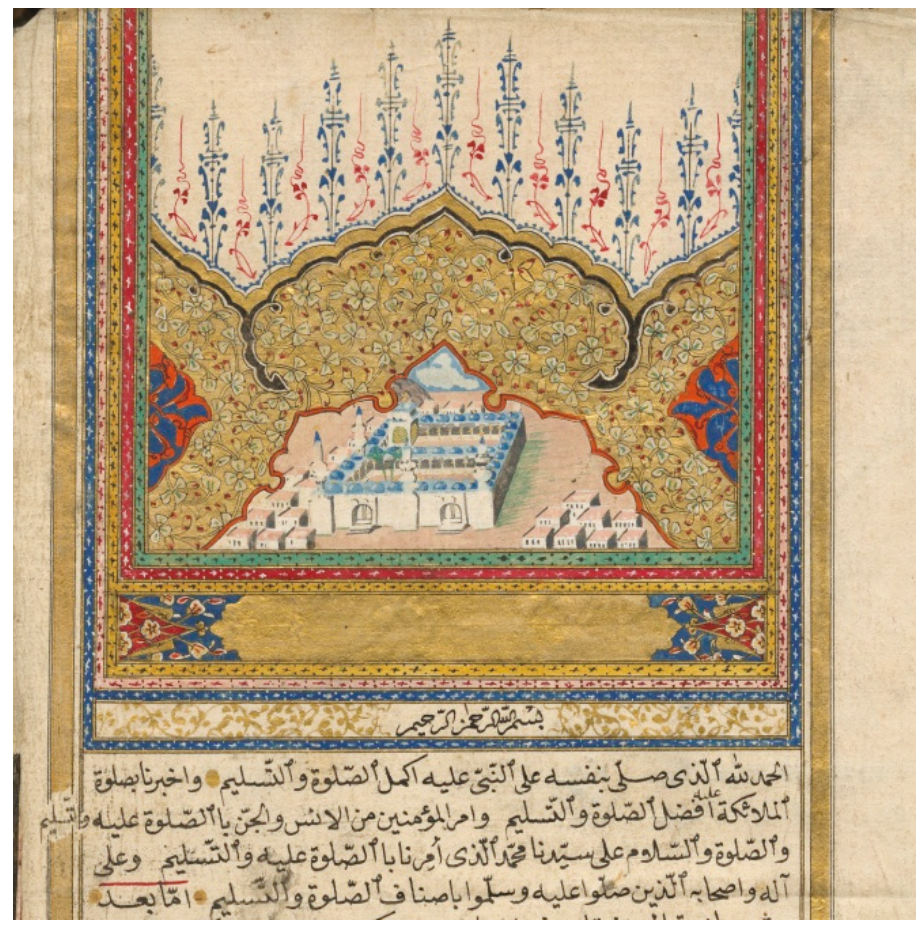

Figure 9. A miniature rendering of Medina surmounts the 'unwān (illustrated headspiece) at the beginning of this Ottoman Turkish translation and expansion of al-Fāsī's commentary on the Dalä'il. Ann Arbor, University of Michigan, Special Collections Library, Isl. Ms. 672, fol.1a. 


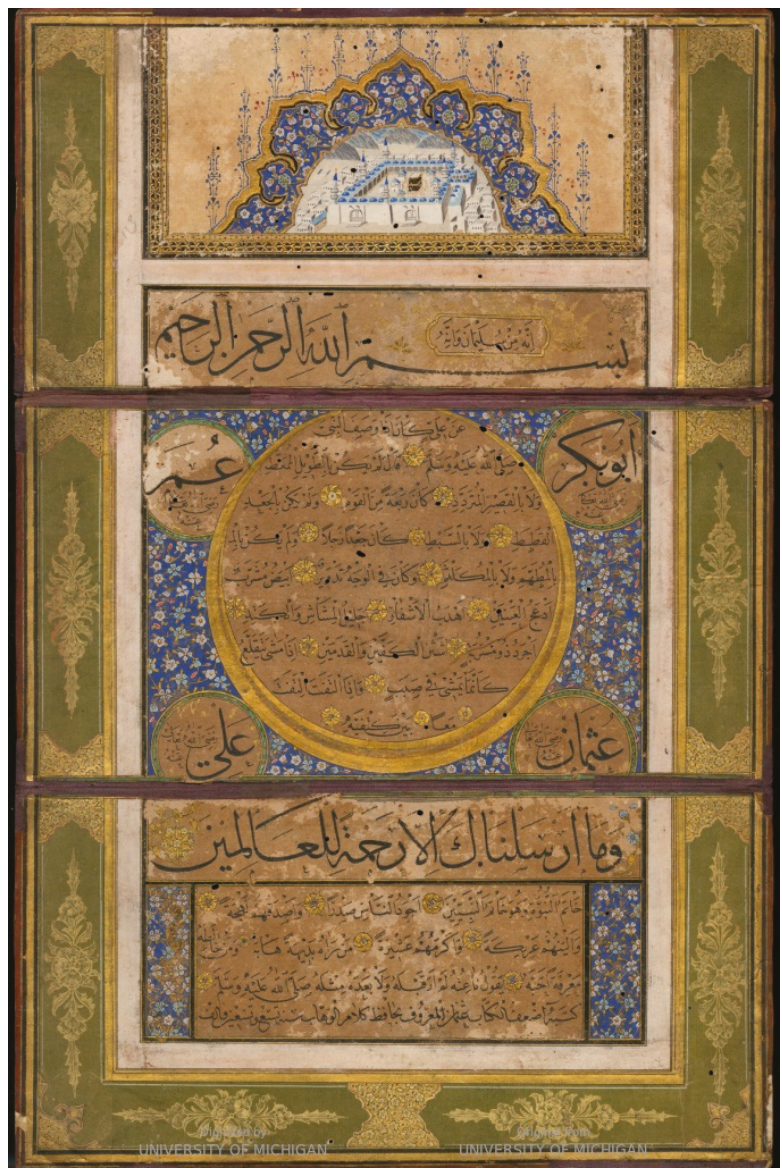

Figure 10. This Hāfiz 'Osmān hilye received in the mid-eighteenth century an illumined headpiece with an image of Mecca, and, at some point in the nineteenth, the green-and-gold border; signs of considerable use and display and ensuing re-mountings are evident across the piece. Ann Arbor, University of Michigan, Special Collections Library, Isl. Ms. 238.

Unlike medieval images of Mecca and Medina or the iconic depictions of the 'relics' of Muhammad, the hilye appears to have been geared towards domestic contexts from the beginning, reflective of the inceasingly domestic context of devotion to Muhammad by the end of the seventeenth century. The following 'hadith' (it is almost certainly an early modern pious fabrication), taken from a mostly Turkish book-form compilation on the Prophet's physical characteristics (Dervīş Ibrahīm 1716/17), suggests both the domestic context and the perceived power of these texts:

There is none who writes out this my [Muhammad's] description, then arranges (yaṣna'uhā) it within their house $(d \bar{a} r)$ except that Satan will not draw near their house (bayt), nor will oppressive power, trial, nor pestilence strike that house, nor will sickness, pain, the envy of an envious person, the magic of a magician, flood, fire, theft, feebleness, nor misfortune. And no worry, sadness, nor anxiety will afflict them, so long as this blessed, beneficious description is in that place (manzil), house, or palace (qașr) wherein they dwell. And whoever recites it or listens to it, it is for them the proof and profound reliance which the Prophet, peace and blessings be upon him, vouchsafes. 


\section{(Hilye-ye Şeriff, pp. 8-12)}

The domestic context is foregrounded here: The hilye protects one's house (whatever sort of house it may be) simply by being there. Like the aural performance of tasliya, it is offered as a means of replicating Muhammad's powerful presence within the walls of one's homes, of opening out domestic space to the eternal presence of the Prophet. Like other examples from the broader genre, this text offers multiple routes to 'activation' of its powers. Whether in textual form such as that in which the above hadith was embedded, or in the calligraphic 'textual icons' popular from the late seventeenth century forward, which could be displayed on one's wall, the hilye acted as a prophylactic for one's home, regardless of whether anyone was looking at it, reading it, or handling it. ${ }^{25}$

The fact that many Arabic-language descriptions of Muhammad were not simply reproduced in Arabic but were also the object of Ottoman Turkish translations, sometimes in a decidedly vernacularizing register, points to a related means whereby these descriptions could activate the Prophetic presence, namely, through the (properly guided) imagination. Closely akin to the practices of 'fixing' the image of one's shaykh in one's heart and thereby establishing an effective spiritual 'link' with him from afar, practices developed by, but not limited, to the Naqshbandī sufi tarīa, descriptions of the Propeht could enable one to 'see' Muhammad by means of the heart (Allen 2019b, pp. 432-34). The reader (or listener) could form his or her own image of the Prophet, which might in turn aid in summoning Muhammad's visit through the medium of the dream-vision. The overlap with the role of tașliya, as disussed above, in summoning the Prophet's presence is obvious enough. Unsurprisingly, by mid-eighteenth century, if not before, descriptions of Muhammad, including entire calligraphic hilyes, had entered into the Dala' il's iconographic repertoire (see Figure 3 for an example) alongside the by then 'canonical' sets of image; at the same time, 'freestanding' hilyes themselves also often took on aspects of the Dalā'il's visual scheme, such as the eighteenth century versions printed using brass matrices, a form of the device that would have been particularly suited to domestic use up and down the social ladder (Perk 2010, pp. 36-37). ${ }^{26}$

The devotional images of the Dala' $i$ - from those of the Rawda to the full-fledged depictions of Mecca and Medina to embedded hilyes - elicited responses that ranged from the highly tactile to the visual and imaginative. Particularly when placed within the pages of books, these devotional images invited physical, bodily reponses. Reading the written labels (indicating names of mosque and tomb structures, gates, and various other significant sites) of these depictions and imaginatively engaging with them required, as Sabiha Göloğlu has noted, no small degree of bodily interaction simply by virtue of either turning the manuscript or turning one's self in order to literally read the writing and to follow the layout of the image (Göloğlu 2018, pp. 151-56). Most significantly, as Christiane Gruber has discussed in detail, both representational and more abstract depictions of Muhammad were for many early modern Muslims, Ottoman and otherwise, objects of tactile devotion, as they rubbed, kissed, even scraped off paper and pigment as means of activing the baraka contained therein (Gruber 2010; Gruber 2019, pp. 269-85). Yet, while in the neighboring Maghrib the images within the Dala' $i l$ were frequently objects of tactile veneration all through our period, as clearly visible in Figure 11, relatively few Ottoman Dalá'ils show such signs of intense tactile engagement. Significantly, neither do early modern hilyes, whether of the free-standing, wall-mounted sort or of those included in devotional books such as the Dalá $i l^{27}$ Instead, the emphasis appears to have been

25 Yet another devotional innovation from the eighteenth century, the pious hadith-tuğra, which combined the venerable calligraphic emblem of the Ottoman sultan, the tuğra, with short hadith, and which was developed by none other than Sultan Ahmed III, should also be seen as part of this trend towards 'iconic,' domestically suited objects of devotion intended for visual and imaginative participation (Keskiner 2013).

26 Other extant examples of these curious and understudied objects include one held at the Walters Museum of Art, W54.510.

27 In some eighteenth century examples, the neutral space surrounding the images of the holy places in the Dala $\bar{a}^{\prime} i l$ has been filled in with devotional verse, quite possibility replicating, in the inscriber's understanding, the pious wall-writing discussed above, replicating the experience of a public holy place within the confines of an intimately experienced manuscript. In a lovely copy of the Dalā'il completed in 1794, someone-perhaps the scribe or the artist or, more likely, an owner-added 
upon the visual and imagination-facilitating aspect of these images, their power unlocked primarily through the devotee's gaze. Even as tactile forms of veneration and engagment remained popular in other contexts, in the core Ottoman lands, the images of the Dala' $i l$ became primarily objects of visual and imaginative engagement.

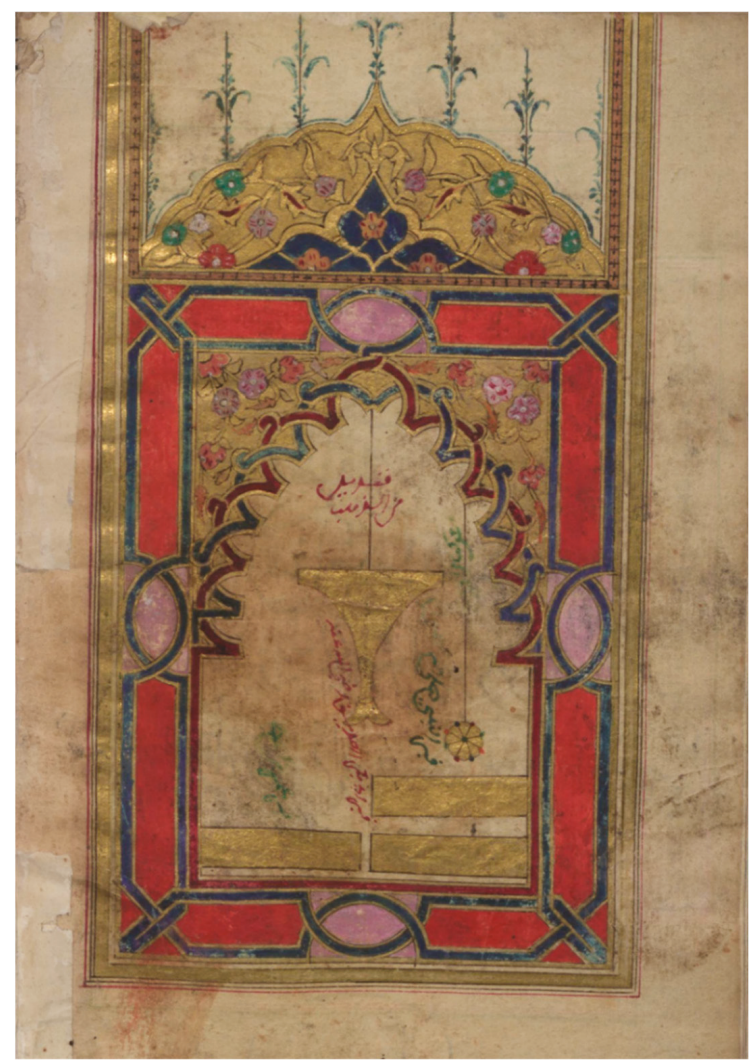

Figure 11. A probably eighteenth century depiction of the Rawda, from a Maghribi manuscript copy of the Dalä'il, demonstrating the highly schematized style that remained popular in North Africa-here reduced to almost pure geometric abstraction-alongside evidence of extensive tactile veneration, all contained within a framing that combines long-standing Maghribi motifs with a headpiece and floral fill highly typical of Ottoman manuscript illumination. University Library of Leipzig, Cod. Arab. 114.

Our commentator al-Fāsī provides a good picture of such overlapping layers of meaning and use these various images could receive as well as the transformations of the early modern period. While himself resident in the Maghrib, al-Fāsī drew upon opinions-concerning the Dala' $i l$ and other, related matters-which he found in writings by (usually anonymous) 'Easterners' (mashäriqa), that is, inhabitants of the Ottoman Arabic-speaking provinces. His discussion of the purpose of the imagery in the Dalā'il begins with a citation from the Mamluk jurist Tāj al-Dīn al-Fākihānī (d. 1331) who, despite his opposition to the emergent practice of mawlid festivities (Katz 2007, pp. 70-71), wrote approvingly that 'one who cannot make pious visitation to the Rawda [itself] may go and piously visit the likeness

Turkish devotional verse in a tiny but neat naskh hand, slanting towards the images. Beinecke Library, Yale University, Arabic MSS suppl. 616, 14r-14v. 
(mithāl) [thereof], witnessing it with desire and kissing it so that love and desire increase in him' (al-Fāsī 1865). He gives as his example, not an image of the tomb itself as we might expect, but the image of Muhammad's sandal-print, one of the oldest such devotional images (Gruber 2019, pp. 276-85).

In the passage cited from al-Fākihānī, reflecting late medieval devotional culture before the Dalā'il, two major differences stand out: One, devotees are envisioned going to visit a pious image, the verb used normally referring to pious visitation to a tomb or other holy place. The imagery is public. Second, the primary mode of participation the passage suggest is the tactile, not the visual and imaginative, which are decidedly secondary. By contrast to the late medieval context visible in the Mamluk jurist's words, the proliferation of the Dalä'il and related texts and objects meant that for many early modern Muslims, no special trip was necessary: Devotional imagery could be brought into one's home. While bodily engagement remained a part of Ottoman devotional repertoires, the visual and imaginative emerged as just as, if not more, important, at least in the context of images of the holy places of the Hijāz. Al-Fāsī goes on to provide his own explanation for why images are included in the Dala' $i l$ : First, he notes that the Dalä'il acts as compendium uniting all the various ways Muslims had sought to describe Muhammad, from sìra literature to accounts of his miracles to descriptions of his physical characteristics (sham $\left.\bar{a}^{\prime} i l\right)$. Depicting his tomb-and by extension, other places closely associated with his life- 'is among the things connected with that.'

Al-Fāsī goes on to discuss, drawing upon anonymous contemporaries (in all probability from the Ottoman world) the practice of imaginatively summoning up the image of Muhammad himself. The devotee should picture 'before his eyes' Muhammad in his humanity, clothed in divine light, so that the devotee can 'stamp his image, God bless him and give him peace, within his spiritual self (rūhānīya) and become intimately acquainted with [that image],' to great spiritual benefit. In order to strengthen this practice, one might also picture one's self gazing at Muhammad while sitting-in one's mental picturing-before 'his blessed tomb,' an image that the devotee can keep constantly before himself and which will spur him to greater commitment and contact with the Prophet. However, what if the devotee has never been to Medina and does not know what the Rawda and Muhammad's tomb looks like? Then, he can gaze upon the images within the Dalā'il, al-Fāsī writes, and having familiarized himself with them use them in his practice of visualization. Such images are especially useful for the 'commonality.' However, citing an anonymous 'Easterner,' al-Fāsī also notes that these images can be rendered in gold leaf and brilliant colors as a way of honoring Muhammad and of strengthening visualization practices, a likely reference to the proliferating diversity and complexity of images in the Ottoman world from the seventeenth century forward (al-Fāsī 1865, pp. 137-38).

Here, the stress is on the individual viewer using the images as mental templates for constructing, as it were, the topographic ground for encountering Muhammad, transforming the inner 'space' of one's heart into the sacred space of Mecca and Medina, their significance here having less to do with their role in the hajj and more to do with their connections with Muhammad. If tactile responses were invited by the correspondence of the image on the page (or other medium) with prototype, here the correspondence was of image in the mind and heart with the prototype, the image on the page a means of forming that inner image. Not unlike the role that descriptions and images of Jerusalem could play for medieval and early modern Christians (East and West) in their devotions to Christ, the imaginative landscape of the holy cities mattered most because it facilitated personal desire and encounter (Mecham 2005). All of this devotional practice could take place as easily within the confines of one's home as anywhere else, even in Mecca and Medina themselves, with devotional images providing a potent and, al-Fāsī stresses, widely accessible means of doing so. In his slightly later Ottoman Turkish commentary, Qara Dāvudzāde's explanation tracks largely alongside that of al-Fāsī, though he focuses entirely on the visual and imaginative, as we might expect based on the physical evidence from the Ottoman world. The images of the holy places stir up desire for Muhammad, he writes, for those who cannot visit the holy places in person but who can gaze upon their representations. By bringing the distant holy places home these images provided the potent topography of encounter with the Prophet to men and women for whom physical pilgrimage was 
not a possibility (Qara Dāvudzāde 1750, pp. 241-42). The holy places provide yet another means of facilitating Muhammad's presence for ordinary believers, men and women alike.

\section{Conclusions: Recapitulation and Considering Points of Connectivity}

Looking back over our vista of Ottoman devotional life as it intersected with different iterations of the domestic, it will be helpful to reconsider the linkages between the two realms covered, that of the saint's shrine and of household devotion to Muhammad. First, one striking commonality that I have touched on only in passing is the degree to which both the 'domesticated' space of the shrine and the temporary space of devotion in the domestic household were both accessible, with something like rough equality, to men and women. While our analysis of the space of the shrine only touched on gendered aspects, the free movement of women in and out of shrines, often in conditions of unsegregated mixing, was noted by critics and supporters alike. Not only did this sort of gender mixing heighten the domestic aspect of the shrine in its function as the home of the saint, we may speculate that Ottoman observers themselves may have made sense of such mingling as permitted by the unique nature of the physical vicinity of the saint. In the case of devotion to Muhammad, the importance of women as devotees alongside men is laid out explicitly in the prescriptive literature, with the implication that the kind of devotion represented by the Dalä'il al-khayrät did not require special, communal space but was as easily carried out at home as anywhere else. Such accessibility was not limited to spatial requirements. The ritual material of devotion to Muhammad included much that could be easily memorized, even for those lacking literacy, while the imagery associated with the Dalā'il did not require literacy at all, only some awareness of what was depicted and what one ought to 'do' with that imagery. Also, where the images of the holy places (and things) of the Hijāz, of the 'relics' of Muhammad, and the written descriptions of the Prophet's characterstics had in earlier periods been of much more limited and restricted access, early modern transformations permitted their introduction into household settings and hence at least potentially for women's use. In neither the shrine nor in domestic devotion did 'domesticity' preclude the participation of men, nor ought we to see either as being gendered 'feminine' as opposed to 'masculine.'

Second, one of the most fundamental and direct points of contact between these two regimes of practice lay in whay they 'did' with holy places, and how different sorts of holy sites interacted in Ottoman Muslim imaginations. Whether it was the tomb of Muhammad in far-off Medina or the shrine of a neighborhood saint all but unknown a few neighborhoods over, these holy places served as the powerful topography of personal encounter with holy persons. In both shrine and household devotion, such encounters, the cultivation of presence, and the formation of love in the devotee were foremost, the language and practice of the veneration of the saints ultimately on a continuum with that of devotion to Muhammad. Acts of wall-writing or the depositing of votives ${ }^{28}$ served to anchor an individual's person presence in the 'home' of the holy person (including for those fortunate enough to visit the actual physical tomb of the Prophet in Medina), establishing a connection between the saint's 'household' and the home of the devotee, one often reinforced by regular visits to the saint's shrine. Home and shrine were joined by various circuits, towards shared ends. Devotion to Muhammad drew upon some of those same logics: Where aural, text-based devotion aimed at summoning the presence of Muhammad through effacious words and inward dispositions, the imagery of Mecca and Medina helped to forge links between the distant holy places and the homes (and other physical locations, we should keep in mind, from mosque to madrasa to palace) in which the images were kept and used, both by the mere prophylactic presence of those images and through tactile and, particularly in the core Turkish-speaking Ottoman provinces, visual and imaginative participation. This linking of the home to the holy places in turn went hand-in-hand with the summoning of the Muhammadan presence within

28 Another, related avenue for investigation, with its own deep historical roots and broad global analogues. 
the home. ${ }^{29}$ In a sort of nesting relationship, the tomb-shrine of Muhammad in Medina, so central to devotional life and to the configuration and justification of other shrines, was itself deeply domestic in that it overlay a literal house site, one which became the 'home' of the Prophet's physical remains. 'Artefacts' and 'icons' of Muhammad (and, to a subordinate degree, other major holy figures) as found in the hilye or in other forms worked in a similar way to images of the holy places, whether through tactile or visual engagement, or both. Through this entire complex of devotional practice, domestic space could possess possible transformations and resonances otherwise associated with the space of the mosque, madrasa, or sufi lodge, or the holy places themselves. All of these acts of devotion were deeply sensory, both in practice and in imagination, incorporating hearing, sight, touch, smell, and, though not addressed here, taste as well. Not only were the bodies of devotees marked by devotion, devotees left their traces upon the material stuff of devotion: In our examples, most significantly the walls of shrines and the pages of devotional texts.

Even as the 'domesticated' saint's shrine and regimes of household devotion fed into and referenced one another in constitutive ways, neither unfolded solely within the milieu of Ottoman Muslims or in total isolation from the devotional and cultural lives of others. Not only did the elaboration of shrine visitation and of devotion to Muhammad involve, in ways both closely shared and subtly divergent, both Arabic and Turkish speaking Muslims (and, though not investigated here, other linguistic and cultural communities of Muslims within the empire), it is clear that Ottoman Christians (and, future research might reveal, others outside of 'normative' Ottoman Islam as well) shared in major aspects of these devotional cultures. One particularly striking example is the proskynetaria genre of Greek texts (with almost identical parallels in Armenian), so-called 'guidebooks' to the central holy places of Christianity, but whose text and imagery almost certainly had uses akin to the visual repertoire of the Dalä'il and other Islamic early modern works of devotion, as templates for imaginative 'spiritual' travel and ensuing sacred encounters (Arakelyan 2017). In a 1693 version of the much copied and printed Proskynetarion of Chrysanthus of Bursa, the images of the holy places are rendered in a style very much akin to contemporary and earlier Ottoman Muslim architectural images, reflective of the often intimately shared cultural milieus of differing religious communities and suggestive of similar devotional uses as the imagery of the Dala' $i l$ (Figure 12). While it is beyond the limits of this article to explore in depth, it is notable that just as depictions of Mecca and Medina drawing upon the cavalier perspective (such as seen in Figure 13) prolifered in the eighteenth century, in part driven by the availability of non-Muslim European models, something similar happened with Chrysanthus' Proskynetarion, as seen in Figure 14, which draws much more decidedly upon Western European artistic conventions, though in neither this nor the images of the Dalä'il is it simply a question of outright imitation, but adaptation to internal needs. Did this genre of Christian devotional imagery undergo a similar shift in emphasis from the tactile to the visual and imaginative? How much do these artistic shifts owe to changing devotional styles, and how much did changing devotional styles owe to broader cultural changes? Comparative study of analogous texts and bodies of practice, Muslim and non-Muslim, might help to more satisfyingly answer such questions.

29 It is worth noting here that from a phenomenological perspective, this linkage of domestic space to the Muhammadan presence rich holy places of the Hijāz was rendered all the more 'effective' due to the relative paucity of images of any sort within an Ottoman home; while the eighteenth century would see wall paintings (including paintings of Mecca and Medina, clearly associated with our investigations here) become more common, these would have marked generally well to do households, which would have still remained relatively 'image poor' compared to many other parts of the early modern world. 


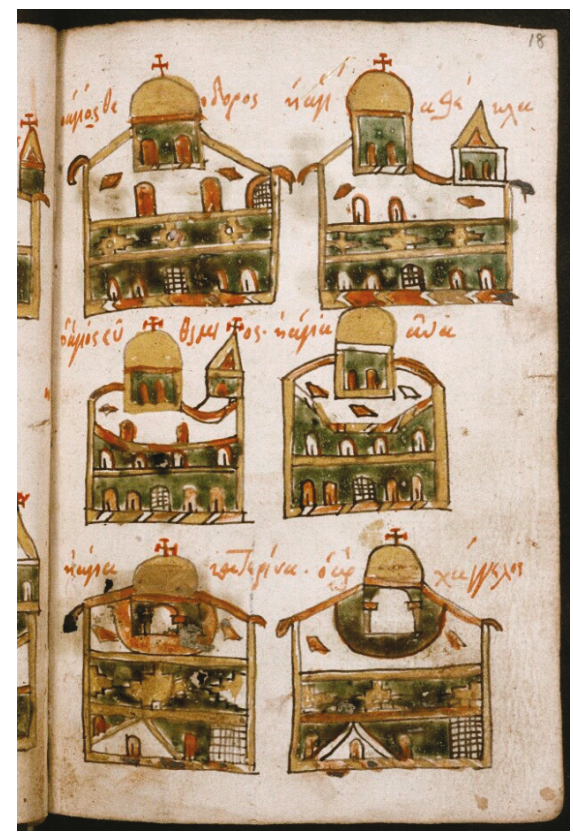

Figure 12. A leaf from a 1693 copy of the Proskynetarion of Chrysanthus of Bursa, showing the relatively schematic style in use at the time, not unlike that in use in many Dalā'il copies contemporary to it. Bodleian Library MS. Canon. Gr. 127, 18r.

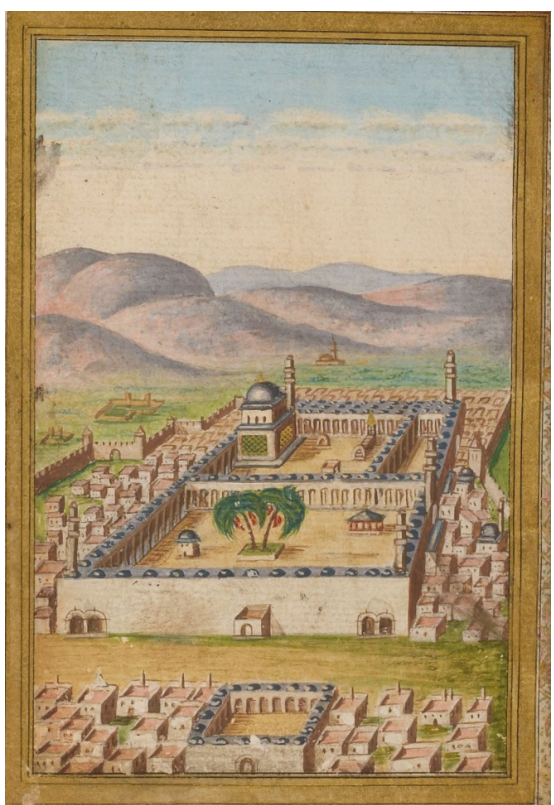

Figure 13. A fine example of the cavalier perspective in depictions of Mecca and (pictured here), Medina, from a 1738-1739 copy by one Muhammad al-Shahrī. Bibliothèque nationale de France, Département des manuscrits, Arabe 6859, f. 22v. 


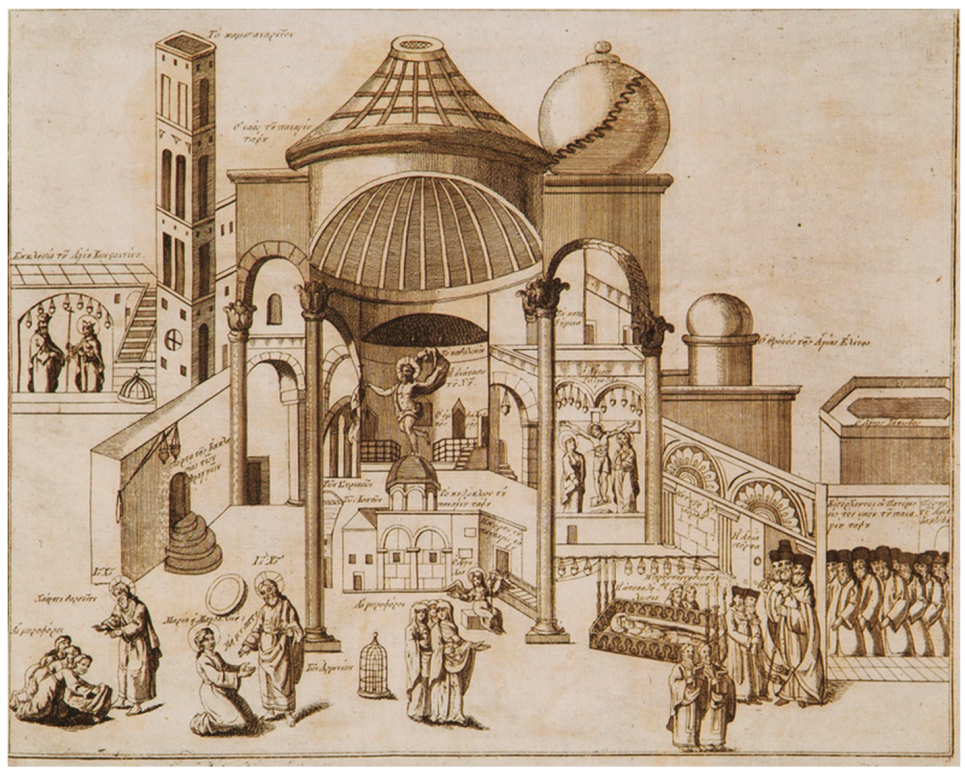

Figure 14. The Church of the Holy Sepulchre, from Chrysanthus of Bursa, Proskynetarion (Vienna, Schrämbl, 1807), showing an adaptation of perspectival view with iconographic scenes interspersed within the architecture.

In their interactions with holy places, Ottoman Christians clearly shared many presuppositions and ways of imagining sacred space with their Muslim neighbors. Pious wall-writing is one of the most visible such commonalities. At the Church of the Holy Sepulchre, one can still see such pious etchings in the great Crusader-era entrance portal, the vast majority of them, whether Armenian, Syriac, Greek, or otherwise, of Ottoman-era provenance (Brock et al. 2007; Tchekhanovets and Tamara 2016); based on my own observations, local Christian communities in and around Jerusalem continued writing prayers and other short texts in the iconography of the main church of the Monastery of the Cross, well into the nineteenth century. Other examples could no doubt be found even with sporadic survivals and the terrible fates visited upon many a holy place over the last century. Shrine wall-writing was not only multi-confessional, but can also be seen in the wider context of public wall writing and, even more broadly, attitudes towards the nature of text and writing itself; there is a clear linkage between the acceptability of wall-writing and the ways in which pre-modern audiences across the world conceived of things like the nature of texts, of authorship, and whether a text was ever truly 'complete' without need of marginalia and commentary unauthorized by an 'original' author. Holy places, while public and communal, did not preclude their being intimate and almost familial in nature-here Ottoman communities of all sorts agreed.

Other routes of contact and commonalities with the rest of the Islamicate world and beyond into Christian Europe could no doubt be discerned with further investigation. For instance, in an English travelogue from 1838, the author Julia Pardoe mentions seeing a fine model of Mecca and Medina within the monumental türbe of Sultan Süleymān, depicted in a drawing by W. H. Barlett included in the volume (Figure 15. Other models of holy places, from Ottoman mosques to Mecca's Ka'ba, are known (Necipoğlu-Kafadar 1986, pp. 237-39) though this one stands out for its size, complexity, and similarity to scale models known in considerable numbers from early modern Europe (Puff 2014, 
pp. 83-124). ${ }^{30}$ That this was a work of devotional art, in dialogue with the images of the Dalä'il and other texts and objects, seems all but certain; but much else is, for now, murky, including its likely relationship to scale models at use for other purposes in Western Europe, or whether smaller models of a devotional nature may have existed in households as well as in shrines and mosques.

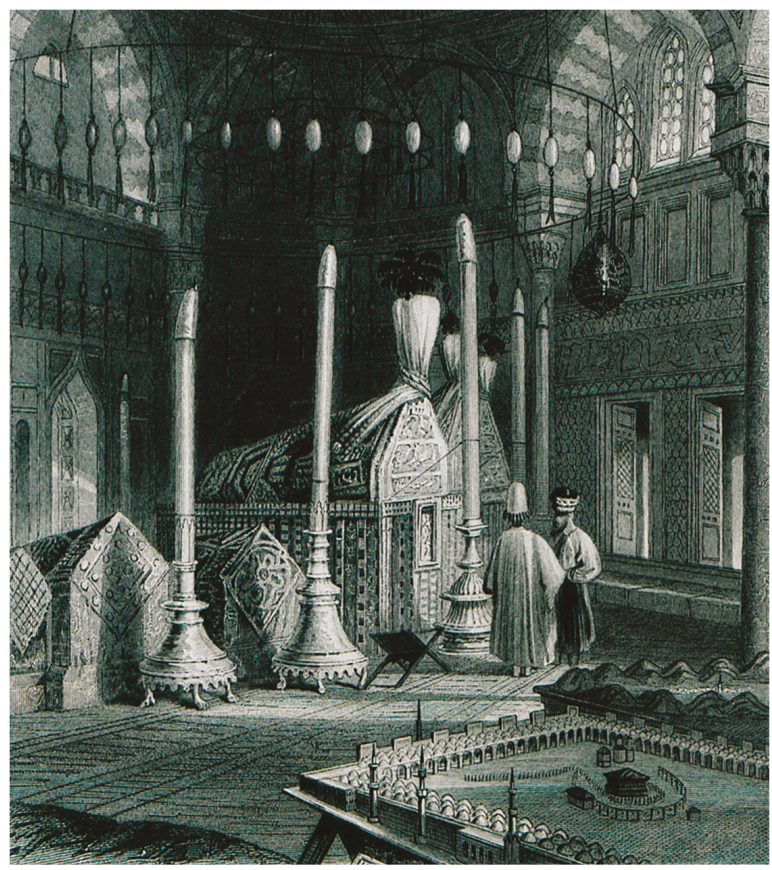

Figure 15. Scale models of Mecca (foreground) and Medina (visible just behind and to the right of the Mecca model) within the türbe of Sultan Süleymān, as depicted in the 1830s. From Julia Pardoe, The Beauties of the Bosphorus; by Miss Pardoe, Author of The City of the Sultan, illustrated in a Series of Views of Constantinople and its Environs, from original Drawings by W.H. Bartlett (London: George Virtue, 1838).

What is clear, from this curious object as from the wider practices of shrine visitation, veneration of Muhammad, and so forth, is the continuing dynamism of Ottoman Islamic devotional culture and the often unexpected connectivities of the global early modern world at levels beyond the merely economic and political. This interconnectivty and dynamism might help to make sense of some of the changes to Ottoman devotional culture, which we have descried here but have not really explained in causative terms: The increased centrality of the visual and imaginative, I have suggested, owed something to other popular 'technics' of devotion, though pointing to such parallel developments only goes so far. A truly satisfying explanation would need to put the developments in devotional culture, domestic, communal, and everywhere in between, in sustained contact with histories of other developments in Ottoman cultural and social life. That the imagery of the Dalä'il and associated texts (and those of non-Muslims) was shaped by artistic and imaginative trends of the Ottoman Baroque is quite likely (Rüstem 2019), but a more precise locating of devotional culture within such wider eighteenth century trends would repay further investigation. Indeed, our exploration of the intersections of

30 Compare also models of the Church of the Holy Sepulchre, and one of the Church of the Nativity, made by seventeenthand eighteenth-century Ottoman Syrian or Palestinian craftsmen: British Museum 1983,0107.1, OA.10338, OA.10339, and SLMisc.151. 
the domestic and the devotional in the early modern Ottoman world has only begun to address the many possibilities - much material remains to be examined, and other routes to be followed, with the promise of uncovering integral, if often overlooked, aspects of everyday life for Ottoman believers and the spaces they shaped and inhabited.

Funding: No external funding was received for the writing of this article.

Acknowledgments: The author would like to thank first of all Salvador Ryan for his great patience and advice in seeing this article through, and my two anomymous reviewers for laboring through my sometimes convoluted prose yet still offering many helpful insights and criticisms, helping me to change tack at some significant points; thanks is also due to, among others, Sabiha Göloğlu, Christiane Gruber, and Ashley Dimmig for discussions and suggestions concerning these topics. I benefited from presenting some of this material to an audience here at the University of Maryland, from whom I received helpful questions and feedback. Thank-you as well to members of Gloria Dei Church in Philadeliphia and Prince George Winyah Church in Georgetown, South Carolina for discussions and insight into the early modern pious (and perhaps not-so-pious!) graffiti in their historic churches.

Conflicts of Interest: The author declares no conflict of interest.

\section{Appendix A}

\section{An Excerpt from the Dalä'il al-khayrāt:}

'O God, I believe in Muhammad though I do not see him, so do not forbid me the sight of him in the Garden! And grant me provision with his companionship, place me in his religious community, give me to drink a fresh, quenching, healthgiving drink form his Basin, no thirsting ever afterwards. Verily you are powerful over everything! O God, cause the spirit of Muhammad to impart to me life and peace! O God, as I believe in him though I do not see him, so do not forbid me the sight of him in the Garden! O God, accept the great intercession of Muhammad, and elevate his exalted degree, and fulfill his request in the Other World - he is the foremost-as you fulfilled for Abraham and Moses. O God, blessings upon Muhammad and the Family of Muhammad, as you blessed Abraham and the family of Abraham, and blessing upon Muhammad and the family of Muhammad, as you blessed Abraham and the family of Abraham. You are praiseworthy and effecious!'

(Dalā'il al-khayrāt wa-shawāriq al-anwār fì dhikr al-ṣalāh 'alá al-Nabī al-mukhtār, al-Jazūlī 1863, pp. 45-46)

\section{References}

\section{Primary Sources}

al-Bakrī, Mușțafá. 2009. Bur' al-isqām isqām fī ziyārat Barzah wa-al-Maqām. Beirut: Markaz Dirāsāt al-Adab al-'Arabī. al-Bakrī, Aḥmad ibn Zayn al-'Ābidīn. 2015. The Merits of the Bakri Lords: An Anthology. Edited by Mustafa Mughazy. Translated by Adam Abdelhamid Sabra. Beirut: Dār al-Mashriq.

al-Bakrī, Mușțafá. 2015. al-Khațra al-thāniyya al-unsiyya lil-rawḍa al-dāniyya al-qudsiyya. Edited by 'Abd al-Raḥmān Muhammad Mughrabī. Ramallah: Jāmi'a al-Quds al-Maftūḥa.

al-Fāsī, Muhammad al-Mahdī ibn Aḥmad. 1865. Mațāli' al-masarrāt bi-jalā' Dalā'il al-khayrāt. Cairo: [no publisher name given].

al-Fāsī, Muhammad al-Mahdī ibn Aḥmad. 1989. Mumti' al-asmā' fī dhikr al-Jazūlī wa al-tabbā' wa mā lahumā min al-atbā.

al-Ghazzī, Muhammad ibn Muhammad. 2012. Intimate Invocations: Al-Ghazzī's Biography of 'Abd al-Ghanī al-Nābulusī (1641-1731). Edited by Samer Akkach. Leiden: Brill.

al-Ișbahānī, Abū al-Faraj. 2000. The Book of Strangers: Mediaeval Arabic Graffiti on the Theme of Nostalgia. Translated by Patricia Crone and Shmuel Moreh. Princeton: Markus Wiener Publishers.

al-Jazūlī, Muhammad ibn Sulaymān. 1863. Dalā'il al-khayrāt wa-shawāriq al-anwār fì dhikr al-ṣalāh 'alá al-Nabī al-mukhtār. Cairo: Al-Mațba'ah al-Kāstalīyah. 
al-Kurdī, Ṭaha ibn Yahyá. 2007. Riḥlat al-shaykh Ṭāhā ibn Yahyá al-Kurdī: Al-tarbīya wa-al-sulūk fī tarājim mashāyikh al-taṣawwuf alladhīn iltaqāhum al-mu'allif fī riḥlatihi al-'ilmīya wa-al-rūḥīya. Edited by 'Alī Najm 'Īsá. Beirut: Dār al-Kitāb al-'Ilmīyah.

al-Nābulusī, 'Abd al-Ghanī. 1979. Hullat al-dhahab al-ibrīz fì riḥlat Ba'labakk wa-al-Biqā̄' al-'azī. In Riḥlatān ilá Lubnān. Edited by Șalāḥ al-Dīn Munajjid and Stefan Wild. Beirut: Dār al-Nashr Frānts Shtāynar.

al-Nābulusī, 'Abd al-Ghanī. 1990. al-Haḍra al-unsīya fì al-rihla al-qudsīya. Edited by Akram Hasan 'Ulābī. Beirut: Al-Maṣādir.

al-Nābulusī, 'Abd al-Ghanī. 1998. al-Haqīqa wa al-mijāz fì rihlat balād al-Shām wa Miṣr wa al-Hijā̄z. Edited by Akram Hasan 'Ulābī. Damascus: Dār al- Ma'rifa.

al-Sha'rānī, 'Abd al-Wahhāb ibn Aḥmad. 2005. al-Ṭabaqāt al-kubrā: Al-musammá Lawāqị al-anwār al-qudsīya fì maāqib al-'ulamā' wa-al-șūfìya. Cairo: Maktabat al-Thaqāfah al- Dīnīya.

Asiye Hatūn. 1994. Rüya mektupları. Edited by Cemal Kafadar. Istanbul: Oğlak.

Dervīş Ibrahīm bin Hafīz Halīl. 1716/17. [Hilye-ye Şerīf]. Ann Arbor: Special Collections Library, University of Michigan, p. 1008.

Evliyâ Çelebi. 1988. Evliyâ Çelebi in Diyarbekir: The Relevant Section of the Seyâhatname. Edited and Translated by Martin van. Bruinessen and Hendrik Boeschoten. Leiden: Brill.

Evliyâ Çelebi. 1996. Evliyâ Çelebi Seyâhatnâmesi. Beyoğlu, İstanbul: Yapı Kredi Yayınları Ltd. Şti.

ibn 'Ațīyah, 'Alwān ibn 'Alī. 2003. Asná al-maqāṣid fī ta'zīim al-masājid. Beirut: Dār al-Kutub al-'Ilmīya.

İbrâhîm Hakk1, Erzurûmlu. 1914. Ma'rifetnâme. Edited by Yusuf Ziya Kırımi. Istanbul: Matbaa-yı Ahmet Kâmil.

İbrâhîm Hâs. 2013. Hasan Ünsî Halvetî ve Menâkıbnâmes. Edited by Mustafa Tatc1. Bağcılar, İstanbul: Kırkambar Kitaplı̆̆ı.

Peçevî, İbrāhīm. 1864-1866. Tarîh-i Peçevî. Istanbul: Matbaa-yi Âmire.

Qara Dāvudzāde, Mẹ̣med Efendi. 1750. Tawfīq muwaffiq al-khayrāt li-nūl al-barikāt fì khidhmat al-sha'ādāt. Ann Arbor: Special Collections Library, University of Michigan, p. 672.

\section{Secondary Sources}

Abid, Hiba. 2017. Un concurrent du Coran en Occident musulman du X e /XVI e à l'aube du XII e /XVIII e siècle: Les Dalā'il al-khayrāt d'al-Jazūlī. Journal of Qur'anic Studies 19: 45-73. [CrossRef]

Aksoy, Şule, and Rachel Milstein. 2000. A Collection of Thirteenth-Century Illustrated Hajj Certificates. In M. Ŭğur Derman Festschrift. Edited by Irvin Cemil Schick. Istanbul: Sabanci Üniversitesi İletişim Merkezi, pp. 101-34.

Allen, Jonathan Parkes. 2019a. Up All Night Out of Love for the Prophet: Devotion, Sanctity, and Ritual Innovation in the Ottoman Arab Lands, 1500-1620. Journal of Islamic Studies 30: 303-37. [CrossRef]

Allen, Jonathan Parkes. 2019b. Self, Space, Society, and Saint in the Well-Protected Domains: A History of Ottoman Saints and Sainthood, 1500-1780. Ph.D. dissertation, University of Maryland, College Park, MD, USA.

Arakelyan, Mikayel. 2017. An Illuminated Armenian Manuscript 'Guidebook' to the Sacred Shrines in the Holy Land from the End of the Seventeenth Century. Études Arméniennes Contemporaines 9: 217-37. [CrossRef]

Aubin-Boltanski, Emma. 2005. Salāh Al-Dīn, un Héros à l'épreuve: Mythe et Pèlerinage en Palestine. Annales. Histoire, Sciences Sociales 60: 91-107. [CrossRef]

Aubin-Boltanski, Emma. 2013. Objectiver une Présence Sainte: Le Cas de la Tombe de Moïse en Palestine. Archives de Sciences Sociales Des Religions 58: 201-19. [CrossRef]

Blecher, Joel. 2013. Hadīth Commentary in the Presence of Students, Patrons, and Rivals: Ibn Hajar and Șahīị al-Bukhārī in Mamluk Cairo. Oriens 41: 261-87. [CrossRef]

Brock, Sebastian, Haim Goldfus, and Aryeh Kofsky. 2007. The Syriac Inscriptions at the Entrance to Holy Sepulchre, Jerusalem. ARAM Periodical 19: 415-38. [CrossRef]

Brown, Peter. 1981. The Cult of the Saints: Its Rise and Function in Latin Christianity. Chicago: University of Chicago Press.

Campo, Juan Eduardo. 1991. The Other Sides of Paradise: Explorations into the Religious Meanings of Domestic Space in Islam. Columbia: University of South Carolina Press.

Chekhab-Abudaya, Mounia, Amélie Couvrat Desvergnes, and David J. Roxburgh. 2016. Sayyid Yusuf's 1433 Pilgrimage Scroll (Ziyārātnāma) in the Collection of the Museum of Islamic Art, Doha. Muqarnas 33: 345-407. [CrossRef] 
Cornell, Vincent J. 1998. Realm of the Saint: Power and Authority in Moroccan Sufism. Austin: University of Texas Press.

De La Puente, Cristina. 1999. The Prayer Upon the Prophet Muhammad (Tasliya): A Manifestation of Islamic Religiosity. Medieval Encounters 5: 121-29. [CrossRef]

Dennerlein, Bettina. 2018. al-Fāsī family. In Encyclopaedia of Islam, THREE. Edited by Kate Fleet, Gudrun Krämer, Denis Matringe, John Nawas and Everett Rowson. Leiden: Brill.

Elias, Jamal J. 2012. Aisha's Cushion: Religious Art, Perception, and Practice in Islam. Cambridge: Harvard University Press.

Faini, Marco, and Alessia Meneghin. 2019. Domestic Devotions in the Early Modern World. Leiden: Brill.

Göloğlu, Sabiha. 2018. Depicting the Holy: Representations of Mecca, Medina and Jerusalem in the Late Ottoman Empire. Ph.D. dissertation, Köç University, Istanbul, Turkey.

Gruber, Christiane. 2010. A Pious Cure-All: The Ottoman Illustrated Prayer Manual in the Lilly Library. In The Islamic Manuscript Tradition: Ten Centuries of Book Arts in Indiana University Collections. Edited by Christiane Gruber. Bloomington: Indiana University Press, pp. 117-51.

Gruber, Christiane. 2014. The Rose of the Prophet: Floral Metaphors in Late Ottoman Devotional Art. In Envisioning Islamic Art and Architecture: Essays in Honor of Renata Holod. Edited by David Roxburgh. Leiden: Brill, pp. 223-49.

Gruber, Christiane. 2019. The Praiseworthy One: The Prophet Muhammad in Islamic Texts and Images. Bloomington: Indiana University Press.

Hanna, Nelly. 2003. In Praise of Books: A Cultural History of Cairo's Middle Class, Sixteenth to the Eighteenth Century. Syracuse: Syracuse University Press.

Katz, Marion H. 2007. The Birth of the Prophet Muhammad: Devotional Piety in Sunni Islam. London and New York: Routledge.

Katz, Marion H. 2019. Commemoration of the Prophet's Birthday as a Domestic Ritual in Fifteenth-and Sixteenth-Century Damascus. In Domestic Devotions in the Early Modern World. Edited by Marco Faini and Alessia Meneghin. Leiden: Brill, pp. 167-81.

Keskiner, Philippe Bora. 2013. Sultan Ahmed III's Hadith-Tughra: United the Word of the Prophet and the Imperial Monogram. İstanbul Araştırmaları Yıllığı 2: 1-15.

Krstic, Tijana. 2011. Contested Conversions to Islam: Naratives of Religious Change in the Early Modern Ottoman Empire. Palo Alto: Stanford University Press.

Maury, Charlotte. 2010. Ottoman Representations of the two Sanctuaries: From Topographical Diagrams to Perspectival Views. In Roads of Arabia: Archaeology and History of the Kingdom of Saudi Arabia. Edited by Ali Ibrahim Al-Ghabban, Béatrice André-Salvini, Françoise Demange, Carine Juvin and Marianna Cotty. Paris: Musée du Louvre, pp. 547-59.

Maury, Charlotte. 2013. Depictions of the Haramayn on Ottoman tiles: Content and Context. In The Hajj: Collected Essays. Edited by Venetia Porter and Liana Saif. London: British Museum, pp. 143-59.

Mecham, June L. 2005. A Northern Jerusalem: Transforming the Spatial Geography of the Convent of Wienhausen. In Defining the Holy: Sacred Space in Medieval and Early Modern Europe. Edited by Andrew Spicer and Sarah Hamilton. Aldershot: Ashgate, pp. 139-60.

Michot, Yaha M. 2015. Sufi Love and Light in Tillo: Ibrāhīm Haqq1 Erẓurūmī (d. 1194/1780). The Muslim World 105: 322-67. [CrossRef]

Milstein, Rachel. 2006. Futuh-i Haramayn: Sizteenth-century illustrations of the Hajj route. In Mamluks and Ottomans: Studies in honour of Michael Winte. Edited by David J. Wasserstein and Ami Ayalon. New York: Routledge Studies in Middle Eastern History, pp. 166-94.

Mulder, Stephennie F. 2014. The Shrines of the 'Alids in Medieval Syria: Sunnis, Shi'is and the Architecture of Coexistence. Edinburgh: Edinburgh University Press.

Necipoğlu-Kafadar, Gülru. 1986. Plans and Models in 15th- and 16th-Century Ottoman Architectural Practice. Journal of the Society of Architectural Historians 45: 224-43.

Padwick, Constance. 1961. Muslim Devotions: A Study of Prayer Manuals in Common Use. London: SPCK. Perk, Halûk. 2010. Osmanlı tılsım mühürleri: Halûk Perk koleksiyonu. Istanbul: Zeytinburnu Belediyesi.

Petersen, Andrew. 2018. Bones of Contention: Muslim Shrines in Palestine. Singapore: Palgrave Macmillan. Puff, Helmut. 2014. Miniature Monuments: Modeling German History. Berlin: De Gruyter. 
Ragazzoli, Chloé. 2018. Scribbling Through History: Graffiti, Places and People from Antiquity to Modernity. London: Bloomsbury Publishing PLC.

Rippen, Andrew. 2012. Tașliya. In Encylcopedia of Islam, 2nd ed. Edited by P. Bearman, Th. Bianquis, C. E. Bosworth, E. van Donzel and W. P. Heinrichs. Leiden: Brill.

Rogers, J. M. 2010. The Arts of Islam: Masterpieces from the Khalili Collection. London: Thames \& Hudson.

Rusli, Nurul Iman, Heba Nayel Barakat, and Amira Salleh. 2016. Dala'il al-Khayrat: Prayer Manuscripts from the 16th-19th Centuries. Kuala Lumpur: Islamic Arts Museum Malaysia.

Rüstem, Ünver. 2019. Ottoman Baroque: The Architectural Refashioning of Eighteenth-Century Istanbul. Princeton: Princeton University Press.

Sabra, Adam. 2006. Illiterate Sufis and Learned Artisans: The Circle of 'Abd al-Wahhab al- Sha'rani. In Le développement du soufisme en Égypte à l'époque mamlouke. Edited by Richard J. McGregor and Adam Sabra. Cairo: Institut français d'archéologie orientale, pp. 153-68.

Sabra, Adam. 2016. Building a Family Shrine in Ottoman Cairo: Five Waqf Endowments by Fādila daughter of Shaykh Muhammad al-Bakrī, 1619-1629. DYNTRAN Working Papers, n 11, April 2016. Available online: https://dyntran.hypotheses.org/1065 (accessed on 27 January 2020).

Şahin, Haşim. 2013. Yahyâ Efendi, Beşiktaşlı. TDV İslâm Ansiklopedisi.

Sirriyeh, Elizabeth. 2005. Sufi visionary of Ottoman Damascus: 'Abd al-Ghan̄̄al-Nābulusī, 1641-1731. New York: Routledge-Curzon.

Sotheby's London. 2015. Abduljalil Ibn Muhammad Ibn Uzum al-Muradi al-Qairawani (d.1553), Tanbih al-Anam fi Mad'h Taha, copied by Al-Hafiz Abdul Rahman, Turkey, Ottoman, dated 1177 AH/1763 AD. In Arts of the Islamic World, 22 April 2015. London: Sotheby's, Lot no. 78.

Stern, Karen B. 2018. Writing on the Wall: Graffiti and the Forgotten Jews of Antiquity. Princeton: Princeton University Press.

Tchekhanovets, Yana, and Pataridze Tamara. 2016. Revisiting the Georgian Inscriptions on the Portal of the Holy Sepulchre Church in Jerusalem. Le Muséon 129: 395-422.

Winter, Michael. 1982. Society and Religion in Early Ottoman Egypt: Studies in the Writings of 'Abd Al-Wahhäb Al-Sha'rānī. New Brunswick: Transaction Books.

Witkam, Jan Just. 2007. The battle of the images: Mecca vs. Medina in the iconography of the manuscripts of al-Jazūlī's Dalā'il al-Khayrāt. In Theoretical Approaches to the Transmission and Edition of Oriental Manuscripts: Proceedings of a Symposium Held in Istanbul, March 28-30, 2001. Edited by Judith Pfeiffer and Manfred Kropp. Würzburg: Ergon in Kommission, pp. 67-82.

Wollina, Torsten. 2019. Between Home and Sufi Convent: Devotional Book Use in Early Modern Damascus. In Domestic Devotions in the Early Modern World. Edited by Marco Faini and Alessia Meneghin. Leiden: Brill, pp. 147-66.

Yürekli, Zeynep. 2012. Architecture and Hagiography in the Ottoman Empire the Politics of Bektashi Shrines in the Classical Age. Farnham: Ashgate Publising.

(C) 2020 by the author. Licensee MDPI, Basel, Switzerland. This article is an open access article distributed under the terms and conditions of the Creative Commons Attribution (CC BY) license (http://creativecommons.org/licenses/by/4.0/). 
Article

\title{
Devotions in the Ancient Way of Offices: Medieval Domestic Devotion in the Seventeenth Century
}

\author{
Jantina Ellens \\ Department of English, McMaster University, Hamilton, ON L8S 4L8, Canada; ellensjc@mcmaster.ca \\ Received: 2 September 2019; Accepted: 18 September 2019; Published: 23 September 2019
}

\begin{abstract}
Devotions in the Ancient Way of Offices was first published as a Catholic primer for worship between intimates, then reformed for individual Protestant worship, and then reformed again for Protestant worship between intimates. Each adaptation engages the so-called "ancient" quality of its offices, primarily medieval, as authorization for the kinds of domestic worship it promotes. I examine how the author and adapters of the text authorize their creative and adaptive devotional texts through a nostalgic interpretation of medieval worship practices as uniquely representative of the worship practices of the early church. While confessional debates had polarized discussions about the lineage of the church, this text represents a trend in seventeenth-century Protestant devotional primers attempting to reconcile spiritual divisions by re-introducing Protestant believers to pre-Reformation practices of domestic devotion.
\end{abstract}

Keywords: medieval; breviary; devotion; recusant; nonjuror; schismatic; women; early modern; restoration

The Protestant response to the Catholic John Austin's popular Devotions in the Ancient Way of Offices first published in 1668 and adapted for Protestant worship in 1686 and 1700, highlights the continuing import of "ancient," i.e., medieval, devotional methods in the seventeenth century. ${ }^{1}$ Austin's text creatively adapts the so-called "ancient way of offices" from the Catholic breviary for a seventeenth-century recusant community, but the model that his text provides for structured worship between intimates held much broader appeal. The text was popular across confessional boundaries and was republished in four more editions before Theophilus Dorrington took it upon himself to reform the text for Protestant worshippers in 1686. Dorrington drastically simplified the offices and published his devotional text as Reform'd Devotions. Dorrington's adaptation was popular, but some objected to his redaction of the interpersonal emphasis of Austin's liturgy. In 1700, Susanna Hopton, with the aid of her editor George Hickes, brought a second reformation of Austin's Devotions to print. Hopton's Devotions in the Ancient Way of Offices returned the text to a near original state by retaining Austin's method and removing only the most blatant references to Catholic beliefs. Hopton's reformation was even more popular than its predecessor.

Hickes authorizes Hopton's adaptation by defending the observance of devotional offices as "an ancient practice of Devotion, which was in use among the Jews and the Primitive Christians, among whom the distinction of Hours for Prayer was not the effect of Superstition, but a rational Institution, in which they agreed as it were by common Inspiration, as the best means of advancing Piety, and Devotion" (Hopton 1700, p. A8v). Hickes makes this defense of the liturgy's ancient history because Dorrington, the text's previous reformer, had removed the liturgical elements of Austin's offices after declaring them to be a Romish innovation that counteracted true devotion. Dorrington's text sought to draw out only the "Sence with what those Rules suggested to be truth" (Dorrington 1686, p. A6v) by

1 (Austin 1668), (Dorrington 1686), (Hopton 1700). 
appealing to "the directions of the Holy Scripture, and the Articles of our Church which are drawn from thence, according to the usual Interpretation made of it by the most pure and primitive Ages of Christianity" (p. A6r). Dorrington believed only the sense of devotion communicated by Austin's text to be inherited from an ancient and True Christianity and rejected almost all other aspects of Austin's text. Ironically, both adaptations claim the authority of ancient and primitive forms of worship as the grounds for their adaptive process but with opposing results. Dorrington rejects the adaptation of pre-Reformation devotional practices for Protestant worship as an attempt to fracture the authority of the established church's weekly worship, but Hickes embraces communal devotion as ideally suited to re-unify an English church split by schism.

Hickes and Dorrington's disagreement over the "ancient" and "primitive" qualities of the faith promoted in the adaptations is a continuation of the argument incited by Austin naming his adaption of the catholic breviary method an "ancient way." The character of the "True," "primitive," or "apostolic" church and its practices were at the heart of disagreements between the Roman and English churches in the early modern period. The early church was understood to be the purest manifestation of the True Church on earth and the Roman church had been believed to be the sole heir of that tradition until the Protestant Reformation. Austin's references to ancient traditions signal an attempt to separate the practice of medieval devotional methods from the stigma of late Catholicism by reconnecting the breviary genre to the ancient church. By naming the observance of devotional offices an "ancient" method, Austin advanced the view that pre-Reformation devotional genres and the communities they fostered were an authoritative expression of universal Christian worship. In the second edition of Austin's Devotions in the Ancient Way of Offices, Austin's friend, John Sergeant, also a well-known advocate for the English recusant community, suggests that Devotions was popular because its ancient method was universally accessible to all believers (Austin 1672). These men attempt to build consensus between Roman Catholic believers and their Protestant adversaries by appealing to a like-minded desire for communities of faith.

Austin's Devotions in the Ancient Way of Offices, its adaptations, and the paratextual links made between them demonstrate an attempt to parse the spiritual heritage of the early church in the devotional practices of the Roman Church for a Protestant audience. The authorization of Devotions in the Ancient Way of Offices for Protestant worship hinges on a discussion of the extent to which medieval faith communities are uniquely representative of a universal Christian church as instituted by the apostles. Dorrington, a strong advocate for the established church, considered the kinds of alternate faith communities supported by Austin to be a threat to the authority of the established church. His adaptation of Austin's "ancient way," Reform'd Devotions, removes all trace of the communitarianism of Austin's liturgy in favor of emphasizing the individual's responsibility to reinforce their attachment to the universal (read, established) church through the performance of set prayers and meditations in private. Dorrington vehemently rejects what he believes to be the blatant Catholicism of Austin's method and believes it utterly separable from its universal "Sence" (Dorrington 1686, p. A6v).

Hickes and Hopton were Protestant like Dorrington but they were also non-jurors who embraced Austin's model for worship between intimates as a means to encourage dissident communities of faith. ${ }^{2}$ Hopton had converted to Catholicism during the interregnum but returned to the church of England in the early 1660s after becoming thoroughly versed in the controversial writings of both Catholics and Protestants. In contrast to Dorrington's adaptation, Hopton's Devotions in the Ancient Way of Offices returns the text to its liturgical roots by restoring its offices to near original state. Her meticulous reformation of Austin's text illustrates her esteem for his method, but her multiple conversions also highlight her vested interest in reconciling with the Church of England's Roman past. She attempts to harmonize her Protestant beliefs with an affinity for pre-Reformation methods by emphasizing an

2 Non-juring clergy were relieved of their livings when they refused to swear the oath to William III and Mary II after James II and VII was deposed, but they often continued to serve independent congregations in the belief that they had been ordained by God. 
alternate history of medieval devotional techniques in which the Roman church is the faulty purveyor of those traditions, not their origin. In doing so, she finds comfort and support for the marginalized faith community of which she is a part. Hickes and Hopton, both staunchly anti-Catholic, become unlikely promoters of pre-Reformation devotional practices out of an affinity for the intimate communities of faith which those practices promote.

While scholarship has often focused on how confessional debates over the lineage of the church fractured its unity, this study explores how the adaptation of the Roman breviary for seventeenth-century worship attempted to reunite the body of Christ by reintroducing believers to pre-Reformation modes of worship. I examine the descriptions of the church's "ancient" and "primitive" character in the works of Austin and his editor, John Sergeant; in Austin's adaptions by Theophilus Dorrington and Susanna Hopton; and in the preface by Hopton's editor, George Hickes, in order to explore how appeals to the authority of the ancient church express which kinds of communities of worship are believed to be good and valid. In this debate over the "ancient" quality of the offices, sides are not drawn according to one's affiliation with the Protestant or Catholic church, but by one's reverence for or aversion to the established church.

John Austin converted to Catholicism some time before 1640 and soon after began agitating for religious toleration (Blom and Blom 2004). In his Christian Moderator series, Austin poses as a member of the Anglican Church considering whether Independents ought to be granted freedom of worship. As if by surprise, the author discovers that his arguments in favor of freedom of conscience can also be extended to Catholics. Warming up to his argument, the speaker wonders "who live more peaceably with their neighbours ... who deal more justly with all men, then they? Who are more constant in their Religion, and more scrupulous in the observances of their Law, then they? Who suffer for their Faith more then they; nay at this time, who besides them?" (Austin 1651, p. 8). He concludes that those who have suffered so much in quiet humility should be allowed to conduct their faith in peace. Austin's Devotions in the Ancient Way of Offices seeks to nurture the faith of a recusant community more directly. The impact of Austin's Counter-Reformation context can be seen in how his text defends the usefulness of the Roman church's ancient heritage by making it new again for contemporary believers. On the one hand, the text conserves the monastic tradition by following the example of the daily office quite closely. The text, over 500 pages in length, provides Matins, Lauds, Vespers, and Compline for each day of the week, as well as for various festivals. Each service is made up of versicles and responses, Bible readings, and metrical hymns, prayers, and psalms; but, as Alison Shell discusses in her chapters "Intimate Worship: John Austin's Devotions in the Ancient Way of Offices" (Shell 2012) and "Seraphic Discourse, Mystical Bodies" (Shell 2015), Austin's use of scripture is distinctly creative. Many of Austin's psalms were "original psalms," in which passages from the Psalms or other areas of scripture intermingle with original reflections (Shell 2015). Austin's original psalms are especially notable for the way that they encourage believers reciting them to a heightened awareness of the performativity of their worship. Summarizing the scholarship of Philip H. Pfatteicher, one of the few scholars to study Austin directly, Shell highlights the impact of the formal structure of Austin's original psalms: "the psalms direct the congregation to recite a biblical text, then articulate the orthodox response to it, and finally adjure the reader to mean what they have just said" (Shell 2015, p. 223). ${ }^{3}$ In this way, Austin's text reveals him to be uniquely aware of the weaknesses of liturgy and guarded against accusations that set forms of worship lend themselves to mindless repetition (Shell 2012, p. 260).

Devotions in the Ancient Way of Offices provides a fascinating reinterpretation of Catholic liturgy given that, only a century before, John Christopherson, Catholic dean of Norwich, had objected to communal liturgy in the vernacular because it made believers negligent in their prayers. ${ }^{4}$ In contrast to this earlier renunciation of communal liturgy, Austin reworks the traditions of the monastic offices

\footnotetext{
See also (Pfatteicher 1968).

See "Common Prayer" in (Targoff 2001).
} 
to nurture the underground communities of Catholic believers through communal worship. The first edition of Devotions in the Ancient Way of Offices opens immediately with "Directions," which provides a template for worship between two believers. Austin writes,

When one says his pray'rs alone, the circumstances are free to be govern'd by his own devotion. But if two say together 'tis convenient they agree on some rules: for which purpose the following are propos'd; yet so as to be alter'd by their own discretion as they please.". (b1r)

He provides ample allowance for believers to contemplate their relationship with God privately by punctuating the directions with the admonition "secretly" or "thus far secretly," but he also discusses the execution of responsive readings and hymn singing. Shell suggests that the directions might have also served to re-educate Catholic believers whose worship was "of necessity, undertaken at a distance from mainstream Catholic tradition" ("Intimate" 269). Austin's "Directions" reveal that much of the focus of his liturgy is on making do. His instructions encourage believers to conduct the liturgy according to their discretion within their private Oratory or some other private or semi-private space and using a liturgy conducted primarily in English. Shell suggests that this form of worship hinges on an understanding of paradoxical spiritual intimacy- "a quasi-monastic ideal which also, pragmatically, made the most of small congregations" ("Intimate" 271). By developing worship practices that supported the development of spiritual intimacy, Austin relies on the timeless qualities of the medieval tradition reworked within a period of persecution to bind together a new generation of faithful believers.

The relevance of Austin's approach was demonstrated by its widespread popularity. A preface to the second edition of Austin's Devotions in the Ancient Way of Offices, written by John Sergeant, reveals that the text had circulated in manuscript for several years before Austin was forced to bring it to press to avoid an adulterated copy going public first. In his preface, Sergeant describes the widespread appeal of the text:

'Tis a Book which Catholiks use with great Devotion and Benefit. The moderate Protestant will find nothing in it he can with reason dislike, nor the passionate Zealot which he can justly traduce. 'Tis the most Substantial part of Divinity render'd usefully Practical. 'Tis the ripe Production of an aredently enflam'd Will exprest by an excellently clear Understanding; yet so, that only the Heart seems to speak and not the Brain. In a word, 'tis the Best Matter deliver'd in the Best Manner. (Austin 1672, p. A2v)

Sergeant's preface makes clear that the text's popularity is not faddish, but longstanding, and well-founded on the quality of its author's devotion, and he highlights the ecumenicism of Austin's devotions as an illustration of its quality. He affirms the possibility of an ancient, universal, Christian experience to promote Catholic devotional practices among Protestant readers.

Sergeant is not just paying lip service to the text's reputation: Devotions in the Ancient Way of Offices was reprinted again in 1684 and 1685 before being reformed by Dorrington and Hopton (among others) whose adaptations of the text also enjoyed significant popularity (Blom 1982; Smith 2010). Later adapters also maintained respect for the devotion that inspired the original work. George Hickes describes Austin as "the divine Author (so I cannot help but call him)" (Hopton 1700, p. A3r) and develops a significant case in defense of the sincerity of Roman Catholic believers' devotion inspired by Austin's devotion. Despite Sergeant's assurances, the recurrence of reformed editions of Devotions in the Ancient Way of Offices makes clear that Austin's Catholicism stained the book's reception among Protestant believers. In the preface to the 1686 reformation of the text, Dorrington argues, "I am justified in the reforming of this Book, and purging out those fore-mention'd Doctrines, by the Authority of our Nation, which did, for the sake of them, a few years ago condemn the Book to a publick burning" (Dorrington 1686, p. A7r). The burning of the book demonstrates that not all Protestants were convinced of its ecumenicism. At least some, including Dorrington, saw Austin's liturgy as a continuing threat to English Protestantism. 
Like Austin, Dorrington authorizes his edition according to ancient practices but flips the ancient and innovative associations of its devotion and liturgy. Dorrington rejects the suggestion that Devotions in the Ancient Way of Offices employs an ancient mode, baldly describing it as "a Book of Devotions dispos'd into the Form and Method of the Roman Breviary" (p. A6r). The problem with the breviary, Dorrington explains, is that it emulates the bewildering mix of old and new doctrines of its church. Describing Austin's text, he writes "therein were the Truths of Christian Religion frequently mixed, as in the Belief of that Church, with those erroneous Doctrines which in latter Ages have been added to Christianity" (p. A6r). Dorrington remedies the breviary's misappropriation of Truth by retaining only those foundational truths that are indisputably Christian. Dorrington qualifies these truths as those which follow "the directions of Holy Scripture, and the Articles of our Church which are drawn from thence, according to the usual Interpretation made of it by the most pure and primitive Ages of Christianity" (p. A6v). Dorrington's repeated contrast of Roman "form and method" with the Truth of the "primitive Ages of Christianity" and the "truly ancient and Apostolick Church" illustrate his conception of the individual devotion of post-Reformation English believers as radically at odds with the corrupted liturgies of the Roman church. For him, medieval church practices like the observance of hours and monastic-like antiphonal readings are Roman innovations that are necessarily separable from the universal Christian devotion of the apostolic tradition, because the universal Christian experience instituted by the early church is a matter of one's heart and mind, not a particular set of practices. This reasoning is apparent in his defense of Reform'd Devotions's ecumenicism as even more universal than the original's. He argues that, "the leaving out those principles renders the book more generally useful, since now it is become so to those of our Church, while they will meet with nothing in it, but what they can assent to: And it may still, if they please, be useful to those of the other Communion, since the peculiar principles, which they have receiv'd are onely left out, and the remaining matter is what all sober Christians may agree in" (p. A9v). Disagreeing with Sergeant, who argued that even the most "passionate Zealot" can disagree with the universal appeal of "Best Matter deliver'd in the Best Manner," Dorrington finds both the matter and manner of Austin's breviary to be problematically Catholic.

Dorrington acknowledges that some might question the possibility of separating universal Christian principles from the Catholic form of Austin's breviary. To them, he responds,

It were a mistake therefore to imagine, that we must needs be beholden to any peculiar or distinct principles, not held by the truly ancient and Apostolick Church, for such a production as this. I think it may appear by the following book, that those principles are not necessary as a Foundation, nor any ways advantageous to the superstructure. (p. A10r)

Dorrington contracts the four offices of matins, lauds, vespers, and compline into two, an office for the morning and one for the evening. He also excises a number of the liturgical elements like the versicles, antiphon, and other responsories. Instead, Dorrington's offices consist of three meditations each, interspersed with hymns and petitions. But even though Dorrington objects to Devotions in the Ancient Way of Offices's use of the offices, he does not deny its stylistic appeal. Dorrington explains that he was motivated to preserve the text because "there was a great deal of it very good Sence, and that comps'd in a very devout strain, and an ingenious style, and mixt with several curious Hymns" (p. A7r); but, ironically, the few elements which he preserves unadulterated from Austin's text, such as Austin's original hymns are, objectively, the most innovative. Shell remarks that "for much of Elizabeth I's reign it was, in practice, easier for an English writer to write secular verse than the wrong kind of religious verse" (Shell 2012, p. 265). ${ }^{5}$ There is some evidence of hymn writing by earlier seventeenth-century writers, but the evidence also suggests that their use was still limited to private singing. Austin's hymns, based on scripture but written in a "highly allusive style" (Shell 2012, p. 266) and written for

5 See also (Shell 1999). 
communal worship, were groundbreaking; but while Dorrington finds the affect of Austin's hymns irresistible, he again resists the hymns' communalism by constraining the use of them to private worship. The result is a text that emphasizes scriptural reading and individual meditation rather than responsive recitation of scripture and communal worship. Describing "Directions in the Use of it," Dorrington emphasizes meditation as the best means to affect devotion. He argues, "To revolve, and over and over consider, and reflect upon some divine Truths, that they may make impression on their minds, and raise them suitable Affections: This tends to make the temper of the mind, and the course of a Mans life conformable to Truth, which is the end every man should aim at in his private Devotions" (pp. A10r-A10v). For Dorrington, communal liturgy distracts believers from the real work of individually conforming one's heart to divine truth. Dorrington's message was well received. Alongside a fifth edition of Austin's Devotions in the Ancient Way of Offices in 1687, Dorrington's Reform'd Devotions enjoyed five editions in the fourteen years between its first publication and 1700, proving that its simplicity held appeal.

However, the second reformation of Austin's Devotions in the Ancient Way of Offices indicates that Dorrington's rejection of communal worship was not as universally acceptable as he had assumed it would be. Dorrington had concluded the justification of his text with the pronouncement, "I thought it was worthy of a Reformation, and as well too good to be thrown away whole, as too bad to be used whole" (p. A7r), but the criticism of his text that appears in George Hickes's preface to Susanna Hopton's edition of Devotions in the Ancient Way of Offices suggests that Dorrington had underestimated the appeal of the medieval methodologies he rejected. Hickes tells of devout readers who,

chose to mark with their Pencils, whatever was amiss in the unreform'd Devotions, that they might use them for their own private Benefit in the Author's own Method, rather than in that of Mr. Dorrington, which in their judgement was not so enflaming, nor fitted for the great and delightful Benefit of mutual and alternate Devotions, for which the divine Author (so I cannot but call him) seemed principally to contrive his Book, though it is no less fit for solitary Devotions, than that of Mr. Dorrington is.

(Hopton 1700, p. A3r)

Where Dorrington had concluded that the communal worship practices of Austin's text were too closely related to Roman Catholic practices and therefore suspect, Hickes champions Catholic adaptation of the ancient practice of set hours of devotion as the fruit of some piety in the Roman church (p. A12r). Hickes counters the concern that set hours of devotion promote superstitious recital of psalms, prayers or hymns by highlighting the ancient wisdom motivating its practice. He argues that he recommends the use of set hours of devotion "to restore the ancient practice of Devotion, which was in use among the Jews and the Primitive Christians, among whom the distinction of Hours for Prayer was not the effect of Superstition, but a rational Institution, in which they agreed as it were by common Inspiration, as the best means of advancing Piety, and Devotion" (p. A8v). He defends choral response or alternate reading of scripture and the antiphons on the same grounds, pointing out that choral response is preferred by the Reformers as well as Austin, according to the tradition of the early church and scripture. He writes, "The Primitive Church had them both from the Synagogue; and there are many Examples of both to be found in the Book of Psalms" (p. A10v). Building upon Austin's discussion of the ancient character of the offices, Hickes returns pre-Reformation devotional practices to Protestant worship by reconstructing them as a rational early Christian institution crafted upon the foundation of scripture.

Austin's counter-Reformation tactics also held appeal for Hickes. In the twenty-first century, Hickes is known as one of the foremost Anglo-Saxon scholars of the early modern period, but he was also a deprived bishop and a prominent non-juror who came to see himself as primarily responsible for the last remnant of the true apostolic church. Though he claimed his linguistic research was bipartisan, his biographer suggests Hickes makes a political statement when he includes a Saxon coronation oath as historical evidence of divinely ordained kingship. Theodor Harmsen argues that 
such a claim would have "invalidated, in his view, Whig historians' arguments about the 'original contract' between the king and his people as a basis of good government, a contract which justified resistance (or even active rebellion) if a king proved a tyrant" (Harmsen 2004). Hickes faced increasing persecution for his political outspokenness in the latter half of his life and, as he became increasingly marginalized, he became more enamored with devotional practices that promoted intimate worship between like-minded believers. He says as much when he praises the "Heaven-like Fellowship of alternate Devotion, to so much advantage as to Religious Societies, of whose Rise and Progress the World hath lately had an Account, by the Reverend Mr. Woodward, Minister of Poplar" (Hopton 1700, p. A4r). Josiah Woodward had published An Account of the Rise and Progress of the Religious Societies in the City of London in which he catalogues a number of societies dedicated to the reformation of manners in restoration London (Woodward 1697; Spurr 2004). As in the recusant communities, the fostering of devotionality within these societies became a form of spiritual problem solving, in which communal liturgy helped to retrain believers who may have become separated from official forms of church governance. For Hickes, the restoration of social worship represented a return to the "best and purest Ages of Christianity;" but alternate worship also resembled "the Worship and Devotions of the great Choire of Saints and Angels in the Church Triumphant, that most glorious Jerusalem, which is above" (p. A3r). In this context, the adaptation of pre-Reformation devotional practices takes on an apocalyptic aura that promised to reinvigorate the English church's failed reformation and usher in a new age of devout worship.

Despite Hickes's investment in the promises held in trust by Devotions in the Ancient Way of Offices's mode of worship, he did not reform the text himself. Devotions in the Ancient Way of Offices was adapted by his friend and sometimes patron, Susanna Hopton. Hickes does not name the text's reformer in the first edition of the book, but does describe her as having "a mighty genius for Divinity; and though never bred in Scholastick Education, yet by Conversation with learned Clergy-Men and reading the best Divinity Books hath attained to a Skill in the Sacred Science, not much inferior to that of the best Divines" (Hopton 1700, p. a4r). Throughout his description, Hickes refers to the reformer as "one who," an awkward construct which becomes more understandable once, years after the publication of Hopton's Devotions in the Ancient Way of Offices, he finally reveals "the reformer" to be a woman. Hickes reveals Hopton's identity in a letter that he appends to A Second Collection of Controversial Letters Relating to the Church of England, and the Church of Rome (Hopton 1710). In the introduction to the letter "written by a Gentlewoman of Quality to a Romish Priest upon her Return from the Church of Rome to the Church of England," Hickes reveals that the author who penned this letter had just passed away and in doing so released him to publish both her letter and her identity as the second reformer of Devotions in the Ancient Way of Offices.

In addition to revealing her identity as Devotion's second reformer, Hopton's letter also provides insight into her approach to the reforming of the Catholic text in the defense of double-conversion which she pens to her former confessor. Hopton had converted to Catholicism some time after the outbreak of the civil wars but became disillusioned and returned to the church of England around 1660. Soon after her conversion, she wrote a letter to her former confessor defending her return to the church of England. In her letter, Hopton harmonizes her second conversion with her first by describing the second as the completion of a sanctifying process initiated by her engagement with Rome but perfected by her return to Protestantism. Francis Dolan's research has demonstrated that women faced significant risk when converting to Catholicism (Dolan 1999). Conversion made her vulnerable to accusations of inconstancy and spiritual promiscuity. A second conversion enhanced the potency of these accusations. These accusations must have been on Hopton's mind when she urges her reader to not "impute my Change to the inconstancy of my Sex" (Hopton 1710, p. 119). In order to prove her constancy, she argues that she, too, has the "highest degree" (p. 119) of contempt for inconstancy and she combats her liability by reinterpreting her first conversion as a misdirection of her commitment to an "ancient Catholick and Apostolick Truth" (p. 119). She argues that she became waylaid because, in a period of "Mists and Clouds of Error" (p. 119), her former confessor took advantage of "the Eclipse 
of the Church of England, and my own Youth; which was too weak to discern her as she is now, and then really was in her self" (p. 119) and he convinced her of the more catholic qualities of the Roman church. She writes,

There you made me believe I should find Unity without Division, Light for Darkness; Truth, even the ancient Catholick and Apostolick Truth, instead of Errors; Certainty and Satisfaction instead of Uncertainty and Doubts; and wholesome Food instead of Poison. And encouraged with these assurances, I entred in the simplicity of my Heart, into the Field of your Church, in which you persuaded me to expect nothing but pure Wheat without Tares. (p. 120)

Hopton's letter highlights, again, the weight attached to the apostolic tradition within discussions about the validity of the Roman and English churches. An examination of Turberville's controversial works makes it clear that he turned her desire for a connection with the ancient church against her. In the subtitle of Turberville's A Manual of Controversies which he dedicates to Hopton, he promises to demonstrate "the Truth of Catholique Religion, By Texts of holy Scripture, Councils of all Ages, Fathers of the first 500 years, Common Sense and reason" (Turberville 1654). The frontispiece also quotes Deuteronomy 32:7, admonishing "Remember the Old days, think upon every Generation: Ask thy Father, and he will declare to thee; thy Elders, and they will tell thee." Turberville clearly appeals to the ancient qualities of the Roman tradition to defend his religion and deploys its significance in his proselytizing.

Unfortunately, upon conversion, Hopton finds herself "greatly disappointed" (Hopton 1710, p. 120). She admonishes Turberville, "I have found Plenty, great Plenty of Tares there, which grow so thick, that in truth they almost choak the good Seed of God's most holy Word" (p. 120). Motivated by her constant commitment to apostolic truth, she eventually rejects the present Roman church as a misbegotten iteration of the universal church based on its failure to deliver on the promises of ancient Catholicism. She argues,

I have done what I could to find out the Doctrines and Practice of the ancient Catholick Church, and have followed her Guidance in understanding the Scriptures, which I believe to contain all Verities necessary for Salvation. And I here declare, and profess that I believe all that the Universal Church taught and believed as Matters of Faith for the first Five Hundred Years; and particularly I believe all that is in the Confession of Faith made by Pope Gregory the First, in which I can find none of the Twelve new Articles that are in the Creed of Pope Pius IV. But I find that the present Church of Rome doth not agree with the Universal Church of God for the first Five or Six Hundred Years. She hath not Antiquity, Universality, and Succession of her side, nor can I believe her to be the Catholick Church. (p. 126)

Hopton's response addresses the highlights of Turberville's Manual point by point, negating its adherence to scripture, then the councils and the fathers of the first five or six hundred years, and all with a clear and reasonable tone. Her letter suggests that in returning to the church of England she has become even more catholic than when she was affiliated with the church of Rome because, now, she has truly aligned herself with the apostolic church, instead of its pretender.

And yet, she must still also account for the many years that she belonged to the Roman faith. Hopton reconciles those convictions to her present spiritual state through an examination of the parable of the weeds. In several of his parables, Jesus figures the gospel as a seed that must be sown in the hearts of believers. True believers are encouraged to demonstrate the flourishing of the seed of the gospel in their hearts through demonstrations of thankfulness that produce the attributes of the fruit of the Spirit: love, joy, peace, patience, kindness, goodness, faithfulness, and self-control. Jesus contrasts the fruitfulness of those who receive the gospel with the infertility of the unfaithful. In the parable of the weeds, Jesus describes a man who sows his fields with good seed. Then, while he sleeps, his enemy sows weeds among the fields. When the servants discover the treachery, they ask their master if they should tear out the weeds but the master declines saying, "Nay, lest while ye go about to gather the 
tares, ye pluck up also with them the wheat" (Matt. 13:29 (KJV)). Instead, he instructs them to allow both to grow until harvest when the instruction will finally be given to the reapers "Gather ye first the tares, and bind them in sheaves to burn them: but gather the wheat into my barn" (Matt. 13:30). During the Reformation, this parable becomes crucial to discussions about the character of the true church: each church insisted that the others were the weeds and worthy to be burned; yet, the parable also confirmed that until the final day of reckoning, the weeds and wheat were intermingled and might be indistinguishable from each other, providing the theoretical justification for considered borrowing between traditions. Hopton applies this justification to her heart: as a passive actor, it is not culpable for the sewing of the weeds, and she is commended for waiting a period of time in order to be able to test between the fruit of the weeds and the wheat. By describing her conversion in terms of a field sewn with weeds and wheat, one that is growing and being harvested, and replanted, Hopton reimagines the process of conversion not as a dramatic change, but as a kind of adaptation and renewal.

We can see this methodology at work in her approach to carefully adapting the Catholic Devotions in the Ancient Way of Offices. The Wren Library at Trinity College, Cambridge UK holds a copy of Austin's Devotions (classmark C.20.33) signed "Susanna Hopton her book." The text is extensively annotated with underlining and marginal comments, as well as square brackets and marginal crosses marking doctrinally-suspect material. Julia Smith suggests that the annotations were made initially for Hopton's private use as they do not always correspond with her published revisions and the text is not fully marked up as a copy-text; however, the latter half does contain instructions for an amanuensis on material to be included or left out, indicating that it served to some extent in the preparation of her text for publication. ${ }^{6}$ Hopton is very light-handed in the editing of the text, but although she returns Austin's voice to the edition, she does not return the text to its catholic roots. Hopton's letter highlights the teaching of transubstantiation as a particularly egregious error in Catholic doctrine and her reform of Devotions in the Ancient Way of Offices reiterates that concern. In a hymn contemplating communion's significance during Thursday Matins, Hopton brackets two verses in Austin's version which reflect on its "mysterious Dictates" (Austin 1672, p. 145). She replaces the verses that meditate on transubstantiation with ones that celebrate how "That what appears in form but Bread, / By Consecration's Holy made" (Hopton 1700, p. 193), but leaves the rest of the verses as she found them. These adaptations fit her letter's attempt to realign a formerly Roman text with Protestant beliefs through a slight modification of the direction of its "Way." In her letter, she defends her constancy:

No, I humbly thank my God, my love to that hath always been constant, though I have varyed in the Opinion of Things that I thought best secured my eternal Happiness. Heaven was always the mark I ever aimed at; and though through mistake of the wrong for the right Way, I have for some time gone astray, yet my Heart was ever fixed there, and in the love and search of Truth. (Hopton 1710, p. 119)

For Hopton, the mistake of Catholicism comes in the varying of "Opinion of Things" that misdirects one's good love of truth. Reconciliation occurs through the appropriate redirection of the heart's way.

That Hopton cannot believe her Catholic devotion to be at fault becomes apparent in her support for the ancient and apostolic virtues of communal devotion. Hopton maintained an extensive network of theological correspondence in which she advocates for the support of intimate devotional communities. The entry for Susanna Hopton in Orlando: Women's Writing in the British Isles from the Beginnings to the Present describes an undated letter "sent by Mrs Hopton in her own name to her Bro[the]r Mr Geers a Serjeant at Law," which survives in a collection of Nonjuring letters as Bodleian MS Eng. hist. b. 2, ff. 176-80 (Brown et al. 2006). In the letter, Hopton takes Thomas Geers to task for failing to stay loyal to the deprived nonjuring community in England. She had sent Geers a copy of John Kettlewell's

6 See (Smith 2010). 
Of Christian Communion, to be kept on in the unity of Christs Church, and among the professors of truth and holiness fitted for persecuted or divided or corrupt states of churches when they are either born down by secular persecutions or broken with schisms or defiled with sinful offices and ministrations (Kettlewell 1693) which discusses the responsibilities of the adherents of the true church to preserve the true church by continuing to worship together rather than participating in schismatic worship. Kettlewell defends his use of apostolic precedent to support his arguments, suggesting, "For we all know, that our Holy Religion doth not begin with us, and that we are not the First Christians, but only their Successors, and that too at a great Distance. We all profess to be their Followers, and should think we have best provided for our own Safety, when we have taken the Way to be found in their Company" (Kettlewell 1693, p. A3v). Geers disagreed with Kettlewell and Hopton, but Hopton's continued advocacy on behalf of the nonjuring community and the spiritual significance of their worship communities, in addition to her reformation of a work of communal devotion, highlights her investment in domestic worship practices as a means to sustain a remnant of the apostolic church in a time of schism. ${ }^{7}$ In this way, she relies on pre-Reformation devotional practices to authorize a nonjuring definition of church unity based on their presumed reconstruction of early church practices. Despite the marginality of Hopton's personal beliefs, her text was well received as demonstrated by its widespread use. It became a standard component of the seventy-two-volume library dispatched to parishes by the Trustees for Erecting Parochial Libraries between 1710 and 1713 (Smith 2010). Several of its hymns were excerpted in collections like John Wesley's Collection of Psalms (Blom 1982). Hopton was also valorized in several collections of notable women in the eighteenth and nineteenth centuries (Smith 2010). That her text was so popular reinforces the authoritativeness of Austin's adaptation of the breviary and its interpretation of ancient faith communities and the pertinence of Hickes's and Hopton's introduction of it to Protestant believers plagued by schism. Even Dorrington eventually published Family Devotions for Sunday Evenings, in which he provides a series of sermons to be read, not as a replacement for Sunday worship, but as an educational supplement for those invested in the "Recovery of Religion among us" (Dorrington 1693, p. A3v).

In the twentieth century, the attribution of Hopton's texts has come into question (Wade 1932; Ross 2005; Smith 2009), but questions about her authorship do not negate the texts' illumination of the significance of medieval worship practices in seventeenth-century religious debates. These attempts to reclaim early church faith practices through the recuperation of medieval devotional methods in the seventeenth century illuminate another facet through which to understand how faith, politics, and history interact in seventeenth-century confessional debates. After examining the character of "ancient" faith in Austin's text and its adaptations, I began to notice references to the primitive church in many texts at the center of debates about the character of the English church. When John Cosin was commissioned to produce an English Protestant Book of Hours to help sustain the Protestant faith of the ladies of the Carolinian court, he compiled A Collection of Private Devotions (Cosin 1627) based on "the practice of the ancient chvrch, called The Hovres of Prayer. As they were after this maner published by Authoritie of Q. Eliz[abeth] ... Taken Out of the Holy Scriptures, the Ancient Fathers, and the diuine Seruice of our own Church." Likewise, in an address to Charles I, Archbishop Laud defended high church ceremonialism by arguing that the Church of England derives its authority from her perseverance in "Church Government, as it hath beene in use in all Ages, and all Places, where the Church of Christ hath taken any Rooting, both in, and ever since the Apostles Times" (Laud 1639, p. ${ }^{*} 1$ r). In Laud's opinion, high-church forms of governance demonstrate the church's allegiance to the "Ancient Catholike Faith" (p. *1r). Appeals to apostolic tradition also appear in debates between supporters of liturgy and prayer ex tempore. Following the publication of the liturgically lukewarm Directory for Public Worship in $1645^{8}$, Jeremy Taylor, a supporter of the episcopacy, published A Discourse

7 Hickes also published several letters arguing with Geers, who he became acquainted with while Hickes hid from authorities in Hopton's home. See (Hickes 1716).

8 (Parliamentary Subcommittee 1645). 
concerning Prayer Ex Tempore in which he defends liturgy by describing it as an apostolic gift passed down through the ages in order to perfect devotion through daily worship (Taylor 1646). Several years later, in a defense of English liturgy in A Collection of Offices or Forms of Prayer in Cases Ordinary and Extraordinary, Taylor celebrates early English reformers who, in the course of their reforming during the reign of King Edward, "did rather retain something that needed further consideration, then reject any thing that was certainly pious and holy" (Taylor 1657, p. A6v). He continues, "onely in which the Church of Rome had prevaricated against the word of God, or innovated against Apostolic tradition, all that was par'd away" (p. A7v). Taylor's appreciation for early reformers' light-handed approach to removing popish invention from the liturgy suggests a perceived continuity between the apostolic tradition and the practices of the Church of Rome. His invocations of the apostolic tradition indicate an attempt to reclaim those practices from the Roman Catholic tradition by appealing to the perceived timelessness of their methods. Thus, adaptations of Austin's recusant re-introduction of pre-Reformation devotional practices texts follow a tradition of attempting to parse the Christian inheritance in Roman traditions, a tradition which I have only begun to touch on in this article. More research is required. As is obvious from the tangled interactions between church and state governance in the seventeenth century, calls for church unity were not unpolitical, but it is interesting to note that, at least in the adaptations of Devotions in the Ancient Way of Offices, spiritual intimacy was seen as a primary means through which to affect the ancient unity which its adapters nostalgically craved.

Funding: This research was funded in part by a Yates Scholarship and Edna Elizabeth Ross Reeves Scholarship from McMaster University.

Acknowledgments: Many thanks to the staff of the Wren Library, Trinity College, Cambridge University, UK. Thank you, also, to Cathy Grisé for her advice on drafts of this paper.

Conflicts of Interest: The author declares no conflict of interest.

\section{References}

Austin, John. 1651. The Christian Moderator: or, Persecution for Religion Condemned. London: Available online: http://gateway.proquest.com.libaccess.lib.mcmaster.ca/openurl?ctx_ver=Z39.88-2003\&res_id=xri: eebo\&rft_id=xri:eebo:citation:99865774 (accessed on 13 September 2019).

Austin, John. 1668. Devotions in the Ancient Way of Offices. Paris: Available online: http://gateway.proquest.com.libaccess.lib.mcmaster.ca/openurl?ctx_ver=Z39.88-2003\&res_id=xri: eebo\&rft_id=xri:eebo:citation:10140745 (accessed on 20 September 2019).

Austin, John. 1672. Devotions. First Part. In the Antient Way of Offices. Roan: Available online: http://gateway.proquest.com.libaccess.lib.mcmaster.ca/openurl?ctx_ver=Z39.88-2003\&res_id=xri: eebo\&rft_id=xri:eebo:citation:99834091 (accessed on 20 September 2019).

Blom, J. M. 1982. The Post-Tridentine English Primer. Meppel: The Catholic Record Society, pp. 145-49.

Blom, J., and F. Blom. 2004. Austin, John [pseud. William Birchley] (1613-1669), religious writer. In Oxford Dictionary of National Biography. Available online: https://www-oxforddnb-com.libaccess.lib.mcmaster.ca/ view/10.1093/ref:odnb/9780198614128.001.0001/odnb-9780198614128-e-908 (accessed on 14 August 2019).

Brown, Susan, Patricia Clements, and Isobel Grundy, eds. 2006. Susanna Hopton entry. In Orlando: Women's Writing in the British Isles from the Beginnings to the Present. Cambridge: Cambridge University Press, Available online: http://orlando.cambridge.org/public/svPeople?formname=r\&person_id=hoptsu\&heading=c (accessed on 22 March 2018)

Cosin, John. 1627. A Collection of Private Devotions. London: Available online: http://gateway.proquest.com.libaccess. lib.mcmaster.ca/openurl?ctx_ver=Z39.88-2003\&res_id=xri:eebo\&rft_id=xri:eebo:citation:38159601 (accessed on 15 August 2019).

Dolan, Frances. 1999. Whores of Babylon: Catholicism, Gender and Seventeenth-Century Print Culture. Ithaca: Cornell University Press.

Dorrington, Theophilus. 1686. Reform'd Devotions, in Meditations, Hymns, and Petitions. London: Available online: http://gateway.proquest.com.libaccess.lib.mcmaster.ca/openurl?ctx_ver=Z39.88-2003\&res_id=xri: eebo\&rft_id=xri:eebo:citation:690988263 (accessed on 20 September 2019). 
Dorrington, Theophilus. 1693. Family Devotions for Sunday Evenings. London: Available online: http://gateway.proquest.com.libaccess.lib.mcmaster.ca/openurl?ctx_ver=Z39.88-2003\&res_id=xri: eebo\&rft_id=xri:eebo:citation:11759571 (accessed on 20 September 2019).

Harmsen, Theodor. 2004. Hickes, George (1642-1715), bishop of the nonjuring Church of England and antiquary. In Oxford Dictionary of National Biography. Available online: https://www-oxforddnb-com.libaccess.lib. mcmaster.ca/view/10.1093/ref:odnb/9780198614128.001.0001/odnb-9780198614128-e-13203 (accessed on 6 August 2019).

Hickes, George. 1716. The Constitution of the Catholick Church, and the Nature and Consequences of Schism. London: Available online: http://find.gale.com.libaccess.lib.mcmaster.ca/ecco/infomark.do?\&source= gale\&prodId=ECCO\&userGroupName=ocul_mcmaster\&tabID=T001\&docId=CW120785821\&type= multipage\&contentSet=ECCOArticles\&version=1.0\&docLevel=FASCIMILE (accessed on 13 September 2019).

Hopton, Susanna. 1700. Devotions in the Ancient Way of Offices. Edited by George Hickes. London: Available online: http://gateway.proquest.com.libaccess.lib.mcmaster.ca/openurl?ctx_ver=Z39.88-2003\&res_id=xri: eebo\&rft_id=xri:eebo:citation:13705144 (accessed on 11 August 2019).

Hopton, Susanna. 1710. A Letter written by a Gentlewoman of Quality to a Romish Priest upon her Return from the Church of Rome to the Church of England. In A Second Collection of Controversial Letters Relating to the Church of England, and the Church of Rome. Edited by George Hickes. London: pp. 118-52. Available online: http://find.gale.com.libaccess.lib.mcmaster.ca/ecco/infomark.do?\&source=gale\&prodId=ECCO\& userGroupName=ocul_mcmaster\&tabID=T001\&docId=CW117534321\&type=multipage \&contentSet $=$ ECCOArticles\&version=1.0\&docLevel=FASCIMILE (accessed on 20 September 2019).

Kettlewell, John. 1693. Of Christian Communion to Be Kept on in the Unity of Christs Church among the Professors of Truth and Holiness. London: Available online: http://gateway.proquest.com.libaccess.lib.mcmaster.ca/openurl?ctx_ ver=Z39.88-2003\&res_id=xri:eebo\&rft_id=xri:eebo:citation:9863663 (accessed on 7 August 2019).

Laud, William. 1639. A Relation of the Conference, between William Lawd, then, Lrd. Bishop of St. Davids; now, Lord Arch-Bishop of Canterbury: and Mr. Fisher the Jesuite by the Command of King James of ever Blessed Memorie. With an Answer to Such Exceptions as A. C. Takes against it. London: Available online: http://gateway.proquest.com.libaccess.lib.mcmaster.ca/openurl?ctx_ver=Z39.88-2003\&res_id=xri: eebo\&rft_id=xri:eebo:citation:99848401 (accessed on 17 August 2019).

Parliamentary Subcommittee. 1645. A Directory for Publique Worship of God throughout the Three Kingdoms of England, Scotland, and Ireland Together with an Ordinance of Parliament for the Taking Away of the Book of Common-Prayer, and for Establishing and Observing of This Present Directory throughout the Kingdom of England and Dominion of Wales. London: Available online: http://gateway.proquest.com.libaccess.lib.mcmaster.ca/openurl?ctx_ver= Z39.88-2003\&res_id=xri:eebo\&rft_id=xri:eebo:citation:9527387 (accessed on 20 September 2019).

Pfatteicher, Philip H. 1968. John Austin: A Liturgist Worth Remembering. Worship 42: 299-302.

Ross, Jan. 2005. General Preface. In The Works of Thomas Traherne. Inducements to Retirednes; A Sober View of Dr Twisses, His Considerations; Seeds of Eternity, or, The Nature of the Soul; The kingdom of God. Cambridge: D. S. Brewer, vol. 1, pp. ix-liv.

Shell, Alison. 1999. Catholic Poetics and the Protestant canon. In Catholicism, Controversy and the English Literary Imagination, 1558-1660. Cambridge: Cambridge University Press, pp. 56-104.

Shell, Alison. 2012. Intimate Worship: John Austin's Devotions in the Ancient Way of Offices. In Private and Domestic Devotions in Early Modern Britain. Edited by Jessica Martin and Alec Ryrie. Farnham: Ashgate, pp. 259-80.

Shell, Alison. 2015. Seraphic Discourse, Mystical Bodies: John Austin's Original Psalms. In Mysticism and Reform, 1400-1750. Edited by Sara S. Poor and Nigel Smith. Notre Dame: University of Notre Dame Press, pp. 220-40. Smith, Julia J., ed. 2009. Thomas Traherne: Select Meditations. Manchester: Carcanet Press.

Smith, Julia J., ed. 2010. Introductory Note. In Susanna Hopton, I and II. Farnham: Ashgate, pp. ix-xxiii.

Spurr, John. 2004. Woodward, Josiah (1657-1712), Church of England clergyman and moral reformer. In Oxford Dictionary of National Biography. Available online: https://www-oxforddnb-com.libaccess.lib.mcmaster.ca/ view/10.1093/ref:odnb/9780198614128.001.0001/odnb-9780198614128-e-55600 (accessed on 15 August 2019).

Targoff, Ramie. 2001. Common Prayer. In Common Prayer: The Language of Public Devotion in Early Modern England. Chicago: University of Chicago Press, pp. 14-35. 
Taylor, Jeremy. 1646. A Discourse Concerning Prayer Ex Tempore, or, by Pretense of the Spirit. In Justification of Authorized and Set-Formes of Lyturgie. Available online: http://gateway.proquest.com.libaccess.lib.mcmaster. ca/openurl?ctx_ver=Z39.88-2003\&res_id=xri:eebo\&rft_id=xri:eebo:citation:99861779 (accessed on 19 August 2019).

Taylor, Jeremy. 1657. A Collection of Offices or Forms of Prayer in Cases Ordinary and Extraordinary. London: Available online: http://gateway.proquest.com.libaccess.lib.mcmaster.ca/openurl?ctx_ver=Z39.88-2003\&res_id=xri: eebo\&rft_id=xri:eebo:citation:99863582 (accessed on 20 August 2019).

Turberville, Henry. 1654. A Manuel of Controversies. Doway: Available online: http://gateway.proquest. com.libaccess.lib.mcmaster.ca/openurl?ctx_ver=Z39.88-2003\&res_id=xri:eebo\&rft_id=xri:eebo:citation: 43077723 (accessed on 17 August 2019).

Wade, G. I. 1932. Preface. In The Poeticall Works of Thomas Traherne. London: Dobell.

Woodward, Josiah. 1697. An Earnest Admonition to All, but Especially to Young Persons. London: Available online: http://gateway.proquest.com.libaccess.lib.mcmaster.ca/openurl?ctx_ver=Z39.88-2003\&res_id=xri: eebo\&rft_id=xri:eebo:citation:99895372 (accessed on 21 August 2019).

(C) 2019 by the author. Licensee MDPI, Basel, Switzerland. This article is an open access article distributed under the terms and conditions of the Creative Commons Attribution (CC BY) license (http://creativecommons.org/licenses/by/4.0/). 


\title{
Article \\ The Saint in the Woods: Semi-Domestic Shrines in Rural Sweden, c. 1500-1800
}

\author{
Terese Zachrisson \\ Department of Historical Studies, University of Gothenburg, 40530 Gothenburg, Sweden; \\ terese.zachrisson@gu.se
}

Received: 9 May 2019; Accepted: 8 June 2019; Published: 17 June 2019

\begin{abstract}
In the seventeenth century, a common saying in parts of rural Sweden when discussing someone lacking in piety was that they went to neither church nor cross. This reflects the practice of placing shrines in the fields, along the roads and in the woods as a communal semi-domestic complement to official church space. In the remote woodland areas of Sweden, the distance between parish churches could be considerable, and many parishioners were not able to attend church on a regular, weekly basis. At these sites, parishioners could kneel and make their prayers as a complement to church service. However, they could also be used as points of contact in communicating domestic issues with the divine, with votives being left at the shrines by those hoping for deliverance from disease and difficult childbirths. In the post-reformation period, such sites were regarded with suspicion by the higher ranks of the clergy, and were often considered "idolatrous" and "superstitious". Yet, they seem to have filled an important religious need among their laity that made it possible to interact with the divine on sites bordering the domestic and the public space of the church.
\end{abstract}

Keywords: sacred space; materiality; popular piety; lived religion; Lutheranism

\section{Introduction}

[ ... ] In Pelarne parish, fourteen or fifteen years ago, there was a large wooden cross on the lands of Hult, along the road to Fastnefall, now downfallen; and it is said that formerly, the old and the sick have gone there, fallen to their knees and held their worship. (Stahre 1992, p. 184)

The statement above was written by a vicar in Småland in eastern Sweden in 1667 in a report to the College of Antiquities. Mentions of such sites are not uncommon from early modern Sweden. The vicar mentions that the cross was primarily used by "the old and the sick", which implies that the cross was a form of semi-domestic alternative or complement to regular church attendance. In many parts of rural Sweden, the distance to one's parish church could be considerable and roads were often in a poor state, which limited the ability to attend service regularly-and these difficulties doubtlessly increased for those in old age and failing health (Kuha 2015, pp. 19, 22-23). To fulfil their religious needs, the laity could invest other sites than official church space with sacredness, sites that were convenient in their closeness to home while still maintaining something of the otherness of the holy.

Space offers an excellent opportunity to investigate religious cultures and in recent years, scholarly interest in spatial aspects of religious thought and practice have greatly increased (notable works include Alexandra Walsham, 'The Reformation of the Landscape', 2011 (Walsham 2011); Andrew Spicer and Will Coster (eds.), 'Sacred Space in Early Modern Europe', 2005 (Coster and Spicer 2005)). In general, though, the focus of scholars has been the "grand" spaces-towns, cathedrals and Western and Central Europe-while the rural village chapels of Eastern and Northern Europe have received less attention. The focus of this article is on these "small" spaces: devotional sites created and maintained by the laity in rural areas of Sweden. The study is carried out mainly through a close 
reading of seventeenth-century acts from the College of Antiquities, early modern topographical works and visitation records. In cases where the objects of these shrines have been preserved, the objects themselves will also be used as a source of analysis.

Most commonly, these shrines consisted of a simple wooden cross, but could also include saint's sculptures and small chapels. They could be found in a variety of settings: on farmland properties, in the woods, on churchyards and crossroads. They were also often found in close proximity to holy wells. Holy wells and healing springs are the perhaps most well-known and subsequently the most researched example of landscape sites that bordered on domesticity. The overshadowing emphasis on miracles and healing makes the character of the visits to holy wells somewhat different from that of crosses, sculptures and other small shrines in the landscape, though this difference is by no means absolute-holy wells could be visited for prayer and crosses and images could be visited for healing as well as the reverse (Ray 2014; Christian 1989, pp. 93-97). The wells and springs themselves will, however, not be the focus of this particular piece, though they will be discussed on occasion in relation to the manmade shrines erected on its grounds.

In multi-religious areas, such as the Holy Roman Empire, the presence or absence of crucifixes, shrines and images could function as a way of claiming and confessionalising landscapes and public space (Freist 2009, pp. 214-15; Louthan 2005, pp. 286-96). This was likely not the case in early modern Sweden, which, with the exception of the easternmost part of Finland, did not border any areas where other faiths were commonplace. Thus, roadside shrines probably did not explicitly signal Catholicism in the same manner as these sites did in continental Europe, which could be a reason behind the viability of the custom of constructing and maintaining such shrines even in the post-reformation period.

The timeframe of the following study is deliberately kept elastic. This is due to the fact that many of the sources discussing the sites in question are vague in their descriptions of when a certain practice occurred or when an object was in place. Furthermore, even when specified, in certain cases there might be reason to suspect that these timeframes have been modified to suit the individual author's purposes. This could be done as a rhetorical way of placing fairly recent practices in a distant "pagan" past, thus emphasising the author's perception of pre-reformation religion as non-Christian. Timeframes could likely also be altered or kept vague for a different reason. To a vicar, admitting that illicit practices were continuously occurring in his parish might pose a risk in calling his office into question, and it could thus be safer to discuss questionable practices in the past tense. Geographically, this article investigates these practices in the Kingdom of Sweden as of its borders at the end of the seventeenth century. This included Sweden proper, as well as Finland and the provinces of Jämtland, Scania, Härjedalen Bohuslän, Halland and Blekinge, which had been acquired from Denmark and Norway earlier in the 1600s.

Religious traditions in the Scandinavian Middle Ages differed little from the beliefs and practices of other parts of Northern Europe (Laugerud 2018, pp. 56-58), where we are more well-informed on the complex web of natural and manmade access-points to the sacred that characterised the religious landscape. Small prayer houses, crosses, sculptures and other shrines were scattered across Europe, fulfilling a variety of religious needs-most notably penance and healing. In remote areas where parishes were large, priests few and the churches far between, local holy sites may have been of greater importance than regular church attendance to these communities (Walsham 2011, pp. 49-50). Because of the general scarcity of sources from medieval Sweden, mentions of these outdoor places of worship are few. When combined with records from other parts of Scandinavia though, a somewhat coherent context may be sketched. For instance, a twelfth-century Icelandic homily mentions such sites, in that it advices the faithful to hastily head to either "church or cross" in the morning, to kneel and sing the Pater Noster. Crosses could be erected by pious laypeople or by the Church, and in the former cases the crosses could be blessed by the local bishop. Their primal function was probably that of private worship for local residents and travellers, especially pilgrims passing on their way to the tomb of St. Olaf in Nidaros. Some were erected in the woods or along roads while others were placed in small chapel-like structures (Gardell 1931, pp. 4-7). Though the medieval Church initiated, maintained and 
promoted many shrines in the landscape, some could still be met with suspicion and disapproval. When local cults initiated by the laity could not easily be brought under clerical control, such sites were often deemed superstitious (Walsham 2011, pp. 66-79). Such non-official sites are largely unknown in a Scandinavian context, but considering the general shortage of preserved pre-reformation sources in the region, there is no reason to take this as proof of the absence of such shrines in the medieval period. There is, however, an important difference between wayside shrines in the medieval period and those in the post-reformation era. Devotions at these sites in the middle ages were usually firmly incorporated in the ecclesiastical sphere. They were visited on certain dates of the liturgical calendar, and sometimes even Mass was celebrated at portable altars in conjunction with these visits (Timmermann 2012, p. 393). Post-reformation shrines, on the other hand, seem to have been kept and used exclusively by lay believers, and as such, they bordered more heavily on the domestic than the public spheres of worship.

Officially, the Kingdom of Sweden broke its ties to the Roman Catholic Church in 1527 and adopted Lutheran teachings. In reality though, changes in ecclesiastical law and liturgy were introduced step-by-step throughout the sixteenth century. During the 1544 'riksdag' in Västerås, several traditional devotional practices, such as the veneration of saints, pilgrimages, masses for the dead and others, were officially abolished (Alin and Hildebrand 1887, pp. 390-91). Among these changes, the banning of pilgrimage was probably the factor that had the greatest consequences for wayside shrines-though pilgrimage in various forms continued to be a part of popular religious repertoires throughout the early modern period (Weikert 2004, p. 151; Malmstedt 2014, p. 103). In the first Lutheran church ordinance, authored by Archbishop Laurentius Petri in 1561 (printed in 1571), Petri criticised the abundance of places of worship, stating that "unnecessary" churches, chapels and altars, had all led to a "great abuse" (Nordström 1872, p. 99). While generally tolerating images in the churches, the ordinance also stressed that all "superfluous" images that were sought by "ignorant folk" were to be "taken off the road" [sv. taghas bortt aff wäghen] (Nordström 1872, p. 101). This has sometimes been interpreted as specifically referring to the outdoor crosses, saint's sculptures and other shrines that were placed along the roads (Piltz 2016, pp. 76-77, 82), but it could also be a figure of speech and thus referring to "abused" images in general.

According to historical scholar Johannes Messenius (1579-1636), Archbishop Laurentius Petri had himself ordered the demolition of a famous miraculous crucifix that was placed at the Well of the Holy Cross [sv. Helga Kors Källa] in the parish of Svinnegarn (Messenius 1703, 98v). However, apart from this story, we have very little evidence on how the process of removing such shrines played out in practice, or how far-reaching such initiatives were in reality. As will be discussed below, many shrines seem to have been preserved-sometimes even in locations where the local clergy surely must have been aware of their existence, such as churchyards and in connection to chapels. For instance, in 1707, the vicar of Särna in the province of Dalarna told the College of Antiquities that there was a moss-grown wooden cross of about two metres of height just outside of Idre parish church. The use of the cross was still in living memory, and parishioners had told the vicar that people previously made their worship there, "crawling on their hands and knees" (Ståhle 1960, p. 166). That the cross was still in place in this central location, and that it had been in use up to at least some years following the reformation, was apparently not viewed as problematic in this context. However, as will be discussed in the following section, attitudes towards these shrines varied.

\section{Votive and Devotional Crosses}

From the parish church of Liden in the province of Medelpad an unusual early 16th-century wooden cross is preserved (Figure 1). It is painted with an image of St. Martin on one side and of St. Margaret on the other. The cross seems to have played a role in popular religion even in the post-reformation period, as indicated by the multitude of engravings covering its surface, consisting of initials, house marks and the year 1641 . These engravings were possibly made in order to tie oneself or one's household to the cross, or to that which the cross represented, and the cross was apparently used in this manner after the reformation as well as before. In a 1776 letter to Härnösand cathedral chapter, 
the local vicar describes a nearly identical cross preserved in Liden's annex parish Holm. Instead of St. Martin and St. Margaret, this cross is said to have had paintings of the Virgin Mary and St. Joseph instead. Due to the unusual design - to my knowledge, the Liden cross is the only example of such a cross in Sweden-they seem to have been manufactured as a pair for the two churches. Another possibility is that the vicar was actually discussing the Liden cross, and that the images of Martin and Margaret had been reinterpreted as that of Mary and Joseph in a post-reformation context. In an inventory from 1831 the cross is described as "an old cross with painted images of humans on both sides, with unknown letters, that seem to be a remnant of paganism" (ATA: Holm 1831). Due to the mention of the house marks, his statement seems to support the idea that this was in reality the preserved Liden cross. House marks often took a runic form, and this could easily have been the unknown "pagan" letters referred to in the inventory. There is, of course, a possibility that the Holm cross, if a separate object, was also covered with similar marks, though this is somewhat unlikely due to the rarity of such inscriptions on images.

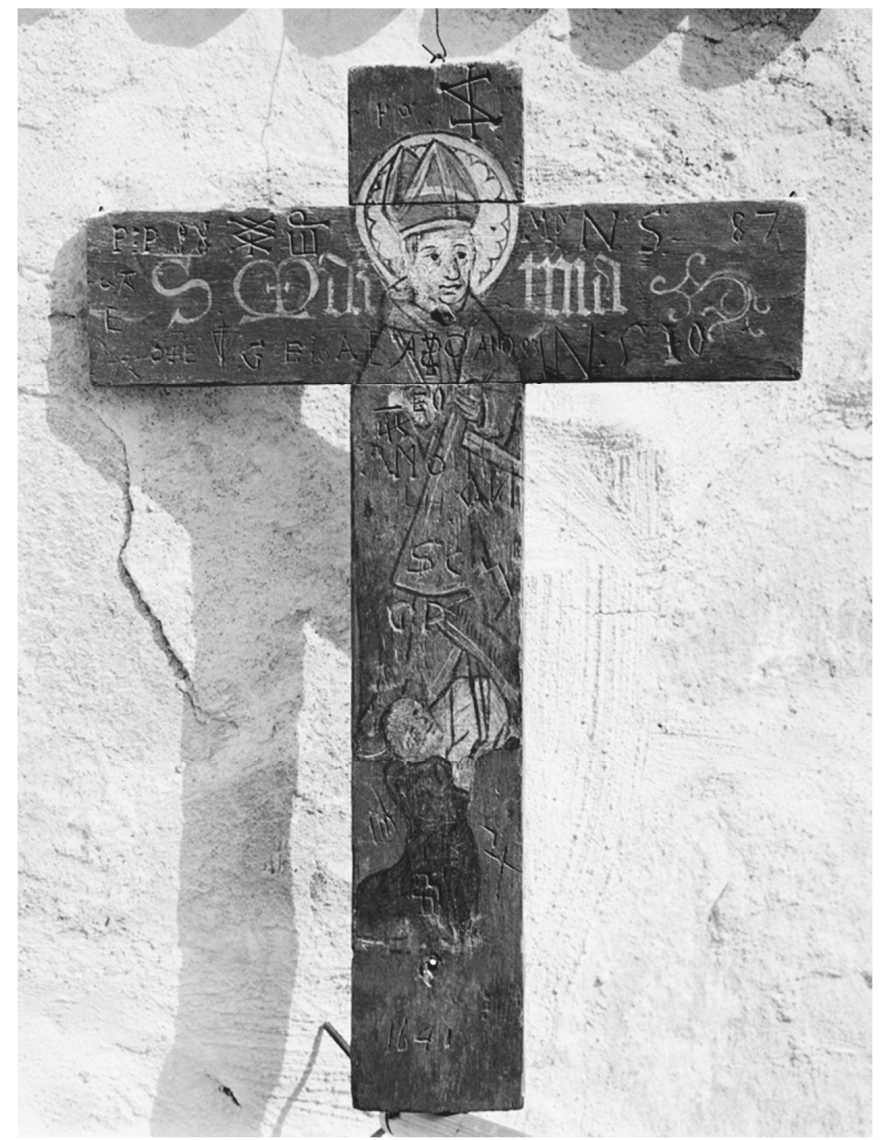

Figure 1. Votive cross depicting St. Martin from Liden parish. Photo: Ivar Andersson, Swedish National Heritage Board.

Regardless, the vicar continues his 1776 letter with saying that he had heard from his parishioners that "during the Papacy", the cross had been placed in some sort of small structure at a placed called Korsmobacken in the woods between the Liden and Holm churches, and that it had functioned as "an 
offering stead" for those unable to walk the distance to either of the churches (HLA: Domkapitlet i Härnösand E III:76, Holm 1776-09-11). The cross was probably manufactured in the sixteenth century, placing this devotional custom on the "eve of the reformation", and it is unlikely that the practice was ended abruptly as the new teachings were introduced. Rather, that parishioners had clear memories of how the cross had been used, even in the late eighteenth century, suggests that such customs continued into at least the seventeenth century, as indicated by the year carved into the cross along with the house marks. Similar statements, that project such practices to a vague "popish" or "pagan" past, are rather common in the sources. The fact that such objects existed in the pre-reformation period calls for little doubt; however, such statements could also have been made in order to disguise a more recent practice, and thus to evade possible criticism and sanctions from the cathedral chapter.

Though no longer part of officially sanctioned religious practices, wayside crosses continued to be a feature of the post-reformation landscape. Some of them, in particular memorial crosses, even survive to this day. These memorial crosses were often said to have been raised at sites where someone had met a violent end, in particular sites of murders and riding accidents. (Säve 1873, pp. 4-20). Wayside crosses primarily serving a mnemonic function like these were likely never demolished in part because they were not tied to practices that the early modern Church perceived as threatening. Crosses used for devotional purposes are preserved to a much lesser degree because they were often constructed out of wood rather than stone, and likely also because they were associated with religious traditions that were frowned upon by the early modern Church. However, they certainly still existed in many parts of the country by the seventeenth century, and sometimes as late as the nineteenth century.

In 1666, in accordance with the Gothicist movement of the Swedish empire, the crown issued a decree for vicars and local officials to submit reports of historical monuments found in their parishes. What actually constituted a "monument" seem to have been subject to a wide range of interpretations and the acts submitted vary greatly in geographical distribution, length and quality (Baudou 1995, pp. 164-68; Bringéus 1995, pp. 92-93; Zachrisson 2017, pp. 98-104). Still, these inventories, dating between 1667 and 1693, are invaluable sources for early modern folklore and material culture, and many wayside crosses are mentioned in these acts. For instance, in the parish of Tolg in Småland, the vicar of the area wrote of an old wooden cross by the name of "Three Lords" or "Lord Three" [sv. Herra Tre], that never seemed to rot (Stahre 1992, p. 153).

An expression that seems to have been commonplace in early modern Sweden as well as in the other Scandinavian countries, when discussing someone lacking in piety was that he or she went to "neither cross nor church". This suggests that the practice of visiting wayside crosses for devotional purposes had been far more common than what may be attested from preserved sources from the medieval period (Gardell 1931, p. 7; Bø 1964, p. 183). For example, one vicar, in writing to the College of Antiquities in 1683, tells us of the use of this expression in his parish:

Up and by the old Dalby church [ ... ], there has been a large wooden cross, six cubits high or long, outside of the gate of the church, and by the same cross there was a collection box. On Sundays and holidays where there was no service in the church, some of the peasants still went to the cross and made offerings in the box on behalf of their sick back at home. But if someone was negligent in their worship, other peasants would reproach him, saying that he goes to neither cross nor church, hence this expression has come in use in this area. (Ståhle 1969, p. 186)

In Funäsdalen and Hede in the province of Härjedalen, remnants of a votive crosses [sv. Offerkors] could still be seen according to an antiquarian report from 1684, written by the vicar of the parish. At their bases, coins placed there as offerings had been found. However, the vicar was also quick to finish the sentence with a redeeming "—but not nowadays" (Ståhle 1960, p. 245).

The practice of not only preserving, but also raising new crosses continued well into the post-reformation period, though it was regarded with suspicion by the clerical élite. When writing about the customs of the province of Hälsingland in the early 18th century, local pastor and schoolmaster 
Olof Broman wrote, with what appears to be a sigh of relief, that the custom of "raising crosses in woods, by the roads, on beaches and other places" was not as common as before (Broman 1911-1949, p. 29). These crosses were used for a kind of semi-domestic devotion, as they were somewhere in the middle between the private and the public, between the familiar grounds of the home and the official space of the parish church. Records from the 17th and 18th centuries show that people approached these sites with bodily acts of reverence, such as kneeling, bowing and making the sign of the cross. Some sources also discuss how people interacted with these crosses in the pre-reformation period. An antiquarian report of 1668 mentions a cross placed in a pasture in Södra Åsarp parish, Västergötland, where "the simpleminded people read their rosaries during the Papacy" (Ståhle 1969, p. 202).

In addition to the saying "neither church nor cross", another common expression in these cases is the verb "krypa till korset", which literally means "crawling towards the cross" The expression has since been incorporated into everyday modern Swedish, meaning to admit one's fault or to "come crawling back", but in these cases, it was used in a more literal sense. In the acts from the College of Antiquities, many sites figure where people claim such crosses had been used in penitential acts. In 1624, the vicar of Viby parish in Scania ${ }^{1}$ tells of a hill called Korshøjen [litterally "Cross-hight/hill"] where people had previously "crawled to the cross" (Tuneld 1934, p. 166). In Tuna parish in Hälsingland, a report from 1686 states that a "votive board" [sv. Offertavla] had been placed by a crossroad, where the author uses the same expression for the penitential acts that had previously been carried out at the site (Ståhle 1960, p. 201). A later comment further elaborates the ordeal that sinners underwent at the cross in Tuna, which perhaps has more to say of 18th-century anti-Catholic imagination than the actual traditions of the medieval era. According to Olof Broman, writing in the early 18th century, the site was still called Taveltäkten [literally "board-quarry"], because of the board shrine once placed there. The image consisted of a cross, lined with sharp rocks at its foot, and Broman continues to tell us that:

[... ] those that were guilty and to be punished, or those wanting to show their repentance, had to crawl among these sharp stones on their bare knees, until their skin was shed and the blood was flowing. Because of this there is an expression: 'Go to Tuna and learn your manners!'. (Broman 1911-1949, p. 243)

The demonization of pre-reformation religious practices occasionally went so far as to, likely deliberately, misinterpret them as pre-Christian. On a hill outside the small village of Valparbo, Uppland, there had once been a pinewood cross, and when reporting this to the College of Antiquities in 1672, an official described the shrine as a place where "the pagans have made their offerings and committed their idolatrous worship", all while maintaining that there were still men alive in the parish that remembered the now-ruined cross (Ståhle 1960, p. 91).

In some areas, the practice of visiting wayside crosses for worship continued into the eighteenth century. One instance is mentioned briefly in a parish description from Bottnaryd in Småland, authored by Vicar Petrus Blidberg in 1742. The cross, which by the time of the description recently had been removed, had stood at a place called Korsängen [litterally"Cross-Meadow"], and the peasants had "always fallen to their knees and made the sign of the cross" before it when passing by on their way to church (SSLB Wilskman, vol. II). As such, this cross was clearly not just a complement or alternative to regular church attendance, but rather incorporated into weekly Sunday worship.

In 1727 Bishop Jesper Swedberg of Skara visited the parish of Broddetorp, Västergötland (GLA: Broddetorp C:2). During the visitation he was informed that at a place called Korstorp (literally "Cross-thorpe"), several crosses had been erected, and that "many superstitious people" went there to leave their offerings. In this case, the offerings consisted of items of clothing, which were hung from the crosses. The land on which the crosses stood was owned by the vicar of the parish, but was tenured to a local farmer. This implies the crosses must have been fairly close to the tenant's dwelling,

1 The antiquarian acts from Scania are of an earlier date than the other provinces', since Scania was a part of Denmark up until 1658 , and the Danish crown had issued an earlier antiquarian project, similar to the project launched in Sweden by 1666. 
since such plots of land were rarely large. It was decided that the farmer was to be ordered to remove the crosses, in order to not let "such superstition" continue, and the vicar owning the land was to appear before the cathedral chapter. What is especially curious about this case is that it took place in a region that, though distinctively rural, cannot be called peripheral. Located among the fertile plains of south-western Sweden, the parishioners of Broddetorp would have had no trouble finding a parish church within walking distance. These shrines could apparently fill multiple functions, both incorporated in regular worship as in the previous case, and more unofficial ones that probably served religious needs that were not always fully catered for by the institutional Church.

The practice of bringing votives to crosses in the landscape is also documented from Finland, (Arffman 2017, p. 259). ${ }^{2}$ Most such crosses seem to have been in communal use, but there are some cases where they were raised and kept by a single individual or household. In relation to a 1670 visitation in Kuopio parish in Savolax, two neighbours had revealed that a farmer named Michel Owaskainen had made a shrine for himself in the woods. There he had raised a tall cross, which he was said to "worship". When this came to the attention of Dean Cajanus of Kajaani, the dean decided that Michel himself was to "cut the cross down, and ruin its foundations", and that he was not to be admitted to communion until he had done so. Apparently, Michel refused to cut his cross down. The case was mentioned briefly once again during the following year's visitation, in that the local vicar was ordered to investigate the matter further. What came of this investigation, and whether Michel eventually submitted to the dean or not, we do not know, since the case is not discussed in later records (Häkli 2015, p. 201).

In 1705, the brothers Per and Christopher Åmunds in Roma parish on the island of Gotland had raised a cross on their farm property, which was brought to light during a visitation by the superintendent. The brothers had previously had much ill fortune with their animals, and the cross was believed to prevent similar misfortune in the future. The superintendent declared their action as "superstition and idolatry", but the custom was in reality quite common on the island (Wikman 1947, p. 111). A detailed account on such a gårdskors (litterally "farmhouse-cross") is preserved from the late 18th century. In the farmyard of a prosperous farmer in Källunge parish, a large crucifix of about five metres of height was in place in the 1780s. Each morning the entire household, from master to milkmaid, gathered by the cross. There they made their morning prayers while someone read a prayer of confession, "or at the very least an Our Father". The children were kneeling by a rod or board at the foot of the cross, while the adults stood around with their hands folded, the menfolk baring their heads. Though the cross clearly belonged to this particular household, day labourers and servants from other farms sometimes went there with their children to pray too. When interviewed by nineteenth-century antiquarian P.A. Säve, an old man remembered how he, as a child, would ask the mistress of the house for bread, and that she used to chase him out of the kitchen telling him to go to the cross first, for a reminder of who was the real provider of all good gifts (Wikman 1947, pp. 111-12).

As mentioned briefly in the introduction to this article, crosses were often raised at holy wells and other locations that were already invested with notions of sacrality. Even though the emphasis at these sites were the wells and springs themselves, crosses on the grounds further marked the sites' sacred character. Such well-side crosses are known from several Swedish holy wells, such as St. Thorsten's Well in Vättlösa and the Holy Cross Well [sv. Helge korss kella] in Mortorp (KoB Dahlb. III:49 Ex. I. Stahre 1992, p. 161)]. In 1719, when discussing two springs in Torpa in Västergötland dedicated to the Virgin Mary, topographer Nils Hufwedsson Dal mentions that next to the springs several crosses had been "raised by those that have benefited from the water or regained their health" (Dal 1719, pp. 29-30). The practice of building a cross to show one's gratitude is also evidenced from early modern Denmark, as in the case of the well-known St. Helen's Well [da. Helle Lenes Kilde] in Tibirke (Schmidt 1926, pp. 46-48). Votives in the form of clothing and human hair were sometimes left hanging from

2 Finland was, up until 1809, a part of the Kingdom of Sweden. 
these wellside crosses. In the parishes of Lyngby and Kiaby in Scania, an antiquarian report from 1624 mentions several wooden crosses erected at S. Ellenis Kilde ("St. Helen's well”) and at Hellig Trefolldigheds killde ("Holy Trinity Well"), where those seeking healing at the sites had hung pieces of cloth and tufts of hair (Tuneld 1934, pp. 60, 159-60). Pieces of cloth were also hung upon the crosses raised among the holy birches of St. Ingemo in Dala, Västergötland (Ståhle 1969, pp. 216-17), and strands of human hair were found on the crosses surrounding S. Nicolaj kilde (St. Nicholas' Well) in Ödsmål, Bohuslän, as noticed by bishop Jens Nilssøn in the 1590's (Nilssøn 1885, pp. 164-65).

\section{The Illicit Chapels in Jämtland and the "Vebomark God"}

Though crosses were by far the most common form of these semi-domestic places of worship, they occasionally took other forms, such as lay-controlled small chapels and sculptures originally acquired for use in churches, transferred to a domestic or landscape setting.

On the 18th of February in 1621, an unusual case was brought before the regional court in Offerdal, in the province of Jämtland. Oluf Nielssen, vicar of Offerdal parish, had written to the bishop of Nidaros demanding a case of idolatry to be prosecuted. The "idolatry" in question centred on three "illicit chapels" in the area. When the parish church had been cleared of medieval sculptures following the Protestant reformation, the parishioners with the help of a former church warden, had managed to salvage some of the sculptures. The images had then been placed in three small chapels. Two of the chapels are mentioned as being located in the woodlands, and one on the properties of a farm named Landö. It is not clear whether these shrines were built by the laity themselves for this purpose, or if they were abandoned chapels that had previously been kept and administered by the pre-reformation Church. Vicar Nielssen stated in court that he, on many occasions, had ordered the peasantry to tear these chapels down, but that they had refused and insubordinately responded that if he wanted them gone, he would have to find someone else to do the deed.

During the trial, a man named Halvard Jonsen was brought forth, and he retold the story of how his late wife Magdalene, "out of ignorance" had made a promise during a difficult childbirth in 1598. If she were to be safely delivered, she vowed to give something to the image of St. Lawrence in the Landö chapel. Her prayer was answered and when she had recovered from the birth, she proved true to her word and walked a distance of 40 kilometres to the chapel, where she hung a neckerchief on the image. Another witness, Simen of Landön, then appeared and confessed that his mother-in-law Bente had chosen to leave the sculpture of St. Lawrence a part of her inheritance, likely textiles. Upon Bente's death, her daughter had fulfilled her mother's wish and "carried it there and hung it on the aforementioned image".

The second of the three chapels was called Korset (The Cross). According to a witness who had apparently functioned as an unofficial church warden for this chapel, the means gathered from the offerings had been used to renovate or expand the chapel—which suggest that in addition to everyday objects, money was also donated. On the Korset chapel, the peasantry had pleaded with vicar Nielssen that they needed to keep it to "make their prayers in" (no. thill Att Bede dieris böner Udj), to which the vicar simply had responded with a laconic "no". The trial was concluded with the burning of the "clothed" images, that had apparently been brought along to court, and the men of the parish were ordered do demolish the chapels themselves. Those still alive that were guilty of practicing superstition in any of the chapels were to stand kyrkoplikt-a form of public humiliation punishment in which the offender would publicly confess their sin in church before the entire parish (Hasselberg 1933, pp. 18-20). These chapels were clearly outside the control of the institutional Church, and were kept and maintained solely by the laity. All named persons stated to have made offerings at the chapels, were conveniently already dead by the time of the trial, which probably reflects the parishioners' unwillingness to betray their neighbours more than anything else. The fact that the images had been hung with items of clothing seems to have been an aggravating aspect in the case, with the court record mentioning in on several occasions. 
Whether some of the wayside crosses discussed in the previous section were actually sculpted crucifixes is difficult to determine, since the words 'kors' and 'krucifix' were often used interchangeably in pre-modern sources (Lange 1964, p. 175), but there are some examples mentioning proper sculptures being used by the laity for semi-domestic devotions. Three cases of images of saints that functioned as woodland shrines in a similar fashion as the wayside crosses are known from the sparsely populated woodlands of Västerbotten, northern Sweden. In his dissertation on the midnight sun, translated into English in 1698, theologian and cartesian philosopher Johan Bilberg recounts his visit to Bygdeå parish church in Västerbotten:

[... ] there was the Picture of a certain Religious Person which was placed in a Wood half a Mile and more from thence; where formerly those who were Remote from Church, are said to have met together to Prayers, on Holy-Days; but after the most happy Times of Religion being purged from the Heresies of Papists, as that sort of Worship ceased in our Country, so the Picture was laid in a certain place of the Temple, in detestation of the Memory of that Matter. (Bilberg 1698, p. 86)

A 1731 topographical dissertation on the area by Jonas Ask also mentions the "image of a holy man", this time in Lövånger parish church. According to the parishioners, the image had been used in the same manner as the one in Bygdeå (Ask 1928, p. 76). Even in the early twentieth century, the image was remembered in the parish, referred to as the "Vebomark God" (sv. Vebomarksguden) in folklore records from the area, as the image had been placed in a hollow tree in the Vebormark forest. Since 1924, a sculpture said to be the Vebomark God is kept in the collections of Västerbotten's museum (Figure 2). The image is severely damaged, with its hands and face cut off, which makes it difficult to established what saint it once represented, though the figure seems to be wearing the dress of a deacon (Burman 2014, p. 51). Unfortunately, the sculpture is yet to be dated, which could have provided a more precise timeframe for when the Vebomark God was in use as an outdoor shrine.

A similar case from the same province is attested as late as in the 1800s, when artist and antiquarian Nils Månsson Mandelgren visited Skellefteå. He was told that up until 1823, a wooden image had been placed in a hollow tree in the Gärdsmark forest. Allegedly, this image had been "worshipped" by the local peasantry, and Mandelgren referred to this behaviour as "pure Catholicism" (Lund University Folklore Archives, Mandelgren: 5: Härnösand: Skellefteå).

Much further south, in Västergötland, another image bore a similarly suggestive name as the Vebomark God-the Grain God of Vånga (sv. Kornguden i Vånga). The use of this image, a thirteenth-century apostle (Figure 3), first caught the attention of scholars when an elderly couple from Vånga visited a local museum in the 1870s. When confronted with the sculpture the man had exclaimed: "Look Wife-They have the Grain God here!". When questioned he told the curator of the museum that the sculpture was known as the Grain God in the parish, and that before the image was transferred to the museum in the 1860s, in springtime, the villagers used to smuggle the sculpture out of the church at dawn to carry it across the fields in order to secure a good harvest (Svanberg 1985, p. 259). Whether or not this tradition had developed from pre-reformation practices is unclear, since the earliest mention is found in a legal protocol from 1760, where a witness in an unrelated case was recorded absent from court due to being out "carrying the Grain God" for two days (Bergstrand 1972, p. 18). Folklore about the Grain God is rather abundant from the late nineteenth- and early twentieth centuries, and while the records often bear the marks of local humour and tall tales, the sheer amount of the stories suggests that the sculpture was indeed being carried in procession at least up to the middle of the nineteenth century (Svanberg 1985, pp. 259-60). 


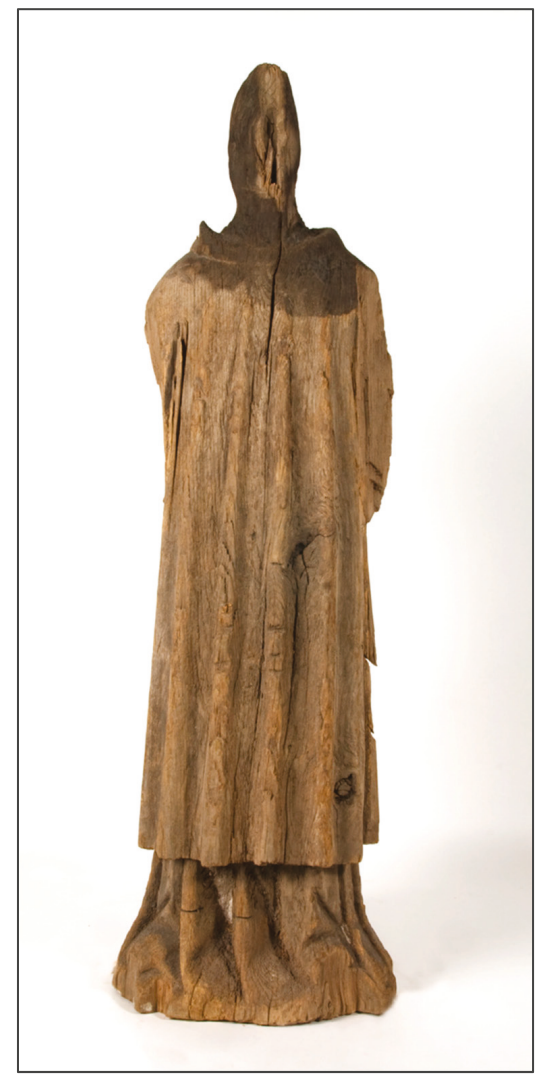

Figure 2. "The Vebomark God", possibly a holy deacon, from Lövånger parish. Photograph by Pekka Nyman, Västerbotten's museum.

Other sculptures could be temporarily removed from their more permanent placements in churches and chapels to the actual homes of laypeople. This seems to have occurred in particular in association with childbirth. The axe of St. Olaf is one such example. Writing in 1760, travelling topographer Carl Frederic Broocman wrote of such an axe in the parish of Västra Husby, Östergötland. By the mid-eighteenth century, the sculpture of St. Olaf in the parish church was missing the axe that the Norwegian royal saint was usually depicted with. According to Broocman, this was the result of the continuous use of the axe by women in labour:

Of this image's axe, it is said, that the peasantry has used it for birthing mothers, in the belief that its touch and placement would ease and quicken the birth; and that they have used it in this manner for so long, that it has subsequently gotten worn down and ruined. (Broocman 1760, p. 380)

Similarly, a sculpture of the Virgin, normally placed upon the "Women's altar" (sv. Kvinnoaltare) in the church of $\mathrm{Od}$, was reportedly lent to pregnant women across the surrounding area (Hvarfner 1959, p. 36). A corresponding practice is also described from the pre-reformation era, where the Christ

3 Women's altar was a post-reformation term used for the altar of Our Lady, upon which a new mother was expected to place an offering of foodstuff in the churching ritual (Gustavsson 1972, pp. 121-22). 
child could be borrowed from Madonna sculptures in such cases. The strength of this tradition could possibly be evident from the fact that the child was often sculpted separately from the Virgin for easy removal (Stolt 1996, pp. 407-19). Such cases, while only rarely figuring in the sources, demonstrate the portable character of the sacred, and how the holiness of church space could be transferred to a domestic setting.

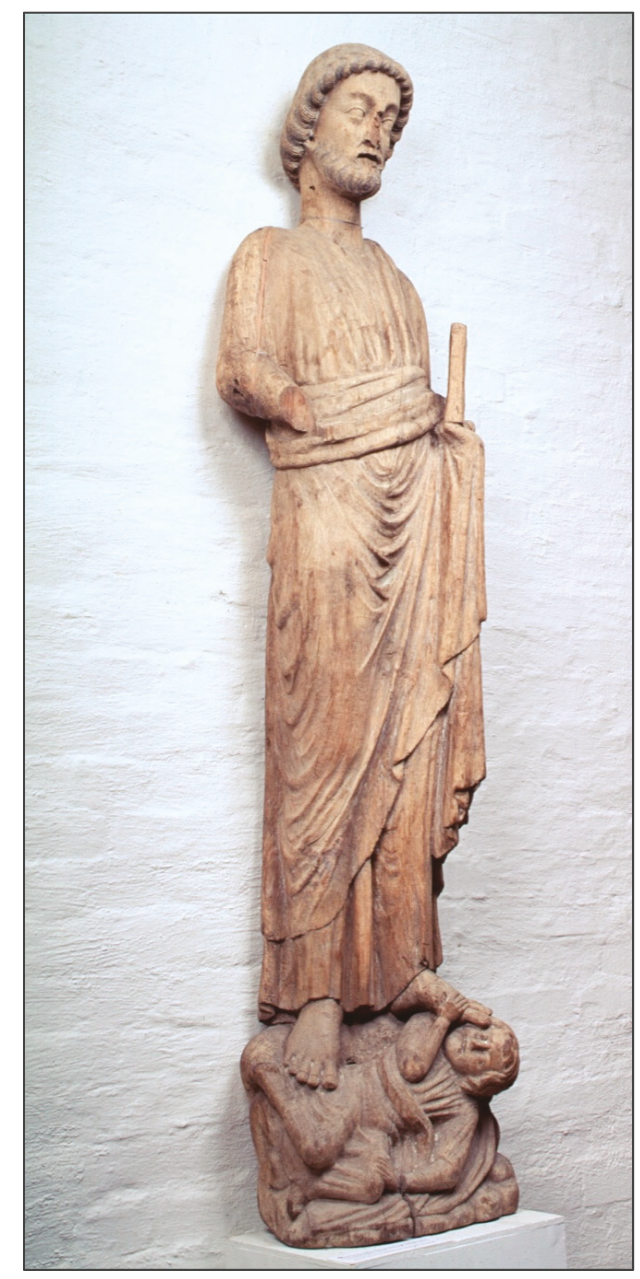

Figure 3. "The Grain God of Vånga", a 13th century apostle from Norra Vånga parish. Photograph by Lennart Karlsson, The Swedish History Museum.

\section{Concluding Remarks}

From the medieval period, most of the sacred sites in the landscape that we have knowledge of today are those that were administered by the Church. In general, those of a more domestic, private character are unfortunately lost to us, but this should by no means be taken as evidence that such shrines were not present in the sacred landscape of the middle ages. Tales of such medieval sites were frequently told in the seventeenth and eighteenth centuries, and were recorded by the antiquarians, clergymen and scientists of the era. To some degree, these stories surely reflect early modern prejudice 
towards a "superstitious" and "popish" pre-reformation culture, but they likely also contain elements of genuine memories from a not-too-distant past.

Achim Timmermann has suggested that in the late medieval period, such shrines functioned as thresholds, where mundane everyday spaces such as forest clearings, roads and pastures could temporarily transform into the true, spiritual landscape, providing glimpses of the path leading to God (Timmermann 2017, p. 264). Perhaps this was also the experience of those that visited similar sites in the early modern era, though the available sources provide no such insights. However, these sites and objects were clearly important to the people that built, used and maintained them-so important that they on occasion defied the Church in order to keep them. In addition to prayer, people interacted with the sacred at these shrines by the use of bodily gestures such as kneeling, bowing and making the sign of the cross. They also frequently left votive gifts, often consisting of everyday domestic objects like clothing, at the sites. Votives were particularly important when petitioning for aid, and the issues mentioned in these cases are distinctively domestic in their character. When visiting such shrines, parishioners hoped for intervention in securing their health, a safe delivery, a good harvest and the welfare of cattle. In some cases, shrines were built within the bounds of farms and villages, while in others they were placed along roads and in the woods. Sometimes they could also be found in churchyards or just outside of the lychgate, functioning as an extension of church space that could be utilised when there was no service or the church itself was locked. There are several cases that show that both the maintenance of and the creation of new semi-domestic sacred sites continued well into the early modern period. If we are to look for explanations as to why these outdoor shrines remained in place for such a long period of time, despite the fact that the institutional Church disapproved of them, we might consider the physical environment in which these shrines were built. In the dense woodlands of the northern and eastern part of the realm, the distance to proper church buildings could call for complementary sites of worship closer to one's home. With the reformation, a number of small chapels that had previously served such communities had been demolished or taken out of use, prompting parishioners to either maintain these buildings themselves, such as in the case from Jämtland, or to create new sites. But as the cases from Scania and Västergötland demonstrates, these sites could also be found in agricultural areas where the access to parish churches was not an issue. This suggests that these sites provided a complementary layer to religious life in more than purely spatial terms.

As to the question of what to make of this religious culture, and whether such traditions are to be interpreted as surviving features of pre-reformation piety or as distinctly Lutheran traditions, the issue is complex, and the answer, I would argue, lies somewhere in between. In areas where Catholic, Lutheran and Reformed communities lived side by side, such as in the principalities of the Holy Roman Empire, the physical environment functioned as a way to accentuate difference (Freist 2009, pp. 214-15). This might have also been the case in the parts of Eastern Finland bordering Orthodox Russia-at the very least, the presence of a distinct group with a different faith worried Swedish authorities (Toivo 2016, pp. 112-24). However, as for the rest of the kingdom, areas with different faiths were remote. In the seventeenth century, the discussions on religious tolerance that were underway in other parts of Europe were largely absent in Sweden-apart from foreign dignitaries and merchants, it had no significant religious minorities (Ljungberg 2017, pp. 7, 22). It is possible that the general absence of the religious Other made the limits of Lutheranism more flexible in this area. Though the use of outdoor shrines sometimes worried local clergy and visiting bishops, no large-scale efforts to root out such traditions were ever attempted. In general, most clergymen seem to have chosen to turn a blind eye to practices bordering the limits of evangelical teachings, and as argued in the introduction of this article, the association of such shrines with Catholicism were likely not as strong in Sweden as in parts of Continental Europe. In traditions with a similar underpinning logic, such as offerings to sculptures and holy wells, local clergy sometimes even promoted and took part in these practices (Zachrisson 2018, pp. 112-13).

Other studies have shown that the cult of the saints and other traditional religious practices persisted in various forms, both official and un-official, throughout the seventeenth, and sometimes 
into the eighteenth century in Scandinavia (Laugerud 2018; Malmstedt 2002; Nyman 2002) The use of outdoor shrines and wayside crosses was no different, though it was not a part of the official religious repertoire. The tradition of visiting outdoor shrines clearly has its origins in pre-reformation piety, but the intentions of participants in these visits likely underwent change throughout the rather vast timeframe of this study. There are, however, few credible indications that these visits took place in any other context than a Christian one, and the allusions to heathenry sometimes made by clergymen likely reflect an insistence on the illegitimacy of said practices rather than an actual parallel religious tradition with roots in pre-Christian times. That the religious culture of rural communities still heavily relied on physical focal points for interaction with the sacred seems apparent. The need for additional sacred space, as a semi-domestic complement to parish churches did not necessarily diminish in the wake of the reformation.

Funding: This research was funded with a grant from the Birgit \& Gad Rausing Foundation.

Conflicts of Interest: The author declares no conflicts of interest.

\section{References}

\section{Archival Sources}

ATA = Antiquarian Topographical Archives of the Swedish National Heritage Board: Gemensamma handlingar, F1 Antikvarisk-topografiska dossierer. Medelpad, Holms socken, Kyrkan, Inv. 1831.

KoB = National Library of Sweden: Dahlb. III:49 Ex. I. https://suecia.kb.se/suecia/pdf/47/8468047.pdf Lund University Folklore Archives: Mandelgren Collection, Series 5, Härnösands stift: Skellefteå kyrka.

GLA = Regional State Archives in Gothenburg: Broddetorp Parish Records, C:2, visitation record of 1727.

HLA = Regional State Archives in Härnösand: Härnösand Cathedral Chapter, E: III:76, Holm September 111776.

SSLB = Skara Stift- och Landsbibliotek: Sven Wilskman's Collection, De Singulis in Diocesi Scarensi Parichiis, vol. II.

\section{Printed Sources and Literature}

Alin, Oscar, and Emil Hildebrand, eds. 1887. Svenska riksdagsakter jämte andra handlingar som höra till statsförfattningens historia under tidehvarfvet 1521-1718, Band 1 (1521-1544). Stockholm: Norstedt.

Arffman, Kaarlo. 2017. Resistance to the Reformation in 16th-Century Finland. In Lived Religion and the Long Reformation in Northern Europe c. 1300-1700. Edited by Sari Katajala-Peltomaa and Raisa Maria Toivo. Leiden: Brill, pp. 255-74.

Ask, Jonas Elias. 1928. Om Umeå stad och närliggande pastorat. En gradualavhandling av år 1731 av Jonas Ask. Översättning från latinet med ett förord av rektor J. V. Lindgren. Med 3 kartor och 7 illustrationer. Västerbotten: Västerbottens läns hembygdsförenings årsbok 1928: 38-78.

Baudou, Evert. 1995. Politik, vetenskap och folkliga föreställningar. Olika syn på rannsakningarna-Exemplet Norrland. In Rannsakningar efter antikviteter-ett symposium om 1600-talets Sverige. Edited by Evert Baudou and Jon Moen. Stockholm: Kungl Vitterhets Historie och Antikvitets Akademien, pp. 155-74.

Bergstrand, Carl-Martin. 1972. Brott och straff i 1700-talets Västergötland. Gothenburg: Carl-Martin Bergstrand.

Bilberg, Johan. 1698. A Voyage of the Late King of Sweden and Another of Mathematicians Sent by Him: in Which are Discover'd the Refraction of the Sun Which Sets Not in the Northern Parts at the Time of the Solstice, Varition of the Needle, Latitudes of Places, Seasons Ec. of those Countries: By Command of the Most Serene and Most Mighty Prince, Charles XI, King of Swedes, Goths and Vandals/Faithfully Render'd into English. London: Printed for Edward Castle.

Bringéus, Nils-Arvid. 1995. Antikvitetsrannsakningarna som folkloristisk källa. In Rannsakningar efter antikviteter-ett symposium om 1600-talets Sverige. Edited by Evert Baudou and Jon Moen. Stockholm: Kungl Vitterhets Historie och Antikvitets Akademien, pp. 79-95.

Broman, Olof. 1911-1949. Glysisvallur och övriga skrifter rörande Helsingland, Bd. 1. Uppsala: Gestrike-Helsinge nation. 
Broocman, Carl Fredric. 1760. Beskrifning öfver the i Öster-Göthland befintelige Städer, Slott, Soknekyrkor, Soknar, Säterier, Öfwer-officersboställen, Jernbruk och Prestegårdar med mera. Norrköping: Carl Fredric Broocman.

Burman, Mattias. 2014. Medeltida kyrkor. Västerbotten: Västerbottens läns hembygdsförenings årsbok 4: 46-57.

Bø, Olav. 1964. Kors. In Kulturhistoriskt lexikon för nordisk medeltid från vikingatid till reformationstid. Edited by Ingvar Andersson and John Granlund. Malmö: Allhem, vol. 9, pp. 183-85.

Coster, Will, and Andrew Spicer, eds. 2005. Sacred Space in Early Modern Europe. New York: Cambridge University Press.

Christian, William A., Jr. 1989. Local Religion in Sixteenth-Century Spain. Princeton: Princeton University Press.

Dal, Nils Hufwedsson. 1719. Boerosia, urbs, per regna septemtrionis, mercature nobilis. Then vti the Nordiska Riken för sin köp-handel wälbekante Boeråås stad, beskrifwen af Nils Hufwedsson Dal. Stockholm: Sahl-M. Laurelii Enkia.

Freist, Dagmar. 2009. Crossing Religious Borders: The Experience of Religious Difference and its Impact on Mixed Marriages in Eighteenth-Century Germany. In Living with Religious Diversity in Early-Modern Europe. Edited by Scott Dixon, Dagmar Freist and Mark Greengrass. Farnham: Ashgate, pp. 203-21.

Gardell, Sölve. 1931. Om kors och korshus i medeltidens Bohuslän. Gothenburg: Elanders Boktryckeri Aktiebolag.

Gustavsson, Anders. 1972. Kyrktagningsseden i Sverige. Lund: Folklivsarkivet i Lund.

Hasselberg, Gudmar, ed. 1933. Jämtlands domböcker och landstingsprotokoll. 1, Jämtlands domböcker 1621-1628. Östersund: Jämtlands läns fornskriftsällskap.

Hvarfner, Harald. 1959. Od, Molla, Alboga, Öra: Fyra socknar - fyra kyrkor, ett pastorat. Skara: G. Hvarfner.

Häkli, Esko. 2015. Biskops- och prostvisitationsprotokoll från det äldre Wiborgska stiftet: På basis av Albin Simolins samling kompletterade och utgivna av Esko Häkli. Helsinki: Finska kyrkohistoriska samfundet.

Kuha, Miia. 2015. Popular Religion in the Periphery. Church Attendance in 17th Century Eastern Finland. Perichoresis 13: 17-33. [CrossRef]

Lange, Bernt C. 1964. Kors. In Kulturhistoriskt lexikon för nordisk medeltid från vikingatid till reformationstid, Vol. 9. Edited by Ingvar Andersson and John Granlund. Malmö: Allhem, pp. 173-77.

Laugerud, Henning. 2018. Reformasjon uten folk: Det katolske Norge i før-og etterreformatorisk tid. Oslo: St. Olav forlag.

Ljungberg, Johannes. 2017. Toleransens gränser: Religionspolitiska dilemman i det tidiga 1700-talets Sverige och Europa. Lund: Lund Univeristy.

Louthan, Howard. 2005. Breaking images and building bridges: The making of sacred space in early modern Bohemia. In Sacred Space in Early Modern Europe. Edited by Will Coster and Andrew Spicer. New York: Cambridge University Press, pp. 282-301.

Malmstedt, Göran. 2002. Bondetro och kyrkoro: Religiös mentalitet i stormaktstidens Sverige. Lund: Nordic Academic Press.

Malmstedt, Göran. 2014. In Defence of Holy Days: The Peasantry's Opposition to the Reduction of Holy Days in Early Modern Sweden. Cultural History 3: 103-25. [CrossRef]

Messenius, Johannes. 1703. Joihannis Messenii Scondia Illustrata, tomus IX. Historiam sanctorum E præsulum, sive prælustrium hominum, Scondix conversionem sibi vendicantium, complectens. Stockholm: Johan Peringskiöld.

Nilssøn, Jens. 1885. Biskop Jens Nilssøns Visitatsbøger og Reiseoptegnelser 1574-1597. Christiania: A. W. Brøggers Bogtrykkeri.

Nyman, Magnus. 2002. Förlorarnas historia: katolskt liv i Sverige från Gustav Vasa till drottning Kristina. Stockholm: Veritas.

Nordström, Johan Jakob, ed. 1872. Kyrko-ordningar och förslag dertill före 1686. Första Afdelningen. Stockholm: P.A. Norstedt \& Söner.

Piltz, Anders. 2016. Örat tar över: Trons avmaterialisering på 1500-talet. In Doften av rykande vekar: Reformationen ur folkets perspektiv. Edited by Fredrik Heiding and Magnus Nyman. Skellefteå: Artos \& Norma.

Ray, Celeste. 2014. The Origins of Ireland's Holy Wells. Oxford: Achaeopress Achaeology.

Schmidt, August F. 1926. Danmarks helligkilder: Oversigt of literaturfortegnelse. Copenhagen: Schønbergske vorlag. Stahre, Nils-Gustaf. 1992. Rannsakningar efter antikviteter Bd 3 Öland, Småland, Blekinge, Halland, Skåne, H. 1 Text. Stockholm: Kungl Vitterhets-, Historie- och Antikvitetsakademien.

Stolt, Bengt. 1996. Mariaskulpturer med förlorade Jesusbarn. In Maria i Sverige under tusen år: Föredrag vid symposiet $i$ Vadstena 6-10 oktober 1994, vol. 1:2. Edited by Sven-Erik Brodd and Alf Härdelin. Skellefteå: Artos, pp. 407-19. 
Ståhle, Carl Ivar. 1960. Rannsakningar efter antikviteter. Bd 1, Uppland Västmanland, Dalarna, Norrland, Finland, H. 1, Text. Stockholm: Kungl Vitterhets-, historie- och antikvitetsakademien.

Ståhle, Carl Ivar. 1969. Rannsakningar efter antikviteter Bd 2 Södermanland, Närke, Värmland, Västergötland, Östergötland, Gotland, H. 1 Text. Stockholm: Kungl Vitterhets-, historie- och antikvitetsakademien.

Svanberg, Jan. 1985. Kornguden i Vånga: En bild i långvarigt bruk. Taidehistoriallisia tutkimuksia—Konsthistoriska studier 8: 256-64.

Säve, P. A. 1873. Kors på Gotland. Svenska fornminnesförenings tidskrift 2: 1-21.

Timmermann, Achim. 2012. Highways to Heaven (and Hell): Wayside Crosses and the Making of Late Medieval Landscape. In The Authority of the Word: Reflecting on Image and Text in Northern Europe, 1400-1700. Edited by Celeste Brusati, Karl A. E. Enenkel and Walter Melion. Leiden: Brill, pp. 385-442.

Timmermann, Achim. 2017. Memory and Redemption: Public Monuments and the Making of Late Medieval Landscape. Turnhout: Brepolis.

Toivo, Raisa Maria. 2016. Faith and Magic in Early Modern Finland. New York: Palgrave Macmillan.

Tuneld, John. 1934. Prästrelationerna från Skåne och Blekinge av år 1624. Lund: Gleerup.

Wikman, K. Rob. V. 1947. Gårdskors och bönkors. Fataburen: Nordiska museets och Skansens årsbok 1947: 105-22.

Walsham, Alexandra. 2011. The Reformation of the Landscape: Religion, Identity, and Memory in Early Modern Britain and Ireland. Oxford: Oxford University Press.

Weikert, Monica. 2004. I sjukdom och nöd: Offerkyrkoseden i Sverige från 1600-tal till 1800-tal. Gothenburg: University of Gothenburg.

Zachrisson, Terese. 2017. Mellan fromhet och vidskepelse: Materialitet och religiositet i det efterreformatoriska Sverige. Gothenburg: University of Gothenburg.

Zachrisson, Terese. 2018. Images, 'Superstition', and Popular Piety in Post-Reformation Sweden. Mirator 19: 108-18.

(C) 2019 by the author. Licensee MDPI, Basel, Switzerland. This article is an open access article distributed under the terms and conditions of the Creative Commons Attribution (CC BY) license (http://creativecommons.org/licenses/by/4.0/). 


\title{
Article \\ The Cult of Old Believers' Domestic Icons and the Beginning of Old Belief in Russia in the 17th-18th Centuries
}

\author{
Aleksandra Sulikowska-Bełczowska \\ Institute of Art History, University of Warsaw, Krakowskie Przedmieście 26/28, 00-927 Warszawa, Poland; \\ a.sulikowska@uw.edu.pl
}

Received: 23 August 2019; Accepted: 11 October 2019; Published: 14 October 2019

\begin{abstract}
The aim of this paper is to present the cult of icons in the Old Believer communities from the perspective of private devotion. For the Old Believers, from the beginning of the movement, in the middle of the 17 th century, icons were at the center of their religious life. They were also at the center of religious conflict between Muscovite Patriarch Nikon, who initiated the reforms of the Russian Orthodox Church, and the Old Believers and their proponent, archpriest Avvakum Petrov. Some sources and documents from the 16th and 18th centuries make it possible to analyze the reasons for the popularity of small-sized icons among priested (popovtsy) and priestless (bespopovtsy) Old Believers, not only in their private houses but also in their prayer houses (molennas). The article also shows the role of domestic icons from the middle of the 17th century as a material foundation of the identity of the Old Believers movement.
\end{abstract}

Keywords: icons; private devotion; Old Believers; Patriarch Nikon; archpriest Avvakum

\section{Introduction}

When I visited an Old Believers' prayer house i.e., molenna for the first time I was amazed by the uncommon organization of the space. It was in 1997, in Vidzy—a village in northwestern Belarus (Sulikowska 1998, pp. 43-45) inhabited by a multiethnic and multicultural community, a large part of which was a group of Old Believers ${ }^{1}$. They belonged to a faction of priestless Old Believers (bespopovtsy), thus in their prayer house there was no division into the nave and the presbytery, which is typical for the Eastern rite. Without the clergy, bespopovtsy do not perform the Eucharistic liturgy (the liturgy of the faithful) and in their molenna there is no altar, and no barrier separating the sanctuary from the nave. However, Old Believers used the word "the iconostasis" to denote several rows of icons that were placed on the molenna's eastern wall (Iwaniec 1977, p. 150). Therefore, the iconostasis in molenna of priestless Old Believers does not have its usual function and it does not correspond to the Orthodox Church canonical order. Icons are placed also on a so-called altar in the eastern part of the space and on the small partitions in front of it (barriers of kliros) (Figure 1). The Old Believers' prayer houses, just like other Eastern churches, are always full of icons but most of them are domestic icons, i.e., icons of relatively small size and of specific iconography with a definite predominance of images of the Mother of God, Christ, and angels or saints, especially St. Nicholas the Miracle Worker. Such icons were the most popular among the Old Believers and, as a consequence, the vast majority of the objects of their

1 Old Believers (Russian: старообрядцы, староверы) are supporters of a religious movement that began in the mid-17th century as a result of opposition to reforms initiated by Patriarch Nikon (1652-1666). The Old Believers rejected the new customs and preserved the old rite, being conservative in both doctrine, including art and aesthetic tastes, and morality. In the 17 th and 18 th centuries Old Believers divided into different groups, so called soglasiia, tolki (согласия, толки). Persecuted by both the Orthodox Church and the state, Old Believer communites migrated to various parts of the world. 
worship in the contemporary museum collections are domestic icons or small-sized metalwork-icons and crosses.

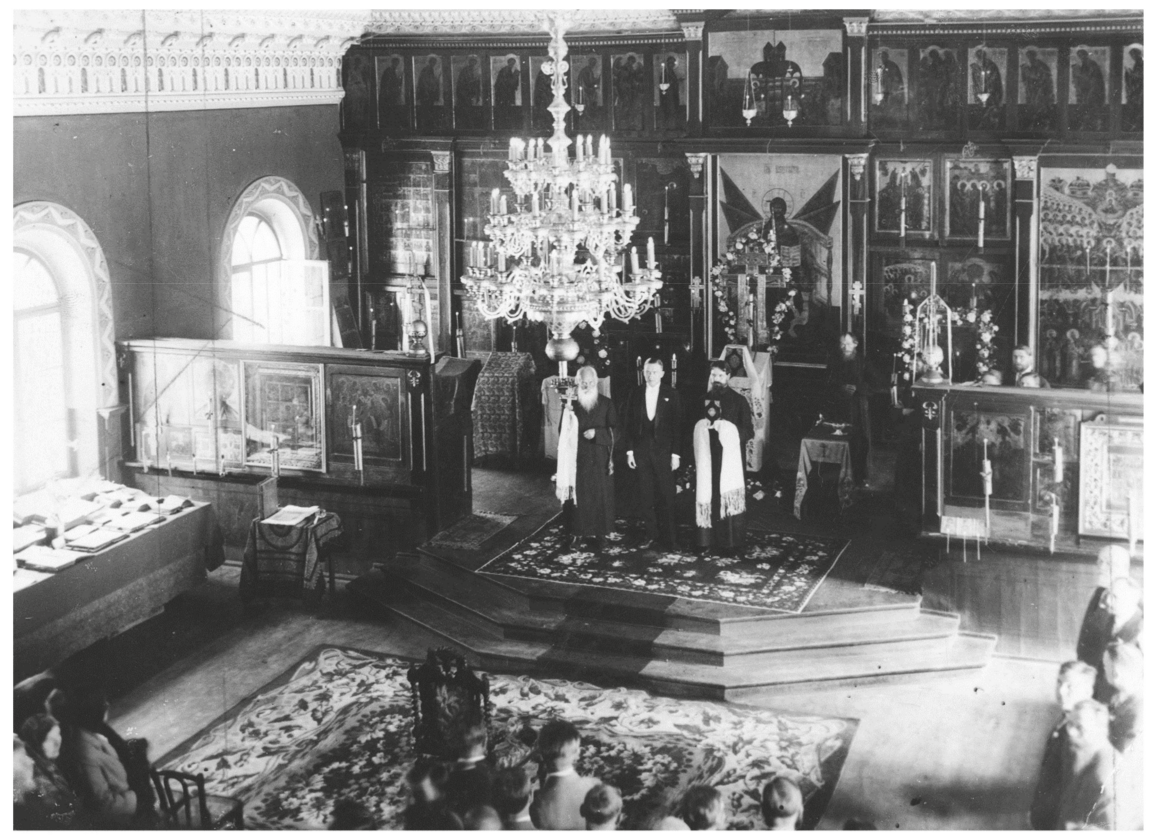

Figure 1. Molenna of priestless Old Believers in Vilnius (waiting for President Ignacy Mościcki), 1930, Warsaw, National Digital Archives.

Research on the Old Believers and their art has been conducted in Russia since the 19th century (Robson 1995, pp. 3-7). Subsequently, scientific interest has expanded internationally, in particular following the publication of the English translation of Oleg Tarasov's monograph Icon and Devotion. Sacred Spaces in Imperial Russia (see Tarasov 2002 for further bibliography). However, various aspects of the Old Believers' culture, especially icons, require further research. Hitherto, the Old Believers' domestic icons have not been discussed in detail. Roy R. Robson has shown the role of religious practices, especially of the liturgical life, prayer houses and "icons of ancient piety" in forging the Old Believers' identity up to the beginning of the 20th century (Robson 1993, pp. 713-24; Robson 1995, p. 127). The purpose of this paper is to describe the status and the function of those "icons of the ancient piety", most of which were the domestic icons in Old Believer communities, and to highlight the reasons for their unique status, from the mid-17th to the mid-18th century. I will also raise a question concerning the reasons for their predominance. We can identify such factors as the mobility of the Old Believers and the traditions that arose at a time when Old Believers were separated from Russian Orthodox society. The background is the dissimilarity between the Old Believers and other Orthodox groups, not only from the official Church of Russia (before and after mid-17th century), but also with the Byzantine tradition. I will discuss broadly the iconographic themes included in the domestic icons of the Old Believers, a subject that deserves a more extensive study, that is beyond the scope of this article.

\section{The Rise of the Old Believers Movement and Patriarch Nikon against Domestic Icons}

The beginning of the Old Believers movement in the middle of the 17th century was a reaction to the reforms of the Church initiated by the Moscow Patriarch Nikon (1652-1666) during the reign of tsar Aleksey Mikhailovitch (1645-1676). The reforms from 1653 consisted of changes of rituals, symbols of 
Russian Orthodoxy and its religious books (Lobačev 2003, pp. 113-29). The most important changes included the spelling of the name "Jesus" (Іисусъnot Ісусъ; therefore, ІИС XС instead of IC XС in the Christological monogram (Figures 2 and 3), allowing the so-called Greek (four-pointed) cross instead of the Orthodox (eight-pointed) one (Figure 3). The reform codified the position of the fingers while blessing oneself, the reduced number of bows during the rites, and the form of these bows (bowing to the waist instead of bowing to the ground), as well as the direction of the procession around the church, "against the sun" instead "towards the sun" (Zenkovskij 1995, pp. 185-257; Staroobrjadčestvo 1996, p. 197). A large part of Muscovite society understood these changes as a blasphemously overturning and destruction of God's world order. Some believers of the Moscow Church began to see Nikon as an enemy of Orthodoxy, predecessor of the Antichrist or even as Antichrist himself (Lobačev 2003, pp. 269-70; Sulikowska-Bełczowska 2017, p. 71). The persecution of those who opposed the reforms, initiated by the Patriarch and the tsar, began shortly afterwards. The Old Believers' archpriest Avvakum Petrov (1620 or 1621-1682) and many others were imprisoned, banished and even killed (Zenkovskij 1995, pp. 322-39).

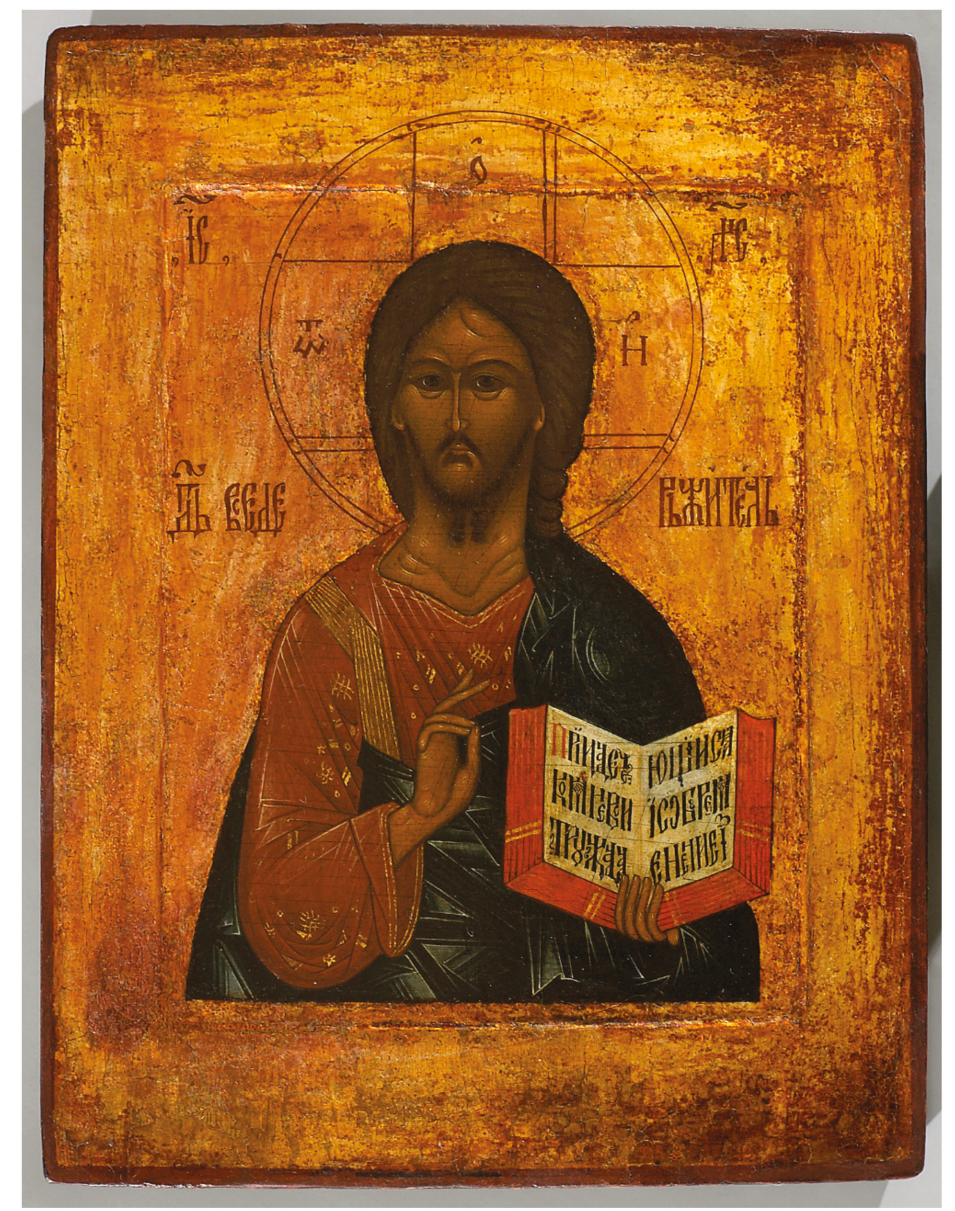

Figure 2. Christ Pantokrator, Russia, workshop of the Old Believers, end of 17th-beginning of 18th c., tempera on wood, $31 \times 24 \mathrm{~cm}$, National Museum in Warsaw, photo: National Museum in Warsaw. 


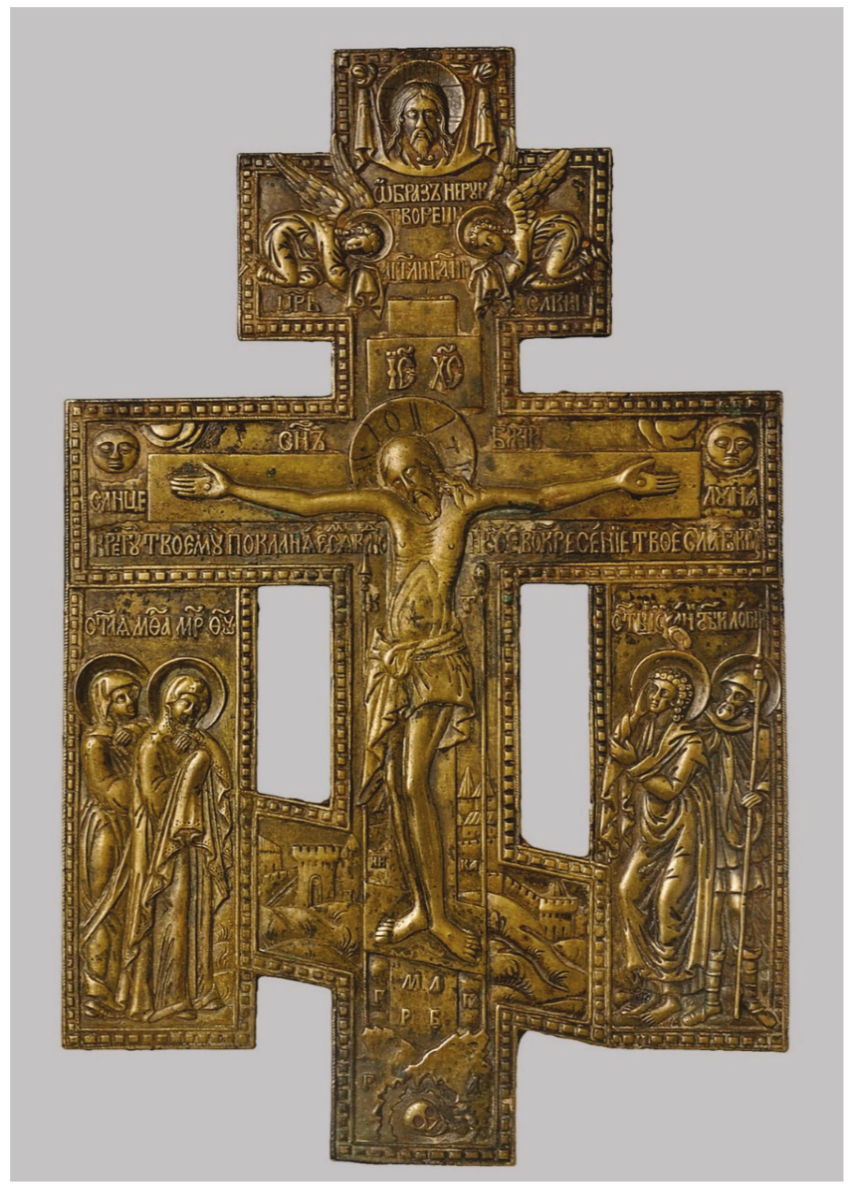

Figure 3. Crucifixion of Christ, Northern Russia, workshop of priestless Old Believers, beginning of 19th c., brass, $23.2 \times 15.1 \mathrm{~cm}$, National Museum in Warsaw, photo: Zbigniew Doliński.

The symbolic day for the new times became the feast of the Triumph of the Orthodoxy, in March 1655-two years after the beginning of the reforms, after the liturgy in the Cathedral of Dormition on the Moscow Kremlin, in the presence of Macarius, the Patriarch of Antioch and Gavrilo, Metropolitan of Serbia, when Nikon gave a homily against icons of the Western style (Zenkovskij 1995, p. 219). Paul of Aleppo, the Syrian archdeacon who travelled to the Eastern Slavs in the middle of the 17th century as a companion of Macarius, in his memoir, The Journey of Patriarch Macarius of Antioch to Russia in the Mid-17th Century, noted that, after the liturgy, Nikon for a long time, spoke about icons, citing extensively the Church Fathers (PAP III 1898, p. 135). The homily was directed against the new kind of icons, based on Western models (the so-called fryazhskye pisma). Finally, the Patriarch ordered that old and new icons be brought-some of them were painted in Moscow workshops inspired by French or Polish paintings and collected from various Moscow houses, "wheresoever they might be, even if it were in the houses of high dignitaries of the state" ${ }^{2}$ to be brought to him (PAP III 1898, p. 136; cf. Andreyev 1961, pp. 40-41; Kozlov 1976, pp. 107-8). Then the new icons were destroyed by the

2 “В какомъ бы домЂ ни находили ихъ, даже изъ домовъ государственныхъ сановниковъ”. 
Patriarch. According to Paul of Aleppo, Nikon took icons "in his right hand, [and] one after another, he showed them to the people" (PAP III 1898, p. 137) and every time, taking an icon, he said: "That icon [was taken] from the house of dignitary [here the name], son of [the name]"3 (PAP III 1898, p. 137). Afterwards, the Patriarch "threw them and smashed them on the floor of the church" (PAP III 1898, p. 137; cf. Lobačev 2003, p. 152). Then Nikon ordered that the remains of the broken icons be burned; however, as a result of the tsar's objection (burning of icons or their parts could be considered a sacrilege), he refrained from that intention. Ultimately, the fragments of the icons were buried (PAP III 1898, p. 137; cf. Zenkovskij 1995, p. 220). Finally, Patriarch Nikon and Patriarch Macarius declared an anathema against those who painted icons "of the Western style" and those who had such icons in their houses ${ }^{4}$ (PAP III 1898, p. 137; cf. Zenkovskij 1995, p. 220). Subsequently, in the summer of 1655, Nikon returned to the issue of new images. He ordered the eyes of the "Western" icons to be gouged out and to be carried by strelts $y^{5}$ throughout the streets of Moscow (PAP III 1898, p. 136; cf. Andreyev 1961, p. 41; Kozlov 1976, pp. 107-8;), who shouted: "From now, whoever paints icons in this way, will be similarly punished" (PAP III 1898, p. 136).

All these actions concerned objects that were widely venerated in Russia. Paul of Aleppo noted that: "All Muscovites are very attached to their icons. They pay no heed to the beauty of the image or the skill of the painter, all icons are the same for them" (PAP III 1898, p. 136; Andreyev 1961, p. 38). According to the Syrian clergyman, in Moscow "in everyone's house there is a countless multitude of icons, adorned with gold, silver and precious stones, ( ... ) and it happens not only at Boyars, but at peasants in the villages, since their love and faith towards the icons is very great"6 (PAP III 1898, p. 32; cf. Tarasov 2002, p. 38). He further noted: "in each cell there is an iconostas with images, and not only within, but also outside, above the door, even above the staircase door, for such is the custom among the Muscovites, that they hang icons on all doors of their houses, their cellars, their kitchens and their store-rooms" $^{7}$ (PAP II 1897, p. 150; cf. Tarasov 2002, p. 39). The 17th century is noted as a time for the creation of many high-quality and sophisticated icons were created. Brjusova referred to the icons of that time as the art of "small form", intended for private devotion (Brjusova 1984, p. 12), such as icons from the so-called Stroganov school (Brjusova 1984, p. 16-59). The icons of the Western style were present and venerated not only in Muscovite houses, but also in the churches. However, it was against private icons that the Patriarch directed his speech and actions in the first years of his rule.

It is very possible that Nikon's stand against domestic icons was the reason why for many believers his acts against icons were understood as the renunciation of religion or as an iconoclastic offense (Dab-Kalinowska 1990, p. 11). The icons had been humiliated by Patriarch Nikon, and Paul of Aleppo believed that soon after that incident Moscow suffered an epidemic punishment (PAP III 1898, p. 136; Lobačev 2003, pp. 152-55). Moreover, at the beginning of August 1655, the Muscovites saw a solar eclipse (PAP III 1898, p. 136). For the Russians, Nikon's treatment of icons was "a big sin" (PAP III 1898, p. 136) - the plague and eclipse of the sun were understood as signs of God's wrath (PAP III 1898, p. 136). The fact that the domestic icons were the main subject of oppression was particularly painful and severe and could be treated as an element of repression that was turned towards opponents of the new order in the Church.

The leader of the movement that rejected Nikon's changes, archpriest Avvakum, spoke out sharply against the reforms of the official Church, but also against the icons of the new style. In his famous

\footnotetext{
“Эта икона изъ дома вельможи такого-то, сына такого-то”.

“Патріархи предалианафемЂ иотлучилиот Церкви и тЂхъ, кто станеть изготовлать подобныеобраза, и тЂхъ, кто будетъ держать ихъ у себя".

Streltsy were the units of firearm infantry.

6 “У всякаго въ домъ имъется безчисленное множество иконъ, украшенныхъ золотомъ, серебромъ и драгоцънными каменьями, ( . . ) и это бываетъ не уоднихъ бояръ, но и у крестьянъ въ селахъ, ибо любовь и вЂра ихъ къ иконамь весма велики".

7 “Въ каждой кельъ есть иконостасъ съобразами, и не только внутри, но и снаружи надъ дверью, даже надъ дверью лЂстницы ибо таковъобычай у москвитовъ, что они вешаютъ иконы на всъхъ дверяхъ своих домовъ, подваловъ, кухонь и лавокъ".
} 
statement On the Holy Icons he criticized "Western" icons and their sensual character. According to Avvakum, in the new icons the Savior is "painted with the plump face, red lips, curly hair, fat arms and muscles, thick fingers and likewise the legs with thick hips, and altogether [they] make him look like a German, bigbellied and fat" (Pustozerskaja proza 1989, p. 101; cf. Andreyev 1961, p. 43). Avvakum called such images "heretical" or "pagan", denying "old tradition" (Pustozerskaja proza 1989, pp. 101-2; cf. Crummey 1970, p. 13) and deemed them unworthy of devotion and ritual bows. In some sense, his views were similar to those of Nikon. Both of them objected to the icons of the Western manner and "German tradition" (Pustozerskaja proza 1989, p. 103). In the middle of the 17th century both Nikon and Avvakum were opposed to the new trends in contemporary Orthodox painting and were not interested in "beauty" of the icons but in their "truthfulness" (Andreyev 1961, pp. 37-38). In his homily On the Holy Icons, Avvakum commands that such icons be ignored: "better pray to heaven or to the East, but do not pray in the front of the icons like that"8 (Pustozerskaja proza 1989, p. 103). However, for Avvakum and other Old Believers, destruction of images was unacceptable. After the events of 1655, when Nikon authorized the destruction of icons, a large number of Moscow's citizens, and in particular the Old Believers, began to think that he was behaving like an iconoclast. It is noteworthy that Nikon's actions had been directed against domestic icons, which became "martyrs" of his reforms, even if they were far from the Orthodox canon. Owing to the enormous importance of icons for the Russians, their "martyrdom" contributed to the increase of aversion to Nikon's reforms and ideas.

As early as the end of the 17th century, there were two factions of the Old Believers: those who recognized priests (popovtsy), and those who became priestless (bespopovtsy) with subsequent divisions occurring over time (Zenkovskij 1995, pp. 424-85). The icons of different groups varied from each other, sometimes in a very significant way. Icons of bespopovtsy from the North of Russia or present-day territories of Belarus, Estonia, Latvia, Lithuania and Poland are more traditional, archaic and "ascetic" and icons of popovtsy, both from the Polish-Lithuanian Commonwealth (Vetka) and the Urals are more innovative, lighter-toned and decorative, and sometimes even based on Western prints. Despite these differences the attitude to the icons among all the Old Believers remained the same, as well as writings about icons read by different factions of the faithful. Old Believer sources, Diakonovy otvety (Diakon's Answers: ОтветыАлександра диакона на Керженце, 1719 (Figure 4) and Pomorskye otvety (The Pomorian Answers: Ответы пустынножителей на вопросы иеромонаха Неофита, 1723), the most important texts of the Old Belief, which can be understood as their "declarations of faith", contain fragments about icons and their veneration (Staroobrjadčestvo 1996, pp. 87, 228-29; Sulikowska 2004, p. 36), but do not define precisely either an "Old Believer icon" or "Nikonian icon". Diakonovy otvety contains some brief notes about icons and listed examples worthy of veneration, namely those "painted by Apostle Lucas" (Mother of God of Vladimir and of Tikhvin) and others, on which the monogram of Christ had a form: IC XC (Otvety Aleksandra 1906, p. 86). The text criticized "new kinds of icons", based on "Latin patterns" (Otvety Aleksandra 1906, p. 88), as sensual and indecent, because they showed holy women without headscarves and with tanned hair (Otvety Aleksandra 1906, p. 89). The more extensive chapter On the Holy Icons from Pomoskie otvety opens with a critique of novelties introduced into icon painting by Patriarch Nikon. According to the source "Nikon changed icon painting", which was previously protected by the Orthodox Church (Pomorskye otvety 1911, p. 340). It contains references to acts of the Moscow Council of a Hundred Chapters (1551) and much older sources, such as the acts of the council in Trullo, the writings of Simeon of Thessaloniki, as well as more recent accounts-the writings of Patriarch Joakhim. They all recommend painters to refer to "old good models" (Pomorskye otvety 1911, pp. 341-42; Sulikowska 2004, p. 36). Neither Diakonovy otvety nor Pomoskie otvety make a distinction between private icons and those belonging to the community.

8 “И ты на небо, на Восток, кланяйся, а такимобразом не кланяйся”. 

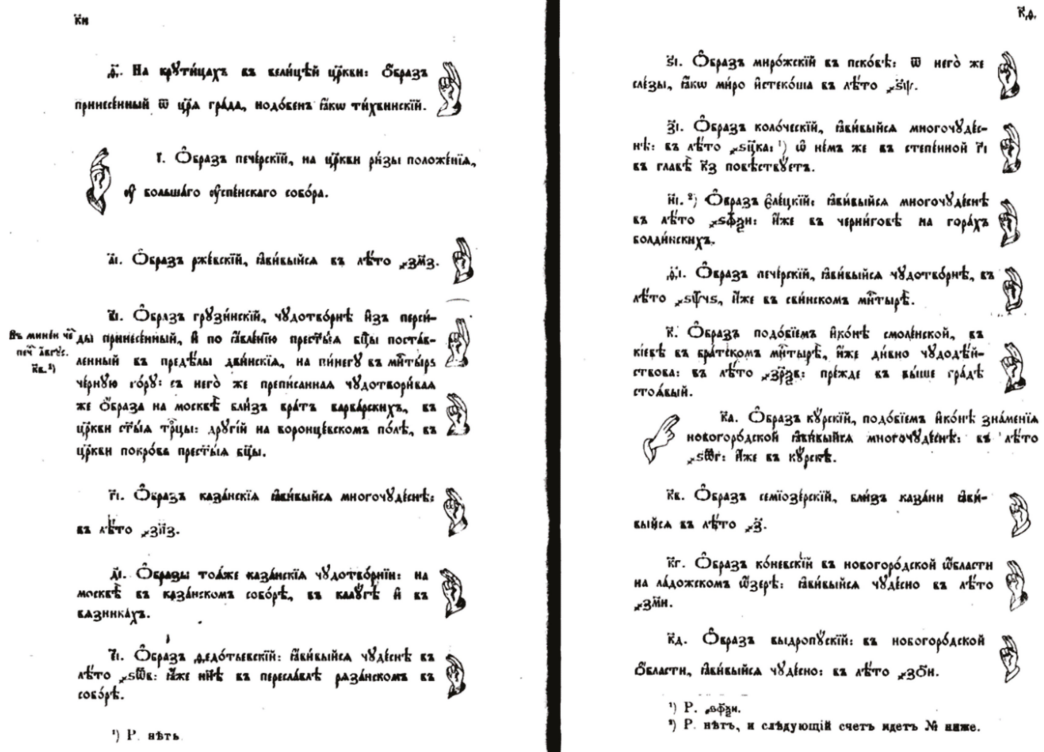

Figure 4. Diakonovy otvety (ОтветыАлександра Діакона, 1719), Бесплатное приложение к журналу“Старообрядец” [Besplatnoe priloženie k žurnalu “Staroobrjadec”], 1906, pp. 30-31.

\section{Domestic Icons in the Space of the Church}

In June 1657 tsar Aleksey Mikhailovitch decided to establish a special committee to deal with the issue of domestic icons (Kämpfer 1982). However, this also concerned private icons ${ }^{9}$ that, for various reasons, were placed in churches (Kotkov 1974, p. 296). At the time, the icons were increasingly taken from the churches to houses ${ }^{10}$ on a massive scale (Kotkov 1974, p. 309), but they were often later they were restored to their original location. Sometimes icons were taken from the church to the house for a special occasion and then they were given away. It also happened that metal covers were taken from these icons by people from the lower classes (Kotkov 1974, pp. 302-3; cf. Kämpfer 1982). The anxiety of the authorities rose. Those who were interrogated could not or did not want to say what the purpose of these actions was and whether someone was urging them to do so ${ }^{11}$ (Kotkov 1974, pp. 309, 311; cf. Kämpfer 1982). The investigation focused on private icons and, despite the ambiguities of the events, it seems that all these actions were related to Nikon's reforms. Already in 1655, a similar controversy had arisen, when Patriarch Nikon ordered that private icons be removed from the western walls of churches. People brought them to the church and prayed to them, addressed them, and placed them on the western wall, not towards the iconostasis (Dab-Kalinowska 1990, p. 12).

This pious practice is mentioned by travelers who visited Russia in the mid-17th century, e.g., the ambassador Augustine Mayerberg, who noticed the custom of bringing private icons to the church and turning to them (with one's back to the altar) during the service (Putešsstvie v" Moskoviju 1874, p. 171). Nikon decided that the presence of private icons in the church was inappropriate, because the people bowing to their icons led to a messy and disrupted the liturgy. However, the custom proved to be lasting. The acts of the Holy Synod of 1723 document the continuity of these practices, as they mention

\footnotetext{
“Иконы свое моление".

"И увидели де как несут из цркви шбразы по домом"

“А по какому указу кто из цркви выносил шбразы того де мы не въдаем”; “Мы де не видали каконе ис цркви бжие млсрдие ікон свое моление выносили и длњ чево хто будет иконы выносил і мы того не слыхали”.
} 
brass icons brought from houses (ikony chastnykh lic ${ }^{12}$ ) to churches (Polnoe sobranie postanovlenij 1875, p. 31). These customs in the 18 th century still escape straightforward interpretations. However, they seem to illustrate the situation of tension between the public and private spheres of worship, which the authorities intended to control. The goal of the official Church from the time of Nikon was to introduce "Byzantine" order in the Church, which was undermined by the faithful approach to their private icons.

It can be presumed that the bringing of private icons to the church and keeping them there for some time strengthened their sacred status. However, it could also be due to the opposite conviction that the space of the church itself gains something though the presence of these domestic icons. Private icons were symbols of an old tradition and had additional, sentimental family value. This surely resulted from the question of understanding hierarchy and sanctity. It is also worth noting that the official Church was reluctant to support such practices. The moving of icons between the house and the church in official Orthodoxy, and which took place in the 17th and even in the 18th century, has an analogy in the practice of the Old Believers, who began collecting domestic icons in their prayer houses. This could be due to practical reasons. The accounts of foreigners relate that Russians did not go to churches very often and that private devotion was central to their religious practices (Bushkovitch 1992, p. 48), and they almost never parted with their private icons (Brjusova 1984, p. 11). According to Domostroy, written after the middle of the 16th century, in every Russian house there were at least a few icons and wealthy people would have a whole iconostases in their homes (PLDR 1985, pp. 76-77; Brjusova 1984, p. 11), which is why in Eastern Slavdom the number of domestic icons was very large. Domostroy recommends: "Each Christian must, in all the rooms, hang by seniority holy images, adorning them beautifully, and place light-holders in which candles are to be lit during prayers in front of the holy images, and are then extinguished after the service, are covered with a curtain for the sake of cleanliness from dust, for correct order and for their preservation"13 (PLDR 1985, pp. 76-77; Tarasov 2002, pp. 38-39; cf. Kaiser 2011, p. 126). An earlier 16th-century account of Antonio Possevino, about the Russians who came to Italy, confirms the importance of private icons: "While we were staying with the Governor of Arimini, the Muscovites removed the holy images and set up tiny ikons of their own, painted in the Muscovite manner" (Possevino 1977, p. 24). As we can see, the Russians, having embarked on a journey, had their icons with them. Possevino noticed not only that "every single Muscovite wears a cross on his breast" (Possevino 1977, p. 40), but also that Russians "consider it a disgrace to confuse [a] holy ikon with profane articles of clothing" (Possevino 1977, p. 40). This principle was always maintained by the Old Believers.

However, for the Old Belief, because of the situation of believers, the icons could change their meaning. Avvakum in his Life mentions long, even all-night long and full-of-tears ("until eyes were swollen") prayers in front of icons. The prayers were motivated by the fear of the world, fear of death, unclean thoughts, sexual temptations and sins, and almost always were directed to domestic, personal icons (Žitie protopopa Avvakuma 1979, pp. 22, 23). Avvakum suggested also to the believers, who had no other option, for their cult to be concentrated around their domestic icons: "If you do not find a priest at last $(\ldots)$, if you are traveling or whatever happens. ( . . L Light the candle in front of the icon of the Saviour and cover the box with a handkerchief, pour a little water into a small pot and take a spoon with a piece of the body of Christ with a prayer, incense all and cry ( ... ). Then bow to the front of the icon, pray for forgiveness and stand, kiss the icon, cross yourself, receive communion with a prayer ( ... ). Even if you die imminently, it will be good"14 (Žitie protopopa Avvakuma 1979, pp. 37-38). This

12 “Иконы частныхъ лицъ".

13 “В дому своемъ всякому християнину во всякой храмине святыя и честныяобразы написаны на иконахъ, по существу ставити на стенах, устроив благолЂпно со всякимъ украшениемъ, и со светилники, въ них же свъщи пред святымиобразы возжигаются на всякомъ славословии божии, и по пЂнии погашают, завБсою закрывются всякия ради чистоты иот пыли, благочиния радии и брежения".

14 “Аще священника, нужды ради, не получиш (.. ), аще в пути или на промыслу ( . . ) предобразом Христовым на коробочку постели платочик и свечку зажги, и в сосуде водицы маленько, да на ложечку почерпи и часть тела 
passage shows that the concept of icons was formulated by the first generation of the Old Believers. Icons played a symbolic role in domestic devotion creating the space where the faithful could enact the full liturgy modelled on their experience of church. For the societies of bespopovtsy, who had no clergy, the role of icons would be more important (Zenkovskij 1995, pp. 438-66; cf. Staroobrjadčestvo 1996, p. 47), and icons became witnesses of the prayers of the faithful and defined the boundaries of the sacred sphere. The sacred was spatially bound to the icons.

\section{Icons in Old Believer Communities: Private and Public}

All groups of the Old Believers migrating from place to place, took their own icons. Nicolai Leskov, a 19th-century Russian writer, to whom we owe accurate descriptions of Old Belief culture, in his great novel The Sealed Angel wrote: "We travelled about from job to job ( . . ) just as the Hebrews of old wandered in the wilderness with Moses, and we even had our own tabernacle with us and we were never parted from it. I mean to say that we took our own "blessing from God" along with us" (Leskov 1984, p. 8). In addition, then: "We all loved these holy things of ours with a passionate love, and together we burned the holy oil before them. In addition, at the expense of our whole group we kept the horse and a special vehicle in which we took this blessing from God along with us in two large trunks wherever we went" (Leskov 1984, p. 8). It is worth noting that the Old Believers were persecuted by both the Russian state and the official Church. For this reason, the Old Believers rejected the both tsar's decision and church law, and private icons, along with books, became a link with tradition.

From the mid-17th century the Old Believers migrated to the peripheral areas, increasingly distant from the power of Church and tsar (Robson 1995, p. 23-24). All groups met with repression, which also concerned icons used by the Old Believers. The period of group migrations could be the time when icons changed their status from being private icons to being the belonging to the entire community. According to the oral tradition of the Old Believers, if the icons were requisitioned from the molenna by officials, people brought their own icons to the church, as a gift for all the community (vkladnye $\left.i k o n y^{15}\right)$. In the Pokrov cathedral on the Rogozhskoye Cemetery, one of the largest centers of priested Old Believers, more than 400 icons from the 14th-18th centuries came from the private houses (1956, p. 7). Moreover, the faithful in Vetka and other centers continued the tradition of donated (vkladnye) icons (Nečaeva 2002, p. 49). Private icons in Old Believers' molennas were included in the already existing iconographic program, but this one was usually quite imprecise.

Mobility of communities caused also the spread of small, portable metalwork objects: icons, triptychs and crosses, which could be easily moved from place to place (Karpenko 2006, p. 5). On the 31st of January 1723 the Holy Synod prohibited the use of brass icons (Polnoe sobranie postanovlenij 1875, p. 31; Dąb-Kalinowska 1990, p. 73; Karpenko 2006, p. 5). As Synod noted “domestic brass icons" had become very common all over Russia, in many cathedrals and parish churches, and they were to be taken away from there back to the homes of their owners (Polnoe sobranie postanovlenij 1875, p. 31). In the beginning of the 18th century the Holy Synod also received several denunciations related to non-canonical icons, i.e., "icon of the Saviour with three hands", "sepulcher icon of St. Nicholas" (Opisanie dokumentov II 1878, p. 440) and also "the Old-Rite icons" in Russian churches (Opisanie dokumentov II 1878, p. 440). Icons and other sacred objects i.e., lestovki, podruchniki (Sulikowska 1998, p. 45) used by the Old Believers clearly differed from those of the official Church—to such an extent that for the controlling officials they could be indications that some community practised the Old Rite (Sbornik pravitel'stvennyh svedenij 1861, pp. 3-4). Already in the 19th century private Old Believers' icons, as well as their private chapels were eliminated or, at least, considered suspicious (Crummey 1970, p. 216; Shvezov 2004, pp. 19, 128).

Христова с молитвою в воду на лошку положи и кадилом вся покади, поплакав, глаголи (... ). Потом падше на землю предобрвазом прощение поговори и восставобразы поцелуй и, прекрестясь, с молитвою причастися ( ... ). Хотя и умрешь после тово, ино хорош".

15 "Вкладные иконы". 
Because of their life amidst hiding and persecutions, the Old Believers could not fully develop in the public sphere. Relations between members of the community were usually very close and they concerned not only the religious sphere, but also family and economic relations (Sbornik pravitel'stvennyh svedenij 1861, p. 33). According to the oral Old Believers' tradition, because of persecutions they were forced to pray in secret, like the early Christians, in private houses, even in forests and caves (Iwaniec 1977, p. 150). Their churches in Imperial Russia were usually small and unobtrusive, often located in private houses or even chambers (Iwaniec 1977, p. 150), not only due to the state regulations (Ogarev 1862, pp. 21, 38; Shvezov 2004, pp. 101-2; cf. Robson 1995, pp. 51-57), but also because of the established traditions of the group.

Icons were stored in the molennas and in the private houses, always kept in designated spaces for the sacred items: icons, crosses, books and others (cf. Robson 1995, pp. 76-77). Krasny ugol, understood as the main "beautiful" corner of the room, could be named the "domestic iconostasis" and also "High Jerusalem", or even "window to Heaven" (Tarasov 2002, p. 39; Kaiser 2011, p. 137). It was a "domestic church", the center of the chamber and the center of the Old Believers' house (Sulikowska 1998, p. 45), the model for which was the monastery (cf. Zabelin 1992, p. 141). In the houses of the wealthy Old Believers, they sometimes became very large and were composed of many icons, very similar to those in molennas.

The image of the world of the Old Believers was based on models of the Muscovite culture of the 16th and 17th century. That model assumed a division of the world into sacred and profane parts. The way of life was based on Domostroy, which calls a house a temple, where all the family should pray, standing in the front of the icons (PLDR 1985, pp. 76-79; cf. Bushkovitch 1992, p. 48). This also concerned the status of icons and the popularity of icons of small format, i.e., domestic icons. In rich houses krasny ugol may even turn into a domestic church (Tarasov 2002, p. 39). For the Old Believers, the presence of icons sanctified the members of the community (Sulikowska 1998, p. 45).

It seems that among Old Believers' domestic icons, some topics appeared exceptionally often. According to the tradition, a typical krasny ugol contained the cross (Crucifixion of Saviour (Figure 3) and usually images of the Mother of God as a "woman icon" (Figures 5 and 6) and Christ as a "man icon" (Figure 2) (Nečaeva 2002, pp. 85-86). An image of the Savior could be replaced by the icon of St. Nicholas the Miracle Worker (Figure 7). The icons of Christ usually depicted him as the Pantokrator, whilst the icons of St. Nicholas pictured him blessing, with the book and flanked by the images of Christ with the book and the Virgin with the omophor. The images of the Mother of God varied, although most often they were images mentioned in Diakonovy otvety (Figure 4) and Pomorskye otvety. They are icons of Vladimir, of Tikhvin, of Blachernae, of the "Sign", of Jerusalem, of Peter, of Smolensk, of Theodor (Figure 6), of Kazan (Figure 5), of Pechersk, of Svensk and many others (Otvety Aleksandra 1906, pp. 25-42; Pomorskye otvety 1911, pp. 31-51). Typical for private devotion are icons with an image of the Guardian Angel (Figures 6 and 7). Many of these icons belonged to the most popular images of the Virgin venerated in Russia before the middle of the 17th century, especially four of them-Vladimir, of Tikhvin, of the "Sign", of Smolensk. They were copies of the icons playing the roles of palladia-in religious and political life and were popular especially before the mid-17th century (Bushkovitch 1992, pp. 100-27). Both the 18th-century popovtsy books (i.e., Sbornik Mokhovikova or sbornik from the village Perevoz) and their icons (Boeck 2018, pp. 38-40; Pozdeeva 1975, pp. 57-61) reflect the dominance of Marian images. Among the Old Believers there were also icons of unusual iconography i.e., Archangel Michael, Archistrategos of Heavenly Hosts (Figure 8), the Mother of God Looks like Fire (Ognevidnaya) (Figure 9) and other inspired by the Apocalypse. Most of the Old Believer icons are small-sized domestic icons, and even "great topics", i.e., the Resurrection and the Twelve Great Feasts of the Church, Pokrov (Figure 10) or Hexaemeron were conceived to fit the reduced scale. Preservation of old icons, or painting them in an old way, was important for the communities, as a way of preserving their old tradition. 


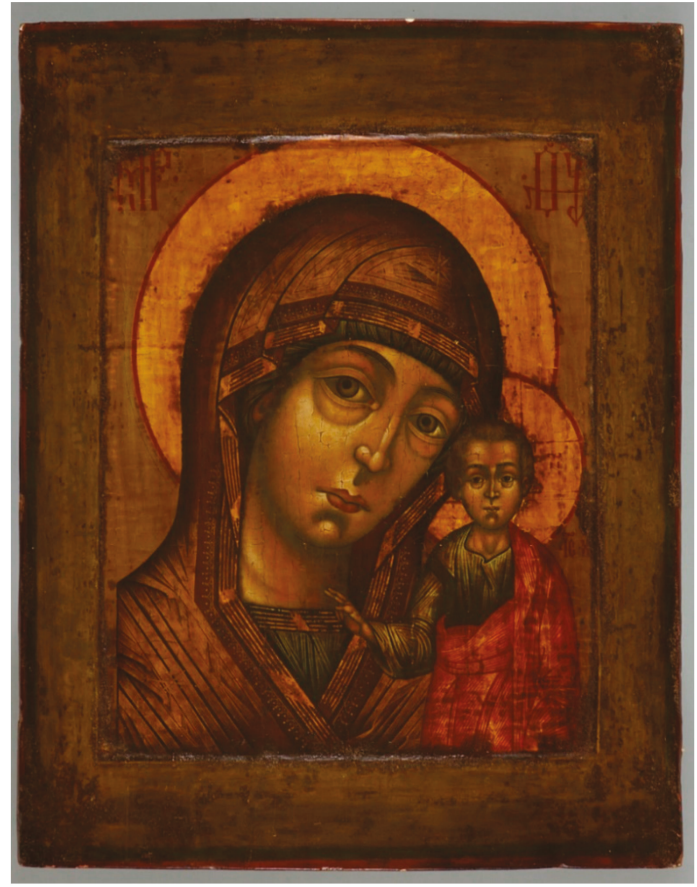

Figure 5. Mother of God of Kazan, Northern Russia, workshop of the Old Believers, beginning of 18th c., tempera on wood, $36.3 \times 28.7 \mathrm{~cm}$, National Museum in Warsaw, photo: Zbigniew Doliński.

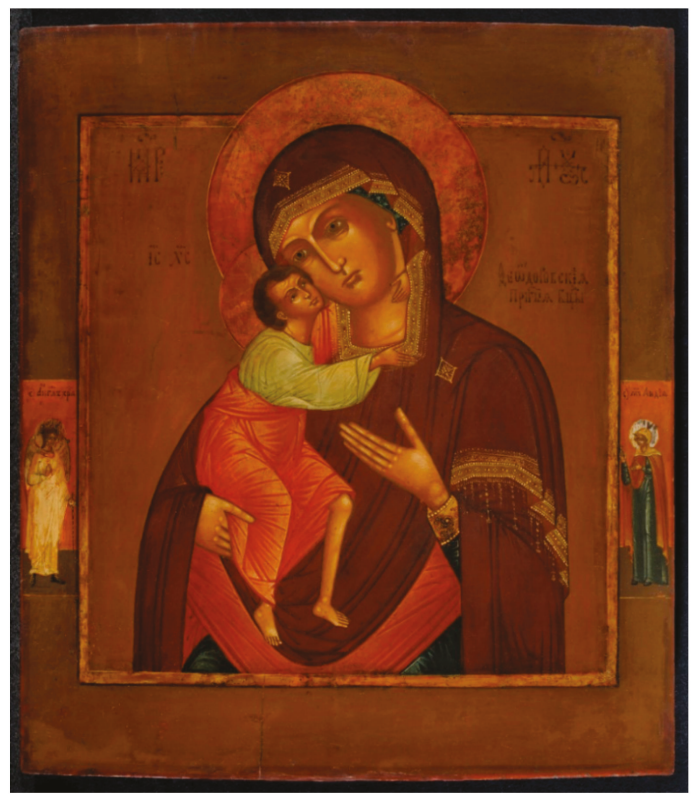

Figure 6. Mother of God of Theodor, Russia, workshop of the Old Believers, end of 18th c., tempera on wood, $35 \times 31.2 \mathrm{~cm}$, National Museum in Warsaw, photo: Małgorzata Kwiatkowska. 


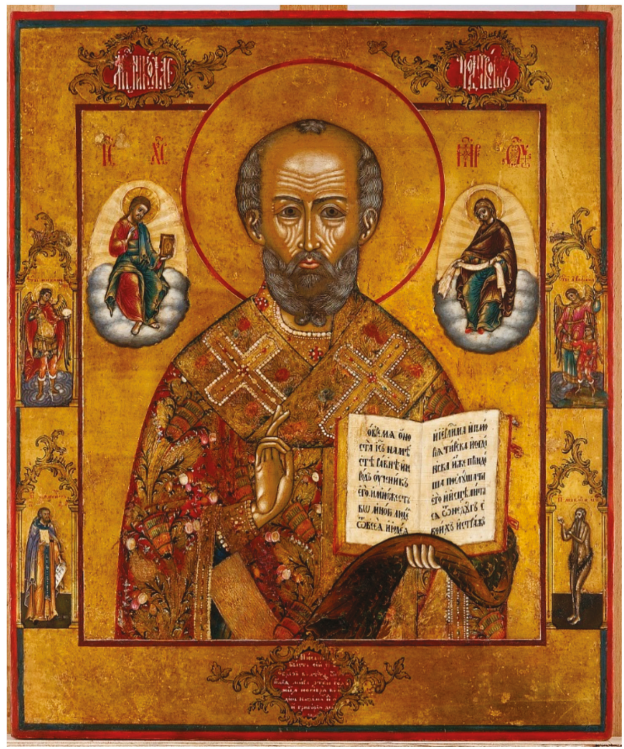

Figure 7. St. Nicholas Miracle Worker, Russia, Nevansk, workshop of the Old Believers, 1820, tempera on wood, $33.8 \times 27.9 \mathrm{~cm}$, National Museum in Warsaw, photo: Małgorzata Kwiatkowska.

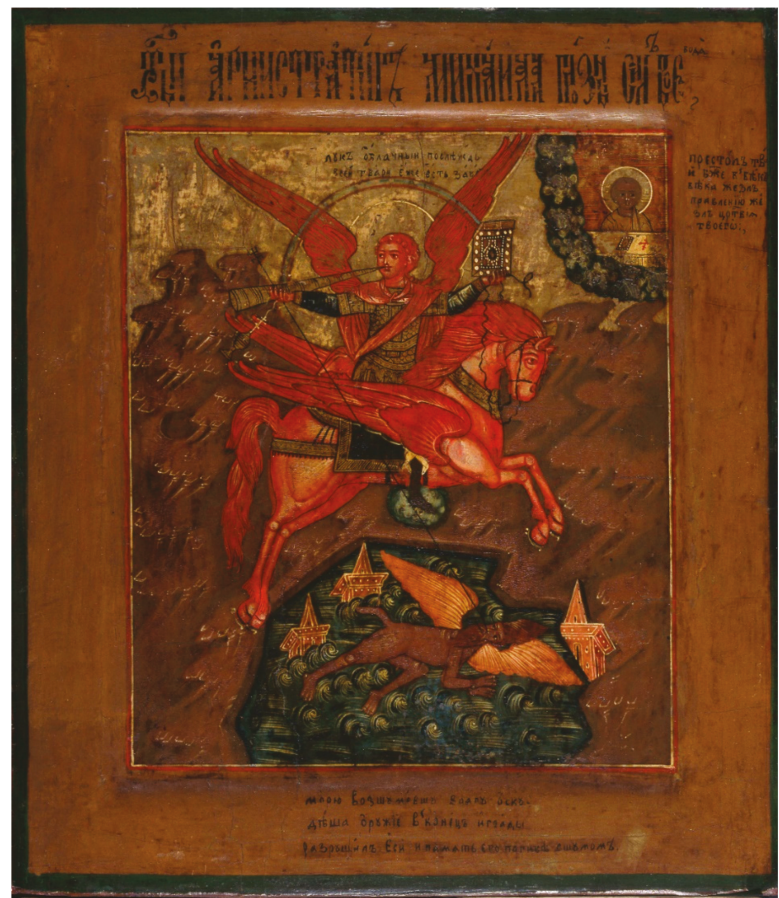

Figure 8. Archangel Michael, Archistrategos of Heavenly Hosts, Northern Russia, workshop of priestless Old Believers, beginning of 18 th c., tempera on wood, $42.5 \times 37$, National Museum in Warsaw, photo: National Museum in Warsaw. 


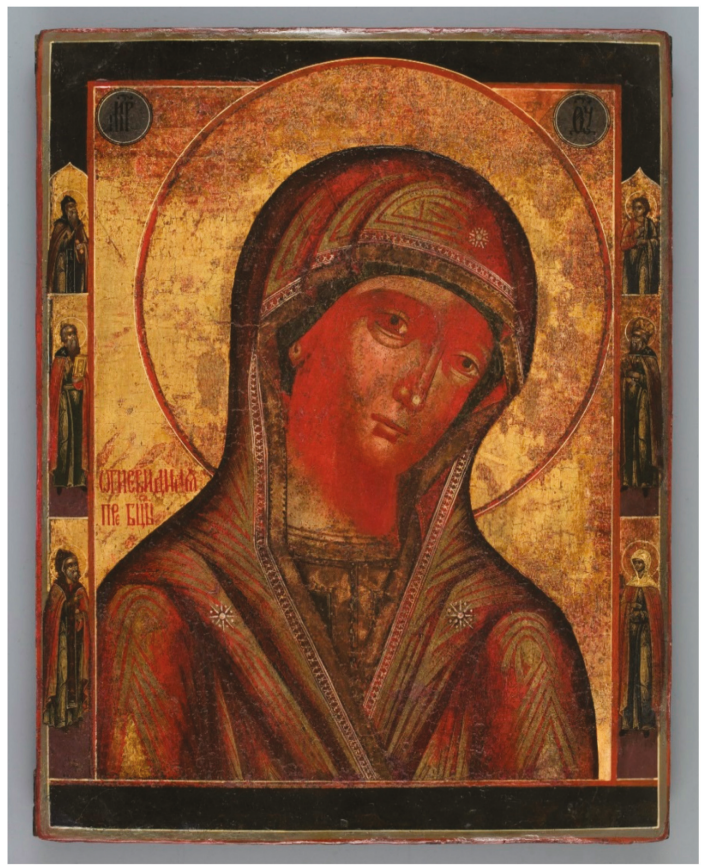

Figure 9. Mother of God Looks like Fire (Ognevidnaya), Belarus, Vetka, workskop of priest Old Believers, beginning of 19th c., tempera on wood, $35.5 \times 27.6 \mathrm{~cm}$, National Museum in Warsaw, photo: Piotr Ligier.

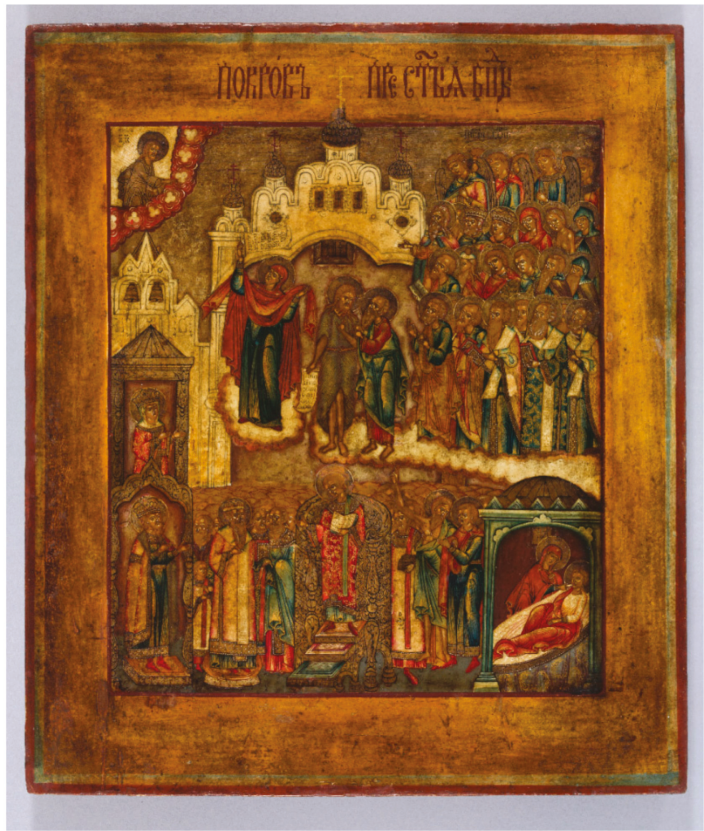

Figure 10. Pokrov of Mother of God, Russia, Volga region, workshop of the Old Believers, 18th c., tempera on wood, $31.5 \times 27 \mathrm{~cm}$, National Museum in Warsaw, photo: Krzysztof Wilczyński. 
Is it possible to compare religious customs of the Old Believers to Byzantines or Russians before and after the mid-17th century? In Byzantium, as Henry Maguire wrote, "it is difficult to distinguish between a private as opposed to a public cult of the Virgin as reflected in the material culture ( . . ) , because objects could move from one sphere to the other" (Maguire 2000, p. 279), and as a private cult he considered mainly small personal items such as jewelry or pectoral icons and crosses (Maguire 2000, pp. 279-88). Paul Bushkovith noticed that in Russia the 16th and 17th centuries brought changes that "reflected a shift in religious experience from one basically public and collective, which stressed liturgy and miracle cults, to a more private and personal faith" (Bushkovith 1992, p. 3). It seems that among the Old Believers after the mid-17th century the private sphere became a particularly sacred realm. Some instructions of the Russian Holy Synod, i.e., about the domestic brass icons, point to the common problems in Russia, not only in Old Believer communities (Polnoe sobranie postanovlenij 1875, p. 31 ). It seems that objects could move also in the Russian tradition, as well as in that special tradition of the Old Believers. However, in the latter's case, the migration of icons arose as a result of religious, political and social circumstances, which derived from the problems faced by the Old Believer communities.

\section{Conclusions}

It seems that neither priested and priestless Old Believers did not clearly distinguished between domestic and "public" icons. Small-scale icons were used not only in private devotion but also in collective rituals. A typical practice was to move domestic icons and place them not only on analogia, but also in an iconostases (on the western wall of the molenna). This was not because the Old Believers did not recognise the difference between the two spheres, but rather because their godliness was not public, but private. Among bespopovtsy even molenna - as a prayer house-played the role of a private space. The attitude of the Old Believers towards icons resulted from the Moscow tradition of the 16th and 17th centuries. The period of rule of Patriarch Nikon and his actions against icons led the Old Believers to perceive icons as martyrs for the "old rite". In the second half of the 17th century, personal religious experience may have been considered sufficient and appropriate for the pious life. However, Old Believers' normative writings in the beginning of the movement did not connect their identity with specific kinds of devotion, either private or public. They also did not postulate a distinction between the official Church and Old Believers' icons. This distinction resulted only from the "modernization" of the official art, which took place from the time of Peter I (1682-1725) onward. The Old Believers rejected Western-style icons and instead valued highly icons based on the 17th-century art of the "small form" from the Stroganov, Moscow or Povolzhe schools, intended for private devotion. Towards the end of the 17th and in the first half of the 18thcentury the situation of the Old Believers confirmed their attachment to small, old or old-looking icons, crosses and other religious artefacts. The dominant role of domestic icons was associated with the situation of migrating, hiding and separated groups. Staying in the private sphere allowed the Old Believers to remain outside the control of the state and the official Church. The presence of "icons of the ancient piety" or "icons of the fathers" gave a guarantee of ties with the old tradition.

Funding: This research received no external funding.

Conflicts of Interest: The author declares no conflict of interest.

\section{References}

\section{Primary Sources}

Leskov 1984. Leskov, Nikolay. The Sealed Angel and Other Stories. Ed. and transl. K. A. Lantz. Knoxville: The University of Tennesse Press. 
Kotkov 1974. Котков, Сергей И. Московская реч в начальный период становления русского национального языка. Москва: Наука. [Kotkov, Sergej I. Moskovskaja reč v načal'nyj period stanovlenija russkogo nacional'nogo jazyka. Moskva: Nauka.]

Opisanie dokumentov II 1878. Описание документов и дел, хранящихся вАрхивеСвятейшего Правительствующего Синода. Т. II, ч. 2 (1722 год). СанктПетерсбург: Синодальная Типографія. [Opisanie dokumentov i del, hranjaŝihsja v Arhive Svjatejšego Pravitel'stvujuŝego Sinoda. T. II, č. 2 (1722 god). SanktPetersburg: Sinodal'naja Tipografija.]

Opisanie dokumentov IV 1880. Описание документов и дел, хранящихся вАрхивеСвятейшего Правительствующего Синода. Т. 4 (1724 год), СанктПетерсбург: Синодальная Типографія. [Opisanie dokumentov i del, hranjaŝihsja v Arhive Svjatejšego Pravitel'stvujuŝego Sinoda. T. 4 (1724 god). SanktPetersburg: Sinodal'naja Tipografija.]

Otvety Aleksandra 1906. ОтветыAлександра Діакона (на Керженце) поданныя НижегородскомуепископуПитириму въ1819 [a mistake in the title; it should be: 1719] году. Бесплатное приложение к журналу“Старообрядец”. [Otvety Aleksandra Diakona (na Keržence) podannyja Nižegorodskomuepiskopu Pitirimu v" 1819 [a mistake in the title; it should be: 1719] godu. Besplatnoe priloženie k žurnalu "Staroobrjadec".].

PLDR 1985. Памятники литературы Древней Руси, т. 7. Середина XVI века. Ред. Л. А. Дмитриева, Д. С. Лихачева. Москва: Художественная литература. [Pamjatniki literatury Drevnej Rusi, t. 7. Seredina XVI veka. Red. L. A. Dmitrieva, D. S. Lihačeva. Moskva: Hudožestvennaja literatura.].

Possevino 1977. The Moscovia of Antonio Possevino SJ. Transl. H. F. Graham. Pittsburg: University of Pittsburg Press.

Polnoe sobranie postanovlenij 1875. Полное собраніе постановленій и распоряженій по въдомствуПравославнагш ИсповъданіА Россійской Имперіи, т. III, 1723. СанктПетерсбург: Сvнодальная Типографія. [Рolnoe sobranie postanovlenij i rasporjaženij po vedomstvu Pravoslavnago Ispovedania Rossijskoj Imperii, t. III, 1723. SanktPetersburg: Sinodal'naja Tipografija.].

Pomorskye otvety 1911. ПоморскїеотвЂты. Напечатаны съ подлинника. Москва: ТипографїяП. П. РАбушинскаго. [Pomorskye otvety. Napečatany s" podlinnika. Moskva: Tipografija P. P. Ryabušinskago.].

PAP II 1897. ПутешествіеАнтіохійскаго Патрі $a$ рха Макарія въ Россію въ половинъХVII вЂка описанное его сыномъархидіакономъПавломъАлеппскимъ. Пер. Г. Муркоса, т. II. Москва: Унверитетская Типографія. [Putešestvie Antiohijskago Patriarha Makarija v" Rossiju v" polovine XVII veka opisannoe ego synom" arhidiakonom" Pavlom" Aleppskim". Per. G. Murkosa, t. II. Moskva: Unveritetskaja Tipografija.].

PAP III 1898. ПутешествіеАнтіохійскаго Патріарха Макарія въ Россію въ половинъХVII вЂка описанное его сыномъархидіакономъПавломъАлеппскимъ. Пер. Г. Муркоса, т. III. Москва: Унверитетская Типографія. [Putešestvie Antiohijskago Patriarha Makarija v" Rossiju v" polovinie XVII vieka opisannoe ego synom" arhidiakonom" Pavlom" Aleppskim". Per. G. Murkosa, t. III. Moskva: Unveritetskaja Tipografija.].

Putešestvie v" Moskoviju 1874. Путешествіе въ Московію барона Августина Майерберга ( ... ) къ царю и великому князюАлексђю Михайловичу. Москва: Изд. Императорскаго Общества Исторіи и Древностей Россійскихъ при Московскомъ Университетъ. [Putešestvie v" Moskoviju barona Avgustina Majerberga ( . . ) k" carju i velikomu knjazju Alekseju Mihajloviču. Moskva: Izd. Imperatorskago Obŝestva Istorii i Drevnostej Rossijskih" pri Moskovskom" Universitete.]

Pustozerskaja proza 1989. Пустозерская проза. Сост., пред. М. Б. Плюхановой, ред. В. М. Живов. Москва: Московский рабочий. [Pustozerskaja proza. Sost., pred. М. B. Pljuhanovoj, red. V. M. Živov. Moskva: Moskovskij rabočij.]

Sbornik pravitel'stvennyh svedenij 1861. Сборник правительственных свЂденійо раскольниках. Сост. В. Кельсіевымъ, вып. 2. Лондонъ: Trübner \& Co. [Sbornik pravitel'stvennyh svedenij o raskol'nikah. Sost. V. Kel'sievym", vyp. 2. London": Trübner \& Co.].

Žitie protopopa Avvakuma 1979. Житие протопопа Аввакума им самим написанное и другие его сочиниения. Иркутск: Восточно-Сибирское книжное издательство. [Žitie protopopa Avvakuma im samim napisannoe $i$ drugie ego sočinienija. Irkutsk: Vostočno-Sibirskoe knižnoe izdatel'stvo.].

\section{Secondary Sources}

Andreyev, Nicolai. 1961. Nikon and Avvakum on Icon-Painting. Revue des études Slaves 38: 37-44. [CrossRef] 
Boeck, Elena N. 2018. More Numerous Than the Stars in Heaven. An Early Eighteenth-Century Compendium of Mariology. In Framing Mary. The Mother of God in Modern, Revolutionary, and Post-Soviet Russian Culture. Edited by Ammy Singleton Adams and Vera Shvezov. DeKalb: Northern Illinois University Press, pp. 37-57.

Brjusova, Valerija G. 1984. Russkaja živopis' 17 veka. Moskva: Iskusstvo, [Брюсова, ВалерияГ. 1984. Русская живопись17 века. Москва: Искусство.

Bushkovitch, Paul. 1992. Religion and Society in Russia. The Sixteenth and Seventeenth Centuries. New York and Oxford: Oxford University Press.

Crummey, Robert O. 1970. The Old Believers and the World of Antichrist. The Vyg Community and the Russian State, 1694-1855. Madison: The University of Wisconsin Press.

Dąb-Kalinowska, Barbara. 1990. Między Bizancjum a Zachodem. Ikony Rosyjskie XVII-XIX Wieku. Warszawa: Państwowe Wydawnictwo Naukowe.

Drevnye ikony staroobrjadčeskogo kafedral' nogo Pokrovskogo sobora pri Rogožskom kladbiŝe v Moskve. 1956. Red. Vasilij F. Korolev, Kirill A. Abrikosov, Mihail I. Tjulin. Moskva: Staroobrjadčeskaja Arhiepiskopija Moskovskaja i vseja Rusi, [Древные иконы старообрядческого кафедрального Покровского собор $a$ при Рогожском кладбище в Москве. Ред. Василийф. Королев, КириллА. Абрикосов, Михаил И. Тюлин. Москва: СтарообрядческаяАрхиепископия Московская и всея Руси.].

Iwaniec, Eugeniusz. 1977. Z dziejów staroobrzędowców na ziemiach polskich XVII-XX w. Warszawa: Państwowe Wydawnictwo Naukowe.

Kaiser, Daniel H. 2011. Icons and Private Devotion Among Eighteenth Moscow Townfolk. Journal of Social History 45: 125-47. [CrossRef]

Kämpfer, Frank. 1982. Verhöre über des Entfernen von Ikonen aus den Kirchen. Ein Vorgang aus dem Moskau des Jahres 1657. In Unser ganzes Leben Christus unserm Gott überantworten: Studien zur ostkirchlichen Spiritualität: Fairy von Lilienfeld zum 65. Geburtstag. Hrg. v. Peter Hauptmann; Kirche im Osten 17. Göttingen: Vandenhoeck \& Rupchert, pp. 295-302. Available online: http://www.frank-kaempfer.de/Neuer\%20PDF\% 20Ordner/Ikonen-Verh\%F6re\%201657.pdf (accessed on 20 July 2019).

Karpenko, Elena. 2006. "Obraz litoj, starinnyj". Mednoe hudožestvennoe lit'e XII-XX vv. Nacional' nogo hudožestvennogo muzeja Respubliki Belarus'. Minsk: Belprint, [Карпенко Елена. “Образ литой, старинный”. Медное художественное литьеХII-XX вв. Национального художественного музея Республики Беларусь. Минск: Белпринт.].

Kozlov, O. F. 1976. Delo Nikona. Voprosy istorii 1: 102-14, [Козлов, О. ф. Дело Никона. Вопросы истории. № 1: 102-14.].

Lobačev, Sergej V. 2003. Patriarh Nikon. Sankt-Peterbur: Iskusstvo-SPB, [Лобачев, СергейВ. Патриарх Никон. Санкт-Петербург: “Искусство-СПБ”.].

Maguire, Henry. 2000. The Cult of the Mother of God in Private. In Mother of God. Representations of the Virgin in Byzantine Art. Edited by Maria Vassilaki. Athens: Skira, pp. 279-89.

Nečaeva, Galina G. 2002. Vetkovskaja ikona. Minsk: Izdatel'stvo “Četyre Četverti”, [Нечаева, Галина Г. Ветковская икона. Минск: Издательство “Четыре Четверти”.].

Ogarev, Nikolaj P. 1862. Gonenie za veru. Obŝee veče 3. 1 sentjabrja 1862 g [Огарев, НиколайП. 1862. Гонение за веру. Общее вече. № 3, 1 сентября1862 г.].

Pozdeeva, Irina V. 1975. Arheografičeskie raboty Moskovskogo Universiteta v rajone drevnej Vetki i Staroduba (1970-1972 gg.). Pamjatniki Kul'tury. Novye Otkrytija, 52-69, [Поздеева, Ирина В. Археографические работы Московского Университета в районе древнейВетки иСтародуба (1970-1972 гг.). Памятники Культуры. Новые Открытия, pp. 52-69.].

Robson, Roy R. 1993. Liturgy and Community among Old Believers, 1905-1917. Slavic Review 52: 713-24. [CrossRef]

Robson, Roy R. 1995. Old Believers in Modern Russia. DeKalb: Northern Illinois University Press.

Shvezov, Vera. 2004. Russian Orthodoxy on the Eve of Revolution. Oxford: Oxford University Press.

Staroobrjadčestvo. 1996. Lica, sobytija predmety i symvoly. Opyt ènciklopedičeskogo slovarja. Moskva: Cerkov, [Старообрядчество. Лица, события предметы и сымволы. Опыт энциклопедического словаря. Москва: Церков.].

Sulikowska, Aleksandra. 1998. Domowy ikonostas. Ikony staroobrzędowców i kult wizerunków w czasach współczesnych. Konteksty. Polska Sztuka Ludowa LII: 43-45. 
Sulikowska, Aleksandra. 2004. "Katalog" ikon Matki Boskiej w starowierskch źródłach XVIII wieku'. In Ikony. Przedstawienia maryjne z kolekcji Muzeum Narodowego w Warszawie. Edited by Aleksandra Sulikowska. Warszawa: Muzeum Narodowe, pp. 34-41.

Sulikowska-Bełczowska, Aleksandra. 2017. Old Believers and the World of Evil: Images of Evil Forces in Old Believer Art. Ikonotheka 27: 70-91.

Tarasov, Oleg. 2002. Icon and Devotion. Sacred Spaces in Imperial Russia. Translated by R. Milner-Gulland. London: Reaktion Books.

Zabelin, Ivan E. 1992. Domašnyj byt russkih caric v XVI i XVII stoletijah. Novosibirsk: Nauka, [Забелин, Иван Е. Домашный быт русских цариц вXVI иXVII столетиях. Новосибирск: “Наука".].

Zenkovskij, Sergej A. 1995. Russkoe staroobrjadčestvo. Duhovnye dviženija semnadcatogo veka. Moskva: Cerkov, [Зенковский, СергейА. Русское старообрядчество. Духовные движения семнадцатого века. Москва: Церков.].

(C) 2019 by the author. Licensee MDPI, Basel, Switzerland. This article is an open access article distributed under the terms and conditions of the Creative Commons Attribution (CC BY) license (http://creativecommons.org/licenses/by/4.0/). 
Article

\title{
Devotion, Paintings, and the House: The Collections of Ercole and Giuseppe Branciforti, Princes of Scordia (Palermo, 1687-1720)
}

\author{
Valeria Viola \\ Department of History of Art, University of York, Heslington, York YO10 5DD, UK; vv565@york.ac.uk \\ Received: 17 December 2019; Accepted: 8 January 2020; Published: 10 January 2020
}

\begin{abstract}
This paper interrogates familial devotion and its relationship with parts of the house other than the chapel. In detail, it aims to problematize the issue of the devotional/non-devotional use of paintings inside the house by moving the focus from this dual opposition to the active role of canvases, broadly defined. Informed by Jacques Derrida's and Pierre Bourdieu's writings, this paper argues for the structural nature of the paintings inside the house and their meaningful correlation with both the arrangement of the domestic interior and the practices of people experiencing those spaces. To do this, the paper challenges the overwhelming attention paid by early-modern scholars to Northern and central Italy and investigates a precise case study, i.e., Palazzo Scordia in Palermo (Sicily). The research draws upon primary sources and amongst these, upon two detailed inventories of furniture referring to two subsequent generations of an aristocratic clan residing in Palermo between the seventeenth and the eighteenth century, i.e., Ercole and Giuseppe Branciforti, princes of Scordia.
\end{abstract}

Keywords: domestic devotion; Palermo; Spanish Sicily; aristocratic palaces; collections of paintings; catholic devotion; Bourdieu's habitus

\section{Introduction}

The theme of domestic devotion in the early modern age has received increasingly wide-ranging attention in the last few years. ${ }^{1}$ Scholars have explored both domestic spaces exclusively designated for devotion, such as chapels and oratories (Hirschboeck 2011; Lillie 1998; and Mattox 1996), and devotional items located in other parts of the dwelling (Campbell et al. 2013; Musacchio 2008; and Anderson 2007). Particularly, the role of the house as a site for devotional practices of the family has been argued through an extensive examination of coeval treatises, religious literature, depictions of interior environments, and domestic objects listed in inventories. Indeed, a high number of religious paintings, crucifixes, and reliquaries emerge from inventories of Italian early-modern families, listed in different rooms of the house. The large quantity of items with possible devotional association led some scholars to presume that houses were entirely imbued with a sort of Christian spirituality, in continuity-albeit with the proper differences - with religious institutional environments outside the domestic, such as churches, monasteries, and convents. This perspective has been relevantly fuelled by the writings of Margaret Ann Morse (Morse 2018, 2013a, 2013b, 2007). As a result, the domestic has been finally freed from its long-relegation in the secular sphere. In this sense, the interdisciplinary project Domestic Devotions: The Place of Piety in the Italian Renaissance Home (2013-2017), conducted by the University of Cambridge, undertook significant research on domestic devotion by producing a 3-day conference in July 2015,

1 This paper results from the thesis, which I wrote during my PhD programme at the Art History Department of the University of York, under the supervision of professor Helen Hills. The research gave priority to Italian, English, and Spanish-speaking scholarship, including translations from other languages into these three. 
an exhibition in 2017, and four volumes (Brundin et al. 2018; Corry et al. 2018; Faini and Meneghin 2018; Corry et al. 2017). However, another issue arose from this result, that is, the problematic idea that the presence of religious images could bestow, per se, a devotional use on domestic objects and on any domestic space that housed them. Conversely, apart from rosaries or prayer books, the use of which is rather clear, the devotional use of a biblical scene engraved on a knife or on a hairbrush is not equally straightforward. This indeterminacy results from the fact that inventories report more quantitative than qualitative data (Nalle 2008, p. 256).

Notwithstanding, this paper does not aim to solve the question of the devotional/non-devotional use of images, but to problematize it by moving the focus from this dual opposition to the active role of canvases-broadly defined-inside the house. Informed by Jacques Derrida's and Pierre Bourdieu's writings, this paper argues for the structural nature of the paintings inside the house and their meaningful correlation with both the arrangement of the domestic interior and the practices of people experiencing those spaces. To do this, a precise case study has been investigated, i.e., Palazzo Scordia in Palermo (Sicily), thus challenging the overwhelming attention paid by early-modern scholars to northern and central Italy, which has been decried by the results of the project Domestic Devotions (Faini and Meneghin 2018, pp. 1-2; Corry et al. 2017, p. 7). Despite the lack of extensive research on early-modern Sicily, the collections of paintings seem to have been quite frequent in the greatest aristocratic abodes in Palermo since the end of the sixteenth century (Piazza 2018, p. 118). Amongst these, the collections examined here cannot be considered outstanding or particularly influential. This paper resists both the search for exceptionality and the generalization of the results. Rather, it aims to investigate the specificities of the selected case study, as it offers the occasion to relate with relative certainty the palace articulation, two inventories of paintings, and two precise historical figures, i.e., Ercole Branciforti Campolo Russo et Spatafora (ruled 1658-1687) and his son Giuseppe Branciforti et Morra (ruled 1697-1720), respectively, the second and third princes of Scordia. More than this, the collections of the two nobles marked an important period in the ascent of their family and in its establishment in the city, thus allowing us to explore intersections between architecture and issues of devotion, gender, and rank.

\section{The Route towards the Chapel}

Palazzo Scordia results from the connection of two different buildings, set in the contrata Seralcadi, a district of Palermo (Figure 1). ${ }^{2}$ The original nucleus on the vanella del fico (the current via Trabia) in the mid-sixteenth century was a simple compound of houses, most probably set around the current courtyard (Nobile et al. 2000, pp. 29-38). The addition on via Maqueda was begun between 1600 and 1602, when this new road was created (Fagiolo and Madonna 1981, p. 45). Ercole Branciforti's post-mortem inventory, dated to 24 October 1687 and grouping his possessions in apartments and rooms, reveals that the piano nobile, the first and primary floor of the palazzo, was then divided into three apartments: the quarto della Galleria (the apartment of the gallery), the quarto grande (the big apartment) and the casa piccola (the small house). ${ }^{3}$ The quarto grande, located in the oldest nucleus of the palace, was where the prince primarily dwelt with his wife. Whilst the main anterooms remained within or close to the quarto della Galleria, the prince's bedroom was in the northern extreme of the quarto grande preceded by two anterooms and followed by the chapel. Arguably, the location for a chapel

2 In 1683, the words palazzo grande (the big palace) and palazzo picciolo (the small palace) were used to describe the family abode. ASP, Notai defunti, Not. Sardo Fontana Honofrio, vol. 2048, fols. 1378r-v. In 1702, the palace was still indicated as "duo palatia magna simul coniunta" ("two palaces joined together"). ASP, Notai defunti, Not. Sardo Fontana Honofrio, vol. 2076, fol. 1834r.

3 ASP, Notai defunti, Not. Sardo Fontana Honofrio, vol. 2049, fols. 462r-509r. In his last will, the prince of Scordia was very clear about when, how, and under the surveillance of whom to compile the list. ASP, Notai defunti, Not. Sardo Fontana Honofrio, vol. 2048, fol. 1383r. 
resulted from the position of the main dwelling and its correlated activities. ${ }^{4}$ This proximity remains true also in cases where the use and importance of the chapel went beyond domestic boundaries. For example, Guarino Guarini's Shroud Chapel (1668-1694) was connected to both Turin's Cathedral and the apartments of the dukes of Savoy (Beldon Scott 1995, p. 628).

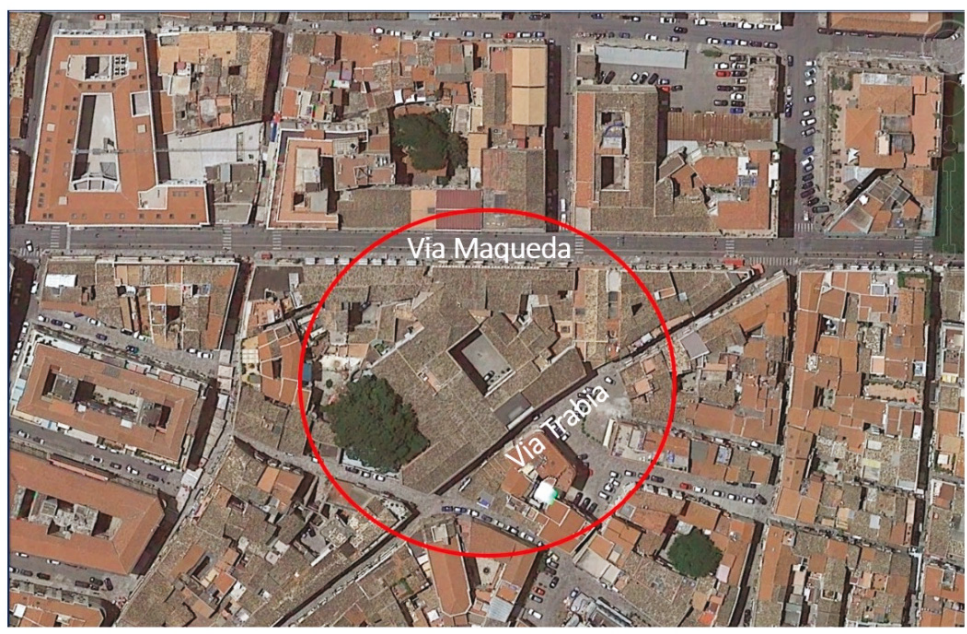

Figure 1. Palazzo Scordia. Manipulation of a Google Earth image.

In Palazzo Scordia, the main chapel was easily accessible from the bedroom of the prince. Conversely, the route from the anterooms to the chapel was through several narrow corridors (Viola 2019, pp. 286-309). The location was in line with the suggestions about building given by the Sicilian theologian and architect Giovanni Biagio Amico (Trapani, 1684-1754) in his 1750 treatise L'architetto pratico (Amico 1997, p. 63). In the second volume, dedicated to palaces and secular dwellings, Amico listed the main rooms on the piano nobile and located the chapel close to the bedroom (Amico 1997, p. 64). The statement "[o]ra gli appartamenti giusta il costume di Sicilia si dipongono cosi" ("now, the apartments according to Sicilian custom are arranged, as follows." Amico 1997, p. 66) suggests that the author benefitted from direct knowledge of most residences already built in the area between Palermo and Trapani, where he lived and worked. This arrangement is anything but new: for instance, it is close to the sequence anteroom-bedroom-private oratory-private chapel that Sabina de Cavi argues to be repeatedly proposed in Spanish royal apartments (including the viceroy's apartments in Naples), after Juan de Herrera's articulation of Philip II's Escorial (De Cavi 2008, p. 168). ${ }^{5}$ What is more striking is that the bedroom was deemed by Amico as a sort of watershed between anterooms and backrooms, constituted by a single room or divided in two rooms, the camera di parata and the real bedroom behind it. One could also add a further passage through the narrow spaces (gabinetti) flanking the bed-alcove, pushing the backrooms even further away. This complexity makes the boundary between anterooms and backrooms blurred and fuzzy. At any rate, the bedroom area was never thought to be off-limits, as family members, servants, and the closest friends continually crossed it. Amico himself reports the presence of servants who could be asked to sleep in the backrooms, or to cook there. He also mentions the existence of nannies, which implies the presence of children

4 In aristocratic palaces of early-modern Italy, this frequently excluded the ground floor, which was mostly used for services such as stables and warehouses, but could easily include upper floors, if inhabited by members of the family, in addition to the piano nobile.

5 In this case, oratory and chapel are not synonyms, the former being a non-consecrated religious space from which to watch the services celebrated in the latter. 
(Amico 1997, p. 67). To sum up, from Amico's description a difference between anterooms and backrooms emerges as realized not through a clear-cut separation but a nuanced gradation implying the crossing of numerous subsequent thresholds. This crossing enabled the palace inhabitants to distance the outside world, even though they did not isolate themselves completely from it.

In the house, the position of the paintings with a religious theme seems to follow the gradualness of this transition from the anterooms to the backrooms. The 1687 inventory, compiled after the death of Ercole Branciforti, allows us to relate the articulation of the house with the location of the paintings, thus revealing that in the main dwelling, the number of religious depictions increases when approaching the bedroom area and the chapel. At the time of Ercole's death, there was only one depiction of a biblical scene in the Sala out of 225 canvases hanging on its walls $(0.44 \%)$ and 1 out of 31 in the first anteroom $(3.22 \%)$, whilst the second anteroom accommodated 9 depictions of Christ, the Madonna, and other Saints out of 14 paintings with various subjects (64.28\%), and 3 out of 4 paintings had a religious subject in the bedroom (75\%). ${ }^{6}$ Unsurprisingly, in the chapel there were only religious images $(100 \%)$. This suggests a connection between these paintings and the location of the chapel. According to Caroline Anderson, along with the subject depicted, "another way in which an artwork's meaning was elicited was through its placement" (Anderson 2007, p. 79). Anderson's doctoral thesis focuses on the importance of the belongings and domestic spaces in forming confessional identities in Florence between 1480 and 1650. Examining a large number of inventories, she compared the paintings' locations to what was prescribed by Silvio Antoniano's Dell'Educazione Cristiana e politica de' figlioli (1609) and Giulio Mancini's Alcune considerazioni appartenenti alla pittura come di diletto di un gentiluomo (1617-'21). For Mancini, paintings "should be distributed, in an orderly manner, and in specific principal places that took into account their subject matter" (Anderson 2007, p. 80). Particularly, he prescribes the use of the bedroom area for religious depictions (Mancini 2005, p. 49). Anderson's findings confirm that Mancini's advice more or less mirrored the contemporary practice in Florence and that sacred images frequently were in camere, being the generic term camera defining both the bedroom and the rooms close to it (Anderson 2007, pp. 79-80). This offers an interesting insight into the association between religious paintings and what Mancini calls " $\mathrm{i}$ luoghi ritirati" ("withdrawn places") even though not a univocal interpretation. Mancini himself confuses the rules he has just proposed by suggesting for the bedroom, besides the religious subjects, also lascivious depictions that can foster the excitement of the couple (Mancini 2005, p. 48).

\section{Devotional or Non-Devotional?}

At this point, the question resulting from these data would be whether the increase in religious paintings along the path to the chapel was also marked by an increase in their devotional use. The fact that Mancini prescribes the bedroom and rooms close to it to be the most suitable place for "le cose di devotione" ("items for devotion") in every type of house leads us to dwell further on the meaning of the word "devotion." To do this, however, it is necessary to consider both theoretical indications and common practices. As to the former, the work of the theologian Lodovico Antonio Muratori (1672-1750), although published in a later period, is particularly informative as well as connected to the context of the case study under investigation. ${ }^{7}$ In 1747 , Muratori stressed that the moment a person is christened they enter into a pact of love with God, a sort of contract between two parties (Muratori 1747, p. 3). The mortal party "obliges and devotes himself to a regular and affectionate homage to his Creator, and to a total obedience to His will and laws" (Muratori 1747, pp. 3-4). This commitment is called "devotion" and is usually put into effect through acts of mercy and worship, which can be facilitated

6 This seems to be in line with results of other investigations. For Brundin, Howard and Laven, "the bedchamber was the room most likely to contain religious objects and images" (Brundin et al. 2018, p. 64).

7 Muratori's ideas about devotion were summed up and spread by a Sicilian treatise (Di Maria 1772). Yet, his relationship with the city started earlier, as Muratori's outspoken opposition to the city's cult of the Immaculate Conception provoked debates in Palermo since the 1714 publication of his De ingeniorum moderatione in religionis negotio (Turco 2006, p. 433). 
by objects. Muratori argues that even if love, respect, and obedience should be obvious consequences of the agreement, material objects are needed in order to excite the devotion of the uneducated as the sepulchers of Saints and their relics did, because "[b]ooks are not for them; to move them they need material objects, that should catch their eyes and ears" (Muratori 1747, p. 331). He then explains the role of images by way of examples, at the same time warning against idolatry. So far, his contribution is anything but new, as Muratori himself recalls Gregory the Great (540-604)'s definition of pictures as "books for the ignorant" (Muratori 1747, p. 331). ${ }^{8}$ However, he adds later that "even people eminent for their intelligence or holiness, praying in front of the sacred image of crucified Jesus, feel their imagination helped by that most pious object and their mind moved to holy thoughts and affection." (Muratori 1747, p. 332). In this way, Muratori includes people of various social and cultural levels in the same discourse; nevertheless, warning all of them against drifting from devotion to superstition. ${ }^{9}$ To sum up, for Muratori, devotion is articulated by an action, typically a prayer or an act of mercy, that makes the relationship between God and the devotee effective. In other words, the painting of the Madonna della Grazia, placed on the chapel altar in Giuseppe Branciforti's time, is more likely to be a devotional image than the depiction of David beheading Goliath, hanged in his father's gallery among dozens of other paintings with different topics, simply because a gallery was unlikely to be used for praying.

Despite the warnings of theorists like Muratori against superstition and idolatry, it has been argued that the images were also associated with a role independent of any voluntary act of the faithful and considered acting by themselves. According to Corry, vision was perceived as capable of affecting people's life. That is why, for instance, "the fifteenth century Lombard humanist Maffeo Vegio suggested that parents keep an appropriate religious picture in view during conception to ensure the birth of a well-formed child and advised parents not to let children see images of the devil" (Corry 2017, pp. 67-68). Muratori himself describes the role of images as active, when he admits that images, as well as processions, acts of mercy, and pilgrimages, could "move devotion" ("muovere la divozione"). This vague expression implies a broad range of uses: images can act as a focus for either group or individual worship and they might also provoke responses, thus encouraging the practice of devotional acts, e.g., as they showed edifying episodes or figures to be imitated, such as the Good Samaritan or Saint John preaching in the desert. ${ }^{10}$ These uses can imply both the conscious and the unconscious participation of the faithful. Extending Muratori's thought further, it could be asserted that images might be reminders of the ongoing pact with God, in the sense that they could remind the faithful that God protects their family in return for prayers and acts of mercy. The encouragement towards these devotional practices could also occur independently of the devotee's awareness. Yet, the reasoning can go further, as images themselves might guarantee God's protection even in the absence of a response from the faithful. Although Muratori would hardly have agreed with this, his contemporaries may have thought in this way. Caroline Walker Bynum's work on late medieval art posits that for all the emphasis theorists put on seeing beyond the images, people kept considering them a "locus of the divine" per se (Bynum 2011, p. 65). Bynum's oeuvre, focusing on the pervasive Christian concern with materiality, argues that devotional images often drew the viewer to themselves, as holy matter instead of guiding him/her beyond themselves, and this regardless of the viewer's education or social status (Bynum 2011, p. 267). Reflecting on the active role of images, David Freedberg argues for the effectiveness of religious images, which could become objects of devotion spontaneously, thus motivating different responses, that is, different actions and behaviours of the beholder (Freedberg 1989, p. 96). Following Freedberg's thought, Brundin, Howard and Laven argue

\footnotetext{
A brilliant summary of the centuries-long debate about images in the Cristian world can be found in Bynum (2011), pp. 44-52. Muratori expressed his fears mainly in De superstitione evitanda (1727). This book supports a Christocentric devotion, exclusively founded on the Bible and on tradition.

10 On the assumed supportive role of images for spiritual improvement, see Corry (2018), pp. 320-321.
} 
that the reading of religious images had an unstable subjective dimension, since the image could act on the faithful on several levels and with different results (Brundin et al. 2018, pp. 175-90).

I do not want to dispute these arguments but only observe that precisely because of the relativism resulting from this view, it is appropriate to consider the cautious warning of those-like Silvia Evangelisti-who argue that the capacity of objects to act as devotional tools closely depended on their specific use and on the meaning they had for their users, which can be rarely fully grasped from inventories (Evangelisti 2013, p. 395). This is particularly problematic in the case of paintings, since in inventories, the depicted subject mattered less than the gilded frame of a painting or its relative size (Musacchio 2008, p. 211). In other words, this perspective runs the risk of fuelling an unconstructive and infinite discussion on what was devotional and what was not. As Elizabeth Carroll Consavari realized about Venetian collections (Carroll Consavari 2013, p. 153), the second risk is to isolate devotion from other decisive factors, an approach that can lead to an underestimation of the weight that the images had in the active interaction with the beholder. Elements such as the taste, political view, and social position of those who purchased, positioned, and observed the canvases were tightly intertwined with their religious beliefs and could influence their response. Likewise, isolating a painting from others hanging in the same room, because they are not religious in theme, prevents us from perceiving their collective action on the observer.

In order to explore the relationship between the paintings and the choices of their owner, Pierre Bourdieu's concept of habitus can be useful. For Bourdieu, the habitus is "the system of structured structures predisposed to function as structuring structures" (Bourdieu 1995, p. 72). In other words, even if apparently determined by the achievement of a future aim, practices are determined by the interiorization of antecedent models (Bourdieu 1995, p. 73). Consequently, the acts of purchasing, displaying, or contemplating specific paintings, were-consciously or unconsciously-produced by the habitus of a ruling class which invested its economic and cultural capitals to perpetuate its distinction from "the other" through a luxury lifestyle (Bourdieu 1996, pp. 283-95) and the appropriation of cultural goods, like paintings (Bourdieu 1986, p. 246) ${ }^{11}$ Bourdieu's habitus does not imply a mechanical repetition of models (Bourdieu 1995, p. 95). Rather, it ceaselessly adjusts to the demands inscribed as potentialities in any situation by engendering thoughts and actions consistent with different conditions. Regarding the case study, this means that, although the general objective remained that of aristocratic distinction, the purchase of the canvases and their location in the house could vary according to conditions that were susceptible to change, such as personal preferences and family strategies.

The complexity and instability of the relationship between the paintings and those who bought/positioned/observed them emerges significantly when owners and users of the house changed. The two inventories, which were compiled after the deaths of Ercole Branciforti and of his son, Giuseppe Branciforti et Morra, in a span of little more than thirty years (1687 and 1720), allow us to analyse this relationship.

\section{Paintings as Ornament of the House (Ercole)}

Having inherited titles and fiefs from his father Antonio in 1658, Ercole Branciforti showed a particular dedication to the family residence in Palermo that his father had abandoned for a long time. This dedication was part of a plan to establish the lineage in the city after the family power had been secured by a stable feudal authority in the countryside. After the revolt of Messina against the Spanish authority (1673-1678), its repression, and the deliberate degradation of the city by the Spaniards, Palermo became the stable location of the Spanish viceroy court and, therefore, the most attractive place for Sicilian aristocracy (Ligresti 1992, pp. 84-85). Analogously to what occurred in greater European courts, the proximity to the viceroy, even if it put the aristocrats under his control,

11 It must be noticed that in the early-modern society this otherness could be generated by a difference of rank as well as by other discriminating factors, such as gender and age. 
allowed them to take advantage of his manifestations of favour and to maintain, through the complex court etiquette, the power ensuing from them (Elias 1980, pp. 97-100). Additionally, according to Ronald G. Asch, the court temporarily worked also as a bulwark against the ascent of the new urban nobility that struggled to make its way in a world of conspicuous consumption (Asch 1991, p. 4). Consequently, it is understandable that Ercole paid all the debts related to the house in Palermo and started to renovate it. ${ }^{12}$ In his 1683 will, Ercole reported that he had done "a lot of good works" ("molti benfatti") on the larger of the two buildings, even if it had not been completed by that time. ${ }^{13}$

The realization of a sort of familial iconographic programme was part of Ercole's renovation, since the Sala and the Galleria at the piano nobile were already full of paintings by $1687 .{ }^{14}$ The huge Sala, which was the first room to be seen by visitors to the piano nobile, housed 225 paintings of different size and with various subjects. ${ }^{15}$ Among the canvases, there were many iconographies related to classical mythology, some scenes from Dante's Inferno, several landscapes, portraits of famous philosophers and humanists such as Aristotle and Petrarch, and one painting depicting a scene from the Old Testament, Susanna and the Elders. Featuring subjects drawn from the recent and distant past might have been a way to bestow a cultured aura on the house. Considering the openness of anterooms and the Sala, "dov'è lecito venir ad ognuno" ("where it is licit to come to everyone") even Mancini suggests hanging there images like portraits or historical depictions (Mancini 2005, p. 49). For Anderson, the reason for this suggestion was to impress and influence visitors waiting in the Sala or negotiating business with the Master in the anterooms (Anderson 2007, p. 80).

The importance attributed by Ercole to this operation emerges from his own words when, in his last will, he left his heir the choice of selling home furnishings "with the exception, however, of the paintings, which must always remain as ornament to the house." 16 Even in the case of relatively mobile elements, like paintings, the word "ornament" cannot refer solely to the embellishment of the house. Jacques Derrida's reading of Kant's Critique of Judgement (1760) warns against the reiterated attempts of drawing a line between the work of art (ergon) and its accessories (parergon), between the internal meaning of architecture and its external circumstances (Derrida and Owens 1979, p. 26). The philosopher highlights how ornament disturbs this attempt, because it is neither simply inside nor simply outside the work. Rather the parergon "has traditionally been determined not by distinguishing itself, but by disappearing, sinking in, obliterating itself, dissolving just as it expends its greatest energy" (Derrida and Owens 1979, p. 26). Paintings articulated the family identity in every room of the palace, as the owner perceived and fashioned it. ${ }^{17}$ The "Casa" that Ercole mentioned was the family abode but also, in the broader sense, the Scordia casato, a relatively young lineage in comparison with the other powerful branches of Branciforti.

Ercole's purchase of the paintings was intrinsically connected to the structural works on the palace conducted by him, with the aim of decorously settling the family down in Palermo, which had just become the most important city in Sicily. The entire environment of the Sala, including an amazing frescoed vault, was a sort of introduction to the family and to its relatively recent claims to high nobility. A painting depicting Sicily probably made the identification of the family's fiefs

12 The effort must have been considerable, since the amount of Antonio's debts (ca. 21,000 onze) was more than the whole annual income of the Scordia family (ca. 15,000 onze) estimated at the beginning of the eighteenth century. ASP, Corte Pretoriana, n. 5874, fol. 81r. Candela (1996), p. 38.

13 ASP, Notai defunti, Not. Honofrio Sardo Fontana, vol. 2048, fols. 376r-389r.

14 ASP, Notai defunti, Not. Sardo Fontana Honofrio, vol. 2049, fols. $1479 \mathrm{v}-1484 \mathrm{v}$.

15 Ibid., fols. $1479 \mathrm{v}-1481 \mathrm{r}$.

16 "[E]sclusi però li quadri, li quali sempre debbiano restare per ornamento della Casa." Ann Matchette wrote that "testamentary mandates of barring the sale of furnishing or collections by heirs have been deployed by scholars in order to advance an argument that objects, identity, and family honour were inextricably linked." Matchette (2006), p. 705.

17 Renata Ago argues that inalienable items were supposed to be capable of prolonging the memory of the testator among the living. Yet to talk about the deceased, they had to belong to his/her social sphere to be socially acknowledgeable, like the family palace or the collections inside it. By passing from one generation to another, these goods implemented continuity and transmission of family's identity/identities. Ago (2013), pp. XVII-XIX. Amongst these kinds of items, the scholar lists also the paintings. Ibid., pp. 137-56. 
possible for any visitor. ${ }^{18}$ More than this Ercole fashioned a cultural identity supporting the title purchased by his father Antonio, claiming a noble spirit beside the riches of his properties. In this respect, the only biblical depiction present in the Sala, Susanna and the Elders, is more likely to be just another item contributing to Ercole's image rather than a strictly devotional object. Yet the inventory reveals something else going on in the anterooms.

Pictures like Susanna and the Elders in the Sala or the Carità Romana in the second anteroom could exhort female members of the family to virtuous behaviour, but also allude to the recurrent phenomenon of couples in which husbands were much older than wives. It is worth noticing that a depiction of Susanna was also in the Sala of the casa piccola so that it welcomed the visitor at both the two different entrances of the first floor. These suggestions were part of a patriarchal cultural system, so familiar to be embedded in everyday life. It is hard to determine how conscious was the acceptance of these cultural tropes. Yet, given the prominent positions of these paintings in the house, it is possible to argue that these images were deliberately displayed to an outside audience to stress the belonging of the Master to this cultural system. As a male counterpart, three depictions of King David were distributed in the rooms of the quarto della Galleria, together with five portraits of the kings of Spain hanging in the first anteroom, i.e., at the entrance of the apartment, which was most probably used for public events and had to display the family's loyalty. ${ }^{19}$ Instead, the portraits of the family's ancestors were hung beside Ercole's own portrait in the first anteroom of the quarto grande, where Ercole himself dwelt.

As already seen, in the three apartments, from the first anteroom to the backrooms the number of religious paintings slightly increases. Among these, there were many depictions of Jesus Christ and male Saints, such as Saint John and Saint Sebastian. In comparison, the depictions of the Virgin Mary are scarce, just eight, and, of these, only five in the main dwelling. Even fewer are the depicted female characters: the casa grande had only one painting with a female subject, a depiction of a "female saint martyrized by a tyrant," in the main bedroom. The violence of the theme seems increased by its proximity to a depiction of battle and the scene of Christ expelling the merchants from the temple (the only episode of the Gospel showing Christ angry), hung in the same room. Although the chapel was already located close to the bedroom and furnished with eleven religious images, it does not seem to have affected the choice of the paintings for the bedroom. Neither were located there the lascivious depictions suggested by Mancini. Rather, an atmosphere of male domination must have transpired from its pictures. Obviously, daily practices do not always correspond to theoretical indications, such as Mancini's, but these indications often reflect a widespread practice. At issue here is the effect that a divergence from this practice could provoke. If all these paintings were reflections of an individual attitude towards life and people, in the case of the main apartment, they would be related mostly to the chief of the household, Ercole, who lived there and most probably bought the paintings, since the palace was almost abandoned before his time. It is definitely not a fully reliable method to understand an individual's personality, but it gives a vague clue about the environment of the inside and about the relations between the two sexes in a space that would have framed their intimacy. Surely, the sight of the only depiction of the Virgin Mary present in the bedroom must have been a source of relief for the wife.

\section{Roots in the City's Devotion (Giuseppe)}

A change occurred in the Scordia palace under Ercole's heir. Giuseppe did not alter the Sala arrangement and left many paintings in the anterooms as his father located them, but variously changed the other rooms, moving extant paintings and purchasing new canvases. A large number

18 ASP, Notai defunti, Not. Sardo Fontana Honofrio, vol. 2049, f. 1480v.

19 Sicily was under the rule of the Spanish crown for a long period, i.e., the House of Aragon from 1282 to 1516 , the House of Habsburg from 1516 to 1700, and the House of Bourbon from 1700 to 1713. 
of paintings were set in what the inventory compiler called "camerini" (small rooms), which may be identified with the backrooms between the courtyard and the additional structure on via Maqueda. ${ }^{20}$ Excluding 16 geographic maps, 24 out of 55 paintings set there had a religious subject. Amongst these, depictions of the Virgin and female Saints, like Agatha, Ann, Catherine, Mary Magdalene, outweighed the male Saints. It is remarkable that a small depiction of Saint Rosalia, patroness of Palermo since 1624, finally appears. Lacking family payment registers, the purchase of new paintings by Giuseppe's mother, Giovanna Morra, could not be excluded, as she shared with her stepson the tutelage of the legitimate heir and the administration of family assets for ten years after Ercole's death. However, the list refers to Giuseppe's possessions at the time of his death and this means that in any case, he approved this arrangement. Collecting paintings was a widespread custom for aristocrats to enhance the palace's magnificence and the casato's glory. As Edward L. Goldberg argues in relation to Leopoldo Medici's collection, a specific selection of items could also be used to distinguish the contribution of one member of the family amongst those of his predecessors (Goldberg 1983, p. 23). According to Goldberg, Leopoldo de' Medici tried to isolate his voice from previous patrons of his family through a relevant specialization of his interests. In this case, the diversification, established by Giuseppe's contribution apparently aimed to further root the family in the cultural and religious life of the city.

In this sense, a significant parallel emerges from the comparison of the princes' personal attitudes towards religious matters as they can be inferred from Ercole's and Giuseppe's last wills. Dated to 1683 and 1715, respectively, and arguably the richest sources about these two figures, these documents are highly conditioned by the perspective of the paterfamilias and consequently focused primarily on the issues of the preservation of wealth and the perpetuation of the family (Matchette 2006, p. 705). Nevertheless, some spiritual concerns of the two princes emerge between the lines. Above all, they kept alive the connection with the main fief of Scordia through acts of mercy, namely annual donations to the Franciscan convent founded by Antonio, dowries given to two needy female orphans from Scordia on Saint Roch's day, and the custom to be buried in the church of the above-mentioned convent. It is not unusual to find within wills pious bequests to religious institutions "for which the testator wished to display a devotional preference" (Rusconi 1992, p. 310). Yet this act suggests also how much their devotion was connected to the establishment of the family's authority in the fiefs. However, a slight difference in attitude emerges between Ercole and Giuseppe. Although Giuseppe followed his father's example with charitable acts in Scordia, he also included in his will the possibility for his vassals to file a complaint, within two months of his death, for any wrongs suffered in order to receive compensation in cash. ${ }^{21}$ Leaving aside any doubts about the efficacy of this arrangement, in any case, it shows how Giuseppe apparently distanced himself from the despotic attitude towards peasants that had been typical of the heads of the family since his grandfather's rule (De Mauro 1868, pp. 186 and 191). Most probably, he needed to adapt to the new conditions of the family in the polished environment of the city. Similarly, Giuseppe showed a different attitude towards Palermo. The general recommendation of Ercole's soul to the Saints of the Heavenly Court was replaced by Giuseppe's specific invocations to precise Saints in his will. Among these, Saint Francis of Paola, Saint Rosalia, and the Immaculate Conception of Mary are noteworthy, since these cults were tightly connected to the city of Palermo more than to the town of Scordia.

\section{Conclusions}

In line with investigations on other contexts, this case study has illustrated that the path approaching the chapel from the rest of the house was characterized by the crossing of numerous consecutive thresholds marked by an increase in religious images hanging on the walls. However,

20 It cannot be discounted that the small apartment on via Maqueda was occupied by Ercole's widow, until her death in 1701.

21 ASP, Notai defunti, Not. Sardo Fontana Francesco, vol. 2190, fol. 299r. 
not only the devotional use of these paintings remains mostly obscure and unverifiable, but its investigation seems also to limit the exploration of the relationship between the depictions and people in the house.

After years in which the early-modern house has been assessed as a secular place alien to devotional practices, the risk is now to go towards the opposite extreme; that is, the univocal interpretation of the house as a "devotional place," thus neglecting the complexity and the instability of meanings that spaces, images, and objects sustained in the domestic environment. To avoid this, I have proposed to interpret the purchase and location of the paintings in the house considering Bourdieu's habitus as the source of these moves. This allows us to investigate the arrangement of religious paintings beyond their strictly devotional use, including questions of power, lineage, gender, taste, and so on. Furthermore, the comparison between the two different collections of canvases showed that the relationship between the paintings and the inhabitants of the house could change. The differences emerging from Ercole's and Giuseppe's collections illuminate two different attitudes towards family and the city: although produced by the same attempt of distinction, their actions adapted to different conditions.

Funding: This research received no external funding.

Conflicts of Interest: The author declares no conflict of interest.

\section{References}

Archival Documents from the State Archive of Palermo

ASP (Archivio di Stato di Palermo)

ASP, Notai defunti, Not. Sardo Fontana Honofrio, vols.2048, 2049, 2076,

ASP, Notai defunti, Not. Sardo Fontana Francesco, vol.2190

ASP, Corte Pretoriana, n.5874

Ago, Renata. 2013. Gusto for Things. A History of Objects in Seventeenth-Century Rome. Chicago and London: University of Chicago Press.

Amico, Giovanni Biagio. 1997. L'architetto pratico in cui con facilità si danno le regole per apprendere l'architettura civile. Libro secondo. Palermo: Città di Palermo Assessorato al Centro Storico. First published 1750.

Anderson, Caroline Corisande. 2007. The Material Culture of Domestic Religion in Early Modern Florence. Ph.D. dissertation, University of York, Heslington, UK.

Asch, Ronald G. 1991. Court and Household from the Fifteenth to the Seventeenth Centuries. In Princes, Patronage, and the Nobility. The Court at the Beginning of the Modern Age c. 1450-1650. Edited by Ronald G. Asch and Adolf M. Birke. London: German Historical Institute London, pp. 1-38.

Beldon Scott, John. 1995. Seeing the Shroud: Guarini's Reliquary Chapel in Turin and the Ostension of a Dynastic Relic. The Art Bulletin 77: 609-37. [CrossRef]

Bourdieu, Pierre. 1996. Distinction. A Social Critique of the Judgement of Taste. Translated by Richard Nice. Cambridge and Massachusetts: Harvard University Press. First published 1945.

Bourdieu, Pierre. 1995. Outline of a Theory of Practice. Translated by Richard Nice. Cambridge: Cambridge University Press. First published 1977.

Bourdieu, Pierre. 1986. The forms of capital. In Handbook of Theory and Research for the Sociology of Education. Edited by J. Richardson. Westport: Greenwood, pp. 241-58. First published 1983.

Brundin, Abigail, Deborah Howard, and Mary Laven. 2018. The Sacred Home in Renaissance Italy. Oxford: Oxford University Press.

Bynum, Caroline Walker. 2011. Christian Materiality. Cambridge and London: MIT Press.

Campbell, Erin J., Stephanie R. Miller, and Elizabeth Carroll Consavari, eds. 2013. The Early Modern Italian Domestic Interior 1400-1700: Objects, Spaces, Domesticities. Farnham: Ashgate.

Candela, Simone. 1996. I piemontesi in Sicilia 1713-1718. Caltanissetta-Roma: Sciascia.

Carroll Consavari, Elizabeth. 2013. Il Mare di Pittura: Domestic Pictures and Sociability in the Late Sixteenth-Century Venetian Interior. In The Early Modern Italian Domestic Interior 1400-1700: Objects, Spaces, Domesticities. Edited by Erin J. Campbell, Stephanie R. Miller and Elisabeth Carroll Consavari. Farnham: Ashgate, pp. 191-204. 
Corry, Maya. 2017. The Madonna, Christ, and the Saints. In Madonnas \& Miracles. Edited by Maya Corry, Deborah Howard and Mary Laven. Cambridge: Philip Wilson Publishers, pp. 47-90.

Corry, Maya. 2018. Delight in Painted Companions: Shaping the Soul from Birth in Early Modern Italy. In Domestic Devotions in Early Modern Italy. Edited by Maya Corry, Marco Faini and Alessia Meneghin. Leiden: Brill, pp. 310-41.

Corry, Maya, Deborah Howard, and Mary Laven. 2017. Madonnas E Miracles. Cambridge: Philip Wilson Publishers.

Corry, Maya, Marco Faini, and Alessia Meneghin, eds. 2018. Domestic Devotions in Early Modern Italy. Leiden: Brill. De Cavi, Sabina. 2008. Il Palazzo Reale di Napoli (1600-1607). Un Edificio 'spagnolo'? In "Napoli è tutto il Mondo." Neapolitan Art and Culture from Humanism to the Enlightenment. Edited by Livio Pestilli, Ingrid D. Rowland and Sebastian Schütze. Pisa and Rome: Fabrizio Serra Editore, pp. 147-69.

De Mauro, Mario. 1868. Notizie storiche sopra Scordia Inferiore. Catania: Stabilimento tipografico di C. Galatola nel R. Ospizio di Beneficienza.

Derrida, Jacques, and Craig Owens. 1979. The Parergon. October 9: 3-41. [CrossRef]

Di Maria, Francesco. 1772. Dilucidazione del trattato della regolata divozione de' cristiani di Lamindo Pritanio, e confutazione di alquanti errori degli eretici de' nostri tempi intorno alla stessa divozione. Opera di f. Francesco di Maria d'Avola ministro provinciale de' Cappuccini di Siracusa. Siracusa: nelle stampe di d. Francesco M. Pulejo impress. della Cam. e dell'ill.mo Senato.

Elias, Norbert. 1980. La società di corte. Translated by Giuseppina Panzieri. Bologna: Il Mulino. First published 1969.

Evangelisti, Silvia. 2013. Material Culture. In The Ashgate Research Companion to the Counter-Reformation. Edited by Alexandra Bamji, Geert H. Janssen and Mary Laven. Farnham and Burlington: Ashgate, pp. 395-416.

Fagiolo, Marcello, and Maria Luisa Madonna. 1981. Il Teatro del Sole. La rifondazione di Palermo nel Cinquecento e l'idea della città barocca. Palermo: Officina.

Faini, Marco, and Alessia Meneghin, eds. 2018. Domestic Devotions in Early Modern World, 1400-1800. Leiden: Brill.

Freedberg, David. 1989. The Power of Images. Studies in the History and Theory of Response. Chicago and London: University of Chicago Press.

Goldberg, Edward L. 1983. Patterns in Late Medici Art Patronage. Princeton: Princeton University Press.

Hirschboeck, Martin. 2011. Florentinische Palastkapellen unter den ersten Medici-Herzögen (1537-1609). Verborgene Orte frommer Selbstdarstellung und konfessioneller Identität. Berlin: I Mandorli.

Ligresti, Domenico. 1992. Per un'interpretazione del Seicento siciliano. In L'Italia degli Austrias. Monarchia Cattolica e domini italiani nei secoli XVI e XVII. Edited by Gianvittorio Signorotto. Cheiron 17-18: 81-105.

Lillie, Amanda. 1998. The Patronage of Villa, Chapels and Oratories near Florence: A Typology of Private Religion. In With and without the Medici: Studies in Tuscan Art and Patronage 1434-1530. Edited by Eckart Merchand and Elison Wright. Aldershot: Ashgate, pp. 19-46.

Mancini, Giulio. 2005. Riflessioni sulle Regole per comprare, collocare e conservare le pitture. Edited by Cristina De Benedictis and Roberta Roani. Firenze: Edifir. First published 1621.

Matchette, Ann. 2006. To have and have not: the disposal of household furnishings in Florence. Renaissance Studies 20: 702-16. [CrossRef]

Mattox, Philip Emerson. 1996. The Domestic Chapel in Renaissance Florence, 1400-1550. Ph.D. dissertation, Yale University, New Haven, CT, USA.

Morse, Margaret Ann. 2007. Creating sacred space: The religious visual culture of the Renaissance casa. In Approaching the Italian Renaissance Interior. Edited by Marta Ajmar-Wollheim, Flora Dennis and Ann Matchette. Oxford: Blackwell, pp. 95-128.

Morse, Margaret Ann. 2013a. From Chiesa to Casa and Back: The Exchange of Public and Private in Domestic Devotional Art. In Reflection on Renaissance Venice. A Celebration of Patricia Fortini Brown. Edited by Blake De Maria and Mary E. Frank. Milan: 5 Continents, pp. 143-53.

Morse, Margaret Ann. 2013b. The Venetian Portego: Family Piety and Public Prestige. In The Early Modern Italian Domestic Interior 1400-1700: Objects, Spaces, Domesticities. Edited by Erin J. Campbell, Stephanie R. Miller and Elizabeth Carroll Consavari. Farnham: Ashgate, pp. 89-106.

Morse, Margaret Ann. 2018. Domestic Portraiture in Early Modern Venice: Devotion to Family and Faith. In Domestic Devotions in Early Modern Italy. Edited by Maya Corry, Marco Faini and Alessia Meneghin. Leiden: Brill, pp. 117-38. 
Muratori, Lodovico Antonio. 1747. Della regolata Divozione de' cristiani. Venezia: Stamperia di Giambatista Albrizzi. Musacchio, Jaqueline Marie. 2008. Art, Marriage, and Family in the Florentine Renaissance Palace. New Haven: Yale University Press.

Nalle, Sara T. 2008. Private Devotion. Personal Space. Religious Images in Domestic Context. In La imagen religiosa en la Monarquía hispánica. Usos y espacios. Edited by María Cruz de Carlos Varona, Pierre Civil, Felipe Pereda and Cécile Vincent-Cassy. Madrid: Casa de Velázquez, pp. 255-72.

Nobile, Marco R., Giovanna D'Alessandro, and Fulvia Scaduto. 2000. Costruire a Palermo. La difficile genesi del palazzo privato nell'età di Carlo V. In Lexicon. Storie e architetture in Sicilia. Palermo: Caracol, pp. 29-38.

Piazza, Stefano. 2018. Dalla quadreria alla cineseria: lo sviluppo della Galleria di palazzo nella Sicilia del Seicento e Settecento. In La "Galleria" di Palazzo in Età barocca dall'Europa al Regno di Napoli. Edited by Vincenzo Cazzato. Galatina: Mario Congedo Editore, pp. 116-25.

Rusconi, Roberto. 1992. Women Religious in Late Medieval Italy: New Sources and Directions. In Women and Religion in Medieval and Renaissance Italy. Edited by Daniel Bornstein and Roberto Rusconi. Chicago: The University of Chicago Press, pp. 305-26.

Turco, Giuseppe. 2006. L'Immacolata e gli Agostiniani siciliani. In La Sicilia e l'Immacolata. Non solo 150 anni. Edited by Diego Ciccarelli and Marisa Dora Valenza. Palermo: Biblioteca francescana, pp. 431-34.

Viola, Valeria. 2019. Excess without Display. The Chapel of Palazzo Scordia in Palermo (c. 1680-1760). In La Sovrabbondanza nel Barocco. Edited by Valeria Viola, Rino La Delfa and Cosimo Scordato. Palermo: Euno-Sikè, pp. 286-309.

(C) 2020 by the author. Licensee MDPI, Basel, Switzerland. This article is an open access article distributed under the terms and conditions of the Creative Commons Attribution (CC BY) license (http://creativecommons.org/licenses/by/4.0/). 
MDPI

St. Alban-Anlage 66

4052 Basel

Switzerland

Tel. +41616837734

Fax +41 613028918

www.mdpi.com

Religions Editorial Office

E-mail: religions@mdpi.com www.mdpi.com/journal/religions

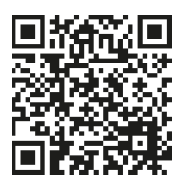



MDPI

St. Alban-Anlage 66

4052 Basel

Switzerland

Tel: +4161 6837734

Fax: +41 613028918

www.mdpi.com 